


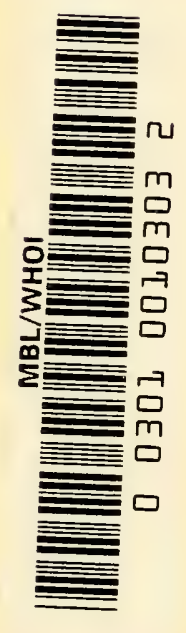





\section{LABORATORY TECHNIQUE IN BIOLOGY AND MEDICINE}





\title{
LABORATORY TECHNIQUE \\ IN \\ BIOLOGY AND MEDICINE
}

\author{
BY \\ E. V. COWDRY
}

Research Professor of Anatomy and Director Wernse

Cancer Research Laboratory, Washington

University, St. Louis

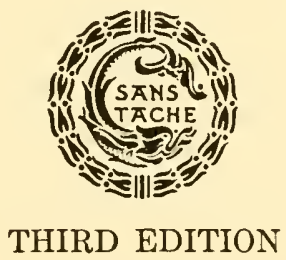

BALTIMORE

THE WILLIAMS \& WILKINS COMPANY 1952 


\title{
COPYRIght, 1952 \\ The Williams \& Wilkins Company \\ Made in the United States of America
}

\author{
Published 1943
}

Second Edition 1948

Third Edition 1952

First Edition known as

Microscopic Technique in Biology and Medicine

\section{Composed and Printed at the}

WAVERLY PRESS, INC.

FOR

The Williams \& Wilkins Company

BAltimorz, MD., U. S. A. 


\section{PREFACE TO THE THIRD EDITION}

The request by The Williams \& Wilkins Company to prepare a Third Edition so soon after the Second Edition was published (1948) was unexpected. It would have been a simple matter for them simply to reprint the Second Edition in whatever number they thought that they could sell within a reasonable period. This, however, would have been a short-sighted policy for there have been great advances in laboratory technique since the material for the Second Edition was assembled in 1947 prior to publication in 1948, which advances should be included. In fact the advances made, or reported, in the four years 1948, 1949, 1950 and 1951 are probably greater than those accomplished in any previous 10 year period. Though some of the modifications of standard techniques that proved useful during the war as well as altogether new techniques were published before 1948, many, while known to specialists in various fields, had not been gathered together in convenient form from which laboratory workers as a whole could select those most likely to be helpful in their particular problems. It is hardly necessary to note that the vast amount of new work in the four years mentioned, reflecting the appreciation of the value of research in medicine and biology gained in the war, and implemented by an unprecedented outpouring of funds for research, has resulted in the discovery of new and better means of revealing the structure of organisms from the highest to the lowest in health and disease. The electron microscope has found its way into about 300 laboratories. Satisfactory methods for cutting the extremely thin sections required have been devised. There is a rapprochement between what one can see at very high magnification and increasing knowledge of molecular structure and orientation determined by several methods. The phase microscope likewise has been produced in quantity in this country. The quality of moving pictures of living cells has thereby been greatly improved. Thanks to the Atomic Energy Commission radioactive isotopes have been made available. Plastics have been introduced in great variety. Microscopic localization of enzyme activities has been advanced. Microchemistry has leaped ahead. Quantitative analyses of extremely small amounts of material reduce the gap between chemistry and microscopy. The separation and collection en masse in a condition suitable for analysis of certain cellular components has been most helpful. And so on almost without end.

Obviously no single individual can authoritatively present these new techniques, as well as myriads of others of great value, because he cannot have personal experience with all of them. Partly to compensate for such limitations I have included descriptions of some of the key techniques written by the investigators who introduced them, or by others who have had extensive experience in their use. Most of these descriptions are new while others are revisions of accounts given in previous editions of this book. The name of each contributor 
is given followed by the address (so that questions can be asked if desired) and the date (so that it can be seen at a glance when it was last checked for improvements). To all of these friends I am very grateful. Proofs of their contributions have not been submitted to them for corrections, because there are so many such accounts-most of them very brief-and the material presented by them was clear and concise. For typographical errors the responsibility is to be shared by me and the publisher. Most of the text I have written or revised and in doing so I have relied heavily on the reference books listed on page xxxi.

It is hardly necessary to explain that the policy is to provide brief accounts of techniques, and leads to others, and to make this information quickly available by alphabetical arrangement. It is better to give some data not required by experts than it is to write mainly for well-informed but limited groups. Obviously the said experts must approach fields other than their own as beginners handicapped by unfamiliarity with specialized technique and equipment. Because the several biological and medical sciences have so much to offer each other in the way of laboratory technique this exposure of opportunities may facilitate inter-specialty cooperation. My thanks are due to Mrs. Theresa Bresnahan for help in preparing the manuscript.

E. V. COWDRY

St. Louis 


\section{COOPERATION}

My friends have generously contributed techniques written by them as follows:

G. Adolph Ackerman, Hinsman Hall, Ohio State University, Columbia 10, Ohio, May 24, 1951.

\section{Auer Bodies.}

Paul M. Aggeler, University of California Medical Center, San Francisco 22, California, November 15, 1951.

\section{Blood Platelets.}

Glenn H. Algire, U. S. Public Health Service, Bethesda Maryland, June 15, 1950.

\section{Transparent Chamber Techniques.}

James W. Bartholomew, Department of Bacteriology, University of Southern California, Los Angeles 7, California, July 9, 1951.

\section{Gram Stains Mechanism.}

H. W. Beams, Department of Zoology, University of Iowa, Iowa City, Iowa, September 271951.

Ultracentrifuges.

R. Bogoroch, Department of Anatomy, McGill University, Montreal, Canada, September 12, 1951.

\section{Radioautographic Technique (With C. P. Leblond).}

L. R. Boling, Department of Anatomy, Washington University School of Dentistry, St. Louis 10, Missouri, December 16, 1950.

\section{Teeth Blood Vessels.}

Teeth Decalcification.

Geoffrey Bourne, Department of Histology, London Hospital Medical School, London, England, November 5, 1951.

Golgi Apparatus.

Mitochondria.

Marian Pfingsten Bryan (Mrs. William T. K. Bryan), Department of Otolaryngology, Washington University School of Medicine, St. Louis 10, Missouri, September 20, 1951.

\section{Ear Cell Smears. \\ Nasal Cell Smears.}

E. J. CARey, Department of Anatomy, Marquette University School of Medicine, Milwaukee, Wisconsin, 1942 (now deceased).

\section{Carey's Method for Motor End Plates.}

Christopher Carruthers, Division of Cancer Research, Washington University, St. Louis 10, Missouri, May 12, 1950.

Oxidation-Reduction Potential.

Vitamins.

Polarographic Technique. 
Jane E. Cason, Department of Pathology, Medical College of Alabama, Birmingham, Alabama, January 27, 1951.

\section{Mallory-Heidenhain Rapid One-step Stain for Connective Tissue.}

Robert Chambers, Department of Biology, Washington Square College of New York University, New York, New York, May 15, 1950.

\section{Micromanipulation.}

E. R. Clark, Department of Anatomy, University of Pennsylvania, and the Wistar Institute of Anatomy and Biology, Philadelphia, Pennsylvania, November $28,1951$.

\section{Transparent Chamber Technique.}

Barry Commoner, The Henry Shaw School of Botany, Washington University, St. Louis 5, Missouri, November 27, 1951.

\section{Microspectrophotometry.}

A. H. Coons, Department of Bacteriology and Immunology, Harvard Medical School, Boston, Massachusetts, August 31, 1951.

\section{Antigen Localization.}

W.P. Covelu, Departments of Anatomy and Otolaryngology, Washington University School of Medicine, St. Louis 10, Missouri, October 26, 1951.

Ear.

E. W. Dempsey, Department of Anatomy, Washington University School of Medicine, St. Louis 10, Missouri, February 26, 1951.

\section{Cholinesterase.}

\section{Dehydrogenase.}

Enzymes.

Esterases.

Nucleases.

Phosphatases.

W. T. Dempster, Department of Anatomy, University of Michigan, Ann Arbor, Michigan and R. C. Williams, Department of Bicchemistry, University of California, Berkeley, California, June 9, 1950.

Shadow Casting.

O. H. Duggins, Department of Anatomy, Washington University School of Medicine, St. Louis 10, Missouri, May 18, 1950.

Hairs (With Mildred Trotter).

F. Duran-Reynals, Department of Microbiology, Yale University School of Medicine, New Haven, Connecticut, October 8, 1951.

\section{Spreading Factors.}

W. R. Earle, National Cancer Institute, Bethesda, Maryland, July 10, 1951.

\section{Tissue Culture.}

LÁrus Einarson, Normal-Anatomisk Institut, Aarhus Universitet, Aarhus, Denmark, February 27, 1951.

Gallocyanin-Chromalum Staining of Basophilic Cell Structures. 
Joseph A. Falzone, Department of Anatomy, Washington University School of Medicine, St. Louis 10, Missouri, October 26, 1951.

Desoxyribose Nucleic Acid, Method for Determination In Isolated Hepatic Nuclei.

Differential Centrifugation of Cell Particulates.

Honor B. FELl, Strangeways Research Laboratory, Cambridge, England, June 8,1951 .

Organ Culture in Vitro.

F. H. J. Figge, Department of Anatomy, University of Maryland Medical School, Baltimore, Maryland, October 10, 1951.

\section{Porphyrins.}

Hematoporphyrin.

H. I. Firminger, Pathology Section, National Cancer Institute, Bethesda, Maryland, February 9, 1951.

Carbowax Embedding.

E. D. Gardner, Department of Anatomy, Wayne University School of Medicine, Detroit, Michigan, June 15, 1950.

Articular Nerve Terminals.

David Guick, Department of Physiological Chemistry, University of Minnesota Medical School, Minneapolis 14, Minnesota, October 17, 1951.

Linderstrøm-Lang, Kaj. U., and Holter Heinz, Histochemical Advances.

Morris Goldman, Department of Parasitology, School of Hygiene and Public Health, Johns Hopkins University, Baltimore, Maryland, January 29, 1951.

Iron Hematoxylin Single Stain.

G. Gomori, Department of Medicine, University of Chicago, Chicago, Illinois, May 7, 1950.

Gomori's Method for Reticulum and Acid Phosphatase.

A. R. Gopal-Ayengar, Barnard Free Skin and Cancer Hospital, St. Louis, Missouri, September 10, 1946 (now Tata Memorial Hospital, Bombay).

Chromosomes.

Hyaluronic Acid.

H. S. N. Greene, Laboratory of Pathology, Yale University School of Medicine, New Haven, Connecticut, September 11, 1951.

Anterior Chamber Transplantation.

Joan Haberman, Parkland, Washington, March 10, 1951.

Anethole Clearing Agent.

J. D. Hamilton, Department of Medical Research, University of Western Ontario, London, Canada, February 13, 1951.

Cell Measurement, The Elliptometer.

N. L. Hoerr, Department of Anatomy, School of Medicine, Western Reserve University, Cleveland 6, Ohio, November 28, 1951.

Frozen-Dehydration Method.

Isolation of Mitochondria. 
R. D. Нотснкiss, The Rockefeller Institute for Medical Research, New York 21, New York, November 14, 1951.

Polysaccharides.

M. H. KNISELy, Department of Anatomy, University of South Carolina, Charleston, South Carolina, June 27, 1950.

Quartz Rod Technique for Illuminating Living Organs.

N. B. Kurnick, Department of Medicine, Tulane University, New Orleans 12, Louisiana, January 31, 1951.

Aceto-Orcein-fast Green.

Edward L. Kuff, Department of Anatomy, Washington University School of Medicine, St. Louis, Missouri, December 18, 1951.

Nucleic Acid-Dye Interactions.

A. I. Lansing, Department of Anatomy, Washington University School of Medicine, St. Louis 10, Missouri, October 5, 1951.

\section{Collagenic Fibers.}

Elastic Fibers.

A. Lazarow, Department of Anatomy, Western Reserve University School of Medicine, Cleveland, Ohio, November 28, 1951.

Separation of Cell Components by Differential Centrifugation.

C. P. Leblond and R. Bogoroch, Department of Anatomy, McGill University, Montreal, Canada, September 12, 1951.

Radioautographic Technique.

R. D. Lrllie, Division of Pathology, National Institute of Health, Bethesda, Maryland, May 8, 1950.

Azure or Toluidin Blue Eosin.

A. M. Lucas, U. S. Regional Poultry Research Laboratory, East Lansing, Michigan, August 10, 1951.

Cilia (with M. S. Lucas).

M. S. Lucas, Department of Biological Science, Michigan State College, East Lansing, Michigan, August 10, 1951.

Cilia (with A. M. Lucas).

Plastics.

B. J. Luyet, Department of Biology, St. Louis University, St. Louis, Missouri, January 15, 1951.

Revival after Ultra Rapid Cooling.

C. C. Macklin, Department of Anatomy, University of Western Ontario, London, Ontario, Canada, November 28, 1951.

Alveolar Epithelium.

Alveolar Fluid.

Alveolar Foam Cells.

Alveolar Pores.

Ammoniacal Silver.

Aquax.

Bronchiolar Epithelium.

Gash Irrigation Recovery Method for Lung Cells $\left(\mathrm{G}^{1}\right)$.

Heart Failure Cells.

Lungs, Uncollapsed, Fixation.

Pneumonocytes.

Silver Lineation on Pulmonary Alveolar Walls. 
Carmine Dusting. Dust Cells.
Tissue Phagocytes.

Vacuoloids.

Wash-out Recovery Method.

Paul Masson, Department of Pathology, University of Montreal, Montreal, Canada, October 24, 1951.

Masson's Trichrome Stain.

Morris Moone, Barnard Free Skin and Cancer Hospital, St. Louis, Missouri, December 10, 1951.

Fungi.

Norman Moskowitz, Department of Zoology, University of Pennsylvania, Philadelphia, Pennsylvania, January 24, 1951.

Protein Silver for Staining Protozoa.

J. L. O'Leary, Department of Neuropsychiatry, Washington University School of Medicine, St. Louis 10, Missouri, May 8, 1950.

Golgi-Cox Method.

Golgi Method, Quick.

O'Leary's Brazilin Method.

Jean Oliver, Department of Pathology, State University of New York, Brooklyn 2, New York, September 4, 1951.

Kidney.

Donald L. Opdyke, Department of Anatomy, Washington University School Medicine, St. Louis 10, Missouri, November 8, 1951.

Keratohyalin Granules, Separation and Analysis.

Robertson Pratt, University of California, College of Pharmacy, San Francisco, January 29, 1951.

Triphenyltetrazolium Chloride.

Eugene Roberts, Division of Cancer Research, Washington University, St. Louis 10, Missouri, July 15, 1951.

Paper Chromatography.

T. B. Rosenthal, Department of Anatomy, Washington University School of Medicine, St. Louis 10, Missouri, June 6, 1951.

Fluorescence Microscopy.

Radioactive Isotopes.

Electron Microscopy.

C. H. Sawyer, Duke Hospital, Durham, North Carolina, December 16, 1950. Safranin-Light Green.

Francis O. Schmitt, Department of Biology, Massachusetts Institute of Technology, Cambridge, Massachusetts, May 19, 1950.

Polarization Optical Method.

Gordon H. Scotт, Department of Anatomy, Wayne University School of Medicine, Detroit, Michigan, January 16, 1951.

Altmann-Gersh Frozen Dehydration Method.

Microincineration.

Cryostat. 
W. M. Shanklin, American University of Beirut, Beirut, Lebanon, March 30, 1951.

Pineal.

Silver Diaminohydroxide after Sensitizing with Sodium Sulfite for Neuroglia.

R. E. Stoweld, Department of Oncology, University of Kansas Medical Center, Kansas City 2, Kansas, January 19, 1951.

Photoelectric Microphotometer.

Charlotte M. Street, Department of Anatomy, Cornell University Medical College, New York, New York, May 21, 1951.

\section{Papanicolaou Techniques in Exfoliative Cytology.}

Lloyd E. Thomas, Department of Biochemistry, University of Missouri School of Medicine, Columbia, Missouri, July 8, 1951.

\section{Arginine Reaction.}

Mildred Trotter and O. H. Duggins, Department of Anatomy, Washington University School of Medicine, St. Louis 10, Missouri, May 8, 1951.

\section{Hairs.}

L. F. Wicks, Veterans Administration Hospital, Jefferson Barracks, Missouri, February 1, 1951.

\section{Hydrogen Ion Indicators.}

Piccolyte Resins.

G. B. Wistocki, Department o Anatomy Harvard Medical School, Boston 15, Massachusetts, March 8, 1951.

\section{Placenta.}

R. C. Williams, Department of Biochemistry, University of California, Berkeley, California, June 9, 1950.

Shadow-Casting (with W. T. Dempster) 


\section{PREFACE TO THE FIRST EDITION}

What appeared altogether impossible twenty-five years ago has in several cases been attained by improvements in technique. Who would have believed at that time that ultramicroscopes would now be manufactured in quantity, built without any optical lenses, and capable of revealing a world of structures quite beyond their ken? Who would have thought that a whole series of different atoms could be tagged and their distribution to the several tissues, when introduced into the body, accurately measured? Who would have anticipated the significant and unexpected new developments which have been made in polarization optical methods? Had we been told twenty-five years ago that the cell itself can be broken up into parts several of which can be collected in quantity and chemically analyzed, we would have been incredulous. All this and more has been achieved as a result of team work between the biological and physical sciences. And we may believe that more surprises are in store.

Yet some of us individually are still extraordinarily conservative in the methods we use. The possibilities of improving old techniques, of replacing some of them by new ones and of relying more upon microchemical and physical procedures are not explored as they should be. The purpose of this book is to extend the horizon by exposing in an introductory way a few of the many opportunities awaiting workers in biology and medicine interested in the minute structure of living things. Success will depend upon ability to anticipate and meet the needs of those likely to consult it. Definite information about specific matters is likely to be more in demand than general statements. The latter are limited to a few pages and deal with "choice of methods" and "organization of laboratory."

Some may turn to the names of the structures in which they happen to be most interested at the moment-Nissl Bodies, Nerve Fibers, Capillaries and so forth-on the off chance of finding some useful hints as to methods better adapted for their microscopic study, the most likely experimental errors and so on. Because the range of cells, parts of cells, tissues, organs and systems is obviously so immense, mention is only possible of a small proportion of them so that much depends on the selection made.

Others may seek information under the headings of elements such as Iron, Potassium and Calcium, of enzymes like Pepsin and Phosphatase and of many other components of living material. It is difficult to draw the line but most of those that can be localized microscopically are mentioned, likewise techniques for the determination of permeability, viscosity, $\mathrm{pH}$ and other properties of tissues.

It often happens, however, that data are required about a particular technique, which the workers are using or expect to use, and which is known to them by the names of those who discovered it, as for example the methods of Giemsa and 
Mallory. Consequently information also must be supplied under various names though this is usually less satisfactory than under subjects. A very annoying handicap is the host of synonyms for dyes. Being ignorant of chemistry, I have with confidence listed those given by Dr. H. J. Conn. Many more will be found in The Colour Index of the Society of Dyers and Colourists.

Since all are busy people, time is a factor and they will wish to dig out what they want as directly and quickly as possible. It is for this reason that everything is listed alphabetically. Obviously this book can be nothing more than a brief entré to microscopic technique. Therefore, numerous references to the literature are supplied for follow up. Again to save time, these are given each in its appropriate place, thus avoiding the necessity of turning the pages and locating them in a large bibliography. But no attempt is made to trace the techniques to their original exponents and to apportion credit for numerous modifications. Often the most recent and accessible reference is provided relying on the author to state history fairly. Evidently, in order to keep up to date as to methods, the reader must repeatedly consult the latest issues of many journals. Stain Techn.; J. Lab. \& Clin. Med.; J. Tech. Meth.; Bull. d'Hist. Appl.; and Zeit. f. mikr. Tech. are particularly valuable.

Finally I wish to thank my colleagues for their help, particularly Drs. L. R. Boling, C. Carruthers, William Cramer, Morris Moore, J. L. O'Leary, W. L. Simpson, R. E. Stowell, Lester Wicks and Dr. H. J. Conn, Chairman of the Biological Stain Commission, who very kindly read the manuscript and made several useful suggestions. 


\section{CHOICE OF METHODS}

The selections will depend on several considerations. The first is what one wants to discover. Many investigators in biology and medicine wish to obtain more information about structural components of the body whether gross, microscopic or submicroscopic in size. They desire to obtain further data on the physical and chemical properties of these components whether solid or fluid in different physiological stages of activity and in disease in both man and in animals. They are interested in subjects, not personalities, so that in this alphabetically arranged presentation the names of individuals are seldom listed. Emphasis is given to subjects. After all the problems continue while the names of generation after generation of individuals fade out.

Those who perchance may consult this book will need help at two levels. It may be a simple question of the nature of some dye, or the composition of some solution, or the making of a well known test, or it may be the selection of a technique to be employed in a comprehensive series of experiments. In the latter case it involves a major decision. It is necessary not only to choose the technique most likely to lead to the answer but also give some thought to the equipment required and the training demanded for its proper use.

A few leading references to the most recent utilization of the technique in question may be helpful. But to discuss the history of its development and to assess priority is not attempted. A complete discussion of the literature may indeed constitute a handicap by providing an excuse for doing nothing. To be stuffed with information may give a feeling of frustration.

Some chemical and physical procedures are well within the reach of people who are neither chemists nor physicists while others will simply lead them beyond their depth into futility. It is equally true that well trained chemists and physicists are likely to fail to realize the complexity of vital processes and to make little progress through ignorance of physiology and pathology. Consequently one should never hesitate to seek advice from friends in other departments.

\section{To Examine Directly in Vivo}

The ideal arrangement is to look into the body and to study its parts as they function without causing any disturbance. With protozoa and certain small transparent invertebrates this is relatively simple. The web of a frog's foot is thin and can easily be looked through without seriously interfering with the frog. Some other parts of the bodies of various aquatic lower forms lend themselves to direct examination in vivo; but there are definite limitations in such a study of what is going on in the human body. It is possible to peer into the various apertures but to get close enough to the living tissues to use high magnifications is not feasible. The cornea and lens of the eye are transparent and 
much valuable information can be secured by direct examination of the retinal blood vessels. Even here their distance from the surface is considerable and magnification is therefore limited. As far as we know at present the best that can be done is to take advantage of a discovery, made by Lombard (W. P., Am. J. Physiol., 1911-12, 29, 335-362) that the epidermis can be rendered transparent by the addition of a little highly refractile oil without noticeably injuring it or disturbing the underlying tissues. By this means the blood vessels of the dermal papillae in the fold of skin over the nail bed, which are very near to the surface, can be studied directly at fairly high magnification and over long periods of time thus permitting the making of excellent pictures. See review of literature by Wright, I. S. and Duryee, A. W., Arch. Int. Med., 1933, 52, 545-575.

That the lymphatics in the human skin can be made visible in vivo by the injection of small amounts of Patent Blue $\mathrm{V}$ has been demonstrated by Hudack, S. S. and McMaster, P. D., J. Exp. Med., 1933, 57, 751-774. The vessels in the ears of living mice can readily be seen without any surgical procedure. It is even possible to directly watch the dye, Chicago blue, after intravenous injection elsewhere in the body, leak out into the tissues especially through the walls of the venules (Smith, F. and Rous, P., J. Exp. Med., 1931, 54, 499-514). Ideas as to the relative hydrogen ion concentrations of some of the tissues visible from without can be secured by the injection of Hydrogen Ion Indicators (Rous, P., J. Exp. Med., 1925, 41, 739-759). The opportunities are many especially in animal experimentation.

Another way to examine structure in vivo is to record the structure by x-ray photographs and to magnify the photographs, see Microradiographic examination.

\section{To Examine through Windows in Vivo}

The construction of windows in the skin or body wall through which the tissues can be examined in vivo is a less ideal technique because it involves surgical interference with the body. In the most used of these techniques a hole is made through a rabbit's ear from one surface to the other. A glass chamber is then sewed into the hole in such a way that a blood vessel is included between a thin layer of glass (serving as a cover glass) and a thicker one serving as a slide. After a time the epidermis adheres to the edges of the chamber and blood vessels, nerves and other tissues grow into it where they can be studied under oil immersion objectives. This technique was first reported by Sandison (J. C., Anat. Rec., 1924, 28, 281) working under Dr. E. R. Clark at the University of Pennsylvania. It has since been very greatly improved (Clark, E. R., et al., Anat. Rec., 1930, 47, 187-211 and Abell, R. G., and Clark, E. R., Anat. Rec., 1932, $53,121-140)$ by the introduction of "round table" and "moat" chambers.

To place a window in the wall of the skull and to observe what is going on within has been done with more or less success on several occasions. The technique devised by Forbes (H. S., Arch. Neurol. and Psych., 1928, 19, 75) permits direct observation at low magnification of the blood vessels over the cerebral 
convolutions with so little injury that their behaviour in various experimental conditions can be investigated (see also Clark, E. R., and Wentsler, N. E., Proc. Assoc. Res. Nerv. and Ment. Dis., 1937, 18, 218-228). Through a window in the thoracic wall Wearn and his associates (Wearn, J. T. et al., Am. J. Physiol., 1934, 109, 236-256) have similarly studied the pulmonary arterioles and capillaries. They employed a fused quartz cone to conduct light to the tissue.

Other investigators have availed themselves of the natural window, the cornea, through which what goes on immediately within it in the anterior chamber of the eye can be observed. Several tissues have been successfully transplanted into this chamber. Perhaps the most dramatic is the behavior of transplanted uterine mucosa in the rhesus monkey. In it the menstrual changes can be seen in detail and the influence of hormones noted (Markee, J. E., Contrib. to Embryol., Carnegie Inst. of Washington, 1940, 28, 219-308). For some kinds of work the fact that the tissue fluid (aqueous humor) in this chamber differs from others in the same animal by the absence of certain species specific growth inhibiting factors is a priceless asset. Thus Greene (H. S. N., Science, $1938,88,357-358$ ) was able to grow pieces of human cancers, which ordinarily quickly die in other species, in the anterior chambers of the eyes of some mammals. The existence of a barrier protecting this fluid against the entry of antibodies from blood plasma and thus making possible the growth of tumor transplants, while all other tissues are resistant to their growth, has recently been emphasized (Saphir, O., Appel, M. and Strauss, H. A., Cancer Res., 1941, 1, 545-547).

In order to view the less accessible living tissues, techniques have been devised that include opening the body and partly withdrawing the organ so that it can be placed on the stage of a microscope but with circulation and nerve supply intact and adequate regulation of temperature and humidity. Particularly fruitful has been the direct observation through oil immersion objectives of secretion by acinous cells of the Pancreas by Covell (W. P., Anat. Rec., 1928, 40, 213-223) and of islet cells by O'Leary, (J. L., Anat. Rec., 1930, 45, 27-58). Thus the influence of drugs on the secretory process can now be followed in minute detail.

Knisely (M. H., Anat. Rec., 1936, 64, 499-523; 65, 23-50) has perfected a technique for the study of the living Spleen at somewhat lower magnification. The essential features are slight displacement of the spleen so that it can be transilluminated by light delivered through a quartz rod. This allows for the first time direct examination of the behavior of the venous sinuses. Undoubtedly the Quartz Rod technique will be of great service in providing light for similar examination of other organs.

\section{To Study the Arrangement of Parts in the Body}

Since the body is structurally so very complex it is often illuminating to view parts of it in their normal shape and size but unobscured by all the neighboring components. There are several ways by which this can be accomplished. 
The first method of Reconstruction from serial sections is well known. Briefly stated the particular tissue, organ or system is outlined, as it appears in section after section, at the desired magnification on sheets of material of uniform and carefully selected thickness. The outlined areas are then cut out and when superimposed they constitute a reconstruction of the original structure. This technique is tedious but it may reveal topographical relations that can be discovered by no other means.

The second kind of technique is to make casts of vascular, respiratory and other lumina. Woods' metal, formerly used for this purpose, has now been almost displaced by Celloidin and other substances. The surrounding tissue is freed from the cast by digestion in concentrated hydrochloric acid and gentle brushing away in a stream of water. Very beautiful Corrosion preparations of the lungs and kidneys have been obtained by this method which should be more widely employed.

The third is by Maceration to soak the organs, without previous preparation, in fluids that either digest away the tissues which it is desired to eliminate or loosen their connections with those under investigation, which, latter, can then be individually examined. Techniques of this sort are the only available means for the isolation of individual seminiferous and renal tubules. Oliver's researches on the kidney illustrate the value of reconstruction and maceration in pathology. Only three other examples will be submitted. Thyroid follicles can be isolated by maceration (Jackson, J. L., Anat. Rec., 1931, 48, 219-239). Their study as individuals provides data as to size and shape only obtainable otherwise by the tedious examination of serial sections. The Epidermis is so tightly bound to the underlying dermis that separation is extremely difficult; but, after treatment of skin with dilute acetic acid, the attachment is loosened and the epidermis can readily be removed as a complete sheet of tissue which can be stained, made transparent and examined as a whole mount. Opportunities are thus afforded for the detection of regional differences which might not be located even by painstaking study of sections and the making of mitotic counts is greatly facilitated. By macerating in the same fashion the nasal mucous membrane covering the septum can also be removed for study. Perhaps still other epithelial sheets can be similarly isolated. However such sheets are of little value for chemical analysis because of the action of the acetic acid. Fortunately it has been found that the epidermis may also be quickly loosened by simply heating the skin to $50^{\circ} \mathrm{C}$. when it can be peeled off like the covering of a scalded tomato (Baumberger, J. P., Suntzeff, V. and Cowdry, E. V., J. Nat. Cancer Inst., 1942, 2, 413-423).

There is still another alternative. Instead of simply omitting the unwanted material by reconstructing only the structures chosen for demonstration, or of removing the material by corrosion or maceration, it can be left in and rendered transparent so that it does not obstruct the view. After marking the particular structures by vital dyes or other means the whole tissue is cleared by the method of Spalteholz or Schultze. These techniques give admirable results in the study 
of Cartilaginous Skeletons, Ossification centers, Blood Vessels and so on almost without end.

\section{To Emplox the More Routine Method of Fixation and Staining}

Here there is wide latitude of choice. For some purposes thin Snears are just fixed and stained without resort to sectioning. In the case of the denser tissues which must be cut in sections one first has to decide which of many Fixatives is likely to give the best results. Then, whether fixation is to be by immersion or injection has to be determined.

The purpose of fixation by vascular injection is to bring the fixative into close contact with the tissues as they exist in the freshly killed animal without subjecting them to mechanical trauma or disturbing their topographic relations one to another. In choosing this procedure it is well to remember: (1) That it is usually necessary first to wash out most of the blood by perfusion with physiological salt solution for otherwise the fixative often clogs the vessels. This washing unfortunately also facilitates chemical change.

(2) That, even when it is not done, the concentration of the fixative about the cells is gradually increased and at different rates, rapidly in highly vascularized tissues (kidney, liver, etc.) and very slowly in avascular ones (epidermis, cornea and cartilage). The time for chemical change before fixation is therefore variable depending upon the tissue. (3) That the pressure may bring about an unnatural swelling of the tissues so located that they can enlarge, especially the abdominal organs as compared with brain and bone marrow which are confined within rigid walls.

Fixation by immersion is the usual and easiest method. If small pieces or thin slices are used the preservation is quicker and more uniform than by vascular injection. The cells are suddenly killed while active. The factor of slow death at uneven rates, present in supravital examinations, does not have to be reckoned with; but many precautions are required. Under Fixation is given a general account of the procedure. Under the several organs, Lungs, Small Intestine, Skin, etc., some special suggestions are provided. There are many fixatives to choose from. For routine purposes Zenker's Fluid as originally described or in one of its numerous modifications is suggested. Bouin's is also a very popular fixative especially among dermatologists. Formalin is an excellent one. It is good practice to set aside some tissue in formalin for examination as may be needed later. Both formalin and alcohol are the most useful fixatives preliminary to microchemical determinations. When preparations must be made very quickly, Alcohol Formalin and Carnoy's Fluid are suggested (see also Frozen Sections). For microincineration, formalin-alcohol is ordinarily employed; but the Altmann-Gersh method of freezing and drying, by which contact with fixatives is altogether dispensed with, is much less open to criticism. Osmic acid containing fixatives penetrate poorly and are therefore only useful for very small pieces of tissue. Regaud's fluid with subsequent mordanting in bichromate is the best for mitochondria. Heat fixation is useful for blood cells. Fixation in various vapors is called for in special cases. Sec Fixatives. 
After fixation some Washing of the tissue in water is necessary unless it has been fixed in alcohol, Carnoy or similar mixtures. The next step is Dehydration and a choice must be made between slow and rapid methods. Sometimes a substitute for alcohol is indicated. If Imbedding is to be in celloidin Clearing in a xylol-like fluid is omitted and heating is unnecessary. There are many ways of clearing preliminary to paraffin imbedding. In Sectioning the thickness depends upon the purpose in view. Thick sections may be as necessary as thin ones and serial sections are often required. In the Mounting of sections on slides the use of water must occasionally be avoided. Numerous techniques are applicable to the sections and are given individually later either under the heading of the substance or structure to be demonstrated or under the name of the technique or its introducer. For choice see Staining.

Many beautifully stained sections of well fixed tissue are of but little value, because the investigator failed to note the exact location in the organ or tissue whence they were excised and omitted to have the sections cut in the most favorable plane.

\section{To Mark Selected Individual Cells or Tissues in Vivo FOR LATER EXAMINATTON}

In this connection we at once think of the vital stains, trypan blue, carmine, India ink (carbon) and hundreds of others, which, when injected into the body, are phagocytosed by the reticulo-endothelial cells (or macrophages). Pieces of tissue can then be excised and the accumulations of stains can be studied within the still living cells, that is supravitally, for unless cultured the cells are slowly dying. But, if desired, the tissues can be fixed and the results observed at leisure in sections.

It has long been known that bone laid down in the presence of Madder fed to the animals is marked by the madder and can thus be distinguished from bone deposited beforehand and afterwards. In the same way dentine can be marked in vivo with Alizarin Red S.

Another example of in vivo marking is the deposition of Prussian Blue. Thus a slightly hypertonic solution (potassium ferrocyanide $0.5 \mathrm{gm}$., iron ammonium citrate, $0.5 \mathrm{gm}$. and aq. dest. $50 \mathrm{cc}$.) injected into the subarachnoid space of the spinal cord is useful in the localization of the pathways of drainage of cerebrospinal fluid, because of the marking secured when the tissues are fixed in $40 \%$ formalin plus $1 \%$ concentrated hydrochloric acid by the deposition of Prussian blue (Weed, L. H., J. Med. Res., 1914, 26, 21-117).

The tissues of animals recently killed or under anesthesia can be selectively marked with various dyes by Perfusion of the blood vessels with dilute solutions of dyes. The outstanding methods in this group have been devised by Bensley (R. R., Am. J. Anat., 1911, 12, 297-388) for histological analysis of the epithelial components of the pancreas and stomach. Dilute solutions of the dyes in physiological saline are injected into the thoracic aorta of an animal killed by bleeding. Pieces of pancreas and gastric mucous membrane are then removed and examined 
fresh. Neutral red picks out the Islets of Langerhans of the panereas, pyronin the duct system of the pancreas, naphthol blue the parietal cells of the Stomach and so on. In the same way Nerve Fibers can be marked for subsequent study by vascular perfusion with methylene blue and degenerating nerve fibers in poliomyelitis (and presumably in other conditions) ean be sharply differentiated from uninjured ones by the fact that they take up neutral red (Covell, W. P. and O'Leary, J. L., Arch. Neurol. \& Psych., 1932, 27, 518-524). It has long been known that the best way to mark renal glomeruli is to perfuse in the same fashion with a dilute solution of janus blue. The glomeruli stand out clearly in the fresh kidney by their deep blue color in a red background (Cowdry, E. V., Contrib. to Embryol., Carnegie Institution of Washington, 1918, No. 25, 39160). A similar selective staining in less brilliant colors is obtainable with janus green. Relatively permanent preparations can be made of some of these specimens.

The same dyes, and many others, can also be applied in dilute solutions to cells freshly removed from the body and which are still living. Such methods have become very popular in hematology. However, the cells thus colored live only for a limited time and it is important to cut short the observations before they are vitiated by approaching death.

It is feasible to employ a wide variety of Tracer Techniques, that is substances can be traced through the body by the markings given to them. The largest group is made up of Radioactive Isotopes. Because of their radioactivity they, and substances in which they are chemically combined, can be quantitatively measured by a Geiger Counter. Wherever they go in the body, they are apparently accepted by the tissues and play their rôles in metabolism in the same way as if they were not radioactive. Thus Radiocalcium is found to be stored almost entirely in bone and the amount taken in in a given time is an indication of the amount of nonradioactive calcium given out in conditions in which the total amount of calcium is not changed. The turnover of calcium can therefore be estimated. Radioiodine tends to be stored in the thyroid, and, again, when the total amount of iodine does not change, the amount stored in a given time balances the amount lost and is a measure of the iodine replacement.

By the technique of Autoradiography the exact location of the radioelements can be determined by holding a section of the tissue in contact with a photographie film. The images on fine grained films can then be magnified. Consequently, by selection of radioelements based on information as to where they are stored in largest amounts and by their use, heavy radiation can be brought to bear upon several kinds of tissues leaving others influenced but little or not at all. An excellent account of Isotopes in Nutrition Research is given in Borden's Review of Nutrition Research, 1945, 6, Nos. 8 and 9.

\section{To Employ Culture Methods}

The common feature in these techniques is to plant cells, tissues or organisms in new and different fluid environments and to observe their behavior therein. 
Thus cells can be grown in Tissue Cultures of chemical composition suited to their requirements. Mixed cultures are those containing several types of cells and pure eultures those containing but one sort. This technique affords'unrivalled opportunities for experimentally changing the fluid environments of cells. for the study of nutritional factors, growth stimulating and growth inhibiting factors, and the influence of cells on one another. Individual cells can be observed at high magnification and the phenomena of motility, phagocytosis, mitosis, cell death, etc. can be recorded by moving pictures so that the analysis of form and function is possible with a high degree of accuracy.

The limitation of the method is the obvious one that the fluid environments are artificial and must be changed at intervals to keep the strains of cells alive. Consequently tissue cultures are unsatisfactory for the investigation of intercellular materials, like fibers, hyaline deposits and so on. Moreover the cells cannot properly organize to form tissues and organs as they do in vivo since they are isolated from normal influences by other tissues of the body. But they make the effort. Methods have recently been advocated for the culture of organized tissues, bones, teeth, etc. (Fell, H. B., J. Roy. Micr. Soc., 1940, 60, 95-112).

In selecting the technique of tissue culture for the solution of any problem it is well to remember that considerable equipment and several years training are required to realize its full usefulness. For this reason valuable time will be saved by learning the technique from an expert.

The new and highly productive technique of analysing cellular responses by their observation in Motion Pictures offers more attractive leads when applied to living cells in tissue cultures than to cells viewed in other situations. In tissue cultures they can be photographed at high magnification, both by direct illumination and in the dark field, because they occur as individuals or as thin clumps in the fluid. Moreover, their behavior can be followed in successive photographs over long periods of time and it is possible directly to observe how this is modified by a host of different influences experimentally brought to bear on them. For teaching Motion Pictures are helpful, but can be used too much. Easy come, easy go is true of instruction. Unless learning is combined with some sort of effort it will be of very transitory value.

Transplantation of tissue from its original location to a new and different position, such as the Anterior Chamber of the Eye, is also a culture method of value in the solution of certain problems. The factors that condition the growth and the behavior of the transplant are of importance.

Some organisms can best be grown, and viruses inereased in amount, by implanting them into the Chorioallantoic Membrane of chick embryos. This technique has abundantly proved its worth. The feasibility of culture in this membrane depends essentially on the lesser development of growth inhibiting factors in young tissues than in older ones.

Viruses will "take" and inerease in amount in some locations better than in 
others. Intracerebral and intratesticular inoculations are often made and, again, young animals are in general most susceptible.

The culture of Bacteria and Protozoa has for generations been a fine art based on meticulous study of their needs. These relatively simple organisms provide wonderful material for the investigation of the most basic of vital phenomena.

\section{To Investigate Composition by Chemical Means}

This cannot be done blindly-by just taking a chunk of tissue and analysing it. The investigations must be guided by knowledge of the structure and function of the materials analysed. Blood can, for example, be collected in sufficient volume for routine chemical analysis; but the results will differ depending upon whether it is arterial blood, portal venous blood from the intestines, or venous blood from the extremities. Analyses of whole skin are practically worthless because the skin is a structure made up of two parts: avascular epidermis of ectodermal origin and underlying dermis made up of connective tissue differing in vascularity, fiber, fat, tissue fluid and gland contents in various regions of the body. Only since a technique has been devised whereby whole Epidermis freed from dermis can be obtained in a condition suitable for analysis, not having been exposed to any fluids, has progress been possible.

Results of direct chemical analysis of any tissue may be misleading unless interpreted in terms of its structural make up and of what has happened to it since it existed in vivo. Among the experimental errors to be guarded against are variability in sacrificing the anımal, or the manner of death of the patient, in excision of tissue allowing more or less blood and other fluids to drain out or evaporate, in time and in temperature, in age, sex, and in conditions before death.

The extracellular and intracellular fluids or phases, are large in volume, when all are taken together, but difficult to get at directly. To obtain data "the deducive histochemical method" is suggested. This is described by Lowry, O. H. and Hastings, A. B. in Cowdry's Problems of Ageing, 1942, 728-755.

Those wishing to analyse extremely small volumes of fluid which by contrast can be collected for direct determinations cannot do better than to familarize themselves with the techniques elaborated by A. N. Richards and his associates at the University of Pennsylvania for the study of glomerular urine.

By the useful technique of Microincineration minerals which are not volatilized at high temperature can be directly studied in the tissues in the positions which they previously occupied in living organisms. They appear as shining particles when viewed by the Dark Field Microscope. Microincineration is truly a microchemical method for the localization of structure which is microscopic in its fineness.

Quite a number of Microchemical Reactions capable of demonstrating the precise location in the cells of minerals, fats, carbohydrates and proteins are available. 
By a Photoelectric Microphotometer it is possible to estimate quantitatively reactions like that of Feulgen for Thymonucleic Acid which give distinctive colors and numerous stains which are specific for tissue components and can be standardized in their action. But the data obtained are relative, that is it can be said that the reaction is say 60 per cent greater in one specimen than in another. The absolute amount of the component demonstrated per gram of tissue cannot yet be arrived at.

Several Enzymes (phosphatase, dopa-oxidase, arginase) can now be microscopically identified and their position within cells determined. By close comparison of enzymatic properties with the cellular composition of tissues, the localization of many others can be inferred.

In the case of these and other microchemical methods the treatment of the tissue after excision and before the special procedures are commenced is of consequence. Even in the preparation of routine frozen sections, and much more so when the specimens are fixed, dehydrated, cleared, imbedded and sectioned, there are many opportunities for the loss of chemical substances and of change in their position in the tissue and within cells. The best way to hold the components in the positions they occupy in the living state is to instantaneously freeze the tissue and dehydrate in vacuum while still frozen, thus avoiding all fixatives, by the Altmann-Gersh technique. Moreover, the reagents used in testing must contact all the tissue equally for unequal contact may well be followed by stronger reactions in some areas than in others.

Quite recently chemical analysis has been accurately focussed, not merely on cells, but on parts of cells. Nuclei, Mitochondria and many other cellular components including even Chromatin Threads can now be collected en masse by Centrifugation of broken up cells and analysed. This is a departure of consequence.

Finally standard qualitative chemical methods are often applicable on a microscopic basis. The reader wishing to do so may well consult Chamot, E. E., and Mason, C. W., Handbook of Chemical Microscopy. New York: John Wiley \& Sons, 1940, vol. 2, 439 pp. Another book that will be found of service, especially for analysis on microscopic slides, is Benedetti-Pichler, A. A., Introduction to the Microtechnique of Inorganic Analysis. New York: Jolnn Wiley \& Sons, 1942, $302 \mathrm{pp}$. Sometimes one is held up by having to deal with some unfamiliar chemical substance in which case aid may be secured from the large and comprehensive "Dictionary of Organic Compounds" edited by Heilbron and published in 3 volumes, 1934, 1936 and 1938, by Oxford University Press, New York. No attempt is made in this dictionary to include dyes but thousands of other organic compounds are conveniently arranged in alphabetical order. If the wanted material is some sort of medical preparation seek information in the following reference books. (1) New and Nonofficial Remedies, 1946. Chicago: Am. Med. Assoc., 770 pp.; (2) The National Formulary. 
VII. Washington: Am. Pharmaceutical Assoc., 1942, 690 pp.; (3) The Pharmacopoeia of the U. S. XII. Easton: Mack Printing Co., 1942, 880 pp.

\section{To Employ Physical Techniques in the Investigation of Composition}

Chemistry is, at rock bottom physics so that the distinction here made is convenient but without validity. Hydrogen Ion Indicators and OxidationReduction Potential could come under either heading.

Histospectrography is a quick and reliable method to gain information on the presence or absence of many minerals. It is a kind of survey technique, for the absorption lines of many elements can be obtained in a single spectrogram. The density of the lines can be determined photometrically but data obtained on concentration of a particular element are relative (more in one tissue than in another) but not absolute (in mgm. per gm. of tissue). Ultraviolet Absorption Spectra have been employed to advantage by Caspersson and others in the intracellular determination of certain components but the technique requires elaborate and costly instrumentation. It gives promise, however, of being of great value in the solution of fundamental problems.

Utilization of physical techniques in biology and medicine is now the order of the day and the limitations thereof cannot be envisaged. In this elementary survey only a few others can be mentioned briefly in passing as examples. By Electrophoresis measurements the electric charge on particles can be determined. The Polarization Optical Method is of surpassing value and Fluorescence Microscopy, supplemented by fluorescence spectrography, is coming into its own. Surface Tension measurements can be made in numerous ways. Particle size can be measured by a flock of different techniques from which the one must be chosen that best suits the material. The simplest way is to compare the objects with rulings of a micrometer slide. Diffraction methods are labor saving and often preferable. Filters of different porosity are available so that the sizes of particles passing through can be roughly gaged. To employ Ultracentrifugation techniques are among several other possibilities. There are now Microscopes of many varieties to choose from.

The Electron Microscope is a physical tool which can be used only by a specially trained individual, and it has the limitation that the cells and other materials must be very thin, sections not more than about $\frac{1}{5}$ of a micron. See Burton, E. F. and Kohl, W. H., The Electron Microscope. New York: Reinhold Publishing Corporation, 1946, 325 pp.

In biology and medicine it is clearly evident that the techniques of physics and chemistry are so revealing that some knowledge of these basic sciences is necessary. A little knowledge can however be a dangerous thing often leading to half baked conclusions. Cooperation with real physicists and chemists is essential and team work must take the place of isolated individual endeavor, moreover a laboratory of whatever kind must be well organized to be effective. 
An untidy laboratory is not a sign of industry but an indicator of carelessness, and sometimes a source of actual danger to the occupants.

\section{To Detect Deviations from Normal}

The Normality of a tissue or organ is often in doubt. There is no single technique capable of yielding an unqualified answer. Since some properties may be normal while others are abnormal (pathological) we need first to be told the property under consideration. If it is, for instance, the amount of contained pigment, this can be said to be normal when it is the amount usually present in a particular tissue under the same conditions. By the word "usually" is intended in the majority of cases, that is in 51 per cent or in any higher percentage. The phrase "same conditions" means that the conditions likely to influence the amount of pigment are so nearly alike as to be not responsible for any difference observed between the property of the tissue where normality is in question and that of others of the same kind. Thus, we could say with reasonable assurance that the amount of pigment is normal if it is that usually demonstrated by the same technique in tissues of the same kind of animals of the same species, sex and age living under the same conditions. Judgment is necessary in specification of possibly modifying conditions which will depend to some extent on the property under consideration and on the number of observations necessary to establish the percentage within the limits of probability. It would not do to compare the amount of pigment in the specimen, the normality of which is in question, with that in too few others. This is the statistical definition of normality which is not universally accepted but which is useful and easily understood.

Only a few samples of the various kinds of technique have been mentioned in this survey as a kind of menu from which to make a selection or to obtain clues to other methods that may fit the case. Many of them are very ingenious and were only discovered after wisely conceived attempts to overcome practical difficulties. This overcoming of obstacles is a pleasant experience. It calls for actual work and experiment and appeals to many of our best minds. The techniques may be regarded as keys by which scientific treasure can be unlocked. Unused they are worthless. 


\section{STANDARDIZATION OF STAINS*}

In the use of stains one encounters a multitude of namcs, many of which are synonyms, and it is difficult to be sure of their meaning. Two comprehensive dye indexes have been published. One, "Schultz' Farbstofftabellen", is now in its 7 th edition (1928 to 1939) but confusion is created by the fact that the index numbers of the dyes given in it do not correspond to those in the earlier editions. The other, the "Colour Index of the Society of Dyers and Colourists", was edited by F. M. Rowe and published in 1924 . It was followed in 1928 by a supplement, but there has been no second edition. This Colour Index gives (1) the commercial name, or much more frequently names for there are so many synonyms; (2) the formula, (3) the preparation, (4) the discoverer and (5) the properties of a vast assemblage of dyes. It is the standard of reference in the United States and other English-speaking countries. When one wishes to be specific it is customary to list after the dye used its colour index number, for example vital red, C. I. No. 456.

The most recent Year Book of the American Association of Textile Chemists and Colorists, New York: Hawes Publishing Co. 1945, 743 pp. is often of assistance. It provides an alphabetical list of over 6,000 American made dyes with classification, manufacturer and Colour Index Number if any. A listing of American made Dyes arranged by Colour Index Numbers is also useful. For example, if one is interested in Orange II, CI. 151, it will be seen that this is available under 26 names from 12 different makers. In another place the foreign prototype names of dyes without Colour Index numbers are listed alphabetically with the corresponding American dyes and their manufacturers so that the available American substitutes for foreign dyes can be found. This Year Book is unfortunately often lacking in medical school libraries but it is usually on hand in the better Public Libraries like that of St. Louis.

Much aid is given to investigators by the Biological Stain Commission and particularly by its distinguished Founder, Dr. H. J. Conn. This commission is concerned with the inspection and standardization of stains, not with their manufacture as is sometimes supposed. It was found in 1920 , while the postwar embargo on dyes was still in effect, that American scientists were being supplied with dyes from three or four different stain companies and that their products were not sufficiently uniform to be reliable. Accordingly, through the cooperation of the National Research Council and of several national scientific societies, the Commission on Standardization of Biological Stains (now the Biological Stain Commission) was established. The Commission is now an independent organization but includes in its membership representatives of eight societies with which it cooperates. The work of the Commission is twofold. First, by cooperation of biologists and chemists it gathers information

* Kindly revised by Dr. H. J. Conn. 
concerning the nature of dyes as related to their use in microscopic technique; secondly, by working with the manufacturers and dealers it endeavors to see that the supply of available stains in America is of the highest possible quality as judged by their performance in actual laboratory use. The first of these purposes has inspired a useful book on "Biological Stains" by Conn, now in its fifth (1946) edition, and at the same time has led to the publication by the Commission of a quarterly, "Stain Technology." The second object is being brought about by the certifying of stains.

The certification plan has been adopted because of the great difficulty of drawing up any chemical or physical standards to determine which stains are satisfactory and which are not. If such standards were formulated, it would be possible to prepare specifications with which manufacturers of stains would be expected to comply. In the early work of the Stain Commission an attempt was made to draw up such specifications and they were published, in provisional form, for a few stains in the first edition of "Biological Stains." Full specifications are given in the current edition and in the National Formulary.

Instead of drawing up specifications, therefore, the Stain Commission instructs the manufacturers of stains to submit samples to it of every batch manufactured of any of the stains that are on the certification basis. The Commission submits these samples to certain definite tests which have now been formulated and published (see Conn, pp. 246-276). The methods in question include chemical, spectrophotometric, and biological tests, and only those dyes are certified which are satisfactory in all these tests. Such dyes the manufacturers are allowed to sell with a special label on the package indicating approval by the Stain Commission.

The certification label on any bottle of stain means, therefore, that: (1) a sample of the batch bearing the label has been submitted to the Commission for testing and a portion of the sample is permanently on file in the chairman's office; (2) the sample proves true to type, as judged by spectrophotometric tests; (3) its dye content is up to specification and is correctly indicated on the label; (4) it has been tested by experts in the procedures named on the label and has been found satisfactory by them; and lastly, (5) no other batch can be sold under the same certification number except by such a flagrant breach of confidence on the part of the company as to risk losing the good will of the Commission. At present (1950) the following stains have been placed on the certified list. In descriptions of their use the names should be followed by C.C., indicating that the products were Commission Certified, for instance, alizarin red S (C.C.).

Eight companies in the United States are now submitting their stains to the Commission for certification before putting them on the market. It must be realized, however, that no one of these concerns necessarily manufactures all the stains which it thus submits; but in the case of any stain which is manufactured elsewhere, the company takes responsibility for its performance as a biological stain, on the basis of tests made to show its adequacy, and in many instances carries out a certain degree of purification or other processing before 
Alizarin red S

Anilin blue, water soluble

Auramine $\mathrm{O}$

Azocarmine $\mathrm{G}$

Azure A

Azure B

Bismarck brown $Y$

Brilliant cresyl blue

Brilliant green

Carmine

Chlorazol black E

Congo red

Cresyl violet

Crystal violet

Eosin, bluish

Eosin, yellowish

Erythrosin B

Ethyl eosin

Fast green FCF

Fuchsin, acid

Fuchsin, basic

Giemsa stain

Hematoxylin

Indigo carmine

Janus green B

Jenner's stain
Light green, S.F., yellowish

Malachite green

Martius yellow

Methyl green

Methyl orange

Methyl violet $2 \mathrm{~B}$

Methylene blue chloride

Methylene blue thiocyanate

Methylene violet

Neutral red

Nigrosin

Nile blue A

Orange G

Orange II

Orcein

Phloxine

Pyronin $\mathrm{P}$

Resazurin

Rose bengal

Safranin O

Sudan III

Sudan IV

Sudan black B

Tetrachrome stain (MacNeal)

Thionin

Toluidine blue $\mathrm{O}$

Wright's stain

putting the stain on the market. One of these companies puts on the market every stain now on the certification list. Two other companies submit samples of over half the stains thus listed, while the other companies merely request certification of one or two dyes in which they specialize. No dyes have yet been certified by the Stain Commission submitted by any foreign concern except for one located in Montreal. Cooperation among the Americas is increasing (Conn, H. J., Stain Techn., 1942, 17, 5-6).

In several recent editions of the National Formulary, published by the American Pharmaceutical Association, a section has been included in which formulae of staining solutions are given. Originally there was no agreement between these formulae and the ones recommended by the Stain Commission. Beginning in 1937, however, it was decided that the National Formulary Committee and the Biological Stain Commission should cooperate in this matter. Accordingly, the chairman of the latter was made a member of the former and a member of the National Formulary was put on the Board of Trustees of the Commission. This interlocking membership is assurance that the work of preparing staining formulae for each edition of the National Formulary is carried on in close cooperation with the Stain Commission. This cooperation has resulted in two important steps:

1. Specifications of the most important stains now on the certification basis have been published in the National Formulary $(1942,1946)$. These specifica- 
tions are partly chemical and spectrophotometric, but also contain detailed statements as to how the stains should be tested as to their behavior for biological purposes and state the results to be expected from these tests. In every case these specifications have been made to harmonize with the tests as actually performed by the Stain Commission.

2. The formulae given in the National Formulary, in "Biological Stains" and in the "Manual of Methods for the Pure Culture of Bacteria," published by the Society of American Bacteriologists, have been compared and critically studied with the object of making them identical in all three.

The years since the second World War have seen more progress in stain standardization than during any preceding similar period. Dr. E. H. Stotz, Biochemist, of University of Rochester Medical School, has been made an officer of the Stain Commission and is now in charge of its research and assay laboratory at Rochester, N. Y. This laboratory is making a comprehensive survey of nearly every stain sample that has ever been submitted for certification, making a systematic comparison between their physical and chemical characteristics (notably spectrophotometric) and behavior in staining. If it is ever going to be possible to draw up specifications that correlate with staining properties, such a survey should furnish the necessary data. 


\section{ABBREVIATIONS}

$1 \mu$ (Greek letter for micron $)=1 / 1000$ th part of a millimeter $(\mathrm{mm})=.0.001 \mathrm{~mm} .=10^{-3}$ $\mathrm{mm} .=10,000 \AA=$ approximately $1 / 25,000$ th of an inch.

$1 \mathrm{~m} \mu$ (millimicron) $=1 / 1000$ th part of a micron $=1 / 1,000,000$ th part of a mm. $=10^{-6} \mathrm{~mm}$. $=0.001 \mu=10 \AA$.

$1 \AA$ (Angstrom unit) $=0.1 \mathrm{~m} \mu=0.0001 \mu=10^{-7} \mathrm{~mm}$.

$1 \mu \mu$ (micromicron) $=1 / 1,000,000$ th part of a micron $=1 / 1,000,000,000$ th part of a mm. $=$ $10^{-0} \mathrm{~mm}$. $=0.000,001 \mu=10^{-2} \AA$.

$1 \mathrm{Kg}$. = approximately $2.2 \mathrm{lbs}$.

$1 \mathrm{gm} .=10^{-3} \mathrm{Kg} ., 0.001 \mathrm{~K} ., 1000 \mathrm{mgm} ., 1,000,000 \mu \mathrm{g}$.

$1 \mathrm{mgm}$. $=10^{-6} \mathrm{Kg} ., 10^{-8} \mathrm{gm} ., 1000 \mu \mathrm{g}$.

$1 \mu \mathrm{g} .=1 \gamma=10^{-0} \mathrm{Kg} ., 10^{-6} \mathrm{gm} ., 10^{-3} \mathrm{mgm}$.

$\mathrm{m} \mu \mathrm{g}=1 / 1,000,000 \mathrm{mgm}$.

$N \mathrm{NaCl}$ is normal solution of sodium chloride, see Normal Solution.

$M \mathrm{HCl}$ is molecular solution of hydrochloric acid, see Molecular Solution.

$M=$ mole.

$\mathrm{mM}=$ millimole.

$\mathrm{ME}=$ milligram equivalent

$1 \mathrm{ml}$ (milliliter $=1 / 1,000$ th part of a liter $=1 \mathrm{cc}$. (approx.) that is $1 \mathrm{cc} .+0.000027 \mathrm{cc}$. at $40^{\circ} \mathrm{C}$.

$\mu^{2}=1 / 1,000 \mathrm{~m}^{1}$

$1 \mathrm{ml}$ (milliliter) $=1 / 1,000$ th part of a liter $=1 \mathrm{cc}$. (approx.).

CI 76 means that the number of a dye is 76 in the Colour Index of the Society of Dyers and Colourists.

CC. given after a dye signifies that it has been certified by the Biological Stain Commission

The following publications are simply referred to by author, or senior author, or editor's name and page number (cf. Conn, p. 26).

Bensley, R. R. ANd S. H., Handbook of Histological and Cytological Technique, Univ. Chicago Press, 1938, 167 pp.

Bourne, G., Cytology and Cellular Physiology, Oxford: Clarendon Press, 1942, 296 pp. (Second Edition, 1951, 524 pp.)

Cons, H. J., Biological Stains, Geneva, N. Y.: Biotech Publications, 1940, 308 pp.

Cowdry, E. V., Textbook of Histology, Philadelphia: Lea \& Febiger, 1938, 600 pp., 3rd Edition, 1950, $640 \mathrm{pp}$.

Craig, C. F., Laboratory Diagnosis of Protozoan Diseases, Philadelphia: Lea \& Febiger $1942,349 \mathrm{pp}$.

Downer, H., Handbook of Hematology, New York: Hoeber, 1938, 3136 pp.

Emig, W. H., Stain Technique, Lancaster : Science Press Printing Co., 1941, 75 pp.

Grasser, O. (Editor), Medical Physics, Chicago: Year Book Publishers, 1944, 1744 pp.

Glick, David, Techniques of Histo- and Cytochemistry. New York: Interscience Publishers, Inc., 1949, $531 \mathrm{pp}$.

Lee, Bolles, The Microtomists' vade-mecum. Philadelphia: P. Blakiston's Son \& Co. (Tenth Edition, Edited by J. B. Gatenby and T. S. Painter, 1937, 784 pp. Eleventh Edition edited by J. B. Gatenby and H. W. Beams, 1950, 753 pp.)

Lillie, R. D., Histopathologic Technic. Philadelphia: Blakiston, 1948, 300 pp.

Lison, L., Histochemie Animale, Paris: Gauthier-Villars, 1936, 320 pp.

Mallory, F. B., Pathological Technique, Philadelphia: Saunders, 1938, 434 pp.

McClung, C. A., Microscopical Technique, New York: Hoeber, 1938, 698 pp., 2nd Edition by Ruth McClung Jones, 1950, 790 pp. 



\section{TECHNIQUES}

A-V Bundle, see Todd, T. W., Cowdry's Special Cytology, 1932, 2, 1173-1210.

Abopon. For mounting amyloid stains (Leib, Am. J. Clin. Path., 1947, 17, 413).

Absorption. Every solid surface attracts other substances more or less. This holding is referred to as absorption. The finer the structure of the solid the greater the combined surface area of the constituent particles and consequently the greater the degree of absorption. An interferometer is an instrument employed to measure change in concentration by absorption. There are many other ways of obtaining this information. See Water Absorption and fat absorption after previous coloration of fat with Sudan III or Sudan black (see Vital Staining). See X-ray Absorption.

Absorption Spectra. Methods are available for the determination of absorption spectra of cell structures. Caspersson (T., J. Roy. Micr. Soc., 1940, 60, 8-25) has described apparatus for absorption from intracellular objects larger than 1 micron such as Nissl bodies. This line of investigation is just developing and is likely to be productive of important results. See Histospectroscopy. Acacia, properties as a macromolecule (Hueper, W. C., Arch. Path., 1942, 33, 267-290). See V. Apáthy's Syrup.

Acanthocephala, see Parasites.

Acarina, see Parasites, Ticks.

Acetic Acid (L. acetum, vinegar). Widely used as a component of fixatives. The undiluted solution is often termed "glacial acetic acid." This contains 99.5\% $\mathrm{CH}_{3} \mathrm{COOH}$. Causes a distinctive swelling of fresh collagenic fibers. Employed in dilute solution to destroy red blood cells so that whites can be examined. In $1 \%$ solution separates epidermis from dermis. See Epidermis.

Acetic-Osmic-Bichromate fixative of Bensley. $2 \%$ osmic acid, 2 cc.; $2.5 \%$ aq. potassium bichromate, 8 cc.; glacial acetic acid, 1 drop. Excellent for mitochondria but very small pieces of tissue must be used because the fluid penetrates poorly. The best stain is Anilin-Fuchsin Methyl Green, see also Copper Chrome Hematoxylin.

Acetin Blue R (CI, 560)-Induline Alcohol Soluble $-a$ basic dye of light fastness 4 . Paraffin sections of plant tissues color dull light blue (Emig, p. 58).

Acetic-Carbol-Sudan III, see Sudan III.
Aceto-Carmine (Schneider's). Add $10 \mathrm{gms}$. carmine to 100 cc. $45 \%$ aq. glacial acetic acid. Dissolve with heat and bring up to boiling. Cool, filter, and store as stock solution. Used for smears this combines fixation with staining; but it causes a swelling of some cellular elements and is not recommended.

Aceto-Orcein-Fast-Green.-Written by Dr. N. B. Kurnick, Dept. of Medicine, Tulane University, New Orleans 12. January 31,1951 - This modification of La Cour's aceto-orcein stain-fixative for chromosomes permits a one-step differential staining of tissues. The introduction of fast green and $\mathrm{NaCl}$ (to prevent overstaining by the former) provides a green counterstain for the reddish-brown chromatin. The intensity of this counterstain may be modified by varying the salt concentration (increasing the salt concentration reduces the intensity of green staining), but the method described here has proved satisfactory for most materials. The stain mixture may be used as a stain-fixative, as for dipteran salivaries, some plant materials, and for the study of isolated chromosomes and nuclei, or as a stain following other fixatives. Flood material for few minutes in following solution: $27 \mathrm{ml}$. $1 \%$ orcein in $45 \%$ acetic acid, $3 \mathrm{ml}$. $1 \%$ fast green in $95 \%$ alcohol, $2 \mathrm{ml} .2 \mathrm{M} \mathrm{NaCl}$; cover with cover slip, press out on filter paper, if desired. Paraffin sections must be brought to water before staining Permanent mounts may be prepared by rinsing the stained material successively in $70 \%, 95 \%, 100 \%$ alcohol, xylene, and mounting in Clarite. Cytoplasm, collagen, and nucleoli are green, chromatin is reddish-brown (Kurnick, N. B., Cold Spring Harbor Symp. Quant. Biol., $1947,12,191$; Kurnick, N. B. and Ris, Hans, Stain Tech., 1948, 23, 17-18).

Acetone, see Dehydration of Tissues, as fixative for Phosphatases and Lipases.

Acid Alcohol is used for the differentiation, or decolorization, of certain stains. It is usually made by adding 1 cc. hydrochloric acid to 99 cc. $70 \%$ ethyl alcohol. It is also employed for cleaning cover glasses.

Acid Alizarin Blue (1) G.R. (CI, 1048). An acid anthraquinone dye called for in Buzaglo's Method which the author proposes as substitute for Van Gieson.

(2) B.B. (CI, 1063) likewise an acid anthraquinone dye little used, if at all. 
Acid Alizarin Green G (CI, 1049), a direct mordant dye of color fastness 1. Use for staining blue green and green algae and paraffin sections of animal tissues after mordanting in $1 \%$ aq. ferric alum is described (Emig, p. 63).

Acid Blue B (CI, 736), an acid dye of light fastness 5 gives light, fugitive and indistinct coloration of tissue (Emig, p. 52).

Acid Blue G (CI, 712)-Brilliant Acid Blue $\mathrm{V}$-an acid dye of light fastness 5 (Emig, p. 52).

Acid Bordeaux, see Bordeaux Red.

Acid Congo R, see Vital Red.

Acid Dyes, see Staining.

Acid Fast Bacilli. Of these the organisms of tuberculosis and leprosy are the most important.

1. In smears apply Carbol Fuchsin gently heat $3-5 \mathrm{~min}$. or stain room temperature $15 \mathrm{~min}$; decolorize $95 \%$ ethyl alcohol containing $3 \%$ of conc. hydrochloric acid until only slight pink color remains; wash in water; counterstain sat. aq. methylene blue or Loeffler's Alkaline Methylene Blue; wash and dry.

2 . In sections the organisms can be stained red in paraffin sections after almost any fixation (formalin-Zenker preferred). First color with Harris hematoxylin. Wash in water and perhaps decolorize a little in Acid Alcohol. Wash again. Stain with warmed carbol fuchsin $1 \mathrm{hr}$. or more. Decolorize in acid alcohol. Wash carefully in water plus few drops ammonia. 95\% alc., abs. alc., xylol, balsam. A critique of the methods has been published (Fite, G. L., Am. J. Path., 1938, 14, 491-508). To color the organisms blue, fix 3-5 days or more in equal parts $10 \%$ formaldehyde and $95 \%$ alcohol. Stain sections in new fuchsin $0.5 \mathrm{gm}$.; phenol crystals, $5.0 \mathrm{gm}$.; alcohol methyl or ethyl, $10 \mathrm{cc}$. + aq. dest. to make $100 \mathrm{cc}$. at $60^{\circ} \mathrm{C}$. over night, 12-24 hrs. or at room temperature $24-48 \mathrm{hrs}$. Longer for $M$. leprae. Freshly distilled aq. formaldehyde $5-30 \%, 5 \mathrm{~min}$. (Note that this formalin must not be alkaline and that it is safer to have it faintly acidified.) $2 \%$ hydrochloric acid in $95 \%$ alcohol, $5 \mathrm{~min} .1 \%$ aq. potassium permanganate 2-5 min. (until brown). $2 \%$ aq. oxalic acid, 1 min. Harris' hematoxylin 2 min. Stain in acid fuchsin, 0.1 gm.; picric acid, $0.5 \mathrm{gm}$; aq. dest. to make 100 cc. Without washing, dehydrate in alcohol, clear in xylol and mount in balsam. Nuclei, brown; connective tissue fibers, red; muscle, yellow; acid fast bacilli, dark ultramarine blue. Good for photography (Fite, G. L., J. Lab. \& Clin. Med. 1939, 25, 743-744; re- vised by G. L. Fite, U. S. Marine Hospital, Carville, La. May 13, 1946.).

3. Mr. J. M. Albrecht employs the following method in our laboratory. Deparaffinize $5-6 \mu$ sections of $10 \%$ formalin or Regaud fixed tissues. Wipe off excess water around sections and cover with strip of filter paper. Flood filter paper with carbol fuchsin (Phenol crystals, $8 \mathrm{gm}$; basic fuchsin, $4 \mathrm{gm}$.; $95 \%$ ethyl alcohol, 20 cc.; aq. dest., 100 cc.). Steam for $3 \mathrm{~min}$. and then allow to stand for $30 \mathrm{~min}$. adding more stain if necessary. The filter paper prevents deposition of ppt. of dye on sections. Flush off stain with aq. dest. Partly differentiate in $1 \mathrm{cc}$. conc. hydrochloric acid in 100 ec. $70 \%$ alcohol, sections becoming deep pink. Wash in aq. dest. Stain Harris' Hematoxylin $10 \mathrm{~min}$., wash in aq. dest. Complete differentiation of both fuchsin and hematoxylin in 50 cc. $70 \%$ alc. $+4-5$ drops hydrochloric acid, sections becoming light pink. Wash in aq. dest. Neutralize in 6 drops conc. ammonia + 50 cc. aq. dest. Wash, dehydrate, clear and mount as usual.

4. In frozen sections (Kírajian, A. A., Am. J. Clin. Path., Techn. Suppl., 1943, $7,45-47)$. Transfer frozen sections of leprous tissue to slides. Dehydrate, blot with filter paper, dip in celloidin. Blow over surface till dry. Wash in tap water. Apply Carbol Fuchsin steaming gently for 3 min. Pour off and wash in tap water. Differentiate with $1 \mathrm{gm}$. arsenic acid in 100 cc. $60 \%$ alcohol applied by medicine dropper. Again wash in tap water and counterstain with Loeffler's methylene blue $2 \mathrm{~min}$. Wash in tap water, dehydrate with 3 applications of anhydrous isopropanol or absolute ethyl alcohol. Apply immediately equal parts anhydrous isopropanol or abs. alcohol and beechwood creosote. Agitate slide removing excess blue color. Blot with filter paper, clear with xylol and mount in damar.

See Tubercle and Leprosy Bacilli, Fluorescence Microscopy, also paper by Richards, O. W., Kline, C. K. and Leach, R. E., Am. Rev. Tuberc., 1941, 44, 255-266. 'Efficiency of Ziehl-Neelsen and fluorescence techniques compared. The latter superior (Van Dyke, A. E., Am. J. Clin. Path., Techn. Suppl., 1943, 7, 6-8.) For acid fast bacilli in urine see Kelso, R. E. and Galbraith, T. W., Am. J. Clin. Path., Techn. Suppl., 1943, 7, 8-11.

Less is known about the conditions that determine acid fastness than those which determine Gram positiveness (see Gram Stain). The facts are well stated for mycobacteria in general and 
especially for the Tubercle Bacillus by Dubos, R. J., The Bacterial Cell. Harvard Univ. Press, 1945, 460 pp. There is present in the tubercle bacillus mycolic acid which is acid fast even after isolation in the pure state; but the property of acid fastness is lost by the bacilli under conditions that do not destroy this acid. These conditions involve destruction or impairment of structure of the organisms by mechanical, chemical or enzymatic means. Apparently the cell surface must be intact. Dubos quotes Yegian et al. as showing that tubercle bacilli stained in absence of electrolytes are uniformly colored rods, that addition of electrolytes causes a beaded appearance and that treatment with ethyl alcohol restores uniform solid staining to beaded organisms which means that the change from beaded to uniform state is a reversible process. This dependence of microscopic appearance on experimental conditions of technique is obviously a matter of great consequence in leprosy as well as in tuberculosis. The investigator has to check carefully by study of living unstained bacilli.

Acid Fuchsin (CI, 692) - acid magenta, acid rubin, fuchsin $\mathrm{S}, \mathrm{SN}, \mathrm{SS}, \mathrm{ST}$ or S IIICommission Certified. Since this is a sulfonated derivative of basic fuchsin, and, because there are 4 possible primary basic fuchsins, Conn (p.118) points out that at least a dozen primary acid fuchsins are possible and samples are usually mixtures of several. Acid fuchsin is employed is so many ways that to enumerate them would be both futile and unnecessary. See New Fuchsin.

Acid Green, see Light Green SF yellowish. Acid Green O, see Naphthol Green B.

Acid Hemalum, see Hemalum.

Acid Magenta, see Acid Fuchsin.

Acid Orange II, Y or A, see Orange II.

Acid Phosphatase, see Phosphatase.

Acid Phloxine GR, see Chromotrope 2R.

Acid Rubin, see Acid Fuchsin.

Acid Violet. Several triphenyl methane dyes come under this heading. Conn (p. 132) says that the term "acid violet" is too indefinite for identification. This is unfortunate because dyes bearing this label have been used in several combinations as in Bensley's Neutral Safranin acid violet. Bailey, P., J. Med. Res., 1921, 42, 349-381 and Maurer, S. and Lewis, D. D., J. Exp. Med., 1922, 36, 141-156, working in Bensley's laboratory, used it for the pituitary. Acid violet is one of the stains employed by Weiss, E., J. Inf. Dis., 1928, 43, 228-231 to stain flagella and spirochetes (J. Lab. \& Clin. Med., 1928-29, 14, 1191-1193).

Acid Yellow, see Fast Yellow.

Acid Yellow R, see Metanil Yellow.

Acidity, see Hydrogen ion indicators.

Acidophilic, see Staining.

Acids, see under first name, Acetic Acid, Hydrochloric Acid, etc

Ackerman, see Auer Bodies.

Acridine Dyes. As the name suggests they are formed from acridine which is related to xanthene. Examples: acriflavine, neutral acriflavine and phos. phine. Phosphine $3 \mathrm{R}$ is employed as a fluorochrome for lipids.

Acridine Orange (CI, 788), a basic dye of light fastness 1 to 2 . Gives clear brown or dark orange coloration of plant tissues of exceptional fastness. Technique described (Emig, p. 55).

Acridine Red $3 \mathrm{~B}$ is, according to McClung, Microscopical Technique, 1950, p. 73, not an acridine dye but a pyromin closely related to Pyronin Y. It has been employed by Gomori, G., Am. J. Path., 1936, 12, 655-663 mixed with methyl green to reveal calcium salts and phosphatase activity.

Acriflavine (Cl. 790). A yellow fluorchrome. It is useful as a vital stain for nuclei. Farr, R. S., Anat. Rec., 1946, 94, 16, has employed acriflavine hydrochloride to label transfused leucocytes and to determine how long they remain in the circulation.

Actinomyces. Mallory's stain for actinomyces in sections (Mallory, p. 279). For the organisms, fixation in alcohol or in $10 \%$ formalin is preferable; but for the lesions, Zenker's fluid is better. Stain deparaffinized sections in Alum Hematoxylin 3-5 min. After washing in water stain in $2.5 \%$ aq. phloxine or in $5 \%$ aq. eosin in paraffin oven, $15 \mathrm{~min}$. After again washing, stain in Stirling's or Ehrlich's aniline crystal violet (see Anilin Crystal Violet), 5-15 min. Wash in water and treat with Gram's lodine, 1 min. Wash in water, blot and destain in aniline oil until no further color comes out. Rinse in xylol and mount in balsam. Branched forms, blue; clubs, pink to red.

Actinosphaerium, see $\mathrm{McCl}$ (ung, Microscopical Technique, 1950, p. 469.

Addis Count to provide quantitative data on number of red blood cells and casts in the urine is critically described by C. J. Gentzkow and H. A. Van Auken in Simmons and Gentzkow, p. 32.

Adenosinase. A method for analysis of adenosinase in lymphocytes and polymorphonuclear leucocytes (neutrophiles) is given by Barnes, J. M., Brit. J. Exp. Path., 1940, 21, 264-275. 
Adenylpyrophosphatase. The technique of localization of this important enzyme in cytoplasmic granules has been described and used in extracts of chick embryos by Steinbach, H. B. and Moog, F., J. Cell and Comp. Physiol., 1945, 26, 175-183. These authors are, however, not sanguine about the feasibility of its localization by histochemical methods (Science, 1946, 103, 144) as reported by Glick and Fischer, Science, $1945,102,429-430$. However, MalngwynDavies, E. D. and J. S. Friedenwald, J. Nat. Cancer Inst., 1950, 10, 1379, recently reported at the Histochemical Society that specific localizations were achieved when unfixed frozen sections were incubated in muscle adenosine triphosphate substrates.

Adermin, see Vitamin B6.

Adhesiveness, or stickiness of cellular surfaces is a phenomenon of great importance in connection with movement, phagocytosis embryological development and other processes. There is no standard technique to measure it, except in special circumstances as when it is manifested by agglutination of bacteria and sedimentation of red blood cells. The way leucocytes stick to the endothelial wall of a small blood vessel, shown by Motion Pictures, is impressive. Adhesion tests have been introduced as means of diagnosis of various trypanosomes. A fine general discussion of this phenomenon is provided by Beams and King in Calkins, G. N. and Summers, F. M., Protozoa in Biological Research. New York: Colombia University Press, 1941, $1148 \mathrm{pp}$.

Adrenal. For routine purposes fix in Zenker's Fluid and stain paraffin sections with Hematorylin and Eosin. There are many techniques for Lipids. The Chromaffin Reaction is of ten used for adrenalin but Cramer, W., J. Path. \& Bact., 1937, 44, 633, considers blackening with osmic acid vapor as more specific. Silver methods for vitamin $\mathrm{C}$ are difficult to apply but are apparently reliable. They are given under Vitamins. The Schultz cholesterol test gives excellent results. A selection may be made from several methods for Reticular Fibers. Corner, G. W., Contrib. to Embryol., Carnegie Inst., 1920, $9,87-93$, employed for reticulum the Bielschowsky-Maresch silver method exactly as specified by Ferguson, J. S., Am. J. Anat., 1912, 12, 277-296. The Bodian protargol method for nerve fibers has been adjusted to the adrenal by MacFarland, W. E., and Davenport, H. A., Stain Techn., 1941, 16, 53-58, also Cajal's chloral hydrate method. If one contemplates ultracentrifugation and the demonstration of the Golgi apparatus consult Guyer, M. F., and Claus, P. E., Anat. Rec., 1939, 73, 17-27.

Method proposed by Bennett, S. H., Am. J. Anat., 1940, 67, 151-227 for ketosteroid cortical hormone said by Gomori, G., Proc. Soc. Exp. Biol. \& Med., $1942,51,133-134$ not to be specific but to indicate merely location of lipids having keto or aldehyde groups. A technique for microscopic study of living grafts of adrenal cortex (Williams, O., Anat. Rec., 1945, 91, 307).

Adrenalin, see Chromaffin Reaction.

Aerosol, a detergent used in preparing bacteria for staining (Sineszko, S. F., Science, 1942, 96, 589).

Affixatives are materials used to fix sections to slides. See Albumen-Glycerin.

Agar, as matrix for cutting plant material with freezing microtome (Evenden, W. and Schuster, C. E., Stain Techn., $1938,13,145-146$ ). Lillie (p. 42) says that infiltration of tissues from water in $2 \%$ aq. agar at $55-60^{\circ} \mathrm{C}$. for $2-4 \mathrm{hrs}$, is useful for holding friable tissues and exudates in place before cutting frozen sections. The Agar does not color appreciably with the usual stains.

Age Changes are as manifold as life itself. Some are detectable by structural modifications while others can only be measured by decrease in performance. Many old tissues can easily be distinguished from new ones as for example Bone. Some accumulate definite products with age like Lipofucsin. The age of tissue and of cellular components, that is the time they endure, can be determined by attaching Tracer Substances to them so that their rates of Replacement can be measured. With the passage of time colloids age, become less elastic and more granular. Old Elastic Fibers can be distinguished from young ones. Now that the ultra structure of Collagenic Fibers has been revealed by the electron microscope we may hope for more accurate means of estimating their condition in relation to age. Numerous physical techniques, including the Polarization Optical Method, may well bring to light significant age changes. Obviously many methods of chemical analysis and of enzyme activity provide data on the modes of run down of vital activities.

\section{Aggeler, see Blood Platelets.}

Agonal Changes are particularly difficult to avoid in villi of small intestine. They are evidenced by a ballooning of the epithelial cap most marked when absorption of ordinary food stuffs is active. The ballooning phenomenon can be produced in the living animal by 
ligating arteries of supply or by employing fixatives which induce forcible contraction of smooth muscle (Macklin, C. C. and M. T., Chapter on Intestinal Epithelium in Cowdry's Special Cytology, N. Y., Hoeber, 1932, 1, 235).

Albert's Stain for Diphtheria Bacilli, which see.

Albumen-Glycerin for mounting paraffin sections. Egg white $50 \mathrm{cc}$., glycerin 50 cc., sodium salicylate $1 \mathrm{gm}$. This is "Mayer's Albumen". Shake together and filter during several days. See also Starch Paste and Masson's Gelatin Glue.

Alcohol. Unless indicated to the contrary the word "alcohol" as employed in this book refers to the ethyl variety. Alone it is a good fixative preliminary to tests for Amyloid, Copper, Fibrin, Glycogen, Gold, Hem ofuscin, Hyaline, Iron, Lead, Palladium, Phosphatase, Potassium and Thallium, which see. It is also employed in the demonstration of Nissl bodies by Gallocyanin, of mucus by Mucicarmine, of proteins by the Romieu Reaction, etc. In combination with other chemicals al cohol is also much used as a fixative, see Alcohol Formalin, Carnoy's Fluid and many others.

Alcohol of $70 \%$ is a good preservative and celloidin blocks can be stored in it. Absolute alcohol is supposed to contain not more than $1 \%$ by weight of water. It is considered to be 100 per cent. A very rough test for absolute alcohol is to mix with it a few drops of turpentine. If it becomes milky it contains too much water. To make a lower per cent from a higher one by dilution take the number of cc. corresponding to the percentage required and add aq. dest. to make in cc. the percentage of the alcohol diluted. Thus to make $30 \%$ from $70 \%$ take $30 \mathrm{cc}$. of $70 \%$ and add aq. dest. to make $70 \mathrm{cc}$. Alcohol is the best dehydrating agent for tissues. It is sometimes not easy to purchase absolute al cohol so that it must be prepared. Take say 10 liters of $95 \%$ alcohol, add 400 gms. freshly ignited calcium oxide. Leave, with occasional shaking, 24 hrs. until most of the water is absorbed by the oxide. Pour off fluid (leaving oxide at bottom of container) and distill using appropriate precautions. Keep the "absolute" as nearly so as possible by using a tight glass stopper for the bottle, or in place of the stopper an absorption tube containing calcium chloride so that any water in entering air will be absorbed and will not reach the alcohol. See Dehydration, also Amyl, n-Butyl, Tertiary Butyl, Isopropyl, n-Propyl and Polyvinyl Alcohols.
Alcohol-Formalin is a fixative containing 9 parts of absolute alcohol and 1 part of formalin. Since it penetrates quickly and dehydration can be commenced in absolute alcohol immediately after fixation, skipping the lower grades of alcohol, permanent preparations can be made within a few hours' time. For routine purposes $3-6 \mathrm{hrs}$. fixation will suffice but as a preliminary to Microincineration $24 \mathrm{hrs}$. is recommended. Alcohol-formalin is recommended for Fibrin, Glycogen, Indigo-Carmine stains and Peroxidase. It is employed with acetic acid in Bodian's Method for nerve fibers.

Aldehyde Green (CI, 676a)-Aniline Green, Benzaldehyde Green-a basic dye of light fastness 4 , employed as counterstain for Biebrich Scarlet, Acid Fuchsin. On xylene and sclerenchyma gives rather brighter shade than Malachite Green (Enig, p. 48).

Aldehydes. The bound form of aldehyde has been called "plasmalogen". From this the loosely bound "plasmal" form is developed on treatment with mercuric chloride or by acid hydrolysis (the Schiff Reaction for aldehydes). This is the basis of the Feulgen reaction for Thymonucleic Acid. According to Oster, K. A. and Oster, J. G., J. Pharmacol. and Exper. Therap., 1946, 87, 306-312, the fuchsin sulphurous acid reagent employed in this reaction when "true" is specific for aldehydes while other carbonyl compounds sometimes give a "pseudo" reaction. Oster, K. A. and Mulinos, M. G., J. Pharmacol. and Exper. Therap., 1944, 80, 132-138 report that the purple of the "true" reaction can be decolorized with dilute sodium hydroxide and restored with hydrochloric acid, whereas the reddish tint of the "pseudo" reaction cannot be restored in this way after decolorization (see Glick p. 65). See Thymonucleic Acid (desoxyribonucleic acid) and Carbonyl Compounds.

Aldolase. Triose phosphate was used as a substrate for the Gomori phosphatase procedure by Allen, R. S. L. and G. J. Bourne, J. Exp. Biol., 1943, 20, 61-64. The phosphate ion liberated by enzymic activity was precipitated in an alkaline medium. The enzyme was not inhibited by fluoride in concentrations which blocked alkaline phosphatase activity. The $\mathrm{pH}$ of the mixture was not stated, but was presumably about 9.5. The method was not completely satisfactory because of spontareous precipitates; nevertheless, concentrations of enzyme were noted in striated and cardiac muscle, kidney and liver. Little or none was seen in the lung. 
Aldolase + Isomerase are known as Zymohexase.

Algire, see Transparent Chamber Techniques.

Alizarin (CI, 1027) a little used acid anthraquinone dye.

Alizarin No. 6, see Purpurin.

Alizarin Blue RBN, see Gallocyanin.

Alizarin Carmine, see Alizarin Red S.

Alizarin Cyanine R (CI, 1050), an acid mordant dye which is not stable in solution, and on heating yields reddish ppt. (Emig, p. 64).

Alizarin SX, or anthrapurpurin, use same as Alizarin Red S.

Alizarin Green G (CI, 917), an acid mordant dye of light fastness 1. After mordanting in $1 \%$ aq. ferric alum stain for $30 \mathrm{~min}$. at $50^{\circ} \mathrm{C}$. in $0.1 \mathrm{gm}$. of dye in 100 cc. $1 \%$ aq. ammonium acetate. The green color obtained is the clearest given by a mordant dye. Additional directions are supplied (Emig, p. 59).

Alizarin Line Test for new bone and vitamin D (Martin, G. J., J. Lab. \& Clin. Med., 1940, 26, 714-719). See Line Test.

Alizarin Purpurin, see Purpurin.

Alizarin Red S (CI, 1034)-alizarin red water soluble, alizarine carmine-Commission Certified. By far the most used of all the alizarin stains. An important ingredient in Benda Method. Much superior to Madder for the staining of bone and dentine laid down while it is in the circulation. Schour has employed it extensively. The technique is described in detail by him and his associates (J. Dent. Res., 1941, 20, 411-418). He employed an Alizarin red S (CI, 1034) obtained from Coleman and Bell Co. The effective dose for rat, rabbit, guinea pig, cat, monkey and human infant is between $50-100 \mathrm{mg}$. per Kilo. conditioned by species, age and weight. For newborn white rats he recommends 0.2 cc. $2 \%$ Alizarin and for rats weighing $100-200 \mathrm{gms}$. $\frac{1}{3}-1$ cc. given intraperitoneally. Colors are retained in specimens fixed in $10 \%$ neutral formalin or in $95 \%$ alc. As in the case of Madder staining of bone, tissues can be cleared and examined as whole preparations, or ground sections can be prepared for microscopic study. Decalcification spoils the color. Age factor in alizarin staining (Ercoli, N. and Lewis, M. N., Anat. Rec., 1943, 87, 67). See Ossification and Line Test.

Alizarin Red Water Soluble, see Alizarin Red S.

Alizarin Sapphire BN (CI, 1054) of NAC, a direct mordant dye of light fastness 2 (Emig, p. 64).

Alkali Blue 6 B (CI, 703), an acid dye of light fastness 4 to 5 and of little value for permanent preparations (Emig, p. 51).

Alkali Green (CI, 665), an acid dye of light fastness 5 gives very fugitive pale dull green color (Emig, p. 47).

Alkaline Methylene blue, see Loeffler's.

Alkaline Phosphatase, see Phosphatase and Kidney.

Alkalinity, see Hydrogen Ion Concentration. Allantoin, colorimetric method, Borsook, J. Biol. Chem., 1935, 110, 481-493.

Allen's Fluids are modifications of Bouin's often containing urea. They are excellent for chromosomes. See McClung.

Allergy, see Pollens.

Allochrome Procedure, a differential method segregating tissues, collagen, reticulum and basement membranes into two groups (Lillie, R. D., Am. J. Clin. Path., 1951, 21, 484-488). The term is derived from $\mathrm{G}$. allóchroos, a different or changing color, since collagen and some related substances during the procedure changes from red to blue. See Lillie's paper for details of this Periodic Acid Schiff, picro-methyl blue technique.

All oxan Reaction. 1\% alcoholic solution of alloxan gives red color with $\alpha$ aminoacids. Romieu (M., Bull. d'Hist. appl., 1925, 2, 185-191) employs a cold neutral solution. Giroud (A., Protoplasma, 1929, $7,72-98)$ uses heat but states that great care is necessary in interpretation. See Lison, p. 129.

This reaction is described as follows by Serra, J. A., Stain Techn., 1946, 21, 5-18. Fix tissue as given under Ninhydrin Reaction. "An alcoholic 1\% solution of alloxan gives with amino acids and proteic compounds a pink coloration, after a long time at room temperature, or rapidly if the reaction is activated by heating in a boiling water bath. In our experience, this test is relatively insensitive; besides this, the coloring formed diffuses easily, so that the reaction can be indistinctly localized. With fixed materials the reaction is weak.

"The test must be carried out in neutral solutions; this is attained by addition of a phosphate buffer, as described for the ninhydrin. This reaction is not specific for amino acids and proteins, as it is also given by compounds with free $\mathrm{NH}_{2}$ and perhaps $\mathrm{SH}$ groups (see Winterstein, 1933)."

Almkvist-Christeller, see test for Mercury. Alpha Naphthol, see Oxidase.

Alpha Particle, see Gross, J., Bogoroch, R., Nadler, N. J., and Leblond, C. P., Am. J. Roentgenol. Rad. Therap., 1941, $65,420-458$.

Alphazurine 2G see Patent Blue. 
Altmann's Fluid. Equal parts of 5\% aq. potassium bichromate and $2 \%$ aq. osmic acid. Employed in his method as well as for staining with Copper Chrome Hematoxylin. It gives good surface fixation but penetrates very badly.

Altmann's Method of anilin fuchsin and picric acid for mitochondria. Fix small pieces not more than $2 \mathrm{~mm}$. in diameter 24 hrs. in Altmann's Fluid. Wash for $1 \mathrm{hr}$. dehydrate, clear imbed in paraffin and cut sections $4 \mu$. Pass down to water. Stain in anilin fuchsin $(20 \%$ acid fuchsin in anilin water) $6 \mathrm{~min}$. Blot with filter paper. Differentiate and counter stain by flooding the sections with 1 part sat.alc. picric acid and 2 parts aq. dest. Rinse rapidly in $95 \%$ alc., dehydrate in abs. alc., clear in xylol and mount in balsam. The mitochondria are stained crimson against a bright yellow background. Altmann's magnificent original plates should be examined (Altmann, R., Die Elementarorganismen und ihre Beziehungen zu den Zellen. Leipzig: Veit Co., 1894, 160 pp.). If these are not available seo Meves, F., Arch. f. mikr. Anat., 1913, 82, (2), 215-260.

Altmann-Gersh frozen-dehydration method (Gersh, I., Anat. Rec., 1932, 53, 309337).-Account written by Dr. Gordon H. Scott, Dept. of Anatomy, Wayne University School of Medicine, Detroit, Mich. This method has proved to be of much value in the preparation of tissues for microchemical procedures. It has also been used as a preliminary treatment for tissues destined for examination by the electron microscope (Wyckoff, R. W. G., Science, $1946,104,21-26)$. Tissues are frozen in liquid nitrogen or in liquid oxygen and dehydrated in vacuo at low temperatures. The tissue sample remains frozen at such a temperature that little or no chemical change can take place. It is believed that the only significant revision in cellular organization takes place during the freezing process. This is occasioned by possible shifts in proteins, etc., during ice crystal formation. Some users of the method believe that it is possible to freeze small tissue samples at speeds which will actually prevent ice crystal formations. Efforts in this direction have been made by freezing in cooled iso-pentane (technical) (Hoerr, N. L., Anat. Rec., 1936, 65, 293317; Simpson, W. L., Ibid., 1941, 80, 173-189).

For many reasons it has been found desirable to dehydrate at lower temperatures than were first thought necessary. Now the standard procedure is to dehydrate in vacuo from $40-65^{\circ} \mathrm{C}$. Apparatus of special design has been constructed a number of times to meet various needs. In general the principles are the same. What is needed is a vacuum system with ligh pumping speed and with provision for keeping the frozen tissue at constant temperature. Several of these have been described, each with its adaptation to the needs of the case.

For general use in histochemistry the device described by Packer and Scott (J. Tech. Methods, 1942, 22, 85-96) and by Hoerr and Scott (Medical Physics, Otto Glasser, 1944, Year Book Publishers) is both easy to operate and reliable. It has the distinct advantage that tissues can be infiltrated with paraffin"without"exposure to air. This apparatus can also be used for the preparation of tissues for electron microscopy. For this use only the dehydration device described by Wyckoff is probably more suitable.

Recently developed equipment will permit drying frozen tissues in 5 hours. (Stowell, R. E., Stain Techn. 1951, in press)

Alum. The alums are double salts of sulphuric acid. Aluminum potassium sulphate, or potassium alum, unless otherwise stated is the one used in making up hematoxylin solutions. Aluminum ammonium sulphate, or ammonia alum, should not be used as a substitute unless called for. Ammono-ferric sulphate, or iron alum is used as a mordant and differentiator in the iron hematoxylin technique and for other purposes. The crystals are of a pale violet color. Their surfaces oxidize readily and become useless. The surface should be scraped off. Only the violet crystals are of any use. Alum-Carmine (Grenacher). Boil 1-5\% aq. ammonia alum with $0.5-1 \%$ powdered carmine. Cool and filter. Does not penetrate very well and hence is not suitable for staining large objects in bulk. But it is useful and does not overstain (Lee, p. 140).

Alum-Cochineal, see Cochineal.

Alum Hematoxylin. Many hematoxylin solutions contain alum, see Delafield's, Ehrlich's, Harris', Mayer's.

Aluminium Chloride Carmine (Mayer). Dissolve $1 \mathrm{gm}$. carminic acid and $3 \mathrm{gm}$. aluminium chloride in $200 \mathrm{cc}$ aq. dest. Add an antiseptic as formalin or $0.1 \%$ salicylic acid. Employ in same way as carmalum. Gives blue violet color. Very penetrating but not so specific for chromatin as carmalum (Lee, p. 142). 


\section{Alveolar Epithelium of Lungs}

1. Gold sodium thiosulphate (Bensley, R. D. and S. H., Anat. Rec., 1935, 64, 41-49). Inject a mouse intravenously through the tail vein with $100 \mathrm{mg}$. of gold sodium thiosulphate in 1 cc. aq. dest. The mouse dies in about $20 \mathrm{~min}$. from asphyxia. Fix pieces of lung in $10 \%$ neutral formalin, dehydrate without washing in water, clear and imbed in paraffin. Deparaffinise sections and stain in $1 \%$ aq. toluidin blue (tested for polychromatism) and examine in water. The epithelium is raised by increase in volume of ground substance which is stained metachromatically pink while the cells and their nuclei are blue. The color of the ground substance can be changed to blue by alcohol and back again to pink by water. To mount protect against reversing action of alcohol by treating with equal parts freshly prepared $5 \%$ aq. ammonium molybdate (Kahlbaum or Merck) and $1 \%$ aq. potassium ferrocyanide. Dehydrate, clear in xylol and mount in balsam. (Revised by R. D. and S. H. Bensley, Dept. of Anatomy, University of Chicago, Chicago, Ill., April 18, 1946.)

2. Silver nitrate (Bensley, R. D. and S. H., Anat. Rec., 1935, 64, 41-49). Use guinea pigs. Silver Citrate sol. (which see) is injected into lung substance by hypodermic syringe, the roots of the lung being first ligated, until the lung is moderately distended. Cut out pieces, fix in $10 \%$ formalin, imbed in paraffin or celloidin, section, develop with dilute photographic developer and counterstain or examine unstained. The margins of the cells are blackened. For the most delicate results a slow acting, fine grain developer such as the following should be used: phenyl hydrazine hydrochloride, $1 \mathrm{gm}$., sodium sulphite (anhydrous), $10 \mathrm{gm}$.; aq. dest., 100 cc. Caution: Phenyl hydrazine hydrochloride is extremely toxic to some people producing skin reactions. (Revised by R. D. and S. H. Bensley, April 18, 1946.)

Alveolar Fluid. Method for collecting, Terry, R. J., Anat. Rec., 1926, 32, 223$304 ; 1936,64,75$.

Alveolar Foam Cells.-Written by C. C. Macklin, Dept. of Histological Research, The University of Western Ontario, London, Canada. November 28, 1951.-These represent nonphagocytic pneumonocytes which have become free in the alveoli and air tract of the lungs. They may be obtained by the "gash-irrigation" and "washout" techniques (which see). Macklin found in them refractile vacuolelike bodies or vacuoloids (which see) readily demonstrable in fresh mounts by brightor dark-field illumination (Proc. 6th International Congress of Experimental Cytology, Stockholm, 1947, published 1949 , pp. 383-387). In ordinary sections these bodies appear empty or with a very small granule within them. When foreign particles appear in them they are known as "Dust Cells" (which see). Mitochondria in foam cells are mainly in the frothy perivacuoloidal cytoplasm (Macklin, C. C., Biol. Bull., 1949, 96, 173-178).

Alveolar Phagocytes of Lungs, see Dust Cells.

Alveolar Pores of the lung.-Revised by C. C. Macklin, Dept. of Histological Research, The University of Western Ontario, London, Canada. November 28,1951 -Formalin $(10 \%)$ and Zenkerformalin are among the fixatives suggested. The fixative is injected into the trachea or bronchus at a gravity pressure of 4-6 inches until the lungs are moderately distended. During this operation they are covered with physiological salt solution. The lungs are then immersed in fixative for days or even weeks. Slices about $1 \mathrm{~cm}$. thick are cut, imbedded in soft paraffin and sections are made at $100 \mu$ or more. Resorcin-fuchsin and other stains may be used. The blood in the capillaries is a useful guide. The pores can be identified by their rounded edges (Macklin, C. C., Arch. Path., 1936, 21, 202-216).

In lungs fixed by immersion of the flayed intact thorax (IIT) or perfusion of the pulmonary capillaries of the intact thorax (PIT) the pores, in thick sections, appear as short narrow tunnels with funnel-shaped entrances joining neighboring alveolar spaces. Their extremely thin walls are composed of parts of the contiguous capillaries. (See "Fixation of the Uncollapsed Lung" and "Dust Cells".) Thus seen a pore is the empty sheath or vagina from which a process of a pneumonocyte (septal cell, alveolar wall cell, etc.) has been withdrawn. When such a process cannot be withdrawn because it is bulbous the cell is seen as a dumbbellshaped structure with the thin connecting part in the pore. In its functional state this vagina is occupied by a part of a pneumonocyte, and the latter is thus placed where nutrition from the capillaries is constantly available. Any good fixative suffices to show pores by these methods. When pieces of lung tissue are fixed by immersion the ensuing contraction usually closes the pores to that they cannot be seen 
(Macklin, C. C., Jour. of Thor. Surg., $1938,7,536-551$, Macklin, C. C., The Lancet, Feb. 24, 1951, 432-435). Sce also Loosli, C. G., Arch. Path., 1937, 24, 743 , and Loosli, C. G., Amer. J. of Anat., $1938,62,375$. When the entire fresh collapsed lungs are fixed by filling them with preservative via the trachea, or when parts of the lung are so filled via a bronchus, the alveolar walls are compressed and flattened, and here the pores appear as holes punched in thick paper; and if the distention has been great the morphology of the pore and its relation to the environing capillary is not obvious (Macklin, C. C., Trans. Roy. Soc. Can., Sect. V, 1934, 28: p. 28; J. Anat. [pt. 2] 1935, 69, 188; J. Assoc. Am. Med. Coll., 1935, 10, 265; Arch. Path., 1936, 21, 202). Pores are well seen "en face" in frozen sections which have been dried on the slide.

The foregoing description refers to true pores. A second type of communication between adjoining alveoli occurs in "medical emphysema" or alveolar ectasia and is due to the wasting of the alveolar sidewalls or bases. These openings may be very large and numerous, and the walls are then said to be riddled with them. Pathologists are familiar with this type of "false pore". The true type of pore is usually undiscernible in the collapsed lung tissue examined by pathologists.

Alveolar Size, in the lungs. For techniques used in determining alveolar size in ten mammalian types see the papers of W. S. Hartroft and C. C. Macklin in the bibliography of the 26th Lewis Linn McArthur Lecture of the Frank Billings Foundation; by C. C. Macklin in Proc. of the Institute of Medicine of Chicago, $1950,18,78-95$.

Alveolus of the Lungs. The morphology, in sections, can best be demonstrated in small animals by immersion of the fresh skinned intact thorax in a fixative, such as Bouin's fluid. See Fixation of the uncollapsed lung.

Alzheimer's Modification of Mann's eosinmethyl blue for neuroglia and degenerate nerve fibers as given by Mallory (p. 245) is abbreviated. Fix thin slices, 14 days, in Weigert's Neuroglia Mordant $+10 \%$ of formalin. Wash 8-12 hrs. in running water. Mordant $10 \mu$ frozen sections 2-12 hrs. in sat. aq. phosphomolybdic acid. Wash 2 changes aq. dest. Stain in Mann's Eosin Methyl Blue 1-5 hrs. Wash quickly in aq. dest. until color "clouds" are no longer given off. Treat with $95 \%$ alcohol until gray matter becomes light blue and white matter pink or bright red. Dehydrate quickly in absolute alcohol, clear in xylol and mount in balsam. Normal axis cylinders, purple or deep blue; degenerating ones, red; neuroglia fibers, dark blue; and neuroglia cytoplasm, pale blue. Mallory states that change from blue to red staining of axis cylinders occurs as soon as 48 hrs. after expcrimental lesion.

Amanil Garnet H., see Erie Garnet B.

Amaranth (CI, 184)-azo rubin, Bordeaux, Bordeaux SF, fast red, naphthol red S, $\mathrm{C}$ or $\mathrm{O}$, Victoria rubin $\mathrm{O}$, wool red-An acid mono-azo dye used long ago by Griesbach, H., Zeit. wis. mikr., 1886, 3, 358-385 to color axis cylinders.

Amebae. The techniques that have been and can be employed for the organisms are almost endless. In brief, these are their examination in the living condition unstained using ordinary, phase and dark ficld microscopes, implemented or not by supravital stains. As a beginning, the method of Meyers is suggested: washed amebae in a clean vessel are allowed to settle to the bottom. They are then fixed in warm Bouin's Fluid, concentrated in a centrifuge tube and stained with Grenacher's borax carmine and Indulin (Meyers, E. H., Trans. Am. Micr. Soc., 1933, 52, 58). For showing cytoplasmic components of Amoeba Proteus, see Mast, S. O. and Doyle, W. L., Arch. f. Protistenk., 1935, 86, 155 . To determine the density of amcbae a starch Density Gradient is recommended by Lovtrup, S. C. rend. Lab. Carlsberg, Sér. Chim., 1950, 27, 137-144. For determination of permiability of nucleus see Monné, L., Proc. Soc. Exp. Biol. \& Med., 1935, 32,1197 . The technique for enucelation of fresh water amebae advised by Holter, H. and Kopac, M. J., J. Cell. and Comp. Physiol., 1937, 10, 423 is recommended. See Entameba.

Amethyst Violet (CI, 847)-heliotrope B, iris violet-It is a basic azin dye of little importance to histologists.

Amine Oxidase. Oster, K. A. and Schlossman, N. C., J. Cell. Comp. Physiol., $1942,20,373-378$. As explained by Glick, p. 93, the method is based on detection of aldehyde formed by amine oxidation. The Fuelgen sulphurous acid agent (see Thymonucleic Acid) is employed for microscopic visualization of aldehyde. Binding of naturally occurring aldehydes and "plasmal" is accomplished with bisulphite so that they do not invalidate the method. Because the color produced diffuses, localization is subject to criticism.

Place frozen sections of fresh tissue in $2 \%$ aq. sodium bisulphite at $37^{\circ} \mathrm{C}, 24$ hrs. wash thoroughly. Control sections immersed in Feulgen agent should remain colorless showing that free 
aldehyde has been bound. Incubate these sections in $0.5 \%$ tyramine hydrochloride in $\mathrm{M} / 15$ phosphate buffer $\mathrm{pH}$ 7.2 at $37^{\circ} \mathrm{C} .24 \mathrm{hrs}$. Incubate other control sections in same solution minus tyramine. Immerse both test and control sections in Feulgen agent. Examine when quickly produced blue color seems to be at maximum. Foci of enzymatic activity, blue in convenient contrast with reddish purple of "plasmal" (see Aldehydes).

Amidonaphthol GR, see Azophloxine GA. Amino Acids, see Alloxan Reaction, also Schmidt, C. L. A., The Chemistry of the Amino Acids and Proteins. Springfield, Charles C Thomas, 1938, $1031 \mathrm{pp}$. See paper chromatography added by Roberts.

Aminoacridines, some are strong antiseptics, do not stain skin (Albert, A. and Ritchie, B., J. Soc. Chem. Ind., 1941, $60,120)$.

Amitosis is direct nuclear division by constriction without formation of a chromatin thread. No special technique required. Study of embryonic membranes and of bladder of mouse (Dogiel, A. S., Arch. f. Mikr. Anat., 1890, 35, 389-406) is suggested.

Ammonia Carmine (Ranvier). A suspension of carmine in water, with slight excess ammonia, is allowed to evaporate in air. If it putrefies so much the better. Dissolve the dry deposit in aq. dest. and filter (Lee, p. 145).

Ammoniacal Silver for branchioles-Written by C. C. Macklin, Dept. of Histological Research, The University of Western Ontario, London, Canada. November 28,1951 -This is useful to demonstrate the two types of epithelium of the finest bronchioles, as in the albino mouse. There are two stock solutions. Solution 1: Five grams of silver nitrate are dissolved in $300 \mathrm{ccms}$. of distilled water and dilute aqua ammoniae is added until the precipitate is nearly redissolved; filter; add water to make 500 ccms. Solution 2: One gram of silver nitrate is dissolved in a small quantity of water and poured into half a liter of boiling water. Rochelle salt ( $0.83 \mathrm{gm}$.) is dissolved in a small quantity of water and added to the boiling solution, which is then boiled for half an hour till a gray precipitate gathers at the bottom of the flask. Filter hot; add water to make $500 \mathrm{ccms}$. In using, equal parts of the two solutions are mixed. The separate solutions, kept in the dark, remain good for a month or two.

The etherized animals are exsanguinated, the lungs collapsed by carefully nicking the diaphragm and filled via the trachea with the ammoniacal silver solution. At the end of two minutes the lungs are emptied, refilled with $10 \%$ formalin, and the trachea is tied. The material is placed in $10 \%$ formalin for a day or more. Frozen, paraffin, or celloidin sections are cut and exposed to direct sunlight or weak photographic developer. Flattened frozen sections are useful in affording surface views of lengths of bronchiolar epithelium. The following description is from C. C. Macklin (Canad. Jour. of Research, D, 1949, 27, 50-58-Bibliography): "Two definite types of cells, dark and light, are revealed in the terminal bronchiolar epithelium by this supravital silverization. The dark cells are ciliated and scattered among the light unciliated cells as singles, pairs, triads or larger groups to form a striking and characteristic surface pattern. The cuticles of the dark cells, far outreached by the neighboring light cells, appear en face as dense crowds of small uniform golden brown granules. Viewed laterally these show as pairs in vertical alignment, and make two layers with a thin lighter band between. The sides and bases are indicated by deposits of black grains. Near the sharply marked margin of the definitive epithelium the dark cells are shorter, more dispersed, and formed like truncated pyramids with narrow densely impregnated apices on some of which are single delicate points instead of discrete cilia. The light cells have bulging villuslike free ends and make flutings on the contiguous dark cells. Small silverized particles sparsely stipple their air surfaces, and rounded plaques crown their summits. A similar dicellular picture is found in the terminal bronchiolar epithelium of the golden hamster."

Ammonium Molybdate, as mordant for Mann's stain and Weigert-Pal (Perdrau, J. R., J. Path. \& Bact., 1939, 48, 609610. Recommended by Bethe as a fixative for supravital methylene blue, see Lillie, p. 245.

Amoeba, see Entameba.

Amphinucleolus (G. amphi on both sides). A nucleolus which is double consisting of both acidophilic and basophilic parts, the former is usually a central core and the latter plastered on its surface.

Amphioxus, as an ancestor of vertebrates of interest to all, see the technique of Conklin, E. G., J. Morph., 1932, 54, 69. Amphophilic, see Staining.

Amyl Acetate, as solvent for imbedding tissues (Barron, D. H., Anat. Rec., 1934, 59, No. 1 and Suppl., 1-3); as a clearing agent for embryological material (Drury, H. F., Stain Techn., 1941, $16,21-22)$. 
Amyl Alcohol. Merck lists 3, commercial, normal and tertiary. It mixes with 95\% alcohol and with xylol. Hollande (A. C., C. rend Soc. de Biol., 1918, 81, 223-225) was the first to recommend amyl alcohol as a substitute for absolute alcohol in the dehydration of specimens stained by the Romanovsky and Giemsa techniques.

Amyl Nitrite. McClung (p. 620) says that this may serve as a dilator of peripheral capillaries when a complete injection of small blood vessels is required. Add it to the ether at time of anesthetization.

Amylase, micromethod for (Pickford, G. E. and Dorris, F., Science, 1934, 80, 317319). This was later used with marked success by Dorris (F., J. Exp. Zool., $1935,70,491-527$ ) in a study of relation between enzyme production and histological development of gut of amblystoma. An extract is made, adjusted to proper $\mathrm{pH}$, applied to slides coated with a starch-agar solution and incubated. The slides are then washed, the coating fixed in formalin and colored with dilute iodine solution. Sites of amylase activity are clear or pink staining spots. For necessary details, see author's description. van Genderen and Engel (H. and C., Enzymologia, 1938, $5,71-80$ ) localized this enzyme by analysis of horizontal sections through the intestinal wall. It was found that it is present in rabbits in maximum amounts in Brunner's glands. Holtér and Dogle (C. R. Lab. Carlsberg, Sér. Chim., 1938, $22,219-225$ ) observed that in amebae it is concentrated in association with the mitochondria which they assume to be carriers of amylase. See Barnes, J. M., Brit. J. Exp. Path., 1940, 21, 264-275 for identification of amylase in lymphocytes and polymorphonuclear leucocytes.

Salivary amylase digests glycogen in sections, but does not alter mucus, cartilage matrix and other mucins which give similar histochemical reactions. Lillie, R. D., Stain Tech., 1947, 22, 6770 , recommends a commercial preparation of diastase for this purpose, but Dempsey and Wislocki prefer saliva, since the commercial preparations apparently contain traces of mucinase which attacks mucus, cartilage and mast cell granules.

Amyloid (G. amylon, starch and eidos, resemblance), a substance which accumulates in pathological conditions in the tissue fluids between cells particularly in chronic infections. Methods for its detection are fully described by Mallory and Parker (McClung, pp. 417-419). From numerous tests the following are selected :
1. Iodine and sulphuric acid: Stain section lightly with Lugol's iodine. Place in $1-5 \%$ aq. or conc. sulphuric or hydrochloric acid. Color of amyloid changes quickly from red through violet to blue or it may become deep brown.

2. Methyl-violet: Treat frozen sections of fresh, formalin or alcohol fixed tissue with $1 \%$ aq. methyl violet, 3-5 min. Wash in $1 \%$ aq. acetic acid, and remove acid by washing carefully in water. Examine in glycerin or water. Amyloid is violet and tissue blue. Colors will be retained longer if sections are mounted in Levulose Syrup.

3. Iodine green: Fresh or hardened sections are stained $24 \mathrm{hrs}$. in $0.3 \%$ aq. iodine green. Wash in water and examine in water or glycerin. Amyloid is stained violet red and tissue, green.

4. Mayer's stain: Transfer paraffin sections immediately after cutting to $0.5 \% \mathrm{aq}$. methyl violet or gentian violet at $40^{\circ} \mathrm{C}$. for $5-10 \mathrm{~min}$. Rinse in water and differentiate in $1 \%$ aq. acetic acid for 10-15 min. Wash thoroughly in water. Change to $\frac{1}{3}$ sat. aq. alum and wash it off in water. Place section on slide and let water evaporate. Remove paraffin, clear in xylol and mount in balsam. Crystal violet and iodine green can be employed in the same way.

A Congo red test has been described (Taran, A., J. Lab. \& Clin. Med., 1936$37,22,975-977)$ and a polysaccharide has been isolated from amyloid bearing tissues which closely resembles chondroitin-sulphuric acid obtained from infantile cartilage (Hass, G., Arch. Path., 1942, 34, 92-105).

As pointed out by Highman, B., Arch. Path., 1946, 41, 559-562 the staining methods for amyloid are in general satisfactory when employed by skilled workers. However, when stained sections are mounted in glycerin Apathy's syrup, or some such medium, they tend to fade quickly, or the stain diffuses out into the surrounding tissue, or mounting medium, and the nuclei are seldom sharply colored. Highman therefore recommends staining of deparaffinized sections of formalin fixed tissues in iron hematoxylin $5 \mathrm{~min}$., washing in water, staining in $0.5 \%$ crystal violet or methyl violet in $2.5 \%$ aq. acetic acid, washing again in water and mounting in Lillie's Apathy's syrup modified by addition of $50 \mathrm{gm}$. potassium acetate or $10 \mathrm{gm}$. sodium chloride to $100 \mathrm{cc}$. of syrup. He also gives a technique for mounting in clarite.

See Mallory-Heidenhain rapid one step stain. 
Amniotic Fluid, technique for study of absorption from, Wislocki, G. B., Bull. Johns Hopkins Hospital, 1921, 32, 93.

Anaplasma is a small spherical body found within red blood cells in anaplasmosis diseases. There are two types $A$ marginale and $A$ centrale depending upon whether the bodies are situated near the margin or in the centers of the cells. The bodies are supposed to be parasites consisting of nuclear material with little if any cytoplasm. Anaplasmosis is important economically as a group of tick borne diseases of domestic animals. For demonstration stain blood smears by the methods of Giemsa or Wright.

Anethol is anise camphor suggested as a medium in which to soak tissues before making frozen sections (Stephanow, Zeit. wiss. Mikr., 1900, 17, 181).

Anethole Clearing Agent.-Written by Joan Haberman, Parkland, Washington. March 10,1951-Anethole is a useful agent in clearing segments of brain tissue because of its refractive index. The basic principle is that homogeneous tissue, which has been bleached and dehydrated, may be cleared by placing in an oil of similar refractive index was discussed by Groat, R. A., Stain Techn., 1941, 16, 111-117. Brain tissue consists primarily of protein and lipids and is therefore not homogeneous as are other types of tissue such as muscle. Since protein forms the framework of the brain, it necessitates that the lipids be removed to obtain a homogeneous tissue.

The lipid composition of the brain tissue must be known in order to perform an adequate extraction. According to Koch, as quoted by A. P. Mathews, Physiological Chemistry. Baltimore: Williams and Wilkins Co., 1939, the lipid composition is as follows: phospholipids $-27.3 \%$ of dry matter; cerebrosides $-13.6 \%$ of dry matter; cholesterol $-10.9 \%$ of dry matter. The phospholipids, according to G. A. Hill and $K$. Kelly, Organic Chemistry. Philadelphia: Blakiston Co., 1943, are composed mainly of :lecithin-48 p.p.m., soluble in ether and in alcohol; cephalin-200 p.p.m., soluble in ether, insoluble in alcohol; sphingomyelin -56 p.p.m., insoluble in ether, soluble in hot alcohol. These are all insoluble in acetone. The cerebrosides are soluble in hot alcohol, insoluble in ether. Cholesterol can be extracted with ether and alcohol. (The data on solubilities are taken from Harrow, B., Textbook of Biochemistry. Philadelphia: W. B. Saunders Co., 1943).

Brain tissue must first be dehydrated by using the alcohol series starting at
$35 \%$ or higher if one wishes to risk more shrinkage and changing every few hours depending upon the size of the tissue. The lipids are then removed by placing the tissue in $100 \%$ alcohol at 60 degrees centigrade for 1-2 days with frequent changes in solution. The remaining lipids are extracted with ether for 2 days or longer depending upon the size of the tissue. The procedure results in a tissue that consists largely of protein and gives a refractive index of 1.560 as determined on the Abbé refractometer.

By placing the tissue in an oil of very similar refractive index it will soon become transparent when brightly illuminated. Anethole $\left(\mathrm{C}_{10} \mathrm{H}_{12} \mathrm{O}\right)$ having a refractive index of 1.5614 was found to be the oil of choice. It is colorless, very slightly soluble in water, soluble in alcohol and ether, and is liquid at ordinary temperatures. It can be obtained from the Eastman Kodak Company, Rochester, New York.

After the tissue has been placed in anethole the container should be left uncovered so that the displaced ether may escape.

The transparency of the tissue depends upon the completeness of the lipid extraction. Larger pieces of tissue such as $4 \times 6 \times 3 \mathrm{~cm}$ must be kept in $100 \%$ alcohol for a week and in ether for 1-2 weeks. Frequent changes of solution are advisable. This block of tissue will still appear only translucent when cleared in anethole. However, sections of $\frac{1}{2}$ to $1 \mathrm{~cm}$. in thickness cut from this block will be sufficiently clear to insure visualization of injected vessels etc. under the binocular with good illumination. It is probable that a large piece of tissue could be cleared satisfactorily if the alcohol and ether extraction were carried out by means of an intermittent siphoning distillation extractor.

Angina, see Vincent's Angina.

Angstrom Unit. $1 \AA=0.1 \mathrm{~m} \mu=0.0001 \mu=$ $10^{-7} \mathrm{~mm}$.

Anhydrase, see Carbonic Anhydrase.

Anilin Blue Alcohol Soluble, see Spirit Blue.

Anilin Blue, WS (CI, 707)-China blue, cotton blue, marine blue $\mathrm{V}$, soluble blue $3 \mathrm{M}$ or $2 \mathrm{R}$, water blue (Wasserblau) $-\mathrm{A}$ mixture of trisulphonates of di-phenyl rosanilin and tri-phenyl pararosanilin. Conn (p. 135) explains that this designation (like acid fuchsin) applies not to a single compound but to a group of dyes. Anilin blue is, nevertheless, the best stain for Collagenic Fibers and is employed for many other purposes. 
Anilin Crystal Violet 1. Ehrlich's. Shake up $5 \mathrm{cc}$. anilin oil with $95 \mathrm{cc}$. aq. dest. Filter and to $84 \mathrm{cc}$. of filtrate add $16 \mathrm{cc}$. sat. alc. crystal violet. Leave $24 \mathrm{hrs}$. before using. After about 10 days staining potency decreases (Mallory, p. 89).

2. Stirling's. Crystal violet, $5 \mathrm{gm}$.; abs. alc., 10 cc.; anilin oil, 2 cc., aq. dest., 88 cc. Keeps well (Mallory, p. 90 ).

See Anilin Crystal Violet and Gentian Violet.

Anilin-Fuchsin Methyl Green method for mitochondria. This technique is based on Altmann's method. It was used by Benslcy to stain tissues fixed in his Acetic-Osmic-Bichromate fluid. Cowdry recommends instead fixation in the better penetrating Regaud's fluid.

Fix small pieces in freshly prepared Regaud's fluid (3\% aq. potassium bichromate 4 parts, commercial formalin 1 part). Ordinarily it is not necessary to neutralize the formalin before hand by saturating it with magnesium carbonate. Keep in ice box and change the fluid every day for 4 days. Pour off fixative and mordant in $3 \%$ aq. potassium bichromate 8 days changing every second day. Wash in running water over night or in several changes of water. Dehydrate in alcohol, clear in xylol, imbed in paraffin and cut sections about $4 \mu$ thick. Pass mounted sections through xylol and alcohol to water. Dry the slide with a cloth except area covered by sections. Pour on anilin acid fuchsin and heat to steaming over a spirit lamp. (To make this saturate 125 cc. aq. dest. with anilin oil by shaking the two together. Filter and add 15 gms. acid fuchsin to $100 \mathrm{cc}$. of filtrate. Allow to stand 24 hrs. before using. It lasts about a month.) Allow to cool and stain about 6 min. Pour stain back into bottle. Remove most of remainder, except from sections, with a cloth or filter paper. Rinse in aq. dest. about $1 \mathrm{~min}$. Allow $1 \%$ aq. methyl green, added with a dropper, to flow over sections and counter stain them. This usually takes about 5 sec. but the time must be determined by trial. Wash off excess methyl green in $95 \%$ alcohol, dehydrate quickly in absolute, clear in toluol (or xylol) and mount in balsam. The mitochondria are stained crimson and the nuclei green. For colored illustrations see Cowdry, E. V., Contrib. to Embryol., Carnegie Inst. of Washington 1917, No. $11,27-43$. If the methyl green does not stain intensely enough treat the scctions, before coloration with fuchsin, with $1 \%$ aq. potassium permanganate 30 sec. followed by $5 \%$ oxalic acid
30 sec. and wash in water. More methyl green can be retained by blotting the sections after staining in it with filter paper and by then passing directly to absolute alcohol. If the time of fixation and mordanting is reduced much below that specified the fuchsin itself may not color with sufficient intensity. Such preparations hold their colors for a year or more unless they have been unduly exposed to sunlight, or the balsam is acid.

Anilin Fuchsin Picric Acid, see Altmann's method for mitochondria.

Anilin Fuchsin Toluidine Blue and Aurantia, see Champy-Kull method for mitochondria.

Anilin Gentian Violet usually credited to Ehrlich. Rarely is its composition given exactly the same by any two people. The "emended formula" (Soc. Am. Bact.) is A: $2.5 \mathrm{gm}$. crystal violet ( 85 per dye content) $+95 \%$ ethyl alcohol, 12 cc. B : anilin oil 2 cc. + aq. dest. 98 cc. (shake, leave few minutes, filter). Mix A and B. (McClung, p. 137.)

Anilin Oil. A good product is easily obtainable. It is much used in the making of stains (cf. anilin fuchsin) and to clear tissues from $95 \%$ alcohol and even sections from $70 \%$. Lee (p. 71) says that it should not be employed after fixation in osmic acid and that unless removed by chloroform or xylol it will give the tissues and mounting medium a brown coloration.

Anilin Red, see Basic Fuchsin.

Anilin-Safranin (Babes). Aq. dest., 98 cc.; anilin oil, 2 cc.; excess of safranin $O$. heat in flask in hot water bath at 70$80^{\circ} \mathrm{C}$. Cool, filter and use filtrate. This is an excellent nuclear stain, but it must be freshly prepared every month or so. It colors very promptly. Differentiation is in $95 \%$ alc. or even in Acid Alcohol.

Anilin Yellow, see McClung, 1950, p. 439. Anterior Chamber TransplantationWritten by Harry S. N. Greene, Laboratory of Pathology, Yale University School of Medicine, New Haven. Sept. 11,1951 - The anteriol chamber of the eye possesses many advantages over other bodily regions as a transplantation site. The technique of transfer is simple, a high percentage of takes is obtained, and the growing tissue can be followed by direct visual observation or even subjected to microscopic examination. A further advantage derives from the fact that the chamber supports the growth of heterologous embryonic or cancer tissue whereas in other bodily sites such tissues generally fail to survive.

The technique varies somewhat with 
the size of the animal used. Animals larger than mice are immobilized on an operating board with tie strings in such a manner that the desired eye presents to the operator. The cornea is anaesthetized by contact with a $5 \%$ aqueous solution of cocaine administered with a medicine dropper. An incision is made at approximately the midpoint of the upper border of the limbus utilizing a sharp double-edged corneal knife. The knife is directed slightly forward so that the blade enters the anterior chamber without damage to the iris. The pressure necessary to pierce the cornea is sufficient, even with a sharp knife, to rotate the eyeball beneath the lower lid and conceal the operative field. A short, quick thrust of the knife, however, results in an adequate opening and the temporary obscurity is inconsequential. Withdrawal of the blade is accompanied by the escape of a small amount of aqueous humor, but unless the iris has been cut by a misdirected knife, there is no bleeding.

Transfer of the tissue is effected by means of a trocar commensurate in diameter with the corneal incision. The trocar should be equipped with a tight-fitting plunger and a short bevelled mouth with all edges filed to smoothness. A small fragment of tissue is placed in the mouth of the trocar and forced into the barrel, a step greatly facilitated by withdrawing the plunger to produce suction. The tip of the trocar is inserted through the incision a short distance so that the bore is entirely within the chamber and the fragment is expelled. It is important that all manual pressure about the eye and head of the animal be released before withdrawing the trocar to prevent escape of the fragment. Finally, the fragment is forced into the inferior angle of the iris by applying light pressure along the corneal surface with a blunt instrument. The incision is not closed.

The knife should be sharp and of such width that a stab wound will admit the trocar. The use of a narrow knife necessitates side cutting to obtain a sufficient opening and this may be attended by iris damage with consequent bleeding. Pointed surgical blades or single-edged corneal knives are undesirable, for the triangular cut produced heals slowly and may result in herniation of the iris.

The fragments of tissue to be transplanted should be cut with sharp instruments and should not exceed $1 \mathrm{~mm}$. in diameter. The careful selection of fragments is of the utmost importance in the heterologous transplantation of human cancer. In addition to essential parenchyma, all human cancers contain stroma and many are infiltrated with desmoplastic connective tissue. Such tissues are adult in nature and will not grow on heterologous transfer. In fact, their presence in quantity will give rise to a foreigh body reaction in the alien host and result in death of the transplant. It is essential, therefore, that selection be based on the content of tumor parenchyma and some knowledge of gross pathology is necessary for such a differentiation. Frozen sections obtained from different areas of the tumor mass are of considerable aid in some cases. It is obvious that necrotic tumor or normal tissue adjacent to the tumor are not suitable materials for transfer.

The placing of the fragment in a wedged position in the inferior angle of the iris is important for early vascularization. Occasionally, fragments so placed work loose shortly after operation and an examination with the required readjustment is desirable before returning the animals to the colony. Fixation occurs within a few hours and further check is unnecessary.

A slight modification of the technique is desirable when applied to mice or to newborn animals of larger species. General anesthesia is preferable and may be effected with ether or nembutal. Both hands are employed in the operation and preliminary loading of the trocar is necessary. The trocar is made by shortening the bevel at the tip of a 20-gauge hypodermic needle. A suitable, tight-fitting plunger can be manufactured or obtained simply be selecting a wire stylet of proper size from the stock supplied with the needles and applying a knob of plastic material to one end.

The mouse is held loosely in the left hand and the lids of the right eye forced apart with the thumb and index finger. Slight pressure with the fingers causes the eye to protrude sufficiently to allow adequate exposure for the operation. An incision is made at the upper border of the limbus using a double-edged knife of the same caliber employed in larger animals.

The trocar, held between the thumb and middle finger of the right hand, is inserted into the chamber through the incision and the fragment expressed by pushing the plunger with the index finger. In order to prevent extrusion and escape of the fragment through the incision, all pressure exerted on the animal by the left hand should be re- 
leased before withdrawing the trocar. Trouble may be encountered in preventing the escape of soft, slippery tissues such as embryonic brain. This difficulty may be circumvented by incising the iris as well as the cornea at the limbus and directing the trocar behind the superior half of the iris, through the pupil and into the inferior portion of the anterior chamber. With withdrawal of the trocar, the fragment is almost invariably caught at the pupillary border and retained in the chamber.

The tissue concerned may be transplanted immediately or stored at icebox temperature for several days before use. It is essential that the material be free of infection and that surgical sterility be maintained throughout all manipulations.

Aside from careful technique, the success or failure of anterior chamber transfer depends on the nature of the tissue used and the species of the recipient host. Adult, embryonic and cancer tissues grow on homologous transfer, while benign tumors and precancerous tissues fail to survive, and heterologous transfer is successful only in the case of embryonic tissue and cancer. In the selection of recipient species for the heterologous transplantation of cancer, it should be noted that transfer between species with the same type of Vitamin C metabolism (man and guinea pig) is comparatively easy, while transfer between species with different types (man and mouse) is difficult.

Takes are first recognized by increase in size and vascularization of the transplanted fragment. The time required varies within wide limits; 1 day in the case of homologous embyronic tissues and 3 months in the case of a heterologous glioblastoma multiforme. In the former instance, the growing transplant may fill the chamber in a week while in the latter, 6 to 8 months may be required. Serial transfer is readily effected with fragments of the first generation growth. For details see Greene, H. S. N., Cancer Res., 1943, 3, $809-822 ; 1917,7,491-501$, and Yale J. Biol. \& Med., 1950, 6, 611-620.

Anthracosis. The deposition of carbon, usually in lungs and mediastinal lymph nodes, distinguished by its resistance to solvents and bleaching agents and by its blackness. See Carbon.

Anthrapurpurin, see Alizarin SX.

Anthraquinone Dyes. Derivatives of anthracene through anthraquinone. Acid alizarin blue $\mathrm{GR}$ and $\mathrm{BB}$, alizarin, alizarin red $\mathrm{S}$, purpurin.
Antibiotics, influence on dehydrogenase. Systems of bacteria, see 'Triphenyltetrazolium Chloride.

Anticoagulant Solutions have been very carefully studied by Leichsenring, J. M., et al., J. Lab. \& Clin. Med., 1939-40, 25, $35-44$. They found that $1.6 \%$ potassium oxalate prepared from dried salt is most nearly isotonic for human blood. Wintrobe, M. M., Clinical Hematology, Philadelphia, Lea \& Febiger, 1942, 792 pp. advises $0.06 \mathrm{gms}$. of ammonium oxalate and $0.04 \mathrm{gms}$. of potassium oxalate for $5 \mathrm{cc}$. of blood. He dissolves 1.2 $\mathrm{gm}$. ammonium oxalate and $0.8 \mathrm{gm}$. potassium oxalate in $100 \mathrm{cc}$ aq. dest. and adds $1 \mathrm{cc}$. formalin to prevent deterioration. Then he measures out with a burette $0.5 \mathrm{cc}$. into each of the containers and lets it dry before taking in to each $5 \mathrm{cc}$. of fresh blood. Heparin is also advised but it is much more expensive. $0.075 \mathrm{gm}$. will prevent coagulation of 5 cc. of blood. See citrate.

Antigens, Histochemical Identification OfWritten by A. H. Coons, Dept. of Bacteriology and Immunology, Harvard Medical School, Boston, Áugust 31, 1951-The localization of antigenic substances in tissue cells can be carried out by the use of specific antibody conjugated with fluorescein. The method furnishes a means for localization and identification with all the specificity of immune reactions. Suitably prepared tissue sections containing an antigenic substance which it is desired to study are flooded with a solution containing antibodies against the substance previously conjugated with fluorescein isocyanate. The antibody molecules precipitate over those sites in the tissue section containing the specific antigen, the excess of fluorescent proteins is washed away, and the tissue section mounted in glycerol. When examined under the fluorescence microscope, the brilliant yellow-green fluorescence of fluorescein is visible over those areas where the immune reaction has taken place.

The preparation and assay of immune sera is described in standard works on immunology. Two such recent ones are Kabat, E. A., and Mayer, M. M., Experimental Immunochemistry, Thomas, Springfield, Illinois, 1948; Boyd, W. C., Fundamentals of Immunology, 2nd Edition, Interscience, New York, 1947. Whenever possible, it is best to start with a purified antigen, since antibodies against any impurities present in the material injected may be represented by antibody in the resulting serum. These antibodies against biological impurities may react in tissue 
sections with antigenic components other than that under investigation. However, it is possible to remove such interfering antibodies by absorbing the anti-serum with material containing the antigenic impurities, but not containing the antigen it is desired to study. Pains should be taken to secure as high a titer of antibody as is feasible. Prolonged courses of immunization and the use of adjuvants may be necessary.

Concentration of antibodies. It is of ten advantageous to concentrate the globulin fraction of such immune sera, either by precipitation of the globulins with half-saturated ammonium sulphate, or the use of low temperature, buffers, and alcohol after the method of Nichol and Deutsch (J. Am. Chem. Soc., 1948, 70, 80). If ammonium sulphate is used, the ammonium ion must be carefully dialyzed from the final globulin solution as otherwise it will interfere seriously with subsequent procedures.

Conjugation with fluorescein isocyanate. The derivatives of fluorescein leading to the isocyanate are not as yet conmercially available. Fluorescein amine may be synthesized and converted to the isocyanate for conjugation to protein by the procedures described by Coons and Kaplan (J. Exp. Med., 1950, 91, 1).

Conjugation of antibody solution with fluorescein isocyanate. The protein content of the serum or isolated globulin fraction to be conjugated must be determined. It should be at least $1.7 \%$. A convenient amount of protein for one run is from 300 to $600 \mathrm{mgm}$. The amount must be known.

Fix a small beaker in an ice-bath and equip it with good mechanical stirring. Put reagents by volume into it in the following order:

Saline $(0.9 \% \mathrm{NaCl})$. to make $100 \%$.

Carbonate - bicar-

bonate buffer $(0.5$

M, $\mathrm{pH} 9.0$ ).

Dioxane (distilled

from and stored

over sodium)..

$15 \%$

Acetone

$7.5 \%$ (minus $2 \mathrm{ml}$ )

When the solution is $4^{\circ} \mathrm{C}$. or below, and the isocyanate solution is ready, add

Protein solution, such that the protein concentration in the final mixture is $1 \%$.

For example, there are $30 \mathrm{ml}$. of a concentrated antibody solution containing $2.0 \%$ total protein. It is desired to conjugate $500 \mathrm{mgm}$. ( $25 \mathrm{ml}$.), holding the remainder for control pur- poses. The reaction mixture:

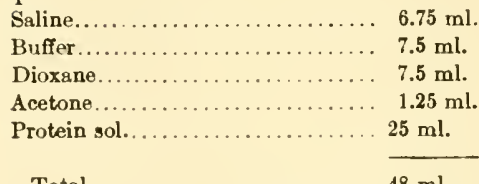

An amount of one of the two fluorescein amine isomers such that there is $0.05 \mathrm{mgm}$. of amine per mgm. of protein (in the example, $25 \mathrm{mgm}$.) is dissolved in dry acetone and treated with phosgene. This procedure should be carried out in a good chemical hood with forced exhaust. Phosgene is led out of the tank through concentrated sulphuric acid, thence to a vessel containing 15 $\mathrm{ml}$. of dry acetone and fitted with a dropping funnel, thence to an empty vessel which serves as a trap, thence through $20 \%$ sodium hydroxide to destroy the excess phosgene, and finally through a trap with a controlled leak to a water suction pump. The whole reaction train should be maintained at a pressure slightly below atmospheric by means of the controlled leak. Phosgene is turned on and allowed to bubble through to remove air and to saturate the acetone in the reaction flask. At the end of a few minutes, the amine dissolved in 5 ec. of dry acetone is added slowly from a dropping funnel to the vessel containing the acetone saturated with phosgene. When all the aminecontaining solution has been added to the reaction vessel, phosgene is allowed to continue bubbling through. During this time the color of the solution in the reaction flask slowly changes from a fluorescent green to a pale yellow. A small amount of heat is generated during the reaction of the amine with phosgene. At the end of about $15 \mathrm{~min}$ utes the reaction flask is transferred to a vacuum still, immersed in a water bath at about $45^{\circ} \mathrm{C}$., and the acetone boiled off under reduced pressure. Small pieces of dry anthracite can be used as antibumping chips. This step removes the phosgene still dissolved in the acetone. When the reaction flask is warm and dry, a greenish brown gum is visible on the wall; this should be dissolved in $2 \mathrm{ml}$. of acetone. This solution is added in toto, drop by drop, to the stirred, chilled, buffered protein solution. (This additional acetone brings the total acetone concentration up to $7.5 \%$.) Stirring is allowed to continue for 16 hours in the cold. The solution is poured into a cellophane sac and dialyzed against repeated changes of saline buffered with 0.01 molar phos- 
phate at $\mathrm{pH} 7.0$ in the cold until the dialysate outside the sac shows fluorescence of less than 1 part in 20 million. This can be roughly determined by eye using a known solution of fluorescein as a standard.

The antibody solution may be further purified by one or more precipitations with half saturated ammonium sulphate followed by re-solution and dialysis, and by precipitation with cold acetone (Coons, et al., J. Immunol., 1942, 45, $159)$ or by $40 \%$ alcohol (Marshall, J. Exp. Med., 1951, 94, 21). Merthiolate (Eli Lilly Co.) should be added as a preservative (1:100 of a $1 \%$ solution).

Despite these chemical purification procedures, substances remain in such fluorescein-protein conjugates which stain some tissue elements. It has been found necessary to shake such conjugates with dry acetone-powder derived from animal tissues, usually liver powder of the species whose tissue it is proposed to study. Such powder may be prepared by homogenizing the tissue in a Waring blendor, washing several times with distilled water in the centrifuge, suspending the product in saline and precipitating it with four volumes of acetone. The acetone precipitate can be harvested on a Buchner funnel and washed with dry acetone to remove water. Such a powder is added to a small aliquot of conjugate in the proportion of $100 \mathrm{mgm}$. of powder to each ml. of conjugate, the paste allowed to stand for an hour at room temperature, and the powder separated in the centrifuge. The yield is higher if the centrifugation is carried out in the cold at $18,000 \mathrm{rpm}$. Two such absorptions are often necessary to remove "nonspecific staining." Merthiolate should be added again as above.

The preparation of tissue sections for use with fluorescent antibody. The problem of preparing tissue sections retaining the antigenic activity of the material sought varies with the antigen in question. The bacterial polysaccharides which have so far been studied survived fixation in Rossman's picric acid-alcohol-formalin followed by paraffin einbedding. Such sections are deparaffinized and hydrated and then stained with the appropriate antibody solution. Care must be taken not to wash out the antigen during the procedures preceding the flooding of the section with labeled antibody. In the case of bacterial polysaccharides, it is necessary to remove the picric acid in $70 \%$ alcohol, in which these polysaccharides are insoluble.

In the case of less stable materials, for example proteins, fixation of the tissue-block is unsatisfactory; sections must be prepared from unfixed material and fixation carried out on the individual section. There are two methods available for the preparation of such sections from unfixed tissue, one that of Linderstrøm-Lang and Mogensen, the other that of Altmann-Gersh, and others.

With either of these two methods, it is necessary to fix the tissue section in some appropriate reagent before applying labeled antibody solutions lest the material looked for be dissolved out of the section during exposure to conjugated antibody. A certain amount of experimentation is necessary with each new antigen in order to find the appropriate fixative. For mumps and influenza $A$ virus and the virus of infectious canine hepatitis, acetone is satisfactory. This is used at room temperature for 15 to 30 minutes followed by drying of the section in an incubator. For proteins, $95 \%$ ethanol by volume (start with absolute ethanol) at $37^{\circ} \mathrm{C}$. for 30 minutes is satisfactory. For $\mathrm{ACTH}$ in the anterior lobe of the hog pituitary, absolute methanol has been shown to be a satisfactory fixative (Marshall, J. Exp. Med., 1951, 94, 21). In general, these organic solvents are best removed by evaporation.

These fixatives and exposure-times are cited as examples only. Any antigen-antibody system chosen for study must be investigated from this as well as from other points of view.

The use of labeled antibody solutions on tissue sections. A small drop of fluorescein-antibody solution is placed over the tissue section, and allowed to react for from 10 minutes to 48 hours, depending on the system under study. Thirty minutes is usually satisfactory. During the reaction, evaporation must be minimized. Usually the reaction can be carried out at room temperature, although incubator temperatures or refrigerator temperatures can be employed. Following exposure to the fluorescent antibody solution, the section should be placed in a Coplin jar containing $0.9 \%$ saline buffered at pII 7.0 with 0.01 molar phosphate, and washed with very gentle motion for 10 minutes. At the end of this time, the slide should be wiped dry except for the area of the section and the section itself mounted under a cover slip in glycerol containing a trace of buffer at $\mathrm{pH} 7$ (commercial glycerol is slightly acid), and examined under the fluorescence microscope.

Control of specificity of staining. 
Because of the ever-present hazard of "nonspecific" staining, careful controls of specificity must be carried out at every step. When the antigenic material sought is foreign to the tissue under investigation, control is relatively simple since the conjugate should fail to stain normal tissue sections. Immunologically specific staining can be greatly diminished or altogether prevented by pretreatment of parallel sections with unlabeled homologous antibody, thereby blocking the antigen. However, replacement of unlabeled by labeled antibody occurs and the time of staining must usually be decreased in order to carry out this control successfully. Failure to stain the tissue under investigation with heterologous labeled antibody is also a suitable control, although the degree of purification of conjugates varies and it is difficult to establish that any two conjugates are strictly comparable. There are so many possible variations that controls appropriate to each situation must be carefully planned.

Fluorescence microscopy. tence microscopy is descr ence microscopy is described section. The amount of $\mathrm{de}-$ posited antibody is quite small, and the fluorescence therefore faint. Light sources adequate for the study of brightly fluorescent materials present in high concentration are not appropriate for use with labeled antibody. Either a 10 amp. carbon arc or a high pressure water cooled mercury vapor arc (General Electric Co., AH-6) are satisfactory. At this level of bombardment, fluorescence of optical elements becomes important. It is necessary to use a quartz condenser since glass ones often fluoresce in the ultraviolet beam. Definition is improved at the cost of intensity by the use of a cardioid darkfield condenser.

Photography. Findings revealed by this method can be recorded photographically using $35 \mathrm{~mm}$. film (either high speed panchromatic film or fast green-sensitive film, e.g., "Photoflure", Eastman). Exposure times vary with the amount of fluorescent antibody deposited and the autofluorescene of the background, varying at "high dry" $(400 \times)$ from 3 to 10 minutes. Focusing on ground glass is very difficult because of the low degree of intensity of the projected image, but can be carried out by the use of a magnifying lens focused on a clear area in the ground glass.

Anyone attempting to carry out these procedures is advised to consult the original papers to which reference has been made (cf. Federation Proc., 1951, 10, 558).

Frozen Sections by the Method of Linderstrom-Lang and Mogensen (Compt.-Rend. Trav. Lab. Carlsberg, série chim., 1938, 23, 27; Coons ,et al., J. Exp. Med., 1951, 93, 173). The principle of this method is quite simple. It involves the quick-freezing of tissues at low temperatures, and cutting them while frozen in a cryostat maintained at -20 . This cold chamber should be equipped with adequate lighting, an insulated window through which the operator can see, and gloved armholes through which he can work. Sections are cut on a good rotary microtome, the knife of which is equipped with a glass guide to keep the sections from curling as they are cut. Convenient improvements in the method are described by Coons, et al. Tissues may be quick-frozen by placing thin slices on the side-walls of test tubes which are then stoppered and plunged but not submerged into alcohol cooled to the temperature of dry ice. They can then be stored in a deep freeze below -20 , or put in the cold cryostat for immediate sectioning. Long storage results in the growth of ice crystals. The tissue is mounted by putting a small drop of water on a previously chilled tissue holder and touching the tissue to it. Within a few seconds the tissue block will be tightly frozen to the holder. Care must be taken to avoid ice formation near the cutting area. The knife blade should be set at an angle of approximately $20^{\circ}$ from the plane of movement of the tissue block. The glass guide should be adjusted so that its upper edge is parallel to and at the height of the cutting edge of the knife. Each section as cut is removed with forceps from the knife blade, placed gently on a cold slide, and thawed by placing a finger under the section. It can then be dried in the air stream from a fan at room temperature. Appreciable movement of tissue components during this momentary thawing has not been troublesome, although it could be serious in some situations.

An adhesive is necessary on the slides; the one in use by the authors quoted has been formalinized gelatin. Acid cleaned, dry glass slides are dipped in $0.5 \%$ gelatin, and placed on end to drain and dry. When dry, they are dipped in $10 \%$ formalin, drained, and allowed to dry. They can then be stored for long periods until required.

Good sections $10 \mu$ thick are easy to cut by this method, and with practice 
sections about $4 \mu$ thick can be cut as a routine.

Antirachitic Vitamin, see Vitamin $\mathbf{D}_{3}$. Antiscorbutic Vitamin, see Vitamin C. Antimony Trichloride, see Carr-Price Reaction.

Aorta, see Arteries and, for an account of technique for measuring clastic properties, Saxton, J. A., Arch. Path., 1942, 34, 262-274.

Aortic Paraganglion (Glomus aorticum). Technique for blood supply and innervation is provided by Nonidez, J. F., J. Anat., 1936, 70, 215-224. Negative results in application of the chromaffin reaction to the rabbit and guinea pig are described by the same author, Am. J. Anat., 1935, 57, 259-293. Carotid glomus is very similar.

v. Apáthy Syrup has been modified by Lillie, R. D. and Ashburn, L. L., Arch. Path., 1943, 36, 432-435. Dissolve acacia (gum arabic) $50 \mathrm{gm}$. and cane sugar $50 \mathrm{gm}$. in aq. dest. $100 \mathrm{cc}$. shaking frequently at $55-60^{\circ} \mathrm{C}$. Add aq. dest. to make up original volume and merthiolate (sodium ethylmercuritheosalicylate) $15 \mathrm{gm}$., or thymol 100 gm. to act as a preservative. This syrup is recommended as a mounting medium for frozen sections stained with Sudan III, or other alcohol soluble dyes.

Aposiderin, see Lillie, p. 127.

Aquax-Written by C. C. Macklin, Dept. of Histological Research, The University of Western Ontario, London, Canada. November 28, 1951-A watersoluble wax for embedding purposes produced by George T. Gurr, 136, New King's Road, London, S.W. 6. It has been found useful for the demonstration of osmiophil granules in pneumopneumonocytes (which see) after levulose mounting (See Levulose Syrup). Its merit is the retention, in sections, of materials which tend to diffuse away in alcohol, clearing fluids and paraffin.

Aqueous Humor, see Anterior Chamber of Eye.

Arachnids, sectioning is facilitated by methods intended to soften Chitin. See also Fleas, Ticks.

Archelline 2B, see Bordeaux Red.

Argentaffine gastrointestinal cells (enterochromaffin cells). Rare even in duodenum. Occur singly, usually in deepest parts of crypts and may be free from epithelium. Cytoplasmic argentaffine granules are of small size, often closely packed together and acidophilic. It is said that they cannot be found in bodies autopsied as late as $4-5 \mathrm{hrs}$. after death (Hamperl, H., Ztschr. f. Mikr.-anat. Forsch., 1925, 2, 506-535).
Fluorescence Microscopy brings out the argentaffine cells sharply because the cytoplasmic granules fluoresce a yellow color. This color is not abolished by fat solvents and is attributed to pterins (McClung, Microscopical Technique, 1950, p. 682). See Eros, G., Zentralbl. f. Allg. Path. Anat., 1932, 54, 385; Jacobson, W., J. Path. \& Bact., 1939, 49, 1).

Two specific methods are advised by Jacobson, W., J. Path. \& Bact., 1939 , $49,1-19$. For both fix in $10 \%$ formolsaline, or $10 \%$ neutral formol, dehydrate in alcohol, clear in cedarwood oil or in methyl benzoate $+2 \%$ celloidin and imbed in paraffin. In the first wash deparaffinized sections $10 \mathrm{~mm}$. in 2 changes glass-dist. water. Transfer for 12-24 hrs. to Fontana's sol. prepared by adding $\mathrm{NH}_{4} \mathrm{OH}$ to $5 \% \mathrm{AgNO}_{8}$ until ppt. is dissolved, then $\mathrm{AgNO}_{3}$ drop by drop until fluid exhibits slight presistent opalescence. Wash in glass-dist. water, 1 min., $5 \% \quad \mathrm{Na}_{2} \mathrm{~S}_{2} \mathrm{O}_{3}, 1 \mathrm{~min}$. and tap water $10 \mathrm{~min}$. Counterstain with carmalum. Dehydrate, clear and mount in balsam. Granules of argentaffine cells appear black. In the second more rapid method dissolve small amount p-nitro-methyloxybenzene diazotate in aq. dest. producing light yellow solution alkalinize with a little $\mathrm{Li}_{2} \mathrm{CO}_{3}$. After about $1 \frac{1}{2}$ min., when pH $10-11$ is reached, color has changed to dark orange-yellow. Immerse sections brought down to aq. dest., in this 30-40 sec. Then wash in aq. dest., $1 \mathrm{~min}$. Granules of argentaffine cells appear dark red in yellow background. Counterstain with hemalum if desired.

Since Dawson, A. B., Anat. Rec., $1944,89,287-294$ has found that a larger number of argentaffine cells are demonstrable in the rat's stomach by Bodian's technique than are reported after silver impregnations like those of MassonHamperl, it is important to try the Bodian Method in the manner suggested by Dawson. Sharples, W., Anat. Rec., $1945,91,237-243$ used the Bodian Method successfully in study of human stomach.

Argentaffine Reaction. This, according to Lison (p. 147) is given by polyphenols, aminophenols and polyamines in ortho and para position. It is a reduction of ammoniated silver hydroxide into metallic silver. He recommends Masson's method for sections : Fix in Bouin's fluid or other fixative. Deparaffinize sections and wash $2 \mathrm{hrs}$. in aq. dest. Treat for 36-40 hrs. in Fontana's fluid in darkness and in a sheltered place. Wash in much aq. dest. Tone with $0.1 \%$ aq. gold chloride (few minutes). Fix in 
$5 \%$ aq. sodium hyposulphite. Counterstain with alum carmine, mount in usual way. To make Fontana's fluid add ammonia drop by drop to $5 \%$ aq. silver nitrate until ppt. formed is exactly redissolved; then carefully drop by drop $5 \%$ aq. silver nitrate until appearance of persistent cloudiness and the liquid does not smell of ammonia. Decant before employing. See also Clara, M., and Canal, F., Zeit. f. Zellf. u. Mikr. Anat., 1932, 15, 801-808; Clara, M., Ergeb. d. Anat. u. Entw., 1933, 30, 240-340.

Arginase. It is possible to localize arginase in the cytoplasm and nuclei of liver cells by Behren's technique (Zeit. Physiol. Chem., 1939, 258, 27-32). Finely ground tissue is dried to powder in frozen condition. It is then suspended and centrifuged in different mixtures of benzene and carbon tetrachloride. The nuclei only are found in the lowest layer, next comes nuclear debris and above this cytoplasmic debris. His analysis showed arginase present in the same concentration in the nuclei as in the cytoplasm. Blaschko and Jacobson (Bourne, p. 217) remark that this is the first instance of the demonstration of an enzyme in the cell nucleus.

Arginine Reaction. The method of Serra, J. A., Stain 'Techn., 1946, 21, 5-18 is detailed by him as follows: Prepare tissue as described under Ninhydrin Reaction.

" 1 . Before the reaction the pieces or sections are hardened with $10 \%$ formaldehyde during 12-24 hours, the formalin being afterwards well washed out. (If the fixative contains formalin this step can be omitted.)

"2. Immerse the pieces for 15 minutes in a mixture consisting of $0.5 \mathrm{ml}$. of diluted $a$-naphthol; $0.5 \mathrm{ml}$. of $\mathrm{N} \mathrm{NaOH}$; and $0.2 \mathrm{ml}$. of $40 \%$ aqueous urea solution. The diluted $a$-naphthol is prepared at the moment of use by diluting a stock solution ( $1 \%$ crystallized $a$ naphthol in $96 \%$ alcohol) 1:10 with $40 \%$ alcohol. The watch glass containing the liquids is placed in an ice-bath and the temperature of the reaction fluid inside it must be $0.5^{\circ} \mathrm{C}$.

" 3 . After 12-15 minutes add $0.2 \mathrm{ml}$. of a $2 \%$ solution of $\mathrm{NaOBr}$. This reagent is allowed to act for 3 minutes and the solution must be well stirred during this time. The $2 \% \mathrm{NaOBr}$ must be freshly prepared by pouring $2 \mathrm{~g}$. (or approximately $0.7 \mathrm{ml}$.) of liquid bromine into $100 \mathrm{ml}$. of $5 \% \mathrm{NaOH}$, with agitation and cooling.

"4. Add another $0.2 \mathrm{ml}$. of $40 \%$ urea solution, stir, and inmediately afterward,
“5. Add another $0.2 \mathrm{ml}$. of $2 \% \mathrm{NaOBr}$ and stir well. The coloration attains its maximum after 3-5 minutes and would last only for a short time if it were not stabilized. To stabilize the coloration:

" 6 . Take the pieces out of the reaction mixture and immerse in pure glycerin for 2-3 minutes and then transfer to fresh glycerin. Repeat the operation another two or three times. The passage through 4 glycerin baths is sufficient to stabilize the coloration for some months, even if the pieces are left at room temperature. (We have not mentioned this improvement in any previous publication.)

"Besides this procedure, which we may call the normal method, there is also another nethod which results in stronger colorations and very satisfactory preparations. To accomplish this, after step 5 the pieces are taken off the reaction liquid and immersed in $\mathrm{NaOBr}$ solution for not more than 3 minutes. Afterwards the coloration is stabilized in glycerin, as in the normal procedure. The pieces are mounted and observed in pure glycerin.

"This reaction is specific for guanidine derivatives in which only one $\mathrm{H}$ atom of one amino group is substituted by a radical of the alkyl or fatty acid type. In proteic compounds it is specific for arginine. As all proteins hitherto analyzed possess arginine in their molecules, the reaction may be used to demonstrate the presence of proteins in general, other compounds with a reactive guanidine group being rare. The test may also be used to characterize the basic proteins."

Arginine Reaction. The method of Thomas, Lloyd E., Stain Techn., 1950, 25, 143 148, has been modified as follows (unpublished) written by L. E. Thomas, Dept. of Biochemistry, University of Missouri, Columbia. July 8, 1951-Bouin's fixative has given the best results. Formaldehyde $(4 \%)$ is almost as good. Carnoy's $(6: 3: 1)$ may be used. After fixation prepare paraffin sections.

1. Remove the paraffin from the slide with xylene and pass it through the alcohols to $70 \%$.

2. Place the slide in a $0.3 \%$ aq. of 8-hydroxyquinoline (oxine) in a Coplin jar for $15 \mathrm{~min}$. at room temperature. This solution is prepared by diluting with water a $1.0 \%$ stock solution of oxine in absolute alcohol.

3 . Move the slide with a quick motion (not allowing it to drain) into a $0.15 \mathrm{~N}$ sodium hypochlorite solution $(0.015 \mathrm{~N}$ with respect to potassium hydroxide). This solution is prepared by standardiz- 
ing Clorox or other suitable hypochlorite solution and using it as a stock solution. Leave the slide in the hypochlorite solution exactly $60 \mathrm{sec}$. at room temperature, holding it stationary.

4. Move quickly (without draining) into a solution containing per $100 \mathrm{cc}$.: $15 \mathrm{gm}$. urea, $70 \mathrm{cc}$. tertiary butyl alcohol and potassium hydroxide to make the concentration of the latter $0.015 \mathrm{~N}$. Move the slide gently in the solution for 10 sec., then transfer to another jar of the same solution for $2 \mathrm{~min}$.

5. Transfer to $100 \%$ tertiary butyl alcohol, moving the slide gently for 10 sec., then place in a second jar of the same reagent for $3 \frac{1}{2} \mathrm{~min}$.

6 . Pass through three changes of xylene for 10 seconds, 1 minute and 2 min., respectively.

7. Mount with Permount containing aniline. This reagent is prepared by dissolving $0.1 \mathrm{cc}$. of aniline in $100 \mathrm{cc}$. of xylene. One volume of this solution is mixed with four volumes of Permount.

Reagents 2, 3 and 4 must be made fresh daily. It is best not to let any water accumulate in the tertiary butyl alcohol.

The three arginine methods are all based on the Sakaguchi reaction, which, in biological materials, is specific for arginine, galegine and agmatine. The latter two are extremely rarely encountered and so for most purposes, it is specific for arginine. The histochemical methods are specific for protein-bound arginine, since free arginine is inevitably removed by the procedures.

Argon, see Atomic Weights.

Argyrophilic Fibers. Because of their affinity for silver, Reticular Fibers are often called argyrophilic.

Arneth Count of lobes of granular leucocytes as a basis for estimation of their relative age. See Lencocyte Counts.

Arsenic 1. Use 10\% neutral formalin in aq. dest. after test with hydrogen sulphide shows absence of trace of metals. To 100 cc. add $2.5 \mathrm{gm}$. copper sulphate. Fix small pieces of tissue 5 days. Wash $24 \mathrm{hrs}$. in running water. Imbed in paraffin. Direct examination of section after removal of paraffin shows arsenic as well defined green granules of hydroarsenite of copper (Scheele's green). If neutral acetate of copper is employed in place of the sulphate the green granules are of acetoarsenite of copper (Schweinfurth's green).

2. Fix pieces of tissue 12-24 hrs. in abs. alc. 50 cc.; chloroform, 50 cc.; pure hydrochloric acid, 3 cc. saturated by passage of pure hydrogen sulphide. In sections the arsenic ppt. appears as yel- low granules: Double coloration with hematein-eosin is possible. Both techniques have been devised by Castel (P., Bull. d'Hist. Appl., 1936, 13, 106112). He has described the histologic distribution of the arsenic. See, however, paper by Tannenholz, H. and Muir, K. B., Arch. Path., 1933, 15, 789-795 who employed a somewhat similar method and were unable to conclude that the yellow crystals were in fact those of arsenic trisulphide. They considered them more probably a sulphur-protein combination.

Consult the detailed account of Osborne's method for arsenic given by Heuper, W. C., Occupational Tumors and Allied Diseases. Springfield: Thomas, 1942, 896 pp. (p. 50). This releates particularly to localization of arsenic in the skin.

The distribution to the several tissues of radioactive arsenic injected intravenously into rabbits as sodium arsenate has been investigated by duPont, $\mathrm{O}$., Irving, A. and Warren, S. L., Am. J' Syph. etc., 1942, 26, 96-118. It is important to determine whether the results conform with those given by the microchemical techniques.

Arsphenamines. The specificity of the silver reaction of Jancsó, N., Ztschr. f. d. Ges. exper. Med., 1929, 65, 98 is questioned by Gomori, G., J. Mt. Sinai Hosp., 1944-45, 11, 317-326 since it may demonstrate other reducing substances beside the arsphenamines.

Artefacts, see Artifacts.

Arteries. If one wishes an elastic artery take a large trunk near the heart such as the aorta, innominate or subclavian; if, on the other hand, a typical muscular artery is required select one further afield like the radial or external carotid. Arterial walls are seldom examined microscopically in vivo because they are relatively large and difficult to get at without injury. An exception in man is the retinal artery which can be seen by ophthalmoscopic examination. To closely observe excised pieces of arteries is all too frequently neglected. The tissue elements are so tightly bound together that to tease them apart for study at high magnification is rather unsatisfactory. However, when the adventitial adipose and connective tissue is stripped off from a fresh specimen, the remainder of the wall can very advantageously be made translucent by treatment with pure glycerin for 1-2 hrs. as described by Winternitz, M. C., Thomas, R. M. and LeCompte, P. M. in their book "The Biology of Arteriosclerosis", Springfield: Thomas, 1938, $142 \mathrm{pp}$. Since the color of the blood is preserved within 
the intramural vessels their arrangement can be studied (see Vasa Vasorum). Fatty substances can also be located because they are not removed by the glycerin.

Chief reliance is ordinarily placed in the appearance of arterial walls when seen in sections of fixed tissue. It is important to remember that, when carry ing blood during life, the lumina are larger and the walls less folded than in the fixed condition. The difference has been graphically demonstrated by Galloway, R. J. M., Am. J. Path., 1936, 12, 333-336. His figures should be examined. For routine purposes fixation in Formalin-Zenker followed by Mallory's Connective Tissue Stain supplemented by Resorcin Fuchsin or Orcein for elastic tissue is satisfactory. Special methods may be needed for Lipids; and for minerals, see Calcium, Iron and Microincineration. Innervation, likewise, is to be studied by methods employed to demonstrate Nerve Endings in other tissues. See Vasa Vasorum. Much literature on techniques is given by various authors in Cowdry, E. V., Arteriosclerosis, New York: Macmillan, $1933,617 \mathrm{pp}$. The investigation of arterial walls is apt to be one sided limited only to structure and composition demonstrated microscopically. It is high time that these lines of study are supplemented by accurate measurement of the physical properties of pulse wave velocity, sound production, elasticity and so on of the same vessels by methods described by Bramwell in the above mentioned volume.

Arterioles, capillaries and venules, in contrast to the much larger arteries and veins, can readily be examined in experimental animals microscopically in the living state. Since they are linked together a single preparation by Sandison's rabbit ear method shows all three, or they may be viewed in the living tadpole's tail or other transparent tissue of lower forms. For convenience, however, it seems best to briefly mention the microscopic techniques for each separately. There is much to choose from. Information is frequently demanded on the condition of the arteriolar walls. This can best be supplied by staining paraffin sections of Formalin-Zenker fixed material with Mallory's Connective Tissue stain or with Masson's Trichrome stain which is closely related to it. Weigert's Resorcin Fuchsin is satisfactory for elastic tissue. The Silver Citrate technique is capable of yielding valuable data on arterioles and capillaries. Because arterioles contain a higher percentage of muscle than any other blood vessel their appearance will vary greatly with the degree of contraction or relaxation of muscle. According to Kernohan, J. W., Anderson, E. W. and Keith, N. M., Arch. Int. Med., 1929, 44, 395-423 in fixed preparatious from normal persons the average ratio of thickness of arteriolar wall to width of lumen is $1: 2$.

Arteriovenous Anastomoses are direct connections between arteries and veins without intervening capillaries. No special histological technique is required for their demonstration in sections but one should look for them where they are particularly numerous, as in rabbits at the tip of the nose (diameter, $80-100 \mu$ ) and in humans in the palms of the hands, the soles of the feet and near the ends of the fingers where their diameter is about $35 \mu$ (Grant, R. T. and Bland, E. F., Heart, 1930, 15, 385-411). The best way is to study them in vivo (Clark, E. R. and E. L., Am. J. Anat., 1934, 55, 407-467).

Arteriosclerosis. The arteries in this condition show changes well demonstrated by Mallory's Connective Stain and its modifications as well as by Weigert's Resorcin Fuchsin. In addition, techniques for Lipids, Calcium and Iron are indicated. Methods for the measurement of physical properties of arteries might well be applied to arteries most and least prone to develop arteriosclerosis. These are summarized by Bramwell, C., in Cowdry's Arteriosclerosis. New York: Macmillan Co., 1933, 617 pp.

Artifacts. Webster defines an artifact as being "in histology, a structure or appearance in a tissue or cell due to death or to the use of reagents and not present during life." The degree of artifact is proportional to the difference between the structure existing normally in the living body and the structure in the condition directly studied.

1. In the case of living tissues, observed with blood and nerve supply intact, there is a possibility of artifact. It is at a minimum in the Rabbit Ear Chambers and rather more to be reckoned with when tissues must be displaced in order to supply the necessary illumination. With increase in time modifications due to changes in light, temperature, hydrogen ion concentration, etc. are likely to also increase.

2. In living cells removed from the body and examined in Tissue Cultures the possibility of artifact is again at a minimum; but, though the cells in successive generations in suitable media go on living indefinitely, their environments are different from those existing 
within the body. When after Vital Staining or Supravital Staining still living cells are examined in approximately isotonic media, there is a grave danger of artifact if the study is prolonged because the cells are slowly dying.

3. In fixed tissues the degree of divergence from the normal living condition is obviously much greater than in the case of still living ones. However death has been sudden so that artifacts due to gradual death are eliminated. If the technique has been carefully standardized the same fixative applied to the same type of cell in the same physiological state is likely to yield similar results. Among common artifacts are: 1 . The shrinkage and increased affinity of cells near the surface for stains due to allowing the surface of the tissue to dry before fixation. 2. The glassy appearance of nuclei and cytoplasm sometimes occasioned by overheating in imbedding or in spreading out sections. 3. Naterial within blood vessels faintly resembling organisms caused by coagulation of blood proteins. 4. Extraneous substances either present in the albumen fixative used to mount the sections or deposited as dust from the air. Careful focussing is required. See Agonal and Postmortem changes, Ice Crystal Artifacts. A paper by Bensley, R. R., Exp. Cell Res., 1951, 2, 1-9 on "Facts versus Artifacts in Cytology: the Golgi apparatus" is illuminating. See Agonal and Postmortem changes, Ice Crystal Artifacts, Normality, Nucroscopic.

Articular Nerve Terminals.-Written by Dr. E. D. Gardner, Wayne University School of Medicine, Detroit. June 15, 1950-Gardner, E. D., Anat. Rec., $1942, \quad 83, \quad 401-419$, adapted silver methods to the demonstration of nerve terminals in the knee joints of mice 1-60 days old. Subsequently, J. Comp. Neur., 1944, 80, 11-32, similar methods were applied to the knee joints of $33-$ and 46-day-old cat fetuses. The Bodian method was used, but the necessary protargol seems no longer to be available. It appears, however, that similar results can be obtained with Romanes' staining method ( $\mathrm{J}$. Anat., 1950, 84, 104-115). A variety of fixatives are satisfactory, particularly Bouin and aceticformol-alcohol. Decalcify in $20 \%$ sodium citrate and $50 \%$ formic acid, equal volumes, prior to embedding. Sections are placed overnight at $56^{\circ} \mathrm{C}$. in the following solution: Distilled water $50 \mathrm{ml}$., $0.1 \%$ silver nitrate 2.9 $\mathrm{ml}$., and $0.1 \%$ sodium chloride $1.0 \mathrm{ml}$. To this are added a few drops of very weak ammonia sufficient just to turn phenol red paper pale pink. Subsequent treatment is similar to that of the Bodian method. Silver methods are excellent for following nerves in thin, serial sections, for tracing nonmyelinated fibers and for small nerve endings. But large, proprioceptive endings such as occur in the posterior region of the knee joint capsule are best demonstrated by methylene blue. Excellent results may be obtained by using $0.05 \%$ methylene blue in normal saline. It may be perfused, injected into the joint cavity or pieces of capsule can be removed and placed in methylene blue at $37^{\circ} \mathrm{C}$. for $10-15$ minutes. They are then exposed to air for 10-15 minutes, placed in $8 \%$ ammonium molybdate in normal saline for at least 1-2 hours and then dehydrated rapidly in cold alcohols. Whole mounts are made after clearing in xylol. This method shows better than any other the grouping and distribution of large endings, and the preparations are also satisfactory for small fibers, particularly those in vascular plexuses.

Artificial Fever, influence on adrenal (Bernstein, J. G., Am. J. Anat., 1940, 66, 177-196). See Cramer, W., Fever, Heat Regulation and the ThyroidAdrenal Apparatus. London: Longmans, Green \& Co., 1928, 153 pp.

Asbestos, see Iillie p. 135.

Ascorbic Acid. Colorimetric method of Lowry, O. H., Lopez, J. A. and Bessey, O. A., J. Biol. Chem., 1945, 160, 609615 for blood serum in volumes of 10 $\mu l$ up. Pijoan, M. and Gerjovich, H. J., Science, 1946, 103, 202-203 advise caution in use with tissues. See titrimetric technique of Glick, D., J. Biol. Chem., 1935, 109, 433-436. See Vitamin C.

Ascorbic Acid, see Vitamin C.

Aspirated Sternal Marrow, method for preparing smears and sections (Gordon, H., J. Lab. \& Clin. Med., 1910-41, 26, 1784-1788).

Astra Violet, see Leishmania.

Astrocytes. These cells make up one of the two divisions into which neuroglia is usually divided. They also pass under the names of "Classical neuroglia" and "macroglia." They are star shaped cells with processes radiating in all directions some of which form perivascular expansions on the surface of small blood vessels. The most selective methods for their demonstration usually involve some form of metallic impregnation. For details see Chapter by Penfield and Cone in McClung's Microscopical Technique, 1950, p. 407 and Lillie's Histopathologic Technic, 1948, p. 237. In this book see Neuroglia.

Atabrine, anti-malarial agent; fluorescence microscopical localization of atabrine 
in the body (Jailer, J. W., Science, $1945,102,258-259$.

Atheroma, see methods for Calcium, Collagenic Fibers, Elastic Fibers, Lipids, etc.

Atomic Weights. See G. E. F. Lundell, Chairman, Report of Committee on Atomic Weights. J. Am. Chem. Soc., $1949,71,1141-1142$.

\section{International Atomic Weights} 1949 Revision

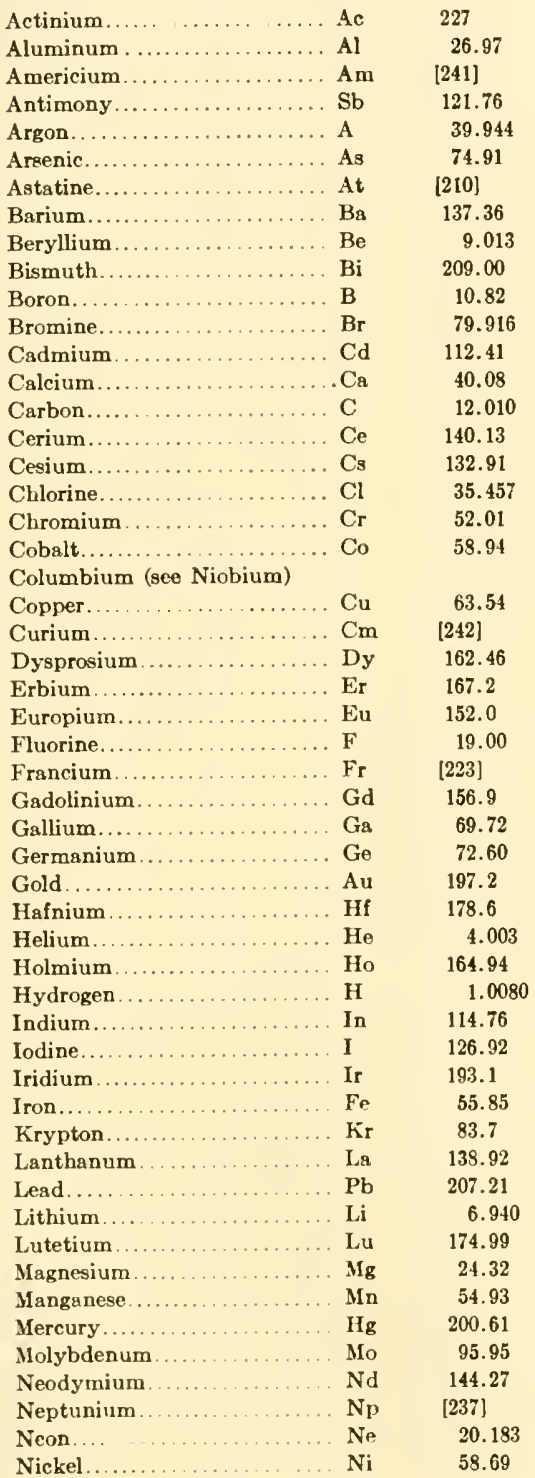

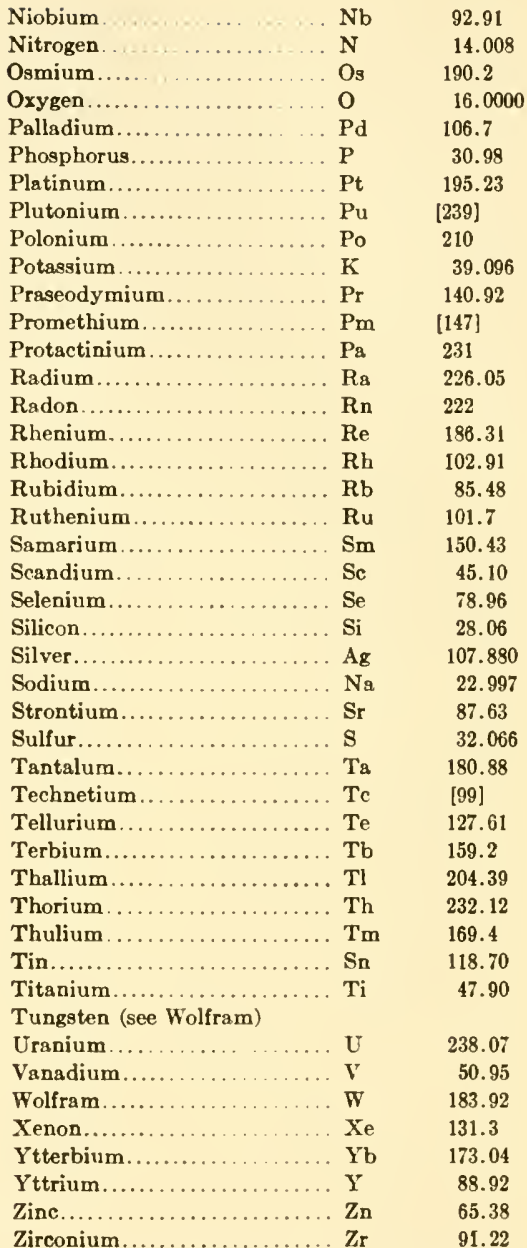

A value given in brackets denotes the mass number of the most stable known isotope.

Auditory System, see Ear.

Auer Bodies. - Written by G. Adolph Ackerman, Hinsman Hall, Ohio State University, Columbus 10, Ohio. May 24, 1951-Auer bodies are rod-like structures occasionally present in the cytoplasm of leukemic myelocytes and monocytes; they are less than 1 micron in width and vary in length from 1-10 micra. Although the number of Auer bodies observed within a single leukemic cell is frequently limited to one or two, more than fifteen have been observed. Auer bodies are formed by the coascervation of the cytoplasmic granules in young leukemic cells. As the cell matures, the Auer bodies undergo dissolution into cytoplasmic granules. 
1. Unstained moist preparations: A drop of blood or bone marrow is placed on a clean cover slip, inverted on a slide and rimined with vaseline or suitable substance to prevent drying. The preparation is examined using the phase microseope. Auer bodies appear as discrete eytoplasmic structures.

2. Supravital technic: The supravital technic is carried out in the same manner as indicated above (No. 1.) for unstained moist preparations with the exception of the fact that the slide has been previously eoated with neutral red and janus green. Preparations may be examined with the bright field, dark field or phase mieroseope. Under the bright field mieroscope, the Auer bodies appear as discrete structures which stain with neutral red. The color of the Auer bodies varies from a deep red (acidic reaction) in young cells to tan (less aeidic reaction) in the more mature leukemic cells. The staining reaction of the Auer bodies with neutral red elosely parallels that of cytoplasmic granules. Auer bodies stain blue-purple in supravital preparations prepared with brilliant cresyl blue (Dameshek, W. Arch. Int. Med., 1932, 50, 579).

3. Romanowsky stains: The Romanowsky dyes may be used to demonstrate Auer bodies. Due to the solubility of the azurophilic component of the Auer body in water and aleohol, this technic is not as satisfactory as the supravital and the unstained moist teehnies.

4. Sudan black B technic: Dry blood and bone marrow films are fixed with either formalin vapor or with a $1 \%$ solution of formalin in $95 \%$ ethyl aleohol for $20-60$ seconds. The dried-fixed films are placed in a saturated solution of Sudan black B (National Aniline Co., New York) in $70 \%$ ethyl alcohol for 20-30 minutes. The solution of Sudan black must be prepared at least 2 weeks prior to using. The blood films are then dipped in $70 \%$ ethyl alcohol and rinsed in $50 \%$ ethyl aleohol and washed in distilled water, dried and mounted with "permount." Auer bodies are sudanophilic and appear black or black-brown. This technie is an excellent method for demonstrating Auer bodies.

5. Periodic Acid-Schiff (PAS) reaction: Dried films are fixed with formalin vapor for 20-60 seconds. The fixed preparations are immersed in an aqueous $0.5 \%$ solution of periodic acid for 5 minutes, washed thoroughly with distilled water and immersed in Sehiff's reagent 10 minutes, followed by 3 successive ehanges in sulfurous acid, 3-5 minutes each, washed in distilled water, dried and mounted with "permount."
Auer bodies are PAS-positive appearing a moderate pink.

6. Toluidine blue and Thionin: Dried films may be fixed with formalin vapor, $4 \%$ aqueous basic lead acetate or other suitable fixatives. Preparations are washed, dried and immersed in a dilute solution $(0.2-0.5 \%)$ toluidine blue or thionin, washed in distilled water, dried and mounted with "permount." Auer bodies stain lavender following the use of this technic.

7. Plasmal reaction: Dried films are fixed with formalin vapor for $20-60$ seconds, washed in distilled water and placed in a $1 \%$ aqueous solution of mercuric chloride for 5 minutes, washed in distilled water and placed in Schiff's reagent; the films are placed in three successive changes of sulfurous acid, washed in distilled water, dried and mounted with "permount." In staining control preparations, the same procedure is followed with the exception of the faet that distilled water is substituted for mercuric chloride. If the control exhibits a positive reaction, the length of exposure to Schiff's reagent must be reduced. Following the plasmal reaction, the Auer bodies appear a very pale pink, and may best be identified by means of the phase microscope followed by bright field observation of the stained bodies.

8. $M$. nudi reaction: Dried blood and bone marrow films may be fixed either with formalin vapor or $1 \%$ formalin in $95 \%$ ethyl alcohol, washed with distilled water and dried. The fixed films are immersed in a mixture containing equal parts of $1 \%$ alkaline solution of alpha-napthol and $1 \%$ aqueous solution of p-aminodimethylanaline (Eastman Kodak, P2147) for 1-5 minutes, washed in distilled water and mounted in water. Auer bodies stain deep blue following the M. nadi reaction. Preparations fade rapidly.

9. Sato and Sekeya Peroxidase Technic: Dried blood and bone marrow films are placed in a $0.5 \%$ aqueous solution of copper sulfate for 30 seconds, washed briefly in $50 \%$ ethyl alcohol and placed in a benzidine-hydrogen peroxide mixture $(0.1 \mathrm{gm}$. benzidine in $100 \mathrm{ml}$. distilled water and 2 drops of $3 \%$ hydrogen peroxide) for 2 minutes, washerl with distilled water, dried and mounted with "permount". Counterstaining with $1 \%$ aqueous safranin is optional. Auer bodies are peroxidase-positive and appear blue or yellow.

Auer bodies are soluble in water, saline and many organic solvents and are readily digested by ribonuclease. Digestion by ribonuclease may be pre- 
vented by immersing the Auer bodies in an aqueous solution of lanthanum acetate for 2 hours prior to exposure to the enzyme. Wright's stain may be used to demonstrate the basophilic properties of the Auer rods. Auer bodies are not demonstrable following the BauerFeulgen, Lugol's aqueous iodine, Gomori acid and alkaline phosphatase and lipase reactions.

Auerbach's Plexus. Supravital staining by injecting methylene blue through the aorta is apparently improved by addition of hydrogen acceptors. Sehabadasch, A., Bull. d'Hist. Appl., 1936, $13,1-28,72-89,137-151$ advises $0.03-$ $0.05 \mathrm{gm}$. per liter of $p$-amidophenol, 0.02-0.07 of $p$-phenylenediamine, $0.02-$ 0.05 of pyrocatechine or $0.05-0.9$ of resorcin. The methylene blue must be of high quality and free from metallic salts. He obtained in $5 \mathrm{~min}$. intense staining of the plexus in a cat which received $1200 \mathrm{cc}$. of fluid of the following concentration : aq. dest., 1000 cc.; $\mathrm{NaCl}$, $7 \mathrm{gm}$.; resorcin, $0.15 \mathrm{gm}$. and methylene blue (chlorzink free, Hoecht) $0.2 \mathrm{gm}$.

Aural Smears, see Ear Smears.

Auramin (CI, 655)-canary yellow, pyoktanin yellow, pyoktaninum aureumThis basic diphenyl methane dye may be of use in fluorescence microscopy. Auramine $\mathrm{O}$ is Commission Certified. It is one of the substances which arrests mitosis in the metaphase, an action which has been carefully studied by Ludford, R. J., Arch. f. Exper. Zellf., 1935-6, 18, 411-441. Tubercle bacilli treated with auramin give golden yellow fluorescence (Hageman, P. K. H., Munch. Med. Woch., 1938, 85, 1066).

Aurantia (CI, 12)-imperial yellow-An acid nitro dye employed in ChampyKull method. Aurantia is explosive and it can cause severe dermatitis. All those using it should be warned of the danger.

Aurin or rosolic acid (CI, 724).

Autoradiography, see Radioactive Isotopes.

Axenfeld Reaction. Giroud (A., Protoplasma, 1929, 7, 72-98): Add to preparation few drops of formic acid, then 3-4 drops $0.1 \%$ aq. gold chloride and heat slowly. A rose color appears, then violet. Lison (p. 129) says that the reaction is very little characteristic of proteins since analogous reactions are given by creatine, urea, uric acid, glycogen. Its employment is contraindicated.

Axis Cylinders. These are the cytoplasmic cores of the nerve fibers. Mitochondria can often be seen in them unstained and after supravital coloration with Janus Green. The best method to demonstrate mitochondria in fixed tissues is Anilin Fuchsin Methyl Green after Regaud fixation. Silver methods show Neurofibrils. Alzheimer's modification of Mann's eosin-methyl blue method is recommended to show early degenerative changes. De Renyi, G.S., Cowdry's Special Cytology, 1932, 3, 1370-1402 has fully described use of methods of microdissection. See Ama. ranth.

Azan Stain, see Heidenhain's.

Azidine Blue 3B, see Trypan Blue.

Azidine Scarlet R, see Vital Red.

Azins. Azin dyes are those formed from phenazin. Two benzene rings are joined by 2 nitrogen atoms forming a third ring. Examples: amethyst violet, azocarmine $\mathrm{G}$, indulin alcohol and water soluble, Magdala red, neutral red, neutral violet, nigrosin water soluble, phenosafranin, safranin $\mathrm{O}$.

Azo Blue (CI, 463)-benzoin blue $\mathrm{R}$ and direct violet $\mathrm{B}-$ This acid dis-azo dye is one of those microinjected vitally into cytoplasm against the nucleus of amebae to ascertain whether the nucleus can be vitally colored (Monné, L., Proc. Soc. Exp. Biol. \& Med., 1934-35, 32, 1197-1199). Butt, E. M., Bonynge, C. W. and Joyce, R. L., J. Inf. Dis., 1936, 58, 5-9 report that azo blue can be substituted for India ink in the negative demonstration of capsular zones about hemolytic streptococci.

Azo-Bordeaux, see Bordeaux Red.

Azocarmine G (CI, 828)-azocarmine GX, rosazine, rosindulin $\mathrm{GXF}$ - This basic azin dye is used in place of acid fuchsin in Heidenhain's Azan stain. Azocarmine $\mathrm{B}$ is $\mathrm{CI}, 829$.

Azocarmine GX, see Azocarmine G.

Azo Dyes. Chromophore $-\mathrm{N}=\mathrm{N}$-uniting naphthalene or benzene rings. See Mono-azo, Dis-azo and Poly-azo Dyes. Lipophilic substitutions in, and slight curative effect claimed in tuberculosis and leprosy (Bergmann, E., Haskelberg, L. and Bergmann, F., J. Am. Chem. Soc., $1941,63,2243$.

Azo-fuchsin. Seven are recognized in the Colour Index. Acid mono-azo dyes related to Bordeaux red and orange $G$.

Azolitmin, see Hydrogen Ion Indicators.

Azophloxine G. A.- Fast crimson GR., Amidonaphthol G-an acid Azo dye.

Azo Reaction for phenols. Formation of azo color by action of diazonium salt on tissue phenol (Lison, p. 140). See Lison, L., C. Rend. Soc. de Biol., 1933, $112,1237-1239$ ).

Azo Rubin, see Amaranth.

Azure Dyes. These are basic thiazin stains of great usefulness. The description given by Conn (pp. 76-80) should be consulted. It is here summarized. Azure I (Giemsa) is a trade name for a secret preparation apperently a variable 
mixture of Azure A and B. Azure II is an intentional mixture, in equal parts, of Azure I and methylene blue. It is the main constituent of Giemsa's stain.

1. Azure $A$ is asymmetrical dimethyl thionin and has been Commission Certified for some time. It is considered as the most important nuclear staining component of polychrome methylene blue by MacNeal, W. J., J. Inf. Dis., 1925, 36, 538-546. This dye has been used as a nuclear stain following eosin and after phloxine, see Phloxine-Azure (Haynes, R., Stain Techn., 1926, 1, 68-69, 107-111).

2. Azure $B$ is the tri-methyl derivative of thionin. It is specified by Jordan, J. H. and Heather, A. H., Stain Techn., 1929, 4, 121-126 as a stain for Negri bodies. Roe, M. A., Lillie, R. D. and Wilcox, A., Pub. Health Reports, $1940,55,1272-1278$ recommend its inclusion in Giemsa's stain.

3. Azure $C$ is mono-methyl thionin. French, R. WV., Stain Techn., 1926, 1, 79 has described a method for its use followed by Eosin $Y$ and orange II in staining sections of formalin fixed material; but Haynes, R., Stain Techn., $1927,2,8-16$ doubts whether it is significantly better than Azure $A$ and thionin.

Azure or Toluidin Blue-Eosin.-Written by Dr. R. D. Lillie, Division of Pathology, National Institute of Health, Bethesda, Md. May 8, 1950.-Prepare a $1 / 1000$ solution of Azure A, Azure C or Thionin (85-90\% dye content) or a $0.15 \%$ solution of Toluidin Blue $(60 \%$ dye content) and a 1/1000 solution of Eosin Y or Eosin B (a redder shade).

Bring paraffin sections to water as usual, including an iodine, thiosulfate sequence for material fixed with mercuric chloride mixtures. Stain 1 hour in stock azure, Toluidin Blue or Thionin 4 cc., stock eosin 4 cc., McIlvaine buffer of desired $\mathrm{pH}$ level $2 \mathrm{cc}$, acetone $5 \mathrm{cc}$. and distilled water 25 cc. Rinse, dehydrate with acetone, clear with $50: 50$ acetone xylene and 2 changes of xylenc, mount in synthetic resin (polystyrene, permount, clarite, HSR or the like). The procedure has been used for technicon staining. The stain mixture is made fresh weekly in this case.

For neutral formalin or Orth fixations, use $\mathrm{pH} 4.0-4.5$, for acid formalin pH 4.5 is better, for Zenker or Helly pH 5.0, for Bouin pH 5.5-6.0 (less satisfactory than others as the picric acid seems to interfere), for Carnoy, alcohol and similar fluids 4.8-5.5.

Color values are deep blue for nuclei, bacteria, and rickettsiae, violet to purple for mast cell granules and carti- lage matrix, lighter blues for cytoplasms, varying pinks for muscle, erythrocytes, fibrin, necrotic cytoplasm and oxyphil inclusion bodies.

This has been modified somewhat from Histopathologic Technic, Iillie (Blakiston, Phila., 1948), which gives further details.

Azure II Eosin and Hematoxylin (Maximow, A., J. Inf. Dis., 1924, 34, 549), gives, in addition to coloration of chromatin by hematoxylin, a granule stain something like that provided by Giemsa's method. Make up: (1) azure II eosin: A. cosin water soluble yellowish, 0.5 gm.; aq. dest., 500 cc. B. azure II, $0.5 \mathrm{gm}$.; aq. dest., $500 \mathrm{cc}$. Mix $10 \mathrm{cc}$. A, 100 cc. aq. dest., and 10 cc. B. (2) hematoxylin (Delafield's) 1-2 drops, aq. dest., $100 \mathrm{cc}$. to make a pale violet solution.

Formalin-Zenker fixed tissues (sections, smears, spreads) are stained upright in hematoxylin washed in aq. dest. and counter-stained with azure II eosin 24 hrs. each. Transfer to $95 \%$ alc., differentiate and dehydrate in abs. (2 changes); clear in xylol and mount in balsam. Care must be taken to use pure aq. dest. The proportions of A and $B$ can be varied slightly to suit the tissue. In order to hold the azure II eosin colors the balsam should be neutral or nearly neutral as when Giemsa's stain is employed.

To appreciate the beauty of this method see numerous colored illustrations marked "ZF, Häm, EAz" of a great many organs and tissues by Maximow, $\mathrm{A}$. Section on Bindegewebe und Blutbildende Gemebe in Möllendorff's Handb. d. mikr. Anat. d. Menschen, 1927, 2, (1) 232-583

Babes' see Anilin-Safranin.

Bacillus Typhosus, technique for dark field study of flagella (Pijper, A., J. Path. \& Bact., 1938, 47, 1-17). See 9 plates by author.

Bacteria. Methods employed for the microscopic identification of bacteria and to demonstrate their structure are legion. The Committee on Bacteriological Technique of the Society of American Bacteriologists has prepared a useful leaflet entitled "Staining Procedures" published in Geneva, N. Y. (Fifth Edition 1934) to supplement their "Manual of Methods for the Pure Culture of Bacteria" (1923). A detailed account of Bacteriological methods by $\mathrm{H}$. J. Conn, F. B. Mallory and Frederic Parker, Jr. is contained in McClung's Microscopical technique to which reference should also be made. Bergey's "Manual of Determinative Bactcriology" (Baltimore: Williams \& Wilkins, 1948), which 
is a key to identification of bacteria, is often useful.

Motility, agglutination, lysis under influence of bacteriophage, ingestion by leucocytes and many other phenomena can best be observed by examination of living bacteria by direct illumination or in the darkfield. Smears, usually fixed by heat, are, however, most of ten used. A choice must be made from many well known stains including: Anilin Gentian Violet, Loeffler's Methylene Blue, Giemsa, Gram and Carbol Fuchsin. Others are best listed under the particular structures to be demonstrated Spores, Flagella, Capsules. In some cases search for bacteria in Milk, Soil, Cheese, Sputum, etc. is indicated. When bacteria are so few in number that they may be missed, or large numbers are required separated from the tissues for chemical analysis, Concentration methods may be useful. Accurate localization of bacteria requires their study in sections. See Giemsa's stain, Gram-Weigert stain, Goodpasture's stain (MacCallum's modification), Mallory's Phloxine-Methylene blue and Acid Fast Bacilli. The darkfield examination of stained preparations is said to be an advantage (Goosemann, C., J. Lab. and Clin. Med., 1935-36, 21, 421-424). Appearance when viewed at high magnification with electron microscope (Mudd, S., Polevitsky, K., and Anderson, T. F., Arch. Path., 1942, 34, 199-207). See Fluorescence microscopy, Negative Strains, Dead bacteria, Tubercle bacilli, Leprosy bacilli, Mitochondria and Bacteria in same cells, Rickettsia, Gonococcus, Diphtheria Bacilli, Bacterium Tularense, Bacterium Monocytogenes.

Bacteria. Biochemical Tests. Given in greater detail by H. R. Livesay in Simmons and Gentzkow, 387-389.

1. Indicators of $\mathrm{pH}$. Incorporate in basic culture of medium measured amounts of $0.02 \%$ aq. phenol red, $0.04 \%$ aq. bromcresol purple, or $0.1 \%$ aq. bromthymol blue. Their $\mathrm{pH}$ ranges and colors are given under Hydrogen Ion Indicators.

2. Indol test. Use Bohne's reagents. To 5 day culture in $1 \%$ aq. peptone add 1 cc. ether, shake and settle. Let $1 \mathrm{cc}$. of following run down inside tube: pdimethylaninobenzaldehyde, $4 \mathrm{gm}$.; $95 \%$ ethyl alcohol, 380 ec.; conc. hydrochloric acid, $80 \mathrm{cc}$. If after 1 min. no color develops add 1 cc. sat. aq. potassium persulfate. Positive, pale pink to deep magenta.

3. Ilosvay's Nitrate reduction. To 5 day culture at $37^{\circ} \mathrm{C}$. in broth $+0.1 \%$ $\mathrm{HNO}_{3}$ add 1 cc. of following solution.
Dissolve $1 \mathrm{gm}$. a-naphthylamine in 22 cc. aq. dest. Filter and add $180 \mathrm{cc}$. of dilute acetic acid (sp. gr. 1.04). Then $1 \mathrm{cc}$. of sulfanilic acid $(0.5 \mathrm{gm}$. in $150 \mathrm{cc}$. dilute acetic acid). Positive, pink, red or maroon; negative, no color.

4. Ammonia. To 5 day peptone water culture add $0.5 \mathrm{cc}$. Nessler's Reagent. Positive, brown; negative, faint yellow.

5. Hydrogen sulfide. Inoculate organisms on lead acetate agar made by sterilizing extract broth containing $4 \%$ peptone $+2.5 \%$ agar and equal volume $0.1 \%$ aq. basic lead acetate. Positive, brown or black; negative, no color.

6. Reductase. To a $24 \mathrm{hr}$. broth culture add 1 drop $1 \%$ aq. methylene blue. Incubate at $37^{\circ} \mathrm{C}$. Positive, complete decolorization; weakly positive, green color; negative, no decolorization.

7. Catalase. Pour 1 cc. $\mathrm{H}_{2} \mathrm{O}_{2}$ over $24 \mathrm{hr}$. agar slant culture incubated at $37^{\circ} \mathrm{C}$. holding tube on incline. Positive, gas bubbles; negative, none.

8. Methyl red. To 4 day culture in glucose phosphate medium at $37^{\circ} \mathrm{C}$. add 5 drops $0.04 \%$ methyl red in $60 \%$ alcohol. Positive, red; negative, yellow.

9. Voges-Proskauer. To 4 day culture in glucose phosphate medium at $37^{\circ} \mathrm{C}$. add 5 cc. $10 \%$ aq. $\mathrm{KOH}$. After 18-24 hrs. positive, pink fluorescence; negative, no color.

10. Oxidase. To surface of colony add loop full or 1-2 cc. fresh $1 \%$ aq. dincthylparaphenylenediamine hydrochloride. Positive, color change from pink to maroon to black.

Bacteria Flagella. Electron microscopic technique reveals the ultrastructure of bacterial flagella as composite structures made up of a central coiled trypsin resistant filament and a peripheral sheath probably nonresistant (De Robertis, E. and Franchi, C. M., Exp. Cell Res., 1951, 2, 295-298).

Bacteria. Media. The following are brief summaries of culture media as described by H. R. Livesay in Simmons and Gentzkow, 388-403.

(Glucose phosphate. Witte or Difco proteose peptone, $0.5 \mathrm{gm}$.; $\mathrm{K}_{2} \mathrm{HPO}_{4}, 0.5$ gm.; glucose, $0.5 \mathrm{gm}$; aq. dest., 100 cc.; $\mathrm{pH}$ 7.5.)

ileat extract broth (routine). Add to 1000 cc. aq. dest., beef extract, $3 \mathrm{gm}$.; peptone, $10 \mathrm{gm}$; sodium chloride, $5 \mathrm{gm}$. Dissolve by stirring with heat (water bath $65^{\circ} \mathrm{C}$.). Make up weight loss with aq. dest. and make $\mathrm{pH} 7.2-7.4$. Boil over flame, cool to $25^{\circ} \mathrm{C}$, again make up weight loss, clarify and check $\mathrm{pH}$. Place in flasks or tubes, autoclave 15 lbs., $15 \mathrm{~min}$. 
Meat extract broth (for water anal. ysis). As above, using beef extract, $3 \mathrm{gm}$.; peptone, $5 \mathrm{gm}$.; aq. dest. 1000 cc. $\mathrm{pH}$ 6.4-7.

Meat extract agar (routine). Dissolve 20-30 gms. powdered agar in 1000 cc. meat extract broth stirring over flame and titrate to $\mathrm{pH} 7.4$. Cool to $50^{\circ} \mathrm{C}$., add stirred eggs, heat gently till egg material is firmly coagulated. Remove coagulum with fine wire mesh strainer, filter through cotton, make up filtrate to original weight with aq. dest. and make $\mathrm{pH} 7.2-7.4$. Tubes or flasks. Autoclave 15 lbs., $15 \mathrm{~min}$.

Meat extract agar (for water analysis). Add $15 \mathrm{gm}$. best quality agar to $1000 \mathrm{cc}$. of above meat extract agar and make $\mathrm{pH}$ 6.4-7.

Meat infusion broth. Mix $500 \mathrm{gms}$. ground fat-free beef, or veal round, in 1000 cc. aq. dest in ice box $18-24 \mathrm{hrs}$. Heat over small flame in Arnold sterilizer, $1 \mathrm{hr}$., add $5 \mathrm{gm}$. sodium chloride and $10 \mathrm{gm}$. peptore. Dissolve our flame, filter, add aq. dest. to 1000 cc., titrate to $\mathrm{pH} 7.4$, tube or flask, and autoclave $15 \mathrm{lbs} ., 15 \mathrm{~min}$.

Meat infusion agar. Add $20 \mathrm{gm}$. agar to $1000 \mathrm{cc}$. Meat infusion broth and continue as in making meat extract agar, $\mathrm{pH}$ to 7.4 .

Gelatin, Nutrient. Add $120 \mathrm{gm}$. gelatin to $1000 \mathrm{cc}$. meat extract broth in double boiler, weigh, dissolve by heat, titrate to $\mathrm{pH} 7.4$ and add aq. dest. to make original weight. Add $1 \mathrm{egg}$ clarified by mixture with small volume of aq. dest., heat slowly till egg is coagulated, filter through cotton and sterilize filtrate in $10 \mathrm{cc}$. portions in tubes in Arnold $20 \mathrm{~min} .3$ successive days.

Huntoon's hormone. Add $500 \mathrm{gm}$. fresh finely ground beef heart, $10 \mathrm{gm}$. peptone, $5 \mathrm{gm}$. sodium chloride, 1 whole egg, $20 \mathrm{gm}$. agar (Bacto) to 1000 cc. aq. dest. in enamel-ware dish, heat and stir constantly. Make $\mathrm{pH} 8$. Cover, place in Arnold $1 \mathrm{hr}$. Remove, separate clot from sides and return to

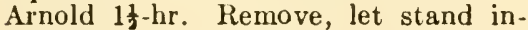
clined, room temperature, $10 \mathrm{~min}$. Renrove clear part and filter it through fine wire sieve into tall cylinders. Allow to stand 15-20 min. and skim off fat. Clear further by passing through glass, or asbestos wool, or by centrifuging. Tube in $10 \mathrm{cc}$. lots, sterilize in Arnold $30 \mathrm{~min}$. on 3 successive days.

Glucose agar. Add $10 \mathrm{gm}$. glucose to 1000 ec. meat extract or meat infusion agar and dissolve by slowly heating. Adjust $\mathrm{pH}$ to that of original agar. Pour in tubes, or flasks, and sterilize in Arnold 3 successive days.
Blood agar. Add 5-10\% of sterile defibrinated blood (preferably horse) to meat infusion or meat extract agar which first has been melted and cooled to $45^{\circ} \mathrm{C}$. Pour into plates or into tubes and slant, then incubate to prove sterility.

Chocolate blood agar. Add 5\% of sterile defibrinated blood to meat in. fusion agar at $50-55^{\circ} \mathrm{C}$. $\operatorname{mix}$ avoiding bubbles, slowly increase to $75^{\circ} \mathrm{C}$. Pour into plates, or into tubes and slant, then incubate to prove sterility.

Serum agar. Add 100 ec. sterile normal horse serum to $1000 \mathrm{cc}$. melted meat infusion agar, pour into plates, or tubes, and slant, then incubate to prove sterility.

Liver infusion agar (for $\mathrm{Br}$. abortus). Mix $500 \mathrm{gm}$. ground beef liver with 500 gm. aq. dest. in cool place $24 \mathrm{hrs}$., strain through cheesecloth and collect $500 \mathrm{gm}$. resulting infusion (1). Add $20 \mathrm{gm}$. agar and $500 \mathrm{gm}$. aq. dest. and autoclave 15 lbs. pressure, $30 \mathrm{~min}$. (2). Dissolve $10 \mathrm{gm}$. peptone and $5 \mathrm{gm}$. sodium chloride in No. 1, beef infusion (3). Add aq. dest to 2 and 3 combined to make up weight lost by evaporation, adjust $\mathrm{pH}$ to 7 and cool to $50^{\circ} \mathrm{C}$. Add $10 \mathrm{gm}$. egg albumin (first dissolved in $10 \mathrm{cc}$. aq. dest.), heat to $100^{\circ} \mathrm{C} .1 \frac{1}{2}$-hrs., strain through fine wire sieve, filter through clean glass wool, adjust $\mathrm{pH}$ to 7 , tube in $15 \mathrm{cc}$. lots and autoclave at $15 \mathrm{lbs}$., 30 min. When required melt and pour plates, or make slants.

Trypagar. Put $500 \mathrm{gm}$. fat free, finely ground beef or veal ground in 1000 cc. aq. dest. in container adding $20 \%$ aq. $\mathrm{NaOH}$ until slightly alkaline to litmus. Cook at $75^{\circ} \mathrm{C}$., $5 \mathrm{~min}$., cool to $37^{\circ} \mathrm{C}$. and add $0.5 \mathrm{gm}$. trypsin (Bacto). Incubate $37.5^{\circ} \mathrm{C}$., 5 hrs. If trypanization is complete 5 cc. liquid $+5 \mathrm{cc}$. in $\mathrm{NaOH}+1$ cc. dil. aq. $\mathrm{CuSO}_{4}$ will give pink color. If not incubate again $1 \mathrm{hr}$. and re-test. When complete, slightly acidify with glacial acetic acid, slowly bring to boiling point and hold $15 \mathrm{~min}$. Filter through wet paper, add $20 \mathrm{gm}$. agar and $5 \mathrm{gm}$. sodium chloride. Dissolve agar with heat, clear with an egg, adjust to $\mathrm{pH} 7.6$ and autoclave $15 \mathrm{lbs}$., $15 \mathrm{~min}$.

Veal infusion brain broth (for Streptococci and anaerobes). With large bore pipette insert about $50 \mathrm{cc}$. ground fresh calf brain in bottom $200 \times 25 \mathrm{~mm}$. tube and add $35 \mathrm{cc}$. veal infusion broth pH 7.6. Autoclave 15 lbs., $20 \mathrm{~min}$. Remove 10 ce. test reaction, $\mathrm{pH} 7.4-$ 7.6 being satisfactory, if a change has taken place adjust to $\mathrm{pH} 7.6$ and estimate from titration of this $10 \mathrm{cc}$. amount needed to bring to this figure 
bulk and correct the whole. Fill tubes with similar amounts, then incubate at $37^{\circ} \mathrm{C}$. to prove sterility.

Robertson's (for Anaerobes). To $500 \mathrm{gm}$. ground fat, fascia and blood vessel-free fresh beef heart, add $10 \mathrm{gm}$. peptone and $1000 \mathrm{cc}$. aq. dest., bring to boil and adjust to $\mathrm{pH} 8$. Continue simmering $1 \frac{1}{2}$-hrs. and again adjust reaction. Separate broth from meat, place former in flasks, autoclave 15 lbs., 15 min. Dry meat on filter paper in oven $56^{\circ} \mathrm{C}$. 48 hrs. Place desired amounts of meat plus $10 \mathrm{cc}$. broth in tubes. Autoclave cool, remove broth and retitrate. Adjust to desired $\mathrm{pH}$, finally fill tubes same quantity meat and broth and autoclave 15 lbs. $30 \mathrm{~min}$. Final $\mathrm{pH}$ should be 7.4-7.6.

Calcium carbonate broth (for Pneumococci). Dissolve $10 \mathrm{gm}$. glucose in $1000 \mathrm{cc}$. meat infusion broth by heating, make $\mathrm{pH}$ 7.6. Place clean marble chips $\left(\mathrm{CaCO}_{3}\right)$ in bottom of tubes pour in broth, sterilize in Arnold 15 min. 3 successive days.

Blood culture (Kracke). Add 500 $\mathrm{gm}$. finely ground fat-free beef heart muscle to $1000 \mathrm{cc}$. aq. dest. in ice box over night. Press through 4 layers gauze cloth, heat extract to boiling, filter through small mesh wire gauze. Add $250 \mathrm{gm}$. ground beef brain to 500 cc., treat in same way but do not filter this suspension. Mix 800 cc. extract, $110 \mathrm{cc}$. suspension, $1 \mathrm{gm}$. sodium citrate, $10 \mathrm{gm}$. dextrose (Bacto), $10 \mathrm{gm}$. proteose peptone (Difco), 2 gm. disodium phosphate and $4 \mathrm{gm}$. sodium chloride and place $50 \mathrm{cc}$. lots in tubes or flasks. Autoclave $15 \mathrm{lbs} ., 20 \mathrm{~min}$.

Bile (For typhoid group). Combine 900 cc. ox bile, 100 cc. glycerol and 20 gm. peptone by heating over water bath. Pour in bottles or small flasks and autoclave.

Brilliant green lactose bile. Dissolve $10 \mathrm{gm}$. peptone and $10 \mathrm{gm}$. lactose in 500 cc. aq. dest. add 200 ce. fresh ox bile, or $20 \mathrm{gm}$. dehydrated ox bile dissolved in 200 cc. aq. dest., the latter having pH 7.4. Add 13.3 cc. $0.1 \%$ aq. brilliant green and aq. dest. to make $1000 \mathrm{cc}$. Filter through cotton, place in fermentation tubes, sterilize after which pH by potentiometer (not colorimeter) should be 7.1-7.4.

Levine's eosin methylene blue agar (Standard for water analysis). Dissolve $10 \mathrm{gm}$. peptone, $2 \mathrm{gm}$. $\mathrm{K}_{2} \mathrm{HPO}_{4}$, and $15 \mathrm{gm}$. agar in 1000 ce. aq. dest. by boiling. Add aq. dest. to compensate for evaporation and distribute measured amounts in flasks. Immediately before use to each $100 \mathrm{cc}$. add $5 \mathrm{cc} .20 \%$ aq. lactose (sterile), 2 cc. $2 \%$ aq. eosin and 2 cc. $0.5 \%$ aq. methylene blue. Mix, pour into Petri plates, harden and incubate to prove sterility.

Endo's (Standard for water analysis). Add $5 \mathrm{gm}$. beef extract, $10 \mathrm{gm}$. peptone and $30 \mathrm{gm}$. agar to $1000 \mathrm{cc}$. aq. dest. in container and weigh. Boil till dissolved, restore lost weight with aq. dest., place in vessel with straight walls and autoclave $15 \mathrm{lbs} ., 15 \mathrm{~min}$. Let agar harden, remove en masse to clean paper, cut away and discard debris from bottom. Melt clean agar, make $\mathrm{pH}$ 7.88.2 , pour in $100 \mathrm{cc}$. or larger amounts and autoclave $15 \mathrm{lbs} ., 15 \mathrm{~min}$. To each $100 \mathrm{cc}$. of this stock agar add 5 cc. $20 \%$ aq. C.P. lactose (sterilized by fractional method), 0.5 cc. $10 \%$ basic fuchsin in $95 \%$ alcohol (from filtrate of supernatant fluid having let stand $24 \mathrm{hrs}$.). Mix carefully, pour into sterile Petri dishes, let agar set at room temperature and harden over night in incubator. Check sterility.

Agar, sodium desoxycholate. Dissolve $10 \mathrm{gm}$. peptone in $1 \mathrm{Kg}$. water, bring to $\mathrm{pH} 7.3-7.5$ with sodium hydroxide, boil few minutes and pass through filter paper. Add 12-17 gm. agar. After soaking $15 \mathrm{~min}$., melt by boiling. To each 1000 cc. add 6 cc. $1 N$ sodium hydroxide plus ferric ammonium citrate, $2 \mathrm{gm}$. dipotassium phosphate and $1 \mathrm{gm}$. sodium desoxycholate. Titrate with phenol red indicator to $\mathrm{pH} \quad 7.3-7.5$ and add $3 \mathrm{cc}$. $1 \%$ aq. neutral red. Sterilize in flowing steam only sufficient to kill vegetable cells (15 min. enough for tubes with 10-15 cc. medium).

Selenite-F enrichment. Use monosodium and disodium phosphates in exact proportions which experiment shows that with particular lot of peptone and brand of sodium selenite will give $\mathrm{pH}$ 7.0-7.1. Dissolve with heat $10 \mathrm{gm}$. these phosphates (anhydrous), $4 \mathrm{gm}$. this sodium hydrogen selenite (anhydrous), $5 \mathrm{gm}$. peptone, $4 \mathrm{gm}$. lactose in aq. dest. to make $1 \mathrm{Kg}$. Boil.

Russell's double sugar agar, Mix $1000 \mathrm{cc}$. melted meat extract agar, $40 \mathrm{cc}$. $25 \%$ aq. lactose (sterile) and 4 cc. $25 \%$ aq. glucose (sterile) and adjust to $\mathrm{pH}$ 7.2 . Add 50 cc. $0.02 \%$ aq. phenol red, filter if necessary, tube and autoclave 8 lbs., $25 \mathrm{~min}$. Slant with deep butt. Check reaction of medium with known $E$. coli and E. typhosa.

Simmons' citrate agar. Dissolve 5 gm. sodium chloride, $0.2 \mathrm{gm}$. $\mathrm{MgSO}_{4}$, $1.0 \mathrm{gm}$. $\left(\mathrm{NH}_{4}\right) \mathrm{H}_{2} \mathrm{PO}_{4}, 2.28 \mathrm{gm}$. sodium citrate $\left(2 \mathrm{H}_{2} \mathrm{O}\right)$ in $1000 \mathrm{cc}$. aq. dest. and add $20 \mathrm{gm}$. agar. Heat to dissolve agar, make $\mathrm{pH} 7.2$, and add 10 cc. $1.5 \%$ alcoholic bromthymol blue. Filter 
through cotton, tube, autoclave $\mathbf{1 5}$ lbs., $15 \mathrm{~min}$. Slant with deep butt. Check reaction of medium with known, $E$. coli., A. aerogenes, S. schottmuelleri and E. typhosa.

Jordan's tartarate agar. Dissolve by heating $20 \mathrm{gm}$. agar, $10 \mathrm{gm}$. peptone, $10 \mathrm{gm}$. sodium potassium tartarate, $5 \mathrm{gm}$. sodium chloride in $1000 \mathrm{cc}$. aq. dest. Adjust $\mathrm{pH}$ to 7.4 and add $12 \mathrm{cc}$. $0.2 \%$ alcoholic phenol red. Tube in $10 \mathrm{cc}$. lots, autoclave $15 \mathrm{lbs}$, $15 \mathrm{~min}$. Check reaction of medium with known $S$. aertrycke, S. enteritidis, $S$. paratyphi and $S$. schottmuelleri.

Lead acetate agar (for $\mathrm{H}_{2} \mathrm{~S}$ test). To 100 cc. sterile meat extract agar add following sterile Seitz-filtered solutions: 4 cc. $25 \%$ aq. glucose, 4 cc. $25 \%$ aq. lactose and 1 cc. $0.5 \%$ aq. lead acetate. Tube aseptically and incubate to prove sterility. Check reaction of medium with known S. paratyphi and S. schottmuelleri.

Dieudonne's alkaline blood agar (for Vibrio comma). Make $700 \mathrm{cc}$. nutrient agar and neutralize to litmus about $\mathrm{pH}$ 6.8. Mix 150 cc. defibrinated beef blood and $150 \mathrm{cc}$. in $1 N \mathrm{KOH}$ and steam in Arnold $30 \mathrm{~min}$. Add this to blood agar. in proportion of 3 to 7 . Pour Petri plates, let harden uncovered (but protected by paper) placing strips sterile filter paper between dish and protection to take up ammonia and moisture. Incubate $15 \mathrm{hrs}$. at $37^{\circ} \mathrm{C}$. before use.

Carbohydrate broth (for fermentation tests). Inoculate $1000 \mathrm{cc}$. infusion broth with active $E$. coli and incubate $18 \mathrm{hrs}$. at $37.5^{\circ} \mathrm{C}$. Boil few minutes to kill organisms. Put in large mortar 20-30 gms. purified talc. While grinding add broth and thoroughly mix. Pass through wet filter paper till clear. Titrate and adjust $\mathrm{pH}$ to 7.3 . Weigh broth and add $1 \%$ of desired ferment. able substances dissolved in a little hot water. Then add 45 ce. $0.04 \%$ aq. bromcresol purple per liter. Sterilize in Arnold 20 min. on 3 successive days, or autoclave $7 \mathrm{lbs} ., 10 \mathrm{~min}$.

Lactose broth (Standard for water analysis). Add $0.5 \%$ lactose to $\mathrm{nu}$ trient extract broth and adjust reaction to $\mathrm{pH}$ 6.4-7. Autoclave $15 \mathrm{lbs}$. 15 min. restricting total heat exposure to $30 \mathrm{~min}$.

Clark and Lubs. Dissolve 5 lbs. each of peptone, dextrose and dipotassium phosphate in 1000 cc. aq. dest. using heat. Filter through paper, add water lost, tube in $10 \mathrm{cc}$. lots, sterilize in Arnold 20 min., 3 successive days.

Bendick's saccharose peptone-water (for Vibrio comma). Add $1 \mathrm{gm}$. anhydrous sodium carbonate to $1000 \mathrm{cc}$. peptone solution ncutralized to phenolphthalein. Boil, filter and to filtratc add $5 \mathrm{gm}$. saccharose $+5 \mathrm{cc}$. sat. phenolphthalein in $50 \%$ alcohol. Tube $10 \mathrm{cc}$. lots, sterilize in Arnold $15 \mathrm{~min}$., 3 successive days.

Dunham's peptone solution (for indol test). Dissolve 10 gms. bacto-tryptone (Difco) +5 gm. sodium chloride in $1000 \mathrm{cc}$. aq. dest. with heat. Make $\mathrm{pH} 7.6$ and filter if necessary, tube $10 \mathrm{cc}$. lots, autoclave $15 \mathrm{lbs} .15 \mathrm{~min}$.

Nitrate broth (for nitrate reduction test). Dissolve 10 gms. peptone +1 $\mathrm{gm}$. potassium nitrate (nitrite-free) in $1000 \mathrm{cc}$ aq. dest. (ammonia-free) with heat. Filter through paper, tube $10 \mathrm{cc}$. portions and sterilize in Arnold $20 \mathrm{~min}$., 3 successive days.

Bromcresol purple milk. Remove cream and heat remainder in cylinder in Arnold $20 \mathrm{~min}$. Again skim off fat and to each liter remaining add 40 cc. $0.04 \%$ aq. bromeresol purple. Tube 10 cc. lots, sterilize in Arnold, 20 min., 3 successive days. Prove sterility by incubation.

Loeffler's (for C. diphtheriae). Collect beef blood in large glass vessels and let clot without moving. Loosen clot from wall with sterile glass rod and place in refrigerator. To 3 parts clear serum removed by pipette add 1 part meat infusion broth containing $1 \%$ glucose $\mathrm{pH}$ 6.8-7. Mix by stirring, inspissate on slant raising temperature gently to approximately $85^{\circ} \mathrm{C}$. Maintain temperature till coagulated firmly. Sterilize in Arnold 20 min., 3 successive days, paraffinize cotton plugs and test sterility.

Hiss' serum-water (for fermentation tests). Add 3 parts aq. dest. to 1 part clear serum, mix, heat in Arnold 15 min. Add $1 \%$ desired carbohydrate dissolved in small quantity hot aq. dest. Add 50 cc. $0.02 \%$ aq. bromthymol blue to each 1000 cc., tube, sterilize in Arnold 20 min., 3 successive days and prove sterility by incubation.

Glycerol agar. Add $30 \mathrm{cc}$. pure glycerol to $1000 \mathrm{cc}$. melted infusion agar, adjust to $\mathrm{pH} 7.2$, tube, autoclave 15 lbs., $15 \mathrm{~min}$. and slant.

Petroff's (for $M$. tuberculosis). Infuse $500 \mathrm{gm}$. beef or veal in $500 \mathrm{cc}$. $15 \%$ aq. glycerol. After 24 hrs. put in sterile press and collect extract in sterile vessel. Place washed eggs in $70 \%$ alcohol, $10 \mathrm{~min}$. Take out with sterile tongs, flame and remove contents to sterile vessel. To 2 parts egg add 1 part meat extract. Add $1 \%$ alcoholic gentian violet to make final concentration $1: 10,000$. Mix and continuc as with Loeffler's medium. 
Cystine blood agar (for $P$.tularensis). To $1000 \mathrm{cc}$. beef or veal infusion broth ardd $15 \mathrm{gm}$. agar, $10 \mathrm{gm}$. peptone and $5 \mathrm{gm}$. sodium chloride. Autoclave $15 \mathrm{lbs} ., 15 \mathrm{~min}$. Before use add $1 \mathrm{gm}$. cystine (or cystine hydrochloride) and $10 \mathrm{gm}$. glucose. Dissolve by heating in Arnold and sterilize $30 \mathrm{~min}$. Cool to $50^{\circ} \mathrm{C}$. add $50 \mathrm{cc}$. sterile horse blood, tube aseptically in $10 \mathrm{cc}$. lots, slant and incubate to prove sterility.

Noguchi's leptospira medium. Combine sterile $80^{\circ} \mathrm{C}$. $0.9 \%$ aq. sodium chloride, 100 cc. fresh rabbit serum, 100 cc. $2 \%$ aq. agar (melted pH 7.4) and 10-20 cc. rabbit hemoglobin (1 part blood, 3 parts aq. dest.). Tube aseptically in $10 \mathrm{cc}$. lots. Incubate to prove sterility.

Tryptone glucose extract milk agar. Combine $15 \mathrm{gm}$. agar, $3 \mathrm{gm}$. beef extract, $5 \mathrm{gm}$. tryptone, $1 \mathrm{gm}$. glucose and 1000 cc. aq. dest. by boiling over free flame. Make up volume lost with aq. dest., adjust to $\mathrm{pH} 7$, add 10 cc. skim milk, place measured volumes in fasks or tubes and autoclave $15 \mathrm{lbs} ., 15 \mathrm{~min}$.

Tellurite (for $C$. diphtheriae). Melt infusion agar, or $0.2 \%$ dextrose agar, and cool to $50^{\circ} \mathrm{C}$. To each $10 \mathrm{cc}$. add 1 cc. citrated, or defibrinated, blood +1 cc. sterile $2 \%$ aq. potassium tellurite, mix and pour into Petri dishes.

Bismuth sulfite agar (Wilson and Blair for E. typhosa). Mix $20 \mathrm{gm}$. agar, $5 \mathrm{gm}$. beef extract and $10 \mathrm{gm}$. peptone in sufficient hot aq. dest. to make 1000 cc. Dissolve by autoclaving 15 $\mathrm{min}$. Store in refrigerator. (A). Dissolve 6 gms. bismuth ammonium citrate scales in $50 \mathrm{cc}$. boiling aq. dest. (1), 20 $\mathrm{gm}$. anhydrous sodium sulfite in $100 \mathrm{cc}$. boiling aq. dest. (2), and 10 gms. (lextrose in 50 cc. boiling aq. dest. (3). Mix 1 and 2, boil and add 10 gnss. anhydrous disodium phosphate while boiling. Cool and add 3 . Add water to restore lost weight. Store in closely stoppered pyrex container in dark at room temperature (B). Dissolve $1 \mathrm{gm}$. ferric citrate in $100 \mathrm{cc}$. aq. dest. using heat and add $12.5 \mathrm{cc} .1 \% \mathrm{aq}$. brilliant green. Store likewise in pyrex vessel in dark. With $1000 \mathrm{cc}$. hot (A) thoroughly mix 200 ce. (B) and 45 cc. (C). Immediately pour into porous-top petri dishes each 15-20 cc. After 2 hrs. at room temperature store in refrigerator and use within 4 days.

Chocolate agar (for Neisseria). Grind strips lean meat of $5-6$ beef hearts. To each $500 \mathrm{gm}$. add $1000 \mathrm{cc}$. tap water, infuse in refrigerator over night, strain and press through course gauze. Add $10 \mathrm{gm}$. proteose peptone No. 3 (Difco) per liter, heat to $50^{\circ} \mathrm{C}$.
$1 \mathrm{hr}$. and boil $10 \mathrm{~min}$. Strain through gauze, dissolve $5 \mathrm{gm}$. sodium chloride per liter and titrate to $\mathrm{pH} 7.6$. Boil lightly 10 min. pour off measured quantities in flasks, autoclave 15 lbs., 15 min. Cool to $60^{\circ} \mathrm{C}$., add $5 \%$ human or horse blood, heat slowly on water bath to $80-85^{\circ} \mathrm{C}$. rotating to get even mixture. Cool to $55^{\circ} \mathrm{C}$. and plate.

Bacterial Pigments. These cannot be measured microscopically but a method has been devised for doing so with spectrophotometer and photoelectric colorimeter (Stahly, G. L., Sesler, C. L. and Brode, W. R., J. Bact., 1942, 43, 149-154).

Bacterial Polysaccharides. Solutions of reduced bases and leuco bases of pentaand hexa-methyl triamino-triphenyl. methane and tetramethyl diaminotriphenylmethane and certain other triphenylmethanes react with staphylococcal polysaccharides and may be useful in their detection (Chapman, G. H. and Lieb, C. W., Stain Techn., $1937,12,15-20$ ).

Bacteriophage Localization by Electron Microscopy, see Hennessen, W., Zeit. f. wis. Mikr., 1951, 60, 172-180.

Bacteriostatic Titration of Dyes. (Reed, M. V. and Genung, E. F., Stain Techn., $1934,9,117-128$ ).

Bacterium Monocytogenes. Intravenous injections of this organism in rabbits produce a marked increase in the number of circulating monocytes and therefore provide an important experimental method (Murray, E. G. D., Webb, R. H. and Swan, M. B. R., J. Path. and Bact., $1926,29,407-439$ ).

Bacterium Tularense in sections. Add 10 cc. sat. aq. nile blue sulphate and 6 cc. $1 \%$ aq. safranin to $60 \mathrm{cc}$. aq. dest. Stain sections over night. Wash quickly, dehydrate in alcohols, clear in xylol and mount (Foshay, L., J. Lab. \& Clin. Med., 1931, 17, 193-195).

Balances. Ordinary balances need no description but for weighing very small amounts special balances are essential. See review of literature by Gorbach, G. , Mikrochemie, 1936, 20, 254-336. The torsion balances of Roller-Smith Co., Bethlehem, Pa., are sensitive to approximately $2 \mu \mathrm{gm}$. The quartz fiber balances are still more sensitive. See Glick, pp. 189-191.

Balantidium. Celloidin embedding and sectioning (Scott, M. J., J. Morph. \& Physiol., 1927, 49, 417).

Balsam for mounting sections is usually satisfactory as purchased. To make, mix equal parts dry balsam and sodium bicarbonate and grind in mortar. Add sufficient xylol to make clear solution. 
After few days filter and heat gently (avoiding flame) to bring to suitable consistency. The best mounting medium when ncutrality is essential is Clarite or the cedar oil used for oil immersion objectives. The latter sets more slowly than balsam and it is ordinarily not necessary to employ it. See Mounting Media.

Barber and Komp thick film for malaria plasmodia (Barber, M. A. and Komp, W. H. W., Pub. Health Rep., 1929, 44, 2330 ) is described by Craig, p. 290-291 as the most used and satisfactory of the thick film techniques. His account of the method abbreviated: Place large drop blood on clean slide and smear with neelle over area about half size of that usually covered by a thin blood smear. Dry in incubator at $37^{\circ} \mathrm{C} ., 1-1 \frac{1}{2} \mathrm{hrs}$. Stain in 1 part good Giemsa and 6 parts neutral or slightly alkaline aq. dest., about $30 \mathrm{~min}$. Partly decolorize in aq. dest. $5 \mathrm{~min}$. (If films have background deep blue and leucocytes almost black they may be worthless; but leaving in aq. dest. longer may help). Drain thoroughly, dry and examine.

Barium, spectrographic analysis of, in retina (Scott, G. I1. and Canaga, B., Jr., Proc. Soc. Exp. Biol. \& Med., 1940, 44, 555556). Barium chloride and formalin are advised as fixative for Bile Components. Barium sulphate emulsion injections are recommended by Woollard, H. H. and Weddell, G., J. Anst., 1934-35, 69, 25-37 to demonstrate arterial vascular patterns. The emulsion should be of such consistency that it cannot easily be forced beyond the small arterioles by a pressure of 1.5 atmospheres. Fix tissues by hypodermic injection of formalin and subsequent immersion in it. Take $\mathrm{x}$-ray photographs of the radiopaque barium. See Cretin's Test for Calcium and barium under Calcium 5 .

Bartholomew, see Gram Stains Mechanism. Bartonella, Try Giemsa's Stain for sections. Basal Bodies of cilia (Wallace, H. M., Science, 1931, 74, 369-370). Fix in Zenker (containing acetic) or in Zenkerformalin $(90 \mathrm{cc}$. Zenkers +10 cc. $10 \%$ formalin). Mount paraffin sections $5 \mu$ thick. After very light staining with hematoxylin and thorough washing in tap water dip in $0.5 \%$ aq. eosin (Grubler's wasserlich. If not available, use Eosin Y.) $\frac{1}{2} \mathrm{~min}$. and wash quickly in large volumes of water. Make up stain by adding 9 parts sat. aq. methyl violet (Grubler's 6B only. If not available, use C.C. which is $2 \mathrm{~B}$.) to 1 part abs. alcohol $33 \mathrm{cc}$.; aniline oil 9 cc. + methyl violet in excess. Stain is best $3-8$ days after mixing but the two solutions can be kept separatcly. After staining sections for $2 \mathrm{hrs}$. wash well in tap water, treat with Lugol's iodine 10-15 min. and repeat the washing. Blot with filter paper. Differentiate in 1 part aniline oil +2 parts xylol. Wash in several changes of xylol and mount in balsam. Basal bodies deep purple, nuclei dark blue. Good also for intracellular bacteria and fibrin.

Basement Membranes of epithelia. Suggest Mallory-Heidenhain's connective tissue stain as modified by Schleicher, E. M. Am. J. Clin. Path., 1943, 7, 35-39 and Schiff Reagent method as detailed by McManus, J. F., Am. J. Path., 1918, 24, 643 .

Basic Brown, G, GX, or GXP, sce Bismark Brown Y.

Basic Dyes, see Staining.

Basic Fuchsin - anilin red, basic rubin, and magenta (CI 676 or 677)-Commission Certified. The tri-amino tri-phenyl methane dyes bearing this name are mixtures of pararosanilin, rosanilin and magenta II in varying proportions. They are employed for a great many purposes. Basic fuchsin in a cytological technique for anterior pituitary is described by Faire, W. R., and Wolfe, J. M., Anat. Rec., 1944, 90, 311-314. New fuchsin (CI 67S) is a different compound. It is the deepest in color of 4 dyes and pararosanilin is the lightest.

Basic Lead Acetate used as fixative for Tissue Basophiles.

Basic Rubin, sce Basic Fuchsin.

Basket Cell Arborizations, technique for, Cajal, S. R., Trav. Lab. Rech. Biol. Univ. de Madrid, 1926, 24, 217.

Basophila Erythroblasts, see Erythrocytes, developmental series.

Basophile Leucocyte (mast-leucocyte, blood mast cell). Least numerous granular leucocyte; percentage about $0-1$; slightly smaller $(8-10 \mu)$ than other types; nucleus spherical or slightly lobated, faintly staining and centrally placed; specific granules only slightly refractile, basophilic, large, variable and less numerous than in other types; function unknown. This cell is difficult to study in fresh preparations of peripheral blood because it is so scarce. Smears colored by the usual methods (Giemsa, Wright, etc.) are satisfactory. The basophilic granules appear to be particularly soluble in water. Doan and Reinhart (C. A. and H. L., Am. J. Clin. Path., 1941, 11, Tech. Suppl. 5, 1-39, with beautiful colored plates) recommend supravital staining with neutral red and janus green. There is difference of opinion as to whether the oxidase and peroxidase reactions are positive (Michels, N. A. in Downey's 
Hematology, 1938， 1, 235-372). See Tissue Basophiles.

Basophilic, see Staining.

Bauer Negative and positive substances, for method see Glycogen.

Beams, see Ultracentrifuges.

Bell's Method for fixing and staining of fats as described by the Bensleys (p. 114). Intracellular fats are mobilized by heat to form droplets which are chromated and later stained. Consequently the preparations show these fats, in addition to other microscopically visible fat, but not their true distribution in the cells. Fix for 10 days at $45-50^{\circ} \mathrm{C}$. in $10 \%$ aq. potassium bichromate $100 \mathrm{cc}$. $+5 \mathrm{cc}$. acetic acid. Imbed and make paraffin sections as usual. Pass them down to absolute alcohol. Stain with freshly prepared Sudan III $10 \mathrm{~min}$. Rinse off in $50 \%$ alcohol and pass to water to arrest action of alcohol. Counter-stain with Delafield's Hematoxylin. Wash in water, differentiate in acid alcohol, wash in water again and mount in Glycerine Jelly.

Benda's Method of crystal violet and alizarin for mitochondria. Fix in Flemming's fluid 8 days (see Flemming's Fluid). Wash in water $1 \mathrm{hr}$. Then half pyroligneous acid and $1 \%$ chromic acid, 24 hrs. $2 \%$ potassium bichromate, 24 hrs. Wash in running water $24 \mathrm{hrs}$. Dehydrate, clear, imbed in paraffin and cut sections at $4 \mu$. Pass down to water and mordant in $4 \%$ iron alum $24 \mathrm{hrs}$. Stain amber-colored sol. sodium sulphalizarinate made by adding sat. alc. sol. to water, $24 \mathrm{hrs}$. Blot with filter paper and color in equal parts crystal violet sol. and aq. dest. ('The sol. consists of sat. crystal violet in $70 \%$ alc. 1 part, alc. 1 part and anilin water 2 parts.) Warm until vapor arises and allow to cool 5 min. Blot and immerse in $30 \%$ acetic acid $1 \mathrm{~min}$. Blot, plunge in abs. alc. until but little more stain is extracted, clear in xylol and mount in balsam. The mitochondria are stained deep violet in a rose background. The colors are more lasting than in Altmann preparations. This is one of the classical techniques of histology but it is difficult. For colored iliustrations see Duesberg, J., Arch. f. Zellforsch., 1910, 4, 602-671.

Benda's stain for fat necrosis. See Fischler's modification.

Bensley's Neutral Safranin. For mitochondria and secretion antecedents especially in the pancreas. Fix in $2.5 \%$ aq. potassium bichromate, 100 cc.; mercuric chloride, 5 gms. 24 hrs. Wash, dehydrate, clear, imbed and section. To prepare stain slowly add sat. aq. acid violet to sat. aq. safranin $\mathrm{O}$ in a flask until ppt. ceases when a drop of mixture on filter paper gives not an outside red rim of safranin but a solid neutral color. Filter. The filtrate should be as nearly as possible colorless. Dry ppt. on filter paper and make of it a sat. sol. in absolute alcohol. Pass sections through 2 changes each of toluol and absolute alcohol, then down through lower alcohols to aq. dest. (Bleach chrome and osmium fixed tissues in permanganate and oxalic acid, as described under Anilin Fuchsin Methyl Green and mercury fixed ones in Lugol's solution, 10 sec. finally washing in aq. dest.) Dilute alcoholic neutral safranin with equal volume aq. dest. and stain 5 min.2 hrs. Quickly blot with filter paper. Plunge into acetone and immediately pass to toluol without draining. Examine and if further differentiation is needed treat with oil of cloves. If this is not enough rinse in abs. alc., flood momentarily with $95 \%$ alc. and pass back through absolute to toluol. Wash in 2 changes toluol and mount in balsam. This is a difficult method but the results are worth it. (see Bensley, R. R., Am. J. Anat., 1911, 12, 297-38s).

Benzamine Blue $3 \mathrm{~B}$, see Trypan Blue.

Benzene-Azo- $\alpha$-Naphthylamine. A monoazo dye used by Carter, J. S., J. Exp. Zool., 1933, 65, 159-179 as a vital stain for Stenostomum.

Benzo Blue 3B, see Trypan Blue.

Benzo New Blue 2B, see Dianil Blue 2R. Benzo Sky Blue, see Niagara Blue 4B.

Benzoazurine G (CI, 502), a direct dis-azo dye of light fastness 4 sometimes polychromatic (nuclei red, cytoplasm blue to blue-violet). Applied after treating blue-green algae with copper sulphate, spores orange red, vegetative cells dark blue or violet (Emig, p. 41).

Benzene ring compounds distinguished from pyrrols by Nitro Reaction.

Benzoin Blue R, see Azo Blue.

Benzopurpurin 4B (CI, 448)-cotton red, diamin red, dianil red, Sultan and direct red, all $4 \mathrm{~B}-\mathrm{An}$ acid dis-azo dye no longer used.

Benzoyl Perioxide treatment is recommended by McClung, Microscopical Technique, 1950 , p. 71 for revival of staining capacity of old smears and spreads.

Benzyl Benzoate is employed in the Spalteholz Method of clearing.

Benzyl Violet. Conn (p. 132) states that this term relates to a group of violets which are pararosanilins, some acid and some basic, with benzyl substitution in one or more amino groups.

Berberian's Method. Berberian, D. A., Arch. Dermat. \& Syphil., 1937, 36, 11711175 , has developed a method for stain- 
ing fungi in epidermal scales and hair, which differentiates epithelial cells, blood cells, bacteria and 'mosaic fungi'. The following account was written by D. A. Berberian, American University of Beirut, Beirut, Lebanon, June 22, 1946:

Fix small pieces of scales or hair on a slide with $50 \%$ aq. glacial acetic acid by drying in an incubator. Defat, clear, hydrate, and wash off the acid as follows: Cover the preparation with ether 2-3 times, 20-30 sec. each; flood twice with absolute acetone, $30-60 \mathrm{sec}$. each; and then flood consecutively with absolute, 95, 70 and 50 per cent alcohol. Stain for 3-5 min. with Martinotti's solution (aq. dest., 75 cc.; lithium carbonate, $0.5 \mathrm{gm}$.; and toluidine blue, $1 \mathrm{gm}$. After the stain dissolves, add 20 cc. glycerin and 5 cc. $95 \%$ alcohol). Wash gently in water and differentiate with $0.5 \%$ acetic acid. Dehydration is best carried out by 3-4 changes of absolute acetone kept 2-3 minutes each time. Pass through xylol and mount in Euparol or any other neutral mounting agent. Success of preparation depends largely on proper differentiation, dehydration and de-acidification. See Fungi.

Berberine Sulphate. An alkaloid used as a fluorochrome for malarial parasites (Metcalf, R. L. and Patton, R. L., Stain Techn., 1944, 19, 11-22).

Bergamot Oil is sometimes used for clearing because it will mix with $95 \%$ alcohol.

Berlin Blue is another name for Prussian Blue (a metallic pigment). It is employed for microchemical detection of Iron. Kremer, Zeit. f. wiss. mikr., $1938, \mathbf{5 4}, 429-432$ suggests proceeding as follows: Fix in absolute alcohol. Deparaffinize $10 \mu$ sections. Bleach in $3-5 \%$ $\mathrm{H}_{2} \mathrm{O}_{2} 3-5$ days. Wash carefully in aq. dest. Quickly darken in $\left(\mathrm{NH}_{4}\right)_{2} \mathrm{~S}$. Transfer to $\mathrm{K}$ ferrocyanide and $\mathrm{HCl}$. Iron gives blue color.

Curiously enough when injection of blood vessels is demanded this mineral pigment is usually called for as Berlin blue. Thus the Bensleys (p. 153) give directions for making up Tandler's Berlin blue gelatin. Soak and melt 5 gms. pure gelatin in $100 \mathrm{cc}$. aq. dest. Add sufficient Berlin blue to give desired color and then 5-6 gms. potassium iodide and a crystal of thymol as a preservative. Inject this mass, which is fluid above $17^{\circ} \mathrm{C}$. Fix tissues in $5 \%$ formalin which preserves it even through decalcification.

Beryllium. In various forms and dosages in production of osteogenic sarcomata in rabbits (Hoagland, M. B., Grier,
R. S., and Hood, M. B., Cancer Res., 1950, 10, 629-635.

Best's Carmine. Grübler's carminum rubrum optimum, or some other good carmine, $2 \mathrm{gm}$.; potassium carbonate, $1 \mathrm{gm} . ;$ potassium chloride, 5 gm.; aq. dest., 60 cc. Boil gently until color darkens, cool and add 20 cc. conc. ammonia. Allow to ripen $24 \mathrm{hrs}$. This is stock solution. Used to stain Glycogen. See Bensley, C. M., Stain Tech., $1939,14,47-52$.

Beta Particles, influence in making radioautographs, see McClung's Microscopical Technique, 1950, p. 707.

Beyer Brown, a diazo dye, stains in aq. or alcoholic solution like a good Ehrlich's hematoxylin (H. G. Cannan, J. Roy. Micr. Soc., 1941, 61, 88-94).

Biaxial Bodies, see McClung's Microscopical Technique, 1950 , p. 605 .

Bichromate-Chromic-Osmic mixture, see Champy's Fixative.

Biebrich Scarlet, water soluble (CI, 280)croceine scarlet, double scarlet BSF, Ponceau B, scarlet B or EC-An acid dis-azo dye much used in histology. See Bowie.

Biebrich Scarlet and Picro-Anilin Blue, as a differential stain for connective tissue and muscle (Lillie, R. D., Arch. Path., 1940, 29, 705). Deparaffinize sections of material fixed in formalin, Zenker's or Orth's fluid. Stain for $\mathbf{5}$ min. in following: Dissolve $1 \mathrm{gm}$. hematoxylin in $95 \%$ alc. and 4 cc. $29 \%$ aq. $\mathrm{FeCl}_{3}$ in 95 cc. aq. dest. +1 cc. conc. hydrochloric acid. Mix and use while fairly fresh. Wash in tap water. Stain for $4 \mathrm{~min}$. in $0.2 \mathrm{gm}$. Biebrich scarlet +100 cc. $1 \%$ aq. acetic acid. Rinse again in aq. dest. Stain for $4 \mathrm{~min}$. in $0.1 \mathrm{gm}$. anilin blue W.S. (C.C.) + 100 cc. sat. aq. picric acid. Wash for 3 min. in $1 \%$ aq. acetic acid. Dehydrate in acetone or alcohol. Clear and mount in salicylic acid balsam. Connective tissue, glomerular basement membrane and reticulum, deep blue; muscle and plasma, pink; erythrocytes, scarlet. (Checked by R. D. Lillie, National Institute of Health, Bethesda, Md., April $22,1946$.

Bielchowsky Silver Methods. These are designed for the nervous system and consist essentially of formalin fixation, silver impregnation, washing, treating with ammoniacal silver solution, washing and reduction in formalin. Several useful modifications are detailed by Addison (McClung, pp. 463-466). See Nervous System, Silver Methods.

Bile. This frequently comes in for microscopic examination of centrifuged sediment. Stitt (p. 761) says that one must be on the lookout especially for: (1) 
Pus cells (neutrophiles), scattered through the specimen and bile stained, which, when occurring in fair numbers, indicate cholecystitis. Unstained pus cells associated with mucus are generally from the mouth. (2) Bile colored epithelial cells and cellular debris suggest chronic cholecystitis. (3) Cholesterin crystals are identifiable as opaque or translucent, flat, rhombic plates or irregular masses. (4) Large amounts of light brown granules or dark blackbrown ppt. of calcium bilirubinate are suggestive of gall stones. (5) Tiny gall stones (bile sand) are identifiable by their concentric lamination. Negative findings are not, he is careful to point out, conclusive of absence of lesions.

Bile Capillaries. 1. Hematoxylin staining. Clara, M., Zeit. f. mikr. Anat. Forsch., $1934,35,1-56$ advises treatment of celloidin sections of pieces of liver fixed in Alcohol Formalin, formalin-absolute alcohol-acetic acid $(20: 80: 1)$ and other mixtures by the Stölzner Holmer technique and his own method. According to the former, mordant the sections in liquor ferri sesquichlorati (try $10 \%$ aq. ferric chloride) 30-45 min. Wash quickly in aq. dest. Stain in ripe $0.5 \%$ aq. hematoxylin, 20-30 min. Wash quickly in water. Differentiate in much diluted liquor ferri sesquichlorati. Wash again quickly in water. Blue with dilute aq. lithium carbonate. Wash in spring water (tap water will do). Dehydrate, clear and mount. According to Clara, mordant the sections in equal parts $\mathrm{A}$ and $\mathrm{B}$ at $40-50^{\circ} \mathrm{C}$. for $24 \mathrm{hrs}$. $(\mathrm{A}=$ potassium bichromate, $2.0 \mathrm{gm}$.; chrome alum, $1 \mathrm{gm}$., aq. dest., $30 \mathrm{cc}$. $\mathrm{B}=$ ammonium molybdate, $2.5 \mathrm{gm}$.; chromic acid, 0.25 gm. ; aq. dest., 100 cc.) Wash briefly in aq. dest. Stain in Kultschitzky's Hematoxylin. Wash in spring water. Dehydrate, clear and mount in balsam. See Clara's illustrations.

2. Rio Hortega silver carbonate method adapted by MeIndoe, A. H., Arch. Path., 1928, 6, 598-614. Fix small pieces normal human liver at least 20 days in $10 \%$ formalin. Heat gently but do not boil and cool several times thin frozen sections for $20 \mathrm{~min}$. in silver bath until they are uniformly of a golden brown color. (To make the bath combine 30 cc. $10 \%$ aq. silver nitrate and 10 cc. sat. aq. lithium carbonate. Wash ppt. repeatedly with doubly distilled water, decanting washings. Add $100 \mathrm{cc}$. doubly distilled water to ppt. Dissolve $\frac{1}{2}-\frac{3}{2}$ of it by adding ammonia water drop by drop. Filter supernatant fluid into opaque bottle and store in dark where it can be kept 2-4 weeks. For use take
$5 \mathrm{cc}$. of this stock solution and add $5 \mathrm{cc}$. aq. dest. and 2-3 drops pyridine.) Wash quickly in aq. dest. Place in $20 \%$ neutral formalin, 1 min. Fix in $2 \%$ aq. sodium thiosulphate, $\frac{1}{2}-1$ min. Wash thoroughly in tap water, 2-3 days adding a little neutral formalin. Dehydrate in $95 \%$ and abs. alc., clear in carbolxylol and mount in balsam. Canaliculi, black.

Bile Components in hepatic cells. Place small pieces of liver in $3 \%$ aq. barium chloride for 6 hours; fix 18 hours in $10 \%$ formalin; dehydrate rapidly in alcohol, clear in benzol and embed in paraffin. The bile components, precipitated by barium chloride, can be stained with acid dyes especially the acid fuchsin in Mallory's connective tissue stain (Forsgren, E., J. Morph., 1929, 47, 519-529).

Bile Pigments. Histochemical reaction. Fix in $10 \%$ formalin or in alcohol. Prolonged fixation is contraindicated. Fix paraffin sections to slides with egg albumen. Deparaffinize and immerse in 2 or 3 parts Lugol's solution and 1 part tincture of iodine, 6-12 hrs. Wash in aq. dest. and cover with sodium hyposulphite (5\% aq.) 15-30 sec. until decolorized. Wash in aq. dest. and stain with alum carmine 1-3 hrs. Wash in aq. dest., dehydrate in acetone, clear in xylol and mount in balsam. Bile pigment granules emerald green (Stein, J., C. R. Soc. de Biol., 1935, 120, 11361138). See Gmelin's Test.

Bilharzial Cercariae. For intra-vitam staining examine in serum plus a little neutral red. For permanent preparations fix in hot lactophenol (equal parts lactic acid, carbolic acid, glycerin and aq. dest.). Stain with alcoholic boraxcarmine. Mount in following: dissolve by boiling gum tragacanth 3 parts and gum acacia 1 part in aq. dest. 100 parts. Add equal parts lactophenol and use filtrate. (Marshall, A., Lab. J., 1937, $7,565-569)$.

Bilirubin, a reddish bile pigment which is isomeric or identical with Hematoidin and which by oxidation can be converted into the green Biliverdin, see Bile Pigments, Urobilin and Van den Bergh Test.

Biliverdin, a green bile pigment produced by oxidation of Bilirubin. See Bile Pigments.

Bindschedler's Green (CI, 819). A basic indamin dye easily reduced to a substituted diphenylamine. See use as a Redox dye in study of metabolism of tumor tissue (Elliott, K. A. C. and Baker, Z., Biochem. J., 1935, 29 (2), 2396-2404).

Binnennetz, see Golgi Apparatus.

Biological Standards. Vitamins, antitoxins, 
hormones and other substances adopted internationally are critically considered by Irwin, J. O., J. Hyg., 1950, 48, 215238.

Biotin, sec Vitamins.

Bird's Eye Inclusions. Some of these bodies, and the so-called Plimmer's Bodies, seen in cancer cells are apparently greatly enlarged Centrosomes. Methods and results are given by LeCount, E. R., J. Med. Res., 1902, 7 (N.S. 2), 383-393.

Birefringence, see Polarization Optical Method.

Bismark Brown Y (CI, 331)-basic brown, $\mathrm{G}, \mathrm{GX}$, or GXP, Excelsior brown, leather brown, Manchester brown, phenylene brown, Vesuvin-A mixture of basic dis-azo dyes of different shades. Quite widely employed, see Blaydes, G. W., Stain Techn., 1939, 14, 105-110 for use with plant tissue. See Weissenberg's method, as described by McClung, 1950 , p. 184, for the preservation in sections of Bismark brown employed for supravital staining of eggs.

Bismiocymol (see Pappenheimer, A. M. and Maechling, E. H., Am. J. Path., $1934,10,577-588$.

Bismuth. Microchemical detection of :

1. Method of Christeller-Komaya. Make frozen sections of formalin fixed tissues. $\mathrm{A}=$ quinine sulphate, $1 \mathrm{gm}$.; aq. dest., 50 cc.; nitric acid, 10 drops. $\mathrm{B}=$ potassium iodide, 2 gm., aq. dest., 50 cc. Immediately before use mix equal parts $A$ and $B$ and add 2 drops nitric acid, C.P. After treating sections with this for $1 \mathrm{~min}$. wash very quickly in 10 cc. aq. dest. +2 drops nitric acid. Mount section on slide. Dry, counterstain with gentian violet. Bismuth appears as dark brown grains (Lison, p. 98). See Komaya, G., Arch. f. Dermat. u. Syph., 1925, 149, 277-291 (good colored figures) and Califano, L., Zeit. f. Krebsf., 1927-28, 26, 183-190.

2. Another modification of the Komaya method is given by Castel, P., Arch. Soc. d. Sci. Med. et. biol. de Montpellier, 1934-35, 16, 453-456 as follows: Dissolve $1 \mathrm{gm}$. quinine sulphate in $50 \mathrm{cc}$. aq. dest. with aid of a few drops of sulphuric acid. Dissolve $2 \mathrm{gm}$. potassium iodide in 50 cc. aq. dest. Mix, apply to section, gives red ppt. of salts of bismuth in form of iodobismuthate of quinine or double iodide of bismuth and quinine. See Pappenheimer and Maechling's (Am. J. Path., $1934,10,577-588$ ) study of nuclear inclusions in the kidney.

3. Wachstein, M. and Zak, F. G., Am. J. Path., 1946, 22, 603-611 have in turn improved the Castel method. See
Glick, Techniques of Histo- and Cytochemistry, 1949 , p. 31 .

Reagents: A. Modified Castel: After dissolving $0.25 \mathrm{gm}$. brucine sulphate in 100 cc. aq. dest. add $2 \mathrm{gm}$. aq. dest. Store in brown bottle and filter before using. B. Diluted Castel: One part A to 3 parts aq. dest. C. Levulose: Dissolve $30 \mathrm{gm}$. in $20 \mathrm{cc}$. aq. dest. at $37^{\circ} \mathrm{C}$. for $24 \mathrm{hrs}$. and add 1 drop of B. D. Counterstain: Add 1 cc. $1 \%$ aq. light green S. F. (Hartman-Leddon Co., Philadelphia) to $100 \mathrm{cc}$. of B and filter before use.

Technique: Treat frozen sections or deparaffinized formalin fixed sections on slides for few sec. with several drops $30 \%$ hydrogen peroxide (Superoxol, Merck) thus removing black sulfide. Wash in tap water and treat with $A 1$ hr. Transfer to $B$ and shake slightly to detach precipitates. Counterstain in $D 4 \mathrm{~min}$. Blot and mount in $C$. Bismith appears as an orange yellow deposit.

Bismuth Pigmentation. Histochemical identification as advised by Wachstcin, M. and Zak, F. G., An. J. Path., 1946, 22, 603-611 depends on ability of hydrogen peroxide to decolorize bismuth sulfide instantaneously and of a slightly modified Castel reagent to change bismuth sulfate into an orange red deposit.

Treat deparaffinized, or frozen, sections with few drops superoxol $(30 \%$ hydrogen peroxide, Merck) from dark bottle kept in refrigerator. In a few seconds black of bismuth sulfide disappears. Wash thoroughly in tap water and place in Coplin jar containing modified Castel reagent made as follows: Dissolve $0.25 \mathrm{gm}$. brucine sulfate (Merck or Eastman Kodak) in $100 \mathrm{cc}$. aq. dest. plus 2 or 3 drops concentrated sulfuric acid. Then add $2 \mathrm{gm}$. potassium iodide, keep in a brown bottle and filrer before use. After $1 \mathrm{hr}$. transfer sections to another jar containing some of reagent diluted with 3 parts aq. dest., and shake gently to remove precipitates. Remove most of fluid from sections by blotting and cover with levulose solution made by dissolving $30 \mathrm{gm}$. levulose in $20 \mathrm{cc}$. aq. dest. at $37^{\circ} \mathrm{C}$. for 24 hrs. to which drop of diluted Castel reagent has been added. To counterstain color $4 \mathrm{~min}$. with freshly filtered $100 \mathrm{cc}$. nondiluted reagent plus $1 \mathrm{cc}$. $1 \%$ aq. light green S F (Hartman-Leddon Co.).

Method also can be used for study of fresh tissues and gross specimens. Add conc. hydrogen peroxide drop by drop to pigmented area. Decolorization is rapid if bismuth sulfide is present. Wash thoroughly in running water to 
remove excess hydrogen peroxide. Apply modified Castel reagent to surface and examine for orange ppt. See description by authors of distribution of bismuth pigmentation in the tissues and comparison with other pigments.

Bismutose, a compound of bismuth and albumen which on application becomes concentrated in the area of the Golgi apparatus (Kredowsky, Zeit. f. Zellf., $1931,13,1)$.

Biuret Reaction. Described as follows by Serra, J. A., Stain Techn., 1946, 21, 5-18: Prepare tissue as described under Ninhydrin Reaction. "The pieces are immersed in strong $\mathrm{NaOH}$ or $\mathrm{KOH}$ solution in a watch glass and some drops of a $1 \%$ aqueous solution of $\mathrm{CuSO}_{4}$ are then added with stirring. A blueviolet coloration indicates the presence of peptides or proteins.

"The reaction is given by the peptide linkage when the peptides are composed of at least three amino acids. The color is more reddish with the simpler peptides. For cytological or histological work, the reaction has the disadvantage of requiring a strong alkaline reaction, which tends to dissolve the protoplasm. To avoid a serious dissolution the tissues must be hardened, for instance with formalin $(10 \%$ formaldehyde during 24 hours, followed by a thorough washing). This reaction has also the disadvantage of being insensitive."

Blastomeres of eggs, see separation of in McClung's Microscopical Technique, 1950, p. 557.

Blastonycosis. The differentiation of $Z y$ monema (Blastomyces) dermatitidis, the cause of blastomycosis, from Cryptococcus hominis, the cause of cryptococcosis or torulosis, is best accomplished by wet India ink technique of Weidman, F. D. and Freeman, W., J.A.M.A., 1924, 83, 1163. Stir suspected material in a drop of india ink, place on a clean slide and cover. Use a small drop so as to form a thin film. Work rapidly before the ink dries out. In blastomy cosis the wall of the organism is thick and presents a double-contoured appearance. Cryptococcus hominis is surrounded by a thick mucoid capsule which, against a dark background, shows up as a clear halo surrounding the fungus. Spinal fluid usually dilutes the ink making a lighter background. See Fungi.

Bleaching of tissue fixed in osmic acid mixtures can be brought about by immersing in dioxan for as long as 3 days. Sections require only a few minutes (Asana, J. J., Stain Techn., 1940, 15, 176).

Blendors, Micro-Waring for low temper- ature use (Sorof, S. and Cohen, P. P., Exp. Cell Res., 1951, 2, 299-300).

Blood. Microscopically considered blood is the field of the hematologist (see Downey's Hematology, N. Y., Hoeber, 1938 in 4 volumes). Any conception of the formed elements of the blood is artificial and inadequate unless it is based upon knowledge of their appearance and behavior in vivo. To examine circulating blood in the web of a frog's foot is helpful but it is better to use mammals. In the latter, the methods devised by Covell and O'Leary for study of the living Pancreas are recommended for blood cells also. Probably the best technique is that of Sandison for direct examination of contents of small blood vessels and capillaries in transparent chambers inserted into rabbits' ears. Living blood cells can be observed in vitro at high magnification in Tissue Cultures; but, of course, circulation is lacking.

When blood cells are removed from the body and mounted on slides in approximately isotonic media, they can be studied for a short time before they become seriously injured and die. Examination in the dark field and after Supravital Staining may be helpful. It is important in interpreting the results to remember that the conditions are very abnormal, that the cells are often more flattened than in vivo, and that the actual speed of movement is not that seen, but is that observed di. vided by the magnification because the distance travelled per unit of time naturally appears greater than it actually is. The motion picture technique has great potentialities.

Examination is usually limited to fixed and stained Blood Smears but valuable data can also be secured from sections. Normal values for blood cells during first year of life (Merritt, K. K., 1933, 46, 990-1010). For details, see Blood Protein (coagulated), Bone Marrow, Chylomicrons, Erythrocytes, Erythrocyte Counts, Fibrin, Hematoidin, Hematoporphyrin, Hem ofuscin, Hem oglobin, Hemosiderin, Leucocytes, Leucocyte Counts, Lymphocytes, Monocytes, Platelets, Parhemoglobin, Reticulocytes, Sulfmethem oglobin.

Blood Agar, see Bacteria, Media.

Blood Cell Volume. Dry Evans Blue (Merck) at $100^{\circ} \mathrm{C}$. Dissolve $400-800$ mg. in 1 liter aq. dest. Put $0.5-1$ cc. in tube $3-4$ cc. capacity and evaporate to dryness at $70^{\circ} \mathrm{C}$. Collect blood to contain 2.0-2.5 units heparin or $0.2 \%$ ammonium oxalate. Centrifuge and transfer $1 \mathrm{cc}$. plasma to tube containing dye. Remove 0.1 cc. dyed plasma to 
$9.9 \mathrm{cc}$. saline in photoelectric colorimeter tube. Make blank without plasma. Compare in Evelyn or Klet-Summerson colorimeter using filter to pass only light of about $620 \mathrm{~m} \mu$. Calculate as directed for the colorimeter (Shohl, A. T. and Hunter, T. H., J. Lab. \& Clin. Med., 1941, 26, 1829-1837). See also earlier cell opacity method (Shohl, A. T., J. Lab. \& Clin. Med., 1939-40, 25, 13251332).

Blood Flow, technique for local measurement of, using radioactive sodium (Semple, R., McDonald, L. and Ekins, R. P., Am. Heart J., 1951, 41, S03-809).

Blood Grouping techuique does not properly come in the scope of this book; but since it is involved in fundamental medical and biological problems the following leading reference is given: Schiff, F., and Boyd, W. C., Blood Grouping Technic. New York: Interscience Publishers, Inc., 1942, $248 \mathrm{pp}$.

Blood Platelets-Written by Paul M. Aggeler, University of California Medical Center, San Francisco 22, California. November 15, 1951-It is believed by most authorities today that the platelets originate from megakaryocytes, chiefly in the bone marrow but perhaps also in the lungs. The platelets are thought to be detached fragments of the cytoplasm of mature megakaryocytes. In man the platelets usually vary between 2 and 3 microns in length, although microplatelets of less than one micron and macroplatelets as long as 25 to 50 microns have been observed. They vary in thickness from 0.5 to 1.0 micron. In rapidly fixed blood they usually assume the shape of an oval disc or lentil although a variety of forms may be encountered. In unfixed blood, even in the presence of an isotonic anticoagulant many degenerative forms may occur. These may appear shrunken or "exploded" and there may be numerous spinelike projections from the surface of the platelet. In dry smears of imperfectly fixed blood stained with Wright's stain the platelets appear to be divided into two zones: the clear blue hyalomere and the chromomere made up of purple staining granules. This separation into two zones is probably an artefact produced by changes in distribution of the granular material of the platelet after leaving the circulation, for when blood is rapidly fixed the granules are evenly distributed through the body of the platelet. Macroplatelets found in the blood in periods of abnormal blood regeneration often take a deeper stain and the granules are coarse and do not show, even in slowly dried preparations, the clear separation between hyalomere and chromomere.

Platelets are found in the circulating blood, particularly in the capillaries of the liver and lung, in the bone marrow and in the spleen, both in the sinuses and between the cells of the pulp. They are not found in the lymph or in the thoracic duct. More platelets are found in arterial than in venous or capillary blood.

Physiological decreases in the platelet count are said to occur during the first day of menstruation and increases have been found after violent exercise and following a change to a high altitude. An increased platelet count (thrombocytosis) may be found in infectious diseases, trauma, fractures, asphyxiation, surgical operations, acute blood loss, chronic myelocytic leukemia, Hodgkin's disease and erythremia. A decreased platelet count (thrombocytopenia) is the basic defect in idiopathic thrombocytopenic purpura and also occurs secondarily in certain acute infectious diseases of the blood and blood forming organs, diseases of the spleen, allergies, sensitization reactions to certain drugs and chemicals, and following the use of certain toxic agents such as benzol or ionizing radiations.

The platelets are thought to survive in the circulation of the normal subject for from three to five days. There is recent evidence to suggest that in idiopathic thrombocytopenic purpura they are destroyed at a much more rapid rate. The reduction of the platelet count in this disease had previously been thought to be due either to failure of production of the platelets or to abnormally rapid removal from the circulation by phagocytosis in the spleen. The mechanism of reduction of the platelet count in secondary thrombocytopenic purpuras may be: (1) destruction of megakaryocytes as in ionizing radiation; (2) splenic inhibition of maturation of megakaryocytes as in congestive splenomegaly or; (3) a direct inhibition of maturation of megakaryocytes as in sensitivity reactions to drugs.

The platelets are concerned in the coagulation of the blood, in retraction of the blood clot, in the formation of thrombi and in the maintenance of capillary continuity. The most significant property of platelets is the readiness with which they agglutinate in shed blood or when exposed to a foreign surface. Agglutination is generally followed by fusion and lysis.

Direct Methods: The principle of all 
direct methods of platelet counting consists in the accurate dilution of a measured quantity of blood with a suitable fluid. The diluted blood is placed in a counting chamber and the number of platelets in a circumscribed volume is counted. These methods of counting platelets involve the use of the same apparatus as is employed in the erythrocyte count. The Thoma diluting pipette is generally used. It consists of a capillary tube graduated in tenths, which opens into a bulb containing a glass bead. The bulb, when filled to the mark 101 above it, will hold 100 times the quantity of fluid contained in the 10 divisions of the capillary tube. The counting chamber is a heavy glass slide, with two central ruled platforms. They are separated from each other by one moat, and from elevated shoulders on each side by transverse moats. These lateral shoulders are so ground that a cover-slip resting on them lies exactly $0.1 \mathrm{~mm}$. above each platform. A ruled area of $3 \mathrm{~mm}$. by $3 \mathrm{~mm}$. ( $9 \mathrm{sq} . \mathrm{mm}$.) is engraved oul each side. This area is divided into 9 large $1 \mathrm{~mm}$. squares. The central square is divided into intermediate sized squares of $1 / 25$ sq.mm., each of which is further divided into smaller squares of $1 / 400 \mathrm{sq}$. mm.

In making a count, blood is drawn by suction into the Thoma pipette to the 0.5 mark. The blood adhering to the outside of the pipette is wiped off and the diluent is drawn in until it fills the bulb and reaches the mark 101 . While drawing in the diluent, the pipette is revolved between the finger and the thumb, in order to mix the blood thoroughly with the diluent. After the desired quantity of solution has been drawn into the pipette, it is held horizontally and shaken for one-half minute, or it may be placed in a mechanical shaking device, in order to secure thorough mixing. The coverglass is next placed on the chamber. Several large drops of fluid are expelled from the pipette and discarded, and a small quantity of diluted blood is allowed to run under the coverglass. The platform should be completely covered with fluid, and none should run over into the moats.

The preparation is allowed to stand 10 minutes to allow settling of the cells, then the number of platelets seen in the entire central $1 \mathrm{~mm}$. ruled area is counted. This number is multiplied by $2000[1 / 10$ (depth) $\times 1 / 200$ (dilution)].

There may be errors due to uncleanliness of the glassware, inaccurate calibration of pipettes or chambers, imperfect dilution, failure to mix the blood and diluting fluid thoroughly, imperfect filling of the chamber and improper sampling, such as might occur if a free flow of blood is not obtained from the finger or ear when capillary blood is employed, or from inproper sampling if venous blood is used. The above sources of error can be avoided by an experienced technician, so that their contribution to the inaccuracy of the platelet count is minimal. However, there are additional sources of error when this method, which was originally devised for erythrocyte counting, is used for the enumeration of platelets. Olef, I. (J. Lab. \& Clin. Med., 1935, 20, 416) has listed the fol. lowing: 1) clumping of the platelets during drawing of the blood into the pipette, 2) the impossibility of visualizing the smaller platelets with the high dry lens, 3) the failure to visualize some of the platelets in the 100 nicron space that exists between the bottom of the counting chamber and the overlying coverglass, 4) the sticking of the platelets to the side of the pipette and to the parts of the hemacytometer, and 5) the confusion of foreign particles or precipitates with platelets.

Platelet clumping, the adhesion of platelets to glassware and the occurrence of morphological changes in the platelets can be prevented only by the immediate mixing of the blood with a diluting fluid which contains both an anticoagulant and a fixative. It is not generally appreciated that degenerative changes occur quite rapidly in platelets, even in the presence of an anticoagulant, unless a fixative is also incorporated in the diluting fluid. The anticoagulants which have been employed are sodium, potassium or ammonium oxalate, sodium citrate, sodium metaphosphate, sodium sulfate, magnesium sulfate, heparin, peptone and gelatin. Of these, 3.8 per cent sodium citrate appears to be the most satisfactory, since it is isotonic with the blood plasma, does not form erystals or precipitates with the plasma, and is not highly conducive to bacterial growth. The fixatives most commonly employed are mercury bichloride, osmic acid, solution of formaldehyde, and methyl or ethyl alcohol. Of these, the solution of formaldehyde is preferable because it does not produce clumpiug of the erythrocytes, does not form protein precipitates with the plasma, and does not produce secondary changes in the platelets. In order to avoid hemolysis from the formation of formic 
acid, the diluting fluid should be made fresh every week and only 38 per cent neutral formaldehyde, U.S.P. should be employed. A concentration of 0.1 per cent of formaldehyde in the diluting fluid is sufficient. Higher concentrations may hemolyze the erythrocytes and form precipitates with the plasma.

It is generally conceded that the incorporation into the fluid of a dyc, such as brilliant cresyl blue, methyl violet, methylene blue, toluene red, crystal violet and nile bluc, is superfluous, since it does not assist in the differentiation of platelets from artefacts and may even itself be the source of artefacts. Diluting fluids containing no anticoagulant, such as Tyrode's or Ringer's solution, produce many artefacts and, in addition, cause rapid disintegration of platelets unless a fixative, such as solution of formaldehyde, is incorporated in the solution. A satisfactory diluting fluid, therefore, is the one proposed by Rees, II. M., and Ecker, E. E. (J. A. M. A., 1923, 80,621 ) without the brilliant cresyl blue dye. This solution is prepared by adding $0.22 \mathrm{ml}$. of neutral formaldehyde ( 38 per cent U.S.P.) to $100 \mathrm{ml}$. of 3.8 per cent sodium citrate. The solution should be kept in a well-stoppered bottle in a refrigerator and should be filtered each time just before use. Tocantins, L. M. (Arch. Path., 1937, 23, 850) also recommends sterilizing the solution in order to reduce the number of artefacts caused by bacterial contamination.

Despite the use of a satisfactory diluting fluid, the adhesion of platelets to each other and to the walls of the pipette is almost impossible to avoid when capillary blood is used. Drawing the diluting fluid to the 0.5 mark on the pipette and subsequently drawing the head of the column of fluid to the 1.0 mark while aspirating the blood into the pipette is unsatisfactory, since there is little opportunity for the blood to $\mathrm{mix}$ with the diluting fluid within the narrow confines of the capillary stem of the pipette. Furthermore, even the very short time required to draw the exact quantity of blood into the pipette and to mix it with the diluting fluid is sufficient in many instances to allow marked clumping of the platelets to occur. The clumping of platelets can best be avoided by mixing the blood with the diluting fluid before it is drawn into the capillary pipette. This can be accomplished by the use of venous blood. The blood may be drawn into a silicone-lined syringe and then immediately transferred to a tube containing the diluting fluid or, preferably, a given amount of blood is lrawn into a syringe containing a measured amount of diluting fluid. Needles varying in size from No. 18 to No. 26 have been employed without any apparent effect on the quality of the preparation. A satisfactory method for obtaining venous blood is as follows. Place exactly $1 \mathrm{cc}$. of diluting fluid in a 5 cc. syringe. Attach a sterile dry No. 24 needle and aspirate $1 \mathrm{cc}$. of blood from the vein, without stasis, by withdrawing the plunger to the 2 cc. mark. Withdraw the needle from the vein and aspirate the blood contained in it into the syringe. Remove the needle and thoroughly mix the diluted blood in the syringe and expell it into a collecting bottle. It is important to use a syringe whose plunger and needle fit snugly, in order to avoid the leakage of any air into the syringe while aspirating the blood. A 1 to 200 dilution of the blood can be made by drawing this mixture to the 1.0 mark on the Thoma pipette and subsequently filling it to the 101 mark with the diluent. If this procedure is carried out carefully, it is unnecessary to resort to methods, such as those suggested by Aynaud, M. (Compt. Rend. Soc. Biol., 1910 68, 1062) and Preiss, W. (Zeitsch. Ges. Exp. Med., 1932, 84, 1932) in which no attempt is made to obtain an accurate initial dilution in the syringe, and consequently the final dilution of blood in the counting chamber must be determined by establishing the ratio of an erythrocyte count done on the platelet preparation with an independent erythrocyte count done in the usual manner.

There is still another limitation in the accuracy of the direct platelet count, imposed by the relatively small concentration of platelets in the counting chamber. The dilution of 1 part blood in 200 parts of fluid is required because with any greater concentration the platelets would be obscured by the erythrocytes. However, since there are only approximately 5 per cent as many platelets as erythrocytes present, the statistical error is much greater than that of the erythrocyte count, even if all the platelets in the entire 1 square $\mathrm{mm}$. central ruled area are counted. Attempts have been made to overcome this difficulty by increasing the concentration of platelets in preparations from which the erythrocytes have been eliminated. In some methods, such as that of Brecher, G. and Cronkite, E. P. (J. Applied Physiol., $1950,3,365)$ the erythrocytes are 
hemolyzed. However, the use of hemolytic diluting fluids, such as potassium cyanide, urea, or 1 per cent ammonium oxalate is to be discouraged since hemolyzed erythrocytes may be the source of artefacts. Furthermore, if such diluting fluids contain no fixative, dissolution of some of the platelets may also occur. Another means of attaining a higher concentration of platelets in the counting chamber is to do the platelet count on plasma. In Reimann's modification of Thomsen's method (J. Exper. Med., 1924, $40,553) 0.9 \mathrm{cc}$. of blood is drawn into a tuberculin syringe containing 0.1 cc. of 10 per cent sodium citrate. The needle is removed and the open end of the syringe is closed with a piece of rubber tubing fastened to the barrel with rubber bands. The plunger is removed and the syringe is placed in a vertical position. After sedimentation has occurred, a 1 to 20 dilution of the supernatant plasma is made with physiologic sodium chloride solution. The platelets in the mixture are then counted in the counting chamber in the usual manner. If the platelets seen in five intermediate sized squares (80 small squares) are counted, the number is multiplied by 1000 [1/5 (area) $\times 1 / 20$ (dilution) $\times 1 / 10($ depth $)]$, in order to find the number of platelets per cubic millimeter of plasma. A further procedure has been designed to determine the number of platelets per cubic millimeter of blood. The syringe containing the blood is centrifugalized for 20 minutes at 2000 r.p.m. The relative amount of plasma and packed cells is recorded and the number of platelets per cubic millmeter of blood is determined thus:

$\begin{aligned} & \text { Number of } \\ & \text { platelets per } \\ & \text { cubic milli- } \\ & \text { meter of } \\ & \text { blood }\end{aligned}=\begin{aligned} & \text { Number of } \\ & \text { platelets per milli- } \\ & \begin{array}{l}\text { meter of } \\ \text { plasma }\end{array}\end{aligned} \times \frac{\begin{array}{l}\text { Amount of plasma } \\ \text { mixture }\end{array}}{\text { motant of }}$

To the result, 10 per cent of the total number is added to offset the original dilution in the syringe. The determination of the platelet count in plasma by this method has the advantage of increasing the concentration of platelets per unit volume in the counting chamber approximately ten times. However, there is some question as to the stability and uniformity of the suspension of platelets in plasma during the time required for sedimentation of the erythrocytes. Furthermore, the determination of the relative plasma and packed cell volumes by the method given is inadequate, since even at a centrifugalization speed of 3000 r.p.m. at least 15 per cent of the plasma is retained in the packed cell mass.

In order to avoid the errors inherent in the Thomsen method and still in. crease the concentration of platelets in the counting chamber, methods have been devised for eluting the platelets from the whole blood. In the method of Villariño and Pimentel (Am. J. Clin. Path., 1942, 12, 362) a 1 to 2 dilution of venous blood with Aynaud's fluid is made in the syringe. Four cc. of pooled eluate from $0.2 \mathrm{cc}$. of this mixture are obtained by four separate centrifugations for 1 minute at 1500 r.p.m. Only an insignificant number of platelets can be recovered by further washing. A similar method has been devised by Scheff, G. I. and Ralph, P. H. (Am. J. Clin. Path., 1949, 19, 1113) using an angle centrifuge at a speed of 700 r.p.m., and dark field illumination. Although it would appear that practically all of the platelets are recovered by these methods since only an insignificant number can be recovered by further washing and only a very few can be found in stained smears of the washed sediment, there is some question as to whether some of them may not have been destroyed during the process of repeated centrifugalization.

Regardless of the source of the specimen, type of diluting fluid or concentration of platelets per unit volume of fluid in the chamber, there are certain difficulties in differentiating platelets from artefacts when high dry magnification and bright field illumination are used. Under these circumstances, a platelet is defined simply as "a small refractile body". According to Tocantins, L. M. (Ârch. Path., 1927, 23, 850) "Only forms from 1 to 3 microns or larger in size, rod or comma-like if seen sideways, and thin, translucent and disclike if flat on the floor of the counting chamber, should be counted. Granules 0.8 micron in diameter or smaller, jerkily moving about more or less actively, globules of oil, irregularly shaped debris floating on the upper layers of the fluid, strings of cocci, and other minute objects may be distinguished from platelets after a little practice. The error of counting too few platelets may be equaled only by the error of counting every particle in the field as a platelet." 'That experienced observers differ significantly, even when using the same method, is shown by the following results of cutaneous platelet counts on normal adult subjects, using the Rees and Ecker method. 


\begin{tabular}{|c|c|c|c|c|}
\hline Author & $\begin{array}{c}\text { Number } \\
\text { of } \\
\text { Subjects }\end{array}$ & Mean & $\mid \begin{array}{l}\text { Stand- } \\
\text { ard } \\
\text { Error } \\
\text { of Mean }\end{array}$ & $\begin{array}{l}\text { Stand- } \\
\text { ard } \\
\text { Devia- } \\
\text { tion }\end{array}$ \\
\hline $\begin{array}{l}\text { Tocantins, L. M., } \\
\text { Arch. Path., 1937, } \\
23,850, \ldots \ldots \ldots \ldots\end{array}$ & 40 & 250,000 & 7458 & 58,500 \\
\hline $\begin{array}{c}\text { Aggeler, P. M., How- } \\
\text { ard, J. and Lucia, } \\
\text { S. P., Blood, 1946, } \\
\text { 1, } 472 \ldots . . . . . . .\end{array}$ & 64 & 409,000 & 8500 & 68,000 \\
\hline $\begin{array}{l}\text { Sloan, A. W., J. Clin. } \\
\text { Path., 1951, 4, 37. }\end{array}$ & 80 & 241,000 & & 50,000 \\
\hline
\end{tabular}

The author (Aggeler) is aware of the fact that the platelet counts reported by him and his associates could be higher than those observed by Tocantins and Sloan because certain artefacts were consistently counted as platelets. On the other hand there is no proof that the latter authors did not mistake some of the platelets for artefacts. It is begging the point to demonstrate that any single observer may obtain consistent results. This means only that he is constant either in identifying platelets as platelets, platelets as artefacts, or artefacts as platelets. It does not prove that the particular observer is always identifying platelets as platelets and artefacts as artefacts. Furthermore, the fact that several observers using the same method may obtain reasonably consistent results does not prove the validity of the method, since both observer may be committing the same error. The ultimate cause for the great variation in the reported results of different observers using the same method is that platelets cannot be distinguished from artefacts with certainty in any method in which the magnification and resolution is not considerably better than that which can be achieved with the high dry lens and bright field illumination. Some improvement can perhaps be gained by the use of dark field illumination, as recommended by Scheff and Ralph (Am. J. Clin. Path., $1949,19,1113)$ or by the use of phase contrast illumination, as advocated by Brecher, G. and Cronkite, E. P. (J. Applied Physiol., 1950, 3, 365).

Nothing is gained by the use of oculars giving a magnification greater than $10 \times$ since, in the absence of any mprovement in resolution, the increased magnification only serves to increase the confusion. Attempts have been made by Helber, E. (Arch. f. Klin. Med., 1904, 81, 316) and by Maixner and Von Decostello (Med. Klin., 1915, 11, 14) to increase magnifica- tion and resolution by the use of the Zeiss D water immersion lens. With this equipment, magnification of $1000 \times$ was attained. Approximately the same magnification with better resolution can be achieved with an oil immersion lens. However, with these lenses, it is necessary to use a specially constructed counting chamber of $0.02 \mathrm{~mm}$. depth and a cover slip not more than $0.25 \mathrm{~mm}$. in thickness. Because of the reduced depth, a 1 to 40 dilution of blood is used in order to obtain the same number of cells per unit area of the counting chamber as are present when using a 1 to 200 dilution in the $0.1 \mathrm{~mm}$. depth chamber. The author and his associates have found no difficulty in distinguishing artefacts from platelets with a chamber and coverslip of this type* used in conjunction with a Spencer binocular microscope equipped with $1.8 \mathrm{~mm} ., 97 \times$ (oil immersion) N.A. 1.25 , medium dark contrast phase objective, standard condenser lower element, standard N. A. 1.25 condenser top element, $97 \times$ annular diaphragm and $10 \times$ Hugenian eyepieces. Even the morphologic characteristics of small platelets were clearly visible. Most of the platelets appeared as flat, round or oval discs, about 2 microns in diameter and 0.5 microns thick. The cytoplasm showed a uniform, fine granulation. Some of the platelets had a slightly irregular shape, most had a single short slightly curled filament, others had more than one filament, and a few had none. Occasionally two platelets attached by a long streamer of cytoplasm were observed. Rarely was an "explosive" degeneration form of platelet seen, and none of the platelets had the multiple sharp spinelike projections which always appear when no fixative is employed in the diluting fluid. The principal artefacts observed were bits of amorphous debris which could be distinguished by their irregular shape and heavy granulation; erythrocyte fragments, which were colored yellow, had no granulation and were intensely refractile; and clumps of bacteria, which were identified by their regularly spaced heavy granules separated by fine dark filaments. Unfortunately, while the problem of differentiation of platelets from artefacts is solved by this system of microscopy, extreme differences in the platelet counts on aliquots of the same venous blood specimen were encountered, so that the advantage of

- Manufactured by the American Optical Co., Buffalo, N. Y., through the courtesy of Mr. C. E. Guellich, Manager of Product Sales, Scientific Division. 
better visualization of the platelets was nullified. The variations in the platelet count appear to be due to differences in the volume of fluid contained in the chamber caused by upward lifting or downward bending of the coverslip. Before this technique can be perfected, it will be necessary to develop methods for insuring scrupulous cleanliness of the glassware. In addition, a radical change in the design of the chamber to prevent downward bending of the coverslip due to intense capillary attraction will be required.

With regard to the established direct methods of platelet counting, therefore, it appears that most of the sources of error can be minimized, except that of accurate identification of the platelets. This error can be made a constant for a given method by an experienced observer, but may lead to large inconsistent variations in platelet counts done by inexperienced individuals.

Indirect Methods: One of the principal advantages of indirect methods of platelet counting is that microscopic objectives giving higher magnification and greater resolution can be employed. Furthermore, the blood can be mixed immediately with the diluting fluid and the platelets fixed before it is necessary for them to come in contact with any foreign surface, except perhaps momentarily with the skin or a venipuncture needle. In all indirect methods of platelet counting unknown quantities of blood and diluent are mixed. The number of platelets seen per 1000 erythrocytes is multiplied by the number of thousands of erythrocytes found in an independent erythrocyte count done in the usual manner. There are three methods of determining the ratio of platelets to erythrocytes: in a counting chamber, a wet slide preparation or a dry smear.

Methods, such as that of Kemp, G. T. and Calhoun, H. (Brit. Med. J., 1901, 2,1539 ) in which capillary blood is employed and the ratio of erythrocytes to platelets is determined in the counting chamber, are subject to the same error involved in identifying the platelets as is inherent in all direct methods of platelet counting.

Methods such as those of Aynaud, M. (Compt. Rond. Soc. Biol., 1910, 68, 1062) and Preiss, W. (Zeitsch, Ges. Exp. Med., 1932, 84, 810) referred to above, in which venous blood is used are sometimes referred to as indirect methods. They are not, since in these methods, the ratio of the erythrocyte count in the platelet preparation to the erythrocyte count done in the usual manner only serves to establish the dilution of blood employed in the platelet count. The number of platelets counted per unit volume is an absolute not a relative value. The additional step of establishing the ratio of the two erythrocyte counts could be avoided by accurate measurement of the degree of dilution of the blood in the syringe. These methods are also subject to the errors involved in identifying platelets with the high dry lens in the standard counting chamber.

Some of the objections to the indirect method of platelet counting in which the ratio of platelets to erythrocytes is determined in a wet preparation or a dry smear have been summarized by Tocantins (Arch. Path., 1937, 23, 850). 1) The mixture of blood and diluting solution is seldom, if ever, uniform and not the same each time. 2) Platelets and erythrocytes are not distributed evently through the preparation, since no provision is made for shaking before counting. 3) The method has defects intrinsic in any determination done indirectly, that is, in relation to another equally changeable element. 4) The greatest source of error, however, is in the assumption that platelets and erythrocytes keep an even proportion in numbers toward each other between the two main steps of the method. The markedly different physical properties (adhesiveness, specific gravity, size and others) of platelets and erythrocytes lead to continuous changes in this ratio. 5) The proportion of platelets to erythrocytes varies at the same time in different portions of the circulation and this variation is even more marked within short spaces of time in capillaries than venules.

The term "indirect platelet count" has come to imply that the count is done on capillary blood. Curiously enough it seems never to have occurred to anyone to do an indirect platelet count on accurately diluted venous blood. This would overcome some of Tocantins' objections since it would allow for a constant uniform dilution of the blood, shaking of the diluted blood before mixing and the determination of the erythrocyte count on the same specimen as is used for the platelet count.

Olef (J. Lab. \& Clin. Med., 1935, 20 , 416) has also raised objections to the technique employed in certain indirect methods of platelet counting. In those in which a drop of blood is allowed to fall into the diluting fluid on a glass slide or contained in a special vessel (Pratt, J. H. J. A. M. A., 1905, 45, 1999). 
the undiluted blood is allowed to come into contact with the surface of the skin and with the external air, thus allowing the platelets to clump. In methods in which the finger is punctured through a drop of fluid (Fonio, A., Deutsche Ztschr. f. Chir., 1912, 117, 176) the first drop of blood must be used. This procedure is said not to yield accurate counts because the blood contains disintegrated products of crushed and injured cells and is also diluted by an admixture of lymph.

With regard to methods in which the blood and diluent are mixed on the finger, Olef stated, "This technic involves a number of inaccuracies. During the process of stirring the mixture there is unavoidable contact of blood and skin with resulting destruction and clumping of platelets. If the blood is flowing freely, as it should if a correct count is to be obtained, the blood and diluting fluid form a very large drop in which it is rather difficult to obtain a uniform distribution of the blood and which frequently rolls off the finger, especially in women in whom the surface at the tip of the finger is small. Furthermore, freely flowing blood mixed with only one drop of preserving fluid yields preparations too thick for accurate platelet counting. Dameshek does not stir the blood-diluent mixture at all; he places a drop of the preserving fluid over the puncture wound after the first drop or two of blood has been wiped away, than allows some blood to escape into the overlying drop of diluent and by applying a cover slip to the mixture carries some of it away. This procedure is inaccurate because the platelets, being very light, quickly rise to the surface of the drop of diluting fluid before the considerably heavier erythrocytes have become uniformly distributed. The fluid on the cover slip, therefore, contains a relatively larger number of platelets than red cells." Olef has also pointed out that all glassware must be scrupulously clean since hemolysis may occur with soiled glassware. For use in wet preparations, he advocates a 1 to 5 dilution of blood, since in very thin preparations both the platelets and erythrocytes are likely to be destroyed, whereas in thick preparations the erythrocytes may obscure some of the platelets. Olef's objection to dilutions of blood of greater than 1 to 5 on the grounds that this may cause dissolution of the platelets is not valid if a fixative is contained in the diluting fluid.

In Olef's method, the first drop or two of blood is wiped away. A drop of diluting fluid is then placed over the puncture wound before the blood reaches the surface of the skin, and the hand is quickly turned over so that the palmar surface is clirected downward. After a sufficiently large drop has escaped, the entire mixture is applied to the surface of a small quantity (three to four drops) of diluting fluid contained in a paraffin cup. The entire drop on the finger, consisting of approximately equal parts of blood and diluent, drops off into the cup. The contents of the cup are then stirred gently with a wooden applicator, the end of which is coated with paraffin. The mixture is allowed to stand for a minute or two, stirred again, and then is transferred by means of a clean paraffin-coated applicator to a glass slide. Usually three preparations are made. A coverslip is placed over each drop and after the preparations have been allowed to stand for ten to fifteen minutes, a relative platelet-erythrocyte count is made, using the oil immersion lens. While this method has certain advantages over other indirect methods, it does not overcome the principal objection raised by Tocantins, i.e., that the platelets and erythrocytes may not keep an even proportion in numbers toward each other between the two main steps of the method. That they do not can be easily demonstrated by counting both the erythrocytes and platelets in successive microscopic fields in different parts of the same preparation. It will be found that the platelets maintain a fairly even distribution despite large differences in the erythrocyte concentration. This leads to variation in the ratio of platelets to erythrocytes in different parts of the same preparation. The observer must arbitrarily choose areas to be counted, but because of unconscious bias in selection he will tend to count only those which he thinks have an average distribution of erythrocytes. However, in this type of preparation it is impossible to determine what the average distribution is. This bias in sampling greatly increases the subsampling error. The uneven distribution of erythrocytes occurs regardless of whether the blood is capillary or venous, or whether it has been thoroughly shaken in a pipette or simply mixed with a stirring rod before the preparation is made. This maldistribution of erythrocytes cannot be avoided since it is caused by physical phenomena which oceur during the very act of placing the coverslip on the drop of blood.

Dry slide preparations made from a 
TABLE 1

Characteristics of various direct methods of platelet enumeration

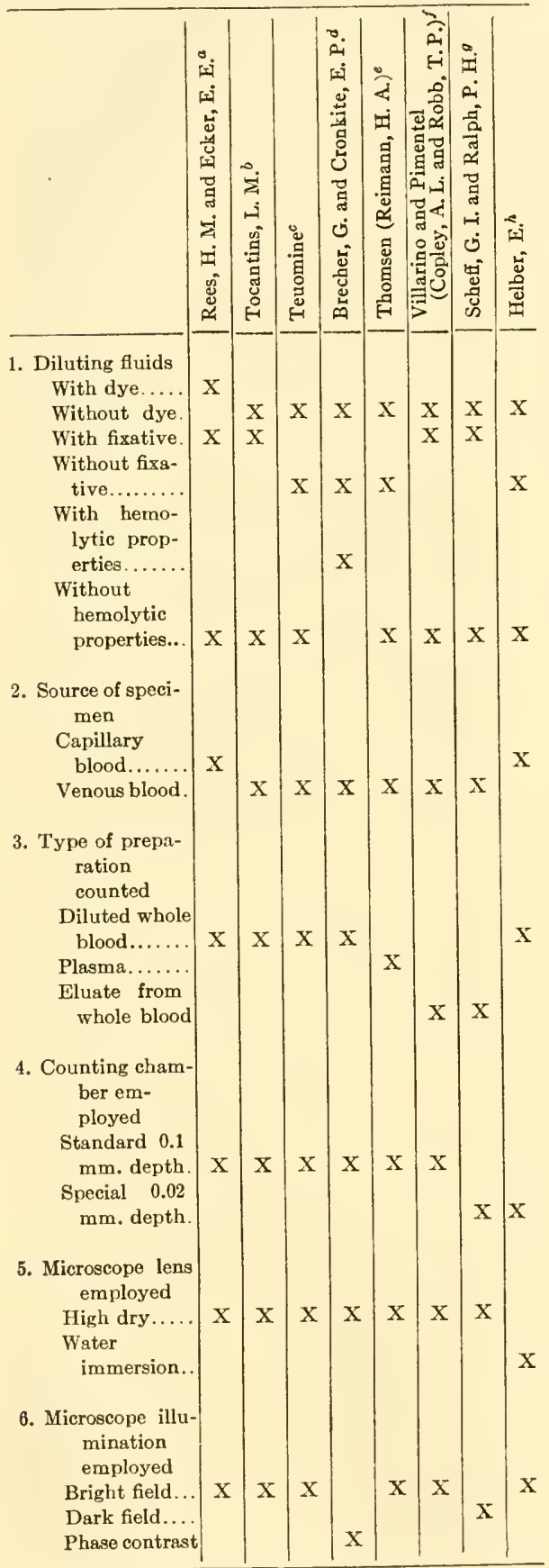

1 to 2 dilution of blood, stained in the usual manner, and examined with the oil immersion lens have the advantage of a somewhat more uniform distribution of erythrocytes and platelets, but there is question as to whether some of the platelets and/or erythrocytes may not be destroyed during the process of making the smears.

The above multiplicity of methods, and numerous other variations not mentioned, is not the result of idle invention but rather of a persistent effort on the part of each investigator to overcome the errors inherent in the methods of his predecessors. In many instances however, the successful solution of one difficulty has only served to give rise to other sources of error. The impossibility of achieving a uniform distribution of erythrocytes and platelets makes it unlikely that a satisfactory indirect method can be devised. On the other hand the inadequate visualization of platelets with the high dry lens makes all direct methods in which the standard $0.1 \mathrm{~mm}$. depth counting chamber is employed unreliable. The only solution to the problem appears to be the perfection of a technique for using an oil immersion lens in conjunction with a chamber of $0.02 \mathrm{~mm}$. depth and phase contrast lighting.

There is, at the present time, no standard procedure for the enumeration of blood platelets. Numerous methods have been devised, which vary in the following manner: 1) Manner of counting-direct or indirect;2) type of diluting fluid-with or without a variety of dyes, fixatives and anticoagulantswith or without hemolytic properties; 3) source of specimen-capillary or venous blood; 4) material on which the count is made-whole blood, plasma or eluate from whole blood; 5) type of counting chamber-standard $0.1 \mathrm{~mm}$. depth or special $0.02 \mathrm{~mm}$. depth with thin coverslip; 6) microscopic magnification and resolution-430X (high dry) to $1000 \times$ (water or oil immersion); 7) microseopic illumination-bright field, dark field or phase contrast (see tables 1 and 2).

Reports of average normal platelet counts in man have varied from 200,000 per cu. mm. (1) to 800,000 per cu. mm.,

a J. A. M. A., 1923, 80, 621 .

${ }^{b}$ Arch. Path., 1937, 23, 850.

c S. Gynec. et Obst., 1927, 15, 436.

d J. Applied Physiol., 1950, 3, 365.

- Exper. Med., 1924, 40, 553.

$f$ Am. J. Clin. Path., 1912, 12, 362.

- Am. J. Clin. Path., 1949, 19, 1113.

h Arch. f. Clin. Med., 1904, 81, 316. 
TABLE 2

Characteristics of various indirect methods of platelet enumeration

1. Diluting fluids With dye.... Without dye. With fixative. Without fixative.........

With anticoagulant...

Without anticoagulant..

2. Source of specimen

Capillary blood....... Venous blood

3. Type of prepsration counted

Dry smear (stained)...

Wet preparation..........

Counting chamber...

4. Microscope lens employed

High dry..... $\mathrm{x}$

Oil immersion

5. Microscope illumination employed

\begin{tabular}{l|l|l|l|l|l|l|l|l}
$\begin{array}{c}\text { Bright field... } \\
\text { Dark field... } \\
\text { Phase contrast }\end{array}$ & $\mathrm{X}$ & $\mathrm{X}$ & $\mathrm{X}$ & $\mathrm{X}$ & $\mathrm{X}$ & $\mathrm{X}$ & $\mathrm{X}$ & $\mathrm{X}$ \\
\hline
\end{tabular}

a Arch. Path., 1937, 23, 850.

b J. A. M. A., 1923, 80, 621.

c J. A. M. A., 1905, 45, 1999.

${ }^{d}$ Arch. Int. Med., 1932, 50, 579.

- Deutsche Ztschr. f. Chir., 1912, 117, 176.

f J. A. M. A., 1921, 76, 427.

- Brit. Med. J., 1901, 2, 1539.

${ }^{h}$ Lancet, 1929, 1, 992.

depending upon the methods employed. The range of reported normal platelet counts in man has varied from a minimum of 130,000 per $\mathrm{cu}$. mm. (2) to a maximum of 900,000 per cu. mm. (3) Since all methods so far devised are subject to inherent errors, there is no sound basis for a choice between the mutually exclusive standards of normality which have been reported.*

Blood Protein. Coagulated blood protein within the vascular lumina of stained sections of fixed tissues is an artifact in the sense that its appearance has been greatly modified by the technique. It is sometimes made up of particles of quite uniform size and has been mistaken for masses of microorganisms; but it does not exhibit both acidophilic and basophilic staining reactions suggestive of cytoplasmic and nuclear components.

Blood Smears. These should be made on slides rather than on cover glasses for several reasons. A larger film of blood is thereby provided for examination. Smears on slides are easier to make and to handle. They can be studied without covering them whereas a smear on a cover glass cannot be moved about on the stage of the microscope unless it is mounted smear side down on a slide. The colors are often more permanent in smears on slides which are not covered with cover glasses. A good way is to spread a thin film of immersion oil over them. This dries much more quickly than balsam or any other medium under a cover glass.

Slides of good quality with ground edges and scrupulously clean are necessary (Cleaning Glassware). A finger tip or ear lobule is first cleaned with $95 \%$ alcohol. As soon as the surface has dried a small puncture is made with a previously sterilized needle. Special needles with lance shaped cutting ends are better than ordinary pointed ones. A small droplet of blood should appear on slight pressure. The first is wiped away with sterile gauze and the second and following ones are used. Unless the blood is very strongly pressed out, the differential count of white cells will not be affected. Some advise holding the fingers in hot water beforehand to produce a temporary hyperemia in them but this is seldom advisable. A droplet of size sufficient to produce a smear of the desired thickness (determined by trials) should be touched to the surface of a slide about $3 \mathrm{~cm}$. from one end conveniently placed on a table. Immediately the end of a second slide, with its edge

- Original observations and confirmation of published data contained in this paragraph were done under a contract (No. AT-11-1-GEN-10, Project 2), between the United States Atomic Energy Commission and the University of California. 
squarely across the first slide is brought in contact with the blood on the remote side of the drop from the nearest end of the first slide. The blood spreads quickly along this edge toward the sides of the slide on the table which is steadied with the left hand. The end edge of the second slide is slowly but steadily pushed the length of the first slide and the blood is drawn out in a thin layer after it. The angle of inclination of the second to the first slide determines the thickness of the smear. It is well to make the first smear at an angle of about 45 degrees; increase it for a thicker smear and decrease it for a thinner one. In the making of smears it is important to have plenty of elbow room. To make good smears is a fine art and a credit to the individual.

Blood smears, whether simply dried by waving in air or thereafter fixed by gently heating, retain their staining properties for a few days but they should be colored without undue delay. However they can be kept unstained or stained if protected by dipping in melted paraffin (Queen, F. B., Am. J. Clin. Path., Techn. Suppl., 1943, 7, 50). It is both wasteful and undesirable to cover the whole slide with stain. Part of the slide will have to be used for record written with a diamond pencil. Therefore draw two lines across the slide near each end with a wax pencil or a piece of paraffin. The stain added with a dropper will cover only the intervening part. For stains see Giemsa, Wright, Ehrlich, Oxidase, Peroxidase and Gordon's Silver Method.

For electron microscopy of blood cells, see Bessis, M., Blood, 1950, 5, 1083-1098.

Blood Species Characteristics. References to the literature on the blood of many different kinds of animals and data on their differential counts, total counts, hemoglobiu concentrations and so on are often found of great service (Wintrobe, M.M., Clinical Hematology. Philadelphia: Lea \& Febiger, 1942, 703 pp.).

Blood Vessels. These comprise structures of different sorts, existing in a wide variety of environments, which can be investigated from many angles. Consequently to present examples of available techniques under the expected headings involves a lot of mind-reading. The blood vessels of the Skin are of course the most accessible. Detailed methods for their direct and indirect study are presented by Sir Thomas Lewis (The Blood Vessels of the Skin and their Responses. London: Shaw \& Sons, 1927, 322 pp.).
But to microscopically examine all the blood vessels of any particular organ is not possible in the living state because of lack of accessibility, thickness and other mechanical obstacles. Resort is therefore made to various devices for viewing the vessels by themselves unobscured by surrounding tissue. The unwanted tissue is removed by corrosion when the vessels are demonstrated by Neoprene injection. It is simply passed over when $x$-ray photographs are examined after the vascular injection of radiopaque substances like Bismuth Sulphate and Diotrast. It is rendered transparent when the vessels are filled with easily visualized materials such as Carmine or Berlin Blue, or is relatively colorless after their walls are selectively stained by Janus Green, Silver Citrate or Silver Chloride Dichlorfluoresceinate. See red lead and glue method for blood vessels of nerves (Epstein, J., Anat. Rec., 1944, 89, 6569).

Though the larger blood vessels are too thick and cumbersome for microscopic study in vivo, this is not so with the smaller ones. Indeed excellent moving pictures can be made of them. A film entitled "Control of Small Blood Vessels" by G. P. Fulton and P. R. Lutz of Boston University is very helpful. The supravital method of studying Nerve Endings with methylene blue must be combined with careful dissections (Woollard, H. H., Heart, 1926, 13, 319-336) in order to gain an impression of the innervation of blood vessels. See Arteries, Arterioles, Capillaries, Sinusoids, Venous Sinuses, Venules, Arteriovenous Anastomoses, Veins, Vasa Vasorum, Valves, Perfusion. See Quartz Rod Technique.

Bodian Method. For staining nerve fibers in paraffin sections (Bodian, D., Anat. Rec., 1937, 69, 153-162; MacFarland, W. E. and Davenport, H. A., Stain Tech., 1941, 16, 53-58). The following details of this very useful technique have been supplied by Dr. J. L. O'Leary. Fix by vascular perfusion, with $80 \%$ alcohol containing $5 \%$ formol and $5 \%$ acetic acid, or by immersion in $10 \%$ formalin or Bouin's fluid. For boutons terminaux, perfuse tissue with $10 \%$ chloral hydrate and extract tissue with alcohol for several weeks. Run paraffin sections (15 $\mu$ or less) to aq. dest. Place in $1 \%$ Protargol (Winthrop Chemical Co.) with 4-6 gms. of metallic copper per $100 \mathrm{cc}$. (This can be used only once.) Wash in redistilled water 1 change. Transfer for $10 \mathrm{~min}$. to : hydrochinone, $1 \mathrm{gm}$.; sodium sulfite, $5 \mathrm{gm}$.; aq. dest., $100 \mathrm{cc}$. Wash in redistilled 
water 1 change. Tone in $1 \%$ gold chloride with 3 drops of glacial acetic acid per $100 \mathrm{cc}$, 5-10 min. Wash in redistilled water 1 change. If sections do not have a light purple color place in $2 \%$ oxalic acid until the entire section has the slightest blue or purplish tinge. Pour off as soon as tissue gets slightly blue. Remove residual silver salts in $5 \%$ sodium thiosulfate $5-10 \mathrm{~min}$. Wash, dehydrate, clear and mount. Note: the Coplin jars used must be cleaned in Cleaning Fluid. The Bodian method has been adjusted for the demonstration of melanin by Dublin, W. B., Am. J. Clin. Path., 1943, 7 (Technical Section), 127.

Boedeker's Method, see Enamel matrix. Bogoroch, see Radioautographic Technique. Boling, see Teeth, Decalcification.

\section{Bollinger Bodies, see Borrel Bodies.}

Bone. A good account of methods is provided by Shipley (McClung, pp. 344-352). Examination without decalcification involves the cutting and grinding of thin sections. The instruments used by dentists for the making of sections of undecalcified teeth are of the greatest service and should be purchased or borrowed. If they are not available Grieves' method for dental tissues is suggested. In order to determine the structure of bone with organic material removed, Shipley advises cutting away all soft parts after which the bone may or may not be split. Place in tap water, or in a $2 \%$ aq. gelatin, to which a loop full of culture of $B$. coli has been added. After 5-6 days wash in running water $24-48 \mathrm{hrs}$. in a stink cupboard. This will dissolve and wash away all organic material. Sterilize the bone by boiling or immersion in alcohol. Saw into sections, grind these to the necessary thinness and polish. Dehydrate in ether. Dry thoroughly and mount in balsam. Routine examination includes some method of fixation, decalcification and staining. Hematoxylin and eosin are recommended, likewise phosphomolybdic acid hematoxylin and Mallory's connective tissue stain.

For different structural components special techniques are required. Bone corpuscles may be isolated by putting a thin section of bone in concentrated nitric acid for a few hours to a day. Then place the section on a slide, cover. Pressure on the cover glass will squeeze out ellipsoidal bone cells with their processes (Shipley). Bone lamellae can be peeled off easily when decalcified bone has been allowed to simmer in water for several hours (Shipley). Lacunae and canaliculi. The easiest method is to impregnate sections of ground bone with $0.75 \%$ aq. silver nitrate for 24 hours. Wash, polish the sections on a fine hone to remove precipitated silver, dehydrate in alcohol, clear in xylol and mount in balsam. The lacunae and canaliculi appear black in a yellowish brown background. To impregnate thin sections with acid fuchsin, dry them after extraction with alcohol. Place them in watch glasses in a $20 \% \mathrm{aq}$. sol. of acid fuchsin in a desiccator connected with a suction apparatus. Extract air for about an hour and close the dessicator. After 24 hrs. the solution will have dried. Rub off ppt. on a hone, pass through xylol and mount in damar or balsam (Shipley).

Linings of lacunae and canaliculi. (Schmorl's method modified by Shipley.) Employ a fixative not containing mercury. Decalcify in Müller's fluid, wash in running water, embed in celloidin and section not over 10 microns. Stain in thionin solution alkalinised by 2 drops ammonia. Transfer with glass needle to sat. aq. phosphotungstic or phosphomolybdic acid. Leave until blue, gray or green. Place in water until sky-blue. Ammonium hydroxide 1 ce. and aq. dest. 10 cc., 3-5 min. Several changes $90 \%$ alcohol. $95 \%$ alc. Clear in carbolxylol and mount in damar (or balsam). This method is suggested for bones of children.

Processes of young osteoblasts in growing bone. Shipley suggests following treatment of slices of bone of a rickety animal. $4 \%$ aq. citric acid 20-30 min. in the dark. Rinse in aq. dest. $1 \%$ aq. gold chloride in the dark, 20-30 min. $3 \%$ formic acid in the dark, 48 hrs. Rinse in aq. dest. and preserve in pure glycerin. Make frozen sections, mount in glycerin and ring with damar, balsam, paraffin or cement. Keep specimens in dark when not is use.

To determine relative age of deposition the following method has proved useful in senile osteoporosis. Saw sections of bone not more than $0.5 \mathrm{~cm}$. thick and fix in $4 \%$ formalin $2-4$ days. Decalcify in $6 \%$ isotonic formalin, 40 cc., $\$ 5 \%$ formic acid, 60 cc., and sodium citrate, $5 \mathrm{gm}$. changing every second day for, say, a week, that is until they become flexible and can be penetrated by a fine needle. Embed in celloidin (slow method). Prepare stain by dissolving $30 \mathrm{gm}$. potassium alum in 1 liter hot water and by adding $1.5 \mathrm{gm}$. hematoxylin crystals. Cool and add 1 gm. chloral hydrate. Ripening in sunlight to rich dark color is hastened by addition of crystal of potassium hydroxide. Stain celloidin sections about 2 days 
checking by microscopic examination until some areas are definite violet azur, others lighter or colorless. Wash in tap water 24 hrs. Stain in 100 cc. aq. dest. $+2-3$ drops $1 \%$ aq. eosin 1-2 days (uncolored areas become dark rose color). Dehydrate, clear in xylol and mount in balsam. Old bone azur; new bone bright rose (Belloni, L., Arch. Ital. Anat. e Istol. Path., 1939, 10, 622). See Madder staining of new bone, Alizarin Red S staining of dentine, various tests for Calcium, and Ossification, Line Test for vitamin D potency. Polarized light is excellent for the demonstration of bone camellae.

The micro-diffraction technique permits obtaining diffraction patterns of small areas such as a single Haversian system. Using it Enström, A. and Zetterström, R., Exp. Cell Res., 1951, $2,268-274$, found that the orientation of mineral salts is relatively unchanged in different physiological conditions.

Bone Marrow. Microscopic examination of bone marrow in vivo has not been achieved because of the obvious technical difficulties involved. The best that can be done is to study still living cells removed from bone marrow unstained or supravitally stained. The methods are essentially the same as for blood. From humans samples can be obtained by sternal puncture (Young, R. H. and Osgood, E. E., Arch. Int. Med., 1935, 55, 186-203, and many others). Primitive cells of the erythrocytic and leucocytic series can only be identified when hemoglobin and specific granules respectively appear within them. Microchemical tests for Hemoglobin should be more used. For the granules the methods of Giemsa, Wright, Ehrlich and others are the best available. Special techniques have been described for Megakary ocytes particularly in relation to platelet formation. The normal megakaryocyte concentration is aspirated human bone marrow is described by Ebaugh, F. G. Jr. and Bird, R. M., Blood, 1951, 6, 75-80. To demonstrate the vascular pattern special methods are required (Doan, C. A., Johns Hopkins Hosp. Bull., 1922, 33, 222-226). To reveal the nerve supply is particularly difficult. Glaser (W., Ztsch. f. Anat. u. Entw., 1928, 87, 741-745) has described a fine network accompanying the vessels but Doan and Langworthy (Downey, p. 1852) were less successful. Sternal bone marrow during first week of life (Shapiro, L. M., and Bessen, F. A., Am. J. Med. Sci., 1941, 202, 341351). Bone marrow of normal adults (Plum, C. M., Acta Med. Scand., 1941, $107,11-52)$. See chapters by Sabin and
Miller and by Doan in Downey's Handbook of Hematology, New York, Hoeber, 1938, 3, 1791-1961 for details. A method for studying numerical and topographic problems in the whole femoral marrow of rats and guinea pigs, with the use of undecalcified sections (Mayer, E. and Ruzicka, A. Q., Anat. Rec., 1945, 93, 213-231). A technique for the quantitative estimation of mast cells in bone marrow is advanced by Mota, Ivan, Blood, 1951, 6, 81-83.

Borax Carmine (Grenacher). Make con. sol. of carmine in borax (2-3\% carmine in $4 \%$ aq. borax) by boiling for $30 \mathrm{~min}$. Allow to stand 2-3 days with occasional stirring. Dilute with equal volume $70 \%$ alc., again allow to stand and filter. Much used for staining tissues in bulk. They are colored for days if necessary, transferred directly to acid alc. (70\% alc. $100 \mathrm{cc}$, hydrochloric acid 4 drops) in which they assume a bright red transparent appearance. Then wash in alcohol, mount as whole specimens or embed in paraffin and cut sections. Borax carmine can also be employed to stain sections (Lee, p. 146).

Borax Ferricyanide, see Weigert's.

Bordeaux, see Amaranth.

Bordeaux Red (CI, 88)-acid Bordeaux, archelline $2 \mathrm{~B}$, azo-Bordeaux, cerasin $\mathrm{R}$, fast red $\mathrm{B}, \mathrm{BN}$ or $\mathrm{P}-\mathrm{An}$ acid mono-azo dye very widely employed in histology.

Bordeaux SF, see Amaranth.

Boron, see Atomic Weights.

Borrel Bodies (Bollinger bodies) in fowl pox. References to earlier staining methods and directions for applying the microincineration technique with figures showing the comparative results are given by Danks, W. B. C., Am. J. Path., 1932, 8, 711-716. See microincineration of Molluscum bodies (Van Rooyen, C. E., J. Path. \& Bact., 1939, 49, 345-349).

Borrelia Vincenti, see Vincent's Angina.

Borrel's Stain. Fix in osmic acid, 2 gm.; platinum chloride, 2 gms. ; chromic acid, $3 \mathrm{gm}$.; glacial acetic acid, $20 \mathrm{cc}$. and aq. dest., $350 \mathrm{cc}$. for $24 \mathrm{hrs}$. Wash in running water several hours. Dehydrate, clear, embed and section. Stain sections in $1 \%$ aq. magenta $1 \mathrm{hr}$. Then in sat.aq. indigo-carmine, 2 parts and sat. aq. picric acid, 1 part. Wash in alc., dehydrate, clear and mount. The above has been partly taken from Lee's Vade Mecum, p. 433. Other more convenient fixatives will do equally well. The stain has been used for the Borrel bodies in fowl pox.

Botanical Technique. Many of the methods used in animal histology are applicable also in plant histology and vice versa. Details are given in a chapter by W. R. 
Taylor in McClung, p. 155-245. See Plants.

Bouin's Fluid. Sat. aq. picric acid, 75 cc.; formalin, 25 cc.; acetic acid, 5 cc. For mammalian tissues fix $24 \mathrm{hrs}$., wash in water, dehydrate and embed in the usual way. This is the most generally useful of all fixatives containing picric acid. Almost any stain can be used after it. The picric acid need not be altogether washed out because it serves as a desirable mordant. Giemsa's stain gives good coloration of protozoan parasites after fixation in Bouin's fluid (Cowdry, E. V. and Danks, W. B. C., Parasitology, $1933,25,1-63)$. The use of this fixative is specified under Argentaffine Reaction, Bodian's Method, Elementary Bodies, Foot's Method, Gold, Johnson's Neutral Red Method, Laidlaw's Method, Liebermann-Burchardt Reaction, Mas son's Trichrome, Purkinje Cells, Tapeworm Proglottids, etc. It is a fixative rapidly gaining in popularity and there are naturally many modifications. The application of Davenport's silver technique to Bouin fixed tissues is described by Foley, J. O., Stain Techn., 1938, 13, 5-8.

The cytological action of Bouin's fluid has been investigated at the University of Pennsylvania. Three formulae are particularly recommended by McClung and Allen (McClung, p. 561). (1) Allen's fluid: sat. aq. picric acid, 75 cc.; formalin C.P., 15 cc.; glacial acetic acid, $10 \mathrm{cc}$; urea, $1 \mathrm{gm}$. (2) The same plus $1 \mathrm{gm}$. chromic acid. (3) The original formula plus $2 \mathrm{gms}$. urea and $1.5 \mathrm{gms}$. chromic acid. For details regarding use in study of cell division, shrinkage, etc. see Allen, Ezra, Anat. Rec., 1916, 10, 565-589.

Bourne, see Golgi Apparatus, Mitochondria.

Boutons Terminaux. For this special type of nerve ending the methods given under Nerve Endings are useful, particularly that of Bodian. These terminal buttons or swellings can be visualized and their behavior determined in living tadpoles by techniques introduced by Speidel, C. C., J. Comp. Neurol., 1942, $76,57-73$. Several special methods for their demonstration in fixed tissues are recommended by Gibson (McClung, 1950, pp. 389-398). Distinction between normal and degenerating in the inferior olive of the cat made by silver methods (Blackstad, T., Brodal, A. and Walberg, F. Acta Anat., 1951, 11, 461-477).

Bowie's Ethyl Violet-Biebrich Scarlet stain for pepsinogen granules (Bowie, D. J., Anat. Rec., 1935-36, 64, 357-367). Dissolve $1 \mathrm{gm}$. Biebrich scarlet in $250 \mathrm{cc}$. aq. dest. and 2 gms. ethyl violet in 500 cc. Filter the former through a rapid filter paper into a beaker and then the latter into the same beaker. The end point of neutralization is when a little of the mixture placed on filter paper does not show any scarlet color. Collect the ppt. of neutral dyc by filtering and dry it. To make stock solution dissolve 0.2 gm. in 20 cc. $95 \%$ alcohol. To make staining solution add 1-5 drops to $50 \mathrm{cc}$. of $20 \%$ alcohol. Stain paraffin sections of Regaud fixed gastric mucosa in this for $24 \mathrm{hrs}$. Wipc dry around edges and blot with smooth filter paper. Differentiate by covering section with cqual parts clove oil and xylol. This takes about $30 \mathrm{~min}$. and should be observed under microscope. Pass through several changes of xylol, rinse in benzol and mount in benzol balsam. Pepsinogen of pepsin-forming cells, violet; and parietal cells, red. Bowie also makes a crystal violet-orange $\mathrm{G}$ stain which does not differ materially from Bensley's Neutral Gentian.

Brandt's glycerin jelly. Melted gelatin, 1 part; glycerin $1 \frac{1}{2}$ parts plus few drops carbolic acid to serve as a preservative. See Kaiser's glycerin jelly under glycerin.

Bryan, see Ear Cell Smears, Nasal Cell Smears.

Brazilin (CI, 1243) is a substance produced from red wood of Brazil. Its formula is like that of hematoxylin minus 1 hydroxyl group and in its use, as well as its origin, it resembles hematoxylin. Ripening may be required for both. Thus we have an iron brazilin method (Hickson, S. J., Quart. J. Micr. Sci., 1901, 44, 469-471) and O'Leary's Brazilin for myelin sheaths. See also Brazilin-Wasserblau technique of Bensley.

Brazilin-Wasserblau for secretion antecedents of thyroid gland (Bensley, R. R., Am. J. Anat., 1916, 19, 37-54) as described later by the Bensleys (p. 80) is: To make up the Brazilin stain dissolve $0.05 \mathrm{gm}$. in a little aq. dest. with aid of heat and add this to 100 cc. $1 \%$ aq. phosphotungstic acid. Ripen by addition of 2 drops hydrogen peroxide. Solution should not be employed after 3 days. Run paraffin sections of formalin-Zenker fixed thyroids down to aq. dest., mordant briefly in a fresh aq. ammonium stannic chloride, and stain in above solution 1 or more hrs. Wash in water and treat for $1-5 \mathrm{~min}$. with aq. dest., 100 cc. $+1.0 \mathrm{gm}$. phosphomolybdic acid and $0.2 \mathrm{gm}$. Wasserblau (anilin blue). Wash quickly in water, dehydrate in absolute alcohol, clear and mount. Sec colors in R. R. Bensley's plate. Nuclear chromatin, red; secre- 
tion antecedents in pale blue droplets; mitochondria, reddish-purple; connective tissue, blue; erythrocytes, orangered; etc.

Brazilwood. The true brazilwood is of the tree, Caesalpina echinata and its varieties. It yields a dye stuff formerly much used after an aluminum mordant for fabrics, except silk, to which it gave a bright red color. After potassium bichromate as a mordant the color obtained was purple red. The term "brazil" is from the arabic word "braza" meaning fiery red. Leggett writes that increased use of brazilwood in Europe resulted from the delivery of Asiatic brazilwood directly to Lisbon made possible by Vasco da Gama's discovery of an all water route from India around the Cape of Good Hope and, further, that three years later a Portuguese expedition bound for India missed the mark and landed on the north eastern bulge of South America where the voyagers found many brazilwood trees so they called the land "Terra de Brazil" (Ieggett, W. F., Ancient and Medieval Dyes, Brooklyn: Chemical Publishing Co., Inc., 1944, 95 pp.).

Breast, see Mammary Glands.

Brilliant Alizarin Blue(CI, 931), a basic dye of light fastness 3 to 4 . Gives darker color than New Methylene Blue (Emig, p. 61)

Brilliant Blue C, see Brilliant Cresyl Blue.

Brilliant Congo R, see Vital Red.

Brilliant Congo Red R, see Vital Red.

Brilliant Cresyl Blue (CI, 877)-brilliant blue $\mathrm{C}$, cresyl blue $2 \mathrm{RN}$ or BBSCommission Certified. This basic oxazin dye is used for making Platelet Counts and for many other purposes.

Brilliant Dianil Red R, see Vital Red.

Brilliant Fat Scarlet B, see Sudan R.

Brilliant Green (CI, 662)-diamond green, ethyl green, malachite green $\mathrm{G}$, solid green JJO-Commission Certified. This di-amino tri-phenyl methane dye is used to color culture media.

Brilliant Pink B, see Rhodamine B.

Brilliant Ponceau G, see Ponceau 2R.

Brilliant Purpurin $\mathbf{R}$ (CI, 454). An acid dis-azo dye. Conn (p. 62) says that this is the dye which Gutstein, M., Zeit. f. Ges. Exp. Med., 1932, 82, 479-524 called "brilliant purpur $\mathrm{R}$ " and used as a vital stain for yeasts.

Brilliant Vital Red. Use in determination of plasma volume is justified, since the dye is not taken into the erythrocytes (Gregersen, M. I., and Schiro, H., Am. J. Physiol., 1938, 121, 284-292). See Vital Red.

Brilliant Yellow (CI, 364), an acid dis-azo dye of light fastness 3 apparently of little use as a stain for paraffin sections. In acid solutions colors resinous tissues bright yellow, and in alkaline solutions, blue-green algae a clear yellow (Emig, p. 39).

Bromcresol Green. See Hydrogen Ion Indicators.

Bromeresol Purple. See Hydrogen Ion Indicators.

Bromine. According to Lison (p. 110) bromine has not been investigated histochemically in animal tissues. For its detection in plants consult Mangenot, H. G., Bull. d'Hist. Appl., 1927, 4, $52-71$.

Bromphenol Blue. See Hydrogen Ion Indicators.

Bromphenol Red. See Hydrogen Ion Indicators.

Bromthymol Blue. See Hydrogen ion Indicators.

Bronchial Aspirates, see Papanicolaou Techniques.

Bronchiolar Epithelium-Written by C. C. Macklin, Dept. of Histological Research, The University of Western Ontario, London, Canada. November 28, 1951-For the dark and light cells, as revealed by supravital silverization, see Ammoniacal Silver. For a means to demonstrate the continuation of the network of surface silver lines from the bronchiolar epithelium to the alveolar walls, in en face views, see Silver Lineation. Mitochondria in the "villus" cells are abundant and often apparently merged (Macklin, C. C., Anat. Rec., 1949, 103, 550; Rev. can. de Biol., 1949, 8, 328; and Proc. Instit. of Med. of Chicago, 1950, 18, 78-95-the 26th Lewis Linn McArthur Lecture). They are well demonstrated by Altmann's method of anilin fuchsin and picric acid (which see). Tumor formation in the bronchiolar epithelium of rats that have been subjected to urethane (which see) has been described by Rosin (Cancer Res., 1949, 9, 583).

Bronz Bromo, see Eosin $Y$.

Brown Salt R, see Chrysoidin Y.

Brownian Movement. Calculation of cytoplasmic viscosity through measurement of displacement of particles in Brownian movement gives results not very different from determinations by the centrifugation method (Danielli in Bourne, p. 31).

Brucella Ring Test, see Triphenyltetrazolium Chloride.

Buffalo Garnet R, see Erie Garnet B.

Buffers. For many purposes it is essential to use solutions buffered at a certain $\mathrm{pH}$. Details concerning numerous buffers are given by Clark, W. M., The Determination of Hydrogen Ions. Baltimore: Williams \& Wilkins, 1928, 717 pp. 
French, R. W., Stain Techn., 1930, 5, 87-90 (see correction, 1932, 7, 107-108) recommends Sorensen's phosphate mixtures and Palitzsch's borax-boric acid mixtures each over certain ranges of $\mathrm{pH}$. He emphasizes the fact that the addition of buffer salts is known to have a decided influence in some cases on the behavior of the dyes irrespective of $\mathrm{pH}$. See also Clark and Lubs Buffers, also Veronal Acetate Buffers.

Petrunkevitch, A., Anat. Rec., 1937, $68,267-280$ explains that aqueous solutions of stains at certain $\mathrm{pH}$ 's are more selective than alcoholic ones and that the greatest differentiation is obtained with the former ones with $\mathrm{pH}$ suitably adjusted by addition of $\mathrm{HCl}$ or $\mathrm{NaOH}$. Next in desirability come stains dissolved in acetate, phosphate and borate buffers. Citrate buffers are in his experience less suitable because a more diffuse staining results while phthalate buffers should not be used. $\mathrm{He}$ gives specific directions for the preparation of solutions at $\mathrm{pH}$ of maximum staining of acid fuchsin, aniline blue, aurantia, benzoazurine, eosin $Y$, light green, metanil yellow, methylene blue, orange $G$, toluidin blue, Wrights stain and eosin methylene blue.

For safranin $O$, see Sawyer, C. H., Stain Techn., 1940, 15, 3-7 and for hematoxylin, malachite green and eosin $\mathrm{Y}$, Craig, R. and Wilson, C., ibid, 1941, 16, 99-109. Levine, N. C., ibid, 1940, 15, 91-112 contributes useful data on buffered stains in relation to isoelectric point of cell components. Obviously the maximum intensity of staining depends not only on pH but also on properties of substances stained and their treatment from beginning to end of the technique. Lillie, R. D., Stain Techn., 1941, 16, 1-6 employed McIlvaine citric buffers in order to improve Romanowsky staining (see Toluidine Blue Phloxinate) after various fixatives. See McJunkin-Haden Buffer. Use of buffered thionin as Nissl stain (Windle, W. F., Rhines, R. and Rankin, J., Stain Techn., 1943, 18, 77-86). For buffering in connection with silver impregnation see Davenport, H. A., McArthur, J. and Bruesch, S. R., Stain Techn., 1939, 14, 21-26; Silver, M. L., Anat. Rec., $1942,32,507-529$. When accuracy is essential check the rctual $\mathrm{pH}$ of the solution to which buffers have been added by the glass electrode method which anyone can learn to use in a few hours and which gives the answer very quickly. See Hydrogen Ion Indicators.

Bundle of His, see Todd, T. W., Cowdry's Special Cytology, 1932, 2, 1173-1210.
Burettes. For accurate volume determinations use Microliter Burettes.

Burns. Methods of experimental production, vital staining with trypan blue, and histological changes (Ham, A. W., Ann. Surg., 1944, 120, 689-697.

Butter Fat, reactions in tissue to fat stains after various fixations (Black, C. E., J. Lab. \& Clin. Med., 1937-38, 23, 1027-1036).

Butyl Alcohol, see n-Butyl and Tertiary Butyl.

Buzaglo's Connective Tissue Stain. (Buzaglo, J. H., Bull. d'Hist. Appl., 1934, $11,40-43)$. This method is intended to replace that of Van Gieson. Solutions required: (1) Gallocyanin (Hollborn, 2264). Boil $0.1 \mathrm{gm}$. in 100 cc. $5 \%$ aq. chrome alum for $10 \mathrm{~min}$. After cooling make up to $100 \mathrm{ec}$. with aq. dest., filter and add a little formalin to filtrate. (2) Orcein (Hollborn, 2466). Dissolve 1 gm. in $100 \mathrm{cc}$. acid alcohol (70\% alcohol, $100 \mathrm{cc}$. + $1 \mathrm{cc}$. hydrochloric acid standard). (3) Acid alizarin blue (Hollborn, 2559). Boil for $10 \mathrm{~min} .5 \mathrm{gm}$. in $100 \mathrm{cc}$. $10 \%$ aq. aluminum sulphate. After cooling make up to $100 \mathrm{cc}$., filter and add formalin. (4) Alizarine-viridine (Hollborn, 2035). Dissolve $0.2 \mathrm{gm}$. in 100 cc. aq. dest. acidulated to $\mathrm{pH} 5.8$ with hydrochloric acid. He advises fixation in formalin, Maximow's fluid, Susa or Hoffker (of which he does not give composition). Pass sections (presumably paraffin) down to aq. dest. Stain nuclei in gallocyanin as deeply as possible 5 times, 24 hrs. Rinse twice in aq. dest. Stain elastic fibers in orcein, then aq. dest., 3 times, 5 min. Stain muscle in acid alizarin blue, $7 \mathrm{~min}$., aq . dest. twice. Differentiate in $5 \%$ aq. phosphomolybdic acid 25-30 min., aq. dest. twice. Stain collagen in alizarine viridine $7 \mathrm{~min}$. Blot with 4 layers filter paper. $95 \%$ alc. $96 \%$ alc. Carbolxylol, 2 changes xylol. Balsam. Nuclei, dark blue; elastic fibers, red brown; muscle and epithelium, pale blue violet; collagen, mucus, cartilage, shades of green; myelin sheaths, rose; axis cylinders, dark blue; erythrocytes, red brown.

Cabot Rings. Ring-shaped formations in erythrocytes which color red with Wright's Blood Stain. The color in the concavity of the ring is the same as that in the rest of the cell. Occasionally observed especially in pernicious anemia and lymphatic leukemia.

Cadmium. The chloride is employed in fixation of Golgi apparatus prior to silver impregnation (Aoyama, F., Zeit. wiss. mikr., 1929, 46, 489-491). See comment by Baker (Bourne, p. 19) on this and use by Ciaccio of cadmium nitrate to 
render phospho- and galactolipines less soluble. Bourne (p. 106) refers to Joyet-Lavergne's claim that cadmium lactate reacts with glutathione in the cell producing a cadmium glutathione compound which is microscopically visible.

Cajal's. Properly the name should be listed as Ramon y Cajal. 1. Brom-formolsilver method for neuroglia. Details supplied by Dr. J. L. O'Leary. Fix small fresh pieces, 3-15 days, in: aq. dest., 85 cc.; formalin, 15 ec.; ammonium bromide, $2 \mathrm{gm}$. Cut $25 \mu$ frozen sections and return to: aq. dest., $50 \mathrm{cc}$.; formalin, 6 cc.; ammonium bromide, $3 \mathrm{gm}$. for $4-6 \mathrm{hrs}$. at $30-38^{\circ} \mathrm{C}$. or for $8-10$ hrs. at room temperature. Wash for a few seconds in aq. dest. Place in the following fluid in a porcelain dish and heat over the flame: aq. dest., 10-15 cc.; ammoniacal silver oxide, 5 cc.; pyridine C.P., 4-5 drops. (To prepare silver oxide solution: Take 10 cc. $10 \%$ silver nitrate, add 12 drops $40 \% \mathrm{NaOH}$. Collect the ppt., wash 5-6 times with aq. dest., then add ppt. to a beaker containing 60-70 cc. aq. dest. Redissolve with least quantity of ammonia necessary. If too much ammonia is added, results are bad.) Remove when sections have reached a tobacco brown color. Wash through 2 changes aq. dest. not more than 5 sec. in all. Reduce in $5 \%$ formalin for $2-3 \mathrm{~min}$. Tone with $0.2 \%$ aq. gold chloride and fix in $5 \%$ aq. sodium hyposulfite. After washing carry to $95 \%$ alcohol, carbolxylol, xylol balsam. See Microglia and Oligodendroglia.

2. Chloral hydrate method as described by Willard, D. M., Quart. J. Micr. Sci., 1935-36, 78, 475-485 for innervation of adrenal. Fix for $24 \mathrm{hrs}$. in : chloral hydrate, $2.5 \mathrm{gm}$.; $95 \%$ alcohol, 40 cc.; aq. dest., 40 cc.; pyridine, 20 cc. Wash in aq. dest. until smell of pyridine disappears. $97 \%$ alcohol, $24 \mathrm{hrs}$. Wash again in aq. dest. and transfer to $2.5 \%$ aq. silver nitrate at $37^{\circ} \mathrm{C}$. for 9-12 days (longer times better for nerve cells). Wash for $1 \mathrm{~min}$. in aq. dest. Reduce for 12-24 hrs. in: liydroquinone, $1 \mathrm{gm}$.; neutral formol, 10 cc.; aq. dest., $90 \mathrm{cc}$. Dehydrate rapidly, embed in paraffin and cut $15-30 \mu$ sections. Nerve fibers, black; background, yellow.

Cajal Silver Methods. These depend mainly on silver impregnations reduced by photographic developers such as hydroquinone. They have all been very greatly improved by a preliminary fixation and in other ways and have played a leading rôle in neurology. See Ranson pyridine method and other modifications given by Addison (Mc-
Clung, pp. 452-463). Many techniques spring from a combination of Cajal and Bielchowsky methods.

Calcareous deposits. Vital staining with Alizarin Red S (Ham, A. W., Arch. Path., 1932, 14, 613-626).

Calciferol, see Vitamin $\mathbf{D}_{2}$.

Calcium. There is no absolutely specific microchemical test for calcium in sections. A critical account by Cameron (G. R., J. Path. and Bact., 1930, 33, 929-955) affords instructive reading.

1. von Kossa test. Sections are transferred from aq. dest. to $10 \%$ silver nitrate and exposed to bright light for 30 min. or more. Wash carefully in aq. dest. Mount in glycerin, or dehydrate clear and mount in balsam. Inorganic material in most cases calcium phosphate or carbonate is deep black. See comments of Gomori, G., J. Mt. Sinai Hosp., 1945, 11, 317-326. Test has been adapted to investigation of bone by McLean, F. C. and Bloom, W., Anat. Rec., 1940, 78, 333-359.

2. Alizarin. Sections from aq. dest. are stained in $1 \%$ aqueous alizarin $\mathrm{S}$ (sodium alizarin sulphonate) or in $1 \%$ alcohol tetra-hydroscy-anthraquinon (or anthrapurpurin) for an hr. or more. They are then differentiated in 1 part concentrated ammonia and 9 parts absolute alcohol. This is followed by rapid washing in acid alcohol (hydrochloric acid 5 cc., $95 \%$ alcohol 95 cc.). It may be desirable to alternate alkali and acid alcohols 2 or 3 times. Wash thoroughly in aq. dest.; dehydrate clear and mount. The alizarin forms a fast compound with earthy salts especially calcium more easily in young than in old bones. Substances may exist in the tissues that inhibit the combination (see Bone, Madder staining).

3. Hematoxylin. This is not, as is generally supposed, a stain for calcium though it may color calcium as well as other materials when mordanted with chromium salts or alum. According to Cameron, in bone, "staining with hematoxylin is dependent on the essential ground substance and the presence of certain heavy metals especially iron chromium and aluminum; it has no direct relation to calcium salts." $\mathrm{He}$ thinks that areas of pathological calcification which stain deeply with alum hematoxylin do so because of the presence of free iron.

4. Fluorescence x-radiation. Used for thin sections of undecalcified bone. It is not feasible to magnify much but the method is said to be almost specific for calcium (Dershem, E., Proc. Nat. Acad. Sci., 1939, 25, 6-10).

5. Cretin, A., Bull. d'Hist. Appl., 
1924, 1, 125-132 has proposed a blue color reaction with trioxymethylene and gallic acid. In comparison with calcium strontium and barium show green, magnesium rose and iron brownish violet. See Lillie p. 251 for details.

6 . With magnesium, but free from all other minerals in muscle, by electron microscope (Scott, G. H. and Packer, D. M., Anat. Rec., 1939, 74, 17-45).

7. Sulfuric and oxalic acid. Lillie (p. $251)$ refers to the granular, opaque and white appearance of unstained calcium deposits. If the material is mounted under a cover glass in water and sulfuric acid is drawn through by removal of water from one side with filter paper the deposits dissolve but colorless monoclinic calcium sulphate (gypsum) crystals are formed while if $5-10 \%$ oxalic acid is used typical cubic calcium oxalate crystals appear. Carbonates, on the contrary, are dissolved by running through acetic acid with the formation of gas bubbles. Phosphates are dissolved by the acetic acid without gas bubbles. The development of these crystalline calcium salts is of course specific.

8. Microincineration. To distinguish between the dense white ashes of calcium and magnesium it is suggested that a "microdrop" of $0.1 \mathrm{~N}$ hydrochloric acid be added, plus a similar drop of $0.1 \mathrm{~N}$ sulphuric acid in order to produce needle like calcium sulphate crystals (Moreau, P., Bull. histol. appl. physiol. et path. et tech. microscop., 1931, 8, 245-248). As follow up on the above microchemical methods a curvette colorimetric by Sendary, J. Jr., J. Biol. Chem., 1942, 144, 243-258; 1944, 152, $539-556$ and a titrimetric technique by Sobel, A. E. and Kaye, I. A., Ind. Eng. Chem., and Ed., 1940, 12, 118-120 are possibilities. By the latter as little as $4 \mu \mathrm{gm}$. of calcium can be measured in a $5 \mathrm{ml}$. burette. See Glick, p. 273.

Calcozine Red CG, see Rhodamine CC.

Camphor, see Sandarac.

Camsal is a mixture of camphor and salol used by McClung in making Sandarac.

Canada Balsam, see Balsam.

Canary Yellow, see Auramin.

Cancer. Because cancer and other malignant tumors can develop in so many organs and tissues that contain intermitatic or reverting postmitotic cells (Cell Classification), techniques designed to compare the malignant cells with their non-malignant prototypes are altogether too numerous to mention. They will be found under the several tissues: Pancreas, Connective Tissue and so forth.
There is no known technique which will reveal a structure or a substance in cancer cells wholly absent in normal cells of the sort from which the particular cancer colls have originated. Neither can the reverse be demonstrated, that is something absent in cancer cells and present in normal ones. Available methods are only capable of demonstrating quantitative differences in properties exhibited by normal and malignant cells. Propertics of cancer cells have been systematically reviewed by Cowdry, E. V., Arch. Path., 1940, 30, 1245-1274. Yet the Dopa Reaction is of service in diagnosis of Melanocarcinoma.

Frequently it is helpful to excise cancers and transplant them into other situations such as the Anterior Chamber of the Eye where they can conveniently be studied. The Tissue Culture method is of great service, likewise Motion Pictures made of cancer cells. The most recently developed line of investigation is by Radioactive Isotopes. See also Papanicolaou Techniques.

Candida Albicans. Method for demonstrating this parasite with fat soluble dyes in frozen sections by Fuentes, C., J. Bact., 1946, 51, 245-246.

Cannulas. Glass cannulas are required for insertion into blood vessels in the Per. fusion technique. To make one of about the size for guinea pig's thoracic aorta file and break $6 \mathrm{~mm}$. outside, $4 \mathrm{~mm}$. inside diameter soft glass tubes into pieces about $15 \mathrm{~cm}$. long. (Pyrex of this size will do. It requires a little more heating.) Take one of these, place middle in gas flame rotating it so as to heat it evenly. When fairly soft remove from the flame, draw the ends apart to a distance of about $50 \mathrm{~cm}$. and hold until solid. File and break in the middle. With a little practice this will give two tubes, each tapering evenly from the $6 \mathrm{~mm}$. outside diameter to about $2-3 \mathrm{~mm}$. within a distance of approximately $3 \mathrm{~cm}$. Next bring the tube where it has a diameter of $2-3 \mathrm{~mm}$. near to a fine flame, like that of a small alcohol lamp. Let it get soft and pull just enough to produce a slight narrowing to be used later to prevent the thread employed to tie the cannula in the vessel from slipping. Then fracture with file and break off the thin end about $4 \mathrm{~mm}$. beyond the constriction and distant from the wide part of the tube. If this break can be made at an acute angle to the length of the tube, so much the better; because then one rim of the small end of the tube will project out beyond the rest which will facilitate its insertion into the vessel to be cannu- 
lated. When the break is made across the tube, at right angles, the rim on one side can be ground down on a water stone so as to produce a similarly projecting lip. In either case it is necessary to remove sharp cutting edges from both ends of the cannula by smoothing in a flame. The $6 \mathrm{~mm}$. wide body of the cannula should be $3-4 \mathrm{~cm}$. long for convenient attachment of rubber tube. Obviously larger cannulas are required for larger vessels. Those for Microinjection are very much smaller, made of hard glass and do not require to be tied in.

Capillaries. In living humans these can best be seen in the skin by the method of Capillaroscopy. Render the epidermis at the root of the finger nail translucent by addition of a drop of highly refractive oil and examine directly at fairly high magnification the capillary loops in the dermal papillae. It is possible to record their changes by making moving pictures through a long period of time. See review by Wright, I. S. and Druryee, A. W., Arch. Int. Med., 1933, 52, 545-575. See also Gingiva.

In living mammals the most favorable site in which to watch capillaries at high magnification is in the transparent chambers of the Sandison's Technique. For shorter periods they can be studied in the displaced but living pancreas by the methods of Covell, W. P., Anat. Rec., 1928, 40, 213-223 and O'Leary, J. L., ibid, 1930, 45, 27-58. Some changes in Permeability of living capillaries are evidenced by the trypan blue capillary permeability test. If microdissection is intended and a shift to the tongues and nictitating membranes of frogs is made consult $Z$ weifach, B. W., Anat. Rec., 1934, 59, 83-108, and Am. J. Anat., $1937,60,473-514$. The results have been recorded in moving pictures. Supravital staining of the tissues just mentioned with janus green (Bensley, R. R., and Vimtrup, B., Anat. Rec., $1928,39,37-55$ ) affords beautifully clear views of the muscular elements of arterioles grading into capillaries. See Perivascular Cells, Rouget Cells.

For investigations on the topographic arrangement of capillaries arterial injections with Carmine, Berlin Blue or some other easily recognizable material followed by clearing by the Spalteholz method may be helpful. When however any fluid is injected, under great pressure, into a fresh, relaxed tissue that can easily swell there is a chance that an exaggerated idea of the capillaries will be conveyed. In resting muscle for instance a large proportion of the capillaries are collapsed (Krogh).

The structure of the endothelial capillary wall is relatively uncomplicated. The outlines of the endothelial cells are nicely revealed in pink by the Silver Chloride Dichlorfluorescineate technique or in black by simply treating with silver nitrate. Nuclear and cytoplasmic structure can be brought out by methods used for other tissues. Nerve fibers closely accompany most capillaries. The existence of actual nerve endings on the wall is debated. The most convincing looking preparations of human tissues have been secured by Stöhr, Ph., Zeit. f. Zellf. u. Mikr. Anat., $1926,3,431-448$ who employed a modification by Gros of the Bielchowsky silver technique (see particularly his Fig. 2). See Sinusoids.

Capillaries of brain. Lepehne-Pickworth peroxidase method simplified by Campbell and Alexander (Mallory, p. 257). Fix for 1-3 weeks in $10 \%$ formalin. To make required solution dissolve $0.1 \mathrm{gm}$. benzidine in $0.5 \mathrm{cc}$. glacial acetic acid and add 20 cc. aq. dest. Dissolve 0.1 gm. sodium nitroprusside in $10 \mathrm{cc}$. aq. dest. and add benzidine solution. Add aq. dest. to $100 \mathrm{cc}$. In case a ppt. forms filter it out. Solution must be fresh. Cut frozen sections $200-300 \mu$ and wash in aq. dest. $1 \frac{1}{2} \mathrm{hr}$. Change to above described solution for $\frac{1}{2} \mathrm{hr}$. at $37^{\circ} \mathrm{C}$. agitating often. Wash in aq. dest. 10 sec. and transfer to $100 \mathrm{cc}$. aq. dest. + 2-3 drops $30 \%$ hydrogen peroxide for $\frac{1}{2} \mathrm{hr}$. at $37^{\circ} \mathrm{C}$. shaking frequently. Wash in aq. dest. and dehydrate in $70 \%$, $95 \%$ and absolute alcohol. Clear in xylol and mount in balsam. Blood vessels black in almost colorless background. This method has the advantage of not involving vascular perfusion. See comparison of injection and red cell staining methods for quantitative study of capillaries of central nervous system (Drummond, S.P., Anat. Rec., 1944, 89, 93-106). Microinjection of capillaries, Chambers, R. W. and Kopac, M. J., McClung's Microscopical Technique, 1950 , p. 530.

Capillary Fragility Tests. Discussion (Goldman, L. and Corrill, E. M., J. Invest. Dermat., 1945, 6, 129-147).

Capillary Respirometry. The development of the techniques is described by 'Tobias, J. M., Physiol. Rev., 1943, 23, 51-75. A differential respirometer is described by Cunningham, B. and Kirk, P. L., J. Gen. Physiol., 1940, 24, 135-149. Whole problem is discussed by Glick, pp. 314-326.

Capri Blue (CI, 876), a basic dye of light 
fastness 3. $0.1 \mathrm{gm}$. in $95 \mathrm{cc}$ aq. dest. +5 ec. $5 \%$ aq. ammonium alum +0.5 cc. acetic acid stains plant tissues blue or black. Can be employed in preference to Cyanine. Should stain well after Erythrosin (Emig, p. 58).

Capsule stain. 1. Hiss' method for smears (McClung, p. 145). Dry organisms in ascitic or serum medium on slides. Stain, slightly heated in 5-10 cc. satu. rated alc. gentian violet or basic fuchsin made up to 100 cc. aq. dest., few sec. Wash off dye with $20 \% \mathrm{aq}$. copper sul. phate crystals. Dry by blotting. See also: Huntoon, F. M., J. Bact., 1917, 2, 241. See Pasteurella.

2. IV. H. Smith's method for sections (Mallory, p. 275). Cover deparaffinized sections of Zenker fixed material with Anilin Crystal Violet (either Ehrlich's or Stirling's). During few seconds warm by passing slide through flame 2 or 3 times. Wash in Gram's Iodine solution followed by formalin (commercial). Decolorize in $95 \%$ alc. Quickly wash again in Gram's iodine. Cover with aniline green eosin and heat as before. To make this shake 1 part aniline green with 200 parts $3-6 \%$ aq. eosin yellowish W.S. and after 1-2 hrs. remove ppt. by filtering. Wash in aq. dest. Dehydrate in $95 \%$ and abs. alc., clear in xylol and mount in balsam. Bacterial capsules, red; Gram positive bacteria, blue. Mallory says that a stronger iodine may be desirable (iodine, $1 \mathrm{gm}$., potassium iodide, $2 \mathrm{gm}$. ; aq. dest., 100 cc.) and that the times must be suited to each preparation.

3. Churchman's (S. Bayne-Jones in Simmons and Gentzkow, p. 385). Flood air-dried films with Wright's stain and leave until almost evaporated to dryness. Original blue of stain is replaced by pinkish color. Wash quickly in water, or in Clark and Lubs buffer $\mathrm{pH}$ 6.4-6.5. Do not blot but dry with fan. Body of organisms, blue; capsular material, purplish-pink; often surrounded by capsular membrane or peripheral zone, decp purplish-pink.

Capsule Substance. This obviously is under investigation in many sorts of cells and the methods introduced for one kind may well be of service for others. See Cell Membrane for physical properties, thickness, etc. See Adhesiveness and Acid Fast Bacilli. Under Gram Stains is a description of the mechanism of their action which includes data obtained by use of the enzyme, ribonuclease, on the nature of walls of Gran positive bacteria. Under Enzymes, see enzymatic destruction of capsules of pneumococci.

Carbanthrene Blue GD (CI, 1113), Carban- threne Brilliant Orange $R K$, Carbanthrene Jade Green (CI, 1101), Carbanthrene Red BN (CI, 1162) Carbanthrene Red BN (CI, 1162) and Carbanthrene Violet 2R (CI, 1104) all of NAC are referred to by Emig, p. 64.

Carbohydrates, see Starch.

Carbol-Anilin Fuchsin methylene blue method for Negri bodies (Goodpasture, E. W., Am. J. Path., 1925, 1, 547-582). Fix in Zenker's fluid 24 hrs. (not Helly's fluid). Color for 10-30 min. in mixture made by adding $1 \mathrm{cc}$. of pure phenol and $1 \mathrm{cc}$. of anilin oil to $100 \mathrm{cc}$. of stock $0.5 \%$ basic fuchsin in $20 \%$ alcohol. Wash in running water, blot with filter paper and decolorize with $95 \%$ alcohol until sections become pink. Then wash in water and stain with Loeffler's methylene blue, 15-60 sec. Wash again in water. Dehydrate and destain for few sec. in absolute alcohol, clear in xylol and mount in balsam. Negri bodies, crimson; background, blue. Also excellent for Borrel Bodies.

Carbol-Crystal Violet. Because the solutions as prescribed in Nicolle's original formula for carbol gentian violet tend to gelatinize, the following formula is recommended by Conn, IH. J., Stain Techn., 1916, 21, 31-32: Mix solution of $0.4 \mathrm{gm}$. crystal violet C. C. in $10 \mathrm{cc}$. $95 \%$ ethyl alcohol with solution of $1 \mathrm{gm}$. phenol in 100 cc. aq. dest.

Carbol-Fuchsin. The original formula of Ziehl has been much modified. ZiehlNeelsen is sat. alc. basic fuchsin, $10 \mathrm{cc}$; $5 \%$ aq. carbolic acid, 90 cc. Verhoeff (F. H., J.A.M.A., 1912, 58, 1355) advises basic fuchsin, 2 gm.; abs. alc., 50 cc.; melted carbolic acid crystals, 25 cc. McClung (p. 136) suggests mixing $10 \mathrm{cc}$. $3 \%$ basic fuchsin $(90 \%$ dye content) with 95 cc. $5 \%$ aq. phenol. The inportant thing is the character of the fuchsin not its concentration relative to carbolic acid. Carbol-fuchsin is employed in stains for Acid Fast Bacilli. Deipolli, G. and Pomerri, G., Mon. Zool. Ital., 1938, 49, 123-124 have advised its use as follows for Nissl Bodies. Fix small pieces in $95-98 \%$ alcohol or in $10 \%$ formalin water or in physiological saline $24 \mathrm{hrs}$. or longer. Stain deparaffinized sections 3-4 min. in carbolfuchsin (basic fuchsin, $0.2 \mathrm{gm}$; conc. phenol, 1 cc.; $95 \%$ alc., 2 cc.; aq. dest. 20 cc.) 2.5 cc.; aq. dest., 100 cc.; glacial acetic acid, $0.5 \mathrm{cc}$. Wash rapidly in aq. dest. and destain in : aq. dest., $100 \mathrm{cc}$; formalin, 1 cc.; glacial acetic acid, 1 cc. Wash in aq. dest., dehydrate in alcohols, clear in xylol and mount in neutral balsam. Nissl bodies and nucleoli dark red, rest unstained.

Carbol-Thionin, see King's. 
Carbol-Xylol. Xylol saturated with carbolic acid crystals. After using it for clearing celloidin sections, wash quickly in xylol before mounting them in balsam.

Carbon from inspired air occurs abundantly in lungs and bronchial lymph nodes. It may be transported to the great blood filters (spleen and liver) where it is distinguishable by its black color and by its insolubility in conc. sulphuric acid which dissolves all other body pigments. Fine suspensions of carbon are of great service as vital stains to demonstrate phagocytosis. See Higgins' Ink and Lampblack.

Carbon Monoxide, Measurement of, see Scholander, P. F., and Roughton, F. J. W., J. Biol. Chem., 1943, 148, 551563 , or Glick, p. 334.

Carbonic Anhydrase. This can be localized in the oxyntic (or parietal) cells of the fundus of the stomach. Davenport, H. W., Am. J. Physiol., 1940, 128, $725-728 ; \quad 129,505-514$ employed an adaptation of Linderstrøm-Lang's technique and observed that in rats and cats the parietal cells contain 5 to 6 times as much of the enzyme as red blood cells while the peptic cells are free from it. A microspectroscopic method for demonstration of carbonic anhydrase within erythrocytes depends on the action of methemoglobin as an indicator which changes both its color and pattern of absorption spectrum with change of $\mathrm{pH}$ from 6.5-9.5 (Keilin, D. and Mann, T., Nature, 1941, 148, 493-496). For data on the distribution of this enzyme in lower forms, see Blaschko and Jacobson (Bourne, p. 200).

Carbonyl Compounds, water insoluble aldehydes and ketones, see critical statement by Glick, pp. 69-72. Bennett, H. S. (Äm. J. Anat., 1940, 67, 151-228) regarded his phenylhydrazine reaction for carbonyl compounds as indicative of ketosteroids in the adrenal cortex. Gomori, G. (Proc. Soc. Exper. Biol. \& Med., 1942, 51, 133-134) however does not agree unless there is additional evidence. Glick is of the same opinion that in the absence of such evidence the carbonyl reactions only indicate lipid aldehyde or ketone. According to Albert, S. and Leblond, C. P. (Endocrinology, 1946, 39, 386-400) it is plasmalogen instead of ketosteroid which is demonstrated by the phenylhydrazine reaction.

1. Bennett's phenylhydrzine reaction. Place frozen sections of fresh tissue from microtome into $M / 10$ acetate buffer, $\mathrm{pH}$ 6.0-6.5. If sections of fixed tissue are employed place in water. Add $1 \%$ iodine in alc. drop by drop till pale straw yellow color persists. Let stand 15 min. Add $1 \%$ aq. sodium thiosulphate drop by drop till color is lost and a small amount more is added. Let stand $5 \mathrm{~min}$. Wash sections repeatedly in aq. dest. Transfer sections to buffered phenylhydrazine solution just prepared by mixing equal volumes of $2 \%$ aq. phenylhydrazine hydrochloride and the acetate buffer and by removing oxygen by gently bubbling through carbon dioxide for $15 \mathrm{~min}$. This solution is to be poured into glassstoppered bottles so that with the sections added no air bubbles remain under the stopper. Control slides are taken through the same procedures but minus the phenylhydrazine treatment.

2. Albert and Leblond's 2,4-Dinitrophenylhydrazine reaction. Saturate 2,4-dinitrophenylhydrazine (No. 1866 Eastman Kodak Co.) in $30 \%$ alc. and bring $\mathrm{pH}$ to neutrality by addition of $0.2 \mathrm{~N}$ sodium acetate. This is the reagent. Fix tissue in formalin neutralized with magnesium carbonate for $48 \mathrm{hrs}$. and wash in running water for 24 hrs. Cut frozen sections 10-15 $\mu$ and transfer to $17 \%$ alc. $4 \mathrm{hrs}$. Place them in the reagent over night and wash in $17 \%$ alc. $20 \mathrm{~min}$. Change to aq. dest. and mount in glycerol gelatin (see Glychrogel). Yellow color indicates positive reaction.

3. Seligman, A. M. and Ashbel, R. (Cancer, 1951, 4, 579-596): Frozen sections, 10 to $20 \mu$, are cut from formalinfixed tissues and attached to slides by air-drying, after which the formalin is washed out with several changes of water. Sections are incubated for one or more hours at room temperature in a $0-1 \%$ solution of 3 -hydroxy-2-naphthoic acid hydrazide in $50 \%$ aldehydefree alcohol and $5 \%$ acetic acid. Remove excess reagent by washing for $2 \mathrm{hrs}$. in $50 \%$ alcohol followed by 2 hrs. in several changes of water.

A blue pigment is produced at the sites of carbonyl reactivity by immersion in a solution prepared from equal volumes of absolute alc. and an aqueous solution containing two parts water and one part $1 / 15 \mathrm{M}$ phosphate buffer (pH 7-2), followed by the addition of tetrazotized o-dianisidine in powered form $(50 \mathrm{mg}$. for $50 \mathrm{cc}$. of solution).

The development of a blue color reaches a maximum in one or two min. The sections are washed in several changes of water (acidified with a few drops of acetic acid) and are mounted in glycerogel.

Carbowax Embedding-Written by Dr. H. I. Firminger, Pathology Section, National Cancer Institute, Bethesda, 
Maryland. February 9, 1951-Carbowax (Carbide and Carbon Chemical Division, 30 East 42nd Street, New York 17, N. Y.), a water soluble wax, is an excellent embedding medium for tissues. It circumvents the long dehydration process necessary for embedding in paraffin or celloidin and yet sections prepared by this method show excellent preservation of cytological detail. Sections of Carbowax embedded tissue are easy to cut at from 1 to $10 \mu$ in thickness which makes this embedding method well suited both for the study of minute cytological details and for routine histological examinations. In contrast to paraffin and celloidin embedded tissues, lipids can be studied in sections of the same tissue block (Firminger, H. I., Stain Tech., 1950, 25, 121-123). Any type of fixation may be used and almost all staining procedures can be performed on Carbowax sections with the exception of osmic acid. Staining with osmic acid is better after the conventional methods.

Carbowax is not miscible with fats and penetrates adipose tissue, brain or spinal cord only very slowly. Such tissues can sometimes be embedded after long periods of infiltration in the oven with resultant distortion or by previous removal of lipid with fat solvents. Other disadvantages are the difficulty in mounting ribbons on the slide because of the solubuility of the Carbowax in the aqueous bath used for floating the sections, and the difficulty of making sections stick on the slide during the staining procedure. Blank et al. (Blank, H. and McCarthy, P. L., J. Lab. and Clin. Med., 1950, 36, 776781) recommend placing sections on an aqueous bath containing gelatin and potassium dichromate, floating sections onto a clean slide and drying thoroughly. An alternate procedure which avoids exposure to chromates (undesirable for certain staining procedures) is to cover the sections with thin colloidin prior to staining. Blank has also suggested mounting ribbons directly on a slide wet with the gelatindichromate solution.

The solution recommended by Blank et al. for floating and affixing sections to slides is made by dissolving $0.2 \mathrm{gm}$. of potassium dichromate and $0.2 \mathrm{gm}$. of gelatin in $1000 \mathrm{cc}$. aq. dest. The mixture is boiled in daylight for five minutes, cooled and filtered.

Embedding in Carbowax ordinarily employs a mixture of Carbowax " 4000 " and Carbowax " 1500 ". The exact proportions of the components must be varied to suit climatic conditions.
Pure Carbowax " 4000 " may be necessary in hot climates. In cooler climates one can use a mixture composed of 85 gms. of Carbowax " 4000 " and 15 gms. Carbowax "1500". For use, the Carbowaxes are combined in the proper proportions, heated to $175^{\circ} \mathrm{C}$. for 30 scconds and placed in an oven at $56^{\circ} \mathrm{C}$. This solution should be kept in the oven. If the temperature of the oven should drop and the Carbowax mixture solidifies, it requires reheating above $56^{\circ} \mathrm{C}$. to remelt it. To embed:

1. Place fixed tissue in small Stender dish containing the above Carbowax mixture in oven at $56^{\circ} \mathrm{C} .1-3 \mathrm{hrs}$.

2. Agitate dish and contents every 10 min. to aid penetration. All tissues, including lung tissue, should sink before further embedding.

3. After sufficient infiltration remove tissue and place in fresh mixture in another Stender dish.

4. Cover dish and place in icebox to harden at $5^{\circ} \mathrm{C}$. to avoid crystal formation.

5. Trim block with hot knife.

6. Block on hot object holder; then cool object holder with ice, taking care that water or ice do not come in contact with block.

7. Cut sections ( 1 to $10 \mu$ ). If satis factory ribbons are not obtained the procedure is not working properly.

8. (a) Float sections on a water bath at room temperature to which 2 drops of a detergent or the first ribbons of Carbowax have been added to prevent "explosion" of the sections by the surface tension. Stain sections before mounting on slide; or, float sections onto the slide from the water bath, cover carefully with a few drops of a mixture of equal parts of absolute alcohol and ether. Pour off excess and cover with thin collodion; harden in $70 \%$ alc. or water. Stain.

(b) Or, place sections on a bath containing $0.02 \mathrm{gm} . \%$ gelatin and $0.02 \mathrm{gm}$. $\%$ potassium dichromate. Float onto clean slide, permit to dry thoroughly and stain.

Carey's method for motor end plates is an adaptation for his study of their ameboid motion (Carey, E. J., Anat. Rec., 1941, 81, 393-413) of Wilkinson's (H. J., Med. J. Austral., 1929, 2, 768-793). Modification of Ranvier's gold chloride technique. -Written by the late Dr. E. J. Carey, Dept. of Anatomy, Marquette University School of Medicine, Milwaukee, Wis.

1. Remove any muscle from rat or chameleon from its origin to insertion while the animal is under ether or nemobutal anesthesia. Using a very sharp 
knife cut the muscle quickly into primary pieces, $0.5 \mathrm{~cm}$. long, and $0.5 \mathrm{~cm}$. thick, following the long axis of the muscle fibers. Then cut the primary pieces longitudinally into thin strips 1 to $2 \mathrm{~mm}$. wide.

2. Soak strips in freshly prepared filtered lemon juice for 5 to $10 \mathrm{~min}$. until they become clear or translucent. Rinse in cold tap water 4 to 5 times.

3. Place strips in $1 \%$ aq. gold chloride at $30^{\circ} \mathrm{C}$. using at least 10 times the volume of gold chloride solution to each volume of muscle. While muscle is in gold chloride solution, stir at least once a min. The time for the optimum impregnation of gold varies in the different muscles of the same animal at a relatively constant rate, for example, the sternocleido-mastoid muscle of the normal rat requires $16 \mathrm{~min}$; the pectoralis major, adductors of the thigh, and biceps femoris, $13 \mathrm{~min}$.; and the gastrocnemius, tibialis anterior, and the intercostal muscles $10 \mathrm{~min}$. After these muscles have been in the gold chloride solution for the proper length of time, they assume a yellowish-tan color and have a firm consistency. It is highly important that this variability in the reaction of different muscles in the same animal to gold inpregnation be realized. This may have been one of the factors that led to the discarding of the gold technique because it could not be rigidly standardized.

4. Pour off gold chloride solution and rinse the tissue with tap water until the water remains clear. Then place muscle in $25 \%$ aq. formic acid in the dark 16 to $24 \mathrm{hrs}$. Too little time gives incomplete reduction of the gold and too long time excessive softening and maceration.

5. Quickly rinse in tap water 5 or 6 times to remove as much of the formic acid on the surface of the muscle as possible. Even small amounts of formic acid in the preserving fluid may cause ultimate maceration of the tissue.

6 . Store the muscles until they are teased in a mixture of $\frac{1}{3}$ glycerine and $1 \frac{1}{2} 70 \%$ alcohol. (The muscles have been preserved in a good condition for teasing in this mixture for 7 years.)

7. To tease the muscle cut from one edge with a flat bladed teasing needle a piece $\frac{1}{2} \mathrm{~mm}$. thick and the full length of the muscle fiber of short muscles. The edge of the teasing needle may be flattened by hammering the needle after it has been placed in a Bunsen flame until the needle is red hot. Orient this strip of muscle in a drop of ylycerine on a clean, 1 x 3 slide. Gently add a clean cover slip. Lightly press down with the teasing needles, using a gentle lateral movement at right angles to the long axis of the muscle fibers. The muscle fibers, by this means, are gently rolled out so that the preparation is one muscle fiber thick. Check with microscope. Such a preparation will keep without any sealing of the cover slip for at least 7 years. Any of the usual cements, however, used for glycerine mounts, may be used to make the preparation permanent. We have successfully used clarite.

8. When cross or longitudinal sections are desired reduce the gold by placing muscle in a mixture of formalin $10 \%$ for $i$ ts hardening effect, and in formic acid $3 \%$ for the reduction of the gold. The gold may, likewise, be reduced by strong electric light for 16 to $24 \mathrm{hrs}$. The routine method for celloidin embedding is then used. After the tissues have been cut in sections, the nuclei can be counterstained by various techniques.

Carmalum (Mayer). Dissolve, if necessary with heat, $1 \mathrm{gm}$. Carminic acid and 10 gms. ammonia alum in $200 \mathrm{cc}$. aq. dest. Filter and to filter add $1 \mathrm{cc}$. formalin as a preservative. The tissues stained should not be alkaline (Lee, p. 141).

Carmine has been very widely used as a stain. Most of the formulae for staining of fixed tissues were proposed 40 or more years ago chiefly by Ranvier and Mayer. Now aniline dyes are more popular but carmine is still of great use for staining small animals in toto, for staining tissues in bulk which are later sectioned, as the best counterstain for blue vital dyes like trypan blue, as the most specific stain for Glycogen and for Mucus in the form of mucicarmine, for coloring gelatin used to inject blood vessels and as a vital stain. Karsner, H. T. and Swanbeck, C. E., J. Med. Res., 1920, 42, 91-98 employed 15-25 cc. of fairly thick suspension for intrapleural injections in cats. At present carminic acid is available and can be employed instead of powdered carmine. The only advantage is that the acid is of more uniform composition. See Acetocarmine (Schneider), Alum Carmine (Grenacher), Aluminum Chloride-Carmine (Mayer), Ammonia Carmine (Ranvier), Best's Carmine for gly cogen, Borax Carmine (Grenacher), Carmalum (Mayer), Lithium Carmine (Orth), Mucicarmine for mucus, Para-Carmine (Mayer), Picro-Carmine. (Ranvier). Many more carmine combinations are given by Lee (pp. 139-149).

Carmine Dusting of the Lungs-Written by C. C. Macklin, Dept. of Histological Research, The University of Western Ontario, London, Canada. November 28,1951 -Mice or other mammals are 
exposed in a closed compartment to air laden with the dust of dry powdered carmine. Agitation is by fan or blast from air-main. Atropinization facilitates entry into the lung alveoli. One hour suffices to mark the alveolar phagocytes (phagocytic pneumonocyteswhich see) with red particles. The cytological picture varies with the time elapsing after cessation of the dusting (Macklin, C. C., The Lancet, Feb. 24, 1951, 432-435). See Dust Cells.

Carmine-Gelatin Injections of blood vessels. Methods have been reviewed by Moore, R. A., J. Tech. Methods, 1929, 12, 5558 . He proposes a more accurate technique for preparation of the gelatin mass. Allow $80 \mathrm{gms}$. gelatin to take up $200 \mathrm{cc}$. cold water and heat to complete the gel. Suspend 20 gms. carmine in 100 cc. water and add ammonia until dissolved. Mix the gelatin and carmine solutions and add $15 \mathrm{gms}$. potassium iodide to reduce gelation point to less than $25^{\circ} \mathrm{C}$. Place in water bath at $25^{\circ} \mathrm{C}$. and immerse a prepared platinum electrode in it. Pass electroly tic hydrogen from a tank over the electrode and agitate the gelatin with a motor stirrer. Read electrical potential by balancing against a standard cell. Add acetic acid cautiously until reading of voltage corresponds to $\mathrm{pH} 7.2$.

Two other techniques are listed by Moore: 1. Dissolve $40 \mathrm{gms}$. carmine in $40 \mathrm{cc}$ strong ammonia and add water. Allow to stand 12-24 hrs. and filter through paper. Boil filtrate until it is ammonia free. Precipitate the carmine as a colloidal gel by adding $95 \%$ alcohol. Filter, wash well with alcohol and dry material collected. Dissolve $2 \mathrm{gm}$. in 5 cc. water and add 5 cc. 100 percent gelatin in water thus making the product $20 \%$ carmine and $50 \%$ gelatin (Bensley, R. R., personal communication to Dr. R. A. Knouff). 2. Triturate 40 gms. carmine Merck NFIV with 40 cc. strong ammonia and add water to $200 \mathrm{cc}$. After standing $24 \mathrm{hrs}$. filter through paper. Boil filtrate down to $100 \mathrm{cc}$., add water to $200 \mathrm{cc}$. and repeat. Add $70 \mathrm{gms}$. gelatin dissolved in water and make up with water to 1 liter (MacCallum, D. B., Am. J. Anat., 1926, 38, 153-175).

Carmoisine, see Chromotrope $2 \mathrm{R}$.

Carnoy-Lebrun fixative for insects and ticks. Equal parts chloroform, absolute alcohol and acetic acid saturated with mercuric chloride. See Slifer-King Method.

Carnoy's Fluid in abs, alc., 6 parts; chloroform, 3 parts; and glacial acetic acid, 1 part. Also known as Van Gehuchten's mixture. A very quick fixative. Do not wash in water but in $95 \%$ alc. It is employed for many purposes. See
Fibrin, Foot's Method, Glycogen Neurofibrils.

Carotene (Carotin), put green leaves in sat. aq. KOH, 1 part; $40 \%$ ethyl alcohol, 2 parts and tap water 3 parts in wide mouthed bottle with tight glass stopper to prevent absorption of $\mathrm{CO}_{2}$ from air or seal with vaseline. Kecp several days in dark until tissue is yellow and fluid is green. Change pieces to aq. dest. several hours. Remove small pieces, dry on slide with filter paper. Add 1 drop conc. $\mathrm{H}_{2} \mathrm{SO}_{4}$. It turns green, then blue. Under microscope carotene crystals appear dark blue (Steiger, A., Microkosmos, 1941, 8, 121-122). Carotene is a precursor of Vitamin A.

Carotinalbumins. Combinations of carotinoid pigments with protein. Rather uncommon. As an example Lison (p. 245 ) cites the blue carotinalbumin in the carapace of the lobster which on boiling is split into a protein and a red carotinoid.

Carotinoids. Pigments which are nonsaturated and nonnitrogenous hydrocarbons. Entirely different chemically from fats, they are nevertheless only present in vivo as solutions within lipoids. They generally appear yellow, orange or brown in unstained frozen sections mounted in syrup of levulose. Lison (p. 244) indicates that tissues containing these pigments can sometimes be embedded in paraffin, because they are only slowly soluble in cold alcohol. They are however more quickly soluble in chloroform, acetone petroleum ether and toluol. According to Lison (p. 245) they are always easily identifiable by the fact that when treated with concentrated sulphuric acid they turn intense blue before being destroyed. Treated with solution of iodine-iodide (say Gram's, Lugol's) they give a black green or brown color. When treated with solution of chromic acid they lose their color more or less quickly. See Lipids, tabular analysis, also Carotin.

Carr-Price Reaction for vitamin A. When frozen sections of liver are plunged directly into a solution of antimony trichloride in chloroform and immediately examined therein mitochondria take bright blue color which fades within 30 min. (Bourne, G., Austral. J. Exp. Biol. \& Med. Sci., 1935, 13, 238-249). Antimony trichloride is said not to be specific for vitamin A since it also gives blue color with carotinoid pigments (Bourne, p. 106). Sterols yield by this reaction a red color (Raoul, Y. and Meunier, R., J. Pharm. Chim., 1939, 29, 112-118). 
Carruthers, see Oxidation Reduction Potential, Vitamins.

Cartesian Diver Manometry, see detailed description of apparatus and technique by Glick, pp. 342-393.

Cartilage. This is one of the most awkward tissues of the body to examine in the living state because of the mechanical difficulties involved in separating its component parts sufficiently thinly for examination at high magnification in approximately isotonic media. But the differentiation of cartilage in tissue cultures has been studied to advantage (Fell, H. B., Arch. f. exper. Zellf., $1929,7,390-412$ ) and an account of the direct investigation of living cartilage in Sandison transparent chambers inserted in the ears of rabbits (Clark, E. R., and E. L., Am. J. Anat., 1942, 70, $167-200$ ) sounds very promising. The varieties of cartilage (hyaline, articular, elastic and fibrous) depend upon the quantitative and qualitative differences in the three chief components-cells, fibers and ground substance.

When the cartilage is fixed to bone, which is also to appear in the sections, it is obviously necessary to employ decalcification, see Bone. Otherwise cut thin slices, $2-4 \mathrm{~mm}$. thick, and fix by immersion. Fixation by perfusion is not a great help because cartilage is practically avascular. The choice of fixatives and stains will depend upon what it is desired to demonstrate. For routine purposes Zenker's Fluid is satisfactory followed by coloration of paraffin sections with Hematoxylin and Eosin or Mallory's Connective Tissue stain. But many prefer Celloidin sections. Resorcin Fuchsin is recommended for the elastic fibers of the matrix. Since the fibers are somewhat obscured by the ground substance in hyaline cartilage dark field and polarized light may be useful as employed by Lubosch, W., Zeit. f. mikr. Anat., Forsch., 1927, 11, 67-171. A paper by Dawson, A. B., and Spark, C., Am. J. Anat., 1928, 42, 109-137 also contains useful information. If it is desired to show the Golgi apparatus in the cells follow the technique used by Fell, $\mathrm{H}$. B., J. Morph., 1925, 40, 417-459. See Chondriotin Sulphuric Acid and Phosphatase as components of cartilage. The specific staining of cartilage cells with crystal violet has been reported by Hass, G. M., Arch. Path., 1942, 33, 174-181. The characteristic basophilia of the ground substance is the basis for the following excellent method for the demonstration of cartilage in whole mounts.

Van Wijhe's methylene blue (Noback,
G. J., Anat. Rec., 1916-17, 11, 292-294). This, by demonstrating cartilage in blue in transparent whole mounts, supplements very nicely the vital coloration of growing bone by Madder feeding or Alizarin injections. Use embryos, or bones of young animals like rats or mice, long bones, ribs, chrondocranium, etc. Fix in $10 \%$ formalin a day or more. $1 \%$ hydrochloric acid in $67 \%$ alcohol several days or a week. Same solution $+0.25 \%$ methylene blue or toluidin blue 1 or 2 weeks until thoroughly stained. Decolorize in Acid Alcohol. Change alcohol when it becomes much colored or every 1 or 2 days. Continue until only the cartilage retains deep blue color. Wash several days in $82 \%$ alc. Dehydrate in $95 \%$ and abs. Equal parts abs. and benzene. Benzene change twice. Leave in this or mount in xylene damar which is better than balsam because of its light color.

Radioactive gold distribution in cartilage (Ekholm, R., Acta Anat., 1951, Suppl. 15 to 11, 75 pp., a first rate study especially on the knee joint).

Cartilaginous Skeleton of mammalian fetuses. A modification of the Wijhe, Lundvall and Schultze techniques used in the Department of Embryology, Carnegie Institution of Washington is given by Miller, C. H., Anat. Rec., $1921,20,415-419$. Wash formalin fixed material over night in water plus few drops ammonia. Transfer to $70 \%$ alcohol and leave 7-14 days changing alcohol daily for first five. Stain for 3-10 days in: toluidin blue (Grubler), 1 gm.; $70 \%$ alcohol, 400 cc.; and hydrochloric acid, 4 cc. Decolorize for 7-10 days until decolorizer is but slightly tinged with the dye in: $70 \%$ alcohol, 100 cc. plus hydrochloric acid, 1 cc. Then $80 \%$ and $95 \%$ alcohol, 3 days each. Transfer to $2 \%$ potassium hydroxide, in aq. dest. and leave 2-3 days until cleared. Change to $20,40,60$, and $80 \%$ glycerin in aq. dest. 2 days or more in each. Store or mount in pure glycerin plus few crystals of thymol. Obviously length of times depends chiefly upon size of specimen. This staining of cartilage with toluidin blue can be combined with the coloration of bone with Alizarin Red $\mathbf{S}$ to make very contrasty preparations (Williams, T. W., Stain Techn., $1941,16,23-25$ ).

Carycinel Red is 1-amylaminoanthraquinone, an oil soluble dye, recommended by Lillie, R. D. Stain Techn., 1945, 20, 73-75 as a stain for fat which it colors deep red. Employ as described for Coccinel Red.

Caryospora, see Coccidia.

Caspersson, see Absorption Spectra. 
Caseation (L. caseus, cheese). This change follows local Necrosis. It is characterized by grayish or light yellow cheesy masses of tissue which look amorphous and have lost their original structure. Identification is morphological. Almost any good staining method is satisfactory. In some cases fibrin is present.

Cason, see Mallory-Heidenhain Rapid OneStep Stain for Connective Tissue.

Catalase. Method for demonstration in elementary bodies of vaccine virus (Macfarlane, M. G., and Salaman, M. H., Brit. J. Exp. Path., 1938, 19, 184; Hoagland, C. L. et al., J. Exp. Med., 1942, 76, 163-173). See Holter, H. and Doyle, W. L., J. Cell Comp. Physiol., $1938,12,295-308$.

Cataphoresis. Most solid particles suspended in water move under electric stress. A positively charged one moves toward the cathode and a negatively charged one toward the anode. Microcataphoretic cells are employed to determine and measure the movement which obviously has an important bearing on bacterial agglutination. Electrophoresis is a better term than cataphoresis. (Holmes, H. N. in Glasser's Miedical Physics, 257-263) see Coagulation.

Cataract, see Optic Lens.

Cathepsin. A method for analysis of cathepsin in lymphocytes and polymorphonuclear leucocytes (neutrophiles) is given by Barnes, J. M., Brit. J. Exp. Path., 1940, 21, 264-275.

Cebione, see Vitamin C.

Cedar Oil, see Clearing, Immersion Oils and Mounting.

Celestin Blue B (CI, 900)-coreine 2R-A basic quinone-imine dye employed by Proescher, F. and Arkush, A. S., Stain Techn., 1928, 3, 28-38 and by Lendrum, H. C., J. Path. \& Bact., 1935, 40, 415416 as a nuclear stain.

Cell Classification according to manner of life. Intermitotic cells live from the mitosis which gives them birth to the mitosis by which they divide to produce two other cells. They thus cease life as individuals by division not by ageing, degeneration and death. There are 2 kinds of intermitotic cells: First, the vegetative intermitotics some of which continue a sort of vegetative life constituting a reservoir of undifferentiated cells on which the body can draw in some cases as long as it lives. They are found in the epidermis bone marrow and other places. Second, the differentiating intermitotics, which exist in series, one building up a certain degree of differentiation, which, when it divides, it passes on to its daughter cells. The progeny of these daughter cells differentiate still further and pass on this higher level of specialization to their successors. Good examples are myeloblasts and myelocytes in leucocytogenesis. But the first differentiating intermitotic in any line of differentiation is produced by division of a vegetative intermitotic. One of the daughter cells of this division, or in some instances both daughter cells from mitosis of a dividing vegetative intermitotic, achieve no further differentiation than their parent cells, for otherwise the reservoir of vegetative intermitotics would not be maintained but would differentiate itself out of existence.

Postmitotic cells, on the other hand, are cells whose lives are postmitotic in the sense that they perform their duty, age and die. They are the culminations of the various lines of differentiation. Again, two sorts are recognizable: First the reverting postmitotics, which are capable of full functional activity and usually go on to death, yet, on occasion, some of which can revert and divide. Hepatic and renal cells are examples. Second, the fixed postmitotics, which are different insofar that they are incapable of mitosis so that aging and death is for them inevitable as for instance nerve cells of adults, sperms and polymorphonuclear neutrophile leucocytes. In contrast with the other 3 kinds these fixed postmitotics have lost the potentiality of malignant transformation (Cowdry, E. V., Problems of Aging. Baltimore, Williams \& Wilkins, 1942, 626-629).

Cell Components can be examined by techniques too numerous to list including Staining, Supravital and Vital Staining, Impregnation, Microdissection, Micromanipulation, Microinjection, Centrifugation, many Microchemical Reactions, and Indicators by at least 6 different kinds of Microscopes. Methods for many of these components are given under Capsule Stains, Mitochondria, Zymogen, Nissl Bodies, etc.

Cell Division, see Mitosis, Amitosis and series of papers on chemistry of cell division (Mauer, M. E. and Voegtlin, C., Am. J. Cancer, 1937, 29, 483-502).

Cell Enlargement, see Giant Cells.

Cell Injury detected by fluorescence (Herick, F., Protoplasma, 1939, 32, 527-535). See Dead Cells.

Cell Measurement, The ElliptometerWritten by Dr. J. D. Hamilton, Dept. of Medical Research, University of Western Ontario, London, Canada. February 13, 1951-The geometric shape of the section of many histological structures is circular or elliptical. The 
cross sections of cell nuclei, the boundaries of many cells such as sea urchin eggs, nerve cells, the central zone of cells undergoing division, ovarian folliculi, glomeruli, blood vessels and ducts, may all be described in terms of their geometrical parimeters.

A quantitative measurement of the area, eccentricity, and geometric center of these structures may often be made by means of an elliptometer (Hamilton, J. D. and Barr, M. L., Stain Tech., 1948, 23, 123).

Essentially the elliptometer method provides a means of fitting an ellipse of light to a camera lucida drawing of the cell or structure. Alternatively in principle, the camera lucida drawing board may be replaced by a ground glass screen and an ellipse of light of suitably controlled intensity, angle, and size matched to the structure as viewed directly in the ocular of the microscope. In both methods, by direct graphic means, the axes of their equivalent ellipse or ellipse of best fit is then determined.

In the instrument a variable circular iris such as a camera diaphragm, is used to define a cone of light arising from a point source. Point sources of light provided by a zirconium or concentrated arc lamp are quite suitable and make possible the construction of the apparatus without the use of a condensing lens systen to define a point source. The cone of light falls upon a screen upon which is mounted a grid of graph paper. An alternative to the above design uses a lens between the illuminated diaphragm and the screen. The lens provides an image of the diaphragm in the perpendicular plane of the screen.

The screen may be rotated by means of an attached arm which rotates in a sleeve bearing lying parallel to the horizontal lines of the grid. By tilting the screen out of the vertical plane it is thus possible to create a family of ellipses. The minor axcs will be controlled and fixed by the diameter of variable diaphragm. The points at which the ellipse touches the horizontal and vertical grid lines may be determined and the axes calculated by addition.

In practice the camera lucida drawing is placed flat on the screen and the elliptometer aperture and screen angle adjusted to give the best fit. The two ends of the major axes are marked on the drawing for future reference, and the grid values noted.

Derived Measurements: a) Area: If A, and $B$, are the major and minor diam- eters of the ellipse then the area is $\mathrm{K} \pi \mathrm{A}, \mathrm{B}, / 4 \mu^{2}$ where $\mathrm{K}$ is a proportionately constant depending on the grid system used and the magnifications entering into the camera lucida tracing. In our experience with motor nerve cells (Barr, M. L. and Hamilton, J. D., J. Comp. Neur., 1948, 89, 93) the areas determined by elliptometer and planimeter measurements agree within five per cent on an absolute basis in comparing individual cells, and the statistical parameters of populations examined by the two methods of measurement are of equal value when control and experimental populations are compared. Area measurement using the elliptometer are more rapid and less fatiguing than planimeter measurements.

b) Center and Foci: The center and foci of elliptic sections may be readily determined by using a rule or by graphic methods. If the plane of section is transverse, sagittal, or coronal, a reference line running dorsal-ventral or antero-posterior or medio-lateral may be drawn through the center of the elliptic figure. The orientation of the ellipse or the location of inclusions or other features of the figure, may then be measured with respect to the axes of the body as a whole. By this type of orientation and definition for example, it has been possible to show that the axone hillock of motor cells of the dorso-lateral group of the ventral horn arise predominantly in the ventral medial quadrant of the cell.

c) Eccentricity: The eccentricity is the ratio of the minor to the major axis $B / A$. The eccentricity of individual sections may possess some value as a descriptive measurement. The average eccentricity of populations may be of value in following the time course of changes of shape, as for example in hypertrophy, cell division, or blastula development.

d) Cell Shape and Value: Consider an ellipsoid having major, intermediate, and minor diameters, $\mathrm{A},>\mathrm{B}, \div \mathrm{C}$, lying at random in space related to the plane of section. The eccentricities of sections drawn from an ellipsoidal population, homogenous in respect to shape (but not necessarily size) will range from a maximum $\mathrm{C} / \mathrm{B}$ (which may be unity if the body is an ellipse of revolution) to a minimum C/A. This distribution of eccentricities is true even if the plane of section does not pass through the center of the ellipsoid.

The eccentricity data may be used to secure an estimate of the shape and 
volume of the average body. According to experience, eecentricities are placed in several classes, from 1.0 to 0.4 , say, with elass intervals of 0.2 . From the first elass, 1.0 to 0.8 , an estimate of the eecentrieity $\mathrm{C} / \mathrm{B}$ ean be made, since ellipses of this class are primarily of the type C, B, partieularly if $\mathrm{B}$ and $\mathrm{C}$ are approximately equal and somewhat less than A. Similarly eccentrieities of the last elass interval 0.6 to 0.4 will provide an estimate of $\mathrm{C} / \mathrm{A}$. Combining these two estimates the average ratio $\mathrm{B} / \mathrm{A}$ may be determined.

The minor axes of sections of the first and last eecentricity groups are representative of the $\mathrm{C}$ or minor axes of the space figure. Hence an estimate of the average value of $\mathrm{C}$ may be made, and using the eccentricity ratios already determined the average values of $A$ and $B$ ean be ealculated. The average volume $\overline{\mathrm{V}}$ equals $\mathrm{k} \pi \overline{\mathrm{ABC}}$ can then be calculated.

Cell Membranes do not require any special technique for their demonstration. Almost any good fixative will do and they can be stained a host of different colors. There is however some difference in the interpretation of what we see with the microseope. The essential component of the walls of all cells is called the plasma membrane. This conditions permeability and its integrity is essential to the life of the cell. It is said to consist of a continuous layer of lipoid moleeules (phosphatides, sterols, fats) not more than 2-4 moleeules thick on which proteins are adsorbed, the lipoids give permeability and the proteins elasticity and great mechanical strength. The evidence is critically presented by Danielli (Bourne, pp. 68-98). He says that it is improbable that the lipoid layer is ever thicker than $10 \mathrm{~m} \mu$ and that the whole membrane is between $1 \mu$ and $1 \mathrm{~m} \mu$ thiek. Consequently in many cases we cannot expect to visualize the plasma membrane itself directly with visible light because the theoretical limit of visibility is a partiele size of $0.25 \mu$. However the position of the plasma membrane is made clear by the difference in properties of the eytoplasm which it limits and the fluid without and also in the dark field by the light reflected from its surface. In addition it is often backed internally by a thin layer of cytoplasmic cortex (ectoplasm) which is typically free from cytoplasmic granules. The plasma membrane may be supplemented externally by special membranes such as the myelin sheaths about nerve fibers. There are many special techniques for its investigation. Some are briefly referred to under
Lysis, Permeability, Surface Tension and Wetting Properties, Nuclear Membrane, Pinocy tosis.

Cell Shape. The shape of epithelial cells, and of all cells for that matter, is determined by perfectly clefinite causes. Obviously those suspended in fluid tend to be spherical (lymphocytes) unless their internal organization conditions some other shape (erythrocytes). Contact with a surface generally promotes flattening on that surface. Epithelial cells are sessile. The study of their morphology is not eomplicated by motility. When disposed in a single layer and subjected to lateral pressure from their neighbors they take a distinetive shape which has been analyzed in a convincing way by F. T. Lewis (Am. Scientist, 1946, 34, 357-369, and many earlier papers). In sections of the layer parallel to the surface it may be seen that most of the cells are six-sided, or hexagonal. They form a mosaic, the charaeter of which can easily be remembered by students forced to dream of the benzene "ring" with its 6 carbon atoms. By drawing many such ehemical symbols side by side a similar mosaic is formed. As Lewis points out, the intersections are three-rayed not fourrayed as might be the ease if the crosssections were squares. Mechanieally this is a great advantage. When the deithelium is stratified provision must be marle for contact with cells on all sides. Nature adheres to the same three-rayed intersection and molds the eells in that shape which provides the smallest surface area for closely erowded bodies. Lewis found that this could be determined mathematically as a 14-sided figure and by careful reeonstruction of actual cells proved that they were all primarily tetrakaidecahedral in shape. Examination of his elear illustrations will be more helpful than pages of description. The same architectural principles apply to many other cell aggregates, like fatty tissue for example. No longer is the histologist justified in vaguely referring to such cells as polyhedral. Evidently in the construction of epithelial surfaces the cells are fitted together in a much more effeetive way than bricks in the building of a wall. Except for the reference, the above paragraph is quoted from the Second Edition of Cowlry's Histology, Philadelphia: Lea \& Febiger, 1938. See technique for three-dimensional study of cell shape in plants, Holtzman, D. H., Am. J. Bot., 1951, 38, 221-234.

Celloidin Imbedding. Celloidin is a kind of generie term eovering various cellulose compounds, nitrocellulose, soluble 
gun cotton, etc., employed for imbedding. The collodions are solutions of pyroxylin made as specified in the U.S.P. Pyroxylin U.S.P. XI consists chiefly of cellulose tetranitrite (Merck Index, p. 465). Obviously a purified, nonexplosive form of pyroxylin is necessary. There are several in the market of which Parlodion (Mallinckrodt) is the one used in our laboratory. The Bensleys (p. 37) use as celloidin "RS $\frac{1}{2}$ sec. low viscosity nitrocellulose 30 per cent solvent in absolute alcohol" obtained from the Hercules Powder Co., Gillespie, N. J. To make $20 \%$ stock solution they dissolve 140 gms. nitrocellulose in $250 \mathrm{cc}$. ether and $210 \mathrm{cc}$. absolute alcohol. This requires $4-5$ days shaling occasionally. It is diluted with ether alcohol to make 10 and $5 \%$ solutions respectively. Nitrocellulose is much used especially in neurological technique. It is abbreviated L.V.N. Some advantages over "celloidin" are claimed for it by Davenport, H. A., and Swank, R. L., Stain Techn., 19\%, 9, 137-139.

Celloidin imbedding is less popular than it used to be owing to certain advantages of Paraffin Imbedding reinforced by the mania for speed. But celloidin imbedding is in some respects superior. It yields sections in which the affinity of the tissue components for dyes is often greater. Clearing of the tissue in xylol and similar fluids is not required and it need not be subjected to heat. The tissue usually shrinks less and seldom becomes so brittle. Brain specimens can easily be cut in celloidin even after long mordanting. When sections are required of large pieces of tissue in which cavities, such as the lumina of the paranasal sinuses, alternate with stout bony walls this method is indicated because the celloidin in the spaces gives more support than paraffin (see also Double Imbedding).

The slow method, which is the best, requires for tissue slices not more than $5 \mathrm{~mm}$. thick, at least 1 day each in $95 \%$ alcohol, absolute alcohol, and in half absolute and ether. This is followed by 1 day in thin celloidin (about 4\% dried strips of celloidin-Parlodion, Mallinckrodt-dissolved in equal parts absolute alcohol and ether) and 1 or more weeks in thick $8 \%$ celloidin. The tissue, with some celloidin about it, is then mounted on a fiber block, hardened in chloroform 1-2 hrs. and stored in $80 \%$ alcohol.

Mallory (p. 60) gives the following as a rapid method. Fix thin tissue pieces 12-18 hrs. in Formalin-Alcohol. Then $95 \%$ alcohol, 2 changes, 2 hrs.; absolute alcohol, 2 changes, 3 hrs.; alcohol-ether, 3 hrs.; thick celloidin 12-15 hrs.; mount and harden in chloroform, 1 hr.; $80 \%$ alcohol.

A still quicker technique has been proposed (Richardson, G. D., J. Tech. Meth., 1934, 13, 81) : To make celloidin solution, add 1100 cc. absolute ethyl alcohol to $8 \mathrm{oz}$. celloidin (dried in air) and leave over night. Add $1100 \mathrm{cc}$. ether. Let stand several days. It is ready when celloidin is dissolved. Fix tissue in $10 \%$ formalin, 2 hrs.; acetone, 2 hrs.; oil of cloves $\frac{1}{2}-2$ hrs. or until clear; celloidin 6 hrs. at room temperature or $\frac{1}{2}-3 \mathrm{hrs}$. in water bath at $55^{\circ} \mathrm{C}$. (being careful to keep away from flame). Block and harden in chloroform $\frac{1}{2}-2 \mathrm{hrs}$.

Another so called hol celloidin method is proposed with all steps in the technique at an elevated temperature (Koneff, A. A., and Lyons, W. R., Stain Techn., 1937, 12, 57-59). Fix pieces not thicker than 2-3 mm. in 10\% neutral formol, Bouin or Susa. Wash in aq. dest. several changes (1 hr. each) at room temperature. Dehydrate at $50^{\circ} \mathrm{C}$. $70,80,95$ and abs. alc. 2 changes $\frac{1}{2} \mathrm{hr}$. each. Equal parts abs. alc. and ether $1 \mathrm{hr}$. Infiltrate at $56^{\circ} \mathrm{C}$. in (1) $10 \%$ nitrocellulose (R.S. $\frac{1}{2}$ second, viscosity $\frac{8}{20}-\frac{4}{2}$, Hercules Powder Co.) in equal parts abs. alc. and ether, $1 \mathrm{hr}$. (2) $25 \%$ in $45 \mathrm{cc}$. alc. $+55 \mathrm{cc}$. ether, over night. (3) $50 \%$ in 40 cc. alc. +60 cc. ether 2-3 hrs. Then transfer tissue to microtome block moistened with ether-al cohol. Add 50\% nitro-cellulose and the tissue. Harden in 2 changes chloroform during $1 \mathrm{hr}$. Then pass through 3 changes $80 \%$ ethyl alcohol and cut. The authors mention fixation in "Carnoy II" and removal of mercury with iodized alcohol in case a fixative containing mercuric chloride was employed. Obviously every precaution must be taken to avoid explosion.

Store celloidin blocks in $80 \%$ alc. See special methods for imbedding Teeth and Bone.

Celloidin Injections of lungs. For smaller vessels and bronchi use : acetone, $100 \mathrm{cc}$; celloidin, $4 \mathrm{gm}$.; and camphor, $3 \mathrm{gm}$. For larger vessels and bronchi employ: acetone, $100 \mathrm{cc}$., sheet celloidin, $20 \mathrm{gms}$., and camphor, $15 \mathrm{gm}$. In place of sheet celloidin old $x$-ray films can be used if first the emulsion is removed by washing in warm water and they are then dried and cut into strips. If colors are desired employ oil paints. If Roentgenograms are to be made of the corrosion specimens add $10-12 \%$ sodium iodide or barium sulphate to a $30 \%$ suspension. In case of the vessels wash out blood first by 
forcing physiological saline solution into vena cava thence through right heart and via pulmonary arteries to lungs evacuating by pulmonary veins. Allow injected lung to stand in running water over night thus hardening celloidin. Immerse in concentrated hydrochloric acid to digest away tissues leaving celloidin cast. This usually takes 24 hrs. Wash thoroughly in gentle stream of water. Mount dry or mount wet in solution made up as follows: Boil for 10 min. 100 cc. aq. dest. +20 cc. glycerin. When cool add formalin to $2 \%$ and filter until clear (Marquis, W. J., J. Tech. Methods, 1929, 12, 59-64). See illustrations of Marquis and arrangement of pressure bottles. A celluloid corrosion technique for the kidney is described by N. W. Baker, J. Tech. Methods, 1929, $12,65-68$.

Celloidin Sections. Cut side of celloidin block to smooth plane surface. Moisten this and surface of microtome block holder with alcohol-ether. Add drop thick celloidin. Press together, harden in chloroform and cut in $80 \%$ a,lcohol on a sliding microtome with knife at an angle. Keep surface of knife and block wet with $80 \%$ alcohol from overhead dropping bottle. (A method has been described for treating block with cedar oil and cutting dry with rotatory microtome, Walls, G. L., Stain Techn., 1936, $11,89-92)$. Sections are usually cut at a thickness of $10-16 \mu$. (It is possible to arrange the sections serially but it is a tedious business. If serial sections are needed, paraffin should be selected in place of celloidin.) The sections unmounted can be stained without removing the celloidin after which they are to be dehydrated and cleared before mounting. The object is not to remove the celloidin but to sof ten it. The following mixture is recornmended by Lee (p. 108) in place of xylol, toluol or benzol : creosote, 40 cc.; Bergamot oil, 30 cc.; xylol, $20 \mathrm{cc}$. and origanum oil, $10 \mathrm{cc}$.

Cellosolve is ethylene glycol monoethyl ether. It mixes with water, acetone, alcohol, ether and dissolves many oils, waxes, etc. Employed by Lendrum (A.C., J. Path. \& Bact., 1939, 49, 590$591)$.

Cellulose, microchemical reaction for. Solution A : Dilute $20 \mathrm{cc}$. of $2 \%$ iodine in $5 \%$ aq. potassium iodide with $180 \mathrm{cc}$. aq. dest., add $0.5 \mathrm{cc}$. glycerin and mix by shaking. Solution B: Saturate 15 cc. aq. dest. with lithium chloride at $80^{\circ} \mathrm{C}$., cool and use supernatant solution. Tease out section or fibers. Apply 2-3 drops " $A$ " by glass rod and leave 10 sec. Blot with filter paper and dry. Add drop "B", cover and examine. Cellu- lose blue, green, yellow depcnding upon its source (Post, E. E. and Laudermilk, J. D., Stain Techn., 1942, 17, 21-26. See Polysaccharides.

Cements. W. C. Tobie (in Simmons, and Gentzkow, p. 356) gives two useful types:

Vacuum wax for ordinary vacuum seals not subjected to high temperature is made by melting together equl parts of beeswax and rosin. It is pliable and easily removed with hot water.

Acid resisting cement is made by mixing asbestos powder and sodium silicate solution (water glass) into a paste of desiled consistency. Will dry in $24 \mathrm{hrs}$.

For ringing specimens mounted in glycerin, etc. sce Kronig's Cement and Mounting Media.

Centigrade temperature to Fahrenheit

1. Above $0^{\circ} \mathrm{C}$. multiply by 9 , divide by 5 , add 32 . Example: $37^{\circ} \mathrm{C} .=37 \times 9=$ $333 \div 5=66.6+32=98.6^{\circ} \mathrm{F}$.

2. Between -17.77 and $0^{\circ} \mathrm{C}$. multiply by 9 , divide by 5 subtract from 32 . Example: $-12^{\circ} \mathrm{C} .=12 \times 9=108 \div 5=$ $21.6 ; 32-21.6=10.4^{\circ} \mathrm{F}$.

3. Below $-17.77^{\circ} \mathrm{C}$. Multiply by 9 , divide by 5 , subtract 32 . Example: $-18^{\circ} \mathrm{C} .=18 \times 9=162 \div 5=32.4-$ $32=0.4^{\circ} \mathrm{C}$.

Central Body, see Centrosome.

Centrifugation. To even sketch in outline the techniques that come under this heading is difficult because the centrifugation of so many materials and tissues is helpful and the instruments vary from simple hand driven machines to powerful ultracentrifuges which may weigh several tons and which certainly require experts to care for them. See Svedberg, T. and Pedersen, K. O., The Ultracentrifuge, Oxford, Clarendon Press, 1940, $478 \mathrm{pp}$.

The centrifuge has long been of help in the displacement of certain components of cells (especially marine eggs) in order to determine their functional rôles. It has also proved invaluable in the investigation of cytoplasmic and nuclear Viscosity, which see.

In recent years centrifugation has opened a new chapter in microchemistry by the part which it has played in the collection of cellular components in sufficient volume for analysis. Pioneer work was done with the liver. The Bensleys (p.6) give instructions which are in part as follows. First perfuse the abdominal organs of a guinea pig with about 1000 cc. $0.85 \%$ aq. sodium chloride (see Perfusion). This removes a good deal of the blood. Excise liver and grind up thoroughly in a mortar. Place the resulting thick fluid in large centri- 
fuge tubes, add about twice the volume of $0.85 \%$ aq. sodium chloride and balance the tubes with more as may be necessary. If complete separation of mitochondria is desired centrifuge for $1 \mathrm{~min}$. at 3000 r.p.m. which results in stratification. In first and lowest stratum, at the bottom of the tubes, will be found liver cells, cell debris and connective tissue elements; in the second, nuclei and red blood cells; in the third mitochondria and small cell fragments; and in the fourth and uppermost, free fatty droplets. The materials in any of these layers can then be collected by drawing up in a pipette, suspended again in salt solution and purified by further centrifugation.

For the isolation of ellipsin (structural protein) and mitochondria see Bensley, R. R. and Hoerr, N. L., Anat. Rec., $1934,60,251-266$ and $449-455$. Since it is in the mitochondrial fraction resulting from centrifugation that vitamin $A$ is found the Goerners have greatly extended the usefulness of the method in a series of studies on tumors (Goerner) A., J. Biol. Chem., 1937-38, 122, 529538 and A., and M. M., ibid, 1939, 128, 559-565). The technique has been further improved by Claude (A., Science, 1938, 87, 467-468; Cold Spring Harbor Symposia on Quantitative Biology 1941, 9, 263-270) who used 18000 r.p.m. See, particularly, standardized techniques in his 1941 paper. Beams, H. W. and King, R. L., Anat. Rec., $1940,76,95-101$, and in a series of other papers, have greatly contributed to the use of ultracentrifugation in the solution of biological problems. See Lucas, A. M., Am. J. Path., 1940, 16, 739-760 on intranuclear inclusions.

Centrifuge Microscope. By this ingenious combination of microscope and centrifuge it is possible to observe living cells with the highest dry objectives while they are actually being centrifuged. Cells or organisms to be examined are placed in isotonic media of appropriate density in special slides constructed so that the centrifugal force derives them into approximately the focus of the objective. The clearness of the 2 dimensional image is not conditioned by the speed of rotation. The slide is fixed into the centrifuge head remote from the axis of rotation. Strong light is focussed by condensing lens from above onto the slide. A prism below the slide in the centrifuge head directs the light toward the axis of rotation directly through an objective. When received at the axis of rotation it is directed upward by reflecting prisms into an ocular in position above the center of the centrifuge head. Not only can stages in displacement of intracellular components be watched but permanent records are easily made in the form of motion pictures. Since its introduction by Harvey and Loomis in 1930 several structural improvements have been achieved. A commercial design is made by Bausch and Lomb Optical Company (Harvey, E. N., in Glasser's Medical Physics 1944, 147).

Centriole, see Centrosome.

Centrosomes (G. Kentron, center; soma, body), sometimes called a "central body", is a minute spherule which is a dynamic center of some sort involved in cell division. It is sometimes called a centriole though Conklin (Cowdry's General Cytology, pp. 542 and 544) says that a central body, the centriole, appears within the centrosome during mitosis. When the centrosome is double, that is consists of two minute bodies side by side, it is designated a diplosome. About the centrosome, or diplosome, there is usually a clear area which is known as a centrosphere. The centrosome, or centriole plus the clear area is called the cytocentrum. For terminology see Wilson, E. B., The Cell. New York: Macmillan Co., 1925, 1232 pp. For functional significance see Fry, H.J., Biol. Bull., 1929, 57, 131-150. Giant centrospheres in degenerating cells are described by Lewis and Lewis (Cowdry's General Cytology, p. 427) and multiplication of centrioles in striated muscle tumors by Wolbach, E. B., Anat. Rec., 1928, 37, 255-273.

Centrosomes are not easily demonstrated in tissue sections. The technique originally used by Heidenhain (Arch. f. mikr. Anat., 1894, 42, 665) appears to be the best. It consists of fixation in a Sublimate Acetic, or Sublimate Alcohol Acetic, and of staining the sections 24 hours in a dilute aq. sol. of Bordeaux red or of anilin blue followed by iron hematoxylin in the usual way. The centrosomes are stained black or gray with a tinge of red or blue. In glandular epithelial cells look for them in the cytoplasm between the nucleus and the lumen.

To reveal centrosomes in non-dividing nerve cells is difficult, probably because they are seldom present. Hatai (S., J. Comp. Neurol., 1901, 11, 25) was able to stain them in certain nerve cells of adult rats. He fixed in sat. mercuric chloride in formalin, 30 cc.; glacial acetic acid, $50 \mathrm{cc}$. and physiological salt solution, 15 cc. for 6-12 hrs., then washed, 4-5 hrs. in running water, imbedded in paraffin, stained in sat. aq. toluidin blue or thionin, dehydrated, 
cleared and mounted the scctions. Rio Hortega (P., Trab. Lab. Invest. Biol. Univ. Madrid, 1916,14, 117) has obtained beautiful silver preparations of centrosomes. Addison (McClung, 1950 p. 378) advises fixation in Flemming's Fluid or in Allen's chromic-urea modification of Bouin's Fluid followed by staining with Heidenhain's Iron Hematoxylin.

A detailed investigation of the effects of a great many fixatives on the mitotic figure in chaetopterus eggs has been made by Fry (Fry, HI.J. Biol. Bull., 1933, $65,207-237$ ). He concluded (1) that acetic acid, picric acid, formaldehyde and alcohol and certain combinations of them are most useful as fixatives (2) that anesthetics like chloroform and ether and inorganic fixatives are to be avoided; (3) that the fixatives must be diluted to about $10 \%$ of the original concentration with aq. dest. or better with sea water. Comparable information for human tissues is lacking.

Cephalin, a phosphatide, is a compound of phosphoric acid, glycerol, 2 fatty acid molecules and amino ethyl alcohol. It differs also from lecithin in being only very slightly soluble in alcohol, see Lipoids.

Cerasin R, see Bordeaux Red.

Cerasin Red, see Sudan III.

Cerebrosides are galactosides, that is compounds of fatty acid, galactose and sphingosine, without phosphorus, soluble in benzene, pyridine and hot alcohol and almost insoluble in ether, see Lipoids.

Cerebrospinal Fluid. Total cell count is best made in a Fuchs-Rosenthal counting chamber. In making smears for the differential count it may be necessary first to add a little albumin fixative to the slides to get the cells to stick (C. J. Lind in Simmons and Gentzkow, p. 91).

Ceresin Imbedding. Ceresin is purified ozokerite, a mixture of hydrocarbons, with melting point $61-78^{\circ} \mathrm{C}$. used as a substitute for beeswax and for other purposes. Waddington, C. H. and Kriebel, J., Nature, 1935, 136, 685 advise for hard objects like feathers addition of ceresin to a paraffin of slightly lower melting point than that usually employed. The whole, when cooled, has a very fine texture. See the methyl benzoate celloidin ceresin method of 'Espinasse for imbedding hard objects in a suitable condition for sectioning as described by Lee (p. 96) and Waterman, H. C., Stain Techn., 1939, 14, 55-62. Ceresin can be obtained from Shell Oil Co., melting point, $82-85^{\circ} \mathrm{C}$.

Cerium, see Atomic Weights.

Ceroid. This is a wax like endogenous pig- ment (G. keros, wax + eidos, resemblance). It is greenish yellow to golden brown in color, fluorescent, acid fast, stains with fat dyes as well as with methyl green, and is relatively insoluble in fat solvents. Ceroid has been separated from the tissues of rats, collected en masse and analyzed by Moore, $\mathrm{T}$. and Wang, Y. H. (Brit. J. Nutr., 1947, 1, 53-64). Mason, K. E. and Emmol, A. F. (Anat. Rec., 1945, 92, 33-59) consider its demonstration in muscle to be reliable indication of vitamin $\mathrm{E}$ deficiency. Grenados, H., Mason, K. E. and Dam, H. (Acta Path., 1947, 24, 86-95) present evidence in rats of a metabolic relationship between vitamin $\mathrm{E}$ and unsaturated fatty acids. A helpful summary of the distribution of ceroid pigment in human tissues is supplied by Pappenheimer, A. M. and Victor, J. (Am. J. Path., 1946, 22, 395-412). The properties of ceroid pigment in relation to experimental necrosis in rats and mice, as revealed by many histochemical techniques, are presented by Lee, C. S. (J. Nat. Cancer Inst., $11,339-347)$. The important point is made that there are easily noticeable differences between the ceroids of these two species. An interesting technique for the study of ceroidlike substances is to produce them in vitro and in vivo from certain lipids and erythrocytes as has been done by Hartroft, W. S. (Science, 1951, 113, 673-674).

Cerotine Ponceau 3B, see Sudan IV.

Cerulein MS (CI, 783)-Anthracene Green, Coerulein MS-a mordant dye of light fastness 3 to 4 gives unsatisfactory coloration of animal tissues. Directions for plants (Emig, p. 55).

Cervical Swab-Smears, see Paparnicolaou Techniques.

Cesares-Gil flagella stain evaluated, Thatcher, L. M., Stain Techn., 1926, 1, 143-144.

Cesium, spectrographic analysis of, in retina (Scott, G. H. and Canaga, B., Jr., Proc. Soc. Exp. Biol. \& Med., 1939, 40, 275).

Cestoda, see Parasites, Taenia.

Cevitamic Acid, see Vitamin C.

Chalkley ratio method for determining relative volumetric composition of tissue sections (Chalkley, H. W., J. Nat. Cancer Inst., 1943, 4, 47).

Chambers, see Micromanipulation.

Champy-Kull's Method of anilin fuchsin, toluidine blue and aurantia for mitochondria. Fix in Champy's fluid $(3 \%$ potassium bichromate, 7 cc.; $1 \%$ chromic acid, 7 cc. ; $2 \%$ osmic acid, 4 cc.) $24 \mathrm{hrs}$. Wash in aq. dest. Place in pyroligneous acid, 1 part and $1 \%$ chromic acid, 2 parts 20 hrs. Wash aq. dest. 30 min.; 
mordant $3 \%$ aq. potassium bichromate, 3 days. Wash running water $24 \mathrm{hrs}$., dehydrate, clear, imbed and section at $4 \mu$. Remove paraffin from sections. Stain with anilin acid fuchsin (acid fuchsin 10 gms., anilin water $100 \mathrm{cc}$.) heated over spirit lamp and allow to cool 6 min. Rinse in aq. dest. Counterstain in $0.5 \%$ aq. toluidine blue 1-2 min. Rinse in aq. dest., then $0.5 \%$ aurantia in $70 \%$ alcohol $20-40$ sec. Differentiate in $95 \%$ alcohol, dehydrate, clear and mount. Mitochondria red, nuclei blue and ground substance yellow.

Champy's Fluid is 3\% potassium bichromate, 7 parts; $1 \%$ chromic acid, 7 parts; and $2 \%$ osmic acid, 4 parts. It is an excellent fixative for cytologic details.

Charcot-Leyden's Crystals. Octahedral phosphate crystals found in stools of persons infected with Endameba histolytica and in a variety of other conditions. See description and illustration by Craig, p. 58 .

Cheese. Bacteria in, see Hucker, G. J., N. Y. Agric. Exp. Sta. Tech. Bull'. 1921, 87 (McClung, p. 147).

Chelidonium, see Fluorescence Microscopy, shows "secondary" fluorescence.

Chicago Blue, see escape from venules after intravenous injection (Smith, F. and Rous, P., J. Exp. Med., 1931, 54, 499514).

Chick, see Chorioallantoic Membrane.

China Blue, see Anilin Blue.

Chitin. This is a keratoid, or keratin-like, substance which is an important part of the exoskeleton and hard parts of invertebrates ( $\mathrm{G}$. chiton, a coat). It is soluble in hot concentrated sulphuric and hydrochloric acids; relatively insoluble in alcohol, ether, dilute acids and alkalies. There are several color reactions for chitin. Lillie (p. 147) applies to chitin, thoroughly washed after treatment with potassium hydroxide, $33 \%$ aq. zinc chloride to which $3-5$ drops conc. aq. iodine potassium iodide have been added to each $10 \mathrm{ce}$. Chitin becomes brown on the surface, violet within. Chitin is colored red violet by a solution containing: iodine, 50 mg., potassium iodide $50 \mathrm{mg}$., calcium chloride $16 \mathrm{gm}$. and aq. dest. $4 \mathrm{cc}$.

1. A method for softening of chitin in formalin fixed insects (Murray, J. A., J. Roy. Micr. Soc., 1937, 57, 15). Fix primarily in $10 \%$ formalin in $0.8 \%$ aq. sodium chloride, or indefinitely. Fix secondarily and dehydrate in equal parts absolute alcohol, chloroform and glacial acetic acid + corrosive sublimate to saturation (about 4\%). Warm together equal parts chloral hydrate and phenol until they fuse and form an oily liquid which is fluid at room temperature. Leave speci- mens in this 12-24 hrs. or longer. Clear in chloroform, xylol or carbon disulphide. Imbed in paraffin.

2. According to Hennings (see Lee, p. 597) fixation of insects in the following mixture softens the chitin sufficiently to permit the making of paraffin sections: nitric acid, 16 cc.; $5 \%$ aq. chromic acid, 16 cc.; sat. corrosive sublimate in $60 \%$ alcohol, 24 cc.; sat. aq. picric acid, 12 cc.; and abs. alc., 42 cc. Fixation is $12-24 \mathrm{hrs}$. followed by washing in iodine alcohol. An older method is to soften chitin by treatment with a solution of hypochlorite of soda (Lee, p. 249). See Diaphanol, N. Butyl Alcohol, Insects, and Ticks.

Chloral Hydrate, as a fixative for peripheral nerves (Bank, E. W. and Davenport, H. A. Stain Techn., 1940, 15, 9-14). Chloral hydrate is also recommended as a macerating medium for the separation and isolation of epithelial and lining cells by the Bensleys (p. 5). According to their instructions remove small pieces alimentary tract of pithed or freshly killed frog and leave them in $5 \%$ aq. chloral hydrate $12-48$ hrs. Then tease with fine needles and examine. See Cajal's chloral hydrate method.

Chlorazol Black E (CI, 581) of British Dyestuffs Corporation-Erie black G X 00 (National Aniline and Chemical Company), Pontamine black E (I. E. Du Pont deNemours \& Co.)-an acid poly-azo dye. First described as a new biological stain by Cannan (H. J., Nature, 1937, 139, 549). Review of its uses (Cannan, H. J., J. Roy. Micr. Soc., 1941, 61, 88-94). As a vital dye (Baker, J. R., Nature, 1941, 147, 744). Stains chromatin black, cytoplasm greenish gray after Zenker fixation (Darrow, M. A. Stain Techn., 1940, 15, 67-68). As an acetocarmine auxiliary stain for chromosomes (Nebel, B. R., Stain Techn., $1940,15,69-72)$. As a simple connective tissue stain (Levine, N. D., and Morril, C. C., Stain Techn., 1951 16, 121-122) it is excellent for arterial elastic tissue.

Chlorazol Blue 3B, see Trypan Blue.

Chlorazol Fast Pink used as anticoagulant in experiments designed to influence growth of transplants of lymphosarcomas (Williams, W. L., Cancer Research, 1946, 6, 344-353). Toxicity as an intra vitam stain is presented by Williams, W. L. and Hodge, H. C., Anat. Rec., 1943, 87, 181.

Chlorazol Paper Brown B. A sat. aq. sol. of this dye has been employed to stain plant tissues. It is differentiated in $1 \%$ aq. nitric acid. Acetone is used for dehydration and Euparal for mounting (Verdcourt, B., Stain Teclin., 1947, $22,155-156$ ). 
Chlorazol Pink Y, see Thiazine Red R.

Chloride. In 1908 Macallum reviewed the older literature and described his silver test for chloride (Macallum, A. B., Ergeb. d. Physiol., 1908, 7, 552-652). The possibility, which has not yet been finally answered, is that at some stage in the technique there is a shift in the position of chloride. The mere application of the silver reagent may conceivably withdraw chloride from the cell. For these reasons prior treatment of the tissue by the Altmann-Gersh freezing and drying method which reduces the chance of movement of chloride to a minimum is recommended.

1. Gersh (Gersh, I., Anat. Rec., 1938, 70,311-329) gives details of the procedure on which the following instructions are based. Tissues frozen in liquid air, dried in vacuum, embedded in paraffin and sectioned at $15 \mu$ are mounted near one edge on chemically clean large cover slips by simply pressing down with a finger, just melting over a flame and pressing down again. Immerse coverslips with attached sections in anhydrous petroleum ether (b.p. $20-40^{\circ} \mathrm{C}$.) freshly distilled over sodium in a watch glass covered by another at all times except during actual manipulations. This removes the paraffin. Remove and burn off the ether quickly by a flame and allow to cool to room temperature. Then treat two coverslips with attached sections differently.

A. Cover for few seconds with drop of $60 \%$ aq. silver nitrate diluted with sufficient quantity of conc. phosphoric acid to prevent precipitation of rather large concentrations of phosphates and then saturate with silver chloride. After filtering $2-3$ drops aq. dest. are added to every $10 \mathrm{cc}$. before using.

B. Cover similarly with: $60 \%$ aq. silver nitrate saturated with silver phosphate and silver chloride and dilute after filtering in the same way.

Decant fluids from both coverslips. Add to each 1 drop chemically pure glycerin and mount with section plus glycerin down on chemically clean slides. Expose both to carbon are radiation for same length of time but at a distance not to warm the specimens. Examine immediately the reduced silver by direct illumination or in the dark field. A. shows specifically only the chloride and B. the same amount of chloride plus maximal concentrations of phosphate and some carbonate.

2. Dichlorfluorescein method (Bensley, R. D. and S. H., Anat. Rec., 1935, 64, 41-49). For the lung of a rabbit. Inject $1 \%$ aq. dichlorfluorescein intravenously until the animal becomes quite yellow. Then kill it and inject $10 \%$ aq. silver nitrate or Silver Citrate solution either intratracheally or directly into the lung substance by a hypodermic syringe until the lung is moderately distended. In about 20 min. the color reaction reaches its maximum. The silver ehloride becomes pink owing to adsorption of the dichlorfluorescein on the positively eharged silver chloride molecule. 'Then fix pieces of lung in $10 \%$ neutral formalin and make frozen sections. Exarnine immediately for best color reaction. Dehydrate the sections, clear in absolute alcohol and iso-safrol and mount in balsam. The color reaction is not permanent but is masked and finally lost by the browning and blackening of the silver. It is not a true mierochemical test; but it does detect the presence of chlorides though they are mobilized by the silver and tend to move to the periphery of the cell. The alveolar epithelial cells are outlined by pink stippling and their cytoplasm is also stippled and the nuclei are richly stippled. Mesothelial and endothelial cells are brilliantly and completely outlined in pink. The technique was first suggested by David M. Ritter.

The loeation of chloride is a matter of great importance. Lowry, O. H. and Hastings, A. B. in Cowdry's Problems of Ageing, Baltimore: Williams \& Wilkins, $1942,936 \mathrm{pp}$. cite the following as evidence for the extraccllular position of chloride in skeletal muscle :

(1) Direct microscopic studies showing that chloride is exclusively extracellular (Gersh, I., Anat. Rec., 1938, 70, 311-329).

(2) Perfusion experiments showing that chloride can be removed without apparently affecting the intracellular phase (Amberson, W. R. et al., Am. J. Physiol., 1938, 122, 224-235).

(3) Variations in amount of chloride and in acid base balances of tissues can only be accounted for by assuming an extracellular position for cliloride (Hastings, A. B. and Eichelberger, L., J. Biol. Chem., 1937, 117, 73-93).

(4) Isolated tissues equilibrated in vivo against solutions of varying chloride concentrations retain chloride in proportion to the concentration in the medium but at a very much lower level (Fenn, W. O., Cobb, D. M. and Marsh, B. S., Am. J. Physiol., 1934, 110, 261272 ; Eggleton, M. G. and P. and Hamilton, A. M., J. Physiol., 1937, 00, 167$182)$.

(5) Conclusion that in many tissues for all practical purposes all radioactive sodium and radioactive chloride remain 
outside the cells (Manery, J. F. and Bale, W. F., Am. J. Physiol., 1941, 132, 215-231; Manery, F. W. and Haege, L. F., ibid, 134, 83-93).

See, however, Heilbrunn, L. V. and Hamilton, P. G., Physiol. Zool., 1942, $15,363-374$ for demonstration of chloride in muscle fibers.

If chloride is always extracellular in all tissues it is possible accurately to measure the amount of extracellular fluid and a new chapter in histochemistry is opened. Lowry and Hastings give an example. If rat muscle is found to contain 10.5 milliequivalents of chloride per kilogram of tissue and the serum of the same animal 105.2 milliequivalents of chloride per kilogram of serum water, in view of the Donnan effect on chloride distribution it can be calculated that a kilogram of extracellular fluid contains 109.7 milliequivalents of chloride. Consequently the sample of muscle contains $\frac{10.5}{109.7} \times 1000=96$ gms. of extracellular fluid per kilogram. When the extracellular fluid contains collagenic and elastic fibers, collagen and elastin must be determined and the necessary corrections made as well as for blood and fat when these are present. When the intracellular phase is chiefly composed of a single type of cell as in skeletal or cardiac muscle the further evaluation of intracellular components is not difficult. Taking every known precaution, evidence can apparently be collected of the relative composition of extracellular and intracellular phases.

If it is desired to determine chloride in very small amounts of fluid, as in the analysis of glomerular urine, a method described by Westfall, B. B., Findley, T. and Richards, A. N. (J. Biol. Chem., 1934, 107, 661-672) is suggested. Glick (p. 200) says this permits chloride determination in a fraction of a $\mu \mathrm{l}$ of fluid containing $1 \mu \mathrm{gm}$. or less of the choride. The technique of Sendroy, J. (Jr., J. Biol. Chem. Chem., 1942, 142, 171-173) is adequate for $10 \mu \mathrm{l}$ of serum. There are in addition several titrimetric methods for chloride which should likewise be considered as possibilities. See Glick, pp. 281-283.

Chlorophenol Red. See Hydrogen Ion Indicators.

Chlorophyll. The green pigment of plants is a mixture of 2 substances chlorophyll $a$ and $b$, of which many derivatives are known. In man several fluorescent chlorophyll porphyrins are identifiable in feces and urine. A detailed account of chlorophyll is provided by Rothe- mund, P., in Glasser's Medical Physics, 1944, 154-180.

Chloroplasts. Isolation and collection en masse from spinach leaves by centrifugation (Menke, W., Zeit. f. Physiol. Chem., 1938-39, 257, 43 . See Glick.

Chloroprene, see Neoprene.

Chlorothymols, as preservatives of gelatin, glues, starches, etc. (Law, R. S., J. Soc. Chem. Ind., 1941, 60, 66).

Chocolate Blood Agar, see Bacteria, Media.

Cholesterol (esters) $=$ cholesterides. In unstained frozen sections mounted in syrup of levulose they show no color of their own; but the Liebermann-Burchardt Reaction in frozen sections of formalin fixed tissue is positive. Digitonine Reaction in similar sections yields a complex in which the esters, if present, will color with Sudan III and lose birefringence in polarized light. See Lipids tabular analysis, see Schultz test for cholesterol and its esters. Technique for determination in buffer is given by Nieman, C. and Groot, E. H., Acta Physiol. et Pharmacol. Neerland., $1950,1,488-501$.

Cholesterols (free). In unstained frozen section mounted in syrup of levulose, they show no color of their own. Liebermann-Burchardt Reaction in frozen sections of formalin fixed tissue is positive: blue, purple or violet then becoming green. Digitonine Reaction in similar sections yields strongly birefringent crystals and rosettes which do not stain with Sudan III. See Lipids, tabular analysis.

Choline. See Florence's Reaction for Seminal Stains.

Choline Deficiency. Use of fluoreseence nicroscopy in (Popper, H. and Chinn, H., Proc. Soc. Exp. Biol. \& Med., 1942, 49, 202-204). See Vitamin B complex.

Cholinesterase-Written by E. W. Dempsey, Dept. of Anatomy, Washington University, St. Louis, February 26, 1951 -Important since it is implicated in nervous transmission. It is present in high concentration in the neuromuscular junction and in the electric organ of eels, and has also been detected in the axis eylinder of nerves. Anfinsen, C. B., O. H. Lowry and A. B. Hastings (J.' Cell. and Comp. Physiol., 1942, 20, 231-237) have developed a method whereby the same section of rat brain cortex can be stained for microscopic examination and thereafter used for enzyme measurement. Recently, a number of techniques have been proposed for its detection on slides. Gomori, G. (Proc. Soc. Exp. Biol. and Med., 1948, 68, 354-358) reported that long-chain fatty acid esters of choline were hydrolyzed by tissue sections, and 
that the fatty acids could then be visualized. However, hydrolysis of such substrates is slow and probably caused by a non-specific esterase. Koelle, G. B. and J. S. Friedenwald (Proc. Soc. Exp. Biol. and Med., 1949, 70,617-622) proposed the use of acetylthiocholine iodide as a substrate, and obtained beautiful localizations within the central nervous system. These authors have also differentiated between the true and pseudocholinesterases by means of specific inhibitors (J. Nat. Cancer Inst., 1950, 10, 1364). Seligman, A. M., M. M. Nachlas, L. H. Manheimer, O. M. Friedman and G. Wolf (Ann. Surg., 1949, 130, 333-341) have proposed a naphthyl derivative of acetylcholine as a substrate. After hydrolysis, the naphthyl moiety is visualized by diazotization.

Chondriosomes, see Mitochondria.

Chondriotin Sulphuric Acid. Present in cartilage and bone, stains metachromatically with basic dyes, described in detail by Lison, L., Arch. de biol., 1935, 46, 599-668. See Muccproteins.

Chorioallantoic Membrane. 1. Vital staining of virus lesions in membrane (Cooke, J. V. and Blattner, R. J., Proc. Soc. Exp. Biol. \& Med., 1940, 43, 255-256). Place 1 cc. $0.5 \%$ aq. trypan blue directly on membrane through window in shell. Rotate egg gently and return to incubator, 10-30 min. Small lesions require longer time to stain than large ones. Remove membrane, wash it gently in physiological saline and fix flat in $10 \%$ formalin, a few minutes. Make up glycerin jelly by soaking 5 gms. gelatin in $44 \mathrm{cc}$. aq. dest. Then add $50 \mathrm{cc}$. glycerin and 1 cc. phenol. Heat gently and stir. Flatten membrane on a $2 \times 2.5 \mathrm{in}$. slide, warm glycerin jelly to about $70^{\circ} \mathrm{C}$. Add drop by drop to membrane until well covered. Flame a cover glass and apply with slight pressure until it has begun to set. Remove hardened jelly around edges and seal with balsam. Foci of virus increase are sharply marked by clumps of deep blue stained cells.

2. Cultivation of microorganisms. The membrane has been shown to be an excellent medium for the cultivation of viruses by Goodpasture, E. W., Woodruff, A. M. and Buddingh, G. J., Am. J. Path., 1932, 8, 271-282 and many others. Its usefulness has been extended to Rickettsiae and spirochetes by Goodpasture, E. W., Am. J. Hyg., 1938, 28, 111-119, to fungi by Moore, M., Am. J. Path., 1941, 17, 103-125 and to acidfast bacteria by Moore, M., Am. J. Path., $1942,18,827-847$. 'This method of inoculation has the advantage over laboratory animal inoculation in that lesions will develop in the former within 5-8 days as compared to weeks or months in the latter; most organisms will produce definite and usually characteristic lesions in the chick membrane, whereas they may have no effect on experimental animals, often requiring human subjects; and because the lesions are so readily visible and traceable the chlorioallantois serves well as a means of virulence determination.

The technique is essentially that of Goodpasture and Buddingh (E. W. and G. J., Am. J. Hyg., 1935, 21, 319-360) with some slight changes. Fertile eggs are incubated 12 days in an electrical thermostat-controlled incubator regu. lated to maintain a temperature of $98^{\circ} \mathrm{F}$. The eggs are turned twice daily. $\mathrm{A} \mathrm{cm}$. square window is cut in the shell above the embryo, exposing the chorioallantoic membrane. The position of the embryo is determined by candling. The membrane is then inoculated directly with the fungus and the window is covered with a sterile coverslip and sealed with a paraffin-vaseline mixture (9 parts vaseline, 1 part paraffin). After inoculation, the eggs are set in a bacteriologic incubator and maintained at a temperature of approximately $33^{\circ} \mathrm{C}$., without turning. The membrane is watched daily through the window. When the inoculated area has shown marked change, the shell is cut below the window and the membrane exposed. The chorioallantois is cut with a pair of fine curved-end scissors, removed, fixed in Zenker's solution (with 5\% glacial acetic). After washing, dehydrating, clearing in xylol, and imbedding in paraffin, it is sectioned and stained. Various staining techniques can be used depending on the organism inoculated. In general, for fungi, Loeffler's methylene blue and eosin have given satisfactory results. For experimental technique of growing mouse sarcoma in chorio-allantoic membrane, see Jacoby, F., McDonald, S. and Woodhouse, D. L., J. Path. and Bact., 1943, 55, 409-417.

Chor's Modification of Ranson's pyridine silver method was worked out in our laboratory to show alterations in motor end plates in biceps and triceps of monkeys in experimental poliomyelitis (Chor, H., Arch. Neurol. \& Psychiat., $1933,29,344-357$ ). Fix in $1 \%$ ammonia water ( $28 \%$ Merck) in $95 \%$ alcohol for 24 hrs. Wash in aq. dest., $\frac{1}{2}$ hr. Pyridine, 48 hrs. Wash in 8 changes aq. dest. during $24 \mathrm{hrs.} 2 \%$ aq. silver nitrate in dark at room temperature, $72 \mathrm{hrs}$. Reduce 6-8 hrs. or over night in : pyrogallic acid, 4 gm.; aq. dest., 95 cc.; formalin, 5 cc. Dip in water and trans- 
fer immediately to $95 \%$ al cohol for a few seconds. Place tissue on slide with longitudinal markings of fibers visible. Add a second slide and squeeze gently. Trim edges with sharp knife so that neat, flat blocks result. $95 \%$ alcohol, $30 \mathrm{~min}$. Absolute alcohol, 2 changes, over night. Xylol, 10-12 hrs. until blocks are clear. Imbed in paraffin $8 \mathrm{hrs}$. changing repeatedly each hr. for first five. Cut serial sections $10 \mu$. Mount in neutral balsam. Nerves, dark brown or black; muscle and connective tissue, yellow.

\section{Chrom Blue GCB, see Gallocyanin.}

Chromaffin Reaction (chromic salts $+\mathrm{L}$. affinis, akin). Brown coloration when treated with fixatives containing bichromate. In adrenal medulla adrenalin is revealed by this brown color but the reaction can also be elicitated by potassium iodate and is not altogether specific for adrenalin. Lison (p. 147) advises fixation in Formol-Müller or in $5 \%$ potassium iodate containing $10 \%$ of formol. After the usual fixations chromaffin substances can be demonstrated simply by treating the sections for a few hours with $3 \% \mathrm{aq}$. bichromate or iodate of potassium (Lison). See Vulpian Reaction and Osmic Acid.

Chromatin Filaments. The studies of Claude, A. and Potter, J. S., J. Exper. Med., 1943, 77, 345 and of Mirsky, A. E., and Pollister, A. W., Biological Symposia., 1943, 10, 247-260 indicate that chromatin is almost wholly made up of fibrous nucleoprotein. By extraction, precipitation and centrifugation the chromatin filaments can be collected. They are of very uniform diameter, like chromosomes, are very resistant to deforming mechanical injury, can easily be stained with acetocarmine are given a positive Feulgen reaction. Mirsky and Pollister favor the view "that chromatin is largely, if not entirely, a complex of highly polymerized desoxyribose nucleic acid with a basic protein of either the protamine or histone type."

Chromatin Stains. The most specific stain for basic chromatin is methyl green. Bismark brown is less so. Safranin is useful for chromatin if a red coloration is desired as in the safranin-light green combination. Tests for Iron and Thymonucleic Acid are listed separately. See Idiochromatin, Linin, Chromosomes and Nucleolus.

Chromatolysis of nerve cells investigated by absorption spectra of Nissl bodies (Gersh, I., and Bodian, D., Biological Symposia, 1943, 10, 163-184).

Chromatophores. These, when present in the dermis, are also called melanoblasts, see Dopa Reaction for their demonstration.

Chrome Violet CG (CI, 727). A carboxyl derivative of pararosolic acid.

Chromic Acid is purchased as the red crystals of chromic anhydride which dissolve easily in water forming chromic acid. The crystals should be kept in a bottle with closely fitting glass stopper because they are highly deliquescent. Alone in very dilute solution chromic acid is helpful in Maceration. When applied in aqueous solutions of about $1 \%$ to a slice of freshadrenal it produces a brown color in the medulla known as the chromaffin reaction. In mixtures with other chemicals it was more used as a fixative 50 years ago than today but in Perényi Fluid it is recommended strongly by Lee (p. 32) for embryos, segmenting eggs, etc. It is also a component of Flemming's fluid.

Chromidial Substance, a designation of ten applied to basophilic cytoplasmic material supposed to be of nuclear origin and therefore to resemble the extranuclear chromatin (chromidia) of protozoa. It is nongerminal chromatin or trophochromidia in contrast to germinal or idiochromidia (G. idios, individual, one's own). See Nissl bodies.

Chromolipoids. In contrast to the carotinoids, which are hydrocarbons, the chromolipoids are fats or derivatives of fats themselves colored. They occur frequently especially in nerve cells, interstitial cells of the testicle and in the adrenal, and are easily distinguishable from carotinoids because they do not give the color reactions with sulphuric acid and iodine-iodide. From melanins they are to be distinguished by not dissolving in alkalies, by staining with sudan and scharlach and by not reducing ammoniacal silver nitrate. The following method of Hueck is given by Lison: Stain with nile blue. Treat the sections for $24 \mathrm{hrs}$. with aq. dest. oxygenated $3 \%$ ( = commercial hydrogen peroxide diluted with 12 volumes water). This leaves the chromolipoids blue, the melanins decolorized. Lison concludes that distinction from pigments of hematogenous origin is not so easy because some chromolipoids contain iron. See Lipids, tabular analysis.

Chromophil (G. chrōma, color and phileō, I love), a loose term applied to almost any granule, cell, or tissue which has a pronounced affinity for stains. Basophilic cytoplasmic materials in gland cells and in nerve cells (NissI bodies) are sometimes called chromophil, moreover chromophil reaction is unwisely used to designate the chromaffin reaction of epinephrin producing tissues. 
Chrom-Osmic-Acetic fixative, see Lillie's. Chromosomes.-Written by A. R. GopalAyengar, Barnard Free Skin and Cancer Hospital, St. Louis, Sept. 10, 1946(Now Pata Memorial Hospital, Bombay). These are discrete bodies usually constant in number in the cells of a given species and frequently having distinctive structure into which the chromatin material of a nucleus resolves itself during the mitotic process (see Mitosis). From a chemical standpoint the chromosome is a protein fiber like silk or hair, presumably depending on a peptide chain linkage- $\mathrm{C}-\mathrm{C}-\mathrm{N}-$. On this structural framework the permanent hereditary units, the genes (which may be considered as the atoms of heredity), are located at definite loci. In a sense, therefore, the chromosome may be considered a giant molecule (Darlington, C. D., Nature, 1942, 149, 66-69, Astbury, W. T., Proc. 7th Int. Genet. Congress., 1939 (Camb.), $1940,49-51)$. It is generally stated that the chromosomes of sperm cells consist of basic proteins, such as protamines or histones, in combination with highly polymerized desoxyribose nucleic acid (Mirsky, A. E., Advances in Enzymology, 1943, 3, 1-34).

Smear-Squash technique. The rapid and spectacular advanees in our knowledge of cytology and cytochemistry during the last fifteen years have greatly altered our ideas of chromosome structure and behavior. Progress in this direction has been possible through the introduction of newer techniques, such as smears and squashes, which have largely supplanted the classical methods of paraffin sectioning. In order to obtain the most satisfactory results, it is imperative to employ cytological techniques calculated to bring out fine structures and details as quickly and accurately as possible. Most of the smear-squash methods, despite their extreme simplicity, give superior results because of instantaneous fixation and rapidity of staining and finishing schedules. Moreover, it is possible to examine thin layers of cells or even isolated cclls and their parts. They are especially useful in the analysis of chromosome complexes and associations and in the exact establishment of numerical relations. Squash preparations have special advantages. Individual chromosomes can be recognized more readily and easily, interrelations between chromosomes become clearer, the preparations are almost two-dimensional, which is of undoubted value in chromosome measurement since it obviates errors of measurements due to differential focussing of three dimensional structures. However, there are a few disadvantages resulting from the disturbances of the natural relationships of the chromosomes. These are more than offset by the merits of the method.

Of course a uniform application of the same technique is not likely to prove suitable for every kind of material, but the general principles are valid, requiring a few modifications depending upon the particular type of material. No single method can be recommended which would prove adequate for all stages of development. The methods that have proved particularly satisfactory and have given the best results are the Feulgen-smear-squash technique of Coleman, L. C., Am. J. Bot., 1940, 27, 887-895; Coleman, L. C., Genetics, 1943, 28, 2-8; Hillary, B.B., Bot. Gaz., 1939, 101, 276-300; Hillary, B.B., Ibid, 1940, 102, 225-235; Heitz, E., Ber. d. biol. Ges., 1936, 53, 870-878; Darlington, C. D., and LaCour, L. F., The Handling of Chromosomes, 1942. New York: The Macmillan Company; and McClintock, B., Stain Tech., 1929, 4, 53-56.

In my experience of making preparations for the study of somatic and meiotic chromosomes, the method given in the schedule here has proven most satisfactory. It is essentially a modification of the technique developed by Dr. Coleman and his associates of the University of Toronto and may be applied to a wide variety of materials, both plant and animal. The tissues are fixed in Carnoy, one of the Navashin modifications or Flemming-Heitz. One of the best fixatives for small mammalian tissues is that given by LaCour, L. F., Proc. Roy. Soc. Edin. B., 1944, $62,73-85$. It is a mixture of methyl alcohol (15 parts), 5\% acetic acid (5 parts), formalin (1 part), and water (5 parts). The proportions may need to be varied according to the material. For a study of the morphology of mouse chromosomes the liver tissue of a newborn mouse is particularly good.

Chromosome structure. The nuclear cycle, whether in plants or animals, is an alternation between two stable statesspiralization and despiralization. The metaphase chromosomes usually represent a state of maximum spiralization and the chromosomes in the resting nucleus, the maximum of despiralization or minimum of spiralization. For demonstration of the spiral structure of the chromosomes and unravelling of the coils, fixation should be preceded by some form of pretreatment. Different methods such as hydration, dessi- 
cation, exposure to acid vapors or subjection to dilute solutions of alkalis, treatment with dilute solutions of salts of strong alkalis and weak acids such as $\mathrm{KCN}, \mathrm{NaCN}$, in fact anything that tends to change the $\mathrm{pH}$, have been employed to bring out the real structure (Nebel, B. R., Zeitschr. Zellf. u. Mikr. Anat., 1932, 16, 251-284; Kuwada, Y. and Nakamura, T., Cytologia, 1934, 5, (2), 244-247; Sax, K., and Humphrey, L. M., Bot. Gaz., 1934, 96, 353-362; Huskins, C. L., and Smith, S. G., Ann. Bot., 1935, 49, 119-150; LaCour, L. F., Stain Tech., 1935, 10, 57-60; Oura, G., Zeit. f. Wiss. Mikr., 1936, 53, 36-37; Kuwada, Y., Shinke, N., and Oura, G., Zeit. f. Wiss. Mikr., 1938, 55, 8-16; Coleman, L. C., and Hillary, B. B., Am. J. Bot., 1941, 28, 464-469; Gopal-Ayengar, A. R., Genetics, 1942; Coleman, L. C., Genetics, 1943, 28, 2-8; Ris, H., Biol. Bull., 1945, 3, 242-257). Some of the best results have been obtained by treatment with $\mathrm{K}$ or $\mathrm{NaCN} 21^{-3}$ to $21^{-5}$ mol. solutions for periods varying with the material.

Stain with aceto-carmine, acetic orcein, acetic lacmoid or Feulgen. If Feulgen is used a counter stain with fast green in acetic acid may be used if desired. The cells are squashed on the slide after staining. The amount of pressure needed is determined by experience. The following schedules of treatment for mouse chromosomes may be applied mutatis mutandis in the study of chromosomes from other tissues.

Fix pieces of liver from a newborn mouse in a mixture of methyl alcoholformalin-acetic acid of LaCour for 15 min. Wash in $70 \%$ alc. Transfer small piece of material on to a slide and add first drop of acetocarmine or acetic orcein and then coverslip. Gently tap with the rubber tipped end of a pencil until the cells are loosened up and are more or less one layer in thickness. Squeeze out gently the excess of stain. Apply pressure on the coverslip with thumb or by carefully rolling a round edged pencil over it, taking care to see that the coverslip does not slide during the process. The amount of pressure needed is judged by experience. If air bubbles get in add a drop or two of the stain at the edge of coverslip and repeat the process if necessary. Seal edge of coverslip with beeswax and vaseline. If it is desired to make slides permanent follow McClintock's method (McClintock, B., Stain Tech., 1929, 4, 53-56).

For Feulgen-squash preparations fix material as in the preceding outline. Wash in water thoroughly. Hydrolyse in $\mathrm{N} . \mathrm{HCl}$ at $60^{\circ} \mathrm{C}$. for $6-8 \mathrm{~min}$. Transfer to Leuco-basic fuchsin for $20 \mathrm{~min}$. to $\frac{1}{2} \mathrm{hr}$. Pour off stain and add $\mathrm{SO}_{2}-$ water and allow it to remain for $3 \mathrm{~min}$. Change 2-3 times. Place a small piece of material in a drop of $45 \%$ acetic acid on a slide and add a coverslip. Gently tap and squeeze out excess of stain as described above. Flatten out the cells by applying pressure with thumb or by rolling a round edged pencil over the coverslip. Transfer slide into large petri dish containing dioxan until coverslip floats off. The cells will adhere either to the coverslip or slide. Use dioxan balsam as mounting medium.

Chlorazol black E + acetocarmine (Nebel, B. R., Stain Techn., 1940, 15, 69-72). Fixation in cold Flemming's fluid plus urea (Hance, R. T., Anat. Rec., 1917, 12, 371-382). Microincineration of (Barigozzi, Cl., Bull. d'Hist. Appl., 1938, 15, 213-219). Method of localization of genes by experimental deletions, distribution of protein and nucleic acid, classification, etc. (Painter, T. S., J. Roy. Micr. Soc., 1940, 60, 161-176). Feulgen stain for chromosomes (Mensinkai, S. W., J. Roy Micr. Soc., 1939, 59, 82-112). Aceticorcein is advocated as a new stain-fixative for chromosomes (LaCour, L., Stain Techn., 1941, 16, 169-174). Demonstration of alkaline phosphatase in chromosomes (Krugelis, E. J., J. Cell. \& Comp. Physiol., 1942, 19, 376-379).

Chromotrope $2 \mathbf{R}$ (CI, 29)-acid phloxine GR, chromotrope blue $2 R$, fast fuchsin $\mathrm{G}, \mathrm{XL}$ carmoisine $6 \mathrm{R}$ - An acid mono-azo dye employed by Lendrum, A. C., J. Path. \& Bact., 1935, 40, 415-416 in a study of breast carcinoma and skin lesions as counterstain for celestin blue.

Chromotrope Blue 2R, see Chromotrope 2R.

Chrysamine $\mathbf{G}(\mathrm{CI}, 410)$ an acid dis-azo dye of light fastness 5 of no value as a tissue stain (Emig, p. 40).

Chrysoidin $Y$ (CI, 20)-brown salt $R$, dark brown salt $\mathrm{R}-\mathrm{A}$ basic mono-azo dye suggested by Conn (p. 46) as a substitute in some techniques for Bismark brown. Used as stain for mitochondria and Golgi apparatus viewed in polarized light (Monne, L., Protoplasma, 1939, 32, 184-192).

Chryosomonadina. Fixation and staining for, Doflein, F. (Arch. f. Protistenk. $1922,44,149)$, also Wenrich, D. H. and Diller, W. F., in McClung's Microscopical Technique, 1950, p. 470.

Chrysophenine (CI, 365), a direct dis-azo dye of light fastness 4 to 5 , for paraffin sections too light and fugitive a color (Emig, p. 39).

Chylomicrons (lipomicrons). These tiny fatty droplets are easily demonstrated by 
dark field examination of blood of a person or animal fed butter or cream. The increase begins about $1 \mathrm{hr}$. there after and reaches a maximum at 4 hrs. after which the number of chylomicrons declines. By contrast a carbohydrate meal of rice and sugar or a protein meal of whites of boiled eggs and salt does not result in an increase. For details see Gage, S. H. and Fish, P. H., Am. J. Anat., 1924-25, 34, 1-86; also, Hadjioloff, A., Bull. d'Hist. Appl. 1938, $15,81-98$.

Ciaccio, methods for lipoids. One of the simplest is: Fix small pieces 2 days in: $5 \%$ potassium bichromate, 80 cc.; formalin, 30 cc., acetic acid, 5 cc. $3 \%$ potassium bichromate for 5-8 days. Running water $24 \mathrm{hrs}$. Ascending alcohols, 24 hrs. Abs. alcohol 2 hrs., xylol, $1 \mathrm{hr} .$, xylol-paraffin at $60^{\circ} \mathrm{C}$, $1 \mathrm{hr}$. Paraffin $1-1 \frac{1}{2}$ hrs. Pass sections down to $70 \%$ alcohol, stain $\frac{1}{2}-1 \mathrm{hr}$. at $30^{\circ} \mathrm{C}$. in: $80 \%$ alcohol, 95 cc., acetone $5 \mathrm{cc}$. saturated at $50^{\circ} \mathrm{C}$. with sudan III then cooled and filtered. Rinse in 50\% alcohol, wash in water, counterstain with hemalum. Mount in syrup of Apathy (or glycerin). Lipoids yellow orange. Lison (p. 206) questions specificity for lipoids and gives in addition, with useful comments, several other methods of Ciaccio.

Cilia-written by Alfred M. Lucas and Miriam S. Lucas, U. S. Regional Poultry Research Laboratory and Biological Science Department, Michigan State College, East Lansing, Michigan. August 10,1951 -Ciliary activity can be studied in isolated cells, in rows of cells, and in broad epithelial surfaces. Cilia and flagella are found throughout most of the animal kingdom except in nematodes and arthropods. They perform many functions, such as locomotion of whole organisms, driving fluids through tubes, and propelling sheets of mucus across epithelial surfaces.

The simplest method of study is to collect isolated cells by gently scraping a ciliated surface and observing the vibrations of the cilia in a suitable fluid on a slide under the microscope. However, numerous special techniques are required to answer problems relating to the shape of cilia during active and passive phases of their cycle, ciliary coordination and how much work they can do. The beats of cilia may occasionally be counted by eye; for example, Lucas, A. M., and Doublas, L. C. Arch. Otolaryng., 1935, 21, 285296) reported 2.2 to 5.5 vibrations per second for cilia on epithelium of the turtle trachea, at about $30^{\circ} \mathrm{C}$. More rapid vibrations require special devices.
Martius (Arch. f. Physiol., 1884, 456460 ) who was the first to use the stroboscope, found 10 to 17 vibrations per second for the cilia of the frog's pharynx and values as high as 32 to 42 vibrations per second are given by Hammond, J. C. (Science, 1935, 82, 68-70) for the membranelle of the protozoan, Epistylis. The use of the stroboscope and moving pictures have been the methods most used, not only to determine the frequency of vibrations but also the succession of shapes taken by the cilia or flagella during the cycle of vibration. Martius emphasized a fundamental caution in using the stroboscope that one should reduce the speed until all multiples of the ciliary frequency have been eliminated. Hammond, on the other hand, recommends using a known harmonic of the frequency. The shapes taken by vibrating cilia and flagella have of ten been subject of study. One of the simplest methods is to reduce the rate of movement by cooling the fluid in which they are beating. Gray, J. (Proc. Roy. Soc., B, 1930, 107, 313-332) and Jennison, M. W., and J. W. M. Bunker (J. Cell. and Comp. Physiol., 1934, 5, 189-197) used both photographic and stroboscopic methods to study the form of the isolated, giant compound abfrontal cilia of the mollusc gill. It is a peculiarity of this cilium to swing usually through an arc of only $90^{\circ}$ instead of $180^{\circ}$ as characteristic of most cilia and thus it may be atypical material for study. Gray noted that in these cilia the active stroke was longer than the recovery stroke. Brown, H. P. (Ohio J.Sci., 1945, 45, 247-301) has given an extensive analysis of types of ciliary and flagellar movement. He tested his theories in various practical ways and one was the construction of a mechanical model which would swim through the water. It was composed of a test tube which contained a strong bent wire holding a rubber band. The rubber band as it unwound rotated a wire passing through a cork stopper. The free end of the wire revolving in the water propelled the tube.

Couch, J. N. (Am. J. Bot., 1941, 218, 704-713) recommends the use of dark field to study movement of individual cilia and flagella. The three common types of dark field, the use of a disc stop below the ordinary condenser, the Zeiss cardioid condenser and even moving the diaphragm laterally to give oblique light worked satisfactorily. With dark field are of ten produced shadow effects of one cilia lying above and across others, which sometimes re- 
semble cross-striations and may be confusing.

Metachromism is shown best by ciliated cells arranged in rows. The most studied examples are the lateral cilia of the molluse gill, especially Mytilus and Modiolus, because in these species the gills are primitive and simple. The location and shape of the cells are shown in Modiolus (Lucas, A. M., J. Morph., 1931, 51, 147-193; and J. Morph., 1932, 53, 265-276). Atkins, D. (Quart. J. Micr. Sci., 1938, 80, 331-344) has made a comparative study of these cells for many families and genera of lamellibranchs. Gray, J. (Proc. Roy. Soc., B. 1930, 107, 313-332) observed that the waves were a succession of spikes and semicircles and with the stroboscope, which caused them to appear to stand still, he measured the relative time consumed by the active and recovery phases of the beat. The ratio was about 1 to 5 . A photographic set-up for obtaining a permanent record of coordination of lateral cilia has been described in some detail by Lucas, A. M. (J. Morph., 1932, 53, 243-263). However by this method stroboscopic effects of the movie camera shutter may give false impressions of the rate of wave propagation.

The most commonly used material for studies on ciliary activity is the frog's pharyngeal membrane, yet it is poorly adapted for practically all the problems for which it has been used as will appear later. Many earlier workers set up recording devices by which records of ciliary activity were recorded on smoked kymograph drums; the cilioscribe, devised by Dixon, W. E., and Inchley, O. (J. Physiol., 1905, $32,395-400$ ) is a good example and still has merit for classroom demonstration. The apparatus consists of a vertical spindle against which is gently pressed the ciliated surface of a strip of frog epithelium. The cilia cause the spindle to rotate and this in turn moves a light weight drum on which an interval timer marks rate of movement. The chief difficulty is the variable amount of mucus which collects on the spindle interferes with its smooth rotation.

Many investigators have used the ciliated epithelium of the frog's pharynx as a delicate indicator to show the response of living tissue to a wide variety of chemical substances, therapeutic compounds, and biological extracts, yet it was not recognized until the work of McDonald, J. R., Leisure, C. E., and Lenneman, E. E. (Am. Acad. Ophthal. and Oto-Laryng., 1928, 318-354) that the cilia of the frog's pharynx are under definite nervous regulation. A few years later Seo, A. (Jap. J. Med. Sc. III Biophysics, 1931, 2, 47-75) established clearly that cilia respond to stimuli through the glossopharyngeal nerves and that the motor fibers are carried along the palatine nerves. Methods which had been used thus far involved placing fluids or particles on the surface but two years later Lucas, A. M. (Arch. Otolaryng., 1933, $18,516-524)$ devised a method whereby ciliary activity could be followed directly under very low powers of the microscope focusing on the high-lights reflected from the surface of the moving ciliary waves. Application of this method the same year to the frog (Lucas, A. M., Proc. Soc. Exp. Biol. and Med., 1933, 30, 501-506) demonstrated conclusively that the cilia in the frog's mouth are normally not active and are completely at rest. They become active when particles, no matter how fine and light they may be, are added to the surface; even the addition of clear saline solutions has the same effect. Thus, the normal baseline for ciliary activity in the frog's pharynx is zero instead of some positive value derived by the use of foreign stimulating agents. Recently Steward, W. C. (Am. J. Physiol., 1948, 152, 1-5) although recognizing these facts, sought to establish some arbitrary baseline values for cilia which had been stimulated by dises and weights placed on the mucous surface. His experiments gave a mean velocity of $0.75 \mathrm{~mm} / \mathrm{sec}$. but he found that even very light stimuli on the membrane, well removed from the route covered by the test object, would increase the velocity 142 to 415 per cent. Lucas, A. M. (Am. J. Physiol., 1935, $112,468-476$ ) designed a moist chamber in which threshold levels of the nervecilia and nerve-muscle preparations could be determined simultaneously. He found that the threshold current which would stimulate the cilia by way of the palatine nerve was 15.4 times stronger than that required to activate the muscles of the toe. The behavior of of excised mucous membrane is quite similar to that found in vivo, the cilia are normally quiescent and become active only when stimulated.

What is needed is a different biological material for studies where chemicals are to be tested in which the cilia beat incessantly, as in the oviduct of birds. This was one of the materials chosen when ciliary studies were just beginning (Purkinje and Valentin, Muller's Arch. Anat. and Physiol., 1834, 1, 391-400). They used macerated black pigments 
of the retina suspended in fluid to follow ciliary movement. The oviduct or trachea of turtles or other reptiles would be useful where it is not convenient to control the temperature of tissues from a warm blooded animal. The ciliature of the avian and reptilian oviducts has been described by Parker, G. H. (Phil. Trans. Roy. Soc. B, 1931, 219, 381-419). Umeda, T. (Acta Dermatologica, 1929, 6, 629-646) used ox trachea which provides large ciliated areas. He employed it to study the effects of temperature, sugar solutions, alkaloids, alcohol, x-ray, and ultraviolet light radiation. The underlying tracheal cartilage was removed with the ciliated epithelium in pieces about $2 \mathrm{x}$ $3 \mathrm{~cm}$. and pinned out beneath a layer of Ringer's solution at $38^{\circ} \mathrm{C}$. It was fixed at a slant of 10 to 15 degrees to facilitate the movements of millet seeds placed on the surface. There is obviously need for someone to recheck the reactivity of the frog's pharyngeal cilia against several other kinds of ciliated epithelium and sort out the effects due to the type of biological material used from the real effects of chemicals or other agents being tested.

In the frog's pharynx and in the tentacle of the snail (Merton, H., Pfluger's Arch. Ges. Phys., 1923, 198, 1-28), nerves are responsible for activation of ciliary movement; whereas, in the Ctenophore swimming plates (Göthlin, G. Fr., J. Exp. Zool., 1920, 31, 403-441) and the velar cilia of the nudibranch veliger (Carter, G. S., Brit. J. Exp. Biol., 1926, 4, 1-26) the nerves are responsible for inhibition of ciliary activity. The procedures used by these authors are well adapted to differentiate between inherent ciliary activity and nerve regulation. In this same category should be included the work of Copeland, M. (Biol. Bull., 1922, 42, 132-142) who studied the rôle played by nerves in regulating the ciliary activity on the foot of the snail.

Polarity and ciliary reversal have been problems of considerable academic interest. Coonfield, B. R. (Biol. Bull., $1936,70,460-471$ ) described his operative techniques for reversing rows of swimming plates in Ctenophores and Twitty, V. C. (J. Exp. Zool., 1928, 50, 319-344) and later Luther, W., (Roux Arch. Entw. Organ. 1934, 131, 532-539) reported the relatively simple methods used in reversing small segments of the ciliated epidermis of the amphibian larva. Induction effects on ciliary polarity have been worked out recently by Twitty, V. C. and Bodenstein, D. ( $\overline{\mathrm{J}}$. Exp. Zool., 1941, 86, 343-379). Reversal of tracheal epithelium in dogs was accomplished by Isayam, S. (Zeit. f. Biol., $1924,82,155-156)$ who found no reversal of direction of ciliary beat. Ciliary reversal is a well known reaction in some ciliated protozoa, such as Paramecium and minimal techniques are required for study of its physiology (Oliphant, J. F., Physiol. Zool., 1912, 15, 443-452). Ciliary reversal in Metazoa is rare indeed. Parker, G. H. (Am. J. Physiol., 1905, 14, 1-6) employed simple techniques to demonstrate its existence in the labial cilia of seaanemones. Equally simple procedures were followed by Matthews, S. (J. Exp. Zool., 1928, 51, 209-262) to demonstrate that the seeming reversal of the ciliature of the pelecypod palp did not actually exist. Atkins, D., (J. Marine Biol. A. United Kingdom, 1930, 16, 919-970) observed permanent, natural reversal in frontal cilia of the gill filaments of Mytilus following injury.

Numerous investigators have presented diagrams of the direction of ciliary movement in a field such as Irving, L. (J. Exp. Zool., 1924, 41, 115-124) who plotted the circulation of fluids and particles within the coelom of the starfish. Another good example is the work of Atkins, D. (Quart. J. Micr. Sci., 1936-1937, 79, 181-308, 339-373, 375-421) who worked out carefully the direction of ciliary movements in a great many molluscs and evolved a phylogenetic tree based, in part, on the lateralfrontal cilia (Quart. J. Micr. Sci., 1938, $80,345-436$ ). Also the study made by Meyer, A. (Biol. Zentralbl., 1936, 56, 532-548) on Nephthys hombergli illustrates a careful approach to this type of problem. Barclay, A. B., Franklin, K. J., and MacBeth, R. G. (J. Physiol., $1937,90,347-348$ ) observed that mucus is moved in a clockwise direction up through the mammalian trachea. Hilding, A. (Arch. Otolaryng., 1932, 15, 92-100) plotted the direction of ciliary movement in the human nose by watching the direction of drainage of the mucous sheet with a speculum after dusting with face powder and observed in the posterior two-thirds of the nose a new mucous layer about every 10 minutes and about once an hour in the anterior third. Lucas, A. M. (Am. J. Anat., 1932, 50, 141-177) and Lucas, A. M. and Donglas, L. C. (Arch. Otolaryng., 1931, 20, 518-5 11) used carbon particles to plot the drainage pattern for monkey, rat, mouse, rabbit, cat, cow, and sheep. The potential error resulting from following mucous flow rather than direct observation of cilia is shown on some wound experiments 
on the frog's palate by Lucas, A. M. (Arch. Otolaryng., 1933, 18, 516-524). Hill, L. (Lancet, 1928, 2, 802-805) had previously shown that cuts across the trachea arranged in alternating fashion completely stopped the flow of mucus and also showed a high sensitivity to ultra violet light. A combination of both direct and indirect techniques gives the most accurate results. The double nature of the mucous layer and the three ways in which ciliary movement and mucous flow could act together or independently were suggested by Lucas, A. M., and Douglas, L. C. (Äch. Otolaryng., 1934, 20, 518-541).

Most ciliary activity can be defined in terms of work. Bowditch, H. P. (Boston Med. and Surg. J., 1876, 15, 159-164), one of the first to study this problem, permitted a sheet of ciliated epithelium from the frog to climb up an inclined plane set at various angles and carrying various loads. He cooncluded that cilia are capable of doing only about one-thirty-fifth the work that the heart does in pumping blood. Parker, G. H. (J. Exp. Zool., 1914, 16, 443-446) placed a tube in the osculum of the sponge and ascertained that the pressure developed in the system by the collar cells was only a few millimeters but that the volume turn-over was large. A similar technique was applied by Hecht, S. (J. Exp. Zool., 1916, 20, 429-434) to the large Bermuda ascidian. A $100 \mathrm{gm}$. animal could circulate about 173 liters of sea water in a day. Hecht gives his technique for getting the glass tube into the excurrent siphon. Galtsoff, P. S. (Bull. U. S. Bur. Fish, 1928 (1930) 44, 1-39) has gone farther than anyone thus far in designing apparatus to measure rate of flow of water due to ciliary action. He used the oyster and collected data on feeding and effects of temperature and seasonal and diurnal cycles. Hilding, A. C. (Trans. Am. Acad. Opth. and Otolaryng., 1944, 367378 ) found that the trachea of the hen was particularly well suited to measure the positive and negative pressures developed at the two ends of the tube. He collected mucus from other hens and used it to form a plug at the lower end of the trachea being tested. A negative pressure of $34 \mathrm{~mm}$. and a positive pressure of $32 \mathrm{~mm}$. of water was developed at the two ends of the tube. The techniques used are simple but effective. The procedures devised by White, H. L. (Am. J. Physiol., 1929, 90, 689-704) to measure the pressure developed by the nephostomal cilia in the Necturus kidney is a good example of how the microscopic methods can be applied. In general, in vivo studies of ciliary movement have been rather few but a nice technique was used by Ernst, A. M. (Arch. Internat. Pharmacodyn. et de Thérap., 1938, 58, 207-212) in which periodic roentograms were made of the cat's thorax to show the rate of elimination of "Neobar" powder (Merck) which had previously been blown into the trachea and bronchial spaces. Normally the trachea was cleared in an hour and from this baseline the effect of various anesthetics could be determined. X-ray radiation in itself has very little effect on ciliary activity as shown by Goldhaber, A. and Black, A. (Proc. Soc. Exp. Biol. and Med., 1941, 48, 150-151) who observed that the oral epithelium of the toad transplanted as a tissue culture required $1,000,000$ to $1,400,000 \mathrm{r}$ to stop ciliary activity. The essential details of their x-ray set-up are given.

Numerous papers mention the presence of cilia lining various parts of the central canal of the nervous system and suggestions have been offered concerning the function they perform but only one author (Chu, H. Y., Am. J. Physiol., $1942,136,223-228$ ) to our knowledge has selected a suitable material (translucent stages of amphibian larva) and thus been able to study this activity in situ. He followed the movements of naturally occurring pigment granules within the ventricles and also injected red cells.

Satisfactory methods for examination of ciliary movement in tubular organs such as the lungs are not always easy. Sollmann, T., and Gilbert, A. J. (J. Pharmacol. and Exp. Therap., 1937, 61, 272-285) filled the excised organ with 10 per cent gelatin in Ringers and then chilled it in iced Ringers. Free-hand sections were pinned on rings of cork and studied in shallow dishes containing Ringer's warmed to $37^{\circ} \mathrm{C}$. Cilia were vigorously active up to about 8 hrs.

A method for testing the phagocytic properties of ciliated epithelium was employed by Ropes, M. W. (Contrib. to Embryol. *128, Carnegie Inst. Wash., 1930, 22, 77-90). He used the tracheal mucosa of the rabbit. Inhaled carbon particles were taken up by the cytoplasm and could be recognized in the living ciliated cells.

The older editions of Lee's Vade Mecum suggest boric acid methods for maceration of ciliated epithelia. This technique has been omitted from some of the more recent editions but is mentioned again in the last edition (11th). The method was revived by Goodrich, 
E. L. (Quart. J. Micr. Sci., 19t2, 83, 245-258) as a new technique with slight modifications. Lucas, A. M. (J. Morph., 1931, 51, 147-193) used sodium borate to which was added a trace of iodine or eosin. The cells swelled as they separated. Poska-Teiss, L. (Zeit. f. wiss. Mikr., 1934, 51, 238-243) used Ranvier's $1 / 3$ alcohol method to isolate the cells. Loginoff, W. J. (Anat. Anz., $1911,38,353-361$ ) who worked with horses, cows and sheep, discovered that preliminary fixation of ciliated cells of the trachea for $30 \mathrm{~min}$. in $1 \%$ formalin followed by $1 / 3$ alcohol for 24 hrs. gave well preserved cells which could be stained under the coverglass with picrocarmine.

In vitro explants of ciliated epithelium show, eventually, dedifferentiation and loss of cilia. More often short term studies on surviving cells have been resorted to. Isolated cells from the frog's palate under tissue culture conditions beat continuously since they are no Ionger regulated by the nervous system and are suitable for testing purposes (Ishikara, S., Acta Dermat., 1927 9, 339-364). Verne, J. (Compt. Rendu Assoc. Anat., 1932, 27, 564-578) using well known tissue culture techniques cul. tivated lung tissue of 15 to 17 day old chick embryos. In this case the ciliated epithelium did not undergo metaplasia but remained active. Proetz, A. W., and Pfingsten, M. (Arch. Otolaryng., 1939, 29, 252-262) combined culture of guinea-pig nasal epithelium with motion picture photography.

Umeda, T. (Acta Dermat., 1930, 1, 13-38) tested some 700 dyes for their vital staining characteristics and found that thionin blue and Nile blue gave satisfactory results with frog ciliated epithelium. Vital methylene blue has frequently been used to stain lining ciliated cells. Carter, G. S. (Brit. J. Exp. Biol., 1926, 4, 1-26) observed that it $w$ as the region of the ciliary rootlets which showed granules staining strongly with this dye and Coonfield, B. R. (Biol. Bull., 1936, 70, 400-471) found the greatest staining in Nephthys where the rootlets converged near the nucleus.

Most of the usual histologic or cytologic techniques will demonstrate cilia. Cilia are stained chiefly with acid dyes but may hold Heidenhain's iron hematoxylin if the tissues have been only lightly destained with iron alum. When staining the very short sensory ciliary hairs on olfactory epithelium, the iron hematoxylin without counter stain or only a light counter stain makes them stand out quite well.

A cuticular border is present on some types of ciliary cells and absent on others. It is refractile to most stains and oftentimes appears as a very narrow clear space between the line marking the surface of the cuticle and the underlying cell membrane. If the overlying mucus is stained with mucicarmine, the outer surface of the cuticle is clearly delimited. Frequently, the lower margin of a nonciliated striated cuticular border looks like a row of basal bodies and Heidenhain's iron hematoxylin gives the best differentiation. Likewise it is the stain usually chosen to show flagellate diplosomes and stages in ciliogenesis.

Ciliary rootlets are as difficult to demonstrate in the stained as in the living condition. In order to see them clearly it is important to select the right kind of cell-one which has a clear protoplasm containing a minimum of pigmented opaque or refractile granules. Even living unstained ciliated cells of the mammalian nose when isolated on a slide show clearly the rootlets in the narrow clear zone of protoplasm just beneath the cell membrane and in relatively transparent cells they can be observed converging toward the nucleus as an inverted truncated cone. In the latero-frontal ciliated cells of the molluse gill the cilia form two rows transversely across the surface of the cells so that the rootlets lie in rows one behind the other and under these conditions they show up very easily. Grave, C., and Schmitt, F. O. (J. Morph. and Physiol., 1925, 40, 479-515) recommended the Ehrlich-Biondi triple mixture as a counterstain to Heidenhain's iron hematoxylin and this works very well although sometimes more than one attempt is necessary to obtain a satisfactory mixture of Ehrlich-Biondi stain.

Considerable latitude is permitted in the use of fixatives. Allen's $\mathrm{B}_{3}$ and $\mathrm{B}_{15}$ are good fixatives although the alveolar appearance that picric-containing fixatives give to the appearance of the cytoplasm may be a disturbing factor. Dellinger, O. P. (J. Morph., 1909, 20, 171209) made an informative comparative study of the fixatives and the basic constituents which go into them. He found that $\mathrm{HgCl}_{2}$ emphasized fibrillar structures and enhanced the staining and so fixatives such as Zenker's, with formalin or acetic acid, are of ten used for preservation of ciliated tissues. These are vigorous fixatives and may result in some cell distortion. Dellinger recommended 0.4 to $2 \%$ osmic acid as best for cilia. Thanhoffer, I. von. (Zeit. Anat. Entwickl., 1929, 90, 713-724) employed 
Heidenhain's "Susa mixture" in his study of ciliogenesis. Mihalik, P. von. (Anat. Anz., 1935, 79, 259-268) preferred the "Susa mixture" for the study of intracellular ciliated cysts in the rabbit oviduct. Meves, Fr. (Arch. mikr. Anat., 1897, 50, 110-114) used Hermann's osmic mixture for the study of sperm flagella of the salamander. Kindred, J. E. (J. Morph. and Physiol., 1927, 43, 267-297) recommended Meves' fluid as giving the most constant results in his investigation of eiliogenesis in the pharynx of the frog. Flemming's fluid also gave good results but he did not find $2 \%$ osmic acid satisfactory. Application of Da Fano's modification of Cajal's silver method by Cowdry, E. V. (Anat. Rec., 1921, 22, 289-299) revealed flagella present on the thyroid follicles of the dogfish. The blackened flagellum showed up nicely against a yellowish background. The collars and flagella of sponge choanocytes are difficult to preserve for microscopic study. Robbertson, M. and Minchin, E. A. (Quart. J. Micr. Sci., 1910, N.S. 55, 611-640) found Hermann's fluid satisfactory and stained with Heidenhain's iron hematoxylin and light green.

Cilia and flagella of protozoa can be demonstrated in fresh preparations by first introducing strands of some $\mathrm{ma}$ terial such as cotton fibers or frayed lens paper beneath the cover glass which will limit activity of the organisms. Maier, H. N. (Arch. f. Protist., 1903, $2,73-179$ ) drew $1 \%$ osmic acid and subsequently $5 \%$ soda solution beneath the cover glass to study cilia and this method is still used. Noland, L. E. (Science, 1928, 67, 535) used a gentian violet stain $(20 \mathrm{mg}$. gentian violet to 1 cc. aq. dest; plus 80 cc. sat. aq. phenol; $20 \mathrm{cc}$. of $40 \%$ formaldehycle; 4 cc. glycerin) which he mixed with a drop of ciliates or flagellates in culture. Donaldson, R. (Lancet, 1917, 2, 571-573) demonstrated protozoa in feces by using 0.1 to $0.5 \%$ aq. of eosin, or with iodine solutions of various strengths, or with a mixture of the eosin and dilute iodine. These solutions are also good for demonstrating cilia and flagella.

Fixation of flagella by cupro-picroformol acetic mixture and staining by eosin and phosphomolybdic acid according to A. C. Hollande's (Arch. de Zool. exper. et gen., 1920, 59, Notes et Revue, 75-77) method is satisfactory. This technique is described in detail by Wenrich, D. H. (McClung's Micr. Technique, 1937, Hoeber, p. 547).

Relief staining demonstrates ciliary rows and other surface markings on protozoa: B. M. Klien's silver method
(Zool. Anz., 1926, 67, 160-162) opal blue, china blue, and other stains dried on the protozoa (Bresslau, E., Arch. f. Protist., 1921, 43, 467-480) and nigrosin (Coles, A. C., Watson's Micr. Rec., 1927, 23-25).

Owen, H. M. (Trans. Amer. Mier. Soc., 1947, 66, 50-58) maintains that the "brush" or "flimmer" effect produced on flagella of bacteria by Loeffler's stain is an artifact due to interaction of heavy metals and mordant. He recommends a fixative which does not produce the "brush" on flagella (10 parts, $2 \%$ osmic acid to 1 part $20 \%$ formalin).

Ciliogenesis can be observed occurring in a number of protozoa. Lucas, M. S. (Arch. f. Protist., 1932, 77, 407423) used vom Roth's platinic chloride fixative followed by Heidenhain's iron hematoxylin stain on smears of Cyathodinium from the guinea pig intestine, demonstrated formation of new basal bodies and cilia within cysts which later evert upon the protozoan's surface.

When cilia or flagella are to be studied under the electron microscope, it is necessary, of course, that they be dried under high vacuum. This may introduce distortion. A critique on the advantages, limitations, and possible artifacts of electron microscope techniques has been given by Williams, R. C. (Growth Symposium, 1947, 11, 205-222). Baylor, M. R. B., Nalbandov, A., and Clark, G. G. (Proc. Soc. Exp. Biol. and Med., 1943, 54, 229-232) studied the sperm head and axial filament. They observed that fresh sperm, dried and dehydrated showed a fraying out of the axial filament into its component fibrils whereas stained sperm did not and they conclude that the stain is sufficiently adhesive to prevent the separation. Brown, H. P. (Olio J. Sci., 1945, 45, 247-301), in his study of flagella, gives his technique for preparation of his material in great detail, including the results from his tests on numerous fixatives and stains suitable for use with the electron microscope. Jakus, M. A. and Hall, C. E. (Biol. Bull., 1946, 91, 141144) studied the trichocyst and cilia of Paramecium and used the shadow-casting technique.

Microdissection has been used occasionally to study the mechanisms of ciliary movement and coordination. The techniques of microdissection are well known from various books and articles. It was used by v. Rényi, G. (Zeit. f. Anat. u. Entwick, 1926, 81, 692-709) to determine if cilia, separated from their basal bodies, were contractile. Carter, G. S. (Proc. Roy. Soc. $\mathrm{B}, 96,115-122$ ) made use of this tech- 
nique to demonstrate that the laterofrontal cilia of Mytilus are compound and that the separate components can beat independently of the others. Peterfi, T., and Woerderman, M. W. (Biol. Zentralbl., 1924, 44, 264-268), Grave, C. and Schmitt, F. O. (J. Morph. and Physiol., 1925, 40, 479-515), Lucas, A. M. (J. Morph. 1931, 51, 147-193) and Worley, L. G. (J. Cell. and Comp. Physiol., 1941, 18, 187-197) employed this method to gain information concerning ciliary coordination.

Cinephotomicrograply. A convenient illustrated account of apparatus and methods is given by Tuttle, H. B., in Glasser's Medical Physics, 183-194. See Motion Pictures.

Cinnamon Oil (Cassia oil) resembles clove oil and is particularly recommended by Lee (p. 70) for clearing. Two kinds are given in Merck Index. The U.S.P.XI variety contains $80-90 \%$ cinnamaldehyde.

Citrate of sodium can be used as an anticoagulant in the proportion of $18 \mathrm{cc}$. of $2 \%$ aq. sodium citrate to $100 \mathrm{cc}$. of blood.

Clarite X (Neville Co., Pittsburg) $60 \%$ in toluol is suggested as substitute for balsam owing to its neutral reaction, lack of yellow color and quickness of hardening. Clarite, also called Nevillite $V$, is useful if added to paraffin when one wishes to obtain thin sections when it is not convenient to imbed in a very high melting point paraffin. Wehrle, W., Stain Techn., 1942, 17, 131-132 advises imbedding in a mixture of $90 \%$ paraffin (m.p. $53^{\circ} \mathrm{C}$.) , $5 \%$ bleached beeswax and $5 \%$ clarite and the elimination of elcctrical charge when ribbons are cut by a spark-coil device described by Blandau, R. J., Stain Techn., 1938, 13, 139-141.

Clark and Lubs Buffers (Clark, W. M. The Determination of Hydrogen Ions, Baltimore: Williams \& Wilkins, 1928, 717 pp.). Prepare: (1) A solution containing $M / 5$ boric acid and $M / 5$ potassium chloride made by dissolving 12.368 gms. of $\mathrm{H}_{3} \mathrm{BO}_{3}$ and $14.912 \mathrm{gms}$. of $\mathrm{KCl}$ in aq. dest., and diluting to 1 liter. (2) A $\mathrm{M} / 5$ sodium hydroxide (carbonate free) solution by dissolving 50 gms. of $\mathrm{NaOH}$ in $50 \mathrm{ml}$. (cc.). aq. dest. in a Pyrex flask. Let stand overnight to allow the sodium carbonate to settle, or filter through a Gooch or sintered glass crucible. (Exclude air to prevent formation of more carbonate by atmospheric $\mathrm{CO}_{2}$.) Keep the strong alkaline solution in a paraffin-lined glass bottle. Dilute with aq. dest. which has been boiled to remove the excess $\mathrm{CO}_{2}$ so that the solution is about $1 \mathrm{~N}$. Then make an approximately $\mathrm{M} / 5$ solution of the alkali which can be accurately standardized against potassium acid phthalate.

To make buffer of the desired $\mathrm{pH}$ add to $50 \mathrm{cc}$. of (1) $\mathrm{M} / 5 \mathrm{H}_{3} \mathrm{BO}_{3}-\mathrm{KCl}$ the desig nated amount of (2) $\mathrm{M} / 5 \mathrm{NaOH}$ and dilute to $200 \mathrm{cc}$. with aq. dest. Or combine the two in similar proportions but in larger amounts to minimize error in measurement.

$\begin{array}{rc}\text { pH } & \text { co. of } \mathrm{M} / 5 \mathrm{NaOH} \\ 7.8 & 2.61 \\ 8.0 & 3.97 \\ 8.2 & 5.90 \\ 8.4 & 8.50 \\ 8.6 & 12.00 \\ 8.8 & 16.30 \\ 9.0 & 21.30 \\ 9.2 & 26.70 \\ 9.4 & 32.00 \\ .6 & 36.85 \\ .8 & 40.80 \\ 10.0 & 43.90\end{array}$

Cleaning Glassware. Pulverize $20 \mathrm{gms}$. potassium bichromate. Dissolve this in 200 cc. aq. dest. with aid of a little heat. Add slowly 20 cc. sulphuric acid C.P. Before treating beakers, graduates, bottles, etc. with this acid cleaning solution first wash them in soap and hot water. Rinse in water to remove the soap. Leave in cleaning solution $2 \mathrm{hrs}$. or more. Rinse in running tap water and dry with opening downward on drying racks as in biochemical laboratories if possible in a dust free cupboard. For new slides and cover glasses wash in the same way and after final rinsing in tap water store in frcsh $95 \%$ alcohol in covered dishes until they are required for use when they should be wiped with gauze. For old slides and cover glasses soak in xylol to permit separation and removal of most of balsam. Then leave in waste alcohol several days. Soak for a day or more in strong soap solution. Wash in running water. Clean in cleaning solution. Wash in water and store in $95 \%$ alcohol. Unless strict economy is necessary it is hardly worthwhile to use slides and covers twice especially when the former have been marked with diamond pencils.

Clearing is a process in microscopic technique which is required in three different situations.

1. As the step following dehydration in paraffin imbedding. The tissue becomes translucent but this is not the essential feature of the process. What is neccssary is for the alcohol, which is not a paraffin solvent, to be removed by the clearing agent before the tissue is infiltrated with paraffin. Consequently the agents must mix freely with alcohol on the one hand and with paraffin on the 
other. Of them xylol is by far the most widely used and rightly so. Two changes of half absolute alcohol and xylol within $1 \mathrm{hr}$. and 2 changes of xylol within the next 3-4 hrs. are usually sufficient for slices of tissue 4-6 mm. thick, but the time should not be extended beyond that needed to attain translucency because so doing causes a hardening and a shrinkage of the tissue.

Several other substances can be used in place of xylol. Cedar wood oil is according to Lee (p. 80) the very best clearing agent for paraffin imbedding. It penetrates rapidly, does not make the tissues brittle, and, when not entirely displaced by paraffin, does not seriously interfere with sectioning. First treat the tissue with $\frac{1}{2}$ absolute and xylol for about $2 \mathrm{hrs}$. The time required in the oil of cedarwood is however a little longer than in the case of xylol used alone, say 12 hrs. Some recommend 2 changes of xylol (about $30 \mathrm{~min}$.) after the oil of cedarwood before entering $\frac{1}{2}$ paraffin and cedarwood oil.

Methyl benzoate is now quite popular. Pass the tissue from absolute alcohol through 2 changes of pure methyl benzoate within 12-24 hrs. When it has been definitely cleared remove benzoate by 2 changes of benzol ( $\frac{1}{2}-1 \mathrm{hr}$.) before passing in to paraffin, or half benzol and paraffin.

Chloroform penetrates poorly and should not be employed unless called for. It has the further disadvantage that unless completely removed in the paraffin bath, it will make the final paraffin block soft and unfit for cutting. The usual practice is to clear very small pieces for about $12 \mathrm{hrs}$. in 2 changes, or as long as may be necessary to make them transparent, and in the imbedding to use 4 changes of paraffin.

A more rapid method is to pass directly from the fixative, Bouin or formalin, without washing, to 3 changes of pure dioxan within 4 hrs. and thence into 3 changes of paraffin as advised by Graupner, H. and Weissberger, A., Zool. Anz., 1931, 96, 204-206. Stowell, R. E., Stain Techn., 1941, 16, 67-83 confirms and extends earlier work of Seki which shows that, although xylol shrinks tissues more than dioxan, in placing in hot paraffin, the final shrinkage is greater in tissues after dioxan. When great haste is necessary Mallory (p. 54) suggests acetone $\frac{1}{2}-2$ hrs. ; benzol, 15-30 min.; and paraffin 3 changes, 30-90 min. The shrinkage, however, is very marked and it would probably be better to use Frozen Sections.

By the Altmann-Gersh technique, which is at once very time consuming and very valuable for special purposes, fixation, al coholic dehydration and clearing can be side stepped and the dried tissue directly impregnated with paraffin.

2. As the step following dehydration of sections before mounting. The clearing is of course easier and much quicker owing to the thinness of the tissue. Again xylol comes first and will probably not be displaced though some prefer toluol. It is not necessary to protect against shrinkage and brittleness. When desired, abs. alc. can be omitted and the clearing be done from 90 or even $80 \%$ alc. with terpineol, clove oil, anilin oil, beechwood creosote, Bergamot or some other substance.

3. As a means of rendering clearly visible certain structures in embryos or whole tissues. Clearing is generally done by the Spalteholz method. See Cartilaginous Skeleton and Ossification centers. When glycerin mixtures are employed as Mounting Media they also clear the tissues. See Groat, R. A., Stain Techn., 1941, 16, 111-117 for clearing tissues with mixtures of tributyl and tri-o-cresyl phosphates.

Cloth Red B (Cl 262). A sulfonated azo dye. For formula and influence on mouse tumors, see Stern, K., Cancer Res., 1950, 10, 565-570.

Cloudy Swelling. This is a marked swelling and granulation of the cytoplasm of cells. It is sometimes observed postmortem in acute febrile conditions especially in the kidneys, liver and myocardium. An almost meaningless synonym, often used, is Parenchymatous Degeneration. The extent of cloudy swelling that may occur in vivo and from which the cells may recover is not known. The fatty droplets present can be demonstrated in sudan stained frozen sections of formalin fixed material. Special stains for Fibrin, Myofibrils and Mitochondria may be desirable.

Coacervates ( $I_{\text {}}$. acervus, a cloud or swarm) are masses of particles clumped together (but encased in a little water) by a change in their electrical charge while in colloidal suspension in water or by dehydration with resultant loss of loosely bound water. Hirsch (G. C., Form und Stoffwechsel der Golgi-Körper. Protoplasma Monographs, Berlin, 1939) has likened the Golgi apparatus to a coacervate. See Bensley, R. R., Anat. Rec., 1937, 69, 341-353 for critical consideration of suggestion that mitochondria are coacervates.

Coagulation. A phenomenon frequently encountered in the case of blood and lymph is of wide occurrence and is in- 
fluenced by many factors. Small quantities of many electrolytes cause coagulation of colloids. Some ions are much more powerful in this respect than others and certain "protective colloids" such as gelatin protect colloidal suspensions against coagulating action of electrolytes. Peptization is disintegration of the coagulum into colloidal particles. Water is employed as a peptizing agent in dissolving glue, agar, and similar materials (Holmes, H. N., Glasser's Medical Physics, 257263).

Cobalt Nitrate Silver for Golgi Apparatus. Cocarboxylase, see Method of Westenbrink, H. G. K., Enzymologia, 1940, 8, 97-107, capable of determining as little as $0.01 \mu \mathrm{gm}$. of carboxylase.

Coccidia. These sporozoa include many parasites of great importance not only to physicians and veterinarians but also to cytologists who are interested in their intracellular behavior. Consequently the volume by Becker, E. R., Coccidia and Coccidiosis of domesticated, game and laboratory animals and of man. Ames: Collegiate Press, Inc., $1934,147 \mathrm{pp}$. will contain numerous helpful leads on the coccidis of the digestive tracts of vertebrates and invertebrates. See also McClung, 1950, p. 455 .

Coccinel Red is 1,5-diamylaminoanthraquinone, an oil soluble dye, recommended by Lillie, R. D., Stain Techn., $1945, \mathbf{2 0}, \mathbf{7 3}-\mathbf{7 5}$ as a stain for fat which it colors deep orange red. Make up stock solution of $4.2 \%$ in absolute $(99 \%)$ isopropanol. Dilute this down to 30 or $40 \%$ isopropanol with water and treat frozen sections of normal cat kidney and human adrenal with resulting solution for 10-20 min. This solution is only usable for several hours. Coccinel red is a good counterstain after hematoxylin.

Coccinine (CI, 120), an acid monoazo dye, light fastness 3 to 4 , which colors sections pale pink not equal to Biebrich Scarlet (Emig, p. 31).

Cochineal (CI, 1239). This crimson dye was in use by the Aztecs before the Spanish conquest. It is derived from an insect which feeds on a cactus. So highly prized was it that Montezuma took as yearly tribute from the State of Huaxyacas (Now Oaxaca) 20 sacks of cochineal. The invading Spaniards were not slow to note the superiority of cochineal over Kermes, the crimson stain in use at home (1523 A.D.). Charles V of Spain commanded Cortez to inform him immediately "whether what has been reported is true that Kermes were to be found in abundance in New Spain and, if so, could be sent with advantage to Spain". So cochineal figured largely in the Aztec tributes to Cortez and the industry became a Spanish monopoly. In 1858 A.D. aniline red became a competitor, depressed the sales of cochineal, which, latter as a commercial lye, was definitely replaced when azo dyes came into the market about A.D. 1880 . (Leggett, W. F., Ancient and Medieval Dyes. Brooklyn: Chemical Publishing Co. Inc., 1944, 95 pp.). See Kermes, Lac.

In microscopy cochineal is used mostly for staining in toto of small invertebrates. Mayer's alcoholic cochineal is a popular preparation made, according to Lee (p. 149), by powdering $5 \mathrm{gm}$. cochineal with $5 \mathrm{gm}$. calcium chloride and $5 \mathrm{gm}$. aluminum chloride to which 100 cc. $50 \%$ alcohol and 8 drops of nitric acid (sp. gr. 1.20) are added. Heat to boiling point, cool, shake occasionally during several days and filter. Before staining bring objects to $70 \%$ alcohol, destain if necessary in $70 \%$ alcohol containing $0.1 \%$ hydrochloric acid. Dehydrate, clear and mount in balsam. Nuclei are colored red and other structures a variety of colors from red to deep purple. In some respects it is better than carmine. Neither fade.

Cochlea, see Ear.

Coelestin Blue, see Skyblue.

Coellne, see Skyblue.

Coeruleum, see Skyblue.

Colchiceine, different from colchicine, see Ludford, R. J., Arch. f. exper. Zellf., $1935-36,18,411-441$.

Colchicine, see Mitosis Counts.

Collagenase. An enzyme which specifically destroys collagenous fibers. Bidwell, E. and W. E. van Heyningen, Biochem. J., 1948, 42, 140-151, report on its isolation and purification.

Collagenic Fibers-Written by A. I. Lansing, Dept. of Anatomy, Washington University, St. Louis 10, Mo. October 5,1951 - These are widely distributed in the body, occur in large bundles in tendons and are best viewed in spreads of loose connective tissue. Unlike elastic fibers which are yellow and highly refractile, collagenic fibers are white and poorly refractile, form broad wavy bands which do not branch, have great tensile strength, and are attacked by dilute acids and alkalis. On boiling they yield gelatin. The fact that they pass from the gel to the sol state on alkalinization or warming is the basis for methods of separating epidermis from dermis. Collagenic fibers are readily digested by pepsin in acid solution but resist trypsin. A specific collagenase has as yet not been found. 
Amino acid analyses (see Elastic Fibers) indicate an absence of aromatic amino acids, an abundance of proline, hydroxylysine and particularly hydroxyproline, the latter being almost a diagnostic feature. The isolectric point of collagen is about $\mathrm{pH} 7.0$.

Although tinctorial reactions would imply that reticulum and collagenic fibers differ, recent studies indicate that reticulum is but a finer unit of collagen. Both have a marked affinity for aniline blue. They differ in that reticulum is strongly argyrophilic while collagenic fibers are not; the former is weakly acidophilic (in $\mathrm{H} \& \mathrm{E}$ ) while the latter takes up much eosin.

Electron microscopy and x-ray diffraction studies have done much to reveal the submicroscopic structure of collagenic fibrils (Gross, V., J. Gerontol., $1950,5,343)$. These are apparently composed of parallel polypeptide chains approximately $10 \mathrm{~A}^{\circ}$ in diameter which are bonded together laterally. Electron microscopy reveals considerable fine structure in fibrils. There is a regular periodicity of $640 \mathrm{~A}^{\circ}$ with much intra-period detail.

A protein termed procollagen may be prepared by extraction with citrate at $\mathrm{pH} 4$. Such procollagen can form fibrils with regular periodicity by the addition of monovalent salts or mucoprotein (Highberger, J. H., J. Gross and F. O. Schmitt, Proc. Nat. Acad. Sci., 1951, 37,286 ).

The best stain for collagenic fibers in sections after Zenker fixation is aniline blue in Mallory's Connective Tissue Stain and in Masson's Trichrome Stain. Phosphomolybdic Acid Hematoxylin also gives a fine coloration of collagenic fibers. See Van Gieson, Buzaglo, Biebrich Scarlet and Picro-Anilin Blue of Lillie and Curtis' Substitute for Van Gieson.

Lillie, R. D. (J. Tech. Methods, 1945, No. 25,45 pp.) has performed a very useful service in testing the effectiveness of a large series of dyes as collagenic fiber stains in the Van Gieson, Mixed Masson-Van Gieson and MassonMallory procedures. He found the best to be Naphthol blue-black (Cl, 246), Fast Green FCF, Acid Fuchsin (Cl, 692), Methyl Blue (Cl, 706), Anilin Blue (Cl, 707), Wool Green S (Cl, 737) and Volamine $\mathrm{R}(\mathrm{Cl}, 758)$. For photometric histochemical determination see Stowell, R. E., J. Invest. Derm., 1945, 6, 183-189.

The technique of microincineration as adapted to collagenic fibers is described by Allara, E., Bull. d'Hist. Appl., 1938, 15, 220-242. See Tendons.
Collargol, as negative stain for spirochetes (Harrison, Brit. Med. J., 1912, 2, 1547).

Collodions. There are several. See U.S.P. XI. All are solutions of Pyroxylin.

Colloxylin, see Pyroxylin.

Colophonium, usually dissolved in turpentine is employed to mount sections. Not advised.

Color Estimation. Accuracy in reporting differential stains and micro-chemical reactions yielding colors is highly desirable. The same holds for colors determined by naked eye inspection. A monograph, Ridgway, R., Color Standards and Color Nomenclature, Washington, D. C., 1912 with 53 colored plates, is the accepted standard for comparison. In general, however, it is desirable to achieve some measure of uniformity by limiting oneself whenever possible to use of the terms recommended in the National Formulary VII. Washington: American Pharmaceutical Association, 1942, 690 pp., a publication which is available in most medical libraries:

\begin{tabular}{|c|c|c|}
\hline pink & yellow & greenish blue \\
\hline red & olive-brown & blue \\
\hline reddish orange & greenish yellow & purplish blue \\
\hline reddish brown & olive & bluish purple \\
\hline orange-pink & yellow-green & purple \\
\hline orange & olive-green & reddish purple \\
\hline brown & yellowish green & purplish pink \\
\hline yellowish orange & green & red-purple \\
\hline yellowish brown & $\begin{array}{l}\text { bluish green } \\
\text { blue-green }\end{array}$ & purplish red \\
\hline
\end{tabular}

For accurate measurement of color employ Photoelectric Colorimeter or Photoelectric Microphotometer. See Hemoglobin Estimation.

Color Index, p. xxvii.

Color Preservation in museum specimens. Fix $24 \mathrm{hrs}$. or less in $10 \%$ formalin. Wash in running water 3-6 hrs. Stand in 2\% aq. ammonia 5-10 min. which hastens return of original colors. Running water another hour. Mount for permanent exhibition in mixture made as follows: Filter a sat. sol. antimony trioxide in aq. dest. (about $5 \mathrm{gm}$. per liter). To each $1000 \mathrm{cc}$. filtrate add 100 gm. potassium acetate, $100 \mathrm{gm}$. chloral hydrate and $50 \mathrm{cc}$. glycerin. Stir until completely dissolved (Meiller, F. H., J. Tech. Methods, 1938, 18, 57-58).

Mallory (p. 380) recommends for this purpose the methods of Kaiserling and Jores.

There are 3 Kaiserling solutions :

1. For fixation: Formalin, 40 cc.; tap water, $2000 \mathrm{cc}$; potassium nitrate, 30 $\mathrm{gm}$. and potassium acetate, $60 \mathrm{gm}$. Small specimens require 1-14 days. Large ones can be more uniformly fixed by vascular Perfusion. Sometimes it is advisable to inject fixative into central 
parts of the tissue with a hypodermic syringe and long needle. Do not use too much pressure and be careful not to let any of the fixative spurt back into one's face. Before the next step wash in running water for about 12 hrs.

2. For color restoration: Place the tissue in $80 \%$ ethyl alcohol for $10-60$ min. and watch for optimum coloration. If left too long in the alcohol the colors fade. Rinse in water and transfer to No. 3.

3. For final prescruation: Change to glycerin, 500 cc.; $1 \%$ aq. arsenious acid, 200 cc.; tap water, 2300 cc.; potassium acetate, 250 gms.; thymol, $2.5 \mathrm{gm}$. To obviate difficulty of dissolving the arsenious acid and to sterilize add 25 gms. arsenic trioxide to $2500 \mathrm{cc}$. water + the thymol crystals first ground up in a mortar and place in steam sterilizer for $6 \mathrm{hrs}$. Then add other substances.

There are 2 Jores solutions.

1. For fixation: Chloral hydrate, 50 gms.; artificial Carlsbad salts (sodium sulfate, $22 \mathrm{gm}$.; sodium bicarbonate, 20 gm.; sodium chloride, $18 \mathrm{gm}$.; potassium nitrate, $38 \mathrm{gm}$; potassium sulphate, 2 gm.), 50 gm.; formalin, 100 ce.; tap water, $1000 \mathrm{cc}$. Allow to act $2-14$ days depending on size, wash $12 \mathrm{hrs}$. in running water.

2. For final preservation: Potassium acetate, $300 \mathrm{gm}$., glycerin, $600 \mathrm{cc}$; aq. dest., $1000 \mathrm{cc}$.

Mallory suggests fixation in Jores' first solution and preservation in Kaiserling's third solution.

Colors, Interference, see Interference Colors.

Columbium, see Atomic Weights.

Concentration. 1. Tubercle bacilli in sputum. Nagy (A.H., J. Lab. \& Clin. Med., 1939, 25, 67-71) having critically evaluated several techniques recommends Pottenger's Dilution-Flotation method. Shake equal parts sputum and $0.5 \%$ aq. sodium hydroxide for $10 \mathrm{~min}$. Digest in water bath at $56^{\circ} \mathrm{C}$. for 30 min. Add $1 \mathrm{ml}$. (= $1 \mathrm{cc}$.) hydrocarbon (gasoline or xylene), then $200 \mathrm{ml}$. aq. dest. and shake $10 \mathrm{~min}$. Allow hydrocarbon to collect at top 15-20 min. Take up hydrocarbon layer in rubber bulbed pipette. Keep in vertical position until supernatant fluid separates from hydrocarbon, 5-10 min. Make smears from hydrocarbon and dry. Remove hydrocarbon by washing with ether. Stain with carbol fuchsin $3 \mathrm{hrs}$. or longer. Decolorize with acid alcohol 30 sec. or less. If further decolorization is required employ $10 \%$ aq. sodium sulphite. Counterstain with $1 \%$ aq. picric acid or with methylene blue. The concentration of bacilli is about 33 times. Perhaps a modification of the method could be used for leprosy organisms in emulsions of tissues. See also Pottenger, J. E., Am. Rec. Tuberc., $1939,40,581$. Concentration of tubercle bacilli in spinal fluids (Hanks, J. H and Feldman, H. A., J. Lab. \& Clin. Med., 1939, 25, 886-892). It is often necessary to concentrate for microscopic study objects which are not present in abundance and which might otherwise be overlooked. See examination of Feces for ova of parasites, of Urine for sediment.

2. Leprosy bacilli for chemical analysis. Ravold's method for leprosy bacilli can perhaps be used for others. Relatively large masses of bacilli-laden cells are dissected away from neighboring uninvolved tissue and from necrotic tissue when present in the centers of the nodules. They are placed in a Wueller press without addition of any fluid. On exertion of pressure many of the cells are ruptured and the tissue fluid, together with cytoplasm, nucleoplasm and some entire cells, passes through minute holes in the press and is collected, leaving most of the fibrous elements behind. Then a little saline solution is added and the material is ground up in sand and made up to a volume of about $50 \mathrm{cc}$. The sand is allowed to sediment out at the bottom of a centrifuge tube. The supernatant fluid is then centrifuged at low speed (300 r.p.m.). This throws all the rest of the debris to the bottom while the bacilli remain in suspension. The supernatant fluid, containing the bacilli, is again decanted and centrifuged at high speed (3500 r.p.m.) in an angle centrifuge for $1 \mathrm{hr}$. The supernatant fluid is discarded and the pasty material at the bottom of the tube, made up of bacilli, is diluted and washed by repeated centrifugation in some expcriments with saline solution and in others with distilled water.

Beginning with a large nodule or with several small ones it is a simple matter to collect in 4 or 5 hrs. billions of bacilli. The pasty bacterial mass can be desiccated and weighed in grams. For our experiments we used only the wet bacilli. When viewed en masse they appear dense white with a faint shade of gray. They are not yellow or even yellowish. Examination of a thick smear, made after washing in saline, shows myriads of bacilli without any trace of cellular material. The bacilli retain to a remarkable degree their characteristic morphology, as scen in sections and in smears of fresh tissue, and their acid-fast properties are not 
interfered with. After washing in distilled water until the supernatant fluid gave no precipitate when added to an aqueous solution of silver nitrate, the bacilli do not fuse together but still remain discrete bodies though their shape is different. (Cowdry, E. V., Ravold, A. and Packer, D. M. Proc. Soc. Exp. Biol. \& Med., 1939, 41, 341$345)$. See Floatation Techniques fоr intestinal parusites.

Condenser Manometers in differential pressure measurements (Hansen, A. T. and Warburg, E., Acta Physiol. Scand., $1951,22,211-215$ ).

Congo Blue 3B, see Trypan Blue.

Congo Corinth G or GW, see Erie Garnet B.

Congo Red (CI, 370). Synonyms: Congo, cotton red, A, B or C, direct red C, R or $\mathrm{Y}$. An acid dis-azo dye which is an excellent indicator and a useful stain. Matsuura, S., Fol. Anat. Jap., 1925, 3, 107-110 has obtained very fine coloration of the skin which he has illustrated in colors. Congo red is the basis of Krajian's stain for elastic fibers. See also Blackman, V. H., New Phytol., 1905, 4, 173-174 (uredineae); Merton, H., Arch. Protistenk., 1932, 76, 171-187; Cumley, R. W., Stain Techn., 1935, 10 , 53-56 (negative stain for bacteria), etc.

Connective System. Provides both for the binding together of parts and for their separation one from another by capsules, membranes and other structures (see Cowdry, p. 429-466). It ranges all the way from Loose Connective Tissue and Fatty Tissue through Fibrous Connective Tissue and Tendons to Cartilage and Bone. Neuroglia is a special form of it. In general there are three components, Fibroblasts, Fibers and Tissue Fluid (ground substance). Cells of hematogenous and lymphatic origin may be present since blood vessels and lymphatics run in connective tissue pathways. See techniques under these headings, also Masson's Trichrome Stain, Mallory's Connective Tissue Stain, Phosphomolybdic Acid Hematoxylin, Van Gieson, Buzaglo, MalloryHeidenhain rapid one step stain, etc.

Connective Tissue Cells, preservation of trypan blue and neutral red in those of loose connective tissue. Inject subcutaneously 5 cc. fresh sterile $1 \%$ aq. vital trypan blue (Coleman and Bell Co.) into a mature white rat weighing about $90 \mathrm{gms}$. and wait $48 \mathrm{hrs}$. Make up $0.02 \%$ certified neutral red (National Aniline in $0.9 \% \mathrm{NaCl}$ ). After slight etherization exsanguinate the animal. Inject neutral red into subcutaneous tissue of groin in several places near original puncture. After 3-5 min. re- move small blobs of edematous tissue. Tease these out on clean slides with aid of needles and filter paper. When corners are dry spread is ready for direct observation under cover glass or for fixation. Make up $10 \%$ formalin. Test it by addition of a drop or two of neutral red. If this turns orange add a little $\mathrm{N} / 10 \mathrm{HCl}$ until it becomes red. Fix in this formalin over night or for several days. Rinse in aq. dest. Counterstain in $1 \%$ fast green F.C.F. (Warner-Jenkinson Co.) in $2 \%$ aq. acetic acid for $\frac{1}{2}-1$ min., pass through successive changes dioxan, 3-5 min. each. Agitate slightly. Mount in dioxan employing medium hardened diaphane (Will Corp.), redissolve in dioxan or pass through xylol and mount in balsam. Avoid alcohols. Note blue granules in macrophages and fine red granules in mast cells (Snook, T., Stain Techn., $1939,14,139-142)$. See Connective System.

Contraction Bands, or waves, demonstration of in smooth muscle. Remove intestine of freshly killed cat, expose to air of room or rub with blunt end of scalpel. When preparations are made numerous contraction bands will be seen. Contrast with this intestine of cat killed with chloroform and not excised until rigor mortis begins in which muscle fibers are fully extended (Dahlgren, McClung, p. 430$)$.

Coons, see Antigen Localization.

Copper. 1. Microchemical tests. Fix in formalin or alcohol, use same hematoxylin or methylene blue stain as for Lead. With former copper hemofuscin is blue and hemosiderin (iron pigment) is black, while with latter copper pigment is pale blue and the iron pigment uncolored (Mallory, F. B. and Parker, F., Jr., Am. J. Path., 1939, 15, 517-522). See also Okamoto, K., Utamura, M. and Mikami, G., Acta Sch. Med., Univ. Imp. in Kioto., 1939, 22, 335-360 (illustrated in colors); Mendel, L. B. and Bradley, H. C., Am. J. Physiol., 1905, 14, 313-327 (bromine test for); Claude, A., Cold Spring Harbor Symposia on Quantitative Biology, 1941, 9, 263-270 (copper of respiratory pigment); Hoagland, C. L. et al., J. Exper. Med., 1942, 76, 163-173 (copper and other substances in virus elementary bodies). Waterhouse, D. F., Bull. Council for Scientific and Industrial Research, Melbourne, Australia, 1945, No. 191, 20 pp.: add 1 drop $0.1 \%$ aq. sodium diethyl dithiocarbamate to fresh tissue followed by 1 drop conc. hydrochloric acid which yield yellow product with copper. Iron interferes with this result but the sensitivity for copper is much greater than 
for iron (quoted from Glick p. 23). When search is necessary for traces of copper without need for microscopic localization an emission speetrograph may give the information qucikly, see Histospectrography. If quantitative determinations of copper in small amounts of tissue are required use the polarographic technique elaborated by Carruthers, C., Indust. and Engin. Chem., 1945, 17, 398-399. Details for determination of copper in epidermis are given by Carruthers, C. and Suntzeff, V., J. Biol. Chem., 1945, 159, 647651.

2. As vital stain. Intravenous injections of colloidal solutions of copper in rabbits are described by Duhamel, B. G., C. rend. Soc. de Biol., 1919, 82, $724-726$.

Copper Chrome Hematoxylin (Bensley's) for mitochondria. Fix very small pieces in Altmann's Fluid or in Acetic-OsmicBichromate fixative of Bensley 12-24 hrs. Wash, dehydrate, clear, imbed in paraffin and cut sections at 4 or $5 \mathrm{mi}$ crons. Deparaffinize. Sat. aq. copper acetate, $5 \mathrm{~min}$. Several changes aq. dest., 1 min. $0.5 \%$ aq. hematoxylin, 1 min. After rinsing in aq. dest. pass to $5 \%$ aq. neutral potassium chromate, 1 min. which should turn sections dark blue-black. If they are only light blue, rinse in aq. dest. again place in copper acetate and repeat if necessary several times until no increase in color is obtained. Wash in aq. dest. and treat for few sec. with copper acetate. Wash in aq. dest. and differentiate under the microscope in Weigert's borax-ferricyanide mixture (borax, $1 \mathrm{gm}$.; potassium ferricyanide, $1.25 \mathrm{gm}$; aq. dest. 100 cc.) diluted with twice the volume aq. dest. Wash in tap water, 6-8 hrs. Dehydrate, clear and mount in balsam. The mitochondria appear a beautiful deep blue against a yellowish background. It is important to have good, ripe hematoxylin. It is usually made by dilution from a $10 \% \mathrm{sol}$. in abs. alcohol. This method of staining should be tried after fixation in Regaud's fluid.

Coproporphyrin of megaloblasts in embryos, see Porphyrins. Coproporphrin III can be isolated from human tubercle bacilli by chromatographic and fluorescence analysis (Crowe, M.O'L., and Walker, A., Brit. J. Exp. Path., 1951, 32, 1-6).

Coreine 2R, see Celestin Blue B.

Corinth Brown G, see Erie Garnet B.

Coriphosphine $\mathrm{O}(\mathrm{Cl} .787)$. An acridine dye used as a fluorehrome (Netealf, R. L. and Patton, R. L., Stain Techn., 1944, $19,11-27)$.

Corn Blue B, see Victoria Blue $\mathbf{R}$.

Corn Blue BN, see Victoria Blue B.
Cornea. This is a difficult tissue to prepare in stained sections beeause of its curvature and avaseularity. A valuable silver method is minutely described by Pullinger, B. D., J. Path. and Bact., 1943, 55, 97-99.

Fix anterior and posterior surfaces in $10 \% \mathrm{aq}$. neutral formalin before excision of eye, if possible, by flooding anterior surface with fixative and by injecting fixative into anterior chamber through a hypodermic needle at the same time withdrawing fluid from the chamber likewise by hypodermic. Remove eye, inject fixative into vitreous at same time removing fluid from it. Leave in fixative 24 hrs. Exeise cornea along corneo-scleral margin, detach iris, ciliary body and lens. Fix latter separately and cornea for further 3 days, 4 altogether. Indieate location future seetions by niek in opposite edge.

Transfer cornea to aq. dest. avoiding metal instruments then and thereafter. Wash and leave over night in aq. dest. +3 drops ammonia (S. G. 0.88) per, say, 50 cc. After washing in 2 or 3 changes aq. dest., and pouring off last aq. dest. filter onto cornea through cotton wool moistened with aq. dest. $20 \mathrm{ce}$. del RioHortega's solution. To make this add 300 ee. $5 \%$ aq. sodium bicarbonate to 100 cc. $10 \%$ aq. silver nitrate in brown glass stoppered bottle. Add few drops ammonia waiting each time for smell of ammonia to disappear until almost but not all ppt. is dissolved. They add 250 ce. aq. dest.

Place container with cornea plus filtered solution in incubator at $37^{\circ} \mathrm{C}$. 4 hrs. Pour off solution and wash cornea in several ehanges aq. dest. Then reduce by pouring onto cornea $10 \%$ aq. neutral formalin, $15 \mathrm{~min}$. Cut away "dome" of cornea with knife and support its coneave surface with the lens, freeze and seetion at about $15 \mu$. parallel to surface. Take seetions into aq. dest.; mount at once in glycerin jelly or pass through alcohols to absolute, elear in creosote and mount in balsam. Collagen pale yellow, nuelei and cytoplasm well shown and especially Descemets menbrane.

Cornyebacterium Diphtheriae. Evaluation of methods for staining metachromatic granules (Morton, H. E., Stain Techn., $1942,17,27-29)$. See Gobar, M. A., J. Bact., 1944, 47, 575, also Diphtheria Bacilli.

Coronary Arteries. Their distribution may be demonstrated by injection of the easily recognizable fluids listed under Blood Vessels. Owing however to their great importance it is well to mention two special adaptations of the said fluids. 
Gross (L., The Blood Supply of the Heart in its Anatomical and Clinical Aspects. New York: IIoeber, 1921) employed injections of barium sulphate suspensions in gelatin followed by $\mathrm{x}$-ray photographs; while Spalteholz (W., Die Arterien der Herzwand, etc., Leipsig: Herzel, 1924) used injections of cinnabar and other pigments likewise in gelatin which were later cleared by his method. Ehrich, Chapelle and Cohn (W., C., and A. E., Am. J. Anat., 1931, $49,241-282$ ) found the latter technique preferable. Celloidin injections also give good results. Histological demonstration of the blood supply of the coronaries is described under Vasa Vasorum.

Corpora Amylacea, see Prostate.

Corpuscles, see those of Pacini, Meissner, Krause, etc.

Corrosion Preparations. In them the structures to be demonstrated are left while all the surrounding tissue is corroded and washed away, for instance Celloidin and Neoprene injections.

Corrosive Sublimate, see Mercuric Chloride.

Corti, organ of, see Ear.

Cortin (interrenalin), hormone of adrenal cortex.

Cotton Blue, see Anilin Blue, Methyl BIue. See Fungi.

Cotton Corinth G, see Erie Garnet B.

Cotton Red, see Safranin O.

Cotton Red, A, B, or C, see Congo Red.

Cotton Red 4B, see Benzopurpurin $4 \mathrm{~B}$.

Covell, see Ear.

Cover Glasses, see Cleaning.

Creatine and Creatinone, see colorimetric methods of Borsook, H., J. Biol. Chem., 1935, 110, 481-493 and of Sure, B. and Wilder, V. M., J. Lab. Clin. Med., $1941,26,874-878$.

Cresol Red. See Hydrogen Ion Indicators.

Creosote (Beechwood) is an important clearing agent for celloidin sections. It is a mixture of phenols, mainly guaiacol and creosol.

Cresyl Blue $2 R N$, or BBS, see Brilliant Cresyl Blue.

Cresyl Violet-cresylecht violet (cresyl fast violet)-Commission Certified. A basic oxazin dye. A technique for its use (or that of toluidin blue) in studies on the cytoarchitectonics of the nervous system is proposed by Landau, E., Bull. d'Hist. Appl., 1934, 11, 44-46. As a stain for nerve cells in celloidin sections (Tress, G., and M., Stain Tech., $1935,10,105-106)$. Wash low viscosity nitrocellulose (celloidm) sections of $10 \%$ formalin fixed tissues in aq. dest. Stain for $30 \mathrm{~min}$. at $50^{\circ} \mathrm{C}$. in cresyl violet, $0.5 \mathrm{gm}$.; aq. dest., 100 cc.; glacial acetic acid, 4 drops (filtered before using).
Wash in aq. dest. Differentiate in $70 \%$ alcohol until most of stain leaves celloidin. Completely immerse for $2-5$ min. in: chloroform, 60 cc.; abs. alc., $10 \mathrm{cc}$; and ether, 10 cc. Almost no destaining of cells occurs but stain is removed from background. Differentiate in 100 cc. $95 \%$ alc. +4 drops $1 \%$ aq. hydrochloric acid but stop while cells are a little darker than desired. Neutralize sections in $90 \%$ alcohol + a little sodium bicarbonate. Wash in $95 \%$ alcohol to remove the bicarbonate. Complete dehydration in 2 changes $n$ butyl alcohol. Clear in 4 changes xylol and mount. See Kallichrom. Note: There are two different dyes sold as cresyl violet: (1) The C.C. product (Nat. Anilin, Mfgrs.; see Conn, 1940, p. 93) which is good in biopsy work; (2) The Grübler product (also sold by Coleman and Bell, but not on the market during the war) which is needed in neurological work, c.f. Tress, above.

Cresylecht Violet, intensification of metachromatic properties (Williams, B. G. R., J. Lab. \& Clin. Med., 1934-35, 20, 1185-1187). A new domestic cresylecht violet has been made available by Coleman and Bell and is strongly recommended by Banny, T. M. and Clar, G., Stain Techn., 1950, 25, 195-196.

Cretin's Test, see Calcium.

Crime Detection Techniques. These are of course legion. Many of them are microscopic and involve identification of materials. See for example, Hair, Semen Stains and Hemoglobin. In respect to the latter the object is to determine whether blood is human by pre. cipitin tests and to which group it belongs by detection of agglutinins as is well described by Schiff, $F$. and Boyd, W. C., Blood Grouping Technic. New York: Interscience Publishers, Inc., $1942,248 \mathrm{pp}$. The identification of metals, such as chips from a razor blade, by spectroscopic examination is often conclusive, see Histospectrography. Cracks in metal surfaces can be detected with astonishing delicacy by the Magnaflux. An interesting elementary account of Crime Detection Techniques is provided by Hoover, J. E., Scientific Monthly, 1945, 60, 18-24.

Croceine Scarlet, see Biebrich Scarlet, water soluble.

Crossman's modification of Mallory's connective tissue stain (Crossman, G., Anat. Rec., 1937, 69, 33-38). Deparaffinize sections of Zenker fixed material. Lugol's iodine, $5 \mathrm{~min}$. Rinse in $70 \%$ alcohol, several changes. Wash $10 \mathrm{~min}$. in running water. Overstain nuclei in Mayer's acid Hemalum or Weigert's Iron Hematoxylin. Wash in running 
water $10 \mathrm{~min}$. Stain for $1 \mathrm{~min}$. or more in: acid fuchsin (C.C.), $1 \mathrm{gm}$.; orange G (C.C.), 0.4 gm.; aq. dest., 300 cc.; thymol, $0.2 \mathrm{gm}$.; glacial acetic acid, 3 cc. Rinse in aq. dest. Decolorize in fresh $1 \%$ aq. phosphotingstic or phosphomolybdic acid until arterial media is red and adventitia is colorless. Rinse very quickly in aq. dest. Counterstain in $2 \%$ aq. anilin blue, W.S. (C.C.) 100 cc. + glacial acetic acid, 2 cc. or in $1 \%$ aq. light green SF yellowish (C.C.) $100 \mathrm{cc}$ + glacial acetic acid, $1 \mathrm{cc}$. Rinse in aq. dest. Decolorize in $1 \%$ acetic acid under microscope. Rinse in aq. dest. Dehydrate in 3 changes abs. alc. Clear in 3 changes xylol and mount. Like original method but nuclei brown or black and collagen blue or green depending on counterstain.

Cryostat. Written by Dr. Gordon H. Scott, Dept. of Anatomy, Wayne University, School of Medicine, Detroit, Mich.This apparatus is one which is designed to dehydrate tissues at low temperatures. A detailed description has been given by Packer and Scott (J. Tech. Methods, 1942, 22, 85-96) and by Hoerr and Scott (Medical Physics, Otto Glasser, 1944, Year Book Publishers). Tissues frozen in liquid air or nitrogen are placed in a chamber which is connected with a fast pumping vacuum system. Water vapor which is released $f$ rom the tissues is trapped by $\mathrm{P}_{2} \mathrm{O}_{5}$ as well as by a cold trap. In the Packer-Scott apparatus the relative amount of water vapor in the system is determined by its flow past a spaced pair of ionization gauges between which is placed the $\mathrm{P}_{2} \mathrm{O}_{5}$ trap. When these gauges are in balance it is assumed water vapor is no longer being released in quantity, and therefore the tissues are dry. As soon as the tissues, placed on a container of solid out-gassed paraffin, are dry, the paraffin is melted and the tissues are infiltrated. This latter procedure is accomplished without the necessity of breaking the vacuum. This small but important step provides tissues which have been frozen-dried and prepared for cutting without their having been partially rehydrated by exposure to air at ambient pressure and temperature. See Altmann-Gersh frozen dehydration method.

Cryptococcus Hominis, see Blastomycosis.

Cryptosporidium, see Coccidia.

Crystal Violet as vital stain for fibroblast nuclei (Bank, O. and Kleinzeller, A., Arch. exp. Zellforsch., 1938, 21, 394399). See Anilin Crystal Violet and Gentian Violet.

Crystal Violet-Acid Fuchsin. This is one of R. R. Bensley's neutral stains espe- cially advocated for the demonstration of secretion antecedents in gland cells. The technique is described by the Bensleys (p. 97). To make stain add filtered sat. aq. acid fuchsin to similar solution crystal violet until precipitation is complete. Collect ppt. on filter paper, wash through once with aq. dest. Dry and dissolve in absolute alcohol to saturation. For staining add 5 cc. of above stock solution to 45 cc. $20 \%$ alcohol made from absolute. In this color paraffin sections of FormalinZenker fixed material for $5 \mathrm{~min}$. Blot with filter paper in one hand and add with other hand absolute alcohol from a pipette, flood with absolute. Blot immediately. Add few drops clove oil. When differentiation, observed under microscope, is optimum transfer to pure benzol and mount in balsam.

Crystal Violet and Alizarin, see Benda's Method for Mitochondria.

Crystals. These are encountered in many forms, see Charcot-Leyden, Ice, Sulfonamides, Hemin, Florence, Virchow's, Spermin, Lubarsch, Neumann's, Teichmann's, Mineral residue of Microincineration, Polarization Optical methods. Numerous Microchemical Reactions especially for minerals, yield crystalline materials. Fat crystals are often distinctive as for beef, duck, lard, etc. (Schneider, A. The Microbiology and Microanalysis of Foods. Philadelphia: P. Blakiston's Son \& Co., 1920, 262 pp.). Study of crystals is really a problem for experts. For the best techniques consult Section I on "Identification" in Bunn, C. W., Chemical Crystallography, Oxford University Press, 1946, $234 \mathrm{pp}$. Comparison of the crystals to be diagnosed with some of the 234 figures in the book may result in prompt recognition.

Culture Media, see Bacteria, Leishmania, Protozoa, Tissue Culture, Trypanosomes, NNN Medium.

Curcumine, see Brilliant Yellow.

Curettings, gelatin method for rapid frozen sections of (Meeker, I. H., J. Techn. Meth. \& Bull. Int. Assoc. Med. Museums, 1936, 16, 41-42).

Curtis' Substitute for Van Gieson stain as modified by Leach, E. H., Stain Teclnn., 1946, 21, 107-110. Use any desired fixative. Bring sections to water and treat with iodine and hypo (sodium thiosulfate) if necessary. Stain for 5$10 \mathrm{~min}$. in Weigert's hematoxylin. To make this mix (just before use) 1 part $1 \%$ hematoxylin in absolute alcohol with 1 part of mixture containing $30 \%$ aq. ferric chloride $4 \mathrm{cc}$., conc. hydrochloric acid, 1 cc. $2 \%$ acetic acid 100 cc. and add 2 parts aq. dest. Wash for $5 \mathrm{~min}$. in 
running water. Stain 2-4 min. in Curtis' substitute: $2 \%$ Ponceau S, CI, 282, (National Aniline) 5 cc.; sat. aq. picric acid, 95 cc.; $2 \%$ acetic acid, 2 cc. Rinse in $96 \%$ alcohol, dehydrate, clear and mount. Chromatin, black; cytoplasm, yellow; collagen and reticular fibers, red. Red and yellow colors are said to be purer than those given by the Van Gieson technique and too heavy staining with red is less likely. In original account volumes are given in $\mathrm{ml}$. which are of practically the same value as cc.

Cyanochin of Bresslau, E., Arch. f. Protisterik., 1921, 43, 467, see Cyanosine.

Cyanosine, see Phloxine B.

Cyclohexanone has been recommended for dehydration and clearing instead of absolute alcohol and xylol by Bourdon, P., Bull. d'Hist. Appl., 1942, 19, 55. After dehydrating tissue in $95 \%$ alcohol, 12 hrs.; pass to cyclohexanone, 4 hrs.; then to another lot of cyclohexanone, 2 hrs.; and impregnate with paraffin 2 baths 2 hrs. or less each. For pieces more than $3 \mathrm{~mm}$. thick longer times are necessary. This saturated cyclic ketone has density similar to water, mixes with organic solvents and paraffin and does not harden tissue. From Review by Jean E. Conn in Stain Techn.

Cyclospora, see Coccidia.

Cytocentrum, centrosome plus centrosphere.

Cytochrome. This is the name given by Keilin (D., Proc. Roy. Soc., 1925, B, $98,312-339$ ) to hemin compounds of a reddish color which occur in oxidized or reduced condition in almost all living cells. Blaschko and Jacobson (Bourne, p. 192) have summarized our knowledge about them. They say that the red color of cytochrome can be observed when a slice of brain tissue, from which the blood has been carefully washed out, is suitably illuminated by transmitted light. A thick suspension of yeast and the thoracic muscles of insects are also recommended as material. There are 4 cytochromes: $a, b, c$ and $a_{3}$ recognizable spectroscopically.

Cytochrome Oxidase. Cytochrome is oxidized by cytochrome-oxidase which is identical with indophenol oxidase and Warburg's respiratory enzyme. See study of cytochrome oxidase-cytochrome system in kidney (Flexner, L. B., J. Biol. Chem., 1939, 131, 703-711). See Oxidase.

Cytolipochrome Pigment, see Lillie, p. 127.

Cytophaga Group of organisms, enrichment cultures, pure culture techniques, methods of examination and identification (Stanier, 1R. Y., Bact. Rev., 1942, 6, 143-197).
Cytoplasmic Inclusions caused by viruses. They are more diversified in size, shape and chemical composition than the Nuclear Inclusions. Frequently, as in the case of large Negri Bodies, they contain both acidophilic and basophilic components (Trachoma Bodies). Gly cogen tests for Trachoma inclusion bodies are described by Thygeson, P., Am. J. Path., 1938, 14, 455-462. The techniques mentioned for Nuclear Inclusions may be employed. See description by Goodpasture, E. W. and Woodruff, A. M., Am. J. Path., 1930, $6,699-711 ; 713-720$ of the reactions of fowl-pox inclusions to potassium hydroxide and other chemicals and the nature of the particles. See also Borrel, Guarnieri and Kurloff bodies. Rickettsia are not to be listed as cytoplasmic inclusions but Giemsa's stain is the best for them.

In plant cells, as in animal ones, certain cytoplasmic inclusions are indicative of virus action. They are of two sorts: (1) X bodies, which are rather amorphorus structures, and (2) crystalline inclusions. The latter are best seen in the dark field and in polarized light and are made up chiefly of virus. For technique employed to demonstrate the relationship of virus to inclusion and a critical review of the whole problem of plant viruses, see Bawden, F. C. Plant Virus Diseases, Waltham: Chronica Botanica Co., 1943, 294 pp.

Cytosiderin Pigment, Lillie, p. 127.

Dahlia, see Hofmann's Violet.

Dahlia B, see Methyl Violet.

Damar is gum damar dissolved in xylol and used to mount sections.

D'Antoni's Iodine solution. First make standardized $10 \%$ aq. potassium iodide. Adjust by specific gravity method to exact $10 \%$ concentration. To $100 \mathrm{cc}$. of $1 \%$ aq. potassium iodide made from it add $1.5 \mathrm{gm}$. powdered iodine crystals. Allow to stand for 4 days before using. Recommended for staining intestinal protozoa (D'Antoni, J. S., Am. J. Trop. Med., 1937, 17, 79-84). See McClung, 1950 , p. 450 .

Dark Brown Salt R, see Chrysoidin Y.

Dark-Field Microscope (From account in Cowdry's Histology, 1950). In darkfield microscopy the light comes in at an angle, and does not enter the objective lens at all. It may be thought of as oblique illumination carried to the limit of obliquity. If there is nothing in the field of view the background will of course be perfectly dark; but the presence of objects will result in the reflection of some light laterally into the objective lens. Since only a minute fraction of the illumieation beam of 
light will go toward the formation of an image this system will require a very strong light source as well as a darkadapted eye for observation. If a special dark-field condenser is not available, one can be easily improvised by adding a central opaque stop just below the regular condenser in the following manner:

Select an objective of not over $0.66 \mathrm{~N}$. A. and a condenser of at least $1.25 \mathrm{~N}$. A. and set up the illumination as for bright field, with the slide oiled to the top of the condenser. Stop down the iris diaphragm until it just encircles the bright central disk which is seen through the tube with ocular removed. Now cut from black paper or thin metal a disk of diameter equal to the diameter of this opening in the diaphragm and cement it to a piece of glass that fits the insertion slot under the condenser. This central stop should block all light from entering the objective if it is properly centered below the iris opening. Next open the diaphragm completely, thus permitting a hollow cone of light to focus on the specimen but not to enter the objective. If the microscope is already supplied with a dark-field stop it should be tested in this manner.

The great advantage of dark-field microscopy is that it enables one to perceive the presence of particles far below the theoretical limit of resolution. Even though a particle may be much smaller than the wave length of light it does scatter incident light laterally into the objective and is imaged as a rather fuzzy point of light. We have here an exact analogy of the situation whereby we can "see" dust particles dancing in a strong beam of light when it enters a darkened room. Most of these particles are of a size far below the limit of visual resolution. Another advantage is that small, transparent objects, like chylomicrons, can be seen in dark-field whereas they are invisible in the glare of bright-field illumination.

For the examination of ordinary histological sections little is to be gained by dark-field observation. Nevertheless Ralph, P.H. (Stain Tech., 1942, 17, 7-10) recommends dark-field examination in the study of stained blood smears. It is most helpful however in the search for very small, scattered bodies in a more or less empty medium, such as chylomicrons or spirochetes in blood plasma. Dark field examination is the standard technique for the study of microincineration preparations. The cytological results of 20 years of of microincineration have been summa- rized by Policard, A. (J. Roy. Micr. Soc., 1942, 42, 25-35).

The source of the light must be very, very strong. To obtain the best results do not rely on improvised, or home made, equipment. A special condenser is needed. Probably the highest objective that can be usefully employed is a $3 \mathrm{~mm}$. oil immersion objective with iris diaphragm. See Microincineration, Spirochetes, Chylomicrons, etc.

Davenport's 2-hour method for staining nerve fibers in paraffin sections with protargol. 1946 modification written by Dr. H. A. Davenport of original (Davenport, H. A., McArthur, J., and Bruesch, S. R., Stain Techn., 1939, 14, 21-26). Fix for 1 to 3 days in: Formamide (Eastman Kodak Co.), 10 cc.; paranitrophenol, $5 \mathrm{gm}$.; $95 \%$ ethyl alcohol, 45 cc.; aq. dest., 45 cc. Transfer thru graded alcohols to absolute, then either n-butyl alcohol or xylene and embed in paraffin. Sections are cut and mounted in the usual manner, paraffin removed and the slides run thru graded alcohols to dist. water. Impregnate for $1 \mathrm{hr}$. at $58-62^{\circ} \mathrm{C}$. in a $5 \%$ aq. silver nitrate. Rinse in 3 changes of aq. dest. with 20-30 sec. allowed for each change. The rinse water should cover the slides completely, each slide separate (not back to back) and the water discarded with each change to prevent carry-over of silver nitrate into the protargol. Place the slides in $0.2 \%$ protargol (Winthrop Chemical Co.) for 1 hour at room temperature. Rinse quickly ( 2 sec.) in aq. dest. and reduce for 1 to $2 \mathrm{~min}$. in the following mixture: Sodium sulfite, $5 \mathrm{gm}$.; Kodalk (Eastman K. Co.), 0.5 gm.; hydroquinone, $1 \mathrm{gm}$.; aq. dest., $100 \mathrm{cc}$. Wash in running tap water several minutes and rinse once in dist. water. Tone in $0.1 \%$ aq. gold chloride for 5 to $10 \mathrm{~min}$. Wash again for about $1 \mathrm{~min}$. and reduce in $1 \%$ aq. oxalic acid for $10-20$ sec. Rinse and place in hypo (10\% aq. sodium thiosulfate) for about 1 min. Wash in running water, dehydrate and cover.

Notes: If the stain is too dark, try any or all of the following modifications: rinse longer after the protargol, use $0.1 \%$ protargol, omit the oxalic acid reduction after gold toning. If too pale: double the concentration of the protargol, double the time of either or both silver impregnations, omit rinsing after protargol, double the concentration of kodalk in the reducer, lengthen the time of reduction in oxalic acid. The technic is suitable for mammalian central or peripheral nervous tissue, but for sympathetic fibers in intestine and uterus a moderate degree of success has 
been obtained with material fixed in Bouin's picric-formalin-acetic (aq.). Use clean glassware and fresh solutions!

Dead Bacteria. To distinguish from living try :

1. Proca-Kayser stain (Gay, F. P. and Clark, A. R., J. Bact., 1934, 27, 175189). Fix bacterial smear by drying and flaming. Stain 3-5 min. in Loeffler's alkaline methylene blue. Wash quickly and stain in Ziehl-Neelsen's carbol fuchsin only 5-10 sec. Wash and dry. Living bacteria blue, dead ones purple to red.

2. Neutral red (Knaysi, G., J. Bact., $1935,30,193-206$ ). Add a little neutral red to the medium. Escherichia coli and Schizosaccharomyces pombi are considered dead when tinged even slightly by the stain.

3. Decolorization (Prudhomme, R. O., Ann. Institut Pasteur, 1938, 61, 512518). Living bacilli separated from all tissue decolorize solutions of 1-naph thol2-sodium sulphonate-indo-2-6-dibromphenol, O-cresol-2-6 dichlorophenol and O-chlorophenol-indo-2-6-dichlorophenol. Bacilli killed by $100^{\circ} \mathrm{C}$. for 15 min. do not decolorize them.

The value of these methods is questionable.

Dead Cells. Often it is very difficult to say whether a particular cell was dead or alive when the preparation was made. The appearance of nuclei in Postmortem Degeneration may be a clue. Evans and Schulemann (H. M. and W. Science, 1914, 39, 443-454) remarked upon the extraordinary rapidity with which dead cells take in vital benzidine dyes and the diffuse, uniform coloration that ensues.

In cells supravitally stained with neutral red Lewis and McCoy (W. H. and C. C., Johns Hopkins Hosp. Bull., 1922, 33, 284-293) employed the following criteria for death: "(1) loss of color from the granules and vacuoles; (2) diffuse pink staining of the cytoplasm and nucleus; (3) the appearance of a sharp and distinct nuclear membrane and a change in texture of the cytoplasm and nucleus." Using dark-field illumination W. H. Lewis (Anat. Rec., 1923, 26, 15-29) observed the appearance in dying cells of certain very small brightly shining (white) bodies which he called $d$ or "death granules." These were first in Brownian movement which soon ceased. To quote Lewis: "During the period when the cells were dying, spherical blebs often appeared on both the flat and rounded cells. These were pale grayish sacs with very thin walls and fluid contents in which varying numbers of small white granules in ac- tive Brownian motion were seen. The blebs varied in size and were occasionally as large as a contracted cell. Sometimes the blebs were so crowded with granules that they were milky in appearance. Frequently one would burst, freeing its granular contents into the surrounding fluid medium where they showed Brownian motion until they settled down on the slide."

Luyet's (B., Science, 1937, 85, 106) method for the differential staining of living and dead plant cells may prove of value for animal cells also. He has written the following account: A piece of the lower epidermis of the scale of the onion bulb is peeled off and placed, cutin side down, on a slide. A drop of a .5 per cent, slightly alkaline, aqueous solution of neutral red is deposited on the piece of epidermis and left there for 2 minutes; then it is blotted off and replaced by a drop of a .4 per cent potassium hydroxide solution, which is immediately removed (also with a blotter); then the preparation is washed with tap water. The living cells take with that treatment a bright cerise red color, while the dead cells are of an intense orange yellow. The contrasts are violent. There are intermediate tints which correspond to the dying cells. See Necrosis, Necrobiosis, Survival of tissue.

Decalcification. The removal of calcium so that bony tissues can be cut in sections. There are many methods almost all of which involve acid treatment. It is generally better to apply the decalcifying agent after fixation, particularily so when the agent is a poor fixative. The volume of decalcifier should be about 100 times that of the tissue. The usual, crude, way of testing the progress of decalcification is to stick a fine needle into the bone being careful to avoid the area that will be cut in sections; but less objectionable methods can be used, see Teeth, Decalcification.

Saturated aq. sulphurous, $5 \%$ trichlorlactic, $5 \%$ hydrochloric and equal parts of $1 \%$ hydrochloric and $1 \%$ chromic acids are all fairly good decalcifiers. Lactic, acetic, phosphoric and picric acids are usually unsatisfactory. Shipley (McClung, p. 347) recommends slow decalcification by long immersion in Muller's Fluid through liberation of small amounts of chromic acid from the bichromate. The bones of an adult rat require 21-30 days. The process can be hurried somewhat by using an incubator at $37^{\circ} \mathrm{C}$. Adequate decalcification is detected by slight bending of the bone or 
by the needle method. Over decalcification is not likely.

For rapid decalcification he advises using sat. aq. phloroglucin to which 5-30\% Nitric Acid is added. A somewhat slower formula is: nitric acid, 5 cc.; phloroglucin, 70 cc.; $95 \%$ alc., 1 cc.; and aq. dest., 30 cc. The phloroglucin allows use of stronger acids. $1-2 \%$ aq. hydrochloric acid decalcifies quickly but it causes the tissue to swell. Formic Acid 1-5\% in $70 \%$ alcohol is, according to Shipley, the best decalcifying agent for large masses of bone. With $5 \%$, the decalcification is completed in $4-5$ days. Use $70 \%$ alc. not water, to wash out the acid.

Kramer and Shipley devised a Magne. sium Citrate method of decalcification in neutral solutions. To make the decalcifier dissolve $80 \mathrm{gms}$. citric acid in $100 \mathrm{cc}$. hot aq. dest., add 4 gms. magnesium oxide and stir until completely dissolved. If the magnesium oxide contains carbonate it should be freshly ignited. Cool and add $100 \mathrm{cc}$. ammonium hydroxide (density 0.90 ) and aq. dest. to make $300 \mathrm{cc}$. Allow to stand $24 \mathrm{hrs}$. and filter. Titrate filtrate with hydrochloric acid to about $\mathrm{pH} 7.0$ 7.6 and add equal volume aq. dest. In decalcifying, this reagent should be changed every 3 days. A dog's rib is decalcified in approximately 15 days.

After decalcification, by whatever method, the bone, or the area of calcification, must be thoroughly washed to remove the decalcifer and imbedded in paraffin or celloidin. Some investigators prefer the latter but celloidin sections are not so easily handled. See Bones, Teeth.

The ion exchange resin technique for bone is new and interesting (Dotti, L. B., Paparo, G. P. and Clarke, B. E., Am. J. Clin. Path., 1951, 21, 475-479). Try keeping tissue in cork stoppered bottle containing $10 \mathrm{gms}$. of the resin (Win-3000 supplied by WinthropStearns, Inc. 1450 Broadway, New York 18 ) in 80 ec $10-20 \%$ formic acid for $1-4$ days. The optimum time should be determined for the particular kind of bone and the size of piece. The authors supply helpful figures illustrating the dependence of intranuclear details on time of decalcification.

Degeneration. Because the structural organization of various sorts of cells is, like their function, so very different the types of degeneration leading to death are also different at least in many of their aspects. See Nerve Fiber Degeneration, Cloudy Swelling, Necrosis, Caseation, Parenchymatous Degeneration, Postmortem Changes.
Dehydration is the removal of water from a tissue preliminary to clcaring and paraffin or celloidin imbedding. This is routinely done by treating the tissue after Fixation and Washing by passing it through a series of ethyl alcohols of increasing concentration. Usually the percentages are $30,50,70,80,95$ and absolute. The time depends upon the size and kind of the tissue and the sort of fixative. For slices of tissue less than $3 \mathrm{~mm}$. thick the dehydration can be accomplished in 6-12 hours. The alcohols for large slices fixed say in Zenker's fluid are ordinarily changed every morning and evening, but it is not desirable to leave them in absolute alcohol very long because it makes them brittle. Three to 6 hours should be sufficient. Tissues fixed in alcoholic solutions take a shorter time to dehydrate. After fixation in alcoholformalin or in Carnoy's fluid the tissue can be dehydrated and partly washed in several changes of absolute alcohol skipping the lower grades of alcohol entirely.

When, for some reason, it is desired to eliminate treatment with absolute alcohol the tissues can be passed directly from $95 \%$ alcohol in to Aniline Oil (say $30 \mathrm{~min}$.) which is itself later removed, at least partly, in 5-10 minutes by washing in 2 changes of chloroform. Clearing is continued in chloroform for imbedding in paraffin, or the tissue may be passed from $95 \%$ alcohol, even from $80 \%$, into Terpineol and cleared in half terpineol and xylol. Still another way to avoid absolute alcohol is to transfer from $95 \%$ alcohol to Bergamot Oil which serves as a clearing agent.

Several substitutes for ethyl alcohol as a dehydrating agent are available. Acetone is the best known. This is strongly recommended by $\mathrm{R}$. D. Lillie, p. 43. Four changes of acetone each lasting 40 minutes are suggested for routine work but this ean be reduced to 4 changes each $20 \mathrm{~min}$. Thereafter pass, the blocks of tissue to a paraffin solvent such as benzene, toluene or xylene before placing in melted paraffin. The schedules which he provides (p. 46) for dehydration, clearing and infiltrating with paraffin are useful. See Cellosolve.

Dioxan will not only take the place of the alcohol but also that of the clearing agent so that it is possible to greatly simplify the technique and make the sequence: fixative to dioxan to paraffin. See Dioxan and note as to possible danger to those using it. Cellosolve has also been proposed as a dehydrating agent. Lee (p. 64) says that it is ex- 
pensive, inflammable and quickly takes up water from the air. Whether it is injurious when breathed remains to be determined. On the whole it appears that little is to be gained by such substitutes. However, Cyclohexanone deserves further trial. If alcohol must be avoided it is always possible to fix in formalin and to use frozen sections. By the Altmann-Gersh technique the tissues are dehydrated in vacuo while still frozen.

Dehydrogenase-Written by E. W. Dempsey, Dept. of Anatomy, Washington University, St. Louis. February 26, 1951-Sometimes used synonomously with Oxidase, since it is an enzyme which catalyzes the transfer of hydrogen from one substance to another, and therefore operates to reduce one material and simultaneously to oxidise another. A variety of dehydrogenases are recognized, depending upon the substrate which acts as the hydrogen donor (e.g. succinic, malic, lactic dehydrogenase). Dehydrogenases have been measured in slices or homogenates of tissues, particularly with reference to malignancy (Greenstein, J. P., Biochemistry of Cancer, New York: Academic Press, 1947,389 pp.) and to changes in the reproductive cycle, a review of which is presented in Chapter 4 of The Enzymes, New York: Academic Press, 1950, Vol. 1, Part 1, 724 pp.

Methylene blue and other dyes have been used as hydrogen acceptors in the dehydrogenase systems. The rate of decolorization of the dye serves as an index of activity. Recently, tetrazolium salts, which form colorless solutions but which upon reduction are converted into colored, insoluble formazans, have been used to localize the enzymes in tissue sections or blocks. Rutenberg, A. M., R. Gofstein and A. M. Seligman, Cancer Research, 1950, 10, 113-121 present a review of the use of tetrazolium salts and methods for studying both non-specific and specific dehydrogenase systems in living tissue and in frozen sections. These methods are still somewhat lacking in histological precision, but are providing interesting data showing altered concentrations of the enzyme in states of different physiological activity (Zweifach, B. W., M. M. Black and E. Shorr, Proc. Soc. Exp. Biol. and Merl., $1950,74,848-854)$. See Succinic De: hydrogenase and Triphenyltetrazolium Chloride.

Dehydropyridines. Warburg noted a marked whitish fluorescence in ultraviolet light. Blaschko and Jacobson (Bourne, p. 196) report that the pyridines do not show this fluorescence and that the small granules that exhibit it in sections of living liver tissue may well be dehydropyridines. Their brilliant white fluorescence quickly fades.

Delafield's Alum Hematoxylin. To $400 \mathrm{cc}$. sat. aq. ammonia alum add $4 \mathrm{gms}$. hematoxylin dissolved in 25 cc. $95 \%$ alc. Leave exposed to air and light 4 days. Add 100 cc. methyl alc. and 10 cc. glycerin; filter. Filtrate will slowly ripen. To hasten ripening add $10 \mathrm{cc}$. hydrogen peroxide.

Delta Dye Indicator, see Nitrazine.

Dempsey, see Phosphatases, Esterases, Enzymes, Dehydrogenase, Nucleases.

Dempster, see Shaddow Casting.

Density Determinations. Technique described for amebae is by use Starch Density Gradient (Løvtrup, S., C. rend. Lab. Carlsberg, Sér. Chim., 1950, 27, 137-144).

Dental Enamel, see Enamel.

Dentin. Can be studied in ground sections of undecalcified teeth as well as in paraffin and celloidin sections of decalcified ones (see Teeth). For the latter Hematoxylin and Eosin, Mal. lory's Connective Tissue stain and many others can be applied as in the case of decalcified bone. Hanazawa's (Dent. Cosmos, 1917, 59, 125) methods for the minute structure of dentin are given in detail by Wellings, A. W., Practical Microscopy of the Teeth and Associated Parts, London: John Bale, Sons \& Curnow. Ltd., 1938, 281 pp. Dentin can be advantageously examined after vital staining with Alizarin Red S. Its $\mathrm{pH}$ can be estimated (Grossman, L. I., J. Dent. Res., 1940, 19, 171-172). For determination of rate of mineral replacement see Radioactive Phosphorus; for Korff's fibers, see Teeth, Developing; and for nerve endings, see Teeth, Innervation.

Desmoglycogen, see Glycogen.

Desoxyribonuclease is an enzyme for degrading desoxyribonucleic acid. It has been used for histochemical observations (Catheside, D. G., and Holmes B., Symp. Soc. Exp. Biol., No. 1, Nucleic Acids, 1947, 225-231, Cambridge University Press) to remove this type of nucleic acid from cells.

Desoxyribonucleic acid. Method for determination in Isolated Hepatic NucleiWritten by Joseph A. Falzone, Dept. of Anatomy, Washington University, St. Louis 10, Mo. October 26, 1951This technique may be applied, with slight modifications to a wide variety of tissues and organisms, but is here described for use with rat liver. In principle, the nuclei of a tissue homogenate are isolated and washed by centrifu- 
gation, counted in an hemocytometer chamber, and the Desoxyribonucleic Acid (DNA) extracted with hot perchloric acid and determined by the Dische (diphenylamine) reaction. By this means, the DNA content of a known number of nuclei is determined, making it possible to calculate the average DNA content per nucleus (Boivin, A., Vendrely, R., and Vendrely, C., Compt. rend. Acad. sci., 1948, 226, 1061-1063.

Isolation, Counting, and Washing of Nuclei: Anesthetize the rat, expose abdominal and thoracic viscera, clamp the inferior vena cava just below the liver, sever the portal vein, and perfuse the liver through the hepatic vein or thoracic inferior vena cava, using about $50 \mathrm{ml}$. of cold normal saline, followed by $30-50 \mathrm{ml}$. of cold $2 \%$ citric acid. Remove the liver, which will weigh about $10 \mathrm{gm}$., and dissect away from it any adhering diaphragmatic and connective tissues. Frozen liver may be used, but nuclear yield is reduced, and increased cellular debris makes counting difficult.

The following steps are to be carried out in a cold room at $5^{\circ} \mathrm{C}$. After taking a specimen for histological control, homogenize remainder of the liver in about $50 \mathrm{ml}$. cold $2 \%$ citric acid (Cunningham, L., Griffin, C., and Luck, J. M., Gen. Physiol. 1950, 34, 59-63) using a Waring Blendor with metal head equipped with an ice-brine cooling jacket. The cold room alone does not prevent heating, but with a cooling jacket, the temperature should not exceed $5^{\circ} \mathrm{C}$. Homogenize for 5 minutes, adding a few drops of capryl alcohol to prevent foaming.

The yield is $50-70 \mathrm{ml}$. of a $20 \%$ homogenate, which is filtered through four layers of cheesecloth. Microscopic examination at this stage reveals a uniform suspension of relatively undistorted nuclei, without any lumped or intact cells.

Nuclei which are spun down for analysis cannot be counted directly, due to clumping, but are estimated by first counting the whole homogenate then subtracting from this value the numbers remaining in the various supernates after centrifugation, as described below. Thoroughly shake the homogenate, withdraw four $5 \mathrm{ml}$. aliquots, and dilute each with $10 \mathrm{ml} .2 \%$ citric acid. These are the counting dilutions, each of which is counted twice in the red cell space $\left(0.1 \mathrm{~mm}^{3}\right)$ of an hemocytometer chamber. Eight to ten individual counts, totaling 3000-5000 nuclei, should keep the error within $5 \%$.

Fifty $\mathrm{ml}$. graduated conical centrifuge tubes are used in the following steps. Remove four $10 \mathrm{ml}$. homogeriate aliquots (each containing about $200,000,000$ nuclei as determined above) and carefully layer each over $20 \mathrm{ml}$. of a solution containing $2 \%$ citric acid and $10 \%$ sucrose. Centrifuge for 20 min. at about $500 \times \mathrm{G}$. By use of this layering technique nuclei are both washed and isolated in a single step (Wilbur, K. M., and Anderson, N. G., Exp. Cell Res. 1951, 2, 47-57).

Decant and keep this supernate. Rewash sediment (containing nuclei) in $35 \mathrm{ml}$. $2 \%$ citric acid, centrifuging as above. Combine supernates $1+2$ from each aliquot, measure volumes, and count nuclei without diluting, if more than 10 per chamber $\left(0.9 \mathrm{~mm}^{3}\right)$ are present.

Extraction of $D N A:$ A recent modi. fication of the Schneider procedure is suggested (Schneider, W. C., Hogeboom, G. H., and Ross, H. E., J. Nat. Cancer Inst., 1950, 10, 977-982). Extract sediment once with cold $12 \%$ perchloric acid and twice with cold $6 \%$ perchloric acid, centrifuging for $15 \mathrm{~min}$. at $500 \times \mathrm{G}$ each time. This removes nucleotides and sucrose. Combine these supernates $(3,4,+5)$, measure volumes, and count nuclei as above, adding this value to that of supernates $1+2$, and subtracting the total from that for the whole homogenate. Supernates $3,4+5$ should not be mixed with $1+2$ because cytoplasmic proteins in the latter would be precipitated, with clumping of nuclei.

Extraction of phospholipids with absolute alcohol may be omitted in working with liver and some other tissues. It is necessary for brain.

Extract sediment with $30 \mathrm{ml}$. $6 \%$ perchloric acid at $90^{\circ} \mathrm{C}$. for 15 minutes, cool, and centrifuge. Wash sediment with $10-15 \mathrm{ml}$. cold $6 \%$ perchloric acid and combine these supernates $(6+$ 7) as the DNA extract. This may be stored for 24 hrs. in a deep freeze with little change but longer storage (several weeks) produces unreliable results.

Determination of $D N A$ : The Dische reagent should be prepared with diphenylamine that is colorless or nearly so, with a well defined melting point at $53^{\circ} \mathrm{C}$. Otherwise this compound must be recrystallized before use.

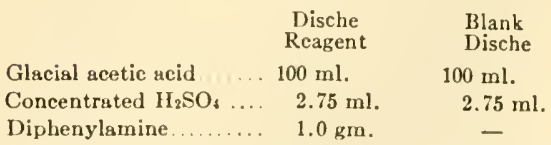

Following the Seibert procedure ( $J$. 
Biol. Chem. 1940, 133, 593-604) set up $108^{\prime \prime} \times 1$, pyrex tubes as below.

$13 \mathrm{ml}$. DNA extract from aliquot $1+6 \mathrm{ml}$. blank Dische

$23 \mathrm{ml}$. DNA extract from aliquot $2+6 \mathrm{ml}$. blank Dische

$33 \mathrm{ml}$. DNA extract from aliquot $3+6 \mathrm{ml}$. blank Dische

$44 \mathrm{ml}$. DNA extract from aliquot $4+6 \mathrm{ml}$. blank Dische

$53 \mathrm{ml}$. DNA extract from aliquot $1+6 \mathrm{ml}$. Dische reagent

$63 \mathrm{ml}$. DNA extract from aliquot $2+6 \mathrm{ml}$. Dische reagent

$73 \mathrm{ml}$. DNA extract from aliquot $3+6 \mathrm{ml}$. Dische reagent

$83 \mathrm{ml}$. DNA extract from aliquot $4+6 \mathrm{ml}$. Dische reagent

$93 \mathrm{ml} .6 \%$ perchloric acid $+6 \mathrm{ml}$. blank Dische

$103 \mathrm{ml} .6 \%$ perchloric acid $+6 \mathrm{ml}$. Dische reagent

Heat tubes 10 minutes at $100^{\circ} \mathrm{C}$., cool to room temperature and read optical density (D) at $600 \mathrm{~m} \mu$. Read unknowns against corresponding blanks set at zero, as below:

tube 5 against tube 1 as a blank $\left(\mathrm{D} \frac{5}{1}\right)$

tube 6 against tube 2 as a blank $\left(D \frac{6}{2}\right)$

tube 7 against tube 3 as a blank $\left(\mathrm{D} \frac{7}{8}\right)$

tube 8 against tube 4 as a blank $\left(\mathrm{D} \frac{8}{4}\right)$

tube 10 against tube 9 as a blank $\left(D \frac{10}{9}\right)$

The nucleic acid concentrations (C) corresponding to the five $\mathrm{D}$ readings are then obtained from a standard curve. The latter is constructed in the usual manner from at least 20 points, by subjecting various concentrations of a standard DNA to the Dische procedure and plotting the resulting D readings against these concentrations. The solvent $(0.01 \mathrm{~N} \mathrm{NaOH}$ or hot $6 \%$ perchloric acid) plus Dische reagent is used as a blank. Beer's law should be closely obeyed in the useful range.

The DNA concentration corresponding to the $\mathrm{D}$ reading of tube 10 against tube $9\left(\mathrm{C} \mathrm{D} \frac{10}{9}\right)$ is the correction for color developed by the reagents alone and is subtracted from the concentrations found for the unknowns.
C D $\frac{5}{1}-\mathrm{C} \mathrm{D} \frac{10}{9}=\begin{gathered}\text { Corrected DNA concentration of } \\ \text { tube } 5\end{gathered}$

C D $\frac{6}{2}-\mathrm{C} \mathrm{D} \frac{10}{9}=\underset{\text { Corrected }}{\text { tube } 6}$ NA concentration of etc.

The volumes of DNA extracts are measured and the total DNA per aliquot thus found. This value is divided by the number of nuclei per aliquot (homogenate nuclei minus supernate nuclei) to obtain the average DNA content per nucleus, most conveniently expressed in $\mu \mu \mathrm{g}(\mathrm{mgm} . \times 10-9)$. This will usually range from 5-10 $\mu \mu \mathrm{g}$., depending upon the purity of DNA employed in constructing the standard curve. The values of the four aliquots should agree within $5 \%$.

If an efficient means could be found of separating the various nuclear types of a given tissue, the value of this technique would be greatly enhanced.

A method for determination of Desoxyribonucleic Acid in isolated nuclei of tumor cells (Dounce, A. L., J. Biol. Chem., 1943, 151, 235-240). Same as DNA and Thymonucleic Acid. The structure of this acid in relation to the cytochemical significance of methyl green-pyronin staining is carefully considered by Vercauteren, R., Enzymologia, 1950, 14, 134-140. See Nucleic Acid.

Destin's fixative. $1 \%$ aq. chromic acid, 99 cc.; formalin, 6 cc.; glacial acetic acid, 2 cc. After standing for a few days it becomes green when it can be used.

Detergents, see discussion of cutaneous detergents by Lane, C. G. and Blank, I. H., J.A.M.A., 1942, 118, 807-817. See Aerosol.

Deuterium is heavy hydrogen. It is an isotope having atomic weight of 2.0135 and the symbol $\mathrm{H}^{2}$. Schoenheimer, R., Harvey Lectures, 1937, 32, 122-144 employed deuterium combined with oxygen as heavy water $\mathrm{H}_{2}^{2} \mathrm{O}$ to mark fatty acids. In his experiments on mice, held on a carbohydrate diet plus heavy water the fatty acids of the body are replaced by new fatty acids containing deuterium. The rate of replacement of fatty acids can therefore be determined. For further experiments along this line see Symposium on Intermediate Metabolism of Fats. Biological Symposia Lancaster: Jaques Cattell Press, 1941. Leading references on deuterium: Cope, O., Blatt, H. and Ball, M. R., J. Clin. Invest., 1943, 22, 111-115; Flexner, L. B., Gellhorn, A. and Merrell, M., J. Biol. Chem., 1942, 144, 35-40; Stern, K. and Dancey, T. E., 
Proc. Soc. Exp. Biol. \& Med., 1941, 48, 619-620.

Deutoplasm, see Paraplasm.

Diacetin (glycerol diacetate) use in flattening paraffin sections (Carleton, H. M. and Leach, E. H., J. Path. \& Bact., $1939,49,572-576)$.

Diamin Red 4B, see Benzopurpurin 4B. Diamine Bordeaux CGN, see Erie Garnet B.

Diaminoacridines have marked affinity for nuclei in vivo. They can be visualized by their fluorescence in near ultraviolet light. Their localization resembles the chromatin pattern as revealed by "nuclear" dyes. These compound are apparently not toxic because regeneration of rat liver cells while they are still within the nuclei takes place at the same rate as in controls (DeBruyn, $R$. S., Anat. Rec., 1950, 108, 279-307).

Di-Amino Tri-Phenyl Methane Dyes. Examples : brilliant green, fast green FCF, light green SF yellowish and malachite green.

Diamond Green, see Brilliant Green.

Diamond Green B, BX or P Extra, see Malachite Green.

Diamond Fuchsin, see Basic Fuchsin.

Dianil Blue H3G, see Trypan Blue.

Dianil Blue 2R (CI, 265)-benzo new blue $2 \mathrm{~B}$, direct steel blue $\mathrm{BB}$, naphthamine brilliant blue $2 \mathrm{R}$-Conn ( $\mathrm{p}$. 63) gives the same formula for this acid dis-azo dye as that supplied by Corner, G. W. and Hurni, F. H., Am. J. Physiol., 1918, 46, 483-486 and Sutter, M., Anat. Rec., $1916,16,164-165$ for dye employed by them in study respectively of corpora lutea and mammary glands but these authors do not employ the name: dianil blue.

Dianil Red 4B, see Benzopurpurin 4B.

Dianthine B, see Erythrosin, bluish.

Diaphane for mounting Giemsa preparations (Coulston, F., J. Lab. \& Clin. Med., $1940,26,869-873$ ).

Diaphanol is according to Lee (p. 598) the trade name for a mixture, formerly obtainable from Leitz, produced by passing chlorine dioxide vapor into ice cold $70 \%$ acetic acid. It should be fresh. He advises against attempts to make it and outlines its use in the softening of Chitin. Rinse well fixed tissues in $63 \%$ alcohol and transfer them to diaphanol until they are softened and bleached. If the diaphanol becomes discolored, repeat. Transfer to $63 \%$ alcohol, dehydrate, clear in tetralin (if not available, benzol) and imbed in paraffin. See use of diaphanol in demonstrating Melanins.

Diazin Black, see Janus Black.

Diazin Green, see Janus Green B.

Diazo Reaction. Serra, J. A., Stain Techn., 1946, 21, 5-18 gives the technique as follows: Prepare tissue as described under Ninhydrin Reaction. "Treat the picces for 2-3 minutes with a saturated aqueous solution of sodium carbonate; afterwards add some drops of the diazo reagent and stir the liquid well. $\mathrm{Ob}$ serve in glycerin. (The coloration develops rapidly and lasts for some days.) Preparation of the diazo-reagent: into a $50 \mathrm{ml}$. flask immersed in an ice bath, pour $1.5 \mathrm{ml}$. of a sulphanilic acid solution (dissolve $0.9 \mathrm{~g}$. of pure sulphanilic acid in $9 \mathrm{ml}$. of concentrated $\mathrm{HCl}$ and add water to $100 \mathrm{ml}$.); add $1.5 \mathrm{ml}$. of a $5 \%$ aqueous solution of $\mathrm{NaNO}_{2}$, shaking the flask meanwhile. After 5 minutes in the ice bath add, also while shaking, another $6 \mathrm{ml}$. of nitrite. After 5 minutes fill up to $50 \mathrm{ml}$. with cooled distilled water. The reagent must be prepared every day and kept in the ice chest.

"The reaction gives an orange or yellow color with the histidine and the tyrosine of the proteins."

Dichlorofluorescein. Structure of, Milligan, R. F. and Hope, F. J., J. Am. Chem. Soc., 1945, 67, 1507-1508.

Dientamoeba fragilis. Technique of staining and points to be considered in diagnosis (Hood, M., J. Lab. \& Clin. Med., 1939-40, 25, 914-918).

Diethylene Dioxide = Dioxan .

Differential Centrifugation of Cell Particulates-Written by Joseph A. Falzone, Department of Anatomy, Washington University Medical School, St. Louis. November 27, 1951-Since the pioneering work of R. R. Bensley and N. Hoerr (Anat. Rec., 1934, 60, 449-455), this technique has proven one of the most versatile and direct in the armamentarium of cytology. In essence it consists of a rather drastic mechanical disruption of large numbers of cells in various media, separation of the result ing particulates by centrifugation, and determination of chemical components and enzymatic activities in these fractions. There are almost as many variations in the technique as investigators, each variation having its peculiar merits or limitations, the one chosen depending upon the particular cell organelle or chemical data desired.

The obvious and inherent weakness of these methods is their tendency to produce morphological and chemical artefacts. For example, when a tissue is homogenized in an aqueous medium, enzymes and other substances may be lost by denaturation or solution, or what is worse, adsorbed to particulates that never contained them in life. Large particles may fragment and sediment with an unrelated small granule 
fraction. With nuclei, at least, these obstacles are in part overcome by the $M$. Behrens technique (Ztschr. f. physiol. Chem., $1932,209,59$ ) by which tissue is frozen and dehydrated at the outset, fractionation being accomplished in nonpolar organic media.

The factor of tonicity is poorly understood. A solution isotonic to erythrocytes may not be so to mitochondria or nuclei. Mitochondria eventually swell and burst in distilled water, but appear well preserved in almost syrupy sucrose solutions. The review of $W$. C. Schneider and G. H. Hogeboom (Cancer Res., 1951, 11, 1-22) should be consulted in this respect. Nuclei, on the other hand, do not appear to behave as osmometers.

Hydrogen ion concentration, of course, is an important variable and should be adjusted according to experimental aims. In using citric acid media, for example, we find a number of $\mathrm{pH}$ dependent effects. At $\mathrm{pH} 4$ or below (2\% citric acid) nuclei are readily isolated in bulk, morphologically free of cytoplasm, with no tendency to clumping, and with an apparently complete complement of desoxyribonucleic acid (DNA). At pH 4-6, agglutination of cytoplasmic particles produces nuclear clumping. At $\mathrm{pH} 6$, nuclei are again dispersed, and contain, in addition to DNA, some enzymatic activity. Above $\mathrm{pH} 6.5$, they disintegrate completely, in contrast to cytoplasmic components, which are better preserved in nearly neutral media (Dounce, A. L., Ann. N. Y. Acad. Sci., 1950, 50, 982999).

It might appear that ideal media would duplicate the tonicity, $\mathrm{pH}$, and electrolyte pattern of cytoplasm, and some attempts in that direction have been made (Wilbur, K. M. and Andreson, N. G., Exp. Cell Res., 1951, 2, 47-57). However, the most versatile media employed to date have been simple iso- or hypertonic sucrose solutions, without buffers or electrolytes. These media, especially if hypertonic, yield excellent morphological preservation of all components, including nuclei, which resemble those of living cells in their optical homogeneity (Schneider and Hogeboom, Ibid.). One wonders if this is really an advantage in the determination of nuclear nucleic acids, where acid precipitation may be desirable, but the question is not fully answered. Bearing on this problem is the fact that many such unbuffered homogenates are slightly acid, due probably to glycolysis, but that liver appears to be an exception in this respect (Wilbur and Anderson, Ibid.).

The separation of cell components by centrifugation has relied more upon difference in particle size, with resulting difference in velocity of fall, than upon any variation in specific gravity. This would be expected from a consideration of Stokes' Law, which states that the velocity of particle fall is proportional to the square of its radius, but only directly proportional to the density difference between particle and medium. Moreover, we are dealing with semipermeable or frankly porous cell particles, whose density is altered with that of the medium, so that very dense (and correspondingly viscous) fluids must be employed to effect separation by flotation. In these cases we may be roughly measuring 'dry weight densities', as in the Behrens procedure. An obvious exception to these considera. tions is that of lipid-rich constituents, which migrate centripetally.

As a result, whole cells are commonly sedimented at very low speeds, nuclei at approximately $500 \mathrm{xg}$ mitochondria at 2000-20,000 x g, and microsomes at 20,000 to over $100,000 \times \mathrm{g}$. The "supernate" is the remaining non-sedimentable fraction, and with the preceding particulates, completes the list of usually studied components. Others, such as chromosomes and melanin granules, will not be considered here. The time of centrifugation or field required varies with the viscosity of the medium, the time ranging from a few minutes to several hours.

A layering technique, as emphasized by Wilbur and Anderson (Ibid.), is the most efficient means of centrifugation, as the mean distance of particle fall is both increased and nearly equalized. This naturally has the effect of both isolating and washing the most rapidly sedimenting fraction at a single step. From this and the above considerations it is obvious that a more efficient centrifuge would be one constructed to hold longer tubes. With such an instrument, employing tubes only double the length of standard models, an even further fractionation of particulates might be achieved, with diminished loss due to washing. Separation of nuclear types suggests itself, and some endeavors in that direction are promising (Marshak, A., Cancer Res., 1950, 10, 232).

Below is a brief outline of some of the more popular and useful techniques, listed according to homogenizing fluids employed. Centrifuging media are usually similar to these, or made slightly 
denser by addition of sucrose, if layering is employed. Temperature should be kept at $5^{\circ} \mathrm{C}$. or below throughout. No attempt at completeness has been made, and some interesting methods, including ultrasonic and enzymatic cell disruption, are omitted.

1) Citric acid, low pH (Dounce, Ibid; Cunningham, L., Griffin, A. C. and Luck, J. M., J. Gen. Physiol., 1950, 34, 59-63).

Procedure: Tissue homogenized several minutes in cold $2 \%$ citric acid in a Waring Blendor. The latter should be equipped with a cooling jacket.

Particulates obtained: Nuclei.

Morphology: Reasonably good. Nuclei plump and unwrinkled, but with fine internal granulation. No clumping or cytoplasmic tags.

Value: Simplicity and use of large volumes of tissue, if desired. Nuclei readily counted and DNA per nucleus determined by the Dische or other suitable analytical technique. The method of choice for this purpose, but of little other value.

Limitations: Water soluble substances, including much protein and probably RNA, are lost; enzymes inactivated.

2) Citric acid, controlled $\mathrm{pH}$ (Dounce, Ibid.).

Procedure: Enough $0.1 \mathrm{M}$ citric acid is added dropwise to partially homogenized tissue in ice water to give a $\mathrm{pH}$ of 6.0. Used with a low speed Waring Blendor or glass homogenizer.

Particulates obtained: Nuclei.

Morphology: Similar to procedure 1.

Value: Suitable for DNA and many enzyme studies.

Limitations: Similar to 1, except that apoenzymes are retained. Coenzymes must often be added in determinations of their activities, however. Adsorption effects must be controlled by repeated washing of nuclei.

3) Sucrose, near pH 7 (Schneider and Hogeboom, Ibid.).

Procedure: Small amounts of tissue homogenized by ground glass apparatus or other "gentle" mechanical means, in a medium of neutral but unbuffered iso- or hypertonic (usually $0.88 \mathrm{M}$ ) sucrose. Probably best to avoid $\mathrm{NaCl}$ and other electrolytes for general purposes.

Particulates obtained: Nuclei, mitochondria, microsomes, supernate. Also melanin granules and particulate glycogen.

Morphology: Excellent, with preservation of all particulates in a form resembling that in living cells.
Mitochondria filamentous, at least in hypertonic sucrose, and stainable with Janus Green B; nuclei homogeneous with several nucleoli.

Value: Versatility, due to the large number of fractions obtained. Suitable for determinations of enzymes, nucleic acids, proteins and lipids of these fractions. The method of choice for microspectrophotometry of stained nuclei, as optical homogeneity is a definite prerequisite for valid results with this method.

Limitations: Solution and adsorption effects again come into play, but many artefacts can be partially ruled out. Nuclei probably not so readily obtained in bulk as with methods 1) and 2).

4) Behrens technique (Behrens, Dounce; Schneider and Hogeboom, Ibid.).

Procedure: Tissue frozen, dried in vacuo, ground in ball mill to disrupt cells, and centrifuged in non-aqueous media, usually hydrocarbons.

Particulates obtained: Nuclei.

Morphology: More seriously distorted than with any other procedure; nuclear membranes wrinkled. Contamination with cytoplasm must be appraised from smears stained with both acid and basic dyes.

Value: Probably the most widely applicable method for nuclei. Procedure of choice for water soluble substances, i.e., coenzymes, vitamins, free amino acids, etc. Results with total protein and $\mathrm{RNA}$ also appear the most reliable of any method. Suitable also for DNA and many enzyme studies.

Limitations: Procedure is laborious. Lipids, lipases, and many other enzymes removed or destroyed.

This brief summary can only suggest the variety of applications of cell fractionation to cytology, as new ones are continaully uncovered. For example, D. L. Opdyke has recently described a valuable technique for the isolation of keratohyaline granules of epidermis, using isotonic saline and a Waring Blendor for homogenization. The particles are sedimented at 25,000 $\mathrm{x} g$ and appear to have all the morphological and staining characteristics of these granules (See Keratohyaline Granules, procedure for isolation).

Differential Leucocyte Count, statistical study of uniformity in (Klotz, L. F., J. Lab. \& Clin. Med., 1939, 25, 424-434).

Diffraction Methods for measuring diameter of red blood cells (Haden, R. L., J. Lab. \& Clin. Med., 1937-38, 23, 508-518).

Digitonine reaction of Windaus for free 
cholesterol. This has been adapted to histochemical use by Brunswick and by Leulier and Noel (A., and R., Bull. d'Hist. Appl., 1926, 3, 316-319). 'Lison (p. 211) recommends a slight change. Immerse frozen sections of formalin fixed tissue in $0.5 \%$ digitonine in $50 \%$ alc. for several hrs. Rinse in $50 \%$ alc., then in water and mount in Apathy's syrup or glycerin gelatin. With crossed nicols (polarizing microscope) one observes appearance of needles or rosettes of the complex cholesteroldigitonide. To resolve this complex stain with sudan. The esters will color and lose their birefringence while the cholesterol will remain uncolored and retain birefringence.

Dilatometric Techniques. These have been adjusted so that they will give the fine quantitative measurements needed in histochemistry by Linderstrøm-Lang, $\mathrm{K}$ Nature, 1937, 139, 713. He reported especially ultra-microestimations of peptidase activity.

Di-Nitrosoresorcinol test for iron, see Iron.

Diodrast, trade name for an organic iodine preparation recommended by Gross, S. W. (Proc. Soc. Exp. Biol. \& Med., $1939,42,258-259)$ for injection into common carotid with later $\mathrm{x}$-ray photographs of the vascular tree. For visualization of ventricular chambers and large arteries see method of Ponsdomenech, E. R. and Nunez, V. B., Am. Heart J., 1951, 41, 643-651.

Dioxan is diethylene dioxide. It mixes with water, ethyl alcohol, many clearing agents and paraffin (slightly). McClung (p. 39) recommends its use to replace ordinary agents like xylol. Dioxan fumes are said to be dangerous to laboratory workers so that it should be used under a hood or in a well ventilated room with container covered when not in use (Magruder, S. R., J. Lab. \& Clin. Med., 1937-38, 23, 405-411).

For fixation the following mixtures are recommended (McClung, p. 39): (1) Sat. aq. picric acid, 5 parts; glacial acetic, 1 part; dioxan, 4 parts. (2) Sat. picric acid in dioxan, 4 parts; glacial acetic, 1 part; absolute alcohol, 4 parts. Graupner and Weissberger (von $H$. and A., Zool. Anz., 1933, 102, 39-44) suggest: dioxan $80 \%$, methyl alcohol $20 \%$, paraldehyde $2 \%$, and acetic acid $5 \%$. See Clearing, Pituitary. See as ingredient of Lison's glycogen method; also dioxan imbedding of Pituitary.

A method for the dehydration, purification and clarification of dioxan so that its use in tissue technique can be continued has been described by Hall, W. E. B., Am. J. Clin. Path., 1943, 7 (Technical Section), 98-100.
Dipeptidase can be localized in chief cells of stomach. See review of methods (Gersh, I., Physiol. Rev., 1941, 21, 242-266).

Di-Phenyl Methane Dyes. Of these only auramin need be referred to.

Diphosphopyridine Nucleotide, see technique of Anfinsen, C. B., J. Biol. Chem., 1944, 152, 285-291.

Diphtheria Bacilli. 1. Neisser's stain (Stitt, p. 863). $A=$ methylene blue, 0.1 gm.; $95 \%$ alc., 2 cc.; glacial acetic acid, 5 cc.; aq. dest., 95 cc. $B=$ Bismark brown, $0.2 \mathrm{gm}$.; aq. dest. (boiling) $100 \mathrm{cc}$. Dissolve and filter. To stain smear pour on $A: 30-60$ sec. Wash. Then $B, 30$ sec. Wash in water, dry and mount. Bacilli brown with dark blue dots at either end. Better results can be secured by adding 1 part of crystal violet (Hoechst) 1 gm.; $95 \%$ alc., 10 cc.; aq. dest., 300 cc. to 2 parts of $A$ before using. Chrysoidin $1 \mathrm{gm}$. in hot aq. dest. 300 cc. is more satisfactory counterstain than Bismark brown. Most American brands of crystal violet are satisfactory.

2. Ponder's stain (Stitt, p. 863). Toluidin blue (Grubler) 0.02 gm.; aq. dest., 100 cc.; glacial acetic acid, 1 cc.; abs. alc., 2 cc. Add small amount to fixed film on cover glass. Invert and mount on slide. Diphtheria bacilli recognizable by metachromatic granules intensely stained, diphtheroids by their strong color in contrast with ordinary cocci and bacilli the bodies of which are only faintly blue.

3. Laybourn's modification of Albert's stain (Stitt, p. 863). $A=$ toluidin blue, $0.15 \mathrm{gm}$.; malachite green, $0.2 \mathrm{gm}$.; glacial acetic acid, 1 cc.; $95 \%$ alc., 2 cc., aq. dest., 100 cc. $B=$ iodine crystals, 2 gm.; potassium iodide, 3 gm.; aq. dest., 300 cc. Let both stand 24 hrs. and use filtrate. Apply $A$ to heat fixed smears 3-5 min. Wash in water. Apply $B$ for 1 min. Wash, blot and dry. Granules of diphtheria bacilli, black; bars, dark green; intermediate parts, light green and all three in sharp contrast.

Diplosome, a double centrosome.

Direct Black, see Chlorazol Black E.

Direct Fast Orange (CI, 326)-Erie Fast Orange (NAC), Erie Fast Scarlet YA (NAC)- a direct disazo dye of light fastness 3 (Emig, p. 38).

Direct Fast Scarlet 4 BS (CI, 327)-Pontamine Fast Scarlet 4 BS of DuPont-, a disazo direct dye of light fastness 3 , can be employed instead of carmine as a general stain. Details of use in study of plant and animal tissues are described (Emig, p. 38).

Direct Garnet R, see Erie Garnet B. 
Direct Green B (CI, 593)-Diazine Green $\mathrm{B}-\mathrm{a}$ direct disazo dye of light fastness 3 to 4 . Recommended as counterstain for Crocein Scarlet $7 \mathrm{~B}$ of invertebrates or paraffin sections, time $5 \mathrm{~min}$. (Emig, p. 43).

Direct Green G (CI, 594)-Alkali Grcen Da direct disazo dye of light fastness 3 to 4. Formula for blue green algae and whole mounts is given (Emig, p. 43).

Direct Red 4B, see Benzopurpurin 4B.

Direct Red, C, R, or Y, see Congo Red.

Direct Sky Blue, see Niagara Blue 4B.

Direct Steel Blue BB, see Dianil Blue 2R.

Direct Violet B, see Azo Blue.

Direct Violet C, see Erie Garnet B.

Dis-Azo Dyes. Azo blue, benzopurpurin $4 \mathrm{~B}$, Biebrich scarlet, Bismark brown $\mathrm{Y}$ and $\mathrm{R}$, brilliant purpurin $\mathrm{R}$, congo red, dianil blue $2 R$, Erie garnet $B$, Niagara blue $4 \mathrm{~B}$, orseillin, trypan blue, trypan red, sudan III, sudan IV, vital new red, vital red, etc.

Dissociation, see Maceration.

Distrene 80 is a polysterene which forms a water clear solution in xylol. It is recommended by Kirkpatrick and Lendrum (J. and A. C., J. Path. and Bact., $1939,49,592-594)$ as a mounting medium giving good preservation of color in microscopic slides. See also Kirkpatrick, J. and Lendrum, A. C., J. Path. \& Bact., 1941, 53, 441.

Dominici's Stain, see Eosin-Orange $G$ and Toluidin blue.

Donaldson's Iodine-Eosin Method, see Iodine-Eosin.

Dopa, Oxidase Reaction for Melanoblasts (Laidlaw, G. F., Anat. Rec., 1932, 53, 399-407). Dopa is short for 3.4-dihydroxyphenylalanin, a substance which when applied in a certain way picks out the melanoblasts by blackening them. Use frozen sections of fresh material or of tissues fixed 2 to 3 hours but not longer in $5 \%$ formalin. Rinse 4 or 5 seconds in aq. dest. and immerse in buffered dopa. (To make dopa stock solution dissolve $0.3 \mathrm{gm}$. dopa powdermanufactured by Hoffmann-La Roche, Nutley, New Jersey-in 300 cc. cold aq. dest. Keep in refrigerator and discard when solution becomes dark red. To make buffers dissolve $11.87 \mathrm{gms}$. disodium hydrogen phosphate $\left(\mathrm{Na}_{2} \mathrm{HPO}_{4}\right.$ $\left.+2 \mathrm{H}_{2} \mathrm{O}\right)-$ or what would be better $9.47 \mathrm{gm}$. anhydrous $\mathrm{Na}_{2} \mathrm{HPO}_{4}$-in 1000 cc. aq. dest. and 9.08 gms. anhydrous potassium dihydrogen phosphate $\left(\mathrm{KH}_{2} \mathrm{PO}_{4}\right)$ in an equal amount aq. dest. Immediately before use buffer to $\mathrm{pH} 7.4$ by adding $2 \mathrm{cc}$. potassium phosphatc solution, and 6 cc. sodium phosphate solution to $25 \mathrm{cc}$. dopa solution). The reaction is slow for $3-4$ hours at room temperature. If solution becomes sepia brown it is likely to overstain. Observe under microscope. Wash in aq. dest., dehydrate and counterstain if desired with alcoholic erystal violet, clear and mount in balsam. Melanoblasts should be black.

This much used method has been criticized by II. Sharlit et al. (Arch. Dermat. and Syph., 1942, 45, 103-111) chiefly on the ground that the incubation for $3 \mathrm{hrs}$. at room temperature may itself increase the amount of melanin present which happened in their experience at $37^{\circ} \mathrm{C}$. See also remarks by Blaschko and Jacobson (Bourne, p. 198) on specificity of the reaction. It is given by phenoloxidases but thus far they have not been found in mammalian tissues.

Dorothy Reed Cells, see Reed-Sternberg Cells.

Double Green, see Methyl Green.

Double Imbedding. 'To facilitate section cutting by making a celloidin block firmer, harden first in chloroform vapor, then in chloroform, transfer to benzol until it becomes transparent and infiltrate with $38^{\circ} \mathrm{C}$. paraffin (Lee, p. 104). See Fleas.

Another method of double imbedding is that of Péterfi (T., Zeit. f. wiss. mikr., 1921, 38, 342-345). As employed in this laboratory it is as follows: Make $1 \%$ and $3 \%$ solutions of celloidin in methyl benzoate which take about a month. Pour some $1 \%$ into a dish. Add absolute alcohol containing the tissue which gradually sinks down into the celloidin. Transfer tissue to $3 \%$ solution, 48-96 hrs. Drop tissue directly into benzol for a few hrs. Then infiltrate and imbed in $40^{\circ} \mathrm{C}$. paraffin about 12-24 hrs.

Double Scarlet BSF, see Biebrich Scarlet, water soluble.

Downey's Fluid, see Megakary ocy tes.

Ducts. These structures lead (L. ducere) the products of glands to the site of discharge. They are of considerable variety. Ordinarily they are easily identified by their morphology in hematoxylin and eosin preparations. But special techniques are required for their visualization in whole mounts of some glands.

In the pancreas for example the system of small ducts (ductules) can easily be demonstrated by perfusion of the pancreas with pyronin-one of the many methods discovered by $R$. R. Bensley. Proceed as described under Perfusion using a solution made up by adding $10 \mathrm{cc}$. of $1 \% \mathrm{aq}$. pyronin to 1000 cc. $0.85 \%$ aq. sodium chloride. When the pancreas has assumed a rose red color the optimum intensity of which 
must be determined by trials, remove a piece of it, tease out a small lobule and examine under low power mounted in $0.85 \%$ aq. sodium chloride. The complicated system of ducts should be sharply delineated by their deep rose red color in an almost colorless background. If there is any question of their identification examine the original figures of Bensley, R. R., Am. J. Anat., $1911,12,297-358$. A double staining of ducts and Islets of Langerhans can be obtained by perfusing in the same way with pyronin solution to $1000 \mathrm{cc}$. of which $6 \mathrm{cc} .1 \%$ aq. neutral red has been added. The islets appear yellow red in contrast to the rose red ductules. See, in addition, ducts in whole mounts of Mammary Glands and in sections of Submaxillary Glands which are of particular interest in detecting the action of salivary gland viruses.

Duggins, see Hairs

Duodenal Fluid. Microscopic study must be prompt because of the presence of cytolytic engymes. Examine sediment after centrifugal concentration as in the case of urinary sediments. Epithelial cells from the entire alimentary tract leading to and including the duodenum may be present, generally bile stained, also a few neutrophiles. A great increase in both or either may indicate inflammatory lesions. A polarizing microscope is helpful, but not essential, in recognizing cholesterol crystals as thin, flat, colorless fragments with chipped edges. The more irregular the crystals the more significant they are of calculi formation. Bilirubin is easily detected as amorphous amber, brown or black material and calcium bilirubinate as bright yellow granular deposits. See Gentzkow and Van Auken in Simmons and Gentzkow, p. 63.

Duodenal Drainage Smears, see Papanicolaou Techniques.

Duran-Reynals, see Spreading Factors.

Dust Cells of the Lungs-Written by C. C. Macklin, Dept. of Histological Research, The University of Western Ontario, London, Canada. November 28, 1951 - These phagocytes develop in the alveolar walls of the lungs of man and other mammals from the primitive pneumonocytes to arrest particles of carbon and other particulate matter which elude the apprehensional mechanism of the respiratory tract; and to dispose of them by conveying them to the ciliary escalator which evacuates them. Collapse of the lungs dislodges them from their normal position in the alveolar wall (Macklin, C. C., Lancet, Feb. 24, $1951,432-435)$ where they are wedged in between capillaries, whence they derive their nutriment. Thus the ordinary human lung material available to pathologists is unfavorable in that it shows phagocytes lying loose which normally are tethered. If the skin and outer musculature be removed from a fresh intact thorax of a small animal such as the mouse and it be at once immersed in a fixing fluid such as Regaud or Bouin's, the lung is quickly fixed in its normal uncollapsed condition. Penetration is facilitated by exposing the lower surface of the diaphragm, but this must not be breached. Sections from such material show as many as $99 \%$ or more of the dust cells morphologically affixed to the alveolar walls, while only $1 \%$ or less are free, and these are regarded as spent and on their way to the exterior. If, however, fresh normal mouse lungs are collapsed and promptly filled with fixing fluid via the trachea, and cut into sections, it is found that only about $5 \%$ of the dust cells remains fixed in the alveolar walls, the remainder now appearing free. An erroneous picture of the relation of the dust cells to the alveolar walls is thus presented. That collapse alone dislodges many of the phagocytes formerly affixed in the alveolar walls is shown by finding undue numbers of free dust cells in sections from pieces of lung tissue which have been fixed by immersion. For larger lungs the method of perfusion of fixing solution through the pulmonary vasculature of the unopeued thorax (Hartroft, W. S., Anat. Rec., 1942, 82, 419) also showed the majority of alveolar phagocytes affixed to the walls rather than free. Thus dust cells are fundamentally mural phagocytes, and are regarded as originated ultimately from endodermal epithelium (Macklin, C. C., Trans. Roy Soc. of Canada, Sect. V, 1946, 40, 93-111). The presence of fluid in the alveoli favors a shedding of mural phagocytes from their walls. No evdence of dust cell entry into the connective tissue or lymphatic channels of the lung was found, contrary to the opinion of many pathologists. On the other hand, dust cells are often found stuck in the mucus overlying the epithelium of the bronchioli and bronchi, and these are probably wornout cells being carried to the glottis by ciliary movement Macklin, C. C., Can. J. of Res., D, 1949, 27, 50-58; Macklin, C. C., Proc. Instit. of Med. of Chicago, $1950,18,78-95)$. They are recoverable from sputum, and then have been referred to by the unsuitable term "heart failure cells" (which see).

In the mouse most alveolar phagocytes are in alveolar angles and have 
processes inserted into tunnels encircled by capillary loops; but a lesser number, resting on connective tissue, occupy sockets. After brief treatment of fresh lungs with ammoniacal silver solution, many of the dust cells are blackened and their mode of insertion into mural vaginae are made clear (Macklin, C. C., Anat. Rec., 1948, 100, 693). After withdrawal of such processes these vaginae or tunnels, now vacated, have become pores Macklin, C. C., J. of Thor. Surg., 1938, 7, 536-551). These mural phagocytes may have as many as four functional air faces or particle receptors of varying area. These of ten conform to the contour of the alveolar wall, but, in presumably active cells, project into the air space. The edges may extend upon adjacent capillaries and have been mistaken for respiratory squames by some workers. They are sister cells of the alveolar granular cells (septal cells, niche cells, etc.) and the term "pneumonocyte" (which see) includes both types. Dust cells may be recovered from fresh lungs by the "gash-irrigation" and "wash-out" methods (which see), and studied in fresh mounts or after being dried and stained like a blood smear. Mitochondria are scarce or absent in them.

One hour after exposure to carmine particles in the inspired air they contain much carmine, which is almost completely absent on the remaining alveolar wall surface, and almost $100 \%$ are inserted into the walls. One week after such dusting, however, only $35 \%$ are mural phagocytes, the rest being free. Since dust cells are constantly being lost to the body they must be replaced correspondingly by the multiplication of sister cells remaining in the walls. Dust cells often show bizarre shapes and a common one is that of a dumb-bell in which the middle narrow bridge occupies a pore. Such cells are probably not in transit from one avleolus to another.

Histocytes of the lung connective tissue often pick up dust particles which have passed the inefficient surface guards of the broncho-alveolar system. These are regarded as quite different from the endodermal alveolar phagocytes. Great amounts of such aberrant dust may accumulate and may give rise to disease processes.

Dyes, see "standardization of Stains" pp. xxvii-xxx of this book and Staining.

Dysentery, see Endamoeba.

Dysprosium, see Atomic Weights.

Ear-Written by W. P. Covell, Dept. of Anatomy, Washington University, St. Louis 10, Mo. October 26, 1951-Micro- scopic examinations of the ear are nearly always made on sections. This is understandable, but it is possible that the study of still living tissues, removed by careful and minute dissections, is a field of considerable promise. The close apposition of epithelial and nervous components to bone necessitates decalcification except in the case of young embryos. The frequent use of celloidin in place of paraffin for imbedding is occasioned by the wide range of diversity in resistance of the organ to the microtome knife, fluid containing lumina being surrounded by hard dense bone. The histological techniques actually in use for the ear are fewer in number and more limited in range than those employed for most other parts of the body. The difficulty experienced in obtaining fresh and normal adult specimens has turned investigation toward human fetuses and the ears of experimental animals.

The commonly used fixatives are either Zenker's fluid, with or without acetic acid, Zenker-formol, $10 \%$ formalin, Wittmaack's solution and Heidenhain-Susa. Mygind, S. H. and coworkers (Acta oto-laryng., 1945, 33, 273280) prefer the Wittmaack's for fixation of hair cells and use the latter for study of such structures as the stria vascularis. The best results are to be obtained by the use of animal material for which prompt fixation by perfusion methods has been done. Kristensen, H. K. (Acta oto-laryng., 1945, 33, 225244) recommends the use of a $6 \%$ gum arabic in physiologic saline for intravital perfusion to precede the fixative. Isolated temporal bones placed in fixative are prone to show autolytic changes in end organs and ganglion cells in one-half hour following death. The literature is filled with autolytic changes described as specific pathologic alterations due to drugs, toxins, poisons, and so forth. Actually many of these are the result of poor penetration of fixatives and elapse of time between autopsy and fixation. In an attempt to overcome the slow penetration of a fixative and prevention of artefacts Guild made use of water from which gases had been exhausted as the medium for fixatives, decalcifying solutions and alcohols.

A variety of decalcificants have been used with the view to preserving finer cell structures of the soft tissues, viz: Formic, trichloracetic, and trichloralactic acids, long immersion in Mullers' fluid and so forth. Nitric acid in 2 to $5 \%$ concentration is generally used for human temporal bones. The lower con- 
centration while it takes longer to act is less likely to overdecalcify. A few investigators recommend the use of $3 \%$ nitric acid in water and a constant temperature $\left(37 \frac{1}{2}^{\circ} \mathrm{C}\right.$.) to hasten the procedure. Degree of decalcification is usually judged by probing with a needle, or a simple test with an indicator such as phenol red. Most small animal bones are decalcified in about 4 to 10 days. However, human temporal bones vary considerably and may take as long as 6 to 8 weeks with weekly change of the solution. After decalcification, thorough washing for 24 hours in running water is necessary following which neutralization in $5 \%$ sodium sulfate may be used and washing in running water repeated.

In order to preserve cytologic detail attempts have been made to circumvent decalcification in strong acids. Small laboratory animals may be perfused with Regaud's solution and following fixation mordanted in potassium bichromate for a considerable length of time. The blocks can be embedded in paraffin and sections made although decalcification is usually incomplete. Mitochondria in hair cells, stria vascularis and spiral ganglion cells can be studied by this method.

In his study of kittens, young rabbits, dogs and rats, Van der Stricht, O., Contrib. to Embryol., Carnegie Inst., $1920,9,109-142$ fixed isolated cochleas in $5 \%$ aq. trichlorlactic acid, Bouin's and Zenker's fluids, mordanted for "many weeks" in 70\% alcohol t a few drops of iodine solution. After the last 2 fixatives he completed decalcification in $2 \%$ nitric acid in $70 \%$ alcohol. Before imbedding in paraffin he stained with Borax Carmine and he colored the sections with Iron Hematoxylin, Congo Red and Light Green. Directions will be found in his paper for the demonstration of mitochondria in the sustentacular and hair cells. A differential stain for hair cells is described by MacNaugh ton, I. P. J., and Peet, E. W., J. Laryng. and Otol., 1940, 55, 113-114 with a fine colored figure of the results.

Celloidin is generally used for imbedding animal and human material. It is not ideal since it is difficult to handle, takes considerable length of time to infiltrate and is expensive. Various nitrocellulose samples have been tried for small blocks of bone with success but usually centers of large blocks, particularly human temporal bones, do not become sufficiently hard. Concentrations of celloidin usually ranging up to $15 \%$ are employed in successive steps. While the material is in
$8 \%, 10 \%$ and $15 \%$ celloidin negative pressure is used in moderate amounts to insure infiltration of celloidin into chambers of the inner ear. This should be done with considerable caution as rupture of the delicate membranes may result. When the specimen is ready for embedding it is amply covered with $15 \%$ celloidin and allowed to remain in the refrigerator (the lower temperature prevents excessive bubble formation) until it can be blocked. Blocking of the material is important and depends upon whether vertical or horizontal sections through the cochlea are desired. This is readily determined by such landmarks as the eminentia arcuata, and external and internal auditory meati.

Sections of large blocks are usually cut at 10 to 15 micra in thickness on a sliding microtome. Every section is numbered and kept for further study if necessary, while every tenth or every twentieth section is put aside for staining as a "tracer" series.

The nerves of the tympanic membrane were successfully stained intravitally by the use of methylene blue; Wilson, J. G., J. Comp. Neurol. and Psychol., 1907, 17, 459-468. Peripheral endings of the cochlear nerve were stained with 1:5000 methylene blue in isolated pieces of the fresh membranous cochlea removed under the dissecting microscope; Covell, W. P., Ann. Oto., Rhino., Laryngo., 1938, 47, 62-67.

The peripheral fibers of the cochlear nerve have recently been demonstrated by Fernandez, C. (Laryngoscope, 1951, 59, (in press)) using Bodian and Cajal silver Methods. The course of the fibers were traced in pieces removed by dissection and mounted on slides. A method for study of Wallerian degeneration in the cochlear nerve by use of the Swank-Davenport osmic mixture is described by Rasmussen, G. L. (Abstr., Anat. Rec., 1950, 106, 120). Ross, E. L. and Hamilton, J. W. (Arch. Otol., 1939, 29, 428-436) allowed mercurochrome to remain in the middle ear cavities of dogs for 20 minutes to 2 hours, fixed the mercurochrome in acid solution, decalcified the bones, and studied the distribution of the dye in frozen sections of the cochlea. The passage of fluorescein after arterial injection was observed by Gisselsson, L. (Acta oto-laryng., 1949, 37, 268-275) in the cochlea. Trypan blue has been utilized to ascertain the effects of trauma on scala media cells of the cochlea (Lurie, M. H., Ann. Otol., Rhin., Laryng., 1942, 51, 712-717). The capillary areas of the cochlea have been further studied in animals by Smith, 
C. (Laryngoscope, 1951, 59, (in press)). The precipitation of Prussian Blue in the small vessels or the lead chromate method of Williams, T. W. (Anat. Rec., $1948,100,115-125)$ gave satisfactory preparations.

A method for graphic reconstruction of the organ of Corti was introduced by Guild, S. R., Anat. Rec., 1921, 22, 141157. This method has been used for ascertaining damage to the organ of Corti and for measurements of the length of the organ of Corti in man by Hardy, MI., Am. J. Anat., 1938, 62, 291311. A simple technique for measuring the length of the basilar membrane is reported by Keen, J. A., J. Anat., 1939$40,74,524-527$. To study the mode of vibration of the basilar membrane Békésy, G. V., J. Acous. Soc. Am., 1948, $20,227-241$, used fresh human temporal bones and suspended fine silver crystals in the cochlear duct to visualize the transparent memarane.

Various methods of reconstruction have been employed particularly for study of development of the ear. See Bast, T. H., Arch. Otol., 1932, 16, 19-38 and others. Casts of the labyrinth have been made of a number of different materials including Wood's metal, wax, rubber and so forth. Cummins, H., J. Comp. Neurol., 1924-25, 38, 399-459 used mercury for this purpose. With monomeric methyl methacrylate (Perapex) Gray, O., J. Laryng. and Otol., 1948, 62, 308, developed a method for making an accurate anatomic cast of the labyrinth. See Endolymph.

Ear Smears-Written by Marian Pfingsten Bryan, Dept. of Otolaryngology, Washington University, St. Louis 10, Mo. September 20,1951-Cytological studies of aural exudates are valuable in helping to understand the patterns of disease pertaining to the middle ear, mastoid and external canal of the ear. The smear technique, more fully appreciated now, although stressed by Dean, L. W. (J. A. M. A., 1932, 99, 543-546), yields reliable information. When this is accurately correlated with clinical symptoms it is of diagnostic value. With repeated consecutive smears the course of an inflammatory reaction in the middle ear can be charted and the bacteria generally observed. The severity and type of infection is often indicated by the number and variety of inflammatory cells. The response of the individual, as evidenced by the number and type of phagocytes is important. The phagocytic power of the polymorphonuclear neutrophilic leucocytes along with the monocytes and other cellular forms can be evaluated.
These, with other distinguishing cytological features, are evident in the following-categories of aural disease: chronic and acute suppurative otitis media, cholesteatoma of the middle ear and mastoid, acute and chronic mastoiditis, external otitis and carcinoma.

Studies of these aural secretions have been sparsely scattered through the literature. Ts'en, Shih-Ping (China Med. J., 1926, 40, 136) reported seven aural smears with case histories and suggested that the differences in cytology were due to the duration of the discharge. He noted that in chronic cases the neutrophiles were more degenerated than in acute ones. Since then it has been found that chronic suppurative otitis media can readily be differentiated from acute suppurative otitis media by smears. The amount of fatty squamous epithelial degeneration is of importance. This type of degeneration is rarely found in acute otitis media. The cytology in these cases was studied by Dean, L. W. Jr. and Pfingsten, M. G. (Ann. Oto., Rhinol. and Laryngol., 1933, 42, 484-496). Chronic suppurative otitis media is also characterized by excessive numbers of mixed bacteria and the neutrophiles usually exhibit marked degeneration. If there is an acute exacerbation of a chronic condition, the typically chronic picture is seen plus the acute one, in which the neutrophiles are many, well preserved, and of ten exhibit phagocytic activity as evidenced by containing bacteria. In acute otitis media all gradations of monocytes are found from those characteristic of the circulating blood to other larger mononuclears, but in chronic otitis media the larger ones are rare.

In some cases of chronic otitis media an increase of eosinophiles has been observed in the exudates. Dohlman, F. G. (Nord. Med. Tidskr., 1943, 17, 224 ) worked on this problem. In 178 cases of varying types of otitis he found in 99 of them some increase in eosinophiles. Koch, Hjalmar (Acta Otolaryngol., 1947, Supp. 62) has also made a complete and detailed study concerned with the finding of eosinophiles in the ear discharges from cases of chronic otitis media. He stated that in the 210 chronic ears examined, there were 52 cases which exhibited an increase in eosinophiles. He concluded that in the eosinophile cases the healing time of the lesion was lengthened and that they were characterized by a very vis. cous secretion varying from clear glass to serum-like in appearance. Hansel, F. K., Allergy of the Nose and Para- 
nasal Sinuses; St. Louis: C. V. Mosby Co. 1936,383 pp.) believes that increase of eosinophiles in affections of the middle ear is due to the chronicity of the infection rather than to an allergic condition comparable to that which appears in the nose. Eosinophiles are frequently noted where there is a continual irritation factor. In aural polyps there may be eosinophilic infiltration in the tissue and if discharge is present eosinophiles may be seen. Proetz, A. W. (Ann. Otol., Rhin. and Laryngol., 1931, 40,67 ) reported in detail an asthmatic infant who had attacks of otitis media accompanied by asthma. The secretion from the ear did not contain eosinophiles. The problem merits careful consideration and further investigation.

Considerable significance in the past has been attached to the microscopic findings of cholesterol crystals and fatty degenerated epithelium in chronic middle ear suppurations. Some have regarded these cytological findings as diagnostic of cholesteatoma of the middle ear and mastoid. Particular care must be taken in these conditions when studying the cytology of the ear, as it has been shown by Dean, L. W., Jr. and Pfingsten, M. G. (Ann. Otol., Rhinol. and Laryngol., 1933, 42, 484-496) that characteristic cholesterol crystals and positive chemical tests for cholesterol can be found in acute otitis media and in external otitis as well as in chronic otitis media. Normal cerumen from the ear may contain more amorphous and crystalline cholesterol than cholesteatoma. It is thus important that the ear canal be thoroughly cleansed and that the exudate studied, be taken from the point of perforation of the drum membrane. It would seem that the finding of cholesterol crystals along with fatty degenerated epithelium in aural secretions is not alone diagnostic of cholesteatoma, but may be suggestive in the light of the clinical symptoms.

Cholesterol crystals show double refraction of polarized light, so are best observed in unstained direct smears of secretions with a microscope equipped with polarizer and analyzer. Since lipoid substances are the only ones, so far as is known, that doubly refract polarized light, this method is simple and convenient for detecting cholesterol in its crystalline state. When the Nicol prisms of the polarizing apparatus are crossed the field appears dark, but cholesterol crystals, when present, are brightly illuminated against the dark background. They are seen in the characteristic flat rhomboid plates, often with irregular edges. The crystals, being very fragile, may be broken into fragments lacking true crystal formation. What the actual association of the crystal formation may be to the fatty degenerated epithelial cells is not known.

Cholesterol is also evident in the secretions of infected ears and in cerumen in the form of liquid crystals observed only with a polarizing microscope. They appear as small luminous spheres with a black maltese cross superimposed on each one. The phenomenon is caused by a molecular formation of cholesterol esters that takes place in a liquid medium. The maltese crosses are the result of interference lines of light rays passing through the crystals. They are sometimes seen within large phagocytic cells. Nothing is known of their significance except that their presence indicates cholesterol.

In mastoiditis the smear is equally useful in helping to evaluate whether the condition is acute or chronic. Some consider the clinical signs and symptoms of the patient sufficient, but in these serious diseases every laboratory aid available is needed to facilitate diagnosis. In acute suppurative mastoiditis, the secretion, if there is ample opportunity for it to drain through the tympanic membrane, may contain large lipoid bone phagocytes, which hint at bone destruction. These same cells are usually found at mastoidectomy in cell pockets of diseased bone containing purulent material where the bone is actively being destroyed. They were first observed in the ear and mastoid by Pfingsten, M. G. in 1934 by using the supravital staining technique as developed by Sabin, F. R. (Bull. Johns Hopkins Hosp., 1923, 34, 277-288). Their identification was reported at that time to the clinical conference for Otolaryngology, Barnes Hospital. A complete report concerning these cells, with case histories, before and after the use of antibiotics, is to be published this year by Bryan, M. P. and Bryan, W. T. K.

Such cells are best studied in the living condition since stains and fixations dissolve the lipoid substance within the cell leaving large empty vacuoles in the cytoplasm giving it a foamy appearance. The cell membrane in either the fixed or living condition may be indistinct and irregular. The nucleus is usually eccentric and sometimes double. The cells are for the most part spherical varying in size from 20 42 microns in diameter. In comparison 
to the size of the cell the nucleus is small and ovoid, of ten obscured by the lipoid globules which completely fill the cytoplasm. Pseudopodia may be present but active motility has never been observed. They stain positively with Sudan III in the fresh condition. The stain dissolves the globules and the cytoplasm fills with the orange dye. These large phagocytes, when not filled with lipoid material, may be seen to phagocytose cellular debris, red blood cells and entire neutrophiles as well as bacteria.

All of the cellular elements should be taken into consideration in order to evaluate the inflammatory response. The display of polymorphonuclear neutrophilic leucocytes is important. It can be demonstrated that $45-65 \%$ of these cells will engulf carmine particles suspended in tyrode or neutral saline solution. If the same preparation is counterstained with dilute neutral red the identical vacuoles containing carbon will react to neutral red dye, indicating that neutral red staining is a fairly accurate criterion for judging phagocytic activity. Hesse, Herr (Ztschr. f. Hals, Nasen u. Ohrenh., 1927-1928, 18, 377-382) studied fresh exudates from middle ear suppurations and distinguished phagocytic and nonphagocytic leucocytes, but he stated that cell death occurred very rapidly. For staining he used the Synderhelmishe dye, a preparation of trypan blue and congo red. The dye has been used here and found to be very toxic for the cells. It does not give sufficient time for observation in the living state. $\mathrm{He}$ made no mention of the giant bone phagocytes.

Other cells encountered in acute mastoiditis include monocytes, lymphocytes, eosinophiles and basophiles. Many variations in the mononuclear cells are found. Besides the typical blood monocytes there are gradual transitions between these cells and the giant phagocytes. Often it is advisable to distinguish between these cells when they are not engorged with lipoid material, but yet are larger than the typical monocyte with many varying shades of neutral red vacuoles and a smaller ovoid eccentric nucleus, rather than the typical horse-shoe nucleus of the monocyte. These are termed "transitional mononuclears", or correspond in appearance to the "clasmatocytes" in the classification of Sabin, F. R., Doan, C. A. and Cunningham, R. S. (Contrib. to Embryol., 1925, 16, 127-162). The lymphocytes may show slight motility in fresh preparations and sometimes a few neutral red inclusions. If Janus Green is used the mitochondria may be observed. Eosinophiles, if seen in these secretions, may exhibit motility in the living state but are never seen to store particulate matter. In acute mastoiditis there are rarely more than a few seen. Basophile cells are also noted in many cases. They are usually few in number and their significance is not known.

In contrast to the various cells associated with active mastoiditis, there are those observed in chronic mastoiditis, namely, large numbers of degenerated neutrophiles, fatty degenerated squamous epithelial cells and masses of mixed bacteria. Bacteria are numerous along with cellular debris. Giant phagocytes are rarely found in the chronic condition unless there is an acute exacerbation of the infection. Many other cellular details require to be correlated with the symptoms of the patient in these diseases.

Cytological studies aid in differentiating the many types of external otitis. Senturia, B. H., Matthews, J. I. and Adler, B. C. (The Laryngoscope, 1950, $60,543-550$ ) have made important contributions showing that the smear gives information as to the causative agents in external otitis, whether they are bacilli, cocci or fungi. Examination of the smear is helpful in distinguishing between suppurative otitis media and external otitis (except the circumscribed type). Senturia states that the ear with a hidden tympanic perforation and a secondary diffuse external otitis may be difficult to differentiate from a diffuse external otitis with exudate covering the tympanic membrane. Cytological examination usually makes the difference clear. Otitis media with perforation shows neutrophiles, lymphocytes, mucus strands, with a few bacteria and epithelial cells. Diffuse external otitis shows a predominance of epithelial cells and bacteria, with perhaps an occasional leucocyte or mucus strand in the secretion. The most striking finding in the exudates of acute and diffuse external otitis is the absence of neutrophiles.

In regard to the early diagnosis of malignant and benign tumors of the middle ear and external canal, the cytology of the discharge should be studied carefully with the staining technique developed by Papanicolaou, G. N. (Science, 1942, 95, 438-439). This is a reliable aid in conjunction with biopsy and it is especially important if biopsy is not possible. Fortunately carcinoma of the middle ear is somewhat 
rare. House, H. P. (Ann. Otol., Rhinol. and Laryngol., 1949, 58, 789-797) reviewed the literature and reported that 201 authentic cases of the disease have been reported. He discussed two cases in which the technique of Papanicolaou aided in diagnosis and indicated, that since the majority of malignancies of the middle ear are superimposed on chronically discharging ears, the method should prove of value in the early diagnosis of carcinoma of the middle ear. Diamont, M. (Acta Otolaryng., $1941,29,77-79)$ pointed out the difficulty even with the Papanicolaou stain of differentiating clinically between granulations in chronic otitis and early malignancy. Smears, however are very useful in these cases in efforts to follow the cytology of the lesions after operative procedures. Subsequent biopsies are not always feasible. The smear technique causes no inconvenience to the patient. It is reliable in the evaluation of $x$-ray and radium therapy since the effects of such treatments are reflected in the cellular response.

It seems important, in order to observe all of the cellular details possible, to use the method of Papanicolaou as well as a good polychrome stain, such as Wright's or Hansel's, especially in the chronic ear conditions, so that the exudate can be studied in both stains. Details of the light staining with Wright's and of the use of buffer solution are described in the staining of Nasal Smears. Short drying of the slide in air, rather than flaming, is recommended for maximum cellular detail. Whenever possible, in order to complete the cytological picture, it is interesting to use the supra-vital technique on living fresh material. There are vast differences in appearance between living and stained cells so that accurate cellular differentiation becomes a complicated problem. In order to insure good results from any of the staining techniques, a fresh sampling of the discharge is imporatnt. It has often been found satisfactory to use a frontal sinus silver cannula with rubber bulb to obtain the secretion as close as possible to the point of perforation of the drum membrane. The discharge may then be released and spread evenly and thinly on a clean glass slide. At myringotomy it is taken directly following the incision of the drum membrane.

Since there are a number of reliable staining methods that are adaptable to the study of aural exudates, the knowledge gained thereby can be correlated with the clinical symptoms of the patient. This laboratory aid is important in otologic examination and stimulates an awareness of the ever changing pathological processes.

Earle, see Tissue Culture.

Ectoplasm. Cytoplasm lying immediately internal to the plasma membrane. It is usually gelled, and, being free from various formed bodies present in the endoplasm, has a clear hyaline appearance.

Egg, inoculation of hen's eggs, see Chorioallantoic Membrane. Egg of helminths, see Floatation Techniques. Transplantation of living fertilized eggs, see account of Placenta.

Ehrlich-Biondi Stain, known also as the Ehrlich-Biondi-Heidenhain mixture, is one of the classical stains.

Add 20 cc. sat. aq. acid fuchsin and 50 cc. sat. aq. methyl green to $100 \mathrm{cc}$. sat. aq. orange $\mathrm{G}$ agitating the fluid while doing so. Add 60-100 cc. aq. dest. The diluted mixture should redden slightly if a little acetic acid is added. A drop placed on filter paper should be bluish green at the center and orange at the periphery. If there is an outside red zone too much fuchsin has been used. Stain sections of sublimate fixed tissues 12-24 hrs. Do not wash in water but dehydrate quickly. Clear and mount. This stain gives beautiful results when properly employed but it is fickle. Many helpful suggestions are given in Lee, p. 179.

Ehrlich's Acid Hematoxylin. Dissolve 2 gm. hematoxylin in $100 \mathrm{cc} .95 \%$ alcohol and add; aq. dest., 100 c.; glycerin, 100 cc.; ammonium (or potassium) alum, 3 gm., glacial acetic acid, 10 cc. Ripen by exposure to air (but not dust) 2 or 3 weeks, or immediately by addition of $0.4 \mathrm{gm}$. sodium iodate.

Ehrlich's Aldehyde Reagent. 2 gms. paradimethylamino-benzaldehyde in $100 \mathrm{cc}$. $20 \%$ aq. hydrochloric acid. See Urobilin.

Ehrlich's Triacid blood stain. This, also, is one of the classic stains, now seldom used. It contains methyl green, orange $\mathrm{G}$ and acid fuchsin; but methyl green is a basic dye so that it is not made up of three acid dyes. Ehrlich explained that it is so called "because in it all the three basic groups of the methyl green are combined with acid dye-stuffs" (Lee, p. 167) with which modern chemists do not agree. Air dried smears are fixed by heat $\left(110^{\circ} \mathrm{C}\right)$ about $2 \mathrm{~min}$.; stained in triacid (Grübler) 5 min.; washed in aq. dest. until no more color is extracted and dried with smooth filter paper. Said to color neutrophile granules and leave azur granules unstained. 
Eimeria, see Coccidia.

Einarson, see Gallocyanin-Chromalum Staining of Basophilic Cell Structures.

Elacin, see Elastic Fibers.

Elastase. An elastolytic enzyme from pancreas has been reported by Baló, J. and I. Banga, Biochem. J., 1950, 46, 384387. This enzyme renders elastic tissue soluble, without the formation of amino acid residues. Consequently, it apparently depolymerizes elastin. Since elastic tissue is highly insoluble, this enzyme should prove most useful both for chemical and histological investigations.

Elastic Fibers-Written by A. I. Lansing, Dept. of Anatomy, Washington University, St. Louis 10, Mo. October 5, 1951-Viewed in fresh unstained spreads of Loose Connective Tissue these fibers are generally yellow and are more highly refractile than collagenic fibers. They are optically homogeneous, branch repeatedly to form networks, are of variable thickness and do not swell in dilute acids. Elastic fibers are resistant to both pepsin and pure trypsin and are slowly digested in commercial grade trypsin. The latter may be due to contamination by elastase, a new, relatively specific enzyme which solubilizes elastic fibers (Baló, J. and Banga, I., Biochem J., 1950, 46, 384). Elastic fibers are also solubilized by prolonged boiling in $0.25 \mathrm{~N}$ oxalic acid (Adair, G. S., Nature, 1951, 167, 605).

Elastin may also be distinguished from collagen by its amino acid composition. As indicated in the accompanying table, collagen is very rich in hydroxyproline while elastin has only small amounts. Not all elastins have the same amino acid compositions. Ligamentum nuchae (cow) differs significantly from elastin of human arteries which differ from one another. Indeed age influences the amino acid composition of elastin from human aortas.

Age influences many of the properties of elastic tissue. Senile elastic tissue of skin is often called elacin. This material is not unlike elastic tissue in old arteries. Age differences in elastic tissue may be summarized as follows:

Elastic fibers are readily demonstrated in sections by the use of one of several fairly specific stains including Weigert's Resorcin-Fuchsin, Verhoeff's Hematoxylin Stain, Unna's Orcein Method, Krajian's Congo Stain. After oxidation elastic fibers are colored red by the Schiff Reagent. Victoria blue and Basic Fuchsin also effectively stain elastic fibers.

When viewed with polarized light elastic fibers are not birefringent but become so when stretched. This would suggest that, although the fibers are optically homogeneous, they possess internal structural orientation. Elec-

Amino Acid Composition of Collagen and Elastin *

\begin{tabular}{|c|c|c|c|c|c|}
\hline Amino Acid & 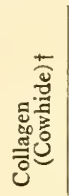 & 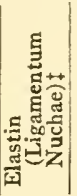 & 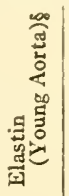 & 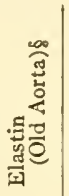 & 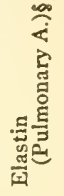 \\
\hline & \multicolumn{5}{|c|}{$g N$ per $100 \mathrm{gN}$} \\
\hline Total N. & 18.6 & 17.1 & 15.71 & 15.56 & 16.00 \\
\hline Glycine. . & 26.2 & 29.9 & 26.10 & 21.30 & 32.00 \\
\hline Alanine... & 9.5 & 18.9 & 23.18 & 21.58 & - \\
\hline Leucine. & $3.2^{2}$ & 8.7 & 4.52 & 4.76 & 4.78 \\
\hline Isoleucine. & $2.1^{2}$ & 4.0 & 2.10 & 2.33 & 2.28 \\
\hline Valine..... & 3.4 & 17.4 & 13.00 & 11.50 & 12.9 \\
\hline Phenylalanine. & 4.2 & 5.0 & 1.73 & 1.97 & - \\
\hline Tyrosine. & 1.4 & 1.61 & 1.45 & 1.76 & 一 \\
\hline Tryptophan... & 0.0 & 0.01 & 0.06 & 0.24 & - \\
\hline Serine........ & 3.4 & .82 & 0.29 & 0.70 & - \\
\hline Threonine. & 2.4 & .96 & 0.65 & 1.13 & - \\
\hline Cystine. & 0.0 & 0.15 & 0.06 & 0.10 & - \\
\hline Methionine... & 0.8 & 0.03 & 0.06 & 0.35 & - \\
\hline Proline....... & 15.1 & 17.0 & 10.10 & 9.20 & 11.0 \\
\hline Hydroxyproline. . & 14.0 & 2.0 & - & - & - \\
\hline Lysine..... & 4.5 & 0.39 & 0.49 & 1.17 & - \\
\hline Hydroxylysine. & 1.3 & - & - & - & 一 \\
\hline Arginine........ & 8.8 & 0.89 & 1.78 & 4.35 & - \\
\hline Histidine.... & 0.8 & 0.07 & 0.15 & 0.75 & - \\
\hline Aspartic acid.... & 6.3 & 0.63 & 0.38 & 1.11 & 0.62 \\
\hline Glutamic acid... & 11.3 & 2.1 & 1.83 & 3.01 & 1.75 \\
\hline Amide $N \ldots .$. & 0.66 & 0.04 & 2.90 & 2.86 & 1.51 \\
\hline Total found & 119.00 & - & 90.83 & 90.17 & \\
\hline
\end{tabular}

- Adapted in part from Gross: J. Gerontol., 1950, 5, 343 .

$\dagger$ Bowes, J. H., and Kenton, R. H.: The amino acid composition and titration curve of collagen. Biochem. J., 1948, 43, 358-365.

$\ddagger$ Neuman, R. E.: Amino acid composition of gelatins, collagens and elastins from different sources. Arch. Biochem., 1949, 24, 289-208.

\&ansing, A. I., Roherts, Eugene, Ramasarma, G. B., Rosenthal, Theodore B., and Alex, Morris: Changes with age in amino acid composition of arterial elastin. Soc. Exp. Biol. Med., 1951, 76, 714-717.

tron microscopy has so far failed to reveal internal structure. Elastic fibers partially digested with elastase do reveal the presence of intertwined fibrils.

Elastic Properties, see Surface Tension. Elastica-Trichrome Stain. In order to demonstrate elastic fibers with equal clearness to the smooth muscular and collagenic fibers, especially in the walls 
of blood vessels, a useful combination of Weigert's elastic tissue stain and Masson's trichrome stain has been worked out by Mendeloff, J., Am. J. Clin. Path., 1943, Tech. Suppl. 7, 65. Deparaffinize sections in usual way, wash thoroughly in water and stain in Weigert's Resorcin Fuchsin mixture for $60 \mathrm{~min}$. Wash quickly in Acid Alcohol, dehydrate and differentiate in abs. alc. till section is only faintly red. Pass

\section{Some Properties of Human Arterial Elastin}

\begin{tabular}{|c|c|c|}
\hline & Young & Senile \\
\hline $\begin{array}{l}\text { Physical } \\
\text { appear- } \\
\text { ance }\end{array}$ & $\begin{array}{l}\text { Straight, anasto- } \\
\text { mosing threads or } \\
\text { ribbons } \\
\text { Glistening, refrac- } \\
\text { tile, water-clear }\end{array}$ & $\begin{array}{l}\text { Frayed, fragmented, } \\
\text { thin strands and } \\
\text { granules } \\
\text { Tend to clump, yel- } \\
\text { low, dull }\end{array}$ \\
\hline Tinctorial & $\begin{array}{l}\text { 1. Not stained by } \\
\text { hematoxylin } \\
\text { 2. Red color with } \\
\text { Congo Red } \\
\text { 3. Resorcin-fuchsin, } \\
\text { orcein "Van } \\
\text { Giesen" positive }\end{array}$ & $\begin{array}{l}\text { 1. Take up hema- } \\
\text { toxylin } \\
\text { 2. Yellow - orange } \\
\text { with Congo Red } \\
3 \text { Stain more } \\
\text { densely with re- } \\
\text { sorcin, etc. }\end{array}$ \\
\hline Chemical & $\begin{array}{l}\text { 1. Mineral-free } \\
\text { 2. Phosphorus-free } \\
\text { 3. Amino acids: } \\
\text { a) trace of as- } \\
\text { partic acid } \\
\text { b) } 1 \% \text { glutamic } \\
\text { acid }\end{array}$ & $\begin{array}{l}\text { 1. Severely mineral- } \\
\text { ized. Contains as } \\
\text { much as } 14 \% \text { cal- } \\
\text { cium } \\
\text { 2. Large amounts of } \\
\text { phosphorus } \\
\text { 3. Amino acids: } \\
\text { a) appreciable } \\
\text { amounts of as- } \\
\text { partic acid } \\
\text { b) } 4 \% \text { glutamic } \\
\text { acid }\end{array}$ \\
\hline Physical & $\begin{array}{l}\text { Dry isolated elas- } \\
\text { tin, has specific } \\
\text { gravity less than } \\
1.30\end{array}$ & $\begin{array}{l}\text { Dry isolated elastin, } \\
\text { has specific gravity } \\
\text { greater than } 1.30\end{array}$ \\
\hline
\end{tabular}

through $70 \%$ alc. to aq. dest. and stain in Harris' Alum Hematoxylin $8 \mathrm{~min}$. Differentiate in water $5 \mathrm{~min}$. Stain in Ponceau acid fuchsin mixture (see Masson's Trichrome) $5 \mathrm{~min}$. Wash thoroughly and place in $3 \%$ aq. phosphotungstic acid, $10 \mathrm{~min}$. Wash again thoroughly in water and stain with light green. Transfer directly to $1 \%$ acetic acid, $3 \mathrm{~min}$. Do not wash but dehydrate, clear and mount in Gum Damar. Elastic tissue, blue-black; smooth muscle, red; collagen, green.

Elastin, see Elastic Fibers.

Electric Tissues of fishes, methods for are given by Dahlgren (McClung, 1950, p. 343).
Electrical Resistance and capacity or Impedence. By employing alternating currents of varying frequencies figures for apparent resistance and capacity can be obtained. Red cells, yeast cells, ova etc. have been investigated. The technique is not microscopic but the data have an important bearing on structure. In view of the wide variety of cells studied it is interesting, as Danielli remarks (Bourne, p. 42), that a definite pattern should emerge of a cell plasma membrane only $10^{-6}-10^{-7} \mathrm{~cm}$. in thickness corresponding to a specific resistance of $10^{10}-10^{11} \mathrm{ohms}$.

Electrodes. See the several varieties described in full with literature references and diagrams by Glick, pp. 183-188.

Electromagnet Technique to determine elasticity (Heilbroun, A., Jahrb. wiss. Bot., 1922, 61, 284), employed by Seifriz, W. and Hock, C. W. (Paper Trade J., $1936,102,36)$ and described by Chambers, R. W. and Kopac, M. J. in McClung's Microscopical Technique, 1950, p. 542 .

Electron Microscopy-Details originally provided by Dr. W. L. Simpson; revised by Dr. T. B. Rosenthal, Dept. of Anatomy, Washington University, St. Louis. June 6, 1951.

1. Transmitted electron beam type. The relationship of the wave length $(\lambda)$ of light employed and to the numerical aperture (N.A.) of a lens system as expressed in the relation R.P. equals

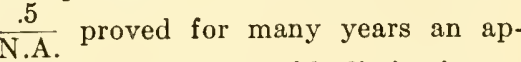
parently insurmountable limitation to the biologist's desire to investigate directly minute structure of cells and tissues. Even when ultraviolet light of $2250 \mathrm{~A}$ was employed the limit of resolution was $0.08 \mu$ in a system of N.A. 1.40. With visible light the limit was approximately $0.2 \mu$. On the assumption that the angle of visual acuity is 1 minute of arc, the greatest magnification that was practical with visible light ranged from 1750 to 2100 times. There is, of course, no limitation to the actual magnification that may be achieved. Increases beyond the limit mentioned, however, do not reveal new structures. As long as this was true there seemed no hope of direct confirmation of the amazing findings made possible by such new methods as $\mathrm{x}$-ray diffraction, ultracentrifugation studies, chemical studies of virus structure, and polarized light methods.

Small wonder is it then that the biologist has grasped with enthusiasm at the possibilities of visualizing ultramicroscopic structure by means of devices that have grown from the fertile 
field of electron optics. Of most general interest is the electron microscope. With this instrument, using the same equation for resolving power, it should be possible to reach a resolution of at least $0.001 \mu$. Thus, an improvement of at least 200 times over the limit with visible light might be achieved. The practical limit on magnification has been placed at from 70,000 to 100,000 times.

Historically the electron microscope is now twenty-four years old. Busch described the first such instrument using magnetic lenses (Busch, H., Arch. f. Elektroteknik, 1927, 18, 583-594). Though many improvements were made in design it was not until 10 years later that the instrument reached the point of being of practical use. Chiefly through the work of Ruska and Borries (numerous papers, 1934-1940) the instrument was developed to the state that it is in at present. In this country an excellent instrument, capable of giving high resolution has been developed and commercially marketed by the Radio Corporation of America. The apparatus is described by Zworykin, V. K. (Science, 1940, 92, 51-53). In this instrument electrons emitted from a hot wire filament are accelerated by a potential of 30,000 or more volts. This beam is condensed and passed through the object which is carried on a wire screen supported nitro-cellulose film. The transmitted electron beam is focussed in a greatly enlarged image by means of two magnetic lenses. The image can be seen on a fluorescent screen or photographed on a sensitive plate.

The conditions under which good results are obtainable are rather stringent. The ideal object must be very small, or capable of being minutely divided without losing its identity; it must maintain its form on drying in vacuum; and it must be rigid enough to resist the heat and disruption caused by exposure to the electronic bombardment. Relatively few biological structures fall into this category; hence early studies were confined to bacteria, viruses, connective tissue fibers, blood cells, spermatozoa, etc.

The great need in histological investigation with the electron microscope has been a method for uniformly cutting tissues no thicker than $0.1 \mu$ and about $1 \mathrm{~mm}^{2}$ or larger in area. With these dimensions a section will yield acceptable electron micrographs. Such a method scems to have been found in a modification of the conventional microtome whereby the advance of the block is reduced about tenfold, either mechanically (Baker and Pease,
J. Appl. Phys., 1949, 20, 480) or by means of a thermal expansion device (Newman, Borysko, and Swerdlow, Anat. Rec., 1949, 105, 267). The tissue must be embedded in celloidin or plastic, but a special blade is probably necessary for the best results. Since 1950 publications have appeared with electron micrographs of nearly all tissues of importance. See Excerpta Med. ica, Sect. I, for listings and abstracts.

Histologists have also borrowed special methods from metallurgists for investigation of hard, opaque structures like bone, tooth, hair, keratinized epithelium, etc. These methods involve the casting of very thin replicas of surface details. Additional contrast is gained by the ingenious process of "shadow casting", either on the original specimen or on its replica. A very thin layer of metal is deposited over the object in a vacuum by evaporation from a hot filament. When done at an acute angle, the elevated portions of the object shadow the depressed areas, thus giving a striking three-dimensional picture (Williams and Backus, J. Appl. Phys., 1949, 20, 98). Methods have also been developed for true stereoscopic images (Heidenreich and Matheson, J. Appl. Phys., 1944, 15, 423). See Shaddow Casting.

The old technique of selective staining has been combined with this newest of procedures in histology. It has been shown that the salts of heavy metals, such as osmium and phosphotungstate, are deposited in a highly localized way on certain protein structures, e.g. muscle fibrils, thus enabling visualization of practically molecular details (Hall, Jakus, and Schmitt, J. Appl. Phys., 1945, 16, 459).

With the proper selection and combination of histological methods now on hand the electron microscopist should have little difficulty in studying any problem in morphology. A practical handbook for the guidance of novice and expert has been prepared by the Royal Microscopical Society: "The Practice of Electron Microscopy", edited by D. G. Drummond and published as Part I of vol. 70, 1-141, 1950 of the J. Roy. Micro. Soc. Papers dealing with details of manipulation are to be found regularly in the Journal of Applied Physics.

2. Emission electron type. The earlicst description of an clectron microscope in this country was of an entirely different type from the new R.C.A. microscopes that give such prodigious magnifications. McMillan and Scott (J. H. and G. H., R.S.I., 1937, 8, 288- 
290) published an account of an electron microscope of simple design that used as a source the electron emission of heated sections of tissues. These were accelerated by a potential of 1000 to 2000 volts, focussed by a magnetic lens onto a fluorescent screen. An improved design (Scott, G. H. and Packer, D. M., Anat. Rec., 1939, 74, 17-29) makes possible magnifications of at least 150 times. By certain modifications the magnification can be increased considerably. It is feasible with this instrument to obtain photographs that record the precise localization of calcium and magnesium salts in tissues. Scott and Packer (ibid, 31-45) showed that the calcium and magnesium of skeletal muscle was confined almost entirely to the muscle fiber's themselves, and that in contracted fibers a great concentration of magnesium appeared in the contraction nodes.

Tissues to be studied with this technique must be preserved in a manner that permits no redistribution of minerals. The satisfactory method is that of Altmann-Gersh.

Electron microscopic technique supplements histospectrography by precisely locating certain elements within tissues and is very useful in conjunction with the technique of microincineration as a means of identifying certain components of the ash seen in sections.

Electrophoresis. Most particles suspended in water carry electricity. If placed in an electric field those possessed of positive charge move toward the cathode and those with a negative charge toward the anode. Obviously therefore the nature of the charge and the speed of movement can be determined by microscopic study of particles suspended in fluid in what is known as a micro-electrophoresis cell. Types of cell and precautions to be observed in their use are described by Moore, D. H. and Abramson, H. A. Glasser's Merlical Physics, 403-407. Their account of the "moving boundary" method of electrophoresis and of the Tiselius apparatus is clear and to the point. This latter method, in contrast witl the microscopic one, affords a technique of great accuracy and sensitivity for separating concentrations and purifying submicroscopic components in blood serum and other complete liquids.

Eleidin (G. elaia, oil) gives to the stratum lucidum its clear, glassy appearance. It may be a dissociation product of keratohyalin. There has been no great improvement on the specificity of the older methods. Mallory (p. 260) gives the method of Buzzi (1889), first cau- tioning that fixation must be in formalin, Orth's or Bouin's fluid. Stain frozen sections of $10 \%$ formalin fixed tissue in sat. aq. picric acid (approximately $1.2 \%$ ) 5 min. Rinse in aq. dest. and counterstain for $1 \mathrm{~min}$. in 1\% aq. nigrosin. Wash in water and then in $95 \%$ alc. (Skip absolute) Clear in terpineol or origanum oil. Mount in balsam: keratin, bright yellow; eleidin, blue black. Ranvier's Picro-Carmine gives a fine red staining of eleidin. See finger Nails.

Elementary Bodies are the smallest particles of viruses. Those of certain viruses are large enough for direct microscopic examination in suitably stained preparations which usually show also the larger Inclusion Bodies if these are present. Various methods designed for Rickettsia are usually satisfactory. Many special techniques have been proposed of which 2 follow :

1. Methyl violet or Victoria blue for smears (Gutstein, M., J. Path. \& Bact., 1937, 45, 313-314). Dry smears on perfectly clean slides in air or incubator. If necessary remove excess protein by rinsing in physiological saline solution followed by aq. dest. Fix in methyl alcohol $1 \mathrm{hr}$. Stain in either of 2 ways: (1) Place slide in Petri dish. Mix equal parts $1 \%$ aq. methyl violet and $2 \%$ aq. $\mathrm{NaHCO}_{3}$. Filter immediately onto the slide, cover dish and incubate at $37^{\circ} \mathrm{C} \cdot 20-30 \mathrm{~min}$. Rinse in aq. dest., dry and mount in cedar oil or liquid paraffin. Elementary bodies light violet. (2) Same except filter onto slide equal parts (a) Victoria blue 4R $1 \mathrm{gm}$., alc. (abs.) 10 cc. and aq. dest. $90 \mathrm{cc}$. and (b) $0.02 \% \mathrm{aq}$. $\mathrm{KOH}$ and leave at room temperature over night. Elementary bodies of vaccinia and other viruses dark blue.

2. Methyl blue acid fuchsin for sections (Nicolau, S. and Kopciowska, L., C. r. Acad. d. Sci., 1937, 204, 1276-1278). Fix in alcoholic Bouin's fluid. Stain 4-5 micron paraffin sections $30-60 \mathrm{~min}$. in: methyl blue (Grübler) 1.5 gm., aq. dest. 65 cc., methyl alcohol 35 cc., glycerin 5 cc., $3 \%$ aq. oxalic acid 5 cc. Wash well in aq. dest. and change to absolute alcohol. Stain $20 \mathrm{~min}$. in: acid fuchsin $1.5 \mathrm{gm}$., aq. dest. $100 \mathrm{cc}$., $3 \%$ aq. oxalic acid 2 cc. Wash directly in absolute alcohol and mount in the usual way. Small particles in cells associated with following viruses: herpes, Borna, Zoster, rabies and pseudo-rabies are stained bright red.

A summary of methods for demonstrating elementary bodies is given by Seiffert, G., Virus Diseases in Man, Animal and Plant. New York: Philosophi- 
cal Library, Inc., 1944, 332 pp. Under favorable conditions some kinds of of elementary bodies are visible at high magnification unstained by both direct and dark field illumination. Supravital stains such as brilliant eresyl blue, neutral red, methylene blue and azur II are recommended. Before staining smears, fixed in a variety of ways, pretreatment with $2.5 \%$ aq. potassium permanganate or $2 \%$ aq. chromic acid is advised. Giemsa stain gives good results but the methods of Paschen, Morosow and Herzberg are suggested by Seiffert. The fluorescence technique of Hagemann consists of staining thin air dried smears with $1 \mathrm{gm}$. primuline dissolved in $1000 \mathrm{cc}$. aq. dest. $+20 \mathrm{cc}$. pure phenol for 15 sec. washing in aq. dest. and observation in ultraviolet light by fluorescence microscope.

New methods for the collection and purification of elementary bodies permit their direct examination at very high magnifications with the electron microscope (von Borries, E. G., Ruska, E. and H., Klin. Woch., 1938, 17, 921 ; Green, R. H., Anderson, T. F., and Smadel, J. E., J. Exp. Med., 1942, 75, 651-656) and their chemical analysis for vitamin catalysts, copper and enzymes (Hoagland, C. L., Ward, S. M., Smadel, J. E., and Rivers, T. M., J. Exp. Med., 1942, $76,163-173)$. See fluorescence of elementary bodies (Turevich, N. I. abstracted in Stain Techn., 1941, 16, 182.)

Ellipsin is structure protein of cells. Methods for its isolation from liver cells of rabbit and guinea pig by grinding fresh tissue, washing, centrifugation and so on are fully described by Bensley, R. R. and Hoerr, N. L., Anat. Rec., 1934, 60, 251-266.

Embedding, see Imbedding.

\section{Embryo Chick, see Chorioallantoic Mem-} brane.

Embryological Methods. In general the techniques which give good results with adult tissues are also satisfactory for embryos; but there are differences as for example in silver impregnations. Moreover greater care is necessary to avoid too sudden changes in the fluids used. Helpful suggestions are given in McClung, pp. 279-286. Application of trichrome staining methods to embryos (Baxter, J. S., J. Anat., 1940-41, 75, 137-140). See demonstration of Cartilaginous Skeleton, Ossification and Spalteholz method. Technique for handling chick embryos (Adamstone, F. B., Stain Techn., 1931, 6, 41-42). Block staining of nervous tissue of embryos with silver (Davenport, HI. A., Stain Techn., 1934, 8, 143-149).
Emission Electron Microscopy, see Electron Microscopy.

Enamel (dental). This can best be studied in ground sections of Teeth. 1. Cuticle. Wash and brush tooth in tap water. $4 \%$ neutral formalin, 24 hrs. Wash tap water, $24 \mathrm{hrs}$. Mallory's anilin blue $(0.5 \%$ aq.) 24 hrs. Again wash and brush in tap water. $10 \%$ aq. hydrochloric aeid, $10 \mathrm{~min}$. As enamel is dissolved delicate opaque white membrane appears. Tease membranc off onto slide coated with egg albumen (AlbumenGlycerin). Blot with filter paper. $5 \%$ aq. sodium thiosulphate or bicarbonate $10 \mathrm{~min}$. Wash in tap water $10 \mathrm{~min}$. Dehydrate in alcohols, clear in xylol and mount in gum damar (McClung, p. 371 ).

2. Rods. Macerate tooth in $5-10 \%$ aq. hydrochloric acid for $24 \mathrm{hrs}$. Remove a little softened enamel and examine (McClung, p. 372). See Chase, S. W. , Anat. Rec., 1927, 36, 239-258.

3. Organic Matrix. Boedeker's method abbreviated from McClung (p. 372). Dehydrate small piece (0.5$1 \mathrm{~mm}$. thick), free from dentin, through alcohols $10 \mathrm{~min}$. each. Methyl alcohol 1-2 hrs. Decalcify in celloidin mixture (parlodion, DuPont) made by dissolving sufficient in methyl alc. C.P. to give thick syrupy solution. To $150 \mathrm{cc}$. of this add drop by drop constantly stirring nitric acid C.P., 10 ec. + methyl alc. 40 cc. Keep tissue in this mixture in glass dish with air tight cover. Organic matrix appears as brown, spongy material in 10-12 hrs. care being taken to leave the dish stationary. After decalcification is completed, 2-7 days, uncover and permit celloidin to harden. Cut out specimen with narrow margin of celloidin. 70 and $40 \%$ alc. $1-2$ hrs. each. Aq. sol. alum, 24 hrs. Running water, 6-12 hrs. Ascending alcohols to $95 \%$ 1-2 hrs. each. Anilin oil, 6-12 hrs. (becomes brown and transparent). Equal parts anilin oil and chloroform, 6-12 hrs. Imbed in paraffin not over $52^{\circ} \mathrm{C}$. Mount $3-10 \mu$ sections, dry and treat with xylol $3 \mathrm{~min}$. Dissolve celloidin in ether-alcohol. Abs. alc. 1 min. Descending alcohols to water. Stain in Iron Hematoxylin.

Improved results may be obtained by slow decalcification and concurrent fixation as described by Sognnaes, J. Dental Res., 1949, 28, 558-564. Teeth are placed in celloidin bags filled with $10 \%$ formalin and immersed in a solution of $5 \mathrm{gm}$. trichloroacetic acid, $2 \frac{1}{2} \mathrm{gm}$. potassium bichromate in $100 \mathrm{cc}$. distilled water. 'Ten centimeter of formaldehyde is added to the solution. Decalcification of enamel is completed after 
one week in a refrigerator at a temperature of $1^{\circ} \mathrm{C}$. Decalcification of dentin is completed by placing the bag in $5 \%$ trichloroacetic acid. Not more than two weeks should be required. Washing and dehydration in ethyl alcohol and butyl alcohol is carefully accomplished with tooth still in the bag. Imbed in paraffin, section and stain by ordinary methods. Constant pressure during decalcification may further improve results (Sognnaes, R. F., J. Dental Res., 1948, 27, 609-622).

4. Cape-Kitchin celloidin decalcification method. Cut DuPont's parlodion into small cubes and dissolve in acetone free methyl alcohol making thick solution. To 200 cc. add 90 cc. methyl alcohol constantly stirring and 9 cc. nitric acid, sp.gr. 1.42. Follow decalcification of enamel in this mixture between crossed nicols of polarizing microscope with $24 \mathrm{~mm}$. objective. Double refraction disappears with decalcification (Bödecker, C. F., J. Dent. Res., $1937,16,143-150)$.

5. Permeability. When the apex of a tooth is immersed in strong alcoholic solution of fuchsin $+\mathrm{NaCl}$ the enamel becomes stained ( $v$. Beust, T., Dental Cosmos, 1912, 54, 659). Another way is to test for penetration of lead, boron and other easily recognizable chemicals (Howe, P. R., Dental Cosmos, 1926, 68, 1021-1033). After intraperitoneal injections of trypan blue blue coloration can be observed in developing enamel only (not adult) as well as in dentin of dogs (Gies, W. J., J. Nat. Dent. Assoc., 1918, 5, 529-531). Marshall (J. S., J.' Dent. Res., 1921, 3, 241-255) employed Naphthamine brilliant blue similarly as a vital stain. See Dentin, vital staining. A "Triple Embedding" technique is described by Brain, E. B., J. Roy. Micr. Soc., 1950, 70, 313-316.

Endamoeba, see Entameba.

\section{Endocervical Smears, see Papanicolaou} Techniques.

Endolymph. To demonstrate its circulation employ method used by Guild, S. R., Am.J. Anat., 1927, 39, 57-81. Introduce solution of potassium ferrocyanide and iron ammonium citrate into cochlear ducts of living guinea pigs under anesthesia. Kill at intervals up to $48 \mathrm{hrs}$. Excise tissue and fix in acid fluid which precipitates Prussian Blue wherever the solution has circulated.

Endometrial Smears, see Papanicolaou Techniques.

Endospore stain for bacteria in blood smears. Smear, air dry and fix by flaming 3 times. $5 \%$ aq. malachite green $5 \mathrm{~min}$., wash in tap water $10-20$ sec. $0.5 \%$ aq. safranin, 10 sec., wash quickly, dry and examine
(Bruner, D. W. and Edwards, P. R., J. Lab. \& Clin. Med., 1939, 25, 543-544).

Enrichment techniques, see Concentration.

Entameba. Craig (p. 35) gives a useful table of diagnostic features of intestinal amebae in man; also, on p. 55, a list of objects that may be mistaken for amebae in unstained and stained preparations; and details as to media for cultivation of which the Boeck and Dobohlav media and the simpler Craig media are the most helpful.

This genus includes $E$. histolytica, the cause of amebic dysentery and $E$. coli and $E$. gingivalis, two apparently harmless commensals. The technique is essentially the same for all three. In searching for $E$. histolytica or $E$. coli take a small amount of fresh feces, mix with physiological saline solution and examine directly. Recognize amebae by large size and movements if slide is kept warm. E. histolytica frequently contains erythrocytes. Mallory (p. 296) advises mixture with Gram's Iodine solution to demonstrate glycogen if present, or mixing with drop $1-2 \%$ formalin, then treatment with drop $2 \%$ acetic acid and coloration with 1 drop $1 \%$ aq. neutral red. E. gingivalis is to be found in decayed teeth. Only $E$. histolytica extensively invades tissues.

1. To make permanent smear preparations (Mallory, p. 296) fix thin smear while moist in $95 \%$ alcohol, 1 part, and sat. aq. corrosive sublimate, 2 parts, for $15 \mathrm{~min}$. Wash for few sec. in water and cover with $1 \%$ alcoholic iodine for $3 \mathrm{~min}$. Wash in aq. dest. until iodine color is extracted. Wash again and stain with Phosphotungstic Acid Hematoxylin, $30 \mathrm{~min}$. Wash in water, dehydrate in 95 and abs.alcohol, clear in xylol and mount in balsam. Nuclei and ectosarc, deep blue; cytoplasm, bluish.

2 . To stain differentially in sections (Mallory, p. 297). Fix in 95\% or abs. alc., and make paraffin or celloidin sections. Stain in $0.25 \%$ aq. thionin $3-5$ min. Differentiate in $2 \%$ aq. oxalic acid, $\frac{1}{2}-1 \mathrm{~min}$. After washing in water, dehydrate in $95 \%$ and abs. alc. Clear in xylol and mount in balsam, except for celloidin sections which require clearing in terpineol, or origanum oil, after $95 \%$ alc. Nuclei of amebae brownish red, those of all other cells, blue. See Iodine-Eosin stain and Walker's Method.

Enterochromaffin Cells. Perhaps the best technique is Bodian's protargol method as described by Dawson, A. B. and Barnett, Julia, Stain Techn., 1944, 19, 115-118. For the influence of pilocarpin on enterochromaffin cells see Hamperl, H., Ztschr. f. Mikr. Anat. 
Forsch., 1925, 2, 506-535. See Small Intestine.

Entomological Techniques, see Mosquito, Ticks, Insects, Arachnids, Parasites.

Enzymes-Written by E. W. Dempsey, Dept. of Anatomy, Washington University, St. Louis. February 26, 1951Their name is legion. At present only a few can be localized with any degree of histological precision, but the number of histochemical methods available is increasing rapidly. There are no better examples of felicitous association between histological and biochemical methods. Four principal kinds of techniques are employed for localization: (1) By spectrographic identification in the tissues-especially the metal-containing Cytochromes and Peroxidase. (2) By close comparison of enzymic activity with cellular composition of tissues. See Glick for detailed methods, derived from the LinderstrømLang procedures-Amylase, Pepsin, Peptidase, Esterase, Protease, Cholinesterase, Lipase, Urease, Carbonic Anhydrase, etc. (3) By separation of cellular components, such as nuclei, granules and mitochondria, from homogenates of tissue. Such separation is accomplished by differential flotation or centrifugation, and is followed by estimation of the enzymic activity of the purified component fractions. Arginase, Adenylpyrophosphatase, Phosphatase, etc. have been investigated by such methods. (4) By the development of characteristic insoluble products within tissues or cells-Aldolase, Acid and Alkaline Phosphatase, Cholinesterase, Cytochrome Oxidase, Dehydrogenase, Dopa Oxidase, Esterase Glucuronidase, Lipase, Peroxidase, Phosphamidase, Sulfatase.

Enzymes are coming into their own as technical tools. Purified enzymes may be used to destroy certain tissue components. Ribonuclease selectively destroys basophilic substances in cytoplasm and nucleus, Desoxyribonuclease similarly attacks nuclear chromatin. Hyaluronidase solubilizes certain metachromatic ground substances, whereas other mucoid materials react only with more potent Mucinases. The connective tissue fibers have long been characterized by their digestibility in Pepsin and trypsin, and recently an Elas. tase has been prepared. A purified Collagenase has been reported. The solubility of glycogen in solutions of salivary Am ylase is an old histochemical procedure. The effects of Lysozyme and other enzymes on the capsules of pneumococci and upon the Gram stain has been summarized by DuBos (The
Bacterial Cell, Harvard Univ. Press, 1945).

Eosin B or bluish (CI, 771)-eosin BN, BW, or DHV, eosin scarlet, eosin scarlet B, imperial red, nopalin G, saffrosin, scarlet J, JJ, V-Dibrom derivative of dinitro-fluorescein. Chemistry of (Holmes, W. C., Melin, C. G. and Paterson, H. R., Stain Techn., 1932, 7, 121-127).

There are several fluorescein dyes and guidance may be needed in the choice of the one best suited for a particular purpose. Conn, H. J. and Holmes, W. C., Stain Tech., 1926, 1, $37-95 ; 1928,3,94-104$ have made a study of color, acidity and chemical structure and Conn ( $p .145$ ) gives further data. Their color increases in depth in this order: eosin $\mathrm{Y}$, ethyl eosin, eosin B, erythrosin B, phloxine and rose bengal. This increase in color is proportional to increase in number of hologen atoms. Their acidity increases in a different order : rose bengal, phloxine, erythrosin, eosin $\mathrm{Y}$ and eosin B. (1) When the eosin is to follow in alcoholic solution a basic dye always in aqueous solution (cf. bematoxylin) the more acid and lighter colors are recommended (eosin Y, ethyl eosin and eosin B. (2) When it is to precede in aq. solution a basic dye (cf. methylene blue) also in aq. solution, use phloxin or erythrosin (see phloxine-methylene blue).

Eosin 10B, see Phloxine B.

Eosin BN, BW, or DHV, see Eosin B or bluish.

Eosin J, see Erythrosin, bluish.

Eosin-Methyl Blue, see Mann's.

Eosin-Methylene Blue has been employed in many combinations for years. But when the acid dye is applied first, phloxine is preferred to eosin. See therefore Phloxine Methylene Blue.

Eosin-Orange G-Toluidine Blue for bone marrow, spleen and connective tissue (Dominici, M. C. rend. Soc. biol., 1902, 54, 221-223). Stain eosin-orange G (eosin B. A. of Hollborn or eosin yellowish of American manufacturers $0.5 \mathrm{gm}$.; aq. dest., 100 cc.; orange G. 0.5 gm.) 7 min. Rinse quickly in aq. dest. Counterstain in $0.5 \%$ aq. toluidin blue 20-30 sec. Rinse again aq. dest. Differentiate in $95 \%$ alc., dehydrate in abs., clear in xylol and mount in balsam. Instead of eosin, $0.5 \%$ aq. acid fuchsin gives a little sharper contrast. In place of toluidin blue $0.1 \%$ Azur A can be employed to advantage. Phloxineorange $\mathrm{G}$ can be tried as a substitute for eosin-orange G. (phloxine $0.12 \mathrm{gm}$., aq. dest. 100 cc., orange G, $0.3 \mathrm{gm}$.). 'The crucial point is the differentiation in $95 \%$ alc. This should be quickly checked 
under the microscope until the time has been determined.

Eosin Scarlet, see Eosin B or bluish.

Eosin Scarlet B, see Eosin B or bluish.

Eosin Y or yellowish (Cr, 768). Tetrabrom fluorescein with some mono- and dibrom compounds. This is the usual kind of eosin employed. Eosin Y and thionin as substitute for Wright's stain (Saye, E. B., Am. J. Clin. Path., Tech. Suppl., 1943, 13, 12).

Eosinophile Leucocyte (acidophilic or coarsely granular leucocyte). Can easily be examined while still living in mounts of fresh blood. The dark field is useful. Most frequently studied in Blood Smears, which see. Mitochondria are readily stainable with Janus Green. For occasional presence of basophile granules and pigment see Downey, H., Folia Haemat., 1915, 19, 148-206. Techniques for rapid experimental increase of eosinophiles in circulating blood are described by Banerji, N., Am. J. Med. Sci., 1933, 186, 689-693; Chillingworth, F. P., Healy, J. C. and Haskins, F. E., J. Lab. and Clin. Med., 1933-34, 19, 486-494; Hajos, K., Németh, I., and Enyedy, Z., Zeit. f. d. ges. Exper. Med., 1926, $48,590-592$. Variations and errors in eosinophile counts of blood and bone marrow are described by Best, W. R. and Samter, M., Blood, 1951, 6, 61-74.

Epidermis. This can be studied in situ with the dermis, see Skin, or it can be examined in 3 ways apart from the dermis.

1. Isolated pieces. Examination of scrapings of the epidermal surface is of limited usefulness in special cases. To cut away a few of the deeper cells, separate them by teasing and to study them in the still living state with or without supravital stains is not particularly helpful. But their microdissection is capable of giving important data on cellular consistency and connections (Chambers, R. and deRenyi, G., Am. J. Anat., 1925, 35, 385-402 and Thanhoffer, L., Zeit. f. Anat. u. Entw., 1933, $100,559-562)$. Their cultivation is possible, see Tissue Culture.

2. Whole mounts for microscopic study (Cowdry's Histology, p. 530). Place excised fresh skin in $1 \%$ acetic acid in ice box for 12-36 hrs. depending upon size, age and region. Wash in tap water, 5 min. Pin skin down with epidermis up and cover with water. Strip off epidermis as a compete sheet. Wash in aq. dest., 5 min. Stain in Harris' hematoxylin, $20 \mathrm{~min}$. Wash in aq. dest., 1 min. Differentiate in 50 cc. $70 \%$ alcohol plus 3 drops hydrochloric acid until epidermis becomes light pink color.
Treat with 50 cc. aq. dest. plus 6 drops ammonia until it becomes blue. Wash in aq. dest. 5 min. several changes. Dehydrate in 50, 70, 95 and 2 changes of absolute alcohol, $10 \mathrm{~min}$. each. Clear in 2 changes xylol, $1 \mathrm{hr}$. each and mount in balsam inner side up.

If the skin is hairy, before excising it, remove hair with scissors and electric razor or depilatory solution. Hair follicles and sebaceous glands, unless particularly large, generally remain attached to the epidermis, but the coiled bodies of the sweat glands are too deeply situated to come off with it. Consequently only their straight ducts are to be seen. Before dehydration, in the above technique the sebaceous glands can be sharply counterstained with Sudan III.

Such whole mounts of epidermal sheets are of value insofar that their study gives a concept of the morphology of the epidermal covering of the body which can be obtained in no other way. For the counting of mitoses they are far better than sections and have been extensively employed for this purpose by Dr. Cooper and her associates in The Barnard Free Skin and Cancer Hospital. See her latest paper (Cooper, Z. K. and Reller, H. C., J. Nat. Cancer Inst., $1942,2,335-344)$. Since the mucous membrane covering the nasal septum can be similarly prepared as a whole mount it is likely that the method may be of service in the study of other sheets of epithelial cells.

3. Sheets of epidermis for chemical analysis. Until very recently the handicap experienced in chemical analysis of the skin has been the difficulty of separating epidermis and dermis by themselves for analysis. All data on the epidermis are of doubtful value because variable amounts of dermis have been included. The method of obtaining pure epidermis by dilute acetic acid separation is unsatisfactory for numerous reasons. Baumberger, J. P., Suntzeff, V. and Cowdry, E. V., J. Nat. Cancer Inst., 1942, 2, 413-424 have discovered that dilute alkali will serve as well as dilute acetic but this also is objectionable from the chemical point of view. They therefore advance a heat method. Place excised skin with dermis down on warm plate such as is used for mounting paraffin sections. Apply temperature of $50^{\circ} \mathrm{C}$. for $2 \mathrm{~min}$. which loosens the epidermis so that it can be easily pushed off with a blunt instrument. Separation is more difficult when temperature is over $51^{\circ} \mathrm{C}$. Epidermises removed in this way for a time continue to consume oxygen and 
are very suitable for chemical analysis. They have been used for epidermal iron and ascorbic acid by Carruthers, C. and Suntzeff, V., J. Nat. Cancer Inst., 1942, $3,217-220$, and for total lipid-protein nitrogen ration by Wicks, L. F. and Suntzeff, V., 3, 221-226.

Epinephrin (adrenin, adrenalin), hormone of adrenal medulla.

Erbium, see Atomic Weights.

Erhlicki's Solution. Potassium bichromate, 2.5 gm.; copper sulphate, 1 gm.; aq. dest., 100 cc. Used for hardening nervous tissues.

Erie Fast Red F D (CI, 419) of NAC is a direct disazo dye of light fastness 3 to 4 . Resembles Congo red insofar that washing in water, or in $95 \%$ alcohol, takes all color out of paraffin sections. In alkaline solutions it colors blue-green algae deep red to reddish brown (Emig, p. 40 ).

Erie Fast Rubine B conc. A sulfonated azo dye. For formula nad influence on mouse tumors, see Stern, K., Cancer Res., 1950, 10, 565-570.

Erie Fast Yellow WB, see Titan Yellow.

Erie Garnet B (CI, 375).- amanil garnet $\mathrm{H}$, Buffalo garnet $\mathrm{R}$, Congo corinth $\mathrm{G}$ or GW, corinth brown $G$, cotton corinth $\mathrm{G}$, diamine Bordeaux CGN, direct garnet $\mathrm{R}$, direct violet $\mathrm{C}-$ an acid disazo dye used for staining frozen sections (Geschickter, C. F., Stain Techn., 1930, 5, 81-86).

Erie Violet BW (CI, 387) of NAC is an acid disazo dye of light fastness 2 to 3 . Directions for use in making preparations of animal and plant tissues are described (Emig, p. 40).

Erie Violet 3R (CI, 394) of NAC is a direct disazo dye of light fastness 3 not as satisfactory for microscopic work as Erie Violet BW (Emig, p. 40).

Eriochrome Azurol V (CI, 720), a mordant dye of acid fastness 3 to 4 . Gives color like that of Niagara Sky Blue. Directions for use (Emig, p. 52).

Eriometer, apparatus advocated by Emmons, W. F., Quart. J. Med., 1927, 21,83 to measure mean diameter of erythrocytes. See Erythrocytometer.

Erythroblasts, see Erythrocytes, Developmental Series.

Erythrocyte Counts do not fall in the scope of this book. It is sufficient to state that they are going out of fashion because of the large experimental error involved and since it is so easy to detect variations in shape, size and maturity of erythrocytes in smears and to measure hemoglobin content of blood by hemoglobinometers. Blum, L. I., Am. J. Clin. Path., 1945, 15, 85 has introduced a rabid photoelectric technique for estimating the number of ery throcytes, See Reticulocytes.

Erythrocytes. For chemical and physical studies erythrocytes are particularly adapted, because they can be collected in enormous numbers free from other kinds of cells and from intercellular substances. In order to determine marked differences in size and shape and hemoglobin content examination of fresh blood with direct illumination, or in the dark field, is probably the best procedure. An interesting photographic method for the stereoscopic visualization of the shape of erythrocytes has been described and illustrated by Haden, R. L., J. Lab. \& Clin. Med., 1936-37, 22, 1262-1263. For more accurate techniques see Wintrobe, M. M. Clinical Hematology, Philadelphia: Lea \& Febiger, 1942, 792 pp. A new anisocytosis index is proposed by van den Berghe, L., and Weinberger, E., Am. J. Med. Sci., $1940,199,478-481$. The refractile body of Isaacs (R., Anat. Rec. $1925,29,299-313$ ) can also be well studied in fresh blood. See Flagella.

Smears, colored by Giemsa or Wright's stain, are satisfactory for Howell-Jolly bodies, Cabot rings, basophilic stippling and polychromatophilia. For resistance to hemolysis in hy potonic sodium chloride solutions, see Daland, G. A., and Worthley, K., J. Lab. \& Clin. Med., 1934-35, 20, 1122-1136. A lysolecithin fragility test is described by Singer, K., Am. J. Med. Sci., 1940, 199, 466-477. For microfragility tests see Kato, K., J. Lab. \& Clin. Med., 1940, 26, 703-713 and for basophilic erythrocytes of the newborn see McCord, C. P., and Bradley, W. R., Am. J. Clin. Path., 1939, Tech. Suppl., 2, 329-338. A thorough investigation of erythrocytes in fetus and newborn has been made by Wintrobe, M. M. and Schumacker, H. B., Jr., Am. J. Anat., 1936, 58, 313-328. A simple method for determination of specific gravity of erythrocytes is described by Reznikoff, P., J. Exper. Med., 1923, 38, 441-444. After hemolysis the stroma remains and can be studied microscopically or chemically. Lipid analyses are particularly significant (Erickson, B. N., et al., J. Biol. Chem., 1937-38, 122, 515-528).

Isolation and collection en masse of nuclei of chicken erythrocytes by Dounce, A. I., and Lan, T. H., Science, 1943, 97, 584-585.

Experiments have been made with radioactive iron as a means of tagging red blood cells (Cruz, W. O., Hahn, R. F., Bale, W. F. and Balfour, W. M. Am. J. Med. Sci., 1941, 202, 157-162) which open up a new field for study of age changes because the cells are 
thereby dated. Stratification of contents of erythrocytes by ultracentrifugation (Beams, H. W., and Hines, E. H., Anat. Rec., 1944, 89, 531). Special methods are given under Hemoglobin, Flagella, Reticulocytes, Cabot Rings, Jolly Bodies, Pencil Red Cells, and Target Cells. For red fluorescent erythrocytes in anemia, see Seggel, K. A., Ergeb. d. inn, Med. u. Kinderh., $1940,58,582$.

Erythrocytes, Developmental Series. The technique employed apparently makes a great deal of difference in the conclusions reached. See Cowdry's Histology, 1938 p. 99.

1. Maximow and Bloom employing mainly permanent preparations list:

Hemocytoblasts: ". . large (up to 15 $\mu$ ) ameboid, non-granular basophil cells of lymphoid nature." Occur extravascularly.

Basophil erythroblasts: The youngest erythroblasts, characterized by the intense basophilia of their cytoplasm. Also called megaloblasts, but "this term is misleading because it was first used for the erythroblasts of pernicious anemia which are cells of quite different nature."

Polychromatic erythroblasts: So-called because after "fixation and staining with the Romanowsky mixture, especially in dry smears, the protoplasm has a mixed color varying from purplish-blue to lilac or gray." This is due to the presence of two substances, a basophile material and hemoglobin.

Orthochromatic erythroblasts or normoblasts: These are smaller "and only slightly larger than the mature, nonnucleated erythrocytes." Since the basophile substance diminishes and the hemoglobin increases, the protoplasm becomes acidophilic "and stains a bright pink with the Romanowsky mixture." They continue to divide mitotically for an unknown number of generations until the nucleus disappears.

2. Sabin and associates relying chiefly on supravital stains list:

Endothelial cells: Occur in special "erythrogenic capillaries."

Megaloblasts: "... a daughter endothelial cell which starts to synthesize hemoglobin." "The megaloblast has maximum basophilia, a moderate number of rod-shaped mitochondria, a trace of hemoglobin, and a nucleus with a minimum of chromatin and conspicuous nucleoli."

Early erythroblasts: "The young erythroblast represents a growth phase, with less rapid division, for the cell is much larger than the megaloblast; it contains the maximum number of mito- chondria. The amount of hemoglobin is still small, but sufficient to give a polychromatophilia, predominately basophilic in methylene blue-azur. The nucleus has a marked increase in chromatin."

Late erythroblasts: This cell "is intermediate in size between the early erythroblast and the definitive red cell. The nucleus has lost the nucleoli but still has massive chromatin.... The increase in hemoglobin is marked and in fixed films the cytoplasm is more acidophilic."

Normoblasts: "The stage of the normoblast is defined as a nucleated red cell after its last cell division. It has a small pyknotic nucleus ready for extrusion or fragmentation."

Erythrocytometer for measuring the diameter of red blood cells. Pijper, A., Med. J. South Africa, 1919, 14, 472 and Lancet, 1935, 1, 1152, deserves great credit for the discovery independently of Thomas Young (1813) of a technique for the measurement of small objects utilizing the principle of diffraction and Zeiss has manufactured an instrument on his specifications. Another, the Haden-Hausser erythrocytometer, is made by C. A. Hausser and Son and is sold by Arthur H. Thomas Co., Philadelphia (Haden, R. L. J. Lab. \& Clin. Med., 1939-40, 25, 399-403)

Erythrosin B, see Erythrosin, bluish.

Erythrosin BB or B extra, see Phloxine.

Erythrosin, bluish (CI, 773)-dianthine B, eosin $\mathrm{B}$, erythrosin $\mathrm{B}$, iodeosin $\mathrm{B}$, pyro$\sin$ B-Fluorescein with 2 iodine atoms. See Eosins.

Escherichia Coli, see Triphenyltetrazolium Chloride.

Ester Wax. An embedding medium designed especially to promote ribboning of sections. It is made up of diethylene glycol distearate, $73 \mathrm{gm}$.; ethyl cellulose, 4 gm.; stearic acid, 5 gm.; castor oil, $8 \mathrm{gm}$.; and diethylene glycol monostearate, $10 \mathrm{gm}$. (Steedman, H. F., Quart. J. Micr. Sci., 1947, 88, 123-133).

Esterases.-Written by E. W. Dempsey, Dept. of Anatomy, Washington University, St. Louis. February 26, 1951Strictly speaking, these are enzymes which hydrolyze the ester linkage derived from any acid, organic or inorganic. Thus, the phosphatases, cholinesterases, glucuronidase, lipase, and sulfatase properly belong among the esterases. However, ordinary usage restricts the term to the enzymes hydrolyzing carboxy esters, particularly those of fatty acids. These esterases apparently exhibit some substrate specificity; at least, the enzymes hydrolyzing esters of short-chain fatty 
acids appear to differ from the true lipases, which aet upon long-chain substrates, and from cholinesterase. Gomori, G. (Proe. Soc. Exp. Biol. and Med., 1945, 58, 362-364, and ibid, $1949,72,697-700)$ has devised a method for localizing lipase. Nachlas, M. M. and A. M. Seligman (J. Nat. Cancer Inst., $1949,9,415-425)$ believe the histochemical methods do not differentiate a true lipase, but only a nonspecific esterase. They deseribe a new and vivid method for this enzyme, based upon the hydrolysis of the acetyl ester of naphthol and staining the liberated naphthol by diazotization. Seligman, A. M., M. M. Nachlas, L. H. Manheimer, O. M. Friedman and G. Wolf. (Ann. Surg., 1949, 130, 333-341) describe the development of speeific methods for a number of hydrolytic enzymes.

Ethyl Eosin (CI, 770). The ethyl ester of eosin Y. Sold of ten as alcohol soluble eosin. See Eosins.

Ethyl Green (CI, 685). This is, like methyl green, prepared from crystal violet but differ's from it insofar that an ethyl group is added instead of a methyl one. For most purposes it is a satisfactory substitute for methyl green.

Ethyl Purple 6B, see Ethyl Violet.

Ethyl Violet (CI, 682)-ethyl purple 6BIt is hexaethyl pararosanilin, a basic dye employed by Bowie, D. J., Anat. Ree., $1924,29,57$ to make a neutral stain with biebrich scarlet for staining islets of Langerhans of fish. Kernohan, J. W., Am. J. Clin. Path., 1931, 1, 399-403 has used in Heidenhain's modification of Mallory's ethyl-violet orange $\mathrm{G}$ after formalin fixation.

Ethyl Violet-Biebrich Scarlet, see Bowie's stain for pepsinogen.

Ethylene Glycol Mono-Ethyl Ether = Cellosolve.

Euchrisine, see Acridine Orange.

Eunematoda, see Parasites.

Euperal is, according to Lee (p. 227), a mixture of camsal, eucalyptol, paraldehyde and sandrac, $n=1.483$ of two sorts colorless and green. Since the green one contains a eopper salt it strengthens hematoxylin stains.

Euporium, see Atomic Weights.

Evans Blue (T. 1824 Eastman Kodak Co.). Used clinically in man for estimation of blood volume. Vital staining of malignant tumors in man (Brunsehwig, A., Schmitz, R. L., and Clarke, T. H., Arch. Path., 1940, 30, 902-910). It is not taken in by red cells and hence is valuable for the determination of plasma volume (Gregersen, M. I., and Schiro, H., Am. J. Physiol., 1938, 121, 284-292. See Blood Cell Volume.
Excelsior Brown, see Bismark Brown Y.

Excretion contrasted with secretion (Cowdry's Histology, p. 259).

Exfoliative Cytology, see Papanicolaou Techniques.

Exogenous Pigments, elassificd by color, Lillie, p. 134

Extracellular fluid or phase, sec Chloride.

Exudates, see Agar infiltration to hold materials in place, also Papanicolaou Techniques.

Eyes. Techniques easily used for other parts of the body require special care in the ease of the eye. When sections through the entire eye are required it is important to see that the fixative chosen penetrates properly and that the normal shape of the organ is retained. Fixation by vascular injection may be helpful but it is not sufficient because so much of the eye is avascular. After removal of the eye from the orbit, whether previously injected or not, and after the dissecting away of unwanted muscular and other tissues, it should be immersed in the fixative. This will harden the outer coats somewhat. After a few minutes small amounts of the fixative should be injected by a hypodermic syringe into both chambers choosing loeations not in the plane of the proposed seetions and providing opportunity for fluid also to leave. Then, with a sharp razor blade, a deep eut should be made to permit free entrance of the fixative. After several hours, more of the tissue on either side of the plane should be cut away. Imbedding in celloidin by the rapid method is preferable to paraffin since it affords much needed support to the less dense parts. Orientation for seetioning is also easier in celloidin because one can see through it fairly well.

If, on the other hand, preparations are needed of small parts of the eye these parts should be carefully dissected out and the paraffin technique employed. Much time will be saved by following the excellent suggestions made by S. L. Polyak, The Retina. Univ. of Chicago Press, 1941, 607 pp. and by G. L. Walls (Stain Techn., 1938, 13, 69-76).

Dr. Polyak in a letter dated April 19, 1946 calls attention to the advisability of soaking celloidin blocks in oil as first described by Apáthy, S., Zeit. f. wis. Mikr., 1912, 29, 464. The same method is well presented by Kranse, R., Enzyk. d. Mikr. Technik., 3rd edit., 1926, 1, 281. For the investigation of permeability, oxidation-reduction potential, enzyme systems, and such properties, see Friedenwald, J. S. and Stiehler, R. D., Areh. Oplith., 1938, 20, 761-786. Useful data are to be found in Kurzes 
Handbuch der Ophthalmologie (Schieck and Brückner, Berlin: Julius Springer, 1930, 1, 882 pp.) The Anterior Chamber is a favourite site for tissue transplantation.

Frozen sections of bird's eyes. (Oakley, C. L., J. Path. \& Bact., 1937, 44, $365-368$ ). Fix in $10 \%$ formol saline 4 days, in Müller's fluid, 6 weeks in incubator, or, in case speed is necessary, in Perdrau's fluid 4 days. Incise large eyes to aid penetration. Wash in running water at least $24 \mathrm{hrs}$. because formalin and bichromate should be completely removed. Cut eye in half being careful not to disturb various structures. $12.5 \%$ gelatin $+1 \%$ phenol over night, $25 \% 24$ hrs. at $37^{\circ} \mathrm{C}$. Employ at least $25 \mathrm{cc}$. for each half eye. Mount with cut surface down in dish containing $25 \%$ melted gelatin. Set overnight in running water or in icebox (not refrigerator). Cut out block, trim away excess gelatin. Harden in large amount $10 \%$ formalin, $2-3$ days, store in $4 \%$ formalin. Before freezing soak $15 \mathrm{~min}$. in tap water. Freeze slowly, over-freeze and then stain usual methods but carefully avoid strong alcohols. They will stand $70 \%$ and $1 \% \mathrm{HCl}$ provided washing in water has been thorough. Use glycerin jelly for mounting. Fluorescence Microscopy of the eye is very revealing, see Evans, J. N. and Singer, E., Arch. Ophthal., 1941, 25, 1007.

Fahrenheit Temperature to Centigrade. Use the following relation:

$$
{ }_{8}^{5}\left(\mathrm{~F}^{\circ}-32\right)=\mathrm{C}^{\circ}
$$

$302^{\circ} \mathrm{F} \pm g^{5}(302-32)=\frac{3}{8}(270)=150^{\circ} \mathrm{C}$.

$.5^{\circ} \mathrm{F} \pm{ }_{8}^{5}(5-32)=8(-27)=-15^{\circ} \mathrm{C}$.

$-13^{\circ} \mathrm{F} \pm \frac{5}{9}(-13-32)=\frac{5}{3}(-45)=-25^{\circ} \mathrm{C}$

Fallopian Tubes (oviducts, uterine tubes). References to many techniques will be found in C. G. Hartman's chapter in Allen, Danforth and Doisy's Sex and Internal Secretions. Baltimore: Williams and Wilkins, 1939, $1346 \mathrm{pp}$.

Falzone, see Desoxyribose Nucleic Acid.

Farrant's Medium. Gum arabic, $30 \mathrm{gm}$.; glycerin, $30 \mathrm{cc}$; arsenous oxide (arsenic trioxide), 0.1 gm.; aq. dest., 30 cc. There are many types of this medium differing slightly in composition, see Gray, P. and Wess, G., J. Roy. Mier. Sci., 1950, 70, 287-291.

Fast Acid Blue $\boldsymbol{R}$ (CI, 760). An acid xanthene dye. Conn (p. 143) says that it is almost the same as violamine $3 \mathrm{~B}$ which contains small amount of a red dye. See Romell, L. G., Stain Techn., 1934, 9, 141-145 under Soil, bacteria.

Fast Acid Green N, see light Green SF yellowish.
Fast Blue B, $\mathbf{O B}, \mathbf{R}$, etc., see Indulin, water soluble.

Fast Blue 3R, see Naphthol Blue R.

Fast Crims on GR, see Azophloxine GA.

Fast Fuchsin G, see Chromotrope 2R.

Fast Green FCF. Commission Certified. Closely related to Light Green SF yellowish and recommended as a substitute because it fades less.

Fast Oil Orange II, see Oil Red $\mathbf{O}$.

Fast Phosphine NAL, see Rheonine A.

Fast Red, see Amaranth.

Fast Red B, BN or P, see Bordeaux Red.

Fast Violet, see Gallocyanin.

Fast Yellow (CI, 16)-acid yellow, fast yellow FY, G, S, BG, etc.-An acid mono-azo dye. Employed by several investigators, see use by Wallart, J. and Houette, C., Bull. d'Hist. Appl., 1934, $11,404-407$ in rapid trichrome hematoxylin, acid fuchsin fast yellow method. They used "Jaune solide G or GG (Ciba).

Fasting. Structural changes in human digestive tract (Cowdry's Histology, p. 305).

Fat Blue B, see Victoria Blue B.

Fat Blue 4R, see Victoria Blue $4 R$.

Fat Ponceau, see Oil Red O.

Fat Ponceau, see Sudan IV.

Fat Ponceau G, see Sudan III.

Fat Ponceau $R$ or LB, see Sudan IV.

Fats, see Lipids.

Fatty Acids, see Lipids, examination with polarized light, also lack of specificity of blue color with Nile Blue Sulphate. A review of the method of tagging fatty acids with radioactive isotopes is given by Bloor (W. R., Physiol. Rev., 1939, $19,557-577$ ).

Feathers, see Ceresin imbedding.

Feces. 1. To demonstrate ova of parasites (Mallory, p. 301). If they cannot be seen when a small bit of feces is mixed with water on a slide attempt to concentrate them. To a small amount of feces add sufficient sugar solution (common granulated sugar, $500 \mathrm{gm}$., water, $360 \mathrm{cc}$., phenol, $1 \%$ ) to almost fill tube. Cover and gently mix contents. Centrifuge at 1000 r.p.m. 5-6 min. Remove material from surface in wire loop and examine microscopically for ova. Another method is to use hypertonic salt solution in proportion to feces of not more than 20:1 in the same way, removing large particles as may be necessary before centrifuging.

2. To find segments and whole adult worms. Wash feces in small amount water through medium mesh screen, collect and examine at low magnification. For identification consult a text book of parasitology.

See Floatation Techniques, Intestinal Protozoa. 
Fell, see Organ Culture in Vitro.

Ferments, see Enzymes.

Ferric Chloride-Osmic Acid for demonstration of Golgi apparatus (Owens, H. B. and Bensley, R. R., Am. J. Anat., 1929, 44, 79-100). Fix and impregnate each piece of tissue $7-10$ days at $37^{\circ} \mathrm{C}$. in ferric chloride, $0.05 \mathrm{gm}$.; $2 \%$ osmic acid, $10 \mathrm{cc}$.

Ferrihemate, see Hematin.

Fettblau -braun, -grün, -orange, -rot and -schwartz. These are lipid stains of Hollborn. For use of hydrotropes (Hadjioloff, A., Bull. d'Hist. Appl., $1938,15,37-41)$.

Feulgen Reaction, see Thymonucleic Acid.

Fiberglass, see Glass Cloth.

Fibers. Many are recognized. See Nerve, Collagenic, Reticular, Elastic, Neuroglia. Muscle fibers are given under Muscle.

Fibrils. These are really small fibers many of which are intracellular. See Neurofibrils, Myofibrils, Epidermal Fibrils, Fibroglia, Myoglia.

Fibrin. Usually easily identifiable in Hem. atoxylin and Eosin preparations. Weigert's (1887) standard differential stain for fibrin may be used as advised by Mallory (p. 193). Paraffin sections of material fixed in abs. alcohol, Carnoy or Alcohol-Formalin can be employed. If the fixative contains chrome salts (Zenker, Helly) treat first with $0.25 \%$ aq. potassium permanganate, $10 \mathrm{~min}$., then $5 \%$ aq. oxalic acid, $20 \mathrm{~min}$. and wash in aq. dest. Stain nuclei with Lithium Carmine. Mix 3 cc. of A : abs. alc., 33 cc.; anilin oil, 9 cc. saturated with methyl violet (crystal violet) with 27 ec. of B : sat. aq. methyl violet. Stain 5-10 min. Drain and blot. Treat sections with Gram's Iodine, 5-10 min. Drain and blot. Differentiate in equal parts anilin and xylol drop by drop until purple ceases to be removed. Blot and remove anilin with xylol. Mount in balsam. Fibrin blue-black, nuclei red.

Fibroblasts. There is no specific stain for fibroblasts. In fresh spreads of Loose Connective Tissue they are fairly conspicuous elements identifiable by their large usually slightly kidney shaped nuclei (possessed generally of a single nucleolus) and tapering cytoplasmic processes devoid of specific granulations. In sections less cytoplasm is scen and it may be impossible in some cases to identify the nuclei with assurance. Recognition is mainly by position and the exclusion of other possibilities. View the beautiful colored plates of Evans, H. M. and Scott, K. T., Contrib. to Embryol., Carnegie Inst., 1922, 47, 1-55 for a comprehensive picture of the responses of fibroblasts to vital stains.
Pure strains of fibroblasts can easily be cultured, their behavior watched and their nutritional and other requirements investigated. See Tissue Culture.

Fibroglia Fibrils. Mallory's Phosphotungs tic Acid Hematoxylin stain for.

Fibrous Connective Tissue. Since this is much denser than Loose Connective Tissue the method of making spreads is not feasible. It can best be examined in sections of Zenker fixed material colored by Mallory's Connective Tissue Stain supplemented by specific stains for Elastic Fibers.

Figge, see Porphyrins, Hematoporphyrins.

Filament-Nonfilament Count. Neutrophilic leucocytes are divided into two classes: filament, in which nuclear segments are connected by delicate strands consisting apparently of nuclear membrane only and nonfilament in which there are no filaments the strand being so coarse that it may be resolved into nuclear membrane plus nuclear contents. The former are mature and the latter are less differentiated cells. According to Pepper, O. H. and Farley, D.L., Practical Hematological Diagnosis, Philadelphia, Saunders, 1933, 562 pp., $8-16 \%$ of neutrophiles are normally nonfilament cells. A shift to the right is a decrease in this percentage. The count is easier to make than the Arnett or Schilling count and is probably of equal value. See also Nonfilament-Filament Ratio.

Filterable Viruses, see Victoria Blue 4B and Virus.

Filters. Choice and use of the various types of filters employed in the study of viruses and bacteria are well described by J. R. Paul (Simmons and Gentzkow, 584-586). There are 4 principal kinds.

Berkefeld. German, from diatomaceous earth. $\mathrm{V}$. pores $8-12 \mu ; \mathrm{N}$, pores $5-7 \mu$; and $\mathrm{W}$, pores $3-4 \mu$.

Mandler. American modification of Berkefeld but made of kieselguhr, asbestos and plaster of Paris. Corresponding grades of pornsity are styled "preliminary", "regular" and "fine."

Chamberland, French, from unglazed porcelain, in 9 grades of porosity.

Seitz. Made of asbestos, in 2 grades $\mathrm{K}$ (coarse) and E. K. which filters out ordinary bacteria.

Elford. Made of collodion.

Firminger, see Carbowax Embedding.

Fischler's modification of Benda's stain for fatty acids and soaps (Fischler, F., Zentralbl f. Allg. Path. u. path. Anat., $1904,15,913-917$ ) has been severely criticized by Lison (p. 203) who concludes that it is of no microchemical value.

Mallory (p. 120) has, however, given a somewhat different description of the 
technique. He explains that since the $\mathrm{Na}$ and $\mathrm{K}$ fatty acid salts (soaps) are soluble in formalin, it is necessary to change them into insoluble $\mathrm{Ca}$ soaps by saturating the $10 \%$ formalin fixative with calcium salicylate. Comparison of stained sections of such material with others fixed simply in formalin shows the presence and absence of the fatty acid salts (soaps). Calcium soaps can be distinguished from fatty acids because they resist solution in a mixture of equal parts abs. alc. and ether or in hydrochloric acid whereas the fatty acids are soluble in this mixture and calcium in hydrochloric acid. The method, as detailed by Mallory, is: Mordant frozen sections of $10 \%$ formalin fixed material in sat. aq. copper acetate $(12.5 \%), 2-24$ hrs. at room temperature. Wash in aq. dest. Stain $20 \mathrm{~min}$. or more in Weigert's hematoxylin made up by mixing $1 \mathrm{gm}$. hematoxylin dissolved in 10 cc. abs. alc. with 1 cc. sat. aq. lithium carbonate (about $1.25 \%$ ) plus 90 cc. aq. dest. several days before use. Differentiate in Weigert's borax-potassium ferricyanide, $(2.5 \mathrm{gms}$. ferricyanide and $2 \mathrm{gm}$. borax plus 100 cc. aq. dest.) much diluted until red blood cells become decolorized. Wash thoroughly in aq. dest. Mount in glycerin jelly or glycerin. Fatty acids deep blue black. Fe, $\mathrm{Ca}$ and hemoglobin may also be stained. Tostain neutralfats inaddition stain with scarlet red after washing out Weigert's fluid, rinse in $70 \%$ alc. and in water and mount in glycerin.

Fite, see his method for Acid Fast Bacilli.

Fixation by immersion is usually the first step in making permanent preparations. Compared with the direct microscopic examination of still living cells removed from the body and placed in approximately isotonic media, it has both advantages and disadvantages. Among the first is the fact that the normal form relations of the tissue components are more faithfully preserved in large pieces by fixation; because it is not necessary to separate the tissue by teasing, or in some other way, into sufficiently small or thin pieces for microscopic study. Moreover, by fixation, the cells are suddenly and uniformly killed, so that the changes resulting from unfavorable fluid environment outside the body, leading slowly or quickly to injury and death, are not encountered. The chief objection to fixation is that the structure is very definitely modified thereby and care must be exercised in reaching conclusions as to living tissues from the study of fixed ones. It is important to restrict these structural changes to those inseparable from the action of the fixative itself, and of the subsequent technique under the most favorable conditions.

Reduce to a minimum the time in which these complicating alterations can occur by prompt fixation. Remove the tissue from an animal under general anesthesia, or immediately after it has been killed, by a method unlikely to injure the tissues. In the case of human tissues removed at operation one should be on the look out for complicating factors. If the tissue is collected at autopsy the autopsy should be made at the earliest possible moment after death. See Postmortem Changes. If delay is unavoidable, keep the body, or the tissue, in an ice box to reduce the speed of chemical change. In case an excised tissue cannot be immediately fixed, place it in a covered glass container with some cotton moistened with physiological saline solution. Do not put it in the solution. Keep the container likewise at a low temperature.

Carefully avoid injury to the tissue from any cause. Letting its surface dry during removal from the body, or at any time before fixation, produces Artifacts. So also does mechanical manipulation. If forceps must be used, do not pinch the part of which the preparation is to be made. It is better to lift the tissues. Scissors tend to squeeze the tissue, but it is necessary to cut with them in some cases. The ideal way is to cut with a sharp razor blade. This is easy with the liver, kidney, brain and other more or less compact organs, but the sweep of a razor blade tends to draw the tissue and cause displacement, especially when the specimen is heterogeneous, some parts being loose connective tissue, others muscle, others gland, etc. When feasible, cut the tissues into slices and lift them into the fixative. For fixatives that penetrate easily (formalin, Zenker's and Bouin's fluids, etc.) make the slices 4-6 $\mathrm{mm}$. thick. For the poor penetrators, in which osmic acid is the principal ingredient (Bensley's A.O.B., Flemming's fluid, etc.), the slices must be not more than $2 \mathrm{~mm}$. thick. In the case of surface tissues (skin, gastric mucous membrane, bladder wall, etc.) fix a strip, flattened on the surface of a piece of wooden tongue depressor or stiff paper card. A volume of fixative at least 20 times that of the tissue fixed is required. Agitate the bottle slightly to prevent the tissue from sticking to the bottom and to ensure penetration from all sides.

It may be desirable to inject the fixative via a large artery supplying the tissue to be examined. This eliminates mechanical injury to the tissue before 
fixation, preserves gross form relations better and is suggested when sections are required of large specimens. Before injecting the fixative wash out some of the blood by Perfusion with physiological salt solution, or at least let the blood drain out from the veins, because, if all is left in, it may clog the arteries and block the entry of the fixative. After fixation by vascular injection it is customary to cut, with a razor blade, suitable slices and to continue the fixation by immersion. Obviously such tissues should not be employed for microchemical analyses because there is a danger of washing out chemical substances. Clearly, also, the speed of fixation depends upon the degree of vascularity. For avascular tissues such as epidermis, cornea and cartilage fixation by injection is not recommended.

After the tissues have hardened a little by immersion in or injection with the fixative, it may be helpful to remove them from the fixative and trim them with a razor blade so that their size and shape will be almost what is needed when they are finally cut into sections. The slices should have smooth upper and lower surfaces including an area which will yield sections that will fit nicely under a $22 \times 22 \mathrm{~mm}$. cover glass unless larger covers are to be used. The shape should be rectangular with opposite edges parallel. In general it is well to have two longer parallel edges and two shorter ones, because a square surface is not so convenient to section as an oblong one. However one must bear in mind exactly what one wishes to demonstrate. This making of uneven surfaces smooth does however introduce an experimental error; because, where much is shaved off, the fixation has penetrated less than where little or no tissue has been removed. After trimming return tissues to a fresh supply of fixative. Tissues fixed in poor penetrators should not be trimmed.

The time of fixation depends upon the tissue, the fixative and the purpose in mind. In general, $24 \mathrm{hrs}$. is suitable. Some fixatives, particularly those containing potassium bichromate and/or osmic acid, are not very stable and for this reason should be renewed. The fixative deteriorates less quickly if the fixation is carried out at a low temperature in an ice box. The speed of fixation is probably also somewhat diminished. The effect of $\mathrm{pH}$ on chromium fixatives has been studied by Zirkle (C., Protoplasma, 1928, 4, 201-227). See results obtained by adding Wetting Agents and Hydroxybenzene Compounds to fixatives. Fixation involving Decalcifica- tion and Mordanting are special cases described under these headings. For choice of fixative see Fixatives.

After fixation Washing may be neecssary, or Mordanting. The tissue may be prepared as a Whole Mount, or Frozen Sections may be made, or it may be dehydrated, cleared and imbedded in Paraffin or dehydrated and imbedded in Celloidin for Sectioning.

Fixatives. The number from which to choose is enormous but the number actually employed is comparatively small. Formalin unquestionably heads the list as being used for a far greater

$\begin{array}{ll}\begin{array}{l}\text { Acetic osmic bichro- } \\ \text { mate }\end{array} & \text { Hischler } \\ \text { Alcohol (ethyl) } & \text { Kleinenberg } \\ \text { Allen } & \text { Lactophenen } \\ \text { Barium chloride and } & \text { Lillie } \\ \text { formalin } & \text { Mann } \\ \text { Basic lead acetate } & \text { March } \\ \text { Bouin } & \text { Maximow } \\ \text { Cadmium chloride } & \text { Mercuric chloride } \\ \text { Carnoy } & \text { Methyl alcohol } \\ \text { Carnoy-Lebrunn } & \text { Muller } \\ \text { Champy } & \text { Orth } \\ \text { Chloral hydrate } & \text { Osmic acid } \\ \text { Destin } & \text { Parabenzoquinone } \\ \text { Diaphanol } & \text { Perenyi } \\ \text { Dioxan } & \text { Petrunkevitch } \\ \text { Downey } & \text { Regaud } \\ \text { Erlicki } & \text { Rabl } \\ \text { Ferric chloride-osmio } & \text { Schandinn } \\ \text { acid } & \text { Silver nitrate } \\ \text { Flemming } & \text { Susa } \\ \text { Formalin } & \text { Tellyesnicky } \\ \text { Formalin-Zenker } & \text { Weigert } \\ \text { Giemsa } & \text { Van Gehuchten } \\ \text { Gilson } & \text { Zenker } \\ \text { Helly } & \text { Zweibaum } \\ \text { Hermann } & \end{array}$

variety of purposes than any other fixative. It penetrates well and is an excellent preservative. It is the only satisfactory fixative for use before the cutting of frozen sections and as a preliminary to certain microchemical reactions. Alcohol comes next in variety of services performed but unfortunately it brings about considerable shrinkage. Both formalin and alcohol are frequently combined with other ingredients.

For routine purposes Zenker's Fluid, either alone or with formalin, is perhaps the most popular fixative. Tissues so fixed give better contrasts of acidophilic and basophilic components than are obtained after fixation in formalin or alcohol by themselves. Bouin's Fluid is also an excellent fixative for general use and is being employed with increasing frequency. It is particularly advocated by dermatologists. Regaud's Fluid is 
the fixative of choice for mitochondria because it penetrates so much better than Osmic Acid containing fixatives. No important new fixatives have recently been devised.

In making the selection one is naturally guided by data concerning the structures which it is desired to demonstrate (see Nerve Endings, Mitochondria, etc.) or the substances to be revealed (Lead, Copper, Oxidases, Lipids, etc.) or the techniques that seem best adapted to the purpose in mind (Mallory's Connective Tissue stain, Weigert's Method, etc.). Some of the more important fixatives are listed, further data being given under each heading.

Flagella. 1. Of bacteria. Loeffler's stain. Mordant in fresh $20 \%$ aq. tannic acid, 10 cc.; sat. aq. ferrous sulphate, 5 cc.; $3-5 \%$ basic fuchsin in $95 \%$ alc., 1 cc. gently heated, $1 \mathrm{~min}$. Rinse in water stain with slight heat in Carbol Fuchsin $1 \mathrm{~min}$. wash and dry. For other flagella stains see discussion in McClung (pp. 143-145) and Shunk, I. V., J. Bact., 1920, 5, 181 ; Galli-Valerio, B., Centralbl. f. Bakt. Orig., 1915, 76, 233; Gray, P. H., J. Bact., 1926, 12, 273. See technique for darkfield study of flagella (Pijper, A., J. Path. \& Bact., 1938, 47, 1-17).

2. Of erythrocytes (Oliver, W. W., J. Inf. Dis., 1934, 55, 266-270). Add $1 \mathrm{mg}$. hirudin to $2-3 \mathrm{cc}$. sterile Ringer's solution in small, sterile test tube. Draw up about 0.5 cc. into a sterile Pasteur pipette fitted with rubber bulb. Apply to drop fresh normal blood from finger. Suck up quickly into pipette and expel into test tube. Incubate at $37^{\circ} \mathrm{C} .40-50$ min. which promotes flagella production. Add small drop to clean slide held at $40^{\circ}$ angle. After the drop has run down slide, let dry completely in horizontal position at room temperature. Mordant in fresh $10 \%$ aq. tannic acid, $50 \mathrm{cc}$; sat. aq. ferrous sulphate, $25 \mathrm{cc}$. and sat. alc. basic fuchsin, 5 cc. which is poured on slide and warmed slightly $20 \mathrm{~min}$. Wash thoroughly in running tap water and dry. Flood with fresh Ziehl-Neelsen $(1 \mathrm{gm}$. fuchsin, $10 \mathrm{cc}$. alcohol $+90 \mathrm{cc}$. $5 \%$ aq. phenol acid) 20 min. not warmed. Wash carefully in running water, blot dry and examine with oil immersion. It will be helpful to examine Oliver's illustrations. (Revised by Wade Oliver, Dept. of Bacteriology, Long Island Medical College, Brooklyn, N. Y., 1946).

The interpretation of observations on bacterial flagella offers many pitfalls. Dubos, R. J., The Bacterial Cell. Harvard Univ. Press, 1945, 460 pp. calls attention to their fineness, the slight affinity of their substance for stains, the use of mordants which adhere to their surface increasing their apparent diameter when stained, and the fact that mechanical agitation alone is sufficient to detach them from the cells. By thus releasing flagella sufficient flagellar material can be collected for immunological study and the action of flagellar antibody on mobile flagella can be followed microscopically. Dubos remarks that the amounts of flagellar material available are too small to permit chemical analysis but we may hope that techniques both of collecting material and of analysis will be so improved as to make this feasible. He refers to numerous papers on electron microscopic examination of flagella as revealing structural details previously unknown.

Details in the structure of Alagellae are revealed by Electronmicroscopy. See Brown, H. P., Ohio J. Sci., 1945, 45, 247; and DeRobertis, E., and Franchi, C. M., Exp. Cell Res., 1951, 2, 295-298. See also Cilia and Polysaccharides.

Flagellates, intestinal. Those commonly found in man are, according to Craig, p. 115, Giardia lamblia, Chilomastix mesnili, and Trichomonas hominis; less frequently seen are Embadomonas intestinalis and Enteromonis hominis. Stains much the same as for Endameba and Leishmania. See Craig for choice of suitable culture medium.

Flame Photometer. Use of this instrument in the analysis of biological materials is critically presented by Wallace, W. M. et al., J. Lab. \& Clin. Med., 1951, 37, 621-629.

Flavins under fluorescence microscope show green fluorescence in liver tissue. See Riboflavin.

Fleas, see method of double imbedding for (Lee, p. 598).

Flemming's Fluid. Weak: $0.25 \%$ chromic acid, $0.1 \%$ osmic acid and $0.1 \%$ glacial acetic acid in aq. dest. Strong: $1 \%$ chromic acid, 15 cc.; $2 \%$ osmic acid, $4 \mathrm{cc}$. ; glacial acetic acid, $1 \mathrm{cc}$. These are classic fixatives now not much used. The Bensleys (p. 45) advocate same ingredients differently made up. A : $1 \%$ aq. chromic acid, 11 parts; glacial acetic acid, 1 part; and aq. dest., 4 parts. $\mathrm{B}: 2 \%$ osmic acid in $1 \%$ aq. chromic acid. Immediately before use, mix 4 parts of A with 1 part of $B$ and employ a volume ten times that of the tissue. Fix 2-72 hrs. and wash in water 24 hrs. See Safranin-Gentian Violet and Orange G method, Mitosis, Benda's Method.

Floatation Techniques. Many methods are available for separating helminth eggs from feces for microscopic examination. 
They are floated out by the use of hypertonic salt and other solutions, sometimes with the aid of centrifugal force (E. C. Faust, in Simmons and Gentzkow, p. 684).

Florence's Reaction. The standard test for choline in seminal stains. As described by Pollak, O. J. Areh. Path., 1943, 35, 140-196: Place one drop semen, or of aqueous extract of seminal stain, on slide. Add drop of reagent (Pot. iodide, $1.65 \mathrm{gm}$.; iodine, $2.54 \mathrm{gm}$.; aq. dest., 30 cc.), cover and examine microscopieally. Dark brown, rhombic crystals appear, about $25 \mu$ long and $S \mu$ wide with bifureated ends resembling swallow tails and Teichmann's hemin crystals. In polarized light these show double contours.

Fluids. Samples of body fluids are often presented for microscopic examination. In a human being containing, say, 100 lbs. of water they are naturally of great variety even under normal conditions. Abnormal fluids are usually described as transudates or exudates. The former compared with the latter are mainly filtrates, are more watery, have lower specific gravity, less albumin, no bacteria and are the result of inechanical forces rather than inflammation. See :

$\begin{array}{ll}\text { Aqueous humor } & \text { Intracellular phase } \\ \text { Cerebrospinal } & \text { Pericardial } \\ \text { Duodenal } & \text { Peritoneal } \\ \text { Endolymph } & \text { Pleural } \\ \text { Extracellular phase } & \text { Synovial } \\ & \text { Tissue }\end{array}$

Fluoran Derivatives. As explained by Conn (p. 144) fluoran is not a dye but a product of phthalic anhydride containing a xanthene ring and a lactone ring with introduced hydroxyl groups and halogen atoms in particular positions. Examples: eosin $B$ and $Y$, erythrosin bluish and yellowish, ethyl eosin, fluorescein, mercurochrome 220 , methyl eosin, phloxine, phloxine $B$, rose bengal.

Fluorescein (CI, 766) is simplest fluoran dye. It stains very poorly but is highly fluorescent. Its sodium salt is called uranin.

Fluorescence Microscopy. Details provided by Dr. W. L. Simpson of The Barnard Free Skin and Cancer Hospital. Supplemented by Dr. T. B. Rosenthal, Dept. of Anatomy, Washington University, St. Louis. June 6, 1951.

Fluoreseence is the property, possessed by many substances, of converting short wavelengths of light into longer wavelengths. In the field of microscopy those structures and substances are of most interest that convert ultraviolet light into light of the visible spectrum, since it is only these sub- stances that can be observed directly. Though fluorescence mieroseopes designed for this type of observation have been available commercially for many years, their use has been limited until recently by their relatively high cost and by the apparent failure of biologists to appreciate the possibilities of this type of observation. Recent technological developments in the glass and electric lamp industries now make it possible to assemble an apparatus for fluorescence microseopy at a cost well within the budget of most laboratories. Evidence of heightened interest in this field is found in the numerous papers concerning fluorescence mieroseopy within the past 10 years. Although several reviews of the subject already exist (Haitinger, M., Fluoreseenz-Mikroscopie, Ákademische Verlagsgesellschift, Leipzig, 1938; Hamperl, H., Virehows Arch. f. path. Anat., $1934,292,1-51$; Sutro, C. J., Arch. Path., 1936, 22, 109-112; and MeClung's Handbook of Microscopical Technique, New York, Paul B. Hoeber Inc., 1937), the technique will be deseribed as it can be used with an assembly of low cost apparatus available in the United States at the present time.

A pparatus required:

1. An intense source of ultraviolet light that is rich especially in the region from 300 to 400 millimicrons. Certain electric arcs using electrodes of special metal alloys (the Haitinger Arc, C. Reichert-Vienna) have been developed for this purpose. More easily available, low in cost, and having an intense output in the desired region, are the medium pressure mercury vapor ares in capillary quartz tubes (the A H 4 lamp of the General Electric Company or Westinghouse Electric Co. and lamps made by Hanovia Chemical Co., etc.).

2 . Filters that remove all or nearly all of the visible light. A considerable selection of glass and liquid filters may be used for this purpose. Since most of the so-called ultraviolet filter's pass also a certain amount of red light, supplemental blue filters must be used with them. A solution of eopper sulfate in a cell or tube of quartz, or of ultraviolet transmitting glass, is satisfactory and readily available. A combination of Shott glass filters U G 2 and B G 14 are recommended by Jenkins (R., Stain Techn., 1937, 12, 167-173). Corning Filters $\$ 5840,5860$, or 5574 used with a copper sulfate solution are satisfactory in our experience. An entirely liquid filter, using solutions of cobalt sulfate and nickel sulfate, is described by Bäckström (H. L. J., Arkiv. för Kemi. 
Mineralogi Och Geologi, 1940, 13A, 1-16).

3. Condensing lenses, if used at all, must be of quartz or ultraviolet transmitting glass.

4. A quartz prism or mirror of polished metal having a high reflecting power for ultraviolet. Aluminum and magnesium-aluminum alloys are best for this. By mounting the microscope and light source horizontally this item can be eliminated.

5. An ordinary microscope that is fitted with a substage condenser of quartz or ultraviolet transmitting glass. Since the ultraviolet light has served its purpose when it has reached the tissue, ordinary glass objectives and eyepieces are used. With some older objectives the balsam of the lenses fluoresces in ultraviolet and causes an unpleasant diffuse light to appear in the microscope that masks the fluorescence of the tissue. This may be eliminated with a darkfield stop that prevents direct rays of ultraviolet light from entering the objective. Newer lenses are free from this fluores. cence and may be used without a darkfield stop. This is desirable since it permits the utilization of a greater portion of the light that strikes the condenser. Popper has reported that the fluorescence of Vitamin A can be observed with an ordinary microscope with glass condenser. Ordinary optical glass transmits sufficiently far into the near ultraviolet that this type of apparatus might be successfully used for strongly fluorescent substances.

6 . Slides for the specimens of ultraviolet transmitting glass. (Corex D glass slides, obtainable from Corning Glass Co. are suitable.)

7. An eyepiece filter that excludes ultraviolet light with a minimum absorption of visible light. This may be of glass (Leitz ultraviolet protecting filter no. $8574 \mathrm{~A}$, Corning Glass Works filters no. 3389 or 3060 ) or, simplest and cheapest, a circle of Wratten 2A gelatin filter cut to fit within the eyepiece (the Wratten 2 filter is not suitable since it fluoresces itself in ultraviolet light).

8. Non-fluorescent media for mounting the section to be examined. $\mathrm{Me}$ dicinal mineral oil, or glycerin is suitable. If immersion lenses are to be usep a non-fluorescing immersion medium must be employed. Sandlewood oil has been recommended for this purpose.

Preparation of tissues: Hamperl (loc. cit.) recommends that tissues for fluorescence examinations be fixed only in a dilute solution of formalin, since metal containing fixatives destroy the fluorescence of some substances. A $5-10 \%$ solution of U.S.P. formalin in aq. dest. is ordinarily employed. Tissues should should not be fixed longer than $24 \mathrm{hrs}$.; certain components of tissue acquire abnormal fluorescence if the time of fixation is prolonged. If fats and other alcohol soluble substances are to be examined, i.e., vitamin $A$, polycyclic organic carcinogens, etc., frozen sections must be made. If these substances are not of interest, the tissue may be dehydrated, cleared, and imbedded in paraffin in the usual manner. High quality reagents are required, because the impurities found in many organic substances themselves fluoresce. All paraffin must be removed since this too fluoresces. The section can be cleared in anhydrous glycerin or pure medicinal mineral oil. Gelatin and celloidin are not recommended for imbedding because of their fluorescence.

Two types of fluorescence may be produced in tissues with this type of apparatus. The first is that seen in tissues that have been subjected to no special treatment and is due to the presence of fluorescent substances in the tissues themselves. This is termed "primary" fluorescence or natural fluorescence and is exhibited by many substances found in animal organisms. In most tissues there are present sufficient quantities of these materials to permit the observer to recognize the general structure of the tissue without recourse to stained control sections studied with transmitted visible light. Hamperl (loc. cit.) describes, in considerable detail, the natural fluorescence of many human tissues. Jenkins (loc. cit.) summarizes the findings in the most common animal tissues. Cornbleet and Popper ( $\mathrm{T}$. and H., Arch. Dermat. \& Syph., 1942, 46, 59-65) review the natural fluorescence of human skin. Popper and his coworkers have contributed a series of papers on the fluorescence of vitamin A in animal tissues (Popper, H., J. Mt. Sinai Hosp., 1940, 7, 119-132. Arch. Path., 1941, 31, 766-S02; Popper, H. and Brenner, S., J. Nutrition, 1942, 23, 431443 ; Popper, H. and Ragins, A. B., Arch. Path., 1941, 32, 258-271). Simpson and Cramer (W. L. and W., Cancer Research, 1943, 3, 362, 515, 604) have used the method to follow the distribution and persistence of methylcholanthrene in skin. Fluorescence color of various tissues and of drugs in tissue sections is described by Helander, S., Acta Physiol. Scand., 1945, 10, Suppl. $29,103 \mathrm{pp}$

Another kind of fluorescence is the "secondary" fluorescence that appears in certain components of the tissue after 
sensitization with dyes and plant extracts. This extends considerably the range of fluorescence microscopy and has been developed chiefly by Haitinger (loc. cit.) in conjunction with Hamperl and Linsbauer. Various fluorescent alkaloids, azo dyes, primulins, auramine, berberine sulfate, chelidonium, rhubarb extracts, etc., are selectively absorbed by certain parts of the cell and cause them to show characteristic fluorescences in ultraviolet light. Such substances are called fluorochromes. Sections of tissue are immersed in such substances for a short period of time before being examined. Examples of the use of these fluorochromes are found in papers by Haitlinger (loc. cit.), Jenkins (loc. cit.), Clark and Perkins (W. M. and M.E., J. Am. Chem. Soc., 1932, 54, 1228-1248), Lewis (M. R., Arch. f. exp. Zellf., 1935, 17, 96-105) and Popper (H., J. Mt. Sinai Hosp., 1940, 7, 119-132). A good account of fluorescence microscopy of insects is given by Metcalf, R. L.. and Patton, R. L., Stain Techn., 1944, 19, 11-27.

Noteworthy applications have been made in the use of acridine derivative dyes. Krebs and Gerlach (Am. J. Roentgenol. Rad. Therap., 1951, 65, 93-98) have demonstrated that acridine orange, C.I. 788, is a sensitive indicator of the viability of cells following thermal and radiational damage. The same dye was used by Zeiger and Harders (Z. Zellforsch., 1951, 36, 62-78) for flurochroming of nerve tissues. In a comprehensive investigation of 24 diaminoacridine stains by DeBruyn, Robertson and Farr (Anat. Rec., 1950, $108,279-307)$ it was found that nuclei of many tissues and organs were vitally fluorochromed without perceptible toxic or degenerative effects.

Primulin is recommended by Schmidt-LaBaume and Jäger (Arch. f. Dermat. Syph., 1939, 179, 531) in an ingenious method for visualizing surface detail of the epidermis.

A most interesting method of demonstrating the distribution of the right and left coronary arteries is given by Peterson and Gibson (Med. Radiogr. Photogr., 1951, 27, 14-17), who inject the vessels with plastics containing red and green fluorescing pigments. The heart is then encased in a plastic shell, following which the tissues are digested by KOH. Finally, the shell holding the arterial cast is filled with the transparent plastic. Under ultraviolet illumination the arterial system is revealed with extraordinary clarity. The use of fluorescein dyes in the diagnosis of malignancy with special refer- ence to tumors of the central nervous system is described by Hubbard, T. B. and Moore, C. E., J. Nat. Cancer Inst., 1949, 10, 303-314 (good bibliography). The Diaminoacridines are important vital stains for nuclei in the sense that they accumulate within nuclei without toxic action and can there be revealed by fluorescence microscopy (de Bruyn, P. P. H., Robertson, R. C. and Farr, R. S., Anat. Rec., $1950,108,279-307)$. Sce Vitamin A, Tubercle Bacillus, Cell Injury, Uranium, Porphyrins, etc.

Fluorescence Spectra. The technique in some detail is described for 3:4-Benzpyrene by Hieger, I., Am. J. Cancer, 1937, 29, 705-714 who thinks that the photographs of the spectra can well be studied by simple visual examination.

Fluorescent Blue, see Resorcin Blue.

Fluorescent $\mathbf{X}$. A special type of reduced neutral red (Clark, W. M. and Perkins, M. E., J. Am. Chem. Soc., 1932, 54, 1228-1248) employed for tissue cultures (Lewis, M. R., Arch. f. exp. Zelf., $1935,17,96-105$ ).

Fluorine, see Atomic Weights.

Fluorochromes. See Fluorescence microscopy.

de Fonbrune pneumatic micromanipulator can be obtained from Aloe Scientific, 5655 Kingsbury, St. Louis 12, Mo.

Foods. The examination of foods to ascertain their suitability for human consumption involves not only organoleptic tests (use of unaided senses, sight, smell, taste, etc.), but direct microscopic examination and certain cultural, experimental feeding, and other tests. The techniques for adulterations, bacteria, fungi, crystals, spores, parasites and so on are usually the routine ones. However, much time will be saved by knowledge as to what to look for in each case, how to look and the best means of making the observations accurately quantitative (Schneider, A., The Microbiology and Microanalysis of Foods. Philadelphia: P. Blakist on's Son \& Co., 1920, 262 pp.).

Foot's Methods. 1. Rapid silver impregnation of reticular fibers (Foot, W. C., J. Tech. Meth., 1929, 12, 117-119). Fix in $10 \%$ formalin (not necessarily neutral), Zenker's, Bouin's or Helly's fluids, 24 hrs. Make paraffin sections. Remove mercury, if present, with iodine. Treat with $0.25 \%$ potassium permanganate, $5 \mathrm{~min}$. and $5 \%$ oxalic acid, $10 \mathrm{~min}$. Wash in aq. dest. Impregnate $15 \mathrm{~min}$. in following silver solution at $50^{\circ} \mathrm{C}$. : Add 40 cc. $5 \%$ aq. $\mathrm{Na}_{2} \mathrm{CO}_{3}$ to 10 cc. $10 \%$ aq. $\mathrm{AgNO}_{3}$. Let precipitate settle. Decant supernatant fluid. Make up to $50 \mathrm{cc}$. with aq. dest. Shake, repeat sett- 
ling and decanting. Dissolve ppt. in just sufficient $\mathrm{NH}_{4} \mathrm{OH}$, added drop by drop, to almost completely dissolve it leaving a few gray granules. Heat to steaming to drive off excess $\mathrm{NH}_{3}$ and cool to $50^{\circ} \mathrm{C}$. Reduce in $1 \%$ formalin $2 \mathrm{~min}$. Wash in tap water. Tone $2 \mathrm{~min}$. in $0.2 \%$ aq. gold chloride. Wash. Tone in $5 \%$ aq. sodium thiosulphate. Counterstain with hematoxylin-Van Gieson. Reticulum, black; collagenic fibers, vermillion; cytoplasm, yellow; and nuclei, brown.

(2). Silver method for nerve fibers in paraffin sections (Foot, N. C., Am. J. Path., 1932, 8, 769-775). This is a modification of Cajal's technique. Fix in fresh Carnoy's Fluid for 24 hrs. Transfer to absolute alcohol for 1-2 hrs., clear in chloroform and imbed in paraffin. Remove paraffin from sections in usual way. Treat with 2 parts pyridine and 1 part glycerol for 1-12 hrs. Wash in $95 \%$ alcohol and then in aq. dest. to remove most of pyridine. A trace remaining does no harm. Immerse in $10 \%$ aq. silver nitrate at $37^{\circ} \mathrm{C}$. for $12 \mathrm{hrs}$. or so covering container to avoid evaporation. Wash in 2 changes aq. dest. Place in 5\% aq. neutral formalin containing $0.5 \%$ pyrogallol in which sections become yellow-brown, $20 \mathrm{~min}$. Wash under tap. Tone in 1:500 aq. gold chloride, $5 \mathrm{~min}$. (Nuclear precision is improved and glacial impregnation is made less intense if $2 \%$ glacial acetic is added to gold solution). Then place in $2 \%$ oxalic acid containing $1 \%$ neutral formalin for $5 \mathrm{~min}$. Wash at the tap and transfer to $5 \%$ aq. sodium thiosulphate for $5 \mathrm{~min}$. Finally wash again in running water, dehydrate, clear and mount. Foot also recommends Rogers' technique practically as given by him (Rogers, W. M., Anat. Rec., 1931, 49, 81-85) The idea of intensifying the gold toning with oxalic acid he credits to Laidlaw, G. F., Am. J. Path., 1929, 5, 239-247. See general remarks on Silver Methods.

Foraminifera, see Carpenter, W. B. The Microscope and its Revelations, London, 1901 .

Formaldehyde is a gas ( $\mathrm{HCOH}$ ) soluble to $40 \%$ in water producing a solution which is termed commercial formalin or formol. See Formalin.

Formalin (Formol) is a $37 \%$ aq. solution of the gas, formaldehyde. Solutions employed as fixatives and preservatives are made in terms of the percentage of formalin, not of formaldehyde. Thus, a $10 \%$ solution of formalin (formol) is by convention $10 \mathrm{cc}$. of formalin plus $90 \mathrm{cc}$. of water. It is not however $10 \%$ formaldehyde. (Obviously to dilute $10 \mathrm{cc}$. conc. hydrochloric acid with 90 cc. of water would not give $10 \%$ hydrochloric acid because conc. hydrochloric acid is not $100 \%$ so that this practice cannot be extended.) Formerly it was necessary to neutralize commercial formalin in various ways, and it still is for special purposes. The best way to obtain neutral formalin is to distil. Atkins (Lee, p. 61) advises addition of borax to the diluted formalin until it shows a good red color with phenolphthalein or slaty blue with thymol blue. Others simply add a little calcium, sodium, magnesium or even lithium carbonate. Obviously the addition of such minerals greatly complicates the problem when formalin is employed with alcohol as a fixative preliminary to microincineration. Lillie (p. 28) specifies a neutral buffered formaldehyde solution $(\mathrm{pH}$ $70)$ as follows: $37-40 \%$ formaldehyde solution, 100 cc.; water, 900 cc.; acid sodium phosphate, monohydrate, $4 \mathrm{gm}$.; anhydrous disodium phosphate, 6.5 gm. Constant $\mathrm{pH}$ is desired. Unless neutral formalin is specified and the manner of neutralization, it is best simply to use the fairly pure product which now can readily be obtained. Experiments by Davenport, H. A., Stain Techn., 1931, 9, 49-52 show that as a neurological fixative slightly acid formalin is somewhat better than neutral formalin. A few of the many demands for formalin as a fixative will be found under:

$\begin{array}{ll}\text { Alizarin red S } & \text { Giemsa staining } \\ \text { Alveolar pores } & \text { Glia staining } \\ \text { Amyloid } & \text { Gomori } \\ \text { Argentaffine cells } & \text { Gordon } \\ \text { Arsenic } & \text { Grieves } \\ \text { Articular nerve ter- } & \text { Johnson's neutral red } \\ \text { minals } & \text { Kinney } \\ \text { Bile pigments } & \text { Krajian } \\ \text { Bismuth } & \text { Liebermann - Burch- } \\ \text { Bodian } & \text { ardt } \\ \text { Bone } & \text { Madder staining } \\ \text { Buzaglo } & \text { Mallory's connective } \\ \text { Cajal's brom-formol } & \text { tissue stain } \\ \text { Cartilage } & \text { Microglia } \\ \text { Chitin } & \text { Mucus } \\ \text { Chloride } & \text { Nile blue sulphate } \\ \text { Chorioallantoic mem- } & \text { Perdrau } \\ \text { brane } & \text { Pia mater } \\ \text { Christeller-Koyama } & \text { Romieu } \\ \text { Chromaffin reaction } & \text { Schultz cholesterol } \\ \text { Color preservation } & \text { Sebaceous glands } \\ \text { Connective tissue cells } & \text { Silver citrate } \\ \text { Dopa oxidase } & \text { Smith-Dietrich } \\ \text { Fatty acids } & \text { Spirochetes } \\ \text { Fluorescence micros- } & \text { Vorhoeff } \\ \text { copy } & \text { Weigert-Pal } \\ \text { Foot } & \text { Weil } \\ \text { Frozen sections } & \text { Wilder }\end{array}$


In combination with other reagents formalin is also a good fixative ef. Formalin-Zenker in which formalin is substituted for acetic acid, Bouin, Regaud's Fluid and many others. Since, however, formalin is a strong reducing agent mixtures of which it is a part are unstable so that it must be added immediately before use. As Mallory (p. 40) points out, formalin also has certain disadvantages. It is inferior to alcohol as a preservative for iron and other pigments. It of ten changes the color of bile concretions from yellow to grcen and it may produce in the tissues a troublesome brown-black finely divided crystalline precipitate from laked hemoglobin. $\mathrm{He}$ advises removal of this precipitate by treating sections for $30 \mathrm{~min}$. in $75 \%$ alcohol, 200 cc.; plus 25-28\% ammonia water, 1 cc. (Schridde's method), or for 10 min. in $80 \%$ alcohol, 100 cc. plus $1 \%$ aq. potassium hydroxide, 1 cc. (Verocay's method) after either of which they are washed thoroughly in water before placing in $80 \%$ alcohol and staining. When employed as a preservative concentration of formalin should be $4 \%$.

Formalin-Alcohol, see Alcohol-Formalin. Formalin-Zenker. Zenker's fluid modified by substituting $5 \%$ formalin in place of the $5 \%$ acetic acid. It is also known as Helly's fluid and Zenker-formol. This is one of the three major routine fixatives the others being Zenker and Bouin. See Acid Fast Bacilli, Alveolar Pores, Arteries, Basal Bodies, BrazilinWasserblau, Mucus, Goodpasture's Method, Methyl-Green Pyronin. In some cases $10 \%$ formalin is inserted instead of $5 \%$.

Formalose see Formalin.

Formamide of Eastman Kodak Co. is a substance, called a "modifier", which when added in $10 \%$ to $50 \%$ alcohol improves fixation and staining of peripheral nerve (Bank, E. W. and Davenport, H. A., Stain Tech., 1940, 15, 9-14).

Formazan, see Triphenyltetrazolium Chloride.

Formic Acid, see Decalcification.

Formol is a synonym for formalin.

Formol-Müller. This is 1 part of formol to 10 parts Müller's fluid.

Formol-Nitric fixative. 3 parts $10 \%$ formalin and 1 part $10 \%$ nitric acid. This has, according to McClung, proved very valuable for chick embryos.

Formol-saline is the fluid resulting when formalin is diluted with isotonic salt solution (presumably $0.85 \%$ aq. sodium chloride) instead of with aq. dest. It is not advised as a fixative.

Formvar, a plastic occasionally employed to make supporting films in electron microscopy. It is made by Shawinigan Products Corporation, New York, N. Y.

Fowl Pox, see Borrel Bodies.

Fractionation, see collection of cell components under Centrifugation.

Fractures. Vital staining with Alizarin Red S (Schour, et al., J. Dent. Res., 1941, 20, 411-418).

Fragility Tests. Micro for erythrocytes (Kato, K., J. Lab. \& Clin. Med.,1940, 26, 703-713. See Capillary Fragility Tests.

Freezing. Details on the formation of ice within cells are given by Chambers, R. and Hale, H. P., Proc. Roy. Soc., B, 1932, 110, 336. See Revival after.

Freezing and Drying, see Altmann-Gersh.

Freifeld's Blood Stain. 'To 20 cc. tap water add 7 drops Ziehl's Carbol Fuchsin and 5 drops $1 \%$ aq. methylene blue. Stain methyl alcohol fixed blood smears 1 hr. Shows clearly basophilic granules of toxic neutrophiles (Naegeli, O. Zlutkrankheiten und Blutdiagnostik, Berlin, 1931, p. 19, quoted from McClung, 1950 , p. 225).

Frozen Sections. These are of great value when preparations must quickly be made and when methods of alcoholic dehydration before sectioning are contraindicated. They are specified elsewhere in this book under several headings including :

$\begin{array}{ll}\text { Amyloid } & \text { Lipase } \\ \text { Cholesterol } & \text { Lipids } \\ \text { Digitonine reaction } & \text { Microglia } \\ \text { Dopa oxidaso } & \text { Millon's reaction } \\ \text { Gold } & \text { Oxidase } \\ \text { Indigo-carmine } & \text { Pepsin } \\ \text { Krajian's Congo stain } & \text { Spirocheta pallida } \\ \text { Liebermann-Burchardt } & \text { Urease }\end{array}$

To make the sections take recently excised still living tissue, or better fresh tissue fixed for about $30 \mathrm{~min}$. in $10 \%$ formalin. First freeze a little water on the block of a freezing microtome. Then add the tissue and freeze it too plus a drop more of water. Allow block to thaw to optimum consistency, cut sections $15-50 \mu$ thick as desired, and remove them with a camel's hair brush from the microtome knife to formalin, water or physiological saline. When many are required, it may be necessary to freeze several times as the tissue becomes too soft. If thinner sections are wanted resort to Gelatin Imbedding before sectioning.

For quick staining Thibaudeau, A. A., J. Lab. \& Clin. Med., 1933, 19, 20t-209 advises that sections of formalin fixed tissue be stained in Harris' hematoxylin 5-15 sec., rinsed in aq. dest., blued in aq. dest. + few drops $\mathrm{NH}_{4} \mathrm{OH}$, passed up through 70,85 and $95 \%$ alcohol counterstained in absolute alcohol and 
eosin (5 sec.), cleared in carbol xylol, blotted with filter paper and mounted in balsam. Proescher, F., Proc. Soc. Exp. Biol. and Med., 1933, 31, 79-81 recommends pinacyanol as giving excellent color contrasts. Perhaps the simplest method advised by the Bensleys (p. 138) is to stain the sections in Goodpasture's Acid Polychrome Methylene Blue (which see) $1 \mathrm{~min}$. or longer, wash and mount in aq. dest. This colors nuclei dark purple and connective tissue bright rose red. But methylene blue is less permanent than hematoxylin. Adamstone and Taylor (Stain Techn. 1948, 23, 109-116) have developed a modified technique useful for histochemical and routine studies.

For reticular and collagenic fibers in frozen sections proceed as follows (Krajian, A. A., Arch. Path., 1933, 16, 376-378): After fixation in $10 \%$ formalin, cut sections 5-10 microns and wash in aq. dest. Then $10 \%$ aq. $\mathrm{NH}, \mathrm{OH}$ at $60^{\circ} \mathrm{C}$, $15 \mathrm{~min}$. Wash in 3 changes aq. dest. and place in $0.3 \%$ $\mathrm{KMnO}_{4}$ for $5 \mathrm{~min}$. Rinse in aq. dest., decolorize in $1.5 \%$ oxalic acid until brown color has entirely disappeared. Wash 4-5 times in aq. dest. and soak in $5 \% \mathrm{AgNO}_{3}$ at $60^{\circ} \mathrm{C}$. for $1 \mathrm{hr}$. Wash twice in aq. dest. Transfer to ammoniacal silver sol. (to make add 6 drops $10 \%$ $\mathrm{NaOH}$ to 8 cc. $10 \% \mathrm{AgNO}_{3}$. Then add freshly prepared $10 \% \mathrm{NH}_{4} \mathrm{OH}$ drop by drop until almost entirely clear. Dilute to $28 \mathrm{cc}$. with aq. dest.) $16 \mathrm{~min}$. at $60^{\circ} \mathrm{C}$. Wash 3 times quickly in aq. dest. Change to $30 \mathrm{cc}$. formaldehyde $+70 \mathrm{cc}$. aq. dest. $1-3 \mathrm{~min}$. at $60^{\circ} \mathrm{C}$. Wash in tap water. Mount on slide. Dehydrate with a little absolute alcohol and blot into position. Dehydrate more, blot, 3 changes equal parts anilin oil and xylol, xylol, balsam. Reticular fibers jet black, collagenic ones dark brown.

For serial sections of brain (Marshall, W. H., Stain Tech., 1940, 15, 133-138) fix slices 24 hours or longer in 10 or $15 \%$ formalin and then treat them with a $20-30 \%$ alcohol or in $15 \%$ formalin in $20 \%$ alcohol. The object of the alcohol treatment is to avoid formation of hard and brittle ice crystals which fracture the sections as they are made. Cut tissue into blocks about $1.0 \mathrm{~cm}$. thick. Place on a $\mathrm{CO}_{2}$ ice freezing disc which has been covered by a piece of wet blotting paper. (In our laboratory we use a regular $\mathrm{CO}_{2}$ gas freezing disc which has been adapted to a precision sliding microtome.) Freeze the block of tissue slowly throughout. The proper degree of freezing depends on the thickness of the sections to be cut. Marshall recommends a paraffin knife, $20-30^{\circ}$ angle with block, knife set in a line perpendicular to the direction of motion. Remove cut sections by a camel's hair brush to $50 \%$ alcohol and keep them in serial order. Mount sections serially on slides coated with Albumen-Glycerin. Smooth out wrinkles and flatten sections by gentle pressure with blotting paper moistened with $50 \%$ alcohol. Remove slides to a $38^{\circ} \mathrm{C}$. oven for $4-6 \mathrm{hrs}$. when they are ready for staining. (In some cases it may be inadvisable to press the sections flat upon the slide. Thin sections require less drying than thick ones. In any case until one has gained confidence in the use of the technique, the sections should be observed at intervals in the $38^{\circ} \mathrm{C}$. oven. At the least sign of excessive drying (whitening of parts of the section) the sections should at once be removed to the stain.) The Cresyl Violet method of Tress and Tress is recommended.

Frozen-Dehydration Method for histological fixation-Written by Normand L. Hoerr, Department of Anatomy, Western Reserve University School of Medicine, Cleveland, Ohio. November 28, 1951-This Method has distinct advantages over the usual methods of preparation of tissues for microscopy, particularly for histochemical studies and for microincineration. Tissues are frozen as rapidly as possible and then maintained below the freezing point while being dried in vacuo. The method changes tissues little or not at all chemically, and physically only by the removal of water. If the freezing of a block of tissue can be effected rapidly enough the ice crystals which form during the freezing out of free water will be below microscopic size. The constituents of the tissue are then not displaced to an extent appreciable by microscopic examination. To prevent displacement of any constituent of the tissue after freezing, it is necessary that the dehydration be carried out below the eutectic point of the tissue. There is no way of knowing what the eutectic point of such a complex mixture of substances as obtains in animal tissues may be but, since in tertiary and quaternary systems the eutectic point is usually lower than the eutectic points of mixtures of any two of the substances, it is reasonable to assume that the absolute depression of the freezing point in tissues may be well below $-54.9^{\circ} \mathrm{C}$. (the eutectic point of aqueous calcium chloride solution). From our experience, we would say that better cytologic appearance of the tissues-i.e., an appearance comparing most favorably with the appear- 
ance of living tissues-is obtained by dehydrating at temperatures below $-30^{\circ} \mathrm{C}$. If the freezing can be effected rapidly enough, the constituents of a tissue are not appreciably changed morphologically.

Die Elementarorganismen und ihre Beziehungen zu den Zellen, Altmann, R.,/Leipzig, Veit and Co., 1890, introduced the method of freezing tissues and dehydrating them over sulfuric acid in vacuo at temperatures below $-15^{\circ} \mathrm{C}$. Gersh, I. (Anat. Rec., 1932, 53, 309-337) overcame many early diffi. culties and elaborated the technic so that good histologic fixation could be procured on a number of tissues. Packer, D. M. and Scott, G. H. (J. Tech. Methods, 1942, 22, 85-96) have overcome, by a newly designed cryostat, many of the dehydration difficulties. The frozen dehydration method has been of value in the preliminary fixation of tissues for a study with the electron microscope of the localization of their contained salts by microincineration. It has been used for the preliminary preparation of tissues in the study of mitochondria and Nissl substance, of secretion in the stomach and the thyroid gland, and in a histochemical study of the Golgi apparatus. More extended discussions of the process of freezing and of the subsequent process of dehydration, with considerations of the advantages and limitations of the method are given by Hoerr, N. L. (Anat. Rec., 1936, 65, 293-295) and Simpson, W. L. (Anat. Rec. 1941, 80, 173-189).

A number of cryostats have been designed recently, including those by Stowell, R. E. (Stain Technology, 1951, 26, 105-108) Emmel, V. M. (Anat. Rec., 1946, 95, 159-175) Scott, G. H., and Hoerr, N. L. (Medical Physics, 1950, 2, 292-296) Wang, K. J., and Grossman, M. I. (J. Lab. and Clin. Med., 1949, 34, 292-296) and Mendelow, H., and Hamilton, J. B. (Anat. Rec., 1950, 107, 443-451). A good commercial design is available from Scientific Specialties Corporation, Cambridge, Mass., or Euclid Glass Engineering Laboratory, 11310 Wade Park Avenue, Clevelend, Ohio.

Fuchsin NB, see New Fuchsin.

Fuchsin S, SN, SS, ST or S III, see Acid Fuchsin.

Fungi.-Written by Morris Moore, Barnard Free Skin and Cancer Hospital, St. Louis, Mo. November 10, 1951.

1. Skin scrapings and hair. The usual method is to mount the material in an alkali-either sodium hydroxide $(\mathrm{NaOH})$ or potassium hydroxide
$(\mathrm{KOH})$. The latter is preferable and should be used in a $10-30 \%$ solution. For rapid work $40 \%$ is employed but this tends to swell and disintegrate the fungi. A weak solution takes longer to clear the skin. The skin usually clears in $5 \mathrm{~min}$. to $2 \mathrm{hrs}$. in concentrations of $10-30 \%$. A little heat helps. Use subdued light in order to avoid high lights. The fungus is clearly discernible against the irregular nondescript background of skin which is usually clear. Dip infected hairs taken from scalps, particularly those that are oily, in ether or in alcohol (absolute alcohol is preferable to $95 \%$ ) for a moment in order to get rid of the oil which of ten simulates spores in shape and size.

Adamson (H. G., Brit. J. Dermat., $1895,7,201-211,237-244$ ) has recommended clearing with $5-10 \% \mathrm{KOH}$ and staining by the Gram method. Chalmers and Marshall (A. J. and A., J. Trop. Med. Hyg., 1914, 17, 256-265, 289-291) suggest soaking scales in $40 \% \mathrm{KOH}$ for some hours in a watch glass in an incubator at $40^{\circ} \mathrm{C}$. Transfer specimens to watch glass containing $15 \%$ alcohol for $30 \mathrm{~min}$., remove to slide, allow alcohol to evaporate and dry over flame; stain with Anilin-Gentian Violet for $20 \mathrm{~min}$. Treat with Gram's Iodine for $3 \mathrm{~min}$.; decolorize with anilin oil, 30 min.; stain in concentrated alcoholic eosin, 1 min.; wash off eosin with anilin oil or clove oil; treat with xylol and mount in balsam.

Priestley (H., Med. J. Australia, 1917,2 , 471-475) recommends lactophenol (lactic acid, 1 part; phenol, 1 part; glycerol, 2 parts, aq. dest., 1 part) for clearing instead of $40 \% \mathrm{KOH}$; or chloral hydrate crystals, 2 parts; lactic acid, 1 part; phenol crystals, 1 part, may be used. For thick material Langeron suggests: chloral hydrate crystals, $40 \mathrm{gm}$.; phenol crystals, 40 gm.; lactic acid (U.S.P.), 20 gm.; and sodium salicylate, $10 \mathrm{gm}$. Slight heat facilitates clearing. To stain, Priestley recommends treatment with chloroform to remove the fat; boiling, $2-3 \mathrm{~min}$., with formic acid; washing for a few minutes in water and staining with Sahli's methylene blue: after which the tissue is to be washed, differentiated with alcohol if necessary, dehydrated, cleared and mounted in balsam.

Bachman (R. W., Arch. Dermat. \& Syph., 1920, 1, 50-51) recommends the following procedure: Place scrapings in a drop of water on a cover slip, tease thoroughly with a dissecting needle, dry over a flame but do not scorch. Stain for 2 min.; decolorize in $95 \%$ alcohol, 15-30 sec.; immerse in aq. dest., 15-30 sec.; pour off excess, dry by heat, 
and mount in balsam. Spores and mycelium, blue; scrapings, yellow. The dye is sat. alc. gentian violet, 2.5 parts; aq. dest., 17.5 parts; orange $G$ solution, 9 parts; acetic acid, 1 part; $95 \%$ alc., 5 parts. The orange $\mathrm{G}$ solution is orange G, 2 parts; $95 \%$ alc., 20 parts; water, 80 parts. Decolorize with $10-20 \% \mathrm{KOH}$.

The hydroxide method of examination is simple and often rapid, but unless used by one familiar with it the results may be misleading. There is danger of confusion with structures which Becker and Ritchie (J. W. and E. B., Arch. Dermat. \& Syph., 1940, 22, 790-802) have indicated as resembling yeast cells. These artifacts may be removed by treating the material progressively with absolute alcohol, ether, absolute and $95 \%$ alcohol. They have been termed 'mosaic fungus' and have been reported by Greenwood and Rockwood (A.M. and E. M., Arch. Dermat. \& Syph., 1930, 21, 96-107) as degenerate fungi. In fact they are cholesterol crystals. The use of dyes eliminates in great measure such artifacts. However, the use of dyes is not practical with thick sections for which recourse must be had to the hydroxide method.

When the scrapings or scales are thin, or when sputum, pus or exudate are examined, a $1 \%$ aq. methylene blue and glycerin can be used as follows: One drop of the $1 \%$ solution of methylene blue is placed on a clean slide and the material is stirred within it, allowed to stand for approximately $2 \mathrm{~min}$. when a clean cover slip is placed over the mixture and pressed down to flatten out the material and to express the excess solution. The superfluous stain is taken up by filter paper. A drop of glycerin is then placed along one edge of the cover slip and allowed to seep under, displacing the stain and giving a clear background to the stained material. The fungus appears bright blue.

The lactophenol-cotton blue technique was developed in the French laboratories using the formula of Amann (J., Zeit. Wiss. Mikr., 1896, 13,18-21). Lactophenol consists of phenol crystals, $20 \mathrm{gm}$.; glycerin, $40 \mathrm{gm}$.; lactic acid, $20 \mathrm{gm}$. and aq. dest., $20 \mathrm{gm}$. Cotton blue (anilin blue, China blue) is a mixture of the trisulphonates of tri-phenyl pararosanilin (C.I. 706) and of di-phenyl rosanilin. Place a drop of the cotton blue $(0.5 \%$ aq.) on the slide; stir up the material within it and allow to stand for about $2 \mathrm{~min}$. Add cover slip and press down to squeeze out any excess dye, which is taken up by filter paper. Add a drop of lactophenol to the edge of cover slip and allow it to replace the cotton blue which dries out. The stain may be rapidly replaced by holding a bit of filter paper at the edge of the cover slip opposite the lactophenol. The cell wall stains lightly as compared with the darkly colored central portion of the fungus. The tissue elements also stain light blue.

Swartz and Conant (J. H. and N. F., Arch. Dermat. \& Syph., 1936, 33, 291-305) have modified the lactophenol and cotton blue procedure. First put a few scrapings in $5 \%$ aq. potassium hydroxide, heat somewhat and wash in water. Place material in a drop of the combined cotton blue $(0.5 \%)$ and lactophenol. The fungi stain a darker blue than the tissue cells.

Schubert M., Dermat. Wchnschr., $1937,105,1025-1029)$ has modified the Swartz-Conant technique. Soak the scales in $2 \% \mathrm{KOH}$ for $30 \mathrm{~min}$. or until they appear glassy and then wash in aq. dest. 2-10 hrs. Transfer small particles to a slide and add 1 or 2 drops of following stain : cotton blue, $0.25 \mathrm{gm}$.; lactic acid, $10 \mathrm{gm}$.; phenol crystals, 10 gm.; and aq. dest., $20 \mathrm{gm}$. The fungi appear dark blue while the epidermal cells stain lightly. See also Berberian's Method.

2. Sputum, pus and exudates: Examine for fungi after mounting directly on a slide after mixing in $20 \% \mathrm{KOH}$ or on stained smears. The latter are not very satisfactory because smearing tends to disturb the arrangement of the cells but they are useful for detection of mycelium. Many contaminating organisms are generally present in these exudates unless material is secured from fresh lesions opened aseptically. Several examinations may be necessary since the organisms in exudates are seldom numerous. The hydroxide usually dissolves most of the tissue elements and the fungi stand out as refractile bodies. Several of the staining methods employed in the study of hair and scrapings may be used. Of these, the methylene blue and glycerin method is best but the lactophenol-cotton blue technique is likewise advised.

3. Vesicles, blister fluid, spinal fluid or urine: These can also be directly examined. But vesicle, or blister, fluid yields only a small amount of material and for best results, the methylene blue-gly cerin method or the lactophenolcotton blue technique is advised. Urine, or spinal fluid, should be concentrated by centrifugation before examination. The same staining procedures are advocated. See Blastomycosis. 
4. Skin: Unna, Jr. (P., Dermat. Wchnschr., 1929, 88, 314-321) advises the following modification of the Pappenheim-Unna, Sr. method for staining fungi in skin. Fix in absolute alcohol, then run through the alcohols to xylol and imbed in paraffin. Cut sections at $10 \mu$, stain with pyronine-methyl green (pyronine, 9 parts; methyl green, 1 part; $96 \%$ alcohol, 90 parts; glycerol, 100 cc.; $0.5 \%$ phenol to make 1000 cc.), 5-10 sec.; rinse in water; dry with absolute alcohol; and mount in balsam. Fungi, rubin red; leukocytes, green to blue green; nuclei of cells of basal horny layer of the epidermis, red.

Fungi in tissue can be easily stained by Iron-Hematoxylin and eosin. The fungous elements take the hematoxylin stain nicely, although some difficulty may be encountered in distinguishing spherical cells or spores from tissue elements. The Gram method of staining for bacteria has been used with a measurable amount of success since fungi are, in general, gram-positive.

Unna's Alkaline Methylene Blue (Unna, P., Monatsh. f. prakt. Dermat., $1891,13,225-237,286-311$ ), although recommended for staining plasma cells and as a general stain in combination with phloxine or eosin, has been especially adapted for staining organisms in the stratum corneum. It consists of methylene blue, $1 \mathrm{gm} . ;$ potassium carbonate, $1 \mathrm{gm}$.; and aq. dest., 100 cc. The solution stains better after ripening for a week or two and should be diluted 1 to 10 or 1 to 5 before use.

Malcolm Morris (Mallory, F. B. and Wright, J. H., Pathological Technique, Philadelphia and London, 1924, p. 175) in staining various parasites of the skin, avoids the use of potassium hydrate. Place skin in ether, or in 1:1 alcoholether; stain for 5-30 min. in 5\% gentian violet in $70 \%$ alcohol. Then pass through iodine solution, 1 min.; anilin, or anilin plus 2-4 drops of nitric acid; anilin; and xylol (2 changes) to balsam.

5. Other tissues: A number of methods listed for staining Bacteria in tissue can be used successfully for fungi. Mallory's Connective Tissue stain is good for Cryptococcus hominis in brain tissue. Fungus cells, red; thick mucoid capsules, light blue. The Gram-Weigert staining method is also excellent. Organisms, deep violet; nuclei, blue to violet; connective tissue, red.

Actinomyces in sections may be stained successfully with Alum-Hematoxylin followed by strong eosin. Mallory (p. 279) lists 2 methods of which the following gives good results with paraffin sections of formalin or Zenker fixed tissue. Stain in alum-hematoxylin, 3-5 min.; wash in water; stain in a $2.5 \%$ aq. phloxine or $5 \%$ aq. eosin, $15 \mathrm{~min}$. in the paraffin oven; wash in water; stain in Anilin Crystal Violet (try Stirling's), 5-15 min.; wash in water; treat with Granı's Iodine solution, 1 min.; wash in water and blot with filter paper; differentiate in several changes of anilin until no more color comes off ; rinse in several changes of xylol and mount in balsam. The branched organisms stain blue while the hyaline sheaths ("clubs") become pink to red.

The Hotchkiss-McManus or Periodic Acid-Schiff Stain (Kligman, A. L. and Mescon, H., J. Bact., 1950, 60, 415-421) may be used to find fungi in tissue or sections. Tissue fixed, embedded in paraffin and sectioned in usual manner. After deparaffinizing, sections rinsed in absolute alcohol; washed in distilled water; immersed in $1 \%$ aq. periodic acid C.P. (Eimer \& Amend) 5 min.; washed in tap water, $10 \mathrm{~min}$; placed in Schiff reagent, 10 to 15 min.; transferred to 1 normal $\mathrm{HCl}, 5$ min.; washed in tap water, $10 \mathrm{~min}$.; counterstained with $1 \%$ aq. light green, for sec.; dehydrated, cleared and mounted. Fungi stain red to purple. Tissue cell nuclei do not stain.

Schiff reagent prepared as follows: Dissolve $0.5 \mathrm{gm}$. basic fuchsin (Coleman \& Bell) by passing over it $100 \mathrm{cc}$. boiling water; cool to $50^{\circ} \mathrm{C}$.; filter and add $10 \mathrm{cc} ., 1$ normal $\mathrm{HCl}$ and $0.5 \mathrm{gm}$. potassium metabisulfite dry reagent (Eimer \& Amend) to filtrate. Solution becomes colorless to pale straw colored by standing in dark overnight. Add 0.25 to $0.5 \mathrm{gm}$. activated charcoal, shake well and filter immediately to completely decolorize. Refrigerate in tightly stoppered bottle.

For smears or nails: Use thin smears and fine nail scrapings. Material made to adhere to slide with egg albumen. Coplin jar or drop bottle technique employed. Immerse material in 95\% alcohol, 1 min.; cover with $5 \%$ aq. periodic acid, 3 minutes; wash in running water. 2 min.; stain in Schiff reagent, 5 min.; rinse in running tap water, 1 min.; dehydrate in $95 \%$ then absolute alcohol and two changes xylol, $1 \mathrm{~min}$. each. Mount in clarite. See Polysaccharides.

After the fungi have been successfully cultivated on the various mediums recommended (Moore, M., Arch. Dermat. \& Syph., 1936, 34, 880-886) they can be examined microscopically by transferring part of the growth with a sterile platinum or nichrome wire to 
a clean slide. This should be done gently to avoid destruction of the fungous growth. The fungus is teased apart gently in one of several fluids such as water, alcohol, alcohol and glycerine (equal parts) or other mounting fluids. Water has a high surface tension and causes disruption of the growth; while alcohol evaporates rapidly and must be replaced. The following solution serves well : $2 \%$ potassium acetate, 50 ec.; glycerin, 20 ec.; and $95 \%$ alcohol, 30 cc. The preparation is examined with reduced light. The preparations may be stained using one of the several methods listed: lactophenol-cotton blue; methylene blue-glyeerin; or Giemsa-glycerin. See Chorioallantoic Membrane, Actinomycetes.

Furfural. Has been suggested but is not recommended as substitute for formaldehyde (Stowell, R. E. and Stokes, J. M., J. Tech. Meth. and Bull. Internal. Assoe. Med. Museums, 1944, 24, 25-30).

Fuscin (L. fuscus, dusky), a dusky pigment of retinal epithelium usually present in crystalline formations made up of albuminous cores, which determine their shape, plus the adsorbed fuscin material. A relationship to melanin is debated but the pigment is very resistant to chemical attack. It can, however, be bleached completely when exposed to light in vitro. For details see Arey, L. B. in Cowdry's Special Cytology, 1932, 3, 1218.

Fustics. "Young" fustic is a stain obtained from the smoke tree, Rhus cotinus of West Indies and South America giving colors from bright yellow to dark olive now seldom used by dyers. "Old" fustie is obtained from a tree of the mulberry family, Chlorophora tinctora, which grows in the same eountries. It is chiefly employed for woolens giving shades of lemon and old gold (Leggett, W. F., Ancient and Medieval Dyes. Brooklyn: Chemical Publishing Co. Inc., 1944, 95 pp.).

Gadolinium see Atomic Weights.

Galliamine Blue (Cl. 894) can be employed in place of hematoxylin as an iron lake stain for nuclei.

Gallein (CI, 781), a mordant dye of light fastness 1 . Use as solution $0.5 \mathrm{gm}$. in 100 ce. of either $1 \%$ aq. ammonium acetate or $0.1 \%$ sulphuric acid. Small invertebrates should be previously mordanted, $30 \mathrm{~min}$. in $1 \%$ aq. ferrie ammonium sulpha $e$ and rinse in aq. dest. before staining for 1 to $2 \mathrm{~min}$. in the solution at $50^{\circ} \mathrm{C}$. Color blue black. If copper sulphate is employed for mordant color is hematein purple. In paraffin seetions of animal tissues nuelei color blue black in 15 to 20 sec. at $50^{\circ} \mathrm{C}$. Directions are also given for plant tissues and Blue-green algae (Emig, p. 54-55).

Gallium, see Atomic Weights.

Gallocyanin-Chromalum Staining of Basophilic Cell Structures-Written by Lárus Einarson. Normal-Anatomisk Institut. Aarhus Universitet, Aarhus, Denmark. February 27, 1951.-Basophilia is an essential property common to some most important cell structures, and the study of the nature as well as the attainment of a method for quanti. tative estimation of basophily have been among the outstanding general problems in histology and cytology. Here, the nerve cells make an especially favourable object on account of their high degree of cytoplasmic basophily due to the Nissl substance, which contains nuclein, acid and basic proteins respectively (Einarson L. J. comp. Neur., 1935, 61, 101-133).

An accurate estimation of basophily can be achieved by staining with dissolved colour lakes of some synthetic dye-stuffs (Ranvier, 1875; Grenacher, 1879; Rawitz, 1896; Becher, 1921), and the staining by gallocyanin-chromalum affords the most accurate representative of this prineiple of staining by innercomplex dye-metal salts (Einarson, L., Acta path. \& Mierobiol. Scand., 195, 28, 82-102).

The staining solution is made as follows: $5 \mathrm{gm}$. chromalum puriss. is dissolved in $100 \mathrm{ec}$ redistilled water, then $0.15 \mathrm{gm}$. gallocyanin (Grübler-Hollborn or Nat. Aniline Division, New York) is added and the whole is mixed by shaking the bottle. The mixture is warmed up gradually and boiled for 5 min. After cooling at room temperature, filtration through filter paper and addition of redistilled water through the filter until the volume again is 100 cc., the solution is ready for use; its $\mathrm{pH}$ is 1.64. Staining time is $48 \mathrm{hrs}$ at room temperature. After staining washing in aq. dest., alcohols, xylol balsam.

The process by which the lake is formed consists in the formation of a soluble lake-cation (reddish), a slightly soluble lake-sulphate (blue) and a nonsoluble lake-hydroxide; the latter is formed by an immediate hydrolysis of some of the lake under formation. After the solution has been prepared no further hydrolysis occurs until 4-5 weeks later (late hydrolysis), and a gradual sedimentation of lake-hydroxide takes place; at the same time the staining power of the solution be- 
comes weaker. Thus, for safety the solution should not be used for morc than 3 weeks. Lake-sulphate and lakehydroxide are the main constituents of the precipitate filtered off the solution before use. Although such inner-complex salts all have very low solubilities in water, the lake-sulphate, however, reamins dissolved in a sufficient amount to become dissociated into the monovalent lake-cation and an anion according to the equation:

\section{(gallocyanin $\left.\mathrm{Cr}\left(\mathrm{H}_{2} \mathrm{O}\right)_{4}\right)_{2} \mathrm{SO}_{4} \rightleftarrows 2$ galloc

$$
\mathrm{Cr}\left(\mathrm{H}_{2} \mathrm{O}\right)_{4}^{+}+\mathrm{SO}_{4}
$$

In the lake-cation, $\mathrm{Cr}\left(\mathrm{H}_{2} \mathrm{O}\right)_{6}{ }^{+++}$is attached to one gallocyanin molecule by two valency bonds to its $\mathrm{OH}$ and $\mathrm{COOH}$, and by two coordinating bonds to its $=\mathrm{O}$ and $/ \mathrm{O}$ through substitution of two $\mathrm{H}_{2} \mathrm{O}$-molecules from the internal sphere of the Cr-atom. The designations $=\mathrm{O}$ and $/ \mathrm{O} \backslash$ are used for the sake of brevity. $=0$ means the 2-carbonyl-O, and $/ \mathrm{O} \backslash$ means the oxazin-O of the chromophor-group).

The reddish lake-cations, the actual staining compound, unite progressively and selectively with the nucleoproteins of the fixed cell structures to form a blue salt of the lake-cation and the tissue. This proceeds until maximum occupation has taken place; then no further attachment of the stain to the basophilic cell structures is possible. When the staining has attained a certain intensity it will not be further increased, even if the tissue be left in the staining solution for a considerable length of time; in this respect the staining is independent of the staining time (exquisite progressivity); no other histological staining known to me possesses this quality in the same degree. Owing to the great stability of the staining it is also completely unaffected by alcohol and xylol during the procedure of dehydration and mounting. In consideration of these qualities the staining intensity depends directly on the inherent capacity to bind the stain, possessed by the living cell at the moment of fixation.

Briefly the staining consists of :

1. A specific binding of the lakecation to the nucleic acids, in which it reacts with the phosphoric acid groups to form a lake-salt of nucleic acid; within the range from $\mathrm{pH} 0.83$ to 4.35 this specific binding always takes place by means of the free +valency of the lake-cation, regardless of the type of nucleic acid.

2. An adsorption of lake-sulphate to the tissue, i.e., an unspecific costaining of non-basophilic structures, which increases directly with the pII of the dye-solution; it mainly takes place by the basic $7-\left(\mathrm{CH}_{3}\right)_{2} \mathrm{~N}$-group of the dye molecule; by staining at a sufficiently low pH the co-staining is reduced to a minimum.

3. A binding of the lake-cation to the proteins of the basophilic structures, which, however, only takes place in a definite range of $\mathrm{pH}$ on the alkaline side of the iso-electric point of the proteins and in fact is unimportant; on the acid side of the iso-electric point the lake-cation combines with the nucleic acids alone.

As the lake compounds stabilize (buffer) the staining solution towards acids and alkalies its $\mathrm{pH}$ can be varied simply by adding 1 to $10 \mathrm{cc}$ of $\mathrm{N} / 1$ $\mathrm{HCl}$ and $\mathrm{N} / 1 \mathrm{NaOH}$ respectively to each $40 \mathrm{cc}$. of stock solution. In the range from $\mathrm{pH} 1.50$ to 1.80 the specific staining reaches its maximum intensity at the same time as the co-staining remains completely negligible; $p H 1.64$ represents the optimum acidity for the specific attachment of the lake-cations to the nucleic acids, the relative quantity of which can be estimated by photometric or densitometric measurements. The increase of the co-staining first sets in at pH 1.80 to reach its maximum between pH 3.4 and 3.5 whereupon every staining fades away; at pH 4.27 it is barely visible. The co-staining at pH $2.90-$ 3.42 may be used for demonstrating axons and neuroglia (Einarson \& Ringsted 1938, p. 43). After the addi. tion of alkali the solution should not be used longer than 4-8 days.

The usual basic blues preferably stain proteins and other amphoteric electrolytes which display "facultative basophily", i.e., under another set of conditions they are less basophilic or even acidophilic; they are amphophilic substances, and the staining is a mere adsorption of the dye.

Gallocyanin-chromalum indicates the degree of "genuine basophily" which depends directly on the quantity of nucleic acids present; genuine basophily is not in the same way dependent on the external conditions as the facultative one, and its changes reflect the decomposition during neuronal function or regeneration and the subsequent repair of cytoplasmic nucleic acid from the nucleus (Einarson, L., Am. J. Anat., 1933, 53, 141-176).

Gallocyanin (Cl. 883)-alizarin blue RBN, ehrom blue GCB and fast violet. It is a basic oxazin dye.

Gamma $=\frac{1}{1000} \mathrm{mg}$. or $0.001 \mathrm{mg}$. 
Gardner, see Articular Nerve Terminals.

Garven's Gold Chloride method for nerve endings in muscle (Garven, H.S.D., Brain, 1925, 48, 380-441). This is Fischer's modification of Ranvier's technique as used in Golgi's Laboratory. Immerse small pieces of tissue in $25 \%$ aq. pure formic acid and tease a little to assure penetration $10-15 \mathrm{~min}$. Blot with clean cloth. Place in $1 \%$ aq. gold chloride just sufficient to completely cover tissue and shake. Avoid all iron instruments. Cover dish with blue or yellow glass. Leave $20 \mathrm{~min}$. Blot with clean cloth and repeat above treatment with formic acid and gold leaving this time in latter $24 \mathrm{hrs}$. in absolute darkness. Repeat still again. Pass to glycerin and leave in closed vessel in ordinary light. The sharpness of the intensely purple black nerves in a lightly colored background increases with time. Small pieces can then be transferred to aq. dest. and the individual fibers separated. This is facilitated by dissociation in dilute nitric acid. Wash and make final mounts in glycerin. The author used panniculus carnosus of hedgehog, striated muscle of frog and lizard, extrinsic eye muscle of rabbit and human pectoral muscle.

Gas Analysis.- Techniques are given in detail by Glick, pp. 313-412.

Gash-Irrigation recovery method for lung cells (GI).-Written by C. C. Macklin, Dept. of Histological Research, The University of Western Ontario, London, Canada. November 28, 1951.-Fresh collapsed mouse or other mammalian lung is cut through a drop of physiological salt solution, blood serum or other suitable liquid, inverted and drained onto a slide. It is then covered and examined as a fresh mount, or spread, dried, stained and mounted like a blood smear. Liberated phagocytic and granular pneumonocytes (dust and foam cells-which see) are thus obtained (Macklin, C. C., Proc. 6th Intern. Congress of exper. Cytol., Stockholm, 1947; published 1949, 383-387; Macklin, C. C., The Lancet, Feb. 24, 1951, 432-435).

Gastric Contents. Examine microscopically material obtained by stomach tube after test meal as described by Stitt (p. 753). Look for mucus, epithelial cells, leucocytes, Gram positive bacilli in smears.

Gastrointestinal Tract. Immediate fixation is desirable because postmortem changes occur especially quickly. Do not wash first with water but with physiological saline or with the fixative itself. It may be desirable to place the excised pieces, with peritoneal surface down, on wooden tongue depressor or stiff paper. Some flattening is required. The mucous surface must not be allowed to dry. See Small and Large Intestine. See Papanicolaou Techniques.

Gautheria Oil used to be employed as a clearing agent. It has been displaced by the artificial oil, methyl salicylate.

Geiger Counters are instruments for the counting of electrons which provide quantitative data of great importance in this electron age. $\bar{A}$ concise description of the history of counter development and of the Geiger-Müller type is supplied by Rovner, L. in Glasser's Medical Physics, 487-495.

Gelatin-Carmine injections, see Carmine Gelatin injections.

Gelatin Glue, method of mounting sections, see Masson's.

Gelatin Imbedding and Sectioning. This is used when sections are required of loose, friable tissues which easily fall apart. Since the imbedding is directly from water, no alcoholic or other dehydration is required. Probably the best method is that of Zwemer (R. L., Anat. Rec., 1933, 57, 41-44), devised primarily for the study of adrenal lipoids. Wash material fixed in formalin or other fluid in water, $4 \mathrm{hrs}$. $5 \%$ gelatin in incubator at $35-37^{\circ} \mathrm{C} .24 \mathrm{hrs} .10 \%$ gelatin at same temperature, 12-16 hrs. Imbed by placing in $10 \%$ gelatin in Petri dish in refrigerator. Cut out blocks of tissue and fix in $10 \%$ formalin several hours to make gelatin insoluble in water. In this formalin solution tissues can be preserved indefinitely. Before sectioning rinse block in water and trim. Freeze with $\mathrm{CO}_{2}$ until block is uniformly white. Allow to thaw until knife cuts easily. Sections as thin as 5 microns can be obtained. Float onto slide in aq. dest. Drain off excess water and run a drop or two of $1 \%$ gelatin under setion. Again drain off excess. After heating in drying oven at $33-37^{\circ} \mathrm{C}$. place slide in $10 \%$ formalin for $10 \mathrm{~min}$. to fix gelatin. In this formalin solution the mounted sections can be stored. Stain sections in usual way with Sudan, Nile Blue Sulphate, Osmic Acid, Laidlaw's Silver Method, and mount in Glychrogel.

Wright's method as described by Mallory (p.34) is much quicker and is recommended for fragmented tissues such as those from curettings. Make a $10 \%$ solution of gelatin in warm aq. dest. and while still fluid add $0.5 \%$ carbolic acid. Do not overheat. The tissue, unfixed or fixed, preferably in $10 \%$ formalin, is "dried" and placed in a small "pool" of gelatin liquified by heat on a or slide in a glass vessel. This is allowed to solidify in the ice box for 
$2 \mathrm{hrs}$. or more. If necessary, store gelatin blocks in $10 \%$ formalin. Cut out block containing the tissue, freeze and section. Float sections from water onto slide well coated with albumenglycerin and spread. Remove excess of fluid and cover with piece of thin cigarette paper. Blot with fine filter paper till cigarette paper is partly dry. Cover cigarette paper with equal parts anilin oil and oil of cloves for few seconds. Drain and peel off cigarette paper. Remove oil by washing in $95 \%$ alcohol and pass to water when sections are ready for staining. Mallory suggests methods for Amyloid, Fat and staining with Hematoxylin and Phloxine for general purposes.

Gelatin Media, see Bacteria, Media.

Gelatin-Ringer electrode vessel, modification by Kriest, A. C., Acta Physiol. et Pharmacol. Neerland., 1950, 1, 32-34.

Gentian Blue 6B, see Spirit Blue.

Gentian Violet. The problem afforded by this dye, like many others, has been attacked by the Stain Commission. The stain thus referred to bas no constancy. Originally it was a mixture in about equal parts of dextrin and methyl violet, the latter itself a mixture in widely varying proportions of tetra-, penta- and hexa-methyl pararosanilins. Later were placed on the market methyl violets with and without dextrin and crystal violet (the hexa methyl compound) all under the label of Gentian violet. As Conn (p. 124) advises the term Gentian violet should be eliminated and crystal violet used wherever in the past the former has been specified. See Neutral Gentian, Methyl Violet, Crystal Violet.

Geranine G (CI, 127). An acid thiazole dye employed in fluorescence studies on account of color imparted by it under ultraviolet illumination (Conn, p. 70 ).

Germanium, see Atomic Weights.

Giant Cells. There is no special technique for their demonstration. Since the features usually employed in classification are size and nuclear detail and arrangement, Hematoxylin and Eosin, or Iron Hematoxylin the latter followed by various counter stains as for Acid Fast Bacilli are recommended. The following is a much abbreviated classification of Giant Cells from Cowdry's Histology 1938 Edition:

1. Megakaryocytes of bone marrow, granules in cytoplasm, best demonstrated by Giemsa's Stain.

2. Foreign body giant cells formed probably by a fusion of cells of mesenchymatous origin, perhaps of nongranular leucocytes, in response to foreign materials of many kindstubercular giant cells, foam cells in leprosy, lympsocystic giant cells of fish (Weissenberg), and possibly ReedSternberg cells in Hodgkin's disease.

3. Osteoclasts (polykaryocytes) of bone marrow and Langhans' giant cells of placenta are normal inhabitants of these organs. Myeloplague and Myeloplax are other terms for osteoclast. Chorioplague is a plate like giant cell of the chorion. See original account for lack of specific properties of socalled Langhans' cells which designation should be abandoned.

4. Epithelial giant cells are clearly of epithelial origin. Found in epidermis in chicken-pox and other diseases, occasionally in the liver and in kidney in many conditions. Often show nuclear irregularity and evidence of nuclear budding.

5. Hypertrophied cells can be either normal to meet physiological demands, as enormously enlarged smooth muscle cells of pregnant uterus, or due to various pathological conditions. Mauthner's Giant Cell in the fish brain is always of tremendous size in adults.

Giemsa's corrosive sublimate fixative. Sat. aq. corrosive sublimate 2 parts, absolute alcohol 1 part.

Gienısa's Stain. 1. For blood or bacteria in smears. Fix air dried smears in methyl alcohol in a covered dish 3-4 minutes. Remove and blot dry. Dilute stock solution of Giemsa in proportion of 1 drop to 1 cc. aq. dest. and stain for 15 minutes. Then wash in aq. dest., blot and dry. If a precipitate is formed in the smear by the stain, invert the slide, support both ends, and the stain will adhere like a hanging drop, kept away from the ends by lines ruled in wax or paraffin. The $\mathrm{pH}$ of the aq. dest. used to dilute the stain may be altered by adding very dilute acid or alkali. Optimum $\mathrm{pH}$ of 6.4 is given by the McJunkin-Haden buffer. This may be used as diluting medium in place of aq. dest. Usually the azurophile are stained more distinctly and the neutrophile granules less sharply than by Wright's stain. Bacteria and intracellular protozoa are better colored than by Wright's stain. The May-Giemsa, and Jenner-Giemsa and the panchrome stains of Pappenheim are important modifications. They are listed separately. Present situation concerning Giemsa's stain is that American products give equally good results with thin films but the German product appears to be better for thick ones (Conn, H. J., Stain Techn., 1940, 15, $41-43)$. 
2. For sections. Much depends upon the choice of fixative. Formalin, generally employed in $10 \%$ solution, acts as a sort of mordant for the blue component so that the blue coloration is particularly strong. Fixation in Regaud's gives good results particularly with Rickettsia, Zenker's fluid is recommended by Wolbach. When this is used it is necessary to remove the mercuric chloride by treating the sections with Lugol's solution. They are then washed in $95 \%$ alcohol and the last traces of iodine are extracted by $0.5 \%$ aqueous sodium hyposulphite for 10-15 min. The hyposulphite in turn is washed out in running water about $5 \mathrm{~min}$. and rinsing in aq. dest. See Cowdry's colored figures of Rickettsia, J. Exper. Med., $1925,42,231-252$. Bouin's fluid (75 cc. saturated aq. picric acid, 25 cc. commercial formalin and 4 cc. glacial acetic acid) is suggested for intracellular protozoa (East Coast fever parasites) by Cowdry and Danks (Parasitology, 1933, 25, 1-63) because after Giemsa staining it gives the chromatin a desirable purple color (see colored plate). Stain sections placed vertically in staining jars in $1.5 \mathrm{cc}$. Giemsa's solution plus 50 cc. aq. dest., changed during the first hour, overnight. Differentiate in $95 \%$ alcohol, dehydrate quickly in absolute alcohol, clear in xylol and mount in balsam.

If the sections are not blue enough add 1-2 drops $0.5 \%$ sodium bicarbonate and 1.5 cc. methyl alcohol to the stain; or remove excess of mordanting potassium bichromate from Zenker fixation by rinsing $1 \mathrm{~min}$. in $1 \%$ potassium permanganate followed by $5 \%$ oxalic acid $4 \mathrm{~min}$. and thorough washing in aq. dest., or do both. If on the contrary they are too blue mordant in $5 \%$ potas. sium bichromate $15 \mathrm{~min}$., rinse in aq. dest. until no more yellow is removed and stain; or add a little colophonium to the alcohol used in differentiating and dehydrating of the sections, as advised by Wolbach, or again do both.

Usually Giemsa's stain gives satisfactory results without any special precautions. The difficulty is that the colors fade quite rapidly particularly when the balsam is noticeably acid and when the sections are left in direct sunlight. Their period of usefulness can be extended by mounting in cedar oil, used for oil immersion objectives, instead of in balsam. Try Clarite. If a variety of fixatives is employed it may be necessary to suit the stain to the fixative by use of buffers, in which case see Lillie, R. D., Stain Techn., $1941,16,1-6$.
To demonstrate the "nucleoids" of bacteria in smears the technique of C. F. Robinow published as Addendum to Dubos, R. J., The Bacterial Cell. Harvard Univ. Press, 1945, 460 pp. is suggested. Fix smears in osmium tetroxide vapor, treat 7-10 min. with $\mathrm{N} / 1 \mathrm{HCl}$ at $60^{\circ} \mathrm{C}$. and color with Giemsa's solution. By this method nu. cleoids are stained whereas similar bacteria not treated with the acid are uniformly colored by Giemsa. Robinow prefers this staining of nucleoids by Giemsa after hydrolysis to the Feulgen technique.

Gilson's Fluid. Nitric acid (sp. gr. 1.456), 15 cc.; acetic acid, 4 cc.; mercuric chloride, $20 \mathrm{gm}$.; $60 \%$ alc., 100 cc.; aq. dest., $880 \mathrm{cc}$. Used mostly for invertebrates.

Gilson's Mixture is equal parts chloroform and cedar oil.

Gingiva. Capillaroscopy of (McClung, Microscopical Technique, 1950, 328); Eosinophile leucocytes in (Orban, B., J. Dent. Res., 1940, 19, 537-543.)

Glacial Acetic Acid, see Acetic Acid.

Gland Cells contrasted. Endocrine, exocrine, apocrine, merocrine, holocrine, serous, zymogenic and mucous (Cowdry's Histology, p. 257).

Glass Cloth, as a substrate for tissue culture, Warner, D., Hanawalt, C. and Bischoff, F., J. Nat. Cancer Inst., 1949, $10,67-74$.

Glass Electrode. Sisco, R. C., Cunningham, B. and Kirk, P. L., J. Biol. Chem., $1941,139,1-10$ have devised an open cup variety described with diagrams by Glick, pp. 183-184. See Claff and Swenson glass capillary electrode and the Pickford sealed-in capaillary glass electrode also described by Glick.

Glia Staining with Anilin Dyes (Proescher, Fr., Stain Techn., 1934, 9, 33-38). Fix in $10 \%$ formalin or in $90 \%$ alcohol followed by formalin. Wash frozen sections, $10-15$ microns thick, in aq. dest. Stain in sat. aq. victoria blue B (not filtered but poured off from the undissolved dye), 14-24 hrs. Wash quickly in aq. dest., mount with glycerin-albumen, blot and dry in air. Treat with ultraviolet light $30 \mathrm{~min}$. Pass to N/20 iodine few sec. Remove iodine, blot, dry, destain in xylolanilin, clear first in clove oil, then xylol, mount in balsam. Glia blue, nerve cells lightly stained, connective tissue metachromatic violet or colorless. Instead of ultraviolet light stained sections can be treated with $0.5 \%$ potassium bichromatic for $30 \mathrm{~min}$. In place of victoria blue, methyl violet $2 \mathrm{~B}$, ethyl violet or crystal violet can be employed. 
Glick, see Linderstr $\phi m-L a n g, K a j, u_{\text {., }}$ and Holter, Heinz, Histochemical Advances.

Glomus. Aortic and carotid, see Aortic Paraganglion.

Glucose Agar, see Bacteria Media.

Glutathione. Demonstrated by Nitroprusside Reaction. Inhibiting factor in Vitamin $\mathbf{C}$ silver test.

Glycerides, see Neutral Fats.

Glycerine. Much used in histological technique in the making up of stock solutions of hematoxylin, like Delafield's, in Albumen-Glycerin used for mounting paraffin sections, etc. It serves as an excellent clearing agent for the walls of large Arteries so that the intramural vessels can easily be distinguished by the blood in them. With potassium hydrate it is employed to clear specimens in the demonstration of Ossification centers. As a mounting medium for frozen sections glycerin is invaluable. In the form of Brandt's glycerin jelly (which see) glycerin is specified in the technique for Sebaceous Glands and many other structures. To make Kaiser's glycerin jelly (Mallory, p. 100) soak $40 \mathrm{gms}$. gelatin in $210 \mathrm{cc}$. aq. dest. for 2 hrs. Add 250 cc. glycerin, stir and heat gently 10-15 min. Keep in ice box and melt before use. The 5 gms. carbolic acid crystals specified in Kaiser's formula has unfortunately. according to Mallory, a deleterious influence on alum hematoxylin preparations. See also Glychrogel and Lactophenol.

Glychrogel, as a mounting medium for teased preparations, Marchi stained sections, gelatin sections, etc. To make $100 \mathrm{cc}$. dissolve $0.2 \mathrm{gm}$. chrome alum (potassium chromium sulphate) in 30 cc. aq. dest. with aid of heat. Add $3 \mathrm{gm}$. Knox granulated gelatin in $50 \mathrm{cc}$. hot aq. dest. Add 20 cc. glycerin with constant stirring and warm. When thoroughly mixed add crystal of camphor (Wotton, R. M. and Zwemer, R. L., Stain Techn., 1935, 10, 21-22). For use in mounting nematodes (Wotton, R. M., Stain Techn., 1937, 12, 145-146).

Glycogen, the 3 chief microchemical methods have been critically studied by C. M. Bensley (Stain Techn., 1939, 14, 47-52). This account follows her presentation. Since glycogen is labile, immediate fixation of very small pieces of tissue $(2-3 \mathrm{~mm}$.) and agitation of the fixative are necessry. She recommends 9 parts absolute ethyl alcohol +1 part commercial formalin (i.e. $37 \%$ formaldehyde) neutralized with $\mathrm{MgCO}_{3}$. If desired the alcohol in this fixative can be saturated with picric acid. After fixation for say $24 \mathrm{hrs}$. wash in aboslute alcohol, embed in the usual way in paraffin (carefully avoiding overheating) or in colloidin.

1. Best's carmine. Grübler's carminum rubrum optimum or some other good carmine $2 \mathrm{gm} .$, potassium carbonate $1 \mathrm{gm}$. , potassium chloride 5 gm., aq. dest. 60 cc. Boil gently until color darkens, cool and add 20 ce. concentrated ammonia. Allow to ripen 24 hrs. This is stock solution. Mount paraffin sections, bring down to aq. dest. Stain nuclei with hematoxylin as in the $\mathrm{H}$. and $\mathrm{E}$. technique. Transfer to fresh stain (stock solution 10 cc., 15 cc. conc. ammonia and 30 cc. pure methyl alcohol) for $20 \mathrm{~min}$. Rinse in 3 changes methyl alcohol, dehydrate in acetone, clear in toluol and mount in balsam. Glycogen brilliant red.

2. Iodine (Gage). Mount paraffin sections as before, being again careful to avoid unnecessary heat, and bring down to water. Lugol's aq. iodine 10-15 min. Blot with filter paper and dry in air. Mount in yellow vaseline as advised by S. H. Gage (J. Comp. Neur., 1917, 27, 451-465) with minimum of heat. Glycogen reddish brown.

3. Bauer-Feulgen. To make Feulgen reagent dissolve $1 \mathrm{gm}$. basic fuchsin in 100 cc. aq. dest. by heat. Filter while warm and add when cool $20 \mathrm{cc}$. normal $\mathrm{HCl}$. Add $1 \mathrm{gm}$. $\mathrm{NaHSO}_{3}$. Allow to rest 24 hrs., when it should be of pale straw yellow color. Treat deparaffinized sections with $4 \%$ chromic acid for $1 \mathrm{hr}$. or with $1 \%$ chromic acid over night. After washing in running water $5 \mathrm{~min}$., place in Feulgen reagent $10-15 \mathrm{~min}$. Rinse $1 \frac{1}{3} \mathrm{~min}$. in each of 3 changes of molecular sol. $\mathrm{NaHSO}_{3} 1$ part and tap water 19 parts. Wash in running water $10 \mathrm{~min}$. Counterstain nuclei with hematoxylin if desired. Dehydrate, clear and mount in balsam. Glycogen deep reddish violet, nuclei lavender. See Polysaccharides.

Control. Prepare at same time some sections of liver rich in glycogen. Because glycogen is quickly removed by salivary digestion, when sample sections are brought down to aq. dest., spit on them and allow to rest 15-30 min. changing saliva several times. Wash thoroughly in water at body temperature to remove inucus and stain by either of the 3 above mentioned techniques. If the material is then absent in such sections and present in other similarly stained and not digested, it is evidently glycogen. Fixation by the freezing and drying method is even better than with the alcohol, picric, formalin mixture because it is quicker and there is less 
chance for displacement of glycogen in the cells.

See also for glycogen staining of Trachoma inclusions Thygeson, P., Am. J. Path., 1938, 14, 455-462. Glycogen is immobilized in its natural position within the cells by the Freezing and Drying technique (Altmann-Gersh). Compare figures 3 and 4 of Bensley and Gersch (R. R. and I., Anat. Rec., 1933, 57, 205-215) showing results by this and other methods.

A new ammoniacal silver nitrate method for glycogen is described by Mitchell, A. J., and Wislocki, G. B., Anat. Rec., 1944, 90, 261-266. To prepare silver solution dissolve $1 \mathrm{gm}$. silver nitrate in $10 \mathrm{cc}$. aq. dest. and add 11 drops $40 \%$ aq. potassium hydroxide. Dissolve ppt. by adding $26 \%$ ammonia drop by drop and make up with abs. alc. to $100 \mathrm{cc}$. Allow to stand over night before use.

Fix livers of guinea pigs and placentas of same and other animals for $6-12 \mathrm{hrs}$. in sat. picric acid in abs. alc., $90 \mathrm{cc}$. and neutral formaldehyde, $10 \mathrm{cc}$. Wash in abs. alc. several times likewise in chloroform and abs. alc. Transfer to chloroform and embed in paraffin.

Place sections in $0.25 \%$ aq. potassium permanganate, 5-10 min.; rinse in aq. dest. 1-2 min., decolorize in $5 \%$ aq. oxalic acid, 5 min. and rinse again in aq. dest. Place in $2 \%$ aq. silver nitrate, 12-24 hrs., transfer to ammoniacal silver nitrate, $15-30 \mathrm{~min}$., rinse in $4 \%$ neutral formalin, 5-20 sec. and in running water, $1 \mathrm{~min}$. Fix in $5 \%$ aq. sodium thiosulphate, 5-10 min. After washing in running water, 1 min., counterstain in paracarmine (Mayer), dehydrate, clear in xylol and mount in balsam. Glycogen, dense black corresponds with that shown by BauerFeulgen technique. Excellent illustrations.

The recently developed techniques of Gomori (Am. J. Clin. Path., 1946, 16, 177) and Hotchkiss (Arch. Biochem., $1948,31,131)$ can be used on tissue sections or blood smears (Gibb, R. P., and Stowell, R. E., Blood, J. Hematology, $1949,4,569-579)$. The evaluation of methods for the histochemical study of glycogen by Carpenter, A. M., Polonsky, B. and Mesiten, M. U. (Arch. Path., 1951, 51, 480-485) may be helpful.

The best way to separate out glycogen en masse is by centrifugal isolation as employed by Lazarow, A. (Anat. Rec., 1942 , 84, 31-50; Biol. Symposia, 1943, 10, 9-26) for suspensions of fragmented liver cells.

Colorimetric methods for glycogen may afford valuable evidence. See Boettiger, E. G. (J. Cell. Comp. Physiol., 1946, 27, 1-8) and Van Wagtendonk, W. J., Simonsen, D. H. and Hackett, P. L. (J. Biol. Chem., 1946, 163, 301-306) and the critical discussion by Glick, p. 247. See Heatley, N. G. and Lindahl, P. E. (Proc. Roy. Soc., B, $1937,122,395-402$ ) for separation of desmo- and lyoglycogen.

Glycol Stearate. As an imbedding medium (Cutler, O. I., Arch. Path., 1935, 20, 445-446). Pass up through alcohols to equal parts $95 \%$ alc. and glycol stearate in incubator at $56^{\circ} \mathrm{C} .12-24$ hrs. Pure glycol stearate at $56^{\circ} \mathrm{C} .24 \mathrm{hrs}$. Imbed as in paraffin.

Glucuronidase. An enzyme, widespread in occurrence in the mammalian organism, which hydrolyzes esters of glucuronic acid. Glucuronides are important detoxification products. Chemical methods for identification of glucuronidase are available (see Fishman, W. H., Chapter 18, The Enzymes New York: Academic Press). 1950 Vol. 1, part 1, pp. 635-652, Friedenwald, J. S. and B. Becker (J. Cell. and Comp. Physiol., 1948, 31, 303-309) have described a method for localizing glucuronidase on tissue sections, and Seligman, A.M., M. M. Nachlas, L. H. Manheimer, O' M. Friedman and G. Wolf (Ann. Surg., $1949,130,333-341$ ) describe a method involving the hydrolysis of the beta glucuronide of beta naphthol. The liberated naphthol is converted to a dye by diazotization.

Glyoxal. As substitute for formaldehyde in tissue fixation (Wicks, L. F. and Suntzeff, V., Science, 1943, 98, 204; Stowell, R. E. and Stokes, J. M. J. Tech. Meth. and Bull. Internat. Assoc. Med. Museums, 1944, 24, 25-30). Concentrations 2-6\% produce less shrinkage and give better cytoplasmic preservation than $4 \%$ formaldehyde. Glyoxal is only recommended as general substitute for formaldehyde when latter is not available.

Gmelin's test for bile pigments. On addition of nitric acid containing a little nitrous acid, color changes to green, then red and finally blue observable under microscope.

Gold, microchemical detection of : 1 . Method of Borchardt. Modified by Michaelis, O., Biochem. Zeit., 1930, 225, 478-488. Treat sections of formalin or alcohol fixed tissues for $15 \mathrm{~min}$. in a boiling water bath or for $12-24 \mathrm{hrs}$. at $40^{\circ} \mathrm{C}$. with $5 \%$ aq. silver nitrate. Remove ppt. from section with $20 \%$ aq. nitric acid. Gold appears as black granules (Lison, p. 100).

2. Method of Okkels, H., C. rend. 
Soc. Biol., 1929, 102, 1089-1091. Simply produce gold salt in sections by exposing for at least $12 \mathrm{hrs}$. to sunlight or to ultraviolet lamp for same time (Gauthier-Villars, P., C. rend. Soc. de Biol., $1932,109,197-193)$. Lison (p. 100) explains that whatever the technique used it is necessary to prove that the black granules are gold by their insolubility in concentrated acids, solubility in aqua regia (equal parts nitric and hydrochloric acids) and solubility in solutions of potassium or sodium cyanide.

3. Method of Roberts, W. J., Bull. d'Hist. Appl., 1935, 12, 314-361. Fix tissues in $20 \%$ neutral formalin or in Bouin's fluid. Avoid fixatives containing a metal. Wash thoroughly in water. Make paraffin or frozen sections. The latter has the advantage of speed. Make 2 solutions: A. Add $2 \mathrm{gm}$. silver nitrate pure for analysis to $100 \mathrm{cc} .10 \%$ gum arabic in the dark immediately before use. B. Add $1 \mathrm{gm}$. hydroquinone pure to 100 cc. $10 \%$ gum arabic the day before use. Take off the frozen sections in aq. dest. Mix 2 cc. A and 2 cc. B, add 1-3 drops $5 \%$ citric acid, shake 30 sec. Leave sections in this mixture 5-10 min. Then without first washing plunge into $5 \%$ aq. sodium hyposulphite for a few minutes. Wash thoroughly and mount. Gold in cells is covered with black deposit of reduced silver. Said to be more sensitive method than spectrographic analysis. See author's illustrations.

4. A technique for demonstration of gold in abs. alc. or neutral formalin fixed tissues, based upon reaction with $p$ - Dimethylaminobenzylidenrhodanin is described by Okamoto, K., Akagi, T. and Mikami, G., Acta. Scholae Med. Univ. Imp. in Kioto, 1939, 22, 373-381.

5. Tin chloride method (Elftman, H. and Alice G. Stain Techn., 1945, 20, 59-62. After rats and guinea pigs are injected intraperitoneally with aqueous yellow gold chloride fix by injection of neutral formalin through heart. Make paraffin sections. Pass down to water in usual way. Place slides in mixture of 10 parts stock $5 \%$ aq. $\mathrm{SnCl}_{2} \cdot 2 \mathrm{H}_{2} \mathrm{O}$ (with some pieces metallic tin added to retard oxidation) and 1 part conc. $\mathrm{HCl}$ (mixture prepared and filtered just before use) in incubator at $56^{\circ} \mathrm{C}$. for 24 hrs. Wash several changes aq. dest. before dehydrating clearing and mounting in damar. Presence of gold indicated by particles exhibiting purple of Cassius grading into brown. Colloidal gold in red, blue and black may likewise occur. To eliminate disadvantages of occasional precipitates of tin unrelated to gold and possible confusion with bile pigments and others the following technique is proposed by these authors.

6. Fix in neutral formalin, bring down mounted sections to water. Place in $3 \% \mathrm{I}_{2} \mathrm{O}_{2}$ in incubator at $37^{\circ} \mathrm{C}$. for at least 24 hrs. better 3 days. Wash in aq. dest. Run up and mount in damar. Gold thus reduced to metallic form shows range of colors, rose chiefly grading into purple, blue and black.

7. Christeller, E., Verh. deutsch. Path. Ges., 1927, 22, 173 reports, as described by Gomori, G., J. Mt. Sinai Hosp., 1944-45, 11, 317-326, demonstration of gold salts by reduction to metallic gold with $\mathrm{SnCl}_{2}$. Similar to No. 5 .

8. For micro-determination of gold in biological fluids and tissues, see Block, W. D., Ann. Rheumatic Dis., $1944-45,4,39-42$. Use of this technique provides a good check on above described microchemical methods.

Radioactive gold, distribution of within cartilage of knee-joint, Ekholm, R., Acta Anat., 1951, Supp. 15 to 11, $75 \mathrm{pp}$.

Gold Chloride for nerve endings, see Graven's and Carey's methods.

Gold Orange, see Orange II.

Gold Orange MP, see Methyl Orange.

Gold Particles. The particles of gold are held in colloidal state by the protective colloid, sodium lysalbinate, and are employed to stimulate macrophage production by intravenous injections in animals (Simpson, M. J., J. Med. Res., $1922,43,77-144)$.

Goldman, see Iron Hematoxylin Single Stain.

Golgi Apparatus-Written by Geoffrey Bourne, London Hospital Medical College, London, England. November 5, 1951-Most recent books which give details of techniques for demonstration of the Golgi apparatus preface their descriptions with the statement that, as some doubt exists as to the nature of the apparatus, it is difficult to describe techniques for demonstrating it. This doubt is still unresolved and various authors hold widely divergent views as to the structure of the apparatus.

These may be summarized as follows:

Gatenby (Microtomist's Vade Mccum, 11th Ed. 1950) believes that the origiual conception of the Golgi apparatus in vertcbrate cells as a juxta-nuclear argentophil network is still correct. Baker's view (Quart. J. Micr. Sci. $1944,85,1-71-\mathrm{a}$ modification of that expressed by Parat, M. Arch. d'Anat. Micr., 1928, 24, 73) is that the apparatus is composed of a series of neutral red staining vacuoles more or less sur- 
rounded by a zone of dense phospholipid material and in some cases embedded in a zone of diffuse phospholipid. Palade and Claude (J. Morph. 1949, 85, 71) claim that the Golgi apparatus is composed solely of neutral red staining vacuoles which possess a membrane containing an appreciable proportion of lecithin and that the classical Golgi apparatus is formed by myelin figures produced by the action of fixatives on these vesicles. Bensley (Exp. Cell. Res. 1951, 2, 1-19) contends that the Golgi apparatus is composed of vesicles or canals containing a watery solution in which various substances, e.g., polysaccharides are dissolved (Gersh, I., Arch. Path. 1949, 47, 99-109). The canalicular conception of the Golgi apparatus is also claimed by Gatenby and Moussa for the mammalian neurone.

Against these views we must put the fact that no canals have been seen in cells by numerous workers using the phase contrast microscope and as Hibbard, H. (Quart. Rev. Biol. 1945, 20, 1-19) has pointed out Lewis has never seen anything resembling the classical apparatus in a life-time of study of tissue culture cells. Most workers on the Golgi apparatus have tended to range themselves behind one or other of these views. It should be realized, however, that all these points of view about the structure of the apparatus simply continue the controversy which has gone on almost since its discovery. The canalicular conception of the apparatus originated in 1902 with Holmgren's Trophospongium, the neutral red vacuole theory found its origin in Parat's 1928 work and has seen some extension in Cowdry's laboratory by the work of Cowdry, E. V. and Scott, G. H. (Arch. Inst. Pasteur de Tunis, 1928, 233), Covell, W. P. and Scott, G. H. (Anat. Rec., 1928, 38, 377), and of course the argentophil network theory originated with Golgi himself in 1898 (Arch. Ital. de Biol., 30, 60) and in more recent years has been supported by Ludford and particularly by Gatenby. So, it is apparent that our knowledge of the nature of the Golgi apparatus has remained more or less in the same state as it was 30 or 40 years ago.

In view of this discrepancy of views techniques will be given for demonstrating the classical Golgi net and also for demonstrating the apparatus as discrete bodics.

The Classical Golgi Apparatus: Even if the Golgi network is an artefact as some workers believe the conventional
Golgi preparation does give us information about its position in the cell and whether the Golgi material is present in small or in large amount.

Golgi's original technique involved the fixation of tissue in a mixture containing potassium bichromate and osmic acid followed by impregnation with silver. The apparatus with this technique appears jet black against a yellowish background. It is a conspicuous structure consisting of an intricate network of anastomosing strands. This network may closely envelop the nucleus, be concentrated to one side of it or else be scattered rather diffusely throughout the cytoplasm. In glandular cells the apparatus grows in size with the development of secretory granules and strands from it ramify between the various granules.

Kopsch, F. (Sitzungsber. K. Akad. Wissensch, 1902, 40, 929) showed that the Golgi apparatus can be blackened by prolonged treatment with $2 \%$ osmic acid. On this affinity for both silver and osmium all the conventional methods of demonstrating the Golgi apparatus are based. Few cytological reactions are more fickle and inconstant; but when, after many attenipts, the technique is successful, convincing and very beautiful preparations result. Mitochondria can be stained supravitally by some vital dyes but no vital dyes will show up the reticular Golgi apparatus, a fact which is taken by some authors to indicate the non-existence in vivo of such networks. However, neutral red will stain spheres in the region of the apparatus.

With both silver and osmium methods considerable experimentation is necessary in order to obtain the best results. The factors to be varied are principally the composition of the fixative and impregnating substance and the time during which they are allowed to act. During impregnation it is always advisable to keep the tissues in the dark and instructions as to temperature requirements should be carefully followed. When either the silver or osmium solution becomes blackened it should be renewed. It is important for the beginner to start with the most favorable material. The spinal ganglion cells of young mammals such as the rabbit are perhaps the best for this purpose. The acinous cells of the pancreas are also recommended but are somewhat more difficult to handle. All the methods of impregnation outlined below frequently bring to light the mitochondria also. 
Osmic Acid Technique:

1. Mann-Kopsch method (Lee's Microtomists Vade Mecum, 11th Ed. 1950 , edited by J. Bronte Gatenby and H. W. Beams). Fix in Mann's fluid (equal parts of $1 \%$ osmic acid and sat. sublimate in normal saline), for $-3 \mathrm{hrs}$. Wash in aq. dest. $15-30 \mathrm{~min}$. $2 \%$ osmic acid room temperature 10-14 days. Wash in rumning water 3 hrs. or more. Dehydrate clear and embed. In the sections Gatenby was able to extract the blackening step by step with turpentine and thus to improve considerably the preparations.

2. Ludford's modification (Ludford, R. J., J. Roy. Micr. Soc., 1926, 107). Fix mammalian and avian tissues in Mann's corrosive osmic solution $18 \mathrm{hrs}$. Wash aq. dest. 30 min., $2 \%$ osmic at $30^{\circ} \mathrm{C}$. for 3 days. Water at $30^{\circ} \mathrm{C}$. for 1 day, dehydrate, clear, embed in paraffin. A useful variant is to fix in the same way, and wash in aq. dest. Then osmicate at $35^{\circ} \mathrm{C}$. for 3 days, first day in $2 \%$ osmic, second in $1 \%$ and third in $0.5 \%$. Leave in water for 1 day at $35^{\circ} \mathrm{C}$. He recommends various counter stains. See Lee's 11th Ed. (pp. 401410) for a discussion of osmication methods; also Owens, H. B. and Bensley, R. R. (Anat. Rec., 1929, 44, 79) for a careful study of factors influencing the osmic acid changes and for their ferric chloride osmic method.

3. Sjovall's method (Sjovall, E., Anat. Hefte, 1906, 30, 261-291). Fix in 10\% formalin 8 hrs. Wash in aq. dest. $2 \%$ osmic acid at $35^{\circ} \mathrm{C}$., 2 days. Dehydrate, clear and embed.

4. Kolatchew's method (Nassonov, D. N., Arch. f. Mikr. Anat., 1924, 103, 437). Fix in $3 \%$ aq. potassium bichromate, 10 cc.; $1 \%$ chromic acid 10 cc.; and $2 \%$ osmic acid, 5 cc. for 24 hrs. Wash in running water $24 \mathrm{hrs} .2 \%$ osmic acid $40^{\circ} \mathrm{C} .8$ hrs. $3-5$ days at $35^{\circ} \mathrm{C}$. Wash in aq. dest., dehydrate, clear and embed. Osmic methods sometimes impregnate mitochondria as well as the Golgi material particularly if the period in osmic acid is prolonged, care must be taken therefore in interpreting results.

Silver Nitrate Methods: Tissues from young animals usually respond best to silver methods but ganglia from older animals respond very well.

1. Aoyama's method (Baker, J. R., Cytological Technique, 3rd Ed., 1950, p. 194). Fix tissues in Aoyama's fixative (cadmium chloride $1 \mathrm{gm}$., neutral formalin $4 \%, 15 \mathrm{cc}$, aq. dest. $85 \mathrm{cc}$.) for $4 \mathrm{hrs}$. Wash with aq. dest. and then place in silver nitrate solution for 12-14 hrs. Wash with aq. dest. and then place in silver nitrate solution for 12-14 hrs. After 8-12 hrs. (kept in dark or in diffuse light). Wash with aq. dest. and place in Aoyama's reducer (Hydroquinone $1 \mathrm{gm} .$, Neutral Formaldehyde $40 \% 15$ cc., aq. dest. 80 cc., anhydrous sodium sulphite $0.15 \mathrm{gm}$.) for $5 \mathrm{hrs}$. Leave for $\frac{1}{2} \mathrm{hr}$. in running water and then place in $50 \%$ alcohol overnight. Dehydrate and embed.

2. Cajal's method (Carleton, H. M., J. Roy. Micr. Soc., 1919, 321-329). This is one of the many methods devised by Cajal. It is recommended for embryos and young animals. Fix in uranium nitrate, $1 \mathrm{gm}$., formalin $15 \mathrm{cc}$., and aq. dest. 100 cc., $8-24$ hrs. Wash quickly in aq. dest. $1.5 \%$ aq. silver nitrate $24-48$ hrs. Rinse in aq. dest., hydroquinone 2 gm., formalin 6 cc., aq. dest. $100 \mathrm{cc}$, anhydrous sodium sulphite $0.15 \mathrm{gm} .12$ hrs. Wash in aq. dest, dehydrate quickly, clear, embed and section.

3. Da Fano's method (Da Fano, C., J. Roy. Micr. Soc., 1920, 157-161). Here the uranium nitrate is replaced by cobalt nitrate. In other respects the technique is similar. Da Fano has, however, so carefully attempted to control troublesome experimental conditions that the various steps are given in detail. Fix in cobalt nitrate $1 \mathrm{gm}$., aq. dest. $100 \mathrm{cc}$, formalin $15 \mathrm{cc} .6-8 \mathrm{hrs}$. The formalin need not be neutralized unless it is strongly acid. For embryos and delicate tissues where there is danger of shrinkage reduce formalin to 6 cc. Cartilage and small pieces of tissue (not more than $3 \mathrm{~mm}$.) fix half fixation time. Hollow organs (e.g. stomach and intestine) fix $1 \mathrm{hr}$. then cut into smaller pieces. Spinal cord, cerebellum and cerebrum of adults fix 8-10 hrs. (fixation should never go beyond 24 hrs.). Testicle, inject fixative through aorta then immerse testicle in fixative. Wash quickly in aq. dest. and impregnate in $15 \%$ aq. $\mathrm{AgNO}_{3}$ 24-48 hrs. Very small fragments impregnate in $1 \% \mathrm{AgNO}_{3}, 2 \%$ for tissues containing much fat and for spinal cord. Impregnation normally satisfactory at room temperature. If unsatisfactory at $35^{\circ}-37^{\circ} \mathrm{C}$. Wash rapidly in aq. dest. and cut down tissues again to a thickness of $2 \mathrm{~mm}$. or less. Reduce in Cajal's hydroquinone mixture, above mentioned, 12-24 hrs. Wash in aq. dest. $\frac{1}{2} \mathrm{hr}$. Cut with freezing microtome or embed in wax. Golgi apparatus dark brown or black against a yellow background. Tone sections with gold to clear preparation. Pass to water. Then $0.1-0.2 \%$ gold chloride 2 
hrs. Counterstain, dehydrate, clear and mount.

It is of interest that all silver preparations depend upon the treatment of the tissue by fixatives which contain the salt of a heavy metal. Silver, M. I. (Anat. Rec., 1942, 82, 507-529) has pointed out that silver micelles are not adsorbed on to the Golgi material unless the cells have been treated with salts of heavy metals-hence the presence of metals like cobalt and uranium in the fixatives used for silver techniques.

Stains:

1. Baker's Sudan Black Method (Baker, J. R., Quart. J. Micr. Sci., 1944, 85, 1-71). Fix small pieces of tissue in formol calcium (formalin $40 \%, 10$ cc., calcium chloride, anhydrous $10 \%$ aq. 10 cc., aq. dest. 80 cc.) for 3 days, embed in gelatine. Harden block in formalin-calcium-cadmium (formalin $10 \mathrm{cc}$., calcium chloride, anhydrous $10 \%$ aq. 10 cc., cadmium chloride, $10 \%$ aq. 10 cc., aq. dest. 70 cc.) and then wash $3-4$ hrs. in running water. Section on freezing microtome at $15 \mu$. Sections when fixed to the slide are placed in filtered formalin-calcium-cadmium solution until ready for staining. Then wash slides for $3 \mathrm{~min}$. in running water, pass through $50 \%$ and $70 \%$ alc. to a sat. solution of sudan black in $70 \%$ alc., leave for $7 \mathrm{~min}$. Pass through 3 lots of $50 \%$ alc., rinse in aq. dest., counterstain, mount in glycerine jelly or Apathy's medium. This method does not demonstrate the Golgi apparatus as a network but as a series of discrete bodies, a form which is claimed by some authors to represent more nearly the true form of the Golgi apparatus in living cells. Many of the vesicles which are demonstrated by this method also stain with neutral red.

2. Baker's Neutral Red Method (Baker, J. R., Quart. J. Micr. Sci., 1944, 85, 1-71). This is a vital method. Cells are separated by teasing in a salt mixture (sodium Chloride, $0.7 \%$ aq. 100 cc., calcium chloride, anhydrous, $10 \%$ aq., 0.2 cc.). To 3 drops of solution containing a suspension of cells add 3 drops of a solution made up of Neutral red $0.1 \%$ aq., $1 \mathrm{cc}$. and sodium and calcium chloride solution (as above) 9 cc. Final concentration of dye is $0.005 \%$. Mix cell suspension with dye by sucking up with pipette and pass out again. Leave preparation (covered) for 20 min. Put 2 drops of mixture on the glass of a compressorium in such a way as to include some air. Cover edge of compressorium with soft paraffin (e.g. Vaseline). Examine under immersion lens.
Golgi Cox Method for adult nervous system. -Written by J. L. O'Leary, Dept. of Neuropsychiatry, Washington University School of Medicine, St. Louis 10, Mo. May 8, 1950.-Fix pieces $3-6$ $\mathrm{mm}$. thick in following fluid: add $20 \mathrm{cc}$. $5 \%$ aq. potassium bichromate to $20 \mathrm{cc}$. $5 \%$ aq. mercuric chloride. Dilute 16 cc. $5 \%$ aq potassium chromate with 40 cc. aq. dest. and add this to the first two. Do not agitate but leave in fixative until scum forms on surface, usually after $1 \frac{1}{2}-2$ months. When impregnation is nearly complete, wash rapidly, dehydrate through graded alcohols and imbed in low viscosity celloidin (see Celloidin Imbedding). Cut celloidin sections serially at 80 to 120 microns. Arrange in serial order on slides $(80 \%$ alcohol). Blot sections dry and cover immediately with $1 \%$ celloidin. When somewhat dry, bring slides with sections to water. The sections on each slide may thereafter be treated as a unit. Run sections from water into a saturated solution of sodium sulfite. They rapidly turn a yellow gray. Wash over night and dehydrate through graded alcohols to absolute. Coat with the following varnish, applying it repeatedly in thin even layers, and allowing each to dry partially before applying the next (sandarac, 75 gm.; camphor, 15 gm.; turpentine C.P., 30 cc.; oil of lavender, 22.5 cc.; abs. alc., 75 cc.; add castor oil, 7 drops. Mixture dissolves very slowly). Since sections are somewhat opaque, the varnish must dry for several days until abs. alc. has evaporated.

Golgi Methods. Fundamentally these are different from both the Cajal and Bielchowsky techniques which were later developments. They depend upon a preliminary fixation in a potassium bichromate solution often containing formalin and sometimes other substances such as osmic acid. The silver is selective tending to impregnate a few cells completely which become blackened when it is reduced. Except for the occasional demonstration of the Golgi Apparatus these methods do not reveal details of the inner structure of nerve cells like Neurofibrils and Nissl Bodies. They are of great service in the demonstration of many non-nervous tissue components, the parietal cells of the stomach, bile canaliculi of the liver, Rouget or perivascular cells, etc.

Golgi Method, Quick. For brains of newborn animals, and of those 1 day to 30 days old.- Written by J. L. O'Leary, Dept. of Neuropsychiatry, Washington University School of Medicine, St. Louis 10, Mo. May 8, 1950.-It is es- 
sential to determine the age of the animal at which the cell or fiber selected for study is reaching maturity. For example, if new born kittens are chosen, and the area striata is the object of study, the best impregnations of entering fibers are obtained at 12 to 15 days after birth; of short axon cells, at 18 to 21 days; and of pyramids at 21 to 24 days.

Cut slices of brains $3-4 \mathrm{~mm}$. in thickness by quick cuts of a sharp scissors. Fix in: potassium bichromate, $10 \mathrm{gm}$.; osmic acid, 1 gm.; aq. dest., 330 cc. Time of fixation must be determined for each part of the CNS studied. In general the older the animal, the longer it is. After fixation, blot blocks of tissue on filter paper and transfer to a bottle containing $\frac{3}{4} \%$ aq. silver nitrate. After 24 hrs. the reaction is complete. Imbed in celloidin. Subsequent treatment is very important. Place block in $95 \%$ alc. for about $5 \mathrm{~min} .$, remove and blot dry. Place block on paraffin disc mounted on a block holder in the orientation desired for cutting. Using a hot teasing needle, melt paraffin around the block so as to fasten block to paraffin. Be sure that melted paraffin does not creep up on the block. Use knife at $45^{\circ}$ angle to the block. Cut serially $\mathrm{S} 0-100 \mu$. Place each section as cut in order in $95 \%$ alc. using Petri dishes. Be sure not to miss first and last section of the block for these are often more valuable than the entire remainder of the block. Using a spatula, transfer to another $95 \%$ alc. after $5 \mathrm{~min}$. After another $5 \mathrm{~min}$. transfer to oil of cloves, arranging in serial order, by placing each section as it enters oil of cloves near the edge of the Petri dish so that it adheres to the edge. When all sections are transferred the group will be placed around the circumference of the Petri dish. As the sections start to retract from the edge, begin to arrange them in the usual order for serial sections. After clearing (clove oil 5 to $10 \mathrm{~min}$.) transfer in serial order to slides. Blot off excess of clove oil and apply xylol, blot off xylol similarly and apply a thin layer of Damar, using the drop method. Let the slide dry on an even surface adding more Damar as necessary to keep sections protected.

Golgi, Method (Porter, R. W. and Davenport, H. A., Anat. Rec., 1949, 103, 583). Radical departure from prior methods because silver impregnation precedes the potassium bichromate solution. Fix 48 hours at $25^{\circ} \mathrm{C}$. in 90 cc. 0.5 aq. $\mathrm{AgNO}_{3}, 10$ cc. formalin, 0.5 cc. Pyridine. Mix in order given, disregarding slight turbidity. Colorimetric test of fixing solution with bromcresol purple should show 5.5 to 6.0 . Fixation can be done by perfusion, or only by immersion. Cut slices to thickness of 0.5 to $1.0 \mathrm{~cm}$. After fixation rinse blocks with distilled water and place in $2.5 \%$ (aq) potassium bichromate to which 1 cc. of $1 \%$ osmic acid is added for each $100 \mathrm{ec}$. Leave there 3 to 5 days. Dehydrate quickly through alcohols and xylol to soft paraffin. Sections should be cut 50 to 100 microns thick.

Gomori's Methods For Reticulum and Acid Phosphatase.

1. Silver impregnation of reticulum (Gomori, G., Am. J. Path., 1947, 13, 993-1001). Treat deparaffinized sections of formalin fixed material with $0.5-1 \%$ aq. potassium permanganate 1-2 min. Rinse in tap water and decolorize in $1-3 \%$ aq. potassium metabisulphite, 1 min. Wash for several minutes in running tap water. $2 \%$ aq. iron ammonium sulphate (violet crystals), $1 \mathrm{~min}$. Wash in tap water few minutes and then pass through 2 changes aq. dest. Impregnate for 1 min. in following solution: To $10 \%$ aq. silver nitrate add $\frac{1}{6}$ to $\frac{1}{2}$ of its volume of $10 \%$ aq. potassium hydroxide. While shaking add strong ammonia drop by drop until ppt. is completely dissolved. Add carefully silver solution drop by drop as long as resulting ppt. easily disappears on shaking. Finally add equal vol. aq. dest. Can be kept 2 days in stoppered bottle. Rinse in aq. dest., 5-10 sec. Reduce in commercial formalin diluted 5-10 times with tap water. Wash under tap few min. Tone in $0.1-0.2 \%$ aq. gold chloride, $10 \mathrm{~min}$. $1-3 \%$ aq. potassium metasulphite for 1 min. Fix in $1-2 \%$ aq. sodium thiosulphate (hyposulphite) for $1 \mathrm{~min}$. Wash under tap, dehydrate, elear and mount. Reticulum black. Note author's figures of sarcomata (Revisde by G. Gomori May 7, 1946).

2. For Acid phosphatase-Written by G. Gomori (University of Chicago. May 7, 1950-see Stain Techn., 1950, 25,81 .

1. Fix thin slices of tissues in ice cold acetone for 24 hours.

2. Change acetone at roon temperature twice for the next 24 hours.

3. Two changes of benzene, $45 \mathrm{~min}$. each.

4. Embed in paraffin (not above $56^{\circ} \mathrm{C}$. and preferably below), 2 changes, 30 to $45 \mathrm{~min}$. each.

5. Cut sections. Float them on lukewarm $\left(30^{\circ} \mathrm{C}\right.$. $)$ water.

6. Carry sections through xylene and 2 alcohols to dist. water.

7. Incubate in the following solutions 
for $1^{1}$ to 24 hours at $37^{\circ} \mathrm{C}$. :

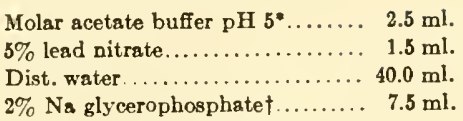

100 cc. of $13.6 \% \mathrm{CH}_{2} \mathrm{COONa} \cdot 3 \mathrm{H}_{2} \mathrm{O}$ plus $50 \mathrm{cc} .6 \%$ acetic acid.

$\dagger$ Commercial grade (mixture of alpha and beta salts).

Shake well, heat to $\pm 60^{\circ} \mathrm{C}$. for about 10 min.; filter.

8. Rinse sections thoroughly first in dist. water and afterwards in 2 to $3 \%$ acetic acid, followed again by dist. water.

9. Immerse sections in a solution of yellow ammonium sulfide (1-12 drops to a Coplin jar) for 1 minute.

10. Wash. Counterstain as desired. For Alkaline phosphatase:

1. Fix thin slices of tissues in $80 \%$ alcohol (or absolute acetone). Dehydrate in $95 \%$ and absolute alcohol (or 2 changes of absolute acetone), embed through benzene or xylene in paraffin. Cut sections around 6 micra thick.

2 . Run slide through xylene and 2 alcohols to distilled water. Incubate for 1 to $2 \mathrm{hr}$. at $37^{\circ} \mathrm{C}$. in the following mixture:

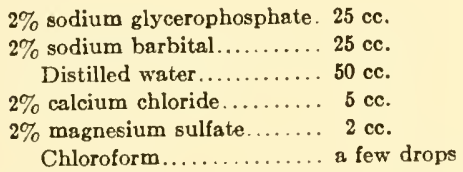

This solution will keep in the ice box for months.

3. Rinse slide thoroughly in repeated changes of distilled water.

4. Immerse slide for 3 minutes in a 1 to $2 \%$ solution of some cobalt salt (chloride, acetate, sulfate).

5. Wash thoroughly under the tap.

6. Immerse slide for 2 minutes in a dilute solution of yellow ammonium sulfide (1-12 drops to a Coplin jarful of distilled water). Wash under the tap.

7. Counterstain as desired; dehydrate, clear and mount.

Attention is called to the earlier demonstration of phosphatase in bone by Robison (R., Biochem. J., 1923, 17, 286-293) and to recent discussion by Blaschko and Jacobson (Bourne, pp. 217-221). The distribution of phosphatase in some normal tissues is indicated in colors by Kabat, E. A. and Furth, J., Am. J. Path., 1941, 17, 303-318. For phosphatase in elementary bodies of vaccinia virus, see Macfarlane, M. G., and Salaman, M. H., Brit. J. Exp. Path., 1938, 19, 184; Hoagland,
C. L. et al., J. Exp. Med., 1942, 76, 163-173. See Kidney.

Gorococcus, methyl green-pyronin stain. To $10 \mathrm{cc}$. absolute methyl alcohol add $1 \mathrm{gm}$. methyl green (dye content 60\%) and $0.2 \mathrm{gm}$. pyronin (bluish certified). Add 100 cc. $2 \%$ aq. phenol and shake 2 hrs. per day for 2 days in a mechanical shaker. Filter and add 20 cc. glycerin, C.P. to filtrate. Fix smoars by passing slides lengthwise through flame 4 or 5 times. Add stain immediately and warm to slight steaming. Wash off stain 20-50 sec. Dry and examine. Gonococci, deep red; other bacteria except these of Neisseria group pale purplish or barely noticeable; nuclei of pus cells green in soft pink or rose cytoplasm (Walton, S. T., J. Lab. \& Clin. Med., 1938-39, 24, 1308-1309).

Goodpasture's Method as modified by MacCallum for bacteria in sections (McClung, p. 152). Fix in Zenker's fluid or in formalin Zenker. Stain thin paraffin sections 10-30 min. in: $30 \%$ alc., 100 cc.; basic fuchsin, $0.59 \mathrm{gr}$. ; anilin oil, 1 cc.; phenol crystals, $1 \mathrm{gm}$. Wash in water. Differentiate in formalin ( $37 \%$ solution of formaldehyde) few seconds until bright red color changes to rose. Wash in water. Counterstain in sat. aq. picric acid 3-5 min. until sections become purplish yellow. Wash again in water. Differentiate in $95 \%$ alc. until red reappears and some of it as well as of the yellow is washed out. Wash in water. Stain in Stirling's gentian violet (gentian violet, 5 gms.; $95 \%$ alc., 10 cc.; aniline oil, 2 cc.; aq. dest., 88 cc.) 5 min. or more. Wash in water. Gram's iodine solution (iodine, $1 \mathrm{gm}$.; potassium iodide, 2 gms.; aq. dest., 300 cc.) 1 min. Blot dry. Clear in equal parts aniline oil and xylol until no color is removed. Clear in 2 changes xylol and mount in balsam. Gramnegative bacteria, red; gram-positive ones, blue; tissue red and blue; fibrin deep blue. See his Polychrome Methylene Blue and Carbol-Anilin Fuchsin Methylene Blue.

Gopal-Ayengar, see Chromosomes, Hyaluronic Acid.

Gordiacea, see Parasites.

Gordon's Silver Method. For blood smears, also shows parasites, Gordon, H., J. Lab. \& Clin. Med., 1936-37, 22, 294298. Dry smears of blood or bone marrow in air and fix in $10 \%$ formalin. Wash in water. $2.5 \%$ aq. iron alum 10 min. or more. 4 changes aq. dest. Dip in $1 \%$ aq. gelatin +1 or 2 drops $2 \%$ sodium carbonate and drain. Wash quickly in aq. dest. Impregnate 5-15 min. in silver solution (Add strong ammonia drop by drop to $5 \mathrm{cc}$. of $10.2 \%$ 
aq. silver nitrate until ppt. is dissolved. Add 5 cc. $3.1 \%$ aq. sodium hydroxide and redissolve ppt. with strong ammonia. With aq. dest. dilute to 100 cc.). Wash in aq. dest. at $60^{\circ} \mathrm{C}$. Reduce in : $10 \%$ formalin 90 cc. $+2.5 \%$ iron alum $10 \mathrm{cc}$. Wash in tap water, dehydrate in alcohol, clear in xylol and mount in balsam.

Gossypimine, see Safranin $O$.

Grafts. Intracoelomic of eye primordium, Joy, E. A., Anat. Rec., 1939, 74, 461486. See Transplantation.

Gram, see Weight measurements.

Gram's Iodine Solution. Iodine, $1 \mathrm{gm}$.; potassium iodide, 2 gm.; aq. dest., 300 cc. A stronger solution may be desirable with only $100 \mathrm{cc}$. aq. dest.

Gram-Pappenheim stain as modified for smears and paraffin sections (Scudder, S. A., Stain Techn., 1944, 19, 39-44).

Gram's Stains for bacteria:

1. In smears. Hucker modification (McClung, p. 138). Stain $1 \mathrm{~min}$. in equal parts $\mathrm{A}$ and $\mathrm{B}: \mathrm{A}=$ crystal violet (85\% dye content, $4 \mathrm{gm}$.; $95 \%$ alc., 20 cc.) $\mathrm{B}=$ ammonium oxalate, $0.8 \mathrm{gm}$.; and aq. dest. $80 \mathrm{cc}$. After washing in water immerse in: iodine, $1 \mathrm{gm}$. potassium iodide, 2 gm., aq. dest., 300 cc. 1 min. Then wash in water and dry by blotting. Decolorize 30 sec. in $95 \%$ alc. gently moving. Blot and counterstain in: 10 cc. sat. safranin in $95 \%$ alc. and aq. dest. $100 \mathrm{cc}$. Wash and dry. Kopeloff-Beerman Modification (McClung, p. 139). Stain $5 \mathrm{~min}$. or more in : $1 \%$ aq. gentian or crystal violet, 1.5 cc. mixed before use with 0.4 cc. $5 \%$ aq. sodium bicarbonate. Rinse in iodine solution made by dissolving $2 \mathrm{gm}$. iodine in $10 \mathrm{cc}$ normal sol. sodium hydroxide and adding 90 cc. aq. dest. and stand 2 min. or more. Blot dry. Add $100 \%$ acetone drop by drop with specimen tilted till no more color is removed, less than $10 \mathrm{sec}$. Dry in air. $0.1 \%$ aq. basic fuchsin, $10-30$ sec. Wash in water and dry. Weiss Modification (Weiss, E., J. Lab. \& Clin. Med., 1940-41, 26, 1518-1519). Make thin, uniform smears and fix over flame. Cover slide with $3 \%$ gentian violet in $20 \%$ alc., 3-5 min. Wash in warm water. Cover 3-5 min. with iodine, 20 gm.; potassium iodide, 40 gm., aq. dest. 300 cc. Wash with warm water. Decolorize in acetone and wash immediately in water. Counterstain quickly in $2 \%$ basic fuchsin in $95 \%$ alc. Wash in water, dry and examine.

The use of colloida! iodine has been suggested to improve the reaction between bacteria and stain (Lyons, D. C., J. Lab. \& Clin. Med., 1936-37, 22, 523-524). Methods for preparing col- loidal iodine are described by Chandler and Miller (W. L. and E. J., J. Phys. Chem., 1927, 31, 1091-1096).

2. In sections. Gram-Weigert method (McClung, p. 152). Fix in Zenker's fluid. Stain paraffin sections lightly in alum hematoxylin and wash in running water. $1 \%$ aq. eosin, $1-5 \mathrm{~min}$., followed by washing in water. Stain $\frac{1}{2}-1 \mathrm{hr}$. in anilin methyl violet made by mixing 1 part of A with 9 of B : A. abs. alc. 33 cc.; aniline oil, 9 cc.; methyl violet in excess. B. Saturated aq. methyl violet and wash in water. Lugol's iodine 1-2 min. and wash in water. Blot; dehydrate and clear in equal parts aniline oil and xylol several changes. Wash with xylol and mount in balsam. Glynn's method. (Glynn, J. H., Arch. Path., 1935, 20, 896-899). To make stain triturate 1 gm. crystal violet and $1 \mathrm{gm}$. phenol crystals in mortar and add 10 cc. absolute alcohol. Before using dilute 10 times with aq. dest., allow to stand 2 days and filter. Stain deparaffinized sections of Zenker (less acetic), Bouin, Helly or $10 \%$ formalin fixed material for $2 \mathrm{~min}$. Drain off but do not wash. Add Gram's iodine, $1 \mathrm{~min}$. Differentiate in acetone until no more color is given off, 10-15 sec. Wash in aq. dest. Counterstain in $0.05 \%$ basic fuchsin in N/500 hydrochloric acid (see Normal Solutions). Drain, do not wash, apply $1 \%$ aq. trinitrophenol, $\frac{1}{2}-1 \mathrm{~min}$. Wash in aq. dest. Dehydrate and differentiate in acetone 10-15 sec., clear in xylol and mount in balsam. Gram + bacteria, violet; Gram-, red; nuclei, light red; cytoplasm, yellow.

3. For organisms in frozen sections by Krajian, A. A., Arch. Path., 1941, 32, 825-827. Stain $7-10 \mu$ frozen sections for 2 min. in Harris' alum hematoxylin. Wash in tap water till blue and destain quickly by dipping 5 to 7 times in acid alcohol. Rinse in tap water and apply following solution for $3 \mathrm{~min}$.-copper sulfate, $7 \mathrm{gm}$.; zinc sul. fate, $4 \mathrm{gm}$. dissolved in $100 \mathrm{cc}$. aq. with aid of heat. Pour off and apply $0.3 \mathrm{gm}$. brilliant green in $10 \mathrm{cc}$. above copper zinc mixture for $5 \mathrm{~min}$. Rinse in water and fortify with $5 \%$ aq. ammonium nitrate for $1 \mathrm{~min}$. Rinse in tap water and stain with carbol fuchsin (Zichl-Neelsen) for $2 \mathrm{~min}$. Rinse in tap water, blot and apply dioxane for $2 \mathrm{~min}$. Pour off and add equal parts creosote and xylol, changing this mixture and agitating to promote even differentiation until background appears clear red. Clear in pure xylol (2 min.) and mount in damar. Gram positive organisms bluish green; gram negative ones red.

The Gram staining technique and 
the interpretation of the findings has been concisely presented by Dubos, $R$. J., The Bacterial Cell. Harvard Univ. Press, $1945,460 \mathrm{pp}$. The Gram + bacteria differ from the Gram - ones in being more acidic and perhaps in possession of lipids with higher content of unsaturated acids. Their Gram positiveness depends on intactness of their cell walls, for erosions of the walls make them Gram negative. When the outer layer of the cell walls is removed by extraction with bile salts they become Gram negative. The Gram positive property can be restored by "replating" the bacteria with the extract of the outer layer. The outer layer apparently contains a protein ribonucleate complex, for Gram positive organisms can be made Gram negative by action of the enzyme, ribonuclease. The quality of the cell membrane conditions not only the entry and retention of stains but the whole manner of life of the cells. See Cell Membrane, Acid Fast Bacilli, and Dead Cells.

Gram Stains Mechanism-Written by James W. Bartholomew, Dept. of Bacteriology, University of Southern California, Los Angeles 7, Calif. July 9, 1951-The literature concerning the mechanism of the Gram stain is voluminous and makes very interesting reading although the exact mechanism is still unknown. The laboratory technician need not be too concerned with the mechanism. It is important for the technician to realize that the technique involved is differential due to relative rather than absolute differences in the organisms studied. Thus, regardless of whether one uses the Hucker, Kopeloff-Beerman, Weiss, or other modification of the Gram procedure, the dependability of the results obtained are greatly influenced by the experience and skill of the operator. One method should be adopted, and practiced, until proficiency and self assurance are obtained.

Gram differentiation is mostly due to one fact, Gram-positive cells resist decoloration after treatment with dye and iodine to a much greater extent then Gram-negative cells. The cause of this resistance has been attributed to 1) cell membrane permeability characteristics, 2) the presence of certain unique chemical compounds, 3) a low isoelectric point of the cell protein, and 4) the presence of a morphological structure, the Gram-positive cortex. Each of these theories, by itself, fails to explain certain experimental data. It is certain that the mechanism of the Gram stain is very complex and several of the above factors probably combine to give the differentiation effect. Cell membrane permeability is hard to ignore since it is well known that rupturing the cell wall immediately results in a Gram-positive cell staining Gram-negatively. The importance of a chemical factor has been well established through the easily demostrable dependence of the Gram-positive state on the presence of magnesium ribonucleate in the cell. The isoelectric point theory applies to the general staining phenomena, but on close inspection looses its significance as a prime factor in the mechanism of the Gram stain. The morphological concepts must await further development in our knowledge of bacterial cell morphology, and better cell sectioning methods, before the contribution of morphological factors to the Gram staining mechanism can be determined.

Currently, an appealing explanation based on published literature could be written as follows. Cell membrane permeability to iodine in alcoholic solution is of first importance. Magnesium ribonucleate and possibly other compounds such as lipoproteins are important in that they influence the cell membrane permeability. Gram-positive cells are less permeable to iodine in alcoholic solution than are Gramnegative cells. On performing the Gram stain a reaction between the dye and iodine, and also possibly the cell protein, takes place within the cell. The alcohol, which is added next, enters the cells and dissociates the dye-iodine precipitate, or dye-iodine-cell protein complex, which has been formed. Since the alcoholic iodine permeates fastest through the cell membrane of the Gramnegative cells the precipitate or complex are dissociated more rapidly in them than in the Gram-positive cells. Hence, the Gram-negative cells are the first to declorize. The counter stain also has a certain decolorization action and it must be employed if a true Gram stain differentiation is desired.

While this concept of the Gram stain mechanism has not been proven, it has the happy quality of combining chemical concepts with cell membrane permeability and it does not rule out possible morphological factors. In the case of the often observed Gram-positive bodies within the cell, a more strictly chemical concept would have to be used to explain them.

As is often the case, the segregation of cells into two distinct groups, the Gram-negative and the Gram-positive, does not coincide with the state actually 
existing in nature. There are many intermediates between the two, and this fact should be kept in mind by the technician. Attempts to create terminology for intermediate groups have not been well received and the classification as it now stands is extremely useful and comparitively simple. Further information is supplied by Bartholomew, J. W. and Umbreit, W. W., J. Bact., 1944, 48, 567; Bartholomew, J. W. and Mittwer, T., Stain Tech., 19t9, 25, 103-110; Dubos, R. J., The Bacterial Cell. Cambridge: Harvard Univ. Press, 1946, pp. 72-\$5; Knaysi, G., Elements of Bacterial Cytology. Ithaca: Comstock Pub. Co., 1951, pp. 267-278; Mittwer, T., Bartholomew, J. W., and Kallman, B. J., Stain Tech., 1950, 25, 169-179.

Gram-Twort Stain. For study of Grampositive and Gram-negative bacteria in sections (Ollett, W. S., J. Path. \& Bact., 1917, 59, 357). Twort oliginally used a neutral red-light green mixture. Ollett, W. S., J. Path. \& Bact., 1951, 63, 166 supplies further details. The stain advised is approximately as follows depending on dye content of the samples employed. For stock solution $0.2 \%$ alc. neutral red, (Cl no. $8259 \mathrm{ml}$. $+0.2 \%$ alc. fast green FCF $10 \mathrm{ml}$. For use dilute 1 vol. stock solution with 3 vols. aq. dest. Employ as counterstain in Gram's method as described (Ollett, 1947) and mount sections in I. P. M. which is Lustron (L 2020), apparently the same as Distreme 80 crystal clear $100 \mathrm{gm}$. dissolved in Dibutyl phthalate $50 \mathrm{ml}$. + monochlorbenzene $300 \mathrm{ml}$.

Gray, R, B, BB, see Nigrosin, water soluble. Green PL, see Naphthol Green B.

Greene, see Anterior Chamber Transplantation.

Gregarines. Technique given by McClung, Microscopical Technique, 1950 p. 455. Fix in picric acid mixtures. Stain smaller ones in Heidenhain's Iron Hematoxylin and larger ones with Hemalum. For Golgi bodies see JoyetLavergne, P., C. R. Soc. de Biol., 1926 , 94, 830 .

Grenacher, see Alum Carmine, Borax Carmine.

Grieves' method for undecalcified dental tissues and bone as outlined by Shipley (McClung, p. 345) is: Fix small pieces in $10 \%$ formalin $24-36$ hrs. or any other desired fixative. Wash in running water 24 hrs. Then pass through 2 changes of aq. dest. $1 \mathrm{hr}$. each. Dehydrate through ascending alcohols beginning with $50 \%$ alc. Equal parts abs. alc. and chloroform, 2 hrs. Chloroform, 2 hrs. $5 \%$ sol. of rosin in chloroform, 2 hrs. $10 \%$ sol. rosin, 2 hrs. Sat. sol. rosin until it becomes transparent. Imbed in melted rosin using one after another the rosins in 3 small glass dishes on a heated copper bar, 1 min. each. The chloroform carried over evaporates. The rosin containing the tissue is allowed to cool. The block is ground very thin by hand on a carboriundum stone and polished on a fine hone all grinding being done under luke warm water. The smooth surface is then mounted on a slide with a little melted rosin after which the surface is ground and polished in the same way and the section is ready for mounting or for staining.

Gross Specimens, see Color Preservation.

Ground Substance (intercellular), see Tissue Fluid.

Growth. Many techniques are now available for the measurement of growth of tissues. Increase in number of cells can be revealed by mitotic counts Mitosis). The amount of bone or of dentine laid down while Alizarin $\mathbf{S}$ or Madder is in the circulation can be estimated. The amount of radioactive isotropes accumulated is a third method (see Radioactive Phosphorus) if the amount increases per unit of time while elimination of the nonradioactive element in question remains the same. Valuable histochemical methods are given by Lowry, O. H. and Hastings, A. B., in Cowdry's Problems of Ageing, Baltimore: Williams and Wilkins, 1942, 936 pp.

Gout, see Urates.

Guanin appears as white granules in retinal tapetum of certain animals including nocturnal ones. Decreases in amount in regions containing more fuscin. For details see Arey, I. B. in Cowdry's Special Cytology, 1932, 3, 1218.

Guanine, see microxide test under Purines.

Guarnieri Bodies, cytoplasmic inclusions in smallpox and vaccinia. See Inclusion Bodies and Cowdry, E. V., J. Exper. Med., 1922, 36, 667-684 for supravital staining with brilliant cresyl blue. For sections Giemsa's stain is excellent.

Gum Damar, see Damar.

Gypsum test, see Calcium 7.

Habermann, see Anethole Clearing Agent. Hafnium, see Atomic Weights.

Hairs-Written by Mildred Trotter and Oliver H. Duggins, Dept. of Anatomy, Washington University, St. Louis, May 8,1951 -The hair shaft (above the surface of the skin), the hair root (below it) and the hair follicle (encasing the root) call for somewhat different techniques.

The shaft may be examined in a dry mount after first washing thoroughly 
and repeatedly in ether-alcohol, or the shaft and root can be cleared and mounted in balsam for repeated study. In case it is too highly pigmented to permit a clear view of its structure first bleach with hydrogen peroxide. Individual cells of the shaft can be isolated by maceration in $40 \%$ aq. potassium hydrate.

Scale counts are made readily after a dry mount has had applied a drop or two of a glycerine-alcohol mixture at the ends of the specimen. This mixture progresses along the shaft by capillary attraction thus bringing into relief the free borders of the scales (Trotter, M. and Duggins, O. H., Am. J. Phys. Anthrop., in press). Determination of the cuticular scale pattern may be made after partially embedding the hair in a glycerine jelly (Eddy, M. W. and Raring, J. C., Proc. Acad. Sci., $1941,15,164-168)$. Study of the cortex (fusi and pigment granules under very high power) and medulla (when present with its clumps of pigment) requires clearing by immersing in some oil the refractive index of which is approximately the same as that of the hair (Hausman, L. A., Sci. Month., 1944, 59, 195-202).

The refractive index of hair may be determined to the greatest accuracy by the double-variation method using both the Becke line and half shadow techniques (Gibb. 'T. R. P., Jr., Optical Methods of Chemical Analysis, pp. 249-250, McGraw-Hill, 1942). Cut hairs into a number of $1 \mathrm{~mm}$. lengths, agitate for 30 minutes in ether-alcohol and mount in oil of a similar refractive index on a temperature cell under a polarizing microscope. True index is found only at crossed Nicols. It is suggested that the index be taken both perpendicular to the lower Nicol $\left(90^{\circ}\right)$ and parallel to the lower Nicol $\left(0^{\circ}\right)$ and that the lower index $\left(90^{\circ}\right)$ be subtracted from the higher index $\left(0^{\circ}\right)$ in order to determine the birefringence. The Becke line method may be followed by the half shadow method for greater accuracy. The limit of accuracy will be approximately +3 in the third place. In addition, it is suggested that the use of the phase microscope for such determinations may increase the accuracy to the fourth decimal place.

Cross sections of a large number of hairs (approximately 150) may be made at one time with very little preliminary preparations by using the "Dr. J. I. Hardy Thin Cross-Section Device" (Gosnell Mfg. Co., Washington, D. C.).

The root and the follicle are to be seen in most sections of hairy skin and require no special technique unless one wishes to study the follicles attached to whole mounts of epidermis. In order to study the growth of a given hair or to determine the cyclic activity of its follicle it is convenient to place a small tattoo mark with India ink in the skin near the mouth of the follicle (Trotter, M., Am. J. Phys. Anthrop., 1924, 7, 427-437).

Distribution of alkaline phosphatase in growth of hair follicle (Johnson, P. L., Butcher, E. O. and Bevelander, G., Anat. Rec., 1945, 93, 355-361). For further details see Trotter, M., Chapter on Hair in Cowdry's Special Cytology, $1932,1,40-65$. Cleaning and mounting of individual hairs (Duncan, F. W., J. Roy. Micr. Soc., 1943, 63, 85-88. Microphotography of keratin fibers of hairs (Stoves, J. L., J. Roy. Micr. Soc., $1943,63,89-90)$. The less pigment in the hair, the greater the fluorescence, so that gray hair is clear white. Hair containing tricopliyton or microsporon fluoresces bright green. See Kinnear, J., Brit. Med., J., 1931, 1, 791-793 on diagnosis of ringworm.

Halides, microscopic localization in tissues by precipitation methods (Gersh, I. and Stieglitz, E. J., Anat. Rec., 1933, $56,185)$.

Halometer, apparatus designed by Eve, F. C., Lancet, 1928, 1, 1070 to measure mean diameter of erythrocytes, see Erythrocytometer.

Halowax, see Paraffin Sections.

Hanging Drop preparations are mostly employed in the examination of living bacteria and protozoa. A drop of the fluid is simply attached to the under surface of a cover glass which is mounted over a depression in a slide. Equally satisfactory results can usually be obtained by simply mounting under a cover glass on an ordinary slide unless the greater depth of the hanging drop is required. When in Microdissection it is necessary to get at the cells from the under surface of the cover glass special chambers and hanging drops are employed.

Harderian Glands, fluorescence in mice (Strong, L. C. and Figge, F. H. J., Science, 1941, 93, 331). Teclnique for rat is given by Grafflin, A. L., Am. J. Anat., 1942, 71, 43-64.

Harleco Synthetic Resin is recommended by McClung, Microscopical Technique, 1950 , p. 24 as a mounting medium somewhat similar to Clarite. It can be obtained from Hartman-Liddon Co., 5821 Market Street, Philadelphia 39, Pa.

Harris Alum Hematoxylin. Dissolve $1 \mathrm{gm}$. hematoxylin in $10 \mathrm{cc}$. absolute alcohol and $20 \mathrm{gms}$. ammonium or potassium 
alum in 200 ce. aq. dest. the latter with the aid of heat. Mix the 2 solutions, bring quickly to boiling and add $0.5 \mathrm{gm}$. mercuric oxide. Solution turns purple. Cool quickly in cold water bath. Mallory (p. 72) recommends adding $5 \%$ of acetic acid.

Heart, see Coronary Arteries, Myocardium. Pericardium, Purkinje Cells and Fibers. Technique and results of "electron histology" of the heart are presented by Kisch, B. and Bardet, J. M., Exp. Med. \& Surg., 1951, 9, 1-47.

Heart Failure Cells-Written by C. C. Macklin, Dept. of Histological Research, The University of Western Ontario, London, Canada. November 28,1951 .- This inapt designation, used by pathologists, applies usually to modified human pneumonocytes which have become free in the lung alveoli, or which are affixed to the moving mucous sheet of the air tract in transit to the mouth region, or which have been hawked up in the sputum. The cells may thus be examined in fresh mounts with or without vital or supravital staining; or in stained dried smears. 'They may also be fixed, embedded and cut into thin sections (Macklin, C. C., The Lancet, Feb. 24, 1951, 432-435). See Dust Cells.

Heavy Water is water in which deuteriun, the heavy hydrogen isotope $\mathrm{H}^{2}$, has taken the place of ordinary hydrogen. See Deuterium which is used as a tracer substance.

Heidenhain's Azan Stain (Heidenhain, M. Ztschr. f. wiss. Mikr., 1915, 32, 361-372). The following details are from Lee (1928, p. 279) : Color sections $1 \mathrm{hr}$. at $55^{\circ} \mathrm{C}$. in $2 \%$ aq. azocarmine plus 10 drops glacial acetic acid in small staining jar. Wash in water. Differentiate in $96 \%$ alc. 100 cc. plus anilin oil 0.1 cc. until cytoplasm becomes pale pink and nuclei clear red. To hurry differentiation add 2 drops anilin oil. Rinse in $96 \%$ alc. containing few drops acetic. Put in $5 \%$ aq. phosphotungstic acid about 2 hrs. until connective tissue is completely decolorized. Wash rapidly in water. Stain $\frac{1}{2}-3 \mathrm{hrs}$. in following solution diluted with equal or double parts aq. dest.: anilin blue (water sol. Grübler) $0.5 \mathrm{gm}$.; orange $\mathrm{G}, 2 \mathrm{gm}$.; acetic acid, 8 cc.; aq. dest. 100 cc. Examine staining under microscope. Wash in water, dehydrate in abs. alc., clear in xylol and mount in balsam. This is a very useful stain. See also McGregor, L., Am. J. Path., 1929, 5, 545-557 for use of this technique particularly as applied to normal renal glomerules. Under Islets of Langerhans is given use of a slightly modified azan method by Gomori.

Heidenhain's Iron Hematoxylin, see Iron Hematoxylin.

Heinz Bodies. These spherical bodies are sometimes seen in erythroeytes especially when examined in the dark field or when colored with Azur 1. They have been referred to as Substantia Metachromatica Granularis and B-substance. The best way to demonstrate them is to use the technique of Figge, F. H. J., Anat. Rec., 1946, 94, 17. Give $0.3 \%$ aq. sulfanilamide to mice as drinking water. Within 4-6 days these bodies will appear in at least $90 \%$ of erythrocytes whence they are cast out into the plasma. They are most readily seen in unstained, unmounted blood smears. They disappear when studied in oil, balsam or other mounting media. Heinz bodies are granules of heme-containing protein denatured by this druy within the cells. They are not produced by sodium sulfathiazole. The Heinz Body phenomenon in erythrocytes is discussed in detail by Webster, S. H., Blood, 1949, 4, 479-504.

Helianthin, see Methyl Orange.

Heliozoa, techniques for, see McClung, Microscopical Technique, 1950, p. 468, also Rumjantzew, A., and Wermel, E. Arch. f. Protistenk., 1925, 52, 217.

Heliotrope B, see Amethyst Violet.

Helium, see Atomic Weights.

Helly's Fluid is Zenker's fluid in which 5\% formalin is substituted for $5 \%$ acetic acid.

Helminthosporia. Stain for nuclei in (Parris, G. K.., Phytopathology, 1944, 34, 700 ).

Helvetia Blue, see Methyl Blue.

Hemalum (Mayer's) Hematin, 1 gm.; $90 \%$ alc., 50 cc.; aq. dest., 1000 cc.; ammonia alum, 50 gms.; thymol, 1 crystal. Keeps better after adding $20 \mathrm{cc}$. glacial acetic acid and making Acid Hemalum. A good nuclear stain when diluted with aq. dest. 1:20. The above formula has been modified by Lillie (R. D., Stain Techn., 1942, 17, 89-90) : hematoxylin, 5 gm.; sodium iodate $\left(\mathrm{NaIO}_{3}\right), 1 \mathrm{gm}$.; ammonia alum $\left(\mathrm{AlNH}_{4}\left(\mathrm{SO}_{4}\right)_{2}+12\right.$ $\left.\mathrm{H}_{2} \mathrm{O}\right), 50 \mathrm{gm}$.; aq. dest., $700 \mathrm{cc}$., gl y cerol, 300 cc., glacial acetic acid, 20 cc. No ripening is necessary. Stain sections formalin fixed material, $2-5 \mathrm{~min}$. Blue 2-10 min. in tap water. Counterstain in $0.2 \%$ aq. eosin $\mathrm{Y}$. Dehydrate clear and mount as usual. This method is quick and gives a sharp stain.

Hematin, identified by luminescence with Luminol. Do not confuse with hematein, see Hematoxylin.

Hematocrit, a tube used to concentrate red 
blood cells by centrifugation and to measure their volume, see Ponder, E. in Glasser's Medical Physics, 597-600.

Hematoidin (hematin + G. eidos, appearance). An iron free pigment produced by phagocytic digestion of erythrocytes or in clots and old hemorrhages, chemical composition similar or identical with Bilirubin. Seen as red or orange rhombic plates or radiating yellow needles, insoluble in ether, water and soluble only with difficulty in alcohol, easily soluble in chloroform. Gives positive Gmelin's test.

Hematoporphyrin (G. haima, blood + porphyra, purple).-Written by Frank H. J. Figge, Dept. of Anatomy University of Maryland Medical School, Baltimore, Md. Contrary to a deeply rooted misconception, this substance is not the pigment as it occur's in hemoglobin, but is artificially produced by the drastic decomposition of hemoglobin in concentrated strong acids. Since it does not occur in nature, such terms as "hematoporphyrinuria" are obsolete. In addition, protoporphyrin, which is the true, unaltered, pigment found in heme compounds, is not excreted as such by the kidney. Protoporphyrin is heme minus iron and has two vinyl group side-chains. Hematoporphyrin is heme minus iron, plus two hydrogen and two hydroxyl groups. Hematoporphyrin is soluble in water, ether, alcohols, dilute alkalies, and acids. For references and additional information, see Porphyrins.

Hematoxylin is the most useful of all dyes in animal histology and pathology (Gr. haimatōdēc, blood like + Xylon, wood). It is an extract of logwood (Haematoxylon campechianum) and is marketed in crystalline form. When the crystals are first dissolved in water or alcohol it is not an energetic stain; but requires to be "ripened" before it can be used to advantage. Ripening is brought about by the formation of oxidation products. Consequently it is recommended that solutions be exposed to light and air. Hematein (not hematin-a blood pigment) is the oxidation product which yields a fine deep blue coloration and is the one most desired. It can be purchased. To make up solutions of hematein instead of hematoxylin is logically sound but there is no way to prevent further ripening (oxidation) with the development of other browner unwanted products and precipitation of dyes. Therefore it is good practice to begin with hematoxylin, to let it ripen naturally over a fairly long period of time or to ripen almost immediately by adding about $5 \%$ hydrogen peroxide, or
$5 \%$ of $1 \%$ aq. potassium permanganate. $10 \%$ solution of hematoxylin in $96 \%$ or abs. ethyl alcohol should always be kept on hand. It attains maximum ripening in about one year, but must be kept in a stoppered bottle for otherwise the alcohol will evaporate. It is diluted to $0.5 \%$ of hematoxylin with aq. dest. for the Iron Hematoxylin technique. See also Delafield's, Ehrlich's, Harris' and Mayer's hematoxylin solutions, likewise Azure II eosin and Hematoxylin. Addition of a drop of Tergitol No. 7 to hematoxylin solution will greatly increase speed of staining but has no other advantage (McClung 1950 p. 136).

Hematoxylin and Eosin is rightly the most used of all staining methods. If the tissues have been fixed in a fluid containing mercuric chloride such as Zenker's fluid deparaffinize sections and treat with dilute iodine in $70 \%$ alcohol for $1-2 \mathrm{~min}$. Wash in aq. dest., bleach in $5-10 \%$ aq. sodium hyposulphite to remove iodine and wash again in aq. dest. Stain with Harris' Hematoxylin (full strength) for 12-15 min. Blue in tap water or in aq. dest. + few drops sat. aq. lithium carbonate, $5-10 \mathrm{~min}$. Stain in $0.2 \%$ aq. eosin, $1 \mathrm{~min}$. Rinse in aq. dest. and $95 \%$ alcohol. Dehydrate in absolute alcohol, clear in xylol and mount in balsam. Nuclei, deep blue; cytoplasm, pink. In place of Harris' alum hematoxylin, which we use, Delafield's Alum Hematoxylin or Ehrlich's Acid Hematoxylin may be employed. The Bensleys (p. 73) dilute 1 part of the last named with 2 parts cold sat. aq. ammonium alum and 4 parts aq. dest. Nuclei, dark blue; cytoplasm, collagenic fibers, erythrocytes, pink; smooth muscle, lavender. $0.2 \%$ aq. erythrosin can take the place of the eosin but the advantage is questionable.

Hemin Crystal Test for blood pigment, Teichmann (Stitt, p. 698). Dissolve in 100 cc. glacial acetic acid, $0.1 \mathrm{gm}$. of $\mathrm{KI}$, of $\mathrm{K} \mathrm{Br}$ and of $\mathrm{K} \mathrm{Cl}$. Add few drops to suspected material on a slide and cover. Gently warm until bubbles begin, then slowly cool and examine for typical dark brown crystals. The test is not very sensitive but positive result is conclusive.

Heinochromatosis, clinical test for, see Iron.

Hemochromogen Crystal Test. Donogány (Stitt, p. 698). Mix 1 drop of suspected fluid, of pyridin and of $20 \%$ aq. NAOH on a slide and allow to dry. Radiating crystals appearing within several hours indicate presence of hemochromogen. 
Hemocytoblasts, see Erythrocytes, developmental series.

Hemoflagellates on tissues may be demonstrated by many methods. (McClung, Microscopical Technique, 1950, p. 464).

Hem ofuscin. Mallory's fuchsin stain. Fix in Zenker's fluid, alcohol or $10 \%$ formalin. Stain nuclei in paraffin or celloidin sections with Iron Hematoxylin. Wash thoroughly in water. Stain 5-20 min. in : basic fuchsin $0.5 \mathrm{gm} ., 95 \%$ alc. $50 \mathrm{cc}$. and aq. dest. $50 \mathrm{cc}$. Wash in water. Differentiate in $95 \%$ alcohol, dehydrate in abs. alc., clear in xylol and mount in balsam in the case of paraffin sections. Celloidin sections are to be cleared in terpineol or origanum oil after $95 \%$ alc. Nuclei blue, hemofuscin granules bright red, hemosiderin and melanin unstained (Mallory, p. 136).

Hemoglobin, histochemical test (Ralph, P. H., Stain Techn., 1941, 16, 105-106). Flood dried blood smear with $1 \%$ benzidine in absolute methyl alc., 1 min. Pour off and replace with $25 \%$ superoxol in $70 \%$ ethyl alc., 90 sec. Wash in aq. dest., 15 sec. Dry and mount in neutral balsam. Hemoglobin dark brown.

Goulliart, M. C. rend. Soc. Biol., $1941,135,1260-1262$ adds to frozen section or dried smear a drop from a bottle containing glacial acetic acid to which has been added less than a week ago a few crystals of potassium iodide. After about 30 min. examination with a polarizing microscope shows tiny boat shaped birefringent crystals of protoiodoheme which later change into Teichmann erystals.

Dunn, R. C., Arch. Path., 1946, 41, 676,677 . Dissolve $1 \mathrm{gm}$. cyanol (National Aniline Division, Allied Chemical and Dye Corporation, 40 Rector Street, New York) in 100 cc. aq. dest. Add 2 cc. glacial acetic acid. Boil gently and bluc color will disappear. Keep for several weeks. Immediately before using filter $10 \mathrm{cc}$. and add $2 \mathrm{cc}$. glacial acetic acid and $1 \mathrm{cc}$. commercial $3 \%$ hydrogen peroxide. Treat frozen or paraffin sections of tissue fixed in $4 \%$ formaldehyde buffered to $\mathrm{pH} 7.0$ after rinsing in water with this fresh cyanol mixture, 3-5 min. Rinse in water, counterstain with $0.1 \%$ safranin in $1 \%$ glacial acetic acid. Wash in water, dehydrate, clear and mount in Clarite. Hemoglobin, blue; nuclei, red and cytoplasm, pink (from Glick, p. 63).

Hemoglobin Estimation is done by comparing blood with a colored paper scale or by a more accurate scale in a hemoglobinometer. The experimental error is at least 5\%. Staining reactions for hemo- globin within cytoplasm (Kindred, J. E., Stain Techn., 1935, 10, 7-20).

Hemolysis. Methods for measuring the velocity of hemolysis depend on the fact that red blood eell suspensions as they hemolyse berome more and more translucent. Techniques differ merely in the ways of measuring the transmitted light. Simple visual photometers and photoelectric ones are described by Ponder, E. Glasser's Medical Physics, 605-612. The same authority explains the "equilibrium methods" for measuring the amount of hemolysis which has taken place if the process has been arrested. One of these is to count the cells remaining, another to determine the amount of hemoglobin set free, etc.

Hemophilus Pertussis. Staining of capsules in air drier smears with $5 \%$ aq. phosphomolybodic acid. Growth on a special medium is advised (Lawson, G. McL., J. Lab. \& Clin. Med., 1939-40, 25, 435-438).

Hemosiderin, soluble in acids and other reagents used in histological technique. After formalin fixation the order of decreasing removal is oxalic, sulphuric, nitric, formic and hydrochloric. Speed of solution is but little influenced by age of pigment (Lillie, R. D., Am. J. Path., $1939, \quad 15,225-239)$. See Iron, Dinitrosoresorcinol method.

To demonstrate hemosiderin microscopically pour on cleparaffinized sections of fresbly fixed tissue 1 part of fresh $2 \%$ aq. potassium ferrocyanide and 3 parts $1 \%$ aq. hydrochloric acid heated to $60^{\circ}-80^{\circ} \mathrm{C}$. Thoroughly wash in several changes of water. Counterstain in $0.1-0.5 \%$ basic fuchsin in $50 \%$ alcohol, 5-20 min. Wash in water. Pass through $95 \%$ and abs. alcohol and xylol and mount in balsam. Nuclei and hemofuscin, red; hemosiderin, blue (J. E. Ash in Simmons and Gentzkow, p. 744). See Iron and Hemofuscin.

Heparin. A method for the histological demonstration of heparin has been described by Jorpes, E., Holmgren, H. and Wilander, O., Ztsch. f. mikr. anat. Forsch., 1937, 42, 279-301. It is based on evidence that Tissue Basophiles contain this substance. See also Anticoagulants.

Heptaldehyde. An agent said by Strong, L. C., Am. J. Cancer, 1939, 35, 401-407, to produce liquefaction of spontaneous mammary tumors of mice. It was not helpful when injected into rat lepromata (Cowdry, E. V. and Ruangsiri, C., Arch. Path., 1941, 32, 632-640).

Hermann's Fluid. 2\% osmic acid, 4 ce.; $1 \%$ platinum chloride, 15 cc.; glacial acetic acid, 1 cc. This resembles 
Flemming's fluid and is a good cytological fixative.

Herring Bodies, see Cushing, H., Proc. Soc. Exp. Biol. \& Med., 1932-33, 30, 14241425.

Hertzberg's Victoria Blue stain for elementary bodies is described by Seiffert, G., Virus Diseases in Man, Animal and Plant. New York: Philosophical Lirary, Inc., $1944,332 \mathrm{pp}$. It is rather like Gutstein's technique (see Elementary Bodies) except that Hertzberg does not make up the stain with potassium hydrate and Gutstein does not destain in $1 \%$ citric acid.

Herxheimer's solution for staining fat: scarlet red (scharlach R, sudan IV), $1 \mathrm{gm}$.; $70 \%$ al cohol, 50 cc.; acetone C.P., 50 cc. See Sudan IV.

Heterophile, see Staining.

Hexamethyl violet, see Crystal violet.

Hexuronic Acid as antiscorbutic factor (Harris, L. J., and Ray, S. N., Biochem. J. 1933, 27, 58-589).

Hickson Purple, a disazo dye, giving in aq. sol. a purple color to leucocytes and a red color to erythrocytes introduced by H. G. Cannan (J. Roy. Micr. Soc., 1941, 61, 88-94).

Higgins' Ink. This was apparently first used as a vital stain by George Wislocki, see Foot (McClung, p. 114). Dilute with equal volume sterile aq. dest. Warm and inject into marginal vein of rabbit's ear 5 cc. daily for 3-4 days, then every 3 days as long as desired. Since the carbon is relatively insoluble it is a simple matter to fix, imbed, section and counterstain. Smaller amounts are to be used for smaller animals, see Vital Stains.

Hirudinea, see Parasites.

Hischler's Fluid, see Golgi Apparatus.

Hiss's Method for capsule staining, see Capsule.

Histamine, improved colorimetric method for estimating (Rosenthal, S. M. and Tabor, H., J. Pharmacol. \& Exp. Therap., 1948, 92, 425). For use in determination of the histamine content of experimental tumors, see Rosenthal, S. M., J. Nat. Cancer Inst., 1949, 10, 89-91,

Histiocyte, a term without value as it simply indicates a "tissue cell," often applied to phagocytic cells of connective tissue.

Histones, see discussion of Saint-Hilaire's method under Purines.

Histoplasma Capsulatum. The halos about this organism and Sporotrichum schencku as seen in $\mathrm{H} \& \mathrm{E}$ stained tissue sections suggest that they are encapsulated. Technique for search for such capsules and its failure to reveal them is described by Kligman, A. M. and
Baldridge, G. D., A. M. A. Arch. Path., 1951, 51, 567-574. The HotchkissMcManus stain, as employed by Kligman, A. M. and Mescon, H., J. Bact., $1950,60,415$ is considered by Kligman and Baldridge to be the best suited one for the demonstration of capsules.

Historadiography is the $\mathrm{x}$-ray photography of tissues. By a special technique Larmaque, P., Bull. d'Hist. Appl., $1937,14,1-16)$ rays emitted at a tension of $50-100 \mathrm{KV}$ having a length of $0,12-0,2$ $\mathrm{A}^{\circ}$ are directed upon a section closely applied to a particularly finely grained emulsion. The absorption of the rays by the section depends upon the density of its parts. Total opacity of the tissue to the rays is marked on the photographic negative by white, permeability by black, and there are usually all grades between the two. Subsequent magnification of about 500 times is possible, but is not advisable. Sections, not more than 4 microns thick, of formalin fixed tissues, are recommended. An illustrated description of the appearance of epidermis, cartilage, artery wall, thyroid and other tissues is provided by Tur. chini (J. Bull. d'Hist. Appl., 1937, 14, 17-28). Historadiography may have many uses in the measurement of densities in different physiological states and in study of the distribution of substances opaque to $\mathrm{x}$-rays experimentally introduced. In some cases great density may accompany high Viscosity.

Histospectrography. This is a very valuable survey method for minerals in tissues. See Policard, A., Protoplasma, 1933, 19, 602-629; Scott, G. H. and Williams, P. S., Anat. Rec., 1935, 64, 107-127; Cowdry, E. V., Heimburger, L. F., and Williams, P.S., Am. J. Path., $1936,12,13-29$. Optic lens and cataracts have been analysed particularly for iron, copper and zinc (Busnel, R. G., Pillet, P. and Tillie, H., Bull. d'Hist. Appl., 1938, 15, 99-109). MacCardle, R. C., Engman, M. F., Jr. \& Sr., Arch. Dermat. and Syph., 1941, 44, 429-440 have employed histospectrography to advantage in determination of skin magnesium. See Absorption Spectra.

Hodgkin's Disease, see Reed-Sternberg Cells.

Hofmann's Violet (CI, 679)-dahlia, iodine violet, primula $\mathbf{R}$ water soluble, red violet, violet $R, R R$ or $4 R N-$ Conn (p. 120) says above names are applied rather indiscriminately to stains varying in shade from methyl violet to basic fuchsin which are mixtures of methylated and ethylated rosanilins and pararosanilins having less than 5 methyl or ethyl groups. He further remarks that a mixture of basic fuchsin and 
methyl violet of the color desired may perhaps be made by the worker himself as a substitute for Hofmann's violet which is in fact the composition of some samples sold as Dahlia and Hofmann's violet.

Hollande's Fixative. Picric acid, $4.0 \mathrm{gm}$; copper acetate, $2.5 \mathrm{gm}$.; formol, $10 \mathrm{cc}$; glacial acetic acid, 1.5 cc.; aq. dest. 100 cc. Recommended for flagellate protozoa (McClung, 1950, p. 445).

Holmium, see Atomic Weights.

Holtfreter's Solution, for use in examination of fresh tissues; $\mathrm{NacCl}, 0.35 \mathrm{gm}$.; $\mathrm{KCl}, 0.005 \mathrm{gm}$.; $\mathrm{CaCl}_{2}, 0.01 \mathrm{gm}$., $\mathrm{NaHCO}_{3} \cdot \mathrm{H}_{2} \mathrm{O}, 0.02$ gm., aq. dest. 100 cc. (Holtfreter, J., Arch. Entio.-Mech., 1931, 124).

Hookworms. To eliminate opacity in mounts of, see Tahmisian, T. N., Stain Techn., 1945, 20, 26.

Hormones. Consult volume entitled New and Nonofficial Remedies published each year by the American Medical Association. See Testosterone, Chromaffin Reaction, Vulpian Reaction, Osmic Acid.

Hotchkiss'Method, see Polysaccharides.

Howell-Jolly Bodies, see Jolly Bodies.

Huber's Toluidin Blue stain for Nissl bodies (Addison in McClung, p. 150). This much used method is suggested for autopsy material. Fix in $95 \%$ alcohol, 100 cc.; trichloracetic acid (Mallinckrodt), $1.5 \mathrm{gm}$.; mercuric chloride (Mallinckrodt), 3 gm. 2-10 days depending upon size of piece of tissue. Change fixative every 2 days for larger specimens. Pour off fluid and store in $95 \%$ alcohol until used. Do not take out mercury with iodine. Stain paraffin sections in toluidin blue 15-18 hrs. (Make up solution by adding $1 \mathrm{gm}$. to $500 \mathrm{cc}$. aq. dest. Heat gently and when it is dissolved add 500 cc. aq. dest.). Pour off stain. Wash in aq. dest. Leave $2 \mathrm{hrs}$. in lithium carbonate. (Make this by adding $5 \mathrm{gm}$. to $1000 \mathrm{cc}$. aq. dest. Boil several minutes. Cool. Filter. To $100 \mathrm{cc}$. filtrate add $900 \mathrm{cc}$. aq. dest.). Differentiate in $70 \%$ alcohol $5-30 \mathrm{~min}$. Leave flat in $95 \%$ alcohol, 5-15 min. Dehydrate in absolute, clear in xylol and mount in balsam.

Humus, see soil.

Huntoon's Hormone Medium, see Bacteria, Media.

Hyalin. This is usually easily recognizable in sections stained with Hematoxylin and Eosin or by Phloxin and Methylene Blue, by its affinity for eosin or phloxin. Phosphotungstic Acid Hematoxylin colors it deep blue. A hematoxylinphloxin method is also recommended by Mallory (p. 207). Fix in alcohol or $10 \%$ formalin and imbed in paraffin or celloidin. Stain in alum hematoxylin, 1-5 min. or more. Wash in tap water and stain with $0.5 \%$ phloxin in $20 \%$ alcohol, 10-30 min. or longer. Wash in tap water and treat for $\frac{1}{2}-1 \mathrm{~min}$. with $0.1 \%$ aq. lithium carbonate. Wash in tap water, dehydrate, clear and mount. In case of celloidin sections, clear in terpineol or origanum oil from $95 \%$ alc. Nuclei, blue; fresh hyalin, intensely red; older hyalin, pink to colorless. A simple thionin stain is also given by Mallory. It is to stain similar sections for $5-10 \mathrm{~min}$. in $0.5 \%$ thionin in $20 \%$ alc. Differentiate and dehydrate in $80 \%$ alcohol. Then $95 \%$ alcohol, terpineol and terpineol balsam. Nuclei and old hyalin, blue.

Hyaluronic Acid.-Written by A. R. GopalAyengar, Barnard Free Skin \& Cancer Hospital, St. Louis. (Now at Tata Memorial Hospital, Bombay.) This is a polymer of acetyl glucosamine and glucuronic acid. It occurs in a polydisperse form in a variety of tissues such as umbilical cord, synovial fluid, vitreous humor, skin, tumors due to virus of leucosis and sarcoma of fowls, and in pleural fluid associated with human mesothelioma. (For an extensive treatment of the subject of acid polysaccharides and a comprehensive bibliography, refer to Karl Meyer's reviews on, "Mucolytic enzymes" in Currents in Biochemical Research, Interscience Publishers, N. Y., 1946; "Mucoids and Glycoproteins" in Advances in Protein Chemistry, Academic Press, N. Y. 1945; "The Chemistry and Biology of Mucopolysaccharides and Glycoproteins" in Cold Spring Harbor Symposia on Quant. Biol., 6, 1938, 91102.) The enzyme, hyaluronidase, depolymerizes and hydrolyses hyaluronic acid. It is a Spreading Factor and has been ably presented, along with other spreading factors, by DuranReynals, F., Bact. Rev., 1942, 6, 197252 ; Meyer, K. and Chaffee, E., Proc. Soc. Exp. Biol. \& Med., 1940, 43, 487489; Meyer, K. et al., Proc. Soc. Exp. Biol. \& Med., 1940, 44, 294-296, and others.

A histochemical method for the demonstration of acid polysaccharides like hyaluronic acid is described by $\mathrm{Hale}$, C. W., Nature, 1946, 157, 802 . The use of metachromatic stains such as toluidine blue while satisfactory for sulphated polysaccharides like chondroitin sulphate is valueless for hyaluronic acid and for related acid polysaccharides which do not stain metachromatically. Fixation of material is an important factor in the retention of hyaluronic acid for subsequent staining. The or- 
dinary aqueous fixatives containing formalin, while eminently suitable for fixing protein components, tend to dissolve the hyaluronic acid. To preserve intact hyaluronic acid it is therefore imperative to employ dehydrating fixing agents like Carnoy. The material after fixation, dehydration and embedding is sectioned in the usual manner and treated with an acid solution of ferric hydroxide. The iron combines with hyaluronic acid but not with the neutral polysaccharides or proteins. The combined iron is then characterized as Prussian blue by treatment with hydrochloric acid and potassium ferrocyanide. A counter stain like fuchsin is recommended in order to bring out sharply the blue stained acid polysaccharides against a background of red stained cells.

The detailed outline of the Hale technique is as follows: Fix small pieces of tissue in Carnoy (Abs. alcohol, 6 pts. + chloroform, 3 pts. + glacial acetic acid, 1 pt., for $\frac{1}{2}$ hr. Dehydrate in abs. alcohol, clear, embed in paraffin and section in the usual manner. Mount sections on clean slides without albumen. Bring sections rapidly to water and flood with a mixture of dialysed iron, 1 vol. and acetic acid (2M), 1 vol., $10 \mathrm{~min}$. (Dialyzed iron may be prepared by adding ammonia water to a concentrated solution of ferric chloride and dialysing the resulting solution until free or nearly free of ammonium salts. It is a dark red liquid easily miscible with water and contains approximately 3.5 per cent $\mathrm{Fe}$, or $5 \%$ $\mathrm{Fe}_{2} \mathrm{O}$, $\mathrm{M}=$ Molecular Solution, which see.) Wash well with aq. dest. Flood with a solution containing potassium ferrocyanide (0.02M) and hydrochloric acid $(0.14 \mathrm{M})-10 \mathrm{~min}$. Wash with water and counterstain with appropriate contrasting dye. Dehydrate rapidly, clear in xylol and mount in Canada balsam.

In order to distinguish hyaluronic acid from other blue staining structures Hale recommends interpolation of another step during the staining process. The procedure suggested involves use of the specific enzyme-hyaluronidase - soon after fixation. The enzyme hydrolyses the hyaluronic acid and prevents the combination of the polysaccharide with iron. Since hyaluronidase is specific, it has no similar action on other polysaccharides.

Hyaluronidase is the spreading factor which increases the permeability of connective tissue by reduction in viscosity and by hydrolysis of Hyaluronic Acid. Commercial preparations of hyal- uronidase from bull testes are available from the Schering Corp., Bloomfield, N. J. Enzyme prepared from certain bacteria apparently have hydrolytic powers different from those of the testicular preparations.

Hydrax is a synthetic resin used as a mounting medium (Hanna, D., J. Roy. Micr. Soc., 1930, 50, 424-426).

Hydrogen Acceptors. These are substances like $p$-amidophenol, $p$-phenylenediamine and resorcin, recommended to strengthen supravital staining of nerve fibers with methylene blue, see Auerbach's Plexus.

Hydrogen Ion Indicators-Written by L. F. Wicks, Veterans Administration Hospital, Jefferson Barracks, Missouri. February 1, 1951.-These are also called acid-base indicators and $\mathrm{pH}$ indicators. They are dye compounds which are themselves weak acids or weak bases, more usually the latter, and have definite ionization constants. According to the old theory of Ostwald, the color of the indicator in solution depends upon the degree of dissociation and the relative ratio of dissociated and undissociated forms. This ratio, and the corresponding shift in color, varies with the concentration of hydrogen ions present, the effect being a composite one. The color change interval will span a certain range, usually of two $\mathrm{pH}$ units or less, and it does not require a shift which crosses neutrality. Of the very many dyes and plant coloring matters which alter color with $\mathrm{pH}$, only a few change sufficiently sharply to be of analytical value.

Acid-base indicators may, of course, be employed for adjusting the $\mathrm{pH}$ of a solution. If direct addition is not desired, small portions of both liquids may be transferred to a spot plate, or the indicator may be applied in the form of test papers. (Some indicators such as litmus are now rarely employed otherwise. This is partly true also for Congo red and nitrazine.)

Indicators may also be used to estimate the reaction of a solution by the application of a series with different $\mathrm{pH}$ ranges. Once roughly determined, there is a procedure ("Gillespie's dropratio method") by which a fairly accurate $\mathrm{pH}$ measurement may be easily made with a single indicator of proper range.

Perhaps the commonest use for hydrogen ion indicators by the analytical chemist (who usually prefers the glass electrode for $\mathrm{pH}$ measurement and adjustment), is as an end point device in acid-base titrations. When titrating a weak acid or a weak base, the choice of 
indieator is very important. That ehosen should be one which has its sharpest color transition near the true equivalence point. For example, when titrating the weak base ammonium hydroxide with hydrochloric acid, the resulting ammonium chloride is an acidic salt, and the indicator selected should be one that shifts below neutrality, methyl red for example. Again, when titrating a weak acid such as lactic acid with sodium hydroxide, the salt formed is alkaline, and the indicator of choice should shift above neutrality, bromthymol blue, for example. In titrating strong acids and strong bases against each other, the selection of indicator is not critical, as here the $\mathrm{pH}$ change near the end point is very great for only a small increment of the added reagent. One should never titrate weak acids and weak bases together, for the results cannot be accurate.

There are several possible sources of error in the use of hydrogen ion indicators. Some are present at all times and others especially so in biological fluids. An occasional indicator, with two groups sensitive to acid or alkali, has two ranges of color transition at perhaps widely separately $\mathrm{pH}$ values. For example, thymol blue shifts from red to yellow at a low range (1.2-2.8) and from yellow to blue at a much higher one (8.0-9.6). A very few indicators exhibit "dichroism" (or "dichromatism") in which the color varies with the depth and concentration of the solution. Bromeresol purple and bromphenol blue are examples.

In attempting to determine the $\mathrm{pH}$ of a very dilute solution, a false result may be obtained by the use of indicators as they are themselves acids or bases. (Recall, for example, that water in contaet with the carbon dioxide of the normal atmosphere has a pH of about 5.7.) For such cases, a very small amount of buffer should be present to offset this effect.

Indicators are intended for aqueous systems, and the presence of other solvents such as alcohol decreases the dissociation constant. Acidic indicators then become more sensitive to hydrogen ions, and basic ones less sensitive. A control solution of the same solvent composition may be used for comparison, however.

Temperature errors are slight over the usual ranges.

Indicators may be altered or destroyed by the presence of oxidizing and reducing agents, and they may unite with heavy metal ions. Fortunately, these are negligible considerations in biological fluids, but greater potential errors exist.

Proteins and their hydrolysis products are usually amphoteric and may combine with the indicator. Congo red, for example, is almost worthless in protein solutions.

The presence of much neutral salt will affect the color of indicator solutions, partly by influencing the light absorption and partly by shifting the ratio between dissociated and nondissociated forms of the indicator.

The use of mixed indicators deserves greater attention than it has yet received. As employed for titrations, they are of two general types. One sort consists of two acid-base indicators which have color transitions in opposite directions, resulting in a very sharp change at a narrow $\mathrm{pH}$ zone. The other kind utilizes for contrast color a dye whieh itself is not influenced by hydrogen ion concentration. The composite color change resulting is usually much sharper than that of the indicator alone. In recent years, there have appeared "universal indicators" consisting of a mixture of half a dozen compounds with a "spectrum" of colors which may vary over the entire $\mathrm{pH}$ range. Such indicators are not very accurate and should be used only as a first rough test on an unknown solution. These mixtures are more common as test papers.

It is not surprising that hydrogen ion indicators have been employed as a sort of vital stain to determine the reaction of various living components.

In 1893 Ehrlich injected neutral red in an attempt to determine the reaction about phagocytosed granules. Since then, other workers have applied other dyes, striving to estimate the approximate $\mathrm{pH}$ of tissues, of the fluids bathing them, and even of individual cells. Alizarin red and litmus have been much used, the later especially with lower organisms. Thus, Steiglitz applied all three dyes mentioned above to estimate the reaction of living kidney (E. J., Arch. Int. Med., 1924, 33, 483-496) and confirmed the contention that alkaline urine can be formed by an acidic cortex. Harvey and Bensley (B. C. H. and R. R., Biol. Bull., 1912, 23, 225-249) used $\mathrm{pH}$ indicators to indicate that gastric fluid does not arise directly within the cells of the mucosa. Margaria (R., J. Physiol., 1934, 82, 496-497) injected bromcresol purple and bromphenol blue, and claimed to have measured $\mathrm{pH}$ changes upon stretching a muscle. Orr (J. W., J. Path. \& Bact., 1937, 44, 
19-27) employed phenol red to estimate alterations in $\mathrm{pH}$ in the skin of tarred mice during carcinogenesis. Chambers and his colleagues have added $\mathrm{pH}$ indicators to tissue cultures (R., Proc. Roy. Soc., B, 1932, 110, 120-124) and have injected them directly into individual living cells (McClung, pp. 62-109). The most enthusiastic investigator to employ the phthalein and sulphonphthalein indicators is Rous (P., Science, 1924, 60, 363: J.A.M.A., 1925, 85, 33-35, and many articles in J. Exp. Med., 1925 to 1927). The literature is extensive but scattered. There are brief reviews by Rous (P., J. Exp. Med., $1925,41,379-411)$ and von Möllendorf (W., Ergebn. Physiol., 1920, 18, 141306). See W. M. Clark in Simmons and Centzkow 161-171.

It is well to question the dependability of data upon $\mathrm{pH}$ of living material as apparently indicated by vital staining methods. Consider the ideal requirements for such a vital stain. It should exhibit a sharp and pronounced color change in the proper $p H$ range. It should be fairly soluble, readily diffusable, strongly colored, of low toxicity and stable in the organism (not readily oxidized or reduced or precipitated by tissue electrolytes). Of the many indicators employed in analy tical chemistry, only a few meet these requirements. Certain errors are to be guarded against in their use. The "salt error" and "protein error" are unavoidably present. In the application of these vital stains changes may take place that will themselves cause a pH change. Among them anesthesia, traima, loss of carbon dioxide from exposed tissues, interference with blood supply, and postmortem change deserve special mention. However crude though the methods may be, these dye indicators are of value in preliminary experiments or where no better procedure is applicable.
The indicator dyes of most promise are certain of the phthalein and sulphonphthalein compounds. They are generally quite soluble, highly diffusable, show marked color shifts and are fairly constant in composition. The dye solutions diffuse quickly when injected, and quickly appear in the urine and stools. For these reasons, fairly large doses given intraperitoneally are more suitable than subcutaneous injections. But it is doubtful, according to Chambers (personal communication), whether the more soluble dyes actually penetrate the walls of most cells.

The following selection of indicators is based upon the reports of Rous and others, and upon experiments with mice carried out at The Barnard Free Skin and Cancer Hospital. Their chemical names can be found in The Merck Index or in any good textbook of chemistry. Some are to be used in $1 \%$ aq. solutions, others in sat. solutions in physiological saline, litmus in either aqueous or agar solution (Rous, P., J. Exp. Med., 1925, 41,379 ), while the remainder, which are acidic (the sulphonphthaleins and methyl red), require to be converted to their corresponding sodium salts because the latter are more soluble in water. Consequently the proper equivalent of sodium hydroxide must be reacted with each compound. Rub up $0.1 \mathrm{gm}$. of the dry dye in a mortar (agate, preferably) with the volume of $\mathrm{N} / 20$ sodium hydroxide solution given in cc. below the dye in the table. Filter, wash out the mortar with several small portions of saline $(0.9 \% \mathrm{NaCl})$ and make all to a volume of $10 \mathrm{cc}$. For a mouse, $0.5-2.0 \mathrm{cc}$. of the dye solution should be injected intraperitoneally.

It should be emphasized that wherever possible, the glass electrode should be employed for direct measurement of $\mathrm{pH}$ in biological fluids rather than indicators. Micro electrodes, including injection types, have been developed.

\section{HXDROGEN ION INDICATORS}

Indicator

Bromphenol blue*

$3.0 \mathrm{~N} / 20 \mathrm{NaOH}$

Sodium alizarin

sulphonate

(Alizarin red)

$1 \%$ aq. or sat.

in saline

Bromcresol green *

$2.9 \mathrm{~N} / 20 \mathrm{NaOH}$

Methyl red *

$7.4 \mathrm{~N} / 20 \mathrm{NaOH}$
pH Range and Colors

yellow $\leftarrow 3.0-4.6 \rightarrow$ blue

yellow $\leftarrow 3.8-5.0 \rightarrow$ pink

yellow $\leftarrow 4.0-5.6 \rightarrow$ blue

red $\leftarrow 4.2-6.3 \rightarrow$ yellow
Value as Vital Stain

Very strong stain, too far on acid side.

Very toxic, weak stain.

Strong stain, persistent, well tolerated.

Unstable in organism, weak stain, fixes on lipoids. 


\section{HYDROGEN ION INDICATORS-Continued}

Indicator

Chlorphenol red*

$4.7 \mathrm{~N} / 20 \mathrm{NaOH}$

Bromeresol purple*

$3.7 \mathrm{~N} / 20 \mathrm{NaOH}$

Bromphenol red

$3.9 \mathrm{~N} / 20 \mathrm{NaOH}$

Methyl violet

$1 \%$ aq. or sat.

in saline

Bromthymol blue*

$3.2 \mathrm{~N} / 20 \mathrm{NaOH}$

Phenol red

$5.7 \mathrm{~N} / 20 \mathrm{NaOH}$

Litmus, purified

(Azolitmin)

$1 \%$ aq. or

in agar sol.

Neutral red

(Toluylene red)

1-2\% aq. or sat.

in saline

Cresol red

$5.3 \mathrm{~N} / 20 \mathrm{NaOH}$

Metacresol purple $5.3 \mathrm{~N} / 20 \mathrm{NaOH}$

Thymol blue*

$4.3 \mathrm{~N} / 20 \mathrm{NaOH}$
pH Range and Colors

$$
\begin{aligned}
& \text { yellow } \leftarrow 4.8-\text { red }-6.8 \rightarrow \text { purple } \\
& \text { yellow } \leftarrow 5.4-6.6 \rightarrow \text { purple } \\
& \text { yellow } \leftarrow 5.4-\text { red }-7.0 \rightarrow \text { purple }
\end{aligned}
$$

blue-violet $\leftarrow 6.0-7.0 \rightarrow$ violet

yellow $\leftarrow 6.0-7.4 \rightarrow$ blue

yellow $\leftarrow 6.6-7.8 \rightarrow$ red

$(6.8-8.4)$

(approx.) red $\leftarrow 6.0-8.0 \rightarrow$ blue

(approx.) red $\leftarrow 6.8-8.0 \rightarrow$ yellow

yellow $\leftarrow 7.2-8.4 \rightarrow$ purple-red

yellow $\leftarrow 7.4-9.0 \rightarrow$ purple

yellow $\leftarrow 8.2-9.4 \rightarrow$ blue
Value as Vital Stain

Powerful stain, well tolerated.

Strong stain but rapidly excreted, is toxic and exhibits dichromatism.

Very strong stain, well tolerated.

Weak stain, toxic.

Weak stain, very toxic to mice, but not for insects.

Rapid, intense stain, very well tolerated

Slow stain, diffuses poorly, usually deposits in granules.

Very weak stain, precipitates out readily in vivo, not toxic if pure.

Somewhat toxic, not a strong stain.

Very weak stain, not very soluble.

Toxic, range too alkaline.
The indicators starred are perhaps of of widest ordinary laboratory use. To these may also be added cresol purple (yellow $\leftarrow 7.4-9.4 \rightarrow$ purple) and phenolphthalein (colorless $\leftarrow 8.0-9.8 \rightarrow$ redviolet). The latter is usually made up in $70-90 \%$ alcohol. It should not be used to titrate ammonia.

Hydrokollag, a particulate material employed for injection of Lymphatic Vessels which see.

Hydrotropes, see Sudan Stains.

Hydroxy Tri-Phenyl Methanes. These are the rosolic acids. Amino groups of triamino tri-phenyl methanes are replaced by hydroxyls making them acidic instead of basic. Examples: aurin (or rosolic acid); red corallin.

Hydroxybenzene Compounds as cytoplasmic fixatives. Details of use of pyrogallol and resorcinol in neutralized formaldehyde solutions are given. The simplicity and rapidity of the procedures and the ease of thereafter cutting sections $1-2 \mu$ in thickness are cited as the advantages special attention having been paid to mitochondria and secretion granules (Huseby, R. A., Proc. Suc. Exp. Biol. \& Med., 1946, 61, 122-125).

Hydroxyquinoline test for iron, see Iron.
Hypophysis, see Pituitary.

Hypoxanthine, see Murexide test under Purines.

Ice-crystal Artefacts in normal and chromatolytic anterior horn cells (Gersh, I., and Bodian, D., Biological Symposia, $1943,10,163-184)$.

Icterus Index is a simple measure of the degree of yellow color of blood plasma, or serum, in comparison with standard potassium bichromate solutions. Make up in tubes of same thickness and bore as hematocrit tubes a series of unit dilutions of the bichromate solution Unit $1=1 \mathrm{gm}$. potassium bichromate in 10,000 ce. aq. dest., Unit $3=3 \mathrm{gm}$. in 10,000 cc., Unit $5=5 \mathrm{gm}$. in 10,000 cc. etc. The plasma of centrifuged blood in hematocrit is compared with these. If it has a color corresponding to, say, Unit 5 of the bichromate solution the icterus index is considered to be 5 . The normal value of the icterus index is usually given as 4-7 units. The measure being that of color, and, since increase in color can be caused by substances other than bilirubin, the index is not a specific measure of bilirubinemia. Lipochromes can increase the index. If the blood is unusually 
concentrated the index is higher although the total amount of bilirubin in the circulation may not be elevated. See much more adequate description by Wintrobe, M. M., Clinical Hematology. Philadelphia: Lea \& Febiger, 1942, $703 \mathrm{pp}$.

Idiochromatin (G. idiōs, one's own, peculiar). The chromatin concerned particularly with reproductive functions such as chromosome formation contrasted with nutritive trophochromatin (G. trophē, food, nourishment). There is no special technique for it.

Illumination. For microscopic work the lighting is of great importance. Direct visible light can best be obtained from various electric microscopic lamps on the market. Only when the light is more intense than that required for routine purposes can it be properly employed for dark field examination or for polarization. Therefore an intense source should be available. The intensity can be reduced to optimum by using an iris diaphragm. When it is desired to deliver light into the body to a position behind living tissues or organs for transillumination the Quartz Rod technique is suggested.

Even to make the light equivalent in quality to that from the white cloud on a bright day, that microscopists used to search for, is quite unnecessary. If the light is too much screened by "daylight" or other glass its intensity will be impaired. Green light was recommended quite enthusiastically about 20 years ago. But it is difficult to secure green light of the necessary intensity and it is unpleasant to work with. Ultraviolet light, which permits higher resolution and is selectively absorbed especially by nucleoproteins, is used occasionally for Ultraviolet Photomicrography. The objects, however, can of course not be seen directly so that to photograph them is a hit and often miss experience, though it is possible to focus on a fluorescent screen. The principal use of ultraviolet light is in the Fluorescence Microscope by which the structures giving off fluorescence can be viewed in a dark background at high magnification.

Imbedding, see Celloidin, Parafiin, GlycolStearate, Rubber Paraffin, Ceresin, Double and Gelatin for imbedding preparatory to sectioning. The Mounting of sections and whole tissucs is a kind of imbedding.

Immersion Oils. A special grade of cedar oil is usually employed for oil immersion objectives. Mineral oil is also sold for this purpose. If a refractometer is not available Lillie (p.6) recommends ad- justment of the index of refraction of the mineral oil by the addition to it of alphabromonaphthalene gradually to the point where a white glass slide immersed in the oil can no longer be seen through the oil. It should be $\mathrm{N} \Delta 1.515$ to 1.520 . For darkfield work he uses a mixture of 4 parts of "heavy" mineral oil with one part of alphabromonaphthalene. Cedar oil as supplied for this purpose may easily get too thick by evaporation. It should never be left to harden on the objective but should always be brushed off with lens paper immediately after use. If it does harden on the objective, condenser or slide wet the lens paper with xylol which will dissolve the oil when it is gently applied. See Lens Paper.

Immunization of monocytes against foreign erythrocytes with phagocytosis of the latter (Bloom, W., Arch. Path. and Lab. Med., 1927, 3, 608-628).

Impedence, see Electrical Resistance.

Imperial Red, see Eosin B or bluish.

Imperial Yellow, see Aurantia.

Impression Preparations, see Smears.

Inanition, see Fasting.

Inclusion Bodies are any substances included in a cell, tissue or organ. There is the implication that the substance is included from without, that is to say, it is of extrancous origin. But the designation is so loosely used as to be almost meaningless. It is applied to droplets of fat, ingested pigments, remnants of phagocytosed materials, bodies developed in cells as a result of virus action and so forth. The virologists have taken over the designation from normal eytology in which it is used less and less. In certain virus diseases inclusions form in the nucleus, in the cytoplasm or in both (Cowdry, E. V. in Rivers' book on Virus Diseases, Baltimore, Williams \& Wilkins, 1928, pp. 113-154).

Since the nucleus is shielded from the environment by the cytoplasm its reactivity is restricted and the materials a vailable for the formation of nuclear inclusions are also limited as compared with those in the cytoplasm. Consequently the composition of nuclear inclusions in virus diseases is more uniform than that of cytoplasmic inclusions. See Nuclear and Cy toplasmic Inclusions

Indamin Dyes. Methylated amino derivatives of indamin. Bindschedler's green and toluylene blue.

India Ink, see Higgins'.

Indian Blue 3 RD, see Naphthol Blue.

Indicators, see Hydrogen Ion and Oxidation Reduction Potential indicators.

Indigo, a fine blue dye produced from the leaves of Indigofera tinctoria, employed 
as a stain and a cosmetic for more than 4000 years, and early adopted officially for the uniforms of American and British sailors, its history reads like a romance. (See, Leggett, W. F., Ancient and Medieval Dyes. Brooklyn: Chemical Publishing Co., Inc., 1944, 95 pp.)

Indigo (Cl. 1177) is now produced artificially as well as from plants.

Indigo-Carmine (CI, 1180)-indigotine IaThis sodium salt of indigosulfonic acid is blue with acid characteristics so that it is a good counterstain for carmine. It has been employed with fuchsin by Shumway, W., Stain Techn., 1926, 1, 37-38. See renal excretion of (Kempton, R. T., Bott, P. A. and Richards, A. N., Am. J. Anat., 1937, 61, 505-521). It was used as a vital stain by Heidenhain who employed 35-60 cc. of $0.4 \%$ suspension for rabbits and $150-1500 \mathrm{cc}$. for dogs (see Foot, McClung, p. 113). The Bensleys (p. 151) advise intravenous injection of $4 \mathrm{cc}$. sat. filtered aq. indigo-carmine per kilogram of body weight. Fix by vascular perfusion with formalin alcohol (neutral formalin, 10 cc.; absolute alcohol, 90 cc.) or by immersion in it. Counterstain frozen sections with Mayer's Acid Carmine or with $1 \%$ anridine red. Another way is to imbed (in paraffin), section, clear and examine with or without this counterstaining.

Indigotine Ia, see Indigo-Carmine.

Indin Blue 2rd, see Naphthol Blue R.

Indium, sec Atomic Weights.

Indo Reaction for phenols. Formation by oxidation of an aromatic paradiamine in presence of tissue phenol of a blue or green indamine. A difficult reaction (Lison, p. 142). See Lison's study of the venom gland of toads (Lison, L., C. Rend. Soc. de Biol., 1932, 111, $657-658$ )

Indol Compounds, see Nitro Reaction, Nitrosamino Reaction.

Indophen ol Blue (CI, 821). This is formed by oxidation of a mixture $p$-aminodimethylaniline and $\alpha$ naphthol. Conn (p. 73) says that this is probably the dye employed for staining fat by Herxheimer, G., Deut. Med. Wochenschr., $1901,27,607-609$.

Indophenol 1. See Oxidation-Reduction.

Indophenol Oxidase, see Nadi Reagent, Cytochrome, Oxidase.

Indophenols. Dyes closely related to indamines. Example: indophenol blue.

Indulin. 1. Spirit soluble (CI,860)-spirit indulin and spirit nigrosin $\mathrm{R}$.

2. Water soluble (CI, 861)-fast blue $\mathrm{B}$, OB, R, etc., soluble indulin $3 \mathrm{~B}-$ An infrequently used acid azin dye. Lynch, J. E., Zeit. f. wis. mikr., 1930,
46, 465-469; Cumley, R. W., Stain Techn., 1935, 10, 53-56.

Indulin Black, sce Nigrosin, water soluble. Infra Red photography shows split appearance of chromosomes (Ganesan, D., J. Roy. Micr. Soc., 1939, 59, 75-78) and gives better definition of epiphyseal layers of normal and rachitic bone (Siegel, L., Allen, R. M., McGuire, G. and Falk, K. G., Am. J. Path., 1939, 15, 273-277). Guardabassi, M., C. rend. Soc. de Biol., 1935, 118, 559-561 has used this technique for alcohol fixed sections of brain of rabid dog sensitized with rubrocyanine to demonstrate structure of Negri bodies. Transmission of infra red light through the skin facilitates photography of superficial veins in the living state. Resolution with this light of relatively long wave length is inferior to that with visible light.

Injection, see Microinjection. Perfusion of blood vessels and Neutral Red method of staining pancreas by vascular injection.

Innervation, determination by dissection (Wharton, L. R., Anat. Rec., 1937, 67, 467-475). Place tissue sheets or thin organs on writing paper. Allow to adhere 5-10 min. Place in 1 part glycerol, 1 part glacial acetic acid and 6 parts $1 \%$ aq. chloroal hydrate, $18 \mathrm{hrs}$. Gly cerol, 1 part; Ehrlich's hematoxylin, 1 part; and $1 \%$ aq. chloral hydrate, 6 parts, 24 hrs. or more. If overstained decolorize in first solution or in $1 \%$ hydrochloric acid in $70 \%$ alcohol. Transfer to glycerol 10 days. Dissect under binocular microscope in fresh glycerol. To make permanent preparations, pass up to $95 \%$ alcohol, then through bergamot oil, 2 parts; cedar oil, 1 part; and pure carbolic acid liquefied by heat, 1 part, to xylol. Mount in balsam. See Nerve Endings.

Inoculation is to introduce materials into the body usually disease producing or antigenic. They are in reality injected and we speak of injecting a host of different substances, see in this connection Microinjection, Perfusion and Transplantation.

Insects. For whole mounts of large insects Stapp, P. and Cumley, R. W., Stain Techn., 1936, 11, 105-106, specify abs. alc., 5-15 days; $95,85,70$, and $50 \%$ each 15 min. Alc. $35 \%, 30$ min. Equal parts $\mathrm{H}_{2} \mathrm{O}$ and $\mathrm{H}_{2} \mathrm{O}_{2}+$ trace $\mathrm{NH}$ OH, 12-24 hrs. Alc. $35,50,85$, and $95 \%$, 15 min. each. Abs, alc. 2-3 changes, 3 days or more. Toluol, 10-21 days. Pass from thin to thick dammar and mount. Perhaps the simplest method for small insects (fleas, ctc.) is simply to drop them in creosote, U.S.P. and after 24 hrs. to mount them directly in balsam 
(Fox, 1., Science, 1942, 96, 478). Sectioning is facilitated by methods designed to soften Chitin, see also Fleas, Ticks. Use of fluorescence microscopy in entomology (Metcalf, R. L. and Patton, R. L., Stain Techn., 1944, 19, 1127). In making preparations of insect tissues one must of course be on the lookout for infecting organisms. A well illustrated volume, giving many technical details, is that of Paillot, A. L'Infection Chez Les Insectes. Imprimerie de Trévoux, G. Patissier, 1933, $535 \mathrm{pp}$.

Interference Colors for daylight, Newton's scale, see McClung's Microscopical Technique, 1950, p. 633 .

Intermitotic Cells, see Cell Classification.

Intestinal Protozoa. 1. Johnson's rapid iron hematoxylin method (Johnson, C. M., Am. J. Trop. Med., 1935, 15, 551). Fix thin smears 10 min. in Schaudinn's fixative containing 5-10\% glacial acetic acid $\left(37^{\circ}-45^{\circ} \mathrm{C}\right)$. Treat for $5 \mathrm{~min}$. with iodine in $95 \%$ alcohol (port wine color). After placing in $70 \%$ alcohol for $5 \mathrm{~min}$. rinse in tap water $1-3 \mathrm{~min}$. Mordant in $4 \%$ aq. iron alum (purple crystals) for $15 \mathrm{~min}$. Rinse in tap water 1-2 min. and stain for $10 \mathrm{~min}$. in $0.5 \%$ aq. hematoxylin (10 cc. $5 \%$ hematoxylin in $95 \%$ alc. plus 90 cc. aq. dest.). Differentiate in $0.25 \%$ aq. iron alum $6-10 \mathrm{~min}$. for flagellates and $12 \mathrm{~min}$. for amoebae. After washing in running water for 3 30 min., dehydrate in alc., clear in xylol and mount.

2. Long method of Heidenhain (Q.M. Geiman in Simmons and Gentzkow, p. 616). Recommended for Balantidium coli and for permanent mounts. This is practically the same except for longer mordanting and staining. See

Iron Hematoxylin and Iron Hematoxylin Single Stain.

Intestine. Difference in appearance of wall when contracted and normally distended (Johnson, F. P., Am. J. Anat., 1912-13, 14, 235-250). Alterations in human mucosa from absorption of fat and from fasting (Cowdry's Histology, pp. 302305). Effect of different dehydration and clearing agents on intestine (Ralph, P., Stain Techn., 1938, 13, 9-15). Rosenberg, L. E., Stain Techn., 1940, 15, 53-56 has given an interesting account of postmortem autodigestion. Mingazzini phenomenon (Macklin, C. C. and M. T., J. Anat., 1926, 61, 144-150). See Large and Small Intestines.

Intracellular Phase, see Chloride.

Intranuclear crystals. Hepatic cells of dogs. Determination of properties (Weatherford, H. L., and Trimble, H. C., Anat. Rec., 1940, 77, 487-502).
Intranuclear Inclusions, see Nuclear Inclusions.

Intravitam Staining, the same as Vital Staining but different from Supravital Staining, which is upon or after, the death of the animal whose tissues are stained.

Iodeosin B, see Erythrosin, bluish.

Iodides. Histochemical localization not feasible (Gersh, I. and Stielglitz, E. J., Anat. Rec., 1933, 56, 185-193). As explained by Glick, p. 34, a precipitating agent to fix iodide will also precipitate protein and thus prevents its own penetration into the tissue.

Iodine, detection of: 1 . Ionized iodine in the form of iodides. Stieglitz (E., J. Pharm. and Exp. Therap., 1924, 22, 89-98) injects 20 cc. $5 \%$ aq. lead nitrate intravenously into an animal to be killed and fixes the tissue in formalin. In the sections, iodine is found in the form of yellow crystals of lead iodide. Methods have been reviewed by Gersh and Stieglitz (I. and E. J., Anat. Rec., 1933, 56, 185-193).

2. Methods for iodine in organic combination appear to be unsatisfactory. The whole subject of iodine has been critically considered by Lison (p. 111113). See Gram's and Lugol's solutions.

Iodine, as a stain is one of the stains used for Glycogen and Starch Grains. It is also advised in the form of Lugol's solution to bring out in frozen sections of nervous tissue certain extremely minute bodies in the cytoplasm and along the processes of nerve cells by Adamstone, F. B. and Taylor, A.B., Science, 1946, 104, 111. See Gram-Pappenheim stain and Gram Stain for bacteria.

Iodine-Eosin stain of Donaldson, R., Lancet, 1917, 1, 571 is highly recommended by Craig, p. 45 for intestinal amebae and flagellates. Saturate one volume of $5 \%$ aq. potassium iodide with iodine crystals and mix with equal volume of sat. aq. eosin (yellow aqueous eosin). Mix small drop with a little feces on slide, cover and examine. Cysts of amebae and flagellates, yellow to greenish yellow in red background; glycogen bodies within eysts, brown.

Iodine Green (CI, 686), closely related to methyl green, only used occasionally.

Iodine-Iodide Solution. This term is employed for almost any solution containing iodine and iodide as Lugol's and Grams.

Iodine Number of lipids, see SchmidtNielsen, K., C. rend. trav. lab. Carlsberg, Sér. Chim., 1944, 22, 87-96 and Kretchmer, N., Holman, R. T. and Burr, G. O., Arch. Biochem., 1946, 10, 101-105. 
Iodine Violet, see Hofmann's Violet. Iris Blue, see Resorcin Blue.

Iris Violet, see Amethyst Violet.

Iron occurs in tissues "masked" in organic compounds which are not ionisable and free in inorganic compounds which are ionisable into ferric and ferrous salts.

1. Macallum's hematoxylin method depends upon the formation of a blue black iron hematoxylinate. The tissue is fixed in $95 \%$ alcohol $24-48$ hours, dehydrated, cleared, imbedded in paraffin and the sections are passed down to distilled water. Contact with iron is reduced to a minimum. The microtome knife must be free of rust. Treat sections with a freshly prepared straw yellow $0.5 \%$ aqueous solution of hematoxylin which must be of the highest purity. Inorganic iron produces the blue-black compound which is relatively insoluble. Dehydrate, clear and mount in balsam in the usual way.

The technique for organic iron is more difficult because it must be unmasked before it will react in this way. The best account is Nicholson, F. M., J. Comp. Neurol., 1923, 36, 37-87. In studying the cytoplasmic iron containing proteins of nerve cells of the medulla of rats, he fixed in $95 \%$ alcohol 48 hours; dehydrated in absolute alcohol 2-5 hours; cleared in cedarwood oil until transparent; imbedded in paraffin (2 changes) and cut sections $7 \mu$. After being deparaffinized, the sections were passed through alcohols to $4 \%$ pure sulphuric acid in $95 \%$ alcohol held at $60^{\circ} \mathrm{C}$. for 5-60 minutes. This liberated the iron. The sections were washed in 95\% alcohol; passed down through graded alcohols to aq. dest., and placed in freshly prepared $0.5 \%$ aqueous hematoxylin, 1-5 minutes in which the blue-black hematoxylinate forms. Then wash in aq. dest. (not tap water). Counterstain in dilute alcohol erythrosin and mount as usual. As a check the nuclear chromatin of sections not treated with the acid alcohol should not be colored black by this hematoxylin solution. Difficulty may be experienced because the color of the unmasked iron is faint. The reaction is a chemical one of great delicacy and requires practice. Pancreatic acinous cells also afford favorable material. Look for cytoplasmic iron in the poles distant from thelumen where the chromidial material, which resembles the Nissl bodies, is most concentrated.

2. Prussian blue reaction. Prepare sections in the same way, deparaffinize and test as described in Lee (p. 291). For ferric salts of inorganic iron wash in aq. dest., $2 \%$ aqueous potassium ferro- cyanide, 3-15 minutes; Prussian blue is formed, wash, dehydratc, clear and mount. For ferrous salts substitute ferricyanide for ferrocyanide in the test. For both use cqual parts of ferrocyanide and ferricyanide. When the iron is organic it is unmasked by treating the sections with $3 \%$ pure nitric acid in $95 \%$ alcohol for 2.4-36 hours at room temperature or at $35^{\circ} \mathrm{C}$. if necessary. Wash in pure $90 \%$ alcohol and in aq. dest. Place in equal parts freshly made of $1.5 \%$ aqucous potassium ferrocyanide and $0.5 \%$ aqueous hydrochloric acid for not more than 5 minutes. Wash well in aq. dest., colored with eosin or safranin, dehydrate, clear and mount.

Hemosiderin gives Prussian blue reaction for inorganic iron. The iron in hemoglobin is not unmasked by these acid alcohols. Brown, W. H., J. Exper. Med., 1911, 13, 477-485, devised special methods for its demonstration. Testing for iron in association with calcium particularly in bone is critically described by Cameron, G. R., J. Path. and Bact., 1930, 33, 929-955. He emphasizes the fact that exposure of tissues and fluids to dust in a city like London is an important source of error.

3. Microincineration yields a mineral residue that contains iron originally both organic and inorganic. Color of the iron oxides, viewed in the dark field, varies according to Policard (C. rend. Acad.d.sc., 1923, 176, 1187) from yellow to deep red. He suggests that perhaps the yellow to brown ash is of organic iron and the red ash is of free iron. See also Marza, V. D., Marza, E., and Chiosa, L. Bull. d'hist. Appliq., 1932, 9, 213. Scott (McClung, p. 758) warns against confusion with carbon.

4. Hydroxyquinoline test (Thomas, J. A. and Lavollay, J., Bull. d'Hist. Appl., 1935, 12, 400-402). Fix in alcohol, trichloracetic acid or neutral formalin. Avoid formol with alkaline water and fixatives containing chromium. Make up reagent by dissolving $2.5 \mathrm{gm}$. 8 -hydroxyquinoline in $4 \mathrm{cc}$. pure acetic acid warming gently. Add quickly aq. dest. to make $100 \mathrm{cc}$. Filter. Wash sections (or smears or cultures) well in neutral aq. dest. Then add few drops of reagent 5-15 min. Pour off reagent. Add to preparation 1 drop $25 \%$ aq. ammonia which produces a ppt. Wash in a stream of neutral aq. dest. If large crystals remain wash more energetically. Stain nuclei with lithium carmine. Examine in neutral aq. dest. or dehydrate in terpinol and mount in vaseline oil. Iron, green black; nuclei, red. Recommended for localization of iron in granules of vitellus, in red blood 
cells, and in connection with microincineration. Said to be better than Prussian Blue reaction for iron.

5. Dinitrosoresorcinol (Humphrey, H. A., Arch. Path., 1935, 20, 256-258). Treat paraffin sections of formalin fixed tissue with $30 \%$ aq. ammonium sulphide, $1 \mathrm{~min}$. Rinse in water and immerse in sat. aq. dinitrosoresorcinol (Eastman) 6-20 hrs. A counterstain can be employed. Humphrey does not say which. $1 \%$ eosin in $50 \%$ al cohol should be satisfactory because the iron containing compounds such as hemosiderin are colored green. Wash, dehydrate, clear and mount.

Intravenous injections of colloidal solutions of iron in rabbits are described by Duhamel, B. G., C. rend. Soc. de Biol., 1919, 82, 724-726.

6. A clinical demonstration of iron in the skin in hemochromatosis involves intradermal injection of equal parts of sterile $0.5 \%$ aq. potassium ferrocyanide and $1 / 100 \mathrm{~N}$ hydrochloric acid. This produces a wheal which turns dark blue in $5 \mathrm{~min}$. A positive reaction can even be obtained after death. (Fishback, H. R., J. Lab. \& Clin. Med. 1939-40, 25, 98-99).

In special cases, as in the analysis of small amounts of epidermis, resort may be had to a quantitative polarographic determination of iron, see Carruthers, C. and Suntzeff, V., J. Nat. Cancer Inst., 1942, 3, 217-220.

Kirk, P. L. and Bentley, J. T., Mikrochemie, 1936, 21, 250-259 advocate a titrimetric method for iron. The difficulty of interference by small amounts of copper has been overcome by Ramsay, W. N. M., Biochem. J., 1944, 38, 467-469. Glick (p. 277) is of the opinion that such methods may be adapted for use with the quantities of material employed in histochemical work.

Iron Hematoxylin of Heidenhain is one of the standard stains. It will give excellent results after almost any good fixation. Zenker's fluid and formalinZenker are suggested. Bring paraffin sections down to aq. dest. Mordant in $5 \%$ aq. iron ammonium sulphate (iron alum, light violet colored crystals, discard the brownish material accompanying them) 12-24 hrs. Rinse quickly in aq. dest. Transfer to $1 \%$ aq. hematoxylin (made up by diluting 1 cc. sat. sol. hematoxylin in abs. alc. with $99 \mathrm{cc}$. aq. dest.) for 12-24 hrs. Differentiate under microscope in $1 \%$ aq. iron alum. Wash thoroughly in tap water. Many counterstains can then be used such as $1 \%$ aq. Bordeaux red, orange G., acid fuchsin, acridine red, or Mucicarmine.
Dehydrate, clear and mount. Nuclei dense blue-black in background of color selected. See Centrosomes, Nuclei, Regaud's Method for mitochondria.

1. Koneff, A. A., Anat. Rec., 1936, $66,173-179$ advises use with anilin blue. Mordant sections $5-10 \mathrm{~min}$. in $5 \%$ aq. iron ammonium sulphate. Rinse quickly in aq. dest., stain 3-15 min. in Harris' hematoxylin. Rinse again in aq. dest. and stain in: anilin blue (Grübler) $0.1 \mathrm{gm}$; ; oxalic acid, $2 \mathrm{gm}$.; phosphomolybdic acid, $15 \mathrm{gm}$. and aq. dest. $300 \mathrm{cc}$. Wash in aq. dest., differentiate in alcohol, dehydrate ( 2 changes of absolute), clear in xylol and mount in balsam. If euperal is used for mounting omit the xylol. Nuclei, violet-brown; cytoplasm, light brown; erythrocytes, dark violet; myelin and muscle brown; elastic fibers, reddish brown to red.

2. Lillie, R. D. and Earle, W. R., Am. J. Path., 1939, 15, 765-770 recom: mend employment of a hematoxylin containing ferric and ferrous iron: (A). Ferric ammonium sulphate, violet crystals, $15 \mathrm{gm}$.; ferrous sulphate, $15 \mathrm{gm}$.; aq. dest., 100 cc. (B). Hematoxylin, 1 gm.; $95 \%$ alcohol, 50 cc., glycerin, C.P., 50 cc. Mix $A$ and $B$ in equal quantities before using. For best general discussion of iron hematoxylin, see Lillie, p. 58.

Iron Hematoxylin Single Stain-Written by Morris Goldman, Dept. of Parasitology, School of Hygiene and Public Health, Johns Hopkins University, Baltimore. January 29, 1951-This stain is useful for the purpose of diagnosing intestinal protozoa occurring in fecal smears. It is not intended to replace the longer and more precise iron hematoxylin methods. The stain is prepared from the following relatively stable solutions: Solution A: $1 \%$ hematoxylin in $95 \%$ alc. (best prepared from a stock $10 \%$ alc. solution of hematoxylin). Solution B: $\mathrm{NH}_{4} \mathrm{Fe}$ $\left(\mathrm{SO}_{4}\right)_{2} \cdot 12 \mathrm{H}_{2} \mathrm{O}, 4.0 \mathrm{gms}$., glacial acetic acid, $1.0 \mathrm{ml}$., concentrated $\mathrm{H}_{2} \mathrm{SO}_{4}, 0.12$ $\mathrm{ml}$., and aq. dest. to make $100 \mathrm{ml}$. Mix equal parts of Solution A and B, filter after several hours and use. Staining time varies from $30 \mathrm{sec}$. when stain is fresh to $3 \mathrm{~min}$. after $24 \mathrm{hrs}$. Discard stain after 3 days. Fecal smears are fixed in Schaudinn's, passed through iodine alcohol to $50 \%$ alcohol, stained, washed in running tap water 5 minutes, dehydrated and mounted. The stock solutions used in this technique may also be used in the Heidenhain iron hematoxylin procedure.

Iron Pigments, see Berlin and Turnbull blue reactions.

Iron, Radioactive. See Erythrocytes.

Isamine Blue is described by Conn (p. 137) 
as a sulfonated naphthyl-rosanilin or naphthyl-pararosanilin. He questions the synonym (alkali blue $\mathrm{XG}$ ) given in the Colour Index. This acid has been much used as a Vital Stain in European laboratories. It is not made in the United States.

Islets of Langerhans of the pancreas. Thele are many techniques for the study of these cellular masses.

1. To study in the living state the method employed by O'Leary, J. L., Anat. Rec., $1930,45,27-58$ is recommended. It consists essentially of partly withdrawing the pancreas from a mouse and of mounting it in such a way that a thin film of tissue can be closely examined with circulation still active. The islet cells can be studied with oil immersion lenses and the changes in them on the injection of insulin noted.

2. To obtain an idea of the distribution, number and size of the islets supravital staining with Neutral Red or Janus Green is indicated, which see.

3. To stain the cell types specifically Neutral Gentian and other stains advised by Lane, Bensley and their followers are available. The Azan Stain suggested by Bloom, W., Anat. Rec., $1931,49,363-371$ (see his beautifully colored plate), has been further investigated by Gomori, G., Anat. Rec., 1939, 74, 439-459 whose technique abbreviated is as follows: Fix thin slices of pancreas in Bouin's fluid 8-10 hrs. Wash in aq. dest. Imbed in paraffin and cut $4 \mu$ sections. Stain $45-60 \mathrm{~min}$. at $56^{\circ} \mathrm{C}$. in azocarmine. (To make dissolve $0.1 \%$ azocarmine in aq. dest. Boil about 5 min. Cool and add $1.0 \mathrm{cc}$. glacial acetic acid to each $50 \mathrm{cc}$. solution. Before use filter at $60^{\circ} \mathrm{C}$. Stain will keep for months.) Rinse quickly in aq. dest. and blot. Destain in $90 \%$ al cohol containing $1 \%$ aniline oil until acinous tissue is al. most wholly decolorized and B cells show red against pink background of $\mathrm{A}$ cells. Rinse briefly and treat with $5 \%$ aq. iron alum for $5 \mathrm{~min}$. or more. Rinse again and stain $2-20 \mathrm{~min}$. in the usual mixture (anilin blue, $0.5 \mathrm{gm}$.; orange $\mathrm{G}, 2.0 \mathrm{gm}$.; + aq. dest. to make $100 \mathrm{cc}$.) diluted with 2-3 times its volume of aq. dest. until under the microscope collagenic tissue becomes decp blue. Rinse and blot. Differentiate and dehydrate in absolute alcohol, clear in xylol and mount in balsam. Cytoplasm of A cells rich orange yellow, of $B$ cells fiery red and of $D$ cells sky blue. The author states that by first staining with Bensley's neutral gentian, decolorizing and restaining by above Azan method it can be seen that there is no gradation between $A$ and $B$ cells.

Isoelectric Points of ccllular structures. Methods for their determination at con. trolled pH's by intensity of staining have been critically evaluated by Levine, N. D., Stain Techn., 1910, 15, 91-112. His conclusion is that no true isoelectric points have yet been established for nucleus, cytoplasm or other tissue elements by these techuiques. See reticulo-endothelial cells (Fautrez, J., Bull. d'Hist. Appl., 1936, 13, 202-206). Isohematein, as a biological stain (Cole, E. C., Stain Techn., 1931, 6, 93-96). Greater tinctorial power than hematoxylin but less selective.

Isomerase with Aldolase are known as Zymohexase.

Isopentane recommended by Hoerr, N. L., Anat. Rec., 1936, 65, 293 for freezing in the Altmann-Gersh technique.

Isopropanal in combination as a new fixative for animal tissues which also dehydrates (Cleverdon, M. A., Science, 1943, 97, 168). Isopropanal, 55 cc.; picric acid, 5 gms., acetone, 30 ce.; glacial acetic acid, 55 cc.; formalin ( $40 \%$ formaldehyde CP), 5 cc. Fix 2 hrs. -4 days depending on size. Store in $70 \%$ isopropanal or imbed in paraffin after first washing in 2 changes nearly absolute isopropanal. Remove picric acid from mounted sections just before staining with $1.5 \%$ ammonium hydroxide in $95 \%$ alcohol.

Isopropyl Alcohol. Has been recommended as a substitute for ethyl alcohol since it mixes with water and xylol. It is said to be less hardening than ethyl alcohol (Bradbury, O. C., Science, 1931, 74, 225) but it is more expensive. See Herman, C. M., J. Lab. \& Clin. Med., 1941, 26, 1788 .

Isorubin, see New Fuchsin.

Iso-Safrol is obviously an isomer of safrol which is given as 3,4-methylene-dioxyallylbenzene in the Merck Index. Isosafrole is listed among Eastman's organic chemicals. It is sometimes recommended as a partly dehydrating and clearing agent (Silver Citrate injection of blood vessels, etc.) but in all likelihood other clearing agents can be used as substitutes.

Isospora, see Coccidia.

Jacobson's Organ, innervation, Bellairs, A., J. Anat., 1942, 76, 167-177.

Jalowy modification of Hortega method for the skin (Jalowy, B., Zeit. f. Zellf. u. Mikr. Anat., 1937, 27, 667-690). To make reagent wash ppt., formed by adding 20 drops $40 \%$ aq. $\mathrm{NaOH}$ to $20 \mathrm{cc}$. $10 \%$ aq. silver nitrate, 10 times with aq. dest. Suspend ppt. in 20 cc.aq. dest. Add ammonia drop by drop till it dis- 
solves. Add 100 cc. aq. dest. and store in dark. Deparaffinize sections of tissue fixed 1-2 days in neutral formalin. Treat with above reagent 5-30 min. at $30^{\circ} \mathrm{C}$. Rinse in aq. dest. and in ammonia water. After treating with 1 part neutral formalin to 4 of aq. dest. wash in running water, dehydrate, clear and mount in balsam. Collagen, yellow to brownish yellow; reticular fibers, black.

Janssen's Iron Hematoxylin recommended in place of Weigert's acid iron chloride, hematoxylin (Lillie, R.D. and Earle W.R. Stain Technol., 1939, 14, 53-54).

Janus Blue can be used in exactly the same ways as Janus green and with equal success.

Janus Dyes. Named after the God, Janus with two faces since they of ten exhibit two colors. Their chemistry and use in histology is described by Cowdry, E. V. Contrib. to Embryol., Carnegie Inst. Washington, 1918, No. 25, pp. 39-148.

Janus green (formerly made by Grubler) is safraninazodimethylanilinchloride. This is useless for staining mitochondria.

Janus green $C$ (Hoechst) is dimethyl saf raninazodimethyl anilinchloride. This likewise is useless for mitochondria.

Janus green $B$ (Hoechst) is diethylsafraninazodimethylanilinchloride. This is the most specific stain for mitochondria and is now supplied by many companies both as Janus Green B and simply as Janus Green.

Janus blue $G$ and $R$ (Hoechst) is diethylsafranin-B-naphthol and stains mitochondria as well as Janus Green B. The marks $\mathrm{G}$ and $\mathrm{R}$ indicate differences in method of manufacture not different dyes.

Janus black D, I, II and $O$ (Hoechst), of these Janus Black $I$ is a mixture of two substances Janus green B and a brown dye. It colors mitochondria by virtue of the former.

Janus gray $B, B B$ (Hoechst) are also safranin derivatives but useless for mitochondria.

Janus yellow $G, R$, (Hoechst) likewise safranin derivatives and no good for mitochondria.

Diethylsafranin is a reduction product of Janus green B. It is a red dye which colors mitochondria specifically but not very strongly.

Janus Green B (Diazingrün) is diethyl safraninazodimethylanilinchloride. Janus green now sold without the qualification $B$ is usually the same substance because it has become well known that the dye required must have the composition indicated. Owing to its toxicity Janus green cannot be injected into living animals like trypan blue and other "vital" stains. It is employed as a supravital stain by simply immersing tissues in it or better by its injection into the vessels of a freshly killed animal the individual cells of which remain for some time alive. Janus green is the best supravital stain for mitochondria. Janus green is also very useful for staining the islets of Langerhans of the pancreas and the renal glomeruli of the kidney when injected intravascularly, see Neutral Red. Both islets and glomeruli are colored deep bluish green against a background at first colorless, or faintly green, and changing to pink by reduction of the dye to diethylsafranin. This permits the counting of islets and glomeruli in pieces of tissue mounted in salt solution and observed at low magnification. When the oxygen is further consumed by the cells the dye is reduced to a second colorless leucobase. It is therefore an oxidation-reduction indicator as well as a specific stain for mitochondria. See Neutral Red-Janus Green stain.

Janus Red B (CI, 266), a basic disazo dye of light fastness 4. Action on parenchyma described (Emig, p. 36).

Jaws, see Teeth and

Jenner-Giemsa method of Pappenheim (see May-Giemsa).

Jenner's Stain for Leishmania as described by Craig, p. 146: To make, mix equal parts $1.2 \%$ water soluble eosin (Grubler or NAC) in acid free aq. dest. and $1 \%$ aq. medicinal methylene blue in a flask. Shake thoroughly and let stand at room temperature $24 \mathrm{hrs}$. Collect ppt. on small filter paper and wash with aq. dest. till filtrate is almost colorless. Dry ppt. and store in dark at room temperature. Dissolve $0.5 \mathrm{gm}$. ppt. in 100 cc. pure methyl alcohol (Merck's Reagent). Cover smears with this 1-2 min. Then add aq. dest. drop by drop till metallic sheen forms on surface. Leave 5-15 min. longer as desired for intensity. Method said by Craig to be less reliable than Giemsa, Leishman or Wright techniques.

Johnson's Neutral Red stain for Nissl bodies (Addison in McClung, p. 450). Ripen $1 \%$ aq. neutral red $1-4$ years. Dilute to $0.25-0.5 \%$ before using. Differentiate and dehydrate in the usual way. Clear in 1 part xylol $+2-3$ parts castor oil. Gives good results in thick sections $(50 \mu)$ and can be employed after silver methods on tissues fixed in alcohol or formalin.

Kirkman, I. J., Anat. Rec., 1932, 51, 323-326 used the following unripened stain after Bouin and formalin fixatives: neutral red (Coleman \& Bell), 1 gm.; aq . dest., 500 cc., $1 \%$ aq. glacial acetic acid, 
2 cc. $10-20 \mathrm{~min}$. is sufficient for counterstaining Weigert-Pal preparations. Then rinse in aq. dest., differentiate in $95 \%$ alcohol, dehydrate in absolute, clear and mount.

Joints. Meniscus (Raszela, F., Bull. d'Hist. Appl., 1938, 15, 186-210).

Jolly Bodies. These structures are nuclear remuants in erythrocytes that are strongly colored by Giemsa's Stain.

Jores' Solution, see under Color Preservation of gross specimens.

Kabunylin, a dye extracted from bectroot. Said to be good for use with picrofuchsin (Fuse and Hino, Arb. Anat. Inst. zu Sendai, 1937, 20, 111-113).

Kaiserling's Solution, see under Color Preservation of gross specimens.

Kallichrom, a combination of cresyl violet and auramin recommended for both plant and animal tissues (Kisser, J., Mikr. f. Naturfreunde, 1931, 9, 95).

Kardos-Pappenheim modification of Giemsa's stain (Kardos, E., Folia haematol., Archiv., 1911, 12, 39). To make the methyl green-orange stain mix $2 \%$ aq. orange $\mathrm{G}$. with concentrated aq. methyl green. Filter, dry the ppt. and dissolve in methyl alcohol. Shake well together 5 drops methyl green-orange, 10 drops of Giemsa's stock solution and 15 cc. aq. dest. The fluid under the foam is used for staining. First fix and stain the blood smear with May-Grünwald mixture 3 minutes; add equal volume aq. dest., 1 minute; pour off and add the methyl green-orange 15 minutes; wash quickly in water and blot dry.

Karo, white corn syrup (Corn Products Co.) is a useful medium for mounting whole insects because they can be transferred to it directly from water or weak alcohol and clearing is unnecessary (Patrick, R., Science, 1936, 33, 85-86).

Karotin, see Carotin.

Karyosome (G. Karyon nut, nucleus + soma, body). A basic staining or chromatin-nucleolus, in contrast to a plasmosome, generally more numerous, smaller and of less regular shape of ten called a net-knot.

Kerasin is a Cerebroside.

Keratin, a scleroprotein contained in hair, nails, horns, epidermis, etc. There are apparently two sorts. Their chemistry is discussed by Giroud, A., Bulliard, H. and Lebond, C. P., Bull. d'Hist. Appl., $1934,11,365-373$. See Orange II, Oral Mucosa.

Keratohyalin Granules, separation and analysis-Written by Donald L. Opdyke, Dept. of Anatomy, Washington University Medical School, St. Louis, Mo. November 8, 1951-Highly refractile cytoplasmic granules of varying size found in the cells of the stratum granulosum of stratified squamous epithelia. They were first described by Langerhans in 1882 and were called "keratohyalin" by Waldeyer who ascribed to them a constant relation to the process of cornification.

The tinctorial properties of these granules may be summarized as follows: They stain beautifully with picrocarmine, have an intense affinity for all of the hematoxylin stains and stain as well with unoxidized hematoxylin as with the aged stain. They stain metachromatically with toluidine bluc, and after staining with crystal violet and iodine they resist destaining by acetone. They do not stain by the Feulgen procedure but give beautifully intense reactions to the Bauer, Iodine, and Best's Carmine technics for glycogen. The reactions with the P.A.S. method of McManus give varying results. The granules are not tannophil; do not osmicate or stain with the Sudan dyes. They are argentophil.

Microincineration studies reveal large concentrations of mineral in the granules as demonstrated by quantities of the white ash typical of calcium and/or magnesium. These ashed sections, as well as unashed sections show intense reactions of the granules to the gallamine blue staining method of Stock, A., J. Roy. Micr. Soc., Series III, $1949,69,20-24$.

Enzyme studies have resulted in disagreement in the literature with respect to ribonuclease digestibility, but the granules are definitely not totally digestible in ribonuclease, hyaluronidase, crystalline trypsin or salivary enzymes. They are readily dissolved by elastase.

The most striking features of these granules, both tinctorially and histochemically, are their similarities to elastic fibers: These common features include their tinctorial reactions to Verhoeff's procedure, congo red, and orcein; their affinity for mineralization with calcium salts; their solubility in the elastolytic enzyme of Balo and Banga (See Elastic Fibers).

References for the above data are: Lansing, A. I., and Opdyke, D. L., Anat. Rec., 1950, 107, 379-398; Smith, C., and Parkhurst, H. T., Anat. Rec., $1949,103,649-674 ;$ and unpublished work of D. L. Opdyke.

The origin of these granules is much debated. Stam, Von F. C., Acta Dermato-Venereol., 1951, 31, 407-411 bclieves them to be extruded nucleoli. Favre of the Bacteriological Institute of Lyon is of the opinion that they are formed by the spiral shaped mitochondria of the basal layer of the epi- 
dermis (Favre, M. Ann. de Dermat. et Syph., 1950, 10 (3), 241-262). The literature 1886 to date includes reports of the origin of keratohyalin granules from nuclear substance, intercellular fibrils, and cytoplasm.

The particulate nature of the granules has been demonstrated recently by their isolation from epidermis (D. L. Opdyke). Epidermis is separated by the heat method of Baumberger, J. P., Suntzeff, V., and Cowdry, E. V., J. Nat. Cancer Inst., 1942, 2, 413-423. The sole of the foot offers an ideal place to obtain keratohyalin granules.

After separating the epidermis from the dermal layers, the proximal side of the epidermal sheet is scraped with a knife, removing all of the epidermis in fine shavings down to the highly transparent, thick stratum corneum. The shavings are then homogenized in a Waring Blendor until no cells remain intact. This homogenate is made in a $0.85 \% \mathrm{NaCl}$ solution of $\mathrm{pH} 7.3$ to which a crystal of thymol is added to minimize bacterial contamination. All procedures are carried out in a cold room. The process of cell fractionation in the micro cup of the Waring Blendor requires about $45 \mathrm{~min}$. If $2 \%$ citric acid is employed as the medium, this time may be reduced to $15 \mathrm{~min}$.

The components of the suspension are then separated by differential centrifugation. Nuclei and whole cells are found to sediment at 2.5 to $3 \mathrm{Kg} / \mathrm{g}$. The keratohyalin granules sediment at $25.2 \mathrm{Kg} / \mathrm{g}$. after $12 \mathrm{~min}$. of centrifugation. These can be resuspended in fresh saline, washed, and reprecipitated. They can be distinguished from mitochondria by their tinctorial properties.

Kermes. This scarlet dye was known in Egypt and farther East at a very early date. Kermes is the Armenian term for a "little worm", variously identified as Coccus arborum and Coccus ilicis. Moses referred to it as "Fola" and "Fola shami". Remember the promise of Jehovah: "Though your sins be as scarlet (Fola) they shall be as white as snow; though they be red as crimson (Fola shami), they shall be as wool". So valuable was Kermes that after the subjugation of Spain by the Romans the people were made to pay half of the tribute in Kermes. At about 1640 a Dutch chemist discovered the similarity of this dye to cochineal. Its history affords interesting reading (Leggett, W. F. Ancient and Medieval Dyes. Brooklyn: Chemical Publishing Co. Inc., 1944, 95 pp.).

Kidney-Written by Jean Oliver, Dept. of
Pathology, State University of New York, Brooklyn 2, N. Y., September 4, 1951-Techniques for the general demonstration of the elements of the renal tissue, epithelial cells, sustaining tissues, blood vessels and nerves are essentially the same as those used for other organs. Masson's Trichrome stain has the advantage of affording a particularly colorful differentiation of the various elements in a single section.

The individual renal organs that make up the kindey, the nephrons, can be isolated in their entirety by maceration and teasing as described by Huber, G. C., Cowdry's Special Cytology, 1932, 2, 935-977. Partly wash out blood by injecting physiological saline into the renal artery. Then follow with hydrochloric acid (conc. $\mathrm{HCl}, 3$ parts and aq. dest. 1 part) using care to protect the eyes. Remove and immerse the organ in the same fluid. After a suitable time, determined by excising pieces, wash a block of tissue with aq. dest. and stain in Hcmolum. Wash in very dilute aq. sodium hydrate. Isolate individual tubules by teasing with fine needles. Wash, and mount in glycerin. With small mammals Huber's results were excellent but he was not satisfied with his human preparations. The method has however been well adjusted to the human kidney by Oliver, J., Architecture of the Kidney in Chronic Bright's Disease, New York: Paul B. Hoeber, 1939 , by a simpler procedure and dis: sected nephrons may be mounted, stained and photographed. A method of montage then affords a demonstration in their natural continuity of the cellular elements of the nephron at high magnification (J. Clin. Invest. 1951, in press).

A clear distinction between glomeruli and the renal tubules is important. It is a simple matter to color the former with 1:5000 Janus blue (which is more satisfactory for this purpose than Janus green) in $0.85 \%$ aq. sodium chloride by vascular Perfusion and to determine their number, size and distribution against a background of unstained or faintly rose tinged tubules in slices of fresh kidney (Cowdry, E. V., Contrib. to Embryol. Carnegie Inst., Washington, 1918, 8, 39-160).

Perhaps in no other organ is it possible to correlate morphological structure with functional activity so closely and by so many methods as in the kidney. See Oliver, J., Am. J. Med., $1950,9,88$ for a general statement of the problem with various examples of such procedures. As an example, the technique for the microscopic study in 
vivo of the surface of the guinea pig's kidney, and for the marking of single tubules with India ink for their later isolation by maceration, is given by Walker, A. M. and Oliver, J., Am. J. Physiol., 1941, 134, 562-595. The micro collection of fluid from single tubules is as the authors state a direct continuation of the researches of A. N. Richards. See Oliver, J., Harvey Lectures, 1944-45, 40, 102-155.

Vital staining of kidney tubules is a valuable method for "seeing" functional activity in morphological form. It is usually carried out by techniques not requiring special adaptation. See Vital Staining. The procedure employed by Oliver, J., Bloom, F. and MacDowell, M., J. Exp. Med., 1941, 73, 141-160 deserves mention because it gives a clear demonstration that the cells of abnormal proximal convoluted tubules can be marked by their inability to concentrate trypan blue which consequently stains the tubule wall diffusely. This is illustrated in colors. Microscopic observations, having a close relation to function, are easily made on the kidneys of lower forms. See the account of contractility of the ciliated necks of renal tubules in Necturus by Lucas, A. M. and White, H. L., Anat. Rec., 1933, 57, 7-11. The functional handling of fluorescent dyes by the amphibian kidney may be observed microscopically in vivo by means of ultraviolet light (Ellinger, P., Quart. J. Exp. Physiol., 1940, 30, 255, and Singer, E., Anat. Rec., 1936, 66, 343). The amphibian kidney also lends itself to the application of the "extra-vital" method where the functional activities (secretion of dyes) of the isolated perfused kidney are correlated with the structural aspect of the process by subsequent histological examination (Oliver, J. and Smith, P., J. Exp. Med., 1931, 53, 785; Oliver, J. and Lund, E. A., Ibid, 1933, 57, 435).

The study of the renal tubules present in tissue cultures is useful in the study of function. Thus Chambers, R. and Cameron, G., Radiology, 1941, 37, 186-193 have found that susceptibility to $\mathrm{x}$-rays is increased when a secretory stimulant is added but that in cultures it is distinctly less than in vivo. See references accompanying this paper.

A method has been devised by Crabtree, C. E., Endocrinology, 1941, 29, 197-203 of measuring by a differential count the number of Bowman's capsules made of cuboidal as contrasted with squamous cells. The count appears to provide an index of age and sex variations in normal mice and of the in- fluence of testosterone propionate on castrated mice. The glomeruli may also be counted in aliquot portions of macerated renal cortex and decreases in their number correlated with the abnormal conditions of disease or senility (Aratabi, M., Am. J. Anat., 1926, 36, 399).

Methods for estimating the distribution of enzymes in the tissue components of the rabbit's kidney are given by Weil, L., and Jennings, R. K., J. Biol. Chem., 1941, 139, 421-432. They depend on topographic correlation between distribution of cell types in $15 \mu$ frozen sections and decomposition of substrates. The techniques are capable of demonstrating catheptic, aminopolypeptidase and esterase activities in all of the epithelial components and of showing that the cells of the proximal and distal convoluted tubules are about twice as active enzymatically as those of the ascending and descending loops of Henle and about 4 times as active as the cells of the collecting tubules. Amylase and dipeptidase activities can also be localized and expressed quantitatively in relative terms.

For the application to proximal convoluted tubules in phlorizin glycuresis of the Kabat and Furth procedure for alkaline phosphatase see Kritzler, R. A. and Gutman, A. B., Am. J. Physiol., 1941, 134, 94-101. See Phosphatase. Lipase may also be shown by the procedure of Gomori, G., Arch. Path., $1946,41,121$.

Techniques capable of revealing very interesting data on the shape of cells of the proximal tubule have been devised and employed by Foote, J. J., and Grafflin, A. L., Am. J. Anat., 1942, 70, 1-20. They can probably be employed to advantage in different functional states and to other than renal cells.

Methods have been elaborated for measurement of the renal filtration surface and data have been supplied for the albino rat (Kirkman, H. and Stowell, R. E., Anat. Rec., 1942, 82, 373-389). The original paper should be consulted. See also the measurements of glomerular number and size in various species by Rytand, D., Am. J. Anat., 1938, 62, 507. A valuable collection of measurements of renal structures will be found in v. MöllendorfHandbuch d. mikr. Anat., 1930, VII-1, $20-34$.

$\mathrm{pH}$ determinations can be made as described by Emmel, V. M., Anat. Rec., $1940,78,361-377$ by means of a capillary glass electrode (Voegtlin, C. and Kahler, H., Science, 1932, 75, 362) and a vacuum tube potentiometer (Hill, 
S. E., Science, 1931, 73, 529). It is significant that increase in activity of the renal cortex immediately follows ligation of the renal artery and that the mitochondria respond by enspherulation and fragmentation within $6 \mathrm{~min}$ utes. The kidney is an organ in which mitochondria must be examined with the utmost promptness. A delay in fixation of 15 minutes at room temperature is sufficient to cause disturbances in the mitochondrial rodlets of the proximal convolution. Material from human autopsies is therefore of questionable value. Fuller, R. H., Arch. Path., 1941, 32, 556-568 could find no relation in a rather large number of cases studied between age, hours postmortem and cause of death (except renal disease) and quantity and distribution of stainable lipoid.

The interstitial framework of the kidney in both normal and pathological conditions is well shown by the silver methods that impregnate "reticular" fibers (q.v.). The "polysaccharide" content of the interstitial tissue may be examined by the use of periodic acid (McManus, J. F. A., Amer. J. Path., $1948,24,643$. See also, Ritter, H. B. and Oleson, J. J., Amer. J. Path., 1950, 26, 639 .

The blood vessels of kidney may be injected with Neoprene (q.v.) and also with radio-opaque suspensions for a radiographic demonstration of microarteriography (Barclay, A. J., Amer. J. Roent., 1948, 60, 1). For a demonstration of the functional status of the renal circulation, the fluorescent dye Vasoflavine may be injected into the living animal and the kidney removed and viewed with ultraviolet light Moses, J. B., Emery, A. J., and Schlegel, J. V., Proc. Soc. Exp. Biol. and Med., 1951, 77, 233).

The cytoplasmic particulates of the renal epithelium, (mitochondria, microsomes and droplets of absorbed protein) can be isolated in sucrose suspensions and examined by standard biochemical procedures. Cf. Oliver, J., J. Mt. Sinai Hospital, 1948, 15, 175.

King's Carb ol-Thionin stain for Nissl bodies (Addison in McClung, p. 450). Stain paraffin or celloidin sections, 2-3 min., in sat. thionin in $1 \%$ aq. carbolic acid. Then wash quickly in aq. dest., differentiate in 95\% alcohol. Pass through equal parts absolute alcohol and chloroform to xylol and mount in balsam.

Kinney's Method for staining reticulum (Kinney, E. M., Arch. Path., 1928, 5, 283). Fix $18 \mathrm{hrs}$. in $1 \mathrm{gm}$. sodium sulphantimonate dissolved in $100 \mathrm{cc} .4 \%$ formalin immediately before using.
Imbed in paraffin, but more than 1 or 2 hrs. in xylol or cedar oil will remove the dark brown stain from the reticulum. Hematoxylin is contraindicated as counterstain because it obscures the color of the reticulum. Other ordinary counterstains can be used. This method works well even with autopsy material. It is recommended particularly for kidney and pancreas. Results are sometimes patchy in the spleen.

Kleinenberg's fixative. Saturated picric acid in $2 \%$ aq. sulphuric acid. Embryos and marine organisms.

Knee-Joint, method for investigation therein of radioactive gold (Ekholm, R., Acta Anat., 1951, Suppl. 15, 11, 75 pp.).

Knisely, see Quartz Rod Technique.

Kolatchew Fluid, see Golgi Apparatus.

Korff's Fibers of dentin, see Teeth, Developing.

Kossa, see his test for Calcium.

Krajian's Congo Stain. Elastic fibers (Krajian, A. A., Arch. Path., 1934, 18, 378380 ). Fix in $10 \%$ formalin, 24 hrs. or more. Cut frozen sections. Wash them in tap water. Place in $2 \%$ aq. aluminum chloride $5-10 \mathrm{~min}$. Wash and stain $10 \mathrm{~min}$. in $8 \mathrm{cc} .4 \%$ Congo red in $5 \%$ aq. sodium citrate +2 cc. glycerin C.P. After washing in tap water transfer to $1 \%$ aq. KI for 10 sec. agitate. After again washing in tap water, stain 5-10 min. in : anilin blue, $1.5 \mathrm{gm}$.; orange G, 2.5 gm.; resorcinol, 3 gm.; phosphomolybdic acid, 1 gm.; aq. dest., 100 . Wash carefully in tap water. Blot sections on slides. Dehydrate in absolute alcohol 2 min.; clear in origanum oil; pass through xylol to balsam. Elastic fibers bright red, fibrin dark blue.

Krause's End-Bulbs. Methylene blue demonstration of in skin of forearm (Weddell, G., J. Anat., 1940-41, 75, 346-367). See Skin.

Krause's Membrane. Special technique for, see Dahlgren (McClung, p. 427).

Kronig's Cement is recommended by Bensleys (p. 41) for ringing preparations mounted in glycerin jelly or glycerin : 7-9 parts colophonium (resin) melted and stirred with 2 parts beeswax.

Kuff, see Nucleic Acid Dye Interactions.

Kurloff Bodies are cytoplasmic inclusions which frequently occur in the non-granular leucocytes of guinea pigs. They show particularly well in smears of the spleen, may attain a size equal to that of the nucleus and can be brilliantly colored supravitally by $1: 2000$ brilliant cresyl blue in physiological salt solution (Cowdry, E. V. chapter in Rivers'book on Viruses, Baltimore, Williams \& Wil. kins, 1928, p. 141). 
Kultschitzky's Hematoxylin is $1 \mathrm{gm}$. hematoxylin dissolved in a lit tle alcohol made up to $100 \mathrm{cc}$. with $2 \%$ aq. acetic acid (Lee, p. 526).

Lac, a crimson dyc obtained from resinous incrustation caused by the insect, Coccus lacca, of Siam, Indo-China and Southern India. This dye, introduced into England about 1790 A.D., became an important article of commerce in competition with cochineal of Mexican origin, but before long proved inferior to cochineal and was no longer imported. The crimson dyes, Kermes, cochineal and lac have played important parts in the history of civilization (Leggett, W. F., Ancient and Medieval Dyes. Brooklyn: Chemical Publishing Co., Inc., 1944, 95 pp.)

Lacmoid, an indicator similar to Resorcin Blue.

Lacteals, see Lymphatic Vessels.

Lactoflavin, see Vitamin $\mathbf{B}_{2}$.

Lactophenol, a fixative for Bilharzial Cercariae. See Lactophenol-cotton blue technique under Fungi.

Laidlaw's Methods. 1. For inclusion bodies (quoted from Pappenheimer, A. W. and Hawthorne, J. J., Am. J. Path., 1936, $12,625-633$, see colored figure, who used it for cytoplasmic inclusions in liver cells). Fix in sat. aq. corrosive sublimate $100 \mathrm{cc} .+5 \%$ glacial acetic acid or in Zenker's fluid without acetic. Imbed in paraffin, cut sections $3 \mu$. Remove paraffin and pass down to water. Weigert's iron hematoxylin (2\%) $5 \mathrm{~min}$. Differentiate in $0.5 \%$ acid alcohol. Rinse in tap water, then aq. dest. $1 \%$ aq. acid fuchsin 5-15 min. Rinse in aq. dest. Mordant in 1\% phosphomolybdic acid 30 sec. Rinse in aq. dest. Differentiate in $0.25 \%$ orange $\mathrm{G}$ in $70 \%$ alc. Dehydrate, clear and mount in balsam.

2. For silver staining of skin and tumors (Laidlaw, G. F., Am. J. Path., 1929, 5, 239-247). Fix in Bouin's fluid or in $10 \%$ neutral formalin for 3 days. (To make the Bouin's fluid he uses, add 100 cc. commercial formalin and $20 \mathrm{cc}$. glacial acetic acid to $300 \mathrm{cc}$. tap water and saturate with picric acid). Fix paraffin sections to slides by Masson's Gelatin Glue. Wash Bouin sections for $20 \mathrm{~min}$. in running water, and formalin ones for $5 \mathrm{~min} .1 \%$ alc. iodine, $3 \mathrm{~min}$., rinse in tap water. $5 \%$ aq. hypo (sodium thiosulphate), 3 min., rinse in tap water. $\frac{1}{2} \%$ aq. potassium permanganate $3 \mathrm{~min}$., rinse in tap water, $5 \%$ oxalic acid, $5 \mathrm{~min}$. Wash in running water, $10 \mathrm{~min}$. Aq. dest. 3 changes in 5-10 min. to clean before adding silver. Heat stock Lithium Silver solution to $50^{\circ} \mathrm{C}$. and stain in oven for $5 \mathrm{~min}$. Pour aq. dest. over both sides of slides. Flood sections frequently for $3 \mathrm{~min}$. with $1 \%$ formalin in tap water. Again rinse both sides of slides with aq. dest. 1:500 yellow gold chloride in aq. dest. in Coplin jar at room temperature, 10 min. Rinse both sides with aq. dest. Pour on $5 \%$ oxalic acid $10 \mathrm{~min}$. Rinse in aq. dest. Pour on $5 \%$ hypo changing as often as it becomes turbid, $10 \mathrm{~min}$. Wash in running water. Counterstain if desired. $\mathrm{De}$ hydrate, clear and mount in usual way. Reticulum, black threads; collagen reddish purple.

Lake Ponceau, see Ponceau 2R.

Lampblack. A colloidal suspension of lampblack is an excellent substance to inject intravenously to demonstrate phagocytosis, especially by monocytes. McJunkin, F. A., Arch. Int. Med., 1918, $21,59-64$, advised adding $0.4 \mathrm{gm}$. of carefully pulverized lampblack to $100 \mathrm{cc}$. $2 \%$ gelatin in aq. dest. Inject intravenously with 5-9 cc. $10 \%$ aq. sodium citrate, as in the case of Higgins' Ink. The method has been slightly modified by Simpson, M. J., J. Med. Res.,1922, 43, 77-144; Wislocki, G. B., Am. J. Anat., 1924, 32, 423-445; and Lang, F.J., Arch. Path., 1926, 1, 41-63.

Lanacyl Blue BB (CI, 210), an acid monoazo dye which colors cell walls and parenchymatous cells light blue but less well than other blue acid dyes (Emig, p. 35).

Lanacyl Violet B (CI, 207), an acid monoazo dye of light fastness 3 . Directions for staining plant tissue and fungous mycelia (Emig, p. 35).

Langerhans, see Islets of .

Lard, reactions in tissue to fat stains after various fixations (Black, C. E., J. Lab. \& Clin. Med., 1937-38, 23, 1027-1036).

Large Intestine. The conditions that influence the appearance of sections are easier to guard against than in the Small Intestine because of the absence of villi and greater uniformity of contents. The pronounced influence of degree of distention is described and well illustrated by Johnson (F. P., Am. J. Anat., 1912-13, 14, 235-250).

Lansing, see Collagen, Elastin.

Latex-Cast Techniques for study of the circulation have been applied to the spleen by Gall, D. and Maegraith, M. G., Ann. Trop. Med. \& Parasit., $1950,44,331-338$, who give good illustrations of results and references to previous work.

Lauth's Violet, see Thionin.

Lead, histological demonstration.

1. Mallory and Parker's method (Mallory, F. B. and Parker, F. J., Am. J. Path., 1939, 15, 517-522): Fix tissues in 95 or abs. alcohol (not formalin). Stain celloidin sections at $54^{\circ} \mathrm{C}$. in : 5-10 gm. 
hematoxylin dissolved in few drops abs. or $95 \%$ alcohol +10 cc. freshly filtered $2 \%$ aq. $\mathrm{K}_{2} \mathrm{HPO}_{4}$ for 2-3 hrs. Wash changing tap water $10-60$ min., dehydrate in $95 \%$ alc., clear in terpineol and mount in terpineol balsam. Lead light to grayish blue, nuclei deep blue. Another method applicable to paraffin sections of Zenker fixed material is to stain in $0.1 \%$ methylene blue in $20 \%$ alc. $10-20 \mathrm{~min}$. Differentiate 10-20 min. in $95 \%$ alc., dehydrate, clear and mount. Phloxine is recommended as a contrast stain before the methylene blue.

2. Chromate method (Frankenberger, Cretin). By simply fixing in Regaud's Fluid lead is precipitated as insoluble yellow lead chromate easily identifiable microscopically. This method is strongly advised by Lison (p. 101). It has been used by Truc (E., Bull. d'Hist. Appl., 1929, 6, 393-399). See Sieber (E., Arch. f. exper. path. u. pharmak., 1936, 181, 273-280) for demonstration of lead in bones.

3. Attempts have been made to identify lead after microincineration by exposure to hydrogen sulphide, because lead sulphide is black, but Gordon $\mathrm{H}$. Scott emphasizes difficulty in distinguishing it from other sulphides and from carbon in imperfectly incinerated specimens (McClung, p. 660).

4. The method of Sieber, E., Arch. f. exper. Path. u. Pharmak., 1939, 181, 273 depending on production of acid resistant brown-black lead sulfide when tissue is treated with acidulated $\mathrm{H}_{2} \mathrm{~S}$ solution is said to be satisfactory by Gomori, G., J. Mt. Sinai Hosp., 1944$45,11,317-326$ when presence of other heavy metals is ruled out.

Methods for chemical determination of lead in biological materials are important as checks on above. Consult Smith, F. L. 2nd., Rathmell, T. K. and Williams, T. L., Am. J. Clin. Path., 1941, 11, Suppl. 5, 653-668.

For a convenient method of giving colloidal lead intravenously to rabbits see Crawford, B. L., Stewart, H.L., Willoughby, C. E. and Smith, F. L., Am. J. Cancer, 1938, 33, 401-422. The authors describe techniques for direct analysis of lead in the tissues.

Leather Brown, see Bismark Brown Y.

Leather Yellow, see Phosphine.

Leblond, see Radioantographic Technique.

Lebowich's soap-wax technique eliminates use of alcohol, xylol and overnight drying of paraffin sections. Takes only 6-8 hrs. (Moritz, C. E., Stain Techn., 1939, 14, 17-20).

L. E. Cells. The discovery of these cells in acute disseminated lupus erythe- matosus by Hargraves, M. M., Richmond, M. and Morton, R., Proc. Staff Meet., Mayo Clin., 1948, 23, 25-28, was a definite advance in diagnostic procedure. The simplest test for L. E. cells is that of Lee, Stanley L., Am. J. Clin. Path., 1951, 21, 492-496. Withdraw 1-2 ec. venous blood into a clear dry test tube. Let clot and remain at room temperature for $2 \mathrm{hrs}$.; with wooden applicator "fish out" the clot. Lecithin, a compound of phosphoric acid, glycerol, choline and 2 fatty acid molecules. It is a phosphatide soluble in alcohol, chloroform, ether and benzene, see Lipoids.

Lee-Brown. Modification of Mallory's aniline blue connective tissue stain (LeeBrown, R. K., and Laidley, J. W. S., J. Urol., 1929, 21, 259-274). Mallory (p. 155) states that the following technique is particularly valuable for the kidney. Treat paraffin sections of Zenker fixed material with iodine to remove mercury. Wash. $1 \%$ aq. phosphomolybdic acid, 30 sec. Wash in aq. dest. 1-2 min. Stain in: aniline blue, $0.5 \mathrm{gm}$.; orange G., 2 gm.; phosphomolybdic acid, $2 \mathrm{gm}$.; aq. dest., $100 \mathrm{cc}$. for $30 \mathrm{~min}$. at $55^{\circ} \mathrm{C}$. Wash in aq. dest $2-5 \mathrm{~min} .1 \%$ aq. phosphomolybdic acid, 30 sec. $95 \%$ alc., abs.alc., xylol, balsam. Glomerular basement membrane and collagen, deep blue; nuclei, orange.

Leishmania Donovani, a search for stains that will color more rapidly than Giemsa revealed Astra violet F. F. Extra, Himmelblau, Magenta Lermont and Navy blue shade, each to be used in fresh $10 \%$ aq. solution (Takasaki, S., Lues, Tokyo, 1938, 16, 127).

Leishmania. Media. Direct microscopic examination of peripheral blood may be negative while detection in culture is feasible. Q. M. Geiman (Simmons and Gentzkow) recommends addition of $10 \mathrm{cc}$. blood to sodium citrate in physiological saline, centrifuge and inoculate few drops buffey coat into tubes of NNN medium, incubatc $22-28^{\circ} \mathrm{C}$. and examine microscopically $10-20$ th day for motile forms. The following media are abbreviated from Geiman's account.

1. Blood agar or NNN (Novy, MacNeal and Nicolle, 1908). Agar, $14 \mathrm{gm}$., sodium chloride, $6 \mathrm{gm}$., aq. dest. 1000 cc. Add $\frac{1}{3}$ vol. sterile defibrinated rabbit's blood cooled to $45^{\circ}-50^{\circ} \mathrm{C}$. Mix, tube long slant. After agar sets, cap with stcrile rubber stoppers. Prove sterility by incubation $37^{\circ} \mathrm{C}$, 24 hrs. Inoculate material to be cultivated on slant and in water of condensation. Incubate $20^{\circ}-25^{\circ} \mathrm{C}$. Transfer every 20 30 days to maintain. 
2. Leptospira (Noguehi, 1924). $0.9 \%$ aq. sodium chloride, 800 parts; fresh rablit serum, 100 parts; $2 \%$ nutrient agar $\mathrm{pH} 7.2,100$ parts, rabbit hemoglobin solution 10-20 parts. (To make this hemoglobin solution take 1 part defibrinated rabbit's blood and 3 parts aq. dest., centrifuge and use clear supernatant fluid.) Tube, prove sterility by incubation before using. Subculture every 30 days. An increase in hemoglobin solution improves growth of Leishmania.

3. Adler's modification of above. Agar, 1 part; Locke's solution eontaining $0.2 \%$ dextrose, 8 parts; fresh rabbit serum, 1 part. For species of Leishmania and Trypanosoma cruzi.

4. Modified, Salle and Schmidt (Cleveland and Collier, 1930). Veal infusion (50 gm. Bacto-veal, Difco + 1000 cc. aq. dest.), 250 ce.; proteose, peptone (Difeo), $10 \mathrm{gm}$; sodium chloride, 5 gm.; aq. dest., 550 ec. Dissolve make pH 7.4 and autoclave. Add $20 \mathrm{cc}$. $50 \%$ aq. glucose (sterilized by filtration or in autoclave $10 \mathrm{lbs} ., 10 \mathrm{~min}$.) and $60 \mathrm{cc}$. horse red cells laked with 2 parts aq. dest. Pour in medium flasks or tubes. Vigorous long lived cultures.

Length measurements:

Millimeters to inches $\times 0.0394$. Inches to $\mathrm{mm} . \times 25.4$. See Micron.

Lens Paper, a specially prepared soft paper indispensible for cleaning immersion oil from objectives.

Leprosy Bacilli. Stain by carbol-fuchsin in smears. See Concentration method for collecting bacilli from lesions. For study in sections, see Acid Fast Bacilli.

Leptospira Medium, Noguchi's, see Leishmania.

Leptospiras, method for isolation from water (Bauer, J. H., Am. J. Trop. Med., 1927, 7, 177-179. See Spirochetes.

Leuco Basic Fuchsin. To make add to 200 cc. aq. sol. fuchsin, $2 \mathrm{gm}$. potassium metabisulphite and 10 cc. N hydrochloric acid. After bleaching 24 hrs. add $0.5 \mathrm{gm}$. Novit, shake $1 \mathrm{~min}$. and filter through coarse paper. Resulting elear solution works nicely in Feulgen technique (Coleman, L. C., Stain Techn., $1938,13,123-124)$.

Leuco-Dyes as vital stains. Make $0.01 \%$ aq. solutions of methylene blue, azur A, thionin toluidine blue and brilliant cresyl blue. Add to 100 cc. $1-2.5$ cc. $\mathrm{N} / 10$ $\mathrm{Na}_{2} \mathrm{~S}_{2} \mathrm{O}_{3}$ and 1-4 ec. $\mathrm{N} / 10 \mathrm{HCl}$. Mix and store at room temperature in dark. To stain, add 1-2 drops of leucobase to the protozoa, blood cells, etc. in physiological saline. Said to give good contrast staining of nucleus and cytoplasm and to be useful in oxidation-reduction determinations (Roskin, G., Arch. Russ.
Anat. Hist. Embr., 1937, 16, 107-109).

Leuco-Patent Blue V, see Lillie, p. 285.

Leucocytes. In the broad sense they include all white blood cells but the term is generally restricted to the "granular" leucoeytes as compared with the "nongranular" ones (Lymphocytes and Monocytes). In a still narrower sense the leucocytes include only polymorphonuclear neutrophiles, eosinophiles and basophiles which are easily found in circulating blood as contrasted with less differentiated leucocytes called Myelocytes and Mycloblasts generally confined to the bone marrow.

For mitochondria within leucocytes supravital staining with Janus green is indicated. In smears Giemsa's stain has a little advantage over Wright's in the fact that it better demonstrates any bacteria that may be present. The May-Giemsa teehnique is most used in Europe. It is, in effect, a double staining because the air dried smears are first treated with the May-Grunwald combined fixative and stain and are later colored by Giemsa's stain. It gives satisfying deep colors. The KardosPappenheim modifieation is suggested when a particularly intense coloration of neutrophilic granules is desired. Ehrlich's triacid stain may likewise be useful because it is said to stain the neutrophilic granules leaving the azur granules untouched.

Leucocytes give strong Peroxidase and Oxidase reactions, which are, however, not specific for them. The Golgi Apparatus (reticular material) can be demonstrated by long treatment with osmic acid or by the Cajal uranium nitrate and silver method (Cowdry, E. V., J. Exper. Med., 1921, 33, 1-11). The demonstration of degenerative leucoey tic changes associated with ageing is described by Lowell (A. L., J. Lab.\& Clin. Med., 1937-38, 23, 791-796), of variability in relation to alterations in meteorologic conditions by Berg (M., J. Lab. \& Clin. Med., 1937-38, 23, 797-803) and of lipoid components by Bacsich (P., J. Anat., 1935-36, 70, 267-272). Chemotactic response and motility can be measured both in tissue cultures (Coman, D. R., Arch. Path., 1940, 30, 896-901) and directly by observing the behavior of leucocytes with relation to bacteria and in temporary mounts (Mallery, O. T. and McCutcheon, M., Am. J. Med. Sci., $1940,200,391-399)$. By the latter method differences in behavior of neutrophiles from seriously ill and normal persons have been reported. Motion pictures are of great assistance in making a thorough analysis of the movements and behavior of leucocytes. Some excellent ones, 
taken by Dr. W. H. Lewis, are available for distribution by the Wistar Institute of Anatomy in Philadelphia. To investigate their behavior after they have left the blood vessels and entered the surrounding tissues is immensely more difficult. The only method that gives promise of important results is to employ for this purpose special chambers in. serted in the ears of rabbits (Clark,E.R. and E. L., Am. J. Anat., 1936, 59, 123173). See Neutrophile, Eosinophile and Basophile Leucocytes.

Leucocyte Counts. 1. Total number white blood cells per c. mm. Over 12,000 a leucocy tosis, less than 5000 , a leucopenia. Average about 7,500.

2. Differential. Smears colored by Giemsa's or Wright's stains are more satisfactory than supravitally stained preparations because the latter are more difficult to handle and the cells are slowly dying and showing more and more deviations from normal. Relative number of different white cells is expressed in percentages, i.e. neutrophiles 55-75, eosinophiles, 2-4, basophiles, $0-1$, lymphocytes 21-31, and monocytes 4-5. Both total and differential counts should be correlated to avoid misconceptions. $60 \%$ neutrophiles in total count of 8,000 amounts to 4,800 neutrophiles per c. mm. $80 \%$ neutrophiles in total count of 4,800 is the same, namely 4,800 neutrophiles per c. $\mathrm{mm}$. although a relative neutrophilic leucocy tosis exists. $60 \%$ neutrophiles in a total count of 16,000 makes on the other hand 9,600 neutrophiles per c. $\mathrm{mm}$. which is an actual neutrophilic leucocytosis. 20\% lymphocytes of 9,000 is the same number per c. $\mathrm{mm}$. as $60 \%$ of 3,000 ; while $30 \%$ of 11,000 is an actual lymphocytosis.

3. Age. Since young neutrophiles have fewer nuclear lobes than older ones counts of the number with from 1-5 lobes were made by Arneth. Today simpler methods are used.

The Schilling is the usual one. It is both a total, a differential and an age count combined. The normal is given

$\left.\begin{array}{lc}\text { Total } & 5,000 \text { to } 10,000 \\ \text { B } & 0-1 \\ \text { E } & 2-4 \\ \text { I } & 0 \\ \text { J } & 0-1 \\ \text { St } & 3-5 \\ \text { S } & 51-67\end{array}\right\}$ Leucocytes

above. $\mathrm{B}=$ basophile. $\mathrm{E}=$ eosinophile. $\mathrm{M}=$ myelocyte (Nucleus large, occupying about half cytoplasmic area, spherical to oval or kidney-shaped, pale staining, chromatin reticulated, nucleoli present. Cytoplasm faintly basophilic with few specific granules which are small, of ten difficult to stain and irregularly distributed). $\mathrm{J}=$ juvenile (A little larger than mature neutrophiles. Nucleus saucer to bean shaped. Stains poorly. Circumscribed basophilic nucleoli). St $=$ stab nuclear (Slightly smaller than juveniles. Nucleus $\mathrm{T} V$ or $\mathrm{U}$ shaped but not divided into segments by filaments and without nucleoli). $\mathrm{S}=\mathrm{seg}$ ment nuclear (Fully differentiated neutrophiles having 2-5 or more segments often joined only by filaments. Nuclei stain intensely.) $\mathrm{L}=$ lymphocyte. Mon $=$ monocyte.

When the numbers of M. J. St. are increased relative to $\mathrm{S}$., it is called a "shift to the left", meaning that immature leucocytes are called into the circulation, which is an unfavorable sign. When the relative number of $\mathrm{S}$ is increased over the others, it is termed a "shift to the right", meaning that only mature leucocytes are called out, which is a favorable sign if it follows a previous shift to the left. Details are given by Wintrobe, M. M., Clinical Hematology, Philadelphia, Lea \& Febiger, 1942, 792 pp. For blood containing gum acacia, see Monke, J. V., J. Lab. \& Clin. Med., $1940-41,26,1664-1667$ and for interference by decreased fragility of erythrocytes see Bohrod, M. G., J. Lab. \& Clin. Med., 1940-41, 26, 1953-1955.

A better method, unfortunately not widely employed, is the filament-nonfilament count. Filaments are neutrophiles in which the nuclear segments are connected by delicate strands apparently made up of nuclear membrane only and nonfilaments are those in which the connections are so wide that they can be resolved into nuclear membrane plus nuclear contents. In 100 neutrophiles there are normally 8-16 nonfilament cells. A greater per cent is a shift to the left. For counts see Krusen, F. H., Am. J. Med. Sci., 1937, 193, 470474.

Leucocytes. Developmental series. The technique employed apparently makes a great deal of difference in the conclusions reached. See Cowdry's Histology, p. 99.

1. Maximow and Bloom employing mainly permanent preparations list:

Hemocytoblasts: ". . . large (up to 15) ameboid, non-granular basophil cells of lymphoid nature." Occur extravascularly.

Promyelocytes: "The oval or kidneyshaped, clear nucleus contains a loose 
chromatin network and several nucleoli. At the indentation of the nucleus there is a distinct cytocentrum. The ameboid protoplasm is slightly basophil, although it of ten shows acidophil areas." Specific granules "are scarce and usually confined to the periphery of the cytocentrum and to the acidophil spots in the cell body." Azurophil granules are present but later disappear. They of ten show mitosis.

Myelocytes: "The protoplasm becomes diffusely acidophil while the specific granules increase in number and fill the whole cell body, except for the cytocentrum. The nucleus keeps its compact form while its previously loose chromatin network becomes coarser and stains darker. The nucleoli are indistinct. Mitoses are common."

Metamyelocytes: After an unknown number of mitoses a generation appears. The nucleus "as soon as it is reconstructed after the last mitosis, shows a beginning polymorphism and has the shape of a horse-shoe." The mature leucocyte is formed from these cells by individual maturation without division.

2. Sabin and associates relying chiefly on supravital stains list:

Reticular cells: They "are small, their cytoplasm is faintly basophilic, as seen in fixed films, and in supravital preparations they show no differentiation of specific substances." Reticular cells "lack the striking rod-shaped mitochondria which characterize the lymphocytic strain... The nuclei have less sharp contours and less chromatin than those of lymphocytes."

Myeloblasts: These differ "through the elaboration of a marked basophilia and of great numbers of small mitochondria.... In supravital technique, the myeloblast has usually no stainable substance except mitochondria ..." but occasionally a few vacuoles reacting to neutral red are present as well as some which are not colored by it.

Myelocytes $A$ : The earliest stage with the specific granules up to 10 "reacts with a single blue granule in the oxydase test."

Myelocytes B: "May be conveniently divided into those with less than half and those with more than half the full quota of granules."

Myelocytes $C$ : These cells contain the full quota. Metamyelocytes: They "show the earliest signs of the nuclear changes toward polymorphism and the first sign of the transformation of the cytoplasm to a phase sufficiently fluid to allow the flowing of granules which is essential for ameboid movement. In passing through these stages, there is a gradual decrease of basophilia of the cytoplasm and in the numbers of mitochondria. The basophilia disappears entirely in the early leucocytes, while the mitochondria persist in small numbers until the stage of senility in the leucocytes."

Leucocytic Index, ratio of number of polymorphs to number of lymphocytes, considered by Turley, L. A. and McClellan, J. T., Am. J. Clin. Path., 1943, $7,87-95$ to be valuable indicator of condition of the patient, a high or rising index being a bad sign and a low or falling one, a favorable sign.

Leucocytic Infiltrations. A convenient way to produce an intense local neutrophilic infiltration is to inject starch as described by Chambers, R. and Grand, C. G., Am. J. Cancer, 1937, 29, 111-115. Cowdry, E. V. and Ruangsiri, C., Arch. Path., 1941, 32, 632-640 made repeated injections of $1 \%$ corn starch suspensions in physiological saline in amounts of $0.1-0.2 \mathrm{cc}$. into leprous nodules of rats.

Leucocytozoa, Protozoa, belonging to the Hepatozoidae, which inhabit the monocytes of dogs, rats, and other animals particularly in the tropics. See, Wenyon, C. M., Protozoology. New York: William Wood \& Co., 1926, 2,1053-1563.

Leucosin, a stored reserve in lower plants (Taylor in McClung, p. 221).

Levitation Method, see Floatation Method.

Levulose Syrup for fluid mounts. Mallory (p. 99) specifies $30 \mathrm{gms}$. levulose dissolved in $20 \mathrm{cc}$. water by warming at $37^{\circ} \mathrm{C}$. for $24 \mathrm{hrs}$.

Lewis-Locke solution, see Locke-Lewis.

Leydig Cells are usually known as interstitial cells of the Testis which see.

Liang uses Schiff reagent for nerve endings (Liang, H. M., Anat. Rec., 1947, 97, 419).

Lieberkühn's Glands, data on size, surface area, number of cells etc. in human large intestine (Policard, A., Bull. d'Hist. Appl., 1939, 16, 261-262).

Liebermann-Burchardt reaction for cholesterol and its esters (cholesterides).

1. Modification of A. Schultz. Expose frozen sections of formalin fixed tissue at least 4 days (more in winter) to strong light, if possible sunlight. Mount. Dry carefully with blotting paper. Cover with few drops equal parts acetic and sulphuric acids. Drain and examine in the reagent. Cholesterol and its esters dark blue or red purple becoming green.

2. Modification of Romieu (M., C. rend. Acad. d. Sci., 1927, 184, 1206-1208) Mount frozen sections of formol or Bouin (less acetic) fixed tissues and dry. Cover with 1 drop conc. sulphuric acid, $3-15$ sec. Stop reaction by adding $2-3$ 
drops acetic anhydride. Wash with several drops of same. Cover and examine immediately. Cholesterol and its esters violet lilac or red purple, becoming green. The above two methods abbreviated from Lison (p. 210) are in his excellent judgment specific for cholesterol and its esters if positive. A negative reaction does not definitely prove their absence. See Swyer, G. I. M., Cancer Research, 1942, 2, 372-375 for quantitative measurement of the color.

Light Blue, see Spirit Blue.

Light Green, see Methyl Green.

Light Green N, see Malachite Green.

Light Green SF yellowish (CI, 670) S-acid green, fast acid green N-Commission Certified. This acid di-amino triphenyl methane dye is a sulfonated derivative of brilliant green and a valuable counterstain for safranin. It is used by Twort, F. W., Brit. J. Exp. Path., 1924, 5, 350-351 as a double stain with neutral red for animal parasites and microorganisms in tissues. Unfortunately light green fades quickly. Conn (p. 110) recommends fast green FCF as a substitute.

Lighting, see Illumination.

Lignin Pink, a monazo acid dye (British Drug Houses Ltd.). Advised $0.5 \%$ aq. solution as a chitin stain and a contrast stain with chlorazol Black E (Cannan, H. G., J. Roy. Micr. Soc., 1941, 61, 88-94).

Lilienfeld-Monti test for phosphorus is not a satisfactory microchemical method. See Bensley's method (R. R., Biol. Bull., 1906, 10, 49-65) and criticism by Lison (p. 118).

Lillie, see Azure or Toluidin Blue Eosin. Lillie's chrom-osmic-acetic fixative. $\frac{1}{2} \%$ aq. chromic acid, 15 cc.; $2 \%$ aq. osmic acid, 3.5 cc.; glacial acetic acid, 3 drops. Used by him for echinoderm eggs.

Lime, see Calcium.

Linderstrøm-Lang, Kaj. U., and Holter, Heinz, Histochemical AdvancesWritten by David Glick, Dept. of Physiological Chemistry, University of Minnesota, Minneapolis 14, Minn. October 17, 1951-Prof. Linderstrøm-Lang, since 1938 the head of the Department of Chemistry of the Carlsberg Laboratory, Copenhagen, Denmark, and Dr. Heinz Holter, his associate and head of the Cytochemical Department, are responsible for some of the most significant advances in the field of quantitative histochemistry as well as of protein and proteolytic enzyme chemistry. Their histochemical work began in 1930 when Dr. Holter came to the Carlsberg Laboratory. Their first ef forts were directed to the development of micro titration techniques that would enable a thousandfold refinement of common macro methods of biochemical analysis without loss of precision. This degree of refinement constituted a temporary compromise since it was their goal to bring reliable quantitative chemical techniques to bear on the study of the single cell, and a hundred thousandfold refinement would be required to achieve this end in the case of mammalian cells. However the one thousandfold refinement did permit work on single microtome sections, protozoa, and ova of certain marine invertebrates.

The first investigation of the series (Linderstrøm-Lang, K. and Holter, H., Compt. rend. trav. lab. Carlsberg, Ser. Chim., 1931, 19, No. 4) described the micro titration apparatus that was devised, (the burettes used were graduated in $0.0002 \mathrm{mI}$. divisions and readings were taken to $0.00002 \mathrm{ml}$.) and subsequently Linderstrøm-Lang and Holter adapted the equipment to the measurement of proteolytic enzyme activity. They employed this method for a study of the distribution of peptidase along the roots and sprouts of barley (LinderstromLang, K. and Holter, H., Ibid., 1932 , 19, No. 6). When one has developed a new tool it is natural that he employ it for diverse purposes to test its range of usefulness. And accordingly, in the next application of their technique, Holter and Linderstrøm-Lang (Zeit. f. Physiol. Chem., 1932, 219, 223-240) investigated the proteinases of Drosera Rotundifolia, an insectivorous plant, and then Linderstrøm-Lang (Ibid, 1933, $215,167-178$ ) made a study of the peptidase content of single eggs of two marine invertebrates, the California sand dollar and Urechis caupo, before and after fertilization. These eggs have a wet weight of only about $5 \times 10^{-7} \mathrm{~g}$.

Linderstrøm-Lang and Holter next extended their technique to a method for the iodometric determination of reducing sugars with a precision corresponding to $25 \times 10^{-8} \mathrm{~g}$. of glucose (Compt. rend. trav. lab. Carlsberg, 1933,19, No. 14), and this was followed by the development of a diffusion method for ammonia sensitive to $14 \times$ $10^{-9} \mathrm{~g}$. of nitrogen (Compt. rend. trav. lab. Carlsberg, Ser. Chim., 1933, 19, No. 20.)

The elegance and general applicability to diverse problems of the techniques and methods being developed began to attract research workers to the Carlsberg Laboratory from other countries, and the series of publications that had evolved under the heading of 
Studies in Enzymatic Histochemistry soon had contributions from $T$. Philipson, who investigated the peptidase activity of single centrifugally separated parts of eggs of Psammechinus miliaris (Ibid, 1934, 20, No. 4), and D. Glick, who elaborated an acidimetric method for lipolytic enzymes (Ibid, No. 5) and applied it to a study of the distribution of esterase in the gastric and duodenal mucosa of the hog (Glick, D. Ibid, No. 11).

The latter was undertaken since Linderstrøm-Lang and Holter had already underway similar investigations of the distribution of pepsin (Ibid, 1935, 20, No. 11), acid (Ibid, 20, No. 11), and peptidase (Ibid, 20, No. 11). A. Søeborg-Ohlsen joined in a collaborative study of the enzyme distribution in the hog stomach as a function of its histological structure (Ibid, 20, No. 11) and the enzyme pattern in the mucosa was revealed to a significant degree.

The need of methods for the chemical determination of additional cellular constituents diverted the Carlsberg Laboratory workers and visiting scientists to the task of elaborating them, and for the visitors this served the additional purpose of providing excellent first hand training and experience in the use of the techniques. With Palmer, A. H. (Ibid, 1935, 21, No. 1), an electrometric chloride titration was developed with a precision of about $14 \times 10^{-9} \mathrm{~g}$. of chloride; Levy, M. (Ibid, $1936,21,101-110$ ) refined the Kjeldahl nitrogen analysis to $3 \times 10^{-8} \mathrm{~g}$. of nitrogen, Linderstrøm-Lang (Compt. rend. trav. lab. Carlsberg, Ser. Chim., 1936, 21, 111-122) worked out an electrometric titration method for sodium plus potassium sensitive to $1 \times 10^{-6} \mathrm{~m}$. equiv., Norberg, B. (Ibid, 1937, 21, 233-241) evolved a titrimetric estimation of potassium isolated as the iodoplatinate that was accurate to $1-2$ $\times 10^{-4} \mathrm{~m}$. equiv., and with Weil, L. (Ibid, 1935, 21, 7-14) micro methods for arginase and urease were established.

Linderstrøm-Lang, K. and Engel, C. (Ibid, 1938, 21, 243-258) employed the method that had been previously developed for reducing sugars for the measurement of amylase activity, the distribution of which they studied in barley, and Holter and Doyle, W. L. (Ibid, 1938, 22, 219-225) subsequently modified the technique to gain greater precision. Meanwhile micro methods for other enzymes were being developed. Holter and Doyle (J. Cell Compt. Physiol., 1935, 12, 295-308) adapted the iodometric method of Stern to the estimation of catalase with a precision equivalent to the decomposition of $2 \times$ $10^{-8} \mathrm{~g}$. of hydrogen peroxide, and Glick, D. (Compt. rend. trav. lab. Carlsberg, Ser. Chim., 1938, 21, 263-268) extended his esterase method to include the measurement of cholinesterase with a sensitivity equivalent to the hydrolysis of $1 \times 10^{-8}$ mole of ester.

As the new methods were made available their application to specific problems followed at once. Thus, Linderstrøm-Lang, K., and Duspiva, F., (Ibid, $1936,21,53-84$ ) studied the digestion of keratin by larvae of the clothes moth, and Duspiva followed this work by investigations of various proteolytic enzymes of clothes- and wax-moth larvae (Ibid, 1936, 21, 177-202), as well as of $\mathrm{pH}$ of the intestinal juice of these organisms for which a micro glass electrode was used (Ibid, 21, 167-176). Holter (J. Cell Comp. Physiol., 1936, 8, 179-200) studied peptidase localization in marine ova, with Kopac (Ibid, 1937, 10, 423437) the localization of this enzyme in amoeba, and with Doyle, W. L. (Compt. rend. trav. lab. Carlsberg, Ser. Chim., 1938, 22, 219-225) the amylase localization in amoeba. Doyle (Ibid, 1938, 21, 291-299) also investigated the catalase and peptidase activity in marine ova. The activation of leucylpeptidase of single Tubifex eggs by magnesium salts was reported by Holter, H., Lehmann-Bern, F. E. and LinderstrømLang (Compt. rend. trav. lab. Carlsberg, Ser. Chim., 1938, 21, 259-262).

Other applications in this period include a study of the distribution of urease in the dog stomach by Linderstrøm-Lang and Søeborg-Ohlsen, A. (Enzymologia, 1936, 1, 92-95), and an investigation of the cholinesterase distribution in the hog stomach before and after administration of drugs that affect gastric secretion by Glick (Ibid, 1938, 21, 269-281). Using a titrimetric dye method previously worked out (J. Biol. Chem., 1935, 109, 433-436) Glick followed the changes in ascorbic acid in different parts of the developing barley embryo up to the ten-day sprout stage (Ibid, 1937, 21, 203-209).

Titrimetric procedures alone had been exploited up to this time. But in the attempt to extend the applicability of this approach to quantitative histochemistry, other analytical principles were tested. Colorimetry, if conducted on the required micro scale, would obviously open a vast new region for exploitation, but the limiting factor in the middle nineteen thirties was the availability of the necessary equipment. At that time, the Zeiss Pulfrich step photometer, that employed cuvettes re- 
quiring $0.2 \mathrm{ml}$. of liquid, was used by Lundsteen, E. and Vermehren, E. (Ibid, 1936, 21, 147-166) at the Carlsberg Laboratory for the determination of phosphorus and phosphatase in 0.05 ml. blood samples. But for many histochemical purposes the liquid volume needed for the colorimetric measurement was still too great. This problem was largely solved subsequently as will be mentioned later.

In 1937 two new analytical techniques were ingeniously developed by Linderstrøm-Lang for micro-biochemical research. One of these was based on the dilatometric principle that in certain reactions a volume change of the total solution occurs which results in a change in specific gravity great enough for measurement. As applied by Linderstrøm-Lang (Nature, 1937, 139, 713714), a density gradient is set up by half-filling a glass tube with a kerosenebromobenzene mixture of appropriate specific gravity, and then filling the remainder of the tube with a similar mixture having a greater proportion of kerosene to give a lower specific gravity. If the liquid in the middle of the tube is stirred, and the tube mounted in a thermostat, a stable vertical density gradient is produced. If into this gradient an aqueous drop of a reaction mixture is placed, it will fall until it reaches the level at which its specific gravity equals that of the surrounding medium. As the reaction within the drop proceeds, the resultant volume change of the drop will alter its specific gravity and the drop will then move to a new level. In some cases the distance the drop will move has been found to be directly proportional to the extent that the chemical reaction has occurred. By employing a cathetometer the movement of the drop can be followed to 0.01 $\mathrm{mm}$. and finer movements can be observed with an ocular micrometer in the telescope of the instrument. After calibration of the gradient with drops of salt solution of known specific gravity, measurements of the excursion of the drop can be used to calculate the amount of reaction products formed in certain instances.

The dilatometric method was employed by Linderstrøm-Lang and $\mathrm{H}$. Lanz, Jr. (Ibid, 1938, 21, 315-338), for peptidase measurements, and a study was made by Holter, Lanz, and Linderstrøm-Lang (Ibid, 1938, 23, 1-9) of this euzyme during the first cell divisions in the egg of Psammechinis miliaris. Linderstr $\not \mathrm{m}$-Lang, Jacobsen, $\mathrm{O}$. and Johansen, G. (Ibid, 17-25) also employed the density gradient tube for the measurement of the deuterium content of heavy water mixtures.

The other new technique that was evolved at this time was based on the principle of the Cartesian diver (Linderstrøm-Lang, K. Nature, 1937, 140, 108-108). The diver is a small vessel, open at one end, that will rise or fall, while submerged in a salt solution, as a result of a change in its gas volume. The volume of the first divers used was about $0.01 \mathrm{ml}$., and a reaction mixture of about $0.001 \mathrm{ml}$. placed in the bottom of the diver was employed for gasometric measurements of enzyme activity or respiration. The measurement was carried out by applying a known positive or negative pressure to the air over the tube containing the submerged diver to bring it to a marked level in the tube. At this level the gas volume in the diver has a fixed value, and by determining the external pressure necessary to bring the gas volume back to this value after expansion (in case of a reaction evolving gas) or contraction (in case of reaction using up gas), the actual amount of gas evolved or consumed can be calculated. In other words the apparatus is a constant volume, variable pressure, unit similar in this respect to the ordinary Warburg apparatus but capable of measuring gas changes of a magnitude a thousand times smaller. Further refinements were developed later and will be mentioned.

The first applications of the Cartesian diver method were to the gasometric measurement of cholinesterase activity in ganglia, Linderstrøm-Lang and Glick (Compt. rend. trav. lab. Carlsberg, $1938,22,300-306$ ), the determination of thiamine and cocarboxylase in yeast, Westenbrink, H. G. K. (Ibid, 1940, 23, 195-212), and the respiration of separated parts of the embryos of Paracentrotus lividus, Lindahl, P. E. and Holter (Ibid, 249-256), and of cells grown in tissue culture, Zamecnik (Ibid, 1941, 24, 59-67).

The following period in the work of Linderstrom-Lang and Holter, although beclouded by the German occupation of Denmark and the accompanying difficulties and distractions, was devoted to the consolidation of the advances already made by further refinements of technique and the extension of the methods to new uses. Meanwhile, Linderstrøm-Lang and Mogensen, K. R. (Ibid, 1938, 23, 27-35) had already described a cryostat for the accurate sectioning of fresh frozen tissue at a constant low temperature and the technique of handling the cut sections to 
prevent their curling etc., and Holter and Lindahl ( $I$ bid, 1940, 23, 249-256) had already studied the distribution of peptidase in the Paracentrotus embryo in continuation of the line of cytochemical work on marine invertebrates that had been instituted earlier.

Considering the disruptive influence of the occupation of Denmark by the Nazis, it is remarkable that the Carlsberg Laboratory workers continued to be productive, but perhaps the very need to have a refuge from the wild events outside served to hold them to their laboratory benches for periods of sweet reasonableness. The record shows that during these years the Cartesian diver technique was subjected to intensive theoretical analysis, Linderstrøm-Lang and Holter (Ibid, 1942, 24, 105-138) and Linderstrøm-Lang (Ibid, 1942 , 24, 249-280; Ibid, 1943, 24, 333395), and laboratory refinement, Holter (Ibid, 1943, 24, 399-478). Zeuthen, E. (Ibid, 479-518) devised a diver with a gas volume a hundred times smaller than the first ones used, and this opened many new possibilities.

Applications of Cartesian diver gasometry at this time included studies on the respiration of the egg and embryo of the ascidian, Ciona intestinalis $L$. Holter and Zeuthen (Ibid, 1944, 25, $33-65)$, the respiration of syncytia formed by abnormal development of Ciona eggs, Andresen, Holter, and Zeuthen (Ibid, 1944, 25, 67-85), and oxygen uptake during mitosis of frog eggs, Zeuthen ( $I$ bid, 1946, 25, 191-228). Holter ( $I$ bid, 1944, 25, 156-167) also developed a colorimetric method for measuring the volume of large amoebae.

Parallel with these developments during the war years were others. Schmidt-Nielsen (Ibid, 1942, 24, 233 247) worked out a titrimetric method for analysis of fat in quantities of $10^{-5}$ g. and later he extended this investigation to methods for the determination of the iodine number in amounts of fat of this order ( $\mathrm{Ibid}, 1944,25,87-96$ ), and he described the extraction and fractionation of fats in $1 \mathrm{mg}$. of tissue (Ibid, 97-105). Previous work on the stomach was also continued by RaskNielsen (Ibid, 1944, 25, 1-32) who studied the peptidase content of the pyloric portion of the hog stomach, and Bottelier, H. P., Holter, H., and Linderstrøm-Lang, K. ( Ibid, 1943, 24, 289-314) investigated the peptidase in the roots of barley.

Further extension to new uses of the microtechniques that had been developed marked the post-war years. Brüel, D., Holter, H., Linderstrøm-Lang,
K. and Rozits, K. (Ibid, 1946, 25, 289 324 ) elaborated a titrimetric method for the determination of total nitrogen with an accuracy of $5 \times 10^{-9} \mathrm{~g}$. nitrogen, Levi and Zeuthen (Ibid, 273-288) adapted the density gradient tube to micro weighing, and later Zeuthen, E. ( bid, 1948, 26, 243-266) constructed a Cartesian diver balance that gave reduced weights to $1 \times 10^{-8} \mathrm{~g}$. The reduced weight is the weight of an object minus the weight of an equal volume of water. This quantity is convenient for expressing the amount of a small biological sample since it is a measure of the quantity of the substance independent of its water content. Zeuthen (Ibid, 1948, 26, 267-276) followed the reduced weight and volume of amoebae during starvation, and Holter and Zeuthen (Ibid, 277-296) studied metabolism and reduced weight of amoebae. A theoretical treatment of the use of the Cartesian diver for respiration measurements on single cell organisms was given by Linderstrøm-Lang (Ibid, 1946 , 25, 229-272)

The main program of the cytochemical department in recent years has been the continuation of Holter's previous work on the biochemistry of unicellular organisms and the developing invertebrate and amphibian embryo. The demand for microanalysis of different constituents during the course of these studies has led to further expansion of method development.

Apparatus that will permit colorimetric analysis of substances at the level required for work on microtome sections, single early embryos, or protozoa was developed more recently by Holter and his coworkers. The volumes of liquid needed for the colorimetry are of the order of $0.010 \mathrm{ml}$. The cuvettes employed to hold these small volumes are pieces of capillary tubing having a lumen of about $1 \mathrm{~mm}$. in diameter and about $10 \mathrm{~mm}$. long. The cuvette is sealed to a microscopic slide with stopcock grease, filled with the colored liquid, and a cover slip is placed on top. This unit is set on the stage of a microscope and a fine beam of filtered light is passed through it. The transmitted light is collected by a low-power objective and sent on to a photocell fixed over the ocular. Galvanometer readings are taken as for any other photoelectric colorimeter. With this equipment Holter and Løvtrup (Ibid, $1949,27,27)$ studied the proteolytic enzymes in amoebae and Krugelis ( Ibid, 1950, 27, 273-290) investigated the properties and changes of alkaline 
phosphatase activity during amphibian development.

Other recent work that has come from the Carlsberg Laboratory includes a micro gasometric method for sulfur compounds such as cystine, for which it is accurate to $1 \times 10^{-9} \mathrm{~g}$. within $2.5 \%$, Holter and Løvtrup (Ibid, 1949, 27, 72-78). Further development of the Cartesian diver technique was effected by using silicone coated divers, Schwartz (Ibid, 1949, 27, 79-92), and Løvtrup (Ibid, 1950, 27, 125-136) modified the diver balance of Zeuthen. In the course of the latter study L $\varnothing$ vtrup designed a microbalance with a sensitivity of $1 \times 10^{-8} \mathrm{~g}$. To conduct studies on the density of microorganisms such as amoebae, a starch density gradient tube was developed by L $\phi$ vtrup (Ibid, $1950,27,137-144)$. From a combination of density and reduced weight measurements the volume of amoebae could be determined with an accuracy of $1 \times 10^{-9} \mathrm{ml}$.

Aside from the classical applications of the quantitative techniques and methods that Linderstrøm-Lang and Holter instituted, their procedures are being employed ever more extensively by an increasing number of scientists in the diverse fields to which quantitative histo- and cytochemistry is of great importance. In fact, the development of the fundamental aspects of all of the life sciences can be expected to be significantly enhanced by their contributions.

Line Test for vitamin $D$. This is the basis for calculating the U.S.P. unit of vitamin $D$ potency. The line test was apparently first introduced by $\mathrm{McCol}$ lum, E. V., et al., J. Biol. Chem., 1922, $51,41-49$. A critique of the test is given by Bills, C. E., et al., J. Biol. Chem., 1931, 90, 619-636. See also Sherman, H. C., The Chemistry of Food and Nutrition, New York: MacMillan, $1941,611 \mathrm{pp}$. A slightly modified technique is proposed and given in detail by Martin, G. J., J. Lab. \& Clin. Med., $1940,26,714-719$. Inject rats intraperitoneally with $1 \mathrm{cc} .1 \% \mathrm{aq}$. sodium alizarin sulfonate at $\mathrm{pH} 8.0$ and give supplements of measured amounts of vitamin $D$ orally. Animals similarly stained but not given the vitamin serve as controls. After test periods of 1 or 2 days, kill the animals, remove radii and ulnae and examine grossly and $\mathrm{mi}$ croscopically for alizarin stained lines at epiphysis. See also use of Alizarin Red S. Both this and the sulfonate are better than Madder because they provide quicker and more intense coloration of bony calcium laid down during the period that they are available in the circulation as accelerated by vitamin $\mathrm{D}$. Linguatulidae, see Parasites.

Linin (L. linum, flax). The acidophilic, thread-like framework of nucleoplasm seen in sections but not in the living nucleus.

Lipase. Frozen sections $30 \mu$ thick and 4.5 $\mathrm{mm}$. in diameter of beef adrenals are extracted in $30 \%$ glycerol + equal volume $1 \%$ methyl butyrate in glycine - NaOH buffer at pH 8.7 ; digested at $40^{\circ} \mathrm{C}$.; enzyme action arrested by addition of $2 \%$ phenol (10 parts) and $0.04 \%$ brom-thymol blue (1.5 parts) to 3.5 times total volume; and end point titrated at $\mathrm{pH} 6.5$ with $0.05 \mathrm{~N} \mathrm{HCl}$. This point is determined by comparing color with standard color of brom-thymol blue in phosphate buffer $\mathrm{pH}$ 6.5. Nearby sections, some stained with hematoxylin and eosin, and others, with Sudan III, are examined histologically. The medulla, which exhibits most lipolytic activity, contains least lipid. Estimations of esterase are also described by Glick and Biskind (D. and G. R., J. Biol. Chem., 1935, 110, 575582). See Barnes, J. M., Brit. J. Exp. Path., 1940, 21, 264-275 for analysis of lipase in lymphocytes and polymorphonuclear leucocytes and Hoagland, C. L., et al., J. Exper. Med., 1942, 76, 163-173 for lipase determinations in elementary bodies of vaccine virus.

An important new technique is described and well illustrated by Gomori, G., Arch. Path., 1946, 41, 121-129:

1. Fix thin slices of fresh tissue in chilled acetone $12-24 \mathrm{hrs}$. in ice box.

2. Dehydrate in 2 changes absolute acetone, $12-24$ hrs. each, room temperature.

3 . Impregnate in $5 \%$ acetylcellulose (Eastman's cellulose acetate "high acetyl, low viscosity, no, 4614") for 24 hrs.

4. Drain off fluid, transfer to 2 changes benzene, $1 \mathrm{hr}$. each.

5. Embed in paraffin $\left(56-62^{\circ} \mathrm{C}\right), 2$ changes, 1 to $1 \frac{1}{2} \mathrm{hrs}$. each. Cut $4-8 \mu$ sections, float on water $\left( \pm 35^{\circ} \mathrm{C}\right)$ and mount on slides. Pass down through xylol and alcohols to aq. dest.

6. Incubate at $37^{\circ} \mathrm{C} 6-12 \mathrm{hrs}$. in $50 \mathrm{cc}$. Solution I +2 cc. Solution II.

Solution I: Glycerin 150 cc., $10 \%$ aq. calcium chloride, 50 cc.; half-molar maleate buffer $\mathrm{pH} 7$ to 7.4 (maleic acid, $5.8 \mathrm{gm}$.; $4 \%$ aq. sodium hydroxide $94 \mathrm{cc}$. + aq. dest. 6 cc.). If maleate buffer is omitted mixture should be adjusted to $\mathrm{pH}$ indicated.

Solution II: $5 \%$ aq. Tween 40, or Tween 60 (Atlas Powder Co., Wilmington, Del.) or Product 81 with about 
$0.02 \%$ merthiolate added. Keep both stock solutions in ice box.

7. Rinse in aq. dest. and transfer to $1-2 \%$ aq. lead nitrate, $10-15 \mathrm{~min}$.

8. Rinse thoroughly in repeated changes aq. dest. and transfer to dilute solution of light yellow ammonium sulfide (few drops to Coplin jar of aq. dest.) whereupon sites of lipase activity become dark brown.

9. Wash under tap and counterstain with hematoxylin and very lightly with eosin.

10. Dehydrate in alcohols; clear in gasoline or tetrachlorocthylene (perchloroethylene) and mount in clarite in these solvents. Avoid toluol and xylol.

Modifications have been suggested by Stowell and Lee (Arch. Path., 1950, 50, 519-537). Consult also the newer technique of Nachlas and Seligman (Anat. Rec. 1949, 105, 677-695) See Esterase.

Lipids. Identification of various kinds in microscopic preparations is extremely difficult. As Lison (p. 192) has shown, reliance cannot be placed in solubility tests. Some bodies, soluble in alcohol, ether, chloroform, carbon tetrachloride and so on, are not fats while some fats show considerable resistance to such solvents. Formalin fixation itself causes marked changes in solubility of fatty bodies (Kaufmann, C. and Lehmann, E., Virchow's Archiv. f. Path. Anat. und Physiol., 1926, 261, 623-648). It is not unusual to find fats slightly soluble or insoluble in microscopic preparations which on chemical extraction are soluble. Results of examination in polarized light must, he states, be interpreted with caution. Glycerides and fatty acids examined in vivo are never birefringent in the dissolved condition. After freezing or treatment with formalin they can become crystalline and birefringent. Cholesterol, in the form of birefringent, rhombic plates, is of rare occurrence in vivo, but easily recognizable. Cholesterides appear sometimes as droplets presenting the black cross of polarization when viewed at low tempcrature. When temperature is increased they lose birefringence and look like droplets of fat. Birefringence is lost as a result of osmication. Coloration with sudan and mounting in syrup of levulos decreases birefringence. Lison gives following tabular method of analysis (abbreviated).

1. In frozen sections, mounted in levulose syrup, without artificial coloration, generally yellow orange or brown.

2. Iodine - iodide solution (like Gram's or Lugol's) gives black-green or brown. Chromic acid solution decolorizes quickly or slowlycarotinoids.

2. Above reactions negative. Sulphuric acid sometimes gives red color-chromolipoids.

1. In frozen sections show no natural color.

2 Liebermann reaction (Schultze or Romieu techniquo) positive: color blue, purplo or violet, becoming green.

3. Digitonine reaction (Brunswick or LeulierNoel technique) gives crystals strongly illuminated between crossed nicols, unstainable by histological methods-free cholesterol.

3. Digitonine reaction gives no crystalline ppt.-cholesterides.

2. Liebermann reaction negative after repeated attempts, no coloration or brown or red color.

3. Mounted in levulose syrup, without artificial coloration, examined with crossed nicols, brightly illuminated and showing cross of polarization-Lipines.

3. Mounted in same way, without artificial coloration, examined with crossed nicols, not illuminated or illuminated but without showing cross of polarization.

4. Smith-Dietrich reaction at $50^{\circ} \mathrm{C}$, positive, color black-Lipines.

4. Above reaction negative. Coloration gray or absent.

5. Lorrain Smith reaction with nile blue sulphate: rose-non-saturated glyceride.

5. Above reaction absent or blue-Saturated or non-saturated glyceride, or fatty acid or Lipine.

The much used Osmic Acid and Sudan staining methods are helpful when other evidence is available as to chemical constitution of substances demonstrated. Fluorochromes are useful for fluorescent visualization of fats (Metcalf, R. L. and Patton, R. L., Stain Techn., 1944, 19, 11-27). See Fatty Acids, Soaps, Neutral Fats (Glycerides), Lipoids, Cholesterol (free), Cholesterol Esters, Myeloidin, Myelin, etc.

In following up almost any problem in biology and medicine one has to resort to chemical methods and seek the help of chemists. A microtitration technique for lipid in amounts of approximately $10 \mu \mathrm{gm}$. in say $1 \mathrm{mg}$. of tissue has been elaborated by SchmidtNielsen, K. C. rend. trav. lab. Carlsberg, Ser. Chim., 1942, 24, 233-247. In compiling a book like this it is interesting to note how many advances have come from the Carlsberg laboratories.

Lipines, see Lipoids.

Lipiodol, reactions in tissue to fat stains after various fixations (Black, C. E., J. Lab. \& Clin. Med., 1937-38, 23, 1027-1036).

Lipochrin is the term applied to certain usually solitary fatty droplets present in retinal cells of several vertebrates 
but absent in guinea pigs and man. For literature see Arey, L. B. in Cowdry's Special Cytology, 1932, 3, 1219.

Lipochrome. Defined by Lison (p. 244) as a solution of a carotinoid in a fatty body, the latter by itself uncolored, often found in nerve, hepatic, cardiac muscle cells and elsewhere. See Carotinoids.

Lipofuscins are fats colored by the carotene dissolved in them found in nerve, hepatic and cardiac muscle cells (Mallory, p. 125).

Lipoids (G. lipos, fat + eidos, appearance). 'This term is taken to mean almost anything even remotely looking like fat. Generally included under it are lecithin, cephalin, sphingomyelin, kerasin, phrenosin, etc. which cannot be identified microchemically in sections. They are referred to as Lipines by Lison. See his tabular analysis under Lipids. See methods of Ciaccio and SmithDietrich.

Lipolytic Enzymes, see Lipase.

Lipomicrons, small droplets of lipid in circulating blood. See Chylomicrons.

Lipophanerosis is fatty degeneration, see Lipids.

Lipoprotein. Method for separation and isolation from liver cells by differential centrifugation, see Lazarow, A., Anat. Rec., 1942, 84, 31-50; Biological Symposia, 1943, 10, 9-26.

Lison's glycogen method (Lison, p. 227). Fix in dioxan saturated with picric acid, 8.5 parts; formalin, 1 part; and acetic acid, $0.5 \mathrm{cc}$. Pass direct through dioxan, dioxan-paraffin, paraffin, imbed, section and stain in the usual way.

Lithium Carmine 1. To make Orth's lithium carmine dissolve 2.5-5 gms. carmine in 100 cc. sat. aq. Iithium carbonate. Boil for 10-15 min. and, when cool, add a crystal of thymol as an antiseptic. Stain sections about 3 min. Differentiate in Acid Alcohol. Wash in water, dehydrate in alcohol, clear in xylol, or toluol, and mount in balsam. Gives sharp bright red stain of nuclei of ten useful in place of the blue of hematoxylin, of methylene blue, etc. It may be used after almost any good fixative.

2. Lithium carmine has also been employed in many classical experiments as a vital stain (Aschoff, L. and Kiyono, K., Folia Haemat., 1913, 6, 213; Suzuki, T., Nierensekretion, Jena, 1912; Kiyono, K., Die Vitale Karminspeicherung, Jena, 1914, etc.). Filter a sterilized concentrated suspension of carmine rubrum optimum ( $5 \mathrm{gm}$.) in cold sat. aq. lithium carbonate and slowly inject $5-10 \mathrm{cc}$. intravenously in rabbits
(Foot, McClung, p. 115). The Bensleys (p. 151) give the following directions. Cook on water bath $100 \mathrm{cc}$. sat. aq. lithium carbonate $+5 \mathrm{gm}$. carmine rubrum (Grubler) for $\frac{1}{2}-1 \mathrm{hr}$. Filter hot. Allow to settle and cool. Filter cold. Sterilize in autoclave and filter again through sterile filter. Inject intravenously once or more. Kill the animal and fix tissues in alcohol, formalin or formalin-Zenker.

Lithium Silver of Hortega as described by Laidlaw (G. F., Am. J. Path., 1929, 5, 239-247): In 250 cc. glass stoppered bottle dissolve $12 \mathrm{gms}$. silver nitrate, C.P. in 20 cc. aq. dest. Add 230 cc. sat. lithium carbonate, C.P. in aq. dest. Shake well. Let settle to about $70 \mathrm{cc}$ ppt. Decant. Wash ppt. with aq. dest. 3 or 4 times. Decant all except 70 cc. ppt. Add ammonia water (26-28\%) shaking until fluid is nearly clear. Add aq. dest. to total vol. of $120 \mathrm{cc}$. Filter through Whatman filter paper No. 42 or 44 or Schleicher and Schüll No. 589 into stock bottle. See Laidlaw's Methods.

Litmus as a vital indicator of acidity and alkalinity in rats and mice (Rous, P., J. Exper. Med. 1925, 41, 379-397). See Hydrogen Ion Indicators.

Liver. In this very large organ, as in the lungs, it is necessary to carefully select the specimens excised for study. It is bad practice to take only slices vertical to the surface including the capsule. The deeper parts should be included. How the weight and structure of the human liver varies with phases of assimilation and secretion as in rabbits (Forsgren, E., Act. med. Scandin., $1931,76,285-315$ ) and in rats (Higgins, G. M., Berkson, J. and Flock, E., Am. J.' Physiol., 1933, 105, 177-186) remains to be determined. Effect of different dehydration and clearing agents on liver (Ralph, P., Stain Techn., $1938,13,9-15)$. A well illustrated account of the influence of fixatives on liver cells is given by Schiller, W., Zeit. f. Zellf. u. Mikr. Anat., 1930, 11, 63-178.

Locke Solution. As given by Craig, p. 69 as a component of culture medium for amebae it is: $\mathrm{NaCl}, 9.0 \mathrm{gm}$.; $\mathrm{CaCl}_{2}, 0.2$ gm.; $\mathrm{KCl}, 0.4$ gm.; $\mathrm{NaHCO}_{3}, 0.2$ gm.; glucose, $2.5 \mathrm{gm}$; aq. dest. $1000.0 \mathrm{cc}$. sterilized in Arnold sterilizer or in autoclave.

Locke-Lewis solution. $\mathrm{NaCl}, 0.85$ gm.; $\mathrm{KCl}, 0.042 \mathrm{gm}$.; $\mathrm{CaCl}_{2}, 0.025 \mathrm{gm}$.; $\mathrm{NaHCO}_{3}, 0.02 \mathrm{gm}$., dextrose, 0.01-0.25 gm.; aq. dest., $100 \mathrm{cc}$. Should be freshly made. Owing to presence of $\mathrm{NaHCO}_{3}$ must not be sterilized by heat.

Loeffler's Alkaline Methylene Blue. As emended Soc. Am. Bact. A. Methylene 
blue (90\% dye content) $0.3 \mathrm{gm} .+95 \%$ ethyl alcohol, $30 \mathrm{cc}$. B. $0.01 \%$ aq. KOH by weight 100 cc. Mix A and B (McClung, p. 137).

Logwood. This source of hematoxylin, the nost important of the older dyes, was discovered by the Spaniards at the Bay of Campeachy in Mexico and was introduced by them into Europe. Much used in Spain in the 16th century logwood was banned in England by Act of Parliament in 1580 and punishment provided for its use. A hundred years later this Act was repealed and since 1715 the tree has been cultivated in Jamaica (Leggett, W. F., Ancient and Medieval Dyes. Brooklyn: Chemical Publishing Co., Inc., 1944, 95 pp.).

Loose Connective Tissue. Subcutaneous tissue of this sort is often chosen for investigation. It may be dissected out and spread on slides. A good way, demanding practice, is to tease the tissue apart, without the addition of any saline solution, so that one edge is parallel to the end of the slide and about 4 $\mathrm{cm}$. from it. This edge is allowed to dry and become affixed to the slide, while the remainder of the tissue is kept moist and is stretched with needles evenly along the length of the slide into a fairly thin film. This spread is then examined in the fresh state, with various solutions added, or it is fixed and stained like a blood smear. Separation of components into a sufficiently thin spread is facilitated by first making a bulla (L. for bubble) under the epidermis by the local injection of fluid (salt solution, serum, etc.).

Sylvia H. Bensley (Anat. Rec., 1934, 60, 93-109) employed a graphic method for demonstration of ground substance. She adapted a culture of paramoecia to $0.6-0.8 \%$ salt solution, injected subcutaneously into a guinea pig, excised the bulla and examined it as a whole mount with cover glass supported at edges. Actively motile organisms suddenly rebounded without coming into contact with microscopically visible structure and none escaped into the surrounding fluid from the bulla. This is evidence of the existence in loose connective tissue of an amorphous ground substance in the physical condition of a gel. She described, and used to advantage, methods for determination of the refractive index, consistency, digestability and tinctorial properties of this substance in several parts of the body.

Methods for the identification of Collagenic and Elastic Fibers, Fibroblasts, Tissue Basophiles and other constituents are described under the respective headings. See also Tissue Fluid.

Lorrain Smith, see Nile Blue Sulphate.

Lubarsch Crystals are tiny formations oceasionally seen post-mortem intracellularily in testis and said to be different from Charcot's and Spermin Crystals.

Lucas, see Clia, Plastics.

Lucidol, a trade name for benzoyl peroxide.

Lucite, disadvantages of as substitute for Canada balsam (Richards, O. W. and Smith, J. A., Science, 1938, 87, 374). It is used in place of Quartz for transillumination by Williams, R. G., Anat. Rec., 1941, 79, 263-270, and in making containers for museum specimens by Snitman, M. F., Arch. Otolaryng., 1942, 36, 220-225.

Lugol's Iodine. Potassium iodide, $6 \mathrm{gm}$.; iodine, $4 \mathrm{gm}$; aq. dest., $100 \mathrm{cc}$.

Luminescence, Bacterial. Technique for inhibition and leads to literature (Johnson, F. H., Flagler, E. A., Simpson, R. and MeGreer, $K$., J. Cells and Comp. Physiol., 1951, 37, 1-14).

Luminol (3-aminophthalhydrazide) made by Eastman Kodak Co. has a marked affinity for hematin yielding brilliant luminescence in ultraviolet light. Hematin in a dilution of $1: 100,000,000$ can be detected thereby. This is a medicological test of great sensitivity but is not limited to human blood (Proescher, F. and Moody, A. M., J. Lab. \& Clin. Med., 1938-39, 24, 11831189 ).

Lungs. To excise properly pieces for fixation requires great skill especially if lesions are present. The slices should be cut with the sweep of a particularly sharp knife to minimize squeezing and the resultant distortion and displacement of fluids when these are present. The contents of small cavities and bronchi may escape unless care is taken to retain them by immediate coagulation by fixation. Owing to regional differences it is important to select representative areas. To demonstrate the fibrin often present in lesions, Weigert's stain is recommended.

Illumination of circulation in lung by quartz rod (Weaver, J. T. et al. Am. J. Physiol., 1934, 109, 236-256). Observation of lung through thoracic window in vivo ('Terry, R. J., Science, 1939, 90, 43-44), See Alveolar Epithelium, Alveolar Fluid, Alveolar Foam Cells, Alveolar Pores, Alveolus, Ammoniacal Silver, Bronchiolar Epithelium, Carmine Dusting, Celluloid Corrosion, Dust Cells, Gash Irrigation, Heart Failure Cells, Pneumonocytes, Silver Lineation, Tissue Phagocytes, Vacuoloids, Wash-out Recovery Method.

Lungs, Uncollapsed, Fixation-Written by 
C. C. Macklin, Dept. of Histological Research, The University of Western Ontario, London, Canada. November 28,1951 -This may be done (1) by prompt immersion of the fresh flayed intact thorax (IIT) in any good fixative which is adaptable to small animals, such as mice; (2) by perfusion of the blood vessels of the intact thorax of the recently exsanguinated animal (PIT); (3) by injection of the fixative into the trachea with the thorax unopened (BF). This is known as bronchial filling. For technique of methods Nos. 1 and 2 see Dust Cells. For method No. 3 a cannula is tied into the trachea and fixing fluid injected in amount equal to one-third to one-half of the volume of the lung in full expiration. Presence of air does not prevent spread. Fixation is rapid. After tying the trachea the preparation is allowed to stand one to twenty-four hours. This method may be combined with No. 1. Methods 1 and 3 may be used without previous exsanguination. In methods 1 and 2 the capillaries of the alveolar walls are fully opened, but in 3 they are only partly so. Methods 1 and 2 possess the advantage of not having loose particles washed into the lower part of the air tract. Other advantages are considered under "Dust Cells". Methods 1 and 2 demonstrate the close and extensive relation of the capillaries of the alveolar wall to the pneumonocytes (Macklin, C. C., Trans. Roy. Soc. of Can., Sect. V, 1916, 40, 93-111).

Lutecium, see Atomic Weights.

Lutein, see Lillie, p. 129.

Luyet, see Revival after Ultra Rapid Cooling.

Lymphatic Vessels. There are many ways of demonstrating lymphatic vessels. The most convenient is to sit in an easy chair and view the splendid moving picture prepared by Dr. Richard L. Webb of the Department of Anatomy of the University of Illinois College of Medicine entitled: "Mesenteric lymphatics, their conduct and the behavior of their valves in the living rat".

Another easy method is to watch absorption of cream in a cat. A fasting animal is fed $\frac{1}{2}$ pint of cream and the abdominal cavity is opened under ether anesthesia a few minutes later. At first sight it may be difficult or impossible to see any lymphatics in the mesentery although a few bean shaped lymph nodes are visible near its base and can be easily felt. Keep the abdominal contents moist with saline. Close the opening. In a little while, when again examined, the lymphatic vessels will be clearly marked in white by the milk fat which has been absorbed by the lacteals and is being transported in them.

A simple method to visualize the pathways of lymphatic drainage from the nasal mucous membrane has been described by Yoffey, J. M., Lancet, 1941, 1, 529-530. Anesthetize a cat. Drop into each nostril 1 cc. $5 \%$ trypan blue (T. 1824) in physiological saline $(0.85 \%$ aq. $\mathrm{NaCl})$. T. 1824 is specified because it is a trypan blue isomer which is deeply colored even in high dilutions but any good trypan blue will do. Dissect away the side of the neck. Lymphatic vessels, deeply stained, will be seen from the nose and pharynx converging to the deep cervical node and from the posterior border of this node a single deep cervical vessel takes origin and proceeds downward in the neck. The technique delineates a functioning system of vessels actually at work.

Lymphatic vessels and capillaries constitute a drainage system provided in largest measure beneath the external surface of the body and the invaginations of this surface in to it in the respiratory, alimentary and urinogenital systems. They are absent in the brain and bone marrow and rare or absent in skeletal muscle. See detailed information concerning the organ or tissue, in which it is desired to demonstrate them, to be found in Drinker, C. K. and Yoffey, J. M., Lymphatics, Lymph and Lymphoid Tissue. Harvard Univ. Press, 1941, 406 pp.

Methods for the injection of lymphatics involve forcing fluid containing particulate matter into areas where there are many lymphatic capillaries. A technique for the observation in vivo of the superficial lymphatics of human eyelids is described by Burch, G. E., Anat. Rec., 1939, 73, 443-446. 0.02 cc. of a dilute solution of patent blue $\mathrm{V}$ is injected intradermally $5-10 \mathrm{~mm}$. beyond the middle of the lid margin. The lymphatics are apparent in about 5 min. and may be observed as long as 75 min. Consult earlier experiments with this dye by McMaster, P. D., J. Exp. Med., 1937, 65, 347-372.

A good way is to utilize the transparent ears of white mice to inject the lymphatics with hydrokollag by means of a microdissection apparatus (Pullinger, B. D. and Florey, W. H., Brit. J. Exp. Path., 1935, 16, 49-61). But the best available technique is closely to examine over long periods of time living non-injected lymphatics in Sandison chambers in the ears of rabbits (Clark, E. R. and E. L., Am. J. Anat., 1937, 62, 59-92. See India ink method for 
renal lymphatics (Pierce, C. E. 2nd., Anat. Rec., 1944, 90, 315-329).

Lyoglycogen, see Glycogen.

Lyons Blue, see Spirit Blue.

Lymphocytes. There is no specific stain for lymphocytes, but identification is usually easy at least for small lymphocytes. To observe motility, mount fresh blood and ring with vaseline to prevent evaporation. Movements usually begin after the neutrophiles have become active. Examination in the darkfield may be helpful. Mitochondria can be demonstrated easier in lymphocytes by supravital staining with Janus Green than in polymorphonuclear leucocytes because they are not obscured by the specific granulations. In the study of smears the characteristic cytoplasmic basophilia of lymphocytes can be brought out by most of the usual stains (Giemsa's, Wright's). The Peroxidase Reaction of lymphocytes is negative, or very strictly limited. Methods demonstrating Cathepsin, Nuclease, Amylase, Lipase, Lysozyme and Adenosinase in lymphocytes are described by Barnes, J. M., Brit. J. Exp. Path., 1940, 21, 264-275. To determine the age of lymphocytes is extraordinarily difficult. Perhaps the nearest approach to this goal is the work of Wiseman, B. K., J. Exper. Med., 1931, 54, 270-294.

Lysis. In histology this term means the solution of a cell resulting from injury to the cell membrane. A choice may be made of several agents productive of this change. As classified by Danielli (Bourne, pp. 74-75) antibodies and polyhydroxylic phenols probably act almost wholly on the protein component of the membrane; lipoid solvents, lecithinase, digitonin, sodium or potas. sium salts of fatty acids and paraffin sulphonates mainly on the lipoid part; and the heavy metals probably on both. $\mathrm{He}$ suggests the probable modes of action. It is therefore possible that these lytic agents may in their action provide clues as to the nature of the plasma membrane. See Cell Membranes.

Lysozyme a heat and acid resistant enzyme produced from egg white and isolated as a basic protein of small molecular weight by Abraham, E. P., Biochem. J., 1939, 33, 622-630. It is present in many animal and plant tissues. A method for its determination in lymphoeytes and polymorphonuclear leucocytes (neutrophiles) is given by Barnes, J. M., Brit. J. Exp. Path., 1940, 21, 261275). The use of lysozyme as a cytological agent in bacteriology is described by Dubos, R. J., The Bacterial Cell. Harvard Univ. Press, 1945, 460 pp.
Observation that a bacterium is susceptible to lysozyme is an indication that it contains as an essential part of its structure a substrate for this enzyme, probably an acetyl amino polysaccharide.

Lyssa Bodies are small Negri bodies which look optically hyaline, see Negri Bodies.

Maceration (I. macerare, to soak) is a very important technique by which tissues are soaked for considerable periods of time in various fluids which loosen the connections between the cells and allow them to be easily separated for microscopic study. This is a method employed by the great masters in histology which is unfortunately not sufficiently used now-a-days.

For nervous tissue Addison (MeClung, p. 439) recommends Gage's dissociator which is $2 \mathrm{cc}$. formalin in $1000 \mathrm{ce}$. physiological salt solution for 2 or 3 days. After this treatment large ventral horn nerve cells can easily be dissected out with the aid of a binocular microscope, stained with carmine, picrocarmine or a dilute anilin dye and viewed as units with parts of their processes attached.

Smooth muscle of the bladder is well dissociated by $10-20 \%$ nitric acid (Dahlgren, in McClung, p. 423). The resulting fibers are suitable for class use.

Thyroid follicles are freed from the surrounding tissue and can be examined individually after maceration in conc. hydrochloric acid 3 parts and aq. dest. 1 part for about $24 \mathrm{hrs}$. and thorough washing in at least 10 changes of tap water (Jackson, J. L., Anat. Rec., 1931, $48,219-239$ ).

Epidermis can be separated from dermis by maceration in $1 \%$ acetic acid, see epidermis.

Kidney tubules. Pieces of kidney fixed in $10 \%$ formalin or in Kaiserling's solution are placed in conc. hydrochloric acid at room temperature until they become sufficiently softened after 2-7 days. The time depends upon size of piece, degree of fibrosis and other factors. There is no advantage in using fresh tissue. When adequately macerated the almost diffluent tissue is washed in repeated changes of aq. dest. in which it may be kept for several days. Dissect out individual tubules with the aid of a binocular microscope (Oliver, J. and Luey, A. S., Arch. Path., 1934, $18,777-816)$.

Seminiferous tubules. Whole human testicles are fixed in formalin. They are then cut into segments $1 \mathrm{~cm}$. thick parallel to direction of the lobules. The tunica vaginalis is not removed but is slit through in one or two places with a 
razor. Each segment is placed in conc. hydrochloric acid, 75 cc., aq. dest. 25 cc. 1-7 days. Heat just below boiling 20-30 min. Tissue shrinks, turns dark brown and sof tens. A sediment collects in the dish. Part of acid is drawn off with a pipette, boiled water is added and the process is repeated until practically all of the acid is removed. The water is boiled to prevent formation of air bubbles along the tubules. It turns the tubules a yellowish white color in which condition they should be isolated by careful teasing. When the tubules cannot be easily lifted away from one another, the maceration is insufficient. When, on the other hand, they break it is a sign of over maceration (Johnson, F. P., Anat. Rec., 1934, 59, 187-199). A similar method was used by Johnson in 1916 to separate the lobules of the pig's liver.

Bone cells and lamcllae. Treat a thin bone section with conc. nitric acid as long as $24 \mathrm{hrs}$. Mount on a slide and squeeze out bone cells by pressure on cover glass. The lamellae can be pealed off easily from a piece of decalcified bone which has been gently boiled in water (Shipley, in McClung, p. 348).

Enamel rods. A piece of dental enamel is dissociated with 5-10\% hydrochloric acid for 24 hrs. When it has become soft, remove a little with a needle to a slide and tease out. Mount in physiological salt solution under a cover glass. Draw through a little carmine stain with a blotter and wash it out with $10 \%$ acetic acid. The specimen can be ringed with paraffin (Churchill, and Appleton, in McClung, p. 372).

Nerve cells. Pieces of gray matter of ventral horn are soaked for 2 or 3 days in 0.02 formalin. The tissue softens, the cells are dissected out and stained with carmine or picro-carmine (Addison, in McClung, p. 439).

MacNeal's Tetrachrome is a blood stain containing eosin, methylene azure A, methylene blue and methylene violet. It is employed like Wright's stain. For details see MacNeal, W. J., J. A. M. A., $1922,78,1122$, and Conn, H. J., Stain Technology, 1927, 2, 31 .

Macrophages. These are the free cells of the reticulo-endothelial system. Almost any method of exposure to relatively non-toxic, finely particulate matter is sufficient to bring them out. The simplest way is to inject mice with trypan blue as described under Vital Staining and to look for the macrophages in spreads of Loose Connective Tissue. Another method, used by
Maximow, is to give rabbits intravenous injections of saccharated iron oxide or India ink and to examine blood from right ventricle in smears (see Cowdry's Histology, p. 69). Lines of division between macrophages and monocytes, if they exist, are difficult to establish. Supravital staining with Neutral Red and Janus Green is useful to demonstrate neutral red granules and mitochondria respectively.

Madder Staining of bone. Madder is a red dye, prepared from the plant Rubia Tinctorum which has been used for thousands of years. It is perhaps the first dye to be used in camouflage in war. With its help Alexander defeated the Persians by staining the clothing of his Greek soldiers red, each garment in a different part so that the Persian leaders at once concluded that all they had to cope with was an already well damaged army. (Leggett, W. F., Ancient and Medieval Dyes. Brooklyn: Chemical Publishing Co., Inc. 944, 95 pp.)

Alizarin and purpurin, formed from madder, are now made synthetically. Madder should be employed for the vital staining of growing bone as described by Macklin (C. C., Anat. Rec., 1917, 12, 403-405; J. Med. Res., 1917. $36,493-507)$. Young rats are suggested as material. Each should eat 1-5 gms. of madder, thoroughly mixed with its food, daily. The calcium deposited in the growing bone while madder is thus made available in the circulation is colored red. Staining is noticeable after 1 day but the feeding should be continued for a week or more.

The ventral ends of the ribs and the epiphyseal lines of long bones are most intensely colored. The bones selected are fixed in $10 \%$ neutral formalin, washed and cleaned in water, dehydrated thoroughly in alcohol, placed in benzene for $24 \mathrm{hrs}$, cleared in oil of wintergreen by the method of Spalteholz and examined with binocular microscope as whole objects.

Chemistry of madder staining is discussed by Dr. Richter (Biochem. J., 1937, 31, 591-595). The substance giving the intense carmine red color is apparently purpurin carboxylic acid. Madder is one of the most classical of stains. Its history extends back through the centuries and has been well reviewed by F. T. Lewis (Anat. Rec., 1942, 83, 229-253). See Line Test.

Magdala Red (CI, 857)-naphthalene pink, naphthalene red, naphthylamine pink, sudan red-According to Conn (p. 102) this basic naphtho-safranin differs from commercial magdala red which is an acid dye belonging to an entirely dif- 
ferent group. He calls attention to its use by Kultschitzky, N., Arch. f. Mikr. Anat., 1895, 46, 673-695) in staining elastic tissue of the spleen. Used as a fluorochrome for Lipids.

Magenta, see Basic Fuchsin.

Magenta II is triamino ditolyl-phenylmethane chloride probably present in most samples of Basic Fuchsin. See Pararosanilin (Magenta O), Rosanilin (Magenta I) and New Fuchsin (Magenta III).

Magnaflux is a useful instrument employed in the FBI Laboratory to detect the occurrence of small cracks and defects in the surface of metallic objects. When, for example, a magnetizable object is placed in a magnetic field, created by the magnaflux, the field is distributed throughout the metal if it is sound. Otherwise, magnetizable pigments become oriented around the breaks in the surface indicating their location (Hoover, J. E., Scientific Monthly, 1945, 60, 18-24). Obviously metallic laboratory equipment can be tested in this way.

Magnesium, Titan yellow method for determination of small amounts in body fluids (Haury, V. G., J. Lab. \& Clin. Med., 1938, 23, 1079-1084).

Methods for detection in plant cells (Broda, B., Mikrokosmos, 1939, 32, 184). (1) Triturate 1 part quinalizarin with 5 parts sodium acetate crystals. Make to fresh $0.5 \%$ solution in $5 \%$ aq. $\mathrm{NaOH}$. Addition of 1-2 drops to paraffin section, then 1-2 drops $10 \% \mathrm{NaOH}$ results after some hours in blue stain. (2) Add to paraffin section 1-2 drops $0.2 \%$ aq. Titan yellow, then 1-2 drops $10 \% \mathrm{NaOH}$ gives rise to brick red stain of magnesium. (3) Add to paraffin section $0.1 \%$ aq. azo blue. Gives, without the $\mathrm{NaOH}$, a violet stain of magnesium. An attempt should be made to adjust these techniques to human tissues in which a magnesium salt has been injected.

By means of a specially constructed electron microscope Scott and Packer (G. H. and D. M., Anat. Rec., 1939 , $74,17-45)$ have accurately localized magnesium and/or calcium in muscle. The method can be extended to other tissues and perhaps to other minerals. Histospectrography gives data on the amount of magnesium relative to the other minerals in the skin of normal and neurodermatitis patients. In the latter there is a magnesium deficiency (MacCardle, R. C., Engman, M. F., Jr. and Sr., Arch. Dermat. and Syph., 1941, 44, 429-440).

If it is desired to supplement microscopic and spectrographic detection of magnesium by quantitative analysis of very small amounts of tissue a tech. nique of microdermination with the polarograph devised by Carruthers, C., Indust. and Engin. Chem., 1943, 15, 412-414 will be useful. It has been employed for analysis of pure epidermis by Carruthers, C., and Suntzeff, V., Cancer Research, 1943, 3, 744-748. See Calcium 5.

The Spectrophotometric determination of magnesium in human serum has been advanced by Craig, P., Zak, B., Iseri, L. T., Boyle, A. J. and Myers, G. B. (Am. J. Clin. Path., 1951, 21, 394-398) through the making of several innovations: (1) destruction of protein by nitric acid digestion, (2) use for stabilizer of a sodium lauryl sulfate-polyvinyl alcohol reagent, (3) choice of a 550 $\mathrm{m} \mu$ wave length for quantitation and (4) the preparation of calibration curves from standards containing urea and electrolytes normally occurring in plasma. It would seem feasible by different calibration and other adjustments to utilize this technique for the estimation of magnesium in fluids other than serum and perhaps in tissue homogenates.

Magnetic Particle Technique to investigate the physical properties of the cytoplasm of living cells as determined by movements of phagocytized particles of various sorts in a magnetic field (Crick, F. H. C. and Hughes, A. F. W., Exp. Cell Res., 1950, 1, 37-80). This appears to be a very promising method.

Malachite, a mineral mined by the Egyptians, and applied as a powder gave a green pigmentation about the eyes. It is said to be the oldest coloring matter known to them (Leggett, W. F., Ancient and Medieval Dyes. Brooklyn: Chemical Publishing Co. Inc., 1944, 99 pp.).

Malachite Green (CI, 657)-diamond green $\mathrm{B}, \mathrm{BX}$ or $\mathrm{P}$ extra, light green $\mathrm{N}$, new Victoria green extra, O, I or II, solid green $\mathrm{O}$, Victoria green $\mathrm{B}$ or WBCommission Certified. A feebly basic di-amino tri-phenyl methane dye quite extensively employed as a counterstain for safranin or carmine.

Malachite Green G, see Brilliant Green.

Malarial Pigment. Produced in erythrocytes by action of the parasites, black and distinguishable from carbon by its solubility in concentrated sulphuric acid. Among distinguishing characteristics given by Lison (p. 254) are solubility in dilute alkalis, argentaffine reaction negative, specific stains for lipids negative, likewise reactions for iron. But Morrison and Anderson (D. B. and W. A. D., Public Health Rep., 
1942, 57, 90-94) find that when the pigment within the parasites (Plasmodium Knowlesi) is extracted in such a way as not to influence the spectra of hemoglobin it can be identified spectrophotometrically as ferrihemic acid, or hematin, which does contain iron.

Malaria Plasmodia. Technique of examination of process of "exflagellation" (Anderson, Ch. W. and Cowdry, E. V., Arch. de l'Inst. Pasteur de Tunis, 1928, 17, 46-72), of quantitative determinations of gametocytes (Cowdry, E. V. and Covell, W. P., Ibid., 147-456) and of demonstrating neutral red granules and Golgi apparatus (Cowdry, E. V. and Scott, G. H., Ibid., 233-252).

For staining the plasmodia in smears, see Giemsa, Jenner, Marino, Nocht, Plehn, Wilson and Wright's stains. A simple method for staining plasmodia in paraffin sections is described with numerous illustrations by Tomlinson, W. J. and Grocott, R. G., Am. J. Clin. Path., 1944, 14, 316-326. The Barber Komp thick film method is strongly recommended for surveys.

Serlin, N. J. and Lissa, J. R., Am. J. Clin. Path., 1912, 6, 8 advise the following method when diagnosis depends on finding gametocytes, or malarial pigment, in peripheral blood. Completely evaporate 1 cc. $1 \%$ aq. potassium oxalate in a $15 \mathrm{cc}$. centrifuge tube. Add 10 cc. venipuncture blood. Mix carefully and centrifuge $30 \mathrm{~min}$. at 2,500 R.P.M. Pipette off all but about $\frac{1}{8}$ in. of supernatant plasma. Smear on 2 slides by wiping buffer layer with stick applicator having non-absorbent cotton tip. Stain by Wright's method. Study of Giemsa stained smears by dark field is suggested (Goosmann, C., J. Lab. \& Clin. Med., 1935-36, 21, 421-424). See Protozoa.

Taylor, D. J., Greenberg, J. and Josephson, E. S. (J. Lab. Dis., 1951, 88, 158-162) describe a useful method for the maintenance of intraerythrocytic forms of Plasmodium gallinaceum in a whole medium on vitro.

Mallory's Connective Tissue Stain. This is name usually given to his anilin blue-acid fuchsin-orange G stain. See also his Phosphomolybdic and Phosphotungstic Acid Hematoxylin Stains. (Mallory, p. 155). Fix in Zenker's fluid. Imbed in paraffin or celloidin. Remove mercury from sections with iodine or $0.5 \%$ sodium hyposulphite. Stain in $0.5 \%$ aq. acid fuchsin, $1-5 \mathrm{~min}$. Drain off stain and put in : anilin blue, water soluble, $0.5 \mathrm{gm}$.; orange $\mathrm{G}, 2 \mathrm{gm}$.; $1 \%$ aq. phosphotungstic acid, 100 cc., 20 min. or longer. Rinse in $95 \%$ alc.
2 or 3 changes until no more stain is removed. Dehydrate in abs. alc., clear in xylol, mount in neutral balsam. For celloidin sections, reduce staining time and pass from $95 \%$ alc. to terpineol and mount in balsam. This is one of the most beautiful of all stains and is very widely used. Collagenic fibrils blue, fibroglia, neuroglia and myoglia fibrils red, elastic fibrils pink or yellow. In McClung, p. 405, Mallory and Parker advise $0.25 \%$ aq. acid fuchsin and staining in the anilin blue mixture for 1-24 hrs. or for $1 \mathrm{hr}$. in paraffin oven at $60^{\circ} \mathrm{C}$. The modifications of this stain are almost endless.

Adaptation to formalin fixed material is often desirable. Kernohan (J. W., J. Tech. Meth., 1934, 13, 82-84) has outlined the following method of doing this by mordanting. Wash formalin fixed tissue in running water or in ammonia water for short time. Place in Weigert's primary mordant-potassium bichromate, 5 gm.; chromium fluoride, $2 \mathrm{gm}$. and aq. dest. $100 \mathrm{cc}$.for 4 days and in his secondary mordant - copper acetate, $5 \mathrm{gm}$.; chromium fluoride, $2.5 \mathrm{gm}$; acetic acid $(36 \%)$, 5 cc.; aq. dest., 100 cc. and formol, $10 \mathrm{cc}$.- for 2 days. Imbed in paraffin in the usual way.

Rexed, B., and Wohlfart, G., Zeit. wiss. Mikr., 1939, 56, 212-215 suggest control of $\mathrm{pH}$ of the acid fuchsin. It is stated that fresh $0.1 \%$ acid fuchsin has $\mathrm{pH} 4.49$ and that increase in alkalinity makes it defective. To prepare one at $\mathrm{pH} 3.29 \pm 0.01$, which is recommended, take acid fuchsin $1 \mathrm{gm}$.; N/10 HCl, 60 cc.; aq. dest. 900 cc.; Störensen's citrate (citric acid crystals, 21 gm.; N/1 $\mathrm{NaOH}, 200$ cc.; + aq. dest. to make 1000 cc.), 40 cc. Most tissues stain in range $\mathrm{pH} 3-4$, red blood cells alone at $\mathrm{pH}$ 5-7.

In 1936, Mallory considered (Stain Tech., 11, 101-102) the most important modifications of his stain to be Heidenhain's Azocarmine (Azan), the LeeBrown and Masson Trichrome methods. See Crossman's modification and Pituitary for special adaptations.

Mallory-Heidenhain Rapid One-Step Stain for Connective Tissue-Written by Jane E. Cason, Dept. of Pathology, Medical College of Alabama, Birmingham, Ala. January 27, 1951-Although innumerable modifications of Mallory's aniline blue collagen stain for tissue sections prevail, this procedure appears to be an improvement over the original and subsequent modifications.

The necessity for adjusting the intensity of the aniline blue and the acid fuchsin in routine staining suggested 
the possibility of combining the two original solutions at a modified ratio. The results proved quite successful, and three definite advantages of this procedure were apparent: 1) Only one step is involved. 2) sufficient and desirable coloration is accomplished in five min. 3 ) The intensity of the color is consistent.

The coloration appears the same as that listed by Mallory: Collagenous fibrils stain intense blue; ground substances of cartilage and bone, mucus, amyloid and certain other hyaline substances stain varying shades of blue; nuclei, fibroglia, myoglia and neuroglia fibrils, axis cylinders and fibrin stain red; nucleoli, red blood corpuscles and myelin stain yellow; elastic fibrils stain pale pink or yellow. This technique is suggested for routine pathologic staining because it is simple to follow, rapid and consistent.

To make stain add to 200 cc. aq. dest.

Phosphotungstic acid crystals (Merck)... $1 \mathrm{gm}$. Orange G (C. I. No. 27) ............. 2 gm.

Aniline blue, W. S. (C. I. No. 707)...... $1 \mathrm{gm}$.

Acid fuchsin (C. I. No. $692 \ldots \ldots \ldots \ldots .3 \mathrm{gm}$.

Formalin-Zenker is the preferred fixative, but Bouin's, formalin, and alcohol have been used with success. Embed tissue in paraffin, and eut sections at $6 \mu$.

1. Deparaffinize in xylene, pass through graded alcohols to tap water. (If formalin-Zenker is the fixative, treat with iodine and sodium thiosulfate.)

2. Stain sections $5 \mathrm{~min}$. in staining solution.

3 . Wash in running tap water 3 to 5 sec.

4. Dehydrate rapidly through $95 \%$ and $100 \%$ alc., clear in xylene, and mount in balsam.

Mammary Glands. These can be studied in sections by methods intended to reveal the particular data sought. For general purposes Hematoxylin and Eosin, Mallory's Connective Tissue Stain, or Phloxine-Methylene Blue is recommended after Zenker fixation. For fat use Sudan Black and Oil Red O on frozen sections after fixation in $10 \%$ formalin or examine in paraffin sections after fixation in Flemming's fluid or some other osmic acid containing mixture.

In the case of the small glands of mice, rats, rabbits and other mammals the method of making whole mounts is invaluable in investigations of the responses of mammary glands to endocrine stimulation. The following is essentially the same technique as that originally described by Turner, C. W. and Gardner, W. U., Agri. Exp. Res.
Stat. Bull., Univ. of Mo., 1931, 158, 1-57: Remove skin and mammary gland. Stretch out and fasten on a cork block with the external surface of the skin down. Fix in Bouin's fluid 24 hrs. Wash in tap water. Dissect away all tissue over the gland which has been tinged light yellow by the picric acid in the fixative. Remove the gland from the skin. Stain in Mayer's Hemalum. Wash in $1 \%$ aq. potassium alum and then in water. Differentiate in $70 \%$ alc. + $2 \%$ of hydrochloric acid until the color has been removed from the connective tissue and the acini and ducts of the glands show up in sharp contrast in a light background. Wash in tap water. Dehydrate in alcohol, clear in xylol, mount in balsam between glass plates and close the edges with sealing wax. Much can be made out when magnified only 2-5 times. Small pieces can be mounted on slides, with edges of cover glasses supported as may be necessary, for examination at higher magnifications. There are many excellent pictures in the paper cited.

For examination of fetal mice, see Turner, C. W. and Gomez, E. T., ibid, $1933,182,1-43$. Valuable data are given in Turner's chapter on mammary glands in Allen's Sex and Internal Secretions, Baltimore: Williams \& Wilkins, 1939, $1346 \mathrm{pp}$. For techniques to reveal secretory phenomena in mammary glands, see Weatherford, H. L., Am. J. Anat., 1929, 44, 199-281; Jeffers, K. R., Am. J. Anat., 1935, 56, 257-277, 279-303. Technique for localizing site of fat formation in mammary glands is given by Kelly and Petersen, J. Dairy Sci., 1939, 22, 7. The differential staining of sections of unpreserved bovine udder tissue is to be found in U. S. Dept. of Agri. Circular No. 514, under authorship of W. T. Miller and H. W. Johnson. A method for obtaining serial slices of whole human breasts is described by Ingleby, H. and Holly, C., J. Tech. Meth., 1939, 19, 93-96.

Manchester Blue (British Drug Houses Ltd), a dis-azo dye of the benzidine series. In either alcoholic or aqueous solution it gives a sharp deep blue effect (H. G. Cannan, J. Roy. Micr. Soc., 1941, 61, 88-94).

Manchester Brown, see Bismark Brown Y. Manchester Yellow, see Martius Yellow. Mandarin G, sec Orange II.

Manganese. Histochemical detection uncertain (Lisorr, p. 9S).

Manganese Dioxide. Drinker, C. K. and Shaw, L. H., J. Exper. Med., 1921, 33, 77-98 employed a suspension of fine particles in acacia water to investigate phagocytic power of endothelium be- 
cause the particles can be seen within the cells and the amounts of manganese in the tissues can be determined by chemical analysis.

Mann's Fixative is equal parts $1 \%$ aq. osmic acid and sat. corrosive sublimate in physiological salt solution $(0.85 \% \mathrm{NaCl})$. It is a good way to apply osmic acid for the blackening of fat.

Mann's Methyl Blue-Eosin Stain. This is used for protozoa and for inclusions caused by viruses. Sections are deparaffinized, stained $12 \mathrm{hrs}$. in $1 \%$ aq. methyl blue $35 \mathrm{cc}$., $1 \%$ aq. eosin 45 cc. and aq. dest. 100 cc. They are then rinsed in $95 \%$ alc., dehydrated cleared and mounted. See Alzheimer's Modification of Mann's method.

Manometer for capillary blood pressure, see Landis, E. M., Am. J. Physiol., $1926,75,548$

Marchi Method. For degenerating nerve fibers. Modification by Swank, R. L. and Davenport, H. A., Stain Techn., $1935,10,87-90$. Details provided by Dr. J. L. O'Leary. Degeneration time of approximately 14 to 20 days. Kill animal by overdose of nembutal or some other barbiturate given intraperitoneally. Open left ventricle, insert cannula into aorta and perfuse with $2.5-5 \%$ anhydrous ( $10 \%$ crystalline) magnesium sulfate solution containing $2-3 \%$ potassium bichromate. Immediately afterwards remove the brain and spinal cord and put into $10 \%$ formalin for $48 \mathrm{hrs}$. Place slices $3 \mathrm{~mm}$. thick directly, without washing, in: $1 \%$ aq. potassium chlorate, 60 cc.; $1 \%$ aq. osmic acid, 20 cc.; glacial acetic acid, 1 cc.; $37 \%$ formaldehyde (Merck's reagent), $12 \mathrm{cc}$. Use about 15 volumes of this fluid to 1 of tissue. Agitate and turn over daily. After staining for 7-10 days, wash in running water, 12-24 hrs., dehydrate in $70 \%$ and $95 \%$ and absolute alcohol and imbed in low viscosity nitrocellulose as described by Davenport, H. A. and Swank, R. L., Stain Tech., 1934, 9, 134-139. See Celloidin Imbedding. Cut $40 \mu$ sections serially, mount on slides, dehydrate to toluol, placing chloroform in absolute alcohol since low viscosity nitrocellulose is soluble in absolute alcohol. Clear in toluol. Mount in clarite $\mathrm{X}$ dissolved in toluol. See these authors (Stain Techn., 1935, 10, 45-52) for artifacts and effects of perfusion in Marchi technique. Rasmussen, G. L., Anat. Rec., 1944, 89, 331-338 has elaborated a very useful cellophane strip method for preparation and study of Marchi serial sections.

Marchi's Fluid. Müller's Fluid, 2 parts; $1 \%$ osmic acid, 1 part. Fix 5-8 days; wash in running water. Employed to blacken degenerated nerve fibers. See Nerve Fibers.

Method, underlying mechanisms involved (Swank, R. L. and Davenport, H. A , Stain Techn., 1934, 9, 11-19; $1935,12,45-52$ ).

Marine Blue V, see Anilin Blue.

Marino's Stain for malaria plasmodia is described in detail by Craig, p. 286 who states that it gives excellent results; but, owing to its complexity, is little used for routine blood examinations.

Marrow, see Bone Marrow.

Marshall Red (British Drug Houses Ltd), a disazo dye. Stain sections in sat. aq. solution $20 \mathrm{~min}$. Rinse in aq. dest. Stain in sat. Victoria Green G in $70 \%$ alcohol $30 \mathrm{~min}$. Rinse in $95 \%$ alcohol, dehydrate, clear and mount in usual way. Myofibrils sage green, nuclei crimson. Advised also for retina (H. G. Cannan, J. Roy. Micr. Soc., 1941, 61, 88-94).

Martius Yell ow (CI, 9)-Manchester yellow, naphthol yellow-An acid nitro dye employed by Pianese (G., Beitr. z. Path. Anat. u. Allg. Path., 1896, Suppl. I, 193 pp.) for investigating cancer tissue in association with acid fuchsin. Conn (p. 44) reports good results in staining of plant tissue with CC product.

Masson's Gelatin Glue. Method for making sections stick to slides (Masson, P., Am. J. Path., 1928, 4, 181-212). Dissolve $0.05 \mathrm{gm}$. sheet gelatin in 20 cc. aq. dest., warming gently. Filter a large drop on each slide on warm plate. Float paraffin sections on drops. When drops spread place slides upright to drain but do not permit drying. Blot and transfer to dish containing formalin (so arranged that vapor only will act on slides) in oven $45-50^{\circ} \mathrm{C}$. For subsequent staining 20 minutes in hot vapor is enough. For silver treatment overnight is suggested.

Masson's Trichrome Stain-Written by Pierre Masson, Dept. of Pathology, University of Montreal, Montreal, Canada. October 24, 1951-The principle established by F. B. Mallory in his famous method of employing acid fuchsin, phosphomolybdic acid, anilin blue, orange $\mathrm{G}$. can be advantageously applied to other acid dyes yielding a more specific staining of the chromatin. It then gives a very instructive combination of tints.

Due to the intensity of the staining, the sections must be thin; $5 \mu$ is optimum. In order to prevent swelling of the collagen and its deformation during the desiccation, the sections must not be left too long $(20-30$ sec.) on the warmed water, or gelatin, especially 
after fixation by picro-formol (Bouin) or formalin. So altered, the collagen does not properly absorb the dyes and differentiates badly. Trichrome stains with iron haematoxylin. All trichromic methods are based upon a common first step: the staining of nuclei by iron alum haematoxylin, followed by differentiation with picric alcohol.

Three solutions are required: $A$. Iron alum, violet crystals, $5 \mathrm{gm}$., aq. dest. $100 \mathrm{cc}$. B. Regaud's hematoxylin solutiin made up by dissolving $1 \mathrm{gm}$. hema toxylin in 60 cc. hot aq. dest. Cool and add $10 \mathrm{cc}$. glycerin and $10 \mathrm{cc}$. of $95 \%$ alcohol. This stain is ready for use at once. C. Differentiating mixture consisting of 2 parts sat. picric acid in $95 \%$ alcohol and 1 part of $95 \%$ alcohol.

Step 1. Place iron alum solution and the hematoxylin solution in staining jars in a water bath heated to $40-45^{\circ} \mathrm{C}$. Mordant deparaffined sections in heated iron alum, $15 \mathrm{~min}$. Rinse with aq. dest. Stain in heated hematoxylin, 15 min. or more. Rinse the uniformly black sections with $95 \%$ alcohol and immerse in picric alcohol. Control the progress of differentiation under the microscope. As soon as the nuclei alone remain colored, wash with running water for $15 \mathrm{~min}$.

If after washing, the background of the preparation, particularly the collagen remains gray, rinse with alcohol and complete picric differentiation. The chromatin is electively black and opaque.

Various authors have proposed to "simplify" this method by staining the nuclei with Weigert's iron perchloride hematoxylin or with alum hematoxylin. I must say that I have long ago tried such modifications before adopting the above method and that I have abandoned them entirely. The red of the ponceau-fuchsin solution superposes itself on the gray or blue color imparted by hematoxylin so that chromatin takes a dull color lacking in specificity. Rather than to use such modifications it would be better to omit all nuclear staining and start with the next step (ponceau-fuchsin and so forth): the results are thus comparable to those obtained by Mallory's original method.

Step 2. Stain in a mixture of acid fuchsin and Ponceau of xylidin BS (J. R. Geigy, S. A. Basel). No other Ponceaux I have used, French, German or American give results comparable to that of Geigy's.

Three solutions are required: $A$. Ponceau, BS (Geigy), 1 gm., aq. dest. 100 cc., glacial acetic acid $1 \mathrm{cc}$. B. Acid fuchsin (National Anilin Co., New
York), 1 gm., aq. dest. 100 cc., glacial acetic acid 1 cc. 1 part of $A$ with 2 parts of $B$. C. Aq. dest. 100 cc., glacial acetic acid 1 cc. D. Aq. dest. 100 ec., phosphomolybdic acid $1 \mathrm{gm}$.

Place sections stained with the iron hematoxylin in the $A B$ mixture, $5 \mathrm{~min}$. Rinse them with $C$. Transfer them to $D$ in a Copeland or Borrel jar at $40^{\circ} \mathrm{C}$. $5 \mathrm{~min}$. or more. The collagen should remain perfectly colorless.

Step 3. Stain the collagen with anilin blue or Fast green. To make the former dissolve $2 \mathrm{gms}$. anilin blue ( $\mathrm{Na}$ tional Anilin Co., New York) in $100 \mathrm{cc}$. warmed aq. dest. Cool and add 1 cc. glacial acetic acid. To make the latter dissolve $1 \mathrm{gm}$. fast green (National Anilin Co., New York) in 100 cc. aq. dest. and add 1 cc. glacial acetic acid. After the phosphomolybdic differentiation (Step $2 D$ ) rinse the sections in acetic water $C$. Pour on them 8 to 10 drops of the anilin blue or fast green solutions 2 to $3 \mathrm{~min}$. Wash in acetic water $C 2$ to $3 \mathrm{~min}$. Dehydrate with absolute alcohol, clear in toluol or xylol, mount in balsam or permount. Total length of this technique is approximately 60 minutes. Chromatin is black, cytoplasms stain in various shades of red, granulations of eosinophiles and mast cells stain ruby red, erythrocytes are black, elastic fibers stain red, collagenic fibers and mucus stain dark blue (with anilin blue) or green (if Fast green is used).

To this relatively fast technique, I prefer a slow one, based on the use of diluted solutions of Ponceau-fuchsin, anilin blue or fast green.

After nuclear staining with iron alum hematoxylin (Step 1) wash and immerse the slides in 1 part $A B$ mixture and 9 parts $1 \%$ acetic acid, $30 \mathrm{~min}$. Rinse in acetic water $C, 1 \mathrm{~min}$. Transfer the sections into $D$ at $40^{\circ} \mathrm{C} .5 \mathrm{~min}$. or more. Rinse in acetic water $C$. Stain Anilin blue solution 1 part (or Fast green solution 1 part) with acetic water $C 9$ parts, 15 to 30 minutes. Rinse in acetic water. Dehydrate with absolute alcohol, clear in toluol or xylol, mount in balsam or permount.

The results are grossly the same as after the rapid method, but more delicate and precise. Moreover, the staining of the collagen is slow and progressive and can be stopped at the most favorable step; in many circumstances an excessive staining of collagen masks some fine details, for example the fine prolongations of the connective tissue cells.

N.B. After staining of collagen with Fast green, it is preferable to mount in 
salicylic balsam or salicylic permount. Spread a drop of saturated solution of salicylic acid in toluol upon a cover slip. Dry. The glass is then covered with a thin crystalline layer of salicylic acid. Put on it a drop of balsam or permount and cover the section taken out of the toluol (or xylol). Salicylic acid instantaneously redissolves in the mounting medium. This addition of salicylic acid is not advantageous after staining with anilin blue.

Mast Cells, see Basophile Leucocytes and Tissue Basophiles.

Mastigophora, Techniques for given by McClung, Microscopical Technique, 1950, p. 469 .

Mastoid Process. Use methods for Bone. Technique for measurements of size of air cell system is given by Diamant, M., Acta Radiol., 1940, 31, 543-548.

Mauveine (CI, 846 ), a basic dye of light fast ness 3 , the first dye made from aniline in 1856. Gives stain of plant tissues like Nethyl violet (Emig, p. 57).

Maximow (see Azure II Eosin Hematoxylin method). He has advised as a fixative $90 \mathrm{cc}$. Zenker's fluid less acetic acid + 10 cc. formalin. This is essentially Formalin Zenker. See Buzaglo's connective tissue stain.

May-Giemsa stain of Pappenheim (Folia Haematol., Arch., 1917, 22, 15). This is the same as Jenner-Giemsa. Fix and stain air dried blood smears about 3 min. in May-Grünwald mixture (sat. sol. methylene blue eosinate in methyl alcohol). Add same amount aq. dest. and leave $1 \mathrm{~min}$. Pour off (but do not wash) and add diluted Giemsa's solution. Stain in this $15-30 \mathrm{~min}$. Rinse aq. dest. 1 min. or until desired color is reached. Blot dry. This is a good modification of the ordinary Giemsa's stain because it gives slightly more intense colors.

May-Grünwald combined fixative and stain is a sat. sol. of methylene blue eosinate in methyl alcohol (Grübler or Hollborn). If methylene blue eosinate is not available make it as originally described by Jenner (Lancet, 1899, No. 6, 370 ). Mix equal parts $1.25 \%$ water sol. eosin and $1 \%$ methylene blue; after 24 hrs. filter; wash ppt. on filter with water; dry and dissolve powder in 200 cc. pure methyl alcohol. It is employed in the May-Giemsa and Kardos-Pappenheim methods for staining blood smears.

May-Grünwald-Giemsa stain in one solution. Strumia (M. M., J. Lab. \& Clin. Med., 1935-36, 21, 930-934) gives directions for combining the stains and for use and notes that a standardized product is prepared by Coleman and
Bell Co. Intensity of coloration is enhanced by the combination.

Mayer's Acid Alum Hematoxylin. The following formula is given by Mallory (p. 73). Dissolve $1 \mathrm{gm}$. hematoxylin in $1000 \mathrm{cc}$. aq. dest. with a little heat if required. Add $0.2 \mathrm{gm}$. sodium iodate and $50 \mathrm{gm}$. ammcnium or potassium alum. When latter is dissolved add 1 gm. citric acid and $50 \mathrm{gm}$. chloral hydrate. Color turns reddish violet. Does not easily over-ripen.

Mayer's Acid Carmine. The Bensleys (p. 131) advise its preparation as follows. Add $4 \mathrm{gm}$. carmine to $15 \mathrm{cc}$. aq. dest. +30 drops hydrochloric acid. Boil until it is dissolved. Add $95 \mathrm{cc}$. $85 \%$ ethyl alcohol. Neutralize with ammonia until the carmine begins to precipitate as seen in a graduate against white paper background. Add 4 more drops ammonia after first precipitation. If this acid carmine stains too quickly, slow it down by dilution with 80-90\% alcohol. This gives a fine red nuclear counterstain for tissues vitally stained with Indigo-Carmine, Trypan Blue and similar dyes.

Mayer's Albumen, see Albumen Glycerin.

McIlvaine Buffers after Stitt from Lillie, R. D., Stain Techn., 1941, 16, 1-6 who employed them to improve Romanowsky staining after various fixatives. See Toluidine Blue Phloxinate Method. (see Molecular Solution) To make M/15 citric acid required dissolve 14.01 gm. mono-hydrated crystalline citric acid in 500 cc. aq. dest. and add enough neutral methyl alcohol C.P. to make total volume 1,000 cc. after careful mixing. To make $\mathrm{M} / 15 \mathrm{Na}_{2} \mathrm{HPO}_{4}$ dissolve $9.47 \mathrm{gm}$. anhydrous $\mathrm{Na}_{2} \mathrm{HPO}_{4}$ in $500 \mathrm{cc}$. aq. dest. and make up to $1,000 \mathrm{cc}$. with methyl alcohol. These, in following proportions listed in cc., give $\mathrm{pH}$ values indicated.

\begin{tabular}{cccl} 
cc. Citric Acid & cc. $\mathrm{Na}_{2} \mathrm{HPO}$ & \multicolumn{2}{c}{$\mathrm{pH}$} \\
1.3 & 0.7 & 3.9 & $(3.873)$ \\
1.25 & 0.75 & 4.0 & $(4.034)$ \\
1.2 & 0.8 & 4.2 & $(4.205)$ \\
1.15 & 0.85 & 4.4 & $(4.44)$ \\
1.1 & 0.9 & 4.6 & $(4.653)$ \\
1.05 & 0.95 & 4.8 & $(4.80)$ \\
1.0 & 1.0 & 5.0 & $(5.042)$ \\
0.95 & 1.05 & 5.2 & $(5.201)$ \\
0.9 & 1.1 & 5.4 & $(5.428)$ \\
0.85 & 1.15 & 5.7 & $(5.696)$ \\
0.8 & 1.2 & 5.85 & $(5.838)$ \\
0.75 & 1.25 & 6.05 & $(6.036)$ \\
0.7 & 1.3 & 6.3 & $(6.29)$ \\
0.65 & 1.35 & 6.5 & $(6.444)$ \\
0.6 & 1.4 & 6.5 & $(6.522)$ \\
0.55 & 1.45 & 6.6 & $(6.60)$
\end{tabular}

Since it is difficult to measure out these 
small volumes accurately at least ten times the volume in each case should be taken and the amount not required simply be discarded. For ordinary purposes employ aq. dest. in place of methyl alcohol.

McJunkin-Haden Buffer has pH 6.4 and is useful in place of aq. dest. for diluting Giemsa, Wright and other blood stains. Monobasic potassium-phosphate, 6.63 gm.; anhydrous dibasic sodium phosphate, 2.56 gm.; aq. dest., 1000 cc. (Haden, R. L., J. Lab. \& Clin. Med., $1923,9,64-65)$.

Meat Extract Broth and other media containing meat, see Bacteria Media.

Mechanical Stages, see Lillie p. 287.

Meckel's Diverticulum. Literature on (Curd, H. H., Arch. Surg., 1936, 32, 506-523).

Media, see Bacteria, Leishmania, Protozoa, Trypanosomes.

Megakaryocytes. These can, like blood cells, be examined in fresh and stained smears of bone marrow. For a determination of their role in platelet formation it is essential to clearly show the granules typical of both. This can best be done in sections of bone marrow prepared by :

1. Wright's method (Wright, J. H., J. Morph., 1910, 21, 263-277). After fixation in sat. mercuric chloride in $0.9 \%$ aq. $\mathrm{NaCl}$, dehydrate in alcohol, follow with acetone, clear first in thick cedar oil and then in xylol, embed in paraffin. Sections deparaffinized are covered with equal parts stain (polychrome methylene blue solution 3 parts and $0.2 \%$ eosin yellowish in methyl alcohol 10 parts) $10 \mathrm{~min}$. A metallic looking scum forms but the stain should not be allowed to precipitate. Stop staining when cytoplasm looks bright red and reticular fibers light red. Wash in water, dehydrate in acetone, clear in turpentine and mount in thick colophonium in pure turpentine oil. See Wright's colored plates. In place of the fixative suggested, Downey (Folia haematol., Archiv, 1913, 15, 25) uses commercial formalin $10 \mathrm{cc}$. and sat. mercuric chloride in $0.9 \%$ aq. $\mathrm{NaCl}$ $90 \mathrm{cc}$.

2. Kingsley's method (Kingsley, D. M., Folia Haemat., 1937, 57, 87-98). Fix in Downey's fluid (given above) 4 parts, saturated picric acid 1 part, $24 \mathrm{hrs}$. Wash in running water, 18-24 hrs. Dehydrate through alcohols up to $70 \%, \frac{1}{2}-1 \mathrm{hr}$. each. $80 \%$ alc. + iodine, overnight. $95 \%$ alc., $45 \mathrm{~min}$. Repeat with fresh alc. $N$ butyl alcohol (technical), $1 \mathrm{hr}$. Repeat with fresh. Paraffin $\left(58^{\circ} \mathrm{C}\right.$.) , $\frac{1}{2} \mathrm{hr}$., then 3 more changes, each $\frac{1}{2}$ hr. Imbed. Prepare stock solutions A: methylene blue (U.S.P. med. $88 \%$ ), $0.065 \mathrm{gm}$.; methylene azure A $(80 \%), 0.01$ gm.; glycerin, C.P., 5 cc.; $\mathrm{CH}_{3} \mathrm{OH}$ (C.P.), 5 cc.; aq. dest., 25 cc.; buffer $(\mathrm{pH}, 6.9), 15$ cc. B: methylene violet (Beruthsen $85 \%$ ), $0.013 \mathrm{gm}$.; eosin, yel. (92\%), $0.45 \mathrm{gm}$.; glycerine, 5 cc.; $\mathrm{CH}_{3} \mathrm{OH}, 10$ cc. ; acetone, C.P., $35 \mathrm{cc}$. The buffer is $40 \mathrm{cc}$. of A $=9.078 \mathrm{gm} . \mathrm{KH}_{2} \mathrm{PO}_{4}$ per liter $+60 \mathrm{cc}$. of $\mathrm{B}=11.876 \mathrm{gm}$. Na $\mathrm{HPO}_{4} \cdot 2 \mathrm{H}_{2} \mathrm{O}$ per liter of aq. dest. Immediately before use mix equal parts of stock stains A and B. After washing deparaffinized sections in aq. dest. stain 8-10 $\mathrm{min}$. Wash off in current of aq. dest. Wash in aq. dest. $100 \mathrm{cc}$. $1 \%$ acetic acid, $0.8 \mathrm{cc}$. Wash again in aq. dest. to remove acid. Blot. Rinse in acetone, $100 \mathrm{cc} .+0.001 \mathrm{gm}$. eosin +4 cc. $1 \%$ acetic acid. Rinse in $n$ butyl alc. + a little eosin. Neutral xylol several changes. Mount in neutral xylol dammar. See Kingsley's plate for colors. Granules dark red. It is important to fix the bone marrow promptly after death or to obtain it by biopsy.

Megaloblasts, see Erythrocytes, developmental series.

Meibomian Glands. Whole mounts of the glands stained with Sudan IV in a transparent background by a method described for Sebaceous Glands.

Meissner's Corpuscles. 'To investigate by supravital staining with methylene blue in skin of amputated fingers, see Weddell, G., J. Anat., 1940-41, 75, 441-446. Skin from general body surface will not do because of rarety of the corpuscles.

Meissner's Plexus, see Auerbach's.

Melanins. Lison (p. 248) gives many differential microchemical properties from which the following are selected. Extreme resistance to most chemicals, not modified by concentrated acids but soluble in concentrated alkalis. They are depigmented by oxydants. Thus, Schultze treats them with diaphanol (chlordioxyacetic acid) for $24 \mathrm{hrs}$. in hermetically sealed container in darkness; and Alfiere treats sections with $0.1 \%$ potassium permanganate $2-24$ hrs.; washes with much water, treats with $0.3 \%$ oxalic acid and again washes. Their power of reducing ammoniacal silver nitrate, Lison regards as very characteristic. MIelanins occur normally in epidermis, hair, choroid of eyes. Greatly increased in Addison's disease. Contain no iron or fat. Difficulties in histological identification (Jacobsen, V. C. and Klinck, G. H., Arch. Path., $1943,17,141-151)$. Use of Bodian method (Dublin, W. B., Am. J. Clin. Path., Techn. Suppl., 1943, 7, 127-128). 
A method for the collection of melanin for analysis by differential Centrifugation is described by Claude, A., Trans. New York Acad. Sci., 1942, II, 4, 79-83.

A very complete account of the melanins has been presented by Gordon, H. (Organizing Chairman): The Biology of Melanomas-Special Publications, New York Acad. Sci., 1948, $466 \mathrm{pp}$.

Lillie (p. 131) cites Alfieri from Romeis as advising for the bleaching of melanin in sections treatment with $0.05 \%$ aq. potassium permanganate until thoroughly brown followed by decolorization in 0.33 aq. oxalic acid and repeating the process if necessary. Obviously stronger solutions could be employed. This removal of melanin might be advantageous in some cases to reveal more sharply other properties of the cells like mitochondrial content. Treatment with $10 \%$ hydrogen peroxide, as suggested by Lillie, is perhaps a better method.

See Dopa Reaction for melanogen in melanoblasts.

Melanoblasts, see Dopa Reaction.

Meldola's Blue, see Naphthol Blue $R$.

Mercuric Chloride (corrosive sublimate) in various combinations is an excellent fixative. It can be used in saturated aq. sol. plus $5 \%$ acetic acid or in saturated alc. sol. with the same amount of acetic acid. See (1) with formalin, glacial acetic and physiological saline for Centrosomes, (2) sat. in $0.9 \%$ aq. sodium chloride for Megakaryocytes, (3) sat. in $70 \%$ alcohol $+5 \%$ acetic for Mitosis, (4) sat. aq. + equal parts $2.5 \%$ aq. potassium bichromate for Neutral Gentian, (5) sat. aq. with equal parts abs. alcohol for Thymonucleic Acid, and (6) with nitric acid for Urea. The mercuric chloride is removed from the sections by Lugol's iodine solution. See also fixatives of Zenker, Gilson, Rabl and Petrunkewitsch. Zinc chloride is suggested as substitute for mercuric chloride in Zenker's fluid (Russell, W. O., J. Techn. Methods \& Bull. Int. Asso. Med. Museums, 1941, 21,47 ).

Mercurochrome 220. Trade name for dibrom-oxy-mercuri-fluorescein. Can be used as substitute for eosin (Baldwin, W. M., Anat. Rec., 1928, 39, 229) but it has little to commend it.

Mercury, microchemical tests for.

1. Method of Almkvist-Christeller. Fix tissues 2 days in sat. aq. picric acid, 100 cc. $; 25 \%$ nitric acid 1 cc., saturated with $\mathrm{H}_{2} \mathrm{~S}$ gas, filtered after 1 day. After fixation wash in running water for 24 hrs. Imbed in paraffin. Mercury appears as black ppt. of sulphide. Lison (p. 102) explains that it is necessary to make parallel tests for iron because this method changes iron into the black sulphide which could be mistaken for the sulphide of mercury. Simonet (M. Arch. d'Anat. Micr., 1929, 25, 372-381) uses instead fixation for $10 \mathrm{hrs}$. in equal parts alcohol and chloroform, $100 \mathrm{cc}$., + nitric acid, 2 cc. the mixture saturated with $\mathrm{H}_{2} \mathrm{~S}$ by bubbling.

2. Method of Brandino (G., Studi Sassari, 1927, 5, 85). Fix in formalin or in alcohol. Treatment of sections with $1 \%$ sol. of diphenylcarbazide which forms with mercury a violet ppt. Gives results with organs of persons killed by mercury poisoning kept in formalin 17 years (Lison, p. 102).

3. Method of Hand et al., J. Lab. \& clin. Med., 1943, 28, 1835-1841. Reagents: (A) Mercurous. 1 cc. thioglycollic acid +9 cc. glycerol. (B) Mercuric. Heat until clear 100 cc. glycerol., +5 gm. tartaric acid, +5 gm. stannons chloride. Add few gms. metallic tin and store in glass stoppered bottle. (C) Iodine. Dissolve $50 \mathrm{gm}$. potassium iodide in 50 cc. aq. dest., add $70 \mathrm{gm}$. iodine and $95 \%$ alc. to make $1,000 \mathrm{cc}$. (D) Chloroauric acid $1 \%$ aq. (E) Control. Let $5 \mathrm{gm}$. tartaric acid stand over night in 100 cc. glycerol. Cut $15 \mu$ frozen sections of tissue. Place on slides and dry. To section on each of 4 slides add 1 drop of one reagent. Add cover glasses. Remove with filter paper excess of reagents. Seal edges of cover glass with commercial gold size, an adhesion intended to hold gold foil on glass. Melted paraffin is less satisfactory but will serve for a short time. After $10 \mathrm{~min}$. examine slides. Metallic mercury visible as small black spherules. These are soluble in $\mathrm{C}$ and form gold amalgam losing glossy surface when treated with $\mathrm{D}$. Reagent $\mathrm{A}$ gives typical yellow crystals with mercurous mercury in addition to globules of mercury when mercuric mercury also is present. Sections treated with $B$ and $E$ are unchanged (adapted from Glick p. 25).

Intravenous injections of colloidal solutions of mercury in rabbits are described by Duhamel, B. G., C. rend. Soc. de Biol., 1919, 82, 724-726.

Mesentery spreads, sections and cultures. Maximow, A., Arch. f. exper. Zellf., 1927, 4, 1-42 (nice colored plates). For microinjection of small vessels of the mesentery see Florey, H., Proc. Roy. Soc. B, 1926 100, 269.

Mesonephric Tubules, cultivation in vitro and method for collection of fluid therefrom (Keosian, J., J. Cell \& Comp. Physiol., 1938, 12, 23). 
Metachromasia, see Metachromatism.

Metachromatism (metachromasia) is the property of certain dyes to stain (G. meta, beyond) the usual color (G. chroma). The action of some impure methylene blues is sometimes cited as an example. Thus polychrome (many colored) methylene blue stains some objects blue and certain granules reddish. This methylene blue is however a mixture of methylene blue and methylene red. The latter dye accounts for the staining beyond. Orcein colors nuclei blue and cytoplasm pink. Safranin stains nuclei in its ordinary solution color (red) and the ground substance of cartilage that of its free color base (orange). Michaelis (Lee, p. 136) thinks that the appearance of the color base is not occasioned by the alkalinity of the objects stained. The red stain of mucin by thionin can be altered to blue by alcohol and be shifted back to red by water. For colored plates showing metachromatic staining of mast cells, see Maximow, A., Arch. f. mikr. Anat., 1913, 83 (1), 247-289. Meta chromasia of acid dyes is increased by adding strychnine, quinine or clupein and of basic dyes by gum arabic or other negatively charged colloid (Bank, O. and Hungenberg de Jona, H. G., Protoplasma, $1939,32,489-516)$. The distinction between "true" and "false" metachromatic staining with toluidine blue is fully described by Sylvén, B., Acta Radiol., 1945, Suppl. 59, 100 pp.

Metacresol Purple. See Hydrogen Ion Indicators.

Metallurgic Microscope. Since the materials routinely studied are opaque the light is reflected vertically down upon them through the objective. This instrument is of little use in biology and medicine.

Metamyelocytes, see Leucocytes, developmental series.

Metanil Yellow (CI, 138)-acid yellow R, orange MNO or MN, soluble yellow OL, tropaeolin G, yellow M-An acid monoazo dye employed in the Masson technique, see Foot, N. C., Stain Techn., 1933, 8, 101-110.

Methacrylate. Plastic for mounting alizarin-red-S preparations. (Holcomb, R. C. and Apterman, P. M., J. Tech. Methods, 1944, 24, 21-24).

Methyl Alcohol, see Elementary Bodies. It is much used in many techniques.

Methyl Benzoate. Refractive index close to that of cedar wood oil. It can be used in place of immersion oil. In addition it is a substitute for absolute alcohol and an excellent clearing agent but it is expensive. See Ceresin imbedding.
Methyl Blue (CI, 706)-cotton blue, Helvetia blue-Widely used. Recommended for connective tissue by Lillie, R. D., J. Tech. Methods, 1945, No. 25, 47 pp. Sec Mann's Methyl-Blue Eosin and staining of Elementary Bodies.

Methyl Blue-Eosin, see Mann's.

Methyl Eosin (CI, 769). The methyl ester of eosin $Y$, see Eosins, choice of.

Methyl Green (CI, 684)-double green, light green-This basic triphenyl methane dye is crystal violet (hexa-methyl pararosanilin) into which a seventh methyl group has been incorporated. Conn (p. 130) points out that this is loosely bound so that some methyl or crystal violet is always present with the methyl green to which circumstance the metachromatic properties of the dye are partly due. Methyl green is not as stable as most dyes and cannot therefore be kept too long in the powdered state. It is very similar to Ethyl Green.

Methyl Green-Pyronin (Pappenheim). Sections of formalin-Zenker fixed tissues are stained about $6 \mathrm{~min}$. in: methyl green $0.5 \mathrm{gm}$.; pyronin Y, $0.5 \mathrm{gm}$.; alc. 2.5 cc.; glycerin 20 cc.; aq. dest. $0.5 \%$ carbolized $100 \mathrm{cc}$. Rinse in aq. dest.; dehydrate in acetone; clear in cedar oil followed by xylol and mount. Optimum time of staining must be determined experimentally. A brilliant stain particularly for lymphocytes and plasma cells. Very useful for spleen and lymph nodes. (Slider and Downey in McClung's Microscopical Technique, p. 342 )

Modification of Scudder (Stain Techn., 1944, 19, 39-41) gives good results on tissue sections and bacteria and has been used for identification of 2 types of nucleic acids (Taft, E. G., Exper. Cell Research, 1951, in press). Dehydration of stained material with tertiary butyl alcohol gives better results than with ethyl alcohol.

Methyl Orange (CI, 142)-gold orange MP, helianthin, orange III, tropaeolin DA slightly acid mono-azo dye widely employed as an Indicator.

Methyl Red (CI, 211). A slightly acid mono-azo dye widely used as an Indicator. See also Carter, J. S., J. Exp. Zool., 1933, 65, 159-179 for vital staining of rabdites of Stenostomum with methyl red.

Methyl Salicylate (oil of Wintergreen) is employed in Spalteholz Method of clearing.

Methyl Violet (CI, 680)-dahlia B, gentian violet, Paris violet, pyoktaninum coeruleum-Exists in various shades $2 R, R$, $\mathrm{B}, 2 \mathrm{~B}, 3 \mathrm{~B}$, etc., depending upon proportions of the mixture of tetra-, pentaand hexa-methyl rosanilins. $R$ indi- 
cates reddish and $\mathrm{B}$ bluish. $2 \mathrm{~B}$ is the one which Conn (p. 123) regards as most satisfactory whenever methyl violet, or one of the redder types of gentian violet, is requested. (It is Commission Certified.) The pure hexamethyl compound is called crystal violet-a dye much in demand. See Hydrogen Ion Indicators.

Methylene Azure (CI, 923). A basic thiazin dye long recognized as a component of Polychrome Methylene Blue. Conn (p. 76) says that the term, methylene azure, should be discarded because it is composed of three components Azure $\mathbf{A}, \mathbf{B}$, and $\mathbf{C}$ which see.

Methylene Blue (CI, 922)-Swiss blueConn (p. 80) says that this basic thiazin dye is theoretically tetra-methyl thionin but the homologues of lower methylation are practically always present; he lists the following grades: methylene blue $\mathrm{BX}, \mathrm{B}, \mathrm{BG}, \mathrm{BB}$, and methylene blue chloride. The last named is Commission Certified and least toxic. Methylene blue Med. U.S.P. is required to be zinc free and is also satisfactory. New methylene blue $\mathrm{N}$ (methylene blue $N N$ ) is a basic dye of the same type but of a slightly greener shade. Conn (McClung, p. 595) states that it was apparently in certain lots of prewar methylene blue. Methylene blue $O$ is the same as toluidin blue $O$ which resembles azure $\mathrm{A}$, a component of methylene azure produced by polychromizing methylene blue. Another of the series is methylene blue GG but it has no particular advantage. Probably no dye, other than hematoxylin and eosin, is more widlely used. The oxidation products of methylene blue are described by Holmes, W. C., Stain Techn., 1926, 1, 17-26 and the influence of $\mathrm{pH}$ on staining of plasma cells and lymphocytes by Kindred, J. E., Stain Techn., 1935, 10, 7-20. Its cytological action has been fully studied by Ludford, R. J., Arch. f. exp. Zellf., 1935. 17, 339-359. It is an excellent counterstain for Acid Fast Bacilli. See Polychrome Methylene Blue, Loeffler's Alkaline Methylene Blue, Nerve Endings, Phloxine Methylene Blue, MacNeal's Tetrachrome, Pancreas, Protozoa, etc. For use of methylene blue as a supravital stain fixed with ammonium molybdate, see Lillie, p. 245.

Methylene Blue NN, see New Methylene Blue N.

Methylene Blue T 50 or T Extra, see Toluidin Blue 0 .

Methylene Blue Eosinate, see May-Grünwald fixative and stain.

Methylene Green (CI, 924). This basic thiazin dye is mono-nitro methylene blue. Conn (p. 86) says that it is occasionally employed as a substitute for methyl green and gives good results as counterstain for eosin.

Methylene Violet. Commission Certified. This feebly basic thiazin dye is, as Conn (p. 86) explains, formed whenever methylene blue is heated with a fixed alkali or alkali carbonate. It may be purified by recrystallization but little is to be gained. The dye is not much used.

Metrial Gland. This is a transitory structure of unknown function in the mouse appearing at approximately the Sth day of pregnancy. Failure of its cells to take up trypan blue seems to eliminate the hypothesis that it is active in phagocytosis (Lobo, B. A., and Atkinson, W. B., Anat. Rec., 1916, 94, 77).

Micelie (dim. of L. Nica a crumb, micella, micellae). Term introduced by Nagcli in 1884 for then hypothetical structural units of the cell.

Michiavello Stain. See Rickettsia.

Microchemical Reactions. For the microscopic identification of particular elements or substances some microchemical reactions are available but it is difficult to sharply distinguish them from other techniques not usually styled microchemical. An attempt is made to list them under the objects demonstrated : Lead, Iron, Vitamin C, Peroxidase, etc. Many are generally known under personal names. See for example: Axenfeld (proteins), Burchardt (gold), Carr-Price (vitamin A), Feulgen (thymonucleic acid), Gmelin (bile pigments), Lilienfeld-Monti (phosphorus), Millon (tyrosin), Romieu (proteins), Schiff (aldehydes), Vulpian (epinephrine), etc.

Microdissection. In the selection of this method for use in any particular problem it is well to bear in mind several considerations. It is of particular value in the direct examination of large cells easily isolated, like sea urchin eggs, and of tissues that exist in thin sheets, like highly vascularized membranes which can be easily approached in the living state without serious injury. The data to be secured relate chiefly to the responses of the cells to the mechanical stimulus of the microneedle, to the character of the connections between fibers, cells and parts of cells as determined by their resistance to attempts to separate them and to the physical consistency of cellular and nuclear membranes and of cytoplasm and nucleoplasm. Moreover individual cells can be isolated by microdissection just as Barber was able to isolate single bacteria by the pipette which he intro- 
duced and which was in fact the inspiration of G. L. Kite's first microdissection apparatus. Today this has been very greatly improved chiefly by Chambers and Peterfi. See detailed account by Chambers under Micromanipulation.

Microelectrodes, see full discussion, McClung, Microscopical Technique, 1950, p. 532 .

Microglia. Method for impregnating with silver in pyroxylin (celloidin) sections (IVeil, H. and Davenport, H. A., 'Trans. Chicago Path. Soc., 1933, 14, 95-96). Wash $15 \mu$ sections in aq. dest. Treat for 15-20 sec. with silver solution (made by adding $10 \%$ aq. silver nitrate drop by drop from a burette to 2 cc. conc. ammonia (28\%) shaking to prevent ppt. formation until about $18 \mathrm{cc}$. have been added and the solution has become slightly opalescent). Transfer to $15 \%$ formalin, moving section rapidly until coffee-brown in color. Pass through 3 changes aq. dest. Dehydrate in alcohol, clear in xylol and mount in balsam.

Microglia and Oligodendroglia. In frozen sections $20-40 \mu$ of formalin fixed material. Immediately place them in aq. dest. + 20 drops ammonia per 100 cc. Thence pass directly to $5 \%$ aq. ammonium bromide $40-50^{\circ} \mathrm{C}$. $10-15 \mathrm{~min}$. Equal parts ammonia, pyridine and aq. dest. 2 min. Then $3-5 \%$ aq. sodium sulfite, $2-3 \mathrm{~min}$. Pass through and shake in 3 changes $1 \mathrm{~min}$. each of following: 8 parts $5 \%$ aq. sodium carbonate, 2 parts, $10 \%$ aq. silver nitrate + ammonia till ppt. Reduce in $1 \%$ formalin less than $1 \mathrm{~min}$. Wash, dehydrate clear and mount (King, L. S., Arch. Neurol. and Psychiat., 1937, 38, 362-361).

Microincineration-Written by Gordon H. Scott, July 26, 19.46 and revised by him January 16, 1951-This method is one which has been used by plant and animal histologists intermittently for over a hundred years. In concept it is simple in that it consists primarily of ashing tissue sections carefully so as to retain the minerals in their position in the fixed tissue. The ashing can be done on glass or quartz slides by a variety of heating processes. Most tissues in the body can be treated by the ashing process with some success. Those which contain large quantities of phospholipids ordinarily do not give as good results as tissues lacking thein.

The method is one which requires some care and the observance of cer- tain very definite precautions if good results are to be had.

Fixation: There are two methods of fixation which can lo used. These are the chemical and the frozen-dehydration. If the cryostat or other suitable devices for frozen-dehydration are not available, fixation by absolute alcohol plus 10 per cent formalin yields reasonably good pictures. This particular fixative is one of the few chemical mixtures which dissolves the minimum of mineral from fresh tissue and adds none to it. Tissues are passed through repeated changes of absolute alcohol to dry them and are then infiltrated with paraffin in the usual manner.

The alternative method, that of frozen-dehydration, is the most suitable for preparation of tissues for microincineration. (See Altmann-Gersh and Cryostat.) This technique yields tissues which, except for the ice crystal formation, have not been altered, to any perceptible degree, either physically or chemically. Dehydration at sufficiently low temperatures maintains an ice-salt equilibrium and no shifting of minerals in the cell results. If the paraffin infiltration is done with care, shrinkage and consequent cellular distortion is avoided.

Methods of examination of the incinerated preparations are several. One of the simplest and best for study and for photography is the dark-field. Of the several types of dark-field, the cardioid condenser probably gives the most uniform results. Illumination from above with the incident light falling on the slide at an angle of $30^{\circ}$ is advised by Policard. This has some advantages over the dark-field but makes the use of higher magnifications difficult if not actually impossible. Cellular details are, therefore, to be observed best by using the cardioid darkfield.

Identification of minerals. Some good results can be achieved by the use of ultraviolet light and with the subsequent fluorescence of minerals. Students should consult reference and textbooks on mincralogy for details of identification.

Calcium and magnesium are characterized in the dark-field by their dense white ash residues. Iron is oxidized during the incineration process and appears as varying tints of red. The amount of this element present can be 
correlated with the color intensity. Silicon is definitely crystalline in character and is recognizable by its property of double refraction in polarized light. This may at times be confusing since all minerals blend to some extent with the glass. Lead and other elements which yield black sulfides can be detected by treating the section with gaseous hydrogen sulfide. Uranium in pathological tissues fluoresces with a unique color under ultraviolet radiation.

Attempts have been made to quantitate the ash residue by photographic means and by the use of a photoelectric cell whose output current is properly amplified. Both methods leave much to be desired both in accuracy and because of the utter relativity of the results obtained. The most useful finding obtained from microincineration, therefore, is the appreciation of the distribution of the total minerals in the cell. Experimental alterations in them can be detected by the technique. See account by Scott in McClung's book and Electron Microscope, Histospectrography and Ultraviolet Photomicrography.

Microinjection. This is an important extension of microdissection whereby various fluids are injected directly into the cytoplasm or nuclei of living cells. It is capable of yielding information on Permeability, Hydrogen Ion Concentration, Oxidation-Reduction Potential which cannot be secured in any other way, but in reaching conclusions due allowance must be made for the fact that cells thus treated are of necessity severely injured. Microinjection with glass pipettes but without an expensive micromanipulator can yield worthwhile results as described by Knower (McClung, pp. 51-61) but for direct work on cells the micromanipulator is essential.

Microliter Burettes are essential in some histochemical techniques. They are of two sorts. In the first a capillary glass tube is calibrated so that volume is indicated by the level of the meniscus. In the second the tube is not calibrated but instead a screw determining the level is provided as a micrometer. The best nicrometer burette was designed by Scholander and has been improved by Scholander, P. F., Edwards, G. A. and Irving, L., J. Biol. Chem., 1943, 148, 495-500. In selecting a microliter burette consult Glick, pp. 255-264.

Micromanipulation-Revised by Robert
Chambers, Dept. of Biology, Washington Square College of New York University, New York. May, 1950Broadly speaking, this term covers two types of operations: delicate free-hand operations in which the only accessory may be a dissecting microscope, and, second, operations conducted by means of micrurgical instruments under high magnifications.

For freehand operations considerable training is required in using a compound microscope because of the inversion of the image. This, however, can be corrected by using the so-called erecting ocular. A decided help to relieve fatigue from too long holding of, a dissecting needle, for instance, is to have the shaft of the needle held in the apex of a pyramid of plastic clay, the base of which has been pressed down on the stage to one side of the microscope. The operator's hand encircles the mound of clay which bends as his fingers guide the needle. The tiring fingers can be released at any time while the needle tip remains in position. Descriptions of excellent methods for injecting minute vessels, such as the marginal vein of chick embryos or lymphatic vessels of frog tadpoles, are as follows: H. McE. Knower, Chapter in McClung's Handbook Microscopical Technique, 3rd ed., New York: Hoeber, 1950; A. L. Brown, Anat. Rec., 1922, 24, 295.

Micromanipulation in its more restricted sense applies to the use of mechanical devices for controlling the movements of the tips of microneedles and micropipettes in the field of high powers of the compound microscope. A full account is given in McClung's Handbook.

Several instruments are now being built. The ones in most general use in this country are those of Chambers, Péterfi and Emerson. The microneedles or pipettes extend into a moist chamber on the stage of the microscope so that their tips can be inserted into hanging drops of fluid suspended from the undersurface of a coverslip which roofs the chamber. The essential condition of an instrument is that the movements be sufficiently smooth and controllable under the highest magnifications of the compound microscope.

Péterfi's instrument was manufactured by Carl Zeiss Co. and is now difficult to procure. Chambers' was manufactured by E. Leitz and has 
undergone many modifications as to its accessory parts. It is now being manufactured by the Gamma Instrument Company, Great Neck, New York. Both instruments are supplied with two main holders each independent of the other for carrying a micronecdle or a micropipette. The advantage of Chambers' is that the two holders are so adjusted as to permit the needles and pipettes to extend parallel to one another on the microscope stage. This permits the insertion of the needles into the moist chamber through one opening, thus increasing the chances of maintaining moisture conditions in the chamber.

More recently an instrument devised by de Fonbrune of Paris is being manufactured by A. S. Aloe Co., St. Louis, Mo. An early form of it is described in L'Illustration, February 15 and 22 , 1941. It depends for its fine movements on hydraulic pressure conveyed through flexible tubing from a hand operated lever. Circus movements in the horizontal plane are performed by rotating the lever, the vertical movement being accomplished by a thrusting action of a plunger in the shaft of the lever. Two such instruments mounted on opposite sides of the microscope permit the use of two microneedles. The instrument has great possibilities but its specific usefulness for the performance of circus movements is fully covered by the Emerson instrument which, incidentally, is sturdily constructed.

The Emerson is a first class machine with a mechanically controlled lever using circus movements in a horizontal plane, also a separate fine adjustment device for the vertical ones. Emerson has two models, the lower priced one having a lever control for both horizontal and vertical fine adjustments. These are being supplied by the J. H. Emerson Company, Cambridge, Massachusetts.

The Chambers instrument is the only one supplied with a microinjection apparatus a description of which is given in the latter part of this article.

Micrurgical instruments lend themselves to several types of operations: (1) Microdissection and injection of animal or plant cells and tissues for studies in cell anatomy and physiology, also cyto-chemistry in which chemical reactions can be obtained by applying chemical agents not only to individual cells but to localized regions within a given cell. (2) Chemical reactions in micro-drops. A very useful method is to deposit the droplets with a micro- pipette in a hanging drop of an inert oil. This prevents evaporation and the sphericity of the droplets in the oil permits quantitative determinations. Application of the technique to certain phases of microchemistry are given by Benedetti-Pichler in his book Introduction to the Microtechnique of Inorganic Analysis, New York: Wiley, 1942. (3) Isolation studies for obtaining pure line cultures (of bacteria, protozoa, etc., breaking of asci and isolation of the liberated spores, etc.). A good isolation technique is given by Reyniers, J. A., J. Bact., 1933, 26, 251.

The movements of the instruments can be controlled in any of three dimensions; the horizontal permits circus movements in one plane. Circus movements in the horizontal plane are best managed with the de Fonbrune and Emerson instruments. The vertical movement is operated by a separate controlling screw. Micro operations also can be performed under relatively low powers of the microscope. However, the operator should realize that the compound microscope, even though it be binocular, is monobjective. This means that the position of an object in the vertical plane can be deduced only by observing whether the object is in or out of focus. The lower the magnifying power of the objective the greater is the depth of its focus. Hence, there may be occasions when the tip of the microneedle and the object to be operated upon are at different levels although both are in focus to the eye.

The mechanical stage of the microscope is a useful adjunct for micrurgy. Particularly for injections, the most satisfactory way is to keep the tip of the micropipette in the center of the field and to perform the operation by raising the tip into the object to be injected after having brought the object into position by means of the mechanical stage.

The manufactured instruments are supplied with instructions as to their usc. Emerson supplies two types, one for coarser movements although it is possible to use this model for remarkably fine operations. The only way to select an instrument is to know what is wanted and then to decide after having the instrument demonstrated to him. All require the use of a good mechanical stage to move the moist chamber which carries the drops containing the tissue to be operated on. All in all, micromanipulation requires not only ability but mechanical aptiturle on the part of the would-be operator. It is one thing 
to have an instrument and a good microscope. It is another matter to build the many accessories, with cement, out of wood, glass or plastic, which the operator may need for his special purposes. Any gadget built may well mean a new discovery.

Tissues and cells to be operated on often require speeial means for holding them in place. Actively moving protozoa can be kept quiet by immersing them in egg albumen or a solution of hemi-cellulose. Strips of the epidermis of onion or tulip bulbs, immersed in varying concentrations of cane sugar, offer good objects for operation on protoplasts under different degrees of plasmolysis, likewise stamen hairs of Tradescantia which show mitotic figures. Similar studies may also be made on the epidermis of the tails of tadpoles. For these, the operator should use frogs' Ringer solution to maintain the proper balance of electrolytes in the medium. Muscle fibers stripped from the semitendinosus of the frog are good material. Urodeles furnish excellent material. An effective means of obtaining red cells undergoing mitosis is to bleed a Necturus or other member of the same order and take a sample of blood after a week or so.

The microneedles and micropipettes are usually made from glass capillary rods or tubes. Serviceable sizes with an outside diameter of 1-2 $\mathrm{mm}$. can be drawn out in a bunsen flame. The needle tips are made over a microflame by heating and pulling the shaft of a capillary held at both ends with the two hands. A serviceable gas microburner for this purpose is a hypodermic needle. When successful, the drawnout tips taper to a point rapidly enough so that the invisibly, fine tip is supported on a relatively rigid shaft. The shaft about $2 \mathrm{~mm}$. from the tip, is bent in the microflame to about a right angle. The other end of the capillary is then inserted into a specially constructed needle-holder and mounted in a micromanipulator so that the tip extends over the microscope stage into a moist chamber. The bent-up tip is adjusted with the screws of the instrument until the tip lies in a hanging drop of fluid suspended from a glass cover-slip serving as the roof of the moist chamber and in the field of the microscope.

Mechanical contrivances for drawing out the end of a glass capillary into a tapering tip are available. The most elaborate one is that of de Fonbrune, the microforge. This is a highly amplified mechanism with a built in microscope and coarse adjustment parts for holding, one, a glass capillary and the other a platinum loop. The mic roscope is adjusted to view the loop of an electrically incandescing fine platinum wire to which one end of the glass capillary is approached and then withdrawn as the glass begins to melt. Then properly done the tip of the capillary is drawn out to a tapering tip.

The principle of the microforge is based on a much simpler device long ago devised by S. L. Schouten and described by him in 1934. This can be readily built in any laboratory equipped with an ordinary microscope and micromanipulator.

A fairly good mechanical device for drawing microtips is being supplied by the Gamma Instrument Company. It depends upon springs to which the two ends of a glass capillary (1-2 mm. diam.) are fastened while the middle of the capillary passes through several coils of a fine platinum or nichrome wire. When the wire is caused to incandesce electrically the glass softens and the taut springs pull the capillary in two, each part with a tapering tip. A more precise needle puller based on the same principle is the Livingston Micro Pipette Puller made by Otto K. Hebel, Swarthmore College, Pennsylvania.

There are several possible ways by means of which a person with some ingenuity should be able to devise from the usual laboratory equipment a simple mechanical device for drawing needle tips. If the glass capillary is of tubing the microtips can be used for microcapillaries.

Injections are performed by breaking the tip of a micropipette against the undersurface of the coverslip while the tip is in view under the microscope. Capillarity draws fluid into the shaft of the pipette when the open tip is inserted into a hanging drop of fluid, be it oil or any given solution. For microinjection, the pipette holder, mounted on the instrument, is attached to a looped, capillary brass tube of which the other end is attached to the nozzle of a syringe. Before mounting the micropipette, the syringe is filled with water previously boiled to be air-free and, by means of the plunger, the water is driven into the brass tubing and the pipette holder after which the micropipette is inserted. Thus, we have a water-filled system extending from the syringe to the base of the micropipette the shaft of which may contain air. Micro-amounts of any given solution are then drawn into or ejected from the tip of the nicropipette by a delicate handling of the plunger of the syringe. 
Considerable deviations are possible in the matter of the microinjection technique. For example, if it is deemed desirable to have no air in the system, the shafts of the capillary tubing, on which the microtips are drawn, may previously be filled with either oil or water. By using some ingenuity the entire microinjection apparatus can be built in the laboratory, the parts required being a Luer syringe, hypodermic needles the shafts of which can be cut off, a strip of flexible brass or copper tubing, glass tubing and of course the operator's constant companion: a stick of deKhotinsky cement or an analogous superior sealing wax.

The instruments are generally supplied in pairs, one part carrying a microneedle for holding the tissue to be injected, the other carrying the micropipette. For microdissection, the instrument carries two needles, each of which can be operated independently.

Wilhelm Pfeffer, to whom we owe the term "plasma membrane" for the limiting boundary of protoplasm, stated, in one of his papers in 1887, that an instrument with which one could operate delicate needles and pipettes in the field of a compound microscope would go far toward the elucidation of the nature of living cells. Pfeffer's dream has been realized in the development of the special field of science called today Micromanipulation or Micrurgy.

Of general interest, and also for many details not described elsewhere, are the following: Barber, M. A., Philippine J. Science, B, 1914, 9, 307; Chambers, R., Anat. Rec., 1922, 24, 1; Péterfi, T., in methodik der wissensch. Biologie, 1928, 1 (4), 5; and Schouten, S. L., Zeit. f. wiss. Mikr., 1934, 51, 421. An excellent book which covers a broad range of the field of Micrurgy is that edited by J. A. Reyniers on Micrurgical and Germ-Free Techniques, C. C. Thomas, 1943, an article on micromanipulation by Chambers, R. and C. G. Grand, Encyclopaedia Britannica, 1948.

Micrometry is the measurement of an object observed microscopically. This can be done either by using an ocular micrometer in which there are lines which can be accurately moved the length of the structure to be measured or by inserting a ruled disc in an ordinary ocular with which it can be compared. Both must be standardized in relation to a micrometer slide generally ruled with lines $10 \mu$ apart. See Cell Measurements.

Micromicron $(\mu \mu)=1 / 1,000,000$ th part of a micron $=1 / 1,000,000,000$ th part of a $\mathrm{mm}_{\circ}=10^{-9} \mathrm{~mm} .=0.000,001 \mathrm{micron}=$ $10^{-2} \AA$. Unfortunately often used syn- onymously with millimicron $(\mathrm{m} \mu)=$ 0.001 micron $=10 \AA$.

Micron (Gr. Mikros, small) expressed by Gr. letter $\mu=$ approximately $1 / 25,000$ inch $=1 / 1000$ part of a mm. $=0.001$ $\mathrm{mm} .=10^{-3} \mathrm{~mm} .=10,000 \AA$ (see Millimicron and Micromicron).

Microphotometer, see Photoelectric.

Microradiographic examination. This consists of magnification of a Roentgen ray image after it has been registered photographically. The essential point is to use film of very fine grain emulsions. Thus the Gevaert Lipmann emulsion permits enlargement 300 times without much loss of detail. In some cases it is helpful before microradiographic examination to increase the absorption of Roentgen rays by "absorption staining" through adding radio-opaque materials such as barium sulp'iate and thorotrast. The application of this technique in the study of biologic materials is described and illustrated by Clark, G. L. and Bick, E. J., in Glasser's Medical Physics, 730-733.

The importance of extremely soft Roentgen rays and the properties of fine-grained emulsions are described by Engström, A. and Lindström, B., Acta Radiol., 1951, 35, 33-44. See also their illustrations of striated muscle fibers and of bone by microradiography.

Microrespirometer to indicate production of carbon dioxide by bacteriophages, viruses and bacteria (Bronfenbrenner, J., Proc. Soc. Exp. Biol. \& Med., 1924, 22, 81-82. See Capillary Respirometry.

Microscopes (From Cowdry's Histology, 1950). While excellence in histological technique is important, knowledge of microscopes and of how to get the best service out of them is also important. There are several kinds from which a choice must be made of the one capable of yielding the information required. The following account of what these instruments are, what their particular use is, what their limitations are, supplemented by leading references to literature on the subject is offered for guidance. It was written for Cowdry's Histology by Dr. T. B. Rosenthal.

The ordinary compound microscope is a precision optical instrument designed to give magnified images of 50 to $1200 \times$. Daylight, or strong artificial light, is used to illuminate by transmission a more or less thin, flat, transparent object. The image appears in its natural form and color, but inverted and reversed in position, at a distance of about 10 inches from the eye. Height and depth are not reversed, however, so it is possible to judge or measure 
the elevations on an object by differential focussing.

Three lens systems, condenser, objective, and ocular cooperate to form the final image. The purpose of the condenser is two-fold: to gather light for illumination, and to focus this light on the object in a cone of the proper dimensions so that the full resolving power of the objective lens can be gained. By means of the objective lens a real, magnified, inverted, reversed image of the object is formed at the upper end of the tube. The ocular further magnifies this primary image to yield a virtual, inverted, reversed secondary image. Total magnification is calculated by multiplying the magnifying powers of the objective and the ocular.

Inspection of the markings on a set of objectives will show their magnifying powers and another optical property called the numerical aperture (N. A.). Usually the $10 \times$ (also called $16 \mathrm{~mm}$ ) objective is marked with the number " 0.25 ;" the $44 \times(4 \mathrm{~mm}$, "high dry"), "0.66;" and the oil immersion, 95X (1.8 or $2.0 \mathrm{~mm})$, "1.25." Numerical aperture, calculated from a geometrical property of the front lens element, is proportional to the theoretical resolving power of the objective and is related to the maximum power of the ocular that can be profitably used with that objective. In order to understand this relation between magnification, resolution, and N. A. it is necessary to consider certain fundamentals.

As successively higher powers of magnification are brought to bear on a tissue section more and more fine detail becomes visible, which is, after all, the only purpose of microscopy. But to fineness of detail a limit is set, not by magnification, but by the nature of light and by the optical properties of lenses. As long as the object to be seen is large compared to the wave length of the light which illuminates it the microscopic image will be sharp. If structural details are so small that their size nearly approaches the wave length of light, the image becomes fuzzy. Although magnification may be secondarily raised by employing stronger eyepieces no further resolution of detail takes place; the image remains fuzzy, and we have what is called "empty magnification."

The formula $\mathrm{R}=\lambda / 2 \mathrm{~N}$. A. gives a general relationship, where $\lambda=$ wave length of light used, N. A. is a given constant of the objective lens, and $R$ is the size of minimum resolvable detail (given in $\mu$ if $\lambda$ is given in $\mu$ ). Taking an average value for $\lambda$ in white light to be $0.5 \mu$ and substituting the N. A. values mentioned above, we have for the $10 \times$ objective, $\mathrm{R}=1.0 \mu$; for the $44 \times, R=0.4 \mu$; and for oil immersion, $\mathrm{R}=0.2 \mu$. Thus, with the best lenses, details lying less than $0.2 \mu$ apart are not discerned.

Another general rule in microscopy states that the maximum total magnification should not be pushed higher than about one thousand times the N. A.; otherwise empty magnification results. With the $10 \times$ objective, $1000 \times 0.25=$ 250. Therefore a $25 \times$ ocular is the maximum. For the oil immersion, a $13 \times$ ocular gives the limiting useful magnification.

It is thus evident that the objective lens is the heart of the microscope. The ocular brings out only the details which already have been resolved by the objective.

The rôle of the condenser may be reviewed in the light of these interpretations. Historically the condenser received its name from its original purpose: it was simply a lens used to concentrate light on the subject. E. Abbe (1840-1905), who contributed a great deal to theoretical and practical microscopy, including the concept of numerical aperture, came to the conclusion that an objective lens could not work at its maximum N. A. unless it were matched with a condenser lens system of equal N. A. Hence the modern condenser is also rated in terms of N.A. and is provided with a variable iris diaphragm to alter its N. A. in order to match with that of the objective.

Since the standard microscope provides all the convenient magnifications and even more resolution than is usually required in routine histology, it is not necessary to take extraordinary pains to collimate (parallel) the microscope with the light source or to worry over the fine points of diaphragm control. However, good photomicrography demands such attention to details, because the plate is much more sensitive to inequalities of lighting than the observer's eye. On the other hand the eye is subject to fatigue unless the proper illumination is employed. In student elasses one commonly finds that the lighting with monocular microscopes is too bright and with binoculars too dim. To minimize visual fatigue the brightness of the microscopic field of view should about equal the brightness of the table-top and be in keeping with the general level of illumination in the room.

The following suggestions for setting 
up the microseope apply to the oil immersion lens. When dry lenses are to be used omit step 2 .

1. Clean the slide with alcohol. Put a drop of xylol on the oil immersion lens and polish it with lens paper. Do the same for the eye piece.

2 . Raise the condenser so that its upper surface is on a level with the stage, or slightly below it. Put a drop of immersion oil on the condenser and lay the slide down. Put a drop of oil on the slide, lower the lens, and focus as usual.

3. Open the diaphragm wide, and removing the eye piece, look down the tube. Adjust the position of the light source and mirror so that the aperture appears symmetrically illuminated.

4. At this point the aperture should look like a bright disk surrounded by a rim of dim illumination. Now, close down the diaphragm until about ninetenths of the area of bright central field remains visible. Replace the eye piece. The N. A. of condenser and objective are now approximately equal.

5. Modify the brightness of the image for visual comfort by altering the intensity of illumination; not by altering the diaphragm. If the image is too bright put tissue paper over the lamp or pull the lamp away and readjust by repetition of Steps 3 and 4. If the image is not bright enough move the lamp in, and if necessary dispense with the substage mirror. As the final step, try a slight change in the position of the condenser, but avoid breaking the oil pool between the condenser and the slide.

Resolving power may be improved somewhat for striated, or periodic, structures by using oblique illumination. Light is sent diagonally from below so that only part of it enters the objective, with the striations (as in muscle) lying across the direction of the light. If the condenser is not laterally movable it should be masked below so that light enters only from one side. By trial and error a favorable orientation of condenser, slide and mask can be found.

The limitations imposed on the resolving power of the conventional microscope cause no inconviences in general histology. It is with the finer details of cytology that need is felt for greater resolution. As is obvious from the formula $R=\lambda / 2 \mathrm{~N}$. A., either an increase of N.A. or a decrease of $\lambda$ will reduce the value of $R$. The best available oil immersion objectives and condensers are rated at $1.40 \mathrm{~N}$. A.
A special lens working at 1.60 has been designed, but the improvement in resolving power over one at 1.40 is not important. Fortunately we can obtain the shorter values of $\lambda$ by employing ultra-violet light and photography (see ultraviolet-microscope).

A word should be said in favor of water-immersion objectives, items formerly found in every laboratory of microscopy but now no longer listed in the catalogues of American manufaeturers. These are made in a series of magnifications, and in resolving power are intermediate between air and oilimmersion objectives. For study of living aquatic organisms and tissue cultures the $10 \times$ and $44 \times$ objectives are very useful since they are made to be lowered directly into the medium. For histological slide material the highpower water-immersion objective is probably not inferior to the oil-immersion, besides being more convenient to use.

It is commonly believed that a binocular, $i$. e., two eyepiece, microscope is superior to the monocular because it affords stereoscopic vision. Actually, it cannot be so since a single objective forms only one primary image regardless of whether it be viewed by one or two eyes. Nevertheless our habit of seeing with two eyes probably creates an illusion of stercoscopic vision with the binocular microscope. On the other hand a binocular microscope employing two objectives on a pair of converging tubes does provide stereoscopic views. The dissecting microscope is built on this pattern, having in addition a set of reversing and erecting prisms so that the final image is normally orientated. Magnifications above $30 \times$ become rather useless for dissection purposes because the depth of focus is greatly reduced. Manipulation is usually by hand.

See Centrifuge, Color Translation, Darkfield, Electron, Phase Contrast, Metallurgical, Polarizing, Reflecting and Ultraviolet Microscopes. Many excellent books on microscopes and photomicrography are available. The following are suggested: Gage, S. H., The Microscope. Ithaca: Cornstock, 1941,616 pp. Shillaber, C. P., Photomicrography in Theory and Practice. New York: John Wiley and Sons, 1944, 773 pp. Wredden, J. H., The Microscope, Its Theory and Application. New York: Grune and Stratton, 1948, 296 pp.

Microsomes (G. mikros, small, soma, body). Term introduced by Hanstcin in 1880 originally to indicate tiny granules-as 
compared with ground substance. Claude, A. Biological Symposia, 1943, 10, 111-129 estimates their size to be 50-300 $\mathrm{m} \mu$ and therefore beyond limits of ordinary microscopic visibility. These microsomes of Claude are obviously not the ones which Hanstein had in mind. According to Claude they are essentially ribose nucleoproteins and phospholipins in definite proportions.

Microspectrophotometry-Barry Commoner, The Henry Shaw School of Botany, Washington University, St. Louis 5. November 28, 1951-Microspectrophotometry is a technique for examination of cells and cell structures designed to yield data on the chemical composition of these objects. The method is based on the fact that given molecular configurations absorb specific wavelengths of ultraviolet, visible or infrared radiation. Under ideal conditions, as in dilute solutions, the molecular group and therefore the substance in which it occurs may be identified from the shape of the absorption spectrum, and its concentration in the sample determined from the amount of absorption at a characteristic wavelength. Unfortunately, cytological objects never offer such simple, readily analyzable situations. Consequently, special steps must be taken to evaluate the absorption spectra of cytological objects, and in most instances, data comparable with those obtained from solutions are not yet attainable. Nevertheless, the technique has thus far proved to be a valuable source of information on cell composition, and if used with care can be advantageously applied to a number of biological problems.

The essential measurement in microspectrophotometry is determination of the reduction in intensity of a light beam after passing through a cytological object. This measurement, made at a series of specific wavelengths, gives the absorption spectrum of the object. The measurements may be made by passing a monochromatic beam through the object; or by passing a heterogeneous beam through the object and then dispersing it into a spectrum. Equipment employing the second of these methods has been described by (Mellors, R. C., Science, 1951, 112, 381-389). Since most microspectrophotometry has employed the first principle of operation, details for this type of apparatus are given below.

1. General. The fundamental parts of a microspectrophotometer consist of a suitable light source, a monochrom- ator, microscope optics, a photoelectric tube which receives the projected image of the object, and appropriate means of measuring the phototube response.

2. Light sources. Ordinary automobile headlight tungsten lamps operated on a storage battery are suitable for work in the visible spectrum. For work in the ultraviolet ranges mercury discharge tubes provide adequate light intensity. High pressure mercury lamps (such as General Electric AH6) emit a continuous spectrum in the visible and ultraviolet superimposed on a number of bright and dark lines. In the infra-red ranges Nernst glowers are suitable.

3. The monochromator. The light beam from source is directed into the entrance slit of a monochromator, thus giving an emergent beam of a determined mean wavelength. The monochromator should supply the condenser of the microscope with a beam sufficient to fill the aperture of the latter.

4. The microscope. The optical arrangement of the microscope must be such as to give a true light image of the object at the plane of the photocell. The requirements for this condition have been presented by Caspersson, T. (Cell growth and cell function, New York: Norton, 1949). For visible work any good apochromatic system of sufficient numerical aperture is adequate. In the ultraviolet range quartz lenses or reflecting objectives must be used. The latter have the advantage of being achromatic in the ultraviolet spectrum.

5. The light detector. The most suitable detector for this type of work is the photomultiplier tube. The tube is supplied with a suitable power source and its output amplified and read off a microammeter. Less conveniently the direct output may be detected with a galvanometer.

The construction of microspectrophotometric equipment has been described in the following papers: Norris, K. P. and Wilkins, M. H. F., Discussions of the Faraday Society, 1950, No. 9, 360-363, Barer, R., Discussions of the Faraday Society, 1950, No. 9, 369378, Mellors, R. C., Discussions of the Faraday Society, 1950, No. 9, 398-406, Thorell, B., Discussions of the Faraday Society, 1950, No. 9, 432-436, Walker, P. M. B. and Davies, H. G., Discussions of the Faraday Society, 1950, No. 9, 461-470, Commoner, B., Ann. Mo. Bot. Gard., 1948, 35, 239-254, Burch, C. R., Proc. Physic. Soc., 1947, 59, 41-46, Grey, D. S., J. Opt. Soc. 
Amer., 1950, 40, 283-290, Mellors, R. C., Science, 1951, 112, 381-389. The problem of instrumental accuracy is discussed by Glick, D., Engström, A. and Malmström, B. G. (Science, 1951, 114, 253-258) and Caspersson, T. (Cell growth and cell function, New York: Norton, 1949).

The light absorption due to the object may be determined in the following ways.

1. Direct method. The microseope is focused on an area of the object slide adjacent to the object itself. The light impinging on the photo-cell is then adjusted to give a fixed response in the detector circuit. Without altering the optical conditions the slide is then moved so that the object is now centered in the field and a reading of the detector response is taken. This measurement compared with the first one yields the per cent of the incident light which is transmitted by the object.

2. Split-beam method. In this method the light beam is split before entering the microscope and a small fraction directed toward an accessory phototube and detector circuit. It is then possible to evaluate the intensity of the light reaching the main phototube in terms of the intensity of the beam directed toward the seconday phototube. This method has the advantage of being independent of random fluctuations in the intensity of the light source. As in the direct method the absorption of the object is determined by comparison with a blank area in the slide.

Interpretation of data: The derivation of valid conclusions from intracellular absorption data is a considerably more difficult task than the experimental work itself; in fact, it is frequently the case that data are obtained for which there are as yet no valid interpretations. The difficulties in interpretation arise from the fact that little is known concerning the physical state of intracellular objects and its effect on their optical properties. Since the rules for analysis of absorption data are based exclusively on the optical properties of homogeneous systems such as gases and dilute solutions, entirely new methods need to be developed for the heterogeneous structures of the cell.

For homogeneous solutions of absorbing materials, the following relations (the Beer-Lambert Laws) are found, within limits, to hold:

$$
\mathrm{D}=\mathrm{k} \cdot \mathrm{c} \cdot \mathrm{d}
$$

where $c$ is the concentration of absorbing material, $d$ is the length of the opti- cal path through the absorbing layer, and $\mathrm{k}$ is the extinction coefficient of the substance in question. D, the optical density, is defined by the expression $\mathrm{D}=\log _{10} \frac{I_{0}}{\mathrm{I}}$, where $\mathrm{I}_{0}$ is the intensity of the incident beam and I the intensity of the transmitted beam. The BeerLambert relationship holds only where all light absorption is due to the trapping of photons by the substance in question, and where each molecule in the light path contributes equally to the absorption.

In microspectrophotometric work, the value of $\mathrm{D}$ for a given optical section through the object is determined experimentally at various wavelengths. This gives the absorption spectrum of the entire object. Since the aim of the measurement is usually the identification and estimation of a specific substance present in the object, the initial spectrum needs to be evaluated in the light of the above relationships.

In order to identify a specific substance from the absorption spectrum of an object believed to contain this substance, the following conditions must be met. 1) It must be known that the object contains no absorbing material other than the substance in question; or the relative contents and absorption spectra of extraneous substances must be determined. Without this information one cannot conclude that a given absorption maximum in the spectrum of the object is due to a specific substance; such a maximum may result from the superposition of the spectra of two or more different substances. 2) It must be known that the absorption of light at any given wavelength is due only to the trapping of photons by the substance in question and is not a consequence of scattering and similar non-specific processes. In general, light scattering rises with increase in frequency but since sharp changes in refractive index occur near the absorption maximum of a substance, scattering effects may sometimes pass through a maximum with variation in wavelength.

If in addition to identification an estimation of the relative amount of the substance present in the intracellular object is to be made, the following further conditions must be fulfilled. 3) The length of the optical path through the object must be known. This is not necessarily identical with the thickness of the object for in heterogeneous systems internal reflections may cause a significant fraction of the entering beam to be reflected through 
a total path length considerably longer than the dimensions of the object. This effect will enhance the absorption of the object and may be variable with wavelength. 4) The relationship between the optical density of an object and the concentration of the specific substance must be demonstrated since it cannot be assumed that the linearity predicted by Beer's Law will hold. 5) The effect of inhomogeneous distribution of the absorbing material in the planes perpendicular to the optical axis must be estimated since relatively small departures from homogeneity will lead to significant alterations in the absorption due to a given amount of material.

If the absolute quantity of a given substance in an intracellular object is to be determined, two further conditions must be met. 6) The specific extinction of the substance in question, that is, the absorption per mole per liter must be known. 7) 'The influences of the conditions noted above on the extinction must be determined quantitatively. If accepted analytical practices are to be followed, this would mean that the absorption due to a known amount of the specific substance when added to the intracellular object be determined or that the amount of the substance found in the object by independent analytical means be correlated with its absorption characteristics.

Unfortunately, presently available techniques can meet the above conditions only in part. The procedures thus far worked out or suggested are noted below.

1. The problem of complex composition of the object. If it can be shown that the composition of the object is uniform throughout its optical depth, then the relative contribution of individual substances to the overall absorption spectrum may be worked out by comparison of the latter with individually determined spectra of the separate components. However, large differences in the height of the maxima frequently make this procedure a difficult one. To demonstrate that this optical uniformity exists, it must be shown that the absorption spectrum of the object is constant for all thickness (obtained, for example, by sectioning). Where this is not possible as in the case of structures enclosed within intact living cells an optical method may be used (Commoner, B., Discussions of the Faraday Society, 1950, No. 9, 449-460). This procedure may be applied to objects in which one component is distributed in an invariant layer in a cell which varies considerably in thickness. In this case it is possible to determine the absorption due to the separate components in situ by measuring the absorption spectra of two regions in the cell which differ in thickness by a known amount. In favorable instances this method may be used to determine the absorption spectrum of the nucleus contained within a living cell. Thus far the procedure has been applied only to certain types of plant cells. Failure to conform with this condition casts doubt on the meaning ascribed to the absorption spectra of structures such as the nucleolus which have been obtained without detailed analysis of the contribution made by over-lying and under-lying material.

2. Non-specific light losses. It has been frequently assumed (Caspersson, T., Cell growth and cell function, New York: Norton, 1949) that light lost due to scattering is related to wavelength according to the Rayleigh equation. Using this assumption and the further assumption that at some specific wavelength range such as $300-350$ $\mathrm{m} \mu$ no specific absorption occurs, the scattering losses are calculated by extrapolation from the readings obtained in this limited range. The usefulness of this method is considerably weakened by the fact that the first assumption has never been demonstrated to be true for intracellular objects and that the second assumption can be true only very rarely. An experimental determination of losses due to scattering may be made by an apparatus described by Caspersson, $\mathrm{T}$. (Cell growth and cell function, New York: Norton, 1949) which measures the light emerging from the object at various angles from the optical axis. Scattering losses may vary considerably with the physical state of the object and are, therefore, very sensitive to fixation procedure, etc.

3. The length of the optical path. Thus far no method for determining this value has been proposed and in practice the only approach to the value of this dimension is the observed thickness of the object. Thus in making this assumption, calculations are exposed to an error of unknown magnitude.

4. The validity of Beer's law. This essential determination has thus far been carried out in only one instance (Commoner, B., Discussions of the Faraday Society, 1950, No. 9, 449-460) in which the cellular material occurred in solution in the vacuole of a mature plant cell. In this case it was possible to alter the concentration of the dis- 
solved material by plasmolysing the cell to various degrees and thereby altering the water content of the solution. This method permits comparison of the optical density of the vacuole at various volumes and provides a direct test of Beer's law. In the case of solid structures such as the nucleus the osmotic method is probably invalidated by the fact that the absorbing material is not freely dissolved throughout the structure.

When the cytological object contains oriented absorbing material in significant amounts, considerable departure from the Beer-Lambert relationship is to be expected (Commoner, B., Science, $1949,110,31-40)$. It may be possible to avoid this difficulty by the use of a polarized incident light beam.

5. The effect of lateral inhomogeneity. This problem has been discussed from a theoretical point of view (Glick, D., Engstrom, A. and Malmström, B. G., Science, 1951, 114, 253-258; Danielli, J. F., Cold Spring Harbor Symp., 1949, 14, 32-39). It is apparent from these discussions that large errors may arise from this effect but at present there is no experimental way of determining their magnitude. In general it would seem essential that objects studied be homogeneous at least with respect to their microseopic appearance.

6. The determination of absolute quantities. In some instances (Caspersson, T. and Schultz, J., Proc. Nat. Acad. Sci., 1940, 26, 507-515) investigators have calculated the absolute content of, say, nucleic acid in a nucleus from measurements of the optical density of the nucleus and the specific extinction of nucleic acid solutions. Such a procedure has never been validated by the customary tests of the analyst.

7. The effect of ultraviolet light on the optical properties of the cell. One of the most serious difficulties encountered in the microspectrophotometric work is the fact that exposure to ultraviolet light is damaging and frequently lethal to the object. At the same time irradiation seriously alters the absorption properties of the cell. It was first shown by Brumberg, E. M. and Larionov, L. P. (Nature, 1946, 158, 663-664) that in living cells undamaged by ultraviolet the optical density of the nucleus does not exceed that of the cytoplasm. When the cell has been killed by ultraviolet radiation the optical density of the nucleus rises sharply whereas the absorption of the cytoplasm drops. Similar changes have now been observed by a number of other workers (Bradfield, J. R. G., Discussions of the
Faraday Society, 1950 , No. 9, 481-490; Walker, P. M. 13. and Davies, H. G., Discussions of the Faraday Society, 1950, No. 9, 461-470). These observations show that 1) absorption measurements of living cells must be made on cells which actually survive the experimental procedure, 2) fixation procedures may seriously alter the apparent distribution of ultraviolet absorbing material and 3) the structural arrangement peculiar to the living nucleus probably has a significant effect on its absorption properties.

Microtome. The freezing, rotatory and sliding microtomes are well known and advertised. The high speed microtome required for cutting especially thin sections for Electron Microscopy is essential for research. See account by G. H. Scott in McClung's Microscopical Technique, 1950 , p. 720 ; also Fullam, E. F. and Gessler, A. E., Rev. Sci. Inst., 1946, 17, 23 and Gessler, A. E. and Fullam, E. F., Am. J. Anat., 1946, 78, 245. Geren, B. B. and McCulloch, D. (Exp. Cell Research, 1951, 2, 97-102) have made adjustments in the Minot rotary microtome by which sections of tissues, prepared by the methacrylate embedding technique of Newman, S. B., Borysko, E. and Swerdlow, M. (Science, 1949, 110,66) can be cut 1/20 $\mu$ in thickness. The edges of some sections are much thinner. Glass knives with water trough (Iatta, H. and Hartmann, J. F., Proc. Soc., Exp. Biol. and Med., 1950, 74, 436) are a great improvement over steel ones.

Microtome Knife, sharpening. There is no easy method. Care and long practice are essential. (See Bensleys, p. 57.) For the usual oil and water stones a ground glass is now sometimes substituted (Uber, F. M., Stain Techn., 1936, 11, 93-98).

Micrurgical Technique (Gr. micros, small + ergon, work) is referred to under the heading of microdissection.

Mikado Yellow G (CI, 622)-Stilbene Yellow-a direct dye of light fastness 3 . Similar to Sun Yellow but lighter in color (Emig, p. 46).

Milk, bacteria in, a modification of Newman technic (Broadhurst, J. and Paley, C., J. Am. Vet. Med. Assoc., 1939, 94, 525-526). To prepare stain add $0.4 \mathrm{cc}$. conc. $\mathrm{H}_{2} \mathrm{SO}$, to 54 cc. $95 \%$ alcohol. Mix with 40 cc. technical tetrachlorethane in flask and heat to $55^{\circ} \mathrm{C}$. but no higher. Add about 1.0-1.2 gm. methylene blue while mixture is still hot. Shake until dye goes into solution. Then add 8.0 cc. $1 \%$ basic fuchsin in $95 \%$ alcohol. Mix, cool, filter and put up in glass stoppered bottle. Spread 
0.01 cc. milk over area of 1-2 sq. cm. on slide. Dry on flat warm surface $5 \mathrm{~min}$. Flood with stain 15 sec. Drain off excess and dry while flat with gentle heat. Wash in cold water till all blue is removed and a faint pink color appears. Dry and examine.

Technique for the rapid detection of Mycobacterium tuberculosis in cows, milk is described by Maitland, M. L. C., J. Hyg., 1950, 48, 397-401.

Millimicron $(\mathrm{m} \mu)=1 / 1000$ th part of a micron $=1 / 1,000,000$ th part of a mm. $=$ $10^{-6} \mathrm{~mm} .=0.001 \mathrm{micron}=10 \AA$ (see Micromicron).

Millon's Reaction. For microchemical purposes it is necessary, as Bensley and Gersh (R. R., and I., Anat. Rec., 1933, 57, 217-233) point out, for the reagent to act without the aid of heat, to give almost immediately with tyrosin in vitro an intense red color yielding red ppt. not changing to yellow within 24 hrs. They give the following directions. Add 600 cc. aq. dest. to 400 cc. conc. nitric acid (sp.gr. 1.42) making $40 \%$ by volume. After 48 hrs. add 1 part to 9 parts aq. dest. Saturate with mercuric nitrate crystals frequently shaking several days. To make the reagent take 400 cc. filtrate, add 3 cc. original $40 \%$ solution plus $1.4 \mathrm{gm}$. sodium nitrite. Mount sections (preferably after freezing and drying technique) to slides without using water. Immerse in reagent in cold. Maximum reaction should be within $3 \mathrm{hrs}$. when sections show noticeable rose color. However use several slides, remove them from reagent in a Coplin jar at intervals, dip immediately in $1 \%$ aq. nitric acid, dehydrate quickly in absolute alcohol, clear in xylol and mount in balsam. Bensley and Gersh found that mitochondria are positive to Million's reagent.

Mineral Oil, reactions in tissue to fat stains after various fixations (Black, C. E., J. Lab. \& Clin. Med., 1937-38, 23, 1027-1036). See Immersion Oils.

Mingazzini Phenomenon in intestinal villi interpreted as an agonal or early postmortem change (by Macklin, C. C. and M. T., J. Anat., 1926, 61, 144-150).

Mites. The techniques given for Ticks and Insects are applicable for making whole mounts. The simple creosote method (see Insects) is recommended.

Mitochondria-Written by Geoffrey Bourne, London Hospital Medical College, London, England. November 5, 1951Granules, rods and filaments existing in the cytoplasm of practically all living cells of plants and animals. They can be studied in living cells unstained and after supravital staining, and in fixed tissues.
They can be seen in living cells even with direct illumination if it is critical. In mammals they probably are best seen unstained in very small pieces of pancreas mounted in normal saline and flattened out by the pressure of the cover glass. The distal poles of the acinous cells, facing the glandular lumen may be identified by densely packed, highly refractile zymogen granules. The proximal poles are nearer the surrounding blood vessels and comparatively free from zymogen granules. With direct illumination a careful search with an oil immersion objective will distinguish the mitochondria as delicate, slightly refractile filaments oriented in general with their long axes parallel with the length of the cell. Wernicke illumination (phase contrast) will permit immediate recognition of these bodies which are shown up clearly by this method. Phase contrast enables mitochondria to be studied with ease in a variety of living unstained cells (H. U. Zollinger, Rev. d'Hematol., $1950,5,696$ ).

The clarity with which mitochondria may be observed with phase contrast in living unstained cells has rendered their supravital staining less necessary. However, on occasions, this may be desirable. When very dilute methylene blue is added to tissue culture cells the mitochondria become stained a brilliant blue (Ludford, R. J., Arch. f. Exp. Zellf., 1935, 17, 339-359). Other vital dyes are diethylsafranin, Janus blue, Janus black 1, Pinacyanol, Rhodamin B. and Janus green B. (diethylsafraninazo-dimethyl-analin chloride).

The latter is used, as an example, as follows:

Place a small drop of $1: 10,000$ Janus Green B in $0.85 \%$ aq. sodium chloride. dye should be added from a $1 \%$ stock The solution in aq. dest. because the power does not dissolve easily in salt solution. In this drop a variety of small pieces of tissue, may be teased, and then covered with a cover glass, and mitochondria may be demonstrated very beautifully in blood leucocytes by adding a drop of freshly drawn blood to a drop of 1 in 10,000 solution of Janus Green B and examining after adding a coverslip. Mitochondria become colored a dark bluish-green after 5-10 $\mathrm{min}$. They first appear in the lymphocytes and then between the granules of the granular cells. R. R. Bensley (Am. J. Anat., 1911, 12, 297-388) has described a method of intravascular staining of mitochondria using Janus Green B. Janus Green preparations of mitochondria are not permanent-they 
bleach in from $30-60 \mathrm{~min}$. to a colorless leucobase. Mitochondria show up brilliantly in living, particularly tissue culture, cells using dark-ground illumination (see Strangeways, T. S. P. and Canti, R. G., Quart. J. Micr. Sci., 1927, 71, 1-14). 'They may be also photographed at high magnification with the electron microscope (R. Claude and E. F. Fullam, J. Exp. Med., $1945,81,51-62$; H. U. Zollinger, Experientia, $1950,6,16-17)$.

Mitochondria can be isolated from tissues by the process of differential centrifugation (R. R. Bensley and N. Hoerr, Anat. Rec., 1934, 50, 251, 499). This technique has been developed by Porter, K. and his colleagues ( $J$. Exp. Med., 1945, 81, 233-246); Claude, A, (Science, 1943, 97, 451-456; J. Exp. Med., 1944, 80, 19); Hogeboom, G. H. and his colleagues (J. Biol. Chem., $1946,165,615-630)$. These authors not only developed the technique of isolation of mitochondria by differential centrifugation and were able to obtain mitochondria which were morphologically identical with those in the living cell, but by a series of chemical studies were able to show that such isolated mitochondria contain the greater part of the respiratory enzymes of the cell, a fact which suggests, not only that the mitochondria may be the main respiratory centers of the cell but that they can function as synthetic centers as well. The conception of the respiratory function of mitochondria was suggested as long ago as 1912 by Kingsbury A. (Anat. Rec., 1912, 6, 39).

Isolation of Mitochondria. Hogeboom, G. H., Schneider, W. C. and Pallade, G. E. (Proc. Soc. Exp. Biol. Med., 1947, 65, 320-321) have described a method of obtaining morphologically intact mitochondria from rat liver. Rat liver is homogenized by the method of Potter, V. R. and Elvehjem, C. A. (J. Biol. Chem., 1936, 114, 495-504) in $0.88 \mathrm{M}$. sucrose. The homogenate is centrifuged 3 times at $600 \mathrm{~g}$. for $10 \mathrm{~min}$. This removes nuclei and intact colls. The supernatant is then centrifuged at $24,000 \mathrm{~g}$. for $20 \mathrm{~min}$. This brings down the mitochondria together with a few microsomes. Mitochondria obtained in this way remain stable in form for several days at $4^{\circ} \mathrm{C}$. There are a variety of methods for making permanent preparations of mitochondria in tissue sections.

1. Altmann's method. Sec Carleton, H. M. and Leach, E. H., Histological Technique, Oxford, 1949. Fix in Champy's fluid or in Flemming without acetic. Postchrome for 3 days (trans- fer tissue direct to $2 \frac{1}{2}-3 \%$ aq. potassium dichromate). Wash 12-24 hrs. in running water, dehydrate, embed and section. Bring sections to water, then: (1) flood slide with aniline fuchsin (aniline water, made by adding 10 cc. of aniline to one half or one litre of hot aq. dest. in a flask and ashing, cooling and filtering, 100 cc., acid fuchsin $12 \mathrm{gm}$.). Warm slide with bunsen flame till the stain steams (but does not boil). Leave for $5 \mathrm{~min}$. (2) rinse rapidly in aq. dest. (3) differentiate in slide jar of picric acid, sat. sol. in ab. alc. 20 cc., $30 \%$ alc. 80 cc. Differentiation should be stopped when the red dye has diffused out of the nuclei and cytoplasm and the mitochondria are bright red. (4) rinse in aq. dest. (5) dehydrate rapidly, mount in balsam.

2. Heidenhain's iron hematoxylin method. Tissues may be fixed in formaldehyde, Helly's fluid, Zenker formaldehyde or Flemming-without-acetic, for 24 hrs. Sections are mordanted in a $5 \%$ aq. iron alum for $\frac{1}{2}$ to $24 \mathrm{hrs}$. according to the nature of the tissue. Rinse in water. Stain in $0.5 \%$ aq. hematoxylin for a period of time equal to that of the iron alum treatment. Rinse in water, differentiate in iron alum solution and control differentiation with microscope until only nuclei and mitochondria are black. Counterstain if necessary, dehydrate, mount in balsam.

3. Cain's method (Quart. J. Micr. Sci., 1948, 89, 229-231). Fix in Helly's fluid, $6 \mathrm{hrs}$. postchrome $48 \mathrm{hrs}$. at $37^{\circ} \mathrm{C}$. in sat. aq. potassium dichromate, wash overnight in running water, embed in paraffin wax and cut sections about $3 \mu$. Bring sections to water treating with iodine ( $\frac{1}{2}$ in $70 \%$ alc.) and then $5 \%$ aq. sodium thiosulphate on the way. Dry slide, except sections, flood with aniline fuchsin, (see Altmann's method) and heat until steaming as for Altmann's technique. Wash off acid fuchsin with aq. dest. Irrigate with alkaline solution (one drop of aq. sodium carbonate in $10^{\circ} \mathrm{cc}$. of aq. dest.). Dif ferentiate $30 \mathrm{sec}$. to $1 \frac{1}{2} \mathrm{~min}$. To stop differentiation dip slide into $1 \% \mathrm{HCl}$. Wash in aq. dest., counterstain $1 \%$ aq. water-soluble methyl blue. Wash aq. dest., dip into $1 \%$ acid 3 sec. only. Wash aq. dest., dehydrate and mount in balsam.

The Bensley-Cowdry acid fuchsin and methyl green method (Cowdry, E. V., Contrib. Carnegie Inst., Wash., VIII, 1918) gives beautiful results. In it the metliyl green is used both as differentiator and counterstain. 
Schridde's method (Ergn. Anat. u. Entw., Bonnet, XX, 1911) can also be recommended as giving beautiful preparations of mitochondria.

4. Pritchard's silver method (J. Anat., 1951,85 , in press). This method, which is dependent upon reduction of silver on the slide, gives very beautiful and precise preparations of mitochondria which show up black. Small pieces of tissue should be fixed in Regaud's or Helly's fluid for 3 days and postchromed for 4 days in $3 \%$ aq. potassium bichromate. Prepare paraffin sections in the usual way, avoiding excessive heat in flattening and drying. Remove paraffin and proceed to aq. dest. Slides placed for $20 \mathrm{sec}$. with agitation into a dilute solution of silver diamino hydroxide (Wilder's Solution diluted with an equal volume of aq. dest. to which is added 2 drops of $8 \% \mathrm{NH}_{4} \mathrm{OH}$ per 50 cc.). Drain quickly and without rinsing immerse in a large volume (e.g. 200 cc.) of very dilute formalin (1/1000 commercial formalin in aq. dest.) agi tating for 10-20 sec., not more. Fresh formalin solution for each section is preferable. Wash in aq. dest. Differentiate carefully under the microscope with $1-2 \%$ aq. potassium ferricyanide until mitochondria show up black against a clear background. Wash in aq. dest., counterstain with $1 \%$ Safranin or Ponceau fuchsin. Regaud's fixative gives the best results but after Helly's fluid the Golgi element is often sharply impregnated as well as the mitochondria.

Mitochondria and Bacteria. Demonstration in the same cells. See Cowdry, E. V. and Olitsky, P. K., J. Exper. Med., 1922, 36, 521-533, Cowdry, E. V., Am. J'. Anat., 1923, 31, 339-343. Stain as for mitochondria with Anilin Fuchsin and Methyl Green. Mitochondria are colored crimson. When the bacilli are acid fast as in leprosy they are colored a dark reddish purple; but when they are not acid resistant they are stained bluish green.

Mitogenic Radiations. It is questionable whether these rays, said to generate mitosis, really exist. A critical and well balanced statement is afforded by Glasser, O., in Glasser's Medical Physics, $760-763$.

Mitosis (G. Mitos, thread). Indirect nuclear division in which the chromatin forms a thread which breaks up into chromosomes.

Material should be freshly fixed, less than half hour after removal. But mitosis can be seen in some tissues 24 hrs. or longer after death, especially if the body is kept at a low temperature but the number is less and the details not so clear as after quick fixation (Mallory, p. 108). Sat. mercuric chloride in $70 \%$ alc. plus $5 \%$ acetic acid, Zenker's fluid, formalin-Zenker, Bouin's fluid and Flemming's strong fluid are satisfactory fixatives but the last named penetrates very badly.

The most beautiful stain for mitotic figures is safranin light green but the mitoses can be more clearly distinguished without the green counterstain. Simply deparaffinise and stain sections in anilin-safranin (Babes), wash quickly in tap water, differentiate in acid alcohol until the resting nuclei are less intensely colored than the dividing ones, wash in $95 \%$, dehydrate in abs. clear in xylol and mount in balsam.

Another excellent method is to apply the Feulgen reaction for Thymonucleic Acid to sections of tissues preferably fixed in Carnoy's fluid or acetic sublimate. This demonstrates thymonucleic acid in the chromatin, and the dividing nuclei, as with safranin, are more deeply stained than the others. This method is displacing the older safranin technique.

To demonstrate mitosis in whole mounts of epidermis place freshly excised skin (circumcision specimen preferred) in $0.1 \%$ aq. acetic acid in the icebox over night. Wash quickly in aq. dest. Strip off the epidermis with needles, stain it like a section with anilin-safranin or with Harris' hematoxylin and mount with the outer surface uppermost. This technique could probably be adapted to relatively flat epithelia of the respiratory digestive, urinary and genital systems.

In order to reveal the maximum num. ber of mitotic figures it is important to study the mitotic rhythm of the particular tissue or organ and take tissues at the peak which in the case of the human foreskin is probably between 9 p.m. and midnight (Cooper, Z. K. and Schiff, A., Proc. Soc. Exp. Biol. \& Med., $1938,39,323-324)$. The relation of alimentation and nutrition to cyclic variations in mitotic activity is presented by Blumenthal, H. T., Growth, $1950,14,231-250$.

To experimentally increase the number of mitosis use colchicine which arrests the process chiefly in the metaphase by causing failure of the mitotic spindle to form and function (Iudford, R. J., Arch. f. exper. Zellf., 1936, 18, 411-441). Consequently as long as the cells are under the influence of colchicine-a matter of a few hours onlymitosis begins as usual; but, since it is not completed, the proportion of mitotic 
figures to resting nuclei is temporarily greatly increased. Sodium cacodylate, auramine and other substances listed by Ludford likewise influence mitosis. For checks on the method of estimating growth by counting arrested mitoses, see Paletta and Cowdry (F. X. and E. V., Am. J. Path., 1942, 18, 291-311). Aisenberg (E. J., Bull. d'Hist. Appl., $1935,12,100-122)$ has found that mitosis of epidermal cells is arrested in the metaphase simply by passing a ligature around a frog's leg and keeping the foot in distilled water. The mitoses accumulate in large numbers but continue when released from the hypotonic environment. Aisenberg (ibid. 1936, 13, 265-286) also discovered low concentration of ethyl alcohol to stimulate mitosis, $0.4-0.8 \mathrm{M}$ to arrest in metaphase, 1.2-1.5 M. to cause gelatinization of mitosis and higher concentrations to kill the cells. Comprehensive data on the influence of Colchicine are supplied by Levine, M., Ann. N. Y. Acad. Sci., $1951,51,1365-1408$.

Moist Chambers, Small for stage of microscope (Chambers, R. and Kopac, M. J. in McClung's Microscopic Technique, 1950). Large in which hands can be used (Holter, H., C. rend. trav. lab. Carlsberg, Sér. Ćhim., 1915, 25, 156167). See diagrams for air-conditioning (Glick, p. 182).

Molecular Film Technique, see Taylor, H. S., Lawrence, E. O., and Langmuir, I., Molecular Films, the Cyclotron and the New Biology, Rutger's University Press, 1942,95 pp.

Molecular Solution is the molecular weight of the substance in grams made up to 1 liter with aq. dest. Thus $M$ oxalic acid $(\mathrm{COOH})_{2} \cdot 2 \mathrm{H}_{2} \mathrm{O}$ is 126 gms. with aq. dest. added to 1 liter; but $N$ oxalic acid is half of this concentration. See Normal Solutions.

The molecular weight expressed in grams is called the gram-molecular weight or mole.

Millimole is $1 / 1000$ of a mole.

Milligram equivalent (milliequivalent). The equivalent weight, the gram-equivalent, or the equivalent of a substance is the weight in grams which in its reaction corresponds to a gram atom of hydrogen, or of hydroxyl, or half a gram atom of oxygen, or gram atom of a univalent ion. Milliequivalent is $1 / 1000$ of the equivalent weight, i.e., the equivalent weight of sodium carbonate is $\frac{1}{2}$ the molecular weight, or 53.0. Therefore, the milliequivalent (m.e.) or the weight in $1 \mathrm{ml}$. of normal solution is $0.0530 \mathrm{gm}$.
Molybdenum, see Atomic Weights.

Mono-Azo Dyes. Amarnth, azo fuchsin, benzene-azo- $\alpha$-naphthylamine, bordeaux red, brilliant yellow $S$, chromotrope $2 R$, chrysoidin Y, fast yellow, janus green $B$, metanil yellow, methyl orange, methyl red, narcein, nitrazine, oil red $O$, orange $G$, orange $I$, orange $I I$, orange $I V$, ponceau $2 R$, sudan $R$, thiazine red $R$.

Monocytes. When "typical" these are easily recognized in stained blood smears and in supravital preparations but there is no technique by which they may always be distinguished from all Lymphocytes and Macrophages. That is, they possess no single feature, like the eosinophile granules of eosinophile leu cocytes, for their certain identification (see Cowdry, p. 66-71). They ingest particulate matter including Trypan Blue and similar vital stains and are therefore to be considered as components of the Reticulo-Endothelial System. Many of their properties can to great advantage be investigated in Tissue Cultures. The best way to demonstrate the remarkably close relation that may exist between monocy tes and contained bacilli is to stain leprous tissue for acid fast bacilli (see Leprosy Bacilli). See Bacterium Monocytogenes.

Monolayer technique is a physico-chemical line of investigation that gives valuable data on the structure of protein and lipoprotein films and consequently on the plasma membrane of cells. See Schulmann (Bourne, pp. 51-67).

\section{Moore, see Fungi.}

Mordant (L. mordere, to bite), a substance, like alum, employed to make a dye bite into the tissue and hold on. The dye combines with the mordant which is itself in high concentration in the structures to be stained. In the Iron Hematoxylin technique the sections are mordanted with iron alum. They are briefly washed in aq. dest. to remove some of the excess mordant. Then they are stained with a dilute aqueous solution of hematoxylin and differentiated in the mordant which draws out most of the hematoxylin until it remains only in the structures which took up the mordant most energetically in the first place and which therefore alone remain colored. Copper salts are also good mordants. See Weigert's mordants.

Morosow's Method for elementary bodies as modified by Fonta and Triboudeau and given by Seiffert, G., Virus Diseases of Man, Animal and Plant. New York: Philosophical Library, Inc, 1944, 332 pp. Dry thin smear in air. Place vertically in aq. dest., 10-15 min. and 
dry again. Cover with mixture of acetic acid, 1 cc.; $40 \%$ formalin (commercial formaldehyde), 2 cc.; aq. dest., 100 cc. Rinse well in aq. dest. and heat till steam rises in mixture of carbolic acid, 1 cc.; tannin, 50 gm.; aq. dest., $100 \mathrm{cc}$. Rinse in aq. dest. $\frac{1}{2}$ min. aud heat slightly 1-2 min. till smear becomes brown or slightly black in silver solution made up as follows: To 20 cc. aq. dest. add "platinum loop" of $25 \%$ ammonia and then drop by drop from pipette of $10 \%$ aq. silver nitrate until an opalescent ppt. appears. About $0.5 \mathrm{cc}$. of silver solution will be needed. After silvering smear rinse well in aq. dest., mount and seal edges with paraffin.

Moskowitz, see Protein Silver for Staining Protozoa.

Mosquito larvae, technique of raising anopheline (Bates, M., Am. J. Trop. Med., 1941, 21, 103-122). Bodian technique for mosquito nervous system (Rogoff, W. M., Stain Techn., 1946, 21, 59-61).

Motion Pictures. The technique of making motion pictures of living cells and organs has proved its worth. The movies can be projected again and again and the sequence of events made very clear. It is important to remember that motile cells do not run around at the speed indicated, because the actual distance travelled is far less than on the screen and the time much greater. The Wistar Institute of Anatomy in Philadelphia is distributor of a comprebensive series of motion picture films on either a purchase or rental basis.

Motor End Plates. The particular morphological type of nerve ending in muscle does not concern us here; but reference can be made if desired to the classification by Hines, M., Am. J. Anat., 1931, 47, 1-55. The methods advocated for histological demonstration are legion. Reference is made to 2 gold techniques (Graven's and Carey's) and to 1 silver method (Chor's). The former can be ul timately traced back to Ranvier and the latter to Cajal. See also techniques described under Nerve Endings.

Mounting Media. The refractive index of the medium is important and a table giving the indices for many substances used is supplied by Lee (p. 218). As pointed out, the greatest transparency is secured when the refractive indices of media and tissues are equal and media of lower index than the tissues give somewhat greater visibility of tissue components, while those of higher index provide less visibility. There are many media to choose from, the refractive indices of which are more or less satis. factory. The selection will depend more upon whether the medium can be employed for the particular tissue and its relative permanence.

For frozen sections and tissues to be mounted from water and aqueous solutions various glycerin mixtures are popular: Lactophenol, Glychrogel, Brandt's and Kaiser's glycerin jellies. Having taken the easiest one to prepare, for their merits are about equal, the tissue is mounted and covered and it is necessary to seal the edges. In the case of temporary mounts a little paraffin applied with a heated scalpel, or wire, will suffice. Lee (p. 230) advocates Peter Gray's sealing medium made up by melting together 4 parts anhydrous lanolin, 1 part Canada balsam (dry) and 8 parts resin which becomes solid on cooling. Apply to edges in the same manner as the paraffin. Kronig's cement is employed in Bensley's laboratory. Duco cement is very worthwhile because it is insoluble in xylol, alcohol and other chemicals used to clean microscopic preparations. Mallory (p. 99) dilutes it with an equal volume of acetone. See Karo.

For sections and tissues which are first dehydrated and cleared the investigator must choose the mounting medium best adapted to his purpose from a considerable number proposed of which the following are given elsewhere in this book: Balsam, Cedar Oil, Clarite, Colophonium, Damar, Diaphane, Euperal, Nevillite, Sandarac, Terpineol Balsam.

The chief desiderata are a medium which will harden fairly quickly, which will not become acid and bring about the fading of anilin dyes and which will not crack or develop granules. Clarite is competing for first place with balsam; because, to make the balsam neutral and keep it so, is a troublesome job. Directions for its preparation are given by the Bensleys (p. 39). But the balsam ordinarily purchased is satisfactory for hematoxylin and eosin and iron hematoxylin preparations except when the latter are counterstained with an anilin dye. The writer used to employ cedar oil (for immersion objectives), in mounting sections stained by Giemsa's method, which is superior to balsam, but it drys slowly and is not better than clarite. Damar has been recommended for stains likely to fade and colophonium for thick sections of the nervous system for which covers are not used; both however appear to be less valuable than clarite.

Museum specimens require an aqueous mounting medium which preserves 
colors. See Color Preservation. See Plastics for museum work.

Mucicarmine for mucus, Mayer's (Mallory and Parker in McClung, p. 417). To make up stain, mix carmine, $1 \mathrm{gm}$.; aluminum chloride, 0.5 gm.; and aq. dest., 2 cc. Heat over flame for $2 \mathrm{~min}$. Color of solution darkens. Add gradually 100 cc. $50 \%$ alc. stirring constantly until dissolved. After 24 hrs. filter. Filtrate keeps well. Stain paraffin sections of absolute alcohol fixed tissue in carmine sol. 5-10 min. Wash in water, dehydrate, clear and mount. Mucus is red. When nuclei also a re colored red, add few drops $1 \%$ aq. sodium bicarbonate to the stain. It is customary to stain cells and nuclei before hand with alum hematoxylin. Bensley (Cowdry's Special Cytology, 1932, p. 203) uses alcoholic chrome sublimate (sat. mercuric chloride and potassium bichromate in $95 \%$ alc.) and increases the content of carmine 5 times.

Mucigen, intracellular antecedent of Mucin.

Mucihematein for mucus, Mayer's, Mallory and Parker in McClung, p. 416). Make up: (A) hematein, $0.2 \mathrm{gm}$.; aluminum chloride, $0.1 \mathrm{gm}$; glycerin, $40 \mathrm{cc}$.; aq. dest., 60 cc. and (B) hematein, 0.2 $\mathrm{gm}$.; aluminum chloride, $0.1 \mathrm{gm}$.; $70 \%$ alcohol, $70 \mathrm{cc}$; nitric acid, 1-2 drops. $A$ is advised except when the mucus swells much in which case use $B$. Stain paraffin sections of absolute alcohol fixed tissue 5-10 min. Wash in water. Dehydrate in $95 \%$ alc. and in abs. Clear in xylol and mount in balsam. Mucus stains blue. The other materials are colorless. Preliminary coloration with carmine is suggested. Bensley (Cowdry's Special Cytology, 1932 , p. 203) used alcoholic chrome sublimate fixation (sat. mercuric chloride and potassium bichromate in $95 \%$ alc.) and increased the content of hematein five times.

Mucin, one of several glycoproteins found in mucus. See Mucus and Mucicarmine, Mucihematein and Mucisudan stains, also Polysaccharides.

Mucinase. Enzyme(s) which hydrolyze mucus or mucoproteins. They are not very well understood, and specific preparations are not yet available. Purified enzymes, capable of differentiating the various mucopolysaccharides one from another would be very valuable.

Mucisudan is a dye of undetermined composition made by hydrolysis of sudan black $B$ with acetic acid and recommended as a new stain for mucin (Leach, E. H., J. Path. and Bact., 1938, 47, $637-639)$

Mucoproteins. A method for histological distinction between the chondroitin sulphuric acid protein of connective tissue mucus and the mucotin sulphuric acid protein of epithelial tissues has been worked out by L. H. Hempelmann, Jr., Anat. Rec., 1910, 78, 197-206. Briefly stated toluidin blue in $1: 280,000$ will stain the former vividly and the latter not at all. Glick, p. 46, notes that confirmation of Hempelmann's work is still lacking.

Metachromatic staining with toluid in blue is specific for mucoid compounds containing polysaccharide esters of sulfuric acid provided that Lison's technique is followed closely in the opinion of Sylvén, B., Acta Radiol., 1945, suppl. 59, 100 pp. Fix tissue (12-24 hrs.) in equal parts $8 \%$ aq. basic lead acetate and $14-16 \%$ formalin. Stain some paraffin sections (30 min.) in $0.1 \%$ toluidin blue in $1 \%$ alc. and others in $0.1 \%$ toluidin blue in $30 \%$ alc. (first allowing these stains to age for several days). Wash in alc. briefly, mount in natural cedar oil. See Michaelis, L. and Granick, S., J. Am. Chem. Soc., $1915,67,1212-1219$ and Hess, M. and Hollander, F., J. Lab. \& Clin. Med., 1947,32 , 905-909 for data about metachromasia. Leach, E. H., Stain Techn., 1947, 22, 73-76 has advocated Bismark brown as a stain for mucoproteins.

Mucus means slime. It is a viscid, stringy material which ordinarily stains with basic dyes and is found in many parts of the body. The chemical composition of mucus is not uniform. It may consist of one of several glycoproteins, called mucins, which are by contrast definite chemical substances. The term mucous is an adjective describing a cell or tissue which produces or contains mucus. Mucigen is the intracellular antecedent of a mucin. Since there are several mucins there are several corresponding mucigens.

Pathologists sometimes divide mucins into two categories, epithelial and connective. The connective tissue type is found in the ground substance of bone, synovial fluid and in other locations. It increases in amount in the myxedema (G. myxa, mucus + oiderna, swelling) of certain thyroid deficiencies as well as in arteriosclerosis and various tumors. The ubiquitous fibroblast is said to be a great former of mucins. Epithelial mucins are produced by epithelial secretory cells. The goblet cells are easily recognized by the fact that the material to be discharged is held in a goblet like expansion of the cell. Other mucous cells can be distinguished from 
serous or zymogenic cells by several criteria :

1. The nuclei instead of being roughly spherical are often, but not always, pressed against the cell membrane remote from the lumen.

2. The mitochondria are usually of smaller diameter and shorter than in zymogenic cells.

3 . The secretion antecedents (Mucigens) of mucous cells are more difficult to see in the fresh state, more labile, and in fixed tissues are metachromatic and can be stained almost specifically with mucicarmine and mucihematein.

See Mucicarmine and Mucihematein of Mayer.

A simple method for mucus has been described by Lillie (R. D., J. Tech. Methods, 1929, 12, 120-121). Sections of tissue fixed in formalin or in Zenkerformol (Helly) are passed to water. In the case of the latter remove mercury with iodine and sodium thiosulphate as usual. Stain 1 min. in $0.2 \%$ aq. toluidin blue. Wash in water. Dehydrate in pure acetone, clear in xylol and mount in balsam. Mucus, reddish violet; nuclei, blue; red cells, yellow or greenish yellow. In the case of old formalin material rinse in $95 \%$ alcohol before the acetone.

McManus, J. F. A., Nature, 1946, 158, 202 , recommends the use of Schiff's Reagent followed by periodic acid. Material fixed in Zenker-formal is dehydrated and embedded in the usual manner and the sections transferred to water after treatment with iodine and hypo and placed in a $0.5 \%$ aq. periodic acid $2 \mathrm{~min}$. The slides are washed in tap water and aq. dest. and kept in Schiff's reagent for 15 minutes; rinsed in Sulphurous Acid, dehydrated and cleared in the alcohol and xylol series respectively and mounted in balsam. According to McManus, the mucus of the goblet cells of the human intestine and bronchus, mucus salivary glands, certain pituitary cells, the colloid of the pituitary stalk and thyroid, granules in some nerve cells in the medulla of the rat and in the human intestine, the basement membranes of the tubular epithelium and of the glomerulus in the kidney were tested by this method and an intense coloration detected in all instances.

Hotchkiss, R. D. deserves credit for reporting in a personal communication to Glick (p. 43) in 1946 the independent discovery of a similar technique to that of McManus employing periodic acid. This is given in detail by Glick.

Muelengrath Test, see Icterus Index.

Müller's Fluid. Potassium bichromate, 2$2.5 \mathrm{gm}$.; sodium sulphate, $1 \mathrm{gm}$.; aq. dest., $1 \mathrm{gm}$. This was formerly much used for long fixation and mordanting of nervous tissue. See Chromaffin Reaction, Decalcification, O'Leary's Brazilian Method, Weigert Method. It is now largely replaced by Orth's Fluid which is really formalin-Müller.

Mumps. Refractile, eosinophilic bodies in red blood cells are very small first 5-6 days. Increase in size and elongate 7-14 days. (Parsons, H. H., Military Surgeon, 1938, 83, 541-543).

Murexide Test, see Purines.

Muscle, to distinguish in sections from connective tissue, Dahlgren (McClung, $p$. 306) suggests Retterer's and Van Gieson's stains, picronigrosine and Unna's orcein to which may be added Mallory's stain. Demonstration of chloride in muscle fibers (Heilbrunn, L. V. and Hamilton, P. G., Physiol. Zool., 1942, 15, 363-374). For contraction bands and wave mechanics, see Carey, E. J., Arch. Path., 1940, 30, 881-892, 1041-1072. A technique for separating nuclei from cytoplasm for analysis is given under Nuclei. If microdissection is contemplated the pioneer paper by Kite, G. L., Am. J. Physiol., 1913, 32, 146-164 should be consulted. The experimental production of myocardial segmentation is described by Saphir, O. and Karsner, H. T., J. Med. Res., 1923-24, 44, 539556. Methods of Maceration are often useful in the isolation of single fibers. Mitoses can only be induced in exceptional cases (Allen, E., Smith, G. M. and Gardner, W. U., Am. J. Anat., 1937, 61, 321). An electron microscopic technique for localization of magnesium and calcium is described by Scott, G. H. and Packer, D. M., Anat. Rec., 1939, 74, 31-45. Muscle gives beautiful fluorescent colors in ultraviolet light with many fluorochromes (Metcalf, R. L. and Patton, R. L., Stain Techn., 1944, 19, 11-27). See Myosin and Purkinje cells and fibers.

Museum Specimens, see Color Preservation.

Mycelia, see Lillie, p. 289.

Mycobacteria, see Ácid Fast Bacteria.

Mycological Techniques, see Fungi.

Myelin, see various methods for demonstration of Nerve Fibers.

Myeloblasts. The recognition of these cells is a fine art; because, by definition, they are so little differentiated that the granules characteristic of the 3 types of leucocytes are absent. For contrasting views, dependent largely on whether supravital staining or fixed and stained preparations are used, see Cowdry's Histology, p. 100, also Leucocytes, developmental series. 
Myelocytes, see Leucocytes, developmental series.

Myeloidin is the term applied to the material of certain spheroidal or cuboidal bodies of wax-like luster present in the bases of retinal pigment cells of monkeys and some other animals but reported as absent in man and said to resemble myelin. For literature see Arey, L. B. in Cowdry's Special Cytology, 1932, $3,1218$.

Myocardium. Method for separation of fiber bundles (Mall, F.P., Am. J. Anat. 11, 211-266).

Myofibrils. The best method is to fix in Zenker's fluid or strong Flemming's mixture and to stain with iron hematoxylin (see Dahlgren in McClung p. 425). Microincineration is useful for the demonstration of minerals.

Myoglia is a fine network of fibers associated with muscle cells well demonstrated by Mallory's Connective Tissue Stain.

Myonemes of trypanosomes, gold chloride method for (Ogawa, M., Arch. f. Protistenk., 1913, 29, 248). See Wenrich, D. H. and Diller, W. F. in McClung's Microscopical Technique, 1950, p. 464 .

Myosin is a protein, present in muscle, the molecules of which are needle-shaped. Cross striations of muscle are thought to depend on their arrangement. In the isotropic (non-birefringent) bands the myosin molecules are believed to be disposed at random and in the anisotropic (birefringent) bands parallel to the length of the fiber (see Bourne, p.30).

\section{Myriapoda, see Parasites.}

Nadi Reagent is dimethyl-paraphenylenediamin $+\alpha$ naphthol. Indophenol oxidase catalyses oxidation of nadi to indophenol blue and that of paraphenylene diamin to diamin.

Nails. These very interesting structures are seldom examined microscopically despite the fact that changes in them may provide significant clues to the condition of other tissues. They are chiefly made up of stratum lucidum thickened by much eleidin. It is a simple matter to macerate cut finger or toe nails in $40 \%$ aq. potassium hydroxide or in concentrated sulphuric acid for a few days and then to isolate the individual cells some of which are nucleated. MacLeod, J. M. H., Practical Handbook of the Pathology of the Skin. London: H. K. Lewis, 1903, 408 pp. gives Heller's method which involves fixation of ungual phalanx for a few days in Muller's fiuid, prolonged washing, decalcification for 4-6 days in 1 part nitric acid and 3 parts of water followed by thorough imbedding in celloidin. The sections can then be stained with hematoxylin, gentian violet, safranin or any other of a number of dyes.

Naphthalene Pink, see Magdala Red.

Naphthalene Red, see Magdala Red.

Naphthamine Blue 3BX, see Trypan Blue.

Naphthamine Brilliant Blue $2 \mathrm{R}$, sce Dianil Blue 2r.

Naphthol Blue Black (CI, 246). Lillie, R. D., J. Tech. Methods, 1945, No. 25, $47 \mathrm{pp}$. has reported that this dye (NAC7080 and DuPont L 6401) gives excellent staining in combination: Stain with Weigert's iron hematoxylin, $6 \mathrm{~min}$. Wash in water and counterstain $5 \mathrm{~min}$. in 3 parts $1 \%$ brilliant purpurin $\mathrm{R}(\mathrm{Cl}$, 454 ) in $1 \%$ aq. acetic acid and 2 parts $1 \%$ azofuchsin $(\mathrm{CI}, 153)$ likewise in $1 \%$ aq. acetic acid. Rinse in $1 \%$ aq. acetic acid and stain $5 \mathrm{~min}$. in $1 \%$ naphthol blue black $(\mathrm{CI}, 246)$ in sat. aq. picric acid. Rinse in $1 \%$ aq. acetic acid, $2 \mathrm{~min}$. Deliydrate and clear in alcohol, alcohol and xylol, xylol and mount in clarite. Collagen, reticulum and basement membranes, dark green; smooth muscle, brown; nuclei brownish-black.

Naphthol Blue R (CI, 909)-fast blue 3R, Indian blue 2RD, Meldola's blue, new blue $R$, phenylene blue-An oxazin dye used by Harvey, B. C. H., and Bensley, R. R., Biol. Bull., 1912, 23, 225-249 as a supravital stain for gastric mucosa. The Bensleys' report that this dye has proved useful in the localization of unsuspected parathyroid and thyroid tissue in experimental animals. After vascular perfusion in a concentration of $1: 40,000$ of $0.85 \%$ aq. sodium chloride the thyroid, parathyroid and lymph nodes become colored intensely blue; whereas other tissues, muscles, salivary glands etc., are colored pale greenish blue.

Naphthol Green, see Naphthol Green B.

Naphthol Green B (CI, 5)-acid green O, green PL, naphthol green-An acid nitroso dye for which a probable formula is given by Conn (p. 42) and which he thinks was the naphthol green used by $\mathrm{v}$. Volkmann, R. and Strauss, F., Zeit. f. Wis. Mikr., 1934, 51, 244-249, and by Mollier, G., Zeit.f. Wis.Mikr., 1938, 55, 472-473.

Lillie, R. D., J. Techn. Methods, 1945, No. 25, 47 pp. recommends naphthol green 13 for connective tissue. Stain sections $6 \mathrm{~min}$. in Weigert's or other iron hematoxylin. Wash thoroughly in water and stain $3 \mathrm{~min}$. in $1 \%$ aq. eosin $Y(C I, 768$. Rinse in water and mordant 4 min. in $10 \%$ dilution of U.S.P. ferric chloride solution. Rinse in water and stain $5 \mathrm{~min}$. in $1 \%$ naphthol green B. Differentiate $2 \mathrm{~min}$. in $1 \%$ aq. acetic acid. Dehydrate in aceton, clear in acetone-xylene and in xylene and mount in clarite xylene or 
in salicylic acid balsam. Connective tissue, green; muscle and cytoplasm, pink.

Y (CI, 2)-fast printing green, Gambine-An acid nitroso dye apparently not used in histology.

Naphthol Orange, see Orange I.

Naphthol Red S, C or O, see Amaranth.

Naphthol Yellow, sec Martius Yellow.

Naphthyl Red (CI, 856), a basic dye of light fastness 5. Only nuclei of mature plant cells colored fugitive red (Emig, p. 57).

Naphthylamiue Brown (CI, 170), an acid monoazo dye which stains plant tissues darker in presence of potassium bichromate (Emig, p. 34).

Naphthylamine Pink, see Magdala Red.

Naples Yellow, an exogenous pigment$\mathrm{Pb}_{3}\left(\mathrm{SbO}_{4}\right)_{2}-$ Lillie, p. 134

Narcein (CI, 152). An acid mono-azo dye. Was used by Ehrlich in combination with pyronin and methylene blue or methyl green to produce a neutral dye (Conn, p. 54). No longer available.

Nasal Cell Smears-Written by Marian Pfingsten Bryan, Dept. of Otolaryngology, Washington University, St. Louis 10, Mo.-September 14, 1951-Since 1927 the study of the cytology of nasal secretions has been recognized as an important aid in the clinical diagnosis and differentiation of certain nasal and sinus conditions. Eyerman, C. H., one of the early investigators (Ann. Otol., Rhinol. and Laryngol., 1927, 36, 808-815), identified the main types of cells found in nasal secretion. Tillotson, R. S. (Arch. Otolaryng., 1929, 10, 262-265) reported on the value of cytologic studies in the diagnosis of sphenoiditis. Sewall, E. C. and Hunnicut, L. (Arch. Otolaryng., 1929, 10, 1-15) discussed cytologic examination of the antrum. Dean, L. W. (J. A. M. A., $1932,99,543-546)$ emphasized the significance of the nasal smear in helping to diagnose allergy of the nose and sinuses. Hansel, F. K. (J. Allergy, 1934, 5,357 ) contributed many observations on the cytology of these secretions, especially in regard to allergy of the nose and paranasal sinuses. There is a good review of the numerons articles in the literature dealing with nasal cytology by Hansel, F. K., Allergy of the Nose and Paranasal Sinuses, St. Louis: C. V. Mosby Co., 1936, p. 333.

The particular emphasis has been on the number of eosinophilic cells in relation to neutrophiles; but, as knowledge has progressed in nasal cytology, the value of the study of smears has been extended. The objectives of nasal and sinus cytology include the following: First, the demonstration of eosinophiles in the secretions in relation to neutro- philes may be helpful in distinguishing allergic conditions from infections, or in establishing the coexistence of both. In the second place the method makes possible detailed observations of the ciliated columnar epithelial cells, exfoliated in the early stages of acute upper respiratory infections. These studies may aid further in differentiating the common cold from allergic rhinitis. Thirdly the smear technique proves to be a reliable help, along with biopsy, in the diagnosis of carcinoma, other tumors and lesions of the upper respiratory tract. Fourth, for the microscopic study of radiation changes produced in cells after treatment with $\mathrm{x}$-ray and radiation, it is invaluable. Fifth, it is a useful tool for working out unknown cellular patterns which may support clinical diagnoses of other nasal and sinus conditions.

For cytological studies, nasal secretion is collected, after blowing the nose, on wax paper or on a cellophane handkerchief. Specimens are preferably taken separately from each side of the nose. The material may also be blown directly on to a clean glass slide, then gently smeared with a probe (not a dry cotton applicator). Thin smears are more suitable for microscopic study than thick ones. In order to obtain a sampling from a normal nose, the nasopharynx is wiped with a small cotton applicator, slightly moistened with saline. For abnormal dry noses, the wiping technique may also be used before application of any drugs. A saline tampon, inserted into the nostril, may stimulate the flow of secretion in order to obtain a specimen. Secretions from the sinuses may be collected by aspiration of the nose, displacement or irrigation of sinuses, or be taken directly from the area during an operation. These secretions, direct from the respective paranasal sinuses, are often of value in locating the sonrce of the infection and in determining the type of cellular response. They thus help to complete the diagnosis. The cytological picture presented in the nose may be somewhat different from the cellular pattern observed when the material is obtained directly from a particular sinus. Both should be studied and correlated whenever possible.

In the differential diagnosis of acute and chronic infections from allergic ones, or in demonstrating the joint existence of both, the numerical proportions of eosinophilic to neutrophilic cells in the nasal smear should be evaluated. The ratio is usually an expression of the nature and stage of the nasal 
condition. Hansel, F. K., St. Louis: C. V. Mosby Co., 1936, gives a useful scheme on page 380 for recording the cytology of nasal secretions similar to that used in the recording of skin tests. In view of the irregularity of distribution of the cells and the amount and type of secretions, it is difficult to determine the cells in percentages. Long personal experience in the observation of smears and in the correlation of them with clinical symptoms is of the utmost value. The smears should be repeated in the same patient in order to obtain a complete picture of the sequence of events. In estimating the numbers of neutrophiles in the secretion, it must be realized that when acute or chronic infections complicate allergic responses, the neutrophiles outnumber the eosinophiles in the secretion about 10 to 1 . In seasonal hay fever, however, uncomplicated by infection, there may be a few neutrophiles along with squamous epithelial cells, but the major cellular response will be eosinophilic. Some cases of hay fever show a response of mononuclears with coarse blue granules filling the cell and nearly obscuring the nucleus. Their actual significance is still unknown. A criticism of Hansel's scheme is that it does not take into consideration the other cellular elements besides eosinophiles and neutrophiles. The type, color, quantity and consistency of the secretion should be included and correlated with clinical symptoms, but the macroseopic appearance cannot be relied upon as an indication of the microscopic cellular contents of the secretion. The smears must also be examined for the presence of microorganisms and the types of epithelial cells should be carefully studied.

Smears from normal noses may or may not show a few scattered neutrophiles. A small amount of thin mucus is usually present with some squamous epithelial cells. Ciliated cells are seen if they have been wiped from the nose with the applicator. In the normal nose they are rarely exfoliated.

In cases of nasal polyps, eosinophiles usually are predominant; but repeated studies of the secretions are necessary to determine whether or not complicating acute or chronic infection is present. If a marked predominance of neutrophiles persists, a chronic infection is present. The cytology of nasal polypi is fully discussed by Walsh, T. E., and Lindsay, J. R. (Arch. Otolaryng., 1934, $20,649)$. Stagnation and secondary infection are frequently present in these cases, since polypi cause obstruction in the nose and a resultant neutrophilic response. A single failure to find eosinophiles in a smear does not rule out the existence of an allergic condition, but repeated failures to demonstrate eosinophiles usually means that an active nasal allergy is not present.

The differentiation of an allergic rhinitis from a common cold may at times be difficult clinically. In these cases microscopic examination of the secretions is helpful; but nevertheless, even with cases of acute upper respiratory infections in non-allergic patients, the eosinophilic response is so marked that it may closely approach the number found in allergic individuals during an infection. In the non-allergic persons the eosinophiles may disappear before the neutrophiles but not in many of them. In allergic persons, the eosinophiles often increase as the neutrophiles disappear, but these differences are uncertain. Acute allergic exacerbations may so closely resemble common colds that in numerous intances, even microscopically, it is difficult to distinguish between them unless repeated consecutive smears are examined throughout the course of the condition. Hilding, A. C. (Tran. Amer. Laryngol. Assoc., 1934, 253-271) carefully studied fresh unstained nasal secretions from common colds in adults and noted that ciliated columnar cells were shed in the exudates during the first few days of the infection. More recently Bryan, W. T. K. and Bryan, M. P. (The Laryngoscope, $1950,60,523-531$ ) applied the staining technique of Papanicolaou to nasal secretions. In a series of upper respiratory infections, in which the secretions were examined at timed intervals during the course of the infection, it was possible to chart a degenerative pattern of change exhibited by the ciliated columnar epithelial cells. These changes suggest virus etiology. In seasonal allergic hay fever the exfoliation of the ciliated columnar cells and their degenerative patterns of change have thus far not been observed. Smears from these cases reveal that large numbers of clumped or grouped eosinophiles are significant when they are not complicated by infection. Ciliated epithelial cells are of rare occurrence, but some basal and squamous epithelial cells are frequently encountered. Consequently exfoliation of the ciliated columnar epithelial cells in acute upper respiratory infections may have diagnostic value.

Within the last few years cytological studies of nasal diseases have been extended by use of the smear technique to include diagnostic information in regard 
to malignancies, other tumors and lesions occurring in this area. Morrison, L. F., Hopp, E. S. and Wu, R. (Ann. Otol., Rhinol. and Laryngol., 1949, 58, 18-31) have employed the smear technique as an adjunct in the diagnosis of exfoliating neoplasms of the nasopharynx. This proved so reliable that a positive smear demanded discovery of the source of the malignant cells. Sooy, F. A. (The Laryngoscope, 1950, $60,964-992)$ in a study of primary tumors of the nasal septum, has also used the smear technique to advantage. Early diagnosis of carcinoma of the maxillary sinus in a series of cases reported by Fitz-Hugh, G. S., Moon, C. N. Jr. and Luptom, C. H. Jr. (The Laryngoscope, $1950,60,376-387$ ) was thus facilitated.

This cell smear method is not only an aid in diagnosis, but also is a convenient means for studying the microscopic course of a lesion during and after treatment. Biopsies are not always possible, but smears are easily obtained without discomfort to the patients. The effects on the cells of $x$-ray or radium therapy comprise a whole new field of cytological research. New knowledge thus gained is of great importance when closely correlated with clinical symptoms.

Satisfactory techniques for staining nasal secretions include the following:

1. Wright's Stain. Slides are dried in air (avoid flaming). Many directions advise staining as a blood film; but two points will improve this technique for nasal work, namely the use of a buffer solution for the diluent and the shortening of the time recommended for blood smears to only 15 or $20 \mathrm{sec}$. of staining. After marking off the ends of the slides with a wax pencil, they are flooded with the dye for 15 sec. The buffer diluent is added to the stain and allowed to $\mathrm{mix}$ well for 15 sec. more. The slides are then washed in buffer solution and placed on end on a blotter to drain and thus to dry more rapidly. This light rapid staining shows cellular detail better in the nasal smear than the usual technique. The slides can be kept and stored for many years without coverslips and thereafter show no signs of deterioration. The cellular details are even better if the slides are stained within $30 \mathrm{~min}$. after being made and dried. The stain is especially good for eosinophiles, neutrophiles and mononuclears. It is not very suitable for differentiating epithelial cells.

2. Hansel's Stain. Color slide 30 sec. with his dye which is an eosin- methylene blue combination and can be obtained directly from him (Dr. F. K. Hansel, 634 N. Grand Blvd., St. Louis 3, Mo.). Then add alkaline water which is made by adding one drop of $1 \%$ potassium carbonate to 60 cc. aq. dest., for $30 \mathrm{sec}$. Wash in alkaline water followed by washing in acid water which is made by adding one drop of $1 \%$ hydrochloric acid in $60 \mathrm{cc}$. aq. dest. Wash again in alkaline water and finally rinse in $95 \%$ ethyl alcohol. This stain is especially good for eosinophiles. The granules are very brilliant and refractile. Other cellular detail may be somewhat dark and indefinite.

3. Giemsa Stain. The preparation from Gradwohl is very satisfactory. The dilution is one drop of stain to $1 \mathrm{cc}$. aq. dest. The slide is flooded with diluted stain for one minute then washed with aq. dest. If overstaining occurs, this may be decolorized with ethyl alcohol. The restulting coloration is excellent for eosinophiles but is not especially recommended for the other cells.

4. Supra-Vital Staining. This technique is particularly useful for studying secretions in the fresh condition when one wishes to observe motility of eosinophiles and neutrophiles, the phagocytic activity of neutrophiles and of mononuclears, as well as the ciliary activity of the exfoliated columnar epithelial cells. One drop of exudate may be mixed with a drop of $1: 15,000$ aqueous Neutral Red or Janus Green, or of both in combination. The technique is fully described by Sabin, F. R. (Bull. Johns Hopkins Hosp., 1923, $34,277-288$ ) who used it to study living human blood cells. The stock solution of Neutral Red contains $100 \mathrm{mg}$. of dye to $10 \mathrm{cc}$. of absolute alcohol. The dilute solution contains $0.4 \mathrm{cc}$. of stock Neutral Red in $10 \mathrm{cc}$. of absolute alcohol. An even dye film is obtained by flaming the slide, then flooding it with the dilute Neutral Red solution or with a mixture of Neutral Red and Janus Green, which is 2 cc. of dilute Neutral Red to 3 drops of saturated solution of Janus Green in absolute alcohol. The slide is quickly drained and placed upright to dry. Fresh exudate is mounted on the slide with coverslip and ringed with vaseline. The cells may last for 2 to $3 \mathrm{hrs}$. if examined under a warm stage. The mitochondria of the cells may be studied when Janus Green is used. This dye is more toxic to the cells than Neutral Red. The ciliated epithelial cells are of particular interest and the motility of the cilia is not impaired. Nuclear staining of the 
cells with either of the dyes is indicative of cell death. The supra-vital staining technique was applied to the study of nasal secretion by Pfingsten, M. G., in 1933 and reported to the E. N. T. clinical conference, Barnes Hospital (unpublished). It is possible to distinguish cells which are stimulated to activity from degenerating cells with this technique. A good azure stain may be obtained by using 10-12 drops of Wright's stain in absolute al. cohol, but this is more toxic than Neutral Red.

5. Papanicolaou Stain. This stain, as developed by Papanicolaou, G. N'. (Science, 1942, 95, 438-439) is the first satisfactory stain and fxation found which permits accurate identification of the different types of epithelial cells found in nasal exudates. Ciliated columnar cells are well preserved and can be readily identified from the squamous and basal types. The nuclear detail of all cells in this stain is very sharp and clear. The eosinophilic granules, however, are not as distinct as in the other stains. Slight modifications can be made to bring out certain details in nasal smears. The chromatin masses may be more distinct when the time in hematoxylin is shortened to 2 to $3 \mathrm{~min}$. The granules of the eosinophilic cells and other acidophilic bodies are brighter red if the time in eosin is increased to $3 \mathrm{~min}$., or, if a trace of phloxine is added ( $0.5 \%$ solution in $95 \%$ alcohol). All cellular details disclosed by the Papanicolaou stain are revealed by Wright's stain, even the patterns of epithelial degeneration, but they are noted with greater difficulty.

It follows that one stain is not sufficient to see all cellular details to the best advantage. It seems important to use several staining methods on the same material to obtain more complete knowledge of cellular responses. The smears, with their cellular patterns, may be considered to be an approximate index of the pathological processes occurring in the tissues.

Nasal Passages. The fluid, when present in unusual amounts can obviously be studied in Smears. Nasal clearance depends upon the movement by the cilia toward the pharynx of a mucous sheet (to which foreign materials become attached) over a layer of fluid in which the cilia act as can be demonstrated by the techniques of Lucas, A. M. and Douglas, L. C., Arch. Otolaryng., 1934, 20, 518-541 and others. Methods for Mucus and Cilia are given under their respective headings. The wall of the nasal passages exhibits marked regional diversity (Hilding, A., Arch. Otolaryng., 1932, 16, 9-18). The nasal mucous membrane covering the septum can be removed in toto by the dilute acetic acid method (see Epidermis) and examined as a whole mount which gives valuable data impossible to secure from the study of sections. Those interested in wound healing would do well to consult a paper by Boling, L. R., Arch. Otolaryng., 1935, 22, 689724. An easy and graphic method for visualization of lymphatic drainage is described under Lymphatic Vessels. For numerous suggestions as to technique see Proetz, A. Applied Physiology of the Nose. St. Louis: Annals Publishing Co., 1941, 395 pp.

Nasal Sinuses. The mechanism of clearance is similar. To make sections of the nasal sinuses, especially the smaller ones, fixation in Formalin Zenker is suggested followed by Decalcification and Celloldin Imbedding. The sections can be stained by the method best adapted to the purpose in mind.

Nasmyth's Membrane, see Enamel cuticle. n-Butyl Alcohol (prophylcarbinol). Recommended by Stiles (K. A., Stain Techn., 1934, 9, 97-100) to replace higher concentrations of alcohol in histological technique especially for lightly chitinized insects but also as a routine for vertebrates. After fixation in Gilson's Fluid pass the tissues through $35 \%$ (ethyl) alcohol $\frac{1}{2}-1$ hr.; 90 cc. $45 \%$ alc. +10 cc. butyl, 2 hrs.; 80 cc. $62 \%$ alc. +20 cc. butyl, 2 hrs.; 65 cc. $77 \%$ alc. +35 cc. butyl, 4 hrs.; 45 cc. $90 \%$ alc. +55 cc. butyl, 6 hrs. to days; 25 cc. abs. alc. +75 cc. butyl, 6 hrs. to over night; butyl 2 changes several hrs. (or store in butyl if desired). To imbed transfer to mixture of butyl and paraffin and to paraffin. $n$ Butyl alcohol is helpful in making permanent preparations of tissues freshly stained with Methylene Blue, which see. It should not be confused with Tertiary Butyl Alcohol.

Necrobiosis was for Minot (C. S., The Problem of Age, Growth and Death. New York, G. P. Putnam's Sons, 1908, 280 pp.) a condition in which the cells continue to live but change their chemical organization so that their substance passes from a living to a dead state. "Here (he says) life and death play together and go hand in hand." The term is current but is of little use because it has no advantage over the word Necrosis for the disorganization of death seldom if ever takes place simultaneously throughout the substance of any living thing. See Dead Cells. 
Necrosis (G. nekrōsis, a killing). The term is usually applied to indicate the local death of a cell or of group of cells, not that of the body as a whole. Death is defined by Webster and others as the "cessation of life" which merely poses the question of what life is. Perhaps the most fundamental vital phenomenon is the oxygen consumption involved in respiration. This may persist in erythrocytes even after the loss of their nuclei (Harrop, G. A., Arch. Int. Med., $1919,23,745-752)$. But cells frozen by special techniques do not respire while frozen. They endure in a state of suspended animation (called vitrification) indefinitely. They are not dead since they retain the structural organization, which, when unlocked by increase in temperature, confers renewed vitality (see Luyet, B., C. rend. Soc. de biol., 1938, 127, 788-789 and many others). Death can therefore be better defined as the disorganization of living matter which makes permanently impossible all vital phenomena. Since the organization of different sorts of living cells is fundamentally different the loss of organization in them is likely also to be different. See various forms of Degeneration. In general necrosis of tissue is of ten evidenced by a breaking up of the nucleus known as caryorrhexis (G. Karyon, nucleus, + rhèxis, rupture) or by its solution, caryolysis (G. lysis, solution). Consequently any good nuclear strain such as hematoxylin or methylene blue is satisfactory. See techniques for Dead Cells, Necrobiosis.

Neelsen, see Carbol-Fuchsin.

Negative Stains are used to show the background in which bacteria and other organisms are present in smears and by contrast thus to reveal them unstained, that is in a negative way. The technique is very simple. Simply mix the fluid containing the organisms with the "stain", smear on a slide, dry and examine. Higgins' India Ink is usually employed; but congo red (Cumley, R. W., Stain Techn., 1935, 10, 53-56) and azo blue (Butt, E. M., Boynge, C. W. and Joyce, R. L., J. Inf. Dis., 1936, 58, 5-9) are among many other materials used. See Azo Blue.

Negri Bodies. 1. Rapid section method (Schleifstein, J., Am. J. Pub. Health, 1937, 27, 1283-1285). Fix in Zenker's fluid, wash, dehydrate in dioxan, embed in paraffin, cut at 4 microns, mount, deparaffinize. Flood slides with 1 drop $1: 40,000 \mathrm{aq}$. KOH in $2 \mathrm{cc}$. stock solution of stain (Rosanilin of Grubler $1.8 \mathrm{gm}$., methylene blue, Nat. Col., 1 gm., glycerol $100 \mathrm{cc}$. and methyl alcohol $100 \mathrm{cc}$.). Steam gentlv $5 \mathrm{~min}$. Rinse in tap water.
Decolorize by gently moving in $90 \%$ ethyl alcohol until color is faintly violet. Pass quickly through $95 \%$ alcohol, absolute, xylol and mount in balsam. Negri bodies deep magenta with dark blue inclusions.

2. Rapid smear method (Dawson, J. R., J. Lab. \& Clin. Med., 1934-35, 20, 659-663). Remove brain to be examined as quickly as possible. Cut several small segments (3-4 mm. thick) from Ammon's horn perpendicular to its long axis and place in Petri dish. Cut away adjacent tissue leaving only the horn. Place a segment, cut surface down, on small end of a new 1 in. cork. With wooden applicator, or match, gently wipe peripheral tissue outward and downward. The segment is thus more firmly attached to the cork and the gray matter containing the pyramidal cells bulges upward. Press this gently against a slide (clean and entirely free from grease) held at one end between thumb and forefinger. Repeat 3 or 4 times, starting at end away from fingers, quickly so tissue does not dry. Immediately immerse in abs. methyl alcohol $5 \mathrm{~min}$. or more. Rinse in running water and stain in $2 \%$ aq. phloxine 2-5 min. Wash off excess stain in running water and color in Loeffler's alkaline methylene blue, $10-20$ sec. Decolorize in $80 \%$ ethyl alc., dehydrate in $95 \%$ and 2 changes of absolute, clear in xylol and mount in balsam. Handle slides with forceps and avoid danger from contact with tissue throughout process. Pyramidal cells blue, Negri bodies bright red to reddish brown. Time including examination $25 \mathrm{~min}$.

Stovall, W. D. and Black, C. E., Am. J. Clin. Path., Tech. Suppl., 1940, 4, 8 recommend control of $\mathrm{pH}$ in staining with eosin methylene blue (see Buffers). Stain with $1 \%$ eosin in $95 \%$ alcohol at pH 6.0 or more alkaline. Negri bodies pale red. The red is much more intense if the $\mathrm{pH}$ is 3.0. Loeffler's methylene blue is best as counterstain at $\mathrm{pH} 5.3$. At $\mathrm{pH} 6.0$ it removes eosin.

Azur B is advised for staining of Negri bodies by Jordan, J. H., and Heather, H. H., Stain Techn., 1929, 4, 121-126; see also Carbol-Anilin Fuchsin methylene blue.

Neisserian Infertion. A differential stain favorable for diagnosis (Scudder, S. A., Stain Techn., 1931, 6, 99-105).

Neisser's Stain for Diphtheria Bacilli, which see.

Nemathelminthes is the phylum of round worms. See Parasites.

Nematodes. See Glychrogel for mounting. See Parasites.

Neodymium, see Atomic Weights. 
Neon, see Atomic Weights.

Neoprene, injection of blood vessels (Lieb, E., J. Tech. Methods, 1910, 20, 50-51). Neoprene is a colloidal, finely divided suspension of synthetic chloroprenc in an alkaline aqueous medium. Instructions for the human kidney. Cannulate renal artery and wash with tap water at slow but constant rate. Ligate grossly leaking vessels. Continue $8-18$ hrs. until organ is pale gray. Cover and keep in ice box 6-7 hrs. or until the next day. Keep specimen at room temperature about one hour before injection. If it feels cold warm it with tap water. Connect cannula with bottle containing neoprene. A special apparatus for maintenance of $150-160 \mathrm{~mm}$. $\mathrm{Hg}$. is advised by lieb but it is probably sufficient to provide gravity pressure by raising the bottle $5 \mathrm{ft}$. or more. Close vessels ejecting the neoprene with hemostats and tie them when vessels are completely filled. Rinse in warm water. If a corrosion specimen is wanted leave kidney in conc. commercial $\mathrm{HCl}$ in tightly covered vessel at $56^{\circ} \mathrm{C}$. over night. Next morning pour off acid and allow stream of water to flow over the cast itself in the bottom of the container. When all debris is removed examine under water with dissecting microscope. Store in $0.3 \%$ Dowicide sol. (American Anode Inc., 60 Cherry St., Akron) to avoid mold. Lieb gives more details and describes combined corrosion, histological and roentgenological methods. Technique should be adapted to other organs. (Revised by Ethel Lieb, May 16, 1916).

Lieb's method has been modified in several respects by Duff, G. L. and More, R. H., J. Tech. Methods, 1944, 24, 1-11. The technique for mounting separately for detailed microseopic examination small sprigs of the renal cortical arteries greatly increases its usefulness.

Neoprene Latex. Employed for injection of coronary arterial system, woll illustrated and with a list of earlier papers (Smith, J. R. and Henry, M. J., J. Lab. \& (lin. Med., 1945, 30, 462-466).

Nerve Endings. These may be demonstrated in many ways. Nothing will aclequately take the place of their study in vivo (Speidel, C. C., J. Comp. Neur., 1942, 76, 57-73); but no method should be used with expectation of satisfactory results the first time. Experimentation is required. Most of the silver methods for neurofibrils show nerve endings. The writer has obtained good results by Bodian's Method applied to paraffin sections of experimental tumors. Graven's Gold Chloride method may bc tried. For silver impregnation of intracellular nerve endings in pars intermedia of pituitary, see Tello, F., Trab. d. Lab. Rech. Biol. Univ. Madrid, 1912, 10, 145-183. Methylene blue is, since the time of Ehrlich, a very popular stain for nerve endings. Addison (McClung, pp. 477-480) has given a full account of the technique. Commission Certified zinc-free methylene blue is suggested. Dye can be applied locally or by vascular perfusion.

1. Local application. Plaee tissue in shallow dish on thin layer of glass-wool moistened with $0.1-0.05 \%$ methylene blue in physiological salt solution. Add enough stain every few minutes to keep tissue moist and covered by film of stain. Beginning after $15 \mathrm{~min}$. examine frequently at low magnification until nerves are colored blue. Fix stain by immersion in cold $8 \%$ ammonium molybdate in physiological salt solution or Ringer's ( $\frac{1}{2} \mathrm{hr}$.). Wash in cold water. Dehydrate in alcohols in refrigerator a little above $32^{\circ} \mathrm{C}$. Either clear in xylol and mount in balsam or imbed in paraffin and section. Cole (E. C., J. Comp. Neurol., 1925, 38, 375-387) proceeded much in this way. He immersed whole alimentary tract of frog in 1:10,000 methylene blue solution for $1 \mathrm{hr}$. and cut it in pieces.

2. Vascular perfusion. Insert cannula in main artery leading to the tissue. Inject 1:10,000 methylene blue in physiological saline until tissue becomes light blue. Leave $15 \mathrm{~min}$. Remove thin pieces or slices. Place in dish and moisten with methylene blue solution. Examine uncovered at low magnification at intervals until nerve fibers and endings are stained. It is essential as in local application not to exclude air from tissue by covering with too much fluid. Fix in ammonium molybdate and continue as described above. For large fetuses use Langworthy's method (O. R., J. Comp. Neurol., 1924, 36, 273-297), for the lungs of rabbits that of Larsell (O., J. Comp. Neurol., 1921, 33, 105-131), for arteriovenous anastomoses Brown's (M. E., Anat. Rec., $1937,69,287-295$ ), and for skin Weddell's (G., J. Anat., 1940-41, 75, 441-416). Staining may perhaps be accentuated by hydrogen acceptors, see Auerbach's Plexus. See Pacinian Corpuscles, Meissner's Corpuscles, Krause's End Bulbs, Motor End Plates, Boutons Terminaux and Synapses.

Nerve Fiber Degeneration. The standard techniques are the Marchi Method by which the lipids produced by degeneration are blackened with osmic acid and the staining of lipoids by Sudan III. 
In addition 3 other much quicker methods are recommended:

1. To stain vitally with neutral red (Covell, W. P. and O'Leary, J. L., J. Tech. Meth., 1934, 13, 92-93). Intensity of staining of degenerating myelin depends upon amount and concentration of the dye. It can be applied in 3 ways: (1) Inject 4 cc. $4 \%$ neutral red in physiological salt solution into marginal ear vein of a rabbit over period of $1 \mathrm{hr}$; (2) Perfuse through aorta with large volume of $1: 1,000$ solution; (3) Immerse finely teased piece of degenerated nerve in $1: 10,000$ solution for about 12 min. Vital staining permits immediate determination of extent and degree of degeneration. See the author's excellent colored figures.

2. To examine by polarized light (Weaver, H. M., J. Lab. \& Clin. Med., 1940-41, 26, 1295-1304). Lay excised nerves without stretching on piece of wooden tongue depressor and fix 24 hrs. or more in $10 \%$ neutral formalin. Cut longitudinal frozen sections 10 microns thick. Float them onto slides from water, mount in neutral glycerin and examine. Weaver gives diagrams to aid in interpretation of findings. See also Pritchett, C. O. and Stevens, C., Am. J. Path., 1939, 15, 241-250; Radhakrishana, Rao, M. V., Ind. J. Med. Res., 1938, 26, 103-106.

3 . To demonstrate early changes in the axis cylinders (cores of the fibers) Alzheimer's modification of Mann's eosin-methyl blue method is strongly recommended by Mallory as showing normal axis cylinders deep blue and degenerated ones, red.

Nerve Fibers. Many excellent methods present themselves: the continuous direct observation of the growth of individual fibers in living tissues of lower animals (Speidel, C. S., Biol. Bull., 1935, 68, 140-161); the microdissection of living fibers (De Renyi, G. S., Cowdry's Special Cytology, 1932, 3, 1370-1402) ; x-ray diff raction studies of the sheaths (Schmitt, F. O., Bcar, R. S. and Palmer, K. J., J. Cell. and Comp. Physiol., 1941, 18, 31-42) and microincineration (Scott, G. H., Proc. Soc. Exp. Biol. \& Med., 1940, 44, 397398). For their demonstration in fixed tissues consult methods of Bodian, Davenport, Golgi, O'Leary, Osmic Acid, Weigert and Weil. The methylene blue technique of staining nerve fibers is given under Auerbach's Plexus. See Nerve Endings, Motor End Plates, Bouton Terminaux. Use of quartz roil illuminator in study of living nerve fibers is described by Speidel, C. C., J. Comp. Neurol., 1935, 61, 1-80 and by Bensley, S. H., Anat. Rec., 1944, 90, 1-11.

Nerve Grafts, methods, histological and otherwise (Sanders, F. K., and Young, J. Z., J. Anat., 1942, 76, 143-166).

Nerve Plexuses, see Auerbach's.

Nerves. A red lead and carpenter's glue method for injection and visualization of blood vessels of nerves (Epstein, J., Anat. Rec., 1944, 89, 65-69). See Pia Mater perivascular nerves.

Nervous System. This, the most complicated of bodily parts, can be investigated microscopically in a great many different ways. It is however shielded from the environment so that there are great obstacles in the way of direct observation in vivo. In mammals the best that can be done is to insert windows in the wall of the skull. A technique for this purpose, designed by Forbes (H. S., Arch. Neurol. and Psychiat., 1928, 19, 75), permits direct study at low magnification of blood vessels with so little injury that their behavior in various experimental conditions can be investigated. It is likely that by the Sandison Technique very significant observations can be made on living, growing nerve fibers of the rabbit. In amphibia Speidel (C. S., Biol. Bull., 1935, 68, 140-161) has been particularly successful in devising methods for study of nerve fibers in vivo.

Another group of techniques is available for marking in vivo and examination of the tissues after removal. Vital Staining has been much used. Some factors that condition the coloration of nerve cells with trypan blue have been described by King, L. S., J. Anat., $1934-35,69,177-180$. The pathways of drainage of cerebrospinal fluid can be marked with Prussian Blue (Weed, L. H., J. Med. Res., 1914, 26, 21-117). Nerve fibers and cells can of course be marked by the in vivo creation of injuries and subsequently examined. To determine the distribution of Radiophosphorus may prove helpful.

For the examination of excised tissues a host of methods present themselves. Consider first the classical techniques from which several others spring.

1. The original Nissl method for internal structure of the nerve cell consisted of fixing in alcohol and of staining sections with methylene blue. It revealed a basophilic material called Nissl Substance. The unfortunate tendency now-a-days is to loosely designate all methods intended to demonstrate 
this substance as Nissl techniques even though resemblance to the original method is lacking.

2. The original Golgi method for the external form of nerve cells depends upon preliminary mordanting of tissue in potassium bichromate solutions, followed by immersion in weak aqueous silver nitrate, and the cutting of thick sections in which occasional nerve cells and processes are outlined with startling clarity by the black deposit of silver chromate. Cajal modified and speeded up the technique by addition of osmic acid to the bichromate solution (see Golgi Method, quick). But the most used modification is the Golgi Cox technique.

3. The original Weigert method for myelin sheaths of nerve fibers depended likewise upon preliminary mordanting in bichromate and the formation of hematoxylin "lakes" when the sections were later stained with hematoxylin. Its most important modification is known as Weigert-Pal. The Marchi method, as modified by Swank and Davenport is based on similar mordanting with bichromate after which they are treated with osmic acid and was designed to reveal degenerated myelin sheaths the lipids of which are unaffected by the mordanting and are blackened while those of the normal sheaths are not.

4. Cajal and Bielchowsky introduced valuable methods for axones, neurofibrils, and nerve endings including synapses. Both techniques as applied to blocks of tissue depend on preliminary "silvering" with weak silver nitrate solution but in those of the former the silver is reduced by a photographic developer generally hydroquinone or pyrogallic acid; while in those of the latter the tissues are first brought into an ammoniacal silver solution and then reduced in formalin. The most useful modification is the Bodian Method of activated protargol. See its evolution under Silver Methods which are of assistance in the study of many other tissues of the body as well as the nervous system.

5. Weigert's neuroglia stain was also a classic, likewise Cajal's gold chloride and sublimate method (1913) which was soon followed by Hortega's carbonate silver method (1917). See recent techniques under Neuroglia.

There are still other techniques to choose from which are not so directly developments of the neurological classics. Nerve cells are closcly mixed with fibers. To isolate them sufficiently for direct study at high magnification in approximately isotonic media involves considerable injury and they cannot be held under observation for long periods because their death ensues fairly quickly. Spinal ganglion cells are the easiest studied. The Maceration technique is not much used for the nervous system but Addison ( $\mathrm{McCl}$ clung, p. 439) states that, if pieces of the anterior horn of the spinal cord are treated with Gage's dissociator $(0.2 \%$ formalin in physiological saline) for $2-3$ days, the nerve cells can easily be dissected out under a binocular microscope, stained and examined more or less as units. Tissue Culture of nerve cells of the adult is not feasible because they are fixed postmitotics (having permanently lost the power of multiplication); but culture of young tissues provides interesting results (Levi, G., Arch. de Biol., 1911, 52, 1-278, profusely illustrated). Nerve Fibers are more easily isolated and their investigation in the fresh state is very profitable. The histological localization of Cholinesterase is now feasible. The measurement of oxidative metabolism in different parts of the nerve cell by reduction of ferric chloride (Gerard, R. W., Assoc. for Res. in Nerv. \& Ment. Dis., Baltimore, Williams \& Wilkins, $1938,18,316-345$ ) can probably be tied up with localization of Oxidases and Peroxidases. Marinesco (G., Arch. Suisse de Neurol. et de Psych., 1924, $15,1-24)$ has published repeatedly on these enzymes in nerve cells. Methods for Pigments and Lipids can easily be applied to the nervous system. For microincineration of nerve cells and fibers see Scott, G. HI., Proc. Soc. Exp. Biol. \& Med., 1940, 44, 397-398. If it is desired to demonstrate mitochondria the Anilin-Fuchsin Methyl Green method is suggested after fixation by vascular perfusion plus immersion. See in addition to above headings: Auerbach's Plexus, Axis Cylinders, Boutons Terminaux, Centrosomes, Cresyl Violet, Golgi Apparatus, Microglia, Motor End Plates, Nerve Endings, Neurofibrils, Neurosecretory Cells, Oligodendroglia.

Neufeld's Quelling Reaction. This is a microscopically demonstrable swelling of the capsules of pneumococei which is of distinct value in typing (L. W. Parr, in Simmons and Gentzkow, p. 426).

Neumann's Crystals, see Charcot-Leyden.

Neurites, see Nerve Fibers.

Neurofibrils. These delicate fibrils and networks can be demonstrated with difficulty mainly by methods of silver 
impregnation in the cytoplasm of nerve cells. In the living nerve cells of selected invertebrates they can also be seen but opinion is divided as to whether they can be detected in the living nerve cells of vertebrates (Cowdry, p. 393).

None of the techniques for neurofibrils are really satisfactory, but, with patience, fairly good results can be secured of adult nerve cells by the following modification (Cowdry, E. V. Internat. Monatssch. f. Anat. u Physiol., 1912, 29, 1-32) of Cajal's technique. Fix pieces not more than $2 \mathrm{~mm}$. thick in Carnoy's 6:3:1 fluid 2-6 hrs. Wash in aq. dest. $24 \mathrm{hrs} .1 .5 \%$ aq. silver nitrate at $39^{\circ} \mathrm{C}$. for 3 days with one change. Rinse in aq. dest. and reduce in pyrogallic acid $1 \mathrm{gm}$.; aq. dest., 100 cc.; formalin $5 \mathrm{cc}$. in the dark, $24 \mathrm{hrs}$. Wash in aq. dest. $1 \mathrm{hr}$. Dehydrate 1 $\mathrm{hr}$. in $95 \% ; 2-4 \mathrm{hrs}$. in abs. changed twice; clear in cedar oil, 2 hrs.; imbed in paraffin 2 hrs. Rinse deparaffinised sections in aq. dest. $0.1 \%$ aq. gold chloride neutralized with lithium carbonate 2 hrs. The sections take a dark purple black color. $5 \%$ aq. sodium hyposulphite $5 \mathrm{~min}$. to bleach out excess of silver. Rinse in aq. dest. dehydrate, clear in toluol and mount in balsam.

The neurofibrils are exaggerated optically by their sharp blue black stain in a colorless background. Moreover they form centers for the deposit of silver which probably increases their bulk. The Nissl bodies can be brought out by staining in the usual way with toluidin blue after washing in aq. dest. following treatment of the sections with sodium hyposulphite. The essential step in this technique is the impregnation with silver. Consequently the time in the silver solution should be varied and perhaps its concentration likewise. To obtain a good preparation without many trials is not to be expected.

Silver techniques for neurofibrils are legion. A book has been written on the subject (Cajal, S. R. and deCastro, F., Elementos de Tecnica micrografica del sistema nerviosa. Madrid, 1933). Special methods are advised for different parts of the nervous system and for animals of different sorts and ages. A very useful synopsis is given by Addison (McClung, 1950, p. 364). See also Seki, M., Ztschr. f. Zellf. u. Mikr. Anat., $1939-40,30,548-566$.

Neuroglia. This is the connective tissue of the nervous system. Like that of the rest of the body it consists of cells, fibers (or fibrils as they are called) and intercellular substance. The last named is inconspicuous and little known. The Neuroglia Fibrils are considered separately. The cells are of three principal sorts: (1) microgliocytes of mesenchymatous origin. These may be resting and extend long, delicate processes or they may be ameboid in which case they look something like lymphocytes being usually identifiable by intensely staining nuclei. (2) astrocytes (star cells) and (3) oligodendrocytes (little tree cells) both of ectodermal origin. A tabular comparison of the three is given in Cowdry's Histology, p. 406. No neuroglial cells possess Nissl bodies. See Cajal's Brom-Formol-Silver Method, the Phosphotungstic Acid Hematoxylin method of Mallory, Weil and Davenport's silver methods given under Microglia and Oligodendroglia and Alzheimer's Modification of Mann's eosin-methyl blue method. See, also Silver Diaminohydroxide after sensitizing with sodium sulfite.

Neurones, see Nerve Cells.

Neurosecretory Cells. A good deal has been written on the subject. The most recent data on location in nervous system and methods are provided by Scharrer, E., J. Comp. Neurol., 1941, 74, 87-92; Scharrer, B., ibid, 93-130.

Neutral Fats. These are glycerides of fatty acids. See Lipids, examination of with polarized light. Colored rose red by Nile Blue Sulphate. See Sudan Stains, Osmic Acid and Oil Red $O$. Neutral Gentian (Bensley, R. R. Am. J. Anat., 1911, 12, 297-388). This gives a very fine deep violet coloration of secretion antecedents of serous (or zymogenic) cells. It has been used particularly for the pancreas and the stomach.

Neutral gentian is the neutral dye obtained when aq. gentian violet (crystal violet) is precipitated by its equivalent of aq. orange $G$ which is added slowly and the mixture agitated. Use solutions almost but not quite saturated. If the right amount of orange $G$ solution is added almost complete precipitation is obtained. If too much is added the precipitate is dissolved in which case add more gentian violet. Excess of orange $\mathrm{G}$ can be detected by the production of a yellow ring of stain about a violet center when a drop of the solution with the precipitate is touched to a piece of filter paper. When satisfied that ppt. is maximal, filter; and dissolve dried ppt. in $20 \%$ alc. until "color of a good haemalum solution is obtained". Allow the solution to stand $24 \mathrm{hrs}$. before use.

Fixatives: Several are advised. Equal parts sat. alc. mercuric chloride and $2.5 \%$ aq. potassium bichromate. 
(2) Potassium bichromate 2.5 gms.; mercuric chloride, 5 gms.; aq. dest., 100 cc. (3) Zenker's fiuid less acetic $90 \mathrm{cc}$, neutral formalin $10 \mathrm{cc}$. or (4) $2 \%$ osmic acid 2 cc.; $2.5 \%$ potassium bichromate $8 \mathrm{cc}$; glacial acetic acid 1 drop. In the case of the last the paraffin sections are treated with $1 \%$ aq. potassium permanganate $1 \mathrm{~min}$.; $5 \%$ aq. oxalic acid $1 \mathrm{~min}$. and are washed thoroughly in water before staining. Stain $4 \mu$ sections 24 hrs. Blot with several layers filter paper. Dehydrate in acetone. Place in toluol. Differentiate in 1 part abs. alc. and 3 parts oil of cloves. Wash in toluol and mount in balsam. Zymogen granules, purple; cytoplasm and nucleus, yellow ; chromophile material, lavender.

Neutral Red (CI, 825) - toluylene red-This weakly basic amino-azin dye is used for many purposes. It is a chloride. Some advocate the iodide as more easily purified but neutral red sold by any reliable manufacturer is satisfactory. Vital neutral red is recommended by Conn. The principal uses of neutral red are to stain:

1. Islets of Langerhans of the pancreas (Bensley, R. R., Am. J. Anat., 1911, 12, 297-388). Add 2 cc. of a previously prepared $1 \%$ aq. neutral red to $300 \mathrm{cc}$. physiological salt solution $(0.85 \% \mathrm{NaCl})$ thus making a concentration of neutral red of $1: 15,000$. Place this, and as much more as may be required in a bottle from the bottom of which a glass tube leads off, or in an ordinary bottle with a bent glass tube to serve as a siphon. The tube is connected with a glass cannula by about 5 feet of rubber tubing. A freshly killed guinea pig is bled from the throat. Insert the cannula in the thoracic aorta and inject the solution by raising the bottle to a height of 4 or 5 feet. Expose the pancreas. Cut the inferior vena cava near the heart so that the blood, followed by the solution, can easily escape. The pancreas will take on a deep rose red color. Remove pieces, mount in physiological salt solution under cover glasses and examine at low magnification. The optimum depth of staining must be determined experimentally. The islets of Langerhans appear as deep yellow red irregular masses of different sizes in a pale red background. After a time the dye is bleached from the background and the islets become more sharply stained.

A wonderfully fine color contrast can be secured when methylene blue is added to the neutral red solution in a concentration of $1: 10,000$ and both are injected in the same way. The islets are stained yellow red and the ducts blue. But it is desirable first to obtain satisfactory results with the methylene blue alone.

2. Parietal cells in the stomach, (Harvey, B. C. H. and Bensley, R. R., Biol. Bull., 1912, 23, 225-249). These are beautifully stained by injection with neutral red as described above.

3. Granules in blood cells. Touch a drop of fresh blood to a little 1:15,000 neutral red on a slide and cover immediately without attempting to mix. When the size of the drop of blood and the amount of stain are properly estimated the cover glass will press out the fluid into a thin film suitable for examination. The specific granules of leucocytes are stained red. In the monocytes red stained granules appear and sometimes increase in size. When the staining is fairly intense, or after a sufficient interval the nuclei of the leucocytes become colored and also a basophilic material in young reticulated red blood cells. Simultaneous coloration with Neutral Red and Janus Green is frequently carried out by hematologists.

Fluorescent $\mathrm{X}$ is a special type of reduced neutral red (Lewis, M. R., 1935, 17, 96-105). See Nerve Fiber Degeneration and Nissl Bodies.

Neutral Red and Janus Green. These are of ten employed together as a supravital stain for blood cells. A recent comprehensive statement of the technique is given by Cunningham and Tompkins (Downey, pp. 555-579). They add 3 drops conc. janus green in absolute alcohol to $1 \mathrm{cc}$. dilute neutral red, which, latter, is $20-30$ drops conc. neutral red in absolute alcohol. This mixture is spread evenly on slides and evaporated. They caution that for exudates, tissue scrapings, leucemic blood, bone marrow and lymph nodes it is necessary to use stronger solutions. Neutral red C.C. (Commission Certified) is satisfactory in place of the neutral red-iodide advised by Sabin. Fresh blood is mounted on the dye deposit, and is ringed with vaseline to prevent evaporation. This technique has had a profound influence on cytology. Obviously it must be cautiously used and observations discontinued as soon as evidences are seen of experimental modifications in the cells. It affords valuable information on the mitochondria and neutral red granules not stainable together by other methods, but it will not supplant the staining of blood smears by the methods of Giemsa, Wright and others. See critical evaluation by Hall (Downey, pp. 643-698). See application in study 
of lymphosarcomata $(\mathrm{Hu}, \mathrm{C}$. $\mathrm{H}$. and Pai, H. C., Arch. Path., 1942, 34, 106116)

Neutral Red Iodide. This is a special form of neutral red prepared by Phillips, M. and Cohen, B., Stain Techn., 1927, 2, 17-18 and recommended by Sabin for the Neutral Red Janus Green method.

Neutral Safranin, or Safranin-acid violet (Bensley, R. R., Am. J. Anat., 1911, 12, 297-388). Make the neutral dye by precipitating sat. aq. safranin $O$ with sat. aq. acid violet. The latter is added slowly and the mixture is agitated gently. The precipitation should be complete so that when it settles the supernatant fluid is of a faintly violet color. Filter and dissolve dried ppt. in abs.alc. Dilute this stock solution with equal vol. aq. dest. allow to stain 30 min. before use. Stain sections, fixed as described under Neutral Gentian, in the same way as with neutral gentian. Nuclei are colored with safranin and secretion antecedents with the acid violet. The method has been used chiefly for the pancreas but it gives fine coloration of nerve as well as gland cells. Unfortunately the colors are not very permanent.

Neutral Stains. As explained by the Bensleys (p. 65) acid and basic dyes are mutually antagonistic. One will extract the other from a section. This can be overcome by having them react on each other to form a molecularly balanced neutral compound insoluble in pure water and which must therefore be employed in alcoholic solution. Because the staining depends upon the hydrolytic splitting of the compound they must be applied at maximum concentration of water consistent with retaining the dye in solution. It is on account of the necessity for dilution with water to promote dissociation that water is added to Wright's blood stain on the slide. These neutral dyes are of particular value in the staining of secretion antecedents by $R$. R. Bensley and his followers, see Neutral Gentian (gentian violet-orange G), Neutral Safranin (safranin-acid violet), Crystal Violet-Acid Fuchsin and Bowie's Stain.

Neutrophile Leucocyte (finely granular leucocyte, polymorphonuclear leucocyte). Most numerous granular leucocyte, percentage 55-75; slightly smaller $(9-12 \mu)$ than eosinophile; nucleus lobated, usually also filamented, stains deeply; specific granules, refractile, neutrophilic, small, uniform and numerous; highly motile and phagocytic. Special methods for their study are far too numerous even to list.
The so-called toxic neutrophiles in certain pathological states differ from normal ones in the staining of nuclei and specific granules (Mommsen, H., Ztschr. exper. Med., 1929, 65, 299). A comprehensive account of neutrophiles is provided by Bunting, C. $\mathrm{H}$. in Downey's Hematology, 1938, 1, 160-177. Because these cells normally constitute by far the majority of leucocytes in the circulating blood, chemical analyses of total leucocytes separated from the erythrocytes relate chiefly to them. The most convenient way is to mix fresh blood with Anticoagulant, centrifuge and take the so-called buffy layer. For lipid analysis of such material, see Boyd, E. M., Arch. Path., 1936, 21, 739-748. Another useful method, described by Haan and employed by Barnes, J. M., Brit. J. Exp. Path., 1940, 21, 264-275, which works nicely with the rabbit but poorly with the cat, is to inject intraperitoneally 200-300 cc. warm sterile saline solution and $4 \mathrm{hrs}$. later to withdraw fluid with a cannula into 5 cc. $4 \%$ sodium citrate. This fluid contains $95-98 \%$ neutrophiles. Barnes has outlined methods for determination of Cathepsin, Nuclease, Amylase, Lipase, Lysozyme and Adenoinase. Since it is possible now to break up cells and to collect by centrifugation masses of Mitochondria and Nuclei, it should be feasible to collect and similarly to analyse the neutrophilic granulations. For technique of measuring motility, chemotaxis and other properties, see Leucocytes.

Neutrophilic, see Staining.

Nevillite $\mathbf{V}$ and No. 1 have been compared with gum damar and Canada balsam as mounting media by Groat (R. H., Anat. Rec., 1939, 74, 1-6). Both are clean, colorless, inert and neutral. $\mathrm{He}$ recommends a $60 \%$ solution of either $\mathrm{V}$ or No. 1 in toluol.

New Blue R, see Naphthol Blue R.

New Fuchsin (Magenta III) (CI, 678)fuchsin NB, isorubin-It is triaminotritolyl-methane chloride. This new fuchsin is sometimes specified for staining of acid fast bacilli.

New Methylene Blue. The Colour Index lists several dyes by this name of which 2 deserve mention: (1) GG (CI, 911) is recommended by the Bensleys ( $p$. 16) as a supravital stain for mast cells and for the thyroid because of its metachromatic capacity. (2) $\mathbf{N}$ (CI, 927)methylene blue NN-Conn (p. 88) says that it may be of some value though it is practically never used in microscopical work. Cowdry tried it and found that it had no particular advantages. 
New Pink, see Phloxine.

New Ponceau $4 R$, see Ponceau 2R.

New Victoria Blue $B$ or $R$, sce Victoria Blue R.

New Victoria Green Extra O, I or II, see Malachite Green.

Niagara Blue 3B, see Trypan Blue.

Niagara Blue 4B (CI, 520)-benzo sky blue, direct sky blue, pontamine sky blue 5BX-A disazo dye, see Varrelman, F. A., Stain Techn., 1938, 13, 115-119. Niagara blue 2B (N.A.C.) is the American prototype of trypan blue for which it can be substituted (Foot, McClung, p. 115)

Niagara Sky Blue 6 B (CI, 518), a direct disazo dye of light fastness 3 . Instructions for employing this useful stain in the examination of plant and animal tissues are given (Emig, p. 41).

Nickel. The microchemical technique of Crétin and Pouyanne (A., and L., Bordeanx chirurgical, 1933, 4, 321-364) employed in a study of the influence of metals on bone deposition, as given by Lison (p. 102), is: Fix in formol, $30 \mathrm{cc}$. "sérum physiologique", 100 cc., and ammonium hydrosulphate 5 drops. Immerse in a solution of ammonium phosphate in order to produce the insoluble double salt: $\mathrm{NH}_{4} \mathrm{NiPO}_{4}+$ $6 \mathrm{H}_{2} \mathrm{O}$. Decalcify. In the sections stain the nickel by an alcoholic solution of pure hematoxylin which forms a lilac colored nickel lake appearing blue when very thick (Lison, p. 102).

Nicotinic Acid. Preliminary detection of it or its amide by fluoresence microscopy (Hirt, A. and Wimmer, K'., Klin. Wochnesdir., $1939,18,765-767)$. Lasting yellow fluorescence. See Vitamin B complex.

Night Blue (CI, 731), a basic dye of light fastness 4 gives beautiful blue-violet coloration of plant tissues but fades (Emig, p. 52).

Nigrosin, water soluble (CI, 865)-gray $\mathrm{R}, \mathrm{B}, \mathrm{BB}$, indulin black, silver gray, steel gray-Commission Certified. This is a mixture. It has been used as a counterstain for neutral red in coloration of Nissl bodies by Bean, R. J., Stain Techn., 1927, 2, 56-59, as a negative stain for bacteria, Treponema, etc. See Picro-Nigrosin.

Nile Blue A, see Nile Blue Sulphate.

Nile Blue Sulphate (C 1.913)-Nile Blue A -This is an important oxazin dye for which purity tests have been established (Conn, p. 270). It was introduced by Lorrain Smith as a fat stain. Briefly the method is to stain fresh tissues, or frozen sections of formalin fixed tissues, for 10-20 min. in a conc. aq. solution of Nile blue sulphate, to differentiate in $1 \%$ aq. acetic acid, wash in water and mount in glycerin. He thought that the neutral fais (glycerides) were thereby colored red and the fatty acids blue, but Kaufmann and Lehmann (C. and E., Virchow's Arch. f. Path. Anat. und Physiol., 1926, 261, 623-648) came to the conclusion that the method was valueless. However Lisson (p. 202) was unimpressed by their evidence. In his opinion the rose (or red) color does signify the presence of a nonsaturated glyceride whereas the blue color is of no significance because of its lack of specificity. He reported that some mixtures of free fatty acids remain uncolored; for those containing saturated fatty acids non-coloration is the rule; while some others, not containing fatty acids, are colored. See Lipids, tabular analysis.

Stone, L. S., Anat. Rec., 1931, 51, 267-273 has advanced a technique for the preservation of supravital staining with Nile blue sulphate the essential feature of which is repeated treatment with phosphomolybdic acid. Zenker's fluid with acetic, 2 hrs. Running tap water, $1 \mathrm{hr}$. $1 \%$ aq. phosphomolybdic acid, 2 hrs. Dehydrate in 50, 70, 80, 95 and abs. alc. each containing $0.1 \%$ phosphomolybdic acid, $30 \mathrm{~min}$. each. Clear in equal parts $0.1 \%$ phosphomolybdic acid in abs. alc. and cedar wood oil, $30 \mathrm{~min}$. Then pure cedar wood oil over night. Embed in paraffin 3 changes 15-20 min. each. Counterstain sections in stain desired applied in abs. alc. containing $0.1 \%$ phosphomolybdic acid. Mount in damar. Balsam will do.

Nile Pink, fat stain prepared from nile blue sulphate by boiling with dilute sulphuric acid (Rettie, T., J. Path. \& Bact., 1931, 34, 595-596).

Ninhydrin Reaction. Berg's (W., Pfluger's Arch., 1926, 214, 243-249) directions : Fix tissues in $10 \%$ formalin, wash in water. Boil section for $1 \mathrm{~min}$. in $2 \mathrm{cc}$. $0.2 \%$ ninhydrin. Wash, mount in glycerin or glycerin jelly. Amino acids, polypeptides and proteins blue or violet. Romieu (M., Bull. d'Hist. Appl., 1925, 2, 185-191) employs a strong solution heated less. See Giroud (A., Protoplasma, 1929, 7, 72-98).

Details are given by Serra, J. A., Stain Techn., 1946, 21, 5-18. He ad' vises that the tissue first be hardened by fixation for an unspecified time in 2 parts $96 \%$ alcohol and 1 part commercial formalin ( $40 \%$ formaldehyde) plus "some drops" of glacial acetic acid in 10 cc. of the mixture. After this it is well washed in running water and in aq. dest. before the frozen sections are made. He also gives a method for paraffin sections. 
The reaction consists of immersing the sections or fresh materials in equal volumes of $0.4 \%$ aq. triketo-hydrindenhydrate (ninhydrin) and phosphate buffer pH 6.98. The ninhydrin solution must be freshly prepared and the phosphate buffer not too concentrated. For the latter he suggests 6 cc. $M / 15$ solution secondary sodium phosphate (11.1S76 gm. $\mathrm{Na}_{2} \mathrm{HPO}_{4} \cdot 2 \mathrm{H}_{2} \mathrm{O}$ per liter) and 4 ce. $M / 15$ primary potassium phosphate $\left(9.078 \mathrm{gm}\right.$. $\mathrm{KH}_{2} \mathrm{PO}_{4}$ per liter). The reaction is carried out, in a covered glass container placed on a boiling water bath. This is allowed to stand 1-2 min. in the vapor after it has reached the boiling point. A blue, or violet, color developing while hot or after cooling indicates the presence of amino acids, fre, or bound in peptides, or proteins.

For microscopic examination mount in pure glycerin squeezing if necessary. The edges can be cemented by using a mixture of $80 \mathrm{gm}$. collophonium and 20 gms. heated lanolin as recommended by Romeis but they nust be studied the same day for the color fades quickly.

Serra carefully states that the reaction is given, not only by all amino acids except proline and hydroxyproline, by peptides and proteins but also by other compounds such as amines, aldehydes, sugars with free aldehyde or keto groups and by ammonia and ammonium salts. "However, with compounds other than amino acids and proteides, the reaction is much less sensitive and sometimes it gives a more reddish color. In general it is easy to exclude the possibility of these compounds being present, by their solubility and localization. It must also be remembered that the intensity of the ninhydrin reaction varies according to the nature of the amino acid and the binding of this in the peptides.

"The coloring formed during the reaction can diffuse and be absorbed by several cell structures. This commonly happens when the heating is exaggerated and when compounds easily soluble are present, for instance after a weak fixation. It is, therefore, recommended to employ fixatives which harden the tissues, as we have said above. To be sure that a secondary impregnation or adsorption of the coloring has not taken place, the following test may be executed: A small weight (some milligrams) of a pure amino acid, such as glycine, is dissolved in distilled water; an equal volume of phosphate buffer of pH 6.98 and a few drops of $0.4 \%$ ninhydrin solution are added; it is boiled slowly and cooled for 20-30 minutes. The ninhydrin employed must be completely consumed-by addition of more amino acid solution. The colored liquid of this reaction is now used to immerse the pieces, with boiling, etc., as for a ninhydrin reaction. If then a certain structure shows a coloration, this means that an absorption or adsorption has taken place and a positive ninhydrin reaction in the same structure does not necessarily demonstrate a proteic or amino acid nature."

Nipple Secretion Smears, see Papanicolaou Techniques.

Nissl Bodies (Tigroid bodies, chromophile granules, chromidia, etc.) are masses of basophilic material easily demonstrable in the cytoplasm of most nerve cells after a wide variety of fixations. Certain types of nerve cells are characterized by the shape, number, size and distribution of their Nissl bodies. Since, moreover, the Nissl bodies ap. pear at a definite stage in the development of the cells and undergo distinctive modifications in physiological and pathological conditions there can be no question that they represent material present in vivo although they cannot be distinguished as such in living nerve cells. Bensley, R. R. and Gersh, I., Anat. Rec., 1933, 47, 217-237 claim that their discovery of well-formed Nissl bodies, stainable with toluidin blue, in sections of tissues frozen in liquid air and dehydrated in vacuo while still frozen is proof of the presence of Nissl bodies in the living state. Wiemann, W., Zeit. f. d. ges. Neurol. u. Psychiat., $1925,98,347-404$ appears to have made ultraviolet photomicrographs of Nissl bodies, and a dense ash, revealed by microincineration (Scott, G. H., Proc. Soc. Exp. Biol. \& Med., 1940, 44, 397$398)$, corresponds with them topographically.

The influence of fixation on the shape (and perhaps to a slight degree on the distribution) of Nissl bodies in nerve cells has never been clearly defined. It is known that the Nissl bodies are much more pronounced after fixation in 95\% alcohol, Zenker's fluid and Carnoy's fluid than they are after fixation in osmic acid, Altmann's fluid and Regaud's fluid. Fixatives of the first group also result in more stainable particles in the nucleoplasm than those of the second. For other details see Hopkins, A. E., Anat. Rec., 1924, 28, 157-163. Influence of staining is also a factor to be reckoned with because of the striking difference in appearance of Nissl bodies when intensely and lightly colored. There are many methods from which to make a choice. 
Some of these are given under Gallocyanin, Gallamin Blue and CarbolFuchsin. See also the methods of Huber, Johnson and King and buffered thionin (Windle, W. V., Rhines, R. and Rankin, J., Stain Techn., 1943, 18, 7786). An apparatus has been devised apparently suitable for obtaining the Absorption Spectra of Nissl bodies.

Nitrates. Make frozen sections of fresh tissues. Cover section on a slide with 1-2 drops hot $10 \%$ "Nitron" in $5 \%$ aq. acetic acid. Place in refrigerator 30 min. to permit nitrates to crystallize and examine in polarized light. Nitron is 1,4-Diphenyl-3,5-endanilo-dihydrotriazol. It precipitates nitrates as insoluble salts (Crämer, G., Zbl. allg. Path., 1940, 74, 241-244).

Nitrazine-nitrazine yellow, delta dye indicator-An acid mono-azo dye suggested as substitute for ponceau de xylidine in Masson's Trichrome Stain.

Nitrazine Yellow, see Nitrazine.

Nitrocellulose for imbedding. Low viscosity nitrocellulose ("Hercules Powder Co.'s R.S. 0.5 second nitrocellulose") does not require to be washed as in the case of celloidin. First add absolute alcohol, break up lumps and add ether. Use $100 \mathrm{gms}$. nitrocellulose, 100 cc. absolute alcohol and $140 \mathrm{cc}$. anhydrous ether. For evaporation a large surface is required in proportion to depth. A precision microtome is needed for sectioning blocks after first hardening in 70-80\% alcohol. Blocks are cut both dry and wet. Serial sections 4 microns thick are obtainable whereas in celloidin the minimum is about 12 microns. Since low viscosity nitrocellulose (L.V.N.) is more readily dissolved than celloidin by absolute alcohol the use of butyl alcohol between 95\% alcohol and xylol is suggested (Davenport, H. H. and Swank, R. I., Stain Techn., 1934, 9, 137-140).

Nitro Dyes. Chromophore- $\mathrm{NO}_{2}$. All strongly acid. Aurantia, martius yellow, picric acid.

Nitro Reaction to distinguish between pyrrols and indols. Treat preparation with a mixture of sulphuric and nitric acids (equal parts). Substances containing the benzene ring (and among them indol compounds) are nitrified and recognizable by their canary yellow color whereas the pyrrols are not nitrified (Lison, p. 162). See Lison, L., J. Physiol. et Path. Gén., 1933, 31, 82-99).

Nitrogen. The titrimetric method of Bruël, D., Holter, H., LinderstrømLang, K. and Rozits, K., C. rend. trav. lab. Carlsberg, Sér. chim., 1916, 25, $289-324$ is recommended.
Nitroprusside Reaction for Glutathione. 1. Mattei and Dulzetto (Atti. e. rend. della Accad. dei Lincei, 1928, 8, 317). Fix in $20 \%$ trichloracetic acid. 'Treat frozen sections $3-4 \mathrm{~min}$. with a fresh solution of sodium nitroprussiate. After quickly drying expose to $\mathrm{NII}_{3}$ vapor. Freeze solidly with ice or solid $\mathrm{CO}_{2}$. Examine frozen on slide at $5^{\circ} \mathrm{C}$. The violet color of sulphydryl rapidly disappears.

2. Joyet-Lavergne (Ph., Bull. d'Hist., 1928, 5, 331-349) Method 1: apply to tissue 1 drop $5 \%$ aq. sodium nitroprussiate, then 1 drop ammonia and examine immediately. Method 2: before applying reagent as above he uses a stimulant $10 \%$ aq. potassium cyanide, 5 min.; or $2 \%$ aq. sodium sulphite, $10 \mathrm{~min}$., or sat. ammonium sulphate, $15 \mathrm{~min}$, or trichloracetic acid, $2-5 \mathrm{~min}$. Method 3 for fixed tissues: fix several hours in abs. alc. or in formol $15 \mathrm{cc}$. + physiological saline sol. $75 \mathrm{cc}$. Tease tissue or make frozen sections. Stimulate with potassium cyanide or ammonium sulphate. Then apply reagent.

3. Giroud and Bulliard (A. and $\mathrm{H}$., Protoplasma, 1933, 19, 381-384). Apply to fresh teased tissues or frozen sections $10 \%$ aq. sodium nitroprussiate alkalinized by about $2 \%$ ammonia. Fix the color by treatment for several seconds with $5 \%$ aq. zinc acetate. Dehydrate, clear and mount in balsan in the usual way. The violet color becomes red but lasts some time especially if kept in ice box. The same technique is possible after alcohol fixation.

Lison (p. 135) has considered the specificity of these reactions and recommends analysis given in an article by Rapkine contained in the last edition of Langeron's Précis de Microscopie. For fresh tissues (pieces, smears, frozen sections) (a) Glutathione reduced. Add to tissue on slide 1 drop $5 \%$ sodium nitroprusside for plants, $2 \%$ for animals. Add a reinforcer such as sat. aq. ammonium sulphate or crystals, then drop of ammonia. Red or violet color. (b) Glutathione total. Treat tissue with $10 \%$ cyanide of potassium, 5-10 min. Then (a). (c) SH radicals fixed to proteins. $10 \%$ trichloracetic acid $15 \mathrm{~min}$. Wash in much water. Repeat several times. For fixed tissues avoid employing absolute alcohol or trichloracetic acid. Use instead formol-saline (above). Then follow as for fresh tissues. Fix colors with zinc acetate as described.

Bourne (G., Austral. J. Exp. Biol. \& Med. Sci., 1935, 13, 238-249) puts frozen sections into hot $5 \%$ aq. acetic acid $30-90$ sec.; drains off the acid; adds $5 \%$ sodium nitroprusside (saturated with 
ammonium sulphate) 2 min., then few drops conc. ammonium hydroxide which turns them purplish blue. For quantitative unreliability of the test for -SH and -S.S- see Hammett and Chapman, (F. S. and S. S., J. Lab. \& Clin. Med. 1938-39, 24, 293-298). See Sulfhydryl Groups.

Nitrosamino Reaction of Lison (p. 161) consists in transforming the amino group present in pyrrol and indol into nitrosamine by action of nitric acid; then by demonstrating the nitrosamine by the reaction of Liebermann.

Nitroso Dyes (quinone oximes). Produced by nitrous acid acting on phenolic compounds. Naphthol green B and Y.

NNN Medium, see Leishmania.

Nocht's Stain for malaria plasmodia is described by Craig, p. 287 as less satisfactory and more time consuming than Wright's.

Nonfilament-Filament Ratio. This is derived from the Filament-Nonfilament Count, the number of nonfilamented neutrophiles being multiplied by 100 and divided by the number of filamented ones. See Stiles, M. H., J. Lab. \& Clin. Med., 1940-41, 26, 1453-1460.

Nopalin G, see Eosin B or bluish.

Normal Solutions. The equivalent of a substance (equivalent weight, the gram equivalent) is the weight in grams which in its reaction corresponds to: a gram atom of hydrogen, or of hydroxyl, or a univalent ion, or to half a gram atom of oxygen. A normal solution contains one equivalent per liter, a 0.05 normal contains 0.05 equivalent.

Hydrochloric acid $(\mathrm{HCl})$, the molecular weight is $\mathrm{H}=1.008+\mathrm{Cl}=35.457$ (see Atomic Weights) $=36.465$. Consequently make up $36.465 \mathrm{gms}$. of $\mathrm{HCl}$ to 1 liter with aq. dest. But it can not be weighed out in this way. Since conc. hydrochloric acid (sp. gr. 1.19) is approximately $12 \mathrm{~N}$, to make a normal solution (approximate) dilute $83.3 \mathrm{cc}$. to 1 liter with aq. dest. The normality can be accurately determined by standardizing with sodium carbonate, or by titration with a solution of sodium hydroxide of known normality.

Sulphuric acid is $\mathrm{H}_{2} \mathrm{SO}_{4}$. Molecular weight calculated in the same way is 98.08. But there are 2 replaceable hydrogen atoms so that in making a normal solution the molecular weight is divided by 2 which means that 65.068 gms. of $\mathrm{H}_{2} \mathrm{SO}_{4}$ is to be made up to 1 liter with aq. dest. A conc. sol. (sp. gr. 1.84 ) is approximately $36 \mathrm{~N}$. To make approximately $1 \mathrm{~N}$ dilute $27.8 \mathrm{cc}$. to 1 liter.

Oxalic acid has the formula $(\mathrm{COOH})_{2}$. $2 \mathrm{H}_{2} \mathrm{O}$ with molecular weight of 126 .
Owing to presence of 2 hydroxyl groups it has 2 hydrogen equivalents and it is necessary to divide the molecular weight by 2 so that 63 gms. is made up to 1 liter with aq. dest.

The alkali sodium hydroxide $(\mathrm{NaOH})$ has 1 hydroxyl group, so that the molecular weight is taken without division. But with disodium phosphate, the formula of which is $\mathrm{Na}_{2} \mathrm{HPO}_{4}$, the hydrogen equivalent is $\frac{1}{2} \mathrm{Na}_{2} \mathrm{HPO}_{4}$, so that the molecular weight is divided by 2. Similarly with the salt $\mathrm{Na}_{2} \mathrm{SO}_{4}$ the molecular weight is halved. For sodium triphosphate, $\mathrm{Na}_{3} \mathrm{PO}_{4}$, the hydrogen equivalent is $\frac{1}{3} \mathrm{Na}_{3} \mathrm{PO}_{4}$, or the molecular weight is divided by 3 .

Normality, Microscopic (From Cowdry's Histology, 1950). Knowledge of the microscopic structure of normal tissues is prerequisite to an understanding of their microscopic structure in abnormal conditions. These latter conditions indeed are defined in terms of the former by merely adding a prefix "differing from," the Latin $a b$. Yet it is extraordinarily difficult to reach agreement on what constitutes justification for the use of the adjective "normal." Discussion tends to drag out almost endlessly.

Definition: We choose here to advocate the statistical definition of normality. According to this: a normal condition is the usual one in members of a homogenous group. By usual is intended in the majority, that is in any number over 50 per cent. By homogeneous is meant in individuals who are alike at least insofar that they are of the same race, sex and age.

Examples: The existence of races, characterized by structural differences, is a fact to be reckoned with. What is normal, or usual, in one may or may not be normal or usual in another. Physical anthropologists deal with such matters but histologists are also involved because it is desirable to explore microscopically differences that are detectable by naked-eye inspection. These racial differences are grafted on a basic and fundamental similarity, for all races of mankind belong to one and the same species. Conspicuous among them are certain features of hair, skin and body build, which are normal for one race, but are not normal for others. The list on careful study, may prove to be a long one.

Between the two sexes there is likewise a fundamental similarity. Many tissues of males and females are indistinguishable. Some others show quantitative differences. The normal number of erythrocytes per cubic millimeter 
of blood in males differs from that in females. And there are qualitative differences in the primary and secondary sex organs. Consequently, as between races, so also as between the sexes what is normal for one is not necessarily normal for the other. One must always bear in mind many known differences and the likelihood of almost innumerable others.

Though a basic similarity exists in the structure and function of the body at all ages in the life of a given individual, some very profound differences clearly obtain, as between different ages. What is normal for individuals of the same race and sex, at, say, age 10 is clearly not normal at age twenty, or at age fifty. It has been said that the boy is not a little man and the senile is not an old boy; he is a different individual but he is constructed in the same general pattern.

No assessment of normality is therefore of any value unless the group is homogeneous in race, sex and age. Even this qualification is only sufficient for a few properties relating to the body as a whole such as weight, height, basal metabolism, urinary excretion and so on.

The body is made up of so many parts that it is frequently essential to consider the normality of each separately maintaining this homogeneity in race, sex and age of the persons having the parts compared. In the vascular system the coronary artery ages much more rapidly than the radial artery. Structure normal, or usual, for the coronary artery at thirty is normal for the radial at about sixty. The skin of the face and hands ages more rapidly than that in less exposed situations as for example the back. What is normal for one at age sixty is definitely not normal for the other.

To be on the safe side still other precautions are indicated. The method employed to determine the normality of the particular property in question should be given, because some other technique might yield higher or lower values. One must also be on the lookout for modifying conditions which should be identified and specified. Thus the normal secretory activity of the sweat glands is modified considerably by changes in the weather. When temperature and humidity are high during the summer, the level of normal activity is different from that in the winter. What is normal at high altitudes is frequently not normal for parts, or functional mechanisms, of individuals of the same race, sex and age measured by the same methods at sea level. To further multiply examples would be wearisome.

Errors in Estimation: Histologists, who would provide a sound basis for work in pathology, should bear in mind the considerations already mentioned and others incident on the very inadequate techniques they employ.

Before attempting to catalogue them, however, it should be freely admitted that, even in the best textbooks, there is a deplorable looseness in descriptions intended to convey information on the normal microscopic structure of the human body. Illustrations of human tissues are often presented without any qualifying data concerning them and others of the tissues of a wide range of lower animals are supplied, likewise without qualifying data, the assumption being made, all too frequently, that they are truly representative of normal human tissues. In many cases they are representative and serve the purpose. And it hardly needs to be said, that for accurate and controlled experiments animal tissues are essential. But it is not difficult to cite cases in which examination of normal animal tissues could convey a misleading idea of normal human tissues of the same kind. Kurloff bodies are a normal component of the spleens of guinea pigs, but not of human beings. The demarkation of hepatic lobules by connective tissue is notably characteristic of certain species. What is normal in this respect for the domestic pig is not normal for man.

Appearance in microseopic preparations of any kind may be technical artifacts and may not indicate any deviation from normal in the tissue or organ from which the preparation has been made. The term artifact is derived from L. ars, art and factus, made. It is something made artificially. Webster defines an artifact as being "in histology", a structure or appearance in a tissue or cell due to death or to the use of reagents and not present during life." The degree of artifact is proportional to the difference between the structure existing normally in the living body and the structure observed in the preparation. Details are given in Laboratory Technique.

In the case of living tissues, observed with blood and nerve supply intact, there is a possibility of artifact. It is at a minimum in the rabbit ear chambers, in which the ingrowing tissue is very thin and can be studied with high magnifications, and rather more to be reckoned with when tissues must be 
displaced in order to supply the necessary illumination. But it is small with relatively thick organs like the spleen and liver transilluminated by Knisely's quartz rod technique and examined at lower magnification. With increase in time modifications due to changes in light, temperature, hydrogen ion concentration, etc. are likely also to increase.

In living cells removed from the body and examined in Tissue Cultures the possibility of artifact is again at a minimum; but, though the cells in successive generations in suitable media go on living indefinitely, their environments are different from those existing within the body. When after vital staining, or supravital staining, still living cells are examined in approximately isotonic media, there is a grave danger of artifact if the study is prolonged because the cells are slowly dying.

In fixed tissues the degree of divergence from the living condition is obviously much greater than in the case of still living ones. However death has been sudden so that artifacts due to gradual death are eliminated. If the technique has been carefully standardized the same fixative applied to the same type of cell in the same physiological state is likely to yield similar results.

1. The folds seen in stained sections of skin are often at least partly due to shrinkage of the collagenic and elastic tissue of the dermis after excision and before fixation. The fixative serves further to exaggerate them (Evans et al., 1943). Some ballooning of the epithelial caps of intestinal villi occurs in vivo. This phenomenon is likewise exaggerated by ligating the arteries of supply in the living animal and by employing fixatives which induce forcible contraction of smooth muscle (Macklins, 1932).

2. Alterations occurring postmortem in tissues before fixation can be confusing. The most frequent one is a breaking up of the tips of intestinal villi, sometimes also caused by mechanical injury during excision or washing out the contents of the gut. In all tissues autolytic and osmotic changes occur after death which profoundly alter their structure after fixation. The rate of autolysis is very rapid in organs like the pancreas rich in enzymes let loose when the action of said enzymes ceases with death to be properly regulated. The rate is slower in the nervous system and especially in the walls of elastic arteries in which the proportion of inanimate components (elastic and collagenic fibers) is high and that of living cells is low.

3. Shrinkage, and increased affinity of surface cells for stains, occasioned by letting the surface dry before fixation should be recognized as such.

4. Differences in the appearance of the peripheral and central parts of tissues, owing to the unequal penetration of the fixative as a whole, or of its components, are sometimes encountered.

5. The displacement of glycogen in the direction of entrance and passage of the fixative, so well illustrated in a book by de Robertis and his associates (1948), suggests the likelihood of similar artifacts in the cytoplasmic localization of other substances.

6 . The glassy appearance of nuclei and cytoplasm, sometimes occasioned by overheating in paraffin imbedding, or in spreading out sections, is easily detected.

7. The presence of formed material within blood vessels, faintly resembling organisms, caused by the coagulation of blood proteins, has led some people astray.

8. The introduction of extraneous substances present either in the albumin fixative used to mount the sections or deposited as dust from the air (bacteria, spores, mineral matter, etc.) is a possibility to be borne in mind. Careful focussing is required to determine whether particulate matter is located below, within or upon the section. In stained smears of blood and other cells precautions against extraneous substances are also indicated.

Interpretation: One has to be almost constantly on guard because the foregoing list of artifacts is by no means complete. It is simply illustrative of some of the conditions that may be met with and must be excluded. Even when appearances, that do not seem to be artifacts, are observed it is not safe to conclude in an unqualified fashion that the tissue, or organ, from which the preparations have been made was in fact normal. On the contrary several conditions require fulfillment before an opinion should be offered.

1. The appearance noted must be present in more than 50 per cent of individual tissues comprising a homogeneous series in the sense already explained. The advice of a colleague versed in statistics is desirable as to the number required in the said series.

2 . In the event that the appearance in question is observed in but small pieces excised from large organs or 
tissues care is indicated that the samples are truly representative of the whole. Examination of samples all from the tail of the pancreas well might fail to reveal conditions prevalent elsewhere in the pancreas and convey a false idea of the normality of the whole. Inadequate preliminary gross examination might lead to the overlooking of small localized modifications, as in beginning cancers, and might result in a verdict of normality based on appearances in poorly selected samples.

3 . If the samples have been taken from organs or tissues which are larger (hypertrophied) or smaller (atrophied) than normal then their examination may likewise result in a misconception of normality. The alterations in volume, which have to be taken into consideration, may be abnormal, or within the range of normal volumetric changes in physiological activity. Among the latter are alterations in the volume of the liver consequent upon the different phases in intestinal absorption. We must face not only changes in volume but also changes in microscopic appearance. The normality of microscopic structure therefore must be specified in relation to physiologic state. What is normal for one stage in the menstrual cycle is not normal for another. What is normal for the gastric mucous membrane during active digestion is not normal while fasting.

4. Since the appearance depends upon the technique employed the qualification is essential that it is normal for, say, intracellular lipids demonstrable by Sudan III, or for whatever is in question. Not all lipids are detectable by this method. It is quite possible that the lipid contents not so revealed are in abnormal quantities.

5 . In returning a verdict that tissues examined microscopically are normal, histologists should still further qualify their conclusions. It is the part of wisdom for them to remember, especially when employing microchemical reactions, that most of them are ignoramuses as to chemistry. A smattering of chemistry is insufficient. It is a kind of wine that goes to the head releasing inhibitions. Not to obtain expert advice from real chemists is foolish.

Consider only the sections of tissues fixed in formalin, or in Zenker's fluid, and stained with hematoxylin and eosin which are examined routinely in so many laboratories. Reflect upon the functional attributes of these tissues while they were alive and to changes to which these specimens give no clues whatsoever. Among these are responsiveness to stimuli, permeability, the entry and outgo of water and of innumerable substances, the integration of thousands of enzymatic activities, the basic submicroscopic structural architecture that makes this possible, and so on almost ad infinitum. It is no wonder that tissues examined by such a crude technique, as well as by other more delicate ones, may look normal, since so much is overlooked, while in fact they are far from normal. Neuroanatomists are among the first to recognize the inadequency of microscopic examinations. They designate abnormalities without structural traces, "functional." Parts of the brain that look normal actually may be far from normal. This qualification probably holds for all parts of the body.

Standards for the comparison are few and disappointing. Some gross weights and measurements are given in Laboratory Technique but these are only for adults and corrections for race are not supplied. For a summary of measurements for children see R. E. Scammon's account in Abt's Pediatrics, Philadelphia: Saunders, 1923, 1, 257444. Anatomische, Physiologische und Physikalische Daten by H. Vierordt, Jena: Fischer, 1906, 616 pp. is still useful. R. Pearl and his associates have published quantitative data on the endocrines in Human Biology, 1935, 7, $350-391$; $555-607.1936, \quad 8,92-125$. 1937, 9, 245-250. For a spleen and thymus see E. B. Krumbhaar in Cowdry's Problems of Ageing, 1942. The Tabulae Biologicae (Amsterdam: Vitgevery, Dr. W. Jurik) is often useful. The last part on the eye (oculus) constitutes vol. 22 of 408 pp. and was published in 1947. The most recent publication on normal values in clinical medicine is that of Sunderman and Boerner (1949). A "Handbook of Biological Data" is in course of organization by the American Institute of $\mathrm{Bi}$ ological Sciences under the direction of E. C. Albritton at the National Research Council, Washington, D. C.

Summary: Histologists must be alert for artifacts, for otherwise there is a danger of describing appearances not present in vivo, or exaggerated or minimized by the procedure employed. They must be good physiologists always mindful of the fact that only a minority of functional states are reflected by microscopic structure. They must remember that their techniques just skim the surface. As experimentalists it is essential for them to gain a knowledge 
of the normal structure of the cells, tissues and organs of the body for otherwise their conclusions based on well controlled experiments on animals and the microscopic observation of parts of the human body in health and disease will be in jeopardy. And they must have a good idea where in the literature they can quickly find helpful accounts of what little has been determined accurately of gross and microscopic normality. It is safe to say that no human body is at any time normal, or usual, throughout its extent, because is is a far too complex organization of multifunctional interlocking mechanisms for uniformity in this respect. Health is not synonymous with an all pervading normality. It is merely a state in which no particular normality is lost to a conspicuously detrimental degree.

Normals, Gross Sizes. What these are is only known in a very hazy way. Yet if the size of an organ is distinctly abnormal this fact must clearly be taken into consideration in evaluating the results of its microscopic study. The best way is to search for papers dealing with the organ in which one is interested in the Quart. Cum. Index Med. The older data are summarized by Vierordt, H., Anatomische Physiologische und Physikalische Daten und Tabellen. Jena: Fischer, 1906, 616 pp. A summary of measurements on infants and children is provided by R. E. Scammon in Abt's Pediatrics, Philadelphia: Saunders, 1923, 1, 257-444. See also Coppoletta, J. M. and Wolbach, S. B., Am. J. Path., 1933, 9, 55-70. Useful quantitative data on the endocrines are supplied by R. Pearl and his associates in Human Biology, 1935, 7, 350-391, 555607 ; $1936,8,92-125 ; 1937,9,245-250$. For spleen and thymus see Krumbhaar, E. B., Cowdry's Problems of Ageing. Baltimore: Williams \& Wilkins, 1942, 139-184. There is a wide range in individual variation. Size may be greater or smaller than the normal or usual without being indicative of disease. Stitt, E. R., Clough, P. W and M. C., Practical Bacteriology, Haematology and Animal Parasitology. Philadelphia: Blakiston, 1938, 961 pp. give these approximate measurements (abbreviated) :

Adrenals-Length, 6-7 cm.; breadth, 3-3.5 cm.; weight, 5-6 gms. each.

Aorta-Length, $42.5-50 \mathrm{~cm}$.; thickness of wall, 1.5-2 mm.; diameter, $1.7-3 \mathrm{~cm}$.; weight, $35-45 \mathrm{gms}$.

Appendix-Length, 9-10 cm.; diameter, $6 \mathrm{~mm}$.; weight, 7-14 gm., quite variable.
Bladder-Capacity, 500 cc. when normally distended; thickness of wall, $2.5 \mathrm{~mm}$.; weight, 30-60 grams.

Brain-Weight, female, 1250-1275 gms.; male, 1365-1450 gms.; length, 16.5 $\mathrm{cm}$.; transverse diameter, $14 \mathrm{~cm}$.; vertical diameter, $12.7 \mathrm{~cm}$; dimensions in female being $1 \mathrm{~cm}$. less.

Fallopian tubes-Length, $7.6-12.6 \mathrm{~cm}$., the right usually the longer; diameter of lumen a verages $2.5 \mathrm{~mm}$.

Gall bladder-Length, 7.5-10 cm.; diameter, 2.5-3 cm.; thickness of wall, 1-2 mm.; capacity, $30-45 \mathrm{cc}$.

Heart-Weight, female, 250-280 gms., male, 270-360 gms.; length, 11.5-14 cm.; breadth, 7.5-10 cm.; thickness, 5-8 cm.; thickness, wall left ventricle, 9-12 mm., right ventricle, $2.5-3 \mathrm{~mm}$.; circumference, mitral orifice, 10.4$10.9 \mathrm{~cm}$; circumference, tricuspid orifice, 12-12.7 cm.; circumference, aortic orifice, 7.7-8 cm.; circumference, pulmonary orifice, $8.5-9 \mathrm{~cm}$.

Intestines-Small intestine, length, 6.75 meters, $2 / 5$ jejunum and $3 / 5$ ileum; diameter from $47 \mathrm{~mm}$. in duodenum to $27 \mathrm{~mm}$. at the end of ileum. Large intestine, length, 180$195 \mathrm{~cm}$.; duodenum, length, 26-28.5 $\mathrm{cm}$.

Kidneys-Weight, left, 150 gms., right, 140 gms.; thickness of cortex, $1 \mathrm{~cm}$.; length, $11.5 \mathrm{~cm}$; breadth, $6.2 \mathrm{~cm}$.; thickness, $3.2 \mathrm{~cm}$.; the left longer and the right thicker.

Liver-Weight, 1440-1680 gms. ; greatest transverse diameter, $20-24 \mathrm{~cm}$., greatest antero-posterior diameter, 10-15 $\mathrm{cm}$., vertical diameter, $12.7-15 \mathrm{~cm}$.

Lungs-Weight, combined, 1020-1290 gms.; weight, male, right lung, 680 gms., left lung, 600 gms.; weight, female, right lung, 480 gms., left lung, 420 gms. ; length, $26-30 \mathrm{~cm}$.; anteroposterior diameter at base, 17.5-20 cm.; transverse diameter at base, 10-12.7 cm.; right lung is shorter, broader and thicker than the left; dimensions in female average $2.5 \mathrm{~cm}$. less.

Mammary gland-Weight in adult, 150200 gms.; weight during lactation, 400-900 gms.

Oesophagus-Length, 25-30 cm.; diameter of lumen, $3 \mathrm{~cm}$; thickness of wall, $8 \mathrm{~mm}$; ; weight, 40 gms.

Ovaries-Weight (each) 4-8 gms., length, $3.8 \mathrm{~cm}$.; breadth, $1.9 \mathrm{~cm}$.; thickness, $1.2 \mathrm{~cm}$.

Pancreas-Weight, quite variable, 60135 gms.; length varies, average 15$20 \mathrm{~cm}$.

Parathyroids-Length, 6-7 mm.; breadth, 3-4 mm.; thickness, 1.5-2 mm. 
Pineal gland-Length, $1 \mathrm{~cm}$.; breadth, $5 \mathrm{~mm}$; thickness, $5 \mathrm{~mm}$.; weight, $0.2 \mathrm{gm}$.

Pituitary body-Length, $8 \mathrm{~mm}$.; breadth, $1.2 \mathrm{~cm}$.; weight, $0.3-0.6 \mathrm{gm}$.

Prostate-Weight, 22 gms.; length 3.1$3.8 \mathrm{~cm}$.; breadth, $3.8-4.5 \mathrm{~cm}$.; thickness, $2.5 \mathrm{~cm}$.

Salivary glands-Parotid, weight, 2530 gms.; sublingual, weight, $2-3 \mathrm{gm}$.; submaxillary, weight, 8-9 gms.

Seminal vesicles-Length, $5 \mathrm{~cm}$.

Spinal cord-Length, $45 \mathrm{~cm}$.; weight, 27-30 gms.; transverse diameter averages $1.2 \mathrm{~cm}$.; antero-posterior diam. eter averages $9 \mathrm{~mm}$.

Spleen-Weight, 155-195 gms.; length, $10-12.5 \mathrm{~cm}$.; breadth, $7.7 \mathrm{~cm}$; thickness, $2.5-3.7 \mathrm{~cm}$.

Stomach-Capacity, 1-2 liters.; thickness of wall, $6 \mathrm{~mm}$.; weight, 125-175 gms.

Testes-Weight, 20-25 gms. each; length, $3.8 \mathrm{~cm}$; breadth, $2.5 \mathrm{~cm}$.; thickness, $2 \mathrm{~cm}$.

Thoracic duct-Length, 37-5-45 $\mathrm{cm}$.

Thymus gland-Weight at birth, 13.7 gms. and increases to $26.2 \mathrm{gms}$. at end of second year when it gradually decreases until gland disappears; dimensions at birth, length, $6 \mathrm{~cm}$.; breadth, $3.7 \mathrm{~cm}$.; thickness, $6 \mathrm{~mm}$.

Thyroid-Transverse diameter, 6-7 cm.; height, $3 \mathrm{~cm}$.; weight, $30-40 \mathrm{gm}$.

Ureters-Length, $28-30 \mathrm{~cm}$., slightly longer on left side and longer in male, diameter of lumen varies, averages $2.5 \mathrm{~mm}$.

Urethra-Male, length, 16-20.6 cm.; prostatic, 2.5-3.1 cm., membranous, $1.5-2.5 \mathrm{~cm}$. , and the anterior, 12-15 $\mathrm{cm}$.; female, length, $3.8 \mathrm{~cm}$.; diameter of lumen averages $7-10 \mathrm{~mm}$.

Uterus-(Virginal) length, $7 \mathrm{~cm}$; breadth, $4 \mathrm{~cm}$.; thickness, $2.5 \mathrm{~cm}$.; weight, $40-50 \mathrm{gm}$.; the dimensions of a multiparous uterus are each increased $1 \mathrm{~cm}$. or more and the weight is increased $20 \mathrm{gms}$.; length of cavity in virgin, $5 \mathrm{~cm}$., in multiparae, $5.7 \mathrm{~cm}$.

Vagina-Length, 7.6-8.9 cm.; posterior wall is slightly longer than the anterior.

Normoblasts (orthochromatic erythroblasts). Stage in formation of erythrocyte between erythroblast and reticulocyte; nucleus spherical or oval, picnotic, of ten excentrically placed. Cytoplasm contains much hemoglobin, not normally present in circulation. See Erythrocytes, Developmental series.

Nucleal Reaction is a microchemical test for Thymonucleic Acid which see, also Feulgen Reaction.

Nuclear Inclusions are characteristic of some virus diseases but in many such diseases they are not found. Only when they are present in large numbers as in yellow fever is it feasible to investigate them in fresh tissucs. Staining reactions, solubility tests and other properties of fresh inclusions are described by Cowdry, E. V. and Kitchen, S. F., Am. J. Hygiene, 1930, 11, 227-299. Methods for their identification in fixed tissues are summarized by Cowdry, E. V., Am. J. Clin. Path., 1940, 10, 133148. For general purposes fixation in Zenker's fluid, paraffin imbedding and coloration with Hematoxylin and Eosin is the most satisfactory. Coloration with Phloxine or Eosin Methylene blue gives more brilliant colors but they fade more rapidly. The nuclear inclusions are typically acidophilic and therefore take cosin and phloxine energetically. When it is desired to reverse the colors use Safranin-Light Green which gives green inclusions and red chromatin. For microchemical methods see Cowdry, E. V., Science, $1928,68,40-41$, see also Specific Gravity determinations. Paper by Lucas, A. M., Am. J. Path., 1940, 16, 739-760.

When the following features are noted in a section it is likely that a virus has been at work:

1. A considerable number of inclusion-laden nuclei which can be arranged in series representing stages in development. This indicates an active process in which the nuclei exhibiting the most advanced alterations were affected first and the others in succession.

2. A change in which the accumulation of acidophilic material, forming the inclusion, is accompanied by margination of basophilic chromatin on the nuclear membrane, a disappearance of nucleoli and ultimate death and disintegration of the cells. This suggests that the inclusion formation is not merely an intranuclear heaping up of material effected without injury.

3. A cellular reaction characterized by hyperplasia, hypertrophy or necrosis.

Nuclear inclusions are of two general sorts-A and B (Cowdry, E. V., Arch. Path., 1934, 18, 527-542). Type A are the most definite and exhibit the properties noted above under 2 . When the basophilic chromatin does not marginate on the nuclear membrane and the nuclear structure does not disintegrate -we have to proceed warily. Such inclusions (type B) are droplet-like masses of acidophilic material surrounded by clear halos. They have been reported in Borna disease, in poliomyelitis and in several other conditions. When observed in routine preparations they are seldom conspicuous structures. 
It is only when strongly stained with fuchsin, for instance, that they catch the eye. Perhaps careful search of tissues not subjected to virus action might reveal similar bodies. Therefore in the case of type $B$ inclusions, insistence on criteria 1 and 3 is desirable. The nuclear inclusions in the liver following severe burns loo very much like those caused by viruses (Belt, T. H., J. Path. and Bact., 1939, 48, 493498).

In plants, as in animals, some nuclear inclusions are indicative of the action of certain viruses, see excellent summarizing account by Bawden, F. C., Plant Viruses and Virus Diseases. Waltham: Chronica Botanica Co., 1943, 294 pp. Since the inclusions usually occur in the form of "thin flat crystalline plates" they should be examined in living cells in the dark field and in polarized light because details of crystalline structure are not so well shown in fixed and stained preparations. The inclusions give the usual protein reactions but are Feulgen negative. They apparently contain virus.

An interesting and well illustrated account of intranuclear parasites is provided by $\mathrm{H}$. Kirby, Jr. in Calkins, G. N. and Summers, F. M., Protozos in Biological Research. New York: Columbia University Press, 1941, 1148 pp.

Nucleases-Written by E. W. Dempsey, Dept. of Anatomy, Washington University, St. Louis. February 26, 1951For many years, cytologists have attempted to apply enzyme preparations which chemically degrade or destroy nucleic acids. Van Herwerden, A., Anat. Anz., 1914, 47, 312-325, and Lison (p. 175) describe the older experiments. Until recently, however, the enzyme preparations were contaminated with trypsin and other proteolytic enzymes, so that the results after digestion of sections with nucleases were difficult to interpret. Kunitz, M., J. Gen. Physiol., 1940, 24, 15-32 described the preparation of crystalline ribonuclease, an enzyme which depolymerizes and therefore solubilizes pentose-nucleic acid. McDonald, M. R., J. Gen. Physiol., $1948,32,39-42$, reported that the last traces of proteolytic activity in this preparation could be removed by heat treatment. Ribonuclease has been widely used to identify many basophilic cytoplasmic components (Nissl substance, ergastoplasm of pancreatic cells, basophilic inclusions in placenta, cytoplasmic basophilia in general), and nucleoli in some but not all cells fail to stain after digestion in the enzyme. Desoxyribonuclease, an enzyme which depolymerizes desoxypentosenucleic acids, has also been crystallized by Kunitz, M., J. Gen. Physiol., 1950, $33,349-362$. This preparation destroys the stainability of chromatin, but has not yet had as wide an application to cytology as had had ribonuclease. These preparations are available through the Worthington Biochemical Laboratories, Freehold, N. J.

Nuclei. To look into the body and study the nuclei of living cells is feasible only up to a certain point. The observation of the Clarks' (E. R. and E. L., Am. J. Anat., 1936, 59, 123-173) that in transparent chambers inserted into the ears of rabbits (Sandison's Technique) the finely granular leucocytes may be followed about and seen to lose their nuclear polymorphism is significant of what can be done. In Tissue Cultures the cells are living under less natural conditions but they grow in thin films and can therefore be observed at high magnification. Careful analysis of moving pictures, showing nuclear form and structure, like those of Dr. W. H. Lewis distributed by the Wistar Institute, can prove very fruitful. By ultracentrifugation data can be obtained bearing on intranuclear Viscosity and the relative Specific Gravity of nuclear components. The techniques of Microdissection and microinjection also offer opportunities for advance. The Vital Staining of nuclei without killing the cells is difficult and not particularly helpful (Acriflavine); but it appears to be feasible in a variety of vertebrate cells with dilute solutions of methylene blue (Russel, D. G., J. Exp. Med., 1914, 20, $545-553)$, in amebae by microinjection (Monne, L., Proc. Soc. Exp. Biol. R. Med., 1934-35, 32, 1197-1199), and in the fibroblasts of tissue cultures with crystal violet (Bank, O. and Kleinzeller, H., Arch. f. exp. Zellf., 1938, 21, 394399). The same can be said for Ultraviolet Photomicrography.

The choice of fixative is important. It is difficult to secure after formalin fixation a brilliant color contrast of basophilic and acidophilic nuclear materials by staining with Giemsa, EosinMethylene Blue and other mixtures of "basic" and "acid" dyes, because the former take very intensely and the latter, lightly. But following Zenker's fluid and other mixtures containing potassium bichromate, which acts as a sort of mordant, these stains color the acidophilic as well as the basophilic components. It is for this reason, and because nuclear inclusions caused by 
virus action are usually acidophlic, that formalin used alone is contraindicated as a fixative.

On the morphological side it is known that nuclei stained in sections after fixation in the usual ways show a diversity, or heterogenicity, of internal structure which cannot be observed by the most careful examination of the nuclei of living cells. In the latter only the nucleolus can generally be distinguished. The so-called linin network, and small irregular particles staining with acid and basic dyes, are not observed. These probably result from the coagulating action of the fixative upon materials present in solution or fairly uniformly distributed in the nucleoplasm. Stained sections of tissues fixed in fluids containing fair amounts of osmic acid (Altmann's Mixture and Bensley's Acetic-Osmic-Bichromate) exhibit, on the contrary, nuclei with quite homogeneous looking nucleoplasm, containing nucleoli, which portray the condition in vivo more accurately. Colored illustrations of the nuclei of liver cells containing inclusions after osmic and non-osmic fixation (Figs. 47 and 20 ) are provided by Cowdry, E. V. and Kitchen, S. F., Am. J. Hyg., 1930, 11, 227-299. This does not mean, however, that the ground substance is always optically homogeneous in vivo.

The shrinkage of nuclei when examined in stained sections is generally more than $10 \%$ of their size invivo. In post-mortem autolysis, particularly of the kidney, one of the first nuclear modifications is shrinkage. The shrunken nuclei may stain intensely with both basic and acid dyes. The acidophilic material in them may even appear to be increased; for it is more concentrated, owing to decrease in volume (oxychromatic degeneration). They are also more spherical and less oval in shape. In early stages this modification can easily be identified by its occurrence in some tubules and not in others. A comparable hyperchromatism of nuclei at the edge of a section accompanied by a flattening of them may indicate that a surface film of tissue was permitted to dry before fixation.

Among the stains Iron Hematoxylin is a favourite because of its sharpness and permanence. Phloxin-Methylene Blue is also recommended. If one desires to reverse the colors and get red nuclei and green cytoplasm Safranin Light Green is suggested. The Safranin-Gentian Violet-Orange G technique gives several beautiful color tones. Recently the Feulgen reaction by which Thymonucleic Acid can be demonstrated has become very popular as the most sharply discriminating nuclear stain. Microchemical studies are now possible which a few years ago were undreamed of. The method of Microincineration reveals some of the mineral constituents (Scott, G. H., Proc. Soc. Exp. Biol. \& Med., 1935, 32, 1428-1429).

The collection of nuclei in bulk for chemical analysis is now feasible (see Centrifugation). Thus nuclei of liver cells can be separated from cytoplasms by centrifugation after treatment with dilute citric acid. Normal liver nuclei do not accumulate $\mathrm{P}_{32}$ while tumor nuclei and regenerating nuclei do (Marshak, A., Federation Proceedings, Baltimore, 1912, 1, (2) 57). A method for separating nuclei from rest of thymus is described by Williamson, M.B and Gulick, A., J. Cell. \& Comp. Physiol., 1942, 20, 116-118. The authors analysed the mass of nuclei for calcium, magnesium and phosphorus. Another method for separating from cytoplasm (Crossmon, G., Science, 1937, 85, 250) is to place drop 5\% aq. citric acid in center of a slide smeared with Mayer's Albumin Glycerin. Add piece fresh muscle. This slowly becomes transparent and infiltrated. The cloudiness of the citric acid is caused by released nuclei. Remove muscle and allow fluid containing nuclei to dry completely. Hold nuclei in place by treating with $95 \%$ ethyl alcohol. Wash in tap water, then in aq. dest., stain with Mayer's Hemalum, blue in tap water, counterstain in eosin, dehydrate, clear and mount.

For a technique to demonstrate sex differences in neuroglial and nerve cell nuclei consult Barr, M. L. (Exp. Cell Res., 1951, 2, 288-290). See Arginase and Diaminoacridines for visualization of nuclei in vivo by their fluorescence.

Nucleic Acids, see Ribonucleic, Thymonucleic and Desoxyribonucleic. Microspectrophotometry.

Nucleic Acid-Dye Interactions-Written by Edward L. Kuff, Dept. of Anatomy, Washington University, St. Louis 10, Missouri. October 18, 1951-Pure nucleic acids are not known to react with acid dyes. The staining of nuclei with acid dyes which can be made to occur in tissue sections is due presumably to interaction of the dye with protein constituents of the nuclei, although this phenomenon has not been adequately studied.

Nucleic acids of both cytoplasmic and nuclear origin react strongly with a 
wide variety of basic dyes, both in the test tube and in tissue sections. In a rough way, it can be stated that the amount of a basic dye taken up by a given nucleic acid-containing structure in the cell is proportional to the amount of nucleic acid present. Attempts at a precise quantitative estimation of nucleic acid content by means of basic dye uptake are in the early stages of development.

The physico-chemical nature of the interaction, in buffered aqueous solution, of rosaniline with yeast and pancreatic pentose nucleic acids (PNA) and with thymus desoxypentose nucleic acid (DNA) has recently been studied (Cavalieri, L. F., Kerr, S. E., and Angelos, A. J. Am. Chem. Soc. 1951, 73, 2567-2578; Cavalieri, L. F. and Angelos, A., ibid., 1951, 72, 4686-4693). The reaction was found to be reversible and stoichiometric. The curves relating amount of dye taken up by the nucleic acid to the concentration of free dye were different for each type of nucleic acid. However, in all cases the data could be explained satisfactorily on the basis of a salt-like linkage between the dye cation and the negatively charged phosphoric acid groups of the nucleic acid, without the assumption of more complicated adsorptive forces. Specific dissociation constants for the dyenucleic acid complexes could thus be calculated. Similar studies using other common histologic dyes are needed before the staining of nucleic acids in tissue sections can be quantitatively evaluated. It should be noted that the dye concentrations used in the above experiments were far below those ordinarily employed in staining. There is some evidence, however, that the dyenucleic acid interaction is stoichiometric at high dye concentrations also.

Mixtures of methyl green and pyronin stain nuclei green and cytoplasmic nucleoproteins red (see Methyl GreenPyronin). This differential staining of the two types of nucleic acid by two different basic dyes has been investigated by N. B. Kurnick (J. Gen. Physiol., 1950, 33, 243-264), who concluded the DNA of the nucleus owed its specific staining by methyl green to its high degree of polymerization. DNA which had been depolymerized by treatment with heat or acid failed to stain well with methyl green but did stain strongly with pyronin or other basic dyes. DNA combined with histone stained less strongly with methyl green than did the free DNA. Under conditions of high dye concentrations, the reaction between DNA and methyl green was said to be stoichiometric (Kurnick, N. B. and Mirsky, A. E., J. Gen. Physiol., $1950,33,265-274)$, and has been made the basis of a quantitative histochemical test for DNA (Leuchtenberger, C., Vendreley, R., and Vendreley, C., Proc. Nat'l. Acad. Sci. 1951, 37, 33-38).

The interaction of nucleic acids with basic dyes, while apparently primarily of an ionic character, can be modified by many factors. Among these are: 1) availability of the phosphate groups of the nucleic acid and their spatial arrangements (polymerization); 2) competition for available phosphate groups by substances other than dye, such as the histones of the nucleus, heavy metal cations of the fixing fluids, or impurities in the dye solution; 3 ) ionic strength, $\mathrm{pH}$, and temperature of the staining bath; and 4) the dye concentration used for staining. To approach any degree of stoichiometry in dye-nucleic acid interactions, all of these factors must be controlled as rigidly as possible.

The absorption spectra of all basic dyes which have been investigated show changes when the dyes are bound to nucleic acids. This fact is of importance if the measured absorption at some particular wave length is to be used to measure the amount of dye present in a cellular structure. It may also provide information as to the structure of the nucleic acids themselves. Such spectral shifts have been studied by Michaelis and Granick (Michaelis, L., J. Phys. and Colloid Chem. 1950, 54, 1-17), who found that basic dyes bound to nucleic acids almost invariably show a shift in their absorption maxima towards the Ionger wave lengths. Binding to nucleic acid was also found to inhibit the development of metachromasia shown by such dyes as thionin and toluidin blue either in concentrated solutions or when bound to other strongly acid substrates such as the acid mucopolysaccharides. Under conditions of excess nucleic acid with regard to the dye, the bound dye was shown to follow the Beer-Lambert Law. Under conditions of high dye concentration with regard to nucleic acid, such as obtain in the usual staining reactions, it has not been shown that the bound dye follows Beer's law. Nucleocytoplasmic Ratio. A histological method for computing this ratio is fully described by Cowdry, E. V. and Paletta, F. X., J. Nat. Cancer Inst., 1941, 1, 745-759; but there are many such techniques. A chemical method has been used to advantage by Dawbarn, M. C., Australian J. Exp. Biol. \& Med. Sci., 1932, 9, 213-226. Her ratio is obtained 
by dividing the nucleic acid nitrogen by the total coagulable nitrogen less nucleic acid nitrogen.

Nucleolinus is a term introduced by Haeckel to indicate a deeply staining granule within a nucleolus. For details see Champy, C. and Carleton, H.M., Quart. J. Micr. Sci., 1921, 65, 589-610.

Nucleolus (L. dim. of nucleus) is a body within a nucleus. There are at lcast three sorts.

1. Plasmosomes. These can be defined as roughly spherical bodies, which can easily be seen in the nuclei of some living cells without the aid of any stains, which stain after appropriate fixation, namely, with plasma or "acid" stains like eosin, (hence the name) and which do not directly contribute material to the formation of chromosomes.

Plasmosomes are not to be confused with cytoplasmic granules called plasmosomes by Arnold many years ago or with plastosomes, a term given by Meves to mitochondria and now fortunately being discarded. They can be referred to as acidophilic or oxyphilic nucleoli, but sometimes they are tinged quite strongly with basic dyes. They are of dense consistency, easily shifted by centrifugal action and are in some cases more resistant to the digestive action of pepsin and hydrochloric acid than karyosomes.

2. Karyosomes, are by contrast intensely basophilic and do contribute material to the making of chromosomes during mitosis. But they are resistant to peptic hydrochloric acid digestion. Wilson (E. B., Heredity, New York: Macmillan 1925 , p. 93) recognizes 3 types, net-knots, chromosome-nucleoli and karyospheres. There is doubt about the existence in vivo of the net-knots in the same shape, size and position as observed in stained sections.

3. Amphinucleoli (G. amphi on both sides) are nucleoli consisting of both plasmosome and karyosome material. Often the acidophilic substance acts as a kind of core and the basophilic substance is close to it or appears to be plastered on its surface. The latter may not occur in the same form in the living nucleus.

The fixation which shows, when the sections are stained, the highest degree of nucleolar detail is not necessarily the best (see remarks about Nuclei,). The Linin network, net-knots and basophilic material marginated on plas. mosomes may result in part from the coagulating action of the fixative on material originally distributed diffusely in the nucleoplasm. Nucleoli which look bubbly, or are surrounded by halos, are to be regarded with suspicion. Fixation in Acetic-Osmic-Bichromate and in other fluids containing osmic acid is indicated but they penetrate poorly. Staining by almost any technique which gives a good color contrast between acidophilic and basophilic materials is satisfactory. The classical stain is with safranin and light green. Eosin and methylene blue, hematoxylin and eosin are recommended, likewise all methods advised for Nuclear Inclusions caused by viruses.

Usually no difficulty is experienced in the identification of nucleoli. However with the plasmosomes there may be some question. In the first place nuclear inclusions type B (Cowdry E. V., Arch. Path., 1934, 18, 527-542) look something like plasmosomes. For example, the nuclear inclusions in Borna disease are acidophilic and may be of the same size as plasmosomes; but, they like others of type B are strongly acidophilic, are seldom tinged with basic stains and are generally surrounded by halos of unstained nucleoplasm. Moreover they are not present in normal animals.

Secondly cells are sometimes encountered in which there is an increase in acidophilic nuclear material often accompanied by nuclear hypertrophy. The material may occur in the form of dense spherules or of masses which are bluntly angular and without halos. Colored illustrations of liver cell nuclei are given by Cowdry, E. V. and Kitchen, S. F., Am. J. Hyg., 1930, 11, 227-299, figs. 43 and 44 . These bodies may be true nucleoli which have undergone hypertrophy or they may be simply accumulations in the nuclei of acidophilic material. The only sure way to tell would be to ascertain whether they comport themselves like true plasmosomes during mitosis but the cells involved have not been seen in division. In other conditions (gliomatous tumors, etc.) cells are found whose nuclei are enlarged and possess roughly spherical, vacuole-like masses of granular acidophilic material. The granules have the appearance of coagula produced by the fixative in a rather thin fluid medium. There is no halo. Such bodies are probably not altered plasmosomes. Their density is much less.

Differential staining. Nucleoli are colored brown after fixation in equal parts of $1 \%$ aq. chromic acid and $10 \%$ formalin and staining of chromosomes by Feulgen Reaction (Bhaduri, P. N., J. Roy. Mier. Sci., 1938, 58, 120-124).

Nucleonucleolar Ratio recommended as an aid in the grading of malignancy with 
review of the literature (Mendes Ferreira, H. E., J. Lab. \& Clin. Med., 1940$41,26,1612-1628)$.

Nucleotides, see Diph osphopyridine Nucle otide and Pentose Nucleotides.

Nutriles, growth promoting (Williams, R. J., Biol. Rev., 1941, 16, 49-80).

Oil Blue NA (Calco) a stain which colors rubber bright blue in various plant species (Whittenberger, R. T., Stain Techn., 1944, 19, 93-102). This dye is also a good stain for fat in animal cells (Lillie, R. D., Stain Techn., 1945, 20, 7-9).

Oil Immersion, see Immersion Oils.

Oil Red IV, see Sudan IV.

Oil Red AS, O, B or 3B, see Sudan III.

Oil Red $O$ (CI, 73). - fast oil orange II, fat ponceau, oil scarlet, orange $R R$, red $B$, Sudan II-an acid mono-azo dye suggested as fat stain by French, R. W., Stain 'Techn., 1926, 1, 79. Proescher's (F., Stain Techn., 1927, 2, 60-61) oil red pyridine stain for fat is to immerse frozen sections of formalin, Mullerformalin (see Muller's fluid) and $5 \mathrm{cc}$. $10 \%$ formalin in 100 cc. sat. aq. picric acid fixed tissues in $50 \%$ aq. pyridine, 3-5 min. Stain 3-5 min in 3-5 gms. oil red $\mathrm{O}$ dissolved in $100 \mathrm{cc} .70 \%$ aq. pyridine C.P. Differentiate in $50 \%$ pyridine several minutes and counterstain for 2-3 min. in Delafield's Hematoxylin. Mount in levulose syrup. For central nervous system differentiate $30 \mathrm{~min}$. in pyridine and use $16 \mathrm{cc}$. Delafield's +2 cc. glacial acetic acid. According to Proescher, oil red $\mathrm{O}$ stains fats and lipids more intensely and quickly than Sudan III or IV.

Oil Scarlet, see Oil Red O.

Oil Soluble Dyes. List with physical properties of each and use as fat stains. Very comprehensive (Lillie, R. D., J. Tech. Methods, 1944, 24, 37-45).

Oil Vermillion, see Sudan R.

Okajima's "omnichrom" stain (Ito, T., Folia Anat. Jap., 1937, 15, 357-359).

O'Leary's Brazilin Method--Revised by James L. O'Leary, Dept. of Neuropsychiatry, Washington University, St. Louis, May 8, 1950-For myelin sheaths. Run paraffin, or celloidin sections of properly fixed and mordanted (Muller's Fluid) tissue to water. After rinsing transfer to $3 \%$ aq. potassium bichromate or in Muller's fluid, 12-24 hrs. Stain in: $10 \%$ Grubler's Brazilin in abs. alc. (1-6 months old), 10 cc.; aq. dest., 100 cc.; acetic acid, glacial, 5 drops. Wash in aq. dest. Differentiate in $0.25 \%$ aq. potassium permanganate 1-5 min. Remove potassium permanganate with Weil's solution (oxalic acid, $2.5 \mathrm{gm}$.; sodium bisulphite, $2.5 \mathrm{gm}$.; aq. dest. 1,000 cc.) Sections should show gray matter light pink, white matter brilliant red. Cell bodies stain in addition to myelinated fibers. If differentiation not complete after first immersion in potassium permanganate followed by oxalic acid-bisulphite mixture, repeat the procedure. Wash, dehydrate and mount. See Golgi-Cox Method and Golgi Method, Quick.

Oligodendroglia. Method for impregnation with silver in pyroxylin (celloidin) sections (Weil, $H$. and Davenport, $H$. A., Trans. Chicago Path. Soc., 1933, 14, 95-96). This resembles their Microglia method. Wash sections in aq. dest. and transfer to aq. dest. containing 1 drop conc. ammonia per $10 \mathrm{cc}$. Treat for $15-20 \mathrm{sec}$. with silver solution made up as for microglia except that $15 \%$ aq. silver nitrate is used and the end point of the titration is reached when about $12 \mathrm{cc}$. of it have been added to the 2 cc. conc. ammonia. Transfer to $10 \%$ formalin and allow section to drop to bottom without moving dish. After the pyroxylin has become deeply stained and the tissue begins to take a brown color, move it with glass rods until it is stained coffee-brown. Use fresh formalin for each section. Pass section through 3 changes aq. dest. Dehydrate in alcohol, clear in xylol and mount in balsam.

Olive Oil, reactions in tissue to fat stains after various fixations (Black, C. E., J. Lab. \& Clin. Med., 1937-38, 23, 1027-1036)

Oliver, see Kidney.

Omentum, spreads of (McClung, p. 336). Transplants of spleen into (Holyoke, E. H., Am. J. Anat., 1940, 66, 87-132.

Oncocytes (G. onkos, tumor, swelling + kytos, cell). These cells are recognizable in ordinary hematoxylin and eosin preparations by their (1) large size, (2) usually single centrally placed picnotic nuclei and (3) the large volume of finely granular eosin staining cytoplasm in proportion to nuclear volume. They are most frequently encountered in the parotid and subrnaxillary glands but they have been reported in many other epithelia including those of the thyroid, parathyroid, pituitary, pancreas, liver, stomach, Fallopian tube, uvula, nose, pharynx, trachea and esophagus. Nohteri, H. (Acta Path. et Micr. Scand., $1946,23,473-483$ ) found them only in individuals over 52 years of age. Hamperl, H. (Virchow's Arch., 1937, 298, $327-375$ ) reported that they are very rarely seen under 50. An excellent cytological account of oncocytes in the salivary glands of a large series of animals of known age is provided by 
Andrew, W. (J. Gerontol., 1949, 4, 95103).

Opdyke, see Keratohyalin Granules, Separation and Analysis.

Opsonocytophagic Index, method for rapid staining of blood smears in (Bondi, A. Jr., J. Lab. \& Clin. Med., 1941, 26, 1811). Derivation of index number in (Foshay, L., LeBlanc, T. J., J. Lab. \& Clin. Med., $1936-37,22,1297-1300)$.

Opal Blue (CI, 689)-Aniline Blue, alcohol soluble, Bleu Lumiere, Gentiana Blue $6 \mathrm{~B}$, Spirit Blue $-\mathrm{a}$ basic dye of light fastness 3 , to be employed in contrast staining with Biebrich Scarlet, Crocein Scarlet and other dyes (Emig, p. 50).

Optic Lens, methods of microincineration and histospectrography as applied to cataracts of various sorts and normal lenses with special attention to copper, zinc and iron (Busnel, R. G., Pillet, P. and Tille, H., Bull. d'Hist. Appl., 1938, 15, 99-109).

Oral Mucosa. Smear method for study of keratinization (Weinmann, J., J. Dent. Res., 1940, 19, 57-71). With end of agate spatula gently scrape area about $1.5 \mathrm{sq} . \mathrm{cm}$. Smear on slide, dry in air and stain for 30 sec. in : sat. alc. gentian violet (or better crystal violet) $10 \mathrm{cc}$. $5 \%$ aq. phenol, 90 cc. Lugol's Iodine, 30 sec. Wash in water until no more color is extracted. Counterstain for 2 min. in sat. safranin $\mathrm{O}$ in $95 \%$ alcohol, 10 cc. + aq. dest., 100 cc. Wash in water $2-3$ sec., dry and mount in balsam.

Orange I (CI, 150). Synonyms: naphthol orange, tropaeolin G or 000 No. 1. An acid mono-azo dye used as an Indicator.

Orange II (CI, 151). Synonyms : acid orange II, Y or A, gold orange, mandarin $G$, orange $A, P$, or $R$, orange extra, tropaeolin 000 No. 2. An acid monoazo dye. Ebbinghaus, H., Centralbl. f. allg. Path. u. Path. Anat., 1902, 13, 422-425 employed gold orange with hematoxylin as a special stain for keratin.

Orange III, see Methyl Orange.

Orange $\mathbf{A}, \mathbf{P}$, or $\mathbf{R}$, see Orange II.

Orange Extra, see Orange II.

Orange G $(\mathrm{CI}, 27)$. Synonym; wool orange $2 \mathrm{G}$. Of slightly different grade according to Conn (p. 47) are orange GG and GMP. An acid mono-azo dye widely used.

Orange MNO or MN, see Metanil Yellow.

Orange R (CI, 161), an acid mononzo dye of light fastness 3-4 action of which on plant and animal tissue is described (Emig, p. 33).

Orange RR, see Oil Red $O$.

Orcein (CI, 1242) is a natural dye produced from lecanora parella (a lichen) and should not be confused with orcin produced from the same plant. It is now prepared synthetically. Its precise for- mula remains to be determined but it is a most valuable stain for Elastic Fibers. Mollier, G., Zeit. f. wis. nikr., $1938,55,472-473$ employed it with iron hematoxylin, naphthol green $\mathrm{B}$ and azocarmine G. Acetic-orcein is advocated as a new stain-fixative for chromosomes (LaCour, I., Stain Techn., 1941 16, 169-174). An acid orcein Giemsa is described for use in dermatology by Pinkus, H., Arch. Dermat. and Syph., $1944,49,355-356$.

Orceille, a purple dye, derived from Lichens growing on the rocks of the Near East and Mediterranean areas, achicved great favor among the ancients being said by Theophrastus and Dioscorides to even excel Tyrian Purple. A Florentine dye trader, Federigo, promoted this dye, built up a thriving business and calling himself Orcelli, founded a large, distinguished and prolific family (Leggett, W. F., Ancient and Medieval Dyes. Brooklyn: Chemical Publishing Co., Inc., 1944, 95 pp.).

Organ Culture in Vitro-Written by Honor B. Fell, Strangeways Research Laboratory, Cambridge, England. June 8, 1951-In most forms of tissue culture, the investigator is not concerned with the original tissue fragment but with the cells which migrate from it and multiply in the medium to form a zone of new growth. The object of "organ culture" is to grow tissue in a differentiated state as an independent organism.

There are several variations of the method, but in general the explants are grown on the surface of a fairly large volume of culture medium with an abundant air supply. Tissue extracts used in the preparation of the medium are never made from young embryos, as such extracts have been shown to inhibit differentiation (Gaillard, P. J., Hormones regulating growth and differentiation in embryonic explants, Act. Sci. et Industr., Paris: Hermann et Cie, 1942). The tissue fragments are transplanted at frequent intervals, partly to restrict outgrowth which disintegrates the histological structure of the explants, and partly because the compact mass of tissue soon exhausts the food material in its immediate neighborhood.

Although as yet the method is practiced in a few laboratories only, many different types of differentiated tissues have successfully been grown (Fell, H. B., J. Roy. Mier. Soc., 1940, 60, 95112; Chapter on "Histogenesis in 'Tissue Culture" in Bourne, G. H., Cytology and Cell Physiology, Oxford:Clarendon Press, 1951). Brachet, A. (C. R. Acad. 
Sci., 1912, 155, 1191), Thomson, G. D. (Proc. Roy. Soc. Med., 1914, 7 and Mareus Beck Lab. Reports, 21), Chlopin, N. (Arch. f. Mikr. Anat., 1922, 96, 435-493) and Maximow, A. (Contrib. Embry. Carnegie Inst., 1925, 16, 49115), showed that small fragments of chicken and mammalian embryos would continue to develop when cultivated in vitro. It has since been found that isolated organ rudiments have a surprising capacity for growth and differentiation in culture. Thus the eye rudiment of a 3-day check embryo forms a retina of almost adult type during cultivation (Strangeways, T. S. P., and Fell, H. B., Proc. Roy. Soc., B, 1926, $100,273-283)$; the rudiments of the long-bones may increase to four times their original length in 11 days, ossify and even undergo considerable anatomical development (Fell, H. B., and Robison, R., Biochem. J., 1929, 23, 767-784, and Fell, unpublished results); the early stages of joint-formation take place in vitro (Fell, H. B. and Canti, R., Proc. Roy. Soc., B, 1934, 116, 316-351); the thyroid rudiment forms vesicles and secretion (Carpenter, E., J. Exper. Zool., 1942, 89, 407-431); embryonic ovaries produce sex cords and ova (Martinovitch, P. N., Proc. Roy. Soc., B , 1938, 125, 232-249, Ibid 1939, 128, 138-143; Gaillard, P. J., Proc. Roy. Neth. Acad. of Sci., 1950, 53). Some organs which are already largely or completely differentiated when explanted, can be maintained in a healthy state for various periods in vitro, e.g. the prostate gland of infant rats (Price, D., Ann. Rep. Strangeways Res. Lab., 1949, 13) and mice (Lasnitzki, Z., Brit. J. Cancer, in press), late foetal mouse bones (Fell, H. B. and Mellanby, E., Brit. Med. J., 1950, 2, 535-539), rabbit lymph glands (Trowell, A. O., Exp. Cell Research, in press), parathyroid (Kooreman, P. J., and Gaillard, P. J., Arch. Chir. Nederl., 1950, 2, 326), pituitary (Martinovitch, P. N., Nature, $1950,165,33-34)$.

It is possible to practice organ culture under very simple conditions. The writer works on an open bench in a small, clean culture room with a sealed window and no outside ventilation; the door of the culture room which opens into a larger laboratory, is left open. A separate culture room, though an advantage, is not necessary, however. No mask or cap is worn. The culture bench is covered with a large sheet of plate glass; a beaker of distilled water is kept boiling on a tripod beside the operator and instruments, pipettes, etc. are frequently rinsed in it. Direct sunlight is excluded by a green, translucent plastic curtain which makes a very pleasant diffused light. Under these conditions the writer of ten works for weeks without losing a single explant from contamination. If occasional colonies should appear, the culture room is thoroughly steamed by boiling a bucket of water over a gas ring for some hours and the walls, ceiling, floor and benches are then wiped with a cloth impregnated with lysol. This procedure usually abolishes any airborne infection.

It may well be that in a city, or in a hot dusty atmosphere, much more stringent precautions may be necessary than these simple arrangements, but it is advisable to try the simple organisation first and only elaborate when strictly necessary.

1. Preparation of tissue. In experiments with early organ rudiments, the most difficult and also the most important part of the technique is to dissect and handle the delicate tissues without damaging the cells. For this it is essential to prepare adequately fine instruments. The writer uses a narrow Graefe's cataract knife and an ordinary sewing needle broken in half and mounted in a thin glass rod.

A No. 1 knife is best but it is much too thick and coarse as it comes from the makers, and for the dissection of early embryos it must be ground to a suitable shape by the worker himself. It is first rubbed on a fairly coarse carborundum stone to render the distal third of the blade as thin and flexible as possible, and is then smoothed and shaped on an Arkansas stone. Throughout the sharpening process the knife is repeatedly examined under a dissecting binocular microscope and when finished, the end third of the blade should be narrow, very flexible and have a perfect point; the flexibility should be carefully graded or the end will snap. In the writer's experience beginners have great difficulty in learning to prepare knives properly.

The needle is mounted by melting the tip of a glass rod and pushing the broken end of the needle into it. The needle should not be stuck too far into the rod or the glass may crack during sterilisation. To sharpen the point, the needle is rotated with the finger and thumb and, at the same time it is rubbed on the end of an Arkansas stone. It should be remembered that where needles are sharpened the stone is spoilt for knives.

When the knives and needles are ready, they are carefully wiped with 
a clean duster, then rinsed in two changes of fresh absolute alcohol and thoroughly dried on a glass cloth. It is convenient to sterilize a knife and a needle together in a single tube. A very firm, deep wad of cotton wool is rammed into the bottom of a test tube, the instruments are slid gently into the tube which is corked with a cotton wool bung and sterilized by dry heat. It is advisable to sterilize the knives and needles point downwards, otherwise moisture may condense on the steel and corrode it.

After use, the instruments should be very carefully wiped to avoid rusting and re-sharpened before being sterilized again. When once the knives have been properly ground, it takes only a few minutes to clean and sharpen them on the Arkansas stone.

The explants are prepared under a dissecting binocular, the stage of which is fitted with a small glass shade to prevent airborne contamination during the manipulation. It is important to select the right magnification and it is often convenient to make the gross dissection (e.g. the removal of a limb or eye from the body) under a low power which provides ample depth of focus, and to complete the process under a higher magnification. The writer uses transillumination for the dissection which is done in a large hollow ground slide containing Tyrode; the tissue should not be left in Tyrode for longer than is absolutely necessary.

It is important to remove from the rudiments as much of the epidermis as possible, otherwise the epithelium envelopes the explant, keratinizes and the imprisoned cells degenerate. To detach the epidermis the very thin, sharp knife blade is slid beneath it and the tissue is then cut by gently stroking the edge of the knife with the point of the needle. It is essential to reduce trauma to a minimum, or the explants will not prosper during subsequent cultivation.

There is an optimum size of explant. If it is very minute it may not thrive in vitro, though sometimes this difficulty may be overcome by placing it in contact with some other tissue (Borghese, E., J. Anat., 1950, 84, 303). If the explant is too large the interior becomes necrotic. There may also be an optimum stage of development at which a given tissue should be explanted. If taken too early it may not differentiate in complete isolation, while if removed from the embryo a few hours later it may attain an almost adult structure. On the other hand, if explanted when differentiation is already well advanced, the tissue may be unable to adapt itself to life in vitro and either degenerate or lose its characteristic structure.

2. Watch-glass method. In the Strangeways Research Laboratory a simple form of moist chamber is employed for organ culture (Fell, H. B. and Robison, R., Biochem. J., 1929 , 23, 767-784). It consists of a Petri dish $80 \mathrm{~mm}$. in diameter and $10 \mathrm{~mm}$. deep, carpeted with a thin layer of absorbent cotton wool in the center of which a round hole is cut. A watchglass $40 \mathrm{~mm}$. in diameter is laid over the hole and the dish is sterilized by dry heat. Ten cubic centimeters of sterile aq. dest. is pipetted into the Petri dish. It is not essential to use exactly the size of watch-glass and Petri dish mentioned; but it is important that the diameter of the Petri dish should be fairly large relative to that of the watchglass for otherwise the culture medium will tend to dry.

The culture medium is dropped into the watch-glass with a pipette; the first drop is always deposited on the cotton wool as this is found to reduce the risk of infection. The type of medium depends on the tissue to be cultivated. Many avian and mammalian rudiments grow well in a mixture of fowl plasma and the extract of an old (12-14 day) chick embryo. Bone rudiments grow very well in a mixture of $3 \mathrm{pts}$. of plasma: $1 \mathrm{pt}$. of concentrated extract of a 14-day chick embryo made with Tyrode containing $1 \%$ glucose, so that the final medium contains $0.25 \%$ glucose. It is important to see that the tissue has a suitable depth of medium beneath it, as large explants soon exhaust and partly liquefy the clot in their neighborhood. For chick bone ludiments of about $1.25 \mathrm{~mm}$. in length the writer uses 12 drops of medium, but by the time they have attained a length of about $5 \mathrm{~mm}$. she adds 20 drops to each watch-glass. Organ cultures are often more fastidious about their culture medium than unorganised tissues. It is therefore advisable to use freshly made embryo extract and plasma which are not more than a week old. Antibiotics are unnecessary.

For many mammalian tissues an admixture of homologous plasma is desirable. Martinovitch, P.N. (Nature, 1950, $165,33-34)$ grows the anterior pituitary of young rats in a mixture of 6 drops of heparinized rat plasma, 3 drops of chicken plasma and 3 drops of concentrated chick embryo extract.

When the organ rudiments are ready 
for explantation, the writer washes them in fresh Tyrode which is then removed and replaced by embryo extract of about half the concentration of that used for the culture medium and lacking the extra glucose. They are sucked into a pipette and deposited on the surface of the clot in the watchglass. The Petri dish is then placed under the shade on the dissecting binocular microscope, the lid removed and the surplus extract carefully sucked off with an extremely fine pipette, care being taken not to damage the clot. Organ cultures do not grow well under a fluid phase, but they quickly become surrounded by a shallow pool of fluid exuded from the clot.

The Petri dishes are not sealed, but under the conditions described, the medium does not dry. If the dishes are laid directly on the incubator shelf, drops of moisture form on the inner surface of the lid, but this can easily be prevented by placing the vessels on planks of wood which are left permanently in the incubator. Should drops form in spite of this precaution, it is probable that the temperature of the incubator is fluctuating unduly.

Many tissues grow and develop well at normal body temperature, but others do better if the temperature is lowered to $34^{\circ} \mathrm{C}$. Martinovitch has kept rat and mouse ovarian explants and rat pituitaries in a healthy differentiated state for several weeks at $34^{\circ} \mathrm{C}$.

The writer transfers explants to fresh medium three, or in the case of large, rapidly growing avian rudiments, four times a week. To make the fourth transplantation, she prepares a double set of watch-glasses on the Friday of each week; one set is employed the same day and the other placed in the refrigerator for use on the Saturday when the explants are again subcultivated. They are then left undisturbed until the Monday. This routine gives excellent results for cultures of avian long bone rudiments.

During incubation, some types of explant become firmly anchored to the clot by migrating cells. To detach the tissue without damage, the clot is pulled away on one side so as to rupture the zone of outgrowth; the explant is then loosened from the underlying clot with the knife and needle, after which the zone of growth on the other side is ruptured, so that the explant now lies freely in the pool of exuded fluid. It is advisable not to rupture both sides of the zone of growth before detaching the explant from the clot; none of the old clot should be left at- tached to the tissue. The explant is sucked into a pipette containing a little Tyrode and deposited in a hollowground slide in a Petri dish. The Tyrode is replaced by the more dilute extract mentioned above and the tissue is placed on a fresh clot in the manner already described.

3. Gaillard's method. Professor P. J. Gaillard (personal communication) uses a rather different technique which has given excellent results in the cultivation of the parathyroid from human infants and of the human fetal ovary.

The explants are cultivated in embryological watch glasses sealed with a small glass plate. They are grown on a very soft clot composed of a mixture of (a) $5-15 \%$ adult human blood plasma containing $0.5 \mathrm{cc}$. of a $0.1 \%$ solution of heparin for $10 \mathrm{cc}$. of blood, (b) $10 \%$ placental vein serum, (c) $65-75 \%$ of Gey's balanced saline solution, (d) human fetal brain press juice. To make the brain press juice, fragments of fetal brain are placed in a Petri dish at $4^{\circ} \mathrm{C}$. for 24 hrs. which facilitates the separation of the tissue fluid; the tissue is then minced with a tissue press, an equal quantity of Gey's saline solution is added and the mixture is centrifuged for $15 \mathrm{~min}$. at $6,000-$ 8,000 r.p.m. The supernatant fluid is decanted into Pyrex glass tubes and stored at $-20^{\circ} \mathrm{C}$. The explants are transferred to fresh medium twice a week.

Parathyroid explants completely liquefy the clot in 3 days. During the first few days in vitro the peripheral zone degenerates and is washed away during transplantation, leaving a healthy ball of tissue which enlarges during subsequent cultivation and may survive for as long as 60 days. The central part of the ovarian cultures degenerates, but the necrotic matter is later resorbed and replaced by healthy tissue growing in from the periphery.

4. Other methods. Recently Trowell has devised a modification of the watchglass method which can be used for metabolic studies and which enables organized tissue to be grown in a fluid medium in any type of gaseous atmosphere required. He has successfully grown rabbit lymph glands by this technique which should be applicable to many physiological problems.

Some rudiments develop well in tubes (Strangeways, T. S. P. and Fell, H. B., Proc. Roy. Soc., B, 1926, 100, 273-283). The medium is placed in a small test tube $\left(2^{\prime \prime} \times \frac{1}{2}\right)$ and allowed to 
clot. The explant is deposited on the surface of the clot and the tulse is corked and incubated in a vertical position. At 2-3 day intervals the explant is removed from the clot with a pipette, washed and re-planted in a fresh tube.

Although large explants thrive best in some form of watch-glass culture, very small rudiments may do better in large hanging drop preparations

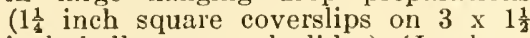
inch hollow ground slides) (Jacobson W. and Fell, H. B., Quart. J. Micr. Sci., 1941, 82, 563-586; Borghese, E., J. Anat., 1950, 84, 287-302). When the tissue is transplanted most of the zone of outgrowth is cut away and the explant is preserved intact.

Application of Organ Culture: The cultivation of organized tissue has an almost unlimited number of possible applications. So far it has been used mainly by embryologists for investigations in developmental mechanics, because explants of embryonic tissue are so readily accessible to manipulation and observation. An important and almost unexploited field, however, is the study of tissue metabolism by this method. Much work has been done on the growth requirements and physiology of unorganised tissue cultures, but very little on the metabolism of the many types of differentiated tissue which can be grown in vitro and which might provide information which would be impossible to obtain from the intact animal or from short-lived tissue slices.

It will be seen from the foregoing description of technique that the necessary procedure and equipment for this kind of tissue culture are not elaborate. All that is needed for success is skill in fine dissection and a sound knowledge of histology and embryology.

Organoids ( $G$. organon, organ + eidos, appearance). The term organoid is not a happy one. It is used to denote the organ-like appearance of some structure that the user fails accurately to describe as in the case of some tumors. Also, certain bodies, such as the mitochondria, are occasionally listed as organoids, or organelles, conveying an unwarranted impression of similarity to the complex organs of the body.

Origanum Oil. With it tissues can be cleared from $95 \%$ alcohol, but care must be taken to obtain a pure product. The kind required consists of carvacrol and cymene terpenes. Ordinary origanum oil is oil of thyme.

Orseillin BB (CI, 284). A little used acid dis-azo dye. See Cohen, I., and Doak, K. D., Stain Techn., 1935, 10, 25-32. For staining fungi (Alcorn, G. D. and Yeager, C. C., Stain Techn., 1937, 12, 157-158).

Orthochromatic Erythroblasts, see Erythrocytes, developmental series.

Orth's Fluid. Potassium bichromate, 2.5 gm.; aq. dest., 100 cc., formalin, $10 \mathrm{cc}$. The $1 \mathrm{gm}$. sodium sulphate originally advised by Orth is omitted as useless. Since the fluid does not keep it should be made up immediately before use. Regaud's fluid, the best fixative for mitochondria, is the same except that the amount of formalin is increased See Lithium Carmine (Orth).

Osage Orange Pigments as brilliant mordant dyes for wool and silk. Wolfsom, M. L., Harris, W. D., Johnson, G. F., Mahan, J. E., Moffett, S. M. and Wildi, B., J. Am. Chem. Soc., 1940, 68, 406418. Should be tried on animal tissues.

Osmic Acid. This is the tetroxide of osmium and has no acid properties. It comes in sealed glass tubes usually each containing $1 \mathrm{gm}$. To make the $2 \%$ aq. sol. of osmic acid gencrally employed, wash the label off the tube with soap and water. After washing repeatedly in aq. dest. rinse in absolute alcohol and dry. Carefully clean the inside of a glass stoppered bottle and of a graduate in the same way. With clean forceps put the tube in the bottle. If it is not easily broken by vigorous shaking it will be necessary to take it out, file one side, break and return to the bottle. Finally add $50 \mathrm{cc}$. of aq. dest, measured in the graduate. The osmic acid slowly dissolves forming a clear light yellow solution. Do not hasten solution by heat. Keep in dark or subdued light. To use a bottle made of colored glass or the outside of which has been blackened is a bad practice because it hides the condition of the solution from the person using it. If there is a blackening of the solution its potency is probably reduced. An indicator of concentration, discovered by Tschngaeff, has been improved by Palmer (R., J. Roy. Micr. Soc., 1930, 50, 221-226).

The fumes of osmic acid are very injurious to the eyes. They are a good fixative for well separated cclls as in smears. They blacken the chromaffin celis of the adrenal charged with epinephrine or its precursor (Cramer, W., Fever, Heat Regulation, Climate and ThyroidAdrenal Apparatus. London: Longmans, Green \& Co., 1928, 153 pp.) Alone, a solntion of osmic acid is a fair fixative for mitochondria and by prolonged action may reveal the Golgi apparatus. See critique by Owens and 
Bensley (H. S. and R. R., Am. J. Anat., 1929, 44, 79-109). But osmic acid penetrates very badly indeed and is best employed in mixtures with other chemicals as in the fixatives of Altmann, Mann, Bensley, Flemming and others. Its chief value is that it blackens many but not all fatty droplets. However it also blackens some materials which are not fatty. Osmic acid plays an important part in the Marchi method for nerve fiber degeneration.

Osmic Acid Method for fat. When reduced to osmium dioxide in the presence of some fats it blackens them as may be seen by the examination of tissues fixed in fluids containing osmic acid (Altmann's, Flemming's etc.) but unless rigidly controlled other substances may be blackened as well or not all of the fats may be shown. See remarks by Owens, H. B. and Bensley, R. R., Anat. Rec., $1929, \mathbf{4 4}, 79-109$. It is best to proceed as advised by Mallory (p. 119). Place frozen sections of tissue fixed in $10 \%$ formalin for $24 \mathrm{hrs}$. in aq. dest. $1 \%$ osmic acid 24 hrs. (or Flemming's or Marchi's solution). Wash thoroughly in running water 6-12 hrs. Abs. alc. for several hours in order to get secondary staining of palmitic and stearic compounds as well as of oleic. Wash in water and mount in glycerin jelly (glycerinalone will do). Fat is black against a yellowish brown background. Non-fatty substances like tannic acid and eleidin of epidermis are also blackened.

For nerve fibers (Dr. J. L. O'Leary, personal communication). Use fresh or $10 \%$ formalin fixed material. Tie a stretch of freshly isolated nerve to short length of glass rod and immerse in $2 \%$ aq. Osmic acid. Leave for $24 \mathrm{hrs}$. Wash 4-6 hrs. in running water. Dehydrate in ascending alcohols and doubly imbed by the Peterfi method as follows: Pour $1 \%$ celloidin in methyl benzoate (which takes about 1 month to dissolve) into a dish. Add absolute alcohol and the tissue. The latter gradually sinks into the celloidin. Transfer to $2-3 \%$ celloidin in methyl benzoate. Leave 2-4 days. Drop tissue directly into benzol. After a few hours in benzol begin infiltration in paraffin at $40^{\circ} \mathrm{C}$. This takes $12-24$ hrs. Change paraffin several times and imbed.

Ossicles, see Ear.

Ossification. Demonstration of in embryos and fetuses up to 18 weeks by staining with alizarin red $\mathrm{S}$ (Richmond, G. W. and Bennett, L., Stain Techn., 1938, 13, 77-79). Eviscerate. Fix in 95\% alcohol 2 weeks or more. Rinse in tap water and put in $1 \%$ aq. $\mathrm{K}_{2} \mathrm{CO}_{8}$ for month or longer. Clear soft partsand make bones clearly visible by placing in $1 \%$ aq. $\mathrm{KOH}$ for 10 days or more. (Specimens fixed in formalin instead of alcohol require about 1 month in $10 \% \mathrm{KOH}$ ) If tissues become too soft harden in equal parts glycerin, $95 \%$ alcohol and water $12-24$ hrs. and continue $\mathrm{KOH}$ if necessary. In last few days reduce $\mathrm{KOH}$ to $0.5 \%$. Wash in running tap water 12 hrs. Immerse in $0.1 \%$ aq. alizarin red $\mathrm{S}$ to which few drops $1 \%$ aq. $\mathrm{KOH}$ has been added for 30-60 min. Wash for $30 \mathrm{~min}$. in running tap water. Remove deep purple color from soft parts by immersing in $20 \%$ aq.glycerin containing $1 \%$ KOH. For small specimens reduce $\mathrm{KOH}$ to $0.5 \%$. This decolorization may require $1-2$ weeks before ossified skeleton remains deep red in transparent background. Dehydrate by passing slowly through $95 \%$ alc., glycerin and aq. dest. in following proportions $10: 20: 70-20: 20: 60-30: 30: 40-$ $40: 40: 20-50: 50: 0$. Seal in specimen jar in the final mixture of alcohol and glycerin.

A rather similar technique leading up to dehydration in absolute alcohol, clearing in toluol and final storage in anise oil saturated with naphthalene is presented by Cumley, R. W., Crow, J. F. and Griffen, A. B., Stain Techn., 14, 7-11. This staining of ossification centers with alizarin red can be combined with the coloration of the cartilaginous skeleton with toluidin blue to make quite brilliant specimens (Williams, T. W., Stain Techn., 1941, 16, 2325).

Ossification, intense glycogenesis during (Gendre, H., Bull. d'Hist. Appl., 1938, $15,165-178)$.

Otoliths, technique for (Johnston, M., J. Roy. Micr. Soc., 1938, 58, 112-119).

Ova, concentration of parasitic ova in Feces.

Ovalocytosis, see Pencil Red Cells.

Ovary. For routine purposes fixation in Zenker's Fluid and coloration by Mallory's Connective Tissue stain or by Masson's Trichrome technique is indicated. Follicular atresia can be beautifully demonstrated by Vital Staining with trypan blue or by other similar dyes, see Evans, H. M. and Swezy, D. R., Memoirs Univ. California, 1931, 9, 119-224. For the utilization of Microdissection in determination of the physical properties of the follicular wall see Thanhoffer, L., Zeit. f. Anat. u. Entw., 1933, 100, 559-562. The interesting fluorescence studies on the ovary by Policard, A., C. rend. Acad. d. Sci., 1924, 179, 1287 are likely to be extended now that the possibilities of Fluorescence Microscopy are better appreciated. Ragins, A. R. and Pop- 
per, II., Areh. Path., 1942, 36, 647-662 have indeed investigated variations in ovarian fluorescence during cyclical changes.

Owen's Blue (British Drug Houses Ltd.), a dis-azo dye similar in composition to Manchester blue. Used best in alcoholie solution (H. G. Cannan, J. Roy. Mier. Soc., 1941, 61, 88-94).

Oxalate Solutions, see Anticoagulant Solutions.

Oxazins. Dyes resembling the thiazins but in which sulphur atom is replaced by oxygen. Examples: brilliant cresyl blue, celestin blue $\mathrm{B}$, cresyl violet, gallamin blue, gallocyanin, Nile blue sulphate, resorcin blue.

Oxidase. Unfortunately, as Lison (p. 263) points out, histologists and biochemists are not always agreed as to terms. The latter include under the designation "oxidases" all enzymes capable of catalysing a reaction of oxidation, for instance the phenolases, purinoxidases, succinoxidase, tyrosinase, etc.; whereas what the former deseribe as "oxidases" are in reality phenolases and thus only a part of the whole group of oxidases. The action of oxidase (or phenolase) in the presence of $\mathrm{O}_{2}$ is the same as a peroxidase in the presence of $\mathrm{H}_{2} \mathrm{O}_{2}$. But the particular oxidases are more delicate and easily modified in their action by variations in temperature, $\mathrm{pH}$ and other factors. The following methods are from Lison, much abbreviated.

1. M. nadi oxidase reaction (Gräff) = oxidase reaction, modification A (W. H. Schultze) and stabile oxidase reaction (V. Gierke). Make 2 solutions: A. Boil $1 \mathrm{gm} . \alpha$ naph thol in $100 \mathrm{cc}$. aq. dest. Add drop by drop $25 \%$ aq. potassium hydroxide until melted $\alpha$ naphthol is dissolved. Cool. Can be kept in dark at least 1 month. B. Obtain good sample dimethyl - $p$ - phenylenediamine furnished in sealed tubes. It blackens quickly when secured in bulk. Gräff advised, as more stable, dimethyl-pphenylenediamine hydrochloride. Make $1 \%$ solution of either in aq. dest. Boil and cool. Keeps 2-3 weeks in dark. Immediately before using take equal parts $A$ and $B$, filter and employ filtrate. Place frozen sections of formalin fixed tissues or smears (after fixing for $2 \mathrm{hrs}$. in formalin vapor or in formol, 10 ee. $+96 \%$ alcohol, 40 cc.) in above mixture of $\mathrm{A}$ and $\mathrm{B}$ in a thin layer at the bottom of a Petri dish. Agitate a little to permit oxygenation of the fluid. Blue granules quickly appear (1-5 min.). Rinse in water and examine. To make more permanent treat with Lugol's iodine diluted one third, 2-3 min., which makes the blue granules brown.
Restore blue by washing in aq. dest. + few drops sat. aq. lithium earbonate. Counterstain with hemalum or safranin, mount in glycerin. Sehmorl advised instead of Lugol's a conc. aq. sol. ammonium molybdate.

2. G. nadi oxidase reaction (Gräff) $=$ labile oxidase reaction (V. Gierke). This more difficult method is for fresh tissues. The nadi reagent is prepared without addition of alkali. The required $\mathrm{pH}$ depends on the cells investi. gated. For animal tissues Lison recommends about $8.2,8.1$ and 7.8 and for plants 3.4-5.9. Directions are given by Gräff (S., Die Mikromorphologisehen Methoden der Fermentforschung, Abderhalden's Iandb., 1936, 4 (1), 93-142).

3. Naphthol reaction of Loele. This is not, in the opinion of Lison, strictly speaking a microchemical reaction, but it is as simple. Place small amount $\alpha$ naphthol in a test tube. Add drop by drop $10 \%$ aq. potassium hydroxide until naphthol is completely dissolved. Add 200 cc. aq. dest. Solution may be used after 24 hrs. It will last about 3 weeks. Frozen sections of formalin fixed tissues treated with this reagent show violet or black granules, which quickly disappear.

Oxidation-Reduction Potential. Written by Christopher Carruthers, Division of Cancer Research, Washington University, St. Louis 10. May 12, 1950.

This very important measurement is particularly well explained by Seifriz, W., Protoplasm, New York: MeGrawHill Book Co., 1936, 584 pp. For a comprehensive developmental treatment of the subject see Clark, W. MI. and coworkers, Hygienic Laboratory Bull., 1928, 151, 1-352.

Oxidation is the process in which a substance loses electrons, and reduction is the process in which a substance takes on electrons. For example when ferric chloride $\mathrm{FeCl}_{3}$ gains an electron it is reduced to $\mathrm{FeCl}_{2}$, or

$$
\mathrm{Fe}^{+++}+\text {electron } \rightarrow \mathrm{Fe}^{++}
$$

Because the ion, $\mathrm{Fe}^{++}$, can lose an electron it is a reducing agent or reductant, and since $\mathrm{Fe}^{+++}$ean gain an electron it is an oxidizing agent or oxidant. The change is reversible

$$
\mathrm{Fe}^{+++}+\text {electron } \rightleftarrows \mathrm{Fe}^{++} \text {. }
$$

When an acid mixture of ferrous and ferric chloride is placed in an electrode vessel it will yield a potential - the oxidation potential. This potential can be measured by placing a noble metal, such as a bright platinum wire in the solution, and measuring the potential against the normal calomel electrode with a potentiometer. The intensity of the oxidizing or reducing action of a 
system is determined by its oxidation potential. The potential produced is determined by the ratio of ferrous to ferric ions, and is given by the relation:

$$
\begin{aligned}
E_{h}=E_{0}-\frac{R T}{F} \ln \frac{\left(\mathrm{Fe}^{++}\right)}{\left(\mathrm{Fe}^{+++}\right)} ; \\
\frac{\mathrm{Fe}^{++}}{\mathrm{Fe}^{+++}}=\frac{\text { (Reductant) }}{\text { (Oxidant) }}
\end{aligned}
$$

$E_{h}$ is the observed difference in electromotive force between the electrode and the normal hydrogen electrode; $E_{0}$ is a constant characteristic for the ferrousferric system (the so-called normal potential); $R, T$, and $F$ have their customary significances. The parentheses represent concentrations of the two components.

Certain groups of organic dyes are likewise able to induce upon electrodes reversible potentials. These organic dyes can be used as indicators of oxidation-reduction, and the following relation holds :

$$
E_{h}=E_{o}-\frac{R T}{n F} \ln \frac{(\mathrm{Red})}{(\mathrm{Ox})}
$$

If the reductant is identified as an ion, or the oxidant as a cation, for two simple cases there would be

$$
\begin{aligned}
& \mathrm{Ox}+\text { electron } \rightleftharpoons \text { Red }^{-} \\
& \mathrm{Ox}^{+}+\text {electron } \rightleftharpoons \text { Red }
\end{aligned}
$$

For equation (1), the relation would be

$$
E_{h}=E_{o}-\frac{R T}{n F} \ln \frac{\left(\mathrm{Red}^{-}\right)}{(\mathrm{Ox})}
$$

The active reductant of equation (1) is the anion of an acid, and its concentration depends not only upon the amount of reductant, but also upon the hydrogen ion concentration. The relation then becomes

$$
E_{0}^{\prime}=E_{0}-\frac{R T}{n F} \ln \frac{\left(\operatorname{Red}^{-}\right)}{(\mathrm{Ox})}
$$

at any constant $\mathrm{pH}$ (For development see Cohen, B., Symposia Quant. Biol., $1933,1,195-204$ ).

The use and interpretation of indicator dyes in biological systems is given by Cohen, B., ibid, 214-223, and Chambers, R., ibid, 205-213. Sources of error are also indicated by Cohen, B., Chambers, R. and Reznikoff, P., J. Gen. Physiol., 1928, 11, 585-612. Most of the following material is taken from the above papers.

On a microscopic basis, the measurements, like those of $\mathrm{pH}$, are made with indicators in which the cells are bathed or which are injected with them. They are applied in sequence and their reactions observed. Methylene blue, for instance, will be oxidized (retain color) or be reduced (lose color) depending upon the relative activity of the processes of oxidation and reduction.

Although it is difficult to accurately measure the amount of indicator injected into cells, it is imperative that the quantity be small. Otherwise too much indicator may be more than the cell can reduce, or be greater than the reducing intensity which the cell can generate. The following indicators from Cohen provide a useful range in potential values:

\section{Name of Oxidant}

$E$ at $\mathrm{pH} 7.0$

Phenol $\mathrm{m}$-sulfonate indo-2,6 dibromo-

phenol........................ 0.273

m-Bromophenol indophenol........... 0.248

o-Chlorophenol indophenol ............. 0.233

Phenol blue chloride............... 0.227

Phenol indo-2, 6 dichlorophenol . . ....... 0.217

o Cresol indophenol.................. 0.195

- Cresol indo-2, 6 dichlorophenol. ........ 0.181

1-Naphthol-2-sulfonate indophenol o-sul-

fonate ........................... 0.135

1-Naphthol-2-8ulfonate indophenol ...... 0.123

Toluylene blue chloride .............. 0.115

Brilliant cresyl blue chloride.......... 0.047

Methylene blue chloride............... 0.011

$K_{4}$ indigo tetrasulfonate............. -0.046

Ethyl capri blue nitrate............. 0.072

$\mathrm{K}_{\mathbf{3}}$ indigo trisulphonate............. 0.081

$\mathrm{K}_{\mathrm{g}}$ indigo disulphonate............... -0.125

Cresyl violet...................., -0.167

$E^{\prime}{ }_{0}$ represents the potential at any given $\mathrm{pH}$ of a system in which the ratio of oxidant to reductant is unity.

In order to get the indicator dyes into single cells the microinjection technique of Chambers is used. Chambers recommends dilute aqueous solutions of the basic dyes, i.e., $0.05 \%$ to $0.1 \%$, and injects successive small doses. Needham, J. and D. M., Proc. Roy. Soc. B, 1926, $99,173-199 ; 383-397$ used $1 \%$ solution since weaker solutions of particular dyes could not be seen under the microscope when injected into cells.

The determinations are carried out aerobically (cells maintained in a micro drop in water-saturated air at atmospheric pressure) and anaerobically (cells held in an atmosphere of purified process nitrogen saturated with water).

For example, under aerobiosis, if all the indicators down to and including methylene blue are reduced at $\mathrm{pH} \mathbf{7 . 0}$ by cells of a particular type; and if ethyl capri blue is only partially reduced (and the rest of the indicators not reduced), the reducing intensity of the aerobic cell is approximately -0.072 volts at $\mathrm{pH} 7.0$. 'The same procedure is followed for cells anaerobically.

To detect the presence of the indicator after decolorization by the cell proto- 
plasm, reoxidation of the reductant can be accomplished by injecting dilute potassium ferricyanide or of potassium dichromate in the anaerobic state, or by exposure to air in the anaerobic state. The recovery of color on oxidation is a necessary control demonstrating that the indicator has been reversibly reduced and not reversibly destroyed.

It is also essential to bring the cell interior into contact both with oxidant and reductant of the indicator. This is necessary to determine whether the indicator, which would shift to the potential of the electromotive system present, is behaving in a truly reversible manner.

The aqueous solutions of the acid dyes, e.g. the various indophenols give the most clear cut results. Upon injection they rapidly diffuse throughout the cell before being reduced. The experimental evidence indicates that the speed of reduction of the indicator dyes decreases as the potential of the in. dicator approaches that of the cell.

In the immersion method slices of tissue are bathed in solutions of the indicator dyes. Here it is not only necessary to distinguish between penetrating and nonpenetrating indicators but also to watch for differences in the rapidity with which cells and certain cell inclusions are stained by the various indicators. For example, indicators containing the sulfonated radicals do not readily penetrate cells, while the nonsulfonated more or less rapidly penetrate.

Fildes, P., Brit. J. Exp. Path., 1929, 10, 151-175 measured the oxidationreduction potential of the subcutaneous tissue fluid of the guinea pig, and also its effect on infection. Guinea pigs were inoculated with indicator dyes $(0.01 \%)$ in both the reduced and oxidized states and he observed whether change had occurred. The injections were made superficially so that the immediate effect could be seen through the shaved skin. The oxidized form of methylene blue remained a strong blue, and the reduced dye assumed a distinct blue color. This indicated that the subcutaneous tissue maintained an oxidation-reduction potential on the positive side of reduced methylene blue.

Then "indophenol 1" (naphthol-2 sodium sulphonate indo 2,6 dibromophenol) in both states was injected and the animals examined. After $40 \mathrm{~min}$ utes the oxidized and reduced forms of the dye were at about the same intensity of blue. Therefore it was concluded that the $E_{h}{ }^{+}$of the subcutaneous tissue was positive to that of reduced indo- phenol 1. The rate of oxidation was slower here than in the case of methylene blue, because the difference in $E_{\mathrm{A}}$ of the tissues and the reduction point of the dye was less.

$$
E_{h}=E_{0}^{\prime}-0.062 \log \frac{a}{100-a} \text { (at } 37^{\circ} \mathrm{C} \text { ) }
$$

where $E_{0}^{\prime}$ is a constant characteristic of the particular system and $a=\%$ reduction.

Finally the dye indicator, "indophenol 2" (phenolindophenol 2, 6 dibromophenol) was injected. The reduced form of the dye remained colorless while the oxidized form faded from 20 to 80 minutes. Addition of ferricyanide failed to restore all the reduced dye, so the results were complicated by decomposition of the dye in the tissues. It was concluded that the $E_{h}$ of the tissue fluids is positive to the zone of complete reduction of indophenol 1 .

The oxidation-reduction potential of the ciliary body was determined (Friedenwald, J. S. and Stieher, R. D., Arch. Ophth., 1938, 20, 761-786) by introducing indicator dyes into the stroma or epithelium of ciliary body under aerobic and anaerobic conditions. After equilibrium had been reached, the degree of bleaching was observed microscopically. Then an oxidizing agent was added, such as ferricyanide, and recovery of the color was noted. The ratio of intensity of color before and after oxidation with ferricyanide gave the potential in the system since it afforded a measure of the ratio of oxidant to reductant of the indicator in equilibrium in the tissue. Aerobically the epithelium had an estimated potential of +0.100 volts, and the stroma -0.130 volts. Anaerobically both had estimated potentials of -0.290 volts.

Lewis, M. R., Barron, E. S. G. and Gardner, R. E., Proc. Soc. Exp. Biol. \& Med., 1930-31, 28, 684-685 compared the power of cancer tumors, tumors produced by viruses and normal tissue to reduce methylene blue. The tissues were cut in a manner similar for tissue respiration, and the pieces were placed in M/15 Sorensen's phosphate buffers at $\mathrm{pH}$ 7.38. Anaerobiosis was maintained by a stream of nitrogen. The time of reduction of the dye by tumors was the same as that of normal tissues.

Voegtlin, C., Johnson, J. M. and Dyer, H. A., J. Pharm. \& Exp. Therap., 1925, $24,305-337$ have quantitatively estimated the reducing power of normal and cancerous tissue. For the anaerobic experiments tissues were sliced about 2 $\mathrm{mm}$. thick and weighed about $0.5 \mathrm{gm}$. Samples of tissue were placed in sterile 
vacuum tubes, and $5 \mathrm{cc}$. of a sterile solution of the indicator in a phosphate buffer solution (M/15 $\mathrm{Na}_{2} \mathrm{HPO}_{4}, \mathrm{KH}_{2} \mathrm{PO}$, Sorensen) of pH 7.6 were added to each tube by means of a sterile pipette. After evacuation of the tubes by a vacuum pump, they were rapidly fixed in a constant temperature bath at $38^{\circ} \mathrm{C}$. on a revolving rack.

The indicator solutions were prepared by adding phosphate buffer to an accurately weighed amount of the dye in a mortar and grinding. The solutions were made up to volume and boiled to sterilize.

The reducing power of tissues was based upon the time needed to reduce anaerobically equimolar amounts of the indicators used (the dye content of each indicator was determined on a moisture free basis). For the indicators used it was found that the optimum concentration for comparative purposes was approximately M/42,533. A more useful concentration of $\mathrm{MI} / 40,000$ was suggested for future work.

All the tissues (brain, carcinomaperipheral portion, heart muscle, spleen, kidney, liver, lung, skeletal muscle and testis) had a reducing power which varied according to the type of tissue having the highest reducing power (with the exception of the necrotic portion of carcinoma). The latter was devoid of reducing power while the viable portion reduced the indicators as rapidly as did some of the normal tissues.

See Recording Autotitrator as described by Jacobsen, C. F. and Léonis, J. C., C. rend. trav. Lab., Carlsberg, Ser. Chim., 1951, 27, 333-339.

Oxyme. Estimation in plant and animal tissues (Yamafuji, K., Kondo, H. and Omura, H., Enzymologia, 1950, 14, 153-156.

Oxychromatic Degeneration. A kind of degeneration in which oxychromatic (acidophilic) material appears in the nuclei. See Luger, A. and Lauda, E., Med. Klin., Berlin, 1926, 22, 415, 456, 493.

Oxydase, see Oxidase.

Oxygen Consumption. A method is described for epidermis separated from dermis by heat (Baumberger, J. P., Suntzeff, V. and Cowdry, E. V., J. Nat. Cancer Inst., 1942, 2, 413-423.

Oxyntic Cells (G. Oxyntos, making acid), an unsatisfactory term for the parietal cells of the stomach because it implies actual manufacture of acid.

Oxyphil (G.oxys., acid + philos, fond) same as acidophilic. The term is commonly applied to the colloid cells of the parathyroid and thyroid which are colored with "acid" dyes such as eosin.
Ozokerite, see Ceresin Imbedding.

Pacinian Corpuscles can best be located by naked eye inspection of the abdominal viscera of a freshly killed cat as small elongated, cigar shaped bodies situated just within the tunica serosa which appear china white because they have a very poor blood supply. Fix in Zenker's Fluid and color with Mallory's Connective Tissue stain for general purposes or employ Bodian's method for nerve fibers.

Pal-Weigert Stain for myelin sheaths as modified by Erhart, E. A., Zeit. f. wis. Mikr., 1951, 60, 155-156 recommended as fast, simple and giving a blue black stain of myelin sheaths in an almost uncolored background. For paraffin, celloidin or frozen sections of material fixed in formalin. Wash sections in water in $4 \%$ aq. iron alum, 2-3 min. Rinse rapidly in water. Stain in aq. dest 80 cc., lithium carbonate $1 \mathrm{gm}$., $10 \%$ alc. hematoxylin for $5-20$ min. at room temperature. Wash in water, counterstain if desired in $1 \%$ aq. neutral red, carmin or cresyl violet. Dehydrate, clear and mount in balsam. See Weigert-Pal.

Palitzsch's Borax-Boric Acid Buffers (Clark, W. M. The Determination of Hydrogen Ions. Baltimore: Williams and Wilkins, 1928, 717 pp.) Prepare: (1) $\mathrm{M} / 20$ borax solution by dissolving 19.0715 gms. $\mathrm{Na}_{2} \mathrm{~B}_{4} \mathrm{O}_{7} 10 \mathrm{H}_{2} \mathrm{O}$ in 1 liter aq. dest. (2) A solution containing $\mathrm{M} / 5$ boric acid and $\mathrm{M} / 20 \mathrm{NaCl}$ by dissolving 12.368 gms. $\mathrm{H}_{3} \mathrm{BO}_{3}$ and 2.925 gms. $\mathrm{NaCl}$ in 1 liter aq. dest. To make buffer of the desired $\mathrm{pH} \operatorname{mix} 1$ and 2 in the proportions indicated.

(2) $M / 5$ Boric

$\begin{array}{ccc}\mathrm{pH} & \begin{array}{c}\text { (1) } \mathrm{M} / 20 \\ \text { Boraz }\end{array} & \begin{array}{c}\text { Acid, } \mathrm{M} / 20 \\ \mathrm{NaCl}\end{array} \\ 9.24 & 10.0 & 0.0 \\ 9.11 & 9.0 & 1.0 \\ 8.98 & 8.0 & 2.0 \\ 8.84 & 7.0 & 3.0 \\ 8.69 & 6.0 & 4.0 \\ 8.60 & 5.5 & 4.5 \\ 8.51 & 5.0 & 5.0 \\ 8.41 & 4.5 & 5.5 \\ 8.31 & 4.0 & 6.0 \\ 8.20 & 3.5 & 6.5 \\ 8.08 & 3.0 & 7.0 \\ 7.94 & 2.5 & 7.5 \\ 7.88 & 2.3 & 7.7 \\ 7.78 & 2.0 & 8.0 \\ 7.60 & 1.5 & 8.5 \\ 7.36 & 1.0 & 9.0 \\ 7.09 & 0.6 & 9.4 \\ 6.77 & 0.3 & 9.7\end{array}$

French, R. W. Stain Techn., 1930, 5 $87-90 ; 1932,7,107-108$ recommended the use of these buffers for the range $\mathrm{pH}$ 
9.2-8.2 but he made them up in a different way.

Palladium. Histochemical detection based on reaction between palladium and $p$ Dimethylaminobenzyl-idenrhodanin in neutral formalin or alcohol fixed tissues (Okamoto, K., Mikami, G. and Nishida, M., Acta Scholae Med. Univ. Imp. in Kioto, 1939, 22, 382-387).

Panchrome is a modification by Pappenheim (Folia haematol., Arch., 1911, 11, 194) of the Giemsa stain. Add $0.75 \mathrm{gm}$. of the panchrome powder (Grübler) to 75 cc. pure methyl alcohol and $25 \mathrm{cc}$. acid free glycerin at $60^{\circ} \mathrm{C}$. After filtering keep in glass stoppered bottle. Use after May-Grünwald fixation as described for Giemsa after methyl alcohol fixation. According to Slider and Downey (McClung's Microscopical Technique, p. 329) it gives better coloration of neutrophilic granules and metachromasia of mast granules than the plain Giemsa's stain but "some delicacy is lost, and the cells are more likely to be muddy."

Pancreas. This organ lends itself very well to microscopic examination in the fresh state. The classic which everyone seeking technical details should consult is Bensley, R. R., Am. J. Anat., 1911, 12, 297-388. The techniques for Blood Vessels and Nerve Endings are those employed generally and are described under these headings. No particular difficulties will be encountered in their adaptation to the pancreas. It may be helpful however to consult Beck, J. S. P. and Berg, B. N., Am. J. Path., 1931, 7, 31-35 on the blood vessels. The same holds for the Connective Tissue components. Epithelial parts of the pancreas can routinely be examined in a preliminary way with the other parts in tissues fixed in Formalin-Zenker and stained with Hematoxylin and Eosin. For details see Zymogen, Ducts and Islets of Langerhans.

Pancreatin digestion method for spleen (Kyes, P., Am. J. Anat., 1901, 1, 3743).

Paneth Cells. Influence of fasting on (Klein, S., Am. J. Anat., 1905-1906, 5, $315-330$ ). To observe storage and discharge phases examine in guinea pigs 24 and $6 \mathrm{hrs}$. after feeding (Klein, S., Am. J. Anat., 1905-06, 5, 315-330). By combining DeGalantha's amyloid stain with mucicarmine, Paneth granules are colored green and mucous granules red (Hertzog, A. J., Am. J. Path., 1937, $13,351-360$ ).

Pantothenic Acid. Detection by fluorescence microscopy in tomato plants (Bonner, J. and Dorland, R., Am. J. Bot., 1943, 30, 414-418).
Papanicolaou Techniques in Exfoliative Cytology-written by Charlotte M. Street, Department of Anatomy, Cornell University Medical College, New York. May 21, 1951-Exfoliative cytology, i.e. the study of cells which have been exfoliated from the epithelial lining of an organ or cavity of the body, is gradually becoming an independent branch of the morphological sciences. Its usefulness in hormonal studies and in cancer diagnosis has been largely responsible for its great expansion in the last two decades. The progress achieved in this field has been primarily due to the introduction of more adequate technical methods for the preservation and staining of cells in smears prepared from various physiological secretions. The techniques described herein will be limited chiefly to those which have been developed by Dr. George N. Papanicolaou in the course of his cytologic studies, and which are now in use in his laboratory in the Department of Anatomy of Cornell University Medical College.

These technical procedures are based on the use of smears which are fixed without drying, in a solution of equal parts of $95 \%$ ethyl alcohol and ether, by which good preservation of exfoliated cells is attained. In such fixed smears the cells are well preserved and when stained, their cytoplasmic and nuclear details are clearly defined. On the contrary, in dried, unfixed smears many of the structural details are lost, there is an apparent increase in the size of the cells and their nuclei, and their affinity for stains is much impaired. Since cells subjected to drying prior to fixation show a preference for acidophilic stains, it is impossible in dried smears to make the proper evaluation of basophilic and acidophilic cells which is so necessary in endocrine studies. Furthermore, in the examination of smears for malignant cells, good nuclear preservation and staining are of utmost importance. For this reason thorough fixation of wet smears is essential. The relative size of cells and the nuclear-cytoplasmic ratio also play an important role in the diagnosis of malignancy. Therefore, drying of smears which results in apparent enlargement of cells and nuclei may give a false impression of malignancy.

The development of Papanicolaou's staining procedures may be divided into two periods. The first includes his animal experimentation and his early work in the human, during which vaginal smears were used as an index for evaluating cytologic changes occurring 
during the normal estrous or menstrual cycle, as well as changes due to various hormonal factors. For his studies in both guinea pigs and the human, he used alcohol-ether fixed smears which were stained first with hematoxylin and then counterstained with aqueous solutions of eosin and water blue (see staining technique I). This method was a simple one technically and resulted in good differentiation between basophilia and acidophilia. It is still considered useful in hormonal studies.

During the second period, modifications in technique were investigated which would be better adapted to the diagnosis of malignant neoplasms. In thick smears where cells are found in compact clusters, overlying one another, or mixed with blood or mucus, a light, transparent, cytoplasmic stain is necessary to give a clearer view of individual cells and their nuclei. Papanicolaou found that this could be achieved by using $95 \%$ alcohol as the solvent for the counterstains. Stains OG6 and EA36 (formulae 2 \& 3 following the staining procedures) were introduced in 1942; EA 65 (formula 4) is a later modification of EA36. When used in combination with a good nuclear stain, these counterstains are particularly well adapted to cancer diagnosis but are also excellent for endocrine studies because of their delicate differential staining qualities. They can also be used in staining tissue which is advantageous in the correlation of smears and sections. (For use of alcoholic counterstains, see staining techniques II, III, IV, V.)

Female Genital Tract. Vaginal, endocervical and endometrial aspiration smears; cervical swab and spatula smears.

Equipment: For vaginal smears: a glass pipette, five inches in length and one quarter inch in diameter, slightly curved about two inches from its blunt tip, and equipped with a tightly fitting strong rubber bulb. The pipette should be made of hard pyrex glass to withstand sterilization. For endocervical and endometrial aspiration smears: a Becton-Dickenson laryngeal cannula equipped with a strong rubber bulb. For cervical smears: a non-absorbent cotton swab or the wooden spatula designed by Ayre (Ayre, J. E., Am. J. Obst. \& Gynec., 1947, 53, 609). For all types of smears: a jar of fixing solution, i.e. equal parts of $95 \%$ ethyl alcohol and ether, and clean slides. When more than one slide is placed in a bottle of fixative, a paper clip is attached to one end of each slide in order to prevent contact between the smeared surfaces. The slides are marked in advanced with the patient's name or case number, type of smear and date. This can be done either with a diamond pencil, or by clipping a small piece of paper or card to the slide. In the latter case, the label should be written with lead pencil, not ink.

Preparation of smears: Smears should not be prepared after a douche, bath, or use of a lubricant. They should be prepared prior to the pelvic examination, if possible.

Vaginal smears: The dry pipette, with bulb compressed, is introduced into the posterior fornix of the vagina and the bulb released. The pipette is rotated carefully during aspiration to obtain samples from different parts of the vagina. The fluid thus obtained in the pipette is expelled on to a slide, and spread in a uniform smear with the side of the pipette. The slide is immersed immediately, before the smear dries, in the alcohol-ether fixative and allowed to remain for at least 15 minutes for thorough fixation of cells before staining. Smears may be left in alcohol-ether for a week or even longer without harmful effect.

Endocervical or endometrial smears: After a speculum is in place, endocervical or endometrial secretion is aspirated by means of the laryngeal cannula, expelled on to a slide, spread uniformly with the cannula, and the slide immersed immediately in alcohol-ether.

Cervical swab smears: Secretion obtained by means of a swab or spatula from the external os, or from any suspicious appearing lesion of the cervix is quickly spread on a slide and fixed in alcohol-ether.

The vaginal smear, because it is composed chiefly of squamous cells which reflect hormonal activity is the best type for hormonal evaluation, and for a study of the menstrual cycle. For the latter, smears may be prepared daily throughout the complete cycle by the patient. Slides, either dated or numbered serially in advance, with clips attached, a pipette and jar of fixative are given to the patient and she is instructed how to prepare them.

For diagnosis of malignancy, a vaginal smear gives a more complete overall picture of the cytology of the genital tract since cells from all parts accumulate in the vaginal pool. Endocervical smears reveal the presence of a cervical carcinoma, endometrial smears are better for diagnosis of fundal, and occasionally, tubal carcinomas. Cervical swab or spatula smears give informa- 
tion of more specific nature but, unless prepared and fixed very quickly, swab smears are apt to be drier than aspiration smears and therefore difficult to interpret.

Smears of the female genital tract are stained by either method I or II.

Urinary Tract.

Collection of specimens: Urine from females must be catheterized to avoid contamination from vaginal secretion. Ureteral specimens are advisable if carcinoma of the kidney pelvis is suspected. In cases of suspected carcinoma of the prostate, separate voided urine specimens are obtained before and after massage. In such cases, prostatic smears can also be prepared by spreading prostatic secretion directly on slides and fixing them in alcohol-ether.

Preparation of smears: Upon collection, the urine specimen (approximately 50 cc. of a bladder specimen; as much of a ureteral specimen as can be obtained) must be mixed immediately with an equal volume of $95 \%$ alcohol for fixation and preservation of the cells. The mixture of urine and alcohol is centrifuged in 50 cc. conical tubes at medium speed for 15-30 minutes (until sediment is well packed), after which the supernatant liquid is decanted. Clean slides, marked in advance with a diamond pencil or water-proof laboratory ink, are coated with a thin film of Mayer's albumin fixative in order to make the cells adhere to the slide. Sediment can be removed from the tube by means of a small spoon-shaped instrument such as a nasal or small bone curette or, if very scanty, by pipetting. It is spread on the slide, using a second clean slide to distribute it in a uniform smear. When the smear starts to dry at its periphery, it is immersed in $95 \%$ alcohol and ether, equal parts. Care must be taken, however, not to allow the smear to become too dry before fixation for the reasons discussed previously. The smears should be left in the alcoholether fixative for at least one hour before staining or they tend to wash off during the staining process. All instruments used in transferring sediment from tube to slide must be carefully cleaned to avoid contamination of the next specimen. If there is sufficient sediment, three or four smears are prepared from each specimen. If there is an abundance of sediment, it should be stirred up in the tube in order to insure representative sampling. If smears cannot be prepared immediately after centrifugation, the super- natant liquid is decanted, the sediment covered with a few cubic centimeters of absolute alcohol and placed in the refrigerator.

Graham et al. (Vincent Memorial Hospital, The Cytologic Diagnosis of Cancer. W. B. Saunders Co., 1950) suggest mixing two or three drops of albumin fixative with the sediment in the centrifuge tube before making smears so that the cells will stick better to the slides. This has been found to be very helpful in urine as well as other sediments. IV.

Urine smears are stained by method

\section{Respiratory Tract.}

Sputum: This must be a deep cough specimen as a salivary specimen is obviously of no value in the diagnosis of carcinoma of the lung. It is ordinarily collected directly in $70 \%$ alcohol to retard the growth of bacteria and fungi as well as to prevent cellular degeneration. However, if smears can be made within four hours after the specimen is obtained, it need not be fixed in alcohol. Since alcohol hardens sputum, smears are more easily prepared from a fresh, unfixed specimen. When alcohol is used for fixation, $70 \%$ is preferable to $95 \%$ because it causes less hardening of the sputum. Before preparing the smears, the specimen is examined for blood-tinged portions. If any are present, they are transferred to slides which have been marked previously and coated with a thin film of Mayer's albumin. If no blood tinged particles are found, samples from different parts of the specimen are to be transferred to the slides. A fairly thin uniform smear is prepared by spreading the material with another elean slide, gently rubbing it between the two slides in order to crush any hard rubbery masses and distribute them evenly. The smear is fixed, without drying, in alcohol-ether fixative for a minimum of one hour. Three or four smears should be prepared. They are stained by method III.

Bronchial aspirates or saline washings of the bronchus are mixed immediately with an equal volume of $95 \%$ alcohol. Because of the scantiness of material obtained by brouchial aspiration, the collection tube should always be rinsed well with alcohol and the rinse solution added to the specimen. Specimens are centrifuged and smears prepared from the sediment by the method given for smears of urine sediment. They are stained by method III. 
Gastro-Intestinal Tract.

Esophageal aspirates are obtained by means of a rubber suction tube passed through the esophagoscope. Due to the scantiness of material usually obtained, the tube should be rinsed with saline and this rinse added to the aspirate. The entire specimen is then mixed immediately with $95 \%$ alcohol.

Gastric specimens should be obtained after at least eight hours of fasting. Because of the action of free acid and digestive juices in the stomach, best results are obtained if the residual gastric contents are first aspirated and discarded. Freshly exfoliated material can then be obtained by either of the following methods. (1) Using a Rehfuss tube with a bucket tip, the stomach is washed repeatedly with Ringer's solution by aspirating and re-injecting the same solution. (2) A gastric balloon can be used which has been specially designed to cause gentle friction of the gastric mucosa, thereby increasing exfoliation. The balloon and the technique for its use in obtaining gastric specimens have been described by Panico, Papanicolaou and Cooper (Abrasive balloon for exfoliation of gastric cancer cells, J. A. M. A., 1950, 143, 1305-11). Gastric specimens must be fixed immediately in an equal volume of $95 \%$ alcohol to prevent further degeneration and digestion of cells.

Duodenal drainage specimens are obtained through a Rehfuss tube, allowing the tip of it to pass from three to six inches beyond the pylorus. The specimen is immediately mixed with an equal volume of $95 \%$ alcohol.

Rectal or colonic washings: In order to obtain specimens which are as free as possible of fecal material, the patient is given a laxative at night, and a hot soapsuds enema the next morning. Saline washings of the rectum or colon are collected two to five hours later and mixed immediately with $95 \%$ alcohol. A special suction apparatus for rectal washings is described by Loeb and Scapier (Rectal washings, technic for cytologic study of rectosigmoid, Am. J. Surg., 1951, 81, 295-302). Colonic washings can be obrained by high colonic irrigation, using normal saline.

Preparation of smears: All specimens from the gastro-intestinal tract must be processed with the least possible delay because of deterioration of the cells. This is particularly important in gastric and duodenal specimens due to the action of the digestive juices. The specimens are centrifuged as soon as received and the supernatant fluid promptly decanted. The addition of ten drops of Mayer's albumin per 50 cc. of specimen before centrifugation will facilitate sedimentation. If smears cannot be prepared immediately, the sediment can be covered with absolute alcohol and kept temporarily in the refrigerator. Smears are prepared in the same manner as urine smears but stained by method III.

Other Sources.

Exudates, pleural, peritoneal, and pericardial. Fifty ce. of $50 \%$ alcohol is added immediately to $50 \mathrm{cc}$. of the exudate and smears prepared from the sediment in the same way as urine sediment smears, but stained by method III. Fifty percent alcohol is used rather than $95 \%$ so that there will be less precipitation of protein material.

Cystic and other fluids or washings. An equal volume of $95 \%$ alcohol is added immediately to the specimen and smears prepared from sediment and stained by method III.

Nipple secretion smears. If secretion can be obtained easily from the nipple by very gentle pressure, a clean slide is brought in contact with the secretion expressed. Care should be taken not to bring the slide in contact with the nipple itself in order to prevent the collection of an excess of epidermal cells. The material is spread uniformly with another slide, the smear is fixed in alcohol-ether and stained by method III.

Method I. (Papanicolaou, G. N., Am. J. Anat., 1933, 52: No. 13, supplement). This is the original Papanicolaou technique for staining vaginal smears in rodents and the human; it can be used to advantage in endocrine work and in eytologic studies of lower mammals.

1. Transfer slicles from alcohol-ether directly to $80 \%$ alcohol and run down through $70 \%$ and $50 \%$ alcohols to aq. dest.

2. Stain in Ehrlich's hematoxylin 5 min.

3 . Rinse in aq. dest. and place in running tap water $15 \mathrm{~min}$.

4. Stain in $0.5 \%$ aq. eosin $3-4 \mathrm{~min}$.

5. Rinse well in tap water.

6. Stain in $0.5 \%$ aq. waterblue $1 \mathrm{~min}$.

7. Rinse in tap water and run up through $50 \%, 70 \%, 80 \%, 95 \%$ and absolute alcohols, absolute alcohol and xylol (equal parts), to xylol, leaving in each solution long enough to clear.

8. Mount, without drying, with a cover slip.

Nuclei-dark purple; cytoplasm of non-cornified cells-pale blue; cytoplasm of cornified cells-pink. 
Method II. This is the teehnique deseribed by Papanicolaou in Seienee, $1942,95,438-9$, with a few minor modifieations. It is recommended for staining smears of the female genital tract which are to be used for either eaneer diagnosis or endoerine studies.

1. Transfer slides, without drying, from alcohol-ether fixative to $80 \%$ alcohol and run down through $70 \%$ and $50 \%$ aleohols to aq. dest., leaving in each solution long enough to clear.

2. Stain in Harris Hematoxylin (1) $6 \mathrm{~min}$.

3. Rinse in aq. dest.

4. Dip in $0.25 \%$ aq. hydrochlorie acid 6 times.

5. Place in running tap water 6 min.

6. Rinse in aq. dest. and run up through $50 \%, 70 \%, 80 \%$, and $95 \%$ aleohols, leaving in each long enough to elear.

7. Stain in OG6 (2) for $1 \frac{1}{2}$ minutes.

8 . Rinse in $95 \%$ ale., two ehanges.

9. Stain in $\mathrm{EA} 36$ (3) for $1 \frac{1}{2} \mathrm{~min}$.

10. Rinse in $95 \%$ aleohol, 3 ehanges.

11. Dehydrate and elear by running through absolute ale., absolute aleohol and xylol (equal parts) to xylol, leaving in each solution long enough for the smears to be thoroughly penetrated and dehydrated.

12. Mount, without drying, with a eoverslip, using permount, gum damar, Canada balsam or another neutral mounting medium.

Nuclei-dark purple; non-cornified cells-pale greenish-blue; eornified eells -variations from pink to orange, depending on the degree of eornification; keratinized cells-intense orange.

Method III. A modifieation of method II. Reeommended for smears of sputum, prostatic secretion and all sediments except urine.

1-8. Same as steps 1-8 or method II. 9. Stain in EA65 (4) $1 \frac{1}{2} \mathrm{~min}$.

10-12. Same as steps 10-12 of method II.

Note: Reetal and colonic washings are washed in running tap water (step 5) $15 \mathrm{~min}$. instead of $6 \mathrm{~min}$.

Method IV. A modification of method III. In this procedure blueing of the nuelei is aceomplished by the use of ammonium hydroxide rather than running tap water, as the latter tends to loosen cells from the slide. It is therefore recommended for smears of urine sediment which is the least adhesive of the sediments. It is also shorter than method II and may be used for other types of smears for that reason. However, in thick smears, it has a disadvantage in that the cytoplasm retains some hematoxylin and therefore does not take a elear eytoplasmic stain.

1. Transfer slides from aleohol-ether to $80 \%$ aleohol and run down through $70 \%$ and $50 \%$ alcohols to aq. dest.

2. Stain in Harris hematoxylin (1) 2 min.

3. Rinse in distilled water, then $50 \%$ ale.

4. Place in a solution of $1.5 \%$ ammonium hydroxide made up in $70 \%$ aleohol for $1 \mathrm{~min}$.

5. Rinse in $70 \%$ aleohol, 2 ehanges.

6. Run up through $80 \%$ and $95 \%$ alc.

7. Stain in OG6 (2) for $1 \frac{1}{2} \mathrm{~min}$.

8. Rinse in $95 \%$ aleohol, 2 ehanges.

9. Stain in EA65 (4) for $1 \frac{1}{2} \mathrm{~min}$.

10-12. Same as steps 10 to 12 in method II.

Method $V$. For sections.

1. Remove paraffin in xylol and run down through descending alcohols to aq. dest. in the usual way.

2. Stain in Harris hematoxylin (1) $2 \frac{1}{2}$ min.

3. Rinse in aq. dest. (2 ehanges), and $50 \%$ and $70 \%$ aleohols.

4. Place in $3 \%$ ammonium hydroxide made up in $70 \%$ ale. for $1 \mathrm{~min}$. (or until sections turn blue).

5. Rinse in $70 \%$ aleohol (2 changes), $80 \%$ and $95 \%$ ale.

6. Stain in OG6 (2) for $1 \frac{3}{4} \mathrm{~min}$.

7. Rinse in $95 \%$ aleohol (2 ehanges).

8. Stain in EA65 (4) or EA36 (3) for $1 \frac{1}{2} \mathrm{~min}$.

9. Rinse in $95 \%$ alcohol (3 ehanges), dehydrate, elear and mount in the usual way.

Formulae for Stains.

(1) Harris hematoxylin is prepared from the standard formula, using ammonium alum. inum sulphate, but omitting the glacial acetic acid. It is diluted with an equal volume of distilled water before using, and filtered in to a dark bottle for storage when not in use. It should be reinforced by the addition of a s mall amount of fresh undiluted stock solution fairly often in order to maintain uniform staining results.

(2) OG6:

Orange $\mathrm{G}-0.5 \%$ in $95 \%$ alcohol . 100 cc. Acid phosphotungstic. .

$0.015 \mathrm{gm}$.

(3) EA36:

Light Green SF (yellowish) $0.1 \%$ in $95 \%$ alcoliol ....... $45 \mathrm{cc}$.

Bismarck Brown $\mathrm{Y}-0.5 \%$ in alcohol................... 10 cc.

Eosin yellowish $-0.5 \%$ in $95 \%$ alcohol ... ............. $45 \mathrm{cc}$

* Original formula for EA36 calls for $0.5 \%$ light green solution but $0.1 \%$ is now used ....... 
Acid phosphotungstic.........

Lithium carbonate, saturated aqueous solution ............

$0.200 \mathrm{gm}$

1 drop

(4) EA65:

Light Green SF (yellowish)$0.05 \%$ in $95 \%$ alcohol..

$45 \mathrm{cc}$.

Bismarck Brown $\mathrm{Y}-0.5 \%$ in 95\% alcohol.

Eosin yellowish (water and alcohol soluble) $-0.5 \%$ in $95 \%$ alcohol...

Acid phosphotungstic, C.P.

Lithium carbonate, saturated solution

$10 \mathrm{cc}$

$45 \mathrm{cc}$.

$0.2 \mathrm{gm}$.

1 drop

National Aniline and Chemical Company certified stains are used for preparing the above stains. If other dyes are used, the amounts may have to be adjusted.

For the preparation of stains OG6, EA36 and EA65, it is advisable, because of the low solubility in alcohol of orange $\mathrm{G}$, Bismarck brown and light green, to make the individual alcoholic stock solutions from aqueous solutions as follows:

Orange $\mathrm{G}, 0.5 \%$ in $95 \%$ alcohol-to 95 cc. $95 \%$ alcohol, add 5 ce. of $10 \%$ aqueous solution of orange $\mathrm{G}$.

Bismarck Brown, $0.5 \%$ in $95 \%$ alcoholto 95 cc. alcohol, add 5 ce. of $10 \%$ aqueous solution of Bismarck Brown.

Light Green, $0.1 \%$ in $95 \%$ alcohol-to 95 cc. $95 \%$ alcohol, add 5 ce. of $2 \%$ aqueous solution of light green.

Light Green, $0.05 \%$ in $95 \%$ alcohol-to 95 cc. $95 \%$ alcohol, add 5 cc. of $1 \%$ aqueous solution of light green or dilute the $0.1 \%$ alcohol solution with an equal volume of $95 \%$ alcohol. By this method, the alcoholic content of the stock stains is less than $95 \%$ but it makes no appreciable difference in the staining results. Some of the stain may precipitate when added to the alcohol, but the solubility may be increased by heating slightly (not over an open flame).

The strength of the light green solution is less in the EA65 than in EA36 because smears of sputum, gastric aspirates, exudates, etc. are apt to be thicker than those of the female genital tract and therefore require a paler basophilic stain. In smears stained with either EA36 or EA65, as soon as the green begins to predominate at the expense of the acidophilic stains, the counterstain should be discarded.

All stains should be stored in dark bottles when not in use. If used constantly, they should be filtered daily and frequently reinforced by the addition of fresh stain. Because of oxidation, hematoxylin should always be filtered daily regardless of the volume of staining.

Vaginal, cervical and endometrial smears may be stained after fixation of only 15 minutes, but other smears will adhere better if left in alcoholether for an hour or more. In the staining process, when slides are transferred from one solution to another, they should be drained as much as possible, particularly during alcohol rinses following the counterstains, and during dehydration. It is also important that slides be passed through solutions slowly and carefully, with minimal agitation of the solutions, so that cells will not be washed from the slides. Besides the loss of cells, there is also the danger of contamination of other smears with "floaters" (cells or clumps of cells which have fallen into one of the solutions). However, these floaters can usually be recognized microscopically since they are on a higher focusing level than the fixed smear. There is also danger of contamination while mounting the smears if the glass rod used for applying the mounting medium touches the smear and picks up loose cells.

Smears which have been fixed but not stained may be mailed to a laboratory for staining by the method suggested by Ayre and Dakin. (Ayre, J. E. and Dakin, E., Canad. M. A. J., 1946, 54, 489-91.) After the smear has been fixed in alcohol-ether for one hour, it is removed from the fixative and, without drying it, two or three drops of glycerine are placed on it. A clean coverslip or slide is placed on top of the smear thereby sealing it from the air. When the slides are received in the laboratory, they are placed in a jar of alcohol-ether until the covering slide or coverslip becomes loose and slips off without disturbing the smear.

It should be stressed that the Papanicolaou smear technique as applied to cancer diagnosis is not a substitute for biopsy or other established methods of diagnosis and that positive smears should be corroborated whenever possible by biopsy or x-ray before any major surgery is performed. However, it can be extremely useful in the discovery of early or hidden lesions. This is particularly true in cervical and bronchogenic carcinomas, where the degree of accuracy of the test is high. It has an additional advantage in that it is a short and relatively simple laboratory procedure and, in most instances, the specimens can be obtained with little discomfort to the patient. 
Too much emphasis cannot be placed on the importance of good technique in the preparation and staining of smears which is absolutely essential for their correct evaluation. Regardless of the amount of training and experience one has had in cytology, it is most difficult and of ten impossible to interpret cells which have been poorly preserved or improperly stained.

Paper Chromatography - Written by Eugene Roberts, Division of Cancer Research, Washington University, St. Louis 10. July $15,1951-$ This technique makes possible the identification and, in some cases, the quantitative determination of minute amounts of numerous substances of biological interest. Impetus was given to the development of these methods by their classical application to the separation of amino acids (Consden, R., Gordon, A. H. and Martin, A. J. P., Biochem. J., 1944, 38, 224 232). Subsequent studies using paper chromatographic procedures have been made of the chemistry and metabolism of such diverse materials as inorganic ions, amino acids, proteins and enzymes, carbohydrates, fats, vitamins, purines and pyrimidines, pigments, growth factors, and antibiotics. The extensive literature dealing with the theoretical and practical aspects of this rapidly expanding subject has been summarized in recent reviews (Clegg, D. L., Analytical Chemistry, 1950 , 22, 48-59; Strain, H. H., Analytical Chemistry, 1951, 23, 25-38) and in an excellent symposium (Biochemical Society Symposia No. 3, Partition Chromatography, Cambridge University Press).

Basically, the technique consists of applying a small amount of solution containing the sample near the edge of a strip or square of filter paper and allowing a suitable solvent to flow along the paper past the sample either by downward or upward migration. The paper is removed and dried. When conditions are properly chosen, the components of the mixture are separated in such a manner that each one occupies a discrete spot on the paper. When the substances of interest are colorless, numerous optical and chemical methods may be applied in localizing them. Radioautography has proven to be an extremely useful adjunct in work with isotopically labeled compounds. The separation of components in mixtures containing substances of similar chemical properties is often facilitated by the use of two solvents (two dimensional chromatography). The sample is placed $6 \mathrm{~cm}$. from one corner of a large sheet of filter paper (i.e., $18 \times 22^{\prime \prime}$ ). It is developed with one solvent, taken out, and dried. The sheet is then rotated $90^{\circ}$ and placed into a solvent of different properties. After drying, the spots are visualized by an appropriate method. For any given pair of solvent systems the substances will occupy characteristic positions on the ehromatograms: The preparation of reference chromatograms with known compounds of a high degree of purity is made under the same conditions. The spots on the unknown can then be identified by comparison with the reference "map". Paper chromatography can be performed successfully in equipment usually found in biological laboratories (test tubes, bell jars, crocks, petri dishes) and does not require training in complicated chemical methodology.

Results of interest to the cytologist have been obtained in the examination of the free (or loosely bound) amino acids in normal rat tissues (Awapara, J., J. Biol. Chem., 1949, 178, 113-116), in normal and neoplastic mouse tissues (Roberts, E. and Frankel, S., Cancer Res., 1949, 9, 645-648), and in isolated hepatic cell nuclei of the rat (Dounce, A. L. Tishkoff, G. H., Barnett, S. R. and Freer, R. M., J. Gen. Physiol., $1950,33,629-642$ ). Amino acids in acid hydrolysates of mitochondria isolated from various tissues have also been studied by this method $(\mathrm{Li}, \mathrm{C}$. and Roberts, E., Science, 1949, 110, 559-560).

Pappenheim, see Panchrome, Kardos-Pappenheim, Methyl Green-Pyronin and May-Giemsa Stains.

Para Red (CI, 44) is useless as a stain (Emig, p. 30).

Parabenzoquinone, as a fixative for mitochondria (Baker, J. R., Nature, 1932, 130, 134; Sircar, S. M., J. Roy. Mier. Soc., 1935, 55, 238-244).

Paracarmine (Mayer). Dissolve $1 \mathrm{gm}$. carminic acid, $0.5 \mathrm{gm}$. aluminium chloride and $4 \mathrm{gms}$. calcium chloride in $100 \mathrm{cc}$. $70 \%$ alcohol. Warm slightly, if required. Allow to settle and filter. Tissues to be stained should not be alkaline or contain much lime (Lee, p. 147).

Paraffin Imbedding. For routine it is more convenient than celloidin imbedding. Thinner sections can be cut and it is easier to make them in series. Paraffin imbedding is quicker and the blocks being dry are easily stored in a smaller space.

After the specimen has been cleared (see Clearing) it is placed in paraffin held at a temperature just sufficiently high to keep it melted. For ordinary 
purposes a paraffin with melting point of $56-58^{\circ} \mathrm{C}$. is employed; but $60-62^{\circ} \mathrm{C}$. is sometimes selected for very thin sections and $52-54^{\circ} \mathrm{C}$. for thick ones. Paraffins of low melting points are described by Waterman, H. C., Stain Techn., 1939, 14, 55-62. When it is desired to give the imbedding medium more firmness than $60-62^{\circ} \mathrm{C}$. paraffin, use is occasionally made of Rubber Paraffins or Ceresin. Under Clarite is described a mixture of paraffin and clarite for use in hot weather when thin sections are demanded. Routine paraffin infiltration is best done in wide mouthed glass bottles or jars in an incubator held at the proper temperature. Excessive temperatures harden and shrink the tissues. The paraffin over each specimen should be changed at least once to insure removal of the xylol or other clearing agent. If this removal is incomplete difficulties will be later encountered in crystallization of the paraffin block and in sectioning. The time necessary for infiltration will depend on the size of the tissue and its penetrability. Five to 6 hours is about the average with limits of 2 to 24 hours in special cases. See special treatment for Teeth and Bone.

For actual imbedding, folded paper containers have now been rather generally replaced by glass dishes. Watch glasses (Syracuse preferred) are satisfactory ; but Petri dishes, the inner sides of which are not quite vertical but slope outward slightly from the base, are better. First smear a little glycerin evenly over the bottom and sides of the dish. Then pour in a little paraffin, a thin layer of which will harden so that when the tissue is placed in the dish, it will not come in contact with the bottom. It is customary to orient the tissue so that the surface to be cut first is next to the bottom of the dish. Quickly pour in more paraffin until the tissue is covered to a depth of say $6 \mathrm{~mm}$. Hold the dish in ice water until the surface of the paraffin has hardened just to the point when on immersion in the iced water the surface will hold its shape and not run. However too rapid cooling of paraffins of high melting point may cause cracks in the surface and even in the depth of the blocks. After a few minutes the paraffin block slips out easily because the glycerin prevented it from sticking. When several different specimens are imbedded in the same dish identify each by partly imbedding near it a small strip of paper bearing its number. Finally some of the paraffin is cut away from each tissue so that it can be conveniently filed away but it is important not to remove too much paraffin.

Paraffin Sections. 1. Blocking. If the specimen is a slice of tissue it was trimmed at the time of fixation into a quadrangular form with each edge and surface parallel to the opposite one. If the specimen is a cross section of a tubular structure the cutting will be more difficult. Heat the metal holder of the microtome, gently press the surface of the paraffin block against it and harden in iced water. The surface of tissue, protected by the most paraffin (which is the upper surface, remote from bottom of the dish, as it was im. bedded), should be next to the holder and as far as possible evenly equidistant from the surface of the holder. Unless there is plenty of paraffin between the tissue and the holder, difficulties will be encountered if it becomes necessary to remount the block on subsequent occasions to cut more sections. Since the slice of tissue is of even thickness its outer surface will be evenly parallel to the sweep of the knife so that the tissue included in a given section will be approximately the same distance from the surface of the block and equally subjected to fixation and subsequent technique.

2. Cutting. The knife should cut from long side to opposite long side. Trim the edges of the paraffin block so that it will have to pass through an even layer of paraffin at least $5 \mathrm{~mm}$. wide both before and after it enters and leaves the tissue. When more paraffin is cut away it may be later needed if more trimming is required to make the sections into straight ribbons. The sides of the tissue should also be protected by layers of paraffin which are parallel and of even thickness. The object of all this is for the knife to cut through the paraffin and tissue squarely and for it to encounter as nearly as possible equal resistance. The resistance of the paraffin at the sides will, however, always be less than that of the paraffin plus the tissue at the center. For this reason it may be necessary to cut away most of the paraffin from the sides.

But all specimens are not rectangular slices of tissue of uniform thickness. Spherical bodies are easy to cut but the sections obtained are very difficult to flatten. Specimens containing large cavities are troublesome because the paraffin in the cavities offers so little resistance. In such cases celloidin imbedding is advised. When a part of the tissue is brittle and the rest soft it is best to orient the tissue so that the knife passes through the soft part first. In 
orientation of fairly large objects a beam of light passed through the paraffin bloek from an are lamp or other powerful souree is of great assistance. For very minute objects a method described by Fry (H. J., Anat. Rec., 1927, 34, 245252 ) is suggested. For refractory tissues, like yolk laden eggs, MeClung ( $p$. 40) suggests hydration. The block is trimmed until the imbedded tissue is exposed when it is soaked in water for several hours. This reduces friability and brittleness and good sections may of ten be obtained.

Temperature and humidity are factors in securing a good ribbon by making one section stick evenly to the next in series. Sometimes a little boiling water near at hand will help but it should not be necessary if the tissue has been properly infiltrated with paraffin of the right melting point which set firmly when cooled. Static eleetricity, causing the ribbon to adhere in a troublesome way to surfaces, is partly dependent upon difference in density of tissue and paraffiv. But the most important factor in obtaining excellent sections is have the mierotome in good working order and the knife sharp (see Sharpening). Lillie (p. 44) says that ribboning consistency of paraffin is sometimes improved by adding to the paraffin before infiltration $10-20 \%$ of beeswax or $3-5 \%$ of halowax. For ordinary purposes seetions should be eut 6 mierons thick. To mount them on slides first smear earefully cleaned slides (see Slides) with Albumen-Glycerin, cover with aq. dest. and gently heat over an alcohol lamp if a slide warmer is not available. Then mark the slides with a diamond point pencil and leave for about $6 \mathrm{hrs}$. in a drying oven at $40-45^{\circ} \mathrm{C}$.

Parafuchsin, see Pararosanilin (Magenta O). Paraganglion, see Aortic.

Paraldehyde is paracetaldehyde, a polymer of acetaldehyde employed in Dioxan fixative and in other ways.

Paraloidin, see Celloidin.

Paramagenta, see Pararosanilin (Magenta O).

Paramylum, a form of carbohydrate store in lower plants (Taylor in MeClung, $\mathrm{p}$. 221 ).

Paramoecia. Direetions for using dyes for intravitam staining of food vacuoles, contractile vacuoles and various other struetures, as well as minimum coneentration for effeetive staining and toxicity, are supplied by McClung, 1950, pp. $437-439$.

Paraplasm is a term supposed to include nonliving cellular components such as glycogen and lipid granules. It is misleading beeause all cellular eomponents contribute in one way or another to vital phenomena. Deutoplasm is synonymous.

Pararosanilin (Magenta O) (CI, 676)-basie rubin, parafuchsin and paramagentaThis is triamino - triphenyl - methane chloride, the ehief eomponent of most Basic Fuchsins.

Parasites. These range all the way from ultramieroseopic viruses to organisms a yard or more long. Mieroscopic teehniques for viruses are given under Cy toplasmic Inclusions, Elementary Bodies, and Nuclear Inclusions. Certain Gram negative intracellular inseet or arachnid transmitted bacteria-like mieroorganisms are called Rickettsia and require special methods for their demonstration. See also Bacteria and Spirochaetales, Fungi, Piroplasma and Protozoa. A seareh for such small parasites involves not only an examination of tissues but also of body fluids including Blood, Feces, Gastric Contents, Urinary Sediment, etc. When the parasites are scarce resort is made to methods of Concentration. Elementary orientation in respeet to the larger animal parasites (metazoa) is provided by the following elassifieation (according to Stiles) from Stitt (p. 387) which has been slightly modified.

1. Body more or less dorsiventrally flattened.... 3 Body in cross section ordinarily round....... 2

2. Body never snnulated, without legs or jaws.... 4 Body annulated (at least possesses mouth parts), breathes usually through tracheal system, adults with jointed legs or other appendages.. 6

3. Intestine present without snus, 1 or 2 suckers, body not segmented. (In liver, lungs, blood, intestine rarely elsewhere-flukes). Trematoda Intestine sbsent, 2 or 4 suckers on head, body of adults segmented, tissue usually contains calcareous bodies, adults (tapeworms) in intestine, larvae (bladder worms) elsewhere Cestoda

Intestine and snus present, sucker on posterior end, body annulated like earthworm, in upper air passages or externally (lecches, blood suckers $) . \ldots \ldots \ldots \ldots \ldots \ldots \ldots . . . \ldots$ Hirudines

4. Intestine absent, armed rostellum present, very rare in human intestine, thorn headed worms Acanthocephala Intestine present, but no armed rostellum

Nematoda 5

5. Intestine rudimentary in adults, no lateral chords, rare in human intestine (hair snakes or horse hair worms)............... Gordiacea Intestine present with lateral chords, common in intestine, muscles, lymphatics, etc. (round worms) ...................... Eunematoda

6. Six legs in adult, wings in most species, larvae annulated, breathe by trachea, adults ectoparasites, occasionally under skin, in wounds, intestine or bladder (insects)......... Insecta 
Eight legs in adult, 6 in larva, head and abdomen coalesced, ectoparasites, may burrow under skin or live in hair follicles (ticks, mites, etc.).

Acarina

Four claws about mouth, larvae encysted in various tissues, adults occasionally in nasal passages (tongue worms)............. Linguatulidae

Numerous legs, occasionally in nasal passages and intestine (thousand leggers).......Myriapoda

See Tapeworm Proglottids, Trematodes, Taenia, Ticks, Insects, Endamœba, Trichinella, Glychrogel.

Parenchymatous Degeneration, see Cloudy Swelling.

Parhemoglobin, a kind of hemoglobin which crystallizes in same fashion but is insoluble in alcohol (Mallory, p. 135).

Paris Blue, see Spirit Blue.

Paris Violet, see Methyl Violet.

Parlodion, a derivative of pyroxylin (see Celloidin).

Paschen's Method for elementary bodies as given by Seiffert, G., Virus Diseases in Man, Animal and Plant. New York: Philosophical Library, Inc., 1944, 332 pp. Dry very thin smears in air. Place slides perpendicular in aq. dest. Ringer or physiological saline, 3-10 min., longer for older specimens. Then dry and place in abs. alc. 1-24 hours. or in methyl alcohol, $10 \mathrm{~min}$. Dry, cover with filtered Loeffler caustic (Hollborn) and heat. Rinse in aq. dest. and color with well filtered Carbol Fuchsin. Rinse in aq. dest. (To destain if necessary dip in abs. alc., then rinse in aq. dest.), blot dry.

Pasteurella, capsules of. A modification of Hiss's method advocated by Jasmin, A. M., J. Bact., 1945, 50, 361-363. Transfer amount of surface culture adhering to a fine, straight platinum wire to loopful physiological saline $+0.5-1 \%$ phenol and $10 \%$ blood serum. Spread thin film on clean polished slide; fix dried film by quickly dipping in methyl alcohol. Drain and flame to remove excess alcohol. Finally color $\frac{2}{3}$ to $1 \mathrm{~min}$. in any regular bacterial stain, wash in water and dry. Capsules appear as clear areas about strongly stained bacteria in lightly colored background.

Patent Blue A (CI, 714)-Brilliant Acid Blue A-an acid dye of light fastness 4 , stains parenchyma blue green with poor definition (Emig, p. 52).

Pectinols are enzyme preparations of 4 grades supplied by Rohm and Haas Co. of Philadelphia. Their primary action is on pectins. McKay, H. H. and Clarke, A. E., Stain Techn., 1946, 21, 111-114 recommend their use to demonstrate chromosomes of root tip smears after colchicine and before staining with carmine.
Pectins, macromolecular properties, test for (Hueper, W. C., Arch. Path., 1942, 33, 267-290). See Ruthenium Red.

Pencil Red Cells are oval or elongated erythrocytes in the condition of ovalocytosis 4 or 5 times as long as they are broad.

Pentose Nucleic Acid is present in cytoplasm, nucleoli and possibly in the chromatin of cells. With proper controls the substance may be identified in cells by use of ultraviolet microspectrophotometry or by Pyroninmethyl Green and ribonuclease. It is found in high concentration in cell, actively producing protein (Stowells R. E., Cancer, 1948, 2, 121-131) and is generally associated with basophilia (Caspersson, T. and Schultz, J., Nature, $1939,143,602-603)$.

Pepsin, microchemical determination:

1. Freeze gastric mucous membrane of freshly killed pig. Keep at $-10^{\circ} \mathrm{C}$. Cut cylinders of tissue $(2.5 \mathrm{~mm}$. in diam eter) with sharp cylindrical borer vertical to surface. Mount cylinders with muscle down on a piece of cardiac mucosa or on stiffened gelatin. Freeze with $\mathrm{CO}_{2}$. Cut sections at 25 microns. Make enzyme determinations on section and correlate with structure in adjacent sections and with known distribution of cell types at different distances from lumen. This shows that chief cells are the source of the pepsin (Holter, H. and Linderstrøm-Lang, K., C. rend. Trav. Lab. Carlsberg, 1935, 20 (11) 1-32).

2. Make extract of tissue, mix with buffers at suitable $\mathrm{pH}$, apply to gelatin surface of Eastman lantern slide plate, incubate, wash gelatin surface, fix in $20 \%$ formalin, stain with acid fuchsin or Delafield's hematoxylin and observe sites of proteolytic activity evidenced by clear spots. Test is positive for 0.0001 $-0.0002 \mathrm{gm}$. stomach of young amblystoma weighed wet. Details of this ingenious technique, also applicable with slight modification for try psin, are given by Dorris (F., J. Exp. Zool., 1935, 70, 491-527). See also Peptidase, Pepsin and Dipeptidase.

Pepsinogen, antecedent of pepsin in body chief cells of stomach. For staining reaction and discharge by vagal stimulation, see Bowie, D. J. and Vineberg, A. M., Quart. J. Exper. Physiol., 1935, 25, 247-257.

Peptidase can be localized in centrifuged marine eggs by direct enzymatic analysis of fragments containing different cytoplasmic components using a procedure essentially the same as that advocated by Linderstrøm-Lang and his associates. It occurs in the hyaline ground substance and is not bound to the granular 
material (Holter, H., J. Cell. and Comp. Physiol., 1936, 8, 179-199). Simitar studies with amebae indicate, likewise, association with cytoplasmic matrix (Holter, H. and Kopae, M. J., J. Cell. and Comp. Physiol., 1937, 10, 423-427). These teehniques are likely to be of wide usefulness. Peptidase has been localized in gastric and duodenal mucosa of the pig by Linderstrøm-Lang and Holter (K. and H., C. rend Trav. Lab. Carlsberg, 1935, 20 (11), 42-56). See also Mauer et al. (J. Nat. Cancer Inst., 1941, 2, 278). An excellent critical discussion of the histologieal distribution of peptidase is provided by Blaschko and Jacobson (Bourne, pp. 207-216).

Anfinsen, C. B., Lowry, O. H. and Hastings, A. B., J. Cell, and Consp. Physiol., 1942, 20, 231-237 have developed a method whereby the same section can be stained for microscopic examination and thereafter used for enzyme analysis. It works also for diphosphopyridine nucleotide and cholinesterase. See Protease.

Perdrau's Modification. Bielschowsky's silver method for reticulum as detailed by Bailey, P. and Hiller, G., J. Nerv. \& Ment. Dis., 1924, 59, 337-361. Fix in $10 \%$ formalin. Wash in running tap water $12-24 \mathrm{hrs}$., then in several changes aq. dest., 24 hrs. more. Cut frozen sections, $15-25 \mu$, and leave in aq. dest. 24 lirs. $0.25 \%$ aq. potassium permanganate, $10 \mathrm{~min}$. Wash in aq. dest. Decolorize in equal parts $1 \%$ oxalic acid and $1 \%$ acid potassium sulphite. Wash in several changes aq. dest. over night. Treat with following solution 40-60 min.: Add 2 drops $40 \%$ sodium hydroxide to 5 ce. $20 \%$ silver nitrate. Just dissolve ppt. with ammonia. Dilute to 50 ce. with aq. dest. and filter. Wash sections rapidly with aq. dest. Reduce in $20 \%$ formalin in tap water, $30 \mathrm{~min}$. Wash in aq. dest. Tone with gold chloride and continue as in Laidlaw's Method. Reticulum, black; collagen reddish. This is intended primarily for nervous system, see Bailey and Hiller's, Fig. 3.

Perényi's Fluid. 3 parts $95 \%$ alcohol, 4 parts $10 \%$ aq. nitric acid, 3 parts $0.5 \%$ chromic acid is according to Lee (p.32) an important fixative for embryos, segmenting eggs, etc.

Perfusion. The technique of washing through the blood vessels with a fluid is one of wide usefulness. It is in general the same but varies somewhat depending upon what is to be perfused. The apparatus consists of a bottle capable of holding at least $1000 \mathrm{ec}$. equipped with an outlet near the bottom or a bent glass tube siphon connected by a rubber tube about 6 fcet long with a glass Cannula. An artery clamp placed about 1 foot from the cannula will serve as a shut off.

If one wishes to perfuse a mouse the best way is to tie a small cannula into the ventricle, if it is the abdominal organs of a guinea pig the following procedure is advised: Fill the animal with chloroform if this anesthetie will not interfere with the results as is seldom the case. Cut carotids and jugular veins to partly exsanguinate the animal. Clip away sternum and most of the ribs. Displace left lung, expose thoracic aorta and free a portion of it from surrounding tissue. Pass moistened ligature thread behind aorta. Make with scissors a small slit in wall of aorta not at right angles to it but directed into it and downward (toward tail) being careful not to cut more than $\frac{1}{3}$ through it. Insert wet cannula into the slit with slight rotatory motion until the constriction in the cannula is about $1 \mathrm{~cm}$. within the aorta. Then bring the two ends of the thread together and tie the cannula in place. Remove clamp from rubber tube and allow fluid to flow in from bottle suspended about 4 feet above cannula, open right auricle to permit free exit of fluid. It may be necessary to clamp inferior vena eava just above diaphragm and increase pressure somewhat. Sometimes it is helpful to vary pressure by opening and closing clamp. After 4 or 5 minutes open abdomen and examine organ which it is desired to perfuse. The absence of blood color in it and the color of the perfusate (if colored) are indicators of completeness of the operation. The pancreas and the liver will swell considerably but this may not be a disadvantage.

Pericapillary Cells, or perieytes, are closely applied to, or wrapped about, the endothelium of blood capillaries. The designation relates to position only and it includes cells of several sorts from much branched Rouget cells to simple fusiform muscle cells and connective tissue cells. Methods of silver impregnation and beautiful illustrations are provided by Zimmernann, K. W., Zeit. f. Anat., 1923, 68, 29-109. The myofibrils in contractile pericapillary cells ean be stained supravitally with janus green, (Bensley, R. R. and Vimtrup, R., Anat. Rec., 1928, 39, 37-55). Valuable data ean be obtained by microdissection of the living tissues (Zweifach, B. W., Am. J. Anat., 1937, 60, 473-657).

Pericardium. Special dissections of bands of fibers in pericardium (Popa, J. 'T'. and Lucinescu, E., J. Anat., 67, 78-107). 
Methods for study of absorption of substances placed in pericardial sac (Drinker, C. K. and Field, M. E., J. Exper. Med., 1931, 53, 143-150).

Peritoneal Fluid. Cells present (Webb, R. L., Am. J. Anat., 1931-32, 49, 283334 ; Folia Haemat., 1933, 51, 445-451).

Periodontium, see method for Teeth and Jaws.

Peritoneum. Outlines of mesothelial cells blackened with silver nitrate (Pumala, R. H., Anat. Rec., 1937, 68, 327-338, good illustrations). Exudate cells stained vitally with lithium carmine (Maximow, A. A., Cowdry's Special Cytology).

Perivascular Spaces of the brain. The Weed McKibben method (Weed, L. H., Am. J. Anat., 1923, 31, 191-221), based on dehydrating the brain by increasing osmotic pressure of the blood and drawing into these perivascular spaces solutions of potassium ferrocyanide and iron ammonium citrate, after injection into the subarachnoid space, and their later precipitation as Prussian blue by fixing tissue in acid formalin, has been modified by Patek, P. E., Anat. Rec., $1944,88,1-24$. In rabbits and cats he injects intravenously 6-8 cc. $30 \%$ aq. sodium chloride during $10 \mathrm{~min}$. and 3-4 cc. particulate suspension of india ink or mercury sulfide in the cisterna magna under atmospheric pressure during $15-20 \mathrm{~min}$. The animal is then killed by bleeding and perfused via the aorta with $10 \%$ formalin. After further fixation of brain by immersion $1 \mathrm{~mm}$. slices are cut and mounted unstained or the tissue naybe imbedded in paraffin in celloidin and $10-50 \mu$ sections colored with gallocyanin or some other appropriate stain. Dogs can also be used as he directs.

Permeability. This is a fundamental property for the study of which there are many microscopic techniques. The idea that what goes in and what comes out through the plasma membrane (see Cell Membralles) always depends upon the character of the particular substance and of the membrane is fallacious. By his method of observing in vivo the ruffle Pseudopodia of macrophages and cancer cells W. H. Lewis (Am. J. Cancer, $1937,29,666-679$ ) has enabled us to see that materials can be drawn into the cy toplasm in invaginations of the plasma membrane which lose connection with the outside so that when the isolated membranous investments disintegrate the materials are liberated in the cytoplasm without ever traversing the intact surface plasma membrane. This is the converse of observations made possible by the direct examination of secreting acinous cells of the pancreas by W. P. Covell (Anat. Rec., 1928, 40, 213-223) which show secretory products leaving the cell in protrusions of the plasma membrane. These later become pinched off, the membranes disintegrate and the product is set free in the lumen. See literature review (Blinks, L. R., Ann. Rev. Physiol., 1942, 4, 1-24). See Spreading Factors.

Peroxidase. This enzyme catalyses oxidation of several oxidizable substrates in presence of peroxide. It is most abundant in plants being usually prepared from horse-radish. In mammals it occurs in mammary glands and in milk. In the peroxidase reaction, so commonly employed in the study of leucocytes, a colored product is formed in the presence of peroxide from a suitable substrate, benzidine or alpha naphthol. Blaschko and Jacobson (Bourne, p. 197) remind us that it is still uncertain that this reaction in leucocytes demonstrates a true peroxidase because it is relatively stable to heat.

1. Alpha naphthol-pyronin (Graham, G. S., J. Med. Res., 1916, 30, 231242). Fix blood smears in 9 parts $95 \%$ alcohol and 1 part formalin freshly prepared, 1-2 min. Wash in water and flood with: alpha naphthol (Merck's "recrystallized" or "Reagent"), 1 gm.; $40 \%$ alcohol, $100 \mathrm{cc}$.; hydrogen peroxide, $0.2 \mathrm{cc}$. for $4-5 \mathrm{~min}$. Wash in dish of running water, $15 \mathrm{~min}$. Stain in: pyronin $0.1 \mathrm{gm}$.; anilin oil, 4 cc.; $40 \%$ alcohol 96 cc., 2 min. Wash in water. Stain in $0.5 \%$ aq. methylene blue (Grübler's BX), $\frac{1}{2}-1$ min. Wash in water, blot, mount in neutral balsam. Fresh smears should be used. When used by a class of students tie droppers to bottles to avoid spoiling solutions by mixing them.

2. Benzidine-methylene blue (Graham, G. S., J. Med. Res., 1918, 39, 1524). Fix as above. Wash in water. Treat 5-10 min. in $0.2 \%$ hydrogen peroxide in $40 \%$ alcohol saturated before using with benzidine, 5-10 min. Wash and stain with methylene blue.

3. Benzidine-safranin (Sato, A. and Shoji, K., J. Lab. and Clin. Med., 1927$28,13,1058-1060$ ). Dry blood smear in air. Flood the slides with solution A $(0.5 \%$ copper sulphate). After 1 minute pour off solution but do not wash or dry slides. Apply solution B (rub up in a mortar $0.2 \mathrm{gms}$. benzidine with a few drops distilled water. Then add 200 cc. aq. dest. and fiter. To filtrate add 4 drops $3 \%$ hydrogen peroxide) for $2 \mathrm{~min}$. Then wash in tap water. Stain with solution C (1\% safranin in aq. dest), 1 min. Wash in 
tap water and dry. Peroxidase granules are colored blue in granular leucocytes and the nuclei orange red.

4. Nitroprusside-benzidine (Goodpasture, E. W., J. Lab. \& Clin. Med., $1919,4,442-444)$. To make the stain dissolve $0.05 \mathrm{gm}$. sodium nitroprusside in 2 cc. aq. dest.; add 100 cc. $95 \%$ alcohol; 0.05 cc. benzidine C.P.; $0.05 \mathrm{gm}$. basic fuchsin and 0.5 ce. hydrogen peroxide. Cover well dried blood smear with known amount of stain, 1 min.; add equal volume aq. dest. plus hydrogen peroxide, 3-4 min.; rinse thoroughly in water and blot dry. Shows many blue granules in granular leucocytes and few in monocytes. Nuclei are colored red. To increase intensity of stain dilute with a little less aq. dest. and stain longer. Method can be used for frozen sections of material fixed in formalin and preserved in $80 \%$ alc. A modification of this stain has been proposed by Beacom (J. Lab. \& Clin. Med., 1925-26, 11, 1092-1093) with hydrogen peroxide omitted and basic fuchsin doubled.

5. Benzidine-Giemsa (Armitage, F. L., J. Path., 1939, 49, 579-580). Fix smears in $96 \%$ alcohol containing $10 \%$ formol freshly made up. Flood with benzidine mixture $(0.75 \mathrm{gm}$. benzidine in 500 cc. $40 \%$ ethyl alcohol. Filter. Add 7 cc. $3 \% \mathrm{H}_{2} \mathrm{O}_{2}$, mix by shaking immediately before using) $2 \mathrm{~min}$. for fresh films, longer for older ones. Wash in $40 \%$ alcohol until definite yellow granules are seen in granular leucocytes. Absolute alcohol and dry in incubator. Counterstain with dilute Giemsa, wash in water, blot and dry.

6. Benzidine for paraffin sections (McJunkin, F. A., Anat. Pec., 1922-23, $24,67-76)$. After fixation of small pieces in $10 \%$ formalin imbed quickly in paraffin; $70 \%$ alcohol, $1 \mathrm{hr}$.; acetone, 30 min.; benzol, $20 \mathrm{~min}$; paraffin, 20 min. Mount thin sections in usual fashion. Deparaffinize in benzol 20 sec., acetone, 10 sec. Water, few seconds. Drain off water, apply mixture ( $80 \%$ alcohol, 25 cc. ; benzidine, $0.1 \mathrm{gm}$.; hydrogen peroxide, 2 drops) diluted with 1 or 2 parts aq. dest., 5 min. Water, 5 min.; hematoxylin, 2 min.; water, 1 min., $0.1 \%$ aq. eosin, 20 sec.; $95 \%$ alcohol, 30 sec.; abs. alcohol, 5 sec. Clear in xylol and mount in balsam.

Note: In above methods a blue counterstain tends to obscure the blue peroxidase reaction.

7. DCPIP-2, 6-dichlor-phenol-indophenol (Jacoby, F., J. Physiol., 1944, 103, Proc. Physiol. Soc. July 29). Fix air dried blood smear in 9 parts abs. alc. and 1 part formol for 2-3 min. Wash in water. Treat smear for $3-5 \mathrm{~min}$. with
$0.5 \%$ aq. 2.6-dichlor-phenol-indophenol to every $5 \mathrm{cc}$. of which 4 drops $\mathrm{H}_{2} \mathrm{O}_{2}$ is added prior to use. Wash in water, blot dry and examine. "Peroxidasepositive" granules, deep purple-violet. No precipitation of crystals and granules on smear. Author suggests $0.5 \%$ aq. neutral red as a counterstain to be applied after treatment with DCPIP. If smear is to be mounted use ncutral balsam. Solution of DCPIP can be stored in ice box for few months.

Peroxydase, sec Peroxidase.

Pétérfi, see Double lmbedding, and Osmic Acid Method for nerve fibers.

Petrunkevitch's Fixatives: Cupric-phenol. Stock solution $\mathrm{A}=\mathrm{aq}$. dest., $100 \mathrm{cc}$.; nitric acid (c.p. sp. gr. 1.41-1.42), 12 cc.; $\mathrm{Cu}\left(\mathrm{NO}_{3}\right)_{2} \cdot 3 \mathrm{H}_{2} \mathrm{O}, 8 \mathrm{gm}$. Stock solution $\mathrm{B}=80 \%$ alcohol, 100 cc.; phenol crystals, c.p. $4 \mathrm{gm}$.; ether 6 cc. Employ 1 part A with 3 parts B. Fix 12-24 hrs. Wash in $70 \%$ alcohol. Cupric-paranitrophenol. $60 \%$ alcohol, $100 \mathrm{cc}$. ; nitric acid (same), 3 cc.; ether 5 cc.; cupric nitrate (same), 2 gm.; paranitrophenol, c.p. crystals, $5 \mathrm{gm}$. Time unspecified. Wash in $70 \%$ alcohol. Said not to harden tissues like ordinary fixatives. May be followed by all common stains. (Petrunkevitch, A., Science, 1933, 77, 117-118).

Petrunkevitch's Fluid is sat. mercuric chloride in aq. dest., 300 cc., abs. alc., $200 \mathrm{cc}$; acetic acid, $90 \mathrm{cc}$; and nitric acid, 10 cc.

$\mathrm{pH}$, see Hydrogen Ion Indicators.

Phagocytosis. There are numerous methods for the demonstration of this phenomenon from which to choose.

1. In Vaginal Smears (which see), made after intercourse, neutrophilic leucocytes can be observed in the act of engulfing individual spermatozoa. C. $R$. Stockard, in Cowdry's Special Cytology, 1932, 3, 1611-1629, has described this remarkable process as seen in the living state. "A leucocyte comes in contact with a spermatozoon which with its tail is longer than the leucocyte. The leucocyte by stretching and contracting finally takes into itself the entire spermatozoon, the tail being wound in a circular fashion within the cell body."

2. In temporary mounts of bacteria and Leucocytes (which sce) phagocy tosis can be followed in detail. Differences in the behavior of neutrophiles from seriously ill and normal persons have been described.

3. Under Vital Staining will be found many techniques which permit the observation of the phagocytosis of inanimate particulate materials by macrophages. A graphic demonstration 
of the immunologic control of phagocytosis of erythrocytes by these cells can be provided by using a method described by Bloom, W., Arch. Path. \& Lab. Med., 1927, 3, 608-628.

Phase Contrast Microscope. By phase contrast one generally means a system of illumination and phase retardation by means of which phase differences of light waves are converted into amplitude differences. Its purpose is to create contrast, thus rendering objects visible which would otherwise be impossible to see in a bright-field because of excessive transparency.

The equipment consists of an annular diaphragm in the front focal plane of the condenser and a diffraction plate of special design placed between the components of the objective lens. Phase contrast microscopes, developed in Europe just before the war, have been commercially available in the United States only recently but already an impressive amount of investigation has been reported on biological material (Bennett, A. H., Sci. Monthly, 1946, 43, 191-193; Richards, O. W., Cold Spring Harbor Svmposium Quant. Biol., 1947, 11, 208-214). Their great usefulness lies in the fact that with their help one can sce more clearly than by other means at high magnification and resolution such external and internal cellular structures as surfaces (Ralph, P. H., Anat. Rec., 1947, 98, 219-223, 489-507); vaginal smears (Culiver, A., and Gluckman, J., J. Obst. and Gynaec., Brit. Empire, 1948, 55, 261-267); centrioles (Buchsbaum, R., Anat. Rec., 1948, 102, 19-27); mitochondria (Ludford et al., J. Roy. Micr. Soc., 1948, 68, 1-9; Zollinger, H. V., Am. J. Path., 1948, 24, 569589); viral inclusion bodies (Angulo, J. J. et al., J. Bact., 1949, 57, 297-303) etc. in the living stage, or in fixed and unstained material.

Lack of contrast has always been a problem peculiar to histology and cytology because the refractive indices of cytoplasm and its inclusions are so nearly the same. The classical method of creating color contrast by selective or differential staining is subject to well-known limitations. Phase microscopy now provides for the first time an effective, reliable method of creating contrast by purely optical means. The chief deterrent to its universal adoption for research is its present high cost, ranging from $\$ 500$ to $\$ 1,000$ for a microscope and accessories. Moreover special adjustments have to be made for the particular tissue to be examined. You cannot simply take the microscope out of the cupboard and get busy in the examination of any old tissue as is customary with the standard bright light microscope. The phase contrast microscope is not of any particular assistance in the study of fixed and stained preparations in which differential tissue transparency is not a factor of primary importance.

Phenol Compounds, see Azo Reaction, Indo Reaction.

Phenolase, see Oxidase.

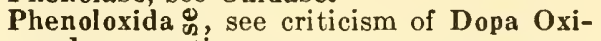
dase reaction.

Phenolphthalein. This compound of phthalic acid with phenol and sulfuric acid is an important indicator. Closely related to it is cresolphthalein.

Phenosafranin (CI, 840) - safranin B extraThis is the simplest of the safranins. It has been used by Moore, E. J., Science, 1933, 77, 23-24 for staining fungi on culture media or in host tissue.

Phenosulfonphthalein, use in renal function tests (Shaw, E. C., in Glasser's Medical Pliysics, 1628-1630).

Phenyl Methane Dyes. The hydrogen atoms of methane can be replaced by phenyl groups and it is possible to add amino groups to the benzene rings. See di-phenyl methanes, di-amino triphenyl methanes, tri-amino tri-phenyl methanes, and hydroxy tri-phenyl methanes.

Phenylene Blue, see Naphthol Blue R.

Phenylene Brown, see Bismark Brown $Y$.

Phloroglucin is 1,3,5-trihydroxybenzene. It is obtained in the form of a yellowish white crystalline powder. It protects the organic components of tissues so that acids can be used in higher concentrations for decalcification. Make sat. aq. sol. phloroglucin and add $5-25 \%$ of the acid.

Phloxine (CI, 774)-erythrosin $\mathrm{BB}$ or $\mathrm{B}$ extra, new pink.

Phloxine B (CI, 778)-cyanosine, eosin $10 \mathrm{~B}$, phloxine $\mathrm{TA}, \mathrm{N}$ or $\mathrm{BB}-\mathrm{Conn}$ (p. 154) explains that this differs from phloxine in possessing 4 in place of 2 chlorine atoms in phthalic acid residue of molecule. This phloxine $B$ is the one ordinarily used. Sec Eosins.

Phloxine Ta, $\mathbf{N}$ or BB, see $\mathbf{P h l o x i n e ~ B . ~}$

Phloxine-Azure. This resembles Mallory's phloxine-methylene blue. Stain sections after Bouin or Zenker fixation in $2.5 \%$ aq. phloxine, $15 \mathrm{~min}$.; wash in water and stain in $0.1 \%$ aq. azure $\mathrm{A}$, $30 \mathrm{~min}$.; wash in water, differentiate in $95 \%$ alc. plus few drops xylene colophonium; dehydrate in abs., clear in xylol and mount. Particularly good for bone marrow. (Haynes, R., Stain Technology, 1926, 1, 68).

Phloxine-Methylene Blue. Mallory (p. 86) recommends that phloxine be employed 
in place of eosin in the following method because it gives (as Conn suggested) a more brilliant color. Deparaffinize sections of Zenker fixed material in usual way. Remove mercury with $0.5 \%$ iodine in $95 \%$ alcohol $5-10 \mathrm{~min}$. and the iodine with $0.5 \%$ aq. sodium thiosulfate (hypo) $5 \mathrm{~min}$. Wash thoroughly in water. $2.5 \%$ aq. phloxine in paraffin over $1 \mathrm{hr}$. or more. Cool stain, drain and rinse in water. Take 5 cc. $1 \%$ methylene blue on $1 \%$ borax, 5 cc. $1 \%$ aq. azure II, add 90 cc. aq. dest., filter onto the sections. Pour on and off several times. After required time differentiate in 100 cc. $95 \%$ alcohol plus $2-5$ cc. $10 \%$ colophony (rosin) in absolute alcohol. Control differentiation under microscope. Dehydrate in several changes abs. alc. Clear in xylol and mount in balsam. Nuclei and bacteria, blue; collagen, etc. bright rose. The method yields beautiful preparations of intranuclear inclusions in yellow fever and is extensively used for many purposes.

Phosphamidase an enzyme capable of hydrolyzing para-chloranilido phosphonic acid, at acid pH (5.6). Gomori, G., Proc. Soc. Exp. Biol. and Med., $1948,69,407-409$, describes the method. Phosphamidase is reported to be especially intense in the grey matter of the central nervous system and in malignant tumors.

Phosphatases - Written by E. W. Dempsey, Dept. of Anatomy, Washington University, St. Louis. February 26, 1951Since the original papers of Gomori, G. (Proc. Soc. Exp. Biol. and Med., 1939, 42, 23-26), and Takamatsu, H. (Trans. Jap. Path. Soc., 1939, 29, 492-498), a host of papers on the localization of these enzymes has appeared. The Gomori and Takamatsu methods involve incubating sections in a solution of organic phosphate, during which free phosphate radicals are liberated. These are instantly precipitated as the calcium or the lead salt, for the alkaline and acid phosphatase reactions, respectively. The insoluble metal phosphate is then visualized by staining with alizarin or silver solutions (Kabat, E. A. and J. Furth, Am. J. Path., 1941, $17,303-318$ ) or by transformation to black cobaltous sulfide. For technical procedures, see Gomori's Method.

The question whether alkaline phosphatase is a single enzyme, or whether several, substrate-specific enzymes exist, is an actively debated topic. Dempsey, E. W. and H. W. Deane (J. Cell. and Comp. Physiol., 1946, 27, 159-179) suggested that there are several different alkaline phosphatases, and Emmel, V. E. (Anat. Rec., 1946, 96,
423-438) has demonstrated that the enzyme of the intestine is easily poisoned by KCN whereas that of the kidney is not. Contrariwise, Gomori, G. (Proc. Soc. Exp. Biol. and Med., 1949, $70,7-11)$ reports no difference in localization of phosphatase when 19 different substrates were employed, although a phosphonate substrate gave different results in the acid range. Later, Gomori, G. (Proc. Soc. Exp. Biol. and Med., 1949, 72, 449-450) found that 5 -nucleotidase was localized differently than is glycerophosphatase. The possible multiplicity of the alkaline phosphatases is therefore unsettled, but the weight of evidence suggests there is more than one enzyme for dephosphorylating mechanisms.

A new chemical approach to the phosphatases appeared when Menten, M. L., J. Junge and M. H. Green (J. Biol. Chem., 1944, 153, 471-477) employed beta naphthyl phosphate as a substrate. After enzymatic hydrolysis, beta naphthol was visualized by coupling with a diazonium salt. This procedure has been greatly improved by Mannheimer, L. H. and A. M. Seligman, J. (Nat. Cancer Inst., 1948, 9, 181-200) and by Seligman and Mannheimer (ibid., 1949, 9, 427-434) for alkaline and acid phosphatase respectively. The present methods give results fully comparable in precision with the Gomori procedures.

The possible occurrence of artifacts of various sorts in the phosphatase methods has been actively debated. Most observers agree that histochemical preparations are of considerable value, but many warn of the possible migration of enzyme or of its products during the technical procedures. The problem therefore becomes one of resolving power-What are the spatial limits of dependability of the phosphatase procedures? Two references on this debated topic are: Martin, B. F. and Jacoby, F., J. Anat., 1949, 83, 351-363, and Leduc, E. H. and Dempsey, E. W., J. Anat., 1951, in press.

Finally attention should be called to the excellent review on phosphatases by Lison, L., Bull. d'Histol., 1949, 25, $23-41$.

Phosphate Ion. A capillary colorimetric technique has been described by Walker, A. M., J. Biol. Chem., 1933, 101, 239-254. He employed it for glomerular urine. See discussion by Sumner, J. B., Science, 100, 413-414. The technique is suitable for even $0.08 \mu$ l containing less than $1 \mathrm{~m} \mu \mathrm{gm}$. phosphate phosphorus with a mean error approximately $0.1 \%$ (Glick, p. 
208). See method of Lowry, O. H. and Lopez, J. Biol. Chem., 1946, 162, 421428 for determination of inorganic phosphate in presence of labile phosphate esters. See Phosphorus.

Phosphate Solutions. A method for the direct observation of the effect of buffered phosphate solutions on a thin layer of living, vascular tissue in moat chambers introduced into the rabbit's ear is described by Abell, R. G., Anat. Rec., 1935-36, 64, 51-73.

Phosphine (CI, 793)-leather yellow, xanthin-a basic xanthene dye used as a microchemical test for nucleoproteins by Schumacher, J., Zentralbl. Bakt., Abt. I. Orig., 1922, 88, 362-366. Phosphine $3 \mathrm{R}$ is fluorchrome for lipids.

Phospholipid Content of white blood cells (Boyd, E. M., J. Lab. \& Clin. Med., 1935-36, 21, 957-962).

Phosphomolybdic Acid Hematoxylin Mallory's, see McClung, p. 406). Fix in Zenker's fluid, imbed in paraffin and remove mercury with iodine. Rinse in water. Phosphomolybdic acid hematoxylin at room temperature 12-24 hrs. or at about $54^{\circ} \mathrm{C}, 2-3 \mathrm{hrs}$. (That is hematoxylin $1 \mathrm{gm} .$, phosphomolybdic acid crystals 2 gm., aq. dest. 100 cc. Requires several weeks to ripen or ripening may be immediate after addition of 5 cc. $1 \%$ aq. potassium permanganate.) Wash in water. Decolorize in $95 \%$ alc.; dehydrate in abs. Clear in xylol and mount in balsam. Collagenic fibers deep blue. To counterstain first color 5-10 min. in $0.5 \%$ aq . acid fuchsin, drain and stain directly in the hematoxylin.

Phosphorescence Microscope, Science (News), 1943, 98, 8 (No. 2547).

Phosphorus. The histochemical detection of phosphorus is a matter of great importance but the techniques are open to much criticism. Lison (pp. 113-120) has reviewed the whole question and advises two techniques as vigorously specific for phosphorus in the ionic form:

1. Angeli, A., (Riv. di Biol., 1933, 10, 702) using plant material treats sections for $20 \mathrm{~min}$. with: ammonium molybdate, $3 \mathrm{gm}$; aq. dest., 20 cc.; $30 \%$ aq. hydrochloric acid, $20 \mathrm{cc}$; reduces in $\mathrm{N} / 50$ stannous chloride, rinses quickly in aq. dest., washes longer in $2.5 \%$ aq. ammonia which results in elements containing phosphorus being colored blue green.

2. Serra, J. A. and Queiros Lopes, A., Portugaliae Acta Biol., 1945, 1, 111122. Reagents: (A) Fixative. Add few drops glacial acetic acid to mixture 2 parts $96 \%$ alc. and 1 part formalin. (B) Molybdate. $0.5 \mathrm{gm}$. ammonium molybdate dissolved in 20 cc. aq. dest. + 10 cc. conc. (30\%) hydrochloric acid diluted to 50 cc. with aq. dest. (C) Benzidine. $25 \mathrm{mg}$. dissolved in 5 ec. glacial acetic acid diluted to $50 \mathrm{cc}$. with aq. dest. Fix tissue in " $A$ " and wash well in water. Treat frozen sections, or small pieces, with " $\mathrm{B}$ " at $10-12^{\circ}$ for $2-3$ weeks and then at $20-25^{\circ}$ for 2-3 days. Cover with drop of " $\mathrm{C}$ " for 3 min. Add 2 drops sat. aq. sodium acetate. Mount in glycerol from a supply containing crystals of sodium acetate. Phosphate is revealed by intense blue color. When these authors digest tissue with nuclease to liberate phosphate from nucleic acid this visualization of phosphate indicates localization of the nucleic acid (partially quoted from Glick, p. 35).

By the titrimetric method of Lindner, R. and Kirk, P. L., Microchemie, $1937,22,300-305$ phosphorus can be detected quantitatively in the range $0.5-10.0 \mu \mathrm{gm}$. The whole subject has been reviewed by Glick, D., J. Chem. Education, 1935, 12, 253-259.

Phosphotungstic Acid Hematoxylin. (Mallory's, see McClung, p. 403) Fix in Zenker's fluid and remove mercury from sections with iodine or $0.5 \%$ sodium hyposulphite. Rinse in water. $0.25 \%$ aq. potassium permanganate, 5-10 min. Wash in water. $5 \%$ aq. oxalic acid, 10-20 min. Wash carefully in several changes of water. Phosphotungstic acid hematoxylin, 12-24 hrs. (To make this dissolve $0.1 \mathrm{gm}$. hematoxylin by heat in $50 \mathrm{cc}$. aq. dest., when cool add $2.0 \mathrm{gm}$. phosphotungstic acid dissolved in $50 \mathrm{cc}$. aq. dest. Requires a few weeks to ripen. Ripening can be done at once by addition of $10 \mathrm{cc} .0 .25 \%$ aq. potassium permanganate). $95 \%$ alc., 30 sec.; dehydrate quickly in abs. Clear in xylol and mount in balsam. Fibroglia, myoglia, neuroglia and fibrin, deep blue; ground substance, cartilage and bone, yellowish to brownish red; coarse elastic fibers, purple.

Mullen, J. P. and McCarker, J. C., Am. J. Path., 1941, 17, 289-291 suggest the following procedure for nervous tissues fixed in formalin. Tissues stored in $4 \%$ aq. formalin for several years give good results. After fixation in $4 \%$, cut blocks $5 \mathrm{~mm}$. or less in thickness. Wash for 6-12 hrs. in running water. Dehydrate to include $95 \%$ alcohol as usual. Complete dehydration in 2 changes $n$ butyl alcohol, $4 \mathrm{hrs}$. each (but absolute alcohol xylol is satisfactory). Imbed in paraffin directly from $n$ Butyl Alcohol (which see).

Treat sections for $2 \mathrm{hrs}$. or longer in following mordant: Dissolve $5 \mathrm{gms}$. chromium chloride (green crystals ob- 
tainable from General Chemical Co., New York) in 100 cc. aq. dest. and add 5 cc. glacial acetic acid. This dark green solution soon becomes purple black but is usable after many weeks. Rinse in aq. dest. Stain, as above, with phosphotungstic acid hematoxylin.

Photodynamic Action of thiazine dyes on vaccine virus may be due to red or infra red rays (Hirano, N. and Sayama, K., Arch. exp. Med., 1936, 13, 324-332).

Photoelectric Colorimeter, construction and use (Hanselman, R. C., Am. J. Clin. Path., 1943, 13, 108-116).

Photoelectric Microphotometer-Written by R. E. Stowell, Dept. of Oncology, University of Kansas Medical Center, Kansas City 2, Kan. January 19, 1951-The original type of apparatus developed by Stowell, R. E. (J. Nat. Cancer Inst., 1942, 3, 111-121) was used to measure the light absorbed as a result of the specific coloration of tissue components. Such equipment consists of a light source, microscope, and photoelectric equipment with amplification and recording of light absorption. Modified equipment is discussed by Pollister, A. W. and Moses, M. J. (J. Gen. Physiol., 1949, 32, 567-577). Among substances measured by these techniques are Feulgen reaction for desoxypentose nucleic acid, pyronin methyl-green stain for nucleic acids and Millon's reaction for tyrosine. For other possible uses of this type of equipment see Stowell, R. E. (J. Invest. Derm., 1945, 6, 183-189).

Photoxylin, see Celloidin.

Phrenosin is a Cerebroside.

Phthalein Indicators. Table giving relative reactions of the several organs and tissues after vital staining (Rous, P., J. Exper. Med., 1925, 41, 739-759). See Indicators of $\mathrm{pH}$.

Physiological solutions. These are intended for the examination of living cells with a minimum of change. Blood serum, or plasma, is an unnatural medium for any living cells except those naturally intravascular as shown by the fact that alone and undiluted it is a poor medium for tissue culture. Physiological saline is for mammals $0.85-0.9 \%$ aqueous $\mathrm{NaCl}$ and for amphibians about $0.65 \%$ aqueous $\mathrm{NaCl}$. For others see Ringer, Ringer - Locke, Locke - Lewis and Tyrode. Normal solutions (which see) are different.

Pia Mater. P'erivascular nerves. Wash out blood by vascular perfusion with saline solution or by rinsing nonperfused tissue with saline. Fix with $10.5 \%$ citric acid in $20 \%$ formalin preferably by injection. Dissect out blood vessels of pia under binocular microscope. Wash in aq. dest. twice and place in $20 \%$ aq. silver nitrate 2 hrs. Pass through 4 changes $20 \%$ formalin in Petri dishes each containing about $100 \mathrm{cc}$. Transfer directly to ammoniated silver nitrate made by adding conc. ammonia $(28 \%)$ drop by drop to $20 \%$ aq. silver nitrate using 3 drops more than amount required to dissolve ppt. Observed under the microscope the nerves "come up" slowly and when they are dark enough transfer to $20 \%$ ammonia water for $1-2$ min. Wash in aq. dest. plus few drops glacial acetic acid. Tone in $0.2 \%$ aq. yellow gold chloride $30-60 \mathrm{~min}$. Wash in water, dehydrate in 3 changes $95 \%$ alcohol, clear in carbol-creosote-xylol mixture and mount in balsam (Penfield, W., Am. J. Path., 1935, 11, 1007-1010); revised by W. Penfield, Montreal Neurological Institute, Montreal, Canada, May 1, 1946.

Pianese Method. Much used a generation ago for study of cancerous tissue. Pianese, G., Beitr. z. Path. Anat., u. Allg. Path., 1896, Suppl. I, 193 pp.

Piccolyte Resins-Written by L. F. Wicks, Veterans Administration Hospital, Jefferson Barracks, Mo. February 1, 1951 - These synthetic terpene resins ( $\beta$ pinene polymers) have been recommended for permanent mounting media (Wicks, L. F., Carruthers, C. and Ritchey, M. G., Stain Techn., 1946, 21, 121-126). Natural resins are quite variable in quality, and with age the poorer samples may turn yellow, crack or develop acidity which fades basic stains. Synthetic resins, being of controlled manufacture, are much more uniform in composition and predictable in behavior. An examination of many such resins was stimulated by the war-time scarcity of Canada balsam.

The requirements for a good mounting medium are abbreviated from the original article. It should possess: 1. Correct refractive index, 2. Freedom from acidity, 3. Clarity, present and future, 4. Solubility in the proper organic solvents, 5. Good adhesion for glass, 6. Reasonable hardening time (and not craze or granulate later), 7 . An approximately right softening temperature, 8. Constant composition, stability, inertness, availability and moderate price.

The Piccolytes meet all the above stipulations, being of correct refractive indices, very low acid numbers, pale, non-yellowing, adherent to glass, and soluble in xylol and toluol. They are also available in a variety of softening points. Unfortunately, despite considerable interest shown in the descrip- 
Fion the resins, the manufacturer (Pennsylvania Industrial Chemical $\therefore$ Corp., Clairton, Penn.) is not interested in packaging for such small specialty sale. However, one of the Piccolytes ("S-115") is on the market under the name of "Harleco Mountant" (Hartman-Leddon Co., Philadelphia). Possibly, one of lower softening point than S-115 would have been a better choice for general use.

Picric Acid is a very important ingredient of several fixatives. It penetrates rapidly and serves to some extent as a mordant like potassium bichromate. See Bouin's fluid. Picric acid staining of chromophobe bodies of Lipschutz (Schiller, Virchow's Arch., 1930, 278, 663-689).

Picro-Carmine (Ranvier). There are many sorts most of them based on Ranvier's original formula: Add carmine (dissolved in ammonia) to sat. aq. picric acid to saturation. Evaporate to $\frac{1}{5}$ original volume, cool, filter out ppt. and evaporate filtrate to dryness. The resultant red crystalline powder is picro-carmine. Make a $1 \%$ aq. sol. for staining. If overstained decolorize with $0.2 \%$ hydrochloric acid. This is an excellent and very popular stain. It colors keratohyalin very brightly (Lee, p. 146).

Picroformaldehyde Formic Acid for fixation (Lillie, R. D., J. Tech. Methods, 1944, 24, 35-36). Formaldehyde (37\% solution), $10 \mathrm{cc}$., formic acid, $5 \mathrm{cc}$. and sat. aq. picric acid, $85 \mathrm{cc}$. is recommended as a substitute for Bouin's Fluid. It decalcifies femurs of mice well in 48 hrs., provides sections adapted to Romanovsky staining, and in general acts like Bouin's fluid.

Picro-Formol, see Bouin's Fluid.

Picro-Indigo-Carmine is a much used stain. Lee (p. 433) advises 3 parts sat. indigocarmine in $70 \%$ alc. and 1 part sat. picric acid also in $70 \%$ alc.

Picro-Mallory. Several fine modifications of Mallory's connective tissue stain using picric acid (McFarlane, D., Stain Techn., 1944, 19, 29-37).

Picro-Nigrosine for muscle. After alcohol or Bouin fixation, stain sections in sat. nigrosine in sat. aq. picric acid. Muscle yellow, connective tissue black.

Picro-Sulphuric fixative, see Kleinenberg's.

Pigments, general reviews: Bergmann, E. Ergeb. d. Physiol., 1933, 35, 158-300; Lederer, E., Biol. Rev., 1940, 15, 273306 (invertebrates). See Aposiderin, Bacterial, Bile Pigment, Bilirubin, Biliverdin, Carotin, Carotinalbumins, Carotinoids, Ceroid, Chromolipoids, Cytochrome, Cytolipochrome, Cytosiderin, Exogenous, Hematin, Hematoidin, Hematoporphyrin, Hem ofuscin,
Hemoglobin, Hemosiderin, Trichosiderin, Iron Pigments, Lipochrome, Lipofuscin (wear and tear pigment), Malarial Melanins, Parhemoglobin, Porphyrins, Rhodopsin, Scheele's Green, Sulfmethemoglobin, Schweinfurt Green, Ultramarine Green and Verdigris.

Pinacyanol (CI, 808)-sensitol red-A basic xanthene dye of the cyanine group. Proescher, F., Proc. Soc. Exp. Biol. \& Med., employed the Eastman Kodak Co. product of which a $0.1-0.5 \%$ solution in absolute ethyl or methyl alcohol for 510 sec. stains frozen sections brightly. Wash in water, mount in glycerine. Chromatin, blue violet; protoplasm, purple; connective tissue, red; elastic tissue, black violet; muscle, bluish violet to purple; amyloid, red; etc. Hetherington, D. C., Stain Techn., $1936,11,153-154$, used pinacyanol as a supravital stain for mitochondria in blood cells.

Pineal. Staining pineal parenchyma by a modified Hortega method after paraffin embedding-Written by W. M. Shanklin, American University of Beirut, Beirut. March 30, 1951-Fix the gland as soon as it is removed in $10 \%$ formalin (Merck blue label 40\%) 3 days at room temperature of about $24^{\circ} \mathrm{C}$. Wash for 2 hrs. in aq. dest. to which 6 drops of ammonium hydroxide are added for each $100 \mathrm{ml}$. Wash again in aq. dest. for a few minutes and dehydrate the gland in $70 \%, 80 \%, 95 \%$ and absolute alcohol, leaving $3 \mathrm{hrs}$. in each. Clear in cedar oil over night, followed by pure xylene for half an hr. Infiltrate with paraffin (melting point $48^{\circ} \mathrm{C}$.) for 3 hrs. Embed in pure paraffin. Cut sections $7-10 \mu$, fix to slides by the albumen-water method and dry thoroughly. Heat the slide gently to sof ten the paraffin and pass through 2 changes of xylene. Pass slides through absolute alcohol, $95 \%$ and $80 \%$ alcohol 1-2 min. in each. Wash in 3 changes aq. dest. for $3 \mathrm{~min}$. each. Place slides in $10 \%$ aq. silver nitrate to which 3 drops of pyridine for each $10 \mathrm{ml}$. are added and keep in the dark at room temperature $24^{\circ} \mathrm{C}$. for $24 \mathrm{hrs}$. Wash in aq. dest. and sensitize by placing in $5 \%$ sodium sulfite $1 \mathrm{hr}$. This step may be omitted, but sometimes this is necessary as it acts as a rejuvenator. Wash in aq. dest. to which 2 drops of pyridine for each $10 \mathrm{ml}$. are added for 1 to 2 min. followed by 2 changes aq. dest. Impregnate in strong silver carbonate at room temperature for 2 to $5 \mathrm{~min}$. Formula: 10\% aq. Silver nitrate (pure crystals) $100 \mathrm{ml} ., 5 \%$ aq. Sodium carbonate (pure) 300 ml., Ammonium 
hydroxide, just sufficient to dissolve the precipitate, Distilled water 100 $\mathrm{ml}$. Dip in aq. dest. for a few seconds, without agitating. Reduce in $10 \%$ formalin (Merck blue label), to which 4 drops of pyridine are added for each $10 \mathrm{ml} ., 1 \mathrm{~min}$. agitating gently. Wash in aq. dest. Tone in yellow gold chloride ( $1 \mathrm{~g}$. to $500 \mathrm{ml}$. aq. dest.) for a few seconds to $1 \mathrm{~min}$. Time should be checked carefully. Fix in 5\% hypo for 1 to 2 min. Wash and counterstain lightly with $1 \%$ erythrosin or safranin. Dehydrate in $80 \%, 95 \%$ and absolute alcohol. Clear in two changes of xylene, mount in neutral Canada balsam and cover with cover slips (see Nassar, T. and Shanklin, W. M., Stain Techn., 1950, 25, 35-38).

Pinocytosis, a term introduced by Lewis (W.H., Bull. Johns Hopkins Hosp., 1931, $49,17-26$ ) to indicate drinking by cells as opposed to phagocytosis, or eating by cells. It means (Lewis, WV. H., Am. J. Cancer, 1937, 29,666-679) microscopically visible drinking, not submicroscopic "sipping" which Meltzer termed "Potocytosis". By this process in tissue cultures proteins and other substances that do not diffuse into the cells are engulfed by wavy ruffle pseudopodia. The cell membrane, which first invests the globulus of fluid taken into the cytoplasm, later disappears and the fluid becomes part of the cytoplasm. Can be best seen in cultures of cancer cells of which an excellent moving picture is available for distribution by the Wistar Institute of Anatomy at Philadelphia.

Piroplasma (L. pirum, pea + G. plasma, a formed thing). Piroplasmas are pear shaped parasites of red blood cells causing diseases of great importance in domestic and other animals but not as yet found in man. They can be colored by any good blood stain. Giemsa and May-Giemsa are recommended.

Pituitary. The microscopic techniques for this conductor of the endocrine symphony are obviously too numerous to mention. Consult each issue of the Quart. Cum. Index Med.

To differentiate 2 classes of acidophiles in the cat a modification of Heidenhain's "azan" modification of Mallory's connective tissue stain is proposed by Dawson, A. B. and Friedgood, H. B., Stain Tech., 1938, 13, 17-21. T. Maxwell, Jr. (ibid, 93-96) proposes a modification especially designed for the basophiles and Koneff, H. H. (ibid, 4952 ) one for the rat. In addition all within the space of a few months, Lewis, M. R., and Miller, C. II., Stain Techn., 1938, 13, 111-114 give following direc- tions to demonstrate 2 types of granular cells in the pars nervosa. Fix in $3 \%$ aq. potassium bichromate 2 parts and half sat. corrosive sublimate in $95 \%$ alc. 1 part, $12-24$ hrs. with 1 change. Dehydrate to $70 \%$ alcohol to which add few drops iodine. Change each day until solution retains color. Dioxan, 8-24 hrs., 3 changes. Dioxan + little paraffin. Paraffin 4 changes. Cut sections 4 microns and deparaffinize. Stain $0.25 \%$ aq. acid fuchsin $30 \mathrm{~min}$. Then 1-24 hrs. in Mallory's stain (aq. dest., 100 cc.; anilin blue, $0.5 \mathrm{gm}$.; orange $\mathrm{G}$, $2 \mathrm{gm}$. and phosphotungstic acid, $1 \mathrm{gm}$.). Differentiate in $95 \%$ alcohol until no more color comes out. Abs. alc., xylol, balsam. To identify microglia in neurohypophysis see Vazquez-Iopez, E., J. Anat., 1942, 76, 178-186. Differential stain for mouse pituitary (Dickie, M. M., Science, 1914, 100, 297-298). Pituicytes by Hortega silver carbonate technique (Shanklin, W. MI., Stain Techn., 1943, 18, 87-89).

Placenta-Written by Dr. George B. Wislocki, Dept. of Anatomy, Harvard Medical School, Boston 15. March 8, 1951-Of all the organs of the body the placenta has been the least investigated by histological, cytological and cytochemical methods. Compared to most other tissues, its study is greatly complicated by the marked and perplexing differences in its structure in various mammals, as well as by the fact that from the moment of implantation up to the time of parturition, it goes through a complex succession of structural changes. The latter are doubtlessly related to differing physiological and nutritional needs, first of the implanting egg, then of the differentiating embryo before the heart has begun to beat, and finally of the growing and maturing fetus. Besides subserving the immediate needs of fetal nutrition and of excretion of fetal waste products, the placenta is also an endocrine organ which produces both steroid hormones and chorionic gonadotropin. Relatively little is known concerning the functions of these hormones, but the placental steroids apparently play an important part in the growth, regulation and maintenance of the pregnant uterus by exerting their influence principally upon the uterine musculature and blood vessels and thereby influencing also the length of gestation and the onset of labor. The role of chorionic gonadotropin is more obscure, but it seems to interplay with the pituitary, as well as with the steroid-producing glands including the placenta itself, 
in maintaining gestation and regulating maternal metabolism.

In view of the manifold functions of the placenta, which surpass in number those of any other organ, it is not surprising to find that it gives a great many cytological and histochemical reactions. Despite the variety of these, none of them is specific for the placenta alone. The placenta in this respect differs, for example, from brain where for various neuronal and glial elements a variety of special cytological techniques has been developed.

For a general understanding of the comparative anatomy and phylogeny of the placenta, consult Grosser $O$. (Frühentwicklung Eihautbildung und Placentation des Menschen und der Säugetiere, 1927) Bergmann, Munich, and Mossman, H. W. (Carnegie Contrib. to Embryol., 1937, 26, 129-246). See also the chapter by E. C. Amoroso on Placentation in the forthcoming 3rd edition of Marshall's Physiology of Reproduction. For the placentation of the Primates consult Hill, J. P. (Phil. Trans. Roy. Soc. Lond. Ser. B., 1932, 221, 45-78), Wislocki, G. B., and Streeter, G. L. (Carnegie Contrib. to Embryol., 1938, 27, 1-66) and Stieve, H. (Ztschr. f. mikro-anat. Forsch., 19.44, 54, 480-543). Various aspects of the topography, growth and vascularization of the human placenta are described by Spanner, R. (Morph. Jahrb., 1935, 75, 374-392) and Ztschr. f. Anat. u. Entwicklungsgesch., 1936, 105, 163242) and Stieve, H. (Ztschr. f. mikroanat. Forsch., 1940, 48, 287-449).

Interest in placental cytology and histochemistry was initiated by Hofbauer (Biologie d. menschlichen Plazenta, 1905, Braumüller, Vienna and Leipzig) who investigated the placental assimilation of iron, protein and fats in man, and by Edwin Goldmann (Beiträge z. klin. Chir., 1912, 78, 1-108) who studied extensively the distribution and significance of glycogen, fat, iron and hemoglobin in the placentas and fetuses of mice and rats.

In the past two decades with the development of many new cytological and histochemical methods, interest in the placenta has been rekindled. The human placenta has been investigated by a variety of histochemical techniques for the demonstration of various proteins, carbohydrates, lipids, enzymes and inorganic compounds. Wislocki, G. B. and Bennett, H. S. (Am. J. Anat., 1943, 73, 335-449) have described the presence in the trophoblastic syncytium of birefringent, sudanophilic droplets which react with phenylhydrazine and which are soluble in acetone, and they concluded that these reactions indicate the site of formation of placental steroid hormones. The Liebermann-Burchardt reaction, the Schiff reaction and the property of autofluorescence have been introduced as further means of characterizing placental steroid hormones in histological sections (Dempsey, E. W. and Wislocki, G. B., Endocrin., 1944, $35,409-428)$. These investigators also describe glycogen, revealed by Best's carmine and Bauer's method, and iron, demonstrated by both the Turnbull blue reaction and microincineration. The distribution of acid and alkaline phosphatases has been explored (Dempsey, E. W. and Wislocki, G. B., Am. J. Anat., 1947, 80, 1-33) by using Gomori's methods with a variety of substrates (glycerophosphate, fructose diphosphate, nucleic acid, adenylic acid and lecithin). Mucopolysaccharides as well as glycogen have been investigated by McManus' periodic acid-Schiff reaction, metachromatic substances by their responses to toluidin blue after basic lead acetate fixation and lipids with sudan black B (Wislocki, G. B. and Dempsey, E. W., Am. J. Anat., 1948, $83,1-30)$. The affinity of syncytium, fibrin and fibrinoid of the human placenta for acid and basic dyes has been studied (Singer, M. and Wislocki, G. B., Anat. Rec., 1948, 102, 175-193). A basophilic substance abundantly present in syncytium and cytotrophoblast and digested by crystalline ribonuclease has been identified as ribonucleoprotein (Dempsey, E. W. and Wislocki, G. B., Am. J. Anat., 1945 , $76,277-301)$. Some of the functional activities of the placental trophoblast, as revealed by histochemical studies, have been summarized (Wislocki, G. B., Dempsey, E. W. and Fawcett, D. W., Obstet. and Gyn. Survey, 1948, 3, 601-614).

Our knowledge of the trophoblastic cell columns and shell and of the basal plate, as well as of the relationships of of these structures to the underlying basal decidua in the human placenta has been materially advanced by the use of cytological and histochemical methods (Wislocki, G. B. and Bennett, H. S., 1913, 1.c.; Wislocki, G. B. and Dempsey, E. W., 1948, l.c.). By these means two types of cytotrophoblast can be distinguished from the elements of the maternal decidua (Wislocki, G. B., Proc. Am. Assn. Anat., 1951).

Tissue cultures of young human placentas containing actively growing cytotrophoblast produce gonadotropic 
hormone, even after repeated transplantation (Jones, G. E. S., Gey, G. O. and Gey, M. K., 1943, Bull. J. H. Hosp., $72,26-38$ ).

The placentas of various mammals other than man have been investigated by multiple cytological and histochemical methods, for example, pig's placenta (Wislocki, G. B. and Dempsey, E. W., Am. J. Anat. 1946, 78, 181-225), eat's placenta (Wislocki, G. B. and Dempsey, E. W., Am. J. Anat., 1946, 78, 1-45), rodent's placenta (Wislocki, G. B., Dempsey, E. W. and Deane, H. W., Am. J. Anat., 1946, 78, 281-345), shrew's placenta (Blarina brevicauda and Sorex fumeus) (Wislocki, G. B. and Wimsatt, W. A., Am. J. Anat., 1917, 81, 269-307), and bat's placenta (Myotis lucifugus lucifugus) (Wimsatt, W. A., Am. J. Anat., 1949, 84, 63-141). The distribution of acid and alkaline phosphatases in the placentas of pig, cat, rodents and man has been the subject of a separate report (Dempsey, E. W. and Wislocki, G. B., Am. J. Anat., 1947, 80, 1-33). By his method for lipase, Gomori (Menstruation and its Disorders, edited by E. T. Engle, 1950, C. C. Thomas) depicts lipase activity in the allantoic and vitelline placentas of mouse and rat. The distribution of saliva-insoluble glycoproteins, stained by the periodic acid-Schiff procedure, is briefly described in the placentas of pig, cat, mouse, rat and man (Wislocki, G. B., J. Natl. Cancer Inst., 1950, 10, 1341).

Goldmann (Beiträge z. klin. Chir., $1909,64,192-265)$, in his classic account of vital staining, described the intravitam deposition of pyrrhol blue in the placenta of the mouse. Wislocki (Carnegie Contrib. to Embryol., 1920, $11,45-60$ and $1921,13,89-101)$, by employing the technique of vital staining, demonstrated the storage of trypan blue in the trophoblast of the placentas of cats, rabbits and guinea pigs, while Gérard (Arch. d. biol., 1925, 35, 269293) and Everett (J. Exp. Zool., 1933, 70, 243-284) carried out further similar experiments in mice and rats.

By using solutions of iron ammonium citrate and sodium ferrocyanide which could be traced in the placenta by converting them into the Prussian blue reaction, Cunningham (Am. J. Physiol., 1920, 53, 439-456 and 1922, 60, 448-460; Proc. Soc. Exper. Biol. and Med., 1923, 20, 343-345) investigated the relative permeabilities of the placentas of cats and rabbits to these two substances.

Everett (J. Exp. Zool., 1935, 70, 243280) has made a series of important observations concerning the activities of the yolk-sac epithelium in the rat by devising a technique for perfusing the uterine circulation with thionin, toluidin blue, methylene blue, brilliant blue, Nile blue sulfate, Janus green, neutral red and trypan blue. This important study included direct microscopic observation of the vitelline epithelium in hanging drops in a constant temperature chamber.

An ingenious technique of tying off the vitelline blood vessels in living rabbit fetuses in utero, for the purpose of ascertaining the route followed by antibodies in their passage across the placental barrier, has been devised by Brambell, Hemmings and Rowlands (P̈roc. Roy. Soc., London, B, 1949, 136, 131-144).

Transplantation of living fertilized eggs and pieces of placenta, for purposes of observing their growth, has also been successfully carried out. Fertilized mouse eggs, introduced into the anterior chamber of the eye, into the ovarian bursa, or under the capsule of the kidney, undergo development, producing large amounts of seemingly normal placental trophoblast (Runner, M. N., Anat. Rec., 1947, 98, 1 18; Fawcett, D. W., Wislocki, G. B. and Waldo, C. M., 1947, Am. J. Anat., 81, 413-444; Fawcett, Anat. Rec., 1950, 108, 71-91). Implants of trophoblast from 9-11 days of development also establish themselves in the eye, although their capacity for growth declines in relationship to their age at the time of implantation (Grobstein, C., J. Exp. Zool., $1950,114,359-373$ ).

Plant Cell Walls, see Polysaccharides.

Plants. Except for pathogenic Bacteria and Fungi, technique for plants does not come within the scope of this book. However much is to be learned, especially in microchemistry, from many methods employed by botanists and the reader is advised to consult Johansen, D. A. Plant Microtechnique. New York: McGraw-Hill, 1910, 523 pp., also McClung, 1950.

Plasma Cells. Since plasma cells (of the Marschalko type) are mainly identified by recognition of a small area near the nucleus which does not stain as intensely as the rest of the cytoplasm with basic dyes, it is important to use a technique which reveals basophilia. In prartice Giemsa's stain, or a good coloration with hematoxylin and eosin, is generally sufficient. Unna used the term "plasma cell" for almost any kind of cell with much plasma including macrophages so that the designation Unna's plasma cell is almost meaningless.

Plasma Membrane, see Cell Membranes. 
Plasma Stains are too numerous to catalogue here. Lillie (p. 66-68) states that they functionally are divisible into two groups: plasma stains proper and those selective for extracellular materials such as collagenic and elastic fibers, bone and cartilage matrix and so on.

Plasma Reaction, see Aldehydes and Schiff Reaction.

Plasmalogen. A component of the cytoplasm which gives a positive Feulgen test (Bourne, p. 22). See Aldehydes.

Plasmodesmata, dehydrogenase activity in, see Triphenyltetrazolium Chloride.

Plasmosin, the gel and fiber forming constituent of the hepatic cell. Method of isolation and properties (Bensley, R. R., Anat. Rec., 1938, 72, 351-369).

Plasmosome. The true nucleolus staining with "plasma" or "acid" dyes, that is to say, red with eosin in the hematoxylin and eosin combination. The plasmosome apparently does not make any direct contribution to chromosome formation. Acidophilic nucleoli are quite different from certain cytoplasmic granules which Arnold called "plasmosomes" and mitochondria termed "plastosomes" by Meves.

Plastics-Written by M. S. Lucas, Dept. Biological Science, Michigan State College, East Lansing. August 10, 1951-Acrylic plastics have been described very early for use as embedding media in biological fields whereas polyester resin plastics, the so-called "coldsetting" plastics, have more recently come into use. Acrylics require very careful handling and the fumes are more toxic than those of the polyester resins. These features have somewhat deterred the general use of acrylics. Finished blocks of both types of plastic are clear and resistant to damage; acrylics are a little clearer but the difference is so slight that it is not noticeable except when a block of acrylic plastic is compared directly with a block of polyester resin. The plastics can be tinted or even rendered opaque on the back by addition of dyes and chemicals. The specimens to be embedded can be opaque or cleared.

Techniques involved in successful embedding of biological and medical specimens are not difficult. However, use of plastics is not as simple as some pamphlets indicate. It is as precise a technique as any used in listology and for this reason many people have mediocre success. Most successful users of plastics feel that the possible uses for them have only begun to be described. It is a good idea for those interested, to secure current pamphlets concerning new methods and also about the development of new and simplerto-use plastics from the several firms listed below as well as others not listed here.

Acrylics

1. The Polychemicals Dept., E. I. du Pont de Nemours and Co., Wilmington, Del. "Du Pont Plastics in Medicine and Surgery", Mimeo. Pp. 9, references.

2. The Plasties Dept., Rohm and Haas Co., Washington Sq., Phila. 5, Pa. "Embedding Biological Specimens in Acrylic Plastic", Mimeo. Pp. 6, references.

3. Description of and Methods for Acrylics in general: "Preservation of Agricultural Specimens in Plastics". Miscell. Pub. No. 679 U.S.D.A., G. R. Fessenden, 1949. Pp. 78, Ill., Lit. Cited, bibliography. Polyester Resin Plastics.

Selectron No. 5026, manufactured by

the Pittsburgh Plate Glass Co. The following are some trade names under which it is retailed.

1. "Bio-plastic"-Wards Natural Science Establishment, Inc., 3000 Ridge Rd., E., Rochester' 9, N. Y. "How to Embed in Bio-plastic", 1950, Pp. 20, 25\&, references, Ill.' "Natural Science Bulletin" Published monthly.

2. "Castolite"-The Castolite Co., Woodstock, Ill. 'Preserving Specimens in Castolite", 1950, Mimeo. Pp. 23, references, Instruction Manual.

3. "Turtox Embedding Plastic"-The General Biological Supply House, 761 E. 69th Pl., Chicago, Ill. "Embedding Specimens in Transparent Plastic", Turtox Service Leaflet No. 33, 1951.

4. "Carolina Embedding Plastic"Carolina Biological Co., Elon College, N. Carolina. Instruction Booklet $50 \dot{c}$.

The pamphlets listed above contain detailed procedures. They also give the history and specifications of the plastic and indicate biological materials that are suitable for embedding. Most of them contain large bibliographies. In addition to the references listed in these pamphlets, certain additional work, can be mentioned.

Albert Jehle (University Museum, Univ. of Pennsylvania, Phila., Pa.) is using dental acrylics as reconstruction material in restoration of skeletons (report in preparation). In some instances he has replaced an entire bone by preparing a plastic cast.

A new technique for light and electron 
microseopy (Newman, S. B., Borysko, E. B., and Swerdlow, M., U. S. National Bur. Standards, J. of Res., 1949, 43, 183-199) uses n-butyl methacrylate for mounting specimens which can be sectioned for study under a mieroseope.

Plastics in the form of sheets find interesting uses. Microscopy with plastic substitutes for cover glasses is discussed by Richards, O. W., Small, J. H. and Collyer, P. W., Stain Teeh., 1944, 19, 59-62. Natural color plant specimens have been mounted between plastic plates by Fessenden (G. R., loc. cit. under Acrylics 3). Preserved flowers can be placed in transparent plastic containers with a desiccant and kept for long periods of time (Specht, R. C., Engineering Progress, Univ. Florida, 1950 IV No. 12, Gainesville).

Polyester resins: Gross anatomical and embryological specimens have been mounted and are deseribed in the following articles:

1. "Embedding Gross Sections in Ward's Bio-plastic", T. H. Romaniak, Ward's Nat. Sci. Bull., 1948, 22, 34.

2. "The Mounting of Human Sections in Bio-plastic," Ward's Nat. Sci. Bull., 1950, 24, 27.

3. "On the Mounting of Anatomical Museum Specimens in Transparent Plastic", Kampmeier, $O$. 'T. and Haviland, R. N., Anat. Record, 1948, 100, 201-231 (Used Castolite on 100 specimens).

4. "The Relationships of Herniated Intervertebral Dises to the Spinal Cord and Spinal Nerves", (Used Castolite). A. F. Reed and H. D Kirgis, Abstract D2, Anat. Ree., $1951,109,146$.

5. "The Blood Vessels of the Jejunum and Ileum in Man and Certain Laboratory Animals", (Used Polystyrene). Abstract D 16, Anat. Ree., $1951,109,144$.

6. Charles H. Glines (with Alexander Barry and Bradley M. Patten at the Anatomy Dept., Sehool of Medicine, University of Michigan, Ann Arbor) has prepared both cleared and opaque embryos following techniques set out in Ward's, "How to Embed in Bio-Plastic". Glines and his co-workers have devised some modifications over methods described by Kampmeier. These data are available in mimeograph form, and were also presented at the 1951 meeting of the Am. Assoc. of Anatomists (Barry, A., Patten, B. M. and Glines, C. H., Abstract D 31, Anat. Rec., 1951, 109,133 ).

Demonstration sets composed of animal types have been prepared in plastic for elass use (Lucas, M. S. report in preparation) in connection with a study of "unknown" specimens (Study 1, 1951, Guide for Laboratory Studies Biological Science, Mich. State College Press, E. Lansing). Each animal is prepared individually in a block and later is set with others into an illuminated box or in to a large block of plastic. This provides an attractive variety of unknown animal types to supplement conventional identifieation studies. Plastic-corrosion specimens prepared by injecting blood vessels of animals with Vinyl Acetate (Vinylite) can be embedded in blocks of polyester resins (Service Bulletin No. 5, 1950, and Natural Science Bulletin 1950, 24, 3, Ward's Natural Seience Est.). Vinylite of the type carried by this firm contains a pigment which will not bleed out of the preparation into the plastic block, whereas, latex-injected vessels will permit dye diffusion. The use of plastic in reconstructions from serial sections is advocated by Boyer, C. C., Anat. Rec., 1948, 100, 191-197.

In soils studies, vinylite resin (The Bakelite Corp., 230 N. Mich. Ave., Chicago 1, Ill.) and cellulose acetate (Hercules Powder Co., Cellulose Products Dept., Parlin, N. Y.) are used to make soil profiles (Berger, K. C. and R. J. Muckenhirn, Soil Sei. Soc. of Amer. Proc., 1945, 10, 368-370; Smith, H. W. and C. D. Moodie, Soil Science, 1947, 64, 61-69). Permanent plastic color standards for rapid soil and plant tissue testing are employed by Lynd, J. Q., and L. M. Turk, J. Soc. Agronomy, 1948, 40, 940-941. Plant materials showing deficiency symptoms can be prepared in plasties as deseribed by Lynd, J. Q., Quart. Bull. Mich. State Coll. Agric. Exper. Station, In press, Art. No. 1238.

A process for embedding flowers in plastic so as to preserve their natural shape and color has been deseribed by Specht, R. C., Engineering Progress at the U. of Fla., 1950, IV No. 12, Gainesville, \$1.

Plastids are by definition simply formed bodies. The term is usually applied to certain cytoplasmic bodies in plants. They may be colorless leucoplastids, chloroplastids containing chlorophyll or chrornoplastids containing other pigments. Chlorophyll thus segregated in these bodies acted on by light plays its part in starch production, as hemoglobin (erythroplastids) acts in transport of oxygen. The chloroplastids are easily visible microscopically. Special 
techniques are only required to reveal the organization of the ground substance, holding the chlorophyll, and their rôles in photosynthesis. A full account is provided by Guilliermond, A., The Cytoplasm of the Plant Cell. Waltham: Chronica Botanica Co., 1941, $247 \mathrm{pp}$. (translated from the French by L. R. Atkinson).

Platelet Counts, see Blood Platelets.

Plastosomes, see Mitochondria.

Platino-Acetic-Osmic mixture, see Hermann's Fluid.

Platinum. Intravenous injections of colloidal solutions of platinum in rabbits are described by Duhamel, B. G., C. rend. Soc. de Biol., 1919, 82, 724-726.

For microchemical detection of platinum reference is made to the method of Okamoto and Associates as described by Glick (p. 26).

Platinum Chloride is the name usually given to hydro-chloroplatinic acid. It is used occasionally as an ingredient of fixatives.

Platyhelminthes is the phylum of flatworms. The two classes of important parasites are the Cestodes and Trematodes. See Parasites.

Pleuropneumonia. Staining of organisms. Stain paraffin sections 4 microns thick of tissue fixed in Zenker, Bouin, absolute alcohol or Carnoy's fluid brought down to water directly in Mallory's phosphotungstic acid hematoxylin (1824 hrs.) with out preliminary treatment with permanganate and oxalic acid. Do not wash but blot nearly dry and dehydrate rapidly in absolute alcohol, clear in xylol and mount in balsam. Organisms in lungs appear as deep blue masses of mycelial threads (Turner, $H$. W., Austral. J. Exp. Biol. \& Med. Sci., $1935,13,149-155)$.

Plehn's Stain for malaria plasmodia is described by Craig, p. 289 as uncertain in its action and is not recommended if other modifications of Romanowsky stain are available.

Plimmer's Bodies, see Bird's Eye Inclusions.

Plutonium. Use of micrurgy in study of chemical properties of first microquantities of plutonium, Seaborg, G. T., Science, 1946, 104, 379 also Chambers, R. W. and Kopac, M. J. in McClung's Microscopical Technique, 1950, p. 543.

Pneumonocytes-Written by C. C. Macklin, Dept. of Histological Research, The University of Western Ontario, London, Canada. November 28, 1951-These elements are also known as pulmonary cells (la cellule pulmonaire), epicytes, septal cells, niche cells, alveolar epicytes, alveolar cells, alveolar wall cells, alveolar granular cells, alveolar epi- thelial cells, residual epithelial cells on the pulmonary alveolar walls, little granular cells, etc., and are demonstrable in mammalian lungs by the usual methods of fixation and staining.

Their most prominent feature is an array of Vacuoloids (which see) in the cytoplasm (Macklin, C. C., Anat. Rec. $1947,97,397$ ). This gives to them in ordinary sections a frothy appearance. Many contain phagocytized particles. The latter are the mural phagocytes or dust cells (which see). They are found inserted into canals in partitional alveolar walls, which, when vacated, are natural pores. In the marginal alveolar walls they are inserted into sockets. Most are lodged in crotches of adjoining alveolar walls. Normally they are $5 \mu$ to $15 \mu$ in maximum diameter, but when stimulated exceed this size.

The form is altered by the fixation technique employed to demonstrate them. After perfusion of the pulmonary blood vessels of the unopened thorax with physiological saline solution followed by a fixative such as Bouin's fluid, the pneumonocytes of partitional alveolar walls often in profile have the shape of a carafe with larger head and attenuated foot, and a connecting stalk which is indented by the environing capillary. In material fixed by immersion of a block of lung tissue the cell often appears rounded with little or no indication that it extends from one alveolus to another. When fixation was by filling of the air spaces with preservative the cell is often extended laterally, that is flattened, and appears wider and shorter than normal. Probably the best preservation of normal form is attained in the mouse by immersion of the unopened thorax, cleared of skin and muscle, in fixing fluid. In such preparations the cell, when situated in an alveolar "corner" may show as many as four separate air faces, and a cytosome often forked in longitudinal section (Macklin, C. C., Trans. Roy. Soc. of Canada, Sect. V, 1946, 40, 93111-bibliography).

H. von Hayek, Anat. Anz., 1942, 93, 149-155, estimated their combined volume in both human lungs to be 150 cubic centimeters; and they would thus, in his view, compose a diffuse intrapulmonic alveolar epithelial organ as large as the spleen of man. Sjöstrand and Sjöstrand (Zeitsch. f. mik.-anat. Forsch., 1938, 44, 370-411) give their combined volume as $10 \%$ of the lung tissue.

From them tumors may originate in man and the lower mammals. Mostofi 
and Larsen (J. Nat. Cancer Inst., 1951, $11,1187-1222$ ) have shown that in Strain A mice, adenomata may arise from them during continuous administration of urethane, $0.1 \%$ in the drinking water. Macklin (J. Thor. Surg., 1938, 7, 536551; Trans. Roy. Soc. of Can., 1946, Sect. V, 40, 93-111; and Biol. Bull., $1949,96,173-178$ ) has admitted a high degree of mitotic potency in the pneumonocyte (epicyte) and has accepted it as a primary center for lung cancer.

Pneumonocytes may become phagocytic and appear with more or less foreign material within the cytoplasm. When such cells become free they take a rounded or oval form and are known as epithelial phagocytes, alveolar phagocytes or Dust Cells (which see), and are quite different from the histocytes of the pulmonary connective tissues. Free pneumonocytes without particulate matter within them are known as Foam Cells (which see). In silver-wash preparations (see Silver Lineation) these alveolar mural cells are encircled each by a heavy goldenbrown line, and the air-faces are sprinkled with particles of the same hue.

Large rounded granules of different sizes are seen in them in frozen sections from Regaud-fixed lungs which have been well mordanted with potassium bichromate and strongly stained with Heidenhain's iron hematoxylin (Macklin, C. C., Can. J. Res., D, 1950, 28, 5-15). Similar granules, but gray or black, may be seen in Aquax (which see) sections from lungs that have been filled while fresh with fixative containing from $\frac{1}{15}$ to $1 \%$ of osmium tetroxide. Similar granules, but of dark brown color, have been described in them by Sjöstrand and Sjöstrand (Zeitsch.f. mik.-anat. Forsch., $1938,44,370-411)$ where the living cells have come into contact with blood and an aldehyde, as pure formalin. They find that this colored substance has properties of hemin.

Polarization Optical Method.-Written by Francis O. Schmitt, Dept. of Biology, Massachusetts Institute of Technology, Cambridge, Mass., May 19, 1950.The examination of tissues and cells with the polarizing microscope gives information about the presence of preferentially oriented constituents, the direction of their orientation, their shape, regularity of internal construction, partial volume and refractive index. Details of the theory and methods by which such information may be obtained are contained in the books and papers of Schmidt, Frey-Wyssling and Schmitt listed at the end of this section.
The polarizing microscope is equipped with a polarizer (nicol prism or polaroid disc) below the condenser and an analyzer in the draw tube above the objective. Between the analyzer and objective is a slot into which may be inserted a compensator or gypsum plate. When the planes of polarization of polarizer and analyzer are perpendicular no light passes through the ocular. If a specimen is now placed on the stage, oriented constituents may become visible on a dark field. The intensity will be maximum when the distinguishing direction of the object, such as a fiber, is oriented at $45^{\circ}$ to the planes of polarization of polarizer and analyzer. Objects having internal regularity of structure may have two descriptive refractive indices, hence show double refraction or birefringence. It is the object of polarized light microscopy to detect, measure and interpret this birefringence.

Birefringence is numerically equal to the difference between the two descriptive refractive indices, $N_{0}$ and $N_{0}$. It is usually determined by the use of a compensator which measures the phase difference expressed as fractional wavelength, $\theta$, or retardation, $\Gamma$, expressed in $m \mu$. Thickness of the specimen, $d$, is also expressed in $\mathrm{m} \mu$. Then birefringence $=\mathrm{N}_{0}-\mathrm{N}_{0}=\frac{\theta \lambda}{\mathrm{d}}=\frac{\Gamma}{\mathrm{d}}$.

Commonly used are the Berek, quarter-wave (Sénarmont) and Köhler rotating mica-plate compensators, in order of increasing sensitivity.

Besides the magnitude of birefringence its sign is of importance in diagnosing the ultrastructure of biological constituents. If the refractive index for vibrations paralleling the distinctive direction, e.g. the long axis of a fiber, is greater than that for vibrations perpendicular to this direction, the birefringence is positive with respect to this direction. If the refractive index relations are reversed the birefringence is negative. Most protein and carbohydrate fibers show positive birefringence while nucleic acid and nucleoproteins usually show negative birefringence. While the sign of birefringence may be determined with compensators, the gypsum Red I plate may be very useful. When this plate is inserted into the compensator slot, the field appears red if the nicols are crossed. Birefringent objects show addition or subtraction colors, such as blue or yellow, respectively, depending on the orientation of the object with respect to the planes of polarizer and analyzer and on the sign of birefringence. Thus a fiber of connective tissue 
or muscle will appear blue in one diagonal position and yellow in the diagonal perpendicular thereto; this is because these fibers manifest birefringence which is positive with respect to the fiber axis. A nerve fiber shows the same colors in its myelin sheath except that the diagonal positions in which it shows these colors are reversed from those of the above case; this is because the myelin sheath manifests birefringence which is negative with respect to the fiber axis.

The birefringence of most biological objects is due to regularity of structure of components considerably smaller than the wavelength of light. To get at the nature of these components, one studies the relation of the birefringence to the refractive index of the medium in which the object is immersed, using consecutively a number of media (usually organic solvents) of varying refractive index. Application of Wiener's theory then makes it possible to deduce the orientation of the submicroscopic particles as well as their internal regularity of structure, refractive indices and approximate partial volumes.

Electron microscope observations have confirmed many of the deductions based on the polarization optical analysis of tissue ultrastructure. This method will continue to be of importance biologically despite the great possibilities of the electron microscopy, for the polarized light method is applicable to tissues in the fresh state. See Schmidt, W.J., Die Doppelbrechung von Karyoplasma, Zytoplasma und Metaplasma, Berlin Geb. Borntrager, 1937. Frey-Wyssling, A., Submicroscopic morphology of protoplasm and its derivatives, Elsevier Publishing Co., Inc., 1948. Schmitt, F. O., The ultrastructure of protoplasmic constituents. Physiol. Rev., 1939, 19, 270. Schmitt, F. O., Tissue structure: polarization optical analysis. In Glasser's Medical Physics, Vol. II, 1950, p. 1128. Bennett, H. S., The microscopical investigation of biological materials with polarized light. In McClung's Microscopical Technique, in press.

Polarized Light is said to be better than Marchi and Sudan III methods for study of myelin degeneration of peripheral nerves (Prickett, C. O. and Stevens, C., Am. J. Path., 1939, 15, 241-250). Used in study of mitochondria and Golgi apparatus (Monné, L., Protoplasma, 1939, 32, 184-192).

Polarizing Microscope. The polarizing microscope in its simplest form is a conventional microscope used with planepolarized light for illumination and with a polarizing ocular (called the analyzer) to detect the presence of birefringence. Or it may be thought of as a polariscope equipped with magnifying lenses. This instrument has been borrowed from the minerologists, who employ it in the study of crystalline materials. Its purpose in histology is to seek substances characterized by their ability to rotate the plane of polarized light (Schmitt, 1939).

A Nicol prism, or a disk of Polaroid, is placed below the condenser. Ordinary light is thereby polarized, but goes on to form an image, exactly as in the bright-field microscope. With an analyzer in the ocular, oriented so that its polarizing direction is parallel to that of the polarizer below, one sees the regular image. With a $90^{\circ}$ rotation of the analyzer, however, the field becomes completely dark, except in those places on the slide where birefringent crystals or crystalloidal materials are found. Since this image in general will not be very bright it is best to observe with the dark-adapted eye and to have a very strong source of light for so much light is lost. Starch grains, cholesterol droplets, certain salt crystals, any organic structures with preferentially oriented molecular aggregates, such as nerve fibers (Prickett, C. O. and Stevens, C., Am. J. Path., 1939, 15, 241-250), striated muscle, bone (Kleemen 1945) plant cell walls, etc. stand out well (Johnson, B. K., Endeavour, 1948, 7 , 57-65). With a more elaborate polarizing microscope one may make quantitative measurements leading to the identification of these materials and to certain conclusions regarding the fine structure of organic forms. See Polarization Optical Methods.

\section{Polarographic Technique-Written by} Christopher Carruthers, Division of Cancer Research, Washington University, St. Louis, 10, Mo. October 5, 1951-The polarographic method of analysis is ideally suited for the quantitative and qualitative measurement of minute amounts of reducible substances. It is especially suitable for concentrations of $10^{-6}$ to $10^{-2}$ molar, and since the analysis can be performed with a very small volume of solution, as little as a drop to a few tenths of a cc, traces of reducible substances can be detected. This method was discovered by Professor J. Heyrovsky of Charles University, Prague (Rev. trav. chim., 1925, 44, 488-498). It is based upon the reducibility, or oxidizability, of a substance. For example, when electrons are removed from the ferrous ion, $\mathrm{Fe}^{H}$, it is oxidized to the ferric 
ion $\mathrm{Fe}^{+++}$, or $\mathrm{Fe}^{++} \rightleftarrows \mathrm{Fe}^{++r}+\mathrm{e}$ (electron). Conversely, when an electron is added to the ferric ion, the latter is reduced to the ferrous state. In the polarographic method the electrolysis is carried out with a small easily polarizable electrode, the dropping mercury electrode, and one large non-polarizable electrode.

In the reduction of cadmium ion to metallic cadmium, for example, the applied potential is gradually changed from 0.0 volts and the current flowing through the solution is measured by means of a galvanometer. From 0.0 volt to about -0.5 volt very little current flows through the solution because the potential at which the cadmium ion is reduced has not been reached. At about -0.53 volt the current begins to increase as the voltage is decreased (negative) and at -0.7 volt the current becomes steady again. Between -0.52 volt and -0.7 volt an $\mathrm{S}$ shaped curve is produced, and a measurement of the mid-point of this curve gives the half-wave potential, a characteristic constant of any substance under controlled conditions while the current obtained gives the amount of cadmium in solution.

In other words, the half-wave potential indicates the nature of the reducible substance while the current observed is a function of its concentration. When the current in microamperes is plotted against the applied voltage, the resulting figure is called a polarogram. The small current flowing through the cell prior to reduction is called the residual current. This is due either to the reduction of traces of oxygen, impurities or to the solution of minute amounts of mercury from the dropping mercury electrode. The current, which is obtained at about -0.7 volt for the reduction of cadmium ions, is termed the diffusion current and it is a function of the concentration. The diffusion current is so named since the ions in solution are reduced as fast as they reach the dropping mercury electrode - that is, the reduction is diffusion controlled. Now if the concentration of the cadmium ions is increased, the diffusion rate will increase in proportion to the concentration and this will be reflected by an increase in the diffusion current. A plot of the diffusion current minus the residual current against the concentration can be used for analytical purposes.

The half-wave potential can be employed to determine the nature of unknown substances. For example, when the electrolysis of a mixture of $\mathrm{Fe}^{++}$,
$\mathrm{Pb}^{++}, \mathrm{Zn}^{++}$and $\mathrm{Co}^{++}$is carried out in a solution of $0.1 \mathrm{~N} \mathrm{KCl}$, the supporting electrolyte, the half-wave potentials in volts v.s. the saturated calomel electrode of these ions are respectively $-0.396,-0.995,-1.20$ and -1.3 . The half-wave potentials are separated sufficiently to permit the determination of each one in a mixture of the four ions, and they enable one to determine any of these ions in an unknown solution.

The principal objectives, therefore, of the polarographic method are two. In the first place the determination of the nature of a substance from the halfwave potential. This aspect of the procedure is very important since it enables one to get at structural alterations in reducible compounds from a single measurement. An application of this aspect of the method in biology (carcinogenesis) is given by (Carruthers, C., and Suntzeff, V. Science $1950,114,103-107)$. Secondly, minute amounts of several reducible substances can be quantitatively determined in a mixture without previous separation by the classical chemical methods.

The equation for polarographic analy. sis was developed by Ilkovik (Collec. tion Czechoslov Chem. Commun, 1934, $6,498-513)$. This equation is ${ }^{i} \mathrm{~d}=605$ $\mathrm{nD}^{1 / 2} \mathrm{Cm}^{2 / 3} \mathrm{t}^{1 / 6}$ in which id is the mean diffusion current (in microamperes), $n$ is the number of electrons involved in the reduction of one molecule of reducible substance, $F$ is the Faraday $(96,000$ coulombs), D is the diffusion coefficient (in $\mathrm{cm}^{2} \mathrm{sec}^{-1}$ ), $\mathrm{C}$ is its concentration (millimoles per liter), $\mathrm{m}$ is the weight of mercury flowing from the capillary per second in (mg.) and t (in sec.) is the time necessary for the formation of one drop of mercury. This equation can be reduced to ${ }^{i} \mathrm{~d}=\mathrm{KC}$ when all the other factors are constant. Hence the concentration of a reducible substance is given by the equation $\mathrm{C}=\mathrm{i} \mathrm{d} / \mathrm{K}$. Since $\mathrm{K}$ can be determined expcrimentally from the electrolysis of a reducible substance at known concentrations, the amount in an unknown can be measured from the diffusion current.

For a general and detailed description of the polarographic method of analysis, the following books are recommended: Kolthoff, I. M., and Lingane, J. J. Polarography, New York: Interscience Publishers, Inc. 1941, 215-254; Muller, O. H., The Polarographic Method of Analysis, Easton, Pa., Chemical Education Publishing Co., 1951, 32-80, 141193; Hohn, H., Chemische Analysen 
mit dem Polarographen, Berlin: Verlag von Julius Springer, 1937, 34-47.

There are several American made polarographs which automatically record current-voltage curves (polarograms). These instruments have stimulated research in the field and have simplified the measurements considerably. The following companies make self-recording polarographs: E. H. Sargent and Co., 155-165 East Superior Street, Chicago 11, Ill.; Leeds and Northup, 4912 Stenton Avenue, Philadelphia, Pa.; Rutherford Instrument Co., 8616 Georgia Avenue, Silver Springs, Md. Manual recording polarographs are also made by Fischer Scientific Co., 2850 S. Jefferson Ave., St. Louis, Mo. and E. H. Sargent and Co., 155-165 East Superior St., Chicago, 11 , Ill.

Training in the polargraphic method of analysis is best achieved by working with the instrument under supervision of an expert, or by taking a course in analytical chemistry in which the bases of the method are taught. The latter training can be obtained at the Chemistry Department of the University of Minnesota, Minneapolis, Minn., or at Harvard University, Cambridge, Mass.

Polarographic analysis has been found useful in many fields of endeavor:

Vitamin B (Thiamin). Lingane, J. J. and Davis, O. L., J. Biol. Chem., $1941,137,567-574$ found that this vitamin gave a well defined wave at -0.25 volt (v.s. the saturated calomel electrode in $0.1 \mathrm{~N} \mathrm{KCl}$ ). The method has not been applied to natural products. Vitamin $\mathrm{B}_{2}$ (Riboflavin) has been found to be reducible by the same investigators (Lingane and Davis, Ibid).

Nicotinic Acid and Nicotinamid. Nicotinic acid is reducible in $0.1 \mathrm{~N}$ $\mathrm{NaHCO}_{8}$, Shitkata, M. and Tachi, I., Bull. Agr. Chem. Soc. (Japan) 1927, $3,95-96$. A detailed investigation of the polarographic behavior of nicotinic acid and related compounds has been published by Tompkins, B. C. and Schmidt, C. L. A. (Univ. Calif. Pub. Physiol. 1943, 8, 229-247).

Pantothenic acid is also reducible (Lingane, J. J. and Davis, O. L., Ibid.). Vitamin $\mathrm{C}$ has been determined in fruits and vegetables polarographically by Gillam, W. S. (Indus. and Eng. Chem. Anal. Ed., 1945, 17; 217-220).

Vitamin $\mathrm{E}$ and related compounds have been extensively investigated by Kolthoff and coworkers, see Smith, L. I., Kolthoff, I. M., Wawzonek, S. and Ruoff, P. M., J. Am. Chem. Soc., $1942,64,447-451 ; 644-648$. This class of compounds was oxidized having half- wave potentials of approximately 0.1 to 0.2 volt.

Vitamin $\mathrm{K}$-Vitamin $\mathrm{K}_{6}$ is also reducible at the dropping mercury electrode, see Knoblock, E., Collection Czechoslov Chem. Commun., 1949, 14, 508-531.

Folic acid can be determined polarographically in small amounts in tablets, see Mader, W. J. and Frediani, H., Amal. Chem., 1948, 20, 1199-1201.

Steroids and Related Compounds. Steroids such as testosterone, progesterone, pregnenol-17-one-3, desoxycorticosterone, etc. which have a carboxyl group conjugated with a double bond are reducible (Eisenbrand, $\mathbf{J}$. and Picher, H., Zeit. Physiol. Chem., $1939,260,83-99)$. The reaction products of 17-ketosteroids with Girard's Reagent $\mathrm{T}$ (trimethyl acethydrazide ammonium chloride) make the method more general in scope (Wolfe, J. K., Hershberg, E. C. and Fieser, L. F., J. Biol. Chem., 1940, 136, 653-687).

Minerals. The polarograph is ideally suited for the determination of minute amounts of most of the metals with the exception of the alkali and alkali-earth metals (Kolthoff, I. M., and Lingane, J. J., Ibid.).

Proteins. Very few pure proteins have been studied polarographically. Proteins containing sulphydryl groups or -S-S-groups can be determined and their purification followed by the shape of the catalytic reduction waves (Carruthers, C., H. Biol. Chem., 1947, 171, 641-651). Proteins and polypeptides containing cystine or cysteine, when dissolved in suitably buffered cobalt solutions, produce a catalytic reaction during electrolysis at the dropping mercury electrode (Brdicka, R. Collection Czechoslov. Chem. Commun., 1936, 8, 366-376). This observation was used by some investigators as a test for cancer since the blood sera of normal individuals and of persons suffering from cancer revealed significant differences in the height of the catalytic waves (Brdicka, R., Nature, 1937, 139, 330; 1020-1021). However, this test proved to be nonspecific (Rusch, H. P., Klatt, T., Meloche, V. W., and Dirksen, A. J., Proc. Soc. Exptl. Biol. Med., 1940, 44, 362-365).

Amino acids. Amino acids, with the exception of cysteine and cystine which give catalytic waves in buffered cobalt solutions, are not reducible.

Many other types of organic compounds are also reducible. For example, carcinogenic hydrocarbons such as 3-methylcholanthrene, 3-4, benzypy- 
rene and 9,10-dimethyl-1,2-benzanthracene can be determined polarographically (Wawzonek, S., and Laitinen, H. A., J. Am. Chem. Soc., 1942, 64, 2365-2368).

Many other types of organic compounds (aldehydes, ketones, acids, nitro nitroso and azo compounds, peroxides, sugars (ketases) and heterocylic compounds), which can be qualitatively and quantatively determined polarographically are given in a review by Wawzonek (Anal. Chem., 1949, 21, 61$66)$, and in the books listed above.

Polaroid. This is a polarizing material made up of extremely minute crystals of quinine sulphate periodide. A nitrocellulose film containing the crystals all oriented in the same direction can be mounted between sheets of glass with a total thickness fo about $3 \mathrm{~mm}$. See Bourne, p. 26.

Pollens. The microscopic identification of the different sorts of pollen, especially the allergens, does not involve any complicated technique. From a good textbook, Feinberg, S. M., Allergy in Practice. Chicago, The Year Book Publishing Co., 1944, 798 pp., one is first guided by data on pollens likely to be in the atmosphere at the particular scason and in the special locality. The next step is to spread on microscopic slides very thin films of white petrolatum. Then expose, for measured time, these in a horizontal position coated side up protected by a suitable covering from rain but not so as to interfere with access of air. Examine directly by direct illumination or in dark field. If staining is necessary apply Calberla's solution as described by Gay, L. N., Curtis, H. and Norris, T., Bull. Johns Hopkins Hosp., 1941, 68, 179-189 (glycerin 5 cc.; $95 \%$ alc., 10 cc.; aq. dest., 15 cc.; sat. aq. basic fuchsin, 2 drops). Most important is detailed microscopic comparison of the grains observed with the illustrations in the following monograph: Wodehouse, R. P., Pollen Grains. New York: McGraw-Hill Book Co., 1935.

Poly-Azo Dyes. Chlorazol black E, sudan black B.

Polychromatic Erythroblasts, see Erythrocytes, developmental series.

Polychrome Methylene Blue. Literally many colored, but actually in this case two colored. It is a methylene blue which contains, in addition to the blue itself, large amounts of azures especially $A$ and $B$. These are redder than methy lene blue and are partly responsible for the metachromatic staining (G. meta, beyond + chroma, color) given by polychrome methylene blue. The color is beyond and different from the simple blue by reason of its marked reddish tint. It is usually better to purchase the polychrome methylene blue rather than to make it. If it has to be made dissolve $1 \mathrm{gm}$. methylene blue in 100 cc. $0.5 \%$ aq. $\mathrm{NaHCO}_{3}$; place in steam sterilizer $1 \frac{1}{2}$ hrs.; cool and filter (McClung, p. 334). It slould be a good methylene blue. Goodpasture's (E. W., J.A.M.A., 1917, 69, 998) recipe for polychrome methylene blue is : Boil 400 cc.aq. dest. $+1 \mathrm{gm}$. methylene blue and $1 \mathrm{gm}$. potassium carbonate for $30 \mathrm{~min}$. Cool and add $3 \mathrm{cc}$. acetic acid and shake dissolving ppt. Boil gently down to 200 cc. volume (5 min.). Cool. Eosinates spectra and staining potency (Lillie, R. D. and Roe, M. A., Stain Techn., 1942, 17, 57-63). See also Lillie, R. D., Stain Techn., 1942, 17, 97-110 for acid oxidation methods of polychroming.

Polyethylene glycols are also known as Carbowaxes. See Carbo Wax embedding. Rinehart, J. F. and Suleiman Abul-Haj, A. M. A. Archiv. Path., $1951,51,666-669$ advise the dehydration and embedding of formation fixed tissues and the staining therein of lipids by Sudan and other dyes. This is a great improvement over the frozen section technique.

Polysaccharides-Written by R. D. Hotehkiss, The Rockefeller Institute for Medical Research, New York 21, N. Y. November 14, 1951-Polysaccharides are oxidized by periodic acid with production of polyaldehydes which are stained with Schiff's fuchsin-sulfite reagent. Only substances with 1,2glycol groupings (almost exclusively carbohydrates, which in fixed tissue preparations are chiefly present as polysaccharides, mucins or mucoproteins) are supposed to react in this way. In the Schiff-aldehyde reactions, it is important to note that the fuchsin combines chemically with the aldehydic substances (Wieland and Scheuing, Ber., 1921, 54, 2527-2555); the dye is presumably therefore fixed at the site of the original polysaccharide. J. F. A. McManus (Nature 1946, 158, 202), R. D. Lillie (Bull. Internat. Assoc., Med. Mluseums, 1947, 27, 23-61), and R. D. Hotchkiss (Arch. Biochem. 1948, 16, 131-141) independently discovered and described the periodic acidSchiff method; the description below is that of Hotchkiss' procedure.

Solutions: Alcoholic periodic acid: $0.4 \mathrm{gm}$. periodic acid, dissolved in 15 cc. aq. dest., add $0.14 \mathrm{gm}$. cryst. Na acetate, then add $35 \mathrm{cc}$. alcohol $95 \%$. Reducing rinse: Dissolve $1 \mathrm{gm}$. KI, 
$1 \mathrm{gm}$. Na thiosulfate hydrate in $20 \mathrm{cc}$. aq. dest. Add, with stirring, 30 cc. $95 \%$ alcohol, and then 0.5 cc. $2 \mathrm{~N}$ hydrochloric acid. Precipitated sulfur is allowed to settle out, but the mixture can also be used immediately. Schiff's reagent: fuchsin-sulfite as used in the reaction for Thymonucleic Acid, or the Bauer-Feulgen reaction for Glycogen; maximum sensitivity and stability are obtained by adjusting the acidity to the minimum amount which allows the reagent to dry in a thin film on a glass slide without becoming spontaneously colored. Preserved in the cold. Sulfite wash: Aqueous bisulfite, e.g. 0.2 gni. $K$ metabisulfite and 0.5 cc. conc. hydrochloric acid to $50 \mathrm{cc}$. water.

Procedure: Fix in usual fixatives; alcoholic or picric acid fixatives are suitable if water soluble polysaccharides such as glycogen are to be demonstrated. Bring to $70 \%$ alcohol. Leave $5 \mathrm{~min}$. in periodic acid, rinse with $70 \%$ alcohol, leave in reducing rinse $5 \mathrm{~min}$., rinse with $70 \%$ alcohol, leave in Schiff's reagent 15 to $45 \mathrm{~min}$., wash 2 to 3 times in sulfite water. Examine or dehydrate and mount as desired. Counterstaining with a basic dye such as malachite green (2 mg. per $100 \mathrm{cc}$. aq. dest.) is satisfactory. Control sections treated same except not exposed to periodic acid.

Modifications: Aqueous, instead of alcoholic, periodic acid and reducing rinse may be used, with increased likelihood of removing soluble polysaccharide such as glycogen or dextrins from the section. McManus uses $0.5 \%$ aqueous periodic acid without acetate buffering (Stain Technology, 1948, 23, 99-108); Lillie uses aqueous sodium periodate containing nitric acid (ibid., 1951, 26, 123-136). Periodate or iodate entrapped, or bound to such components as calcium in the sections, will color the Schiff reagent; the reducing rinse is intended to destroy these iodates chemically. Ordinary washing is used in the McManus and Lillie procedures, and is adequate whenever iodate retention does not prove to be a difficulty in the particular tissue under investigation. The periodate-Schiff reaction may be used subsequent to treatment with enzymes such as diastase (Lillie) or hyaluronidase to further identify the material being stained. Isolated substances can be tested for stainability by "spot tests" with the same reagents to help predict their behavior in tissue sections (see Hotchkiss, loc. cit.).

Mechanism of reaction and specificity: Aldehyde groups newly formed by periodic acid and reacting with Schifr's reagent are almost certainly produced by the breakage of 1,2-glycols or equiv alent 1,2-amino alcohols or 1,2-diamines. Biologically occurring substances containing such groups are the simple sugars, polysaccharides, cerebrosides, inositol compounds, certain hydroxy-amino acids, adrenaline. The specificity of the stain further depends in part upon the removal of low molecular compounds in fixation and washing, leaving the polysaccharides (and mucins, glycoproteins) as probably the only known naturally occurring substances which remain to be stained. The sugar residues contained in nucleic acids and the hydroxyamino acids of the proteins (except perhaps terminal residues or hydroxylysine) are chemically so substituted that they do not react with the periodic acid. A number of other substances which reduce the oxidant (such as tryptophan) do not yield aldehyde groups.

Not all sugars or polysaccharides give equally intense coloration (Hotchkiss, loc. cit.: Jeanloz, R., Science $1950,111,289)$ and the presence or amount of color cannot in every single case be predicted from the known structure. A further difficulty is that in some cases the structure of a complex biological material (e.g. hyaluronic acid) is inferred from the chemical study of a highly purified component isolated from only one or two special sources. In such cases the spot tests mentioned may be used to investigate empirically the behavior of crude, or purified, fractions from the tissue uncler consideration.

A method of acetylating tissue sections has been described (McManus, J. F. A. and Cason, J. E., J. Exp. Med., $1950,91,651-654$ ) by which the 1,2 glycol structures are blocked with acetyl groups and no longer are able to be oxidized by periodic acid. Deacetylation with cold alkali introduces an element of greater specificity, since glycol substances such as polysaccharides can now again be stained, but the amino derivatives of glycols are probably not released.

The mechanism of the Bauer-Feulgen and Casella reactions, in which chromic acid and permanganate are the oxidants is probably similar, but these oxidants tend to destroy the aldehyde groups which they first produce. Lillie (loc. cit. 1951) has made a critical study of the various Schiff reactions for carbohydrates and should be consulted for a review of this subject (see also review by McManus, loc. eit. 1948). C. P. 
Leblond (Amer. J. Anat. 1950, 86, 1-50) has surveyed the staining of rat tissues rather thoroughly. A diseussion of the chemical mechanism and tests of many isolated substances were provided by Hotchkiss (loc. eit.)

Uses and applications: The following substances have been stained with the periodic-acid-Schiff methods: glycogen, stareh, mucins, hyaluronie acid, cellulose, ehitin, agar, amyloid, reticulin, kerasin, colloid of the thyroid and hypophysis, collagen. Structures or regions revealed have ineluded basement membranes, cartilage matrix, ground substance of connective tissue, hyaline easts, hyaline of arteriosclerosis, ovarian cyst and folliele fluid, lens capsule, Descemet's membrane, collagenous retieulum, chromaffin, brush border of the renal tubules, elements in the mitotic figures of tumor tissue, zymogen granules, mold myeelium, flagella in certain algae, plant cell walls, sperm acrosomes. The stain is particularly convenient for demonstrating vividly the pathogenic fungi infecting the less intensely staining animal tissues (Kligman, A. M. and Meseon, H., J. Baet., $1950,60,415-421$ ). In a few cases, material stained in tissues has been correlated, with a view to tentative identification, with such specific glycoproteins as gonadotrophic hormone in the pituitary (Catchpole, H. R., Fed. Proc. $1947,6,88)$, renin in the kidney (Marshall, J. and Wakerlin, G. E., Fed. Proc. 1949, 8, 106-7), accessory reproductive gland secretion in beetles (Anderson, J. M., Biol. Bull., 1950, $99,49-64)$, and hyaluronidase in the sperm acrosome (Leutehtenberger, C. and Schrader, F., Proc. Natl. Acad. Sei., 1950, 36, 677-683).

Polyvinyl Alcohol, macromolecular properties (Heuper, W. C., Arch. Path., $1942,33,271$ ). Use in preparing tissues for staining with Sudan III (Lubkin, V. and Carsten, M., Science, 1942, 95, $634)$.

Ponceau B, see Biebrich Scarlet, water soluble.

Ponceau R, RG, G, 4R, 2RE, NR, J, FR, GR, see Ponceau $2 R$.

Ponceau 2R (CI, 79).-Brilliant ponceau G, lake ponceau, new ponceau $4 R$, poncea $u$ R, RG, G, 4R, 2RE, NR, J, FR, GR, searlet $R$, xylidine ponceau $3 R S$. An acid mono-azo dye which may be the ponceau de xylidine called for in Masson's Trichrome Stain.

Ponceau S (CI, 282) of National Aniline Division of Allied Chemical and Dye Corporation is used by Leach, E. H., Stain Teehn., 1946, 21, 107-110 in Curtis' Substitute for Van Gieson Stain.
Ponceau de Xylidine. The diffieulty is that the Fench "ponceau de xylidine" cannot be secured. It appears to be similar to ponceau $2 \mathrm{R}$ (C.I. 79 ) but the latter does not give regularly good results. I.illic (R. D., Stain Teeh., 1940, $15,17-22)$ suggests the following substitutes for ponceau de xylicline: azofuchsin 3B (C.I., 54), nitrazine yellow and biebrich searlet (C.I., 280). See the Biebrich Scarlet and Picro-Anilin Blue method of Lillie. See Masson's Trichrome.

Ponder's Stain for Diphtheria Bacilli, which see.

Ponsol Red 5 GK (CI, 1131) and Ponsol Red $\mathrm{AFF}$, both of DuPont are referred to by Emig, p. 64.

Pontachrome Brown MW (CI, 101) of DuPont, a monoazo mordant dye, light fastness 4 , action of which on blue green algae is described (Emig, p. 31).

Pontachrome Orange R (CI, 415) of DuPont, a direct disazo dye of color fastness 5 . Gives fugitive eolors only (Emig, p. 40).

Pontacyl Carmine 6B (CI, 57), DuPont, is an acid, monoazo dye which colors sections bluish fuchsia darkened by mordanting with potassium biehromate. Not important in microtechnique (Emig, p. 30).

Pontacyl Carmine 2 G (CI, 31)-Made by DuPont. Light fastness 3. More intense color than azofuchsin. Action on fungous mycelia (Emig, p. 29).

Pontamine Fast Pink BL (CI, 353), a disazo direet dye of light fastness 3 to 4 . Use in acid and alkaline solutions as stain for plant tissues and algae are deseribed (Emig, p. 39).

Pontamine Sky Blue 5BX, see Niagara Blue 4B. Use in measurement of lymph flow (MeMaster, P. D., J. Exper. Med., 1937, 65, 373-392).

Poppy Seed Oil, reactions in tissue to fat stains after various fixations (Black, C. E., J. Lab. \& Clin. Med., 1937-38, 23, 1027-1036).

Porphyrins.- Written by Frank H. J. Figge, Dept. of Anatomy, University of Maryland Medical School, Baltimore, Md. October 10, 1951-There is no specific histo-chemical reaction for porphyrins, but Watson, C. J. and Clark, W. O., Proc. Soc. Exp. Biol. \& Med., 1937, $36,65-70$ believe that it is the protoporphyrin in reticulocytes that stains with brilliant eresyl blue. See Reticulocytes.

Watson and his workers and other investigators have more recently modified their views regarding this point (IVatson, C. J., Blood, 1946, 1, 99-120) Keller and Seggel had demonstrated that the porphyrin containing cells were not identical with reticulocytes 
but could be recognized by their fluorescence. These fluorescytes normally constitute only $0.1 \%$ of the red cells (Keller, Ch. J. and $K$. A. Seggel, Folia Haematol, 52, 241, 1934). No parallellism was found to exist between the reticulocytosis in cases of remission of pernicious anemia and the concentration of porphyrin in the erythrocytes, which reaches a maximum after the reticulocyte peak. Watson, Grinstein, and Hawkinson have confirmed this latter observation (J. Clin. Invest., $1944,23,69-80)$. A very comprehensive review describing the distribution of porphyrins in the human body appeared in a book by Lemberg and Legge, "Hematin Compounds and Bile Pigments" (Interscience Publishers, 1949).

Minute quantities of porphyrins may be detected in tissues or solutions by virtue of the red fluorescence of these substances when they are examined in near ultraviolet light (Wood's light). Königsdorfer, Borst, and Fischer employed a spectral analysis microscope to detect and identify porphyrins in histological material (See Fischer and Orth's Die Chemie des Pyrrols, 1937, press of Paul Dunhaupt, Köthen. It is also available in Lithoprint form: Edwards Bros., Ann Arbor, Mich.). Attempts have been made, Klüver, $H$., Science, $1944,99,482-484$, to identify the type of porphyrin present in tissues and in nervous tissue by means of fluorescence spectra determination. The precise identification and determination of porphyrins involves determination of relative solubility in ether and in acid solutions of various concentrations, absorption spectra, and melting points of the methylesters.

The detection of porphyrins in tissues by means of the visually observed red fluorescence is beset with several pitfalls. Red fluorescence is not a specific test, because occasionally other naturally occurring red fluorescent substances are encountered. The red fluorescence of porphyrins may also be masked in at least two ways:

1. The presence of certain substances which quench the fluorescence of the porphyrin, i.e., protoporphyrin and coproporphyrin are abundant in bone marrow, but the fluoresence is not apparent because of the high concentration of heme compounds and other forms of iron.

2 . The presence of a substance or substances with a blue-green or in other words, a complimentary fluorescence spectrum. As one would expect, porphyrin in such a combination gives rise to a white fluorescence, i.e., urine usually contains substances which fluoresce blue-green. The addition of porphyrin changes this to white fluorescent urine. Urine fluoresces red only when the concentration of porphyrin is very high.

For an excellent account of the chemistry and distribution of porphyrins in tissues and organs, the reader is referred to the review of Dobriner, K., and Rhoads, C. P., Physiol. Rev., 1940, 20 , 416-468. Everett's Medical Biochemistry (1942, Paul B. Hoeber, New York) also contains a good summary of this field. In the following discussion, some of the original references to statements regarding porphyrins have been omitted. These may be found in one of the above reviews or in Fischer and Orth. Most of the porphyrins encountered in nature may be classified as type III or type I of the four series of isomers. This is because protoporphyrin, which belongs to the type III series, is involved in the formation of such important substances as chlorophyl, hemoglobin, myoglobin, cytochromes, catalase, peroxidase, and cytochrome oxidase. Protoporphyrin (and a small amount of coproporphyrin) are usually formed during the synthesis, but as a general rule, porphyrin is not formed during the breakdown of these compounds in the liver.

Intestinal bacteria convert many of these heme compounds to protoporphyrin. Deuteroporphyrin, coproporphyrin III, and mesoporphyrin may all be derived from this. These same porphyrins may also result from the sterile autolysis of hemoglobin or myoglobin (Hoagland, R., J. Agr. Res., 1916, 7, 41-45). It is, therefore, probable that these pigments would be present in thrombotic areas, severely damaged tissues, and necrotic tissues in general.

Hematoporphyrin is an artificial porphyrin resulting from the treatment of reduced hemoglobin with strong acids. Since it does not occur in nature, the name is unfortunate and has given rise to much confusion (see "Hematoporphyrin").

Nornally 20-100 micrograms of coproporphyrin I are excreted daily in the urine. Coproporphyrin, as its name implies, is present in large amounts in the feces, but is also found in the amniotic fluid, meconium, and in the sebaceous glands in certain areas of the skin of the human subject (FischerOrth; Figge, Symposium on Cancer, A. A. A. S., 1945, 117-128). In certain pathological states, large amounts of the ether insoluble uroporphyrins are 
excreted in the urine. Protoporphyrin, which is now known to be the same as oöporphyrin, is excreted in relatively large amounts by female birds. A porphyrin-secreting gland deposits this on the egg shell as it passes through the oviduct. The purpose of this is not known. Protoporphyrin and coproporphyrin develop in abundance in eggs as they are incubated and embryonic tissues and fluids in general have a relatively high porphyrin content. Graflin, A. L., Am. J. Anat., 1942, 71, 43-64 gives the technic for histochemical studies of the protoporphyrin in rat harderian glands. This includes several good illustrations. These glands excrete porphyrins which pass via the naso-lachrymal duct and larynx to the gastro-intestinal tract (Figge and Salomon, J. Lab. \& Clin. Med., 1942, 27, 1495-1501). Most of the porphyrin in the feces of rats is derived from the harderian gland excretions. In addition to rats, mice also excrete relatively large amounts of protoporphyrin via the harderian glands. The variability with respect to the red fluorescence of the harderian glands of mice of strains with different susceptibility to spontaneous mammary carcinoma gave rise to the hypothesis that porphyrins were involved in the regulation of susceptibility to mammary carcinoma (Figge, Strong, Strong, Jr., and Shanbrom, Cancer Res. 1942, 2, 335-342). Hamsters, which are very susceptible to chemically-induced tumors, were also found to have brilliant red fluorescent harderian glands. The occurrence of porphyrins in certain organs and tissues of the human subject which exhibit a high cancer incidence (cervix of uterus, skin, etc.) led to the concept that these substances may act as co-carcinogens in a more general manner than postulated at first (Figge, A. A. A. S., 1945, 117128). Jones, E. G., Shaw, H. N., and Figge, F. H. J., Am. J. Obs. \& Gyn., $1946, \quad 51,467-479$ give technics for demonstrating porphyrin on the cervix of the uterus in the human subject. See Hematoporphyrin.

Postmitotic Cells, see Cell Classification.

Postmortem Change. These are alterations in structure due to autolytic and osmotic changes. The rate of autolysis is very rapid in some organs such as the pancreas which are enzyme producers. It is relatively slow in the walls of elastic arteries in which the proportion of inanimate components (elastic and collagenic fibers) is high. In the case of tissues which cannot be immediately fixed certain precautions should be taken to minimize postmortem change.
See Agonal Changes, Artifacts, Fixation, and Small Intestine, Necrosis, Necrobiosis.

Potocytosis, a term introduced by Meltzer to designate submicroscopic "sipping" of fluid by cells. See Pinocytosis.

Pottenger's Dilution Flotation method, see Concentration of bacteria.

Potassium, Histochemical methods.

1. Policard, A. and Pillet, D., Bulld'Hist. Appl., 1926, 3, 230-235, have suggested that potassium and sodium probably occur as chlorides and that their conversion to sulphates by treating the sections with sulphuric anhydride fumes makes them more stable and better able to withstand the high temperature of Microincineration which see.

2. Marza, V. D., Bull. d'Hist. Appl., $1935,13,62-71$ has modified Macallum's well known technique. Fix small pieces of tissue in $96 \%$ pure alc. in the ice box. Make paraffin sections. To eliminate the possibility of the presence of iron leave fcontrol sections $5 \mathrm{~min}$. in freshly prepared sol. yellow ammonium sulphate. Wash in aq. dest., dehydrate, clear and mount in neutral balsam. There should be no ppt. Make up following solutions: A. Cobalt nitrate, 5 gm.; aq. dest., 10 cc.; glacial acetic acid, $2.5 \mathrm{cc}$. B. Sodium nitrite, $25 \mathrm{gm}$.; aq. dest., 36 $\mathrm{gm}$. To $A$ add 41 cc. of $B$ and use immediately. If delay is necessary keep in ice box and filter before using. Cover test sections with this for 2 hrs. in a closed Petri dish to avoid evaporation. Wash slowly in $50 \%$ alc. to remove every trace of reagent. Plunge in ammonium sulphate solution $3 \mathrm{~min}$. Wash in aq. dest. to remove ammonium sulphate. Dehydrate, clear and mount. Examine illustrated paper by Marza and Chiosa (V. D. and L. T., Bull. d'Hist. Appl., 1935, 13, 153-177) on application of this method to the problem of ovogenesis.

3. Gersh, I., Anat. Rec., 1938, 70, 311-329 has also modified Macallum's method. It involves the making of similar paraffin sections as for Chloride, which see. Transfer these to a fairly large cool room $\left(-1^{\circ}\right.$ to $+1^{\circ} \mathrm{C}$.) and remove paraffin and petroleum ether as for chloride. Cover with $12 \%$ sodium cobalti-nitrite solution of Bülman (Treadwell, F. P., Analytical Chemistry, vol. 1, 4th English Ed. translated by W. T. Hall, New York, John Wiley \& Sons, Inc., 1916, p. 81). Decant fluid, mount in glycerin in same way and examine. Crystals of sodium potassium cobalti-nitrite are just visible with oil immersion lens. They are short yellow rods with rounded ends in a diffuse pale 
yellow background soluble at room temperature.

4. Carer-Comes, O., Zeit. f. wis. Mikr., 1938, 55, 1-6 has advised histochemical demonstration of potassium by Siena orange (K. Hollborn), which is sodium paradipicrylamine. Deparaffinize sections of neutral formalin fixed tissue. Place in Siena orange solution, as received ready for use from Kollborn, 2 min. Then $10 \% \mathrm{HCl} 3 \mathrm{~min}$. Wash twice in aq. dest. $10 \mathrm{~min}$. Blot with filter paper and dry at $37^{\circ} \mathrm{C}$. Mount in thickened cedar oil. Tissues containing potassium, orange; others, pale yellow or unstained.

5. Radioactive potassium can be easily measured in tissues and cells. There is $40 \%$ penetration of red blood cells in vivo (Mullins, L. J., Noonan, W. O. and T. R. and Halge, L., Am. J'. Physiol., (1941, 135, 93-101). See Radiopotassium.

Titrimetric methods for potassium. The one of Norberg, B. (C. rend. trav. lab. Carlsberg, Sér. Chim., 1937, 21, 233-241) is given by Glick, p. 268. Apparently the presence of sodium in amounts 150 times that of the potassium did not interfere. The one of Cunning. ham, B., Kirk, P. L. and Brooks, S. C. (J. Biol. Chem., 1941, 139, 21-28) is effective when amount of sodium is not more than 20 times that of potassium and the quantity of potassium is $2 \mu$ gm. + .

Pratt, see Triphenyltetrazolium Chloride.

Preputial Gland of rats. Useful histochemical methods of investigation and changes following thyroidectomy (Montagna, W., Anat. Rec., 1946, 94, 38).

Pressure. Increase in pressure beyond a certain limit, somewhat characteristic for particular cells (300-1000 atmospheres), brings about a liquefaction of the plasmagel which can be directly observed microscopically or determined by certain measurements like action potential for nerve fibers. Danielli (Bourne, p. 38) has expressed the opinion that the factor causing inhibition of movement may, in all cases, be increased hydration of protein molecules and that the method of increased pressure may be of great value to large scale and micro-biologists.

New technique for differential pressure measurements employing condenser manometers as given by Hansen, A. T. and Warburg, E., Acta Physiol. Scand., 22, 211-215.

Price-Carr Reaction, see Carr-Price Reaction.

Primula R Water Soluble, see Hofmann's Violet.

Primulin (CI, 812)-primuline yellow-An acid thiazole dye used in fluorescence microscopy (Pick, J., Zeit. Wis. Mikr., $1935,51,338-351$ ).

Praseodymium, see Atomic Weights.

Primuline Yellow, see Primulin.

Proflavine, a dye similar to Acriflavine.

Prolactan. Methods for assay (Bates, R. W., Cold Spring Harbor Symposium on Quantitative Biol., 1937, 5, 191-197).

Promyelocytes, see Leucocytes, developmental series.

Prontosil as a vital dye (Carter, W., Science, $1939,90,394)$. It is fluorescent.

Propylcarbinol, see n-Butyl Alcohol.

Prostate. This organ cannot be examined microscopically in vivo and supravital staining has not proved very fruitful. The cutting and staining of sections is the conventional method. It is important that the blocks of tissue fixed be oriented with great care, and that microscopic and gross observations be correlated. For normal size and weight see Moore, R. A., Am. J. Path., 1936, 12, 599-624 and for age changes a chapter by the same author in Cowdry's Problems of Ageing, Baltimore: Williams \& Wilkins, 1942, $936 \mathrm{pp}$. Since the structure of the prostate exhibits so many local differences there is a danger of erroneous conclusions from incomplete examination. In their classic paper on the rat-prostate cytology as testis hormone indicator Moore, C. R., Price, D. and Gallagher, T. F., Am. J. Anat., 1930, 45, 71-107 secured best results after fixation in Bouin's Fluid and staining with Harris' Hematoxylin and Eosin.

Swyer, G. I. M., Cancer Research, $1942,2,372-375$ has checked with satisfactory results the Schultz test for cholesterol by chemical analyses. He has also outlined a method for measuring the color in the Liebermann-Burchardt reaction. For singly refractile fat in the epithelial cells see Gylling, P., Acta Path. et Microb. Scan., 1941, 18, 247-258.

To demonstrate the ducts (Le Duc, I. E., J. Urol., 1939, 42, 1217-1241) in autopsy material lay open prostate by incising length of anterior commissure and express secretion from ducts by gentle massage and careful sponging. Locate orifices of ducts with aid of a dissecting microscope. Inject celloidin solution into them through No. 26 or 27 gauge hypodermic needle fitted with tapering solder tip. Then macerate with hydrochloric acid and remove all except casts of the ducts. See his illustrations.

A method for demonstrating arterial supply is described and illustrated in some detail by Flocks, R. H., J. Urol., 
37, 524-548. Inject internal iliac arteries of a fresh cadaver with equal parts barium sulphate and water at $200-$ $250 \mathrm{~mm}$. mercury pressure. But beforehand cut small branch of superior vesical artery to relieve pressure in prostatic vessels. Remove prostate with sufficient surrounding tissue. Cut gland into 5-6 sections each about $1 \mathrm{~cm}$. thick. Dehydrate in ascending alcohols and clear in oil of wintergreen (methyl salicylate).

Examination of corpora amylacea by various methods is described by Moore, R. A., Arch. Path., 1936, 22, 24-40.

Protactinium, see Atomic Weights.

Protanines, see Saint Hilaires Method discussed under Purines.

Protargol. This is a light brown protein silver compound containing approximately $8 \%$ silver. To demonstrate phagocytosis by the reticulo-endothelial system fine suspensions may be injected intravenously (Askanazy, M., Aschoff Path. Anat., Jena, 1923, 1, 183) but the method is not recommended by Foot (McClung, p. 115). Protargol is also used for staining of paraffin sections (Bank, E. W. and Davenport, H. A., Stain Techn., 1940, 15, 9-14). See Silver Methods, Bodian Method, Protein Silver.

Protease. An enzyme located in leucocytes which can be demonstrated in small quantities of blood has been described (Cooke, J. V., Arch. Int. Med., 1932, 49, 836-845). Pickford, G. E. and F. Dorris (Science, 1934, 80, 317-319) have reported a micromethod for protease. DeRobertis, E. (Ann. N. Y. Acad. Sci., $1949,50,317-335$ ) devised micro methods for the analysis of proteolytic activity in thyroid colloid. Sections were incubated on plates covered with gelatin, which was subsequently stained. Proteolytic activity digested the gelatin, causing the film to stain less intensely than neighboring undigested areas. Similar experiments for localizing protease have been carried out using fibrin film as a substrate.

Protein, see following reactions: Alloxan, Axenfeld, Azo, Indo, Ninhydrin, Nitro, Nitroprusside, Nitrosamino, Romieu, Xanthroproteic.

Proteolytic enzymes. These include protease and many others. See Glick, pp. 302-306.

Protein Silver for Staining ProtozoaWritten by Norman Moskowitz, Dept. of Zoology, University of Pennsylvania, Philadelphia. January 24, 1951-When commercially prepared silver products suitable for staining Protozo: by the Bodian silver technic apparently became unavailable, a substitute for
Protargol was prepared as follows: 0.9 g. of granular gelatin is dissolved by heat in $100 \mathrm{ml}$. aq. dest.; to this $0.1 \mathrm{~g}$. of silver nitrate is added at $60^{\circ} \mathrm{C}$.; this solution is poured into Columbia staining dishes $(10 \mathrm{ml}$.) in which one or two drops of $M / 10$ sodium hydroxide have been added. Copper is not used in the impregnating bath. Smears fixed in Hollande's or Scliaudinn's fixatives are bleached and impregnated for 36 hours or more at $35^{\circ} \mathrm{C}$. Impregnated smears are reduced with a mixture of hydroquinone and sodium sulfite, and toned with gold chloride.

1. Fix smears in the recommended fixative.

2. Wash in $50 \%$ alc. to remove fixative.

3. Harden the smears in $70 \%$ alc. in which they may be stored for not more than a few days.

4. Hydrate smears.

5. Bleach: Treat preparations in $0.5 \%$ potassium permanganate $2 \mathrm{~min}$. Wash in at least two changes of aq. dest., 1 min. each. Treat with $5 \%$ oxalic acid 2 min. Wash in three changes aq. dest.

6. Impregnate smears in protein silver solution for 36 hours at approximately $35^{\circ} \mathrm{C}$. Results in staining can be varied by omitting the $M / 10$ sodium hydroxide or by adding one or two drops of $\mathrm{M} / 10$ nitric acid instead of the recommended alkali.

7. Reduction of silver. Rinse impregnated smears in aq. dest. water and treat for 5 minutes in a reducing bath prepared by dissolving $1 \mathrm{~g}$. of hydroquinone and $5 \mathrm{~g}$. of sodium sulfite in $100 \mathrm{ml}$. aq. dest.

8 . Wash in aq. dest. $1 \mathrm{~min}$.

9. Toning. Immerse preparations for 4 min. in $0.2 \%$ ( $1 \%$ may be used) aq. yellow gold chloride. Staining is affected by the duration of the gold toning.

10. Rinse in aq. dest.

11. Treat with $2 \%$ oxalic acid for 3 min. Variation of time in oxalic acid produces staining differences.

12. Wash $1 \mathrm{~min}$. each in of 3 changes aq. dest.

13. Treat with $5 \%$ sodium thiosulfate for $7-8 \mathrm{~min}$.

14. Wash in running tap water for $\frac{1}{2} \mathrm{hr}$. or more.

15. Dehydrate and mount. The impregnating medium should not be exposed to intense daylight at any time.

Proteinase, determinations (Maver, M. E. Mider, G. B., Johnson, J. M. and Thompson, J. W., J. Nat. Cancer Inst., $1941,2,278$ ).

Protosiderin, see Lillie, p. 127. 
Prothrombin, rapid micro test (Abramson, D. J. and Weinstein, J. J., Am. J. Clin. Path. Technical Suppl., 1942, 6, 1-7) :

1. Make M/40 calcium chloride by dissolving $1.11 \mathrm{gms}$, anhydrous calcium chloride C.P. in 400 cc. aq. dest.

2. Make thromboplastin suspension from brain freshly killed rabbit as described by Quick, A. J. Am. J. Clin. Path., 1940, 10, 222. Dehydrate macerated brain in acetone, dry completely, mix with normal saline $(0.3 \mathrm{gm}$. to 5 cc.) and incubate at $50^{\circ} \mathrm{C} .15 \mathrm{~min}$. The supernatant turbid fluid is thromboplastin. It must be kept in ice box when not in use.

3. Measure separately in microhemopipettes $10 \mathrm{cc}$. of calcium chloride sol., of thromboplastin and of blood.

4. After adding blood, mix thoroughly with fine glass rod, tilt gently from side to side until gelation begins, then time end point by passing rod through mass.

Prothrombin time (Sherber, D. A., J. Lab. \& Clin. Med., 1940, 26, 10581061; and Isenberg, H. D., J. Lab. \& Clin. Med., 1951, 37, 807-809).

Protoporphyrin in Harderian glands, see Porphyrins.

Protozoa, staining in bulk. (Stone, W. S., J. Lab. \& Clin. Med., 1935-36, 21, 839842): Suggested for mucous surface protozoa of man and used at Army Medical School. 'Thoroughly emulsify $20 \mathrm{cc}$. feces in $200 \mathrm{cc} .37^{\circ} \mathrm{C}$. physiological saline solution. Allow to stand for 5 min. and pour supernatant fluid into two $50 \mathrm{cc}$. centrifuge tubes. Centrifuge at 1,850 r.p.m. 5 min. Decant supernatant fluids. Examine residue from one, fresh, and to other add $25 \mathrm{cc}$. Schaudinn's Fixative. Mix and leave 24 hrs. Protozoa in cultures and other fluids are to be concentrated by centrifugation and fixed in the same way. Between each of following steps centrifuge organisms and discard supernatant fluid before adding the next. Wash twice in aq. dest. Wash with $70 \%$ alcohol plus sufficient Gram's iodine to make it light brown color, $10 \mathrm{~min}$. Wash $70 \%$ alcohol $10 \mathrm{~min}$. Stain Harris' Hematoxylin 1-24 hrs. Wash tap water. Destain by adding $20 \mathrm{cc}$. acid alcohol $(1 \% \mathrm{HCl}$ in $70 \%)$ controlled by microscope. When desired definition is reached add sufficient ammonia water (6 drops $\mathrm{NH}_{4} \mathrm{OH}$ to 50 cc. aq. dest.) to neutralize acid and give bright blue solution. Wash in tap water. Dehydrate 10 mins. in each of 5 alcohols: $70,95,95$, abs., and abs. Clear in xylol. Mount in balsam. See author's figures.

Perhaps the best method for concen- trating and sectioning protozoa is that of Lucas, M. S., Science, 1929, 70, 482483. Use a round bottom vial. Let protozoa settle to bottom, pipette off fluid to within $4 \mathrm{~mm}$. of level of top of protozoan mass, then add dilute alcohol. Next change, pipette off, and add stronger alcohol. Alcohol, xylol, pure xylol, melted paraffin (the vial being held under an electric bulb, etc.) several changes of each. Finally lower protozoa with as little paraffin as possible into a specially prepared paper tray and harden.

Levine W. D., Stain Techn., 1939, 14, 29-30 suggests following method to make Methylene Blue stains permanent: Wash methylene blue stained smears of protozoa repeatedly in aq. dest. $15 \mathrm{~min}$. to $1 \mathrm{hr}$. Place in tertiary butyl alcohol 1-2 min. then in 3 or more changes $15 \mathrm{~min}$. each. Pass through xylol to balsam or mount directly in balsam. Other dyes like toluidin blue 0 , nile blue sulfate, eosin $Y$, ponceau $2 \mathrm{R}$ can likewise be retained.

The protargol method of Bodian has been adjusted to protozoa by Cole, R. M. and Day, M. F., J. Parasitology, 1940, 26 Suppl. 29. See also Parasites, Endamoeba Leishmania, Leucocytozoa, Malaria, Intestinal Protozoa. Wenyon, C. M., Protozoology. New York: William Wood, $1926,1563 \mathrm{pp}$. is a convenient book of reference. It gives a fine list of blood protozoa. No investigator can afford to ignore the discussion by Wenrich, D. H., J. Parasitol., 1941, 27, $1-28$ of alterations in the form of protozoa resulting from variations in microtechnique.

Protozoa can be beautifully demonstrated by fluorochromes showing in ultraviolet light various fluorescent colors (Metcalf, R. L. and Patton, R. L., Stain Techn., 1944, 19, 11-27).

Obviously the investigation of protozoa cxtends far beyond their identification in preparations made by various methods. Those dealing with pathogenic protozoa will greatly extend their horizon by consideration of the form and function of these organisms and the ingenious techniques of investigation ably presented in a volume entitled Protozoa in Biological Research edited by Calkins, G. N. and Summers, F. M., New York: Columbia University Press, 1941, 1148 pp. See Intestinal Protozoa, Protein Silver.

Protozoa. Media. The following are recommended for intestinal protozoa by $\mathrm{Q}$. M. Geiman (Simmons and Gentzkow, 617-619):

1. Modification of Cleveland's and Sanders' (for E. histolytica). (1) Dis- 
solve $33 \mathrm{gm}$. Bacto-Entamoeba medium (Difco) in 1000 cc. aq. dest. Pour in test tubes in amounts sufficient to make medium length slants with no butts. Autoclave, slant, harden at room temperature several days. (2) Place few $\mathrm{gm}$. Bacto-Rice-Starch powder (Difeo) in $18 \times 150 \mathrm{~mm}$. culture tube and steril ize with tube horizontal in hot air oven $160-180^{\circ} \mathrm{C} .1 \mathrm{hr}$. Repeat twice at daily intervals being careful to avoid chemical changes in the starch occasioned by higher temperatures. (3) Dissolve $11.23 \mathrm{gm}$. $\mathrm{Na}_{2} \mathrm{HPO}_{4} 12 \mathrm{H}_{2} \mathrm{O}+0.269 \mathrm{gm}$. $\mathrm{KH}_{2} \mathrm{PO}_{4}+8.0 \mathrm{gm} . \mathrm{NaCl}$ in aq. dest. to make $1000 \mathrm{cc}$., autoclave $15 \mathrm{lbs} .20$ min. and cool. Add 10 parts above solution to 1 part sterile horse serum. Cover $\frac{3}{4}$ of each slant with this mixture, add 2-3 loopfuls of the sterile starch, incubate $37^{\circ} \mathrm{C} .24$ hrs. to prove sterility. Final pH should be 7-7.2. Store in refrigerator till used.

2. Boeck and Drbohlav's. Wash 6 eggs in $70 \%$ alcohol and emulsify contents in $75 \mathrm{cc}$. sterile Locke or Ringer. Distribute in $4 \mathrm{cc}$. lots in $15 \times 150 \mathrm{~mm}$. culture tubes, slant in inspissator and heat $70^{\circ} \mathrm{C}$. till mixture solidifies, then autoclave $15 \mathrm{lbs}, 20 \mathrm{~min}$. Slant tubes in autoclave, close doors and ports, turn in steam increasing quickly to $15 \mathrm{lbs}$., for $10 \mathrm{~min}$. Through lower port run in live steam in place of steam-air mixture maintaining constant 15 lbs. pressure. After replacement by steam close lower port and hold $15 \mathrm{lbs}$. another $15 \mathrm{~min}$. Cut off steam and let cool slowly. Cover each slant with 4 cc. 10:1 Ringerhorse serum mixture +2 or 3 loopful sterile rice starch. Incubate $37^{\circ} \mathrm{C}$., $2 \pm$ hrs. to prove sterility.

3. Nutrient agar serum-saline. Cover long slants of nutrient agar (Difco. $1.5 \%$ ) in standard test tubes $\frac{1}{2}$ to $\frac{1}{4}$ with 20:1 sterile Ringer-horse serum mixture. Smaller quantity for intestinal flagellates, larger quantity for Trichomonas vaginalis.

4. Trussell and Plass (for Trichomonas vaginalis). Overlay slants of liver infusion agar (Difco) with a selected mixture as for nutrient agar medium. Adjustment of agar and solution by $1 N \mathrm{HCl}$ and $0.25 \%$ aq. sodium phosphate is suggested, likewise addition of $0.2 \% \mathrm{aq}$. dextrose. Incubate $37^{\circ} \mathrm{C}$., 24 hrs. to prove sterility; store in refrigerator.

The technique of obtaining cultures of protozoa free from bacteria has been described in a comprehensive fashion by G. W. Kidder in Calkins, G. N. and Summers, F. MI., Protozoa in Biological Rescarch. New York: Columbia University Press, 1941, $1148 \mathrm{pp}$. He was concerned mainly with protozoa from natural waters, soil and so forth, closely associated with bacteria throughout their existence. 'The techniques advo. cated are of 3 types: (1) to get rid of the bacteria by simply washing the protozoa in sterile fluid; (2) to serape off the adhering bacteria by causing the protozoa to migrate through semi-solid media and (3) to kill off the bacteria by agents non-toxic for the protozoa. The establishment of sterilized protozoa in culture is an essential prerequisite to investigation of their behavior in response to accessory food factors and nutritional supplements.

Prussian Blue (CI, 128S) is ferric ferrocyanide, a colored salt. It is also known in commerce as Berlin blue, Chinese blue, Paris blue, Milori blue and Steel blue. An aqueous solution of Prussian blue is a good medium for the injection of blood vessels. It contrasts nicely with carmine. The particles of both are sufficiently large to be held within the endothelium. Deposition of Prussian blue is useful in the localization of drainage of Cerebrospinal Fluid (Weed, L. H., J. Med. Res., 1914, 26, 21-117) and in the microchemical demonstration of Iron (Gomori, G., Am. J. Path., 1936, 12, 655-663). See Berlin Blue.

Pulp of Teeth. This can be studied in situ in undecalcified teeth or in paraffin or celloidin sections of decalcified ones. See Teeth. If it is to be examined by itself after removal from the teeth and fixation, attempt to preserve its natural elongated shape. Almost all methods available for other soft tissues are applicable. Wellings, A. W., Practical Microscopy of Teeth and Associated Parts. London: John Bale, Sons \& Curnow, Ltd. 1938, 281 pp. gives many of them. See Teeth, Innervation.

Purines. See critical evaluation of microchemical techniques for purines by Glick, p. 72-73. The difficulty is that the tests are positive for all the purines and specificity is lacking. SaintHilaire's method of precipitating them as insoluble copper salts and the formation therefrom of red ferrocyanide gives positive reactions for protanines, histones and other protein products. Detection by reduction of silver salts is worthless.

The murex test is positive with xanthine, guanine and uric acid but negative with adenine and hypoxanthine (Lison, 1936, p. 186). However, it involves the use of strong acid and alkalis and is thus very drastic. It is included by Glick since it may be useful in some cases though it is no different from the Xanthroproteic Reaction. To a section 
prepared by standard methods add 1 drop conc. nitric acid and warm gently for 30 sec. Drain off acid with blotting paper. Add drop of aq. dest. and remove in same fashion. Expose section to ammonia vapor. Uric acid, guanine, xanthine and its methyl derivatives, purple violet; protein material often yellow orange.

Psittacosis, method for staining elementary bodies (Hornus, G. J. P., Ann. Inst. Pasteur, 1940, 64, 97-116). See other kinds of Elementary Bodies.

Purkinje Cells of heart. Distend entire heart by injecting fixative through 4 cannulae, in aorta, in pulmonary artery, in superior vena cava, in one pulmonary vein and ligating other vessels. Fix in Zenker's or Bouin's fluid. Sinoauricular node is at junction of superior vena cava and right auricle. Cut blocks perpendicular to the node. Color paraffin sections with Masson's trichrome stain or with hematoxylin and eosin for transitions between Purkinje and cardiac muscle cells. The sharpest differential stain for the former is Best's carmine stain for glycogen (Taussig, H. B., J. Tech. Methods, 1934, 13, 85$87)$.

Purkinje Fibers. In excising the specimen the presence of Purkinje fibers is localized by the dimpling in a cross section because in the fresh state the Purkinje fibers contract more than the cardiac fibers (Todd, T. W. in Cowdry's Special Cytology, 1932, 2, 1179). Todd recommends for general purposes Bouin's fixative and Mallory's stain. Safranin light green is good for the intercalated discs (Jordan, H. E., and Banks, J. B., Am. J. Anat., 1917, 22, 285-338). Techniques for bringing out the Purkinje system particularly of mammalian ventricles are described by Abramson, D. I. and Margolin, S., J. Anat., 1935$36,70,250-259$.

Purpurin (CI, 1037)-alizarin No. 6, alizarin purpurin-An acid anthraquinon dye. The bright red color of madder-stained bones is due to purpurin carboxylic acid (Richter, D., Biochem. J., 1937, 31, 591-595).

Pycnosis (G. pyknos, dense) When the substance of a cell, as seen in stained sections is unusually dense it is sometimes said to be pycnotic. The increase in density is usually accompanied by a decrease in size of cytoplasm and/or nucleus and the nucleus may be hyperchromatic, that is have an increased affinity for stains like hematoxylin and methylene blue. Sometimes pycnotic cells occur singly surrounded by others not in the same condition but they may be present in group. Those in the cen- tral nervous system have been called chromophile cells (Cowdry, E. V., Contrib. to Embry., Carnegie Inst., $1917,11,29-41$ ). Information is needed on the cause or causes of pycnosis and on the fate of cells in this condition. Technique for the microspectrophotometric study of pyknosis) of red blood cell nuclei is given by Korson, R., J. Exp. Med., 1951, 93, 121-128.

Pyoktanin Yellow, see Auramin.

Pyoktaninum Aureum, see Auramin.

Pyoktaninum Coeruleum, see Methyl Violet.

Pyridoxine, see Vitamin $\mathbf{B}_{8}$.

Pyronin. There are 2 pyronins: $\mathrm{B}$ (CI, 741 ) and $\mathrm{Y}(\mathrm{CI}, 739)$ also known as $\mathrm{G}$. Conn (p. 140) describes them as closely related to diphenyl methanes since they have one carbon atom attached to 2 benzene rings and exhibit similar tendency to quinone structure. Their formula also resembles that of oxazins except that nitrogen of central ring is replaced by $\mathrm{CH}$ radical. Pyronin $B$ is tetra-ethyl diamino xanthene and $\mathrm{Y}$ is the tetra-methyl compound. Conn (McClung p. 599) advises $Y$ with methyl green in Pappenheim's stain, for the granules of mast cells and the gonococcus in smears of pus. B is satisfactory for most purposes. Only recently has the distinction been made so that most formulae call simply for pyronin. American pyronins are now more concentrated than those imported before 1914. Conn says that allowance should be made for this difference in the proportions of pyronin and methyl green.

Pyronin G is the best supravital stain for the duct system of the pancreas (Bensley, R. R., 1911, 12, 297-388). It is applied by Perfusion a solution of $1: 1000$ in $0.85 \%$ aq. $\mathrm{NaCl}$ being used until the pancreas takes a light rose color. Small pieces are then mounted in salt solution and examined. The ducts from the main ones to the centroacinous cells are sharply stained in red against an almost colorless background. The ducts may be similarly stained by methylene blue in a concentration of $1: 10,000$. To obtain a beautiful contrast coloration Bensley injects with a salt solution containing 1:100 pyronin and $1: 15,000$ janus green. This stains the ducts red and the islets bluish green. The combination of $1: 1000$ pyronin and $1: 15,000$ neutral red also demonstrates ducts and islets but without an equally distinct color contrast. The pyronin method for ducts is one of the most useful techniques both for investigation and for class room demonstration.

Pyrosin B, see Erythrosin, bluish. 
Pyroxylin (collodion cotton, colloxylin, soluble gun cotton, xyloidin, cellodion wood). It is chiefly cellulose tetranitrite. Mainly used in manufacture of Collodions, Celloidin, Paraloidin, Photoxylin, etc.

Pyrrol Compounds, see Nitro Reaction, Nitrosamino Reaction.

"Quad" Stain. A recent modification of this excellent orcein-alizarine-Orange G phosphotungstic and phosphormolybdic acid technique is given in detail by Kornhauser, S. I., Stain Techn., $1945,20,33-35$.

Quartz Fiber Balance and quartz torsion balances, see Balances.

Quartz Rod Technique for Illuminating Living Organs.-Written by Dr. M. H. Knisely, Department of Anatomy, University of South Carolina, Charleston, S. C. June 27,1950 -The general purpose of this technique is to permit direct microscopic study of living internal organ in situ while maintaining experimental conditions which disturb the structures and processes to be observed as little as possible. Like all techniques it has advantages and limitations; there are specific purposes for which it works well, and purposes for which it has not yet worked at all. The method makes it possible to study at 32 to about 600 diameters magnification those living structures whose colors and/or indices or refraction differ from those of adjacent structures. With quartz rods we can illuminate for examination under nearly normal conditions many living tissues and organs which heretofore have been inaccessible. The method depends upon two physical principles:

1. Conducting light from a suitably intense source directly to the structures to be studied by way of a fused quartz rod. Clean, smooth transparent rods conduct light around bends and turns by internal reflection almost like a hose conducts water. With suitably shaped rods brilliant illumination of relatively inaccessible structures is relatively easy. As evidence of intensity, with a 750 watt T-12 tungsten filament bulb and a two foot length of 7 millimeter rod, so much light can be sent into a microscope objective that one can scarcely look into the ocular. Lesser degrees of intensity are of course easily obtainable. Substitutes for quartz rods have been suggested and occasionally used. (Cole, E. C., Science, 1938, 87, 396-398. Williams, R. G., Anat. Rec., 1941, 79, 263-270). We have tested several. No substitute has yet proven as effective for illuminating living tissues as fused quartz itself.
2. Maintaining the normal temperatures of intensely illuminated living structures with a slowly flowing isotonic isothermal wash solution. It is impossible to illuminate a non-transparent structure without heating it at the same time. The color of an object, even a translucent object, as seen by either transmitted or reflected light is due to the patterns of the wave lengths which reach the eye after parts of the incident light are "absorbed", and the word absorbed here means transformed into heat by and within the substance of the object seen. Light filters as commonly used between light source and illuminated object can shelter a specimen from the wave lengths which the filters absorb, but they do not alter the fact that a part of the light energy which passes the filters and falls on the specimen is always transformed into heat within the specimen by the materials of the specimen itself. Hence, in continuously illuminating a living object heat is simultaneously developed in it at a constant rate. If the specimen is small, thin, and very nearly transparent and if its illumination is dim, the small amount of continuously produced heat may be transferred to adjacent objects so rapidly that the temperature of the specimen never rises enough to interfere with its normal functioning. However, in illuminating relatively thick translucent structures such as frog kidney or liver, or mammalian spleens, brightly enough for microscopic study, heat is developed in the illuminated structures faster than it can be removed without assistance. To remove this heat a flowing solution at constant temperature is applied to the illuminated tissue, either through sets of glass tubes, or more recently through hollow tipped quartz rods which deliver both light and flow ing solution precisely to the selected portions of the specimen. The fluid delivered to the tissue must of course be isothermal and isotonic with the fluid which normally bathes it, i.e. plain water at room temperature is used to carry heat from frog skin or tongue, amphibian Ringer's solution at room temperature to carry heat from frog kidney, and mammalian Ringer's at mammalian body temperature to carry heat from monkey omentum. On account of the high specific heat of water the flowing solution can take up the heat as fast as it is produced with but little change in its own temperature; each small portion of flowing solution is warmed but little as it passes through, then leaves the illuminated field. By these physical mechanisms the heat in- 
escapably developed by transformation of light energy is removed as fast as it is produced and in consequence the temperature of the illuminated tissue does not rise.

Thus far in a series of careful tests we have found no visible change in any structure and/or process within any living tissue or organ in response either to a sudden change from dim to intense illumination or to hours of continuous intense illumination, provided the temperature of the illuminated specimen was maintained normal by a continuously flowing solution. In the best experiments the tissue being studied floats on a thin film of slowly moving fluid but does not itself touch the rod which conducts light to it.

For more detailed descriptions of the method see Knisely, M. H., Anat. Rec., 1936, 64, 499-524; McClung, C. E., Handbook of Microscopical Techniques for Workers in Animal and Plant Tissues, New York: Paul B. Hoeber, Inc., 1937, p. 632-642; Knisely, M. H., Anat. Rec., 1938, 71, 503-508; Hoerr, N. L., 1944, see, Glasser, O., Medical Physics, Chicago: Year Book Publishers, Inc., 1944, 625-626.

The limitations and range of applicability and usefulness of this technique may be roughly indicated by a few notes describing some of its current and projected uses. As the method depends upon seeing, its usefulness is continuously limited by the mechanisms whereby we see. As a brief rough statement we "see" by recognizing patterns of color and/or intensity of the light "rays" coming to the retina. The vascular system with its refractile (brightly transparent) vessel walls, plasma and white cells, and its brightly colored erythrocytes is one of the most conspicuous features of living tissues and has thus far in our laboratory received more attention than other living structures. Further, the vascular system is worth intensive study, because from moment to moment continuously under all conditions of health and disease it sets the maximum rates at which oxygen, glucose and other anabolites are carried to and metabolites are removed from, almost every cell, tissue, and organ of the body. For an elaboration of this theme see: Knisely, M. H., Stratman-Thomas, W. K., Eliot, T. S. and Bloch, E. H., J. Nat. Malaria Soc., $1945,4,285-300$.

For microscopic study of the peripheral vascular beds of internal organs, the method is limited by the necessity of an anesthetic, an operation, and the exposure of the surfaces of internal organs to the outer air, an unusual gaseous environment.

The method is most successful when employed to examine structures just below normal anatomical surfaces, rather than just under cut surfaces of tissues. Thus studies have been carried out in frog skin, brain, peripheral nerves, smooth muscles of the gastrointestinal tract, stomach mucosa, mesentery, striated muscles, lung, suprarenal, kidney, and liver, and in mammalian spleen, stomach and intestinal wall, intestinal villi, omentum, mesenteries, liver, and brain surfaces. All these have natural anatomical surfaces which can be exposed without damaging the underlying microscopic structures. In contrast, much as we would like to study mammalian bone marrow, we have not yet found a way to expose a portion of it while preserving its structures and their functioning well enough so that the specimen was worth any serious attention.

The conditions of an experiment limit the phenomena which occur during that experiment. An anesthetized animal obviously does not run or swim about; it cannot perform many obvious wellknown functions of normal unanesthetized animals. By extension, there is no reason to assume that a particular set of experimental conditions do not inhibit, retard, alter, or prevent functions as yet unknown, or one or more phases of the particular functions one is trying to study. When one selects an anesthetic, gives an animal a specific quantity of it, ties the animal down, and operates upon it, he thereby puts that animal's circulatory system into one of its reaction states, and all tests made on the animal from that time on can show only various factors of that reaction state or those deviations from it which are possible under those particular experimental conditions. For example, the circulatory responses to exercise are not occurring in an anesthetized animal whose muscles have been and are in a prolonged state of rest. It cannot be too strongly emphasized that within our experience each experiment, or class of experiments, always acts toward minimizing or preventing known and probably unknown functions. Each time that a new type of experiment has been devised, new kinds or degrees of responses of peripheral vascular beds have been encountered. Each time we have learned how to maintain lesser degrees of anesthesia and/or to do less damaging operations the peripheral vascular beds have exhibited increasingly complex integrated reac- 
tions. For some detailed deseriptions of complex integrated vaseular reactions see Knisely, M. II., Bloch, E. H., and Warner L., K. Danske-videnskabernes selskab. Biologiske skrifter. 1947, 4: (No. 7).

By careful operative techniques some of the common deleterious effects of operations can be prevented. Bloodless sludgeless operations ean be performed on animals from those as small as frogs and mice up to those at least as large as rhesus monkeys. Sufficient care can be taken so that almost no blood is lost; simultaneously eare can be taken to traumatize but very little tissue, thus minimizing the amounts of precipitated-agglutinated blood pouring from traumatized tissues into the general circulation (Knisely, M. H., Eliot, T. S., and Bloch, E. H., "Sludged Blood in Traumatic Shoek", Arehives of Surgery, 1945, 51, 220-236). As (a) hemorrhage and (b) preeipitationagglutination of the circulating blood are two separate factors which ean act alone or in combination in initiating some of the pathologie proeesses which are commonly included under the term "shoek", it cannot be too strongly emphasized that bloodless sludgeless operations must be performed if one wishes to study the cireulatory system when its parts are not participating in shoek reactions.

Living tissues move, and the movements tend to limit the microscopic study of living structures. When an objeet moves under a microseope, each point of its microseopic image moves as many times as far as the object moves as the magnifying power of the lenses employed. Thus, at 100 diameters magnification each point of an image moves 100 times as far as the eorrespond. ing part of the object. Further, the image moves during the same time interval that the object moves, so in each small interval of time the image goes 100 times as far as the objeet: thus at all times during the movement the image is going 100 times as fast as the object. From this example it is obvious that when an objeet moves under a mieroscope each point of the image moves as many times as far and as many times as fast as the object moves, as the magnifying power of the lens system employed. These faetors rapidly inerease the difficulty of observing moving struetures as higher magnifications are used. However, the movements of most tissues do not present as formidable an obstacle as the bare statement of the problem might imply. For as one gains experienee in working with living tissues, many small methods are developed for holding tissues still, and for observing between movements, and one learns to swing his eyes with the image and observe many details sharply even while the tissues are in moderately rapid motion.

The depth in the transilluminated tissue to which one can observe is limited by a number of factors. Most important is the focal length of the lenses employed, the higher the magnifications used the more closely are observations restricted toward surface structures. The natural transparency or translucency of the tissues also limits the depth of observations. Some curious effects result from this, for instance: when smooth muscle is relaxed it is on the transparent side of translucent, but when it contracts it beeomes quite opaque, hence, in this tissue, the maximum possible depths of observations are a funetion of the physiological state of the tissue. For similar and other reasons, such as the amount of blood present at any moment in very vascular tissues, the depth to which one can see in many tissues is partly dependent on the particular set of physiologic processes going on at the time the tissue is studied.

The maximum duration of the observations made in any one animal depends upon the species, the care in maintaining light anesthesia, the care exercised in the initial operation, and the purpose of the study itself. Individual frog kidney glomeruli have often been kept under continuous observation at magnifieations up to 400 (sometimes 600 ), up to as long as $12 \mathrm{hrs}$., without injuring the tissues enough so that the blood began to agglutinate or so that passing white eells ever began to stick to the inner surfaces of the brilliantly illuninated glomerular endothelium. (Clark, E. R. and E. L., Am. J. Anat., 1935, 57, 385-438). For a record of prolonged observations see Knisely, M. H., Stratman-Thomas, W. K., Eliot, T. S. and Bloch, E. H., J. Nat. Malaria Soc., $1945,4,285-300$.

Thus far the limitations of the method have been more eonsidered than the range of its usefulness. The limitations are important and must be clearly recognized and understood by all who plan either to use it or to evaluate reports of work done by means of it. However, as one purpose of this book is to help experimenters seleet methods which may be useful to them, the range of usefulness of the method will now be roughly outlined.

The fused quartz methol, like all others does not have uses which are in- 
dependent of the purposes of those who use it. Methods are always dependent upon purposes. Analytical mechanistic biologists are working on the solutions of many problems including: How are the bodies of the adults of each species constructed? How does each body develop? How does it change with time? How is it constructed while it is alive? How is it constructed so that it can function? What physical and chemical functions does each small part have? During each phase of physiology how does each small part behave? How do the coordinated functions of the small parts summate? How does the function or functions of each small part contribute at each moment to the integrated symphony of the functioning of the whole? Further, what can go wrong with each part? And in addition the clinical sciences continually ask, "What can we do to prevent or help repair whatever can go wrong with each part, with each group of parts, with the integrated functioning of the body as a whole?"

Histological studies are made for a definite purpose, to help collect evidences from which to develop accurate concepts of the structure, functioning and responses of the small parts of living bodies. When we have accurate concepts of the structure and behavior of small parts then we can deal inductively with this information and so build up concepts of the functioning of whole organs. Our current more trustworthy concepts of the structure and function of the kidney were built up by this inductive approach (Vimtrup, Bj., Am. J. Anat., 1928, 41, 123-151; Richards, A. N., Proc. Royal Soc. London B. $1938, \mathbf{1 2 6}, 398-432$ ), which is exactly opposite from trying to deduce the function of microscopic parts from the results of gross experiments performed on whole organs or systems.

Each living animal lives in four dimensions, three of space and one of time. At any moment each feature of an animal's structure exists in the three space dimensions. But many features of the spacial architecture undergo rapid or slow cyclical, intermittent, or progressive changes with time. The chemical and physical characteristics, the shapes, the magnitudes and the positions of structures change as parts of development, of physiology and of pathology. New structures appear and old ones disappear. These are changes along the time dimension. The rates at which changes occur are most important parts of our concepts of the struc- ture and functioning of the small parts of living bodies.

The usefulness of microscopic studies of living organized tissues (as opposed to tissue cultures) becomes most apparent when one recognizes the limitations of histological sections. A histological section is not the original living material. It is only a two dimensisnal slice out of a four dimensional system, minus what had been lost and plus what has been added in its preservation-preparation. No one can possibly begin to appreciate "what has been lost" in the preparation of histological sections unless and until he studies tissues by methods which do not involve any of the steps commonly used in preparing sections.

The spacial dimensions of living tissues are invariably altered in the preparation of histological sections. The alterations in dimensions frequently or usually are as great or greater than the changes in dimension which microscopic structures undergo as parts of their own physiologic processes. Hollow structures, for example, liver sinusoids, collapse during death and fixation, their fixed tissue dimensions becoming less than meaningless. Inowledge of the exact dimensions of structures, the surface areas of vascular networks, the surface areas of glands etc., are urgently needed as a basis for quantitative physiological work. (Krogh, A., Anatomy and Physiology of Capillaries, New Haven: Yale University Press, 1929, p. 46.) It cannot be too strongly emphasized that for strict, mathematical treatment of physjological problems (Bloch, I., Bull. Math. Biophysics, 1941, 3, 121-126, ibid., 1943, 5, 1-14) measurements of the dimensions of microscopic structures taken from fixed tissues, untempered by knowledge obtained from the living, cannot be used. For after the abuse which the tissues undergo in death and fixation, shrinking and swelling in various reagents, and the mechanical distortions caused by the cutting processes (Dempster, W. T., Anat. Rec., 1942, 84, 241-267, ibid, 269-274, Stain Technol. $1943,18,13-24)$, the dimensions of the microscopic parts of a section bear no known or at present knowable relationship to any of the size or sizes which these parts had in life.

In the light of the above paragraphs it becomes apparent that microscopic observations of living organized tissues, illuminated by quartz rods or other techniques, makes available certain classes of information not obtainable 
by other histological techniques. This method permits study of the following: 1. The true dimensions of visible microscopic structures. Further, it permits direct study of changes of dimensions of structures during physiologic and/or pathologic processes. 'The dimensions of visible structures can be measured by ocular micrometers, or by taking motion pictures of the structures and making "cine tracings" of their projected images (Knisely, M. H., Eliot, T. S., and Bloch, E. H., 1945; Knisely, M. H., Bloch, E. H., and Warner, L., cited above). When a set of physiologic processes have been studied throughout their course, the method then permits study of the dimensions of living microscopic structures during defined phases of physiologic processes, or during de. fined physiologic states. (The same can be said of pathologic processes.) The results of this kind of study are quite different from summations of the records of dimensions of tissues taken at unknown phases of physiologic processes and studied and measured after unknown amounts of distortion. For an example which demonstrates this see Knisely, M. H., Bloch, E. H., and Warner, L., cited above.

2 . The rates and changes in rate of visible processes, most of which are quite unknown today. Histological sections reveal steps in processes which have long cycles, such as the endometrial changes during the menstrual cycle. They frequently fail to record as sequences changes which are parts of short cycles, the reasons being (a) that the stages of short-cycle phenomena appear in a collection of sections simply as a frequency distribution of the states of the observed structures and (b) that the dimensions are so altered during death, fixation and sectioning that functional differences are quite obliterated, jumbled, and obscured. Further, all too frequently the series of sections present no real indicator valid for determining the sequence of the steps in short-cycle phenomena. When motion pictures are taken through the microscope the method permits accurate recording and measuring of the rates of very rapid processes. For example, Knisely, M. H., Eliot, T. S., and Bloch, E. H., 1945, cited above, measured the rate of formation of precipitates in blood flowing through crushed tissues, finding that the precipitates formed in from $1 / 8$ th to $1 / 4$ th of a second while the blood flowed from 100 to 150 micra. In the future this method should make it possible to measure, in organized tissues, the rates of many visible phys- iologic, pathologic, pharmacologic and/ or therapeutic processes or responses. It should make it possible to measure the rate of formation of any visible end product of in vivo chemical reactions.

Further, and most important, the study of processes as they occur frequently makes it possible to determine steps in chains of causation. If one assumes that an effect cannot precede its cause in time, then it is possible to be certain that some phenomena do not cause, but rather may be caused by, others.

3 . The method should make it possible to obtain small samples of tissues and/or fluids from defined microanatomical regions, during defined phases of physiologic and/or pathologic processes. Wearn, J. T. and Richards, A. N., Am. J. Physiol., 1924, 71, 209227 , used micro-pipettes to remove glomerular filtrate from the Bowman spaces of frog Malphigian corpuscles. This was a triumph of imagination, insight, and technique. It initiated and provided a firm foundation for the whole modern series of studies of kidney function. The example set by Richards and Wearn should not be lost or ignored. Sinilar studies of samples from defined micro-anatomical structures, taken during defined phases of physiologic and pathologic processes will undoubtedly go a long way toward unravelling many current and future problems. This must be kept in mind as increasingly sensitive and accurate methods are devised for measuring the concentrations of substances in very small samples of rather dilute solutions. The use of special isotopes (initiated by Hevesy) is greatly increasing the abilities of analysts to detect and measure substances in extremely small biological samples. One next necessary step in this growing branch of knowledge nust consist in defining and knowing the micro-anatomical regions from which each sample comes and the physiologic or pathologic states under which each sample is collected, as aceurately as the composition of the sample can now be determined. This seens obvious; obvious also is the fact that in many quarters it seems not yet to be appreciated.

4. The method plus suitable and alequate micro-dissection and micro-injection techniques (Chanbers, R. and Kopac, M. J., in McClung, 2nd ed., pp. 62-109; Buchtal, F., 'Ztschr. f. Wissensch. Mikr., 1942, 58, 126-133) should make it possible to place samples of various substances in defined microanatomical areas, during defined phases 
of physiologic or pathologic processes and watch or otherwise determine the responses of parts of living systems to the newly arrived material. For an extensive example of one such set of studies, see Kinisely, M. H., Bloch, E. H., and Warner, L., cited above.

5. The method permits the study of some kinds of pathologic processes while they are still in reversible stages, that is, in controllable stages. Autopsies and autopsy sections show the final cumulative results of all of the simultaneous and consecutive pathologic and reparative processes which had been going on. That is, they show the preservable, visible part of the accumulated results after some one or more sets of pathologic processes have become irreversible. The microscopic studies of living tissues allow examination of some pathologic processes (a) as they develop, (b) as they proceed at sublethal degrees of intensity, and (c) as they accumulate toward lethal combinations of factors, but are still reversible, that is while the animal's life can still be saved, and (d) as they accumulate into non-reversible stages. Further, the method permits study of the results of experimental therapeutics on visible pathologic processes. For demonstrations and elaboration of this theme see Knisely, M. H., Stratman-Thonas, W. K., Eliot, T. S., and Bloch, E. H., 1945, cited above.

It may seem to some that the above discussion is too critical or unjustly critical of the histological sectioning techniques, or that the author is trying to belittle their use. This I do not believe to be so. The best histologists have always studied sections not for the structure of the dead sections themselves, but rather to determine as closely as possible the structure and functions the tissues had had when last alive. Precision and accuracy in developing concepts from the evidences gathered by a technique can never be greater than the user's understanding of the inherent limitations of that technique. The accuracy of a technique cannot be determined simply by repeating its steps an infinite number of times; its limitations and degrees of accuracy must also be cross-checked by other and, if possible, quite different techniques. Each useful technique delineates one or more aspects of the original tissue more accurately than do other techniques. Obviously the most accurate and comprehensive concepts of micro-anatomy, microscopic physiology and microscopic pathologic physiology can be developed only by synthesis; by putting together in the mind of the student the most accurate of the available individual aspects. For this purpose each technique has special values of its own; for this purpose not enough different techniques are yet available.

Quinoline Dyes. Only pinacyanol is of apparent value to histologists.

Quinone-Imine Dyes. Possess 2 chromophores: indamin- $\mathrm{N}=$ and quinoid benzene ring. They are divisible into Azins, Indamins, Indophenols, Oxazins, Thiazins.

Quinone Oximes, see Nitroso Dyes.

Rabbit Ears, see Sandison's Technique for inserting transparent chambers in.

Rabies, see Negri Bodies.

Rabl's Fluid is sat. aq. mereuric chloride, 1 part; sat. aq. picric acid, 1 part; aq. dest., 2 parts.

Radiation. Methods and results of radiation of normal tissues reviewed (Warren, S. and Dunlap, C. E., Arch. Path., 1942, 34, 562-60s and earlier papers).

Radioactive Isotopes-Written by $T$. B. Rosenthal, Dept. of Anatony, Washington University, St. Louis. June 6, 1951.- The release of radioactive isotopes in large quantities for civilian use (1946) has resulted in a voluminous literature concerned with fundamental concepts of metabolism and with the diagnosis and treatment of malignant disease. Although only a few notable discoveries may be directly attributed to the use of these isotopes (since the basic ideas were derived from earlier work with stable isotopes and conventional biochemical methods), it must be admitted that the relative ease and speed of operations with radioactive isotopes have thrown open a hitherto difficult field. By these means the validity of many old concepts has been confirmed, while others have been conclusively demonstrated to be false. It must be emphasized however that research with isotopes has not displaced the traditional methods in biology and chemistry. Reliable techniques are still requisite, and there is more need for good analytical chemistry than ever before. The radioactive isotope now takes its proper place among the resources of the laboratory as a tool indispensable for certain problems, useful for others, and merely optional for still others.

Mass production of reliable instruments for measurement of radio-activity has extended the use of isotopes to those Iaboratories previously deterred by expense or lack of specially trained personnel. The choice of equipment, i.e., Geiger-Muller counters, proportional counters, scintillation counters, 
ionization chambers, electroscopes, etc. will depend on the nature of the problem and the degree of versatility desired. For complete details see the monographs listed below and the latest manufacturers' catalogs.

Renewed interest in the pioneering technique of the autoradiograph has resulted from the development of new procedures and specially prepared emulsions. Under favorable circumstances it is now possible to achieve intracellular localization of tracer elements. See Gross, Bogoroch, Nadler, and Leblond, Am. J. Roentgenol. Rad. Therap., $1951,65,420-455$ for a review of the potentialities and limitations.

The first step in a biological investigation would naturally be to decide whether the use of isotopes is warranted, on the basis of results expected in relation to the economic investment. The next step is a survey of the literature to ascertain whether isotopes suitable for the purpose are available and what measure of success could be expected on the basis of similar experiments. From these considerations a choice of instruments and procedures would be made. On the other hand, a certain project may be warranted and feasible, but rendered impracticable by technical complications of various kinds.

For a source of materials one would first consult the catalog issued by the Oak Ridge Laboratory of the Atomic Energy Commission. Herein are listed the available stable and radioactive isotopes together with prices, shipping information, and official regulations on clinical applications, health protection, etc. Of the 96 elements, only $\mathrm{He}, \mathrm{Li}$, $\mathrm{B}, \mathrm{Ne}, \mathrm{N}, \mathrm{O}, \mathrm{Mg}, \mathrm{Al}, \mathrm{F}$, Si, and Fa lack radioactive isotopes of suitable halflife, leaving 85 elements whose employment is limited only by the ingenuity and resources of the investigator. In many cases the form of the material may be specified: pure metal, inorganic compound, labelled organic compound, colloid, solution, etc.

In addition a number of commercial firms are engaged in supplying on order and from stock an ever-increasing variety of organic intermediates and compounds of metabolic importance synthesized with tagged atoms in various positions. If only small quantities are needed, the investigator may conduct a biological synthesis from plant or animal sources in his own laboratory.

Although the nuclear reaction pile now supplies the great bulk of radioisotopes, those who have access to cyclotrons or similar machines should inquire about the possibility of getting small amounts of the shorter-lived isotopes.

Studies involving radiation by alphaparticles require the naturally-occurring radioactive elements. The metabolism of $\mathrm{Pb}$, for ex:mple, may be traced by means of those elements isotopic with $\mathrm{Pb}$.

Radioactive Tracers in Biology. 2nd Ed. M. D. Kamen, Academic Press, New York, 1951, $429 \mathrm{pp}$.

Radioactive Indicators. G. Hevesy, Interscience l'ublishers, Inc., New York, 1948, $556 \mathrm{pp}$.

Isotopic Tracers and Nuclear Radiations. W. E. Siri, Ed., McGrawHill, New York, 19.19, 653 pp.

The latter features a selected bibliog. raphy to 1947 of papers on biological and medical applications arranged by elements, together with extensive tables which aid in reviewing previous findings and suggesting new possibilities. The current literature is recorded in the abstract journals and in Nuclear Science Abstracts, published twice monthly by the A.E.C. The "Annual Reviews of Biochemistry" should be consulted for comprehensive reviews of special topics.

Radioautography-Written by C. P. Leblond and R. Bogoroch, Department of Anatomy, McGill University, Montreal. September 12,1951-Radioautography is a technique devised to define the localization of radioactive substances within biological specimens. The usual practice is to place a histological section as closely as possible to a phtographic emulsion, expose for a suitable time interval and subsequently develop as in ordinary photography. In place of light, the rays emitted by the radio-isotope furnish the energy necessary to transform the silver bromide of the emulsion into photolytic silver, which may then be developed by photographic reagents into visible black silver grains. Such grains overlie the sites of deposition of the radioactive substance in the histological sections, thus giving a permanent, visible record of the localization of this substance.

The use of the silver bromide grain as a detector of radioactivity has four advantages over other techniques: 1) the effect of the radiation is cumulative, and, therefore, minute quantities of radioactivity may be detected if exposure is sufficiently prolonged; 2) the radioautographic image is a permanent record; 3) the record is two-dimensional and may be three-dimensional in case of tracks; and 4) the smallness of the 
silver bromide granules makes it possible to localize the radioactivity to a comparably small area in the tissue.

It is, therefore, possible by this method to virtually "sce" a "labeled" element in an animal tissue. Furthermore, the fate of that element in the animal body may be detected by following the element through from structure to structure. The metabolism of any labeled physiological or pharmacological compound can thus theoretically be traced throughout the animal body using the radioautographic method, providing the labeled material, be it a product of synthesis or degradation, is retained in its original site in a tissue during processing for radioautography. The advantage of this method for the progress of histology is apparent, since the significance of known structures will often be revealed by the substance which they incorporate. From the medical point of view the sites of accumulation of the isotopes as well as their length of stay in the body provide useful information as to the radiotoxicity of known amounts of these isotopes. The applications of radioautography to biology and medicine have been fully reviewed most recently by Gross, J., Bogoroch, R., Nadler, N. J., and Leblond, C. P., Am. J. Roentg. and Radium Ther., 1951, 65, 420-458. The use of a-ray-producing isotopes was examined by Y agoda, H., Radioactive Measurements with Nuclear Emulsions. New York: John Wiley and Sons, Inc., 1949.

As with other methods involving the use of radioisotopes, it is assumed that the chemical behavior of a labeled substance is identical with that of its stable counterpart. This will also be true of its biological behavior providing that 1) the amount of radioactivity administered be small enough not to have a significant radio-chemical effect, and 2 ) the amount or weight of material injected be sufficiently small not to produce a significant increase in the amount of this substance present in the circulation. The labeled substance will then truly act as a "tracer" of the normal metabolism.

The success of radioautography depends on 1) the geometrical relationship between specimen and emulsion, the best conditions being a minimal distance between source and emulsion, a thin section ( $5 \mu$ or less), and a thin emulsion coating ( $10 \mu$ or less); 2 ) the features of the emulsion-the silver bromide grains should be uniform (for contrast), of a small size, and in high concentration; and 3 ) the charactcristics of radiations (i.e., energy and intensity) - particles of low energy such as soft $\beta$-rays give a better resolution. For a more detailed discussion of each of these factors see Nadler, N. J., Canadian J. Med. Sci., 1951, 29, 182-194; Gross et al., referred to above; Doniach, I. and Pelc, S. R., Brit. J. Radiol., $1950,23,184-192$.

Exposure time for a radioautograph is influenced by these three groups of factors: 1) Geometrical relations. Thus, minimizing the distance between emulsion and specimen will tend to reduce exposure time, while decreasing the thickness of section and emulsion will tend to prolong it. 2) Properties of photographic emulsions. Emulsions vary in sensitivity to $\beta$-rays. Also, the more contrasty the emulsion is, the narrower is the range of satisfactory exposure time. 3) Character of the radiation. The radiation influences the exposure time not only by its concentration per unit area, but also according to the half life and energy of the isotopes used. With soft radiation, the absorption in the tissue will also increase the exposure time.

It is important to keep the exposure to a minimum, as overexposure results in a more diffuse image with corresponding loss in resolution. Unstained "test" slides developed periodically will indicate the optimum exposure time.

\section{Preparation of Tissues.}

In the case of soft tissues, paraffin or celloidin sections are prepared in the routine histological manner and dehydrated with or without staining. For practical purposes, $5 \mu$ paraffin sections and $10 \mu$ celloidin sections seem to be most convenient. Carbowax (Blank, H., McCarthy, P. L., and DeLamater, E. D., Stain Techn., 1951, 26, 193-197) and frozen dried (Holt, M. W., Cowing, R. F., and Warren, S., Science, 1949, 110, 328-330) sections have also been used.

Bones and teeth may be embedded and cut according to Bloom's method (see Leblond, C. P., Wilkinson, G. W., Bélanger, L. F., and Robichon, J., Am. J. Anat., 1950, 86, 289-341) for bones of young animals or according to Arnold, J. (Science, 1951, 114, 178-180) for hard bones of older animals. Methods employing grinding machines have to be used for teeth as described by Hoecker, F. E. and Roofe, P. G. (Radiology, 1949, 52, 856-865), Laude, P. P., Janes, R. G. and Boyd, J. D. (Anat. Rec., 1949, 104, 11-15), Sognnaes, R. F., Shaw, J. H., Solomon, A. K. and Harvold, E. (Anat. Rec., 1949, 104, 319-330). 
Large specimens of soft tissues may be frozen, ground in the same way as above, or sectioned with a chilled blade or fine electric saw while being kept in the frozen state, and thus applied to the photographic emulsion.

1. Contact method: This technique, the oldest and crudest method, has been used extensively especially in the investigations of the sites of localization of isotopes in hard tissues such as bones and teeth, and in frozen sections of sof $t$ tissues. (See reviews: Gross, J. and Leblond, C. P., Canadian Med. Assoc. J., 1947, 57, 102; Gross, J., Bogoroch, R., Nadler, N. J. and Leblond, C. P., Am. J. Roentg. and Radium Therap., 1951, 65, 420-458.) The method consists essentially of placing a microscopic slide bearing a radioactive tissue in close contact with a photographic plate; and of keeping these in close contact by pressure.

1. A slide containing the histological section is dipped twice into an etheralcohol solution of $1 \%$ celloidin following staining and passage through 95\% and absolute alcohol. The section is then dried overnight to insure hardening of the celloidin.

2. In the darkroom, the histological slide, which should be free from dust particles or granules which may make for uneven contact, is placed gently against a photographic plate or film (x-ray, Eastman Kodak Medium Lantern Slide plate, etc.). Various methods may be used to insure an intimate uniform contact between slide and plate or film. They may be held in a roentgen-ray type pressure cassette or in a printing frame. Naturally, these holders should be light-tight.

3. After exposure, the photographic plate or film is processed according to the routine photographic procedures, i.e., developed in D-72 for 2 min., rinsed in water, fixed in acid fixer for $10 \mathrm{~min}$., washed for $30 \mathrm{~min}$. and dried. To protect the radioautographic image in the emulsion from scratches or abrasions, it is useful to cover the image with a drop of Canada balsam and a histological coverslip. The histological section and the radioautographic image may then be simultaneously examined under a dissection or ordinary microscope.

This method has the advantage of simplicity but the localization at best is rather crude due to low resolution.

2. Mounting method: The one most usually followed is that of floating sections onto a photographic emulsion.
Recent attempts at doing this under dry conditions will be mentioned later.

In the Wet Mounting method (Evans, T. C., Proc. Soc. Exp. Biol, and Med., 1947, 64, 313-315; Endicott, K. M. and Yagoda, H., Ibid., p. 170-172; Boyd, G. A., and Williams, A. I., Ibid., $1948,69,225-232$ ) the tissue section is floated directly onto a photographic film or plate. This insures a more intimate contact between tissue and emulsion and results in a better resolution, i.e., permits a finer localization. This method, however, cannot be used if the radioactive substance in the tissue is water soluble.

1. Unstained strips of paraffin sections of radioactive tissues are floated in a $40^{\circ} \mathrm{C}$. water bath to remove any wrinkles from the tissue. The water is cooled to $18^{\circ} \mathrm{C}$. by the addition of ice cubes at $18^{\circ} \mathrm{C}$. and all subsequent steps are carried out in the darkroom using a Wratten No. 1 safelight.

2. A photographic plate or film (Eastman Kodak Medium Lantern Slide Plate, NTA, NTB 1, 2, or 3 plates, etc.) is slipped under the tissue section, the corner of the tissue being held against the photographic plate with a needle, and the plate with adherent tissue is lifted out of the water. After the excess water has drained off the plate and the emulsion has dried completely, the tissue adheres intimately and permanently to the photographic emulsion.

3. After proper exposure at refrigerated temperatures $\left(4^{\circ} \mathrm{C}\right.$. or lower $)$, the section is deparaffinated in two changes of xylol and hydrated through graded alcohols.

4. The preparations may be developed in D-72 developer for 2 minutes or in D-19 for 10 minutes, rinsed in water, fixed in acid fixer $\mathrm{F} 5$ or in $30 \%$ thiosulphate, and washed in running water for 30 minutes.

5. Unstained sections may be dehydrated, cleared and mounted immediately or they may be stained with either dilute hematoxylin overnight, or metanil yellow (Gross, J. and Leblond, C. P., Canadian Med. Assoc. J., 1947, 57, 102-122; Simmel, E. B., Fitzgerald, P. J. and Godwin, J. T., Stain Techn., 1951, 26, 25-28). For staining prior to development, Doniach and Pelc have used hot carbol fuchsin-neutral red which resists the destaining action of the developing reagents.

Because the mounting method allows for a very intimate contact between tissue section and emulsion, a very very fine localization is possible. 
This method has been used extensively especially in hospitals where a minimum of equipment was desirable. The method, however, does not always give reproducible results because of 1) uneven penetration of developer through the tissue leaving some areas with latent images in the emulsion beneath undeveloped, 2) staining of the emulsion gelatin which obscures histological and radioautographic detail to some extent, and 3) chemical action of the tissue on the emulsion causing in some cases blackening of the emulsion or in other cases insensitization of the emulsion. These possibilities are eliminated in the "coating" methods. It must be emphasized, however, that the preparations obtained by the mounting technique are often more satisfactory under high than under low magnification.

Several "Dry" Mounting methods have been described (Hoecker, F. E. and Roofe, P. G., Radiology, 1949, 52, 856-865; Williams, A. I., Oak Ridge, Tenn., Los Alamos Scientific Laboratory, AECU, 1950, 930; Gallimore, J., Oak Ridge, Tenn., 1951). These eliminate the use of large quantities of water on the tissue prior to exposure, thus preventing loss or displacement of water soluble material. The published results indicate that the method is still in the experimental stage.

3. Coating method: A slide bearing a histological tissue section is covered with either a fluid emulsion or a stripping emulsion removed from its backing. The method as used with fluid emulsion is very satisfactory for research purposes, because of the possibility of controlling the thickness of the emulsion. In "fluid coating":

1. The sections, stained or unstained, are coated with celloidin as in the contact method. The area on the slide to be coated is outlined with a diamond pencil.

2. Using a Wratten No. 1 safelight at a distance of three feet, photographic emulsion is prepared for coating.

a) The most convenient emulsions with which to work are the bulk emulsions, several of which have been kindly given the authors by Ansco Corporation and British Kodak Limited. These emul sions are scooped directly into a $50 \mathrm{cc}$. beaker which is placed in a $37^{\circ} \mathrm{C}$. water bath. Ansco Radioautographic Emulsion A has a large grain size and a very high sensitivity but gives a poor resolution, while British Kodak $\mathrm{NT}_{4}$ bulk emulsion has a small grain size and medium to high sensitivity but gives a good resolution. Unfortunately, none of the bulk emulsions is available on a commercial bais.

b) The next best possibility is to remove emulsion from photographic plates or films such as Eastman Kodak NH Special Spectroscopic plates or Eastman Kodak "Special" Medium Lantern Slide films. Both these emulsions have a medium grain size and sensitivity. The emulsion is hydrated in distilled water $\left(18-20^{\circ} \mathrm{C}\right.$.) for 10 minutes and scraped with a clean edged object (such as a glass slide) into the $50 \mathrm{cc}$. beaker.

c) "Stripping" emulsions, such as Ilford Special Half Tone Stripping Plate, that can be stripped away from a glass or film support have also been used successfully. These may be stripped, hydrated, and melted, or may be treated as above.

d) Another form in which emulsion is available is the "pellicle" which consists of a fairly thick $(250 \mu)$ layer of concentrated silver bromide grains in a matrix of low gelatin content. Although these pellicles have no support, they must be presoaked in distilled water and dupanol $\mathrm{C}$ solution (10 ce of water and $10 \mathrm{cc}$ of $1 \%$ dupanol per $1^{\prime \prime} \times 3^{\prime \prime}$ pellicle) for 24 hours at $18^{\circ} \mathrm{C}$. The beaker containing the solution and the hydrated pellicle is then transferred to a $37^{\circ} \mathrm{C}$. water bath. Eastman Kodak $\mathrm{NTB}_{2}$ and $\mathrm{NTB}_{3}$ pellicles have a small grain size and high sensitivity to $\beta$ radiations, $\mathrm{NTB}_{3}$ being the more sensitive of the two.

All these emulsions are ready to be applied after 15 minutes in the water bath.

To further improve resolution, these emulsions may be diluted with distilled water and dupanol (Gross et al., referred to above).

3. Using a medicine dropper, two drops of melted emulsion are applied per square inch of slide to be coated. These are spread quickly and evenly with a camel's hair brush (kept at $37^{\circ} \mathrm{C}$.) over the outlined area and the emulsion is allowed to gel and dry completely on a leveling table at room temperature.

4. The dry preparations are stored horizontally in light-tight slide boxes (plastic are recommended) which are kept at refrigerated temperatures 
for the duration of exposure. A drying agent (Drierite or $\mathrm{CaCl}_{2}$ ) in the slide box prevents the hydration of the emulsion during exposure, thus reducing the possibility of fogging due to the solution of gases in the emulsion and also causes a sharper image to be produced.

5. After a suitable exposure, the preparations are developed in D-72 developer for one and a half minutes, rinsed, fixed in acid fixer, washed in running water $\left(18^{\circ} \mathrm{C}\right.$.) for $15 \mathrm{~min}$ utes, dehydrated for 2 minutes in each of the following solutions: $95 \%$ alcohol, absolute alcohol, alcohol-xylol, and cleared in three changes of xylol. To prevent artefacts, the preparations are immersed in a $1 \%$ solution of balsam or a $50 \%$ solution of permount for at least one hour. Longer periods of time are recommended for hard tissues. Slides should at all times be kept horizontal.

6. The sections are mounted in balsam or permount under a coverslip and the preparations allowed to dry at room temperature.

For a more detailed description of this method see Gross et al., referred to above.

In "Strip Coating": Instead of using fluid emulsion, the sections may be coated with a Stripping emulsion, such as Ilford Special half tone stripping plate or British Kodak stripping plates for autoradiography. Eastman Kodak NTB stripping films have also been used.

1. The emulsion strip is removed from its glass support by cutting the emulsion along the three edges of the glass plate.

2. The emulsion is then slowly peeled off to about 1 inch from the edge and, with the side that formerly adhered to the glass facing upwards, kept for 5 minutes in a shallow dish containing a $1 \%$ solution of dupanol $\mathrm{C}$ at $18-20^{\circ} \mathrm{C}$.

3. The microscope slide bearing the section is slipped underneath the emulsion and lifted together with the emulsion out of the water.

4. Three edges of the emulsion are folded underneath the slide to insure adherence. (Pelc dips the glass slides in gelatin prior to mounting the section on them. This also insures adherence.)

5. The slides are then stored dry at $1-2^{\circ} \mathrm{C}$. as above.

For more detailed report see Berriman, R. W., Hertz, R. H., and Stevens, G. IV. W., Brit. J. Radiol., 1950, 23, 472
477; Bogoroch, R., Stain Techn., 1951, 26, $43-50$.

The difficulty with the coating methods as described is that the preparations must be stained before they are coated. This procedure in some cases such as $\mathrm{P}^{32}$ in bone removes radioactive material, thus making staining of sections before radioautography inadvisable. The removal of $P^{32}$ is negligible, however, when prestaining is done with safranin or acid fuchsin. By using both stained and unstained preparations at the same time, it is possible to make sure of the extent of the loss. It is also possible to completely prevent loss by using the original coating method first described by Bélanger, L. F., and Leblond, C. P., Endocrinology, 1946, 39, 8-13, in which the sections were stained through the developed and fixed emulsion. This method has recently been successfully used by Arnold, 1951, in the study of calcium ${ }^{45}$ in bone. In this case, however, success may be obtained only if very thin emulsion coats are used and celloidin is not deposited on the sections prior to emulsion coating. The danger of artefacts production must be kept in mind.

3. Inverting Method: In this the sections are coated with liquid emulsions without prestaining of the sections (Bélanger, L. F., Anat. Rec., 1950, 107, 149. Kodak matrix emulsion is used for best results) or with a strip emulsion (MacDonald, A. M., Cobb, J., and Solomon, A. K., Science, 1948, 107, 550-552). After exposure and development the preparation is placed under water and the emulsion, with the section adhering to its undersurface, is lifted from the slide using a razor blade.

The complex is then inverted and fixed on a clean slide, section side up. The free edges are sealed with $1 \%$ celloidin, which is allowed to dry completely. The section may now be stained with ease since the celloidin protects the emulsion from the action of the dyes.

The preparation is mounted under a coverslip using Canada balsam.

4. Wet collodion method: The Wet Collodion method (Gomberg, H. J., Nucleonics (in press)) - an adaptation of the old wet collodion process of photography-involves the deposition of a very thin and dense layer of sensitized silver bromide in a collodion matrix formed by chemical reaction directly on the surface to be studied. After a suitable exposure time in a silver nitrate bath, the affected silver grains are physically developed using 
a ferrous sulfate solution. By using a physical rather than a chemical development, it is theoretically possible to obtain developed grains ranging from $10^{-8} \mu$ to $1 \mu$ in diameter. This method, however, is still in the experimental stage and there are several serious technical difficulties that must be overcome before the method can be used in biological research, e.g., exposures for more than two days have not been successful due to the great accumulation of background fog.

Quantitative Determinations.

Quantitative results have been obtained using 1) densitometric measurements, 2) grain counts, and 3) track counts.

The measurement of photographic densities using a photometer is satisfactory for work at low magnifications. The magnified radioautographic image is projected onto a frosted glass, and estimated as "densities" (which are measured as the log of the intensity of incident over transmitted light). This method has been used by Dobyns, B. M., Skanse, B., and Maloof, F. (J. Clin. Endocrinol., 1949, 9, 11711184) and by Axelrod, D. J. and Hamilton, J. G. (Am. J. Path., 1947, 23, 389412). Much work remains to be done before densities may be translated into concentrations of radio-elements.

The counting of photographic grains in an area outlined by a micrometer placed in the ocular is the most satisfactory method to estimate densities. It may be used with small or large specimens. All the grains in the area of the emulsion overlying a reactive zone may be counted (Pelc) or only the grains at definite levels are recorded. Results have been obtained with the latter method in this Department in collaboration with $\mathrm{N}$. Nadler and a good agreement with theoretical calculations was found.

The results may be expressed in several ways. The density or grain counts in one structure may be compared with that of another structure in the same tissue or animal or they may be expressed in more absolute terms by comparison with densities or grain counts of standards radioautographed at the sane time as the test material and containing known amounts of radioactivity and geometry (Dudley, R. A., and Dobyns, B. M., Science, 1949, 109, 327-328; Nadler, N. J. and Bogoroch, R., Anat. Rec., Supplement, $1951,109,69$ ).

When attempting quantitative comparisons, the geometry of the source, the distance between source and emul- sion, i.e., interspace, the emulsion and section thickness must all be taken into consideration. It must also be remembered that the density remains proportional to the intensity of the source, and thus to the concentration of the isotope, only when the exposure is kept to a minimum. Otherwise the relationship between density and concentration is more complex.

It is necessary to have uniform emulsions of absolute thicknesses (such as stripping films) for densitometric measurements since all the grains in every level of the emulsion are measured. On the other hand, when only definite levels in the emulsion are examined for grain counts, small variations in emulsion thickness are unimportant. The liquid coating method has made it possible to obtain reproducible quantitative results under these conditions.

The counting of tracks per unit area using nuclear emulsions for $a$ and $\beta$ rays may also be used as a quantitative method (Boyd, G. A. and Levi, H., Science, 1950, 111, 58-59).

Qualitative Results.

Space does not permit a review of the interpretation of the many results that have been obtained using the various techniques described above. Such a review with bibliography as previously mentioned has been given by Gross et al. Only a few examples of the more extensive work with this technique will therefore be cited.

Foreign substances such as heavy metals or fission products have been investigated radioautographically especially by the Berkeley group under J. G. Hamilton. The actinide and lanthanide groups of elements, administered as their ions, are found to be localized primarily in bone where they remain for considerable periods of time. The elements related to calcium localize mostly in the growing ends of bone.

The dynamics of phosphate deposition were used to study the growth of bone by Leblond, C. P., Wilkinson, G. W., Bélanger, L. F., and Robichon, J., (Am. J. Anat., 1950, 86, 289-341). By studying coated and inverted radioautographs of serial sections of bones obtained from young rats sacrificed at various time intervals after injection of $\mathrm{P}^{32}$, these authors found that two types of reactions occurred soon after injection, diffuse and localized. The diffuse reaction, extending throughout the bone, was due to exchange between labeled circulating phosphates and the surface phosphates of crystals 
of bone salts. These reactions diminished rapidly in intensity within a short interval of time (one day). The localized reactions, on the other hand, did not decrease significantly with time and were due to a deposition of labeled phosphate salts in the formation of new bone. The displacement of the new bone with time revealed the mechanism of bone formation.

Similarly, using $I^{131}$, Leblond, C. P. and Gross, J. (Endocrinol., 194S, 43, 306-320) followed the synthesis and degradation of the protein, thyroglobulin, in the thyroid gland of the rat. They found that labeled iodide very shortly after injection into normal animals was incorporated into thyroglobulin at the apical portion of the thyroid follicle cells. Later, the labeled thyroglobulin was deposited into the colloid where it was eventually broken down into amino acids, one of which was the hormone, thyroxine (which then diffuses out of the follicle). This study revealed that regardless of the physiological state of the animals, all the cells of the thyroid gland are always functioning in one direction. The rate at which the cells of any one follicle is functioning, however, changes with the physiological state of the animal.

The pathological physiology of the thyroid gland, especially in reference to the detection of thyroglobulin in neoplasms, has also been studied radioautographically by Marinelli, L. D., Foote, F. W., Hill, R. F. and Hocker, A. F., (Am. J. Roentgen. and Radium Therap., 1947, 58, 17-32), Franz, V. K., Quimby, E. H. and Evans, T. C. (Radiology, 1948, 51, 532-552), Dobyns, B. $M$. and Lennon, B., (J. Clin. Endocrinol., 1948, 8, 732-748), and more extensively by Fitzgerald, P. J. and Foote, F. W., (J. Clin. Endocrinol., 1949, 9, 1153-1170).

Still another application of the technique was used by Leblond, C. P., Stevens, C. E. and Bogoroch, R. (Science, 1948, 108, 531) in which they followed the turnover of desoxyribonucleic acid labeled with $\mathrm{P}^{32}$ in newly formed cells. This method not only gives an indication of the rate of formation of cells in different tissues in the rat body but also is a means to demonstrate the displacement and fate of these newly formed cells.

The examples cited are but a few of the important contributions to biology and medicine that have been made possible through the use of the radioautographic techniques as they exist today. Many more contributions can and most likely will be made with these techniques-at present the only means available to obtain minute localization of radioactive substances.

Ranson Pyridine method for unmyelinated nerve fibers (Ranson, S. W., Rev. Neurol. \& Psychiat., 1914, 12, 467-474). Fix in absolute alcohol $+1 \%$ ammonia, $48 \mathrm{hrs}$. Rinse in aq. dest. and treat with pyridine, $24 \mathrm{hrs}$. Wash repeatedly in aq. dest., 24 hrs. $2 \%$ aq. silver nitrate at $35^{\circ} \mathrm{C}$. in dark, 3 days. Rinse in aq. dest. Reduce in: pyrogallol, $4 \mathrm{gm}$; $5 \%$ formalin in aq. dest., 100 cc. Wash and imbed in paraffin. This much used technique gives a fine blackening of unmyelinated fibers. See also Ranson, S. W. and Billingsley, P. R., J. Comp. Neurol., 1918, 29, 313-358; Johnson, S. E., ibid, 1928, 38, 299-314). The latter believes the essential features of the technique to be vascular perfusion with physiological saline solution followed by $1 \%$ ammonia in absolute alcohol.

Ranvier's Gold Chloride method for nerve endings in muscle, see Graven's and Carey's methods. See also Ammonia Carmine and Picrocarmine of Ranvier.

Reconstruction. Stereoscopic $\mathrm{x}$-ray method (Morton, W. R. M., J. Anat., 1940-41, $75,265-266)$; wax plate method as applied to the stapes (Anson, B. J., Karabin, J. E. and Martin, J., Arch. Otolaryng., 1939, 29, 939-973).

Rectal Washings, see Papanicolaou Technique.

Red B, see Oil Red O.

Red Blood Cell, see Erythrocyte.

Red Corallin, (CI, 726). Look up in Colour Index.

Red Violet, see Hofmann's Violet.

Redox dyes are those employed in reduction-oxidation potential determinations, see Oxidation-Reduction Potential.

Reducing Sugars, Titrimetric methods for: Linderstrøm-Lang, $K$. and Holter, H., C. rend. trav. lab. Carlsberg, Sér. Chim., 1933, 19, 1-12; and Heck, K., Brown, W. H. and Kirk, P. L., Mikrochemie, 1937, 22, 306-314.

Reed-Sternberg Cells. Recognition of these cells is helpful in reaching a diag. nosis of Hodgkin's disease. Special technique other than Hematoxylin and Eosin is ordinarily not necessary. Comparison by Jackson, H., Jr. and Parker, F., Jr., New England J. Med., 1944, 231, 35-44 of Reed-Sternberg Cells with certain other multinucleated cells will be helpful. The use of tissue culture technique in their investigation opens many promising leats (Grand, C. G., Proc. Soc. Exp. Biol. \& Med., $1944,56,229-230$ ). Thus, it should be possible to determine their life history and check on the suggestion that the 
hyperchromatic Sternberg Cells are a later development of Reed cells (Bersack, S. R., Am. J. Clin. Path., 1913, 13, 253-259). The cytoplasmic inclusions, reported by Grand, are suggestive of virus action. The claim of Symmers, D., J. A. M. A., 1945, 128, 1248 1249 , that these cells should be called Greenfield Cells in honor of Greenfield's first description in 1878 will probably not be followed. The Phase Microscope can be helpful in the study of Reed-Sternberg cells (Hoffmann, J. T. and Rottino, A., Blood, 1950, 5, 74-78).

Reflecting Microscope. As is well known, magnified images can be produced by mirrors as well as by lenses. Proposals have been made many times to take advantage of the special properties of mirror systems to create a microscope of a pattern analogous to the reflecting telescope. Such an instrument would be completely achromatic, and thus superior to the best refracting microscope objective, the apochromatic, which is corrected for only three colors of chromatic aberration and two colors of spherical aberration. There would be no great loss of light by reflection compared to the considerable loss encountered in lenses by absorption. Furthermore, a reflecting microscope focused by visible light would also be in focus for ultraviolet and infra-red light, thus simplifying the process of photography. The construction of several such microscopes has been described in the literature, but serious consideration was not given to them because their numerical apertures were low and they had but little to offer over the lens type of microscope.

The rising tide of interest in ultraviolet microscopy has stimulated recent developments in this field. Burch in England (Burch, C. R., Proc. Phys. Soc., London, 1947, 59, 41-49) has designed a long focus, reflecting objective with magnification and resolving power equivalent to that of the average "high-dry" lens. This permits one to employ a micro-manipulator and to observe objects behind thick glass walls, such as tissue cultures.

Another design, originating in $\mathrm{Hol}$ land (Bouwers, A., Achievements in Optics, New York: Elsevier, 1946, 135 pp.) has been put into commercial production recently by Van Leer of Pittsfield, Mass. It can be mass produced because, unlike Burch's model, it uses only spherical reflecting surfaces.

Bausch and Lomb have also brought out a special reflecting objective (Grey, D. S. and Lee, P. H., J. Opt. Soc. Am.,
1949, 39, 719-728). This instrument combines both lenses and mirrors in order to gain even higher magnification and resolving power. It is apochromatic from 220 to $800 \mathrm{~m} \mu$, and has a working distance of about $1 \mathrm{~mm}$. A reflecting condenser has been designed to match this objective. The whole outfit costs about $\$ 1,000$. Use of a reflecting microscope for the study of cells is described by Mellors, R. C., J. Nat. Cancer Inst., 1950, 10, 1358-1361.

Refractive Index. Microscopical determination by standard liquids. See paper by Kunz, A. H. and Spulnik, J., Reviewed in J. Roy. Micr. Soc., 1937, 57, 55.

Regaud's Fluid. $3 \%$ aq. potassium bichromate, $20 \mathrm{cc}$; formalin, $5 \mathrm{cc}$. When this is used for mitochondria fix tissue for 4 days changing every day and then mordant in $3 \%$ aq. potassium bichromate for 7 days changing every second day. It is a fluid that can be profitably employed for many other purposes. Of these see Giemsa's Stain, Lead, Masson's Trichrome, Romieu Reaction and Starch Grains.

Regaud's Method of iron hematoxylin for mitochondria. Fix tissues in Regaud's fluid, mordant, imbed and section as described under Anilin Fuchsin Methyl Green Method. Run mounted sections down to water and mordant for $24 \mathrm{hrs}$. in $5 \%$ aq. iron alum. Rinse quickly in aq. dest. (not tap water) and transfer to hematoxylin (made by dissolving $1 \mathrm{gm}$. hematoxylin crystals in $10 \mathrm{cc}$. abs. alc. adding $10 \mathrm{cc}$. gly cerin, 80 cc. aq. dest. and allowing to ripen 3 weeks). If traces of iron alum are carried to the stain they will do no harm, but if too much enters the hematoxylin a dense black precipitate will form and ruin the hematoxylin. On the other hand, if the sections are washed excessively in aq. dest. too much of the alum will be removed and the hematoxylin will not stain as intensely as it should. The happy mean must be determined. The hematoxylin should be used over again about 10 times. Differentiate in 5\% aq. iron alum under low magnification. Wash in running tap water (not aq. dest.) $1 \mathrm{hr}$. This should bring out the blue-black color of the hematoxylin stain. Dehydrate, clear and mount. Various counterstains can be used if desired. Consult Meves'beautiful figures of collagenic fibers stained with fuchsin (Meves, F., Arch. f. Mikr. Anat., 1910, 75, 149-208). This is the most permanent stain formitochondria but lacks the color contrast afforded by anilin fuchsin methyl green.

Reissner's Fiber, staining reactions of 
(Jordan, H., Am. J. Anat., 1925, 34, $427-443)$.

Relief Methods, see Negative Stains.

Replacement of Tissue to take the place of that worn out or lost can now be measured more accurately. Though some signs of youth and age of cells can be detected (Chapter 24 in Cowdry, E. V., Problems of Ageing. Baltimore: Williams \& Wilkins, 1942, 936 pp.), it is not so easy to determine the percentage actually dying as the percentage of new cells produced to replace them by counting mitoses. Using whole mounts of separated human Epidermis from foreskins removed by circumcision Cooper, Z. K., and Schiff, A., Proc. Soc. Exp. Biol. \& Med., 1938, 39, 323324 have discovered that the production of new cells is rhythmic being greatest at night and least by day. To obtain material, as they did every hour of the day and night, of other human tissues seems impossible. If one wishes to investigate rate of cellular replacement in internal less accessible tissues that are replaceable, take advantage of the fact that the drug, colchicine, permits cells to enter mitosis but arrests the process usually in the metaphase. In consequence of this experimental summation many more mitoses can be counted in a given specimen than would be found if cell division had been completed as usual (See Mitosis for the necessary controls). There are no special means for the study of replacement of Fibers but careful use of available techniques will probably yield data as to whether the fibers are newly formed or old and practically useless.

Physico-chemical methods are however promising when backed by histological researches. Thus the new bone formed, during the time that Madder, or better Alizarin Red S, is made available in the circulation can be measured. In adult animals, assuming that the amount of bone remains approximately constant, it can be concluded that the breakdown is at the same rate and in this round about way arrive at a figure for replacement.

Some fats can be conveniently colored with fat soluble dyes which they retain on ingestion and after incorporation in the fatty depots of the body. It should, therefore, be possible to keep animals at a fairly constant weight on a diet containing a certain amount of fat, to substitute for this fat stained fat of the same sort without increasing their weight and to estimate the ratio of stained to unstained fat after a definite interval of time-in other words the replacement. Other possibilities are to employ for the test a fat of melting point quite different from the native body fat of the animals; and fatty acids tagged with radioactive isotopes, see Fatty Acids.

The radioactive isotopes, particularly those of Phosphorus and Iron give somewhat similar clues. The amount of radiophosphorus, for example, accumulating in any particular tissue can be accurately determined. If the supposition is justified that the total amount of phosphorus (radioactive and non-radioactive) remains about the same, then non-radioactive phosphorus must be lost at the rate that the radiophosphorus enters. It is too soon however to predict what this possible line of investigation with the isotopes will show. See Radiophosphorus.

Resazurin, a compound used as an oxidation-reduction indicator.

Resorcin Blue (CI, 908)-fluorescent blue, iris blue-Often called Lacmoid. See Nebel, B. R., Stain Techn., 1931, 6, $27-29$.

Resorcin-Fuchsin, see Weigert's resorcinfuchsin method for elastic fibers.

Respiratory System. This contains very diversified structural components for which no single technique or group of techniques can be offered. But the interpretation of the preparations depends, as in all systems of the body, on the age. A chapter by Macklin, C. C. and M. T., in Cowdry's Problem of Ageing, Baltimore: Williams and Wilkins, 1942, $936 \mathrm{pp}$, gives the necessary background and numerous hints and references to technique. Sce Lungs, Trachea, Nasal Passages and Nasal Sinuses.

Respiratory Tract Smears, sec Papanicolaou Techniques.

Respirometry, see Capillary Respirometry.

Restaining Faded Sections. This is sometimes very desirable. Try technique outlined by Small, C. S., (Amer. J. Clin. Path., Techn. Suppl., 1943, 7, 66-67).

Reticular Fibers. These are more finely divided and tend more to form a reticulum than the collagenic fibers. Yet there may be anatomical continuity between collagenic and reticular fibers and there is reason to believe that the two are fundamentally similar. They are not so conveniently viewed in the fresh condition because to make thin spreads is more difficult. For details see Maximow, A. A., von Mollendorf's Handbuch der Mikroskopischen Anatomie des Menschen, 1927, 2 (1), 232-583. The principal methods for reticular fibers in sections involve silver impregnation (Perdrau, Foot, Wilder, Gomori and Laidlaw), the choice of which will to some extent depend on the kind of 
tissue studied. There are, however, several which are stains (Kinney's Method and Biebrich Scarlet and Picro-Anilin Blue).

Reticular and Collagenic Fibers in frozen sections (Krajian, A. A., Arch. Path., 1933, 16, 376-378). Cut frozen sections $5-10 \mu$ thick of tissue fixed in $10 \%$ formalin and wash in 3 changes aq. dest. After treating with $10 \%$ aq. ammonium hydroxide at $60^{\circ} \mathrm{C}$. for $15 \mathrm{~min}$. (in paraffin oven) wash them again in 3 changes aq. dest. Place in $0.3 \%$ aq. potassium permanganate for $5 \mathrm{~min}$., wash in aq. dest. for a few seconds and decolorize in $1.5 \%$ aq. oxalic acid till brown color has just disappeared. Wash thoroughly in aq. dest. and place in 5\% aq. silver nitrate at $60^{\circ} \mathrm{C}$. for $1 \mathrm{hr}$. Wash in 2 changes aq. dest. and place in ammoniacal silver nitrate solution at $60^{\circ} \mathrm{C}$. for $15 \mathrm{~min}$. (To make this solution add 6 drops $10 \%$ aq. sodium hydroxide to $8 \mathrm{cc} .10 \%$ aq. silver nitrate which gives a brownish black precipitate. Add fresh $10 \%$ aq. ammonium hydroxide drop by drop until only a few small particles of the precipitate remain. Dilute to $28 \mathrm{cc}$. with aq. dest.). Wash sections quickly in 3 changes aq. dest. Treat them with $30 \%$ formalin at $60^{\circ} \mathrm{C}$. 1-3 min., wash in large amount of tap water and mount on slides. Cover sections with a little absolute alcohol and blot into position. Then complete dehydration with absolute, blot, clear in equal parts aniline oil and xylol. Wash in xylol and mount in gum dammar or Canada balsam. Reticulum black; collagen, brown.

Reticulocytes. These are the stages recognized in the red series before the assumption of properties of Erythrocytes. An excellent review of the properties of reticulocytes is supplied by Orten, J. M., Yale J. Biol. \& Med., 1933-34,6, 519-539. Reticulocytes can easily be identified by supravital staining with brilliant cresyl blue. First make a thin film of the dye on slide by allowing a $1 \%$ solution in absolute alcohol, spread evenly, to evaporate. Then mount fresh blood, ring with vaseline and observe. To make relatively permanent specimens, remove the cover glass after 2 min., smear dry and color by Wright's Stain. The supravital staining with cresyl blue is inhibited by certain substances (Heath, C. W. and Daland, G. A., Arch. Int. Med., 1931, 48, 133-145). For a calculation of experimental error in reticulocyte counts, see Marcussen, P. V., Folia Haemat., 1938-39, 61, 49-64 and for fragility tests, see Mermod, C. and Dock, W., Arch. Int. Med., 1935, 55, 52-60. Resistance to hypotonic sodium chloride solutions is described by Daland, G. A., and Zetzel, L., Am. J. Med. Sci., 1936, 191, 467-474.' The protoporphyrin content of reticulocytes can be estimated by the fluorescence technique. Watson and Clarke (C. J. and W. O., Proc. Soc. Exp. Biol. \& Med., 1937, 36, 65-70) have discovered that it is greater than in erythrocytes and that brilliant cresyl blue is precipitated by protoporphyrin which may explain the characteristic staining of reticulocytes by this dye.

Reticulo-Endothelial Blockade. Supposed to be a method whereby $R$. E. cells are so blocked by the ingestion of one foreign material that they are unable to take in another. For experiments with India ink and brilliant vital red and critical statement, see Victor, J., Van Buren, J. R., and Smith, H. P., J. Exper. Med., 1930, 51, 531-548.

Reticulo-Endothelial System. This is by definition made up of the reticular cells of the connective tissues plus certain special endothelial cells chiefly located in the spleen, liver, bone marrow, adrenals and lymph nodes. All have the common property of phagocytosing particulate matter such as trypan blue, carbon, etc. These, and possibly others, may leave their moorings and become free cells when they become known as Monocytes or Macrophages. A better term is the "system of macrophages" (or big eaters) in which emphasis is placed on function not origin. See Vital Staining.

Retina, see Eyes.

Retterer's Stain for muscle. Fix in 10 parts $80 \%$ alcohol plus 1 part formic acid. Stain paraffin sections with alum carmine. Muscle light red, all connective tissue unstained.

Revival of Vinegar Eels after Ultrarapid Cooling.-Written by B. J. Luyet, Dept. of Biology, St. Louis University. January $15,1951-A$ drop of a concentrated vinegar eel suspension, obtained by centrifugation, is deposited on a glass slide and most of the remaining vinegar is blotted off. Then a drop of $30 \%$ ethylene glycol is added to the squirming mass in order to reduce somewhat the water content of the worms. After about 5 minutes the excess ethylene glycol is blotted off, and the eels, still moving actively, are "wiped up," in a thin layer, on very thin pieces of mica (about $5 \mathrm{~mm}$. on a side and about 35 micra thick). The eels, supported on this mica slip, are then immersed in liquid air. After about one minute they are removed, and, by means of a vigorous shake of the hand, the droplet of liquid air which may adhere to the 
mica support is dislodged, whereupon the preparation is abruptly swished in a little water (about 2 cc.) in a watch glass, at room temperature or preferably at $30^{\circ} \mathrm{C}$. (The purpose of the immersion in water is rapid rewarming.) After about 5 minutes one sees, under a low power microscope, several eels begin to move and, after about ten minutes, some 50 out of 200 in the drop become quite active, though they are never entirely normal. See Luyet, B., C. rend. Soc. Biol., 1938, 127, 788-789.

Rhenium, see Atomic Weights.

Rheonine, A, Synonymous with Rheonine $\mathrm{AL}, \mathrm{G}$ or $\mathrm{N}$ and Fast Phosphine NAL, an acridine dye occasionally employed in fluorescence microscopy (Conn, H. $\mathrm{J}$. in McClung's Microscopical Technique, 1950 , p. 98)

Rhodamine B (CI, 749)-brilliant pink B, rhodamine $\mathrm{O}-\mathrm{A}$ basic xanthene dye. It gives a good color contrast with methylene blue in coloration of the spleen (Houcke, E., C. Rend. Soc. de Biol., 1928, 99, 788-789).

Rhodamine $\mathrm{O}$, see Rhodamine $\mathrm{B}$.

Rhodamines. Similar in some respects to pyronins but there is a third benzene ring affixed to central carbon atom and to this in turn is attached a carboxyl in ortho position. Examples: Rhodamine $B$ and fast acid blue R. Rhodamin B (Merck) and 6G IG. have been employed as vital stains. When used with plant cells mitochondria become fluorescent (Strugger, S., Protoplasma, 1938, 30, 85-100).

Rhodanid. Ammonium thiocyanate.

Rhodium, see Atomic Weights.

Rhodopsin (G. rhodon, rose + ops, eye) is a photosensitive visual purple pigment of the rods of the retina easily seen in ordinary microscopic preparations. It is a protein vitamin A combination. Night blindness is an indication of vitamin A deficiency.

Riboflavin (lactoflavin) shows typical green fluorescence in living liver and kidney observed under fluorescence microscope (Ellinger, P., and Koschara, W., Ber. deutsch. Chem. Ges., 1933, 66, 315-317, 808-813, 1411-1414). Detected also in Malpighian tubules of American roach (Metcalf, R. L. and Patton, R. L., J. Cell and Comp. Physiol., 1942, 19, 373-376) and in tomato plants (Bonner, J. and Dorland, R., Am. J. Bot., 1943, 30, 1008-1009). See Hirt, A. and Wimmer, K., Klin. Wochnschr., 1939, 18, 733-740. See Vitamine $\mathrm{B}_{2}$

Rinbonuclease is an enzyme which degredates ribonucleic acid. When used to remove ribonucleic acid from sections for histochemical observations, (Stowell, R. E. and Zorzoli, A., Stain Tech.
1947, 22, 51-61) pure preparation (McDonald, M. R., J. Gen. Physiol. $1948,32,39-42)$ is desirable. See Gram Staining, Nuclei Acids and Nuclease.

Ribonucleic Acid. A type of Pentose Nucleic Acid containing the ribose type of sugar present in yeast. Also known as IRNA. (Ribonucleic Acid) Use of electrotitration technique shows that yeast ribose nuclei acid under the influence of ribonuclease yields a secondary phosphate and a hydroxyl group (of a purine or pyrimidine). See Vandendriessehe, L., C. rend. trave. Lab. Carlsberg, Sér. Chim., 1951, 27, 341-391.

Rickettsia are small, gram negative, bacterialike organisms which are insect transmitted and typically inhabit endothelial cells of vertebrate hosts named after H. T. Ricketts who died of typhus fever while investigating them. They are best stained by Giemsa's method after fixation in Zenker's, Bouin's or Regaud's fluids.

1. Rapidstaining with thionin. Make sat. sol. of thionin in aq. dest. Precipitate by adding $10 \% \mathrm{NaOH}$. Collect ppt. on filter and wash until filtrate becomes neutral. Dissolve ppt. in $2 \%$ phenol. Stain absolute alcohol fixed smears only $30-50$ sec. Drain, wash quickly in absolute alcohol, clear in xylol and mount in cedar oil. Rickettsia, deep violet; cytoplasm, light violet; red cells bluish green (Laigret, J. and Auburtin, P., Bull. Soc. Path. exat., 1938, 31, 790-791).

2. Fuchsin staining method. Smear tissue culture on slide. Dry in air, then by heat. Filter directly on to smear $0.25 \%$ basic fuchsin in phosphate solution buffered to $\mathrm{pH} 7.4$ or in aq. dest. made $\mathrm{pH} 7.2-7.4$ by adding sodium hydrate or carbonate (see Buffers). Stain $4 \mathrm{~min}$. Wash quickly with $0.5 \%$ aq. citric acid. Pour off citric and wash rapidly in tap water. Counterstain in $1 \%$ aq. methylene blue, 10 sec. Rickettsia, red; cells, blue; not recommended for tissue sections (Zinsser, $\mathrm{H}$., Fitzpatrick, F. and Hsi Wei, J. Exp. Med., 1939, 69, 179-190). This is very similar to Michiavello's method described by Cox (H. R., Publ. Health Rep., 1939, 53, 2241-2247) as superior to Giemsa's stain for Rickettsiae of Rocky Mt. Spotted Fever and Typhus groups.

The Michiavello technique las been adapted for sections by Pinkerton (see Harry Plotz, in Simmons and Gentzkow, p. 572). Stain paraffin sections after Regaud fixation overnight in $1 \%$ aq. methylene blue and decolorize in 
$95 \%$ alcohol. Counterstain with $0.25 \%$ aq. basic fuchsin for $30 \mathrm{~min}$. Decolorize quickly (say 3 sec.) in $0.5 \%$ aq. citric acid. Differentiate rapidly in abs. alc. clear in xylol and mount in dammar. Rickettsiae, deep red; surrounding tissue, partly red. Background can be made bluer by washing lightly in aq. dest. after the citric acid treatment and by staining again with methylene blue, before differentiation in $95 \%$ alcohol dehydration, clearing and mounting as advised by Plotz. Plotz gives details of use of Michiavello's stain in demonstration of Rickettsiae in yolk sac cultures.

A fuchsin and methyl violet combination is recommended for typhus fever Rickettsiae by Nyka, W., J. Path. \& Bact., 1944, 56, 264.

See cultivation of Rickettsiae in eggs (Fitzpatrick, F. K., J. Lab. \& Clin. Med., 1946, 31, 45-55), Typhus Fever rickettsiae, and Rickettsia orientalis. A convenient list of pathogenic Rickettsia is provided by Pinkerton, $\mathrm{H}$., Bact. Rev., 1942, 6, 37-78.

Rickettsia orientalis. Rapid method for staining in smears by Clancy, C. F. and Wolfe, D. M., Science, 1945, 102, 483. Air dry smears of infected yolk sac membranes, or of other tissues, and fix by heat. Flood slide with xylol, dry in air current, immerse in 1:5,000 methylene blue and basic fuchsin in aq. dest. for $5 \mathrm{~min}$. Wash, dry and examine. Organisms blue, background pinkish purple. Grams should be diluted from $1 \%$ stock solutions on the day used.

Ringer solution. $\mathrm{NaCl}, 0.85$ gm.; $\mathrm{KCl}$, 0.025 gm.; $\mathrm{CaCl}_{2}, 0.03$ gm.; aq. dest., 100 cc. Lee (p. 731) advises for amphibians same except that $\mathrm{NaCl}$ is 0.65 gm. and $\mathrm{NaHCO}_{3}, 0.02 \mathrm{gm}$. is added to make $\mathrm{pH}$ about 7.0-7.4. If $\mathrm{NaHCO}_{3}$ is present it must not be sterilized by heat.

Ringer-Locke solution. $\mathrm{NaCl}, 0.85 \mathrm{gm}$; $\mathrm{KCl}, 0.042$ gm.; $\mathrm{CaCl}_{2}, 0.025$ gm.; $\mathrm{NaHCO}_{3}, 0.02$ gm.; aq. dest., 100 cc. for cold blooded animals. Lee (p. 73) advises same except that $\mathrm{NaCl}$ is $0.65 \mathrm{gm}$. Should be freshly made. Owing to presence of $\mathrm{NaHCO}_{3}$ must not be sterilized by heat.

Rivanol, a dye sometimes employed in fluorescence microscopy.

Roberts, see Paper Chromatography.

Rocky Mountain Spotted Fever, see Rickettsia.

Roller Tube Cultures. Control of $\mathrm{pH}$ in, see paper by Paff, G. H., Proc. Soc. Exp. Biol. \& Med., 1946, 62, 184-187. Sce Tissue Culture.

Romanowsky Stains contain polychrome methylene blue eosinates. Those of Wright, Leishman and Wilson are wellknown. The Romanowsky effect is the lavender-red coloration by them of the nuclei of lymphocytes, monocytes, protozoa and other materials. Acetone solvents for Romanowsky stains (Kingsley, D. M., J. Lab. \& Clin. Med., 1936-37, 22, 524-531). Polychroming process (ibid, 736-752). Dyes for (ibid, 1264-1273). Large bibilographies.

Romieu Reaction for proteins. Fix in formalin, in alcohol or in Bouin's fluid. Make rather thick sections in paraffin or preferably in celloidin. Cover section with a drop of syrupy phosphoric acid. After few minutes in oven at $56^{\circ} \mathrm{C}$. examine directly. A red or violet color develops in location of proteins. According to Blauchetière and Romieu (A. and B., C. Rend. Soc. de Biol., 1931, 107, 1127) it is due to the tryptophane grouping. See Lison, p. 129.

Rongalite White, said to stain normal but not cancerous cells (Roskin, G., Bull. d'Hist. appl., 1938, 15, 20-23).

Rosanilin (Magenta I) is triamino-tolyldiphenyl-methane chloride, a component of most Basic Fuchsins. Rosanilin with methylene blue for Negri bodies (Schleifstein, J., Am. J. Pub. Health., 1937, 27, 1283-1285).

Rosazine, see Azocarmine G.

Rose Bengal (CI, 779). A xanthene dye of fine color used for several purposes including the staining of Soil Bacteria by Conn (p. 157). Make suspension of soil in 9 times its weight of $0.015 \%$ aq. gelatin. Spread drop on clean slide and dry over boiling water bath. Cover, while still on bath for $1 \mathrm{~min}$., with rose bengal $1 \mathrm{gm}$.; $\mathrm{CaCl}_{2}, 0.01 \mathrm{gm}$.; $5 \%$ aq. phenol, $100 \mathrm{cc}$. Wash quickly in water. Dry and examine. Used to demonstrate injured liver cells by vital staining (Williams, W. U., Yale J. Biol, and Med., 1950, 23, 177-183). See Eosins.

Rosenthal, see Fluorescence, Microscopy, Radioactive Isotopes, Electron Microscopy.

Rosin U.S.P. XI (Colophony, yellow resin, abietic anhydride) used in Grieves' method for undecalcified dental tissues and bone.

Rosinduline GXF, see Azocarmine G.

Rosophenine 10B, see Thiazine Red R.

Rouget Cells, see Pericapillary cells.

Rouleaux Formation of red cells. They are stacked like coins, outlines distinet, usually break up on shaking, diminished on washing with saline solution; whereas agglutinated reds stick together irregularly, outlines indistinct, usually become more compact on shaking, not affected by washing. 
Rubber. To stain rubber in tissues many techniques have been reported by Haasis, F. W., Stain Techn., 1945, 20, 37-38. The work was done in Guayule studies under project of Bureau of Plant Industry. Micromanipulation in study of latex particle of rubber (Hauser, E. A., Ind. Eng. Chem., 1926, $18,1146-1147$ ).

Rubber. To stain rubber in tissues many techniques have been reported by Haasis, F. W., Stain Techn., 1945, 20, 37-38. The work was done in Guayule studies under project of Bureau of Plant Industry.

Rubber Paraffin. Johnson, J. (Applied Micr., 1903, 6, 2662) has recommended $1 \%$ crude India rubber in paraffin colored amber yellow by addition of asphalt heated to $100^{\circ} \mathrm{C}$. $1-2$ days. The supernatant fluid is poured off and used as ordinary paraffin. Double Imbedding in celloidin and paraffin has been suggested. See Beyer, E. M. (Am. J. Clin. Path., Tech. Suppl., 1938, 2, 173-175).

Rubidium, see Atomic Weights.

Russell-Body Cells, Russell bodies and the cytoplasm of plasma cells are probably not hemoglobiniferous because they do not react as do the substances in known hemoglobiniferous cells with reference to isoelectric point of hemoglobin (Kindred, J. E., Stain Techn., 1935, 10, 7-20).

Ruthenium, see Atomic Weights.

Ruthenium Red is ammoniated ruthenium oxychloride, a mineral pigment. Conn (p. 187) says that it is used microscopically as a test for Pectin for which some consider it to be specific.

Ruthenium Tetroxide, as a fixative said to be superior in some ways to osmium tetroxide; but it decomposes readily and penetrates poorly. To prevent decomposition make $1 \%$ sol. in sat. chlorine water (Carpenter, D. C. and Nebel, B. R., Science, 1931, 74, 154-155).

Saffron, a yellow dye obtained from the plant, Crocus sativus. Long cultivated in Persia this plant was introduced into China by the Mongols and throughout the Orient. In the early days of Greece saffron was the official color. Saffron was spread on the streets of Rome to welcome the Emperor and his army. Some monks discovered that by use of an iron mordant and saffron manuscripts could be cheaply made to appear golden. The City of Florence for a time incorporated the saffron blossom in its coat of arms. Later the City of Basle, Switzerland, followed suit and the "Saffron war" resulted in 1374 A.D. This acknowledged imperial color has come down through the ages; witness the yellow roofs of the Imperial and Forbidden Cities in Peking. For a valuable account read Leggett, W. F., Ancient and Medieval Dyes. Brooklyn: Chemical Publishing Co., Inc., $1944,95 \mathrm{pp}$. See also saffron as employed by Vieussens and Leeuwenhoek (Lewis, F. T., Anat. Rec., 1942, 83, 229).

Safirosin, see Eosin B or bluish.

Safranin. In the safranins one nitrogen of the azin group is pentavalent and to this a benzene ring is attached. All are strongly basic. Amethyst violet, azocarmine G, Magdala red, phenosafranin and safranin $\mathrm{O}$ are mentioned.

Safranin Acid Violet, see Neutral Safranin.

Safranin B Extra, see Phenosafranin.

Safranin O (CI, 841) - cotton red, Gossypimine, safranin $\mathrm{Y}$ or A-Commission Certified. A basic azin dye of great usefulness which is sold as a mixture of di-methyl and tri-methyl phenosafranins. Conn (p. 97) explains that the shade depends upon their relative proportion. The red is deeper when there is more of the latter. Safranin O can be employed irrespective of whether safranin $O$ wasserlöslich, or safranin spiritlöslich or safranin gelb is called for. The safranin pur, likewise of Grubler and Co., is in his opinion methylene violet (CI, 842). Safranin $\mathrm{O}$ is one of the finest nuclear stains especially in the Safranin Light Green method. It is also useful in making certain neutral stains (Neutral Safranin). Standardized technique for safranin $\mathrm{O}$ employing buffered solutions is given by Sawyer, C. H., Stain Techn., $1940,15,3-7$.

Safranin Y or A, see Safranin O.

Safranin-Gentian Violet-Orange G. This is Flemming's tricolor stain for nuclei. As described by the Bensleys (p. 88). Fix in Flemming's fluid and bring paraffin sections down to $95 \%$ alcohol. Stain in equal parts sat. safranin in $95 \%$ alcohol and filtered sat. anilin oil in aq. dest., 2-24 hrs. Rinse in aq. dest. and stain in sat. aq. gentian violet (crystal violet), $\frac{1}{2}-2$ hrs. Drop on sat. aq. orange $\mathrm{G}, 30-60$ sec. Drop $95 \%$ alcohol on slide until clouds of color cease coming off. Drop on clove oil and differentiate under microscope. Clear in benzol and mount in balsam. Violet should color diffused chromatin strand; safranin denser part; and orange $G$, the background.

Safranin-Light Green.-Written by C. H. Sawyer, Duke Hospital, Durham, North Carolina, Dec. 16, 1950.-Stain sections 24 hrs. in $2 \%$ aq. safranin $\mathrm{O}$ and wash out the excess safranin in $0.25 \%$ aq. light green (acid violet). Chromatin appears red and acidophilic nuclear in- 
clusions caused by viruses green. A very brilliant stain but the green fades in the course of a month or two. Standardized safranin $\mathrm{O}$ technique advised by C. H. Sawyer (Stain Techn., 1940, $15,3-7)$ is: overstain deparaffinized sections in $0.1 \%$ light green S.F. or fast green FCF in $50 \%$ alcohol adjusted to $\mathrm{pH} 2.4$ with $0.1 N \mathrm{HCl}(100 \mathrm{cc} .=20 \mathrm{cc}$. $0.5 \%$ stain +50 cc. $100 \%$ alcohol + 8 cc. $0.1 N \mathrm{HCl}+22$ cc. aq. dest.) for $4 \mathrm{hrs}$. or more. Destain in Sörensen's buffer $\mathrm{pH} 8,30$ minutes or more. Overstain in $0.1 \%$ aq. safranin $\mathrm{O}$ at least 4 hrs. Rinse in aq. dest. Destain in $0.01 \mathrm{~N} \mathrm{HCl}(\mathrm{pH} 2)$ or in 0.001 $N \mathrm{HCl}$ (pH 3) for light green and fast green respectively, 15 min. After rinsing in aq. dest. dehydrate in 2 changes dioxan, pass through xylol and mount in balsam. As fixatives Sawyer finds Petrunkevitch's paranitrophenol-cupric-nitrate-nitric and picro-formol-acetic better than Bouin's fluid. Zenker's fluid can be employed.

Salmonella, Flagella of non-motile, Edwards, P. R., Moran, A. B. and Bruner, D. W., Proc. Soc. Exp. Biol. \& Med., 1946, 62, 296-298. See Triphenyltetrazolium chloride.

Samarium, see A tomic Weights.

Sandarac mixed with dioxan, camphor and salol is recommended by McClung (p. 40) as a mounting medium in place of balsam.

Sandison's Technique for inserting transparent chambers in rabbit ears (Sandison, J. C., Anat. Rec., 1924, 28, 281). This has been improved by Clark, E. R. et al., Anat. Rec., 1930, 47, 187-211 and by Abell, R. G. and Clark, E. R., Anat. Rec., 1932, 53, 121-140. See modifications by Williams, R. G., Anat. Rec., 1934, 60, 487-491 and by the same author (ibid, 493-499) the latter for insertion into skin. Moore, R. L., Anat. Rec., 1935-36, 64, 387-403) has adapted the chamber for insertion into dog's ear.

Saponin, for hemolysis in centrifugal isolation of nuclei from chicken erythrocytes (Dounce, A. L. and Lau, T. H., Science, 1943, 97, 584-585).

Sarcolemma. Special technique for, see Dahlgren in McClung's Microscopical Technique, 1950, p. 341 .

Sawyer, see Safranin-light green.

Scandium, see Atomic Weights.

Scarlet B or EC, see Biebrich Scarlet, water soluble.

Scarlet B Fat Soluble, see Sudan III.

Scarlet J, JJ, V, see Eosin B or bluish.

Scarlet R, see Ponceau 2R.

Scarlet Red, see Sudan IV.

Schaudinn's Fixative. Sat. mercuric chloride in $0.85 \%$ aq. sodium chloride 2 parts. Add 1 part $95 \%$ ethyl alcohol and enough glacial acetic to make $1 \%$ solution immediately before use. For Protozoa, staining in bulk.

Scheele's Green, an exogenous pigment, copper arsenite.

Schiff's Reaction for aldehydes (Bourne, p. 22) is basis of Feulgen reaction for Thymonucleic Acid.

Schistosomes. When it is necessary to collect at autopsy all parasites irrespective of the stage of development or location, a modification of previous techniques described by Pan, C., and Hunter, III. G. W. (J. Lab. \& Clin. Med., 195, 37, 815-816) is suggested. To infect small mammals with Schistosoma Japonicum see method of Pan, C., Kaufman, E. H., and Hunter, III, G. W., Ibid., 817-819.

Schlesinger's Reagent. Add to 4 gms. zinc acetate in a bottle $95 \%$ ethyl alcohol to make up 100 cc. Shake occasionally and use supernatant fluid. See Urobilin.

Schmitt, see Polarization Optical Method.

Schneider's Aceto-Carmine, see AcetoCarmine.

Schultz, H. Cholesterol Test. Cut frozen sections of formol fixed material. Place sections in a $2.5 \%$ solution of iron alum mordanting for 3 days in low temperature $\left(37^{\circ}\right)$ oven. Rinse the sections after removal from the alum solution in aq. dest. to which are added a few drops of nitric acid (2 to 3 drops per 25 cc.). This removes alum precipitate in the sections. They are then transferred to $2-3 \%$ gelatin solution and mounted in dilute gelatin on the slide. After the mounted sections have completely dried add a few drops of a mixture of equal parts of concentrated sulphuric acid and glacial acetic acid. The appearance of a blue-green color indicates that cholesterol, either in free or ester form, was present in the sections before treatment. Both acids must be of analytical reagent standard and the sulphuric acid at least $98 \%$ pure. The appearance of bubbles in large numbers indicates impure reagents. See Knouff, R. A., Brown, J. B. and Schneider, B. M., Anat. Rec., 1941, 79, 17-38. Revised by R. A. Knouff, Dept. of Anatomy, Ohio State University, Columbus, Ohio, April 24, 1946. Swyer, G. I. M., Cancer Research, 1942, 2, 372-375 has checked in a satisfactory way the Schultz test with quantitative determinations of cholesterol in normal and enlarged prostates.

Schultze's Method for clearing embryos has been modified by Miller. See Cartilaginous Skeleton. 
Schweinfurt Green, an exogenous pigment, cupric acetoarsenite.

Scott, see Altmann-Gersh Frozen Dehydration Method.

Sebaceous Glands. Method for staining in toto (Badertscher, J. A., Stain Techn., 1940, 15, 29-30). Fix fresh skin for 24 hrs. in $10 \%$ formalin, or take skin from dissecting room cadaver and $f x$ in the same way. Make free hand vertical sections 1-2 mm. thick from region possessing the glands. Whole pieces of skin $12 \mathrm{~mm}$. square or larger (without subcutaneous fat) can be used in place of the sections. Pass through 50 to $70 \%$ alcohol. Stain for $12-24$ hrs. in a mixture of 70 parts absolute ethyl alcohol, 20 parts $10 \%$ aq. sodium hydroxide and 10 parts of aq. dest. saturated with Sudan IV. Wash away excess stain by repeated changes of $70 \%$ alcohol until glands become sharply outlined. Clear in glycerin. Mount in Brandt's glycerin jelly (melted gelatin, 1 part; glycerin, $1 \frac{1}{2}$ parts + few drops carbolic acid). Glands scarlet in transparent background. This method may prove useful to bring out the distribution, number, size and other features of sebaceous glands in different conditions as well as at different ages. The same method can be used for Meibomian (tarsal) glands after a little preliminary dissection described by the author.

Another method of staining sebaceous glands in toto employed in the Barnard Free Skin and Cancer Hospital is to separate epidermis from dermis by the dilute acetic acid method (see Epidermis) and stain the epidermal sheet, with sebaceous glands attached, with Sudan III or IV as one would a section for fat. A hematoxylin counterstain is useful.

The technique of Fluorescence Microscopy is useful. Figge, F. H. J., Bull. School of Med. Univ. Maryland, $1942,26,165-176$ has described the remarkable red, white or yellow fluorescence of blackheads which is characteristic of different individuals.

Secretion contrasted with excretion (Cowdry's Histology, p. 259).

Sectioning, see Celloidin, Frozen, Gelatin and Paraffin Sections. Also Bone grinding and Teeth cutting with power lathe.

Selectron, a synthetic resin recommended by $\mathbf{R}$. McClung Jones in McClung's Microscopial Technique, 1950 , p. 152 for embedding embryos. May be purchased from Pittsburgh Plate Glass Co., Grant Building, Pittsburgh, $\mathrm{Pa}$.

Selenium. Intravenous injections of colloidal solutions of selenium in rabbits are described by Duhamel, B. G., C. rend. Soc. de Biol., 1919, 82, 724-726. Sce Radioselenium.

Semen Stains, examination of for spermatozoa. Place picce of soiled cloth not more than $\frac{1}{2}$ inch in diameter on a slide. Add few drops saline solution and scrape surface of cloth with blunt edge of a scalpel. Carry scrapings off with fluid and spread on a slide. Dry and fix with heat. Cover with 4 cc. $1 \%$ aq. Wollschwartz (Grubler) + 0.05 cc. $2 \%$ aq. sulphuric acid, $5 \mathrm{~min}$. Wash in water. Counterstain $6-8 \mathrm{sec}$. with Loeffler's methylene blue diluted with 15 parts aq. dest. Wash in aq. dest., dry and examine. Heads of spermatozoa bright golden or yellowish color, all else gray. Useful in legal medicine (Williams, W. W., J. Lab. \& Clin. Med., 1936-37, 22, 1173-1175). See author's figures. See Pollak, O.J., Arch. Path., $1943,35,140-196$.

Seminal Fluid. To study in sections centrifuge fluid $\frac{1}{2}$ to $1 \mathrm{hr}$. after ejaculation for 20 min. at 3000 r.p.m. Fix centrifugate in $4 \%$ formalin, $48 \mathrm{hrs}$. 2 changes. Take sediment into abs. alc., then 9 parts abs. and 1 part xylol. Gradually increase xylol to 9 parts to 1 part alc. Xylol paraffin $30 \mathrm{~min}$. Then $54^{\circ} \mathrm{C}$. melting paraffin for $3 \mathrm{hrs}$. in incubator at $58^{\circ} \mathrm{C}$. After $3 \mathrm{hrs}$. in $60^{\circ} \mathrm{C}$. melting paraffin embed and section 2-3 microns thick (Joël, K., J. Lab. \& Clin. Med., 1939, 24, 970-972).

Sense Organs, see Eyes, Ear, Pacinian Corpuscles, Meissner's Corpuscles, Krause's End Bulbs, Nerve Endings. Sensitol Red, see Pinacyanol.

Separation of Cell Components by Differential Centrifugation-Written by A. Lazarow, Department of Anatomy, Western Reserve University School of Medicine, Cleveland, Ohio. November 28, 1951-When R. R. Bensley and N. Hoerr (Anat. Rec., 1934, 60, 449455) successfully separated mitochondria from the cell, they initiated a now era in cytochemistry. The cells are disintegrated by passing the tissue through bolting silk (mild homogenization in a glass homogenizer may also be used). This procedure ruptures the cytoplasmic membranes but leaves most of the cell components relatively unaltered. The resulting suspension of cell fragments is subsequently fractionated by differential centrifugation.

The sedimentation of a particle in a centrifugal field is dependent upon its size, its shape, and its density (relative to the suspending media) and upon the centrifugal force. The sedimentation of non-spherical particles is slower than 
that of corresponding spherical particles; this difference is attributable to a viscosity factor and to Brownian bombardment (the larger surface area of the non-spherical particles results in an increased Brownian bombardment and a greater tendency to keep the particles in suspension). If the density of the particle is equal to that of the media no sedimentation will take place regardless of the centrifugal force applied. If the particle is less dense than the suspending media (as lipid particles) it will be subjected to a flotation during centrifugation.

The centrifugal force is defined as the force in dynes acting on a mass of one gram. Since it is customary to express the centrifugal force in terms of the earth's gravitational force, and since the gravitational force acting upon a mass of one gram is 980 dynes, the centrifugal force is given by the equation

$$
\text { Centrifugal force }=\frac{4 \pi^{2} \mathrm{rn}^{2}}{980} \text { where } \mathrm{n} \text { is }
$$

equal to the number of revolutions per second and $r$ is equal to the radius of centrifuge. Thus the centrifugal force is proportional to the square of the number of revolutions and directly proportional to the radius of the centrifuge. [Thus for an angle centrifuge with a radius of $8 \mathrm{~cm}$., the centrifugal force is $12,400 \times$ g. at a speed of 12,000 RPM. For the International centrifuge with a radius of $20 \mathrm{~cm}$. the centrifugal force is $1,400 \times \mathrm{g}$. at a speed of 2500 RPM]

In carrying out the fractionation of the cytoplasmic constituents, a short preliminary centrifugation at low speed removes the intact cell nuclei and larger cell fragments. The mitochondria are subsequently separated from the supernatant by centrifuging at about $1,000-1,500 \times$ gravity for $10-30$ minutes. Less contaminated preparations can be obtained by successively centrifuging the supernatant for several 10-minute periods. The purity of the preparations is checked by microscopic observation of the separated fractions. After the mitochondria have been removed, the supernatant is recentrifuged at about $10,000 \times \mathrm{g}$. for $30-60$ minutes. This removes the submicroscopic particulate components of the cell (microsomes, or ultramicrosomes). There are at least two types of submicroscopic particulate components, a lipo protein nucleic acid complex and a hydrated glycogen-particle (Lazarow, A., Biol. Symposium, 1943, 10, 9, edited by N. L. Hoerr). The supernatant of this high speed centrifugation contains the soluble proteins, metabolites, electrolytes, etc.

When sucrose is used as a suspending media (Hogeboom, Schneider, and Palade, J. Biol. Chem., 1946, 177, 610) the morphology of the mitochondria is better preserved (in salt solution the mitochondria tend to swell and they lose their rod-like shape). By using various buffered citric acid solutions it is possible to make a clean separation of the nuclei (Dounce, A. L. Ann. N. Y. Acad. Sci., 1950, 50, 982 999).

In the past decade considerable progress has been made with regard to the localization of enzymes with the component parts of the cell. In determining the localization of enzymes (etc.) it is necessary to consider both the concentration [i.e., the amount of enzyme per unit of weight or per unit of protein nitrogen] and the relative fraction [i.e., what fraction of the total enzyme of the cell is present in a given fraction]. The cytochrome-cytochrome oxidase system as well as many of the components of the Kreb's tricarboxylic acid cycle are concentrated within the mitochondria (Schneider, W. C., and Hogeboom, G. H., Cancer Res., 1951, 11, 1-22). The submicroscopic particles (ultramicrosomes) are thought to be concerned with protein synthesis.

Serum Agar, see Bacteria, Media.

Setoglaucine O (CI, 658), a basic dye less light fast than Malachite green (CI, 657 ), a constituent of some bacteriological media (Emig, p. 47).

Shadow-Casting-Written by W. T. Dempster, Dept. of Anatomy, University of Michigan, Ann Arbor, Mich., and R. C. Williams, Dept. of Biochemistry, University of California, Berkeley, Calif. June 9, 1950-This is a technique for revealing the surface form and texture of microscopical material in either light or electron microscopy. It is an outgrowth of R. C. Williams' experience with vacuum deposited metal films on astronomical mirrors and of studies on the physics of metallic films. Metal evaporated from a hot filament in a high vacuum is of atomic dimensions, and upon condensation on a perfectly smooth surface it forms a continuous film of uniform thickness. The atomic particles of metal travel in straight line paths from the flament. When these are directed to an obliquely placed surface, obstructions, however small, cast a "shadow" that is metalfree. The technique of condensing metal films obliquely upon specimens 
was first applied successfully to electron microscopy by R. C. Williams and R. W. G. Wyekoff (J. Appl. Phys., 1944, 15, 712-716) who used the technique for determining the heights of minute objects by measuring the lengths of their shadows. It was found by them, however, that the process greatly enhanced the contrast in images of very small objects, or of very small irregularities on larger objects, and this advantage of the technique has come to outglow in importance the original purpose of measuring heights.

Bacteria, viruses and the larger protein molecules have been studied by the use of the shadowing technique (for complete bibliography, see R. W. G. Wyckoff, Electron Microscopy, 1949, Interscience Publishers Inc., New York) as applied to electron microscopy. The advantages of improved contrast are so great that, in the case of the observation of small biological objects and minute chemical aggregates, the technique has improved the practical usefulness of the electron microscope almost ten-fold. Further applications to both electron and visual microscopy involved a method of studying opaque surfaces by colloidin replicas that are shadowed (Williams, R. C., and Wyckoff, R. W. G., J. Appl. Phys., 1946, 17, 23-33). Applications of the method to biological material viewed with the light microscope and an account of the casting apparatus have been presented by W. T. Dempster and R. C. Williams (Anat. Rec., 1946, 96, 27-38).

For the shadowing of non-opaque objects, materials to be observed with the light microscope, the following procedure is followed: Material is affixed to cover slips; smears are thoroughly dried; paraffin is dissolved from sections with solvents. With no further preparation, other than thorough drying, the slips are shadowed with a metal deposit in a vacuum chamber. After this, they are mounted face down on slides with clarite or balsam.

If large, opaque materials are to be examined under the light microscope, a surface replica can be taken by flowing a dilute solution of celloidin over the surface, allowing it to harden, and stripping the celloidin film off after drying. The film is then shadowed with its replicating surface uppermost.

For electron microscopy, regular screen grids with a thin colloidin film over the mesh are used as substrates for suspensions; replicas are placed directly on the mesh. A droplet of suspension containing small biological objects is placed on the substrate and allowed to dry. Salts are then rinsed off with distilled water and the specimen is ready for shadowing; then the specimen may be studied with the electron microscope.

A metal to be used in shadowing for light microscopy should be readily evaporated and it should be relatively opaque when present in a very thin film. Metallic chromium appears to be the best metal to use for light microscopy since it transmits less than $50 \%$ of the incident light in a thickness less than 0.03 micra. For electron microscopy chromium is generally satisfactory for objects as large as bacteria, but, for finer detail, the metals palladium and uranium are superior, as they can be applied to yield satisfactory contrast in thicknesses of only 0.001 micra.

The casting technique is similar for the different metals. Shadow-casting produces a visually structureless deposit which sticks to all surfaces save those directed away from the hot filament and shadow areas due to obstructions. Surfaces perpendicular to straight line paths from the filament get the heaviest deposit; oblique surfaces get less and shadows none. Metal deposited at a rather oblique angle has a distribution much like light from a point source shining obliquely on threedimensional objects. Highlights and shadows are produced. Through the microscope, shadows in the preparations trausmit light and appear bright; highlights are dark. The eye, however, readily adapts to this reversal of tone. Photographic negatives or prints made from glass positives reverse the microscope appearance; highlights then are bright, shadows are dark, and variations of surface texture are shown by gradations of tone.

Electron-microscopic negatives show the same natural appearance of light and dark. Although the electron micrographs are taken by transmitted electrons, in complete analogy with photomierographs, the negative prints give one the impression that one is looking down on the surface of the material being examined.

The apparatus for shadowing consists of a bell jar and a base plate with vacuum tight electrical connections. Electrodes raised above the level of the base plate carry a tungsten filament on which the metal is placed for vaporizing. Cover slips with affixed material (or the grid sereens) are arranged in a semicircle at a predetermined distance from the filament and the metal thereon to be vaporized. The 
height of the filament and the distance from filament to specimens determine the casting angle. Both an oil-diffusion pump and a mechanical pump must be used to produce the degree of vacuum required (at least $10^{-4} \mathrm{~mm}$. Hg.). With a suitable vacuum provided, the filament is heated electrically and a measured weight of metal is vaporized. Preparations are then ready for mounting or examination. A figure of the apparatus and the formula for calculating the appropriate mass of metal for the conditions of shadowing are presented in the Dempster and Williams paper. Wyckoff's book (above) covers completely applications to electron microscopy.

Shanklin, see Pineal, Silver Diaminohydroxide after sensitizing with sodium sulfite.

Sharpening, see Microtome Knife.

Shrinkage caused by fixation, dehydration and clearing of nervous tissues has been measured by King, H. D., Anat. Rec., $1910,4,213-244$ and by Allen, Ezra, Anat. Rec., 1916, 10, 565-589.

Sickle-Cell Trait. A critical study of methods for detection by Diggs and Pettit (L. W. and V. D., J. Lab. \& Clin. Med., 1939, 25, 1106-1111) gives first place to the Moist Stasis technique of Scriver and Waugh. Place a rubber band about proximal part of a finger. Leave $5 \mathrm{~min}$. Puncture and examine fresh blood for sickle cells. According to Hansen-Pruss (O. C., J. Lab. \& Clin. Med., 1936-37, 22, 311-315) the maximum percentage of sickle cells is produced in 4-5 hrs. by supravital staining with brilliant cresyl blue or janus green, while it takes $24 \mathrm{hrs}$. in unstained moist preparations.

The following rapid method of diagnosis is reported by Neuda, P. M. and Rosen, M. S., J. Lab. \& Clin. Med., 1945, 30, 456-458. Mix "cherry-size" piece of feces with 5 cc. isotonic sodium chloride solution. Add $0.1 \mathrm{cc}$. of filtrate to tube of nutrient broth, incubate $24 \mathrm{hrs}$. at $37^{\circ} \mathrm{C}$. To top of suspected blood on slide add drop of culture. Something in broth makes susceptible cells quickly assume sickle form.

Siena Orange (K. Hollborn, Leipsig) = sodium paradipicrylamine, an alleged stain for potassium (Carere-Comes, O., Zeit. wiss. Mikr., 1938, 55, 1-6).

Silicon. Easily recognizable in sections viewed in polarized light. It often occurs as sericite in combination with magnesium, iron and other minerals, see Jones, W. R., J. Hyg., 1933, 33, 307-329. Microtechnique is discussed by Policard, A., and Mastin, E., Bull. d'Hist. Appl., 1933, 10, 22-36. Microincinera- tion is useful but Scott says that an exaggerated idea of amount may be obtained (McClung, p. 659).

Sintered-glass filters, see Cunningham, B., IKirk, P. L. and Brooks, S. C., J. Biol. Chem., 1944, 139, 21-28.

Silver is occasionally found in the tissues particularly after treatment with silver nitrate or argyrol. It appears as brown to black granules or masses, is definitely blackened by ammonium sulphide and may be removed by a mixture of sodium thiosulphate and potassium ferricyanide solutions. Recently a method based on reaction between silver and $p$ dimethylaminobenzylidenrhodanin has been described and illustrated in colors (Okamoto, K., Utamura, M. and Akagi, T., Acta Scholae Med. Univ. Imp. in Kioto, 1939, 22, 361-372). Details are supplied by Glick, p. 26.

Silver Chloride Dichlorfluoresceinate coloration of vascular endothelial cells (Bensley, R. D. and S. H., Anat. Rec., $1935,64,46-49)$. Inject intravenously $0.8 \%$ aq. dichlorfluorescein until animal becomes quite yellow. Kill animal; remove tissues and immerse in $10 \%$ aq. silver nitrate or in Bensley's Silver Citrate solution until salmon pink color develops. Fix in $10 \%$ neutral formalin. Dehydrate in alcohol and Iso-Safrol, clear in iso-safrol and mount in balsam. Endothelial cells outlined in pink. On exposure to light color changes in time the silver becoming brown and black. See demonstration of Chlorides in lungs by this method.

Silver Citrate injection of blood vessels (Bensley, R. D., Am. J. Anat., 1929, 40, 146-169). This method has proved of great value in the investigation of efferent vessels of renal glomeruli. It can be employed to advantage in other situations particularly in association with supravital staining of Pericapillary Cells with janus green. To make up the solution dissolve $4 \mathrm{gm}$. silver nitrate in 100 cc. aq. dest. and remove to dark room. Completely precipitate silver as silver phosphate by addition of sodium phosphate solution. Wash ppt. repeatedly with aq. dest. decanting supernatant lluid. Make up to volume approximately $30 \mathrm{cc}$. Dissolve ppt. by adding 28 gms. pure citric acid (or tartaric acid) in crystals. Dilute with aq. dest. to 1000 cc. and keep in dark.

For use, dilute this stock solution with 3 times its volume $1 \%$ aq. sodium citrate. Kill the animal by bleeding. For kidneys and other abdominal viscera insert into aorta cannula connected by rubber tubing with pressure bottle. First perfuse with $1 \%$ aq. sodium citrate with the pressure bottle 
about $60 \mathrm{~cm}$. above cannula. When clear fluid, free from blood, appears in inferior vena cava, clamp tube and replace citrate solution with silver solution. Raise bottle about $150 \mathrm{~cm}$. above cannula and release clamp. Determine length of time of perfusion by trials. When complete, immediately make frozen sections to determine results and fix other pieces in $10 \%$ formalin for $24 \mathrm{hrs}$. Cut paraffin sections desired thickness. Mount them in usual way, run down to water and develop in light in diluted photographic developer or simply by direct exposure to sunlight or are light. Counterstain in Mayer's Acid Carmine, hematoxylin, acridine red or some other suitable dye. Dehydrate, clear, mount in balsam.

Silver Deposits, methods for removal, Lillie, p. 135.

Silver Diaminohydroxide after Sensitizing with Sodium Sulfite for NeurogliaWritten by William M. Shanklin, American University of Beirut, Beirut, Lebanon. March 30, 1951-Fix small fresh pieces of the central nervous system in formalin ammonium bromide for 4 days at room temperature (Del Rio Hortega, P., Arch. Hist. Normal Y Path., 1942, 1, 165-205, 329-361; 1943, 2, 231-244, 577-601): Formalin (Merck, blue label $40 \%$ ) $70 \mathrm{ml}$., Ammonium bromide $14 \mathrm{gm}$. and aq. dest. $680 \mathrm{cc}$. Wash 10 hrs. in aq. dest. to which 30 drops of strong ammonia water are added for each $100 \mathrm{cc}$. of water, and cover the jar. Wash in two changes of aq. dest. for 1-2 hrs. in each; dehydrate with alcohol. Clear in cedar oil followed by xylene $30 \mathrm{~min}$. Infiltrate with paraffin for 3-4 hrs., embed, section at $10-15 \mu$ and fix to slide by the albumen water method. Remove the paraffin with xylene, dehydrate with alcohol and pass through three changes of aq. dest. 1-2 min. each. Sensitize by placing the slides in $5 \%$ aq. sodium sulfite, 2 hrs. Pass quickly through 3 changes of aq. dest. To prepare silver diaminohydroxide solution place 1 $\mathrm{ml} .28 \%$ ammonia water in a small flask and add 7 or $8 \mathrm{ml} .10 \%$ silver nitrate rapidly. Continue to add $10 \%$ silver nitrate drop by drop shaking between each addition to clear the solution until a faint permanent turbidity remains after the last drop is added. This takes a total of 9 to $10 \mathrm{ml}$. silver solution. Then dilute the resultant solution with an equal volume of aq. dest. (Lillie, R. D., Stain Techn., 1946, 21, 69-72; Histopathologic Technic, Philadelphia: Blakiston Co. 1948). Impregnate by immersion in the silver solution at room temperature for 2 to 5 min.; the time is varied until the optimum is determined. The silver solution will keep for several days but should be changed frequently after use. Dip in aq. dest. 1 or 2 sec. Reduce for $1 \mathrm{~min}$. in $2 \%$ neutral formalin (Merck, blue label) agitating gently. The formalin should be changed frequently. Wash in aq. dest. for $1 \mathrm{~min}$. Tone in yellow gold chloride $(1 \mathrm{~g}$. to $500 \mathrm{ml}$. aq. dest.) a few seconds to one minute. This step must not be prolonged beyond the exact time needed. Fix in $5 \%$ hypo for $1-2 \mathrm{~min}$. Wash in tapwater and counterstain lightly with $1 \%$ picric acid. Dehydrate in alcohol, clear in xylene, mount in neutral bal' sam and cover with cover slips.

This method successfully stains fibrous and protoplasmic astrocytes, microglia and oligodendroglia. By embedding the tissue in paraffin the problem of overformalinization is avoided and the tissues are still suitable for staining years later (See Nassar, T. K. and Shanklin, W. M., Stain Techn., 1951, 26, 13-18).

Silver Electrode of Linderstrom-Lang, Palmer and Holter described by Glick, p. 183.

Silver Gray, see Nigrosin, water soluble

Silver Lineation on pulmonary alveolar walls-Written by C. C. Macklin, Dept. of Histological Research, The University of Western Ontario, London, Canada. November 28, 1951-The following modernization of a very old technique is recommended. Immediately after cessation of the circulation the collapsed lungs of healthy mammals are filled, via the trachea or a large bronchus, with 0.2 per cent aqueous silver nitrate for not more than one minute; they are at once drained. They are promptly refilled with distilled water and evacuated, and this last process is repeated. They are then filled with $10 \%$ neutral formalin in water; the trachea is tied and the preparation immersed in the same fixative for 24 hours or more. The degree of distention should be approximately that of full inspiration (See C. C. Macklin, J. Thor. Surg., 1938, 7, 536-551, for further details and bibliography). Silver citrate may be used instead of silver nitrate (see R. D. Bensley, Am. J. Anat., 1929, 40, 146-169). Blocks are cut out and sectioned while frozen, or after having been embedded in paraffin or celloidin. Flattened frozen sections are best for en face examination of the silver lines on an expanse of alveolar wall. The silver lines are darkened by exposure of the sections to direct sunlight; or, as an alternative, the lungs 
may be briefly filled with $\frac{1}{10}$ strength hydroquinone solution after the silver has been thoroughly washed out and before fixation. Stains such as Mayer's paracarmine may be used. When the silver is thus applied for but an instant it does not iuvolve the underlying endothelium or reticulin fibers. In thick sections the silver-line network of the bronchial epithelium may often be followed directly into that of the alveolar walls, and the two are then seen to form one system. On the alveolar walls the areas circumscribed by the meshes of the net are not uniform. The smaller, more heavily marked, meshes enclose alveolar wall cells (pneumonocytes, septal cells, etc., which see) while the larger meshes, known as bare areas, contain no surface cells. Around them the lines in some animals, as rabbit, are often incomplete.

Silver Methods. General statement. A brief historical review by Silver, M. L., Anat. Rec., 1942, 82, 507-529 shows that progress has been made in the control of these techniques to the point where they yield reliable results with considerable uniformity. Impregnation of blocks of tissue and reduction of the silver in various ways were and still are the bases of the methods of Golgi, Cajal and Bielchowsky which have contributed so much to our knowledge of the Nervous System, which see. But one had to wait until the sections were cut and examined to ascertain the results. Sometimes they were all that heart could desire; at other times the worker faced repeated disappointments. Having labored with the silver impregnation of neurofibrils I have always avoided silver methods whenever others can be employed in their place. Now however with the successful application of reduced silver to sections mounted on slides the technique is brought from the insides of the blocks of tissue which one cannot see into the open, thanks to Rogers, W. M., Pappenheimer, A. M., and Goettsch, M., J. Exp. Med., 1931, 54, 167-169. Another advance was the introduction of protargol as the silver salt for treating sections of the central nervous system by Bartelmez, G. W. and Hoerr, N. L., J. Comp. Neurol., 1933, 57, 401-428. Then, likewise in Bensley's laboratory, Bodian, D., Anat. Rec., 1936, 65, 89-97 employed protargol with hydroquinone as reducer and speeding up results by copper, mercury and acid. Finally Davenport, H. A., McArthur, J., and Bruesch, S. P., Stain Techn., 1939, 14, 21-26 dispense with copper, and, by combining protargol and silver nitrate at optimum $\mathrm{pH}$, reduce staining time of sections of peripheral nerves to $2 \mathrm{hrs}$. In addition, Silver (loc. cit.) by well planned experiments has shown that staining with silver is brought about through adsorption and flocculation of electrically charged silver micelles by suitably charged surfaces. When these newer methods are widely brought to bear upon tissues of the body in normal and pathological conditions a significant service will be performed. Suffice it here to give a few details under Nervous System, Spirochetes, tests for Calcium, Chloride, Vitamin C, Reticular Fibers, Melanin, etc.

It is in some cases desirable to destain silver slides. To do this pass down to running water for $5 \mathrm{~min}$. and treat sections with $0.25 \%$ aq. potassium permanganate to which $1 \%$ of eone. sulphuric acid is added for $15 \mathrm{~min}$. Wash in running water $2 \mathrm{~min}$. Bleach in $5 \%$ aq. oxalic acid 2-5 min. Wash. Repeat the stain omitting preliminary oxidation-reduction, or apply some other technique (Wilson, R. A. J., Am. J. Clin. Path., 1943, Tech. Suppl. 7, 39)

Silver Nitrate is employed in many techniques. It is important to remember that ammoniacal silver nitrate solutions on evaporation yield an explosive compound. Consequently solutions of this sort should never be allowed to dry but should be washed down the sink with plenty of water.

Silver Staining of bone (McCollum, E. V. Simmonds, N., Shipley, P. G. and Park, E. A., J. Biol. Chem., 1922, 51, 41-49).

Silver's rapid silver-on-the-slide method for nervous tissue (Silver, M. L., Stain Techn., 1942, 17, 123-127). A new feature of this technique is the reducing solution.

1. For nuclei, fine fibers and nerve terminals, fix with $10 \%$ neutral or commercial formalin in $1 \%$ aq. sodium chloride with Bouin's fluid or with some other fixatives which he specifies preferably by Perfusion.

Cut frozen sections $10-40 \mu$ or dehydrate slowly, imbed in paraffin or celloidin and cut $2-20 \mu$. Mount paraffin sections on slides and deparaffinize in the usual way. In the case of celloidin sections remove celloidin with several changes acetone and of equal parts absolute alcohol and ether and pass down through alcohols to water.

To make reducing solution dissolve $64 \mathrm{gm}$. Rochelle salts (potassium sodium tartrate) in 500 cc. aq. dest. Boil vigorously. Add $10 \mathrm{cc} .10 \%$ aq. silver nitrate and boil again at least $5 \mathrm{~min}$. Remove from flame. Add $0.3 \mathrm{gm}$. crystalline 
magnesium sulphate and while simmering $0.2 \mathrm{gm} . K_{2} \mathrm{~S}$ (U.S.P.) employing only the brown unoxidized part of 1 piece. Filter while hot and make up filtrate with aq. dest. to 4 liters. This reducer improves slightly with age.

Place mounted paraffin sections or frozen or celloidin sections in equal parts above reducer and $0.5 \%$ aq. protargol (Winthrop Chemical Co., Inc., New York) at $45-55^{\circ} \mathrm{C}$. Staining is progressive and ordinarily takes $2-3$ hrs. Remove and examine. When complete, generally before a grossly visible reduction of silver is evident in the solution, remove, wash in 2 changes aq. dest., dehydrate, clear and mount. More finely myelinated fibers are revealed than are demonstrated by the standard Weigert technique.

2. For myelin sheaths and mitochondria fix with $10 \%$ formalin in $1 \%$ aq. potassium bichromate or with $10 \%$ formalin in $1 \%$ aq. $\mathrm{NaCl}$ again preferably by perfusion, and mordant small blocks of the tissue in 3\% aq. potassium bichromate for 7 days (This mordanting can be dispensed with if tissue is in fixative for more than 1 week.). Wash, dehydrate, imbed (in paraffin), cut 4-20 $\mu$ and mount on slide. Remove imbedding medium and proceed as described above.

Sinusoids are capillaries of large diameter through which the circulation is slower. The endothelial cells of their walls ingest some forms of particulate matter in the blood stream. The best place to demonstrate them is in carmine stained sections of formalin fixed liver of an animal injected intravenously with India ink as described under Vital Staining.

Sizes of Organs. See Normals.

Skin. No other part of the body is similarly spread out for examination in vivo. Much is to be gained by correlation of gross and microscopic study. Alterations in color, moisture, consistency and thickness can easily be detected. Changes in sensitivity and in the number and activity of sweat glands can be determined by appropriate methods. Simple techniques are available for the visualization of Lymphatic Vessels, and the Capillaries in the dermal papillae can be demonstrated microscopically and their behavior recorded in moving pictures. See 'Thomas Lewis' classic, The Vessels of the Human Skin and their Responses. London: Shaw \& Sons, 1927,322 pp. Very important is direct study of the skin with hand lens or binocular microscope.

But examination in sections will always remain the basic method of study. Hair, where present, should be cut short with scissors and removed with an electric razor, an instrument which does not require the use of any soap and does not scrape away the surface. Samples of skin removed at autopsy are satisfactory for some purposes up to about 24 hrs. if the body has been kept cool because autoly tic changes take place comparatively slowly in the skin. But biopsy specimens are much better. The local anesthetic should be injected in a circle about the skin to be excised and the observer should be on the lookout for slight modifications if the sections include the actual area into which it is forced. Obviously the specimen should be lifted, never pinched with forceps.

Because the skin is made up of 2 tissues, avascular epidermis and vascular dermis, closely bound together, differential shrinkage is a troublesome factor. Evans, R., Cowdry, E. V. and Nielson, P. E., have found in this laboratory that, owing to shrinkage or drawing together of the dermis, the folds in the epidermis are accentuated to an extent much greater than is generally realized. This is more marked in young skins than in those of old people and in living skin than in skin excised after long delayed autopsy. It is apparently not feasible to entirely side step this kind of artefact but the tendency of the whole specimen to curl up can be obviated by spreading it out with dermis down on a piece of wooden tongue depressor or stiff cardboard for the first few minutes of fixation. If interest definitely centers in the dermis it should be mounted with epidermis down. But it should not be kept in either position too long because the complete entry of fixative will thereby be prevented. After 3 or $4 \mathrm{hrs}$. the specimen should be trimmed with a new wet razor blade.

Frozen sections are essential for rapid diagnosis, for staining with Sudan and for many other purposes. The technique most used by dermatologists is to fix in Bouin's Fluid and to stain paraffin sections with Hematoxylin and Eosin. After Zenker Fixation, Mallory's Connective Tissue Stain, or Masson's Trichrome Stain, is suitable for muscle and collagenic tissue. Weigert's resorcin fuchsin is recommended for clastic fibers. The Dopa Reaction is required for melanin precursors. For nerve fibers the Bodian method is probably the best. Another silver technique advised for the skin is that of Jalowy.

MacCardle, R. C., Engman, M. F., Jr. and Sr., Arch. Dermat. \& Syph., 
$1941,44,429-440$ give details of spectrographic analysis of skin lesions. See also Microincineration. Ultracentrifugation method for determination of intranuclear viscosity (Cowdry, E. V. and Paletta, F. X., Am. J. Path., 1941, 17, 335-357). Methods of transplantation are described by Kelly, R. W. and Loeb, L., Anat. Rec., 1939, 74, 487-509 and of fluorescence examination by Cornbleet, T. and Popper, H., Arch. Dermat. and Syph., 1942, 46, 59-65. An adaptation of the Sandison technique is recommended by Williams, $R$. G., Anat. Rec., 1934, 60, 493-499. See Sebaceous and Tarsal glands, Hairs, Nails, Feathers.

If it is not desired to investigate a particular area, to which attention has been called by its unusual gross appearance; but, instead, to demonstrate some special component, or response, of the skin one should be guided in selection of the specimen by the location where the component or response is most likely to be found. Thus Meissner's corpuscles are best seen in sections of skin of palmar surface of finger tips. Weddel, G., J. Anat., 1941, 75 (3), 346-367 reports that multiple groups of Krause's endbulbs occur beneath each cold spot in the forearm about $1 \mathrm{~mm}$. inward from the skin surface. Many helpful clues are supplied by Lewis, T., Pain. New York: MacMillan, 1942, 192 pp. $\mathrm{He}$ quotes Strughold as stating that pain spots are aggregated as closely as 200 per sq. $\mathrm{cm}$. in supraclavicular, antecubital, inguinal and popliteal fossae while they are rare $(40-70$ per sq. cm.) on tip of nose and ear, soles and palms (see Nerve Endings). The skin of axillary, pubic and nipple areas is more likely than that of the rest of the body to respond to sex hormones. Adjustments to external environment are to be expected in exposed parts. To search for sweat glands in those mammals which do not possess any is futile. To expect all epidermal layers in thin epidermis is likewise contraindicated.

Fluorescence Microscopy is capable of yielding interesting results in distinction between psoriasis and hyperkeratosis scales (Radley, J. A. and Grant, J., Fluorescence Analysis in Ultraviolet Light. New York : Van Nostrand, 1935). Further indications on fluorescence are given under Hair and Sebaceous Glands.

Now that epidermis can be conveniently separated from dermis it is desirable to give details of technique relating to it under a separate heading. See Epidermis.

Skunk's Stain, see Flagella.
Skyblue (CI, 1286)-coelestin blue, coeline, coeruleum - a mineral pigment, cobaltous stannate, seldom used in medical research.

Slides, see Cleaning.

Slifer-King method, see Ticks.

Slime Forming Bacteria, Conn's method. Stain for about 1 min. with a little heat in Rose bengal 1 gm., $5 \%$ aq. phenol 100 cc., $1 \%$ aq. $\mathrm{CaCl}_{2}, 1$ cc.; then wash quickly and dry (McClung, p. 146).

Small Intestine. Many conditions influence the appearance seen in sections. If fixed while distended with food material, the spaces between the villi are more noticeable, the villi shorter and the muscular layers thinner than when fixed while strongly contracted. See illustrations provided by Johnson, F. P., Am. J. Anat., 1912-13, 14, 235-250 and Contraction Bands. The time after feeding and the character of the food has a marked influence on structure. The cytoplasmic granules of the Paneth Cells are almost all discharged in guinea pigs $6 \mathrm{hrs}$. after feeding. They are present in large numbers after fasting for 24 hrs. (Klein, S., Am. J. Anat., 1905-06, 5, 315-330). Even vitamin B deficiency al ters the distribution of intraepithelial fat (Mottram, J. C., Cramer, W., and Drew, A. H., Brit. J. Exp. Path., 1922, 3, 179-181). According to Hamperl (H., Ztschr. f. Mikr.-Anat. Forsch., 1925, 2, 506-535) Enterochromaffin Cells can no longer be found in humans autopsied as late as 4-5 hrs. after death. The incidence of Contraction Bands in muscle is increased by exposure to air and mechanical manipulation before fixation. Villi are very prone to exhibit Agonal Changes. If the individual has fasted for a long time before death a marked invasion of the mucous membrane by lymphocytes is to be expected. See Fig. 158, Cowdry's Histology. It may extend throughout the gastrointestinal tract being greatest in the stomach and least in the large intestine.

A good way to examine the wall of the small intestine is to push a test tube of appropriate size into the lumen of a segment. This will hold it open and facilitate dissection. Strip off the serosa, then the tunica muscularis, noting the direction of the fibers and leaving only the mucosa. Take small pieces of mucosa and mount in physiological saline inside up and examine at low magnification. Finally with dissecting needles pick out separate villi and study with oil immersion objective. To obtain a clearer concept of individual muscle fibers first macerate the intestine on the tube in $15 \%$ aq. nitric acid for 
2-3 days. Consult Carey, E. J., Anat. Rec., 1921, 21, 189-215 and Goerttler, K., Morph. Jahrb., 1932, 69, 329. See Chloralhydrate Maceration.

Smears. To examine fluids and tissues as thin films so that the components are individually clearly visible is of ten necessary. Careful preliminary cleaning of the slides is necessary. Touch the surface of a slide about $2 \mathrm{~cm}$. distant from the end to a drop of blood immediately on the appearance of the latter from a puncture in the skin. Quickly apply the smooth end of another slide to the drop and the surface of the first slide so that the drop spreads along the line of contact. Then evenly push the second slide, with the blood following it, along the surface of the first slide. The angle at which the pusher is held plus the speed of smearing and the amount of blood will determine the thickness of the film. Ordinarily it should be so thin that the reds are smeared in a single layer. But for certain purposes as in the search for some parasites thick smears are the best (see Blood Smears).

In the case of cells in cerebrospinal and other fluids and of some bacteria and parasites it may be desirable to concentrate the objects by centrifugation because otherwise smears would show too few of them. See Concentration Methods. The precautions detailed above to obtain evenness are sel. dom required. The material simply is transferred to the slide in a platinum loop or glass pipette and spread on it. Smears of lymph nodes and spleen are generally made by drawing "streaking" the freshly cut, moist surfaces along slides. Impression preparations of these tissues are not smears but they serve the same purpose. In making them the surface of slide is quickly pressed against the surface of the tissue and a considerable number of the easily detachable cells adhere to the slide where they are quickly dried, or, while still wet the impression can be fixed in Helly's fluid (i.e. formalin Zenker) as advised by Maximow (see Downey, p. 2001). McClung (p. 262) recommends smears on cover glasses for certain germ cells.

The smears can be fixed by gentle heat, or by methyl alcohol or in special cases in formalin or osmic vapor. Giemsa's stain is the most popular but a great many others are available especially for Bacteria.

Smears cannot be made of fixed cells isolated by Maceration in the same way because they are not present in body fluids which when they dry facilitate sticking of the cells to the slides. It is therefore necessary to spread them on slides previously moistened with a very small amount of Albumen-Glycerin before drying. See Papanicolaou Techniques, Ear and Nasal Cell Smears.

Smith-Dietrich method for lipoids (Dictrich, A., Verh. d. Deut. Path. Ges., $1910,14,263-268)$. Treat frozen sections of formalin fixed tissues 1-3 days in $5 \%$ aq. potassium bichromate at $37^{\circ} \mathrm{C}$. After washing in aq. dest. stain 4-5 hrs. in Kultschitzky's hematoxylin (stock solution $10 \%$ in abs. alc. ripened at least 6 months, $10 \mathrm{cc} .+2 \%$ acetic acid, 90 cc.). Wash. Differentiate over night in Weigert's borax ferricyanide (borax, $2 \mathrm{gm}$.; potassium ferricyanide, $2.5 \mathrm{gm}$.; aq. dest., $100 \mathrm{cc}$.). Wash carefully. Mount in syrup of levulose. Lipoids dark blue. Lison (201) considers the positive staining as characteristic for a lipine (lipoid) if the possible presence of cholesterides and cholesterol is excluded.

Smooth Muscle, see Contraction Bands.

Soap-Wax technique for paraffin imbedding, see Lebowich.

Soaps. Sodium and potassium salts of fatty acids, see Fischler's modification of Benda method.

Sodium. A method for the retention of sodium and potassium in microincinerated tissue has been proposed by Policard, A., and Pillet, D., Bull. d'Hist. Appl., 1926, 230-235. In their opinion these two elements are present as chlorides in the tissue and their conversion to sulphates by treating the sections with sulphuric anhydride fumes makes them more stable and better able to withstand the high temperature of incineration. See Microincineration Radioisotopes.

A good colorimetric method for sodium is reported by Bott, P. A. (J. Biol. Chem., 1913, 147, 653-661). He used it for determinations of sodium in glomerular urine. It works even with $0.3 \mu \mathrm{gm}$. of urine in $0.2 \mu \mathrm{l}$ with errol of about $3 \%$. Such techniques are not advised for people untrained in chemistry.

Probably the best titrimetric method for sodium is that of Linder, R. and Kíirk, P. L. (Mikrochemie, 1938, 23, 269-279) for small amounts of tissuc having $0.13-4.13 \mu \mathrm{gm}$. of sodium. According to Glick, p. 270 the technique of Clark, W. G., Levitan, N. I., Gleason, D. F. and Greenberg, G. (J. Biol. Chem., 1942, 145, 85-100) might be adapted to the required micro level for histochemical investigation.

An ultramicromethod for sodium employing the polarograph has been devised by Carruthers, C., Indust. and 
Engin. Chem., 1943, 15, 70-71. It has been used for analysis of small amounts of epidermis by Suntzeff, V. and Carruthers, C., Cancer Research, 1943, 3, 431-433. If it is only necessary to prove presence or absence of traces of sodium try Histospectrography.

Sodium Alizarin Sulphonate. See Hydrogen Ion Indicators.

Sodium Fluoride effect on teeth (Cowdry's Histology, p. 267).

Sodium Paradipicrylamine, see Siena Orange.

Soil. Bacteria. 1. Conn's Rose Bengal method (McClung, p. 146). To $1 \mathrm{gm}$. soil add gelatin fixative $(0.015 \%$ gelatin in boiling water used after it has cooled) to make $10 \mathrm{cc}$. Place about $0.01 \mathrm{cc}$. on slide to cover $1 \mathrm{sq}$. cm. Dry on boiling water bath. Stain with Rose bengal as for Slime Bacteria. Unless counts are to be made the amount smeared on the slide is not important.

2. Fast acid blue (C.I. 760) is strongly recommended (Romell, L. G., Stain Techn., 1934, 9, 141-145) but it is doubtful whether any manufacturer other than I. G. Garbenindustrie makes the dye. According to the General Dyestuffs Corporation it is contained in violamin 3B. Dry suspension of soil on slide which has been fixed in alcohol with $0.05 \%$ dye in $4 \%$ aq. phenol. Washing is unnecessary. Examine smears in water. Details are given by Romell.

Solantine Red 8 BLN. A sulfonated azo dye. For formula and influence on mouse tumors, see Stern, K., Cancer Res., 1950, 10, 565-570.

Solanylin, a dye extracted from the eggplant (Solanum melongena, var. esculenta) proposed as a substitute for hematoxylin. It will stain nuclei and mucus (Fuse and Suzuki, Arb. Anat. Inst. zu Sendai, 1935, 17, 175-181).

Solid Green JJO, see Brilliant Green.

Solid Green O, see Malachite Green.

Soluble Blue $3 \mathrm{M}$ or $2 \mathrm{R}$, see Anilin Blue.

Soluble Indulin $3 \mathrm{~B}$, see Indulin, water soluble.

Soluble Yellow OL, see Metanil Yellow.

Solutions. In technique several kinds are employed.

1. Physiological solutions are intended to approximate as closely as possible to the tissue fluid environments of cells so that cells examined in them will not be greatly altered thereby. See Physiological Solutions.

2. Normal solutions are, on the other hand, chemical standards made by dissolving definite amounts of substance (easily calculated) in sufficient aq. dest. to make 1 liter. See Normal Solutions. 3. Molar, molecular and grammolecu- lar solutions contain the molecular weight of the substance in grams per liter. They are of the same concentration as normal solutions of substances possessed of one hydrogen or other equivalent and differ from those of substances containing more than 1 such equivalent. See Molecular Solutions.

4. Molal solutions contain the molecular weight of the substance in grams +1000 grams aq. dest. The designation molal is rarely used, molecular is common and normal most frequent.

Sonic Vibrations. Employed as a means for fractionating spermatozoa so that their several parts can later be collected by centrifugation (Zittle, C. A. and O'Dell, R. A., J. Biol. Chem., 1941, 140, 899907). See Ultrasonic Vibrator.

Sörensen's Buffers. Sörenson's phosphate buffers are prepared from Merck's special reagents. Dry salts at $105^{\circ} \mathrm{C}$. overnight and store in a dessicator over $\mathrm{CaCl}_{2}$. $\mathrm{M} / 15$ solutions are used. To make them dissolve the following amounts in aq. dist. and make each solution up to one liter:

$\mathrm{Na}_{2} \mathrm{HPO}$ anhydrous.......... $9.47 \mathrm{gm}$.

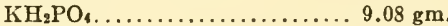

To obtain a solution of the $\mathrm{pH}$ required, mix them in following amounts:

$\begin{array}{ccc}\text { pH } & \text { cc. M/15 } & \text { cc. M/15 } \\ \mathbf{N a} & \text { Na.4 } \text { KO }_{2} \mathrm{PO} & \text {. } \\ 5.6 & 3.0 & 97.0 \\ 5.8 & 5.0 & 15.0 \\ 6.0 & 7.8 & 92.2 \\ 6.2 & 12.0 & 88.0 \\ 6.4 & 18.5 & 81.5 \\ 6.6 & 26.5 & 73.5 \\ 6.8 & 37.5 & 62.5 \\ 7.0 & 50.0 & 50.0 \\ 7.2 & 61.1 & 38 . \\ 7.4 & 71.5 & 28.5 \\ 7.5 & 80.4 & 19.6 \\ 7.8 & 86.8 & 13.2 \\ 8.0 & 91.4 & 8.6\end{array}$

For range $\mathrm{pH}$ 8.2-9.2 see Palitzsh Buffers. See affect of Phosphate Solutions on living cells.

Spalteholz Method for clearing small embryos as suggested by the Bensleys. After appropriate fixation 80 and $95 \%$ alcohol 1 day each. Two changes absolute alcohol, 2 days. Equal parts benzol and absolute alcohol, 1 day. Two changes pure benzol, 1 day. Then Wintergreen oil (methyl salicylate) and benzyl benzoate by weight $5: 1,3: 1$ and $2: 1$ for very young, young and older embryos respectively (under negative pressure in vacuum pump) until cleared. Mount or store in this clearing fluid. In practice it is possible to get good results 
without the negative pressure. This method can be used for many tissues besides embryos. For author's account see Spalteholz, W., Ueber das Durchsichkigmachen von menschlichen und Tierischen Präparaten. Leipzig, 2nd Edition, 1914.

Specific Gravity. It is often desirable to ascertain the relative specific gravities of tissues, cells and parts of cells. See Centrifugation.

Spectrographic Analysis, see Histospectrography and Absorption Spectra.

Spectrophotometric Analysis of tissue staining has been greatly advanced by Stowell, R. E. and Albers, V. M., Stain Techn., 1943, 18, 57-71. Comparison of spectral absorption curves of stains and substances colored by them has demonstrated that data can thereby be obtained on the chemical processes involved. No evidence was found of significant chemical alterations in the chromophox radicals of the stains associated with the tissue staining under the conditions of the experiments.

Spectrophotometric Evaluation of blood stains (Lillie, R. D. and Roe, M. A., Stain Techn., 1942, 17, 57-63).

Spermatozoa, simple method for staining. Make smears of fresh spermatic fluid on slides and dry in air. Fix 3 minutes in $10 \%$ formalin. Stain in Harris' hematoxylin 1 minute, wash in water and dry (Fetterman, G. H., Am. J. Clin. Path., $1942,6,9)$. Microincineration (Policard, A., Bull. d'Hist. Appl., 1933, 10, 313-320). Helpful histochemical methods are detailed by Marza, V. B., Bull. d'hist. appl., 1931, 8, 85-102.

Sperms are excellent material for Electron Microscopy. See Schmitt, F. O. (Biological Symposia, 1943, 10, 261) and Scott, G. H. in McClung's Microscopical Technique, 1950 , p. 723 . The Keilin, D. and Hartres, E. F. (Nature, $1950,165,504)$ device of manifold intensification of absorption spectra in liquid air has been employed by Mann, T. (Biochem. J., 1951, 48, 386-388) for cytochrome determinations in sperm. See Semen.

Spermin Crystals are long prism-like formations produced in dried semen colored brown or violet with iodine or potassium iodide, also known as Boettcher's crystals.

Sphingomyelin, a compound of phosphoric acid, a fatty acid, choline and sphingosine without glycerol, soluble in benzene, pyridine and hot alcohol and almost insoluble in ether, see Lipoids.

Spirit Blue (CI, 689)-anilin blue alcohol soluble, gentian blue $6 \mathrm{~B}$, light, Lyon and Paris blues-A mixture of di-and tri-phenyl rosanilin chlorides. Conn (p. 133) reports that it is a good stain for growing nerve fibers.

Spirit Indulin, see Indulin, spirit soluble. Spirit Nigrosin R, see Indulin, spirit soluble.

Spirochaetales. The organisms of this group often require special methods for demonstration; but within the gastric glands of humans (Doenges, J. L., Arch. Patl., 1939, 27, 469-477) dogs, cats, rats and Macacus rhesus monkeys (Cowdry, E. V. and Scott, G. H., Arch. Path., $1936,22,1-23$ ) they can frequently be seen in ordinary hematoxylin and eosin preparations. Preparations of these benign organisms are therefore easily made and useful as showing intracellular forms within parietal cells. For special techniques see Treponema Pallidum, Warthin-Starry method and Vincent's Angina.

Spleen. Fixatives penetrate the spleen poorly on account of the large amount of blood in it. Consequently it is desirable to fix only thin slices of it, say 3-4 $\mathrm{mm}$. thick. If the spleen is particularly soft to begin with the slices will not hold their shape and it may be necessary to cut parallel to the surface and include the capsule as a support. Direct observation of splenic venous sinuses in vivo (Knisely, M. H., Anat. Rec., $64,499-524 ; 65,23-50$; MacKenzie, D. W., Whipple, A. O. and Wintersteiner, M.'P., Am. J. Anat., 1941, 68, 397-456). Transplants into omentum (Holyoke, E. A., Am. J. Anat., 1940, 66, 87-132). For vascular injections of Malpighian bodies, see Nisimaru, Y. and Staggerda, F. R., J. Physiol., 1932, 74, 327-337. See Kurlof Bodies.

Spodogramme, term used by French histologists for the mineral skeleton of tissue revealed by Microincineration.

Spore Stain, a modification of Dorner's. Make thin film on slide. Cover with blotting paper and add freshly filtered Ziehl's carbol fuchsin. Steam 5-10 min. on hot plate, the blotting paper being moistened with the fuchsin. Decolorize instantaneously with $95 \%$ alcohol and wash in water. Add drop of sat. aq. nigrosine and spread thinly. Dry quickly and examine. Spores red, other parts of bacilli almost colorless against dark background. Said to besimpler, quicker than the unmodified Dorner's method. It is recommended for Bacillus megatherium, $B$. niger, $B$. cereus, $B$. mycoides and some cultures of $B$. subtilis (Snyder, M. A., Stain Techn., 1934, 9, 71-72).

Stain heat fixed film with carbolfuchsin (see Acid Fast Bacilli). Rinse quickly and differentiate in $95 \%$ alcohol. Wash in hot tap water and again rinse in alcohol. Counterstain for 2-5 min. 
with Loeffer's methylene blue. In case of thick films pour off and add more blue. Rinse in tap water and blot dry (S. Bayne-Jones in Simmons and Gentzkow, p. 386).

A modification of Schaeffer's spore stain. Support a small metal tray over asbestos centered wire gauze. Add water and heat to stcaming. Slides with ends resting on either side of the tray should have droplets of water condense on their under surface. Flood properly fixed smear on slide with $5 \%$ aq. malachite green and leave in this way on steam bath 1 min. Drop in cold water, rinse thoroughly and while wet add $0.5 \%$ aq. safranin $30 \mathrm{sec}$. Rinse again in cold water. Spores, green; vegetative cells, red (Ashby, G. K., Science, 1938, 87, $443)$.

Spreading Factors and ground substance of Mesenchyme-Written by F. DuranReynals, Dept. of Microbiology, Yale University, New Haven, Conn. October 8,1951 -A discussion on the nature and function of the spreading factors (S.F.) must be indispensably preceded by a survey on the newer knowledge of the ground substance (G.S.) of the mesenchyme, a knowledge to which, precisely, the discovery of the S.F. has largely contributed.

The importance of the G.S. is easily emphasized: it is impossible to have a complete concept of cell function if this cell is not considered together with its immediate environment, namely, the G.S. or other intercellular matrices which, it is to be hoped, some day will be known. One cannot have a clear idea of the effects on cells of poisons or therapeutic agents; of the portal of entry and of so many other phenomena in infection and defense against infection without a previous knowledge of the initial effect of the invading agent on the G.S. or, conversely, of the effect of the G.S. on the infectious agent.

The G.S. of the mesenchyme is, of course, present in all mesodermic structures, and consequently it pervades the whole animal body. It is present in the skin where it spreads as a continuous sheet underneath the epidermis; and in between the follicular cells surrounding the mammalian ovum. The synovial fluid is a modified G.S., and the joint cavities can be considered as giant intercellular formations. The G.S. is also abundantly in the vitreous humor, in the umbilical cord, and still other structures.

From direct studies by a group of physiologists, the following has been learned about the G.S.:

(a) It is a jelly placed between vessels, cells and fibers, and does not contain spaces;

(b) It does not contain free water under physiological conditions. Water is associated with components of the jelly, which is capable of hydration in different degrees;

(c) Dyes, and presumably metabolites, do not seem to progress through the jelly proper, but following the pathways of the fibrillar structures; and

(d) It shows a resistance to penetration by inoculated fluids, and this resistance is not overcome until a pressure of $8.5 \mathrm{~cm}$ of water has been reached. Therefore, the G.S. is a barrier.

The main, or best known, components of the G.S. are mucopolysaccharides-linked with protein, to which the G.S. owes its viscid consistency. The most significant of the polysaccharides is hyaluronic acid (H.A.)

H.A. is a member of a very important group of substances such as heparin, mucoitin, and chondroitin sulphuric acids, and still others, all of which play preponderant physiological roles.

The G.S. can be identified by means of staining reactions, more or less selective for polysaccharides, such as periodic acid, leuco fuchsin, toluidine blue, Prussian blue, and still others.

One may suppose that since H.A. is so widely present in normal and pathological tissues, its secretion is the work of many mesenchymal cells, including plain fibroblasts, in a manner perhaps comparable to the formation of the acid in the capsule of some bacteria, e.g. streptococcus group A and C. However, a variety of contributions seem to incriminate specialized granule - containing cells, identified or not to mast cells as the elements responsible for the formation of the G.S. in general; or of synovial fluid; or hyaluronic acid in particular. One may wonder whether H.A., so rapidly and abundantly accumulated in some normal or pathological tissues, such as in the sex skin of monkeys and some tumors, is originated by the same cells that secrete the acid present in other tissues. Be it as it may, H.A. formation is controlled by endocrine factors which will be reviewed later.

The S.F. are the agents which act selectively or exclusively on the G.S., changing its physical and chemical characteristics. They were discovered in 1928 by the enhancing effect that extracts from mammalian testes proved to have on infection. The most important, or at least the best known, of the S.F. is hyaluronidase $(\mathrm{H})$ which is present, in mammalian testes, in cer- 
tain invasive bacteria such as staphylococcus, streptococcus, pneumococcus, in animal poisons such as that of rattlesnakes, in secretions of insects such as mosquitoes, etc. Often $H$. is associated with other spreading factors in the animal secretions.

The in vivo effect of $\mathrm{H}$. is shown by the spreading reaction. In this reaction any material injected in the G.S., together with $\mathrm{H}$., spreads rapidly as if ink were dropped on a blotter. The reaction is best shown when a mixture of any H.-containing material and any colored matter is injected intradermally into a rabbit. A control inoculum of the colored material alone remains largely localized where the presence of the injection has left it.

$H$. was identified to a mucolytic enzyme in 1939. The action of the enzyme, or rather of the group of enzymes, has been and still is subject to extensive biochemical study into which we cannot enter. Essentially, H.A. attacked by the specific enzyme contained, for instance, in a simple rabbit testicle extract, is first depolymerized and then split into its components, glucuronic acid and acetyl glucosamine. The first effect manifests itself by a sudden drop in the viscosity of the solution containing the polysaccharide, for instance, a simple extract of umbilical cord.

This reaction in the test tube is the counterpart of the spreading reaction in the animal. In the latter case, therefore, the H.A. of the ground substance is similarly attacked by the injected $\mathrm{H}$. and the jelly is quickly liquefied. What used to be a barrier is now a pathway, and consequently, any material injected together with the enzyme will easily spread throughout the intercellular atmosphere. Mechanical pressure increases the diffusion considerably.

The spreading reaction also takes place, although far less conspicuously than in the skin, in tissues in which connective tissue exists in formations susceptible of being injected. Whether $\mathrm{H}$. attacks components of the blood capillaries resulting in increased capillary permeability is still a debatable point. At any rate, the permeability of the blood capillaries is considerably increased by some factor which is regularly present in the materials containing $\mathrm{H}$.

There are other S.F. which act on the G.S. of the mesenchyme, but their mode of action is far less known than in the case of $\mathrm{H}$. The effect of azoproteins and ascorbic acid seems to be of only a physical, depolymerizing nature. Others, found in several tissue extracts, bacterial cultures and animal secretions, and also simple chemicals, do not affect H.A. in vitro, and their spreading effect may largely be due to edema formation, as shown by the lack of spreading in the skin of the dead rabbit. It may be quite important that, as shown recently, derivations of H.A. have a spreading effect even in the dead rabbit. All these facts emphasize the complexity of the spreading reaction, and suggest that more attention should be paid to S.F. other than H. Such study could prove to be extremely fruitful.

With the above reviewed as a background, we are on a solid basis to explain phenomena of fundamental importance in pathology, such as the invasion of the lung by the pneumococcus; the progressive march of erysipelas; the brutal invasion of tissues following snake bite; the efficacy in the inoculation of infectious agents carried by insect vectors; and so many others.

In the domain of physiology, besides other possible effects we can understand the liberation of the ovum from its surrounding granulosa cells as an event preceding fertilization. H.A. is largely present in the cement holding these cells together, and from the attack of the acid by the enzyme secreted by the spermatozoa, this cement is liquefied and the granulosa cells become dispersed.

In the field of pharmacology, we can also understand the powerful enhancement of the effect of therapeutic agents such as the various solutions administered in clysis, of local anesthetics, of antibiotics, etc. when the agent is injected together with $H$. which will promote its rapid spreading and absorption. Also, the injection of $\mathrm{H}$. in edematous areas subsequent to injury greatly accelerates the absorption of the extravasated fluid, possibly the effect of the enzyme being here of a more complex nature.

If a dye solution is injected intradermally into animals it will sometimes diffuse (although far less than if $H$. is added) depending on different factors such as age, sex, genetic constitution, etc. of the host. This simple experiment indicates that the G.S. has a physiological permeability or tonus, subject to changes. When the permeability is decreased the effect is manifest by a suppression of the spreading effect of H., the opposite being prob- 
ably true when the permeability of the G.S. is increased.

Hormonal effects play a very important part in controlling the permeability of the G.S. Thus, estrogenic, and some adrenal cortical, hormones decrease the permeability of the G.S.that is, increase its barrier valuewhereas chorionic gonadotropin hormone has the opposite effect. Other hormones have been studied in this respect, sometimes with contradictory results.

The mechanism of action of these hormones-a crucial point in our subject-is a matter of conjecture. We have three clear cases in which the hormone has promoted the accumulation of vast amounts of hyaluronic and chondroitin sulphuric acid in tissues of election: estrogens causing the development of the sex skin of monkeys; testosterone inducing the development of the cock's comb; and thyroid stimulating hormone provoking the formation of periorbital tissue in the guinea pig and pretibial myxedematous tissue in man. In all these cases $H$. injected into the tissue causes their quick dissolution or collapse through an effect on H.A.

In the case of the sex skin of monkeys, at least, the accumulation of H.A. increases very much the barrier value of the tissue to the penetration of foreign matter. However, we do not have any evidence that, in the other cases where the permeability of the G.S. has been diminished by endocrine effects, the effect is due to quantitative changes in the tissue polysaccharides. This statement holds specially true in the case of the adrenal cortical hormones because their effect is very rapid, being most marked one hour after intravenous injection. Therefore, it is logical to think that qualitative besides quantitative changes in the polysaccharides are effective in altering the permeability of the G.S.

It would also seem logical to suppose that there is a common denominator to the effects of at least some of the hormones effective on the G.S., and, in view of the newer knowledge on the physiology of the adrenal cortex, one could suspect that some of the adrenal cortical hormones are this common denominator. In favor of this supposition one could quote the fact that a great variety of stimuli which de. crease the permeability of the G.S. are also known to act on the adrenal in the alarm reaction syndrome.

Of special interest in this respect is the case of infection, notably that in- duced by rapidly invading bacteria. Here, shortly after the experimental inoculation of e.g. staphylococcus or streptococcus, the permeability of the G.S. is considerably diminished, and this phenomenon is, in itself, a powerful element in the defense of the organism against infection, specially in its first phases.

Whatever hormones are responsible for the diminished permeability or increased barrier value of the G.S., we do not know how the effect is brought out, although one could theorize on a direct effect on the polysaccharides or on whatever cells of the mesenchyme responsible for the elaboration of these polysaccharides.

These problems are of far reaching importance. For, fluctuations in the permeability of the G.S. of the mesenchyme manifest themselves by parallel alterations in susceptibility or resistance to infection, and to a certain extent it is possible to protect against e.g. vaccinia or tuberculosis by treatment of the host with estrogenic hormones. True, we still do not know the precise mechanisms of the effect above reviewed, but we do know that the harmonious integration of these effects reflects on the G.S., which will maintain its perfectly physiological tonus.

If we know so much about acquired immunity, it is, to a large extent, because the natural immunity has failed. Maintaining this tonus of the G.S. would mean controlling of complex mechanisms leading to natural resistance, that is to health. (See DuranReynals, F., Bact. Rev., 1942, 6, $197-$ 252. Also Symposium on "The ground substance of the Mesenchyme and hyaluronidase" in: Ann. N. Y. Acad. Sci., 1950, 52, 943-1196.)

Sputum. Amount, gross appearance, color and odor (if present) are important. Microscopic examination should first be made mounted but unstained. Look for pus, elastic tissue, pigmented heart failure cells, amebae, fungi, ova of animal parasites, colorless, hexagonal pointed Charcot-Leyden crystals, other crystalline material, etc. Stain smears by methods of Giemsa, Gram and for Acid Fast bacilli. It may be necessary to use Concentration methods. Interpretation of findings requires much experience. Comparison of chlorox and sodium-hydroxide-alum techniques for tubercle bacilli in sputum (Cameron, G. M. and Castles, R., J. Lab. \& Clin. Med., 1946, 31, 361-368). See also Section on Sputum Examination in Osgood, E. S., Laboratory Diagnosis. Phil- 
adelphia: Blakiston Co., 1940,676 pp. See Papanicolaou Techniques.

Staining is the act of giving color to something. It is said to be progressive when the structures colored take up the stain progressively to a greater degree than do others which by contrast are not colored. Thus, in testing for iron by the Macallum method the iron is stained progressively with hematoxylin. Staining is called regressive when many structures are over stained and by decolorization, or differentiation, the color regresses and is retained only by those which hold it most tightly in contrast with which the others are not stained. To demonstrate Nissl bodies in nerve cells the cells are over stained with toluidin blue. By decolorization in alcohol the color is made to regress to the point where the Nissl bodies stand out colored in a cytoplasm no longer blue. See, also vital and supravital staining and acid and basic dyes.

Acid stains are often contrasted with basic ones though the dyes are usually neutral salts. In "acid" dyes it is the acid part, or anion, that is colored and does the staining; while in "basic" dye the reverse holds and it is the basic portion, or cation, that is the coloring agent. For instance, acid fuchsin is a sodium salt of sulphonic acid of fuchsin and it is the acid part which gives the color. Basic fuchsin, on the other hand, is a hydrochloride of rosanilin and it is the base, rosanilin, which stains. A "neutral" dye is a more complex association between a color acid and a color base.

Basic materials may be colored by acid dyes and acid ones by basic dyes, but this does not by any means always hold. A substance staining by an "acid" dye is said to be acidophilic, as for example the specific granules of eosinophile leucocytes which take the "acid" dye eosin. Similarly another material, such as nuclear chromatin is termed basophilic because it colors with toluidin blue which is a "basic" stain. A neutrophilic granule is colored by both the color acid and the color base of a neutral dye. An amphophilic one (G. ampho, both; philos, fond) will stain with either acid or basic dyes or with a neutral dye for it likes both color acids and color bases. Heterophile leucocytes (G. heteros, other, and philos, fond) possess granules which are homologous for the several species but differ in staining reaction for the species (Maximow-Bloom, Histology, 2nd Edit. 1934). See Supravital and Vital Stains.

Stains. The laboratory worker desiring to keep clean can use the methods advised by W. C. Tobie (Simmons, and Gentzkow, p. 358).

Bacteriological stains on hands. Wash in 2 or $3 \%$ conc. hydrochloric acid in $95 \%$ alcohol (by vol.) and then in soap and water. For fabrics, wash in $10 \%$ acetic acid in $95 \%$ alcohol (by vol.) and rinse repeatedly in much water; in case stain remains wash with dilute chlorine, or bromine water, or with filtered chlorinated lime solution (as "HTH" high test hypochlorite) and rinse again in water.

Iodine stains. Remove with aq. sodium thiosulphate and wash in water.

Blood stains. Wash away with $3 \%$ aq. hydrogen peroxide, and rinse in water.

Silver stains occasioned by silver nitrate, argyrol and the like. Treat with hot solution of $5 \mathrm{gm}$. mercuric chloride $+5 \mathrm{gm}$. ammonium chloride in $100 \mathrm{cc}$. water.

Mercurochrome stains. Wash out fresh ones with dilute bromine water or chlorine water or fresh aq. filtered chlorinated lime (HTH). Old ones should be treated with $2 \%$ aq. potassium permanganate followed by $5 \%$ aq. oxalic acid and washing in water.

Biological fluids. Stains and smell of putrefaction caused by them can be removed, as above, by permanganate and oxalic acid.

Standards. See Biological Standards, Normality, Normals.

Starch Grains. The usual microchemical test is to color blue with dilute iodine. Starch grains can also be stained side by side with mitochondria in plant cells (Pea roots, Elodea, etc.). After Regaud fixation stain sections with warmed anilin fuchsin about $5 \mathrm{~min}$. Differentiate in $5 \%$ alcoholic aurantia. Wash in aq. dest. Mordant in $2 \%$ aq. Tannin, $20 \mathrm{~min}$. Wash in aq. dest. and stain in $1 \%$ aq. toluidin blue, gentian violet or methyl green, 5-10 min. Milovidov, (P. F., Arch. d'Anat. Micr., 1928, 24, 8-18). Differentiate in $95 \%$ alc. dehydrate in abs. alc., clear in xylol and mount. Mitochondria red, starch blue, violet or green. Well shown in an excellent colored plate. Armed with illustrations showing the distinctive structural features of starch granules from many species of plants it is ordinarily a simple matter by direct microscopic examination to identify a given sample of starch (Schneider, A., The Microbiology and Microanalysis of Foods. Philadelphia: P. Blakiston's Son \& Co., 1920, 262 pp.). See Polysaccharides.

Starch Paste, as substitute for albuminglycerin mixture in mounting paraffin 
sections. Mix thoroughly $1 \mathrm{gm}$. powdered starch in $10 \mathrm{cc}$. cold water. Pour into 20 cc. boiling water. Add 2 drops dilute $\mathrm{HCl}$ and boil $5 \mathrm{~min}$. constantly stirring to free opalescent sol. from lumps of starch. Add crystal of thymol after paste has cooled. Use as the albumin mixture. Has advantages in staining techniques as it is unaffected by dyes, gives a very light background especially in silver preparations; it is easily made, and sections adhere firmly to slides. R. Spoerri, Science, 1939, 90, 260, see also McDowell, A. M., and Vassos, A. A. Jr., Arch. Path., 1940, 29, 432-434. See account by Bates, J. C., Stain Techn., 19t2, 17, 49-56 on the structure and staining of starch grains in the potato tuber.

Statistical Control in hematology (Lancaster, H. O., J. Hyg., 1950, 48, 402-417).

Steel Gray, see nigrosine, water soluble.

Stereocilia of ductus epididymis are not true cilia. For technique and discussion, see Lucas A. ,M., in Cowdry's Special Cytology, 1932 1, 409-474.

Sternberg Cells, see Reed-Sternberg Cells. Stieve's Fluid. Saturated aqueous mercuric chloride, 76 cc.; formaldehyde solution, $20 \mathrm{cc}$; and glacial acetic acid, 4 cc. Fix for 18 hours and wash in several changes 95 per cent alcohol. A good general fixative recommended by R. E. Stowell.

Stomach, secretory cells of. Use Mucicarmine or Mucihematein for surface epithelial cells and neck chief cells; Bensley's Neutral Gentian for body chief cells and any combination of dyes including a strongly "acid" stain like eosin for the parietal cells, all after Bensley's alcoholic chrome neblimate fixation. The parietal cells can be sharply stained by supravital intravascular injection with Neutral red or Naphthol Blue R. The canaliculi of the parietal cells can be impregnated with silver by a modified Golgi method (Plenk, H., von Mollendor'f Handb. d. Mikr. Anat. d. Menschen. 1932, 5, (2), 235-402). To observe the cytological changes after discharge of strongly acid gastric juice and of juice rich in pepsin inject histamine and stimulate the vagus respectively (Bowie, D. J., and Voneberg, A. M., Quart. J. Exper. Physiol., 1935 25, 247-257). For mitochondria inject Janus Green intravascularly or fix in Regaud's fluid, mordant in potassium bichromate and stain with AnilinFuchsin Methyl Green. See localization of Pepsin.

Stools, see Feces.

Storage of specimens whether microscopic slides, paraffin or celloidin blocks or simply in preservative fluids should be systematic in all laboratories. Every specimen coming in for examination should be given an accession number and the data about it should be inscribed in a book. A book is better than a series of cards because cards can be removed by irresponsible persons and lost. The number, and other necessary information, should be written on the slide with a diamond pencil. This is usually done in pathological laboratories where there is much routine to be attended to. It is equally important in other laboratories devoted primarily to teaching and research even when a number of independent investigators are involved. System pays; lack of a unified system serving several people means loss and waste of valuable material.

Street, see Papanicolaou Techniques in Exfoliative Cytology.

Strength, see Tensile.

Striated Cuticular Border of intestinal epithelial cells is frequently confused with cilia, see Lucas, A. M., in Cowdry's Special Cytology, 1932, 1, 409-474.

Striated Muscle, glycogen distribution (Gendre, H., Bull. d'Hist. Appl., 1938, 15, 265-276). Effect of different dehydration and clearing agents (Ralph, P., Stain Techn., 1938, 13, 8-15). Methods for study of wave mechanics in living state (Carey, E. J., Zeit, W. and Massopust, L., Am. J. Anat., 1942, 70, 119-133.

Styrax, a very highly refractile mounting medium seldom employed in histology (Lee, p. 228).

Subcutaneous Tissue spreads. Making (McClung's Microscopical Technique p. 336).

Sublimate Acetic is a fixative of which the usual composition is 95 parts sat. aq. mercuric chloride plus 5 parts glacial acetic acid. See Laidlaw's method for inclusion bodies. When the saturated solution of mercuric chloride is made in $95 \%$ alcohol the fixative should be called Sublimate Alcohol Acetic. See Mercuric Chloride.

Submaxillary Glands. These can be nicely stained by the supravital methods employed for the Pancreas. Stains for Zymogen and for Mucus are useful. The duct cells are the principal sites of action of the salivary gland virus when this plays an inapparent rôle. The tremendously enlarged duct cells provided with Nuclear Inclusions are of ten seen in the guinea pig's submaxillary and in several other species, see Cowdry, E. V. and Scott, G. H., Am. J. Path., $1935,11,647-657$.

Submicroscopic Fibrils. These by close association may constitute the neurofibrils, spindle and astral fibers, myofibrils, and so on. Use of polarization 
optical methods suggests the orientation of submicroscopic rodlets parallel to the length of the fibers. The electron microscope is capable of demonstrating the component submicroseopic fibrils of collagenic fibrils (Schmitt, F. O., Hall, C. E. and Jakus, M. A., Biol. Symposia, 1943, 10, 261-276).

Submicroscopic Particles. In summarizing work in IR. R. Bensley's laboratory, Lazarow, A., Biol. Symposia, 1943, 10, 9-26 mentions two of these barely visible as shimmering poiuts of light in the dark field: (1) Lipoprotein complex discovered by Claude at the Rockefeller Institute containing fats, proteins and nucleo-protein and when concentrated en masse by centrifugation of cherry red color. Particle size $0.06-0.2 \mu$. (2) Particulate glycogen discovered by Lazarow containing a little protein but no fat. Water content $75 \%$. See Microsomes.

Submicrosopicc Structure of cytoplasm, methods and results (Frey-Wyssling, A., J. Roy. Micr. Soc., 1940, 60, 128-139). Succinic Dehydrogenase. Semenoff, W. E., Zeit, f. Zellforsch. Micr. Anat., 1935, 22: 305-309 as detailed by Glick, p. 96. Treat frozen sections of fresh tissue with 2 cc. 0.05 methylene blue plus 2 cc. $10 \%$ sodium succinate made up to 10 cc. with $\mathrm{M} / 15$ phosphate buffer, pH 7.6-8.0 for $10-15 \mathrm{~min}$. under cover slip with air bubbles excluded and edges sealed with paraffin. Compare with section in control medium nade up without sodium succinate. Fading of dye indicates enzymatic activities, Glick, p. 96. See Dehydrogenase.

Sudan, II (CI. 73)-Oil red O. Physical properties, Lillie, R. D., J. Tech. Methods, 1944, 24, 37-45.

Sudan III (CI, 248)-cerasin red, fat ponceau $G$, oil red $A S, O, B$ or $3 B$, scarlet $B$ fat soluble, Sudan G, Tony red-A weakly acid dis-azo dye, the most popular of fat stains in alcoholic solution. A sat. sol. in $70 \%$ alcohol is used in the same manner as Sudan IV in Herxheimer's solution (see below). Variations in action of sudan stains depending on character of fat and kind of fixation (Black, C. E., J. Lab. \& Clin. Med., 1937-38, 23, 1027-1036).

Staining in aqueous phase (Dufrenoy, J., Stain Techn., 1937, 12, 71-72). Make concentrated solution of Sudan III in 5 cc. methylal (dimethyloxymethane). Add 10-20 ce. aq. dest. The mixture separates into 2 layers: the lower made up of water, methylal and Sudan III and the upper of methylal, Sudan III and water. Whether sections float or sink they take up Sudan III. Another method of staining with Sudan
III in gelatin solution is given by Telford Govan, A. D., J. Path. \& Bact., 1944, 56, 262-264. See Bell's Method for staining fats mobilized by heat.

A promising acetic-carbol-sudan technique for lipids is described by Jackson, C., Onderstepoort, J. Vet. Sci. \& Animal Industry, 194.1, 19, 169-177. To prepare stock solution heat to simmering $2 \mathrm{gms}$. finely powdered Sudan III in $450 \mathrm{cc}$. $95 \%$ alc. Filter hot. Stopper, leave in refrigerator over night and filter cold. Add to any desired anount stock solution $5 \%$ aq. carbolic drop by drop agitating vigorously till alcohol content is reduced to $60 \%$. About $2 \mathrm{cc}$. carbolic to $6 \mathrm{cc}$. stock solution is required. Let stand few hours well corked. Add glacial acetic drop by drop 2.5 drops per cc. of carbol sudan, or 20 drops to the $8 \mathrm{cc}$. in above instance.

Cut frozen sections of formol or formol-saline fixed tissue. Place in $50 \%$ alc. 1 min. Stain in acid-carbolsudan mixture $1 \frac{1}{2}$ hrs. in well stoppered container. Differentiate in $50 \%$ alcohol, containing 5\% acetic acid, 10-60, sec. Wash in aq. dest., 1 min. Counterstain in filtered Delafield's henatoxylin diluted 1:2 with aq. dest. Differentiate in acid water, 10-20 sec. blue in ammonia water $(5 \mathrm{~min}$.) and wash in aq. dest. Finally mount in glycerin-jelly. Method is particularly recommended when existence of socalled "Sudanophobe" lipids is suspected.

Sudan IV (CI, 258)-cerotine ponceau 3B, fat ponceau, fat ponceau $R$ or $L B$, oil red IV, scarlet red-A weakly acid disazo dye also widely used as fat stain sometimes under heading of Scharlach $R$, especially in Herxheimer's Solution. Place frozen sections of formalin fixed tissue in $70 \%$ alcohol for a few sec. Transfer to Herxheimer's solution for 2-5 min. in a covered container to reduce evaporation and precipitation. Rinse in $70 \%$ alcohol. Wash quickly in aq. dest. Counterstain with Harris' hematoxylin. Wash in tap water. Mount in Glycerin. Seal with paraftin, or, if permanency is desired, with Duco or Kronig's cement. As a rule these fat stains do not last more than a few months. Physical properties of Sudan IV (Lillie, R. D., J. Tech. Hethods, 1944, 24,37-45).

Sudan Black B. 'This dye is of English manufacture and is not available in U.S. during the war. Its identity is still uncertain.

1. For fat. Fix tissues 24 hrs. in $5 \%$ formalin in $0.9 \%$ saline or in Zweibaum's fluid. The latter is made by adding 1 part of $2 \%$ aq. osmic acid to 7 parts of a mixture consisting of $3 \%$ potas- 
sium bichromate 6 cc.; $2 \%$ chromic acid, 3 cc.; and aq. dest. 5 cc. Wash in running water $24 \mathrm{hrs}$. In case tissue is delicate and requires support embed in gelatin before cutting frozen sections : $12.5 \%$ gelatin in $1 \%$ aq. phenol filtered, $37^{\circ} \mathrm{C}$., $24 \mathrm{hrs} .25 \%$ solution, same. Embed in fresh $25 \%$ aq. gelatin, cool, trim, harden in $5 \%$ formalin $24 \mathrm{hrs}$. Cut frozen sections, whether first embedded in gelatin or not, 5-10 microns thick. Transfer to aq. dest. and then into $50 \%$ diacetin agitated 30 sec. To make stain, add excess Sudan Black B (I.G.F.) to equal volumes of diacetin and aq. dest., incubate at $55^{\circ} \mathrm{C}$. for 2 days. Cool. Before use filter off amount required. Stain 15 micron sections $2 \mathrm{hrs}$. If speed is necessary warm in paraffin oven. $50 \%$ diacetin $30 \mathrm{sec}$. Counterstain with carmalum. Place in dish of water with care making sections "spin on surface and flatten." Float on to slide and mount in Apathy's medium. Nuclei red, lipids including myelin black (Leach, E. H., J. Path. \& Bact., 1938, 47, 635-637). Diacetin is glycerol diacetate introduced as solvent for scharlach R by Gross (W., Zeit., wiss. Mikr., 1930, 47, 64). Since Leach does not specify what Apathy's medium is, it is suggested that temporary mounts be made in glycerin.

2. For myelin (Lison, L. and Dagnelie, J., Bull. d'Histol. Appl., 1935, 12, 85-91). To stain lipoid granules in leucocytes. Dry blood smear and fix in methyl alcohol, 30 sec. Stain in a jar with sat. Sudan black B in $70 \%$ alcohol, $30 \mathrm{~min}$. Rinse in water and wash $1 \mathrm{~min}$. in $70 \%$ alcohol to remove deposit. Counterstain with sat. alcoholic eosin in $70 \%$ alcohol, 30 sec. Wash and stain in sat.aq. methylene blue $3 \mathrm{~min}$. Rinse, blot dry and examine with oil immersion. Lipoid granules, deep black; nuclei, blue; and erythrocytes, red. (Sheehan, H.L ,J. Path. \& Bact., 1939, 49,580-581).

Sudan Black $B_{1}$ as a bacterial fat stain. Sat. sol. of Sudan black B (Nat. Aniline and Chemical Co.) in $70 \%$ alcohol, or in ethylene glycol stains fat bodies in bacteria deep blue black (Hartman, $T$. L., Stain Techn., 1940, 15, 23-28).

Sudan Blue G, Brown 5 B, Corinth B, as fat stains (Lillie, R. D., J. Lab. \& Clin. Methods, 1944, 24, 35-42). This gives good account of all oil soluble dyes as fat stains.

Sudan Dyes suspended in watery media for use in the staining of fat are described by Telford Govan, A. D., J. Path. \& Bact., 1944, 56, 262-264. While stirring add sat. Sudan dye in acetone drop by drop from capillary pipette to $1 \%$ aq. gelatin containing $1 \%$ acetic acid to development of a deep brick-red color and a milk like consistency. Evaporate acetone for $2 \mathrm{hrs}$. at $37^{\circ}$. Remove sediment by filtration. Cut frozen sections. Transfer them from water to $1 \%$ aq. gelatin for 2-3 min. Stain for $30 \mathrm{~min}$. at $37^{\circ}$ in above described dye suspension. Wash in $1 \%$ aq. gelatin 2-3 min. and thoroughly in water. Mount in glycerin jelly, see Glycerine Jelly or in Karo Syrup.

Sudan G, see Sudan III.

Sudan Hydrotropes. Sudan stains are rela tively insoluble in water. They can be changed to hydrotropes (Neuberg) which are water soluble. The hydrotropes of red lipid stains are of a blue color which changes to red when the dye passes into a lipid or a lipid solvent. This is the basis of a useful technique for lipids (Hadjioloff, A., Bull. d'Hist. Appl., 1938, 15, 37-41).

Sudan R (CI, 113)-brilliant fat scarlet B, oil vermillion-A weakly acid mono-azo dye.

Sudan Red, see Magdala Red.

Sugars, see Reducing Sugars.

Sulfatase. An enzyme capable of hydrolyzing sulfuric acid from its ester linkages. Since cartilage, mucus and many detoxification products contain esterified sulfuric acid, an understanding of the localization of this enzyme would be most interesting. Seligman, A. M., M. M. Nachlas, L. H. Mannheimer, O' H. Friedman and G. Wolf, Ann. Surg., $1949,130,333-341$, describe a method involving the hydrolysis of beta naphthyl sulfate and subsequent diazotization of the enzymatically liberated naphthol.

Sulfhydryl Groups. 1. Prussian blue histochemical reaction for (Chèvremont, $M$. and Frederic, J. Arch. de Biol., 1943, 54, 589-605). Fresh or fixed tissue sections or smears can be used. Formol, formol Ringer (saline) and Bouin are suitable fixatives; but fluids containing sublimate, such as those of Zenker and Helly are contraindicated. The optimum time of fixation is from a few hours to one day. Time of heating during paraffin embedding should be reduced to a minimum. Wash sections carefully in aq. dest. to remove formalin. Plunge sections or smears in 3 successive baths of the following mixture: 1 part fresh $0.1 \%$ aq. ferricyanide of potassium (For Analysis, C.P.) and 3 parts $1 \%$ aq. ferric sulphate (For Analysis, C.P.). The mixture thus prepared has a $\mathrm{pH}$ of 2.4 and, in ordinary light, it is stable for $2 \mathrm{hrs}$; in darkness it lasts longer. The time in the baths is approximately 10-20 min. for frozen sections, 20-25 min. for paraffin sections 
and for blood smears and $1 \mathrm{hr}$. for smears of yeast. If desired, stain the background with Azo carmin. No metal instruments must enter the baths. A positive result is indicated by appearance in cells of blue granules or of a blue colloidal precipitate which gives the impression of being diffuse. After long washing in water preparations can be mounted in Canada balsam after dehydration or in syrup of levulose without dehydration. They last as long as 7 months. Consult original article for histochemical controls and for illustrations of epidermis and other tissues.

2. Another reaction is given as follows by Serra, J. A., Stain Techn., 1946, 21, 5-18: "This reaction has been extensively used for the study of the distribution of the tripetide glutathione. One of the better methods of accomplishing the reaction is that of Giroud and Bulliard (see Lison, 1936), which gives a stable red coloration, while other methods produce a violet color rapidly fading away.

"The pieces are immersed for some seconds (in general an excess of time does no harm) in a $5 \%$ aqueous solution of zinc acetate. Directly afterwards they are treated with a $10 \%$ aqueous solution of sodium nitroprusside, containing about $2 \%$ concentrated ammonia. The pieces acquire a bright red coloration, which attains its maximum in 3-5 minutes. Afterwards they are mounted in pure glycerin for microscopic observation, if necessary with a preliminary washing in distilled water.

"The materials may be studied freshly or after fixation. It must be noted, however, that the majority of the fixatives hinder the reaction. We obtained good results with a fixation in $10 \%$ neutral formaldehyde during 2-15 hours at room temperature. A more prolonged action of this fixative also hinders the reaction; it is recommended, therefore, that if possible 2-4 hours of fixation be used.

"The results of the reaction have different meanings according to the fixation, washings, etc., because the glutathione is partly soluble. When the tissues are treated several times with a $10 \%$ solution of trichloroacetic acid for 15 minutes, the glutathione is dissolved and only "fixed", that is, proteic sulfhydryl groups remain in the preparation. It is still possible not only to demonstrate the existing $\mathrm{SH}$ groups but also to reduce SS groups to SH groups, by means of a pre-treatment of the materials with a solution of $10 \%$ KCN for 10 minutes in a small stoppered bottle (the cyanide solution can be weakly alkalinized with potassium hydroxide, to make its use safe).

"The reaction has been recognized as well localized, but in case of doubt a test of secondary impregnation can be made in the way described for the ninhydrin." See Nitroprusside Reaction.

Sulfmethemoglobin, a greenish compound of methemoglobin and sulphur often encountered in abdominal walls of cadavers, but it may be present in blood where it can be diagnosed by spectroscopic examination (Mallory, p. 135).

Sulfonamides. Great importance of sulfa drugs makes their demonstration in tissues useful. Mackee, G. M., Herrmann, F., Baer, R. I. and Sulzberger, M. B., Science, 1943, 98, 66-68; J. Lab. \& Clin. Med., 1943, 28, 1642-1649.

Fix fresh tissue with dry formaldehyde gas and visualize sulfa compounds as orange precipitates in frozen sections treated with p-dimethylaminobenzaldehyde in acid alcohol solution. In attempting to identify sulfonamides microscopically in urinary sediments the descriptions and diagrams of the various crystals given by C. J. Gentzkow and H. A. Van Auken in Simmons and Gentzkow will be helpful, viz.

Sulfadiazine: (1) free drug, "dark greenish irregularity striated spheres with either fuzzy or clean edges" ; (2) acetyl crystals like "sheaves of wheat with eccentric bindings".

Sulfaguanidine: (1) free drug, rare; (2) acetyl crystals as "thin oblong plates, clear or with fine mesh pattern, often aggregated into cross or star-like clusters"

Sulfanilamide: (1) free drug as large needles with angle of $106^{\circ}$ at ends often in sheaves; (2) acetyl crystals similar needles with square ends.

Sulfapyridine: (1) free drug as stubby prisms; (2) acetyl crystals as "boator petal-shaped forms with rounded edges; start angled crystals; bow ties or burrs; and occasionally as large rosettes."

Sulphathiazole: (1) free drug rare as flattened or 6 sided crystals with angle at end of $84^{\circ} ;(2)$ acetyl crystals may resemble those of free drug but with end angles of $136^{\circ}$ when they look like wheat sheaves with central binding. These may be swollen suggesting 2 half circles fused at center; striated spherulites frequently occur.

Sulfasuccidine crystals absent because of but slight absorption of this drug from intestine.

Sulfonphthaleins. These are compounds of phthalic anhydride and ortho-sulfo- 
benzoic acid. They are most valuable indicators. Examples: brom chlor phenol blue, brom cresol green, brom cresol purple, brom phenol blue, brom phenol red, brom thymol blue, chlor cresol green, chlor phenol red, cresol red, metacresol purple, phenol red, thymol blue.

Sulfur Bordeaux (CI, 1012), Sulfogene Bordeaux BRN (DuPont) and Sulfur Bordeaux BCF (NAC) are direct dyes of light fastness 2. Specifications for staining invertebrates and plant tissues are given (Emig, p. 62).

Sulfur Brilliant Blue (CI, 957), Sulfindone Brilliant Blue CG (NAC), Sulfogene Brilliant Blue 6BS (DuPont), and Sulfogene Brilliant Blue 3 GCF (DuPont) are the best blue direct sulfur dyes of color fastness 2 , the use of which for staining algae and invertebrates is described (Emig, p. 61).

Sulfur Direct Blue (CI, 956), Sulfogene Direct Blue BRS (DuPont), a direct dye of light fastness 2 which does not color blue green algae as intensely or brightly as Sulfur Brilliant Blue, but does present details of cell structure clearly (Emig, p. 61).

Sulfur Green (CI, 1006), Sulfogene Green 2 B (DuPont), Sulfogene Brilliant Green $2 \mathrm{G}$ (DuPont) and Sulfur Green $3 \mathrm{G}$ conc. (NAC), direct dyes of light fastness 2 action of which on plant tissues and invertebrates is described (Emig, p. 62).

Sulfur Orange (CI, 949) and Sulfur Yellow (CI, 948) resemble Sulfur Bordeaux (Emig, p. 61).

Sulphonal Poisoning. Effect on liver cell mitochondria (Grynfeltt, E., and Lafont, R., C. rend. Soc. de Biol., 1921, 85, 406-408).

Sulphur. In inorganic form sulphur is not found in living things except in the thiobacteria. Histochemically one has to consider sulphates and masked sulphur. Macallum has devised a method for sulphates but Lison (p. 121) says that it only gives very rough localization in tissues because the salt is diffusible. For organic, masked sulphur see Sulfmethemoglobin, Glutathione, Radiosulphur.

Sulphur Compounds. Microdetermination by means of the iodine-azide reaction (Holter, H. and Løvtrup, S., C. rend. Lab. Carlsberg, Sér. Chim., 1949, 27, 72-78). They worked with tetrathionate and cystine. $1 \times 10^{-3} \mu \mathrm{g}$ could be determined with an accuracy of $2.5 \%$ (standard deviation).

Sulphurous Acid. This is used for rinsing sections which have been stained with Feulgen or Schiff's reagent. Prepare by dissolving $1 \mathrm{gm}$. potassium or sodium meta bisulphite in $200 \mathrm{cc}$. of tap water to which $10 \mathrm{cc}$. of $N \mathrm{HCl}$ are added.

Sultan Red 4B, see Benzopurpurin 4B.

Sun Yellow (CI, 620), a direct stilbene dye, light fastness 3 . Serves as a mordant to produce green in combinations with blue counterstains. Many combinations of Sun Yellow with blue and red dyes in double, triple and quadruple stains are described (Emig, p. 44-45).

Superchrome Black PV (CI, 170) of NAC, an acid monoazo mordant dye action of which on plant sections and blue green algae is described (Emig, p. 34).

Superchrome Violet B (CI, 169) of NAC, an acid monoazo mordant dye of light fastness 3 of which action on blue green algae is described (Emig, p. 34).

Superchrome Garnet Y (CI, 168) of NAC, an acid monoazo mordant dye of light fastness 3 of which action on blue green algae is described (Emig, p. 34).

Supravital Staining. By this is meant staining upon the living state. In other words stains are applied to cells removed from a living animal, or to cells within a recently killed animal. Thus blood cells are removed from the body and, while still living, are stained supravitally or the stains are applied to still living cells of, say, the stomach within the body of a recently killed animal by vascular injection. The essential point is that the stains act upon living cells but the cells do not go on living, neither does an animal injected intravascularly with a supravital stain. Janus green is our most useful supravital stain. Cells supravitally stained by it die and when it is injected in sufficient quantity into a living animal, the animal dies likewise for it is toxic. Vital stains, on the contrary, do not kill cells and can be safely injected into living animals since they are nontoxic in the concentrations necessary to obtain the desired results. This kind of staining used to be called intravital in contrast to supravital. See Vital Stains.

Supravital stains have been known for a long time but their introduction as essential means of investigation is due primarily to Professor R. R. Bensley of the University of Chicago (Am. J. Anat., $1911,12,297-388)$. He showed their usefulness in demonstrating specifically by vascular injection the different epithelial components of the pancreas and he called attention to the fact that to stain mitochondria specifically it is essential to use janus green having the composition of diethylsafranin-azochmethylanilin, that the dimethyl compound will not work. The supravital staining of blood cells began with the demonstration by Cowdry at Hopkins 
(Internat. Monatschr. f. Anat. u. Physiol., 1914, 31, 267-286), that this particular janus green B as used in Bensley's laboratory stains the mitochondria in human white blood cells specifically. The method was later further developed by Sabin and her associates. Details of techniques are given under janus green, neutral red, brilliant cresyl blue, pyronin, methylene blue, naphthol blue and cyanamin. Useful table giving reactions of types of blood cells (Gall, E. A., J. Lab. \& Clin. Med., 1934-35, 20, 1276-1293). A critique of supravital staining of blood cells is provided by Schwind, J. L., Blood, $1950,5,597-622$.

Suramin, a drug purchasable under term of Naphuride (Winthrop), is only a feeble inhibitor of growth of lymphosarcoma transplants. Its cytotoxic effect is rather similar to that of colchicine on lymphoid tumors (Williams, W. L., Cancer Research, 1946, 6, 344353).

Surface Films, study of by micrurgical technique, Chambers, R. W. and Kopac, M. J. on McClung's Microscopical Technique, 1950 , p. 542 .

Surface Measurements. To determine the surface area of structures of microscopic size involves many techniques some of which are rather complicated. The following references are given to methods and results in a wide variety of instances. Perhaps the particular surface to be measured will be sufficiently similar to one of these to justify employment of the same technique or a modification of it.

Endothelium of vascular capillaries6300 sq. meters-Krogh, A., Anatomy and Physiology of Capillaries, Yale Press, 1929, 422 pp.

Erythrocytes combined-3500 sq. meters-Evans, C. L., Recent Advances in Physiology. Philadelphia: Blakiston, 1926, $383 \mathrm{pp}$.

Filtration surface of both kidneys com bined-1.56 sq. meters-Vimtrup, B. J., Am. J. Anat., 1928, 41, 132-151. See also recent measurements for albino rat by Kirkman, H. and Stowell, R. E., Anat. Rec., 1942, 82, 373-389.

Gastric glands secreting surface2.7 sq. meters-Scott, G. H. (personal communication), see Cowdry's Histology (p. 282).

Lacteal surface in small intestine5 sq. meters-Policard, A., Précis d'Histologie Physiologique. Collection Testut, Paris: G. Doin, 923 pp., after Potter.

Large intestinal crypts- 4.2 metersPolicard, ibid.

Mitochondrial, zymogenic and nuclear surfaces in pancreatic acinous cells of guinea pig-duNouy, P. L. and Cowdry, E. V., Anat. Rec., 1927, 34, 313-329.

Respiratory surface plus nonrespiratory epithelial surface of airways of lungs-70 sq. meters-Wilson, H. G., Am. J. Anat., 1922, 30, 267-295.

Surface Replica Method, see Shadow Casting in Electron Microscopy.

Surface Tension. This, or more correctly interfacial tension, is tension at the surface of a fluid tending to produce a sphere. Surface tension is high for water and low for alcohol. Soap decreases surface tension of water because it concentrates at surfaces. Bile acids lower surface tension of blood serum. According to Gibbs any substance lowering interfacial tension will concentrate at the interfaces. Surface tension is best determined by a Cencodu Nouy tensiometer capable of measuring the force required in lifting a standard platinum ring out of the surface of the liquid. The ring must obviously be held absolutely horizontal and be pulled away slowly (Holmes, H. N., Glasser's Medical Physics, 257263).

Much has been written about surface tension (Reviews: Harvey, E. N., and Danielli, J. F., Biol. Rev., 1938, 13, 319-341 and Danielli, J. F. in Bourne, pp. 69-98). Before measurements can be made on cells it is obviously necessary to isolate them and this entails risk of injury which is much greater in the case of mammalian cells than of the sea urchin eggs usually employed. The following techniques are given as examples :

1. By centrifuging marine eggs elongation can be produced and, when the length exceeds a certain ratio of diameter, the egg divides. Knowing the minimum force required, the difference in density between the 2 halves and the circumference of the cylinder, it is apparently possible to calculate the tension at the surface (Harvey, E. N., J. Franklin Inst., 1932, 214, 1-23).

2. By compressing sea urchin eggs by a minute gold beam the internal pres. sure can be calculated and from this the surface tension (Cole, K. S., J. Cell \& Comp. Physiol., 1932, 1, 1-9).

3. By stretching a cell between the two needles of a microdissection apparatus the force required to secure a given degrce of elongation can be determined and thence the surface tension (Norris, C. H., J. Cell \& Comp. Physiol., 1939, 14, 117-133).

4. Surface tension is probably to some extent at least conditioned by the elasticity of the superficial plasma gel 
layer which brings in the methods and observations of Lewis, W. H., Arch. f. exp. Zellf., 1939, 28, 1-7; Am. J. Cancer, $1939, \mathbf{3 5}, 408-415$ who refers to previous work along this line.

Survival of Tissues after death of the body (Alvarez, W. C., Quart. Rev. Biol., $1937,12,152-164)$. Often determined by measuring how long the tissue continues to respire. Data for whole skin, kidney and liver (Walter, E. M., Sharlit, H. and Amersbach, J. C., J. Invest. Dermat., 1945, 6, 235-238). Schrek, R., Radiology, 1946, 46, 395-410 has made much use of a method for measuring the survival of cells in terms of the percentage which do not stain with eosin (and are presumably alive) in emulsions of cells in a special fluid held at definite $\mathrm{pH}$ and temperature for various lengths of time. See Dead cells, Revival after freezing.

Susa fixative of Heidenhain. Corrosive sublimate, 4.5 gm.; common salt, 0.5 gm.; aq. dest., 80 cc.; formalin, 20 cc.; and trichloracetic acid, 4 cc. Fix about 12 hrs., wash in $95 \%$ alcohol. Has the advantage over most sublimate mixtures that treatment with iodine is not usually required to remove black mercury deposit in the tissues. It has been modified by several people. See Buzaglo.

Swiss Blue, see Methylene Blue.

Synapses, see methods employed by Bartelmez, G. W. and Hoerr, N. L., J. Comp. Neurol., 1933, 57, 401-428.

Synovial Fluid of normal knee joint. Method of examination and results (Coggeshall, H. C., Warren, C. F. and Bauer, W., Anat. Rec., 1940, 77, 129-144).

Syphilis, see Treponema pallidum.

Syrup, see Apathy.

Taenia Echinococcus, a parasite of dogs which produces hydatic cysts in human liver and other tissues. The laminated cyst wall is typical and the heads have double circle of hooks and 4 suckers.

Taenia Saginata. In examination of fresh Feces identify by head with 4 suckers but without hooks.

Taenia Solium. Look in Feces for head with 4 suckers and a circle of small hooks best seen in fresh mounts. The genital system opens at the side and the uterus is only slightly branched.

Tagged Atoms, see Radioactive Isotopes, Deuterium.

Tannic acid iron technique is described by Salazar, A. L., Stain Techn., 1944, 19, 131-135. He advocates it for study of Golgi apparatus and with Giemsa's stain to give sharper differentiation between agranulocytes and granulocytes.

Tantalum, see Atomic Weights.
Tapeworm Proglottids. Orient pieces 4-5 $\mathrm{cm}$. long containing gravid proglottids between glass slides held together by elastic bands. Fix in Bouin's fluid (sat. aq. picric acid, 7 parts; glacial acetic acid, 20 parts; and formalin, 10 parts 10-12 hrs. Wash in running water 2-3 min. Flood with $10 \%$ aq. NaOH (outlines of uterus become visible deep orange). Rinse in tap water. Flood with 5\% $\mathrm{HCl} 1-2 \mathrm{~min}$. Tap water 1015 min. Dehydrate in alcohol, clear in xylol and mount in balsam (Dammin, G. J., J. Lab. \& Clin. Med., 1937-38, 23, 192-194). An oxidation reduction method for stain differentiation is provided by Tapmisian, T. N., Stain Techn., 1945, 20, 11-12. See Parasites.

Target Cells. Erythrocytes looking somewhat like the "bull's eyes" of targets because a central hemoglobin rich area is surrounded by a clear area enclosed by a peripheral ring likewise rich in hemoglobin. Target cells are well demonstrated by Wright's Blood Stain. They are often more numerous when the blood plasma concentration is increased and they show greater resistance than normal erythrocytes to hypotonic salt solutions. Target tissues are those especially influenced by certain hormones which seem to reach them as if aimed at targets.

Tarsal Glands. Whole mounts can be made by the method described for Sebaceous Glands. They are also known as Meibomian glands.

Taste Buds. To demonstrate, choose circumvallate papillae, fix in Bouin's Fluid and stain with Hematoxylin and Eosin. See Arey, L. B. et al., Anat. Rec., 1935-36, 64, 9-25.

Tartrazine (CI, 640), a pyrazolone acid dye of light fastness 4 . This bright yellow dye is useful in coloring foodstuffs, light filters, etc. (Emig, p. 46). Recommended as a substitute for Orange $G$ in Mallory's Stain.

Tatooing Pigments, see Exogenous Pigments.

Teeth. The most comprehensive statement of microscopical technique is contained in A. W. Wellings' "Practical Microscopy of the Teeth and Associated Parts." London: John Bale Sons \& Curnow Ltd., 1938, 281 pp. A chapter by Churchill and Appleton in McClung's Technique is also useful. Teeth can be studied from so many different angles that to outline the techniques in a few words is extraordinarily difficult. Their composition of (1) enamel, the hardest tissue in the whole body, with (2) dentin which is highly mineralized and contains the processes of cells but not their nucleated bodies plus (3) richly cellular 
pulp confers numerous obstacles. The wise histologist or pathologist will save valuable time by at once secking advice from experts in some dental research laboratory. They possess experience and instruments for grinding and sawing both of which he lacks. Teeth of adults can be prepared for examination in 2 principal ways :

1. Without decalcification. Churchill and Appleton (McClung, p. 253) recommend, in place of the usual grinding method, a cutting technique used by Johnston at Yale. After extraction fix the tooth immediately in formalin. Then dry and fix to wooden block by modelling compound. Sections are then made by the cutting wheels of a power lathe. If necessary they are polished on a Belgian stone, dehydrated in alcohol, cleared in xylol and mounted in balsam.

Ground sections of very brittle teeth or teeth with supporting structures intact may be ground after imbedding in methyl methacrylate according to Sognnaes, R. F., Anat. Rec., 1947, 99, 133-144. This method permits sawing of such thin slices before grinding that serial sections may be prepared.

When one wishes to include the soft as well as the hard parts Chase's technique of petrifaction is advised by them. Fix as desired (say $10 \%$ formalin) and wash as required. Transfer to aq. gum arabic or dextrin of syrupy consistency. Freeze on freezing microtome and cut slices with very fine saw (jeweler's). Remove gum arabic by washing in water and stain with carmine or hematoxylin. Dehydrate through alcohols to $95 \%, \frac{1}{2}$ to several hours each depending on size of slice. Acetone $\frac{1}{2}$ hr. or more. Cover with thin celloidin in a container to depth twice or more thickness of slice. Leave container top open very slightly permitting evaporation until celloidin will scarcely flow when container is steeply tilted. Transfer with considerable celloidin to container of heavy lead foil and further evaporate until completely hardened. Grind and polish both sides of slice in presence of water. Remove celloidin with acetone and acetone with xylol. Mount in balsam. Sections obtained by this and the Johnston technique can be examined by direct illumination, in the dark field, in ultraviolet light (Walkhoff, O., Dental Cosmos, 1923, 65, 160-176), in polarized light (Andresen, V. The Physiological and Artificial Mineralization of Enamel. Oslo. Dancke, 1926) and by x-ray for which many references are given (McClung, 381385 ).

2. With decalcification. In the par- affin technique, advised by Churchill and Appleton, clip ends of roots of a freshly extracted tooth or drill hole. Fix in $4 \%$ formalin. Dry with towel and seal openings to pulp with celloidin. Quickly dry. Decalcify in 10\% hydrochloric acid C.P'. 10 days or more testing with needle. Running water, 24 hrs. $95 \%$ alc. 24 hrs. Abs.alc., 5 hrs. Chloroform, 1 hr. Equal parts chloroform and $45^{\circ} \mathrm{C}$. paraffin in glass stoppered bottle on top of oven (oven $58^{\circ} \mathrm{C}$.) over night. $\frac{1}{2} \mathrm{hr}$. each in following paraffins (1) $42-46^{\circ} \mathrm{C}$., (2) $52-56^{\circ} \mathrm{C}$. and (3) $58-$ $60^{\circ} \mathrm{C}$. within oven. Imbed in a mix ture of $235 \mathrm{cc} .52-56^{\circ} \mathrm{C}$. paraffin and 15 cc. beeswax. See Paraffin Sections.

In the celloidin technique (Churchill and Appleton) cut off apex of tooth or drill a hole to pulp through crown. Fix in $4 \%$ formalin, buffered to counteract acid, 45 hrs. for single teeth. (Wash in water) change to $80 \%$ alc. $95 \%$ alc. 2 weeks + depending on size. Abs. alc. 2 weeks $t$, abs. alc. (exposed to anhydrous copper sulphate, see Alcohol) 2 weeks t. Equal parts abs. and ether, 2 weeks $t$. Then 1 month or more in $\frac{1}{2}, 1,2,5,7,10,12 \%$ celloidin (parlodion). Orient and imbed in $12 \%$ in stender dish. Make depth of celloidin twice height of tissue. Place lid of stender dish on tightly. Allow bubbles to rise $24 \mathrm{hrs}$. If bubbles still present move tissue gently so they can escape. Put piece of paper between lid and dish, 24 hrs. + . Evaporate to consistency hard rubber, 7 days + . $80 \%$ alc. 48 hrs. or until beginning decal cification. Trim block leaving sufficient celloidin about tissue to facilitate cutting. $10 \%$ acetic or hydrochloric acid in $70 \%$ alc. changing daily 3 weeks + until needle penetrates easily. When spaces appear in the celloidin drill holes to reach them. Wash $24 \mathrm{hrs}$. in running water; then same time in weak sol. sodium bicarbonate. Wash $24 \mathrm{hrs}$. + in water. 50, 70 and $80 \%$ alc. each 21 hrs. +. $95 \%$ and abs. alc., $\frac{1}{2} \mathrm{hr}$. each. Alc. ether, $0.5 \%$ and $12 \%$ celloidin $5-20$ min. each. Harden in chloroform, 24 hrs. Leave in $80 \%$ until sections are made, see Celloidin Sections.

For small and developing teeth a wider variety of methods is possible see Teeth Developing. To classify examples of all the methods available for old and young teeth and associated structures in a manner expected by the reader is not feasible. In general however there are methods that involve whole teeth which come under Teeth (Blood Vessels, Innervation, Lymphatics) and their response to Alizarin Red staining and exposure to Radioactive Phos- 
phorus. Some techniques are also provided under Teeth and Jaws and parts of teeth: Enamel, Dentin, and Pulp.

Teeth, Blood Vessels (Boling, L. R., Anat. Rec., 1942, 82, 25-32). Revised by L. R. Boling, July 27, 1946. Two suspensions are recommended: (1) cinnabar, 120 gms.; gum arabic, 40 gms.; water, 160 cc. (2) cinnabar (red mercuric sulphide), 80 gms.; corn starch, 40 gms.; $10 \%$ formalin in physiological saline, $125 \mathrm{cc}$. Grind up the mixtures slowly in a glass ball mill for 2 or 3 days, strain through gauze, and use immediately. Anesthetize a cat or dog with sodium pentobarbital. Expose and ligate both common carotid arteries. Perfuse the head with physiological saline through a glass cannula inserted in one carotid. Incise the carotid of the opposite side distal to the ligature and allow it to bleed until clear saline appears when it should be clamped. Open the jugular veins and allow them to drain. As soon as all blood has been washed from the vessels of the head direct the suspension through the same cannula by means of a two way stop cock. Maintain a pressure of $120 \mathrm{~mm}$. of mercury by air pressure. Aid penetration by gentle rhythmic pressure on a hand bulb inserted in the conducting system. When injection of the mass is begun remove the clamp momentarily from the opposite carotid to allow free flow of the mass in all large arteries. This promotes good injections of both right and left sides from the single cannula. After completion of the injection remove the head and place in strong formalin over night, then cut away the sof tissue from the jaws and place the jaws in $10 \%$ formalin in saline solution for several days, wash, and decalcify in $5 \%$ nitric acid. After decalcification dehydrate thoroughly in graded series of alcohol and clear in two changes of methyl salicylate. Dissect away any bone interfering with observation of teeth. This is best done with a dental engine and round bur while the specimen is immersed in clearing fluid. Moisture or heat will cause clouding of the specimen and must be avoided. In addition to the desirable color of cinnabar, is the radiopacity of these injections; the course of all macroscopically visible vessels may be followed in roentgenograms before decalcification. The method also works well on soft tissues. The first mass will pass through all capillaries in a tooth and fill both arteries and veins. Better demonstration of arteries is obtained with the second which has not been found to pass through capillaries. The use of formalin seems to aid in the reten- tion of the mass in the blood vessels and to prevent the formation of gas bubbles in the pulp cavity during decalcification.

Teeth, Decalcification: Details from Dr. L. R. Boling, Washington University (School of Dentistry). Revised by him Dec. 16, 1950.

Decalcification of teeth for the preparation of histological sections presents several problems not encountered with other tissues especially if the surrounding bone and soft tissues are also preserved. The great difference in salt content and organic matrix of enamel, dentin, cementum, bone and soft tissues makes difficult the preservation of one while the others are being decalcified.

Enamel, except in the most immature portions of developing teeth, is entirely destroyed by ordinary decalcification methods. The organic portion of adult enamel may be observed by the slow decalcification of thin ground sections under a cover slip (Chase, S. W., Anat. Rec. 36, 239-258, 1927). The acid, one per cent nitric, hydrochloric or sulphuric, or five per cent chromic, acetic or citric, is run under a propped cover slip over the section. Action may be stopped at any point by substituting water for acid and the remaining material stained and mounted as desire d without disturbance. Boedeker's method of "celloidin-decalcifying" is also said to give good results (Fundamentals of Dental Histology and Embry ology, New York, The MacMillan Co., 1926 , p. 223) and allows sectioning of the organic remainder in any plane. See Enamel.

For the examination of sections of whole teeth without enamel or for teeth in relation to the bone of the jaws five per cent nitric acid in water has been found by most investigators to give consistent results. Hydrochloric acid may be used but causes too much swelling. For delicate objects one to five per cent nitric acid in 70 per cent alcohol may prove superior.

Excellent results have been obtained with the use of formic acid according to the technique of Morse, J. Dent. Res., 1945, 24, 143-153 in the decalcification of single human teeth or teeth and jaws of small animals. Two solutions are made as follows: Solution A: 1 part $90 \%$ formic acid C.P. and 1 part aq. dest., Solution B: 20 grams sodium citrate C.P. and 100 c.c. aq. dest. At the time of use combine equal parts of $A$ and $B$. Change solution daily until decalcification is complete as shown by chemical test. (See below.) When this method is 
used with very large teeth or large blocks of tissue containing bone and teeth, objectionable precipitates may be formed in the depth of the block.

Celloidin imbedding before decalcification helps preserve tissue relationships (See Teeth, celloidin technique). Arnim has perfected a technique of double imbedding for rat jaws and teeth which, though tedious, yields beautiful results. Enamel matrix is frequently preserved. (Anat. Ree., 62, pp. 321330,1935 .) This method has been modified by Burket for larger teeth (McClung, p. 366).

Tooth buds may be decalcified after paraffin imbedding by the following method given by Dr. L. R. Boling in a personal communication. Carefully remove from the tooth bud all surrounding bone. Fix, dehydrate, clear and imbed in paraffin in the usual way. Shave away paraffin and soft tissue from one surface of the specimen so that enamel is exposed. Immerse block in 5 per cent aq. nitric acid until decaleification is complete. Place in 5 per cent aq. sodium sulphate for a few hours. Wash over night in running water and reimbed, handling the tissue as gently as possible in order not to disturb relationship of hard and soft tissues. This method permits demonstration of Golgi apparatus and mitochondria in ameloblasts and odontoblasts in situ. It works best with teeth of small animals easily penetrated by fixative. The paraffin protects the sof t tissues but does not interfere with action of acid on enamel and dentin. (See also Teeth, Developing.)

Successful preparation of decalcified tooth sections depends as much or more on the care of the tissues before and after decalcification than on the actual process. Good fixation of the pulp tissue is difficult but essential to prevent shrinkage. Ten per cent formalin in physiological salt solution may be used for several days or weeks without injury to the soft tissue and allow thorough penetration. Better results are obtained in a short time if the fixative can be perfused through the blood vessels. In the preparation of human or other large teeth, fixation artifacts are minimized if the tooth is ground longitudinally on a flat stone until the pulp is just exposed. Two opposite surfaces may be ground. Grinding should be done on a sharp stone under running water to prevent heating. Cutting of holes through the dentin to the pulp or the amputation of the tips of teeth is often resorted to in order to get better penetration but these meth- ods are apt to disturb the position of the pulp and should be avoided if possible. After decalcification the teeth should be carcfully handled and the dehydration process should be slow to prevent separation of tissues of different densities. The substitution of $n$-butyl alcohol for ethyl alcohol and xylol in dehydration and clearing processes has proven advantageous (Morse, loc. cit.). By this method dehydration may be prolonged with less hardening.

Over decalcification should be carefully avoided because it will partially destroy the dentin matrix, cause separation of tissues of differing consistency and disturb staining reactions. Testing for completion of decalcification by probing with needles or bending and squeezing in the fingers should be avoided at all costs if tissue relationships are desired. The progress of decalcification can be followed radiographically but the end point can not be accurately determined. The best method of testing is that described by Arnim (loc. cit.). Five cc. of the acid used in decalcification is placed in a clean test tube and neutralized with ammonium hydroxide, and .1 ce. of a saturated solution of ammonium oxalate added. If no precipitate forms additional .1 ce. portions of oxalate are added at 15 minute intervals until .4 cc. have been added. If a precipitate is formed the tissue is placed in fresh acid and retested in 24 hours. Formation of no precipitate with $.4 \mathrm{cc}$. oxalate solution after 24 hours in fresh acid is indicative of complete decalcification.

When tissues are found to be not sufficiently decalcified after imbedding the process ean be completed by immersing the celloidin block in acid 70 per cent alcohol or floating the paraffin block, cut surface down, on acid if the dentin is exposed.

Teeth, Developing. 1. Tooth germs. Glasstone (S., J. Anat., 1935-36, 70, 260266) has described a method for the excision of tooth germs from 18-21 day rat embryos and their Cultivation in fowl plasma and embryo extract. The technique of Transplantation of tooth germs of young pups into the abdominal wall has been reported by $\mathrm{C}$. H. Huggins et al. (J. Med., 1934, 60, 199). Bevelander, G., Anat. Rec., 1941, 31, 79-97 obtained fine preparations of Korff's fibers in pig's tooth beginning with $110 \mathrm{~mm}$. stage by fixation in Formalin-Zenker and silver impregnation by Foot's Method.

2. Young teeth. Beams, H. W. and King, R. L., Anat. Rec., 1933, 57, 29-40 fixed the developing molar teeth of white 
rats 1-5 days old in a variety of fluids. They employed the Nass on ov technique for the Golgi apparatus and Regaud's for mitochondria without any special provision for decalcification. In some cases Boling's Decalcification (Teeth, Decalcification) method after paraffin imbedding may prove useful. Dr. Boling states in a personal communication that a modification of Bouin's picroformol fixative may be used for fixing and decalcifying very young tooth buds or teeth and jaws of rats. A mixture of 75 parts saturated aqueous solution of picric acid, 25 parts formalin and 10 to 20 parts glacial acetic acid will decalcify a mature rat jaw and teeth in less than a week. Ordinary Bouin's picro-formol is sufficiently acid to decalcify very young tooth buds in a few days. After decalcification the tissues are handled in the same manner as soft tissues after Bouin fixation except that a longer period is allowed for removal of picric acid. This procedure allows better than average staining of decalcified tissues. Nuclear structure is especially well preserved and little separation of hard and soft tissues is found. The method of microincineration has been adjusted to developing teeth by Hampp, E. G., Anat. Rec., 1940, 77, 273-286.

Teeth, Innervation. Methods described under Nerve Endings require considerable adaptation before they can be employed for the teeth. For obvious reasons methylene blue is particularly difficult to use. From a great many techniques 2 are selected.

1. Van der Sprenkel, H. B., J. Anat., 1935-36, 70, 233-241. Grind dentinal wall of normal human canine tooth down to a thickness of 300-500 microns leaving the cavity closed and the pulp untouched. Saw remainder of tooth into rings (not decalcified). From them cut on freezing microtome cross sections about $40 \mu$ thick and impregnate according to the Gros method. Van der Sprenkel does not give a reference to this method. Perhaps the Gros method, as given by Lee (p. 494) will serve. Treat frozen sections with pyridine. Wash with aq. dest. to remove odor of pyridine. $20 \%$ aq. silver nitrate, in dark, 1 hr. Transfer without washing to $20 \%$ formalin neutralized with magnesium carbonate. Change twice until no more white ppt. is formed. Reduce under microscope in following solution: Add ammonia to $15 \mathrm{cc} .20 \%$ silver nitrate until ppt. formed just disappears. Then add 1 drop ammonia per each cc. silver nitrate solution. After this $20 \%$ aq. ammonia $1 \mathrm{~min}$. or more. $1 \%$ acetic acid, same. Tone in $0.2 \%$ aq. gold chloride treat with sodium hyposulphite, wash, dehydrate, clear and mount. Counterstain with Van Gieson or toluidin blue, if desired before dehydration. See Van der Sprenkel's illustrations.

2. Christensen, K., J. D ent. Res., $1940,19,227-242$ was concerned primarily with determination of the source of the large proportion of unmyelinated and small myelinated fibers in the pulp. His technique is a combination of dissection and the making of histological preparations of cats. First inject arteries with a yellow corn starch mass (composition not specified) and harden tissues in formalin. Expose cervical sympathetic, common carotid and its chief branches, mandibular canal and floor of orbit. Wash dissected areas with aq. dest., and brown nerves with dilute aq. silver nitrate so that they can be easily followed along the walls of the yellow colored vessels. To trace their final path to lower teeth serial sections of inferior alveolar nerve and artery are required and to upper teeth similar ones of internal maxillary plexus and superior alveolar nerves. Wrap canine teeth in cotton, carefully crack with vise and remove pulps. Slightly stretch each pulp along surface of short glass tube attaching the ends to the tube by silk threads to prevent tortuosity of nerve fibers in the final preparations made by the Bodian-Method. Examine the cervical sympathetic ganglia by techniques for Nissl Bodies as well as for nerve fibers before and after degeneration resulting from experimental destruction of dental pulp.

Teeth and Jaws. Sections through (Willman, M., J. Dental Res., 1937, 16, 183190 ). Fix in $10 \%$ formalin, $10-30$ days. Transfer to $95 \%$ alcohol for same time. After decalcification in 5\% aq. nitric acid, change to $5 \%$ aq. sodium sulphate for $24 \mathrm{hrs}$., then wash in running water 24 hrs. Dehydrate through ascending alcohols to $95 \%$, then 2 changes of absolute, $6 \%, 12 \%$ and $25 \%$ celloidin solution, 7 days each. Cut sections with heavy, sledge type of microtome. Remove celloidin from sections with alcohol-ether and pass down to aq. dest. Stain with Harris' hematoxylin and acid alcohol eosin. Mount in dammar. Control decalcification either by testing a second tooth with a needle or by polariscope. See Dental Enamel.

Teeth, Lymphatics. Obviously the work of Fish, E. W., Proc. Roy. Soc. Med., 1926-27, 20 (3), 225-236 ; Bödecker, C. F., and Lefkowitz, W., J. Dent. Res., $1937,16,463-475$ and others relating to 
the "lymph supply" of dentin and enamel does not refer to lymph but to tissue fluid for the spaces are not lined with lymphatic endothelium. For tissue fluid in these situations sec Cowdry, E. V. Problems of Ageing. Baltimore: Williams \& Wilkins, 1942 , p. 593 . An excellent account of techniques designed for investigation of the lymphatic system of teeth and jaws is provided by MacGregor, A., Proc. Roy. Soc. Med., $1935-36,29(2), 1237-1272$. His favorite injection masses were strong solutions of basic lead acetate and acid suspensions of carmine. Before killing and injecting the animals (cats, dogs, guinea pigs and monkeys) he caused them to inhale large doses of amyl nitrite with the idea of dilating the peripheral blood vessels.

Teichmann, see Hemin Crystal Test, Florence Reaction.

Tellurium, see Atomic Weights.

Tellyesniczky's fixative. 5 parts of formol, 100 of $70 \%$ alcohol and 5 of acetic acid.

Tendons. These are dense bands of collagenic fibers interspersed by a few flattened fibroblasts (lamellar cells). Fixatives penetrate the larger ones poorly. Zenker's Fluid and Hematoxylin and Eosin are fairly satisfactory. For mechanical factors in structure see Carey, E. J., Am. J. Anat., 1936, 59, 89-122; Anat. Rec., 1936, 64, 327-341.

Tensile Strength. An ingenious method has been worked out to measure this property of skin (Herrick, E. H., Anat. Rec., $1945,93,145-149$ ).

Terbium, see Atomic Weights.

Tergitol, see Wetting Agents.

Terpineol (or terpinol), a mixture of substances of composition $\mathrm{C}_{10} \mathrm{H}_{16}$ and $\mathrm{C}_{10} \mathrm{H}_{18} \mathrm{O}$ formed by action of dil. $\mathrm{HCl}$ on terpin hydrate. Used as a clearing agent. Can clear tissues from $90 \%$, even from $80 \%$ alc. A good mixture is 4 parts terpineol +1 part xylol.

Tertiary Butyl Alcohol (trimethyl carbinol). Has been recommended as a substitute for ethyl alcohol and clearing agents like xylol in the paraffin technique because it mixes easily both with water and paraffin. It causes but little shrinkage and hardening of tissue. One method (Stowell, R. E., Science, 1942, 96, 165$166)$ is partly to substitute for ethyl alcohol by passing through the following series of mixtures: (1) Aq. dest., 50 cc.; $95 \%$ ethyl, 40 cc.; butyl, 10 cc.; 1-2 hrs. (2) Aq. dest., 30 cc.; $95 \%$ ethyl, 50 cc.; butyl, 20 cc., $2 \mathrm{hrs}$. to several days. Aq. dest., 15 cc.; $95 \%$ ethyl, 50 cc.; butyl, 35 cc.; $1-2$ hrs. (4) $95 \%$ ethyl, 45 cc.; butyl, 55 cc.; 1-2 hrs. (5) Butyl, 75 cc.; abs. ethyl, 25 cc.; 1-3 hrs. (6) Pure butyl, 3 changes 4 hrs, to over- night. (7) Equal parts pure butyl and paraffin oil, 1-2 hrs. Infiltrate in paraffin. Another method (Stowell, R. E., J. 'Tech. Methods, 1942, 22, 71-74) is to entirely substitute $50 \%, 70 \%, 85 \%$ and pure butyl alcohol for the corresponding ethyl alcohols. Stowell provides useful suggestions as to the details of paraffin imbedding. Tertiary butyl alcohol has been recommended for dehydrating material stained with methylene blue and other dyes readily extracted during ethyl alcohol dehydration (Levine, $N$. D., Stain Techn., 1939, 14, 29-30). It may be used as a substitute for ethyl alcohol in the acid fast and Gram stains for bacteria (Beamer, P. B. and Stowell, R. E., J. Lab. \& Clin. Med., 1943, $28,1599-1602$ ). Do not confuse with $n$ Butyl alcohol.

Testis. Methods described elsewhere for the Connective System, Blood Vessels, Nerve Fibers and so on are available. Technique for isolation of scminiferous tubules is given under Maceration. See also Chromosomes. Wagner, K., Biologia Generalis, 1925, 1, 22-51 has employed a method of vital staining with trypan blue which he claims differentiates between interstitial cells and histiocytes or macrophages. Duesberg, J., Biol. Bull., 1918, 35, 175-198, using the Benda Method, obtained preparations of opossums which he thought indicated discharge of material from the interstitial cells into the blood stream. Wagner (loc. cit.) has observed somewhat similar phenomena in other animals, but there has been no satisfactory follow up. For detailed information about interstitial cells see Rasmussen, A. T. Cowdry's Special Cytology, 1932, 3, 1674-1725.

Testosterone, Pollock, Anat. Rec., 1942, 84, 23-27.

Tetrachrome Blood Stain, see MacNeal's.

Tetralin is tetrahydronaphthalene used as a clearing agent after Diaphanol.

Tetrazolium SaIt. Smith, F. E., Science, $1951,113,751-754$ gives uses and limitations. Neotetrazolium gives deep purple to black on reduction and is thus superior to the tetrazolium salt. Blue tetrazolium also has certain special advantages. Sce Triphenyltetrazolium Chloride.

Thallium. Barbaglia's Method. Fix in $95 \%$ alcohol iodized. This precipitates thallium in the form of insoluble crystals of thallium iodide recognizable by their yellow color (Lison, p. 66).

Thiamine. Blaschko and Jacobson (H. and W. in Bourne's Cytology, 1942, p. 196) refer to the work of Ellinger and Koschara in the observation under the fluorescence microscope of green fluorescence 
due to flavin and that on alkalinization this is replaced by a bluish fluorescence which is known to be occasioned by the presence of thiamine, itself identical with vitamin $B$, or aneurin.

See Cartesian diver technique of Westenbrink, H. G. K., Enzymologia, $1940,8,97-107$. Glick, p. 395 thinks that the technique of Schultz, A., Atkin, L. and Frey, C. N., Ind. Eng. Chem., Anal. Ed., 1942, 14, 35-39 based on stimulation of yeast fermentation by thiamine.

Thiazin Dyes. A very useful group of dyes for the histologist. The two benzene rings are joined by $=\mathrm{N}-$ and $-\mathrm{S}-$. Examples: azure A, B and C, methylene azure, methylene blue, methylene green, methylene violet, new methylene blue $\mathrm{N}$, thionin, toluidin blue $\mathrm{O}$.

Thiazine Red $R$ (CI, 225)-chlorazol pink $\mathrm{Y}$, rosophenine $10 \mathrm{~B}-\mathrm{An}$ acid mono-azo dye employed especially as counterstain for iron hematoxylin.

Thiazole Dyes contain thiazole ring with indamine as chromatophore. Geranine $\mathrm{G}$, primalin, thioflavine $\mathrm{S}$, and titan yellow. All of these dyes appear to be useful in fluorescence microscopy. Pick, J., Zeit. f. wis. Mikr., 1935, 51, 338-351 refers to three of them.

Thiazole Yellow, see Titan Yellow.

Thioflavine S (CI, 816). An acid thiazole dye used in fluorescence microscopy.

Thionin (CI, 920)-Lauth's violet-Commission Certified. An extremely useful basic thiazin dye. See Tissue Basophiles, King's Carbol Thionin, etc.

Thiourea. A derivative of urea with sulphur replacing oxygen. As means of activating thyroid gland (Thomas, $\mathrm{O}$. L., Anat. Rec., 1944, 89, 461-469). Effect on organ weights and plasma proteins of the rat (Leathem, J. H., Anat. Rec., 1944, 89, 540).

Thomas, see Arginine Reaction.

Thorium Dioxide is occasionally employed as a vital stain for reticulo-endothelium. Angermann, M. and Oberhof, K., Zeit. f. Ges. Exp. Med., 1934, 94, 121-126 give directions for its administration to rabbits and for determination of its distribution chemically, radiologically and histologically. (Thorotrast)

Thulium. see Atomic Weights.

Thyme Oil N.F. VI. Sometimes misnamed oil of origanum. Contains thymol, carvacrol, cymene, pinene, linalool and bornyl acetate. It is said to be useful for clearing celloidin sections.

Thymol Blue. Sce Hydrogen Ion Indicators.

Thymonucleic Acid is a type of desoxypentose nucleic acid isolated from nuclei of the thymus. Also called desoxyribonucleic acid on DNA for short. (Feulgen or nucleal reaction for). Pass paraffin sections, fixed in equal parts sat. aq. corrosive sublimate and absolute alcohol, through xylol and alcohols to water. Place in a staining jar containing normal $\mathrm{HCl}$ (82.5 cc. $\mathrm{HCl}$, sp. gr. 1.17-1.85 per liter of water) at room temperature for $1 \mathrm{~min}$. Transfer to normal $\mathrm{HCl}$, at $60^{\circ} \mathrm{C}$. and there hydrolyze for $4 \mathrm{~min}$. Treat with the fuchsin sulphurous acid reagent in a staining jar for $\frac{1}{2}-1 \mathrm{hr}$. (This reagent is: One gram of basic fuchsin is dissolved in $100 \mathrm{cc}$. of distilled water with the aid of a little heat. The solution is filtered while still warm and $20 \mathrm{cc}$. of normal $\mathrm{HCl}$ is added to the filtrate. The resulting fluid is then cooled and 1 gm. dry sodium bisulfite $\left(\mathrm{NaHSO}_{3}\right)$ is added. Then, after standing for about $24 \mathrm{hrs}$, the reagent is ready for use and should have a pale straw color.) Pass through a series of 3 jars, eacl containing a solution made by adding $10 \mathrm{cc}$. of a molecular solution of sodium bisulfite (i.e., 104 grams per liter) to $200 \mathrm{cc}$. of tap-water, allowing $1 \frac{1}{2} \mathrm{~min}$. in each and agitating frequently. Wash in tap water for 5 min., dehydrate, clear and mount in balsam. Thymonucleic acid is colored purple or violet and color holds (Cowdry, E. V., Science, 1928, 68, 40-41). Collected references (Milovidov, P., Protoplasma, 1938, 31 (2), 246-266); technique for plant tissues (Whitaker, T. W., Stain Techn., 1939, 14, 13-16). A more recent account is given by Stowell, R. E., Stain Techn., $1945,20,45-58$. Specificity has been considered by Dodson, E. O., Stain Techn., 1946, 21, 103-105. See BauerFeulgen stain for Glycogen.

Dr. A. R. Gopal-Ayengar of the Tata Memorial Hospital, Bombay has supplied details of a modification of the Feulgen technique by Rafalko, J. S., Stain Techn., 1946 21, 91-93. Instead of using $\mathrm{HCl}$ and sulphites, as in the usual method, Rafalko directly charges both basic fuchsin and the bath water with $\mathrm{SO}_{2}$ gas, using $N \mathrm{HCl}$ only for the necessary process of hydrolysis. By this method, he claims to have been able to stain diffuse and small chromosomes, which give negative results with conventional procedure. Three types of organisms were tested: (1) Various small, endosome-containing amoebae; (2) oocytes of parasitic wasps, Habrobracon; and (3) the yeasts Saccharomyces cerevisiac and $S$. carlsbergensis. Fix smears for 2-20 min. Wash in water $20 \mathrm{~min}$. and in aq. dest. 20 min. $N \mathrm{HCl}$ room temperature, 2 min. $N \mathrm{HCl}$ at $60^{\circ} \mathrm{C}$., $8-10 \mathrm{~min}$. Rinse in $N \mathrm{HCl}$ at room temperature. Rinse in aq. dest., Sulphurous acid, 
2 min. Leucobasic fuchsin, $1 \frac{1}{2}-2$ hrs. Sulphurous acid bath, for sufficient time to remove the free untreated leucobasic fuchsin (2-3 changes). Tap water, 10-15 min. Counterstain, if necessary, with aq. or alc. fast green. Dehydrate, clear and mount in the usual manner, or follow Triethyl Phosphate technique.

See Aldehydes for discussion of specificity of Feulgen reaction and Lessler, M. A., Arch. Biochem. and Bioph., $1951,32,42-54$ who thinks that it should be feasible to correlate determinations of color intensity of gelatin DNA preparations of known DNA concentration with that of Feulgen stained nuclei and thus measure approximately the nuclear DNA content. Lessler specifies certain sources of experimental error to be guarded against.

The histophotometric measurement of DNA in the course of embryonic development is described by Lison, L. and Pasteels, J., Arch. de Biol., 1951, 62, 1-43. A biometric investigation of their technique is provided by Martin, L., Arch. de Biol., 1951, 62, $45-64$.

Thymonucleohistone. Technique for dielectric properties, Lars-Göran, Allgén. Acta Physiol. Scand., Suppl. 76, $22,140 \mathrm{pp}$.

Thymus. Isolation en masse of nuclei from Behrens' method as modified by Mayer, D. T. and Gulick, A., J. Biol. Chem., $1942,146,433-440$.

Thyroid. For routine purposes Zenker fixation and hematoxylin and eosin staining of paraffin sections is suggested. If one is interested in the colloid, its appearance after various fixations, its shrinkage patterns and the significance of its acidophilic and basophilic staining are described by Bucher, D., Zeit. f. Zellf. u. Mikr. Anat., 1938, 28, 359-381. The effect on colloid of different agents for dehydration and clearing is described by Ralph, P., Stain Techn., 1938, 13, 915. A method for determination of the volume of colloid is given by Stein, $\mathrm{H}$. B., Am. J. Anat., 1940, 66, 197-211.

The shape of thyroid follicles can be distinguished but imperfectly in sections unless reconstructions are made from serial sections. For an excellent method of viewing entire, isolated follicles see Maceration. The localization of unsuspected masses of follicles, not present in the gland, in the neck tissues of experimental animals can be accomplished by supravital staining with Naphthol Blue.

Many methods are available for the detailed examination of the secretory epithelial cells not requiring their special adjustment to the thyroid gland. See Mitochondria, Microchemical methods, etc. The Brazilin-Wasserblau technique is recommended for incellular secretion antecedents. If the Golgi apparatus is to be investigated consult Welch, C. S. and Broders, A., Arch. Path., 1940, 29, 759-772. A fine beginning has been made in the direct study of vacuoles within the follicles in living mice by transillumination after the fashion of Knisely (Williams, R. G., Anat. Rec., 1941, 79, 263-270). Minute instructions for demonstration of blood vessels and lymphatics and results which are to be expected are given by Rienhoff, W. F., Arch. Surg., 1931, 23, 783-804. For fluorescence sec Grafflin, A. L., J. Morph. and Physiol., 1940, 67, 455-470. Effect of Thiourea on thyroid secretion (Thomas, O. I., Anat. Rec., 1944, 89, 461-469).

Ticks. The following method for softening and sectioning is an adaptation by Miss Slifer of the Slifer-King technique for grasshopper eggs (Slifer, E. H., and King, R. L., Science, 1933, 78, 366-367). Drop animal into dish of Carnoy-Lebrun. After $5 \mathrm{~min}$. place under binocular and puncture with a glass needle. Allow fixative to act for at least $20 \mathrm{~min}$. longer. (Variations in the size of the puncture and in the length of time for fixation should be tried.) Transfer to $70 \%$ alcohol colored a light yellow with iodine over night. If al cohol is colorless next morning let stand a few hours longer. Repeat if necessary. At this point (or somewhat earlier) it is well to make a larger incision in the animal with a scalpel. The viscera should now be well-hardened and should not ooze out through the hole. $70 \%$ alcohol, several hrs. $70 \%$ alcohol containing $1 \%$ phenol, 2 or 3 days. $95 \%$ alcohol 2 hrs. Anilin oil, several hrs. Chloroform (2 changes of $5 \mathrm{~min}$. each). Paraffin about an hour. Imbed and block. Trim block away so that viscera are just exposed, at the point where sectioning is to begin. Place block in water containing $4 \%$ phenol. Be sure that the cut surface is under water and examine occasionally to see that air bubbles do not form on it. After 3 days a swelling of the tissues should be noticeable so that they protrude a little beyond the cut surface of the paraffin. If this has not occurred, cut away a little more and soak several days longer. Trim block, place on microtome and section 5-7 microns. Work rapidly once you have begun. A slight delay between sections will allow the cut surface to dry. If, for any reason, it is neccssary to stop wet a scrap of paper and stick it to the cut 
surface. In case of difficulty in making sections stick to slides try Haupt's gelatine fixative (Stain Techn., 1930, $5,97-98)$. After the sections have been spread, arranged on the slide and albumen (Webb, R. L., Am. J. Anat., 1931-32, 49, 283-334).

Tigroid Bodies (G. tigris, tiger and eidos, appearance). A term applied to Nissl bodies since they sometimes look streaked and spotted like a tiger. See Nissl Bodies.

Tissue Basophiles (tissue mast cells). Some think that these cells are emigrated Basophile Leucocytes and others that they are of extravascular origin. They can easily be studied in fresh spreads of Loose Connective Tissue or omentum. Their granules are readily colored supravitally with brilliant cresyl blue, methylene blue and other stains. Tissue basophiles disintegrate quickly. Maximow, A., Arch. f.mikr. Anat., 1913, 83 (1), 247-289 gives the following metachromatic stain for mast cells. Sections of abs. alc. fixed tissues are stained $24-48$ hrs. in sat. thionin in $50 \%$ alc. Staining can be reduced to $20 \mathrm{~min}$. by adding 4 drops $3 \% \mathrm{Na}_{2} \mathrm{CO}_{3}$ to $20 \mathrm{cc}$. thionin sol. and filtering before use. Maximow gives technique for smears and spreads fixed in formalin Zenker. See his beautiful colored plates. See Toluidine Blue Phloxinate.

Holmgren and Wilander (H. and $\mathrm{O}$., Ztschr. f. mikr. Anat. Forsch., 1937, $42,242-278$ ) recommend fixation in $10 \%$ aq. basic lead acetate and staining with $1 \%$ alc. Toluidin blue. They show that fixation in formalin-alcohol gives very inferior results. In their opinion the metachromatic substance colored is identical with Heparin.

Sylvén, B., Acta Radiol., 1940, 21, 206-212 has followed this matter up by subjecting rats and guinea pigs (in which the basophilic granules are said to be less soluble in water than in most other animals) to Gamma rays. He fixed the tissues in weaker aq. basic lead acetate (4\%) for 24 hrs., stained paraffin sections with $\frac{1}{2} \%$ aq. toluidin blue and other dyes, and reached the conclusion that the radiation brings about liberation of organic sulphuric acids of high molecular weight. It would be natural to investigate the relation if any between heparin and the basophilic granules in buffy coat of centrifuged human blood containing say $0.5 \%$ basophiles and in that of certain turtles in which the percentage is as high as 80 as well as in livers.

Another method of study is to investigate heparin in relation to the characteristic dissolution of basophiles 2 days after the intraperitoneal injection of egg albumen (Webb, R. U., Am. J. Anab., 1931-32, 49, 283-334).

Tissue Culture-Written by Wilton $R$. Earle, National Cancer Institute, Bethesda. July 10,1951-This technique is obviously of great value in biology and medicine. For orientation reference should be made to two recent books: Parker, R. C., Methods of Tissue Culture, 2nd. ed., New York: Hoeber, 1950, Cameron, Gladys, Tissue Culture Technique, New York: Academic Press, 1950. At present (March 1951) the Tissue Culture Association (C/ Dr. Margaret Murray, College Physicians \& Surgeons, W. 168 St., New York) has nearly ready for publication an elaborately cross indexed bibliography containing over 16,000 primary references to papers involving tissue culture. This association also serves as a coordinating organization for tissue culture workers.

By the methods of tissue culture a small clump of cells can be removed from an organism and maintained in a condition of survival or growth for periods ranging from a few hours for some cells to an indefinite number of years for the descendants of others. While so maintained they can be examined microscopically at various magnifications. The differentiation of embryonic organs can be followed (see account in this book written by Honor B. Fell: Organ Culture in vitro). Malignant cells may be grown and studied for an extended interval and their characteristics compared with those of normal cells or with malignant cells in vivo (Lewis, W. H., Arch. f. Exp. Zellf., 1939, 23, 8; Earle, W. R., J. Nat. Cancer Inst., 1943, 4, 165). Cell form, size, internal motion, locomotion and rate and manner of cell proliferation can be routinely studied either visually, or by means of phase interference photography, or by time-lapse cinematography (Fell, $\dot{H}$. B., and Hughes, A. F., Quart. J. Micr. Sci., $1949,90,355)$. The tissue can be vitally stained (Ludford, R. J., 11th Scientific Report, Imper. Cancer Research Fund, 1934, 169) or fixed in situ, and stained for microscopic examination, (Cameron, above cited), for microchemical test, or for electron micrography. Physiological and nutritional studies are possible. The culture medium can be modified by the addition or omission of various nutritional elements or other physiologically active substances (Pogogeff, I. A., and Murray, M. R., Anat. Rec., 1946, 95, 321), and the influence of the altered medium 
on the cells may be studied. The culture medium may be removed and tested to determine what changes the cells have induced in it (Brues, A. M., Rathbun, E. N., Cohn, W. E., J. Cell. and Comp. Physiol., 1944, 24, 155). The tissue growing in culture may be used as a host for the growth and study of bacteria or viruses (Fox, J. P., Amer. J. Hygiene, 1947, 46, 1). Rapidly increasing facility in the handling of many types of cells in culture suggests a constantly increasing usefulness of tissue culture methods in many fields of future scientific work.

1. The Culture Medium and Substrate. A satisfactory isotonic balanced saline solution is necessary for washing cultures and for dilution of plasma and nutrient media. This balanced salt solution can be prepared best from glass distilled water free from fungus or bacterial growth which might give rise to toxic substances. Reagent quality chemicals should be used and heavy metal contaminants in particular should be avoided. Only minor differences exist in the formulae of many of the solutions in current use: Mammalian Ringer (Ringer, S., J. Physiol., 1895, 18, 425), Tyrode (Arch. Intern. de Pharmacodyn. et de Thér., 1910, 20, 205; Gey, G. O. and Gey, M. K., Am. J. Cancer, 1936, 27, 45). Hanks' (Hanks, J. H., J. Cell. and Comp. Physiol., 1948, 31, 235), Simms' (Simms, H. S., and Sanders, M., Arch. Pathol., 1942, 33, 619), Earle's solution (J. Nat. Cancer Inst., 1943, 4, 165). Solutions as amphibian Ringer are used with amphibian cells. Any solution approximating the inorganic salt content of serum, and having a comparable osmotic pressure, can be employed as a diluting and wash solution for routine tissue cultures. For skin epithelium Parshley and Simms (Am. J. Anat., $1950,86,163$ ) have used a diluting solution containing no calcium or bicarbonate, but with increased phosphate, and with aspartic acid added. This, however, apparently has not been tested for longer culture intervals than about a week. About $0.1 \%$ glucose is usually included in balanced saline solutions as a source of carboliydrate. For much tissue culture work the solution used by Earle has the advantage of an alkali reserve, in the form of sodium bicarbonate, comparable to that of serum.

All physiological solutions, such as serum, depending chiefly on sodium bicarbonate for their alkali reserve, can be maintained at a stable $\mathrm{pH}$ within workable physiological limits only when kept in sealed containers with an adequate tension of $\mathrm{CO}_{2}$ in the air overlying the fluid. Sterilization by heat, or by vacuum filtration through a bacteriological filter, causes an alkaline shift, due to loss of $\mathrm{CO}_{2}$, and this causes secondary changes in the solution such as precipitation of the calcium and magnesium salts. Probably the most satisfactory procedure for sterilizing such a solution is to filter by pressure (2-10 p.s.i.) and to store in sealed containers. Before the filtration $\mathrm{CO}_{2}$ should be passed through the solution to bring it to a $\mathrm{pH}$ somewhat more acid than that desired. For routine culture work an initial $\mathrm{pH}$ of about 7.6 in the culture is desirable because elaboration of acid by cells in the culture will carry the $\mathrm{pH}$ to somewhat more acid levels.

While the balanced salt solution may be used for rinsing cultures, and while tissue fragments may be left in it for short periods, these times should be limited as continued action of the balanced saline on the cells is injurious. It is probably wiser to consider the balanced salt solution as having its major use as a diluent for other media such as serum.

For the satisfactory routine maintenance of cultures of most tissue cells a solid support or substrate for their growth and migration is necessary. Various types of substrates have been employed, such as silk thread, spider web, glass wool, lens tissue, cellophane, gelatin and agar, and for simple cultures, the glass surface of the culture dish.

By far the most widely used substrate, and the one which has proven most satisfactory until recently has been a thin layer of plasma or fibrin clot. To prepare the culture with this substrate, the clump of cells to be planted (the implant) is placed in a thin layer (0.1 to $1.0 \mathrm{~mm}$. thick) of plasma or fibrinogen solution, and this is clotted into a solid gel by addition of a little tissue extract or thrombin solution. When of correct consistency the elot is a solid, somewhat elastic, optically clear gel. Its fibrillar structure enables the cells to migrate through it, although they tend to collect at its surfaces.

This type of culture substrate has certain advantages: 1 . The fibrin matrix anchors the cells so that as long as the matrix is intact few cells wash loose and are lost when the culture fluid is renewed. 2. The fibrils of the substrate present effective surfaces for adhesion and migration of the cells. 
3. Consequently, with some cell types at least the fibrillar structure of the clot probably facilitates the final stages of cell division, since final separation of the cells is accomplished by the daughter cells migrating in opposite directions. 4. The freshly prepared fibrin clot contains some serum and possibly other factors which enhance the growth of many cell types.

Chicken plasma is most often used for the preparation of the clot because it is less likely to clot spontaneously and since a gel of good consistency can be more routinely prepared from it; but plasma homologous with the cells is frequently employed, as is also plasma from other animals. Premature clotting of the plasma is usually prevented by addition of a small amount of purified heparin.

Although the plasma or fibrin clot is widely used, it is in many ways unsatisfactory. 1. With certain combinations of cells and media the clot rapidly dissolves and ruins the culture. 2. In the case of very slow-growing cultures the clot may gradually become so opaque as to make optical examination impossible. 3. The chemically undefined nature of the plasma or fibrin clot and the variability of its physical structure interfere with many types of work, such as studies in cell nutrition. 4. Since the cells are embedded in the clot and cannot be separated from it, differential staining, weighing of the cells, or chemical analyses of the cells alone in the absence of the clot, are difficult or impossible.

Perforated cellophane (Evans, V. J., and Earle, W. R., J. Nat. Cancer Inst., $1947,8,103$ ) offers a substrate superior to plasma for many kinds of work. In the cellophane substrate culture the cell clump is placed on the floor of the culture container, is immersed in a nutrient fluid medium, and is covered with a perforated cellophane sheet which holds it in position against the glass floor of the flask. This type substrate is cheap, easily handled, may be sterilized by autoclaving, is relatively inert, and is chemically defined (regenerated cellulose, Earle, W. R., Evans, V. J., Edward, M. F., and Duchesne, E., J'. Nat. Cancer Inst., 1949, 10, 291). Experience to date indicates that when grown on this substrate in an adequate nutrient culture fluid, many cell types, both normal and malignant, continue to proliferate with far greater luxuriance than when embedded in the plasma clot. Loose cells from the implant float loose, reattach to the cellophane or the glass floor of the cul- ture vessel, and rapidly extend the area covered by the cells (Earle, W. R., Evans, V. J. and Schilling, E. L., J. Nat. Cancer Inst., 1950, 10,943). The luxuriance of growth is such that culture flasks of 60 square $\mathrm{cm}$. floor area may often be used for established stock cultures. Frequently this area is covered by cells so rapidly that transplanting is necessary in as short time as 5-10 days. Transplanting of cellophane substrate cultures is most easily accomplished by scraping or shaking the culture. Many cells come loose from the substrate and are transferred as a cell suspension to a new flask. Once a luxuriant cell growth is obtained on cellophane and a heavy cell suspension is prepared from it, the cellophane sheet may in instances be discarded and the cells may be allowed to settle on the glass floor of the flask on which they attach and proliferate (Earle, W. R., Evans, V. J., Sanford, K. K., Shannon, J. E., Jr. and Waltz, H. K., J. Nat. Cancer Inst., in press; Shannon, J. E., Jr. and Earle, W. R., J. Nat. Cancer Inst., in press).

When survival or growth is desired for longer than a few hours, nutrients must be included in the culture medium. In successful instances of cell proliferation over an extended period of time the nutrient materials used have been a tissue extract, most often from embryonic tissue, a serum, or usually a combination of the two. In many instances, particularly in the older literature, where a tissue extract alone has been found satisfactory as a nutrient, serum was actually also present in substantial amounts owing to use of a plasma substrate for the culture.

The tissue extract now commonly employed for routine tissue cultures of cells from many species is made by briefly extracting minced embryonic tissue with an equal volume of balanced salt solution, and by decanting the supernatant fluid after centrifuging. This concentration is known as $1: 1$ embryo extract. An attempt is generally made to eliminate living tissue cells from the solution by freezing in $\mathrm{CO}_{2}$ snow and recentrifuging. The extract loses potency rapidly and should therefore be used within a few days after preparation, but if lyophilized it can be kept for much longer periods (Hetherington, D. C., and Craig, J. S., Proc. Soc. Exp. Biol. and Med., 1939, 42, 831).

One current source of embryo extract is chick embryos of 9-11 days incubation. Where facilities of a local slaughter house are available some workers 
find it convenient to employ beef embryos removed from the uteri by aseptic methods (Gey and Gey, cited above).

At present, whatever the source of tissue, the extract is routinely prepared with rigid asepsis because no means of sterilization has yet proven satisfactory. Filtration through a bacteriological filter results in rapid clogging of the filter and in great reduction in potency. However, it has been recently reported (Bryant, Jay C., Earle, W. R. and Peppers, E. V., at the 1951 Annual Meeting of the Tissue Culture Association, Detroit) that by treating the extract first with hyaluronidase, and then by centrifuging at approximately $40,000 \mathrm{G}$, extract from 9 -day chicks may be rapidly filtered by low pressure through a 03 porosity, $4 \mathrm{~mm}$. wall thickness Selas filter (Selas Filter Co., Philadelphia, Penn.). The resulting extract, used with serum, caused rapid proliferation of mouse strain L fibroblasts for the 17 day interval studied. If these results are confirmed for other cell types and for longer intervals of culture, this method of preparation of embryo extract should substantially facilitate tissue culture work.

Extracts of malignant tissues have often been extremely effective in stimulating growth of some types of cells. A balanced saline extract of Walker 256 rat mammary carcinoma, used with horse serum, has caused rapid proliferation of the Walker 256 carcinoma cells in culture, but no stimulative action was noted on either rat normal mammary epithelium or subcutaneous fibroblasts (Earle, W. R., Arch. f. Exp. Zellf., 1937, 20, 140).

Horse serum has been routinely used in this laboratory over a period of years as a serum component of nutrient fluid. The mixture consisted of $40 \%$ horse serum, 20\% 1:1 extract from 9-day chick embryos and $40 \%$ balanced saline solution. This has given luxuriant long-term growth with such different cells as Walker 256 rat mammary carcinoma (Earle, W. R., Arch. of Path., $1939,27,80$ ), fibroblasts from rats, mice, and humans, and a number of mouse fibrosarcoma strains (Earle, W. R., J. Nat. Cancer Inst., 1943, 4, 165). Not all cells do well in this medium. A high concentration of chicken serum with low embryo extract gave supcrior growth of chicken monocytes. Parker, R. C. (J. Exp. Med., 1932, $55,713 ; 1933,58,97 ; 1933,58,401)$ has found that various strains of fibroblasts required very different concentrations of embryo extract and serum to attain their optimal growth.

Where it can be used horse serum offers certain technical advantages. Eight to 10 liters of horse blood can be obtained from one bleeding without injury to the horse, and after clotting and separation of the blood cells by centrifugation the serum may be sterilized by pressure filtration through a * 03 Selas filter at 5 p.s.i., and stored under refrigeration for a year or longer. Before filtration extreme care should be taken to prevent any fungus or bacterial growth in the serum; toxic substances produced can pass the filter and injure or kill the culture.

Numerous other types of sera have been used in tissue culture. Beef serum would probably be as satisfactory as horse. If local facilities make it available sheep serum could be tried. In hospital centers human cord serum has been available and has been found extremely satisfactory by many workers (Gey, G. O. and M. K. cited above). Dr. Margaret Murray finds that for human tumor material it is superior to horse serum (personal communication).

In exploring the possibilities of growing any cell type, various percentage combinations of embryo extract and serum are among the first media to be tried, after which these combinations may be supplemented by addition of other physiologically active substances.

There has been a great deal of work toward preparing a chemically defined or synthetic culture medium for cells growing in culture (Vogelaar, J. P. M., and Erlichman, E., Am. J. Cancer, 1933, 18, 28; Baker, L. E., Science, 1936, 83, 605; Fischer, A., Astrup, T., Ehrensvard, G. and Oehlenschlager, V., Proc. Soc. Exp. Biol \& Med., 1948, 67, 40; White, P. R., Growth, 1946, 10, 231; Davidson, J. N., Leslie, I. and Waymouth, C., Biochem. J., 1949, 44, 5; Morgan, J. F., Morton, H. J. and Parker, R. C., Proc. Soc. Exp. Biol. \& Med., 1950, 73, 1). Substantial progress has been and is being made in defining the nutritional requirements of cells in culture, but at the present time no chemically defined medium now available appears to be satisfactory for the continued proliferation of any type of tissue cell in vitro. This study of cell nutrition of both the normal and the malignant cell will undoubtedly continue as one of the most interesting and active fields of tissue culture research.

At present our knowledge of the 
media and other conditions prevailing in culture, and of the nutrients and other materials influencing the cells in vitro are all too inadequate. Consequently any extrapolation of cell or tissue behavior from in vitro to normal in vivo conditions must be made with great reserve. Great caution should be observed in undertaking any tissue culture study the significance of which is dependent on in vitro conditions being identical or closely comparable with those in vivo.

2. Types of Cultures. In coverslip preparations the tissue clump is planted in a drop of plasma and nutrient culture medium on a round coverslip of $24 \mathrm{~mm}$. diameter. This coverslip is laid, culture side up, on a coverslip $48 \mathrm{~mm}$. square, and is attached to the larger coverslip through capillarity by allowing a small drop of culture medium to run between them.

A hollow ground slide, charged with a vaseline ring, is then lowered onto the the large coverslip until contact of the coverslip with the vaseline ring on the slide seals the preparation: For coverslips of the size cited a rectangular hollow ground slide $55 \times 80 \mathrm{~mm}$. by 6 $\mathrm{mm}$. thick and with a polished concavity $40 \mathrm{~mm}$. in diameter and about 4.5 $\mathrm{mm}$. deep at its deepest point is excellent. (The more usual Maximow slide is $75 \times 45 \times 8 \mathrm{~mm}$., with a cavity $36 \mathrm{~mm}$. in diameter, and requires a $40 \times 40 \mathrm{~mm}$ coverslip.)

Coverslip preparations can be given a final outer edge-seal of paraffin. By using very thin coverslips, and if necessary, by even omitting the small inner slip, the cells can be critically studied with high numerical aperture lenses. 'This type of preparation is probably the best for routine work with short working distance high resolution objectives.

Since the total amount of culture medium is only 1-3 drops, a tissue clump of very limited size must be used and the reasonably healthy life of the preparation is only a few days. At the end of that time however, the culture may be opened, the inner coverslip with the actual culture lifted out, rinsed in balanced saline; fresh nutrient fluid is added and the whole resealed onto a new outer coverslip and hollow ground slide. By this partial renewal of the culture medium every 2 or 3 days the culture may be carried for extended periods. Pogogeff and Murray (Anat. Rec., 1946, 95, 321) report carrying such cultures of muscle cells for more than a year and a half. When the cell clump gets too large a small frag- ment of it may be re-explanted to a new culture.

Instead of using a plasma substrate for the culture, the cell clump may be placed on the coverslip, a few drops of nutrient fluid added, and a disc of perforated cellophane dropped on the cell clump (Schilling, E. L., Earle, W. R. and Evans, V. J., J. Nat. Cancer Inst., 1950, 10, 883). Or the cell clump may be placed on an inner coverslip in nutrient fluid, and covered with a disc of perforated cellophane. Transfer of the culture to a fresh slide is similar to transfer of the usual double coverslip culture preparation.

Coverslip cultures may be killed and fixed and stained in toto. For even more exacting visual or photographic work the plasma may be omitted and the cells grown or allowed to migrate out directly on the glass coverslip. In migrations under these conditions the cells spread on the glass in extremely thin sheets. These are suitable for critical microscopic study of chromosomes, mitochondria, Golgi apparatus and other cellular components. If grown on thin plastic sheets they can even be fixed and examined with the electron microscope.

Coverslip cultures for short periods of time are recommended in beginning tissue culture work, but when it is necessary to carry them through consecutive changes of media, sterility is difficult to maintain. When dangerous infectious agents are employed, coverslip preparations should be handled with great care to avoid hazard to the operator, as they frequently develop leaky seals and because the thin coverslips are easily broken. Accurate control of culture conditions over long periods of time is more difficult in coverslip preparations than in tube or Carrel flask cultures.

For preparations of high optical perfection, such as are necessary in high resolution microcinematography, the culture is often prepared on a large coverslip as described, and over this a thin glass or metal slide, having a hole through it the diameter of the usual lollow-slide concavity, is placed and sealed. The open top of the preparation is then sealed by means of another coverslip. In this type of mount the nutrient fluid of the culture can form a continuous film joining a small central area of the upper and lower coverslips. While the optical perfection of such a preparation is high, the cell clump used must be small and its life is short due to limited volume of 
culture fluid and limited access of the cells to adequate oxygenation.

In Roller T'ube Cultures (Gey, G. O. and M. K. Am. J. Cancer, 1936, 27, 45) the culture vessel is a test tube usually about $15 \times 150 \mathrm{~mm}$., or in some cases a larger container is used. A thin layer of plasma and nutrient medium is coated over the inner surface of the tube to within $5 \mathrm{~cm}$. of the mouth, and while this plasma layer is still liquid numerous small tissue fragments are embedded in it. After the plasma has clotted, about $1 \mathrm{ml}$. of nutrient solution is added and the tube sealed with a rubber stopper. In the incubator the tube is slipped into a hole in the front end of a drum-shaped frame rotating at about 7-10 r.p.h., so that as the drum rotates about its axis the supernatant culture fluid slowly washes over the clumps of cells embedded in the plasma lining the tube. The fluid is changed every 2 to 4 days. At periods of 9 to 15 days, colonies of cells may be dissected loose from the plasma film lining the culture tube. They are then removed from the culture tube by means of the pipette, cut to convenient size, and subplanted to new cultures.

This type of culture is better adapted than is the coverslip preparation for routine growing of large numbers of cell clumps since each test tube can accommodate 5 to 20 cell clumps, each of them as large as that in a coverslip culture. The fluid can be readily changed with only minimal disturbance of the embedded cultures. Where an extensive series of cultures is carried bacterial infection is usually less troublesome than with slide cultures. Since the tube may be sealed with a rubber stopper, there is less gas $\left(\mathrm{CO}_{2}\right.$ and $\mathrm{O}_{2}$ ) leakage than in the slide preparation (Earle, W. R., U. S. Pub. Health Reports, 1931, 46, 1998). Moreover the rotating mechanism for the roller-tube unit is cheaply and easily constructed and the cost of routine culture tubes (pyrex test tubes) is only a few cents.

But the use of "roller-tube" cultures is not without limitations. The thin layer of plasma clot used is of ten eroded by the cells so that frequent patching of the clot by fresh additions of plasma becomes necessary. This patching interferes with accuracy in control of conditions of the culture and the cultures themselves are not infrequently lost by eroding entirely out of the clot. The curved tube surface, the thick tube wall, and the separation which the tube makes necessary between microscope objective and condenser all complicate microscopic examination and limit it to low magnifications. This handicap can be partially overcome by subculturing to coverslip preparations for detailed microscopic study, but this is objectionable because of listurbance of the culture and because of the poor control of culture conditions in the coverslip preparations. Although each roller tube may contain a number of implants, each of them is usually small so that the total volume of explanted tissue is not large. The consequent necessity of handling many cell clumps makes the initial planting of the cultures relatively slow.

With some cell types, following liquefaction of the plasma film in the roller tube, loose cells scatter over the inner surface of the tubc and proliferate luxuriantly. This gives roller tube cultures which can be handled and transferred by scraping and shaking cells loose, as described below for the cellophane substrate cultures.

Attempts have been made to adapt the cellophane substrate to roller tubes by inserting a loose sleeve of perforated cellophane in the roller tube on top of the cell clumps. Some workers have reported that the cellophane sleeve has a tendency to rotate within the tube and so scrape cells off. Some method must be used to prevent this. Other workers have apparently had extremely satisfactory results with cellophane substrate roller tube cultures. Too little information is at present available to evaluate the method.

Flasks for Carrel Flask Cultures (Carrel, A., J. Exp. Med., 1923, 38, 407) are made in several sizes. A well made "D" 3.5 type flask, as currently used is disc shaped, $3.5 \mathrm{~cm}$. in diameter, with top and bottom blown plane and parallel, each about $\frac{1}{2} \mathrm{~mm}$. in thickness. The sides of the flask are vertical, so that the total separation from top to bottom of the flask is 10.0 to $11.0 \mathrm{~mm}$. A side neck of $10.3-10.8 \mathrm{~mm}$. internal diameter, $1.0-1.3 \mathrm{~mm}$. wall thickness, $32-34 \mathrm{~mm}$. length, projects from the side wall of the flask and slopes upward to form an angle of $140^{\circ}$ with the top surface of the flask. The end of the throat is fire-polished and free from bead or overhang. The entire flask is made of Pyrex glass, and is oven annealed to be strain-free under polarized light.

The cell clump is planted on the bottom of the flask in a layer of medium which consists of $0.6 \mathrm{cc}$. of chicken plasma and 0.7 ec. of some fluid culture medium ( $20 \%$ chick embryo extract, $40 \%$ horse serum and $40 \%$ physiological saline). After this has clotted, 1 to 2 
$\mathrm{ml}$. of the same fluid culture medium is added; the flask sealed with a rubber stopper, and incubated as usual. About 3 times weekly the preparation is unsealed, the old culture medium removed, the solid clot with its contained cells soaked for about $15 \mathrm{~min}$. in isotonic balanced saline, this saline removed, fresh nutrient fluid added, and the flask resealed. At intervals of for instance 28 days, the whole sheet of plasma may be slipped loose from the floor of the flask, poured out of the flask and the cell sheet cut into explants of suitable size. These may be reinoculated to make new cultures in other flasks.

This type of culture, like the "roller tube" culture is well suited for carrying relatively large numbers of cultures over extended periods. Washing of the culture and renewal of the culture fluid can be done quickly. As routinely carried out at the National Cancer Institute, the actual time required for 2 operators to set up apparatus and solutions, wash and renew the nutrient medium on 200 plasma substrate cultures is about $90-110 \mathrm{~min}$. In planting, each culture flask receives one explant of about 3.0 to $4.5 \mathrm{~mm}$. width and $15 \mathrm{~mm}$. length, while the thickness of the explant is only the thickness of the culture sheet of the previous culture generation. Since only one culture is used in each culture flask, transplantation is rapid. Growth from this type explant of a rapidly growing cell strain will often routinely cover the floor of the flask at 28 days.

There are several advantages over the roller tube culture. The plasma clot is usually thicker (though a thin clot can be used) so that there is less trouble from clot erosion; with many cell strains "patching" of the clot with fresh plasma is not necessary. The clot is of such thickness and texture that it can be slipped loose from the flask as a sheet, and slid out onto a sterile glass plate, where the culture can be easily and accurately cut up for subinoculation by means of a Bard-Parker * 11 blade attached to a $\$ 7$ handle. Because a single very thin strip-shaped explant is placed in each flask, actual subplanting of cultures is much more rapid than with the roller tube preparations in general use and the actual amount of tissue is probably greater. If desired, flask cultures may be incubated on slowly rocking shelves but this is necessary only in studies in which the whole surface of the culture must be washed with a moving film of fluid. Cultures can be routinely photo- graphed at magnifications of 200 to 400 diameters and can be examined regularly with up to a $4 \mathrm{~mm}$., $0.65 \mathrm{~N}$.A. achromatic objective. The $5.5 \mathrm{~mm}$. Bausch and Lomb objective of 0.65 N.A. is extremely useful. For higher numerical aperture photographs subinoculation must be made to slide cultures.

The general use of the Carrel flask has been limited. Since the flasks require a high quality of precision glassblowing, they are expensive. D3.5 flasks currently sell for $\$ 2.25$ each. Apparently the dimensional and fabrication specifications of the flask were never published so that many of the flasks sold have been unsatisfactory in use, or have been so fragile as to make their use prohibitively expensive due to breakage. (Satisfactory flasks are now available from Mr. Otto Hopf, Upper Black Eddy, Penn.) In many points their manner of use and accessory apparatus used with them has been inadequately described in text and papers on tissue culture. The writer considers the Carrel flask as the most satisfactory of the three culture methods described for routine qualitative work, and as a method which warrants greater attention than it has received.

The preceding types of tissue culture preparations have all allowed the growth of a very small mass of tissue, or of a very thin sheet of cells adherent to a more or less plane substrate. Another type of three dimensional substrate culture has recently been reported. (Earle, W. R., Schilling, E. L., Shannon, J. E., Jr., 1951 Anuual Meeting of the Tissue Culture Association, Detroit; J. Nat. Cancer Inst., in press). In some iustances this was built from folded perforated cellophane sheets; in others it consisted of a mass of commercially available Pyrex glass chemical absorption tower packing helices of $\frac{1}{8}$ inch lumen, $\frac{1}{2} \mathrm{~mm}$. rod size. The mouse strain $L$ cells studied were inplanted as a cell suspension and adhered to the surfaces of the matrix, while nutrient fluid was periodically circulated through the interstices of the substrate mass. Cell proliferation of $4 \times$ to $8.5 \times$ the original inoculum was obtained with both types of substrates. In one instance an estimated $1145 \mathrm{mg}$. (wet weight) of cells was obtained. In a number of instances the weight exceeded $500 \mathrm{mg}$. per culture.

It appears that the general methods and principles involved in this type culture may already be extrapolated to make practical the growth of far larger masses of tissue cells. Even this type 
of culture, however, must at the present time be considered as merely a step in the development of other and still more useful culture types. Klein (Klein, G., Cancer, 1950, 3, 1052) and others have been able to grow numerous types of malignant tumors intraperitoneally in animals, and by repeated subinoculation in this site have caused the tumor cells to form a dense cell suspension in the ascitic fluid of the animal. It appears quite probable that with further increase of our knowledge of the factors which control the proliferation of cells on these various types of substrates, it may be practical to entirely eliminate the necessity of the substrate, as we now know it. By control of culture conditions it may be possible to routinely grow many types of cells free-floating, or virtually free floating, as a suspension in a nutrient fluid. Already these possibilities are being explored.

Numerous other types of tissue culture preparations are employed for special purposes. A watchglass culture is often used in embryological studies (Fell, H. B., above cited). Porter, K. R., Claude, A. and Fullam, E. F., (J. Exp. Med., 1945, 81, 233) have introduced a special flask, for use in a rollertube unit, designed particularly for electron microscopy. Special flasks designated T-12 and T-60 flasks (Earle, W. R. and Highhouse, F., unpublished) have been designed to handle quantitatively cultures planted from cell suspensions obtained from cellophane substrate cultures. These will be considered in more detail below.

3. The Cells in Cultures. With respect to the types of cells which may be grown in tissue culture at the present time, certain rough but possibly useful generalizations may be made. 1. Nearly any type of cell can be kept alive or in a state of survival, from a few hours to a few days. 2. Cell proliferation is probably not to be expected at all of anatomically incomplete cells, such as the erythrocyte, which lacks a nucleus. 3. Embryonic cells which have not yet assumed a high degree of functional specialization or differentiation, are in general easier to grow than adult, highly specialized or differentiated cells. 4. Many malignant cells are more easily grown than are the normal cells from which they arise. For instance, there has been no satisfactory longterm culture of the normal mammary gland epithelium, although there have been frequent instances of culture of epithelium from carcinoma of the mammary gland. 5. That group of cells which we loosely designate as "fibroblasts", and closely related cells which arise from the mesenchyme or mesoderm are in general reasonably easy to grow, particularly from very young animals. The epithelial tissues, however, especially the highly differentiated secretory epithelia, from the liver or the thyroid, are often far more difficult. 6. It appears quite probable that as our knowledge of the nutrition and endocrine control of specific cell types increases we shall become increasingly able to grow these more highly specialized cells. 7. At present relatively few cell strains, normal or malignant, have been maintained in a state of rapid proliferation for as long as one year. 9. If an easily grown cell type can be used with equal value to one which has never been satisfactorily grown, the easily grown cell is obviously the one of choice. The study necessary to grow luxuriantly a cell type which has never been grown may well take years. Any problem which depends for its success on cultivation of a cell type which has never been satisfactorily grown should be entered into with caution and only after a careful evaluation of whether the results to be obtained justify the expenditure of effort involved.

Until recently it has never been possible to grow a single isolated tissue cell of any type. Consequently it has never been possible to establish a pure culture or strain of cells which could be considered with assurance as made up of only one single cell type. Cultures of such cells as the chick-heart fibroblasts or the malignant mammary epithelium have in instances been cultured and have appeared stabilized for extended periods of years; but there has been no assurance that all cells within such cultures were identical in type or in origin. At best they could be considered as made up of similarly appearing, or comparably reacting cells, as judged by the tests used. Recently, however, methods have been worked out by which a single isolated cell from the subcutaneous connective tissue of a $\mathrm{C}_{3} \mathrm{H}$ strain mouse was successfully isolated and grown (Sanford, K. K., Earle, W. R. and Likely, G. D., J.'Nat. Cancer Inst., 1948, 9, 229). This cell strain has continued to proliferate luxuriantly in horse serum and chick embryo extract for a number of years. (The strain has now been made available to laboratories having facilities for carrying it.) The methorls used have allowed proliferation of a number of other types of isolated cells both 
normal and malignant, and should ultimately allow the growth of large cultures from them. To date, however, (March 1951) technical difficulties have interfered with carrying the proliferating isolated cell of most of these other types through to large cultures.

For accurate cell descriptions which are to be compared with descriptions from other cultures at other times, culture conditions and media should be standardized as accurately as possible. Cultures should be well established yet relatively young, and of comparable size (Earle, W. R. and Thompson, J. W., U. S. Pub. Health Rep., 1930, 45, 2672). Chilling of the cultures, inadequate frequency of change of culture media, severe vibration, all tend to produce aberrant cell shapes. Prolonged exposure to light in the presence of erythrocytes can injure or kill otherwise relatively insensitive cells (Earle, W. R., J. Exp. Med., 1928, 48, 457; 48, 683). 'The central part of a dense culture is often degenerating or necrotic. Cells which have migrated out from an explant frequently exhibit aberrant and "giant cell" forms at the extreme periphery of the culture. Cells located in a plasma substrate at its interface with the supernatant fluid of ten show different structural features from cells at the interface of the plasma with the glass surface of the culture dish (Earle, W. R., Schilling, E. L. and Shelton, E., J. Nat. Cancer Inst., 1950, 10, 865 and 1067 ).

These variations should be recognized by the worker and every effort made to standardize conditions to eliminate them as complicating factors in the records. Photographic records of the culture and of its living cells are extremely satisfactory as records if correctly made. Phase contrast optics are often useful or essential. For following changes in cell activities the time-lapse cinematograph is extremely useful.

4. Tissue Culture and Quantitative Research. When an attempt is made to prepare substantial numbers of replicate cultures for quantitative studies involving changes of cell proliferation rate in culture, numerous difficulties are encountered. If fresh tissue is used, the amount of residual tissue material brought over from the host, and the variability among the explants of an extensive series of cultures of ten raise serious questions as to the significance of results obtained. By using plasma substrate tissue colonies of a well established cell strain, and by bisecting each colony, one half of each colony may be used as a control on the other, but even this type preparation contains the residual materials of the plasma matrix, while the number of control cultures necessary to achieve reasonably accurate conclusions is of ten prohibitive.

If the cell type can be grown satisfactorily on a surface substrate (e.g. cellophane or glass), and if a cell suspension can be prepared from it, this suspension may be handled by special burettes and other accessory equipment, and from the suspension large numbers of replicate cultures having a high degree of accuracy may be rapidly planted (Evans, V. J., Earle, W. R., Sanford, K. K., Shannon, J. E. Jr. and Waltz, H. K., J. Nat. Cancer Inst., $1951,11,907)$.

The term growth has been so loosely used in the tissue culture literature, and particularly in the early literature, as to be confusing and of ten misleading (Essentials of Tissue Culture, Parker, R. C., Cunningham, B. and Kirk, P. L., J. Cell. and Comp. Physiol., $1942,20,343)$. Estimation of change in culture area or diameter has probably been the most widely used and most easily applied index of culture "Growth". Substantial cell proliferation for an extended period of time can be easily recognized, but in the early stages of the life of the culture the method may be grossly inaccurate due to increase in the culture area resultant from cell migration rather than from cell proliferation. Even with older cultures the method is accurate only where cell density per uuit area is relatively constant and if necrosis has not supervened.

Actual observation of cell division is the ultimate evidence of cell proliferation. By determining the number of cells which undergo mitosis in a culture area per unit time relative to the total number of cells in that area, an estimate may be made of the relative frequency of cell proliferation. But such observations are arduous and are subject to a number of possible errors. Cell proliferation rate may be very different in different parts of the same culture, so that the method is valid only for a constant zone or for comparable areas of cultures. If the enumerations are made visually on living cells, the accuracy of enumeration is often very poor while the continuous exposure to light may be injurious. When fixed preparations are counted the method is valid only if the average interval of duration of mitosis is known and if the experi- 
mental conditions do not disturb the duration of mitosis. If such disturbance occurs it nust be compensated for, as must also variation in the proliferation rate with different periods in the diurnal cycle.

By time-lapse cinematography at low magnifications for a period of hours, it is possible to include 100 or more cells in the field, and at the same time to obtain detail adequate to recognize any cell undergoing division. It is consequently practical to determine the percentage of cells which undergo mitosis per unit interval of time with a high degree of accuracy. If at least two or more optical systems are available a control culture and one or more under experimental treatment may be simultaneously recorded. While the method has the disadvantage of requiring expensive equipment, such multiple optical system time-lapse cinematographs promise to be powerful instruments in the quantitative study and comparison of such phenomena as rates of cell proliferation, migration and necrosis in tissue culture preparations.

Where the cells can be grown from a cell suspension planted on the surface of a substrate such as cellophane or glass, the cells mav be treated with a special citric acid diluting solution and the cell nuclei may be enumerated in a hemocytometer (Sanford, K. K., Earle, W. R., Evans, V. J., Waltz, H' K. and Shannon, J. E., Jr., J. Nat. Cancer Inst., 1951, 11, 773). 'While as yet tested for only a few cell types, of which the L strain of mouse cells of single cell origin is the chief one, the method should be applicable to other cell types and should be tried. From the results so far obtained the method is slow, but is both practical and accurate for measuring any change in the total number of nuclei in the culture planted from the cell suspension. It has the advantage that it enumerates only apparently healthy, living nuclei, and it also allows distinguishing and differentially enumerating cells actually in mitosis if this is desired.

Measurement of growth by increase of weight of the culture is usually impractical with cultures of sizes and types now available. If the cells are within a plasma matrix they cannot be separated from it for weighing. Even when the cells are grown on cellophane or glass substrates, difficulties of getting the cells and substrate freed of media and of fluid without changing the weight of the cells makes weight determinations difficult. Determinations of dry weight are similarly complicated.
While optical methods for determining the "growth" of a culture have been tried, optical quality of cultures and of culture media may vary so greatly due to other changes (e.g., clouding or precipitation of the culture media, cell granulation, accumulation of $\mathrm{fat}$ or granules; occurrence of necrosis) that various methods suggested have not proven practical under the widely varied experimental conditions which may prevail.

Many chemical indices of "growth" have been suggested and tried. Some of these have been: rate of utilization of glucose, lactic acid production, change of $\mathrm{pH}$ of the medium, rates of aerobic and anaerobic glycolysis, oxygen consumption, increase in (Kjeldahl) nitrogen content of the culture, increase in desoxyribose nucleic acid or ribose nucleic acid, increase in organic phosphorus and in lipid-free organic phosphorus and the accumulation of radioactive isotopes of some metabolized substance. To a greater or lesser degree these are useful as indices of change of one or more compounds or groups of compounds by the cell or its enzyme systems. Such chemical indices of change are particularly valuable when a number of them can be simultaneously provided in a single study so that results may be correlated. In future work such chemical studies will probably become increasingly significant. However, under the wide range of experimental conditions which may be encountered in tissue culture studies and with our present limited knowledge, it is unwise to rely on any one of these chemical indices as an accurate, quantitative measure of the proliferation of tissue cells or nuclei.

Tissue Culture of Plants is also a fine art. Fortunately an excellent account is available in book form: White, P. R., A Handbook of Plant Tissue Culture. Lancaster: Jaques Cattell Press, 1943, $277 \mathrm{pp}$. The nutrient fluids used are chiefly composed of pure chenicals, blood plasma, embryo juice and so forth are lacking. The temperature of incubation ranges from about $30^{\circ} \mathrm{C}$. down to $5^{\circ} \mathrm{C}$. The tissues are easily killed by high temperatures. The special techniques required in physiology, pathology and morphogenesis are deseribed by White who also reviews the literature. The technique of tissuc culture has proved useful in researches on the disorderly growth of cells from Crown-galls (White, P. R, and Braun, A. C., Cancer Research, 1942, 2, 597$617)$. 
Tissue Eosinophiles. Demonstration is easy by the same techniques as for Eosinophile Leucocytes. In rabbits a marked increase of tissue eosinophiles can be produced in maxillary sinus mucosa by pilocarpinization. This attains a maximum in $5 \mathrm{~min}$. and disappears after $24 \mathrm{hrs}$. (Nemours, P. R., Arch. Otolaryng., 1933, 17, 38-42).

Tissue Fluid. All living cells of the body are aquatic. There is reason to think that the tissue fluids, which they inhabit, are not of uniform composition throughout the body but exhibit regional differences (Cowdry, E. V., Problems of Ageing, Baltimore: Williams \& Wilkins, 1942, 583-625). Except when present in large amounts, these tissue fluids cannot be collected for chemical analysis. Consequently microchemical means are important in determination of their nature. They are often described in the literature as intercellular ground substance. Many methods have been described by S. H. Bensley (Anat. Rec., $1934,60,93-109$ ) for the ground substance of Loose Connective Tissue. See Spreading Factors. A method for quantitative evaluation of tissue fluid lymph cellular ratios has been reported by Allen, L., Anat. Rec., 1945, 92, 279287. See also Cartilage and Bone.

Tissue phagocytes of the lungs (histocytes, histiocytes, etc.)-Written by C. C. Macklin, Dept. of Histological Research, The University of Western Ontario, London, Canada. November 28, 1951-These cells are of mesodermal origin and akin to the phagocytic cells of the general connective tissue. They are made conspicuous by the grains of carbon or other particulate matter which they ingest, which have escaped the phagocytic clearance mechanism of the pulmonary alveolar surfaces, and which have worked their way into the environments of the phagocytes. They are demonstrable by any good fixation and staining technique, and may be made outstanding by the Vital Staining method (which see). They are not to be confused with the Dust Cells (which see) which are of endodermal origin and do not enter the connective tissue.

Titan Yellow (CI, 813)-Erie fast yellow WB, thiazole yellow-An acid thiazole dye used in fluorescence microscopy. See method for Magnesium.

Titanium Dioxide. Huggins, C., Anat. Rec., $1939,74,231-253$ used this compound in a suspension as a vital stain for bone marrow because the amounts taken in by reticuloendothelial cells can be measured. He employed specially purified titanium chloride obtained from
Dr. J. L. Turner and the Titanium Pigment Corporation, 111 Broadway, New York. The method is to make a fine $5 \%$ suspension in $2 \%$ aq. gum acacia by mixing with an electrical mixer for $1 \mathrm{hr}$. After keeping this at $4^{\circ} \mathrm{C}$. for 2 days siphon off the supernatant fluid for use to avoid aggregates which settle to the bottom. Keep this likewise on ice but warm to body temperature before intravenous injection. Inject slowly into ear veins of rabbits, each animal to receive 3-6 injections of $10 \mathrm{cc}$. on consecutive days. The titanium dioxide particles can easily be recognized as a black accumulation in the phagocytes and its amount can be determined chemically in fairly large bone samples by a method detailed by the author.

Tocopherol, see Vitamin E.

Toisson Solution for diluting blood; aq. dest. $160 \mathrm{cc}$; neutral glycerin, $30 \mathrm{cc}$; sodium sulphate, $8 \mathrm{gm}$; ; sodium chloride, $1 \mathrm{gm}$.; methyl violet, $0.025 \mathrm{gm}$.

Toluene Red. Dimethyldiamidotoluphenazin. See Platelet staining solutions.

Toluidin Blue O (CI, 925) - methylene blue $\mathrm{T} 50$ or T extra-Employed very widely. Metachromatic staining with this dye is specific for certain mucoproteins. See Sylvén, B., Acta Radio., 1945, Suppl. 59, $100 \mathrm{pp}$.

Toluidine Blue Phloxinate. Instructions for preparation (Lillie, R. D., Stain Techn., 1941, 16, 1-6). Lillie now recommends Azure Toluidine blue.

Toluylene Blue (CI, 820). A basic indamin dye, homologue of Bindschelder's Green which see.

Toluylene Red, see Neutral Red.

Tolyl Blue 5 R (CI, 289), a disazo mordant dye of light fastness 3 preparation and use of which for plant and animal tissues is described (Emig, p. 37).

Tony Red, see Sudan III.

Torsion Balances, see Balances.

Torulosis, see Blastom ycosis.

Tourmaline, as a polarizer, see Bennett, H. S. in McClung's Microscopical Technique, 1950 , p. 614 .

Toxic Neutrophiles (see Neutrophiles, toxic).

Toxoplasma. These protozoa can be identified microscopically. They can be colored with Wright's or Giemsa's stain in impression preparations (sce Smears). To demonstrate them in sections use Giemsa's stain after Regaud's fixative, eosin-methylene blue after Zenker-acetic or hematoxylin and phloxin after formalin (Pinkerton, $\mathrm{H}$. and Weinman, D., Arch. Path., 1940, 30, 374; Sabin, A. B., Advances in Pediatrics, $1942,1,1)$. It is helpful in diagnosis to compare with standard 
preparations of Sarcocystis and Encephalitozoa.

Trachea. Excellent experimental methods to demonstrate secretion of Mucus are detailed by Florey, H., Carletion, H. M. and Wells, A. Q., Brit. J. Exper. Path., 1932, 13, 269-284. Techniques for Nerve Endings are given under this heading but it would be helpful to consult Larsell, O. and Dow, R. S., Am. J. Anat., 1933, 52, 125-146 who illustrates what one may expect to find. Techniques for Cilia require no special adaptation. Celloidin sections are smoother than paraffin ones.

Tracer Techniques, see Radioactive Isotopes.

Trachoma Bodies. These are easily colored by Giemsa's stain. For demonstration of glycogen in them and other pertinent data see Thygeson, P., Am. J. Path., $1938,14,455-462$.

Evolution forms of Rickettsia trachomatis. Fix smears in iodine alcohol, 4-5 min. Stain in May-Grünwald, 1 part; Giemsa, 1 part; neutral aq. dest. 10 parts for $1 \mathrm{hr}$. Differentiate in $95 \%$ alcohol (Foley, H. and Parrot, L., Arch. Inst. Pasteur d'Algérie, 1938, 16, 283292). See colored plates by the authors.

Transplantation. This technique provides opportunities for important microscopic studies. See Anterior Chamber of Eye, Chorioallantoic Membrane, Organ Culture, Tissue Culture, and Tooth Germs.

Transparent Chamber Technique-Written by Eliot R. Clark, Department of Anatomy, University of Pennsylvania, and Wistar Institute of Anatomy and Biology, Philadelphia, Pa. November 28, 1951-Several types of transparent chambers and windows have been developed, that have been inserted in the outer ears, and in other locations, of rabbits and other animals, with the aid of which it is possible to watch through the microscope-in some types of chambers with the oil immersion lensthe growth and behavior of cells and tissues within the living mammal. The stimulus for this study came from the desire to extend to the mammal the type of prolonged microscopic studies of cells and tissues within the living animal that had been carried out in the tails of living amphibian larvae (cf. F. R. Clark, Anat. Rec., 1909, 3, 183; Am. J. Anat., 1912, 13, 351, and 1918, 23, 37). The first chambers were developed by Sandison, J. C. (Anat. Rec., 1924, 28, 281; Am. J. Anat., 1928, 41, 447), at the instigation of E. R. Clark. His original attempts were with mica chambers, but his successful ones were constructed of celluloid (cellulose nitrate). The latter provided beautiful preparations, in which details of growth and behavior of blood vessels and of other tissues could be seen with highest microscopic magnifications. They remained in the ear a maximum of $4 \frac{1}{2}$ months.

After Sandison transferred from Anatomy to Surgery (1928) a group at the University of Pennsylvania undertook to develop, improve and modify the chamber, and succeeded in developing several types of chambers designed for attacking a variety of problems.

One of the most useful types, which is a modification of Sandison's chamber, is a type named the "round-table" chamber (E. R. Clark, Kirby-Smith, Rex, and R. G. Williams, Anat. Rec., $1930,47,187)$. The "round-table"; chamber, as described in 1930, has been modified as follows: the washers, both celluloid and rubber, have been found unnecessary in "round-table" chambers, though essential in "preformedtissue" chambers, and in "round-table" chambers installed in dogs' ears; the transplant hole in the base of "roundtable" chambers has been omitted, since the Williams removable-top chamber has proven a more satisfactory transplant type; the protective discs have been replaced by splints and shields unattached to the chamber; the celluloid rings of both base and top have been stiffened by gluing on an extra $0.66 \mathrm{~mm}$. thick celluloid, over their medial two-thirds; the edges of the table have been bevelled outward, from above down. This type is especially useful for observation of the growth and behavior of blood vessels, lymphatic vessels, connective tissue, nerves, bone, cartilage, epidermis and blood cells. To date it has been the most used and most imitated of all the chamber types. Published results have appeared largely in the Anatomical Record and the American Journal of Anatomy, from 1930 to the present. In nearly all of them, Eleanor Linton Clark has been joint author.

Construction, installation and methods of study are briefly as follows. The chamber consists of two parts, a base and a top. The base is made of celluloid, $1.8 \mathrm{~mm}$. thick, $3.2 \mathrm{~cm}$. in diameter, at the center of which is a raised round table, $1.3 \mathrm{~mm}$. high, 6.3 $\mathrm{mm}$. in diameter. The top consists of a celluloid ring, also $1.8 \mathrm{~mm}$. thick and $3.2 \mathrm{~cm}$. in diameter, having a central hole $1.4 \mathrm{~cm}$. across. To the ring is glued a mica dise, 75 micra thick using Varian's glue: gum copal, venice turpentine and xylol (Science, 1931, 73, 678 ). Three holes are bored at points equidistant from each other, near the 
outer rim of both the base and the top ring, large enough to take bolts $0.040^{\prime \prime}$ in diameter. The chamber fits the ear best if trimmed to a pear shape. The edges of both base and top are rounded. To the table, close to its outer rim, are glued 4 "buffers" (or separators)squares or octagons of celluloid, $\frac{1}{2} \mathrm{~mm}$. wide and either 42 or 75 micra thick, depending upon the thickness of growing space desired. The purpose of the "buffers" is to leave a non-compressible open space, into which tissues may grow when the top of the chamber is bolted to the base. The bolts are of brass, and may be either headed ( $1 \mathrm{~cm}$. long) or headless $(1.5 \mathrm{~cm}$. long). They are made from brass wire $0.040^{\prime \prime}(1.0 \mathrm{~mm}$.) in diameter, threaded 120 turns to the inch (47 to the $\mathrm{cm}$.). The brass nuts are hexagonal. The central $3 \mathrm{~mm}$. of the bolts is coated with celluloid by dipping in celluloid dissolved in equal parts of absolute alcohol and ether and removing the celluloid from the end sections.

Rabbits used for installation of ear chambers should have ears at least 5 inches long, should be free from "snuffles" and from "ear-mite, and should have no scars in the chamber region. The chamber is inserted in the ear at a place about two-thirds of the distance from base to tip, in the flat portion below the central artery and nerve, with the round-table close to the central artery. With a nearly-sharp, heartshaped dissector, the skin is raised on both sides of the ear, over an area about two $\mathrm{mm}$. beyond the limits of the chamber-care being taken to remove all the dermis, but to leave the vascular subcutaneous layer behind. The central portion of the raised skin is removed to a distance about $4 \mathrm{~mm}$. from the outer edge. A round hole is cut clear through the ear, slightly larger than the top of the table, and the base of the table is placed in position with the table projecting through the hole. The three bolts are forced through the ear following a needle and the end of a probe, the table top is brought down over the three bolts, and the loosened skin is lifted up over the edges of the table on both surfaces of the ear. The nuts are screwed down on the bolts, until the three buffers are clearly seen, but not enough to cause the mica to bulge. The splints and shields (Clark, E. R. and E. I. Clark, Anat. Rec., $1932,51,55$ ) - the former a flat ring of $.66 \mathrm{~mm}$. thick celluloid, the latter a pie-plate shaped piece of $0.24 \mathrm{~mm}$. thick celluloid, shaped by heating in a mould-are installed as follows. Two holes are made in the ear with a leather punch. Through each hole is passed a headless bolt outside of which is a glass sleeve about $4 \mathrm{~mm}$. long. This sleeve protects the ear from the bolt. The hole in the outer splint is large enough so that the splint clears the chamber. The inner splint is trimmed down to a half ring, to fit the ear fold, and the edge rounded. Bits of sterile gauze are pushed into the space around the sleeves. The operations are performed under strict asepsis-aqueous solution of metaphen, 1:500, being the disinfectant of choice, both for preparing the ear and for sterilizing the chambers. Local anesthesia is used-injections of novocaine, $2 \%$, being made across the base of the ear.

Microscopic observations are made with the rabbit lying on its back, held by a special rabbit board (E. R. Clark, Sandison and Hou, Anat. Rec., 1931, $50,169)$, while the ear is clamped to a wooden or bakelite holder, which is held and moved about by the mechanical stage. Camera ludida drawings are made using the Leitz "Zeichenocular *4", which allows the microscope to be tilted at an angle of 45 degrees. The microscope rests on a sheet of heavy plate-glass which is raised at the sides sufficiently to permit a drawing board to move underneath. Illumination is provided by a 6-volt concentrated or ribbon filament lamp, the light passing through water filter or heat-resisting glass. Photographs of whole chamber are made with a bellows camera, using 16 or $32 \mathrm{~mm}$. lenses; photomicrographs with the Leitz "Leica," or similar type camera, that concentrate the light, permitting oil immersion pictures of $x^{\prime \prime}{ }^{\prime \prime}$ to $\frac{1}{25}$ " exposure; and motion pictures including "time-lapse" motion pictures. While oil immersion studies may be made, a most useful lens is the $8 \mathrm{~mm}$., $20 x$ apochromatic objective long workingdistance type with small-tipped nosepiece), since excellent resolution is obtained with even a $25 \times$ compensating ocular. Since the object is about $1 \mathrm{~cm}$. above the top of the microscope stage, illumination is improved by either removing the top lens of the condensor, or by using a supplementary lens placed between the regular condensor and the mirror - as designed by Dr. Poser, for Bausch and Lomb, which sends a beam of parallel rays, instead of rays converging at the thickness of a glass slide and then diverging.

A second type of chamber has been named the "preformed tissue" chamber (E. R. Clark, Kirby-Smith, Rex and 
Williams, Anat. Rec., 1930, 47, 187). It makes possible a microscopic study of the original tissues of the ear, with their original nerve, blood vessel and lymphatic vessel supply.

A third type, termed the "moat chamber", developed by Abell and Clark (Anat. Ree., 1932, 53, 121; and Abell, Anat. Rec., 1937, 69, 14) contains a small space, or "moat", accessible to the outside, in which may be placed any fluid substance, in order to observe, through the micrscope, the effect upon the tissues. The fluid may be withdrawn later, for chemical analysis. This chamber, in Abell's hands, has been used for studies on the absorption of methylene blue, reactions to a phosphate buffer, rate of absorption of urea, rate of diffusion from blood vessels of nitrogenous substances, reaction of blood vessels to foreign protein in a sensitized animal (Abel and Schenck), and behavior of vessels in hypertension (Abel and Page).

A fourth type, named the "removable top" chamber has been developed by R. G. Williams, for the purpose, originally, of obtaining easy access to the living tissue of the chamber, in order that transplants of organs or tissues, or implantation of granular substances, might be made, without seriously disturbing the delicate tissues. It contains a device by which the top cover may be temporarily lifted off merely by unscrewing two or three nuts. This chamber has undergone steady improvement. The original model (R. G. Williams, J. Morph., 1939, 65, 17) was a modifieation of the "moat" chamberconstructed of glass and mica. This was improved (R. G. Williams, Anat. Rec., 1948, 101, 291) by the substitution, for glass, of tantalum - the metal which is practically inert in contact with living tissues-and the replacement of a major part of the relatively large, flat surface that comes in contact with living tissue, by a tantalum gauze, which becomes virtually a part of the ear. Finally Williams and Roberts (Anat. Rec., 1950, 107, 359) have introduced a fifth type of chamber, a radically modified tantalum and mica chamber, which still further reduces the flat surfaces next living tissue, has a Ionger life than any other type of chamber (although many "round-table" and "moat" chambers have survived well over a year), is so little irritating to the ear that outer splints and shields have been found unnecessary, can be installed in much less time than is required for other chambers, gives beautiful pictures of blood capillary growth and behavior, has a removable top for transplanting, could be modified to study preformed tissue, and has been adapted for the introduction or removal of fluids, for which the "moat" chamber was devised. Epidermis, which forms an annoying temporary invader of "round-table" chambers in about one-fourtl of installed chambers, never invades the latest tantalum chambers. On the other hand, up to the present, neither nerves nor lymphaties, which have been studied in "round-table" chambers, have been seen in the new type chambers, although these tissues could probably be brought in by slight modifications of the chamber. Successful autogenous transplants have been made by Williams in one or the other variety of his ingenious chambers, of thyroid, adrenal cortex, spleen, lymph node and testis, many of which have survived and have been available for microscopic study for months, and even for years (ef. Am. J. Anat,, vols. 62, 77, 81, and Anat. Ree., vols. 73, 104, 179).

In other laboratories, Ebert, Florey and Pullinger (J. Path. and Bact., $1939,48,379$ ) have described a "loundtable" chamber, modified chicfly by the substitution of another plastic, "perspex", for celluloid, and the use of a vertical instead of an oblique access hole in the base. They give an excellent description of the entire method of construction and installation of the chambers. Ebert, Ahern and Block (Science, 1949, 110, No. 2868, p. 665) deseribe further modifications, using the same or a similar plastic, namely "plexiglas" (aerylic and methacrylate resin), which include a reduction in outside diameter from 32 to $25 \mathrm{~mm}$. (a dimension also used by Essex); replacement of "buffers" by remote supports (as in Sandison's chambers); replacement of metal bolts and nuts by plastic rods; elimination of splints and shields; and a quadruple punch, guided by a double, transparent template, which is very helpful in installing chambers. Essex, H. E. Methods in Medical Research, Year Book Publishers, 1918, 1, 139) gives again a complete deseription of the construction and installation of a chamber made of "lucite" (methyl methacrylate resin), modelled after the Ebert, Florey, Pullinger style of "round-table" chanber. He studies the ear with the rabbit in its normal position, rather than on its back.

On account of the greater case of construction, greater clearness and freedom from warping, we have tried lucite in our laboratory, but it has been 
found to incite what seemed to us to be definite abnormal or pathological reactions in the living tissue, in the form of excessive extravasations of red and emigrations of white blood cells in the early stages, and later, of large abnormal accumulations of cells next the lucite. Consequently, for "roundtable" chambers we have preferred celluloid, to which the tissues do not appear to react unfavorably. However, beautiful preparations may be obtained with chambers made of methacrylate resin plastics.

Moore, R. L. (Anat. Rec., 1936, 64, 387) (in E. R. Clark's laboratory), has adapted the "round-table" chamber to the ear of the dog. The chamber used was similar in construction to the one used in the rabbit, except that the table was about $2.00 \mathrm{~mm}$., instead of $1.3 \mathrm{~mm}$. high. He also found that, in order to obtain chambers in which the ingrowths from the periphery filled the table area completely, it was necessary to have the two parts of the chamber held more rigidly together than in the rabbits' chambers. This was accomplished by using, in addition to the buffers, the celluloid and rubber washers as advocated in the original "round-table" description (Clark et al. Anat. Rec., $1930,47,187$ ). Of 4 "round-table" chambers installed in ears of dogs, 2 without washers failed to fill the table area, while in both chambers provided with washers, the growth was complete. Quiet, large-eared beagle (or rabbit) hounds were used. Moore also successfully installed a "preformed-tissue" chamber in the dog's ear.

Williams, R. G. (Anat. Rec., 1934, 60,493 ) has installed "round-table", chambers in skin flaps, made in the lateral body-wall of rabbits, and has pointed out that, by the use of the "preformed-tissue" type of chamber, striated muscle (the panniculus carnosus) could be brought under microscopic observation there.

Algire, G. H. (J. Nat'l. Cancer Inst., 1943,4 , p. 1), at the National Cancer Institute, Bethesda, Maryland, has developed methods for the installation and microscopic study of double-walled, transparent chambers in the dorsal skin of mice. The chambers are an adaptation of the "preformed-tissue" chambers of Clark et al. (1930). Recently Algire and Legallais (J. Nat'l. Cancer Inst., $1949,4,225$ ) have described a "round-table" modification, for the study of new forming tissue, and also a method of obtaining access to the chambers. The maximum time of survival of Algire's chambers has been two months. Several cancer studies have been published from Algire's laboratory.

Other parts of the mammal have been brought under long-continued (weeks or months) microscopic observation by the extension of the chamber or window methods. Zintel, H. A. (Anat. Rec., 1936, 66, 437) studied, in rabbits, a loop of small intestine with its attached mesentery, drawn outside the body into a semicircular celluloid container, and maintained there for several weeks. Microscopic studies with transmitted light were possible on the mesentery. Similar chambers have been used on dogs (Abell, R. G. and I. H. Page, Surg., Gyn., and Obst., 1943, 77, 348). Wentsler, N. E. (Anat. Rec., $1936,66,423$ ) has successfully installed single-paned windows, constructed of celluloid, in the skulls of rabbits, through which it was possible to watch, through the microscope, the pial circulation, over a period of 8 months. In Montevideo, Estabile, C. (Proc. Am. Sci. Cong. [of 1940], 1942, 6, 147) has used a window similar to the Wentsler cranial window, to observe the contractions of the musculature of the right atrium, with transmitted light provided by an electric light bulb passed through the internal jugular vein into the atrium. In a personal communication Estabile described to the author the use of the same window to observe the stomach wall in action, with light furnished by a light bulb inside the stomach.

Transparent chambers and windows have provided new methods for microscopic observation of, and for experiment upon cells, tissues and organs in the living mammal. They are of value, in both research and teaching to the physiologist, pathologist, pharmacologist, bacteriologist and surgeon, but especially to the living anatomist, whose field is fundamental to, and in a way encompasses all the others.

Transparent Chamber Techniques-Written by Glenn H. Algire, U. S. Public Health Service, Bethesda, Md. June 15 , 1950-Transparent-chamber techniques seek to make attainable a dynamic, functional approach to problems of histophysiology and cellular biology through microscopic observation of tissues and cells in unanesthetized animals over periods of from several weeks to many months. This technique was first reported by Sandison, J. C. (Anat. Rec. 1924, 28, 281-287; $1928,41,447-474)$, working under Dr. E. L. Clark, in whose laboratory the rabbit ear chambers were later on 
greatly improved (Clark, E. R., et. al., Anat. Rec., 1930, 47, 187-211).

The cartilage of the rabbit ear serves as a natural support for chambers which were constructed first of celluloid and mica and later on of glass and mica, or methyl methacrylate resin (lucite, perspex). An area of skin the size of the chamber is dissected away from the cartilage on both sides of the ear, leaving the central artery intact. The chamber units are inserted close to the main artery, usually with the observation area at the inner surface of the ear, and the skin is drawn over the chamber edges and trimmed to expose the observation area. The chamber is held in position by the use of bolts or pegs and by fitting the cartilage into a space between the upper and lower pieces. Protective covers of celluloid or vinylite are used to prevent injury.

Various types of chambers have been designed for special purposes. The preformed-tissue design used in studies of the original tissues of the ear is restricted to low magnification observations of blood vessels within a relatively thick layer of skin. Introduction of the preformed-tissue chamber into the rabbit ear arouses cellular and circulatory disturbances which require 10-14 days to subside before satisfactory studies can be made.

Greatly improved resolution of cells and tissues at high magnifications was achieved by the round-table designs (Clark, E. R., et al., cited above), in which a central hole is punched through the cartilage and a narrow space of from 40-75 micra thickness is provided for the ingrowth of newly formed blood vessels and connective tissue from the edges of the cartilage. The thickness of the space is controlled by spacers placed between the central round table and the coverslip. Clark, E. R. and Clark, E. L., have applied these techniques to detailed descriptive accounts of the growth, differentiation, and behavior of blood vessels, growth of lymphatics and nerves, development of fat tissues, migration of leukocytes and macrophages, and inflammatory reactions.

Abell and Clark, in devising the moat chamber (Anat. Rec., 1932, 53, 121-140) modified the round-table design to include a well, provided with silver cannulae, for the introduction of chemical solutions into the chamber in contact with the living tissue. This design was used in studies of diffusion of dyes and of effects of chemical agents on tissues. The round-table designs required 3 to 4 weeks for complete vas- cularization of a table area having a diameter of approximately $7 \mathrm{~mm}$.

In the removable top chamber, Williams improved the method for the introduction of cell transplants. These are implanted scveral weeks after insertion of the chamber, when vascularization of the round table is complete. Recently Williams has succeeded in prolonging the duration of the transplant chamber through the use of tantalum gauze (Williams, R. G., Anat. Rec., 1948, 101, 291-298). Growth of blood vessels and connective tissue through the meshes serves to reinforce the attachments between tissue and chamber. The useful duration of some of the tantalum chambers has been approximately 3 years (personal communication).

Ebert, Florey, and Pullinger (Ebert, R. H., Florey, H. W. and Pullinger, B. D., J. Path. and Bact., 1939, 48, 79-94), introduced the use of methyl methacrylate resin (lucite, perspex) in the construction of the chamber and modified an earlier design of the transplant chamber by Clark (cited above), to give access through a removable plug introduced into the central table. This design has been used in studies of tissue reactions in tuberculous infection (Ebert, R. H., Ahern, J. J. and Bloch, R. G. (Proc. Soc. Exp. Biol. Med., 1948, 68, 625-633). Further modifications of this design were published recently which simplify the procedures (Ahern, J. J., Barclay, W. R. and Ebert, R. H., Science, 1949, 110,665).

Essex has made use of a lucite chamber in studies of peripheral nerve injury and repair (Essex, H. E. and de Rezende, N., Am. J. Physiol., 1943, $140,107-114)$, and in studies of leukocytes in leukopenia (Essex, H. E. and Grana, A., Am. J. Physiol., 1949, 159, 396-400). Construction details of this chamber have been given (Essex, $H$. E. in Methods in Medical Research, Year Book Publishers, 1948 1, 139-145).

Chambers have been described for other sites, organs, and species. Permanent cranial windows have been designed, (Wentsler, N. E., Anat. Rec., $1936,66,423-435)$, in which a small glass window set into a celluloid frame is secured to the skull with small silver pegs. A disadvantage of this type of window, as of the lucite disks made by Sohler, Lothrop, and Forbes (J. Pharmacol., $1941,71,325-330$ ), is its small size, having a diameter of $12-16 \mathrm{~mm}$.

Sheldon et al. (Sheldon, C. H. Pudenz, R. H., Restarski, J. S. and Craig, W. Mck., J. Neuro-physiol., $1944,1,67-75)$ describe a technique for 
the preparation of a lucite calvarium, in which the convex portion of the skull of a monkey is permanently replaced by a transparent lucite plate, thus exposing the surface of both cerebral hemispheres. These preparations have been used in studies of superficial cerebral vessels in head injury, drug administration, and oxygen poisoning.

Several attempts have been made to install permanent type chambers for the study of internal organs. Zintel (Anat. Rec., 1936, 66, 437-447) introduced one of the earliest of these for exteriorizing a loop of intestine and its mesentery. A chamber for the pancreas of the mouse has been described (Flory, C. M. and Thal, A., Anat. Rec., 1947, 97, 33-40). Estable (Proc. Soc. Exp. Biol. and Med., 1948, 67, 445-447) has described a technique for biomicroscopic study of the ovary and the Fallopian tube in rabbits. The ovary is transferred to a subcutaneous space, retaining intact its vascular and nerve supply. A transparent capsule is applied for protection during repeated microscopical examination.

Adaptation of the transparent chamber technique to mice (Algire, G. H., J. Nat. Cancer Inst., 1943, 4, 1-11); Algire, G. H. and Legallais, F. Y., J. Nat. Cancer Inst., 1949, 10, 225-243) makes the procedure especially useful in the many phases of cancer research involving inbred strains of mice. In this modification tantalum sutures are used to support a dorsal fold of two skin layers between two plastic (vinylite) splints. One skin layer is incised and retracted to allow the introduction of a lucite ring and attached coverslip which is in contact with the inner surface of the second layer of skin. Both the lucite ring and the supporting splints are held in position by tantalum bolts. This procedure makes accessible to study by transmitted light a layer of tissue approximately 500 micra thick, consisting of peripheral nerves, striated muscle, peripheral blood vessels and lymphatics, subcutaneous connective tissue and fat, hair follicles, and epidermis.

Implantation of normal and neoplastic cells, of embryonic organs, or of carcinogenic chemicals is readily performed during the operation for insertion of the chamber. Experimental studies of the tissue may be undertaken immediately after the operation, and carried on for from 30 to 60 days. Response of the host and the implanted tissues to physical and chemical agents may be studied in terms of cellular and circulatory reactions, including quantitative measurements of blood pressure, dye diffusion, and arterial oxygen saturation. Microscopic resolution is sufficiently good to observe cross-striations in muscle, platelets in circulating blood, and cytoplasmic detail in cells adhering to the coverslip. Additional resolution of cytologic detail has been obtained through use of a round-table design. The access-type consists of a thick (1 $\mathrm{mm}$.) coverslip of lucite through which a $1 \mathrm{~mm}$. diameter hole provides for local application of chemicals at any time after introduction of the chamber. The operative procedure requires less than one hour and two workers can readily handle 10 to 15 animals in daily observations, measurements, and photographic records.

The dynamic, functional aspects of the transparent-chamber approach indicates future increased development and correlations with other methods of biological research.

Trematodes. Make up stain by mixing $1 \mathrm{gm}$. of dried residue on filter paper from Schneider's aceto-carmine with $10 \mathrm{gm}$. ammonia alum in 200 cc. aq. dest. with aid of heat. When dissolved, cool, filter and to filtrate add crystal of thymol. After fixation bring worms to water or to $20 \%$ alcohol. Stain 12-36 hrs. depending on size. Remove to water 2 changes. Dehydrate through 20,35 , and 50 to $70 \%$ alcohol. Place few crystals potassium chlorate in small glass covered dish; add few drops conc. $\mathrm{HCl}$. When chlorine is given off fill dish with $70 \%$ alcohol. If deeply stained differentiate in this chlorinated alcohol. If not or the specimens are small ones add it to the alcohol covering them and agitate. When sufficiently destained remove to fresh $80 \%$ alcohol. Dehydrate in alcohol. Add cedar wood oil to the absolute until mixture is one half oil. Clear in cedar oil and mount in balsam (Gower, W. Carl, Stain Techn., 1939, 14, 31-32).

Treponema Pallidum. The organisms can best be seen in the primary lesions by Darkfield examination. The same method is useful for skin and lymph nodes in the secondary stage but for the tertiary lesions in deep lying tissues sections are desirable supplemented by smears. A negative finding is comforting but does not necessarily signify absence of parasites unless confirmed serologically.

1. Low surface tension stain for smears (Haire, R. D., J. Lab. \& Clin. Med., 1938, 23, 1215-1216). Mix $1 \mathrm{gm}$. Gentian violet (or crystal violet) in 
mortar slowly adding $100 \mathrm{cc}$. hexylresorcinol. Filter and store filtrate in stock bottlc. Stain smears $30 \mathrm{~min}$. Wash in water, dry and examine. Stain on slide must not be heated. Treponemas, light purple.

2. Wright's stain for smears (Mallory, p. 259). To make stain add 1 cc. Wright's stain and 1 cc. $1 \%$ aq. potassium carbonate to $10 \mathrm{cc}$. aq. dest. in test tube and heat to boiling. Spread material thinly on cover glass (not slide) and hold level with forceps. Cover with hot stain 3-4 min. After fluid has turned violet, and a yellow metallic scum has formed over it, pour off and repeat process twice with hot stain. Wash in water, dry and mount in balsam. Treponemas, intenscly violet.

3. Giemsa's stain for smears (Giemsa, G., Deut. med. Wochn., 1909, 35, 17511752) after Mallory (p. 290). Fix smears for $15 \mathrm{~min}$. in absolute alcohol or pass them through flame thrice. Pour on freshly diluted stain (1 cc. aq. dest. + 1 drop stock Giemsa). Steam gently and leave 15 sec. Decant and add immediately fresh diluted stain, warm and let cool 15 sec. Repeat 4 times leaving $1 \mathrm{~min}$. last time. Rinse quickly in running water. Blot. Mount in balsam. Treponemas, dark red.

4. Fontana-Tribondeau silver method for serum (Fontana, A., Dermat.Zeits., 1925-26, 46, 291-293) after Mallory (p. 291). To make silver solution add ammonia water (diluted 1:20) drop by drop to $50-100$ cc. $1 \%$ aq. silver nitrate until a coffee colored clouding takes place. Air dry thin smears of serum. Pour on few drops Ruge's sol. (aq. dest., $100 \mathrm{cc}$; glacial acetic, $1 \mathrm{cc}$; formalin, 2 cc.) and change several times during $1 \mathrm{~min}$. Rinse in running water. Mordant with a little aq. dest., 100 cc.; tannic acid, $5 \mathrm{gm}$.; liquid carbolic acid, $1 \mathrm{cc}$. for 20 sec. warming to steaming. Rinse in aq. dest. Treat with silver solution 30 sec. heating slightly. Wash in tap water. Dry in air. Mount in balsam. Treponemas, brown to deep black.

5. Burri's India Ink method for lesion fluid (Mallory, p. 291). Make 1:4 suspension of India in $\mathrm{k}$ in aq. dest. Sterilize in autoclave, $15 \mathrm{~min}$. Mix this in equal parts with fluid from lesion on slide with platinum loop. Spread thinly. Dry and examine. Treponema (and bacteria if present), white in brown to black background.

6. Quick method for demonstration in fresh autopsy tissues. This is Krajian's modification of Dieterle's method (Am. J. Syphilis, 1933, 17, 127) as amplified in Stain Techn., 1935, 10,
68. Fix tissue $5 \mathrm{~mm}$, thick $10 \mathrm{~min}$. in $10 \%$ formalin, $70^{\circ} \mathrm{C}$. Cut frozen sections 5-7 microns. Place in $2 \%$ aq. sodium cobalti nitrite $5 \mathrm{~min}$. Wash 2 changes aq. dest. Mordant for $15 \mathrm{~min}$. at $70^{\circ} \mathrm{C}$. in uranium nitrate $1 \mathrm{gm}$; $85 \%$ formic acid, 3 cc.; glycerin, 5 cc.; acctone, 10 cc.; $95 \%$ alcohol, 10 cc. Wash quickly in aq. dest. Develop 5 min. in $10 \mathrm{cc}$. of following mixture + 1 drop albumin-glycerin before use (hydroquinone, $0.62 \mathrm{gm}$.; sodium sulfite, 0.12 gm.; acetone, 5 cc.; $40 \%$ neutral formaldehyde, 5 cc.; pyricline, 5 cc.; sat. gum mastic in $95 \%$ alcohol, 5 cc., aq. dest., 30 cc.). Wash few sec. aq. dest. Then warm silver solution 15-25 sec. and wash in aq. dest. Keep all solutions in cool place. (Original gives treatment with $0.75 \%$ aq. silver nitratc at $70^{\circ} \mathrm{C}$. for $1 \mathrm{hr}$. upon the development in hydroquinone mixture.)

7. Levaditi's block silver method (Mallory, p. 293). Fix tissue pieces ( $1 \mathrm{~mm}$. thick) in $10 \%$ formalin, $24 \mathrm{hrs}$. Rinse in aq. dest. 95\% alcohol, $24 \mathrm{hrs}$. Transfer to aq. dest. and leave until tissue sinks to bottom. Fresh 1.5-3\% aq. silver nitrate at $37^{\circ} \mathrm{C}$. in dark $3-5$ days changing 3 times. (The stronger silver is advised for tissues excised during life.) Wash in aq. dest. Reduce 24-72 hrs. in dark at room temperature in: aq. dest., 100 cc.; formalin, 5 cc.; pyrogallic acid, $2-4$ gms. Wash in aq. dest. Dehydrate in 80,95 and absolute alcohol. Clear in oil of cedar wood, imbed in paraffin, mount $5 \mu \mathrm{sec}$ tions on slides, remove paraffin and mount in balsam. Treponemas, black.

8. Heitzman's modification of the Warthin-Starry and Nieto's methods as given by Mallory (p. 293). Cut frozen sections $15 \mu$ or less of $10 \%$ formalin fixed tissue. Place directly in pyridine, $10 \mathrm{~min}$. Wash in aq. dest., 3 changes. $1 \%$ aq. uranium nitrate at $37^{\circ} \mathrm{C} ., 15$ min. Wash quickly in aq. dest., 2 changes. $0.25 \%$ aq. silver nitrate at $56^{\circ} \mathrm{C}$., $15-30$ min. Develop until dark brown in following mixture made immediately beforehand by pipctting into a beaker: (1) 15 cc. $5 \%$ aq. gelatin at $56^{\circ} \mathrm{C}$; ; (2) 3 cc. $2 \%$ aq. silver nitrate; (3) 0.5 cc. $1 \%$ aq. hydroquinone. Remove and thoroughly wash in warm aq. dest. Dehydrate on slide adding by pipette increasing alcohols to absolute. Clear in benzol and mount in balsam. A heavy black ppt. indicates too long development. Treponenmas, black. See Warthin-Starry method.

9. For routine paraffin sections, Steiner, G., J. Iab. \& Clin. Med., 1939, 25, 204-210. Fix in $10 \%$ formalin and make sections 9-10 microns. Remove 
paraffin with xylol. Pass through 2 changes abs. alc. Treat $1-1 \frac{1}{2} \mathrm{~min}$. in $4 \%$ uranium nitrate in abs. alc., 20 cc.; $25 \%$ gum mastic in abs. alc., $40-50$ cc.; abs. alc., 20-30 cc. Wash in at least 3 changes aq. dest. until streaks of gum mastic are removed. $0.1 \%$ aq. silver nitrate in water bath at $100^{\circ} \mathrm{C}$, , 1-1 $\frac{1}{2}$ hrs. Wash in aq. dest. Then through $80 \%$ and $95 \%$ to abs. alc. $10-12.5 \%$ gum mastic in abs. alc. $5 \mathrm{~min}$. Repeat 3 washings described in aq. dest. Reduce 20-30 min. in : hydroquinone, 10 gm.; $12.5 \%$ gum mastic in abs. alc., 1 cc.; aq. dest., 200 cc. (with temperature gradually raised to $100^{\circ} \mathrm{C}$.). Wash thoroughly in aq. dest. Counterstain with hematoxylin and eosin if desired. Dehydrate in abs. Clear in xylol and mount in balsam. The advantages are speed and decrease in confusing silver deposits. See Steiner's illustrations. A technician's experience with Steiner's method has been published (Wilson, R. A. J., Am. J. Clin. Path., 1946, 16, 21-24).

10. Nigrosine has been proposed as a negative stain for treponema (Dienst, R. B. and Sanderson, E. S., Am. J' Public Health, 1936, 26, 910). Comparison of dark field, nigrosine stain and Kahn test in diagnosis (Nagle, N., J. Lab. \& Clin. Med., 1939-40, 25, 660$661)$.

11. Ziehl's fuchsin stain (Perrin, T. G., Am. J. Clin. Path., 1943, Tech. Suppl., 7, 28). Make smears on slides of exudate secured by compressing base of chancre or by scraping surface of ulcer. Dry in air and fix by heat in flame, if desired. Stain $2 \mathrm{~min}$. while heating, or for $6 \mathrm{~min}$. at room temperature, being careful not to let the stain dry. The stain is aq. dest., $10 \mathrm{cc}$.; commercial formalin, 1 cc.; acetic acid, 1 cc.; 'Ziehl's fuchsin (Ziehl's CarbolFuchsin) 4 cc. Wash in water, moving gently, and dry in air. See Vincent's Angina.

Triacid Blood Stain, see Ehrlich's.

Tri-Amino Tri-Phenyl Methane Dyes. These are the rosanilins. Examples: acid fuchsin, acid violet, anilin blue WS, basic fuchsin, benzyl violet, crystal violet, ethyl green, ethyl violet, Hofmann's violet, iodine green, isamine blue, magenta II, methyl blue, methyl green, methyl violet, new fuchsin (magenta III), pararosanilin (magenta $\mathrm{O}$ ), rosanilin (magenta I), spirit blue, victoria blue $B$ and $R$ and victoria blue $4 R$.

Trichinella Spiralis. Mallory (p. 304) gives as a rapid method of diagnosis the squeezing of small picces of jaw muscle or of muscle near tendon of diaphragm between two slides and direct examina- tion at low magnification. A useful device for squeezing the muscle, called a "trichinoscope" has been constructed by Gould, S. E., Am. J. Clin. Path., Techn. Suppl., 1944, 8, 98-100. If trichinellae are calcified or encapsulated specimens can be cleared with acid. In permanent preparations of Zenker or formalin fixed material stained with hematoxylin and phloxine or eosin the parasites are best seen in longitudinal sections of muscle fibers. To demonstrate in migratory phase withdraw blood from vein in arm into syringe containing $3 \%$ aq. acetic acid, centrifuge and examine.

Rapid iodine-silver technique (Kalwaryjski, M. B. E., Wojsk. Przegl. Weteryn., 1938, 9, 123-136). Place thin slices of muscle for $10 \mathrm{~min}$. in iodine, potassium iodide, aq. dest. sol. in following proportions $2: 4: 100$ or $0.5: 1: 100$ or 0.1:0.2:100 Wash in aq. dest. Destain in $2.5 \%$ aq. sodium thiosulphate until muscle is clear. Wash in aq. dest. Equal parts $10 \%$ aq. silver nitrate and strong ammonia until iodine leaves parasites. Wash in aq. dest. Decolorize in $5 \%$ aq. sodium thiosulphate. Wash in aq. dest. and mount in glycerin. Parasites stained dark brown owing to conversion of iodine to silver iodide.

See investigation of larvae with radioactive phosphorus (McCoy, O. R., Downing, V. F. and Voorhis, S. N., J. Parasit., 1941, 27, 53-58).

Trichomonas Vaginalis, technique for, Van Someren, V. D., Vet. J., 1946, 102, 73 ; detailed by Wenrich, D. H. and Diller, W. F. on McClung's Microscopical Technique, 1950 , p. 465.

Trichloracetic Acid employed with mercuric chloride and acetic acid as a fixative (Heidenhain, Zeit. wiss. Mikr., 1909, 25,405 ) also used in 4 or $5 \%$ aq. sol. as decalcifying agent.

Trichlorethylene, as a solvent in histological technique in place of xylol (Oltman, R. E., Stain Techn., 1935, 10, 23-24).

Trichlorlactic Acid used as fixative followed by staining with resorcin fuchsin for cytoplasmic canalicular apparatus (Holmgren, E., Ergeb. d. Anat., 1901, 11, 274-329; Cowdry, E. V., Internat. Monatsschr. f. Anat. u. Physiol., 1912, $29,1-32$ ).

Trichosiderm name suggested for iron pigment from red hair (Flesch, P. and Rothman, S., J. Invest. Dermat., 1945, 6, 257-270).

Trichrome-Stains. There are many such stains. See Mallory's and Masson's. A rapid one is described by Pollak, O. J., Arch. Path., 1944, 37, 294. Com. position of stain: acid fuchsin, $0.5 \mathrm{gm}$.; 
ponceau $2 \mathrm{R}, 1.0 \mathrm{gm}$.; light green $\mathrm{S} \mathrm{F}$, yellowish, $0.45 \mathrm{gm}$.; orange $\mathrm{G}, 0.75 \mathrm{gm}$.; phosphotungstic acid C.P., $1.5 \mathrm{gm}$.; phosphomolybdic acid, C.P., $1.5 \mathrm{gm} . ;$ glacial acetic acid, 3.0 cc.; ethyl alc., $50 \%$ up to $300 \mathrm{cc}$. Add acetic to alcohol and put $50 \mathrm{cc}$. in each of 4 beakers. In first dissolve acid fuchsin and ponceau, in second light green, in third orange and phosphotungstic acid, and in fourth phosphomolybdic acid (the last named by slight warming). Mix and use balance of alcohol to wash out contents of beakers adding them to mixture. Stain keeps well; can be obtained from Will Corporation, Rochester, N. Y. See colored plate by the author.

Triethyl Phosphate in dehydration. Nelsen, O. E., Stain Tech., 1945, 20, 131-132. recommends the use of this compound $\left.\left(\mathrm{C}_{2} \mathrm{H}_{5}\right)_{3} \mathrm{PO}_{4}\right)$ in histological technique, as it displaces water in tissues readily without shrinkage or distortion. Since tissues may be transferred directly into it from water, the tedious alcohol displacement series in the paraffin technique is unnecessary. It is soluble in the alcohols, benzene, ether, chloroform and xylol. Nelsen reports excellent results with smears following the triethyl phosphate method. Following fixation and subsequent staining with Feulgen, the smears are first transferred to equal parts of water and triethyl phosphate, then to triethyl phosphate and finally into xylene before mounting. Fast green may be dissolved in it if counterstaining is desired.

Trimethylcarbinol, see Tertiary Butyl Alcohol.

Triphenyltetrazolium Chloride, A Valuable Reagent for Studies of Reducing Activity in Living Organisms-Written by Dr. Robertson Pratt, University of California College of Pharmacy, San Francisco. January 29, 1951-İn aqueous solutions, 2,3,5-triphenyltetrazolium chloride (TPTC) is colorless, diffusible, and readily transported through plant and animal tissues; but in the presence of living tissue or of some other reducing agents, an in. soluble red formazan is precipitated from solutions of TPTC. This is the basis for use of this compound in histophysiology to determine sites of dehydrogenase or other reducing activity in living organisms, including cultures of bacteria (Pratt and Dufrenoy, Stain Technol., 1948, 23, 137). The compound has practical applications also outside of the field of research (see below).

TPTC has been used successfully in concentrations ranging from 0.05 to $1.0 \%$ in distilled water or in buffers within the biologic range of $\mathrm{pH}$ values. The optimum concentration depends largely on the kind and location of tissue under investigation. Studying sites of reducing activity in stalks of sugar cane, where the compound had to be trans. ported through a considerable distance of vascular tissue and some adsorption undoubtedly occurred on the way, Dufrenoy and Pratt (Am. J. Bot., $1948,35,333$ ) found a $0.5 \%$ solution satisfactory for demonstrating that dehydrogenase activity was nost pronounced in the plasmodesmata and in lipidic parts of the cytoplasm.

In other investigations Pratt and Dufrenoy (Antibiotics, 1949, Philadelphia, Lippincott; J. Bact., 1949, 57, 9 found $0.1 \%$ solutions of TPTC ideal for studying the effects of penicillin and other antibiotics on the dehydrogenase systems of bacteria (Huddleson and Baltzer, Science, 1950, 112,651 ).

Reduction of colorless TPTC to the red, insoluble, formazan occurs over a wide range of $\mathrm{pH}$ values. Reducing sugars do not interfere with the reaction in the normal biologic range of hydrogen ion concentrations, since they do not reduce TPTC below $\mathrm{pH}$ 11.0 (Mattson et al., Science, 1947, $106,294)$. The apparent redox potential of 2,3,5-triphenyltetrazolium chloride is about -0.08 volt (Jerchel and Möhle, Ber. deutsch. chem. Ges., 1944, 77-B, 591). Thus the compound can act as an electron acceptor in reactions involving dehydrogenases of pyridine nucleotides (Jensen et al., Science, $1951,113,65)$.

Other tetrazolium compounds and several derivatives of $2,3,5$-triphenyltetrazolium chloride have been used in the same way as TPTC. One of the most useful of these is $4,4^{\prime}$-bis $(3,5$ diphenyl-2-tetrazolium)-biphenyl dichloride which is colorless in aqueous solution and upon reduction by living cells yields an insoluble, violet or mauve formazan. Other agents which may be used similarly are the 2,5 diphenyl-3( $p$-iodophenyl), the 2,3-di( $p$-iodophenyl)-5 phenyl, and the 2 - ( $p$-iodophenyl $)-3$ - ( $p$-nitrophenyl $)-5$ phenyltetrazolium compounds.

Applications of TPTC and similar compounds outside the fields of histophysiologic and bacteriologic research are in the practical testing of viability of seeds, grains and yeasts; in the dairy industry, for performing the Brucella ring test (Wood, Science, 1950, 112, $86)$; and in the field of antibioties, for rapid microbiologic assaying of preparations of penicillin and other similar agents. Triphenyltetrazolium chlo- 
ride and some of its derivatives may find application also in plant pathology as diagnostic agents or for detection of various bacterial or fungus infections in the tissues of plants (Atkinson et al., Science, 1950, 111, 355).

Stock solutions, prepared in a saturated solution of $\mathrm{NaHCO}_{3}$ are stable in the dark and may be kept for some time, although freshly prepared solutions are preferable. Solutions should be protected from direct sunlight or other strong radiations, especially of the shorter wave-lengths, (Gierlach and Krebs, Am. J. Roentgen. \& Radium Therap., 1949, 62, 559). Similarly TPTC solutions should be shielded from the radiations of radio-active substances (ibid).

Solutions of TPTC may be flooded over living tissues of plants or animals or over cultures of bacteria on solid or semi-solid media for a few minutes and then removed (by decanting or pipetting) whereupon the sites of active reduction are clearly defined by the red deposits of insoluble formazan which is not diffusible. Alternatively, the roots of intact plants or the bases of plant stems may be immersed in solutions of TPTC. The compound is readily absorbed and translocated upward. Subsequent longitudinal sectioning of the plant reveals the sites where the compound has been reduced to the formazan. Stained material can be dehydrated in alcohol and acetone and preserved for cyto-histologic examination. Bacterial cultures on agar or other suitable media can be dehydrated similarly and used for cytologic study.

In bacteriology, 'TPTC may be incorporated in either liquid or semisolid culture media. Relative reducing activity of different organisms under different conditions may be determined by noting relative intensities of color developed in different tubes. In agar media, TPTC has been used to distinguish variants of Salmonella typhimurium, of S. sonnei, of Escherichia coli, and of several species of Brucella on the basis of reducing activity as evidenced by the color of different colonies.

Triphenyltetrazolium chloride has proved of value in cancer studies also and has helped in elucidation of some problems in cancer physiology (Hölscher, Agnew. Chem., 1950, 62, 174).

The reaction involved in reduction of the water-soluble, diffusible, color less tetrazolium salts to the waterinsoluble, non-diffusible, colored formazans is as follows:

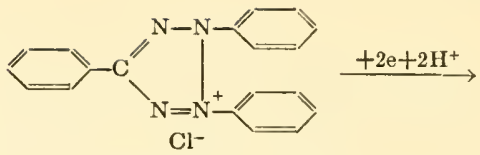

2,3,5-Triphenyltetrazolium Chloride

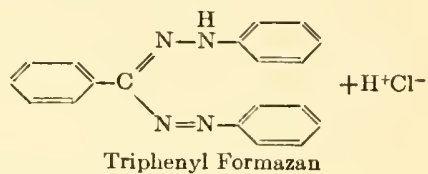

The details of the chemistry of tetrazolium salts and of their insoluble formazans have been reviewed by Benson (Chem. Rev., 1947, 41, 1).

Tropaeolin D, see Methyl Orange.

Tropaeolin G, see Metanil Yellow.

Tropaeolin G or OOO No. 1, sec Orange I. Tropaeolin OOO No. 2, see Orange II.

Trotter, see Hair.

Trypan Blue (CI, 477)-azidine blue $3 \mathrm{~B}$, benzamine blue $3 \mathrm{~B}$, benzo blue $3 \mathrm{~B}$, chlorazol blue $3 \mathrm{~B}$, Congo blue $3 \mathrm{~B}$, dianil blue $\mathrm{H} 3 \mathrm{G}$, naphthamine blue $3 \mathrm{BX}$, Niagara blue $3 \mathrm{~B}-\mathrm{This}$ acid disazo dye is the most popular of all Vital Stains. See also trypan blue capillary permeability test (e Silva, M. R., and Dragstedt, C. A., J. Pharmac. and Exper. Therap., 1941, 73, 405-411).

Trypan Red (CI, 438). So named because of influence on Trypanosome infections (G. trypanon, anger + soma, body). An acid dis-azo dye much used as a vital stain but less satisfactory than trypan blue.

Trypanosomes. The following is based upon Craig's account. Before examining peripheral blood, or cerebrospinal fluid, for trypanosomes it is advisable to concentrate them by centrifugation. They can be well seen in the darkfield. Smears of blood should be made a little thicker than for malaria plasmodia and after being air dried should be stained immediately. The methods of Giemsa and Wright are preferred giving a little more time for the stains to work. For details of structure use iron hematoxylin after Schaudinn's fluid (Craig p. 49). The South American trypanosome, $T$. cruzi, is more easily cultured than either of the African forms, $T$. gambiense or T. rhodesiense. Kelser's medium, described fully by Craig, seems to be the best. See references supplied by $\operatorname{him}(p .199)$ to culture in chick embryoes.

Trypanosomes. Inclusion Bodies are produced in Trypanosomes in vivo by effective doses of antrycide, dimidium bromide and Suramin. Their formation can also be observed in response to 
vital stains under the microscope but in this case they are not permanent. The determination of the chemical nature of these inclusions by Ormerod, W. E., Brit. J. Pharm. and Chemotherap., 1951, 6, 334-341 is a fine example of the application of histochemical methods to protozoa. They contain ribonucleic acid and protein and resemble Volutin granules.

Trypanosomes. Media. Summarized from Q. M. Geiman (Simmons and Gentzkow, $658,661)$.

Brutsaert and Henrard's (A) $6.50 \mathrm{gm}$. $\mathrm{NaCl}$., $0.14 \mathrm{gm} . \mathrm{KCl}, 0.12 \mathrm{gm} . \mathrm{CaCl}_{2}+$ aq. dest. to make $1000 \mathrm{cc}$ (B) $8.0 \mathrm{gm}$. $\mathrm{NaCl}, 0.2 \mathrm{gm}$. $\mathrm{KCl}, 0.2 \mathrm{gm}$. $\mathrm{CaCl}_{2}, 0.1$ gm. $\mathrm{MgCl}_{2}, 0.05 \mathrm{gm}$. NaHP${ }_{2} \mathrm{O}_{4}, 1 \mathrm{gm}$. $\mathrm{NaHCO}_{3}, 1 \mathrm{gm}$. glucose + aq. dest. to make 1000 ec. Sterilize both by filtration and distribute in culture tubes 2 cc. $\mathrm{A}+2.5$ cc. B. Add 2 cc. citrated human blood ( $1 \%$ citrate) and incubate at $37^{\circ} \mathrm{C} .24 \mathrm{hrs}$. to prove sterility. Kieep in refrigerator useful up to 2 weeks. Into a syringe containing $1 \mathrm{cc}$. $1 \%$ aq. sodium polyanethol sulfonate draw up 5 cc. patient's blood. Distribute $0.5 \mathrm{cc}$. to each of 10 culture tubes, incubate $25-28^{\circ} \mathrm{C}$. Examine microscopically for trypanosomes after 10-20 days.

Kelser's. Dissolve $2.5 \mathrm{gm}$. Bactobeef (Difco) in 500 cc. aq. dest. on water bath $55^{\circ} \mathrm{C} ., 1 \mathrm{hr}$. Add $12.5 \mathrm{gm}$. Bacto peptone (Difco) and $3.5 \mathrm{gm}$. sodium chloride by placing flask in boiling water $5 \mathrm{~min}$. Clarify by filtering through cotton and make $\mathrm{pH} 7$ with IN sodium hydroxide. Determine volume and add $1 \%$ Bacto-agar. Dissolve and distribute $5 \mathrm{cc}$. per test tube or 10 cc. per small flask. Autoclave 12 lbs., $30 \mathrm{~min}$. Store for latter addition dextrose and blood or for immediate use add $5 \%$ of $1 \%$ aq. dextrose $(0.25 \mathrm{cc}$. per tube or 0.5 cc. per flask) and $5 \%$ fresh sterile defibrinated guinea pig blood. After thorough mixing slant with short slant or deep butt. Use sterile rubber corks to prevent evaporation. Prove sterility by incubation. Inoculate by adding organisms to slant or water of condensation. On incubation at room temperature $\left(22-25^{\circ} \mathrm{C}\right.$.) growth becomes apparent in approximately 1 week. Subculture at 6-8 week intervals.

Try psin, a gelatin plate method as described under Pepsin but slightly modified is recommended.

Tryptagar, see Bacteria Media.

Tryptophane Reaction. 'The procedure of Serra and Lopes is specified as follows by Serra, J. A., Stain Techn., 1946, 21,
5-18: Prepare tissue as described under Ninhydrin Reaction.

" 1 . Harden the fixed pieces in 10\% formaldehyde for at least 1-5 hours (an unnecessary step if a fixative with formalin has been employed); then wash well.

"2. Immerse for 3-5 seconds in an aqueous solution of sodium silicatc $(d=1.1)$. When the materials arc sufficiently hardened this step may also be omitted; it is recommended, however, that the coloration should be tried both with and without it.

" 3 . Immediately afterwards, immerse the pieces in the Voisenet reagent for 10-15 minutes, in a small glass stoppered bottle. This reagent is composed of $10 \mathrm{ml}$. concentrated $\mathrm{HCl}$ to which is added, with a thorough stirring, one drop of $2 \%$ aqueous formol and one drop of $0.5 \%$ aqueous $\mathrm{NaNO}_{2}$. The reagent is prepared freshly every day and the nitrite solution must also be freshly made.

"4. Mount directly in glycerin and observe, with squeezing, if necessary. As the coloration fades, it is necessary to observe the preparations on the same day.

"The reaction is given by indolic compounds, and in proteins it is specific for tryptophane, which reacts even when bound. The localization of the reaction seems to be satisfactory and the sensitivity is sufficient for it to be used in cytophysiological work." See Romieu Reaction.

Tubercle Bacilli. Stain by Carbol Fuchsin, see Acid Fast Bacilli. Sce Concentration method for sputum. Fluorescence with auramine has been described (Hagemann, P. K. H., Münch. med. Woch., 1938, 85, 1066). Fix smears by flame and stain $15 \mathrm{~min}$. in $1: 1000 \mathrm{aq}$. auramine (Bayer) containing $5 \%$ phenolum liquefactum (liquid carbolic acid). Wash in tap water. Decolorize in ethanol $100 \mathrm{cc}$; $\mathrm{HCl}$ conc., $4 \mathrm{cc}$.; sodium chloride, $4 \mathrm{gm}$. renewing solution after $1 \frac{1}{2}$ min. Wash thoroughly in tap water. Examine without cover glass under fluorescence microscope using apochromatic dry objective and 3 compensating ocular ( $X$ about 180). For visible and red rays employ $3.5 \mathrm{~mm}$. "Uvet" lens and $2 \%$ aq. copper sulphate. Bacilli, golden yellow rods in violet fluorescent background. Kaiserling, C. Dcutsche Med. Wochenschr., 1939, 64, 1354, has described differences in fluorescence of human bovine tubercle bacilli. See Coproporphyrin and Sputum.

Tumor Cells. All of these are not cancer cells but see Cancer for technique. 
Tungstic Acid, a stable soltiuon (Abrahamson, E. M., Tech. Bull., 1940, 1, 75).

Turnbull Blue reaction for iron. Same as Berlin blue except use $\mathrm{K}$ ferricyanide and $\mathrm{HCl}$.

Turpentine. Not advised as clearing agent. See test for Alcohol absolute.

Typhus Fever rickettsiae in lungs of mice. (Nyka, W., J. Path. \& Bact., 1945, 52, $317-324)$. Fix in $10 \%$ neutral formalin. Stain sections in $1: 10,000 \mathrm{aq}$. methyl violet $30 \mathrm{~min}$. to $1 \mathrm{hr}$. Differentiate in acetic acid ( 2 drops glacial acetic in 100 cc. aq. dest.) till cytoplasm is decolorized. Counterstain in 1:10,000 aq. metanil yellow for few seconds. Dehy drate in acetone, clear in xylol and mount in neutral medium (say immersion oil). Rickettsiae, violet.

Tyrian Purple. The ancients prized this dye very highly. Said to have been discovered when a sheep dog of Hercules bit into a shellfish and stained his mouth bright red, this wonderful dye was first produced for local use in Crete about B.C., 1600, and was later distributed by the Phoenicians bringing business to Tyre; hence the name Tyrian purple. Pliny has given a detailed description of its preparation. Factories for extraction of the dye from Murex trunclus were established by the Phoenicians at many points in the Mediterranean basin, chiefly at Tyre, Tarentum and Palermo, and trading points at Cadiz, and in present day Morocco. Tyrian purple became the "royal color" employed by royalty in Persia, Babylon, Media and Syria. The robes of Greek generals were purple, likewise those of their Gods. Jewish tabernacle decorations were colored by a bluish type of Tyrian purple. The sails of Cleopatra's barge were colored purple. According to a decrec by Caesar Augustus none in the Roman Empire but the Emperor and his household could wear purple (Leggett, W. F., Ancient and Medieval Dyes. Brooklyn: Chemical Publishing Co., Inc., 1944, 95 pp.).

Tyrode solution. $\mathrm{NaCl}, 0.8 \mathrm{gm} . ; \mathrm{KCl}$ 0.02 gm.; $\mathrm{CaCl}_{2}, 0.02 \mathrm{gm}$.; $\mathrm{MgCl}_{2}, 0.01$ gm.; $\mathrm{NaH}_{2} \mathrm{PO}_{4}, 0.005$ gm.; $\mathrm{NaHCO}_{3}$, $0.1 \mathrm{gm}$. (giving $\mathrm{pH}$ about 7.5-7.8); dextrose, 0.1 gm.; aq. dest., 100 cc. Solution cannot be boiled but can be passed through a Berkfeld filter.

Tyrosine Reaction. The procedure of Serra and Lopes which gives better results than the Millon Reaction is specified as follows by Serra, J. A., Stain Techn., 1946, 21, 5-18: Prepare tissue as described under Ninhydrin Reaction.

"1. Immerse the objects for $30 \mathrm{~min}$ utes in a few milliliters of the mercuric solution (composition: $\mathrm{HgSO}_{4}, 7.5 \mathrm{~g}$.;
$\mathrm{HgCl}_{2}, 5.5 \mathrm{~g}$.; $\mathrm{Na}_{2} \mathrm{SO}_{4}, 7.0 \mathrm{~g}$.;-dissolved in $85 \mathrm{ml}$. of distilled water to which $12.5 \mathrm{~g}$. of concentrated $\mathrm{H}_{2} \mathrm{SO}_{4}$ is added; after dissolving dilute to $100 \mathrm{ml}$. with distilled water). Perform the treatment in a small glass stoppered bottle, placed in a water bath which is maintained at $60^{\circ} \mathrm{C}$.

"2. After the 30 -minute treatment, cool the bottle in running water and allow to stand at room temperature for 10 minutes.

" 3 . Dilute the mercuric solution in the bottle, by addition of an equal volume of distilled water.

"4. Develop the color, adding now some drops of a freshly-prepared $1 M$ solution of sodium nitrite $\left(6.9 \mathrm{~g}\right.$. $\mathrm{NaNO}_{2}$ in $100 \mathrm{ml}$. of water).

"The coloration attains its maximum in 3 minutes and lasts for some months, though it fades gradually with time. The materials are mounted and observed in pure glycerin, where they can be squeezed or squashed, if necessary.

"The reaction is principally due to the presence of tyrosine in the protein molecule, and is also produced by other phenolic compounds. The method here described gives with tryptophane only a transient coloration, which lasts no more than a few minutes; it is hoped, therefore, that by this procedure this histochemical test reveals only the tyrosine in the proteins."

Turnick, see Aceto-Orcein-Fast Green.

Ultracentrifuges-Written by $\mathrm{H}$. W. Beans, Dept. of Zoology, State University of Iowa, Iowa City. September 27, 1951Few instruments are more essential to certain phases of biological and medical laboratory analysis and research than the centrifuge. However, because of the relatively low centrifugal force generated by this instrument, its usefulness is limited to the displacement of materials within living cells of relatively low viscosity and to biological materials within the test tube that differ greatly in their relative densities. Recent improvement of the electrically driven laboratory type centrifuge has however, resulted in machines capable of generating forces of the order of 10,000 to 30,000 times gravity. It was this type centrifuge that Bensley and Hoerr used to separate and analyze mitochondria (Anat. Rec., 1934, 60, 251), a technique which paved the way for the important discovery that mitochondria are the carriers of all the important oxidative enzymes (Green, D. E., Scientific American, 1949, 181, 48).

The development of the ultracentrifuge has provided a tool for the study of the components of living cells of 
high viscosity, as well as certain of their molecular components after disruption of the cell. In fact it was the latter that led Svedberg and his associates to experiment with machines to develop higher centrifugal force, optical methods of recording the behavior of mixtures during rotation, and suitable mathematical formula for interpreting the molecular weights of proteins (Svedberg, T. and K. C. Pedersen, The Ultracentrifuge. Oxford Univ. Press 1940). Svedberg's many years of experimentation culminated in an oilturbine driven rotor surrounded by an atmosphere of hydrogen to reduce heating and hence, convection which is always a troublesome problem in the high speed centrifuging of mixtures. The safe operating speed of the Svedberg ultracentrifuge is said to be about 67,000 R.P.M., producing about 350,000 times gravity. By means of this instrument Svedberg and his associates have demonstrated that the protein molecule is relatively large. In addition, they have made an important contribution to biology by determining the molecular weights of many of them.

Because the oil-driven ultracentrifuge is relatively complicated and costly, only 9 or 10 of them have been made (Gay, G. W., Scientific American $1951, \mathbf{1 8 4}, 43$ ). In 1930, J. W. Beams developed the air-turbine ultracentrifuge of Henriot and Huguenard to use in connection with some experiments in physics (J. Appl. Phys., 1937, 8, 795). This instrument consists of a cone shaped rotor which is supported and driven by air under pressure from properly directed jets. It is relatively simple (costs less than $\$ 100.00$ ) and has proven most useful for histological and cytological studies. Displacement of materials such as Golgi apparatus, mitochondria, Nissl bodies, neurofibrillae, centrosomes, chromosomes, spindles, acrosomes, plastids, nuclei and nuclear components, intracellular virus bodies, membrane materials, erythrocyte components, secretion products, vitamine $\mathrm{C}$ granules, organizer substances, enzymes, bacteriophage, and sarcoma virus have been observed. In addition, it has been successfully used to study polarity in both plants and animals as well as the relative viscosity of certain cancer cells. Thus, by use of this method, information has been gained concerning both the structure and function of many cellular components and inclusions both inside and outside the cell.

E. N. Harvey has adapted his centrifuge-microscope principle to the air- turbine rotor thus making possible the direct observation of cells in a centrifugal field of 100,000 to 250,000 times gravity (Biol. Bull., 1934, 66, 48).

While the air-turbine ultracentrifuge above described has proved satisfactory for the study of many materials within cells, it is not so suitable for the separation of colloidal solutions within a test tube. This is because of the convection induced by the slight heating of the rotor as it spins in air at atmospheric pressure. This difficulty has been overcome by the development of the air-turbine vacuum-type ultracentrifuge. This machine consists of a large rotor (4 to 7 inch) situated in side a vacuum tight chamber which is driven and supported by an airturbine of the type described above, located outside and vertically above the vacuum tight chamber. The turbine and rotor are connected by a flexible shaft which enters the vacuum chamber through a vacuum tight oil gland. By this means a convection free centrifugal field is established, the intensity of which is limited only by the bursting strength of the rotor spining the vacuum chamber. (Beams, J. W. J. Appl. Phys., 1937, 8, 795; Rev. Mod. Phys., 1938, 10, 245; Bisco, Pickels and Wyckoff, J. Exp. Med., 1936, 64, 39).

The development of this comparatively inexpensive centrifuge has made available to many biophysical and biochemical laboratories an instrument comparable to, if not superior to, the oil-turbine ultracentrifuge of Svedberg. With it investigators have concentrated, separated, and determined the molecular weights of substances such as many different types of virus, antibodies, bacteriophage, cancer agents, hormones, and various protoplasmic constituents.

New uses for the ultracentrifuge in biological research are continually appearing. For example, press reports state that Gofman and associates have separated cholesterol from human blood by use of this machine. Their studies indicate that it may be possible to anticipate certain circulatory diseases, such as high blood pressure and arteriosclerosis, by this technique. In other words, eventually we may have available "diagnostic ultracentrifuges".

The air-turbine tubular-type ultracentrifuge has been used successfully to separate uranium 235 from uranium 238 (Smyth, H. D., Atomic Energy for Military Purposes, Princeton Univ. Press, 1915). This machine was first described by J. W. Beams, and has become a subject of classified military 
research. Efforts to improve the ultracentrifuge are continuing. Beams J. Wash. Acad. Sci., 1947, 37, 221 has described a magnetically supported and magnetically driven ultracentrifuge. This machine in experimental tests obtained rotational speeds of 48 million R. P. M., thus producing a centrifugal force of 500 million times gravity. Further development of the ultracentrifuge will undoubtedly lead to a wider application and more refined techniques for researchers interested in problems involving sedimentation and fractionation of mixtures. See Centrifugation.

Ultrafilter. For filtering small volumes of fluid the ultrafilter of Johnson, H. C. and Kirk, P. L., Mikrochemie ver. Mikrochim, Acta, 1940, 28, 254-257 is recommended by Glick, p. 487. See easily constructed apparatus described by Clark, L. C., J. Lab. \& Clin. Med., $1951,37,481-484$.

Ultramarine Green, an exogenous pigment, a sodium aluminum silicate and sulfide (Lillie, p. 138).

Ultramicroscope, see Darkfield.

Ultrasonic Vibrator. Type used to test effect of ultrasonics on blood elements by Morrow, P. L., Bierman, H. R. and Jenkins, R., J. Nat. Cancer Inst., 1950, 10, 843-859.

Ultrasonics. The division of acoustics comprising sound frequencies beyond the limits of perception by the human ear. Radiation of this sort can be very destructive to living cells. The technique and results are well described by Gregg, E. C., Jr. in Glasser's Medical Physics, 1591-1596.

Ultraviolet Microscope and Color Translation Process. A microscope using ultraviolet radiation instead of ordinary light to form an image is somewhat of a misnomer, for there is nothing to be seen. One must expose a photographic plate sensitive to the ultraviolet rays to record the image. If the ultraviolet is used only to excite fluorescence then of course a visible image is produced as has been described. We are here concerned with images which are not visible.

In accordance with the law, already mentioned, $\mathrm{R}=\lambda / 2 \mathrm{~N}$. A. increased resolving power can be achieved by employing the shorter wave lengths of the spectrum. Considerable improvement of the image is obtained by using monochromatic blue light with the ordinary "achromatic" type of objective; but with highly color corrected "apochromatic" lenses there is little to be gained by so doing. The ultraviolet region of the spectrum of fers wave lengths as short as $0.15 \mu$, most of which are produced very conveniently by modern mercury and hydrogen arcs. Therefore, photography in the ultraviolet should double or even triple the resolving power of a lens if everything else is held constant.

Since ordinary optical glass is nearly opaque to ultraviolet light lens makers must use natural quartz, or fluorite, components throughout the system. This puts rather severe limitations on the lens designer as it narrows his range of possible corrections. For example, an all-quartz objective must be used with ultraviolet radiation of a specified wave length in order to get a good image (Lavin, G. I., Rev. Sci. Inst., 1943, 14, 375-376). Focus is obtained by trial and error. This makes observation somewhat laborious and almost impracticable for living material. Ultraviolet microscopy is, however, used with considerable success in the study of fixed, unstained cells because proteins and nucleic acids show specific absorbtion at $0.280 \mu$ and $0.260 \mu$ respectively (Caspersson, I., J. Roy. Micr. Soc., 1940, 60, 8-25). Thus chromosomes, rich in nucleic acid, reveal themselves in strong contrast and high resolution (Ludford et al., J. Roy. Mier. Soc., 1948, 68, 1-9). After proper calibration one can use the blackening of a plate, or ultraviolet photocell currents, to measure the concentrations of absorbing materials in various structures under examination.

A remarkable advance in ultraviolet microscopy has recently come about with the development of the color translation microscope (Land, E. H. et al., Science, 1949, 109, 371-374), for which an objective lens corrected for three wave lengths in the ultraviolet has been designed. The operator takes a photograph at each wave length. These negatives show somewhat different details because the materila being studied has different ultraviolet absorbing powers at these three wave lengths. The negatives are then treated, as in the conventional process for visual color photography, by assigning to each negative one of the primary colors. The result is a color print representing in an arbitrary way something which is invisible to the eye. Thus, changes in the ultraviolet absorption spectra of the materials are revealed by changes in the apparent colors of details in the print; hence the term "color translation." 
Ultraviolet Microspectrophotometry has been developed by T. Caspersson (Cell Growth and Cell Function. A Cytochemical Study. W. W. Norton and Company, New York, 1950) and permits the determination of absorption curves of light from 240 to 350 millimicrons wavelength in minute parts of cells. Information on steroids, nucleic acids, proteins and iodides can be obtained. Equipment is being developed now in several laboratories to extend the range of measurements from $230 \mathrm{~m} \mu$ to the infra red and the usefulness of these types of technics.

Ultraviolet Photomicrography has certain advantages over visible light photomicrography because the resolving power of the former is greater in consequence of its shorter wave length, and as pointed out by Wyckoff and Louw (R. IV. G. and A. L., J. Exper. Med., $1931,54,449-451$ ), because some proteins absorb ultraviolet more strongly than others, details can be brought out with it not revealed by visible light. This they demonstrate by experiments with $B$. subtilis. It was then found that the substances that strongly absorb ultraviolet light give a positive Feulgen reaction (Wyckoff, R. W. G., Ebeling, H. H., and Ter Louw, A. L., J. Morph., 1932, 53, 189-199) and that they also yield conspicuous mineral ash on microincineration (Scott, G. H., Science, $1932,76,148-150)$-an interesting superposition of three technical methods. The work of the Swedish group is summarized in Caspersson's book (Cell Growth and Cell Function. A Cytochemical Study, W. W. Norton and Co., New York, 1950). Two available articles in the American literature are by Stowell (Arch. Path. 1948, 46, 164-178 and Cancer, 1949, 2, 121-131). The use of other newer types of lenses should be mentioned. See (Mellors, R. C., Berger, R. E., and Streim, H. G., Science, $1950,111,627-632$ for references).

Union Green B, see Janus Green B. Ultropak illuminator of Leitz is helpful for micrological work. The Epi Condenser W of Zeiss is similar. See Chambers, R. W. and Kopac, M. J. in McClung's Microscopical Technique, 1950, p. 508.

Unna's Orcein method for elastic fibers. This is simple and direct. Stain paraffin sections, after almost any fixation, in: orcein, $1 \mathrm{gm}$.; absolute alcohol, $100 \mathrm{cc}$; and hydrochloric acid, 1 cc. for several hours. Wash in $70 \%$ alcohol and sharpen the deep brown coloration of the elastic fibers by removing excess stain from background by destaining under the microscope in $95 \%$ alcohol plus a trace of hydrochloric acid. Wash in $95 \%$, dehydrate, clear and mount. If desired counterstain with methylenc blue.

Dahlgren (McClung, p. 425) advises a modification of this stain for Muscle. After sublimate fixation stain sections 24 hrs. in Wasserblau, 0.25 gm.; absolute alcohol, 60 cc.; orcein, 1 gm.; glycerin, 10 cc.; water, 30 cc. Wash in $70 \%$ alcohol, dehydrate, clear and mount. Muscle, purple; collagenic fibers, blue; elastic fibers, red. It is important in doubtful cases to compare with similar tissue colored by other specific stains before identification of muscle is assured.

Uranin, sodium salt of Fluorescein.

Uranium. Salts injected into tissues can be demonstrated by (1) a method of Schneider (G., Skand. Arch. Physiol., 1903, 14, 383-389). Fix in: $5 \%$ aq. potassium ferrocyanide, 50 cc., sat. aq. picric acid, 50 cc.; hydrochloric acid, 10 cc. Wash in $4 \%$ aq. hydrochloric acid and then in $80 \%$ alcohol acidified with hydrochloric acid. Imbed and cut. The uranium ferrocyanide of potassium is detected by its dark brown color (Lison, p. 103). (2) the Prussian blue reaction for iron as employed by Gérard and Cordier (P. and R., Arch. Biol., 1932, 43, 367-413). According to Lison this method is highly specific.

The possibility of detecting uranium salts in incinerated sections by their fluorescent properties in ultraviolet light has been described (Policard, A. and Okkels, H., Abderhalden's Handb. d. biol. Arbeitsmethoden, 1931, 5, 1815). Gordon H. Scott has been successful when large amounts are present but has called attention to complicating factors (McClung's Microscopical Technique, p. 660).

Urates and Uric Acid. A modification of Courmont-André's method is suggested. Neutralize some formalin with calcium carbonate. Fix tissue in equal parts $1 \%$ aq. silver nitrate and $4.4 \%$ neutral formalin in darkness, 12-24 hrs. Wash in several changes aq. dest., 24 hrs. Imbed in paraffin. Stain sections hematum $10 \mathrm{~min}$; running tap water $\frac{1}{2}-1 \mathrm{hr}$.; $1 \%$ aq. orange $\mathrm{G}$ or eosin $\frac{1}{2}-1$ hr. Wash quickly in aq. dest. Place in $0.5 \%$ aq. phosphomolybdic acid, rinse in aq. dest. and color in $0.12 \%$ aq. light green, 1-10 min. Differentiate quickly in $96 \%$ alcohol, dehydrate in iso-amylalcohol, clear in xylol and mount in balsam. Urates, black; chromatin, blue; protoplasmic inclusions red to orange and collagenic fibers, green. 
Employed by Hollande for bacteriocytes of Periplaneta orientalis $L$ (Hollande, A. C., Bull. d'Histol. Appl., 1931, 8, 176-178).

Urea. Many histochemical techniques have been proposed. Leschke (E., Zeit. Klin. Med., 1915, 81, 14-35) fixes in half sat. sol. mercuric nitrate in $1 \%$ nitric acid for 1 day, then washes in frequently changed aq. dest., imbeds in paraffin and treats the sections with sat. aq. hydrogen sulphide staining nuclei with hemalum. Stübel (H. Anat. Anz., 1921, 54, 237-239) fixes small pieces in $6 \%$ xanthydrol in glacial acetic acid 6-12 hrs., imbeds in paraffin, stains sections by ordinary methods and examines by polarizing microscope. Oliver (J., J. Exper. Med., 1921, 33, 177-186) employs instead a solution containing $2 \mathrm{gm}$. xanthydrol, $10 \mathrm{cc}$. methyl alcohol and $20 \mathrm{cc}$. glacial acetic acid. Lison (p. 169) criticizes these methods severely.

It may be necessary to resort to the capillary tube colorimetric technique of Walker, A. M. and Hudson, C. L., Am. J. Physiol., 1937, 118, 153-166, or to the titrimetric method of Kinsey, V. E., and Robison, P., J. Biol. Chem., $1946,162,325-331$.

Urease. A method for determining the distribution of urease in the gastric mucous membrane (pylorus and fundus) of the dog has been described and used by Linderstrøm-Lang and Ohlsen (K. and A. S., Enzymologia, 1936-37, 1, 92-95). Cylinders of tissue $(2.5 \mathrm{~mm}$. in diameter) are cut vertical to the surface from frozen mucosa. Cross frozen sections ( 25 microns thick) of the cylinders are then tested for urease. This is concentrated in the surface layers containing cells stainable with mucicarmine. Chief cells in the bases of the glands are inactive in both fundus and pylorus and the authors think it very unlikely that the parietal cells contain urease.

For help in the problem of adapting methods for urea to urease see Glick, p. 287.

Uremia. Microscopic demonstration of uremia by precipitation of xanthydrol urea in tissue. A modification of Oestreicher's original method is provided by Brown, A. F. and Krajian, A. A., Arch. Path., 1936, 21, 96-99.

Cut blocks of tissue $2-3 \mathrm{~mm}$. thick. Immerse in fresh xanthydrol solution (xanthydrol, $5 \mathrm{gm}$., glacial acetic acid 100 cc.) at $80^{\circ} \mathrm{C}$. for 2 hrs. Wash in running water, $5 \mathrm{~mm}$. Fix in 1 part formaldehyde U.S.P. and 10 parts aq. dest. at $70^{\circ} \mathrm{C}$. for $15 \mathrm{~min}$. Wash in tap water and cut $5-10 \mu$ frozen sections. Transfer them to slide and pour on several drops "dehydrated alcohol" (presumably abs. ethyl alc.) from a drop bottle and blot. Repeat. Cover by dipping in thin pyroxylin (celloidin) contained in wide mouthed bottle. Fix film of pyroxylin to slide by blowing breath over section and stain in $1 \%$ aq. eosin for several minutes. Wash in water, dehydrate in 3 changes dehydrated alcohol, place in carbol-xylene, clear in 2 changes pure xylene $1 \mathrm{~min}$. each and mount in dammar. Xanthy drol urea crystals appear as closely packed clusters of yellow-green needles.

Uric Acid. Micro colorimetric method, Borsook, H., J. Biol. Chem., 1935, 110, 481-493. See Urates.

Urinary Casts, staining with methyl blue picric acid. To sediment from centrifuged urine add 1 drop $0.5 \%$ aq. eosin. Mix by side to side shaking. After 1-2 min. add 2 drops from 1 cc. $1 \%$ aq. methyl blue +10 cc. sat. aq. picric acid and again mix. Color of sediment should be distinctly bluish green. If it is reddish brown add more methyl bluepicric acid. Transfer to slide cover and examine. The casts should be distinct blue but not too dark. Numerous details are brought out (Behre, J. A. and Muhlberg, W., J. Lab. \& Clin. Med., 1936-37, 22, 853-856). See the author's figures.

Urinary Sediments. The following outline is from Stitt (pp. 707-713) much abbreviated. Concentrate sediment by centrifuging 15 cc. fresh urine 1500 r.p.m. $5 \mathrm{~min}$. but not longer. Decant supernatant urine. Suspend sediment in 2 cc. urine as is the practice in the Naval Medical School. By always using these amounts quantitative differences from normal in individual sediments become apparent. Examine for epithelial cells, leucocytes, erythrocytes, casts, crystalline materials, bacteria and so forth.

Urinary Tract Smears, see Papanicolaou Techniques.

Urine. For microscopic study sediments are divided into classes.

Details with helpful diagrams are supplied by C. J. Gentzkow and H. A. Van Auken in Simmons and Gentzkow, 26-33.

Unorganized components depending chiefly on metabolic activities and changes in content of bladder before urination. See also Sulfonamides. Examine for:

In acid urines

Urates, as pink amorphous materials 
Uric acid, as yellow brown, wedgelike"whetstones", dumb-bell and rosette crystals

Calcium oxalate as "envelope" crystals

Cystine as colorless refractile 6 sided plates

Leucine (ycllow spheroids)

Tyrosine (fine needles)

Hippuric acid (brownish needles or prisms)

In neutral urines

Above components plus

Neutral calcium phosphate (slender pyramidal crystals united at apices forming rosettes)

In alkaline urines

Phosphate deposits (white amorphous)

Ammonium calcium phosphate (coffin lid or feathery crystals)

So-called triple phosphate crystals

Calcium carbonate (spheres, duinbbells or amorphous masses)

Ammonium urate (dark yellow brown cockle burr crystals)

Organized components consisting of cells and their products as well as of casts. Microscopically to identify leucocytes, red blood cells and sperms, when present, is easy. It is necessary to distinguish between cells from renal tubules, transitional cells from bladder and squamous epithelial cells. The casts are of 4 sorts, hyaline, granular, waxy and bloody. See Addis Count.

Detection of acid fast bacilli in urine (Kelso, R. F. and Galbraith, T. W., Am. J. Clin. Path., 1943, Techn. Suppl., 7, 8-11).

Urobilin is a derivative of bilirubin. Schmidt's test for urobilin in feces consists of rubbing up small amount of feces in white dish in sat. aq. mercuric chloride whereupon particles containing this pigment take on a deep red color (C. J. Gentzkow and H. A. Van Auken in Simmons and Gentzkow, p. 82). Wintrobe, M. M., Clinical Hematology. Philadelphia: Lea \& Febiger, 1942, 703 pp. gives several tests for urobilinogen and urobilin.

1. Remove bile pigments, if present from 10 cc. urine (or aq. suspension feces) by addition of 2 cc. $10 \%$ calcium chloride and filtration. Oxidize any urobilinogen not converted into urobilin by adding 1-2 drops of Lugol's Iodine. Then add $10 \mathrm{cc}$. Schlesinger's Reagent, filter let stand 1-2 hrs. Urobilin is indicated by green fluorescence when examined against dark background in bright light.

2. Make dilutions of urine by adding 1 cc. to $10,20,30,40$ etc. cc. of aq. dest.
To 10 cc. of each dilution in test tubes add 1 cc. Ehrlich's Aldehyde Reagent. Urobilinogen is indicated by pink color within $5 \mathrm{~min}$. seen by looking down through mouths of tubes.

Urography, a new technique for study of individual metabolic spectra (Beerstecher, Jr. E., and Sutton, H. E., Texas Reports, Biol. \& Med., 1951, 9, 8-12.

Vaccinia, Cytoplasmic inclusions in, see Cowdry, E. V., J. Exper. Med., 1922, 36, 667-684. Summary of methods used in the investigation of elementary bodies of vaccine virus (Smaclel, J. E. and Hoagland, C. L., Rev. Bact., 1942, 6, 79-110.

Vaccinia, see Guarnieri Bodies.

Vacuoles, food and contractile, sce Paramecia.

Vacuoloids-Written by C. C. Macklin, Dept. of Histological Research, The University of Western Ontario, London, Canada. November 28, 1951-This is a provisional name for numerous clear round regions, often containing a minute central granule, which appear in the pneumonocytes and foam cells (which see) after the usual methods of fixation and staining. They superficially resemble vacuoles and give to the cells a frothy appearance. They are the dominant formations of the cytosomes of these cells. They are often referred to as granules, for after certain techniques many of them are found to have an optically substantial nature, though they ordinarily do not take stains (Brodersen, J., Zeitschr. f. mikros.-anat. Forsch., 1933, 32, 73-83; Macklin, C. C., Trans. Roy. Soc. of Can., 1946, Sect. V, 40, 93-111). They are relatively stable, indenting the nucleus (Macklin, C. C., Canadian J. of Research, D, 1950, 28, 5-15) and are from $0.5 \mu$ to $1.5 \mu$ or more in diameter. In fresh mounts from gash-irrigation and wash-out recoveries (which see) they appeared as clear balls (Macklin, C. C., Proc. 6th Internat. Cong. exp. Cytol., Stockholm, 1947; published $1949,383-387$ ). They seemed to be in inverse ratio to the amount of ingested dust. In the pure form cells they were fairly uniform in size, spheroidal and refractile, gleaming like transparent glass beads; they were not so sharply marked as air bubbles, and had an extremely delicate greenishyellow cast. The contour was evenly curved and sharply set off from the environing cytoplasm. In the fresh condition, with conventional lighting, no structural detail could be made out in the pellucid interior. They were not 
birefringent; and in the dark field the surface was brilliantly illuminated. They did not take colors dissolved weakly in the mounting fluid. When Janus Green B was offered in weak dilution, coccoid mitochondria were observed between the vacuoloids. Fine red-stained granules were found between the vacuoloids in foam cells of frozen sections which had been stained with Sudan IV. Vacuoloids seem to be composed largely of water, but should not be regarded as simple capsules. They should certainly not be confused with myelin figures. When foreign particles are taken into the cell they lodge between, never within, the vacuoloids, and this is true for vital dye accumulations. The possibility of differentiation in vacuoloids is admitted.

In material fixed in Regaud's fluid, mordanted in potassium bichromate, cut into frozen sections and well stained with Heidenhain's iron hematoxylin, some of them appear as dense blueblack balls. Some of them appear as large dense darkbrown spheroids or ovals within pneumonocytes which have been in contact with blood and have been fixed with pure formalin or other aldehyde (Sjöstrand and Sjöstrand, Zeitsch. f. mikros.-anat. Forsch., 1938, 44, 370-411). These workers find that the colored material has properties of hemin. The same coloration of vacuoloids may occur in mice suffering from a virus infection after fixation with Bouin's fluid by immersion of the skinned intact thorax (which see). Some of them, in apparently normal pneumonocytes and foam cells appear black or gray-black after exposure to osmic acid fixation intrabronchially after Aquax (which see) embedding and mounting in levulose (which see). In apparently degenerate pneumonocytes, all of the vacuoloids are impregnated with osmium.

The presence of vacuoloids brings about a lacelike picture in the mitochondria of pneumonocytes, for mitochondria are not admitted to the interiors of the vacuoloids but occupy the cytoplasm between them (Macklin, C. C., Biol. Bull., 1949, 96, 173-178). With Nile Blue Sulphate (Lorrain Smith, which see) the vacuoloids in foam cells which are not stained dark after osmic acid fixation are sometimes colored light red as seen in Aquax sections mounted in levulose.

Macklin spoke in the following words at the 1947 International Cytological Congress: "The function of the foam cell is obscure, and may be concerned with the conditioning of the alveolar wall; as for instance in the maintenance of a proper surface tension. However, the finding of these cells in augmented quantities under pathological conditions in man and rat suggests that they may be implicated in the local body reactions to chronic infection. Amoeboidism seems to be a property. ... It looks as if the antecedent entodermal cell has a dual differential potency, directing it to the left, so to speak, where it may become an irreversible pure foam cell, or to the right, where it may end up as an irreversible pure dust cell. The in-between stages, presumably very useful, may be termed "bireactives." (Macklin, C. C., Proc. 6th Internat. Cong. exp. Cytol., Stockholm, 1947; published 1949, 383-387).

Vaginal Smears, see Papanicolaou Techniques.

Valves. Aortic, staining of elastic tissue in (Wilens, S. L., Arch. Path., 1940, 29, 200-211). X-ray demonstration of valves of veins (Edwards, E. A., Anat. Rec., 1936, 64, 369-385).

Vanadium, see Atomic Weights.

Van den Bergh Test for bilirubin as described by Wintrobe, M. M., Clinical hematology. Philadelphia: Lea \& Febiger, 1942, 703 pp. abbreviated:

1. Qualitative:

(a) Add $1.5 \mathrm{cc}$. conc. hydrochloric acid C.P. to 30 or 40 cc. aq. dest. + $0.1 \mathrm{gm}$. sulphanilic acid which keeps well.

(b) Dissolve $0.5 \mathrm{gm}$. sodium nitrite C.P. in 100 cc. aq. dest. making up fresh every 3-4 weeks.

Make diazo reagent by mixing $5 \mathrm{cc}$. of (a) with $0.15 \mathrm{cc}$. of (b) freshly each day.

Mix 0.25 cc. reagent with $0.2 \mathrm{cc}$. clear plasma or serum ( $2 \mathrm{~cm}$. column in hematocrit). Immediate purplish color attaining maximum in 30 sec. is direct reaction. Color appearing at once but reaching maximum later is biphasic reaction. If no color in $1 \mathrm{~min}$. but on addition of $5 \mathrm{cc}$. alcohol reddish violet color appears reaction is indirect.

2. Quantitative.

(a) Stir and shake 80-90 gms. ammonium sulphate C.P. in 100 cc. aq. dest. until saturated and filter.

(b) Make standard color by dissolving $3.92 \mathrm{gm}$. cobalt sulphate $\left(7 \mathrm{H}_{2} \mathrm{O}\right)$ in 100 cc. aq. dest. over night.

Mix 0.5 cc. diazo reagent with 1 cc. serum or plasma in centrifuge tube. After standing few minutes add $2.5 \mathrm{cc}$. $95 \%$ ethyl alcohol and $1 \mathrm{cc}$. of (a). Mix and centrifuge. In positive reaction uppermost layer is reddish violet alcoholic extract of diazotized bilirubin, 
next layer is flocculated protein and residue is ammonium sulphate. Compare supernatant fluid with the standard (b) in colorimeter. Then:

mm. standard

$\frac{\text { mm unknown }}{\text { ming }} \times 4 \times 0.5$

a mg. bilirubin per $100 \mathrm{co}$.

Van Gehuchten's Mixture, see Carnoy's Fluid.

Van Gieson's Connective Tissue Stain. Paraffin sections of Zenker fixed material are stained with Harris' hematoxylin. Rinse in water. Stain in $1 \%$ aq. acid fuchsin $7.5 \mathrm{cc}$. and sat. aq. picric acid 50 cc., $10 \mathrm{~min}$. Wash in $95 \%$ alc., dehydrate, clear and mount. Muscle yellow, collagenic fibers red, nuclei blue black. A brilliant stain. But it fades quickly and is not so much employed at present as Mallory's connective tissue stain. See Buzaglo's Method, Curtis' Substitute for Van Gieson, Collagenic Fibers, Connective System.

Van Wijhe's method for staining cartilage in whole tissues with methylene blue. See Cartilage.

Vasa Vasorum. Injection with India ink (Winternitz, M. C., Thomas, R. M. and LeCompte, P. M., The Biology of Arteriosclerosis. Springfield: Thomas, 1938, 142 pp.). Filter Higgins Engrossing ink through coarse filter paper and dilute filtrate with 8 times volume of aq. dest. Obtain pressure apparatus consisting of 2 liter metal tank with top and bottom outlets and air pressure gauge. Connect upper outlet with escape valve and high pressure air line and the lower one with rubber tube and cannulac. To inject vasa of coronary arteries place fresh human heart unopened in $0.9 \%$ aq. sodium chloride containing $0.1 \%$ sodium nitrite and a little thymol for $24 \mathrm{hrs}$. at $3-4^{\circ} \mathrm{C}$. Just before injection warm heart to $37^{\circ} \mathrm{C}$., tie cannulae in openings of coronary arteries and clamp or ligate all openings of heart except the aorta. By opening and closing the escape valve the ink in the tank is driven into the coronaries by a pulsating pressure. During first 10 min. maintain the minimum pressure at about $100 \mathrm{~mm}$. of mercury with maximum pressure of pulsations not more than 200. Then increase slowly so that during next $20 \mathrm{~min}$. the minimum pressures vary $500-800 \mathrm{~mm}$. and the maximum $800-1000$. After injection put heart in $10 \%$ formalin for 24 hrs. Dissect out main coronaries. Clear by Spalteholz Method for whole mounts or imbed in paraffin section and color by Masson's Trichrome stain. The authors give special directions for injecting the aorta and vessels of kidneys and amputated legs. Their illustrations afford useful guides to the results expected.

Vaseline in tissues can be distinguished from the normal fats by the fact that the former is colored clear violet and the latter intense blue black by stain. ing for 15 min. with Sudan Black B. Terebenthine, turpineol and methyl benzoate are colored blue black (Gerard, P., Bull. d'Hist. Appl., 1935, 12, 92-93).

Vegetative Intermitotics, see Cell Classification.

Veins, see Blood Vessels and a very fine presentation by Franklin, K. J., A Monograph on Veins. Springfield: Thomas, 1937, $410 \mathrm{pp}$. with hundreds of references to techniques and results.

Venous Sinuses, splenic, direct observation in vivo (Ínisely, M. H. Anat. Rec., 1936, 64, 499-524; 65, 23-50). See Spleen.

Venules. A graphic demonstration of venules in the ears of white mice can be obtained by intravenous injection of Chicago blue because this dye escapes into the surrounding tissue fluid more easily from venules than from capillaries (Smith, P. and Rous, P., J. Exper. Med., 1931, 54, 499-514).

Verdigris. A green progeneous pigment made up of basic copper acetates.

Verhoeff's Elastic Tissue Method (Verhoeff, F. H., J. A. M. A., 1908, 50, 876-877). Gives good results after fixation in Zenker's fluid, formalin alone or after Weigert's mordant for myelin sheaths or Marchi's fluid. It is fairly satisfactory for tissues decalcified with nitric acid. Mercury deposits resulting from Zenker's fixation are removed by the stain: Hematoxylin crystals, 1 gm.; Abs. alc., 20 cc.; Dissolve in test tube with aid of heat, filter and add in order given: $10 \%$ aq. ferric chloride, 8 cc.; Conc. Lugol's solution (iodine, 2 ; potassium iodide, 4; water, 100), 8 cc. Stain sections in above sol. $5 \mathrm{~min}$. or more. Differentiate in $2 \%$ aq. ferric chloride for a few sec. until the connective tissue takes the color of Lugol's solution. Keep sections in motion during differentiation. They can be examined at low magnification in water and if over differentiated can be restained at this stage. Wash in water followed by $95 \%$ alc. to remove the stain of Lugol's solution. Then leave in water $5 \mathrm{~min}$. or more. Counterstain in $0.2 \%$ water sol. eosin in $80 \%$ alcohol. Dehydrate, clear in origanum and mount in balsam. Elastic tissue, black; fibroglia, myoglia, neuroglia, myelin and fibrin, pink. Degenerated elastic tissue (elacin) 
can be distinguished by less intensity of staining and by diffuse outlines.

To differentially stain myelin sheaths fair results are obtained after Zenker's fixative or formalin followed by Marchi's fluid. For best results fix in formalin 4 days, or longer, and mordant in Weigert's potassium bichromate and chrome alum for 4 days. Again it is not necessary before hand to remove mercurial precipitates. Place sections in $3 \%$ aq. potassium permanganate, 30 min. Wash in water and color for 30 min. in the hematoxylin stain described. Wash in water and differentiate in $10 \%$ aq. ferric chloride until the internal elastic membranes of blood vessels are decolorized as determined by examination in water at low magnification. 1-2 min. are required. Wash in water for $5 \mathrm{~min}$., counterstain with eosin and mount in usual way.

Veronal-Acetate buffers, see Michaelis, L., J. Biol. Chem., 1930, 87, 34. Employed for thionin staining of Nissl substance by Windle, W. F., Rhines, R. and Rankin, J., Stain Tech., 1943, 18, 77.

Vestibular Apparatus, see Ear.

Vesuvin, see Bismark Brown $\mathbf{Y}$.

Victoria Blue (1) B (CI, 729)-corn blue $\mathrm{BN}$, fat blue B-(2) R (CI, 728)-corn blue $\mathrm{B}$, new Victoria blue $\mathrm{B}$ or $\mathrm{R}-(3)$ $4 \mathrm{R}$ (CI, 690)-fat blue $4 \mathrm{R}-\mathrm{A}$ useful basic tri-phenyl methane dye. $4 \mathrm{R}$ is quite extensively discussed with other vital stains by Gutstein, M., Zeit. f. d. Ges. Exp. Med., 1932, 82, 479-524. Herzberg, K., Zentralbl. Bakt. I Abt. Orig. 1934, 131, 358-366 employed $4 B$ highly concentrated (Bayer standards, Hollborn), as a stain for filterable viruses (Kikuth, variola, varicella, ectromelia and possibly herpes). Dry smears in air $24 \mathrm{hrs}$. Stain 5-20 min. in $3 \%$ aq. Victoria blue. This dye solution should have been heated to $60^{\circ} \mathrm{C}$. for half an hour, allowed to stand 2 weeks and filtered before use. To increase intensity of stain add $0.3 \mathrm{cc}$. $10 \%$ aq. tartaric acid to $10 \mathrm{cc}$. of stain. Response of different viruses to stain is not uniform. Various counterstains are suggested. The various Victoria blues are not easily disentangled. Victoria blue (variety unspecified) has, according to Lee (p. 187), a special affinity for elastic fibers and mucous cells.

Victoria Green B or WB, see Malachite Green.

Victoria Green G (British Drug Houses Ltd), a triazo dye of benzidine series. In alcoholic solution gives blue green and yellow green colors. Can be used with Marshall red or Hickson purple (H.
G. Cannan, J. Roy. Micr. Soc., 1941, 61, 88-94).

Victoria Rubin O, see Amaranth.

Villi, method for study of movements (King, C. E. and Arnold, L., Am. J. Physiol., 1922, 59, 97-131; King, Arnold and Church, J. G., ibid, 61, 80-92). See Agonal Changes. Changes in shape when intestine is distended (Johnson, E. P., Am. J. Anat., 1912-13, 14, 235250 ).

Vincent's Angina, staining of spirochete. Spread ulcerative material on clean slide. Dry in air and fix with heat. $\mathrm{N} / 20 \mathrm{HCl}, 10$ sec. Running water, 5 sec. Cover with Gram's iodine solution, 5-10 sec. Wash. Cover with anilin gentian violet, 5-10 sec. Wash. Gram's iodine, 5-10 sec. Wash. Anilin gentian violet, 5-10 sec. Wash, blot and examine. Spirochetes deep violet color. Also good for $T$. pallidum (Bailey, H. D., J. Lab. \& Clin. Med., 1937-38, 23, 960).

Violamin $\mathbf{3} \mathbf{B}$, possibly related to fast acid blue.

Violamin $\mathbf{R}$ (CI, 758). Lillie, R. D., J. Tech. Methods, 1945, No.25, 47 pp. has reported that this dye is a good stain for collagen and more light fast than acid fuchsin. Pass sections down to water and stain for $6 \mathrm{~min}$. in Hemalum (Mayer-Lillie). Wash in tap water and stain 4 min. in $0.1 \%$ fast green FCF or in $0.3 \%$ Wool Green S (CI, 737) both in $1 \%$ aq. acetic acid. Wash in $1 \%$ aq. acetic acid and stain 10-15 min. in $0.2 \%$ acid fuchsin, or in $0.2 \%$ violamine $R$, both in sat. aq. picric acid. Wash 2 min. in $1 \%$ aq. acetic acid. Dehydrate in alcohol and alcohol-xylol, clear in xylol and mount in clarite. Connective tissue, red; erythrocytes, green; cytoplasm and muscle, gray-green; and nuclei, brown.

Violet $R$, RR or $4 \dot{R}$, see Hofmann's Violet.

Virchow's Crystals are orange or bright yellow crystals of hematoidin occasionally met with in extravasated blood.

Viruses may now be studied microscopically in several different ways. There is a general but not very satisfactory distinction made between Elementary Bodies of the viruses which may be extracellular and the Inclusion Bodies which may be larger, are intracellular and may contain cellular material perhaps combined with virus. The Chorioallantoic Membrane has proved to be an excellent tissue in which to examine virus action. See further data under above headings. A very comprehensive description is: Rocha-Lima, H., Reis, J., and Silberschmidt, K., Methoden der Virusforschung. Berlin: Ur- 
ban and Schwarzenberg, 1939, 384 pp. The "ultravirus" diseases of insects require special techniques and they should not so often be ignored in obtaining a clear view of the viruses as a whole. The following book is a mine of useful information Paillot, A., L'Infection Chez Les Insectes. Imprimerie de Trévoux, G. Patissier, 1933, 535 pp. The Electron Microscope is of great service in study of viruses.

Botanists have grcatly advanced knowledge of the chemical composition of viruses. Discussion by Bawden, F. C., Plant Viruses and Virus Diseases. Waltham : Chronica Botanica Co., 1943, 294 pp. of data bearing on the purity of virus crystals, paracrystals and liquid crystals shows the use and limitations of present day techniques. His photomicrographs of the virus crystals are interesting. The earlier literature is well summarized.

Viscosity. According to Heilbrunn (L. V., An Outline of General Physiology. Philadelphia: Saunders, 1937), "Viscosity can be roughly defined as the force which tends to hold the particles of a substance together when a shearing force acting on the substance tends to pull it apart." Viscosity is the inverse of fluidity. It is of great importance to histologists to be able to detect and if possible to measure changes in viscosity. When a living cell is examined in approximately an isotonic medium and tiny particles in it begin Brownian Movement a decrease in viscosity is indicated and when the movement ceases an increase is to be expected. Thus Lewis (W. H., Bull. J. Hopkins Hosp., 1923, 34, 373-379) took cessation of Brownian movement of particles in the nucleus viewed in the dark field to mean gelation which is increase in viscosity. A Microdissection method is to insert 2 microneedles into a cell. If they can be pulled apart easily the viscosity is low; if with difficulty, it is high. The idea back of the Ultracentrifuge method is that if two cells of the same sort are subjected to equal centrifugal force and a component, say the nucleus, is displaced more in one than in the other the viscosity of the cytoplasm is greater in the cell showing the least nuclear displacement. But this is not necessarily true. One has to be sure that the nuclei are of equal Specific Gravity. If the more displaced nucleus is of higher specific gravity than the other it will be more subjected than the other to the centrifugal force and its greater displacement will not signify a lower viscosity of the surrounding cytoplasm. Similarly if the specific gravity of the cytoplasm surrounding the more displaced nucleus is less than that in the other cell the greater displacement subjected to the centrifugal force of the nucleus through it will not indicate a lower cytoplasmic viscosity. When a material changes from a sol to a gel its viscosity increases without a change in specific gravity. Consequently in the interpretation of alterations in displaceability of cellular components subjected to centrifugal force one has to be on the lookout for changes in specific gravity and colloidal state. For details in respect to intranuclear viscosity, see Cowdry, E. V. and Paletta, F. X., Am. J. Path., $1941,17,335-357 ; 1942,18,291-311$ ).

Vital New Red. This is an acid dis-azo dye not listed in indexes but Conn ( $p$. 64) calls attention to chlorazol fast pink 4BL (CI, 353) as most nearly resembling it. Vital now red is one of the many dis-azo dyes employed by Evans, H. M., and Scott, K. J., Carnegie Inst. Wash., Contrib. to Embryol., $1921,10,1-56$ to bring out a difference in reaction of the two great groups of connective tissue cells.

Vital Red (CI, 456)-acid Congo $R$, azidine scarlet $R$, brilliant Congo $R$, brilliant Congo red $R$, brilliant dianil red $R$, brilliant vital red-An important acid dis-azo dye frequently used in standard method for determination of blood volume.

Vital Staining. This technique has been contrasted with Supravital Staining. It must be viewed broadly. Any nontoxic coloration of the living body is vital staining. It is not restricted to particulate materials or to colloidal suspensions which are phagocytosed by certain cells. The fat depots of an animal become vitally stained red when the said animal is fed fat colored with alcohol soluble sudan III. Bone formed while madder is available in the circulation is stained red and dentin is vitally stained violet by intravenous injections of $1 \%$ sodium alizarin sulphonate (Gottlieb, B., Ztschr. f. Somat., $1913,11,452)$. The phthalein indicators tint the tissues of living animals faintly but almost all the colors of the rainbow. Bile capillaries of the liver can easily be stained by intravenous injection of sodium sulphindigotate. Many other examples of similar phenomena could be cited. But it is customary to think of vital stains as substances which are regularly taken in by cells of the Reticulo-Endothelial System and by a few others on occasion. 
These include colloidal suspensions of various benzidine dyes (trypan blue, isamin blue, pyrrhol blue, trypan red, etc.), of silver, Higgins ink, lamp black etc.; and of simple suspensions of India ink, carmine, graphite and so on. They are injected intravenously, intraperitoneally or subcutaneously. The literature is enormous. Consult latest issue of the Quarterly Cumulative Index Medicus. For chemistry of Benzidine dyes see Evans, H. M. and Schulemann, W. Science, 1914, 39, 443.

The following experiment is suggested. Give each of a dozen or more white mice $1 \mathrm{cc}$. of $0.5 \%$ trypan blue in sterile aq. dest. intraperitoneally and in the course of a few minutes the beginning of deposition of the dye in the ears will be noted. Give similar doses every second day for 8 days. A few hours after the last draw a little blood from the tail and observe that some of the monocytes have taken up the dye. Then autopsy the mice and study the distribution of the dye in the tissues. The skin, kidneys, adrenals, liver, spleen and bone marrow will be found quite deeply colored while the nervous system has escaped. The heaviest accumulation will be in the peritoneal cavity near the sites of injection and in the loose connective tissue everywhere. Examination of fresh mounts in physiological salt solution will reveal that the dye is concentrated within (1) the epithelial cells of the convoluted tubules of the kidney, of the adrenal and choroid plexus; (2) certain cclls of the ovary and testicle; (3) the macrophages of loose connective tissue and especially of the spleen, liver, bone marrow, adrenals and lymph nodes-fibroblasts are colored less deeply; and (4) the "specific endothelia" of the five organs mentioned. If permanent preparations are desired fix in $10 \%$ formalin and imbed in paraffin.

Vital staining in the narrow sense is used for many purposes. (1) To identify phagocytic cells of the reticuloendothelial system and to see how they behave in normal and pathological conditions. (2) To locate injured cells because some cells that do not ordinarily stain take up the dye when injured. (3) To influence the activity of $R$. E. cells by blocking them with particulate matter. This has not been very successful. See R. E. Blockade (Victor, J., Van Buren, J. R. and Smith, H. P., J. Exper. Med., 1930, 51, 531-548). (4) To measure the absorption by membranes of particulate matter (Wislocki, G. B., Anat. Rec., 1921, 21, 29-33). (5) To distinguish between malignant and non-malignant cells (Ludford, R. J., Arch. f. exp. Zellf., 1933, 14, 42-55). (6) To determine $\mathrm{pH}$ of different organs and tissues by injection with phthalein indicators (Rous, P., J. Exper. Med., 1925, 41, 739-759). (7) To identify calcium salts laid down (Alizarin Red $\mathbf{S}$ and Madder). See method for Reticulo-endothelial system.

It is sometimes very worthwhile to inject simultaneously three materials, for example Higgins' Ink intravenously, trypan blue or Niagara blue intraperitoneally, and lithium carmine intrapleurally (Foot, McClung, p. 116). An interesting experiment is to feed Sudan III or Scharlach (scarlet = Sudan IV) colored lipids. Make solution in olive oil (about 20\%). Introduce by stomach tube into a cat. There is slight staining of fatty tissue within 24 hrs. and maximum in 3-7 days (Hadjioloff, A., Bull. d'Hist. Appl., $1938,15,81-98)$. Try also inducing cat to drink large amount of milk or cream colored with Sudan III or Sudan black, see colored illustrations of Gage and Fish (S. H. and P. A., Am. J. Anat., $1924-25,34,1-81)$. History of vital staining (Conn, H. J. and Cunningham, R. S., Stain Techn., 1932, 7, 81-90, 115-119). See Chorioallantoic Membrane, Carmine, Indigo-Carmine, Manganese Dioxide, Higgins' Ink, Protargol (silver), Lampblack, LeucoDyes, Nuclei, Titanium Dioxide, Thorium Dioxide, Copper, Platinum, Iron, Mercury, Lymphatic Vessels.

Vitanins-Written by C. Carruthers, Washington University School of Medicine, St. Louis. February 15, 1951-Only a few vitamins are susceptible of microscopic localization. Deficiencies in most of them leave structural imprints in the tissues. A list may be therefore useful giving briefly required techniques. Current information is supplied in Annual Reviews of Biochemistry and Physiology.

A useful background is provided by Sherman, H. C. (Chemistry of Food and Nutrition, New York: Macmillan, 1941, 611 pp.). For a summary of tissue changes in vitamin deficiencies see Wollach, S. B. and Bessey, O. H. (Physiol. Rev., 1942, 22, 233-290). For the biochemistry and chemical determination of the vitamins the following are recommended: Gyorgy, P., Vitamin Methods, New York, Academic Press, 1950, 571 pp.; Williams, R. J., Eaker, R. E., Beerstecker, E., and Shive, W., The Biochemistry of B. Vitamins, New York, Reinhold Publishing Co., 1950, 741 pp.; Harrow, B., One Family. Vitamins, Enzymes, Hormones, Minne- 
apolis, Burgess Publishing Co., 1950, $115 \mathrm{pp}$; The Association of Vitamin Chemists, Inc., Methods of Vitamin Assay, New York; Interscience Publishers, Inc., 1947, 189 pp.; Johnson, D. C., Methods of Vitamin Determination, Minneapolis, Burgess Publishing Co., 1949,109 pp.

A. Growth promoting, anti-infective and anti-xerophthalmic vitamin, a polyene alcohol. There are also vitamins $\mathbf{A}_{2}$, $\mathrm{A}_{3}$ and the so-called Sub-vitamin A, and neo-vitamin A, whicl is a sterioisomer of vitamin A. The members of the vitamin A group differ in their maximum absorption in the ultraviolet. Provitamins $A$ are alpha, beta and delta carotene which vary in their vitamin A activity. Vitamin A can be determined by the following methods:

1. Its absorption at $326 \mathrm{~m} \mu$ measured by a spectrophotometer, or photoelectric spectrophotometer, under controlled conditions of analysis.

2. The glycerol dichlorohydrin method of Sobel, A. E. and Werbin, H., Ind. Eng. Chem., Anal. Ed., 1916, 18, 570-573; 1947, 19, 107-112.

3. The Carr-Price reaction which employs the antimony trichloride reagent. This reaction has been applied to determine the vitamin A content of hepatic mitochondria of rats after separation by differential centrifugation. The mitochondria contain 249 to 910 U.S.P. units of vitamin A per $100 \mathrm{mg}$. mitochondrial lipid (Goerner, A. and M. M., J. Biol. Chem., 1937$38,122,529-538 ; 1939,128,559-565)$. This test has also been employed for vitamin $\mathrm{A}$ in serum, the colors being checked against alizarin solutions (Parker, R. C., Methods of Tissue Culture, New York: Hoeber, 1938, 292 pp.). According to Joyet-Lavergne, P., C. rend. Acad. d. Sci., 1935, 201, 1219-1221, vitamin $A$ can be demonstrated in the red blood cells of rays (marine fish) by the Carr-Price test. This same investigator has also applied the Carr-Price reaction to a variety of tissues of several animals, and has found that in every case the mitochondria appear as bright blue bodies (Ann. physiol. physicochem. biol. 1937, 13, 1019-1021), see also Bourne, G. Austral. J. Exp. Biol. Med. Sci. 1934, 13, 239-249. The CarrPrice reaction can localize vitamin A precisely in individual mitochondria according to Jones, J., Lab. Clin. Med., 1947, 12, 700 .

4. Fluorescence microscopy. The fading green fluorescence of vitamin A is used by Popper for the identification and localization of vitamin $A$ in cells (Gyorgy, 1950, p. 89). Tissues are fixed in $5 \%$ formalin and examined as soon as possible. Human tissues are kept in the fixitive not longer than 18 hrs. and animal tissues not longer than 8 hrs. Why this difference should be is not clear. Frozen sections are made, mounted in water and examined at once with a fluorescence microscope. Any green fading fluorescence is considered to be vitamin A and is usually located in fatty material. For a more exact localization of this vitamin, sections may be stained with $1 \%$ aq. methylene blue for 30 sec. This stain interferes only slightly with the vitamin A fluorescence. By Popper's method vitamin $\mathrm{A}$ has been found in the liver, adrenal cortex, testicle, and in the ovary where it undergoes characteristic changes during the mentrual cycle and during pregnancy. It is also present in the intestinal tract during absorption, in lactating breasts, in the retina and in certain pathologic conditions of the kidney. For a review of its distribution in tissues, see Popper, H., Physiol. Rev., 1944, 24, 205-224. According to Popper carotene can be differentiated from vitamin A by its very slowly fading green fluorescence, and anhydro- or "cyclized", vitamin A may be recognized by its dark brown fluorescence, which gradually becomes dull green and finally fades out entirely.

B. Complex contains many factors.

$\mathrm{B}_{1}$. Thiamine hydrochloride (anti-neuritic factor, aneurin). Thiamine can be determined colorimetrically by the method of Hochberg, M., Melnick, D. and Oser, B. L., Cereal Chem., 1945, 22, 83-90. This vitamin can also be determined microbiologically using Streptococcus salwarius, or Lactobacillus fermenti 36 (Gyorgy, 1950, pp. 372-376). The coenzyme derived from thiamine is thiamine pyrophosphate. It is involved in the decarboxylation of alpha keto acids, particularly pyruvic acid, and consequently in the tissue oxidation of carbohydrates. In thiamine deficiency the pyruvate and lactate blood levels are increased, the increase in the former being due to a lack of ability to change pyruvate to lactate without thiamine. No histochemical method is available for this vitamin.

$\mathrm{B}_{2}$. Vitamin $G$ (Riboflavin, Lactoflavin). Riboflavin can be determined chemically by measuring its fluorescence in light of wave length 440 to $500 \mathrm{~m} \mu$ (Gyorgy, 1950, pp. 102-144). Riboflavin can also be determined polarographically in pure solutions. The flavoproteins catalyze the metabolism of such substances as D-amino acids, 
L-amino acids, L-hydroxy acids, aldehydes and purines. In Warburg's "old yellow enzyme", the respiratory enzyme, riboflavin exists as the mononucleotide. The yellowish-green fluorescence of riboflavin has been used for its detection in tissues by Ellinger, P., and Koschara, W., von Euler, H., et al., Hert, A. and Wimmer, K. and Metcalf, R. L. and Patton, R. L. See Glick, D., Techniques of Histo- and Cyto-Chemistry, New York, Interscience Publishers, Inc., 1950, pp. 531 Metcalf, R. L. and Patton, R. L. claim that another form of riboflavin exists which gives a yellow-orange fluorescence.

$\mathrm{B}_{3}$. Pantothenic acid, first designated $\mathrm{B}_{3}$. filtrate factor, factor II, antigrey-hair-factor. This vitamin is a component of coenzyme A involved in the acetylation of aromatic amines and choline, and in the metabolism of fats and carbohydrates. Pantothenic acid is determined microbiologically using Lactobacillus arabinosus 17-5 (Johnson, 1919, pp. 79-80).

$\mathrm{B}_{6 .}$. Group (Pyridoxal, Pyridoxine, and Pyridoxamine). Pyridoxamine is basically as important as pyridoxine, and pyridoxal is even more so. Pyridoxal phosphate is the coenzyme of this vitamin group and is involved in the decarboxylation of amino acids and in transamination. Pyridoxine can be determined chemically by techniques based upon the indophenol test ( $\mathrm{Gy}$ orgy, 1950, p. 239). This method, however, is only suitable for pyridoxine and not for the other two forms so that it is not adaptable for tissue analy. sis. Saccharomyces Carlsbergensis and Neurospora sitophila are employed for the microbiological determination of the $\mathrm{B}_{6}$ group of vitamins. Pyridoxal, pyridoxamine and pyridoxine have approximately the same activity in stimulating growth (Gyorgy, 1950, pp. 406414).

$\mathrm{B}_{12}$. Antipernicious anemia factor (animal protein factor). This vitamin contains 4 per cent of cobalt. It produces a positive hematological response in pernicious anemia in quantities as low as 3 micrograms. This vitamin also improves the hatchability of hen's eggs, the growth and survival of chicks, and the growth of rats. Vitamin $B_{12}$ has been implicated in the following processes: (1) the synthesis of purines and pyrimidines and their derivatives, (2) the synthesis of methionine and folic acid, and (3) the utilization of p-aminobenzoic acid and folic acid. It is related metabolically to folic acid (see The Nutritional and Chemical
Significance of Folic Acid, Lederle, American Cyanamid Co. 1950, 121 pp. Choline, a constituent of phospholipids, lecithins and sphingomyelins, acts as a donator of labile methyl groups and prevents hemorrhagic degeneration of kidneys of young rats deficient in choline. Choline can be determined chemically or microbiologically (Gyorgy, 1950, pp. 243 and 464).

C. Antiscorbutic vitamin (Cebione, Redoxon). Bourne, G., Anat. Rec., 1936, $66,369-385$, has made a critical study of cytological methods for the determination of vitamin $C$. The technique recommended is based on the assumption that the only substance, other than vitamin $C$, capable of reducing an acid silver nitrate solution in the dark is hydrogen sulphide "which is not by any means a common constituent of living tissue, if it occurs at all."

To demonstrate reduced vitamin $\mathrm{C}$, frozen sections of fresh tissue are treated with $5 \%$ aq. silver nitrate, to which $5 \mathrm{cc}$. acetic acid is added for each $100 \mathrm{cc}$., for a few minutes. The vitamin $\mathrm{C}$ granules blacken. After washing in aq. dest. fat may be stained by a solution of Sudan III or Scharlach $\mathrm{R}$ in $90 \%$ alc. and the section cleared and mounted in glycerin.

To reveal both reduced and oxidized vitamin $C$ is more difficult. Bourne advised: Subject fresh tissue to glacial acetic acid vapor for several minutes. Cut into very thin slices and put in atmosphere of hydrogen sulphide for $15 \mathrm{~min}$. All vitamin $\mathrm{C}$ is thereby converted to reduced form. Remove hydrogen sulphide by keeping in partial vacuum for 10 to 30 min., follow by exposure to a strong stream of nitrogen gas for $15 \mathrm{~min}$. Treat with acid silver nitrate solution as described.

If there is reason to believe that glutathione inhibits the reaction, Bourne suggests, after the hydrogen sulphide treatment, to momentarily wash the section, then to plunge into mercuric acetate solution for a few minutes, wash and apply acid silver nitrate solution. See Barnett, S. A. and Bourne, G., J. Anat., 1940-41, 75, 251-264 for methods of demonstration of vitamin $\mathrm{C}$ in chick embryos.

Modification of Giroud and Leblanc silver method (Tonutti, E., Protoplasma, 1938, 1, 151-158). Briefly wash tissue in $5.4 \%$ aq. levulose and $10 \% \mathrm{aq}$. $\mathrm{AgNO}_{3}$ plus 2 drops glacial acetic per cc., up to $30 \mathrm{~min}$. Rinse in aq. dest. 3015 -min. and in $3 \%$ aq. $\mathrm{Na}_{2} \mathrm{~S}_{2} \mathrm{O}_{3}$, 15-30 min. Rinse in aq. dest. 15-30, min. All this is to be done in the dark 
room with red light. Change to $70 \%$ alc. and imbed in paraffin. Counterstain with "Kernechtrot" and light green.

For a description of the localization of vitamin $\mathrm{C}$ in fibroblasts, during the histogenesis of chick embryos, goblet cells, Golgi apparatus, mitochondria, etc. see Bourne, G., Cytology and Cell Physiology, London: The Clarendon Press, 1951, p. 262 et seq.

D. The group of "vitamins $D$ " consists of a number of compounds (Rosenberg, H. P., Chemistry and Physiology of the Vitamins, New York: Interscience Publishers, Inc., New York: 1942, 341 pp.) $D_{1}$ is a molecular compound consisting of vitamin $\mathrm{D}_{2}$ and lumesterol. $D_{2}$-activated ergosterol, calciferol, or viosterol. $\mathrm{D}_{3}$ is activated 7-dehydrocholesterol and $\mathrm{D}_{4}$ is activated 22 dihydrocholesterol. $D_{5}$ is activated 7 dehydrositosterol.

There are at least 10 provitamins $D$. The vitamins $D$ can be determined chemically; but, since the methods are based on conjugated unsaturation of the vitamin $\mathrm{D}$ molecule, no distinction between them can be made (Rosenberg, 1912 , pp. 412-413). The biological methods for the determination of vitamins $D$ are reliable when properly conducted and they are superior to chemical and physical techniques. See the Line Test.

E. Antisterility vitamins of which there are four: alpha, beta, delta and gamma tocopherol. This vitamin can be determined by chemical and biological methods (Rosenberg, 1952, p. 452). Vitamins $\mathrm{E}$ are also antioxidants, the gamma isomer being more effective than the beta isomer which, in turn, is more effective than the alpha isomer which is most active as the antisterility factor.

A pigment, classified as a lipofuscin, develops in the uterus of vitamin $\mathrm{E}$ deficient rats (Elftman, H., Kaunitz, H. and Slanetz, C. A., Annals N. Y. Academy of Sciences, 1949, 52, 72-79). This pigment probably arises from the peroxidation and polymerization of unsaturated fatty acids. Its appearance in the uterus can be prevented by ovariectomy and by maintaining the rats on a vitamin E-deficient diet low in unsaturated fat (Atkinson, W. B., Kaunitz, H. and Slanetz, C. A., Ann., N. Y. Acad. Sci., 1949, 52, 68-71).

Folic acid (pteroylglutamic acid, vitamin $B_{c}$, factor $U^{\prime}, \mathbf{L}$ casei factor, Norite eluate factor). A deficiency in folic acids results in megaloblastic arrest in the bone marrow and the development of a macrocytic anemia. In chicks it is necessary for normal growth, feathering, and egg hatchability. For relationships between folic acid and vitamin $B_{12}$ see The Nutritional and Chemical Significance of Folic Acid, Lederle: American Cyanamid Co. 1950.

F. A Vitagen, Essential fatty acids consisting of linoleic, linolenic and arachidonic. Arachidonic acid is physiologically the most important compound.

H. Biotin (vitamin H, anti-egg-white injury factor). Biotin can be assayed microbiologically (Gyorgy, 1950, p. 61). It is probably a coenzyme for oxalacetate decarboxylase involved in the mechanisms of growth since its content in embryonic and tumor tissue is high.

I. Inositol (mouse anti-alopecia factor). This vitamin is hexahydroxy cyclohexane. Its significance in human nutrition has not yet been established. It is present in relatively large amounts in tissues and its occurrence as a unit structure in lipids may later remove its inclusion as a vitamin. Definite proof that inositol acts catalytically has not been obtained.

$\mathrm{K}$. The antihemorrhagic factor (phylloquinone).

$K_{1}$. is the first form of the antihemorrhagic vitamin isolated by Dam, H., Helv. Chem. Acta, 1939, 22, 310-313. It is 2-methyl-3-phytyl-1,4-naphthoquinone.

$K_{2}$. is the second form isolated by Brinkly, S. B., MacCorquodale, D. W., Toyer, S. A., and Doisy, E. A., J. Biol. Chem., $1939,130,219-234$. It is 2 - methyl - 3 - difarnesyl - 1,4 - napthoquinone. Since the various $K$ vitamins are quinones, or easily oxidized to quinones, most chemical methods proposed for their determination are based on oxidation-reduction titrations or on color reactions utilizing the quinone character of vitamin $\mathrm{K}$ (Gyorgy, 1950 , p. 207 , et seq.).

Nicotinic acid (niacin, nicotin amide and niacin amide). Microbiological methods employing Lactobacillus arabinosus $17-5$, a yeast, Torula cremoris, and a nonpathogenic bacterium, Proteus $H \times 19$, are more sensitive than chemical methods (Williams, pp. 5556. The colorimetric methods which have been widely used in nicotinic acid assays all involve the interaction of this vitamin with cyanogen bromide and an aromatic amine (Williams, 1950, p. 54). The coenzymes, diphosphopyridine nucleotide and triphosphopyridine nucleotide, contain nicotinic acid. About forty different enzyme reactions have been reported to be catalyzed by 
one or the other of these enzymes. The chief metabolites of nicotinic acid which are most abundant in human, swine and dog urine, are $\mathrm{N}^{1}$-methylnicotinamide and 6-pyridone. These can be determined in a fluorometer having a maximum transmission at 365 $\mathrm{m} \mu$ in the ultraviolet (Johnson, 1949, pp. 68-69).

Paraminobenzoic acid. This vitamin is unique among vitamins in that it makes up an integral part of one of the $B$ vitamins, folic acid. Its nutritional significance may depend on its action as a building block of the folic acid molecule. The catalytic action of paraminobenzoic acid probably depends upon this latter property.

P. Permeability factor (citrin), considered to be a minor water-soluble vitamin. The active fraction of vitamin $P$, extracted from lemon peels, is a mixture of glucosides. Rutin is another flavone glucoside isolated from tobacco leaves and buckwheat which resembles vitamin $P$ in structure and activity. It was first used clinically by Griffith, J. Q., Couch, J. F. and Lindauer, M. A., Proc. Soc. Exp. Biol. \& Med., 1944, $55,228-229$ and since has been extensively employed for the control of capillary fragility. This vitamin may act as a biological antioxidant for ascorbic acid, or epinephrine, or it may inhibit hyaluronidase activity.

Volkonsky Method for mitochondria. This is a complicated technique involving staining with anilin fuchsin, aurantia, methylene violet and azure II but can give splendid results. See original account (Volkonsky, M., Bull. d'hist. Appl., 1928, 5, 220-222).

Volume. As explained by Danielli (Bourne, 1951 p. 102), cell volume is a function of the number of contained osmotically active particles unless change is restricted by rigidity of the enveloping membrane. A satisfactory technique for measuring the volume of red blood cells is to determine photoelectrically light absorption of a suspension ( $\mathrm{Ja}$ cobs, M. H., Biol. Bull., 1930, 58, 104). The simplest way to obtain ratio for cytoplasmic and nuclear volumes is to outline nuclei and cytoplasms on kodaloid and determine the weights as has been recently done in carcinogenesis (Cowdry, E. V. and Paletta, F. X., J. Nat. Cancer Inst., 1941, 1, 745-759). The technique, of course, varies with structure involved, for example thyroid colloid (Stein, H. B., Am. J. Anat., 1940, 66, 197-211), fresh endocrine glands (Swinyard, C. A., Anat., Rec., 1939, 74, 71-78). To determine volume and cell numbers in small organs
(Dornfeld, E. J., et al., Anat. Rec., $1942,82,255-259$ ). For influence on tissue volume of various methods of fixation, dehydration and imbedding, see Stowell, R. E., Stain Techn., $1941,16,67-83$.

Volume measurements

1 liter $=2.1$ U. S. pints (1.76 Imperial pints)

1 cc. $=16 \frac{1}{4}$ minims (17 minims B.P.)

1 gallon $=3.79$ liters ( 1 Imperial gallon $=$ 3.79 liters)

1 pint $=473$ cc. ( 1 Imperial pint $=56 \mathrm{~S} \mathrm{cc.)}$

1 fluid ounce $=29.5 \mathrm{cc}$. (1 fluid ounce B.P. $=28.4$ cc.)

1 fluid drachm $=3.7$ cc. (1 fluid drachm B.P. $=3.5$ cc.)

$1 \mathrm{minim}=0.065 \mathrm{cc}$.

For accurate measurements in capillary tube colorimetry and titrametric techniques capillary tubes, pipettes, microliter burettes and other instrumentation is required. These are described in detail by Glick 1949. To measure the volume of irregular aspects $(0.01$ to $1.0 \mu \mathrm{l})$ a method of Holter, H., C. rend. trav. lab. Carlsberg, Sér. chem., 1945, 25, 156-167 is suggested. This is a colorimetric technique, the amount of color displaced by the object being measured.

Volutin. Spherical bodies in fungi, bacteria and other organisms (Taylor in McClung's Microscopical Technique, p. 221). According to R. F. MacLennan, in Calkins, G. N. and Summers, F. M., Protozoa in Biological Research. New York: Columbia University Press. 1941,1148 pp., the term "volutin should either be dropped or definitely restricted to metachromatic granules which respond to Feulgen's stain when used without hydrolysis."

Volocidae, technique for, Hartmann, M., Arch. f. Protistenk., 1918, 39, 1.

Von Kossa, see Calcium.

Vulpian Reaction named after a Parisian physician. Fresh slices of the adrenal immersed in dil. aq. ferric chloride show a green coloration of the chromaffin cells of the medulla. It is a test for tissues producing epinephrine. See : chromaffin reaction and osmic acid.

Walker's Method for intestinal protozoa is recommended as an excellent rapid stream. However, almost equally satis factory results can be obtained by the more tedious method of frequently changing the water. Osmic acid containing fixatives are to be washed in aq. dest. for about an hour. After Regaud's fixative the tissue is transferred to $3 \%$ aq. potassium bichromate without washing in water. Tissues fixed in alcoholic mixtures are to be briefly washed in alcohol before dehydration. For de- 
tails about washing see the individual fixatives.

Wash-out Recovery Method (WO)-Written by C. C. Macklin, Dept. of Histologieal Research, The University of Western Ontario, London, Canada. November 28, 1951-A cannula loaded with physiological saline, serum or other medium is tied into the trachea of a mouse or other mammal and the eontents injected into the fresh lung, withdrawn, and examined as fresh or dried and stained mounts. For most animals it is convenient to wash out a part of a lung through a eannulated bronehus. Granular alveolar and phagoeytic alveolar cells (pneumonocytes) are so recovered. (Macklin, C. C., Proc. 6th Intern. Cong. exp. Cytology, Stoekholm, 1947; published 1919, 3\$3-3\$7). See Dust Cells, Foam Cells, Vacuoloids.

Wasserblau, see Brazilin-Wasserblau.

Water Absorption by slices of liver. The method has been standardized by Sperry and Brand (W. M. and F. C., Proc. Soc. Exp. Biol. \& Med., 1939, 42, 147-150) and may prove useful in the investigation of other tissues.

Water Blue (Wasserblau), see Anilin Blue.

Wear and Tear pigment, see Lipofuscin.

Weigert Method. For myelin sheaths. Kultschitzky modifieation (Romeis, B. Taschenbuch der mikroskopischen technik, ii Auflage Seetion 999, p. 332). Fix in $10 \%$ formalin and mordant in Müller's Fluid, or in Formalin Müller or in Weigert's Quick Mordant. Bring paraffin or celloidin sections to water. Immerse in $3 \%$ aq. potassium bichromate or in Müller's fluid 12 hrs. Stain for 1224 hrs. in : $10 \%$ hematoxylin in abs. ale. (1-6 months old), 10 cc.; aq. dest., 100 ce. Wash and destain in : aq. lithium carbonate, 100 cc.; $1 \%$ aq. potassium ferricyanide, $10 \mathrm{ec}$. until clear differentiation appears between gray and white matter. Wash, dehydrate and mount.

The following is provided by Dr. J. L. O'Leary : Mordanting in the Weigert procedure serves two purposes: (1) It renders the myelin sheath components insoluble in the fat solvents necessary to secure dehydration and imbedding. (2) It distributes the chromate ion in sufficient concentration in the myelin sheaths to ensure the formation of an adequate lake with hematoxylin in the subsequent staining procedure. If paraffin imbedding is to be used, it is absolutely necessary to carry block mordanting to the point where the myelin of all fibers has been rendered insoluble. For this reason paraffin imbedding of material to be used for Weigert staining should be restricted to small nerves and thin pieces of spinal cord, otherwise overhardening results. Here excellent results are to be achieved, the smallest fibers staining as completely as by the osmic acid method. 'T'wo methods are applieable to paraffin imbedded sections, the procedures for which are given subsequently. These are: the Kultschitzky modification of the Weigert method and technique for routine diagnostic work by Craig, p. 55. To make the stain dissolve $1 \mathrm{gm}$. hematoxylin crystals in $300 \mathrm{ec} . \mathrm{aq}$. dest. with aid of a little heat and add $100 \mathrm{ec}$. sat. aq. ammonium alum with a erystal of thymol. Allow to ripen 10 days in flask stoppered with cotton; then keep in dark. Fix smears in Schaudinn's Fixative 5-10 $\mathrm{min}$. Wash thoroughly in several changes aq. dest. Immerse in above hematoxy lin solution 3-5 min. Then pass through 50, 60, 70,90 and $95 \%$ alcohol, at least $5 \mathrm{~min}$, each. After immersing in absolute $10 \mathrm{~min}$. elear in xylol and mount in xylol balsam.

Warburg's Respiratory Enzyme, see Cytochrome-Oxidase.

Warthin-Starry method for spirochaetes in sections has been modified by Faulkner, R. R. and Lillie, R. D. Stain Teehn, $1945,20,81-82$ by the use of a buffered solution. Use Walpole's buffer : 18.5 ce. of solution of $11.8 \mathrm{ec}$. acetic acid in 1000 ce. aq. dest. +1.5 cc. of solution of $16.4 \mathrm{gm}$. sodium acetate in $1000 \mathrm{cc}$. aq. dest. which gives $\mathrm{pH}$ of 3.6. 1 . Pass paraffin sections through xylol and alcohols to aq. dest. buffered to $\mathrm{pH} 3.6$ by addition of $20 \mathrm{cc}$. of above buffer to $480 \mathrm{cc}$. aq. dest. 2. Impregnate $45 \mathrm{~min}$. at $55-60^{\circ} \mathrm{C}$. in paraffin oven in $1 \%$ aq. silver nitrate similarily buffered. 3. Place slides sections up on glass rods pour on developer previously warmed to $55-60^{\circ} \mathrm{C}$. This developer is made by heating 15 ec. $5 \%$ aq. gelatin in above buffered aq. dest. and just before using add $3 \mathrm{ec} .2 \%$ aq. silver nitrate and $1 \mathrm{cc}$. $3 \%$ aq. hydrochinone also made up in the same buffered solution. While developing avoid direct sunlight and cold drafts. Continue $3-5$ min. until sections become golden brown or grayish yellow and developer starts to turn black. Pour off, rinse with warm 55$60^{\circ} \mathrm{C}$. tap water and then with aq. dest. 4. Dehydrate, clear and mount in xylene clarite or balsan. Spirochaetes black. Recommended for syphilitic lesions, yaws and Vincent's stomatitis.

Washing. The surplus of most aqueous fixatives is removed by washing the tissue in water. In the case of Zenker's fluid, for example, wash for 12-24 hrs. in running tap water. A convenient way is to eover the wide mouth of a bottle containing the tissue with gauze 
secured by an elastic band. Water from the tap is allowed to drop on to the gauze or better is led into the bottle through the gauze in a small glass tube. Most laboratories are provided with many such water carrying tubes. The water pressure should be so regulated that the tissue is not bumped about by the O'Leary's Brazilin method. All large blocks of brain or spinal cord should be imbedded in celloidin, the length of time in celloidin and the type of celloidin to be used being determined by the thickness of the sections desired. The following general rules apply to the block mordanting of material to be stained by the Weigert method:

1. If it is advisable to stain nerve cells and myelinated fibers in alternate sections, it is best to forego block mordanting in Müller's fluid. Formalin fixed blocks are imbedded directly in celloidin and alternate sections are stained by Weil's Method and the Gallocyanin Technique.

2 . If only staining by a Weigert procedure is contemplated, the blocks may be mordanted in Müller's fluid for several weeks to several montlis depending upon the size of the block, imbedded in celloidin and stained by the Weigert-Pal method.

3. In special cases (cerebral cortex) the small myelinated fibers are stained completely with great difficulty. Blocks, premordanted or not, are sectioned in celloidin and the sections given long mordanting (one week to one month) in Müller's fluid. Stain by Kultschitzky modification of Weigert or Weigert-Pal.

Weigert's Mordants. (1) Primary, or rapid mordant: potassium bichromate, $5 \mathrm{gm}$.; Fluorchrome, 2 gm.; boiling aq. dest., 100 cc. (2) Secondary, or copper, or neuroglia mordant: boil $2.5 \mathrm{gm}$. Fluorchrome with 100 cc. aq. dest. Take away flame. When boiling has stopped, add 5 cc. glacial acetic acid, then $5 \mathrm{gm}$. finely powdered copper acetate. Stir vigorously until dissolved and cool.

Weigert Pal Method. For myelin sheaths (Dr. J. L. O'Leary, personal communication). Fix in $10 \%$ formalin, 1-2 wks. Wash in running tap water, 3 hrs. Mordant in Müller's fluid $1 \mathrm{wk}$. to $3 \mathrm{mo}$. depending on the size of block. Change Müller's thrice weekly at first, later once weekly. Wash in running tap water, 6$12 \mathrm{hrs}$. Imbed in celloidin. Cut sections $20-100 \mu$ depending upon size of block and detail desired. $0.25 \%$ aq. chromic acid, 3-5 hrs. 3 changes aq. dest. $10 \%$ hematoxylin in abs. alc. ripened and diluted to $1 \%$ with aq. dest. prior to use, 12-24 hrs. 3 changes of aq. dest. Differentiate in Pal's fluid (ox- alic acid, $1 \mathrm{gm}$.; potassium sulphite, 1 gm. ; aq. dest., 200 cc.), alternating with $0.25 \%$ aq. potassium permanganate if differentiation is too slow. Wash in 3 changes aq. dest. Dehydrate in 2 changes $95 \%$ alc. Clear in carbolcreosol-xylol followed by pure toluol. Mount in balsam. Myelin sheaths, deep black; background, unstained. Another variation of the Pal-Weigert method is given by Clark, S. L. and Ward, J. W., Stain Tech., 1935, 10, 53-55. See Johnson's Neutral red for counterstain.

Weigert's Borax Ferricyanide. Borax, 1 gm.; potassium ferricyanide, $1.25 \mathrm{gm}$.; aq. dest., 100 cc. A fluid for differentiation of hematoxylin stain in Weigert's method. Employed also in copper chrome hematoxylin method of Bensley.

Weigert's Resorcin-Fuchsin. Stain for elastic fibers. Given by Mallory, p. 168. Add $2 \mathrm{gm}$. basic fuchsin and $4 \mathrm{gm}$. resorcin to 200 cc. aq. dest. Boil in enamel dish and while boiling, add 25 cc. $29 \%$ aq. ferric chloride. Stir and boil $2-5 \mathrm{~min}$. Cool. Collect ppt. and discard filtrate. Dry ppt. on filter paper and return both to the enamel dish which has also been dried. Add 200 cc. $95 \%$ alcohol, warm carefully, stir and discard filter paper when ppt. is dissolved out. Cool, add $95 \%$ alcohol to $200 \mathrm{cc}$. and 4 cc. hydro. chloric acid. Mixture keeps well. Formalin fixed material is preferred, but most other fixatives are satisfactory. Stain paraffin sections, after removing paraffin, for $20 \mathrm{~min}$. or more in above mixture. Wash off excess in $95 \%$ al cohol and differentiate in Acid Alcohol if required. Wash thoroughly in tap water. Dehydrate, clear and mount. Elastic fibers dark blue black. It is well to stain nuclei with Lithium Carmine (Orth's) before coloring the elastic tissue. Weigert's resorcin fuchsin for elistic tissue has been supplemented by Masson's trichrome for other connective tissue components in a helpful way by Mendeloff, J. and Blechman, H., Am. J. Clin. Path., Techn. Suppl., 1943, 7, 65.

Weight measurements

1 kilogram $=2.2 \mathrm{lbs}$., or $35 \frac{1}{2}$ ounces

1 gram $=15 \frac{1}{2}$ grains

1 pound $=453.6 \mathrm{gms}$.

1 ounce $=28.4 \mathrm{gms}$.

$1 \mathrm{drachm}=3.89 \mathrm{gms}$.

1 grain $=0.065$ gms .

The Troy pounds and ounces are dif ferent but seldom used. For weights of organs, see Normals.

Weil's Method. For myelin sheaths (Weil, A., Arch. Neurol. a. Psychiat. 1928, 20, 392 and Weil, A., Textbook of Neuropathology, 2nd. ed. p. 328. New York 1945. Place celloidin sections of formalin fixed material (not yet mor- 
danted) in aq. dest. Stain for $15 \mathrm{~min}$. at $45-50^{\circ} \mathrm{C}$. in equal parts of $4 \%$ aq. iron alum and $1 \%$ aq. hematoxylin prepared from $10 \%$ sol. in abs. alc. at least 6 months old. (Note: do not filter this stain; do not use it twice; mix fluids just before using.) Wash 2 times in tap water. Differentiate in $4 \%$ aq. iron alum until gray matter or degenerated areas become recognizable. Wash 3 times in tap water. Differentiate over white background to desired degree in : borax, $2.5 \mathrm{gm}$.; potassium ferricyanide, 12.5 gm.; aq. dest., 1000 cc. (For paraffin sections, differentiate just long enough in $4 \%$ aq. iron alum to remove stain from back of slide. Care should be taken not to over-differentiate, for in so doing fine fibers are lost). Wash 2 times in tap water and next in aq. dest., to which $28 \%$ ammonia had been added ( 6 drops to $100 \mathrm{cc}$. of water). Dehydrate in $95 \%$ alc., abs. alc. and ether (equal parts), clear in xylol and mount in balsam or claritex. Revised by A. Weil, Northwestern University Medical School, Chicago, Ill. May 14, 1946.

Weld, a plant, Reseda luteola which yields a yellow dye. The use of this source of yellow coloring matter is said to be of greater antiquity than any other source of yellow dye. It was employed to dye the clothes of the six vestal virgins whose responsibility it was to keep the fire burning in the temple of Vesta in Rome (Leggett, W. F., Ancient and Medieval Dyes. Brooklyn: Chemical Publishing Co., Inc. 1944, 95 pp.).

Wetting Agents. These have been used in experiments designed to increase the rapidity of penetration of fixatives by Chermock, R. L. and Muller, H. E. Science, 1946, 103, 731-732. They found that Tergitol- 4 when added to $10 \%$ formalin, Zenker's fluid and some others improved fixation and staining. Tergitol-08 was also an advantage when employed in Zenker's fluid. The authors give the literature on the subject. Tergitol-7 is recommended in McClung's Microscopical Technique, 1950 , p. 136 to accelerate the staining with hematoxylin. One merely adds one drop to the jar containing the aqueous hematoxylin. To add the tergitol to the mordant before staining with hematoxylin is not helpful.

Wetting Properties. An interesting method for investigating the cell membrane is to measure its wetting properties. The Mudds (S., and E. B. H., J. Exp.Med., 1926, 43, 127-142; J. Gen. Physiol., 1931, $14,733-751$ ) have noticed the responses of cells to a film of oil advancing between slide and cover glass. Erythrocytes are easily wetted by the oil; whereas, when leucocytes are surrounded by the film of oil, the oil does not wet their surfaces but remains separated from them by thin films of saline solution. The Mudd's thought that this indicated that the surface of erythrocytes is lipoid and that of leucocytes protein. Danielli (Bourne, p. 78) has expressed the view that the surfaces of both cells are probably coated with protein, the erythrocytes with serum albumen and the leucocytes with serum globulin. The wetting technique has been employed in a considerable number of experiments. Dawson and Belkin, J. A. and M., Biol. Bull., 1929, 56, 80-86 and Marsland, D., J. Cell. \& Comp. Physiol., 1933, 4, 9-33 worked with amebae and Chambers, R., Biol. Bull., 1935, 69, 331, and Kopac, M. J. and Chambers, R., J. Cell. \& Comp. Physiol., 1937, 9, 331-361 with naked arbacia eggs. See Cell Membranes.

Whole Mounts of tissues which are fairly thick are of ten very useful. See Blood Vessels, Cartilaginous Skeleton, Corrosion Preparations, Epidermis, Insects, Mammary Glands, Nerve Endings, Ossification, etc.

Wicks, see Hydrogen Ion Indicators, Piccolyte Resins.

Wilder's Method of silver impregnation for reticular fibers (Wilder, H. C., Am. J. Path., 1935, 11, 817-819). Fix in $10 \%$ formalin, Zenker or formalin-Zenker. Treat paraffin, celloidin or frozen section in $0.25 \%$ aq. potassium permanganate or in $10 \%$ aq. phosphomolybdic acid for 1 min. Rinse in aq. dest. and transfer to hydrobromic acid (Merck's conc. 34\%, 1 part; aq. dest., 3 parts) for 1 min. This can be omitted af ter phosphomolybdic acid. Wash in tap water and in aq. dest., then dip in $1 \%$ aq. uranium nitrate (sodium free) 5 sec. or less. Wash in aq. dest. 10-20 sec. and place in Foot's silver diamino hydroxide for $1 \mathrm{~min}$. To make this: add $8.1 \%$ aq. $\mathrm{NH}_{4} \mathrm{OH}$ drop by drop to 5 cc. $10.2 \%$ aq. $\mathrm{AgNO}_{3}$ until brown ppt. is just dissolved. Then add 5 cc. $3.1 \%$ aq. $\mathrm{NaOH}$ and sufficient $\mathrm{NH}, \mathrm{OH}$ to just dissolve ppt. Make up to $50 \mathrm{cc}$. with aq. dest. Dip quickly in $95 \%$ alc. and reduce for 1 min. in : aq. dest., 50 cc.; $40 \%$ neutral formalin (neutralized with magnesium carbonate), 0.5 cc.; $1 \%$ aq. uranium nitrate, 1.5 cc. Wash in aq. dest. Tone in 1:500 gold chloride (Merck's reagent), 1 min. Rinse in aq. dest. and treat with $5 \%$ aq. sodium thiosulphate (hyposulphite), 1-2 min. Wash in tap water. Counterstain as desired, dehydrate, clear and mount in balsam. Reticular fibers black. Note author's figures of lymph nodes. 
Wilson's Stain for Leishmania is complicated. Details are provided by Craig, p. 147 in whose opinion it gives no better results than Wright or Leishman stains.

Windaus, see Digitonine Reaction.

Wintergreen Oil (methyl salicylate) is used in the Spalteholz Method of clearing.

Wislocki, see Placentas.

Woad is a blue dye derived from the plant Isatis tinctoria, now only of historic interest, as it was replaced by indigo after over a 1,000 years of supreniacy in Europe. When, nearly 2,000 years ago, Julius Caesar's Roman legions crossed the English Channel they encountered a race of Celtic origin which they called "Picts", or painted people, because they had punctured their skins with flints in many patterns and had rubbed into them anil of the woad plant. The account of this dye by Leggett is interesting reading (Leggett, W. F., Ancient and Medieval Dyes. Brooklyn: Chemical Publishing Co., Inc., 1944, $95 \mathrm{pp}$.$) . Leggett quotes opinion of$ Guest that the word "Britain" is the Latinized form of Brythen, a Celtic term, indicating "painted men".

Woods Metal is now largely replaced by celluloid in the making of corrosion preparations.

Wool Black B (CI, 315), an acid disazo dye of light fastness 3 to 4 staining action of which is briefly reported (Emig, p. 38).

Wool Green S (CI, 737) Lillie, R. D., J. Tech. Methods, 1945 , No. 25, 47 pp. has reported this dye in a good combination for connective tissue. Mordant sections $2 \mathrm{~min}$. in sat. alc. picric acid. Wash 3-5 times in running water and stain $6 \mathrm{~min}$. in Weigert's or other iron hematoxylin. Wash in water and stain $4 \mathrm{~min}$. in 1\% Biebrich scarlet in $1 \%$ aq. acetic acid. Wash in water and mordant $4 \mathrm{~min}$. in $10 \%$ dilution of U.S.P. ferric chloride solution. Wash in water and stain $4 \mathrm{~min}$. in $1 \%$ aniline blue, methyl blue, or wool green $\mathrm{S}$ in $1 \%$ aq. acetic acid. Destain 2 min. in $1 \%$ aq. acetic acid. Dehydrate and clear in acetone, acetone and xylene and in xylene. Mount in clarite in xylene or in salicylic acid balsam. Connective tissue and basement membranes, dark blue or green; muscle and cytoplasm, red.

A substitute for Wright's stain is proposed by Saye, E. B., Am. J. Clin. Path., 1943, Tech. Suppl. 7, 12-13, made up of Eosin $Y$ and Thionin. It is recommended for blood cells and malarial parasites.

Wool Orange 2G, see Orange G.

Wool Red, see Amaranth.

Wound Healing, method for study in vitro
(Bentley, F. H., J. Anat., 1935-36, 70, 498-506).

Wright's Blood Stain. This is a compound stain of the Romanowsky type. The Commission Certified (C.C.) product is available. Dry the smear in air. Cover the area between the wax lines with stain measured by drops from a medicine dropper. After $1 \mathrm{~min}$. add same volume aq. dest., shifting the slide a little from side to side so that it mixes fairly well. A green metallic looking scum forms on the surface. Leave 2 or $3 \mathrm{~min}$. Too long staining produces a precipitate. It may be necessary to use for dilution instead of aq. dest. the McJunkin-Haden buffer. Wash in tap water 30 sec. or more until thin parts of smear become pink or yellow. Dry by blotting with smooth filter paper and examine directly without mounting in balsam and adding a cover glass. Usually excellent results are obtained. If however it is desired to employ buffered solutions especially for sections consult Petrunkevitch, A., Anat. Rec., 1937, 68, 267-280 and Lillie, R. D., Stain Techn., 1941, 16, 1-6. The other most used blood stain is that of Giemsa with its several modifications. Ehrlich's triacid stain is less used nowadays.

X Bodies, see Cytoplasmic Inclusions in plants.

Xanthene Dyes. As the name implies they are derivatives of xanthene. They comprise many indicators and are classified as acridines, fluoran derivatives, phenolphthalein, pyronins, quinolines, rhodamines, and sulfonphthaleins.

Xanthene Dye, see Phosphine.

Xanthine, see Purines.

Xanthoproteic Reaction. Treat section with cold fuming nitric acid. After a few minutes the proteins become colored yellow. Then rinse and expose to ammonia vapor which changes the color to orange. Not specific for proteins because there is also a nitration of aromatic radicals of phenols, alkaloids, etc. The color is often faint but fairly sharp (Lison, p. 127). See also Bensleys (p. 126).

The reaction is described as follows by Serra, J. A., Stain Techn., 1946, 21, 5-18: Fix tissue as given under $\mathrm{Nin-}$ hydrin Reaction. "The pieces are treated for some minutes with concentrated $\mathrm{HNO}_{3}$ until they become intensely yellow. After a washing in distilled water, immerse in a diluted ammonia solution, or expose the pieces to ammonia vapors. The color clianges to orange. The observation can be made by mounting directly in pure glycerin.

"The reaction is due to the presence 
of tyrosine, phenylalanine or tryptophane in the protein molecule, and is also given by all phenolic compounds. Among the peptides, only the protanines do not show a positive reaction. To withstand the treatments, a strong fixation is recommended, though the reaction can also be performed on fresh materials." See Purines.

Xenon, see Atomic Weights.

XL Carmoisine 6R, see Chromotrope 2R.

Xylene Cyanol FF (cyanol FF). Related to Aniline blue, an acid dye of the phenylmethane series employed as ultracellular indicator (Conn, H. J., in McClung's Microscopical Technique, 1950 , p. 103).

Xylidine Ponceau 3RS, see Ponceau 2R. Xyloidin, see Pyroxylin.

Yeasts, vital staining of, see Brilliant Purpurin R. Malachite green-safranin technique for staining spores (McClung, L. S., Science, 1943, 98, 159-160).

Yellow M, see Metanil Yellow.

X-Ray Absorption of tissues provides method of quantitative study of tissue elements and their localization (Engstrom, A., Acta Radiologica, suppl. 53, 1946). This method has been advanced by Engstrom over that described under Historadiography. It has been much used in the quantitative determination of the density of enamel and dentin, see Boedeker, C. F. and Appelbaum, E., Dental Cosmos., 1933, $75,21$.

X-ray Diffraction method for investigating structure of nerve myelin sheath (Schmitt, F. O., Bear, R. S. and Palmer, K. J., J. Cell. \& Comp. Physiol., 1941, 18, 31-42. See, also, Historadiography.

Yaws. Treponema pertenue, 18-20 $\mu$ long, 6-20 uniform spirals. Same technique as for Treponema Pallida.

Ytterbium, see Atomic Weights.

Yttrium, see Atomic Weights.

Zenker's Fluid. Potassium bichromate, 2.5 gms.; mercuric chloride, (corrosive sublimate) 5 gms.; aq. dest., 100 cc.; glacial acetic acid, $5 \mathrm{cc}$. Because this mixture does not keep well make a stock fluid of say 2 liters by adding mercuric chloride to saturation in $5 \%$ potassium bichromate. It will do no harm if more than sufficient mercuric chloride is used and remains undissolved at the bottom of the bottle. The main point is to reach saturation. This will require several hours unless the mercuric chloride is dissolved in the aq. dest. with the aid of gentle heat before adding the bichromate which has been pulverized in a mortar to facilitate solution.

Immediately before use add $5 \%$ of glacial acetic acid. Fix tissues $24 \mathrm{hrs}$. and wash in running water about $12 \mathrm{hrs}$. Dehydrate and imbed in the usual way. Remove mercuric chloride from sections by Lugol's iodine solution 5-10 $\mathrm{min}$. and wash out the iodine in alcohol before staining. This fluid is employed in techniques too numerous to mention. It is called for in case of Mallory's Connective Tissue stain and for demonstration of Tendons, Purkinje Cells, Muscle, Fibrin, Hemofuscin, etc.

Zenker Less Acetic is the stock solution without addition of acetic acid. This will serve as a fixative for mitochondria; because, since it does not contain acetic acid, they are not dissolved. It is, however, not recommended for mitochondria.

Formalin-Zenker or Zenker-Formol is a very useful fixative indeed. Helly's fluid is Zenker with $5 \%$ formalin in place of the $5 \%$ acetic acid. Maximow has used $10 \%$ formalin instead of $5 \%$. It is added, like the acetic acid, just before use. The time of fixation, washing, etc. is the same as for Zenker's fluid.

Ziehl's Carbol-Fuchsin (as emended Soc. Am. Bact.): A. Basic fuchsin, $0.3 \mathrm{gm}$.; $95 \%$ ethyl alcohol, 10 cc.: B. Phenol, 5 gm.; aq. dest., 95 cc. Mix A and B. Much used for the staining of Acid Fast Bacilli.

Zinc. Mendel and Bradley's Method (L. B. and H. C., Am. J. Physiol., 1905, 14, 313-327). Treat paraffin sections with $10 \%$ aq. sodium nitroprussate for 15 min. at $50^{\circ} \mathrm{C}$. Wash carefully in running water. Add cover glass. Introduce under it one drop potassium sulphide solution which causes an intense purple color (Lison, p. 98).

Zinc Chloride, as substitute for mercuric chloride in Zenker's fluid (Russell, W. O., J. Techn. Meth. \& Bull. Int. Assoc. Med. Museums, 1941, 21, 47).

Zirconium, see Atomic Weights.

Zweibaum's Fixative. Add 1 part $2 \%$ aq. osmic acid to 7 parts $3 \%$ aq. potassium bichromate, $6 \mathrm{cc}$., $2 \%$ chromic acid, $3 \mathrm{cc}$. and aq. dest., 5 cc. See Sudan Black B.

Zymogen is substance within cells that produces an enzyme (G. zymē, leaven + gennāō, I produce). It is usually seen in the form of granules. These zymogen granules as they occur in the acinous cells of the pancreas, in the chief cells of the stomach, in the serous (or zymogenic cells of the salivary glands and in other situations can be well stained with Bensley's Neutral Gentian or Bowie's Ethyl Violet-Biebrich Scarlet. They can also be readily studied in living cells and their behavior noted 
as material is discharged from the cells into the lumina of the acini by a method elaborated by Covell, W. P., Anat. Rec., $1928,40,213-223$. The technique consists of carefully mounting the pancreas of a living mouse in such a way that the circulation continues and the influence of pilocarpine can be observed. Zymohexase. This enzyme system is made up of aldolase and isomerase. The former converts hexosediphosphate into dihydrocyacetone phosphate and phosphoglyceraldehyde. The latter catalyses equilibrium between these two products. Allen, R. J. L. and Bourne, G. H., J. Exper. Biol., 1943, 20, 61-64 adapted methods for phosphatase to the demonstration of zymohexase activity. See technique and comments by Glick, p. 86-88.

Zymonema Dermatitidis, see Blastomycosis. 





